NUREG/CR-3620

PNL-4054

Supplement No. 2

\title{
Intruder Dose Pathway Analysis for the Onsite Disposal of Radioactive Wastes: The ONSITE/MAXI1 Computer Program
}

Prepared by W. E. Kennedy, Jr., R. A. Peloquin, B. A. Napier/PNL S. M. Neuder/NRC

Pacific Northwest Laboratory Operated by

Battelle Memorial Institute

Prepared for U.S. Nuclear Regulatory Commission 


\section{NOTICE}

This report was prepared as an account of work sponsored by an agency of the United States Government. Neither the United States Government nor any agency thereof, or any of their employees, makes any warranty, expressed or implied, or assumes any legal liability of responsibility for any third party's use, or the results of such use, of any information, apparatus, product or process disclosed in this report, or represents that its use by such third party would not infringe privately owned rights.

\section{NOTICE}

\section{Availability of Reference Materiais Cited in NRC Publications}

Most documents cited in NRC publications will be available from one of the following sources:

1. The NRC Public Document Room, 1717 H Street, N.W. Washington, DC 20555

2. The Superintendent of Documents, U.S. Government Printing Office, Post Office Box 37082 , Washington, DC 20013-7082

3. The National Technical Information Service, Springfield, VA 22161

Although the listing that follows represents the majority of documents cited in NRC publications, it is not intended to be exhaustive.

Referenced documents available for inspection and copying for a fee from the NRC Public Document Room include NRC correspondence and internal NAC memoranda; NRC Office of Inspection and Enforcement bulletins, circulars, information notices, inspection and investigation notices; Licensee Event Reports; vendor reports and correspondence; Commission papers; and applicant and licensee documents and correspondence.

The following documents in the NUREG series are available for purchase from the GPO Sales Program: formal NRC staff and contractor reports, NRC-sponsored conference proceedings, and NRC booklets and brochures. Also available are Regulatory Guides, NRC regulations in the Code of Federal Regulations, and Nuclear Regulatory Commission /ssuances.

Documents available from the National Technical Information Service include NUREG series reports and technical reports prepared by other federal agencies and reports prepared by the Atomic. Energy Commission, forerunner agency to the Nuclear Regulatory Commission.

Documents available from public and special technical libraries include all open literature items, such as books, journal and periodical articles, and transactions. Federal Register notices, federal and state legislation, and congressional reports can usually be obtained from these libraries.

Documents such as theses, dissertations, foreign reports and translations, and non-NRC conference proceedings are available for purchase from the organization sponsoring the publication cited.

Single copies of NAC draft reports are available free, to the extent of supply, upon written request to the Division of Technical Information and Document Control, U.S. Nuclear Regulatory Com. mission, Washington, DC 20555.

Copies of industry codes and standards used in a substantive manner in the NRC regulatory process are maintained at the NRC Library, 7920 Norfolk Avenue, Bethesda, Maryland, and are available there for reference use by the public. Codes and standards are usually copyrighted and may be purchased from the originating organization or, if they are American National Standards, from the American National Standards Institute, 1430 Broadway, New York, NY 10018. 
NUREG/CR-3620

PNL-4054

Supplement No. 2

Intruder Dose Pathway Analysis

for the Onsite Disposal of

Radioactive Wastes:

The ONSITE/MAXI1 Computer Program

Manuscript Completed: November 1986

Date Published: February 1987

Prepared by

W. E. Kennedy, Jr., R. A. Peloquin, B. A. Napier, Pacific Northwest Laboratory

S. M. Neuder, U.S. Nuclear Regulatory Commission

Pacific Northwest Laboratory

Richland, WA 99352

Prepared for

Division of Waste Management

Office of Nuclear Material Safety and Safeguards

U.S. Nuclear Regulatory Commission

Washington, DC 20555

NRC FIN B2476 



\section{PREFACE}

Pacific Northwest Laboratory (PNL) prepared this document as a supplement to two previous documents that describe the ONSITE/MAXI1 computer software package. These documents are both entitled Intruder Dose Pathway Analysis for the Onsite Disposal of Radioactive Wastes: The ONSITE/MAXI1 Computer Program and are by Napier et al. (1984) and Kennedy et a1. (1986). This document is a second supplement that summarizes our efforts to further modify and improve the ONSITE/MAXI1 computer software package for operation on a personal computer. To facilitate cross-referencing, this document follows the same format as the previous documentation. This version of the software permits the user to optionally select either of two sets of internal dose conversion factors: 1) factors based on the methods published by the International Commission on Radiological Protection (ICRP) in their Publication No. 30 (ICRP 1979-1982), or 2) factors based on the methods published by the ICRP in their Publication No. 2 (1959) (as implemented in the previous versions of the computer program). The pathway-to-human models used in the previous versions of the computer program have not been changed.

This document is written for two audiences, each with different documentation requirements. The first is a scientific or technical audience and includes persons concerned with the mathematical models and computer algorithms. The second is an audience concerned with the details of the computer programming and includes persons that want to exercise the computer program and scenarios for specific onsite disposal applications. Six sample problems are presented and discussed to assist the user in operating the computer program. Summaries of the input and output for the sample problems are included along with a discussion of the calculations performed by hand to verify the correct software operation. Computer listings of the ONSITE/MAXI1 computer program with a complete data base listing are included in the appendices of this document. 



\section{EXECUTIVE SUMMARY}

This document describes how the ONSITE/MAXI1 computer program (Napier et al. 1984; Kennedy et al. 1986) has been enhanced and modified to meet the needs of the U.S. Nuclear Regulatory Commission (NRC) by making the program operable on the IBM PC/XT/AT computer, and by permitting the user to optionally select dose conversion factors based on the dosimetry methods and metabolic data given by the International Commission on Radiological Protection (ICRP) in their Publication No. 30 (ICRP 1979-1982). The original documentation for the ONSITE/MAXI1 software package contains detailed instructiops for operation on the CDC 6600-7600 computer (Napier et al. 1984) and VAX $11-780$ computer (Kennedy et al. 1986). The NRC has established guidance for onsite disposal by subsurface burial in NUREG-1101, Vol. 1 (Neuder 1986). Applications by 1 icensees to the NRC for proposed disposal activities that do not fit the NRC guidance will be evaluated against more complete guidance using a documented technical methodology (Neuder and Kennedy 1986). The ONSITE/MAXI1 computer program described in this document is part of the NRC methodology for estimating radiation doses for human intruder scenarios.

The version of the ONSITE/MAXI1 computer program presented in this document uses the same methodology (with the addition of ICRP Publication No. 30 methods) and produces essentially the same results as earlier versions of the computer program documented by Napier et al. (1984) and Kennedy et al. (1986). Inhalation doses varied from those in earlier versions, because default inhalation parameter values were changed to be consistent with those used in ICRP Publication No. 30. Six sample problems were presented in the two earlier documents. The results of those sample problems were verified by hand-using the equations presented in this document. When those six sample problems are executed using the version of ONSITE/MAXIl presented in this document, the results are the same as those documented in Napier et al. (1984) and Kennedy et al. (1986).

This document contains the user information and instructions for operation of ONSITE/MAXI1 on the IBM PC/XT/AT computer. Additional details and user instructions for program execution on the CDC 6600-7600 computers are presented in Napier et al. (1984), along with detailed instructions for interacting with the ONSITE computer program. Further information on the VAX 11-780 computer and the MAXI time line is found in Kennedy et al. (1986).

The documentation of the ONSITE/MAXI1 computer program is written for two audiences, who have different documentation requirements. The first audience is scientific or technical and includes persons concerned with the mathematical models and computer algorithms. The second audience is concerned with the

(a) IBM PC/XT/AT is a registered trademark of the International Business Machines Corporation, Boca Raton, Florida.

(b) Control Data Corporation, St. Paul, Minnesota.

(c) VAX is a registered trademark of Digital Equipment Corporation, Nashua, New Hampshire. 
details of the computer programming and includes persons who want to exercise the computer program and scenarios for specific onsite disposal applications. Section 2 of this document contains information useful to the first audience and describes the mathematical models and computer software for assessing onsite disposal impacts. Section 3 of this document contains information useful to the second audience and describes the procedures used to operate the software on the IBM PC/XT/AT. Six sample problems that demonstrate the scenario operations of the ONSITE/MAXI1 computer software are also given in Section 3. Section 4 also contains information useful to the second audience and describes the programming structure and hierarchy. It previously was necessary to execute the MAXI2 and MAXI3 computer programs to generate internal dose rate factors that were then used by MAXI1. The major change made to the personal computer version of the software package is the incorporation of the MAXI2 and MAXI3 computer programs for calculating dose from terrestrial and aquatic food pathways (Napier et al 1985; Kennedy et al. 1986) into MAXI1. Complete Tistings of the ONSITE/MAXI1 software package and supporting data libraries are included in the appendices. The organization of this document roughly follows the organization of the previous documentation for the ONSITE/MAXI1 computer software to facilitate cross-referencing. 


\section{ACKNOWLEDGMENTS}

The authors would like to thank all of those individuals at Pacific Northwest Laboratory who have helped make this work possible. The historical development of the MAXI computer program included major contributions from Ed Watson, Glenn Hoenes, and Joe Soldat. We greatly appreciate the guidance, assistance, technical review, and encouragement offered by these individuals in the development of the methods and philosophy applied in this report. We would like to thank Jaime Lara for converting the ONSITE program for execution on a personal computer, and for updating, enhancing, and testing the computer program. We would like to thank Gus Rodriguez for his careful checking of dose conversion factors included in this software package and Joe Soldat and Bill Farris for their helpful technical review of the mathematical models contained in this document. We would also like to thank Kay Hopkins and Carolyn Schauls for their contributions in typing, organizing, and correcting the draft and final manuscripts. Finally, we would like to thank Virginia Brouns for her editorial assistance in the preparation of the manuscript. 



\section{CONTENTS}

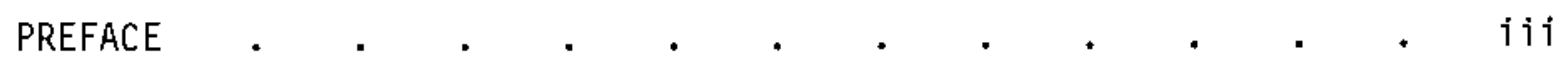

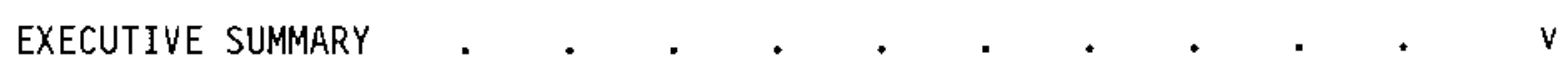

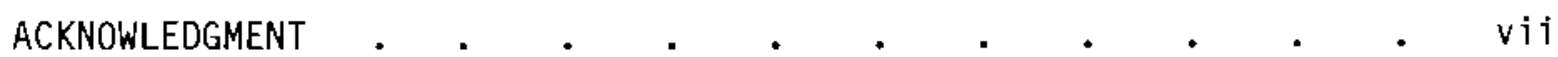

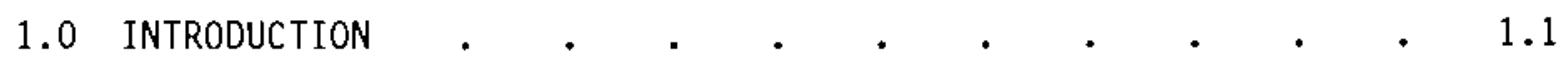

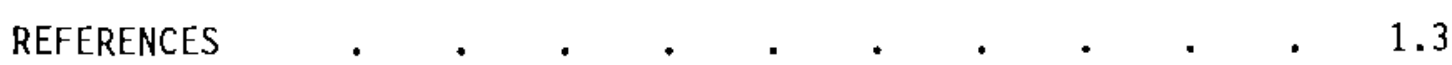

2.0 MATHEMATICAL MODELS AND COMPUTER SOFTWARE FOR ASSESSING

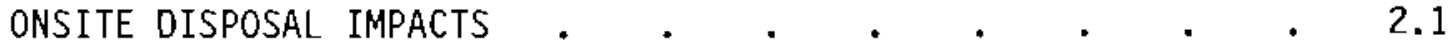

2.1 DEFINITION AND SOLUTION OF THE PROBLEM $\quad \cdot \quad \cdot \quad \cdot \quad \cdot \quad 2.1$

2.1.1 Applying the Pathway Analysis Models . . . 2.2

2.1.2 Onsite Disposal "Environment" Description . . 2.3

2.1.3 Onsite Disposal Scenario Descriptions . . . . 2.6

2.1 .4 Mathematical Models $. \quad . \quad . \quad . \quad . \quad . \quad 2.7$

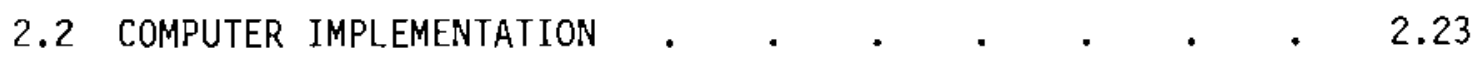

2.2.1 Computer System Requirements . . . . . 2.23

2.2.2 Descriptions of Computer Programs . . . . 2.23

2.2.3 Operation of the ONSITE/MAXI1 Software Package . 2.24

2.2.4 Capabilities, Restrictions and Performance . . 2.25

2.2 .5 Software Package . . . . . . . 2.25

2.3 DATA BASE . . . . . . . . . . . . . . 2.25

2.3.1 Radionuclide Master List - RMDONS . . . . 2.26

2.3.2 Radionuclide Master Data Library - RMDLIB . $\quad$. 2.26

2.3.3 Organ Data Library - ORGLIB . . . . . 2.27

2.3.4 Food Transfer Factor Library - FTRANS . . . $\quad 2.28$

2.3.5 Bioaccumulation Factor Library - BIOAC1 . $\quad$. 2.28

2.3.6 External Exposure Dose Rate Factors - Waste $\quad 2.29$

2.3.7 External Exposure Dose Rate Factors -
Waste Modeled as a Slab Source . . . . 2.31

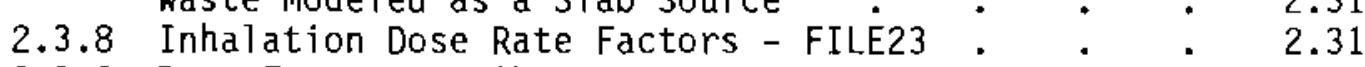

2.3.9 Dose Factors - Library LB30SVBQ . . . . 2.32

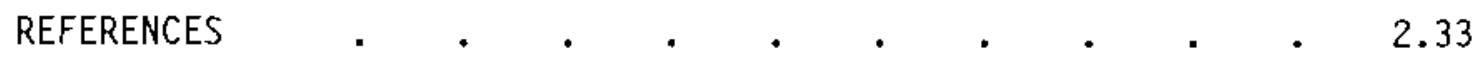




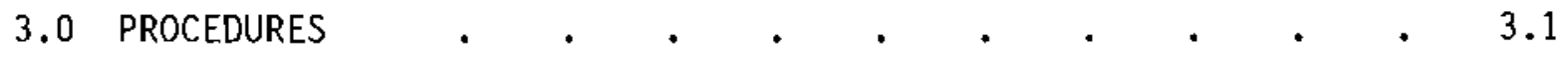

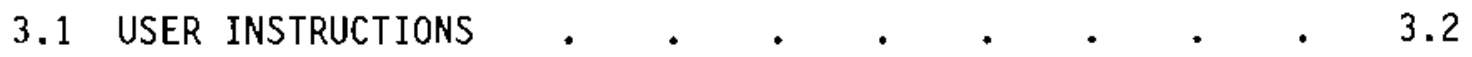

3.1.1 Installing ONSITE/MAXI1 on a Hard Disk . . 3.2

3.1 .2 Interaction with ONSITE: The First Level
of Operation . . . . . . . 3.3

3.1.3 Interaction with the MAXI1 Input File:
The Second Level of Operation $\quad . \quad$ • $\quad 3.4$

3.1 .4 Batch Mode Processing of ONSITE/MAXI1 . . $\quad 3.5$

3.2 SAMPLE PROBLEMS • . . . . . . . . . . . . . . 3.6

3.2.1 Sample Problem One $\quad$. $\quad . \quad$. $\quad . \quad$. $\quad 3.7$

3.2.2 Sample Problem Two $\quad . \quad$. $\quad . \quad$. $\quad . \quad$. 3.15

3.2.3 Sample Problem Three $\quad . \quad . \quad . \quad . \quad . \quad . \quad 3.23$

3.2.4 Sample Problem Four . . . . . . . 3.34

3.2.5 Sample Problem Five . . . . . . . . 3.46

3.2.6 Sample Problem Six $\quad . \quad$. $\quad . \quad$. $\quad . \quad$. $\quad .3 .59$

3.3 INPUT PARAMETERS . $\quad . \quad$. $\quad . \quad$. $\quad . \quad$. 3.68

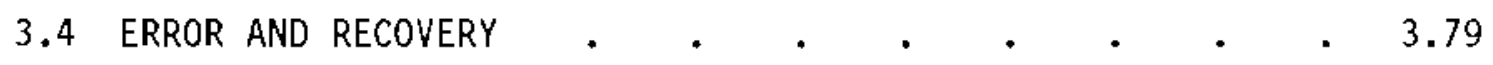

3.4.1 Error and Recovery - ONSITE . . . . . . 3.79

3.4.2 Error and Recovery - MAXI1 . . . . . . 3.79

REFERENCES • . . . . . . . . . . . . 3.82

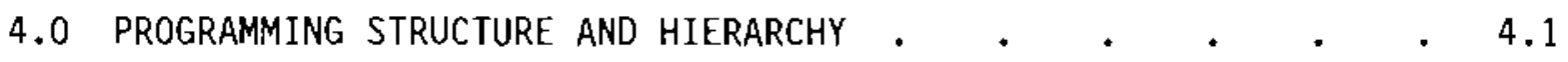

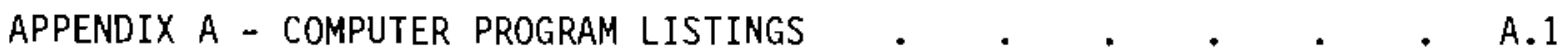

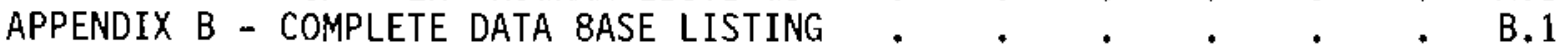




\section{FIGURES}

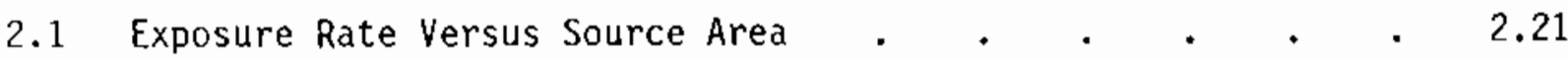

2.2 Exposure Rate Ratio Versus Fractional Hectare of Source Area . 2.21

2.3 Area Correction Factors for External Exposure and the Inhalation Pathway . . . . . . . . . 2.22

2.4 Area Correction Factors for the Ingestion Pathway . . . 2.22

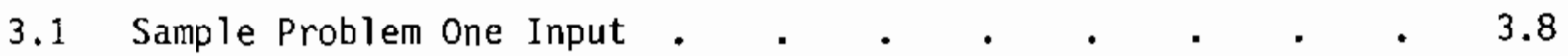

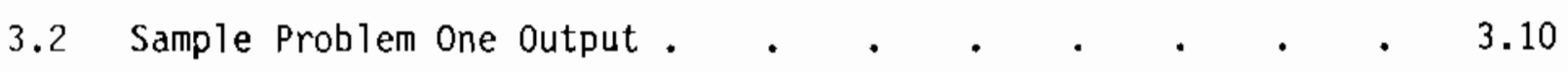

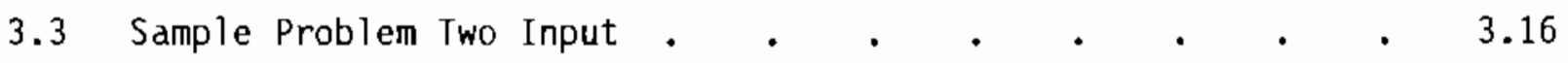

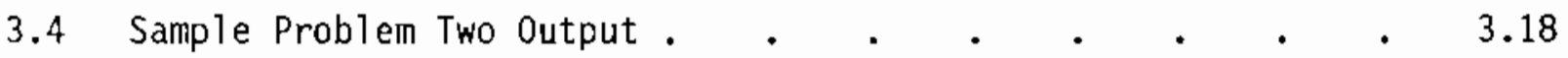

3.5 Sample Problem Three Input . . . . . . . . 3.24

3.6 Sample Problem Three Output . . . . . . . . 3.26

3.7 Sample Problem Four Input . . . . . . . . . . . 3.35

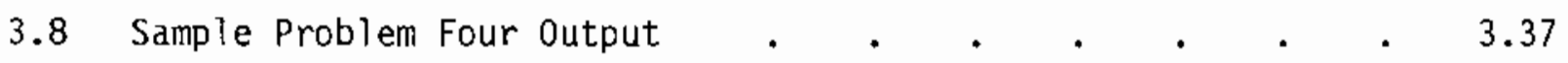

3.9 Sample Problem Five Input . . . . . . . . . . . . 3.47

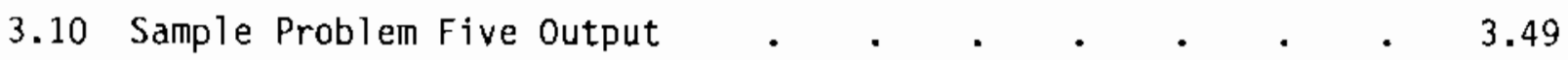

3.11 Sample Problem Six Input . . . . . . . . . 3.60

3.12 Sample Problem Six Output . . . . . . . . . 3.62

4.1 ONSITE Module Heirarchy and Function . . . . . . . . 4.2

4.2 MAXI Module Hierarchy and Function . . . . . . . 4.3 


\section{$\underline{\text { TABLES }}$}

2.1 Parameters Used for Calculation of Radiation Doses

from Consumption of Terrestrial Foods $. . \quad . \quad . \quad . \quad . \quad 2.4$

2.2 Parameters Used for Calculation of Radiation Doses

from Drinking water and Aquatic Foods . . . . . 2.4

2.3 Radionuclides Considered in the ONSITE Disposal Environment . $\quad 2.5$

2.4 External Dose Conversion Factors (mrem/h per $\mathrm{Ci} / \mathrm{m}^{3}$ ) for Selected Waste Form Densities $(\rho)$ and Slab Ihicknesses

with $0.0-\mathrm{m}$ Overburden . . . . . . . . . . . . 2.19

2.5 Dose Rate Factor Files for External Exposure . . . . . 2.29

2.6 ISOSHLD Parameter Values Used for External Exposure

3.1 Scenario Default Parameter Values . . . . . . . 3.77

3.2 Sample Problem Parameter Values $\quad$. . . . . . . 3.78 


\section{$1.0 \quad$ INTRODUCTION}

The ONSITE/MAXI1 computer program (Napier et al. 1984; Kennedy et a1. 1986) is used to estimate radiation doses to humans from a variety of exposure pathways and scenarios. This computer program was originally developed for the U.S. Nuclear Regulatory Commission's (NRC) waste management staff by Pacific Northwest Laboratory (PNL). It has been used by the NRC in estimating humanintrusion scenario doses for evaluating licensee applications for onsite burial of low-activity radioactive wastes. Current NRC regulations require that disposal of radioactive materials by licensees, unless otherwise authorized, be specifically approved by the NRC pursuant to 10 CFR 20.302(a) (1984). This regulation states:

Any licensee or applicant for a license may apply to the Commission for approval of proposed procedures to dispose of licensed material in a manner not otherwise authorized in the regulations in this chapter. Each application should include a description of the licensed material and any other radioactive material involved, including the quantities and kinds of such material and the levels of radioactivity involved, and the proposed manner and conditions of disposal. The application should also include an analysis and evaluation of pertinent information as to the nature of the environment, including topographical, geological, meteorological, and hydrological characteristics; usage of ground and surface waters in the general area; the nature and locations of other potentially affected facilities; and procedures to be observed to minimize the risk of unexpected or hazardous exposures.

To reduce the number of licensee applications that must be reviewed, in 1986 the NRC issued guidance that identifies the categories and limiting conditions for total activity, waste packaging, burial frequency, and other conditions that are likely to be acceptable for subsurface disposal. This guidance is contained in Onsite Disposal of Radioactive Waste: Guidance for Disposal by Subsurface Burial, NÜREG-1101, Vol. 1 (Neuder 1986). Applications for proposed disposal activities that do not fit the NRC guidance will be evaluated against more complete guidance using a documented technical methodology. This methodology is described in NUREG-1101, Vol. 2 (Neuder and Kennedy 1986). The ONSITE/MAXI1 computer program described in this document is part of the Vol. 2 NRC methodology for estimating radiation doses for human intrusion scenarios.

As part of the ONSITE/MAXI1 project, specific human intrusion scenarios used in evaluating doses from onsite disposal of radioactive wastes have been developed (Napier et al. 1984). These scenarios consider various potential combinations of direct exposure to penetrating radiation, inhalation of airborne radionuclides, ingestion of agricultural products raised in contaminated soil, and ingestion of radionuclides in drinking water. They are activated by running the ONSITE/MAXI1 computer software package. As a continuation of the initial work, the ONSITE/MAXI1 software package was enhanced to consider additional optional external exposure dose factors and revisions to the documentation were made to more fully explain the computer software (Kennedy et al. 1986). In addition, scenarios and options for alternative land-use conditions were 
developed that permit the user to select from a meat diet, vegetable diet, or a combination of the two.

As a further extension of the development effort, we have produced a modified version of the ONSITE/MAXIl computer software package that operates on an IBM $\mathrm{PC} / \mathrm{XT} / \mathrm{AT}$ (a) computer. This version also permits the user to optionally select the use of dose conversion factors based on the dosimetry methods and metabolic data given by the International Commission on Radiological Protection (ICRP) in their Publication No. 30 (ICRP 1979-1982) in place of those given by the ICRP in Publication No. 2 (1959) and supplied with the original ONSITE/MAXI1 computer software.

The ONSITE computer program is the interactive user interface that allows the end-user to simply and efficiently create and use the radiation exposure scenarios. The MAXI1 computer program is then used with the scenario information to calculate the maximum annual dose to the exposed individual from selected pathways. Intermediate dose conversion factors for the external exposure pathways, ICRP Publication No. 2 internal dose conversion factors (ICRP 1959), and ICRP Publication No. 30 effective dose equivalent factors for internal inhalation and ingestion (ICRP 1979-1982) are stored in data files. These factors can be found in the ICRP publications or calculated using codes external to the ONSITE/MAXI1 software package. The inhalation dose conversion factors are derived from the model in ICRP Publication No. 2 (ICRP 1959) and are calculated using the Task Group Lung Model (TGLM) (ICRP 1966) as contained in the DACRIN (Houston, Strenge and watson 1974) computer program. Additional metabolic data for the inhalation calculations were obtained from ICRP Publication No. 19 (ICRP 1972). The external dose conversion factors for various waste disposal geometries and densities are calculated using the ISOSHLD (Engel, Greenborg and Hendrickson 1966) shielding program.

This report documents the resulting IBM PC/XT/AT computer version of the ONSITE/MAXI1 computer software package. It is designed to function both as a reference document and as an instructional document. Section 2 contains technical information on the mathematical models and computer algorithms used in the ONSIJE/MAXI1 computer programs. Section 3 contains user information on the procedures for executing ONSITE/MAXI1 computer programs, including a discussion of sample problems and hand calculations performed to verify correct operation of the computer program. Section 4 contains additional details on the structure and organization of the computer programs and is provided for those who wish to modify the computer programs. Listings of the computer programs, data libraries, and dose conversion factors are provided in the appendices.

(a) IBM PC/XT/AT is a registered trademark of the International Business Machines Corporation, Boca Raton, Florida. 


\section{REFERENCES}

Engel, R. L., J. Greenborg, and M. M. Hendrickson. 1966. ISOSHLD - A Computer Code for General Purpose Isotope Shielding Analysis. BNWL-236, Pacific Northwest Laboratory, Richland, Washington.

Houston, J. R., D. L. Strenge, and E. C. Watson. 1974. DACRIN - A Computer Program for Calculating Organ Dose from Acute or Chronic Radionuclide Inhalation. BNwL-B-389, Pacific Northwest Laboratory, Richland, Washington.

International Commission on Radiological Protection (ICRP). 1959. Report of ICRP Committee II on Permissible Dose for Internal Radiation. ICRP Publication No. 2, Pergamon Press, New York, New York.

International Commission on Radiological Protection (ICRP). 1966. "Deposition and Retention Models for Internal Dosimetry of the Human Respiratory Tract." Health Phys. 12:173-207.

International Commission on Radiological Protection (ICRP). 1972. The Metabolism of Compounds of Plutonium and Other Actinides. ICRP Publication No. 19, Pergamon Press, New York, New York.

International Commission on Radiological Protection (ICRP). 1979-1982. Limits for Intakes of Radionuclides by Workers. ICRP Publication No. 30, Part 1 (and subsequent parts and supplements), Vol. 2, No. 3/4 through Vol. 8, No 4. Pergamon Press, New York, New York.

Kennedy, W. E., Jr., R. A. Peloquin, B. A. Napier, and S. M. Neuder. 1986. Intruder Dose Pathway Analysis for the Onsite Disposal of Radioactive Wastes: The ONSITE/MAXI1 Computer Program. NUREG/CR-3620, Supplement No. 1, (PNL-4054), U..S. Nuclear Regulatory Commission, Washington, D.C.

Neuder, S. M. 1986. Onsite Disposal of Radioactive Waste: Guidance for Disposal by Subsurface Burial. NÜREG-1101, Vol 1, U.S. Nuclear Regulatory Commission, Washington, D.C.

Neuder, S. M., and W. E. Kennedy, Jr. 1986. Onsite Disposal of Radioactive Waste: Methodology for the Radiological Assessment of Disposal by Subsurface Burial. NUREG-1101, Vo1, 2, U.S. Nuclear Regulatory Commission, Washington, D.C.

Napier, B. A., G. R. Hoenes, W. E. Kennedy, Jr., and E. C. Watson. 1979. "The Maximum Annual Dose Resulting from Residual Radioactive Contamination." Paper presented at the 24th Annual Meeting of the Health Physics Society, July 8-13, 1979, Philadelphia, Pennsylvania. PNL-SA-7496, Pacific Northwest Laboratory, Richland, Washington.

Napier, B. A., R. A. Peloquin, W. E. Kennedy, Jr., and S. M. Neuder. 1984. Intruder Dose Pathway Analysis for the Onsite Disposal of Radioactive Wastes: The ONSITE/MAXI1 Computer Program. NUREG/CR-3620 (PNL-4054), U.S. Nuclear Regulatory Commission, Washington, D.C.

Standards for Protection Against Radiation, 10 CFR 20 (1984). 



\subsection{MATHEMATICAL MODELS AND COMPUTER SOFTWARE FOR ASSESSING ONSITE DISPOSAL IMPACTS}

This section contains descriptions of the radiation exposure scenarios, mathematical models, computer programs, and data bases for assessing the potential doses to iptruders at onsite waste disposal sites using the ONSITE/MAXI1 IBM $\mathrm{PC} / \mathrm{XT} / \mathrm{AT}$ (a) computer software package. The information in this section is designed to be useful to persons concerned with the mathematical models and computer algorithms. The method used for assessing potential doses to intruders is presented first, followed by a discussion of the computer implementation of that method, and then a description of the associated data base.

\subsection{DEFINITION AND SOLUTION OF THE PROBLEM}

In the Draft and Final Environmental Impact Statements in support of 10 CFR 61 (NRC 1981, 1982), the NRC modeled the potential exposure pathways to man from buried radioactive wastes. In the analysis, the NRC identified four human-intrusion scenarios to account for the actions of humans after the loss of institutional controls (NRC 1981, P. H-15):

- Intruder-Construction Scenario. An individual excavates at a closed disposal site to build a house.

- Intruder-Discovery Scenario. This scenario is a subset of the intruderconstruction scenario and also involves excavation into a closed site. The time over which the excavation proceeds is reduced compared to the intruder-construction scenario.

- Intruder-Agriculture Scenario. An individual lives in a house built on a closed disposal site surrounded by contaminated soil resulting from the intruder-construction scenario. The individual consumes vegetables grown in the contaminated soil.

- Intruder-Well Scenario. An individual uses contaminated water from a well on a closed disposal site.

The disposal limits that result are based on an annual 500-mrem total-body dose to the maximally exposed individual who intrudes after 100 years of site control. Of the four human-intrusion scenarios defined, only the intruderconstruction and intruder-agriculture scenarios affect the determination of disposal limits.

In this report, an approach similar to that applied in the Draft EIS in support of 10 CFR 61 (NRC 1981) is used for the assessment of risks associated with onsite disposal. That is, radiation exposure scenarios are established for the

(a) IBM PC/XT/AT is a registered trademark of the International Business Machines Corporation, Boca Raton, Florida. 
maximally exposed individual (an intruder), and a means of determining the resulting radiation dose is provided.

Five scenarios are identified as being of potential interest in assessing doses to intruders at onsite disposal sites:

1. External Exposure Scenario. An individual is assumed to work in an area previously used for onsite disposal. Surface soil contamination, wastes buried at depths of $0.5 \mathrm{~m}$ or $1.0 \mathrm{~m}$, or entry into a room (or vault) that is used for waste storage or disposal are considered.

2. External Exposure Plus Inhalation Scenario. An individual is assumed to work in an area with limited surface-soil contamination and is exposed to external sources and resuspended dusts.

3. Agricultural Scenario. An individual is assumed to raise his annual diet (or a fraction of it) in soil contaminated by the onsite disposal of radioactive wastes. External exposure and inhalation of resuspended radionuclides in soil are considered.

4. Irrigation/Drinking-Water Scenario. An individual is assumed to use a water supply contaminated by radionuclides from an onsite disposal site for irrigation and/or drinking. In addition, external exposure and inhalation of resuspended radionuclides that are deposited on the surface of the soil by the irrigation water are considered.

5. User-Defined Scenario. The user may construct his own scenario by selecting exposure pathways and defining conditions described in the ONSITE/MAXI1 computer software package.

The following sections contain descriptions of the pathway analys is models used for these scenarios, a definition of the required environmental criteria, descriptions of the scenarios as they are implemented, and descriptions of the mathematical models.

\subsubsection{Applying the Pathway Analysis Models}

The five types of intruder scenarios can be simulated with the pathway analysis models contained in the IBM PC/XT/AT version of the ONSITE/MAXI1 computer software package. The computer programs implement these models in a way substantially different from the one described in the previously documented versions of ONSITE/MAXI1 (Napier et al. 1984; Kennedy et al, 1986). This implementation greatiy reduces the size of the data base and provides greater flexibility during scenario generation. The revised program incorporates the MAXI2 and MAXI3 computer programs into the MAXI1 program.

In the previous versions of MAXI1, an "environment" was defined by the general agricultural and aquatic practices of the geographical area, and the general lifestyle of the intruder. Scenarios were then defined within the context of that environment. A scenario indicated pathways of interest, further defined the life style of the maximally exposed individual, identified the source and 
location of the contamination, and quantified the inventory. A scenario modified the maximally exposed individual's lifestyle assumptions by indicating amounts of exposure and by applying percentage factors to established dose rates. The definition of the environment was an intermediate step that simplified scenario creation and streamlined scenario executions. Dose conversion factors that applied to the defined environment were created by auxiliary programs in the software package (MAXI2 and MAXI3) and stored in the data base where they were accessed during the scenario simulations controlled by the main computer program, MAXI1.

However, defining additional environments, relying on modified pathway parameters, caused the MAXI1 data base to become extremely large and cumbersome. It was decided that the most effective way to implement the ONSITE/MAXI software package on the IBM PC/XT/AT computer was to incorporate the MAXI2 and MAXI3 computer programs into MAXI1, thus substantially reducing the size of the data base. With this program consolidation, the user now has more flexibility when creating scenarios because the user may modify the default values used in defining the environment when constructing a scenario.

The following sections contain discussions of the default values used to define the environment, the mathematical models used for the five intruder scenarios, and the mathematical models that may be used for determining the dose to intruders at onsite disposal sites.

\subsubsection{Onsite Disposal "Environment" Description}

The default environment defined for onsite disposal assumes intruder activity at an onsite low-level waste disposal site. The reference environment is based on a site with an area of 1 ha; however, area correction factors may be included to consider smaller sites. The intruder may be exposed to radioactive contamination via any of the following pathways: external exposure, inhalation of resuspended contaminants, ingestion of farm products grown on a contaminated site, consumption of drinking water from a contaminated well, or ingestion of aquatic food products from a contaminated water source. For external exposure, wastes may be located on the surface, buried at 0.5 - or 1.0-meter depths, or stored in a room-type structure. For inhalation and ingestion, the default parameters used in defining the reference environment are based on those found in Regulatory Guide 1.109 (NRC 1977). The intruder's entire diet for the reference environment consists of vegetables, fruits, and animal products grown on the site. Table 2.1 contains a listing of the intruder's terrestrial food product diet and Table 2.2 contains a listing of the intruder's aquatic food product diet for the reference environment. The intruder is assumed to drink 1.2 liters of water per day from a contaminated well.

Contaminated farm products may result from radioactive wastes located on the soil surface or from irrigation with contaminated water. The radionuclides expected to be of interest in the reference environment are listed in Table 2.3. This list includes 105 radionuclides that might come from the use of radiopharmaceuticals or industrial sources, or from wastes generated as part of the commercial nuclear fuel cycle. 
TABLE 2.1. Parameters Used for Calculation of Radiation Doses from Consumption of Terrestrial Foods

\begin{tabular}{|c|c|c|c|c|}
\hline Food & $\begin{array}{l}\text { Growing } \\
\text { Period } \\
\text { (days) } \\
\end{array}$ & $\begin{array}{l}\text { Yield } \\
\left(\mathrm{kg} / \mathrm{m}^{2}\right)\end{array}$ & $\begin{array}{l}\text { Holdup }(a) \\
\text { (days) }\end{array}$ & $\begin{array}{c}\text { Consumpt } \\
(\mathrm{kg} / \mathrm{yr})\end{array}$ \\
\hline Leafy vegetables & 90 & 1.5 & 1 & 9.5 \\
\hline $\begin{array}{l}\text { Other aboveground } \\
\text { vegetables }\end{array}$ & 60 & 0.7 & 1 & 9.5 \\
\hline Root vegetables & 90 & 9 & 1 & 76 \\
\hline Fruit & 90 & 1.7 & 14 & 42 \\
\hline Wheat and grain & 90 & 0.72 & 10 & 51 \\
\hline Eggs & 90 & $0.84^{(c)}$ & 2 & 19 \\
\hline Milk & 30 & $1.3^{(c)}$ & 2 & $110^{(d)}$ \\
\hline Beef & 90 & $0.84^{(c)}$ & 15 & 39 \\
\hline Pork & 90 & $0.84^{(c)}$ & 15 & 29 \\
\hline Poultry & 90 & $0.84^{(c)}$ & 15 & 8.5 \\
\hline \multicolumn{5}{|c|}{$\begin{array}{l}\text { (a) Time between harvest and consumption. } \\
\text { (b) These rates are obtained from Regulatory Guide } 1.109 \text { (NRC 1977) and } \\
\text { prorated by food category using the fraction of total consumed by an } \\
\text { average individual as calculated from Napier (1981, Table } 8 \text { ). } \\
\text { (c) Yield of animal feeds (i.e., grain or pasture grass). } \\
\text { (d) Units of L/yr. }\end{array}$} \\
\hline
\end{tabular}

TABLE 2.2. Parameters Used for Calculation of Radiation Doses from Drinking Water and Aquatic Foods

\begin{tabular}{|c|c|c|c|c|}
\hline Pathway & $\begin{array}{l}\text { Mixing } \\
\text { Ratio } \\
\end{array}$ & $\begin{array}{c}\text { Holdup } \\
\text { (days) }\end{array}$ & Consumption & $\begin{array}{l}\text { Consumption } \\
\text { Units }\end{array}$ \\
\hline Fish & 1.0 & 1.0 & $6.9^{(b)}$ & $\mathrm{kg} / \mathrm{yr}$ \\
\hline Drinking water & 1.0 & 1.0 & $438^{(c)}$ & $L / y r$ \\
\hline
\end{tabular}

(a) Time between harvest and consumption.

(b) Average rate obtained from Regulatory Guide 1.109 (NRC 1977).

(c) Rate based on scenario assumption of $1.2 \mathrm{~L} / \mathrm{d}$. 
TABLE 2.3. Radionuclides Considered in the ONSITE Disposal Environment

\begin{tabular}{|c|c|c|}
\hline Radionuclide & Radionuclide & Radionuclide \\
\hline $\begin{array}{ll}\mathrm{H} & 3 \\
\mathrm{C} & 14 \\
\mathrm{Na} 22\end{array}$ & $\begin{array}{l}\mathrm{Ag} 110 \mathrm{M} \\
\mathrm{Ag} 110 \\
\text { In111 }\end{array}$ & $\begin{array}{l}\text { Ra224 } \\
\text { Pb212 } \\
\text { Bi212 }\end{array}$ \\
\hline $\begin{array}{ll}p & 32 \\
p & 33 \\
S & 35\end{array}$ & $\begin{array}{l}\text { I } 125 \\
\text { Sb125 } \\
\text { Te125M }\end{array}$ & $\begin{array}{l}\text { U } 234 \\
\text { U } 235 \\
\text { Th231 }\end{array}$ \\
\hline $\begin{array}{l}C 136 \\
K \quad 40 \\
C a 45\end{array}$ & $\begin{array}{ll}\text { Sb } 124 \\
\text { I } & 129 \\
\text { I } & 131\end{array}$ & $\begin{array}{l}\text { Pa231 } \\
\text { Ac227 } \\
\text { Th227 }\end{array}$ \\
\hline $\begin{array}{l}\text { Sc46 } \\
\text { Cr51 } \\
\text { Mn54 }\end{array}$ & $\begin{array}{l}\text { Xe131M } \\
\text { Cs134 } \\
\text { Cs135 }\end{array}$ & $\begin{array}{l}\text { Fr223 } \\
\text { Ra223 } \\
\text { Np237 }\end{array}$ \\
\hline $\begin{array}{l}\text { Fe55 } \\
\text { Fe59 } \\
\text { Co57 }\end{array}$ & $\begin{array}{l}\text { Cs137 } \\
\text { Ba137M } \\
\text { Ce141 }\end{array}$ & $\begin{array}{l}\text { Pa233 } \\
\text { U } 233 \\
\text { Th229 }\end{array}$ \\
\hline $\begin{array}{l}\text { Co60 } \\
\text { Ni59 } \\
\text { Ni63 }\end{array}$ & $\begin{array}{l}\text { Ce144 } \\
\text { Pr144 } \\
\text { Nd144 }\end{array}$ & $\begin{array}{l}\operatorname{Ra} 225 \\
A C 225 \\
U 238\end{array}$ \\
\hline $\begin{array}{l}\text { Zn65 } \\
\text { Se75 } \\
\text { Sr85 }\end{array}$ & $\begin{array}{l}\text { Eu152 } \\
\text { Eu154 } \\
\text { Tb160 }\end{array}$ & $\begin{array}{l}\text { Th234 } \\
\text { Pa234M } \\
\text { Pa234 }\end{array}$ \\
\hline $\begin{array}{l}\text { Sr } 89 \\
\text { Y } 89 M \\
\text { Sr } 90\end{array}$ & $\begin{array}{l}\text { Os } 185 \\
\text { Os } 191 \\
\text { Ir192 }\end{array}$ & $\begin{array}{l}\text { Pu242 } \\
\text { Np238 } \\
\text { Pu238 }\end{array}$ \\
\hline $\begin{array}{l}\text { Y } 90 \\
\text { Nb94 } \\
\text { Mo93 }\end{array}$ & $\begin{array}{l}\mathrm{Hg} 203 \\
\mathrm{Th} 230 \\
\mathrm{Ra} 226\end{array}$ & $\begin{array}{l}\text { Cm244 } \\
\text { Pu244 } \\
\text { U } 240\end{array}$ \\
\hline $\begin{array}{l}\text { Mo99 } \\
\text { Tc99M } \\
\text { TC99 }\end{array}$ & $\begin{array}{l}\text { Rn222 } \\
\text { Pb210 } \\
\text { Bi210 }\end{array}$ & $\begin{array}{l}\text { Pu240 } \\
\text { Pu241 } \\
\text { Am241 }\end{array}$ \\
\hline $\begin{array}{l}\text { Ru } 103 \\
\text { Pd103 } \\
\text { Rh103M }\end{array}$ & $\begin{array}{l}\text { Po210 } \\
\text { Th232 } \\
\text { Ra228 }\end{array}$ & $\begin{array}{l}\text { Cm243 } \\
\text { Pu243 } \\
\text { Am243 }\end{array}$ \\
\hline $\begin{array}{l}\text { Ru } 106 \\
\text { Rh106 }\end{array}$ & $\begin{array}{l}\text { Ac228 } \\
\text { Th228 }\end{array}$ & $\begin{array}{l}\text { Np239 } \\
\text { Pu239 }\end{array}$ \\
\hline
\end{tabular}




\subsubsection{Onsite Disposal Scenario Descriptions}

Five scenarios are identified for onsite disposal in Section 2.1.1. They are designed for use in assessing doses to intruders at onsite disposal sites with default site size of 1 ha. These scenarios are defined within the reference environment described in Section 2.1.2. Detailed descriptions of the scenarios, assumptions, and key parameters are given below.

1. External Exposure Scenario. An individual is assumed to work for $2000 \mathrm{~h} / \mathrm{yr}$ in a l-ha area previously used for onsite disposal. External exposure factor files are supplied to consider surface-soil contamination, buried wastes at depths of $0.5 \mathrm{~m}$ or $1.0 \mathrm{~m}$, or entry into a room (or vault) that is used for waste storage or disposal. For soil contamination, the inventory is modified by a factor of 0.2 to account for dilution. Only total-body dose is calculated. The user selects the location of the waste (e.g., surface, buried, or stored) and supplies the waste inventory. The user may modify the amount of radioactive decay before exposure, the dilution of the waste (during exhumation activities), the reference site size, and the duration of exposure.

2. External Exposure Plus Inhalation Scenario. An individual is assumed to work for $2000 \mathrm{~h} / \mathrm{yr}$ in a l-ha area with surface-soil contamination resulting from a waste exhumation event. Air concentrations of radionuclides are calculated using a resuspension equation by Anspaugh et al. (1975). For the resuspension equation, the age of the contamination at the beginning of the scenario is assumed to be 0.0 and the top 1.0 centimeter of the contaminated surface soil is assumed to be available for resuspension. Doses to total body, bone, lungs, thyroid, and the lower large intestine (LLI) of the gastrointestinal (GI) tract are calculated. The dilution factor (accounting for exhumation activities) applied to the inventory is 0.2 . The user provides the waste inventory. The user may modify the amount of radioactive decay before exposure, the duration of the exposure, the reference site size, and the inventory dilution factor. He or she may also optionally select a mass-loading equation and specify fewer organs. This scenario is similar to the intruder-construction scenario described in the Draft EIS for 10 CFR 61 (NRC 1981).

3. Agricultural Scenario. An individual is assumed to raise his entire diet in a 1-ha area of soil contaminated by the onsite disposal of radioactive wastes. The individual is assumed to be exposed $2000 \mathrm{~h} / \mathrm{yr}$ by external exposure and by inhalation of resuspended radionuclides in soil. Again, the air concentration resulting from resuspension is calculated using the equation by Anspaugh et al. (1975) with parameters presented for Scenario 2. Doses to total body, bone, lungs, thyroid, and LLI are calculated. The user furnishes the waste inventory to which a dilution factor of 0.2 is applied. Modifications can be made to the amount of radioactive decay before exposure, the duration of the exposure, the fraction of the total diet grown on the site, the reference site size, and the dilution of the waste. As in the previous scenarios, the user may substitute the mass-loading model and specify fewer organs. This scenario is similar to the intruder-agriculture scenario described in the Draft EIS for 10 CFR 61 (NRC 1981). 
4. Irrigation/Drinking-water Scenario. An individual is assumed to use a water supply contaminated by radionuclides from an onsite disposal site for irrigation and/or drinking. The user is required to input the concentration of each radionuclide in the water supply. The exposed individual is assumed to irrigate his field at a rate of $150 \mathrm{~L} / \mathrm{m}^{2} / \mathrm{mo}$ during a 6-mo growing season. The site is assumed to be irrigated with contaminated water for 10 years prior to the beginning of the scenario. The individual obtains his entire diet (or a fraction of it) from a 1-ha irrigated field, and drinks 1.2 L of water per day from a contaminated water source. In addition, he is assumed to be exposed $2000 \mathrm{~h} / \mathrm{yr}$ by external exposure and inhalation of resuspended radionuclides that are deposited on the surface of the soil by the irrigation water. Doses to total body, bone, lungs, thyroid, and LLI are considered. Irrigation and drinking water may be from the same or separate water supplies. When providing the inventory, the user is asked for radionuclide concentration in drinking and irrigation water separately. Irrigation or drinking water can be individually simulated by entering zero concentrations for the other pathway. The user may modify the irrigation rate, the length of the irrigation season, the time of irrigation prior to the scenario, the fraction of diet grown with contaminated irrigation water, the consumption of drinking water, the times of exposure, the resuspension equation selection, the reference site size, and the organ selection.

5. User-Defined Scenario. The user may construct his own scenario by selecting exposure pathways and defining conditions associated with each pathway.

\subsubsection{Mathematical Models}

The equations described in this section are arranged to aid understanding of the model as a whole. Consequently, the equations contain both environmentdefined and scenario-defined parameters as distinguished in Section 2.1.1. The origin of the parameters will be discussed when applicable for each equation.

The fundamental relationship for calculating radiation doses to people from any radionuclide exposure pathway is given in Equation (2.1) (Soldat, Robinson, and Baker 1974).

$$
R_{i p r}=C_{i p} U_{p} D_{i p r}
$$

where $R_{i p r}=$ the radiation dose equivalent or committed radiation dose equivalent from radionuclide $i$ via exposure pathway $p$ to organ $r$ (rem)

$c_{i p}=$ concentration of radionuclide $i$ in the media of exposure in pathway $\mathrm{p}$ ( $\mathrm{pCi} / \mathrm{L}$ water, $\mathrm{pCi} / \mathrm{kg}$ food, or $\mathrm{pCi} / \mathrm{m}^{3}$ air); for calculations involving airborne radionuclides, $C_{j p}$ is replaced with the term $x_{j}$, which represents the average ajpborne concentration of radionuclide $i$ 


$$
\begin{aligned}
U_{p}= & \text { usage parameter (exposure rate or intake rate) associated with } \\
& \text { exposure pathway } p(h / y r, L / y r \text {, or } \mathrm{kg} / \mathrm{yr}) \\
\mathrm{D}_{i p r}= & \text { annual dose equivalent factor or the committed effective dose } \\
& \text { equivalent factor for radionuclide } i \text {, exposure pathway } \mathrm{p} \text {, and } \\
& \text { organ } r \text { to convert the concentration and usage parameters to } \\
& \text { the radiation dose equivalent or to the committed effective } \\
& \text { dose equivalent (mrem/pCi) }
\end{aligned}
$$

An analysis of radiation doses from separate exposure pathways requires a determination of the dose equivalent or committed effective dose equivalent factors, the radionuclide concentrations, and the exposure rate or intake rate associated with each exposure pathway. For external exposure, the concentration of radionuclides and the duration of exposure must be quantified. The following sections provide mathematical descriptions of the models used in the analysis of radiation doses using the IBM PC/XT/AT version of the ONSITE/MAXI1 software package.

\subsubsection{Effective Dose Equivalent Factors}

The IBM PC/XT/AT version of the ONSITE/MAXI1 computer program allows the user to select dose calculations based on the internal dosimetry models described by the International Commission on Radiological Protection (ICRP) in either Publication No. 2 (1959) or No. 30 (1979-1982). The ability to produce doses based on ICRP Publication No. 2 is included so that comparisons with the Regulatory Guide 1.109 (NRC 1977) methods can be obtained. The methods based on ICRP Publication No. 30 are included to produce dose estimates that are consistent with the newer dosimetry system and metabolic data. Details concerning these internal dosimetry methods are found in references by the ICRP (1959; 1979-1982).

The methods described by the ICRP in their Publication No. 30 are based on limiting the total risk of health effects rather than on a controlling a "critical" organ risk. The dose equivalent, $H$, at a point in tissue is given by the ICRP (1977):

$$
H=D Q N
$$

where $H=$ the dose equivalent at a point in tissue

$D=$ the absorbed dose

$Q=$ the quality factor to allow for the effect on the detriment of the microscopic distribution of absorbed energy

$\mathrm{N}=$ the product of all other modifying factors that might account for the absorbed dose rate and fractionation.

In addition to the basic dose equivalent, the ICRP defined the committed dose 
equivalent over 50 years of exposure as $\mathrm{H}_{50}$, to a given organ or tissue from a single intake of radioactive material (ICRP 1977):

$$
H_{50}=\int_{t_{0}}^{t_{0}+50 y} H(t) d t
$$

where $\mathrm{H}_{50}=$ the committed dose equivalent

$t_{0}=$ the time of intake.

$H(t)=$ the revellent dose equivalent rate

For stochastic effects, the ICRP recommended a dose limitation system based on the principle that the risk to an individual should be equal whether the whole body is irradiated uniformly or whether there is non-uniform irradiation. This condition is met by the equation (ICRP 1977):

$$
\sum_{T}{ }^{W_{T}}{ }^{H_{T}} \leq H_{W b, L}
$$

where $W_{T}=\mathrm{a}$ weighting factor representing the proportion of the stochastic risk resulting from tissue $(T)$ to the total risk when the body is irradiated uniformily

$\mathrm{H}_{\mathrm{T}}=$ the annual dose equivalent in tissue (T)

$\begin{aligned} H_{w b} L & =\text { the recommended annual dose-equivalent limit for uniform irradiation } \\ & \text { of the whole body. }\end{aligned}$

For the dosimetry system described in ICRP Publication No. 26 (1977), the standard dose calculated is the weighted sum of individual tissue (or organ) committed dose equivalents known as the committed effective dose equivalent. This dose is estimated by the IBM PC/XT/AT version of the ONSITE/MAXI1 computer program using the recommendations of the ICRP (1977, 1979-1982). For the ICRP 30 dose calculations, the ONSITE/MAXI1 computer adds the calculated effective dose equivalent from ingestion and inhalation to the dose equivalent from external exposure to produce the annual effective dose equivalent. 


\subsubsection{Maximum Annual Organ Dose Calculations}

The user of the IBM PC/XT/AT version of the ONSITE/MAXI1 computer program may optionally select to calculate doses using the methodology given in ICRP Publication No. 2 (1959). For this dosimetry system, the ONSITE/MAXI1 computer program calcuiates the maximum annual dose to individual organs. Calculation of the annual dose to an organ requires the dose equivalent from exposure during the year of interest plus that resulting from intake during previous years. The general expression for annual dose calculations is determined here by inspecting the annual dose equations for the first three years of continuous exposure. For the first year, the annual dose to an organ of reference is simply the summation of the radiation dose equivalents from all internal and external exposure pathways. For the second year, the annual dose is calculated by the following expression (Kennedy et al. 1979: Napier et al. 1984):

$$
A_{2}=R_{2}^{*}+\left(R_{1,2}-R_{1,1}\right)
$$

where $A_{2}=$ the annual dose during the second year from all exposure pathways to the organ of reference, mrem

$$
\begin{aligned}
\mathrm{R}_{2}^{*}= & \text { the radiation dose equivalent in the second year to the organ of } \\
& \text { reference from all internal and external exposure pathways from } \\
& \text { intake and exposure in the second year, mrem } \\
\mathrm{R}_{1,2}= & \text { the committed dose equivalent to the organ of reference for the } \\
& \text { first two years from radionucl ides internally deposited during } \\
& \text { intake from exposure pathways in the first year, mrem } \\
\mathrm{R}_{1,1}= & \text { the radiation dose equivalent to the organ of reference for the } \\
& \text { first year from radionuclides internally deposited during intake } \\
& \text { from exposure pathways in the first year (no external component } \\
& \text { to the dose equivalent), mrem. }
\end{aligned}
$$

The expression in parentheses in Equation (2.5) represents the dose equivalent to the organ of reference from radionuclides deposited in that organ during the first year. It is the difference between the two-year committed dose from intake of radionuclides in the first year and the first year's dose from that same intake.

The mathematical expression for the annual dose to an organ of reference in the third year of continuous exposure is (Kennedy et al. 1979):

$$
A_{3}=R_{3}^{*}+\left(R_{1,3}-R_{1,2}\right)+\left(R_{2,2}-R_{2,1}\right)
$$


where $A_{3}=$ the annual dose during the third year from all exposure pathways to the organ of reference, mrem

$R_{3}^{*}=$ the radiation dose equivalent in the third year to the organ of reference from all internal and external exposure pathways from intake and exposure in the third year, mrem.

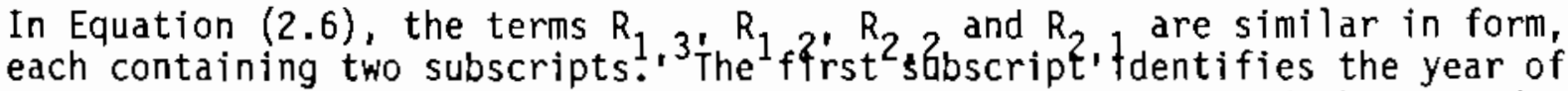
intake or exposure after the start of continuous exposure, and the second specifies the number of years used in calculating the committed dose equivalent. The expression in the first parentheses represents the dose equivalent to the organ of reference in the third year from radionuclides deposited during the first year of continuous exposure (i.e., the difference between the three-year committed dose equivalent and the two-year committed dose equivalent). The quantity in the second parentheses is the dose equivalent in the third year to the organ of reference from radionuclides deposited during the second year of continuous exposure (i.e., the difference between the second-year committed dose equivalent and the first-year committed dose equivalent).

The general expression for calculating the annual dose to an organ of reference during any year after the start of continuous exposure is expressed as (Kennedy et a1. 1979):

$$
A_{t}=R_{t}^{*}+\sum_{i=1}^{t-1} R_{i,(t-i+1)}-R_{i,(t-i)}
$$

where $A_{t}=$ the annual dose during the year $t$ from all exposure pathways to the organ of reference, mrem

$\mathrm{R}_{\mathrm{t}}^{\star}=$ the radiation dose equivalent in year $\mathrm{t}$ to the organ of reference from all internal and external exposure pathways from intake and exposure in the year $t$, mrem.

The summation term in Equation (2.7) represents the dose equivalent delivered to the organ of reference in year $t$ from radionuclides deposited in the organ from intake in all previous years since the start of continuous exposure. This term is valid only for positive integer values of $t$. For $t$ equal to 1 , the summation term is taken to be zero.

The annual dose, $A_{t}$, to the organ of reference is calculated for each value of $t$ from 1 to 50, and the maximum annual dose is determined by inspection. Experience with this method to date indicates 50 years to be a suitable maximum value of $t$; however, higher maximum values are not precluded. Details about the methods of calculating the radionuclide concentrations in various media, $c_{i p}$ in Equation (2.1), are given in Sections 2.1.4.3 through 2.1.4.7. 
No special algorithms or numerical techniques are employed by the ONSITE/ MAXI1 computer programs apart from those required to solve Equation (2.7). However, special formulations for some of the parameters in Equation (2.1) are available in the exposure scenario analysis. Other formulations are directly included in the calculation of the dose conversion factors used by MAXI1. The following sections contain discussions of optional and default equations that are incorporated into the ONSITE/MAXIl software package.

A more complete description of the maximum annual dose, a comparison of maximum annual dose with committed dose equivalents, a comparison with the 10 CFR 61 disposal limits (NRC 1981), and a description of the time line used in the maximum annual dose calculations can be found in Kennedy et a1. (1986).

\subsubsection{Radionuclide Concentration in Terrestrial Food Products}

For ingestion of farm products produced on a contaminated site, the radionuclide concentration in separate food products must be determined by accounting for root transfer from soil, dry deposition from air on leaves, wet deposition from irrigation water, or animal consumption of contaminated forage or feed. The annual diet for the maximally exposed individual and the holdup time between harvest and consumption must also be determined. The dose from any food pathway is given by Equation (2.1). For direct deposition from the air, Equation (2.8) is used to describe the deposition of airborne particulate radionuclides directly onto food products and onto the ground.

$$
d_{i}^{a}=86,400 \bar{\chi}_{i} \quad v_{d i}
$$

where $d_{i}^{a}=\underset{p C i /\left(m^{2}-\text { day }\right)}{\text { deposition rate or flux of radionuclide } i \text { from the air, }}$

$$
\begin{aligned}
86,400= & \text { dimensional conversion factor, seconds/day } \\
\bar{\chi}_{i}= & \text { average air concentration of radionuclide } i \text {, estimated using either } \\
& \text { the mass-loading or resuspension factor as } \mathrm{pC} i / \mathrm{m}^{3} \\
V_{\mathrm{di}}= & \text { deposition velocity of radionuclide } i, \mathrm{~m} / \mathrm{second} \text {, assumed in MAXI1 } \\
& \text { to be } 1 \times 10^{-3} \mathrm{~m} / \mathrm{sec} \text { for all particles. }
\end{aligned}
$$

The MAXI1 computer program permits selection of two methods for calculating the average air concentration. These methods are mass-loading and resuspension analysis. The mass-loading method uses the product of the surface soil radionuclide concentration (in $\mathrm{pCi} / \mathrm{g}$ of soil) and the ąverage mass-loading of dust or particulate material in the atmosphere (in $\mathrm{g} / \mathrm{m}^{3}$ ) to estimate the air concentration $\left(\right.$ in $\left.\mathrm{B}^{\mathrm{Ci}} / \mathrm{m}^{3}\right)$. In the absence of data for a particular site, a value of $1 \times 10^{-4} \mathrm{~g} / \mathrm{m}^{3}$ has been suggested for predictive purposes (EPA 1977; Anspaugh et al. 1975). This value is used in this report for the annual average massloading factor. Annual arithmetic averages around the United States vary from $9 \times 10^{-6}$ to $7.9 \times 10^{-5} \mathrm{~g} / \mathrm{m}^{3}$ (Anspaugh et al. 1975). 
For the resuspension method, the average airborne concentration is the product of a resuspension factor and the surface contamination level as shown in Equation (2.9).

$$
\bar{x}=s_{f} s_{A}
$$

where $\bar{\chi}=$ average airborne concentration, $\mathrm{pCi} / \mathrm{m}^{3}$

$$
\begin{aligned}
& S_{f}=\text { resuspension factor, } \mathrm{m}^{-1} \\
& S_{A}=\text { surface radioactivity, } \mathrm{pCi} / \mathrm{m}^{2} .
\end{aligned}
$$

The MAXI1 computer program permits the use of a time-dependent resuspension factor $\left(S_{f}\right)$ as given by Anspaugh et al. (1975) as:

$$
s_{f}=10^{-4} e^{-\lambda \sqrt{t}}+10^{-9}
$$

where $S_{f}=$ resuspension factor, $m^{-1}$

$$
\begin{aligned}
10^{-4}= & \text { resuspension factor at time } t=0, \mathrm{~m}^{-1} \\
\lambda= & \text { effective decay constant controlling the availability of material } \\
& \text { for resuspension, } 0.15 \text { day } \\
\mathrm{t}= & \text { time after deposition, days } \\
10^{-9}= & \text { resuspension factor after } 17 \text { years, } \mathrm{m}^{-1} .
\end{aligned}
$$

The second term in Equation (2.10) $\left(10^{-9}\right)$ is added based on the assumption that there is no further measurable decrease in the resuspension factor after about 17 years, the longest period for which data are available.

For deposition on plant leaves or soil from irrigation, the deposition rate $\left(d_{i}\right)$ in $\mathrm{pC} i / \mathrm{m}^{2}$-day for radionuclide $i$ from irrigation water onto the ground, is defined by Equation (2.11).

$$
d_{j}^{I}=c_{i w} I
$$

where $d_{i}^{I}=$ deposition rate or flux of radionuclides applied with irrigation water, $\left(\mathrm{pC} i / \mathrm{m}^{2}-\right.$ day $)$

$c_{i w}=$ concentration of radionuclide $i$ in the water used for irrigation, $\mathrm{pCi} / \mathrm{L}$

$I=$ irrigation rate; the amount of water sprinkled on a unit area of field in one day, $L /\left(m^{2}-\right.$ day $)$. 
The total concentration of radioactive material in vegetation resulting from both direct deposition onto plant foliage and uptake of radionuclides previously deposited in the soil is:

$$
\begin{aligned}
c_{i v}=\left\{\frac{\left(d_{i}^{a}+d_{i}^{I}\right) r T_{v}\left[1-\exp \left(-\lambda_{E i}{ }^{t} e\right)\right]}{y_{v} \lambda_{E i}}+\frac{\left(d_{i}^{a}+d_{i}^{I}\right) f_{t} B_{v i}\left[1-\exp \left(-\lambda_{i} t_{b}\right)\right]}{p \lambda_{i}}\right. \\
\left.+\frac{0.15 f_{t} C_{s i}^{B} v i}{p}+\frac{f_{w} C_{t i}{ }^{B} v i}{\rho}\right\} \exp \left(-\lambda_{i} t_{h}\right)
\end{aligned}
$$

where $C_{i v}=$ concentration of radionuclide $i$ in the edible portion of the vegetation, $\mathrm{pCi} / \mathrm{kg}$

$d_{j}=$ previously defined above [see Equation $\left.(2.11)\right], p C i /\left(m^{2}-\right.$ day $)$

$d_{j}^{a}=$ previously defined [see Equation $\left.(2.8)\right], p C i /\left(m^{2}\right.$-day $)$

$r=$ fraction of deposited material retained on the vegetation (dimensionless), taken to be 0.25

$T_{v}=$ factor for translocation of externally deposited radionuclides to the edible parts of the vegetation (dimensionless). For simplicity, this parameter is assumed to be independent of the radionuclide and is assigned values of 1 for leafy vegetables and fresh forage and 0.1 for all other produce, including grain

$\lambda_{i}=$ radiological decay constant for radionuclide $i$, days ${ }^{-1}$

$\lambda_{E i}=$ the effective removal constant for radionuclide $i$, days ${ }^{-1}$; $\lambda_{E i}=\lambda_{i}+\lambda_{W}$ where $\lambda_{W}=$ weathering removal constant for vegetation, days ${ }^{-1}$; taken to be $(0.693 / 14)$ days

$t_{e}=t i m e$ of exposure of aboveground vegetation to contamination during growing season, days

$y_{v}=$ vegetation yield, $\mathrm{kg}$ (wet weight) $/ \mathrm{m}^{2}$

$f_{t}=$ fraction of the roots in the plow layer of soil (dimensionless)

$B_{v i}=$ concentration factor for uptake of radionuclide $i$ from the soil by vegetation $\mathrm{v}, \mathrm{pCi} / \mathrm{kg}$ (wet weight) $\mathrm{per} \mathrm{pCi} / \mathrm{kg}$ soil (wet)

$t_{b}=$ time for buildup of radionuclides in the soil, days; assumed to be 10 years for irrigation 


$$
\begin{aligned}
& p=\text { soil "surface density," } \mathrm{kg}\left(\text { dry soil) } / \mathrm{m}^{2} \text {; a value of } 224 \mathrm{~kg} / \mathrm{m}^{2}\right. \text { is } \\
& 0.15=\text { plow layer, } \mathrm{m} \\
& \mathrm{C}_{\mathrm{S} j}=\text { concentration of radionuclide } i \text { available for plant uptake } \\
& \text { from the waste contained in the plow layer (top } 15 \mathrm{~cm} \text { of soil), } \\
& \mathrm{pCi} / \mathrm{m}^{3} \\
& f_{W}=\text { fraction of the roots that penetrate the waste trenches } \\
& \text { (dimensionless) } \\
& c_{t i}=\text { concentration of radionuclide } i \text { aुvailable for plant uptake from } \\
& \text { the subsurface waste zone, } \mathrm{pCi} / \mathrm{m}^{3} \\
& \rho=\text { bulk soil density of subsurface material, } \mathrm{kg} / \mathrm{m}^{3} \\
& t_{h}=\text { holdup time between harvest and food consumption; days }
\end{aligned}
$$

The first expression inside the braces of Equation (2.12) represents the radionuclide concentration resulting from direct deposition of resuspended material and from irrigation on foljage during the growing season. The second expression inside the braces represents the plant uptake from the soil and reflects the deposition from irrigation. The third and fourth expressions inside the braces represent uptake of radionuclides contained in the top $0.15 \mathrm{~m}$ of soil and below this layer, respectively. Specific values used for the parameters in Equation (2.12) are found in Napier et al. (1980).

Radionuclide concentrations in animal products such as meat, milk, and eggs are dependent on the amount of contaminated forage or feed eaten by the animal. This concentration is:

$$
C_{i a}=S_{i a} C_{i f} Q_{f}+C_{i a w} Q_{a w}
$$

where $C_{i a}=$ concentration of radionuclide $i$ in the animal product, $\mathrm{pCi} / \mathrm{kg}$ or $\mathrm{pCi} / \mathrm{L}$

$S_{i a}=$ transfer coefficient of radionuclide $i$ from daily intake of the animal to the edible portion of the animal product, $\mathrm{pCi} / \mathrm{L}$ (milk) per $\mathrm{pCi} /$ day or $\mathrm{pCi} / \mathrm{kg}$ (animal product) per $\mathrm{pCi} / \mathrm{day}$

$C_{i f}=$ concentration of radionuclide $i$ in feed or forage, $\mathrm{pCi} / \mathrm{kg}$; calculated from Equation (2.12)

$Q_{f}=$ animal consumption rate of contaminated feed or forage, $\mathrm{kg} / \mathrm{day}$

$C_{i a w}=$ concentration of radionuclide $i$ in the water consumed by animais, $\mathrm{PC} i / \mathrm{L}_{i}$ assumed to be the same as the irrigation water, $\mathrm{C}_{i w}$ [see Equation (2.11)] 


$$
Q_{a w}=\text { consumption rate of the contaminated water by the animal, L/day. }
$$

Specific values of the parameters used in Equation (2.13) are given in Napier et al. (1980).

The dose, $R_{I r}$ (in mrem), to an organ of the exposed individual resulting from the ingestion of food products raised in the soil at a contaminated site is calculated using a modified version of Equation (2.1). This general relationship is:

$$
R_{I r}=U_{p} f_{D} \sum_{i=1}^{n} c_{i p} \cdot A_{c}^{I} \cdot D_{i p r}
$$

where $f_{D}=$ the fraction of the total diet grown on the site

$$
\begin{aligned}
A_{C}= & \text { the area correction factor for the ingestion pathway discussed } \\
& \text { in Section 2.1.4.8, }
\end{aligned}
$$

and where $U_{p}, C_{i p}$, and $D_{i p r}$ are for ingestion and are generally defined in Equation (2.1).

\subsubsection{Ingestion of Drinking water}

The dose, $R_{w r}$ (in mrem), from ingestion of water containing radionuclides, is:

$$
R_{w r}=U_{w} \sum_{i=1}^{n} c_{i d w} \exp \left(-\lambda_{i} t_{h}\right) D_{i r} f_{d w}
$$

where $U_{W}=$ annual consumption of contaminated drinking water, $L$

$c_{j \mathrm{~d}}=$ the concentration of radionuclide $i$ in the drinking water (input by user), pCi/L

$\lambda_{i}=$ radiological decay constant for radionuclide $i$, days ${ }^{-1}$

$t_{h}=$ transit time required for radionuclide to reach the point of exposure, days

$D_{i r}=$ radiation dose equivalent factor for ingestion, $\mathrm{mrem} / \mathrm{pci}$

$f_{d w}=$ drinking water cleanup factor (for municipal water supplies). 


\subsubsection{Inhalation}

Dose conversion factors for inhalation used in MAXI1 are obtained directly from ICRP Publication No. 30 (ICRP 1979-1982) or are calculated using the DACRIN computer program (Houston, Strenge, and watson 1974) and stored in the data files. The inhalation dose, $R_{\text {hr }}$ (in mrem), is:

$$
R_{h r}=V T t_{m} \sum_{i=1}^{n} \bar{x}_{i} \cdot A_{c}^{I} \cdot D_{i r}
$$

where $V=$ ventilation rate of exposed individual, $\mathrm{m}^{3} / \mathrm{sec}$

$$
\begin{aligned}
& T \text { = duration of exposure to the airborne radionuclide concentration, } \\
& \text { seconds. } \\
& t_{m}=\text { modification factor applieg } t_{g} T \text { to adjust time of exposure or } \\
& \text { ventilation rate of } 2 \times 10^{3} \mathrm{~m}^{3} / \mathrm{sec} \text { (ICRP 1975) } \\
& \bar{\chi}_{j}=\text { the annual average airborne concentration of radionuclide } i, \mathrm{pCi} / \mathrm{m}^{3} \\
& A_{C}^{I}=\text { the area correction factor for inhalation and external exposure } \\
& \text { pathways as discussed in Section 2.1.4.8. } \\
& D_{i r}=\text { radiation dose equivalent factor for inhalation from the } \\
& \text { DACRIN computer code (Houston, Strenge, and Watson 1974), mrem/pCi }
\end{aligned}
$$

The following terms of Equation (2.16) are found in the MAXI1 computer code:

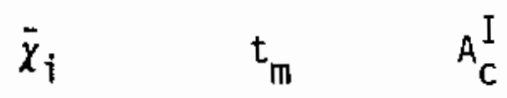

The inhalation dose rate factors calculated by the DACRIN computer code (Houston, Strenge, and Watson 1974) are included in a data library that can be addressed by MAXI1. The following terms in Equation (2.16) are included in those factors.

$$
D_{i r} \quad \text { T }
$$

\subsubsection{External Radiation}

Annual doses, Rer (in mrem), resulting from exposure to contaminated soil or in a reference room (or vault), are calculated using a modified version of Equation (2.1). The modified dose equation for external exposure is: 


$$
R_{e r}=U_{p} \sum_{i=1}^{n} D_{i r} \cdot A_{c}^{E} \cdot C_{i p}
$$

where $A_{C}^{E}=\begin{aligned} & \text { the area correction factor for external exposure and discussed in } \\ & \text { Section } 2.1 .4 .8 \text {, }\end{aligned}$ and where $U_{p}, D_{j}$, and $C_{i p}$ are for external exposure and are generally defined in Equation (2.1).

Dose conversion factors for external exposure are available for several types of exposure conditions. These include surface-soil contamination (using either plane- or slab-source models), subsurface-soil contamination (using slab sources at depths of 0.5 and $1.0 \mathrm{~m}$ from the soil surface), and a finite disk source of contamination deposited on the floor or a wall in a waste-storage room or vault. These dose conversion factors are calculated for a whole-body dose (with a tissue depth of $5 \mathrm{~cm}$ ) located at a point $1 \mathrm{~m}$ above the ground surface. The direct exposure rates encountered by the intruder for various contamination levels in a waste-storage room or vault are calculated using the model developed for decommissioning a reference room at a boiling water reactor (BWR) (Oak et al. 1980). All of the external dose equivalent factors are calculated for the radionuclides of concern using the ISOSHLD (Engel et al. 1966; Simmons et a1. 1967) computer program.

When developing the external-exposure libraries used by the ONSITE/MAXI1 computer program, certain simplifying assumptions were made. Two primary assumptions are evaluated in additional sensitivity studies:

- The effect of waste form density is determined by comparing the external exposure factors, originally calculated for a waste form dęnsity of 1.8 , with factors calculated for densities of 1.0 , and $0.6 \mathrm{~g} / \mathrm{cm}^{3}$.

- The effect of the slab-source thickness is determined by comparing the external exposure factors calculated for 1-m-thick and 3-m-thick slabs.

The sensitivity studies were perfomned using modified input parameters with the ISOSHLD computer program (Engel et al. 1966; Simmons et al. 1967). A summary of the reshlts obtained is shown in Table 2.4 for a constant source strength of $1 \mathrm{Ci} / \mathrm{m}^{3}$ of selected radionuclides. These results show that for a constant source strength, the less the waste density, the greater the calculated dose rate. However, for each waste density there is little dependence on slab thickness.

Based on these results, the ONSITE/MAXIl computer program permits the user to select the waste form density when performing external exposure calculations. The possible waste form densities that ${ }_{3}$ the user may select are $1.8 \mathrm{~g} / \mathrm{cm}^{3}$ (the approximate density of soil), $1.0 \mathrm{~g} / \mathrm{cm}^{3}$ (the density of water), or $0.6 \mathrm{~g} / \mathrm{cm}^{3}$ (the approximate density of noncompacted trash waste forms). No corrections for slab thickness are included in the external exposure data libraries. 
TABLE 2.4. External Dose Conversion Factors (mrem/h per $\mathrm{Ci} / \mathrm{m}^{3}$ ) for Selected Waste Form Densities $(\rho)$ and Slab Thicknesses with $0.0-m$ Overburden

$$
\rho=1.8 \mathrm{~g} / \mathrm{cm}^{3} \quad \rho=1.0 \mathrm{~g} / \mathrm{cm}^{3} \quad \rho=0.6 \mathrm{~g} / \mathrm{cm}^{3}
$$

Slab Thickness:

Radionuclide

${ }^{22} 1_{\mathrm{Na}}^{\mathrm{Cr}}$

${ }_{90}^{57}{ }_{90}^{\mathrm{Co}}$

131 I

$137_{\mathrm{CS}+\mathrm{C}}^{\mathrm{Cs}}$

\begin{tabular}{rr}
$1 \mathrm{~m}$ & $3 \mathrm{~m}$ \\
\cline { 3 - 3 } 514 & 514 \\
12 & 12 \\
327 & 327
\end{tabular}

$\begin{array}{rr}21 & 21 \\ 1000 & 1000 \\ 2 & 2\end{array}$

133

674

241
133

674

241
Slab Thickness:

$1 \mathrm{~m} \quad 3 \mathrm{~m}$

964

28

684

$\begin{array}{rr}70 & 70 \\ 1930 & 1930 \\ 6 & 6\end{array}$

323

1500

573
323
1500
573

Slab Thickness:

$1 m \quad 3 m$

$1600 \quad 1630$

$470 \quad 471$

$1140 \quad 1150$

$\begin{array}{rr}117 & 117 \\ 3200 & 3270 \\ 9 & 9\end{array}$

$539 \quad 541$

$2470 \quad 2500$

935942

\subsubsection{Ingestion of Aquatic Foods}

The concentration of a radionuclide in aquatic foods is directly related to the concentrations of the radionuclide in water. Equilibrium ratios between the two concentrations, called bioaccumulation factors, are taken from Soldat, Robinson, and Baker (1974). The dose, Rafr (in mrem), from consumption of aquatic food, $f_{j}$, containing radionuclide $i$ is:

$$
R_{a f r}=U_{a f} \sum_{i=1}^{n} c_{i w} \exp \left(-\lambda_{i} t_{h}\right) D_{i r}\left(B_{x i}+B_{y i}+B_{z j}\right)
$$

where $\quad U_{a f}=$ annual consumption of contaminated aquatic foods, $\mathrm{kg}$

$C_{j w}=$ the concentration of radionuclide $i$ in the water, $\mathrm{pC} i / \mathrm{L}$

$\lambda_{\mathbf{i}}=$ radiological decay constant for radionuclide $i$, days ${ }^{-1}$

$t_{h}=$ holdup time between harvest and food consumption, days.

$D_{i r}=$ radiation dose equivalent factor for ingestion, mrem/pCi

$B_{x i}, B_{y i}, B_{z i}=$ the bioaccumulation factor for radionuclide $i$, for: $x=$ fish,$y=$ invertebrates, and $z=$ algae, $\mathrm{pCi} / \mathrm{kg} \mathrm{per}$ $\mathrm{pC} \dot{\mathrm{i}} / \mathrm{L}$ 


\subsubsection{Area Correction Factors}

Because of the large variability that may exist in the exposure conditions at any given site, we have defined correction factors that modify the default area exposure pathway assumptions provided in the scenario analysis.

For external exposure, a sensitivity study was conducted for various beta-gamina emitters to determine the exposure rate versus source area. The results of the calculations are shown in Figure 2.1. Since the curves in Figure 2.1 are parallel, there appears to be a uniform correction for reduced site area over a large range of source energies. We have therefore determined the ratio of the exposure rates (for smali to large area sources) and plotted the results versus the source area as shown in Figure 2.2. This figure defines the area correction factors for the external exposure pathway, given in Equation (2.17) as the term $A_{C}$. We have approximated this curve in the MAXI1 computer program as the sum of four line segments as shown in Figure 2.3.

The default values used in the reference environment for the ONSITE/MAXI1 computer program are based on a minimum site area of 1 ha. To account for the limited exposure potential from smaller disposal areas, site area correction factors are required. For example, the amount of agricultural products raised on a site depends upon the intensity of the farming and the types of crops raised. A small site may produce a large fraction of the seasonal fruit and vegetable diet with intensive farming, but be unable to provide enough forage and grain to support a milk cow or other animals. Thus, while a large fraction of the seasonal fruit and vegetable diet may be raised on a small site, the total quantity raised may only equal a small fraction of the total annual diet.

Many of the experiments relating air and soil concentrations were performed on sites of limited size (i.e., sites with areas of 1 ha or less) (Sehmel 1974; Milham et al. 1976; Sehmel and Lloyd 1976). After careful consideration of the potential magnitude of the area correction for inhalation factors, it was determined that the area correction developed for the direct exposure pathway should be applied for the inhalation pathway (Kennedy et al. 1986). These area correction factors for direct exposure and inhalation are shown in Figure 2.3.

In determining area correction factors for the ingestion pathway to be used in the MAXI1 computer program, consideration is first given to the default pathway conditions and then to variable exposure conditions as a function of site area. For the default conditions, the individual's entire diet, as defined in Regulatory Guide 1.109 (NRC 1977), is assumed to be raised on the 1-ha site. This diet consists of fruits and vegetables and meat and animal products. The air concentrations used for the inhalation calculations are for resuspension of radionuclides from large areas of distributed-surface contamination.

For the ingestion pathway, the area correction factors are in the form of a step function as shown in Figure 2.4. This function assumes five steps of potential exposure versus site area ranging from small to large sites. The steps of total exposure are: 1) $10 \%$ for sites with areas less than $50 \mathrm{~m}^{2}$, 2) $5 \%$ for sites with areas between 50 and $\left.200 \mathrm{~m}^{2}, 3\right) 50 \%$ for sites with areas between 300 and $\left.1,000 \mathrm{~m}^{2}, 4\right) 75 \%$ for sites with areas between 1,000 and $10,000 \mathrm{~m}^{2}$, and 5) $100 \%$ for sites larger than $10,000 \mathrm{~m}^{2}$ (or $1 \mathrm{ha}$ ). 


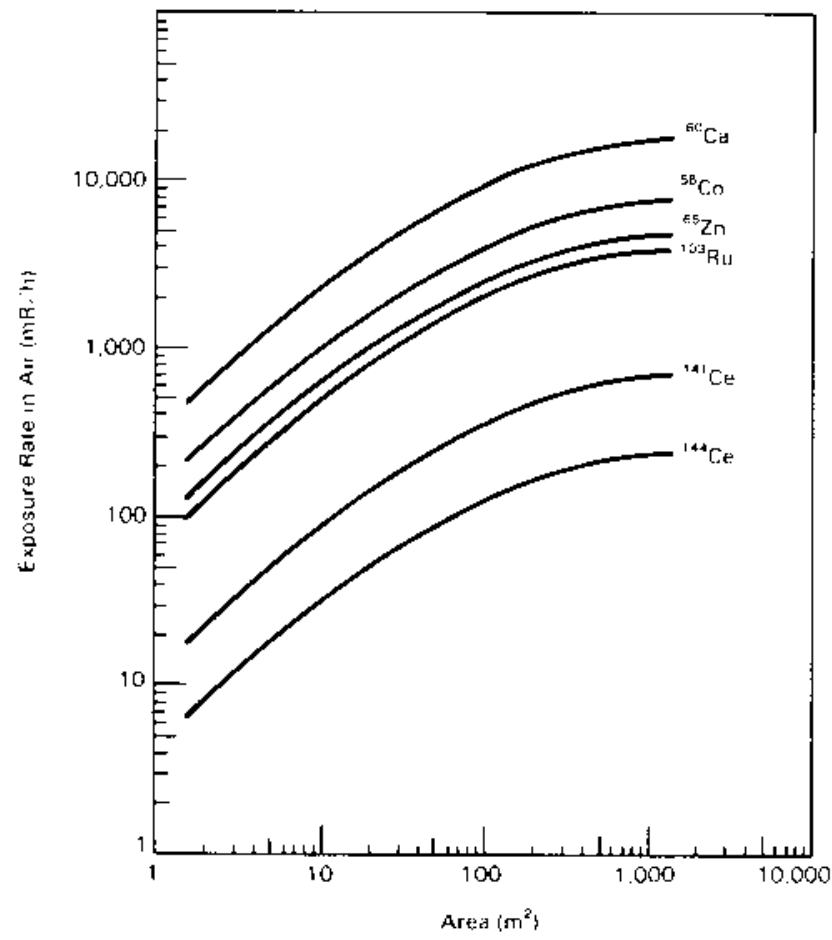

FIGURE 2.1. Exposure Rate Versus Source Area

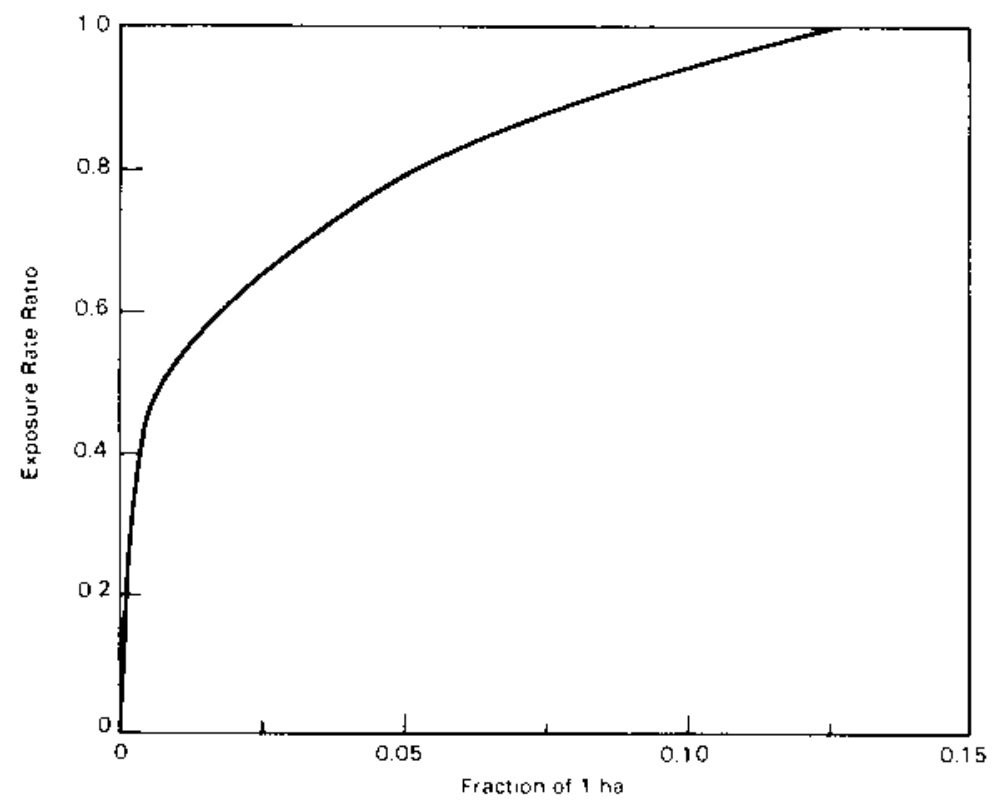

FIGURE 2.2. Exposure Rate Ratio Versus Fractional Hectare of Source Area 


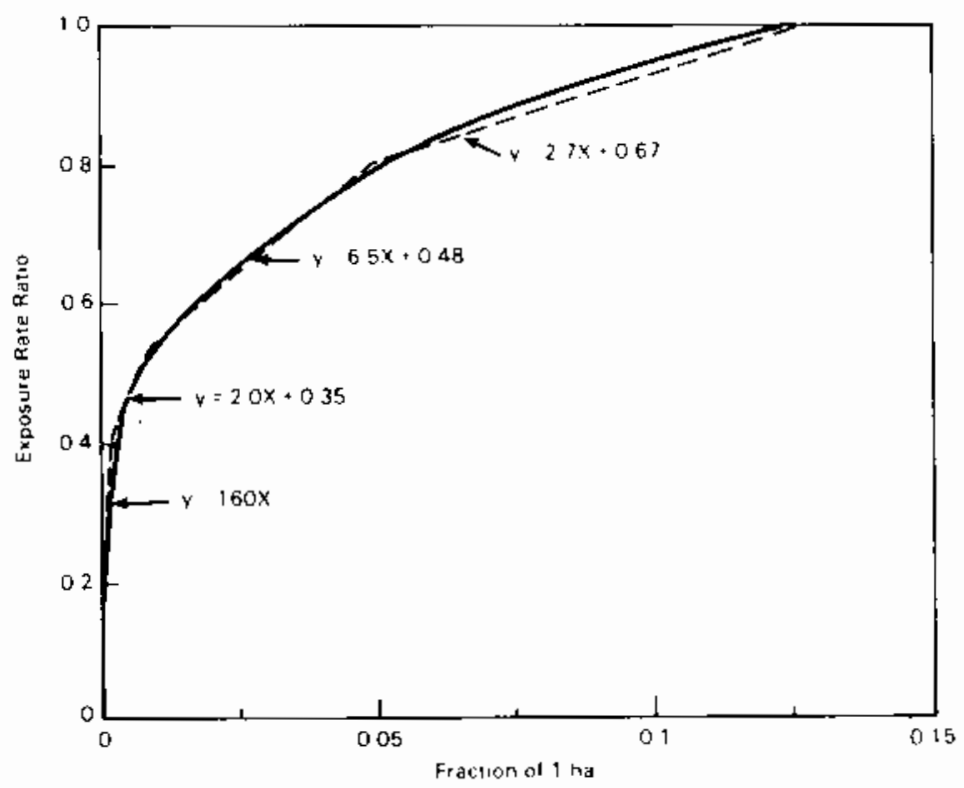

FIGURE 2.3. Area Correction Factors for External Exposure and the Inhalation Pathway

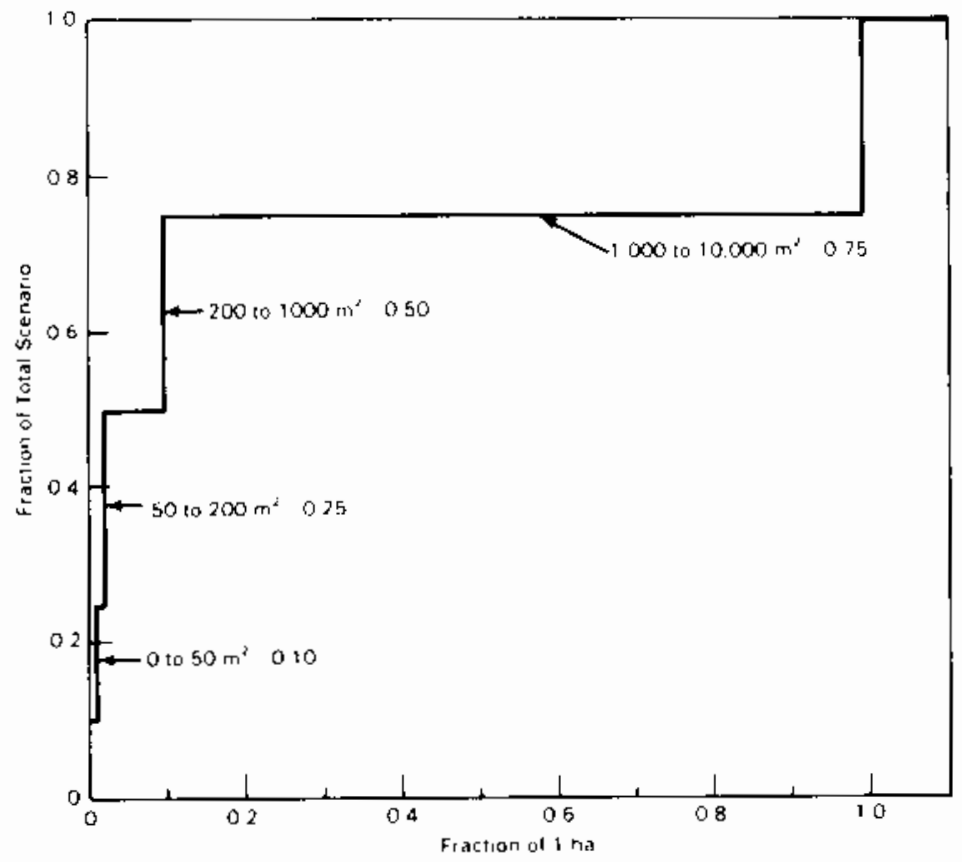

FIGURE 2.4. Area Correction Factors for the Ingestion Pathway 


\subsection{COMPUTER IMPLEMENTATION}

Estimates of the maximum annual radiation doses resulting from the exposure scenarios described in Section 2.1.1 can be made using environment-specific information and computer codes contained in the ONSITE/MAXI1 software package. This version of the package contains two computer programs, ONSITE and MAXI1, as well as an extensive data base. This data base is discussed in detail in Section 2.3. The following sections contain computer system requirements, descriptions of the ONSITE/MAXI1 computer programs, an overview of software operation, capabilities, restrictions, and execution performance of the software.

\subsubsection{Computer System Requirements}

The current version of the ONSITE/MAXI1 software package was designed to execute on the IBM PC/XT/AT computer operating under IBM DOS 2.1 (or greater). The computer must be configured with a minimum of 512 kilobytes of random access memory and a hard disk for peripheial storage. Though not necessary for execution, a math coprocessor will graatly speed program execution.

The ONSITE/MAXI1 software package is available on dual-sided, double-density floppy disks formatted for the IBM PC/XT/AT computer.

\subsubsection{Descriptions of Computer Programs}

The ONSITE/MAXI1 software package contains the ONSITE and MAXI1 computer programs. ONSITE is an interactive user interface that allows the end-user to simply and efficiently create and use the radiation-exposure scenarios. MAXI1 is then used with the scenario information to calculate the maximum annual dose to an exposed individual from selected pathways. The following sections contain descriptions of each of the computer codes.

\subsubsection{ONSITE Computer Program}

ONSITE is an interactive computer program that allows the user to access or create radiation-exposure scenarios used to estimate the dose to human resulting from onsite disposal of radioactive wastes. The software solicits scenario information from the user, controls parameter modification, selects the appropriate data libraries for running MAXI1, and constructs the input file for MAXI1. ONSITE was designed for the novice user of the ONSITE/MAXI1 software package. ONSITE is the only computer program that the user needs to interact with because this interaction automatically produces results from MAXIl. The ONSITE program gives the user the option of changing any parameters of selected pathways used in the radiation-exposure scenarios. Each parameter is described in terms that should be meaningful to the end-user. The dimensional units and the upper and lower bounds of each parameter are displayed for each parameter. Each value entered by the user is tested against upper and lower limits when applicable. If the value is not accepted, the user is asked to re-enter the value. Default (or assumed maximum) values and conditions are activated by a null entry (e.g., simply pressing the 〈return〉 key on the terminal). 


\subsubsection{MAXI1 Computer Program}

The computer program MAXI1 is used to calculate the maximum annual dose (based on the methodology in ICRP Publication No. 2) and the annual effective dose equivalent (based on the methodology in ICRP Publication No. 30) to an exposed individual from a large number of exposure pathways. Exposure pathways that can be modeled include 1) direct external exposure to contaminated soil or building surfaces, 2) inhalation of resuspended material or material that has blown in from an offsite source, and 3) ingestion of contaminated foods, drinking water, and aquatic foods. For the maximum annual dose, the time of the maximum dose to each organ of reference is calculated and the annual dose for that organ is reported.

For the maximum annual dose calculations. MAXIl uses dose conversion factors from the DACRIN computer program (Houston, Strenge, and Watson 1974) for estimating doses from inhalation of airborne radionuclides. The dose conversion factors from DACRIN are based on the International Commission on Radiological Protection's (ICRP) Task Group Lung Mode: (TGLM) (ICRP 1966). For the committed dose equivalent calculations, inhalation dose factors from ICRP Publication No. 30 (ICRP 1979-1982) are used.

MAXI1 calculates the maximum annual dose from ingestion pathways, for either food products or drinking water, based on the dosimetry model of ICRP Committee II as reported in ICRP Publication No. 2 (1959). In the previous version of the ONSITE/MAXI1 software package, ingestion dose factors were calculated by the MAXI2 and MAXI3 computer programs, stored in data files, and then used by MAXI1. In the current version of the program, dose calculations previously performed by MAXI2 and MAXI3 are incorporated into MAXI1. For committed dose equivalent calculations, ingestion dose factors from ICRP Publication No. 30 (ICRP 1979-1982) are used.

For external exposure, dose conversion factors from the ISOSHLD (Engel et al. 1966; Simmons et al. 1967) are used by MAXI1. These factors relate the radionuclide source strength to the dose rate in tissue $1 \mathrm{~m}$ above an infinite plane or slab of contamination. External dose conversion factors are supplied for surface-soil contamination, and for slabs of buried contamination at depths of either 0.5 or $1.0 \mathrm{~m}$. External dose conversion factors from an optional room exposure condition are also available. These factors are intended to model doses to an intruder who may enter a room (or vault) used for storage of radioactive wastes.

\subsubsection{Operation of the ONSITE/MAXI1 Software Package}

Section 3 contains a detailed discussion of the operation of the ONSITE/ MAXI1 software package. A theoretical overview of software operation is presented here. In previous versions of the software package, three levels of operation were defined. In the current version of the package, usage has been simplified resulting in only two levels are operations. The first level is primarily for novice users and the second level is for experienced users. These levels of operation are described in this section. 
The first level of operation user interacts primarily with the ONSITE computer program. The ONSITE program is used to select and establish parameters for a scenario. The user is assisted during scenario creation by a restricted set of parameters and restricted ranges of values for each parameter. The MAXII program is automatically executed at the termination of the ONSITE program. The second level of operation user should be familiar with the mathematical models and software package. This level of operation provides a shorthand method for preparing MAXI1 input. This version of the ONSITE computer program generates an annotated input file for the MAXI1 computer program. The second level of operation user edits the annotated input file for MAXI1 using a standard text editor and then executes the MAXI1 program.

\title{
2.2.4 Capabilities, Restrictions and Performance
}

The capabilities and inherent restrictions of the ONSITE/MAXI computer implementation are discussed in this section. Execution performance of the software package is also presented.

The user is restricted to a inventory of 20 radionuclides. This number includes both the radionuclides entered in the source term and those daughters that will be considered by MAXI1. A large memory version of the ONSITE/MAXI1 software package is available that can handle inventories of up to 50 radionuclides. However, this special version is only available on IBM/AT quad-density floppy disks.

The user is no longer able to specify the number of organs for which maximum annual dose calculations are made. Total body, bone, lung, thyroid, and the lower large intestine (LLI) are included in all cases.

When run on the IBM/AT computer with a math coprocessor installed and processed in batch mode, the set of six sample problems, to be described in section 3 of this document, complete execution in 16 minutes, 34 seconds.

\subsubsection{Software Package}

Information regarding the availability of the software package associated with this NUREG report is available from:

\author{
U.S. Nuclear Regulatory Commission \\ Information Technology Services Support Center \\ Mail Stop P-808 \\ Washington, DC 20555 \\ Telephone: (301) $492-4160$ or FTS $492-4160$
}

\subsection{DATA BASE}

The current version of the data base contained in the ONSITE/MAXI1 software package is substantially different from that included with earlier versions of the package. Previously the MAXI2 and MAXI3 computer programs were used to calculate dose conversion factors that were stored in the data base and then accessed by MAXI1. In the current version of the software package, the 
MAXI2 and MAXI3 programs have been incorporated into MAXIl thus eliminating the need for four of the large data files in the data base.

An understanding of the detailed information that follows is not necessary for the successful execution of ONSITE/MAXI1. The intended audience are those interested in the organization and content of the data base and the second level of operation users.

\subsubsection{Radionuclide Master List - RMDONS}

RMDONS contains those radionuclides considered of importance for assessing the impact of the onsite disosal of wastes for which dose factors are available. RMDONS is used by the ONSITE program to ensure that only radionuclides from the master list are considered and that the user entries conform to the specified nomenclature. A listing of the radionuclides contained in RMDONS may be found in Appendix B. The format of the file is identical to that of RMDLIB. The radionuclide master list for ONSITE/MAXI1, RMDONS, is a subset of the radionuclide master library (RMDLIB) to be discussed in the following section.

\subsubsection{Radionuclide Master Data Library - RMDLIB}

The radionuclide master data library (RMDLIB) contains all radiological decay data used by MAXI1. The radionuclides are organized into decay chains ordered by atomic number under the radionuclides highest in the chain. Calculations of radioactive decay and daughter ingrowth are performed in the MAXI1 subroutine ACHAIN, using the decay chain data of RMDLIB and the decay equations of Bateman (1912). RMDLIB contains 245 entries.

The first record of the library contains 80 characters of descriptive information used as identification in the input data report printed by MAXIl. The balance of the data records have the following information:

\begin{tabular}{|c|c|c|}
\hline Column & 1 & Alphabetic element symbol \\
\hline Column & 2 & Atomic weight, also metastable (m) designation \\
\hline Column & 3 & Radiological half-life, days \\
\hline Column & 4 & $\begin{array}{l}\text { Indicator of relative position in decay chain } \\
\text { (0 is highest position) }\end{array}$ \\
\hline Column & 5 & $\begin{array}{l}\text { Indicator of precursor in decay chain (as identified } \\
\text { in column } 4 \text { of the precursor) }\end{array}$ \\
\hline Column & 6 & Branching ratio for primary precursor \\
\hline lumn & 7 & Indicator of alternate precursor in decay chain \\
\hline umn & 8 & Branching ratio for alternate precursor \\
\hline umn & 9 & $\begin{array}{l}\text { A1ternate specjfication of radionuclide (not used by } \\
\text { ONSITE/MAXI1) }\end{array}$ \\
\hline lumn & 10 & $\begin{array}{l}\text { Flag indicating whether radionuclide is a bone volume } \\
\text { or bone surface seeker }\end{array}$ \\
\hline & & Trans loc \\
\hline
\end{tabular}

Translocation refers to the rate at which radionuclides are transported by body fluids from the lungs to the blood and GI tract after inhalation (sometimes referred to as solubility class). For inhalation calculations, translocation 
classifications are made for each organ based on the usage of the Task Group Lung Model (ICRP 1966). The translocation flags used in RMDLIB refer to the following classes as defined in ICRP (1966):

\section{D - Class D Materials. A maximum clearance half time of less than a day. \\ W- Class W Materials. A maximum clearance half time ranging from a few days to a few months. \\ $Y$ - Class Y Materials. A maximum clearance half time of from six months to a few years.}

The RMDLIB FORTRAN format is (A2, A6, E10.2, 2I2, F7.4, I2, F7.4, A30, $2 A 1$ ). A listing of the material found in the library is located in Appendix $B$.

\subsubsection{Organ Data Library - ORGLIB}

The library ORGLIB contains data used by MAXI1 for calculations of doses to specific organs from ingested radionuclides using the dose methodology given in ICRP Publication No. 2 (1959). Data are arranged in sets by radionuclide. The sets are ordered to be compatible with the RMDLIB chain order. Data in each block are arranged by organ index. The organs for which data are potentially available are:

$\begin{array}{lll}1 \text { - Total body } & 9 \text { - Adrenals } & 17 \text { - Pancreas } \\ 2 \text { - Body water } & 10 \text { - Testes } & 18 \text { - Heart } \\ 3 \text { - Kidneys } & 11 \text { - Ovaries } & 19 \text { - GI tract } \\ 4 \text { - Liver } & 12 \text { - Skin } & 20 \text { - Stomach } \\ 5 \text { - Spleen } & 13 \text { - Brain } & 21 \text { - Small intestine } \\ 6 \text { - Bone } & 14 \text { - Muscle } & 22 \text { - Upper large intestine } \\ 7 \text { - Fat } & 15 \text { - Prostate } & 23 \text { - Lower large intestine } \\ 8 \text { - Lungs } & 16 \text { - Thyroid } & \end{array}$

For most entries, data are included only for total body, bone, lungs, thyroid, and the lower large intestine. The data contained in each record of ORGLIB is as follows:
Column 1
Column 2
Column 3
Column 4
Column 5
Alphabetic element symbol
Atomic weight and metastable designation (m)
Organ index
Biological half-time, days $f_{\text {W }}$, fraction of ingested radionuclide reaching the
Column 6
Column $7,8,9$
$\mathrm{f}_{2}$, fraction from blood to organ of interest (not used by ONSITE/MAXII)
Effective energy absorbed per disintegration in adult organs, MeV. Columns 8 and 9 are only used for lungs.

The FORTRAN format of the organ data library ORGLIB is (A2, A6, I2, 6E8.2). Appendix B contains a listing of the material contained in ORGLIB. 


\subsubsection{Food Transfer Factor Library - FTRANS}

The food transfer factor library, FTRANS, contains factors used in MAXI1 relating concentrations of elements in soil to concentrations in farm products grown on that soil, and relating concentrations in animal feed to concentrations in animal products. Plant concentration factors are taken from URCL-50163, Part IV ( $\mathrm{Ng}$ et al. 1968) and supplemented with radionuclide data as explained in the Handbook of Radiological Protection, Part 1: Data, (Radioactivity Advisory Committee 1971). The literature yielded coefficients of transfer from feed to animal products for a 1 imited number of radionuclides. For those radionuclides lacking data, comparisons were made with the behavior of chemically similar elements in man (ICRP 1959) and animals. In some instances where other data are lacking, transfer coefficients are set to $9.9 \times 10^{-4}$. The most complete listing of transfer coefficients is that for milk (Soldat and Harr 1971). Some experimental data are available on the transfer of several radionuclides for chicken, eggs, and meat (Annenkov et al. 1974). Currently efforts are under way at Pacific Northwest Laboratory to update the food transfer library FTRANS.

Each record in FTRANS contains the following information:

Column 1 Alphabetic element symbol

Column 2 Elemental deposition velocity from air to ground, $\mathrm{m} / \mathrm{sec}$

Column 3 Food transfer coefficients for: plants (dimensionless, pCi per gram plant (wet)/pCi per gram soil (wet); milk (day/L); and eggs, beef, pork, and poultry (day $/ \mathrm{kg}$ )

The FORTRAN format is (A2, 9F9.1). A listing of the material contained in the food transfer library FTRANS may be found on Appendix B.

\subsubsection{Bioaccumulation Factor Library - BIOAC1}

The bioaccumulation library, BIOAC1, contains the factors used by MAXI1 relating the concentration of radionuclides in aquatic biota to the concentration of the radionuclides in the water. These factors are taken from Thompson et al. (1972) and Freke (1965). There are separate factors for fresh and salt water. Also included are factors representing the fraction of chemical elements passing through conventional municipal water treatment plants. The library organization is as follows:
Column 1
Alphabetic element symbol
Column 2
Bioaccumblation factors for fish, crustacea, molluscs, and algae for salt water ( $\mathrm{pCi} / \mathrm{kg} \mathrm{per} \mathrm{pCi} / \mathrm{L}$ )
Col umn 3
Bioaccumulation factors for fish, crustacea, molluscs, and
Column 4 algae for fresh water ( $\mathrm{pCi} / \mathrm{kg}$ per $\mathrm{pCi} / \mathrm{L}$ ) Drinking water clean-up factor (dimensionless)

The FORTRAN format is (A2,9F9.1). Appendix $C$ contains a listing of the material found in the BIOAC1 aquatic bioaccumulation factor library. 
The file assigned to logical unit 2 contains external dose rate factors for waste modelled as a plane source. The dose rate factors are for $1 \mathrm{pCi}$ of radionuclide per $\mathrm{m}^{2}$ of soil surface. These factors relate the radionuclide strength to the dose rate in tissue $1 \mathrm{~m}$ above the plane. Three files, PLANEA, PLANEB, and PLANEC are available based on assumed waste density. The file names, waste densities, and waste configuration description are included in Table 2.5 for easy reference.

The external exposure dose rate factors were created by the ISOSHLD (Engel, Greenborg and Hendrickson 1966; Simmons et al. 1967) computer program.

Table 2.6 summarizes the ISOSHLD parameter values used to approximate a plane source of contamination at the soil surface.

The organization and FORTRAN formats of these files are as follows:

Record 1

Record 2

Remainder of file
Descriptive title, (A80).

Blank

For each isotope (A2, A6, E9.1):

- Element symbol and atomic number as in master radionuclide library

- External exposure dose rate factor

Listings of the files PLANEA, PLANEB, and PLANEC are included in Appendix B. TABLE 2.5. Dose Rate Factor Files for External Exposure

\begin{tabular}{|c|c|c|c|}
\hline File Name & $\begin{array}{l}\text { Logical } \\
\text { Unit } \\
\text { Assignment } \\
\end{array}$ & $\begin{array}{l}\text { Waste } \\
\text { Density } \\
\left(\mathrm{g} / \mathrm{cm}^{3}\right)\end{array}$ & Description \\
\hline $\begin{array}{l}\text { PLANEA.DAT } \\
\text { PLANEB.DAT } \\
\text { PLANEC.DAT }\end{array}$ & $\begin{array}{l}2 \\
2 \\
2\end{array}$ & $\begin{array}{l}1.8 \\
1.0 \\
0.6\end{array}$ & $\begin{array}{l}\text { Infinite plane of soil contamination, } \\
\text { waste thickness of } 15 \mathrm{~cm}\end{array}$ \\
\hline $\begin{array}{l}\text { VOLSA.DAT } \\
\text { VOLSB.DAT } \\
\text { VOLSC.DAT }\end{array}$ & $\begin{array}{l}7 \\
7 \\
7\end{array}$ & $\begin{array}{l}1.8 \\
1.0 \\
0.6\end{array}$ & $\begin{array}{l}\text { Infinite surface slab source, waste } \\
\text { extends to a depth of } 1 \mathrm{~m}\end{array}$ \\
\hline $\begin{array}{l}\text { BURHFA.DAT } \\
\text { BURHFB.DAT } \\
\text { BURHFC.DAT }\end{array}$ & $\begin{array}{l}7 \\
7 \\
7\end{array}$ & $\begin{array}{l}1.8 \\
1.0 \\
0.6\end{array}$ & $\begin{array}{l}\text { Infinite slab source, buried in soil } \\
\text { with an overburden depth of } 0.5 \mathrm{~m} \text {, } \\
\text { waste thickness of } 1 \mathrm{~m}\end{array}$ \\
\hline $\begin{array}{l}\text { BUR1A.DAT } \\
\text { BUR1B.DAT } \\
\text { BUR1C.DAT }\end{array}$ & $\begin{array}{l}7 \\
7 \\
7\end{array}$ & $\begin{array}{l}1.8 \\
1.0 \\
0.6\end{array}$ & $\begin{array}{l}\text { Infinite slab source, buried in soil } \\
\text { with an overburden depth of } 1 \mathrm{~m} \text {, waste } \\
\text { thickness of } 1 \mathrm{~m}\end{array}$ \\
\hline $\begin{array}{l}\text { STOREDA } \\
\text { STOREDB } \\
\text { STOREDC }\end{array}$ & $\begin{array}{l}7 \\
7 \\
7\end{array}$ & $\begin{array}{l}1.8 \\
1.0 \\
0.6\end{array}$ & $\begin{array}{l}\text { Rectangular slab source, } 10 \mathrm{~m} \times 3 \mathrm{~m} \text {, } \\
\text { waste thickness } 1 \mathrm{~m}\end{array}$ \\
\hline
\end{tabular}


TABLE 2.6. ISOSHLD Parameter Values Used for External Exposure Dose Rate Factors

\begin{tabular}{|c|c|c|c|c|c|}
\hline \multirow[b]{2}{*}{$\begin{array}{c}\text { ISOSHLD } \\
\text { Parameter } \\
\end{array}$} & \multirow[b]{2}{*}{$\begin{array}{l}\text { Plane Source } \\
\text { At Surface }\end{array}$} & \multicolumn{3}{|c|}{ Slab Source } & \multirow[b]{2}{*}{$\begin{array}{l}\text { Stored } \\
\text { Waste } \\
\end{array}$} \\
\hline & & At Surface & $\begin{array}{c}0.5-m \\
\text { overburden }\end{array}$ & $\begin{array}{c}1.0-m \\
\text { overburden }\end{array}$ & \\
\hline IGEOM & 5 & 5 & 5 & 5 & 10 \\
\hline ANGL (degrees) & 90 & 90 & 90 & 90 & 90 \\
\hline$x(\mathrm{~cm})$ & 115 & 200 & 250 & 300 & 400 \\
\hline Y $(\mathrm{cm})$ & & & & & 300 \\
\hline SLTH $(\mathrm{cm})$ & & & & & 1000 \\
\hline NSHLD & 3 & 3 & 4 & 4 & 3 \\
\hline $\begin{array}{l}\text { SHIELD 1: } \\
\text { T(1) (cm) } \\
\text { Material } \\
\text { Density }\left(\mathrm{g} / \mathrm{cm}^{3}\right)\end{array}$ & $\begin{array}{l}15 \\
\text { (a) } \\
\text { (a) }\end{array}$ & $\begin{array}{r}100 \\
(a) \\
(a)\end{array}$ & $\begin{array}{l}100 \\
\text { (a) } \\
\text { (a) }\end{array}$ & $\begin{array}{l}100 \\
(a) \\
(a)\end{array}$ & $\begin{array}{c}100 \\
(a) \\
(a)\end{array}$ \\
\hline $\begin{array}{l}\text { SHIELD 2: } \\
\text { T(2) (cm) } \\
\text { Material } \\
\text { Density }\end{array}$ & $\begin{array}{l}95 \\
\text { air } \\
0.00129\end{array}$ & $\begin{array}{l}95 \\
\text { air } \\
0.00129\end{array}$ & $\begin{array}{l}50 \\
\text { concrete } \\
1 . B\end{array}$ & $\mathfrak{c}_{\substack{100 \\
\text { concrete } \\
1 . B}}^{(b)}$ & $\begin{array}{l}295 \\
\operatorname{air} \\
0.00129\end{array}$ \\
\hline $\begin{array}{l}\text { SHIELD } 3: \\
\text { T(3) (cm) } \\
\text { Material } \\
\text { Density }\end{array}$ & $\begin{array}{c}5 \\
\text { skin } \\
1\end{array}$ & $\begin{array}{c}5 \\
\text { skin } \\
1\end{array}$ & $\begin{array}{l}95 \\
\text { air } \\
0.00129\end{array}$ & $\begin{array}{l}95 \\
\text { air } \\
0.00129\end{array}$ & $\begin{array}{c}5 \\
\text { skin } \\
1\end{array}$ \\
\hline $\begin{array}{l}\text { SHIELD 4: } \\
\text { T(4) (cm) } \\
\text { Material } \\
\text { Density }\end{array}$ & & & $\begin{array}{c}5 \\
\text { skin } \\
1\end{array}$ & $\begin{array}{c}5 \\
\text { skin } \\
1\end{array}$ & \\
\hline WEIGHT (Ci) & $1.0 \times 10^{-6}$ & $1.0 \times 10^{-6}$ & $1.0 \times 10^{-6}$ & $1.0 \times 10^{-6}$ & 30 \\
\hline NTHETA & & & & & 6 \\
\hline NPSI & & & & & 5 \\
\hline DELR & & & & & 0.2 \\
\hline
\end{tabular}

(a) External dos

1) $1.8 \mathrm{~g} / \mathrm{cm}_{3}$ (material: concrete)

2) $1.0 \mathrm{~g} / \mathrm{cm}_{3}$ (material: water) $\mathrm{cm}^{3} \mathrm{silica}+0.1 \mathrm{~g} / \mathrm{cm}^{3}$ carbon)

(b) The electron density of soil is approximated by that of concrete. 


\subsubsection{External Exposure Dose Rate Factors - Waste Modeled as a Slab Source}

The file assigned to logical unit 7 contains external dose rate factors for waste modeled 3 as a plane source. The dose rate factors are for $1 \mathrm{pCi}$ of radionuclide per $\mathrm{m}^{3}$ of waste. These factors relate the radionuclide strength to the dose rate in tissue $1 \mathrm{~m}$ away. Files are available to describe four different waste configurations: a slab at the soil surface; slabs with $0.5-m$ and 1.0-m overburden, respectively; and a rectangular slab source. The rectangular slab source approximates waste stored above ground. One of three waste densities may be assumed for each waste configuration. The file names, waste densities, and waste configuration descriptions are included in Table 2.5 for easy reference.

The external exposure dose rate factors were created by the computer program ISOSHLD (Engel, Greenborg and Hendrickson 1966; Simmons et al. 1967). Table 2.6 summarizes the ISOSHLD parameter values used to approximate a slab source of contamination at or on the soil surface or with a soil overburden.

The organization and FORTRAN formats of these files are as follows:

Record 1

Record 2

Remainder of file
Descriptive title, (A80).

Blank

For each isotope (A2, A6, E9.1):

- Element symbol and atomic number as in master radionuclide library

- External exposure dose rate factor

Listings of these files are found in Appendix B.

\subsubsection{Inhalation Dose Rate Factors - FILE23}

The file named FILE23 contains inhalation dose rate factors for $1 \mathrm{pCi} / \mathrm{m}^{3}$ of air. The file is arranged into sets of records for each isotope. Each isotope set contains three solubility class subsets. Each solubility class subset contains a variable number of records, one record per year. Each record contains data on total body, bone, lung, thyroid, and the lower large intestine. The file organization and FORTRAN formats of FILE23 are as follows:

Record 1

Record 2

Record 3
Descriptive titie (A80)

Number of radionuclides in library, (I3)

Master index of organs, as specified for ORGLIB (5I5)

This set of records follows for each radionuclide: 


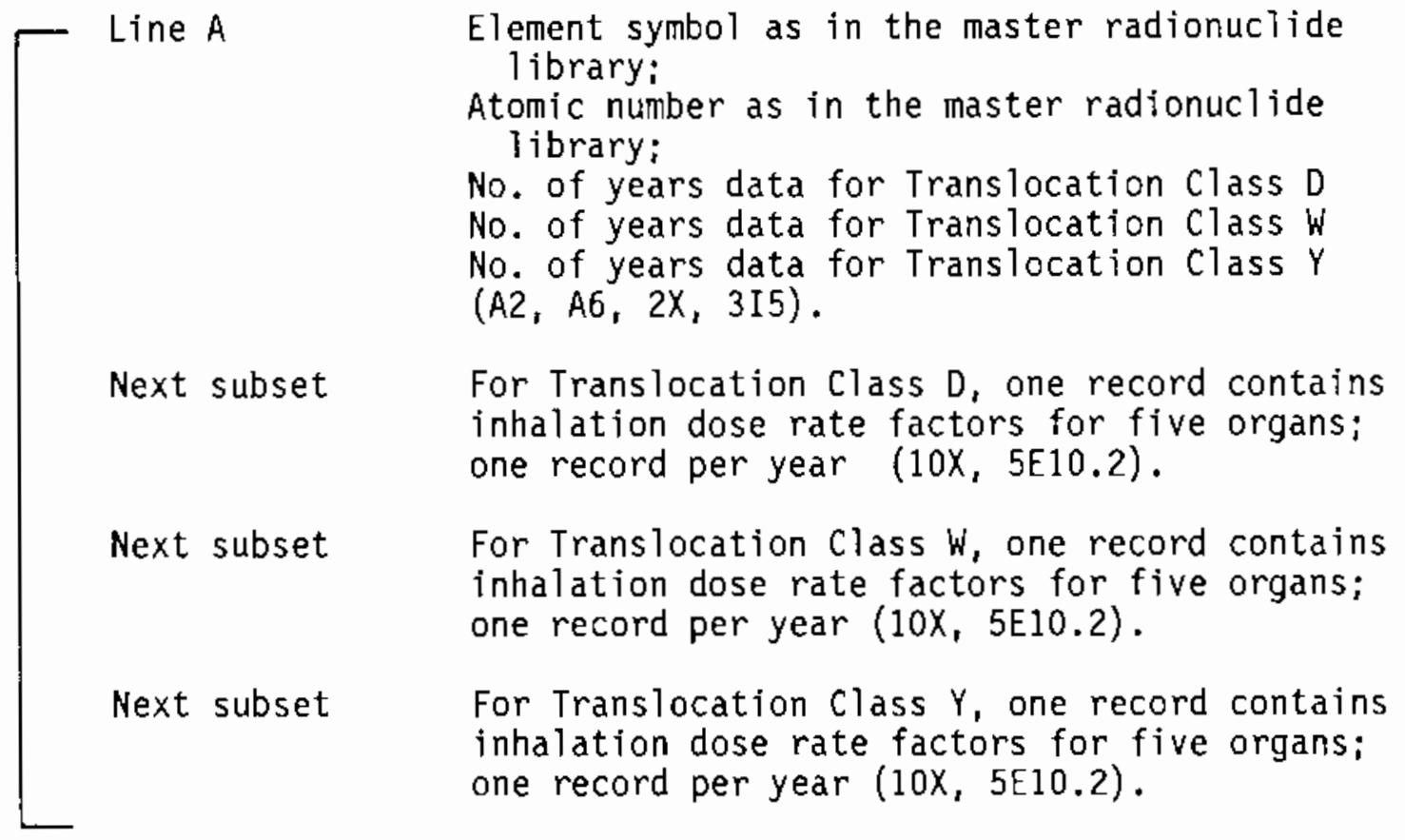

The file contains dose rate factors of 117 radionuclides for five organs for the assumed translocation class and a varying number of years. Dose rate factors are based on a breathing rate of $230 \mathrm{cc} / \mathrm{sec}$. FILE23 is created by a special version of the computer code DACRIN (Houston, Strenge, Watson. 1974). A complete listing of FILE23 is included in Appendix B.

\subsubsection{Dose Factors - Library LB30SVBO}

The library LB3OSVBQ contains comrnitted dose equivalent factors for target organs or tissues in units of Sv/Bq provided in ICRP Publication No. 30 (ICRP 1979-1983). The organization and FORTRAN format of the library is as follows:

Line 1 Descriptive title (A80)

Data sets of the following records for each radionuclide:

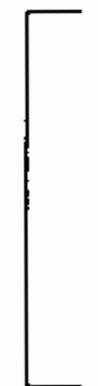

Record $1 \quad$ Element symbol, mass number plus metastable specification, number of organs included in this data set, alternate name for radionuclide (A2, $1 X, A 6,1 X, I 2,11 X, A 20)$

Record 2

Fraction of material absorbed into blood from gut $\left(f_{1}\right)$ for: oral 1 , oral 2, Translocation classes $D, W$, and $Y(3 X, 5(F 7.1,1 X)$

A listing of the material in LB3OSVBQ is included in Appendix B. 


\section{REFERENCES}

Annenkov, I. K., et al. 1974. The Radiobiology and Radioecology of Farm Animals. AEC-TR-7523, U.S. Atomic Energy Commission, Washington, D.C.

Anspaugh, L. R., J. H. Shinn, P. L. Phelps and N. C. Kennedy, 1975. "Resuspension and Redistribution of Plutonium in Soils." Health Phys. 29:571-582.

Bateman, H. 1910. "Solution of a System of Differential Equations 0ccurring in the Theory of Radio-active Transformations." Proc. Cambridge Phil. Soc., $15: 423-427$.

Engel, R. L., J. Greenborg, and M. M. Hendrickson. 1966. ISOSHLD - A Computer Code for General Purpose Isotope Shielding Analysis. BNwL-236, Pacific Northwest Laboratory, Richland, Washington.

Freke, A. M. 1965. "A Model for the Approximate Calculation of Safe Rates of Discharge of Radioactive Wastes into Marine Environments." Health Physics, $13: 743$.

Houston, J. R., D. L. Strenge and E. C. Watson. 1974. DACRIN - A Computer Program for Calculating Organ Dose from Acute or Chronic Radionuclide Inhalation. BNWL-B-389, Pacific Northwest Laboratory, Richland, Washington.

International Commission on Radiological Protection (ICRP). 1959. Report of ICRP Committee II on Permissible Dose for Internal Radiation. İCRP Publication No. 2, Pergamon Press, Elmsford, New York.

International Commission on Radiological Protection (ICRP). 1977. Recommendations of the International Commission on Radiological Protection. ICRP Publication No. 26, Pergamon Press, Elmsford, New York.

International Commission on Radiological Protection (ICRP). 1979-1982. Limits for Intakes of Radionuclides by workers. ICRP Publication No. 30, Parts 1, 2, and 3 (and supplements). Pergamon Press, Elms ford, New York.

International Commission on Radiological Protection (ICRP). 1966. "Deposition and Retention Models for Internal Dosimetry of the Human Respiratory Tract." Health Phys. 12:173-207.

Kennedy, W. E., Jr., E. C. Watson, G. R. Hoenes, and B. A. Napier. 1979. "A Method for Determining Acceptable Residual Radioactive Contamination Levels." Paper presented at the 24th Annual Meeting of the Health Physics Society, July 8-13, 1979, Philadelphia, Pennsylvania.

Kennedy, W. E., Jr., R. A. Peloquin, B. A. Napier and S. M. Neuder. 1986. Intruder Dose Pathway Analysis for the Onsite Disposal if Radioactive Wastes: The ONSITE/MAXI1 Computer Program. NUREG/CR-3620 Supplement 1, National Technical Information Service, Springfield, Virginia. 
Milham, R. C., J. F. Schubert, J. R. Watts, A. L. Boni, and J. C. Corey. 1974. "Measured Plutonium Resuspension and Resulting Dose from Agricultural Operations on an 0ld Field at the Savannah River Plant in the Southeastern United States." In Proceedings of an IAEA/USERDA Symposium on Transuranium Nuclides in the Envi ronment, E. I. du Pont de Nemours \& Co., Aiken, South Carolina.

Napier, B. A., R. L. Roswell, W. E. Kennedy, Jr. and D. L. Strenge. 1980. ARRRG and FOOD - Computer Programs for Calculating Radiation Dose to Man from Radionuclides in the Environment. PNL-3180, Pacific Northwest Laboratory, Richland, Washington.

Napier, B. A. 1981. Standardized Input for Hanford Environmental Impact Statements - Part I. PNL-3509, Part 1, Pacific Northwest Laboratory, Richland, Washington.

Napier, B. A., R. A. Peloquin, W. E. Kennedy, Jr. and S. M. Neuder. 1984. Intruder Dose Pathway Analysis for the Onsite Disposal if Radioactive Wastes: The ONSITE/MAXII Computer Program. NUREG/CR-3620, National Technical Information Service, springfield, Virginia.

Ng, Y. C., et al. 1968. Prediction of the Maximum Dosage to Man from the Fal lout of Nuclear Devices - IV, Handbook for Estimating the Maximum Internal Dose from Radionuclides Released to the Biosphere. Report UCRL-50163, prepared for the U.S. Atomic Energy Commission by Lawrence Livermore Laboratory, University of California, Livermore, California.

Oak, H. D., G. M. Holter, W. E. Kennedy, Jr., and G. J. Konzek. 1980. Technology, Safety and Costs of Decommissioning a Reference $80 i l i n g$-Water Reactor Power station. NUREG/CR-0672, Vol. 1 and 3, prepared for the U.S. Nuclear Regulatory Commission by Pacific Northwest Laboratory, Richland, Washington.

Radioactivity Advisory Committee (H. J. Dunster, Chairman). 1971. Handbook of Radiological Protection, Part I: Data. Department of Employment, Department of Health and Social Security, Ministry of Health and Social Services, Northern Ireland; Number SBN 11360079 8; Her Majesty's Stationery Office, London, England.

Sehme], G. A. 1974. "Particle Resuspension from an Asphalt Road." In Atmospheric-Surface Exchange of Particulate and Gaseous Pollutants, pp. 862-880. CoNF-740921, National Technical Information Service, Springfield, Virginia.

Sehmel, G. A., and F. D. Lloyd. 1976. "Resuspension Rates from a Circular Field Source." In Pacific Northwest Annual Report for 1975 to the DOE Assistant Secretary for the Environment, Part 3, Atmospheric Sciences, pp. 96-98. BNWL-2000, Pacific Northwest Laboratory, Richland, Washington.

Simmons, G. L., et al. 1967. ISOSHLD II Code Revision to Include Calculation of Dose Rate from Shielded Bremsstrahlung Sources. BNWL-236, Supplement 1, Pacific Northwest Laboratory, Richland, Washington. 
Soldat, J. K., and R. D. Harr. 1971. "Radiation Dose Model" in HERMES - A Digital Computer Code for Estimating Regional Radiological Effects from the Nuclear Power Industry (J. F. Fletcher and W. L. Dotson, compilers). Report HEDL-TME-71-68 prepared for the U.S. Atomic Energy Commission by Hanford Engineering Development Laboratory, Richland, Washington.

Soldat, J. K., N. M. Robinson and D. A. Baker. 1974. Models and Computer Codes for Evaluating Environmental Radiation Doses. Report BNwL-1754 prepared for the U.S. Atomic Energy Commission by Pacific Northwest Laboratory, Richland, Washington.

Thompson, S. E., C. A. Burton, D. J. Quinn and Y. C. Ng. 1972. Concentration Factors of Chemical Elements in Edible Aquatic Organisms. Report UCRL-50564 Rev. 1 prepared for the U.S. Atomic Energy Commission by University of California, Lawrence Livermore Laboratory, Livermore, California.

U.S. Environmental Protection Agency (EPA). 1977. Proposed Guidance on Dose Limits for Persons Exposed to Transuranium Elements in the General Environment. EPA 520/4-77-016, U.S Environmental Protection Agency, Washington, D.C.

U.S. Nuclear Regulatory Commission (NRC). 1977. Calculation of Annual Doses to Man From Routine Releases of Reactor Effluents for the Purpose of Evaluating Compliance with 10 CFR Part 50, Appendix I. Regulatory Guide 1.109, Rev. 1, U.S. Nuclear ReguTatory Commission, washington, D.C.

U.S. Nuclear Regulatory Commission (NRC). 1981. Draft Environmental Impact Statement on 10 CFR Part 61, "Licensing Requirements for Land Disposal of Radioactive Waste." NUREG-0782, Vols. 1-4, U.S. Nuclear Regulatory Commission, washington, D.C.

U.S. Nuclear Regulatory Commission (NRC). 1982. Final Environmental Impact Statement on 10 CFR Part 61 "Licensing Requi rements for Land Disposal of Radioactive Waste." NUREG-0945, U.S. Nuclear Regulatory Commission, Washington, D.C. 



\subsection{PROCEDURES}

User instructions and technical details on use of the ONSITE/MAXI1 software package are presented in this section. Instructions are given for installation of the software package on the IBM PC/XT/AT ${ }^{(a)}$ and for user interaction with both of the computer programs. In this section, program commands shown in boldface upper case should be entered exactly as shown and boldface lower case commands are examples of user-specific input. Six sample problems are included with discussions of the hand calculations performed to verify the correct operation of the computer program. Explanations are given where results differ from those presented in earlier volumes of this series. Detailed information. on input parameters is included for reference. Program-generated error messages and corresponding corrective actions for the ONSITE and MAXI1 computer programS are also provided.

Two levels of operation have been defined for the IBM PC/XT/AT version of ONSITE/MAXI1. As with the previous versions of ONSITE/MAXI1 (Napier et al. 1984; Kennedy et al. 1986), the first level of operation is intended for the user who interacts primarily with the ONSITE computer program to create an annotated input file for the MAXI1 computer program. ONSITE is an interactive program that instructs and guides the user in the creation of an environment or scenario. As a user becomes more familiar with the mathematical models and the software package, he or she may progress to the second level of operation. The second level of operation allows the user to edit the annotated input file for MAXI1 using a standard text editor and provides the commands to execute MAXI1.

Previous volumes in this series have focused on the first level of operation. This volume will focus on the second level of operation of the ONSITE/MAXI1 software package because this level is better suited for operations on an IBM $\mathrm{PC} / \mathrm{XT} / \mathrm{AT}$.

The following conventions are used in this manual to distinguish exact user input from instructions and from computer program displays.

boldface

Boldface print designates information entered at the terminal by the user. NOTE: In the appendices, information entered by the user is underlined.

UPPER CASE LETTERS When upper case letters in boldface type are encountered, the user should type in the boldface letters exactly as shown.

lower case letters when lower case letters are encountered in boldface type, the user should substitute urique identifiers.

(a) IBM PC/XT/AT is a trademark of International Business Machines corporation, Boca Raton, Florida. 


\subsection{USER INSTRUCTIONS}

The ONSITE/MAXI1 software package may be installed on an IBM PC/XT/AT computer, or a compatible computer, with 512 kilobytes of random-access memory and 10 megabytes of online-storage (i.e., hard disk) operating under IBM Disk Operating System (DOS) Version 2.1 or greater (IBM 1983, 1985). The ONSITE/MAXI1 software package is distributed on a set of four IBM DOS-formatted dual-density floppy disks. This section contains instructions for installing the software on a hard disk, as well as instructions for both levels of operation of the ONSITE/MAXI1 software package.

\subsubsection{Installing ONSITE/MAXIl on a Hard Disk}

For the ONSITE/MAXIl software package to function properly, the following commands must be in the CONFIG.SYS file in the root directory of the disk drive which is used to boot the system:

\section{FILES $=20$}

\section{BUFFERS $=24$}

Refer to the IBM DOS reference manual (IBM 1983, 1985) for details on installing these commands.

The following instructions are suggestions. Those familiar with the IBM disk operating system may define alternate configurations. However, the software was created to function within and has been tested under the following configuration. $c$ : will be the assumed specification for the hard disk in the following instructions.

To install the ONSITE/MAXIl software package, ensure that you are in the root directory of the hard disk and create two subdirectories with the following commands :

$$
\begin{aligned}
& \text { C: } \\
& \text { MD IMAXI } \\
& \text { MD IMUSE }
\end{aligned}
$$

The subdirectory MAXI1 will be the repository of the computer programs, data base and file buffers included in the ONSITE/MAXI1 software package. MUSE will be a storage area for user application input and report files.

Place the distribution disk labeled ONSITE/MAXI1 Disk 1 into the disk drive specified as $A$ : and type in the following commands:

$$
\begin{aligned}
& c: \backslash \\
& C D \backslash M A X I \\
& C O P Y A:^{\star}, *
\end{aligned}
$$


Place the distribution disk labeled ONSITE/MAXIl Disk \#2 into disk drive A: and type in the following:

\section{$\operatorname{COPY} A: *$ *}

Place the distribution disk labeled ONSITE/MAXI1 Disk \#3 into disk drive A: and type in the following:

\section{$\operatorname{COPY} A:^{\star} \star^{\star}$}

Place the distribution disk labeled ONSITE/MAXI1 Disk \#4 into disk drive A: and type:

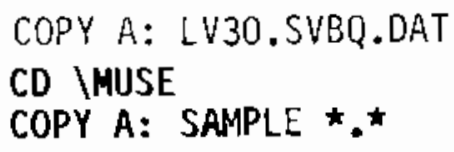

\subsubsection{Interaction with ONSITE: The First Level of Operation}

To establish and execute a scenario in the first level of operation, the user need only type the following cominands:

\section{CD \MAXI \\ ONSITE}

ONSITE is an interactive program that first introduces itself and displays general instructions on use of the program. A menu describing the available scenarios is also displayed. The five scenarios are:

(1) External Exposure Scenario (including Stored Waste Sub-Set)

(2) External Exposure Plus Inhalation Scenario

(3) Agricultural Scenarios

(4) Irrigation/Drinking-Water Scenario

(5) User-Defined Scenario

Additional information on use of ONSITE has been given extensive coverage in earlier volumes of this series (Napier et al. 1984; Kennedy et al. 1986) and is not included here.

ONSITE creates an input file for MAXI1 based on user response to questions and requests a unique filename to store the scenario parameters. For illustrative purposes, let us assume that the user wishes to store the scenario parameter values under the name "myjob." The user would enter:

\section{myjob}

when prompted by the ONSITE program. ONSITE would then create a file named "myjob.IN." Files are stored in the MUSE subdirectory unless a subdirectory path is included when the file name is entered. This MAXIl input file that was created by ONSITE may be viewed or modified with a standard text editor. 
Upon completion of the ONSITE program, the user may execute the MAXI1 program by entering the following command:

\section{COPY \MUSE\mYjob.IN MAXI.IN MAXI}

where myjob is the user-supplied scenario name.

MAXI1 output reports are stored in a file buffer named MAXI.OUT. The contents of MAXI.OUT will be overwritten during the next execution of the MAXI1 program. Consequently, the user may wish to save the execution results under a unique name as follows:

\section{COPY MAXI.OUT \MUSE Imyjob.OUT}

where myjob relates the scenario generated by ONSITE and the MAXI1 results. You may use IBM disk operating system commands to view or print the MAXI output file.

\subsubsection{Interaction with the MAXI1 Input File: The Second Level of Operation}

The ONSITE computer program creates an input file for the MAXI1 computer program based on user responses to questions. Let us assume that you as the user have created several scenarios with the help of ONSIIE and that you now wish to modify a scenario. If you have reached the point where you ask, "But do I have to answer all those ONSITE questions again?": you are ready for the second level of operation. In the second level of operation, the user may create and modify scenarios and environments by modifying MAXI1 input files with a standard text editor. When working with the text editor to change MAXI1 input files, always bear in mind the following:

- The editor must be a standard text editor, not a document processor.

- Always use the editor's "typeover" or "no insert" mode.

- When adding lines to the input file (for the inventory, terrestrial foods, and drinking water/aquatic foods data sets only), be careful that the specified number of entries in the data set equals the number of lines in that data set.

When ONSITE requested a filename for your selected scenario, the program generated a file named filename.IN, where "filename" indicates the eightcharacter unique name you supplied. Select a file associated with a scenario similar to the scenario you wish to create and copy the existing file providing a unique name for the new file as follows:

\section{COPY oldfile.IN newfile.IN}

and then edit the file newfile. IN with a standard text editor. 
Turn to the sample problems in the next section of this volume for an example of the MAXI1 input file format. The MAXI1 input format has been standardized to simplify scenario and environment parameter changes. The file has been annotated to allow the user to make changes without referencing the manual and to acquaint the user with the program names associated with parameters.

The MAXI1 program uses a free format to read the majority of the input file; consequently, the user need not be concerned with column placement and decimal points. Only three sections of the input file vary from this pattern. These three sections contain the inventory data set, the terrestrial foods data set, and the drinking water/aquatic foods data set. In each of these sections, entries must appear in specified columns. To simplify input, column headings are provided; the user is responsible for ensuring that numeric entries are right-justified and character entries are left-justified in these columns. These three sections are also the only places where the input file varies in length. These three sections vary according to the number of entries in each corresponding data set. The value of the parameter on the line preceding each of these sections indicates the number of lines to be read by the MAXI1 program. For example, in the first sample problem under the data set "INVENTORY," the "Number of radionuclides in inventory" entry is set to one. Consequently, the MAXI1 program expects to read one line fgom the input file containing information for one radionuclide, in this case ${ }^{C} \mathrm{Co}$.

After editing the file, copy your newly created input file into the MAXI1 input file buffer named MAXI. IN located in the MAXI subdirectory, and execute MAXI1 with the following commands:

\section{CD $\triangle M A X I$ \\ COPY \MUSE\newfile. IN MAXI.IN \\ MAXI \\ COPY MAXI.OUT \MUSE\newfile.OUT}

The last command copies your output from the MAXI1 output file buffer named MAXI.OUT to a name associated with your input file. You may then use IBM disk operating system commands to view or print your output file.

\subsubsection{Batch Mode Processing of ONSITE/MAXI1}

It may be convenient to process a series of MAXI1 executions in batch mode. Using IBM DOS commands, the batch commands for executing the sample problems are as follows:

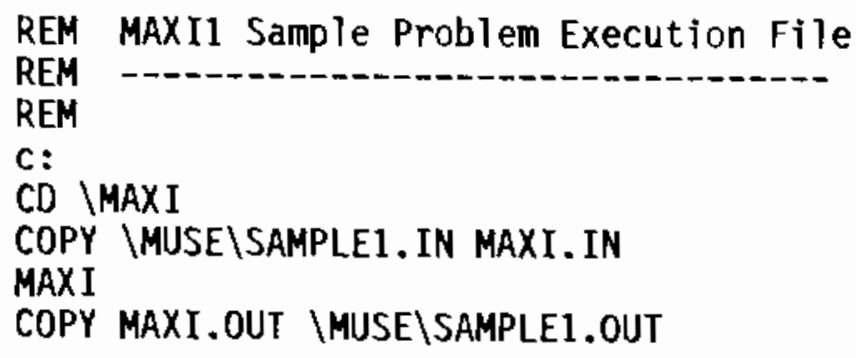




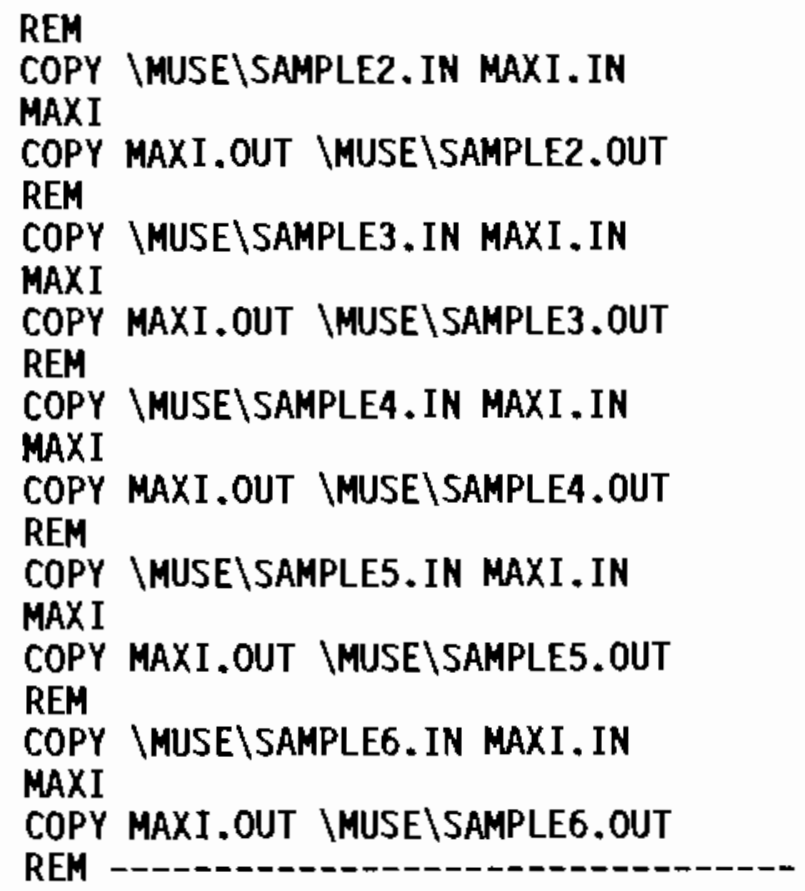

The above command sequence is provided in the file SAMPLE.BAT on the distribution floppy disks.

\subsection{SAMPLE PROBLEMS}

Six sample problems are presented and discussed to assist the user in various types of executions. An attempt has been made to cover a typical range of scenarios for which the code package was intended. The complete set of input and output for each of the sample problems is listed. Input specific to the second level of operation is shown for each of the sample problems. Changes from the previous sample problem are rulous on each of the input files to assist the user in understanding the second level of operation of the ONSITE/MAXI1 software package. A complete discussion of input parameters used in these sample problems is presented in Section 3.3 .

The results of the sample problems documented in Napier et al. (1984) and Kennedy et al. (1986) were verified by hand using the equations in Section 2 . The sample problems presented in this section are the same as those presented in Napier et al. (1984) and Kennedy et al. (1986), with the exception that a

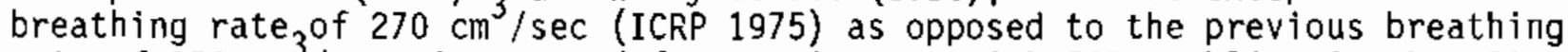
rate of $230 \mathrm{~cm} / \mathrm{sec}$, is assumed for consistency with ICRP Publication No. 30 . The version of the ONSITE/MAXIl software package presented in this document uses the same methodology (with the addition of ICRP Publication No. 30 methods) and data as earlier versions of the software package documented by Napier et al. (1984) and Kennedy et al. (1986) with the exception of the handling of inhalation translocation class (sometimes referred to as solubility class). The earlier versions of MAXI allowed assignment of different classes to each organ. For consistency with ICRP Publication No. 30 (ICRP 1979-19B2), translocation 
class was assumed to be the same across all organs for each radionuclide. In some cases, the assumed translocation classification was also changed. Results of the sample problems in this document are the same as those in Napier et al. (1984) and Kennedy et al. (1986) when inhalation doses are adjusted by the ratio of the prior to current breathing rates, $230 / 270$.

\subsubsection{Sample Problem One}

The first sample problem demonstrates the external exposure scegnario. For this sample problem, a licensee is assumed to dispose of $500 \mathrm{~m}$ of radioactive waste in 3 a bufial area on site. The waste is assumed to contain an average of $0.1 \mathrm{Ci} / \mathrm{m}^{3}$ of ${ }^{60}$ buried to a depth of $0.5 \mathrm{~m}$ over a 1-ha site. The desired result from the sample problem is the dose to an intruder 10 years after the wastes are buried. The MAXI1 input file for Sample Problem One is shown in Figure 3.1 .

The output resulting from this sample problem is shown in Figure 3.2. The calculated maximum annual total-body dose to the intruder resulting from the scenario in this sample problem is about 0.04 rem. The results of the radioactive decay, soil concentration, and total-body dose (for the external exposure pathway) calculations were verified by hand using the equations in Section 2 . 
DO YOU WISH TO CONSIDER:

\section{2}

1.0

1.0

1.0

2000.0

0.0

PLANEA.DAT BURHFA.DAT 270.0

External exposure and root penetration for waste modeled as a slab (volume) source? (0-no 1-yes)

External exposure to surface contamination modeled as a plane source? (0-no 1-yes)

Inhalation of resuspended material? (0-no 1-yes) INHA

Farm product ingestion? (0-no 1-yes)

Aquatic foods ingestion? (0-no 1-yes)

IFOD

Drinking water ingestion? (0-no 1-yes)

IARG

offsite atmospheric source? (0-no, 1-yes)

IWAT

ICRP-26/30 as well as ICRP-2 methodology? (0-no 1-yes)

IAIR

Decay of air release source term? (0-no 1-yes)

Decay of water release source term? (0-no 1 -yes)
Use the One-Year-of-Exposure Model? (0-no 1 -yes)

IEDE

IDKAIR

IDKWAT

Use the One-Year-of-Exposure Model? (0-no 1-yes)

INTRUD

MONITORING AND OUTPUT OPTIONS

Level of detail in reports (0-least to 3 )

Monitor and print hand calculation report

NUMBER OF YEARS AFTER WASTE IS DISPOSED THAT:

Scenario begins:

INVENTORY:

Release Term Input units: (1-pCi 2-uCi 3-mCi 4-Ci) INUT Soil source units $\left(0-m^{\star \star 2} 1-m^{\star \star 3} 2-\mathrm{kg}\right) \quad$ M3M2

Number of radionuclides in inventory:

NIN

\begin{tabular}{lrllll}
$\begin{array}{l}\text { Release } \\
\text { Terms }\end{array}$ & $\begin{array}{l}\text { Soil Source } \\
\text { (per unit) }\end{array}$ & $\begin{array}{l}\text { Irrigation } \\
\text { Aquatic } \\
\text { (per L) }\end{array}$ & $\begin{array}{l}\text { Drinking } \\
\text { Water } \\
\text { (per L) }\end{array}$ & $\begin{array}{l}\text { Atmospheric } \\
\text { Release } \\
\text { (per yr) }\end{array}$ & $\begin{array}{l}E \\
\text { (per }\end{array}$ \\
\hdashline c060 & 0.10 & & & & QI \\
& & & & & QK
\end{tabular}

INVENTORY MODIFICATION FACTORS: (multipliers)

Surface inventory modification factor:

SRDIL

Irrigation/aquatic inventory modification factor:

DILF

Size of site (fractional ha):

FRSIZ

Fraction of total diet grown on site:

RPF2

EXTERNAL/INHALATION EXPOSURE:

Hours of external exposure to contamination $(\mathrm{h} / \mathrm{yr})$ : HREXT Hours of inhalation of airborne contamination (h/yr): HRINH Ext. exp. dose rate factors (plane source) filename: PLANAM Ext. exp. dose rate factors (volume source) filename: VOLNAM Breathing rate $\left(\mathrm{cm}^{* \star} 3 / \mathrm{sec}\right)$ :

BRATE

FIGURE 3.1. Sample Problem One Input 


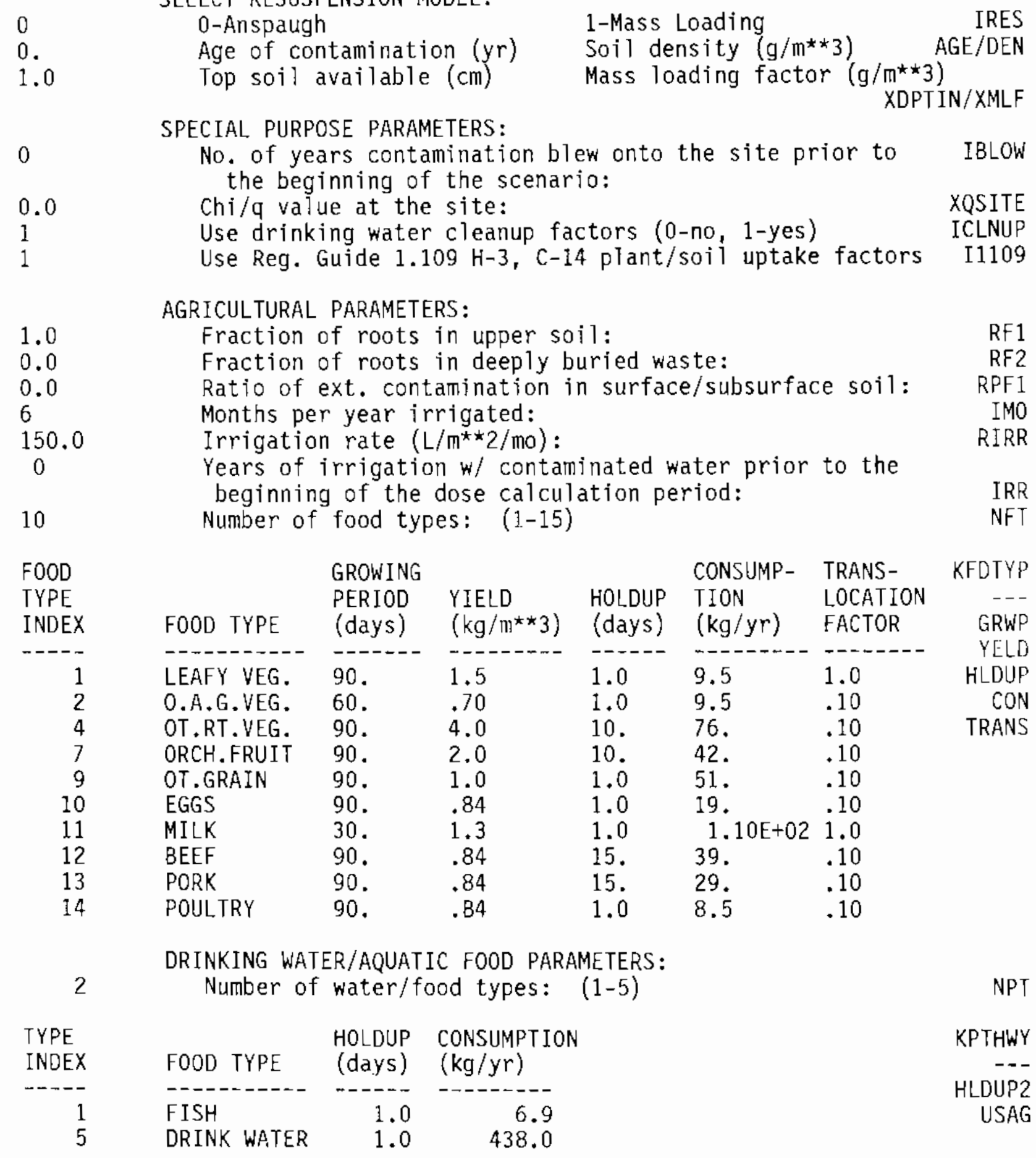

FIGURE 3.1 (Contd) 
ICRP-2 and ICRP-26/30 Maximum Annual Dose Calculation

(MAXI Version IBM 1.0 30-Jun-86)

Case title: SAMPLEl.IN / MAXI Sample Problem One

Executed on: $10 / 2 / 1986$ at $14: 56: 11$

Page 1

PATHWAYS/OPTIONS CONSIDERED:

External exposure/root penetration for waste modeled as slab (volume) source

TITLES OF LIBRARY FILES ACCESSED:

10: RMDLIB - Radionuclide Master Library (24-Sep-86 RAP)

27: OVERBURDEN: 0.5M, SOURCE: 1.0M (DEN: CONCRETE/1.8) MR/HR 8-Ju1-86 RAP

NUMBER OF YEARS AFTER WASTE IS DISPOSED THAT:

Scenario begins: $\quad 10$

Scenario ends:

10
59

INVENTORY:

Release Term Input units: (1-pCi 2-uCi 3-mCi 4-Ci)

Soil source units: $\left(0-m^{\star \star} 21-m^{\star \star} 32-\mathrm{kg}\right)$

Number of radionuclides in inventory:

Release Irrigation Drinking Atmospheric

Terms Soil Source /Aquatic Water Release

$\left(\operatorname{per} m^{\star \star} 3\right) \quad(\operatorname{per} \mathrm{L}) \quad(\operatorname{per} \mathrm{L}) \quad(\operatorname{per} \mathrm{m} 3)$

$\begin{array}{rrrr}-1.0 \mathrm{E}+11 & .0 \mathrm{E}+00 & .0 \mathrm{E}+00 & .0 \mathrm{E}+00\end{array}$

INVENTORY MODIFICATION FACTORS: (multipliers)

Surface inventory dilution factor:

Irrigation/aquatic inventory modification factor:

Size of site (fractional ha):

$i^{2}$

$1.0 \mathrm{E}+00$

FIGURE 3.2. Sample Problem One Output 
Case title: SAMPLE1.IN/MAXI Sample Problem One

Executed on: $10 / 2 / 1986$ at $14: 56: 11$

Page 2

EXTERNAL/INHALATION EXPOSURE:

Hours of external exposure to contamination $(h / y r): \quad 2.0 E+03$

RESUSPENSION PARAMETERS:

Model used:

Average age of contamination on ground at Time Zero $(y r)$ :

Top soil available for resuspension $(\mathrm{cm})$ :

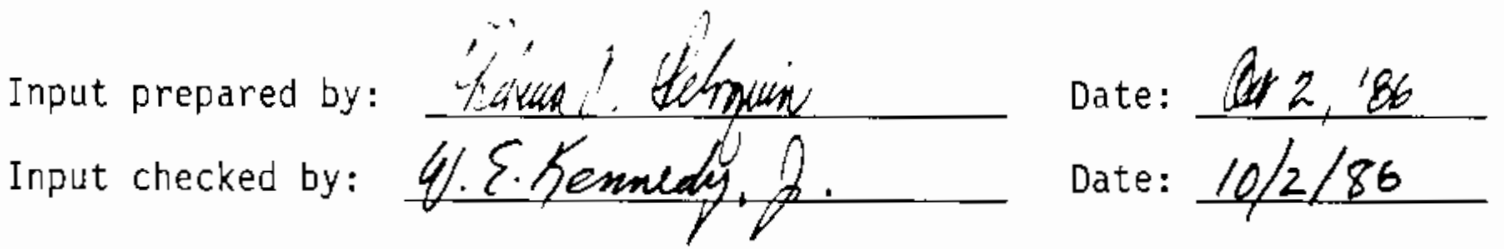

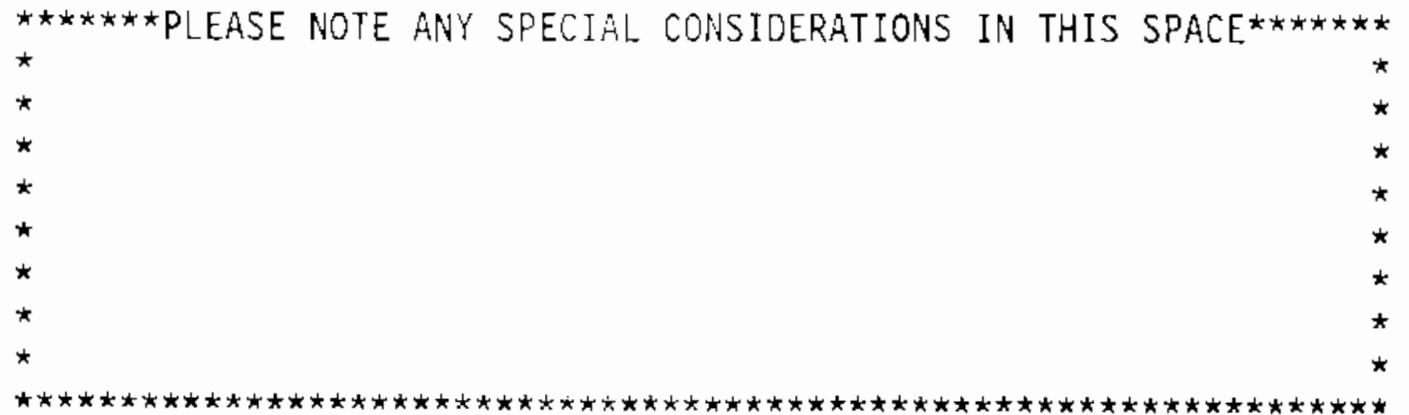

\section{CALCULATED VALUES:}

Ingestion area correction factor:

External/inhalation area correction factor:

1.0

Inhalation exposure modification factor:

1.0

External exposure modification factor:

.00

.23

FIGURE 3.2 (Contd) 
Case title: SAMPLEl.IN / MAXI Sample Problem One

Executed on: $10 / 2 / 1986$ at $14: 56: 11$

SOIL, AIR, AND WATER CONCENTRATION SUMMARY FOR YEAR: 10

\begin{tabular}{lccccc} 
Radio- & Surface Soil & Deep soil & Air & Irrigation & Drink Water \\
nuclide & $\mathrm{pCi} / \mathrm{m} 2$ & $\mathrm{pCi} / \mathrm{m} 3$ & $\mathrm{pCi} / \mathrm{m} 3$ & $\mathrm{pCi} / \mathrm{L}$ & $\mathrm{pC} \mathrm{i} / \mathrm{L}$ \\
\hdashline $\mathrm{CO} 60$ & $.0 \mathrm{O}+00$ & $5.4 \mathrm{E}+09$ & $.0 \mathrm{E}+00$ & $.0 \mathrm{E}+00$ & $.0 \mathrm{E}+00$
\end{tabular}

SOIL, AIR, AND WATER CONCENTRATION SUMMARY FOR YEAR: 59

\begin{tabular}{lccccc}
$\begin{array}{l}\text { Radio- } \\
\text { nuClide }\end{array}$ & $\begin{array}{c}\text { Surface Soil } \\
\mathrm{pCi} / \mathrm{m} 2\end{array}$ & $\begin{array}{c}\text { Deep Soil } \\
\mathrm{pCi} / \mathrm{m} 3\end{array}$ & $\begin{array}{c}\text { Air } \\
\mathrm{pCi} / \mathrm{m3}\end{array}$ & $\begin{array}{c}\text { Irrigation } \\
\mathrm{pCi} / \mathrm{L}\end{array}$ & $\begin{array}{c}\text { Drink Water } \\
\mathrm{pCi} / \mathrm{L}\end{array}$ \\
\hline CO 60 & $-0 \mathrm{E}+00$ & $8.4 \mathrm{E}+06$ & $.0 \mathrm{E}+00$ & $.0 \mathrm{E}+00$ & $.0 \mathrm{E}+00$
\end{tabular}


ICRP-2 and ICRP-26/30 Maximum Annual Dose Calculation

(MAXI Version IBM 1.0 30-Jun-86)

Case title: SAMPLEl. IN / MAXI Sample Problem one

Executed on: $10 / 2 / 1986$ at 14:56:11

Page 4

MAXIMUM ANNUAL DOSE SUMMARY FOR THE YEAR 10 FOR TOTAL BODY

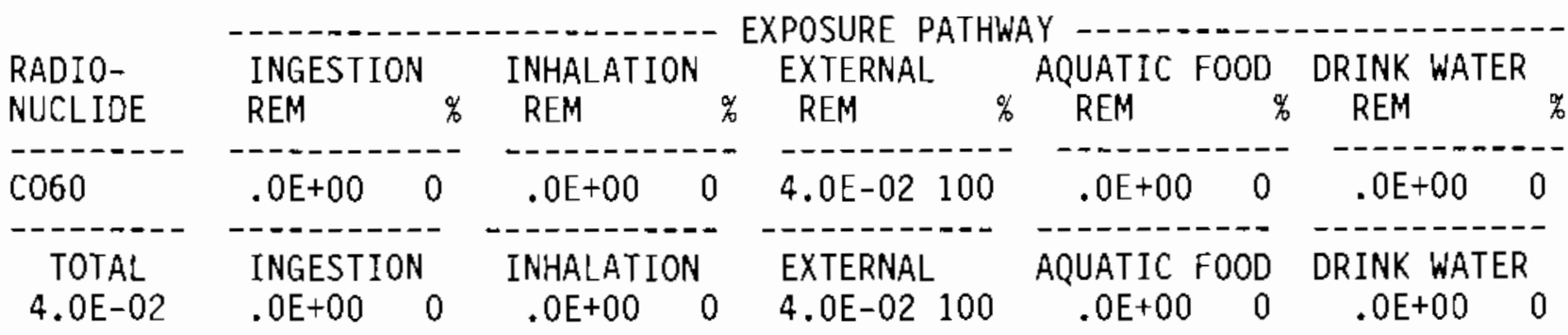

\section{MAXIMUM ANNUAL DOSE SUMMARY FOR THE YEAR 10 FOR BONE}

\begin{tabular}{|c|c|c|c|c|c|}
\hline RADIO- & INGESTION & INHALATION & EXTERNAL & AQUATIC FOOD & DRINK WATER \\
\hline NUCLIDE & REM & REM & REM & REM & REM \\
\hline Co60 & $.0 E+00$ & $.0 \mathrm{E}+00$ & $4.0 \mathrm{E}-02100$ & $.0 E+00$ & $.0 \mathrm{E}+00$ \\
\hline $\begin{array}{l}\text { TOTAL } \\
4.0 \mathrm{E}-02\end{array}$ & $\begin{array}{l}\text { INGESTION } \\
.0 \mathrm{E}+00 \quad 0\end{array}$ & $\begin{array}{l}\text { INHALATION } \\
.0 \mathrm{E}+00 \quad 0\end{array}$ & $\begin{array}{l}\text { EXTERNAL } \\
4.0 \mathrm{E}-02100\end{array}$ & $\begin{array}{l}\text { AQUATIC FOOD } \\
\quad .0 \mathrm{E}+00 \quad 0\end{array}$ & $\begin{array}{l}\text { DRINK WATER } \\
\quad .0 \mathrm{E}+00 \quad 0\end{array}$ \\
\hline
\end{tabular}

MAXIMUM ANNUAL DOSE SUMMARY FOR THE YEAR 10 FOR LUNGS

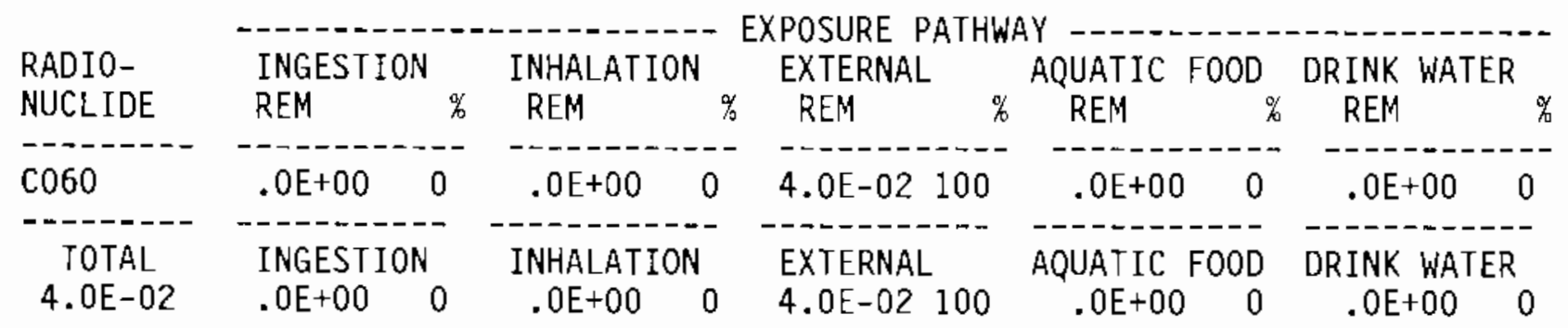

FIGURE 3.2 (Contd) 
ICRP-2 and ICRP-26/30 Maximum Annual Dose Calculation

(MAXI Version IBM 1.0 30-Jun-86)

Case title: SAMPLE1.IN / MAXI Sample Problem One

Executed on: $10 / 2 / 1986$ at $14: 56: 11$

Page 5

MAXIMUM ANNUAL DOSE SUMMARY FOR THE YEAR 10 FOR THYROID

\begin{tabular}{|c|c|c|c|c|c|c|c|}
\hline $\begin{array}{l}\text { RADIO- } \\
\text { NUCLIDE }\end{array}$ & $\begin{array}{l}\text { INGESTION } \\
\text { REM } \%\end{array}$ & $\begin{array}{l}\text { INHALATION } \\
\text { REM }\end{array}$ & $\%$ & $\begin{array}{l}\text { EXTERNAL } \\
\text { REM }\end{array}$ & $\begin{array}{l}\text { AQUATIC FOOD } \\
\text { REM }\end{array}$ & $\begin{array}{l}\text { DRINK WATER } \\
\text { REM }\end{array}$ & $\%$ \\
\hline 60 & $.0 \mathrm{E}+00$ & $.0 \mathrm{E}+00$ & 0 & $4.0 E-02 \quad 100$ & $.0 \mathrm{E}+00$ & $.0 \mathrm{E}+00$ & 0 \\
\hline $\begin{array}{c}\text { TOTAL } \\
4.0 \mathrm{E}-02\end{array}$ & $\begin{array}{l}\text { INGESTION } \\
.0 \mathrm{E}+00 \quad 0\end{array}$ & $\begin{array}{l}\text { INHALATION } \\
.0 \mathrm{E}+00\end{array}$ & $N_{0}$ & $\begin{array}{l}\text { EXTERNAL } \\
4.0 \mathrm{E}-02 \quad 100\end{array}$ & $\begin{array}{l}\text { AQUATIC FOOD } \\
.0 E+00 \quad 0\end{array}$ & $\begin{array}{c}\text { DRINK WATER } \\
.0 \mathrm{E}+00\end{array}$ & ${ }_{0}^{R}$ \\
\hline
\end{tabular}

MAXIMUM ANNUAL DOSE SUMMARY FOR THE YEAR 10 FOR LLI

\begin{tabular}{|c|c|c|c|c|c|c|c|c|c|c|}
\hline $\begin{array}{l}\text { RADI0- } \\
\text { NUCLIDE }\end{array}$ & $\begin{array}{l}\text { INGESTION } \\
\text { REM }\end{array}$ & $\%$ & $\begin{array}{l}\text { INHALATIOI } \\
\text { REM }\end{array}$ & $\%$ & $\begin{array}{l}\text { XPOSURE PA } \\
\text { EXTERNAL } \\
\text { REM }\end{array}$ & $\%$ & $\begin{array}{l}\text { AQUATIC } \\
\text { REM }\end{array}$ & FOOD & $\begin{array}{l}\text { DRINK WATER } \\
\text { REM }\end{array}$ & $\%$ \\
\hline $\mathrm{C} 060$ & $.0 \mathrm{E}+00$ & 0 & $.0 \mathrm{E}+00$ & 0 & $4.0 E-021$ & & $.0 \mathrm{E}+00$ & 0 & $.0 \mathrm{E}+00$ & 0 \\
\hline $\begin{array}{c}\text { TOTAL } \\
4.0 \mathrm{E}-02\end{array}$ & $\begin{array}{l}\text { INGESTION } \\
.0 E+00\end{array}$ & 0 & $\begin{array}{l}\text { INHALATIOI } \\
.0 \mathrm{E}+00\end{array}$ & $\begin{array}{l}v_{0} \\
0\end{array}$ & $\begin{array}{l}\text { EXTERNAL } \\
4.0 \mathrm{E}-021\end{array}$ & & $\begin{array}{l}\text { AQUATIC } \\
.0 \mathrm{E}+00\end{array}$ & $\begin{array}{r}\text { FOOD } \\
0\end{array}$ & $\begin{array}{c}\text { DRINK WATER } \\
.0 \mathrm{E}+00\end{array}$ & 0 \\
\hline
\end{tabular}

FIGURE 3.2 (Contd) 


\subsubsection{Sample Problem Two}

The second sample problem demonstrates the external exposure plus inhalation 3 scenario. For this sample problem, a lifgnsee is assumed to diøpose of $500 \mathrm{~m}^{3}$ of waste onsite containing $0.1 \mathrm{Ci} / \mathrm{m}^{3}$ of ${ }^{60} \mathrm{Co}$ and $0.01 \mathrm{Ci} / \mathrm{m}^{3}$ of ${ }^{90} \mathrm{Sr}$. The wastes are buried near the soil surface with no soil overburden. The wastes are assumed to contaminate a 1-ha site. Default conditions for resuspension are assumed except that the intruder is assumed to be exposed for $3000 \mathrm{~h} / \mathrm{yr}$ instead of $2000 \mathrm{~h} / \mathrm{yr}$. The desired result from Sample Problem Two is the dose to the intruder 10 years after the wastes are buried. The MAXIl input file for Sample Problem Two is shown in Figure 3.3. Changes from the previous sample problem are smax on the Sample Problem Two input file to assist the user in understanding the second level of operation of the ONSITE/MAXI1 software package.

The output resulting for this sample is shown in Figure 3.4. The maximum annua? doses from the scenario in this sample problem are about 16 rem to total body, 24 rem to bone, 31 rem to lung, 16 rem to thyroid, and 17 rem to GI(LLI). The results of the radioactive decay, soil and air concentration, and organ dose (for the external exposure and inhalation pathways) calculations were verified by hand using the equations in Section 2 . 
DO YOU WISH TO CONSIDER:

0

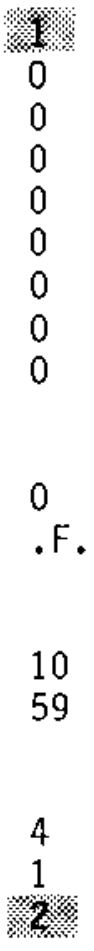

0.2

1.0

1.0

1.0

\% (3) PLANEA.DAT W. 6. . . 270.0
External exposure and root penetration for waste modeled as a slab (volume) source? (0-no 1-yes)

External exposure to surface contamination modeled as a plane source? (0-no 1-yes)

Inhalation of resuspended material? (0-no 1-yes) INHA

Farm product ingestion? (0-no 1-yes)

Aquatic foods ingestion? (0-no 1 -yes)

Drinking water ingestion? (0-no 1-yes)

offsite atmospheric source? (0-no 1-yes)

ICRP-26/30 as well as ICRP-2 methodology? (0-no 1-yes)

Decay of air release source term? (0-no 1-yes)

Decay of water release source term? (0-no 1-yes)

Use the One-Year-of-Exposure Mode1? (0-no 1-yes)

IFOD

IARG

IWAT

IAIR

IEDE

IDKAIR

IDKWAT

INTRUD

MONITORING AND OUTPUT OPTIONS

Level of detail in reports (0-least to 3 )

IOUT

Monitor and print hand calculation report

DEBUG (4)

NUMBER OF YEARS AFTER WASTE IS DISPOSED THAT:

Scenario begins:

IT1

Scenario ends:

IT2

INVENTORY:

Release Term Input units: (1-pCi 2-uCi 3-mCi 4-Ci) INUT

Soil source units $\left(0-m^{\star \star} 21-m^{\star \star} 3 \quad 2-\mathrm{kg}\right)$

Number of radionuclides in inventory:

\begin{tabular}{|c|c|c|c|c|}
\hline $\begin{array}{l}\text { Release } \\
\text { Terms }\end{array}$ & $\begin{array}{l}\text { Soil Source } \\
\text { (per unit) }\end{array}$ & $\begin{array}{l}\text { Irrigation } \\
\text { /Aquatic } \\
\text { (per L) }\end{array}$ & $\begin{array}{l}\text { Drinking } \\
\text { Water } \\
\text { (per L) }\end{array}$ & $\begin{array}{l}\text { Atmospheric } \\
\text { Release } \\
\text { (per yr) }\end{array}$ \\
\hline 060 & 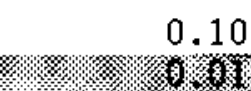 & & & \\
\hline
\end{tabular}

INVENTORY MODIFICATION FACTORS: (multipliers)

Surface inventory modification factor:

Irrigation/aquatic inventory modification factor:

SRDIL

DILF

Size of site (fractional ha):

Fraction of total diet grown on site:

FRSIZ

RPF2

EXTERNAL/INHALATION EXPOSURE:

Hours of external exposure to contamination $(\mathrm{h} / \mathrm{yr})$ : HREXT

Hours of inhalation of airborne contamination (h/yr):

HRINH

Ext. exp. dose rate factors ( $p l a n e$ source) filename:

PLANAM

Ext. exp. dose rate factors (volume source) filename: VOLNAM

Breathing rate $\left(\mathrm{cm}^{\star *} 3 / \mathrm{sec}\right)$ :

FIGURE 3.3. Sample Problem Two Input 


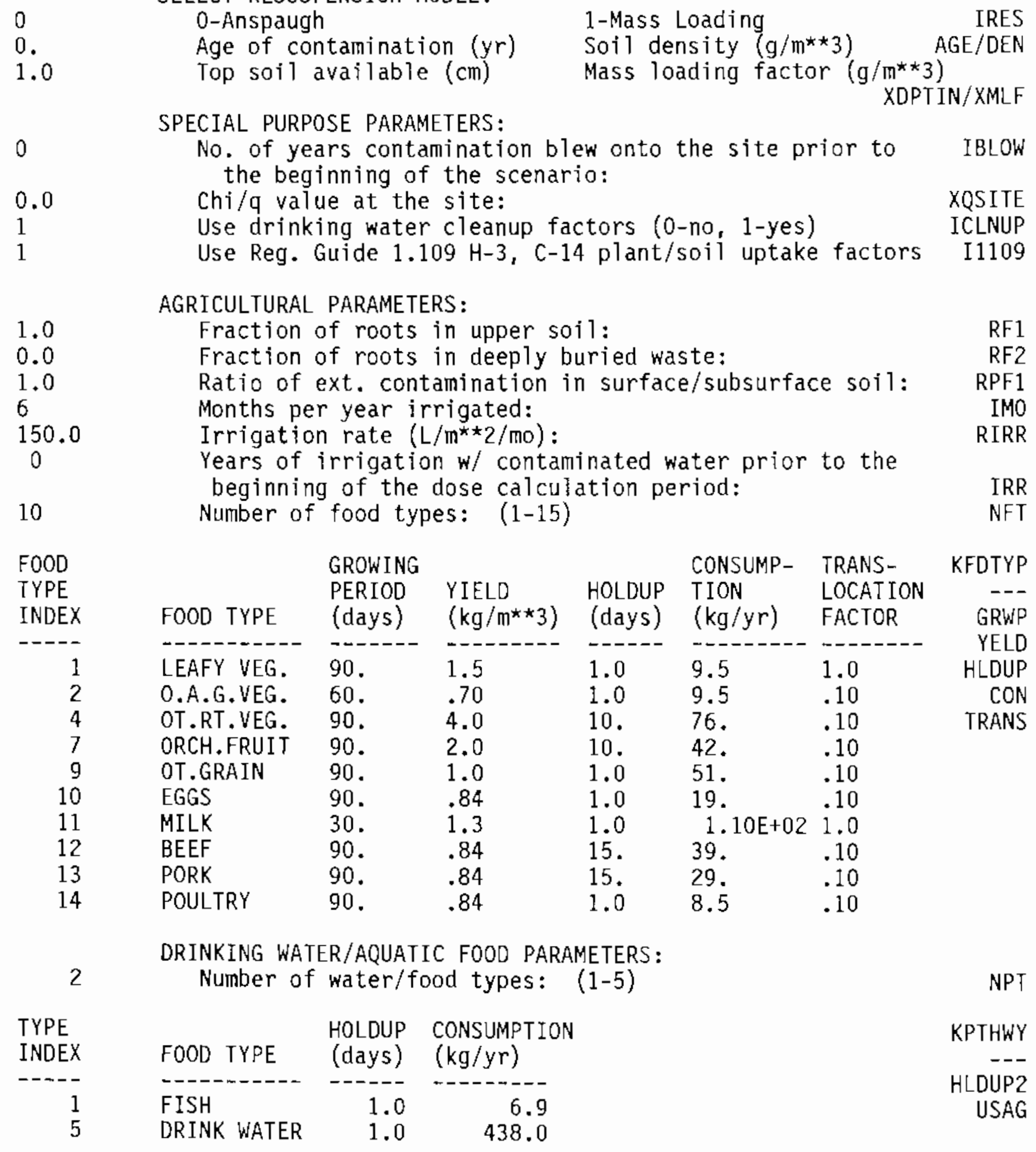

FIGURE 3.3 (Contd) 
(MAXI Version IBM $1.030-J u n-86$ )

Case title: SAMPLE2. IN / MAXI Sample Problem Two

Executed on: $10 / 2 / 1986$ at $14: 56: 41$

Page 1

PATHWAYS/OPTIONS CONSIDERED:

External exposure/root penetration for waste modeled as slab (volume) source Inhalation of resuspended material

TITLES OF LIBRARY FILES ACCESSED:

10: RMDLIB - Radionuclide Master Library (24-Sep-86 RAP)

23: DACRIN (OIFDOS) DOSE INCREMENT FILE ONSITE/MAXI 26-AUg-86 RAP

27: OVERBUROEN: $0.0 \mathrm{M}$, SOURCE: $1.0 \mathrm{M}$ (DEN: CONCRETE/1.8): MR/HR 8-JU1-86 RAP

NUMBER OF YEARS AFTER WASTE IS DISPOSED THAT:

Scenario begins:

10

Scenario ends:

INVENTORY:

Release Term Input units: (1-pCi 2-uCi 3-mCi 4-Ci)

Soil source units: $\left(0-m^{\star \star 2} 1-m^{\star \star} 3 \quad 2-\mathrm{kg}\right)$

Number of radionuclides in inventory:

\begin{tabular}{lrrrr}
$\begin{array}{l}\text { Release } \\
\text { Terms }\end{array}$ & $\begin{array}{l}\text { Soil Source } \\
\text { (per } m^{\star \star 3)}\end{array}$ & $\begin{array}{r}\text { Irrigation } \\
\text { Aquatic } \\
\text { (per L) }\end{array}$ & $\begin{array}{l}\text { Drinking } \\
\text { Water } \\
\text { (per L) }\end{array}$ & $\begin{array}{l}\text { Atmospheric } \\
\text { Release } \\
\text { (per m3) }\end{array}$ \\
\hline C060 & $1.0 \mathrm{E}+11$ & $.0 \mathrm{E}+00$ & $-0 \mathrm{E}+00$ & $.0 \mathrm{E}+00$ \\
SR90 & $1.0 \mathrm{E}+10$ & $.0 \mathrm{E}+00$ & $.0 \mathrm{E}+00$ & $.0 \mathrm{E}+00$ \\
Y 90 & $.0 \mathrm{E}+00$ & $.0 \mathrm{E}+00$ & $.0 \mathrm{E}+00$ & $.0 \mathrm{E}+00$
\end{tabular}

INVENTORY MODIFICATION FACTORS: (multipliers)

Surface inventory dilution factor:

Irrigation/aquatic inventory modification factor:

size of site (fractional ha):

.2

$1.0 \mathrm{E}+00$

FIGURE 3.4. Sample Problem Two Output 
Case title: SAMPLE2.IN / MAXI Sample Problem Two

Executed on: $10 / 2 / 1986$ at $14: 56: 41$

EXTERNAL/INHALATION EXPOSURE:

Hours of external exposure to contamination $(\mathrm{h} / \mathrm{yr}): \quad 3.0 \mathrm{E}+03$

Hours of inhalation of airborne contamination $(\mathrm{h} / \mathrm{yr})$ : $\quad 3.0 \mathrm{E}+03$

RESUSPENSION PARAMETERS:

Mode? used:

Top soil available for resuspension (cm):

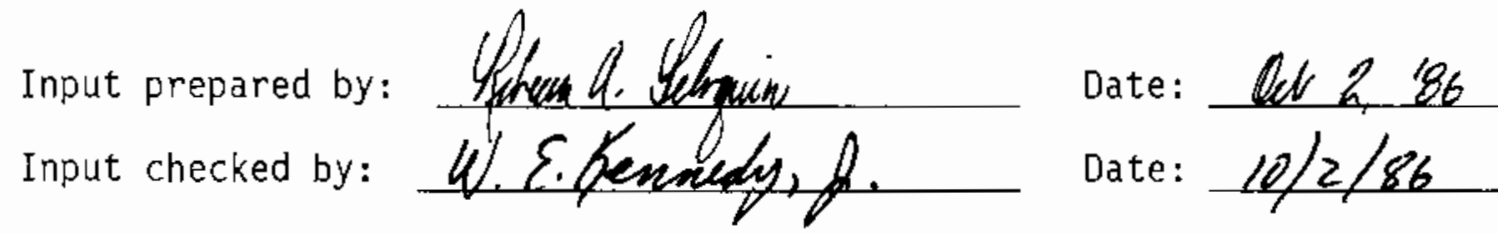

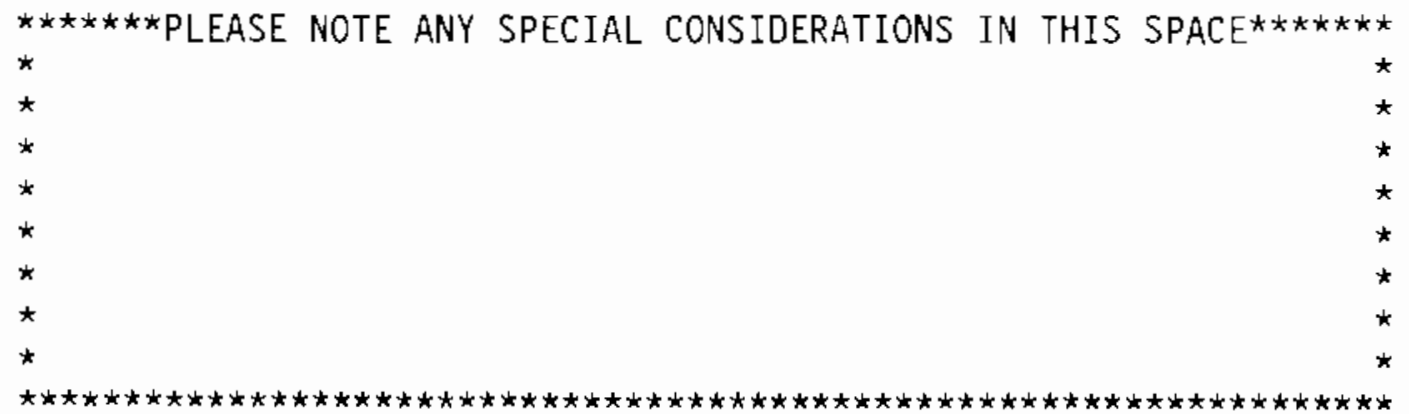

CALCULATED VALUES:

Ingestion area correction factor:

1.0

External/inhalation area correction factor:

1.0

Inhalation exposure modification factor:

.34

External exposure modification factor: 
ICRP-2 and ICRP-26/30 Maximum Annual Dose Calculation

(MAXI Version IBM 1.0 30-Jun-86)

Case title: SAMPLE2.IN / MAXI Sample Problem Two

Executed on: $10 / 2 / 1986$ at $14: 56: 41$

Page 3

SOIL, AIR, AND WATER CONCENTRATION SUMMARY FOR YEAR: 10

\begin{tabular}{lccccc}
$\begin{array}{l}\text { Radio- } \\
\text { nuClide }\end{array}$ & $\begin{array}{c}\text { Surface Soil } \\
\mathrm{pCi} / \mathrm{m} 2\end{array}$ & $\begin{array}{c}\text { Deep Soil } \\
\mathrm{pCi} / \mathrm{m} 3\end{array}$ & $\begin{array}{c}\mathrm{Air} \\
\mathrm{pCi} / \mathrm{m} 3\end{array}$ & $\begin{array}{c}\text { Irrigation } \\
\mathrm{pC} i / \mathrm{L}\end{array}$ & $\begin{array}{c}\text { Drink Water } \\
\mathrm{pCi} / \mathrm{L}\end{array}$ \\
\hline CO 60 & $8.0 \mathrm{E}+08$ & $5.4 \mathrm{E}+09$ & $5.4 \mathrm{E}+03$ & $.0 \mathrm{E}+00$ & $.0 \mathrm{E}+00$ \\
SR 90 & $2.4 \mathrm{E}+08$ & $1.6 \mathrm{E}+09$ & $1.6 \mathrm{E}+03$ & $.0 \mathrm{E}+00$ & $.0 \mathrm{E}+00$ \\
$\mathrm{Y} 90$ & $2.4 \mathrm{E}+08$ & $1.6 \mathrm{E}+09$ & $1.6 \mathrm{E}+03$ & $.0 \mathrm{E}+00$ & $.0 \mathrm{E}+00$
\end{tabular}

SOIL, AIR, AND WATER CONCENTRATION SUMMARY FOR YEAR: 59

\begin{tabular}{lccccc}
$\begin{array}{l}\text { Radio- } \\
\text { nuclide }\end{array}$ & $\begin{array}{c}\text { Surface Soil } \\
\mathrm{pCi} / \mathrm{m} 2\end{array}$ & $\begin{array}{c}\text { Deep Soil } \\
\mathrm{pCi} / \mathrm{m} 3\end{array}$ & $\begin{array}{c}\text { Air } \\
\mathrm{pCi} / \mathrm{m} 3\end{array}$ & $\begin{array}{c}\text { Irrigation } \\
\mathrm{pCi} / \mathrm{L}\end{array}$ & $\begin{array}{c}\text { Drink Water } \\
\mathrm{pCi} / \mathrm{L}\end{array}$ \\
\hline $\mathrm{CO} 60$ & $-1.3 \mathrm{E}+06$ & $8.4 \mathrm{E}+06$ & $8.4 \mathrm{E}-05$ & $.0 \mathrm{E}+00$ & $.0 \mathrm{E}+00$ \\
SR 90 & $7.1 \mathrm{E}+07$ & $4.8 \mathrm{E}+08$ & $4.8 \mathrm{E}-03$ & $.0 \mathrm{E}+00$ & $.0 \mathrm{E}+00$ \\
$\mathrm{Y} 90$ & $7.1 \mathrm{E}+07$ & $4.8 \mathrm{E}+08$ & $4.8 \mathrm{E}-03$ & $.0 \mathrm{E}+00$ & $.0 \mathrm{E}+00$
\end{tabular}

FIGURE 3.4 (Contd) 
ICRP-2 and ICRP-26/30 Maximum Annual Dose Calculation

(MAXI Version IBM 1.0 30-Jun-86)

Case title: SAMPLE2.IN / MAXI Sample Problem Two

Executed on: $10 / 2 / 1986$ at $14: 56: 41$

Page 4

MAXIMUM ANNUAL DOSE SUMMARY FOR THE YEAR 10 FOR TOTAL BODY

\begin{tabular}{|c|c|c|c|c|c|c|c|c|c|c|}
\hline $\begin{array}{l}\text { ZADIO- } \\
\text { UUCLIDE }\end{array}$ & $\begin{array}{l}\text { INGESTION } \\
\text { REM }\end{array}$ & $\%$ & $\begin{array}{l}\text { INHALATIO } \\
\text { REM }\end{array}$ & $\%$ & $\begin{array}{l}\text { EXTERNAL } \\
\text { REM }\end{array}$ & $\%$ & $\begin{array}{l}\text { AQUATIC } \\
\text { REM }\end{array}$ & FOOD & $\begin{array}{l}\text { DRINK WATER } \\
\text { REM }\end{array}$ & $\mathrm{R}_{\%}$ \\
\hline $\begin{array}{r}060 \\
\text { R90 } \\
90\end{array}$ & $\begin{array}{l}.0 E+00 \\
.0 E+00 \\
.0 E+00\end{array}$ & $\begin{array}{l}0 \\
0 \\
0\end{array}$ & $\begin{array}{l}3.4 \mathrm{E}-02 \\
3.4 \mathrm{E}-01 \\
4.3 \mathrm{E}-05\end{array}$ & $\begin{array}{r}9 \\
90 \\
0\end{array}$ & $\begin{array}{l}1.6 \mathrm{E}+01 \\
1.4 \mathrm{E}-04 \\
9.9 \mathrm{E}-03\end{array}$ & $\begin{array}{r}99 \\
0 \\
0\end{array}$ & $\begin{array}{l}.0 \mathrm{E}+00 \\
.0 \mathrm{E}+00 \\
.0 \mathrm{E}+00\end{array}$ & $\begin{array}{l}0 \\
0 \\
0\end{array}$ & $\begin{array}{l}.0 E+00 \\
.0 E+00 \\
.0 E+00\end{array}$ & $\begin{array}{l}0 \\
0 \\
0\end{array}$ \\
\hline $\begin{array}{c}\text { TOTAL } \\
1.6 \mathrm{E}+01\end{array}$ & $\begin{array}{l}\text { INGESTI } \\
.0 \mathrm{E}+00\end{array}$ & $\begin{array}{l}1 \\
0\end{array}$ & $\begin{array}{l}\text { INHALATI } \\
3.7 \mathrm{E}-01\end{array}$ & 2 & $\begin{array}{l}\text { EXTERNAL } \\
1.6 \mathrm{E}+01\end{array}$ & 97 & $\begin{array}{l}\text { QUATIC } \\
.0 E+00\end{array}$ & $\begin{array}{r}O D \\
0\end{array}$ & $\begin{array}{l}\text { RINK WATI } \\
.0 E+00\end{array}$ & ${ }_{0}^{R}$ \\
\hline
\end{tabular}

MAXIMUM ANNUAL DOSE SUMMARY FOR THE YEAR 11 FOR BONE

\begin{tabular}{|c|c|c|c|c|c|c|c|c|c|c|}
\hline $\begin{array}{l}\text { RADIO- } \\
\text { NUCLIDE }\end{array}$ & $\begin{array}{l}\text { INGESTIO } \\
\text { REM }\end{array}$ & $\%$ & $\begin{array}{l}\text { INHALATI } \\
\text { REM }\end{array}$ & $O N$ & $\begin{array}{l}\text { EXTERNAL } \\
\text { REM }\end{array}$ & $\%$ & $\begin{array}{l}\text { AQUATIC } \\
\text { REM }\end{array}$ & $\mathrm{OD}$ & $\begin{array}{l}\text { DRINK WATER } \\
\text { REM }\end{array}$ & $\%$ \\
\hline $\begin{array}{l}\text { C060 } \\
\text { SR90 } \\
\text { Y } 90\end{array}$ & $\begin{array}{l}.0 E+00 \\
.0 E+00 \\
.0 E+00\end{array}$ & $\begin{array}{l}0 \\
0 \\
0\end{array}$ & $\begin{array}{l}.0 \mathrm{E}+00 \\
9.7 \mathrm{E}+00 \\
1.1 \mathrm{E}-04\end{array}$ & $\begin{array}{r}0 \\
99 \\
0\end{array}$ & $\begin{array}{l}1.4 \mathrm{E}+01 \\
1.4 \mathrm{E}-04 \\
9.6 \mathrm{E}-03\end{array}$ & $\begin{array}{r}99 \\
0 \\
0\end{array}$ & $\begin{array}{l}.0 \mathrm{E}+00 \\
.0 \mathrm{E}+00 \\
.0 \mathrm{E}+00\end{array}$ & $\begin{array}{l}0 \\
0 \\
0\end{array}$ & $\begin{array}{l}.0 \mathrm{E}+00 \\
.0 \mathrm{E}+00 \\
.0 \mathrm{E}+00\end{array}$ & $\begin{array}{l}0 \\
0 \\
0\end{array}$ \\
\hline $\begin{array}{r}\text { TOTA } \\
2.4 \mathrm{E}+\end{array}$ & $\begin{array}{l}\text { INGESTIO } \\
.0 E+00\end{array}$ & $\mathrm{~N}_{0}$ & $\begin{array}{l}\text { INHALATI } \\
9.7 \mathrm{E}+00\end{array}$ & $\begin{array}{l}\text { ON } \\
40\end{array}$ & $\begin{array}{l}\text { EXTERNAL } \\
1.4 \mathrm{E}+01\end{array}$ & 59 & $\begin{array}{l}\text { AQUATIC } \\
.0 \mathrm{E}+00\end{array}$ & & $\begin{array}{c}\text { DRINK WAT } \\
.0 \mathrm{E}+00\end{array}$ & 0 \\
\hline
\end{tabular}

MAXIMUM ANNUAL DOSE SUMMARY FOR THE YEAR 11 FOR LUNGS

\begin{tabular}{|c|c|c|c|c|c|c|c|c|c|c|}
\hline $\begin{array}{l}\text { RADIO- } \\
\text { NUCL IDE }\end{array}$ & $\begin{array}{l}\text { INGEST IOI } \\
\text { REM }\end{array}$ & $\%$ & $\begin{array}{l}\text { INHALAT } \\
\text { REM }\end{array}$ & ON $\%$ & $\begin{array}{l}\text { EXTERNAL } \\
\text { REM }\end{array}$ & $\%$ & $\begin{array}{l}\text { AQUATIC } \\
\text { REM }\end{array}$ & F00D & $\begin{array}{l}\text { DRINK WATER } \\
\text { REM }\end{array}$ & R \\
\hline $\begin{array}{l}\text { CO60 } \\
\text { SR90 } \\
\text { Y } 90\end{array}$ & $\begin{array}{l}.0 \mathrm{E}+00 \\
.0 \mathrm{E}+00 \\
.0 \mathrm{E}+00\end{array}$ & $\begin{array}{l}0 \\
0 \\
0\end{array}$ & $\begin{array}{l}1.7 \mathrm{E}+01 \\
3.0 \mathrm{E}-03 \\
8.5 \mathrm{E}-03\end{array}$ & $\begin{array}{r}99 \\
0 \\
0\end{array}$ & $\begin{array}{l}1.4 \mathrm{E}+01 \\
1.4 \mathrm{E}-04 \\
9.6 \mathrm{E}-03\end{array}$ & $\begin{array}{r}99 \\
0 \\
0\end{array}$ & $\begin{array}{l}.0 E+00 \\
.0 E+00 \\
.0 E+00\end{array}$ & $\begin{array}{l}0 \\
0 \\
0\end{array}$ & $\begin{array}{l}.0 \mathrm{E}+00 \\
.0 \mathrm{E}+00 \\
.0 \mathrm{E}+00\end{array}$ & $\begin{array}{l}0 \\
0 \\
0\end{array}$ \\
\hline $\begin{array}{c}\text { TOTAL } \\
3.1 E+01\end{array}$ & $\begin{array}{l}\text { INGESTIC } \\
.0 \mathrm{E}+00\end{array}$ & $\begin{array}{l}N^{N} \\
0\end{array}$ & $\begin{array}{l}\text { INHALAT } \\
1.7 \mathrm{E}+01\end{array}$ & $\begin{array}{l}0 \mathrm{~N} \\
54\end{array}$ & $\begin{array}{l}\text { EXTERNAL } \\
1.4 \mathrm{E}+01\end{array}$ & 45 & $\begin{array}{l}\text { AQUATIC } \\
.0 \mathrm{E}+00\end{array}$ & & $\begin{array}{l}\text { DRINK WATER } \\
.0 \mathrm{E}+00\end{array}$ & $\begin{array}{l}\mathrm{R} \\
0\end{array}$ \\
\hline
\end{tabular}

FIGURE 3.4 (Contd) 
ICRP-2 and ICRP-26/30 Maximum Annual Dose Calculation

(MAXI Version IBM 1.0 30-Jun-86)

Case title: SAMPLE2.IN / MAXI Sample Problem Two

Executed on: $10 / 2 / 1986$ at $14: 56: 41$

Page 5

MAXIMUM ANNUAL DOSE SUMMARY FOR THE YEAR 10 FOR THYROID

\begin{tabular}{|c|c|c|c|c|c|c|c|c|c|c|}
\hline $\begin{array}{l}\text { RADI0- } \\
\text { NUCLIDE }\end{array}$ & $\begin{array}{l}\text { INGESTION } \\
\text { REM }\end{array}$ & $\%$ & $\begin{array}{l}\text { INHALATION } \\
\text { REM }\end{array}$ & $\%$ & $\begin{array}{l}\text { EXTERNAL } \\
\text { REM }\end{array}$ & $\%$ & $\begin{array}{l}\text { AQUATIC } \\
\text { REM }\end{array}$ & $\begin{array}{r}F 000 \\
\%\end{array}$ & $\begin{array}{l}\text { DRINK WATER } \\
\text { REM }\end{array}$ & R $\%$ \\
\hline $\begin{array}{l}\text { CO60 } \\
\text { SR90 } \\
\text { Y } 90\end{array}$ & $\begin{array}{l}.0 \mathrm{E}+00 \\
.0 \mathrm{E}+00 \\
.0 \mathrm{E}+00\end{array}$ & $\begin{array}{l}0 \\
0 \\
0\end{array}$ & $\begin{array}{l}.0 \mathrm{E}+00 \\
.0 \mathrm{E}+00 \\
.0 \mathrm{E}+00\end{array}$ & $\begin{array}{l}0 \\
0 \\
0\end{array}$ & $\begin{array}{l}1.6 \mathrm{E}+01 \\
1.4 \mathrm{E}-04 \\
9.9 \mathrm{E}-03\end{array}$ & $\begin{array}{r}99 \\
0 \\
0\end{array}$ & $\begin{array}{l}.0 \mathrm{E}+00 \\
.0 \mathrm{E}+00 \\
.0 \mathrm{E}+00\end{array}$ & $\begin{array}{l}0 \\
0 \\
0\end{array}$ & $\begin{array}{l}.0 E+00 \\
.0 E+00 \\
.0 E+00\end{array}$ & $\begin{array}{l}0 \\
0 \\
0\end{array}$ \\
\hline $\begin{array}{c}\text { TOTAL } \\
1.6 \mathrm{E}+01\end{array}$ & $\begin{array}{l}\text { INGESTIO } \\
.0 \mathrm{E}+00\end{array}$ & $N_{0}$ & $\begin{array}{l}\text { INHALATION } \\
.0 \mathrm{E}+00\end{array}$ & $\begin{array}{l}1 \\
0\end{array}$ & $\begin{array}{l}\text { EXTERNAL } \\
1.6 \mathrm{E}+01\end{array}$ & 100 & $\begin{array}{l}\text { AQUATIC } \\
.0 \mathrm{E}+00\end{array}$ & $\begin{array}{r}\text { FOOD } \\
0 \quad 0\end{array}$ & $\begin{array}{l}\text { DRINK WATER } \\
.0 \mathrm{E}+00\end{array}$ & ${ }_{0}^{R}$ \\
\hline
\end{tabular}

MAXIMUM ANNUAL DOSE SUMMARY FOR THE YEAR 10 FOR LLI

\begin{tabular}{|c|c|c|c|c|c|c|c|c|c|c|}
\hline $\begin{array}{l}\text { RADIO- } \\
\text { NUCLIDE }\end{array}$ & $\begin{array}{l}\text { INGESTION } \\
\text { REM }\end{array}$ & N & $\begin{array}{l}\text { INHALATIO } \\
\text { REM }\end{array}$ & ON & $\begin{array}{l}\text { EXTERNAL } \\
\text { REM }\end{array}$ & $\%$ & $\begin{array}{l}\text { AQUATIC } \\
\text { REM }\end{array}$ & F0OD & $\begin{array}{l}\text { DRINK WATER } \\
\text { REM }\end{array}$ & R $\%$ \\
\hline $\begin{array}{l}\text { CO60 } \\
\text { SR90 } \\
\text { Y } 90\end{array}$ & $\begin{array}{l}.0 \mathrm{E}+00 \\
.0 \mathrm{E}+00 \\
.0 \mathrm{E}+00\end{array}$ & $\begin{array}{l}0 \\
0 \\
0\end{array}$ & $\begin{array}{l}3.2 \mathrm{E}-01 \\
5.2 \mathrm{E}-02 \\
2.0 \mathrm{E}-01\end{array}$ & $\begin{array}{r}56 \\
9 \\
34\end{array}$ & $\begin{array}{l}1.6 \mathrm{E}+01 \\
1.4 \mathrm{E}-04 \\
9.9 \mathrm{E}-03\end{array}$ & $\begin{array}{r}99 \\
0 \\
0\end{array}$ & $\begin{array}{l}.0 \mathrm{E}+00 \\
.0 \mathrm{E}+00 \\
.0 \mathrm{E}+00\end{array}$ & $\begin{array}{l}0 \\
0 \\
0\end{array}$ & $\begin{array}{l}.0 E+00 \\
.0 E+00 \\
.0 E+00\end{array}$ & $\begin{array}{l}0 \\
0 \\
0\end{array}$ \\
\hline $\begin{array}{c}\text { TOTAL } \\
1.7 E+01\end{array}$ & $\begin{array}{l}\text { INGESTION } \\
.0 \mathrm{E}+00\end{array}$ & $\begin{array}{l}N^{N} \\
0\end{array}$ & $\begin{array}{l}\text { INHALATIO } \\
5.7 \mathrm{E}-01\end{array}$ & 3 & $\begin{array}{l}\text { EXTERNAL } \\
1.6 E+01\end{array}$ & 96 & $\begin{array}{l}\text { AQUATIC } \\
.0 \mathrm{E}+00\end{array}$ & $\begin{array}{r}F 000 \\
0\end{array}$ & $.0 \mathrm{E}+00$ & $\mathrm{R}_{0}$ \\
\hline
\end{tabular}

FIGURE 3.4 (Contd) 


\subsubsection{Sample Problem Three}

The third sample demonstrates the agricultural exposure scenario. For this problem, a licenseg is assumed to dispose of $59 \mathrm{~m}$ gf waste containing 0.1 $\mathrm{Ci} / \mathrm{m}^{3}$ of each of ${ }^{20} \mathrm{Co}$ and ${ }^{3} \mathrm{Cs}$, and $0.01 \mathrm{Ci} / \mathrm{m}^{3}$ of ${ }^{90} \mathrm{Sr}$. Default conditions are assumed except that only 0.05 ha is assumed to be contaminated by the disposal of wastes. The desired result from Sample Problem Three is the dose to the intruder 10 years after the wastes are buried. This sample problem has been expanded in this version of the ONSITE/MAXI1 software package. Both the maximum annual dose (ICRP 1959) and the annual effective dose equivalent, AEDE, (ICRP 1977; ICRP 1979-1982) are to be calculated. The MAXI1 input file for Sample Problem Three is shown in Figure 3.5. Changes from the previous sample problem are samado on the Sample Problem Three input file to assist the user in understanding the second level of software operation.

The output resulting for this sample problem is shown in Figure 3.6. The calculated maximum annual doses from the scenario in this sample problem are about 22 rem to total body, 56 rem to bone, 18 rem to lungs, 13 rem to thyroid, and 17 rem to GI(LLI). Doses to total body, and bone are controlled by ingestion of farm crops, while the doses to lungs, thyroid, and GI(LLI) are controlled by external exposure. The annual external dose was $13 \mathrm{rem}$, and the internal effective dose equivalent was 16 rem. Thus, the annual effective dose equivalent was 29 rem. Results of the following calculations were verified by hand using the equations in Section 2: radioactive decay; soil and air concentration; external dose, inhalation dose, and ingestion dose from consumption of leafy vegetables for both the maximum annual and annual effective dose equivalent. 


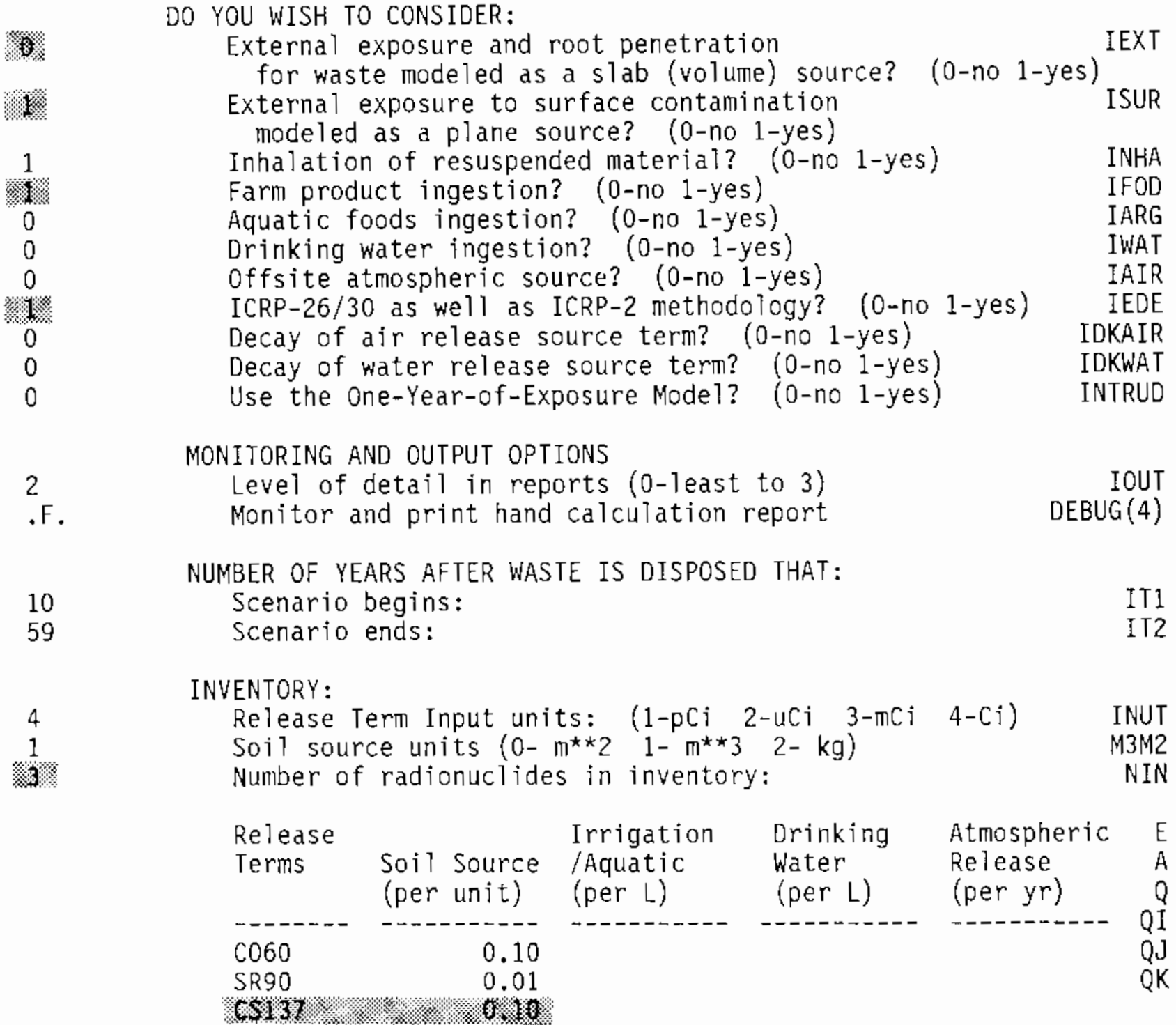

\begin{tabular}{|c|c|c|}
\hline $\begin{array}{l}0.2 \\
1.0 \\
0.0 \\
1.0\end{array}$ & $\begin{array}{l}\text { Surface inventory modification factor: } \\
\text { Irrigation/aquatic inventory modification factor: } \\
\text { Size of site (fractional ha): } \\
\text { Fraction of total diet grown on site: }\end{array}$ & $\begin{array}{l}\text { SRDIL } \\
\text { DILF } \\
\text { FRSIZ } \\
\text { RPF2 }\end{array}$ \\
\hline 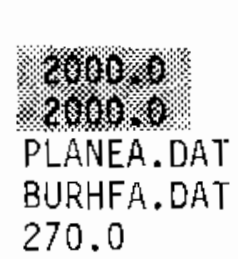 & $\begin{array}{l}\text { TERNAL/INHALATION EXPOSURE: } \\
\text { Hours of external exposure to contamination }(\mathrm{h} / \mathrm{yr}) \text { : } \\
\text { Hours of inhalation of airborne contamination }(\mathrm{h} / \mathrm{yr}) \text { : } \\
\text { Ext. exp. dose rate factors (plane source) filename: } \\
\text { Ext. exp. dose rate factors (volume source) filename: } \\
\text { Breathing rate }\left(\mathrm{cm}^{\star \star} 3 / \mathrm{sec}\right) \text { : }\end{array}$ & $\begin{array}{l}\text { HREXT } \\
\text { HRINH } \\
\text { PLANAM } \\
\text { VOLNAM } \\
\text { BRATE }\end{array}$ \\
\hline
\end{tabular}

FIGURE 3.5. Sample Probiem Three Input 


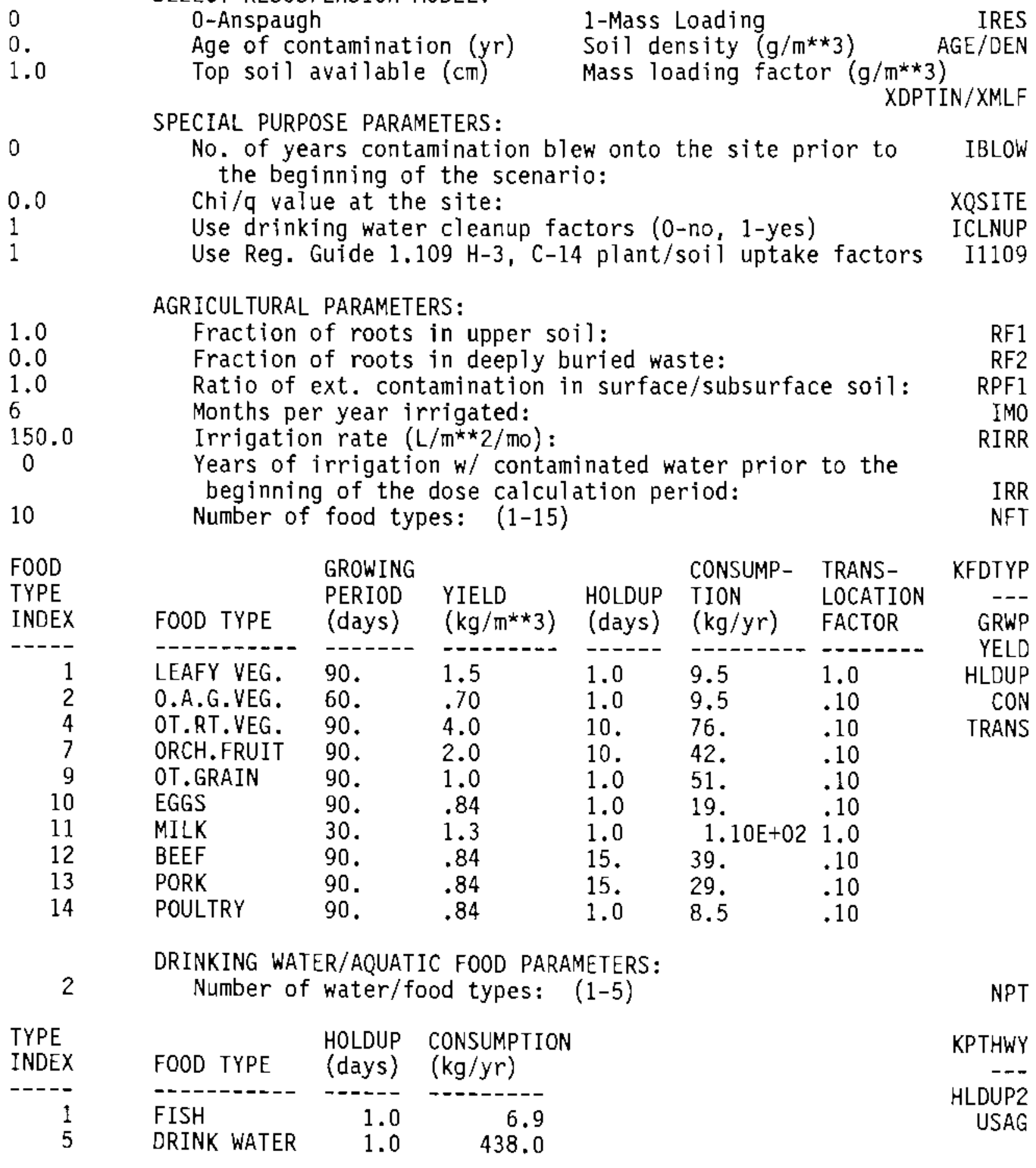

FIGURE 3.5 (Contd) 
(MAXI Version IBM 1.0 30-Jun-B6)

Case title: SAMPLE3.IN / MAXI Sampie Problem Three

Executed on: $10 / 28 / 1986$ at $16: 33: 46$

Page 1

PATHWAYS/OPTIONS CONSIDERED:

External exposure to surface contamination modeled as a plane source

Inhalation of resuspended material

Farm product ingestion

Committed effective dose equivalent (CEDE) calculation

\section{TITLES OF LIBRARY FILES ACCESSED:}

10: RMDLIB - Radionuclide Master Library (24-Sep-86 RAP)

11: ORGAN DATA LIBRARY UPDATED BY RA PELOQUIN 8-Ju?-86

12: FOOD TRANSFER COEFFICIENT LIBRARY (RAP/WTF 04-APR-86 Cl update)

13: Committed Dose Equivalents (Sv/Bq) ICRP Publication 30 (28-0ct-86 RAP)

22: OVERBURDEN: $0.0 \mathrm{M}$, SOURCE: $0.15 \mathrm{M}$ (DEN: CONCRETE/1.8): MR/HR 8-Jul-86 RAP

23: DACRIN (DIFDOS) DOSE INCREMENT FILE ONSITE/MAXI 26-Aug-86 RAP

NUMBER OF YEARS AFTER WASTE IS DISPOSED THAT:

Scenario begins:

Scenario ends:

INVENTORY:

Release Term Input units: (1-pCi 2-uCi 3-mCi 4-Ci)

Soil source units: (0- $\left.m^{\star *} 2 \quad 1-m^{\star *} 3 \quad 2-\mathrm{kg}\right)$

Number of radionuclides in inventory:

\begin{tabular}{lrrrr}
$\begin{array}{l}\text { Release } \\
\text { Terms }\end{array}$ & $\begin{array}{c}\text { Soil Source } \\
\text { (per } m^{\star * 3)}\end{array}$ & $\begin{array}{l}\text { Irrigation } \\
\text { Aquatic } \\
\text { (per L) }\end{array}$ & $\begin{array}{l}\text { Drinking } \\
\text { Water } \\
\text { (per L) }\end{array}$ & $\begin{array}{l}\text { Atmospheric } \\
\text { Release } \\
\text { (per m3) }\end{array}$ \\
\hline C060 & $1.0 \mathrm{E}+11$ & $.0 \mathrm{E}+00$ & $.0 \mathrm{E}+00$ & $.0 \mathrm{E}+00$ \\
SR90 & $1.0 \mathrm{E}+10$ & $.0 \mathrm{E}+00$ & $.0 \mathrm{E}+00$ & $.0 \mathrm{E}+00$ \\
Y 90 & $.0 \mathrm{E}+00$ & $.0 \mathrm{E}+00$ & $.0 \mathrm{E}+00$ & $.0 \mathrm{E}+00$ \\
CS137 & $1.0 \mathrm{E}+11$ & $.0 \mathrm{E}+00$ & $.0 \mathrm{E}+00$ & $.0 \mathrm{E}+00$ \\
BA137M & $.0 \mathrm{E}+00$ & $.0 \mathrm{E}+00$ & $.0 \mathrm{E}+00$ & $.0 \mathrm{E}+00$
\end{tabular}

INVENTORY MODIFICATION FACTORS: (multipliers)

Surface inventory dilution factor:

Irrigation/aquatic inventory modification factor:

Size of site (fractional ha):

Fraction of total diet grown on site:

.2

1.

$5.0 E-02$

1 .

FIGURE 3.6. Sample Problem Three Output 
ICRP-2 and ICRP-26/30 Maximum Annual Dose Calculation

(MAXI Version IBM 1.0 30-Jun-86)

Case title: SAMPLE3.IN / MAXI Sample Problem Three

Executed on: $10 / 28 / 1986$ at $16: 33: 46$

Page 2

EXTERNAL/INHALATION EXPOSURE:

Hours of external exposure to contamination (h/yr):

2.0 0 +03

Hours of inhalation of airborne contamination $(\mathrm{h} / \mathrm{yr})$ :

2. $0 \mathrm{E}+03$

Breathing rate $\left(\mathrm{cm}^{\star \star} 3 / \mathrm{sec}\right)$ :

$2.7 \mathrm{E}+02$

RESUSPENSION PARAMETERS:

Model used:

Average age of contamination on ground at Time Zero(yr):

Top soil available for resuspension $(\mathrm{cm})$ :

Anspaugh

.0

1 .

AGRICULTURAL PARAMETERS:

Fraction of roots in upper soil:

1.00

Fraction of roots in deeply buried waste: $\quad .000$

Ratio of ext. contamination in surface/subsurface soil: 1.00

Months per year irrigated:

Irrigation rate $\left(\mathrm{L} / \mathrm{m}^{\star \star} 2 / \mathrm{mo}\right)$ :

Years of irrigation $\mathrm{w} /$ contaminated water prior to the beginning of the dose calculation period:

Number of food types:

FOOD
TYPE
INDEX
-1
2
4
7
9
10
11
12
13
14

\begin{tabular}{l} 
FOOD TYPE \\
\hline LEAFY VEG. \\
O.A.G.VEG. \\
OT.RT.VEG. \\
ORCH. FRUIT \\
OT.GRAIN \\
EGGS \\
MILK \\
BEEF \\
PORK \\
POULTRY
\end{tabular}

GROWING

PERIOD

(days)

90.

YIELD

60.

90.

90.

90.

90.

30 .

90 .

90.

90 .

$\left(\mathrm{kg} / \mathrm{m}^{\star *} 3\right)$

HOLDUP TION

CONSUMP- TRANS-

$1.5 \mathrm{E}+02$

1.50
.70
4.00
2.00
1.00
.84
1.30
.84
.84
.84

(days)

$(\mathrm{kg} / \mathrm{yr})$

LOCATION

FACTOR

1.
1.
10.
10.
1.
1.
1.
15.
15.
1.

$\begin{array}{rr}9.5 & 1.00 \\ 9.5 & .10 \\ 76.0 & .10 \\ 42.0 & .10 \\ 51.0 & .10 \\ 19.0 & .10 \\ 110.0 & 1.00 \\ 39.0 & .10 \\ 29.0 & .10 \\ 8.5 & .10\end{array}$

FIGURE 3.6 (Contd) 
ICRP-2 and ICRP-26/30 Maximum Annual Dose Calculation

(MAXI Version IBM $1.030-J u n-86$ )

Case title: SAMPLE3.IN / MAXI Sample Problem Three

Executed on: $10 / 28 / 1986$ at $16: 33: 46$

Page 3

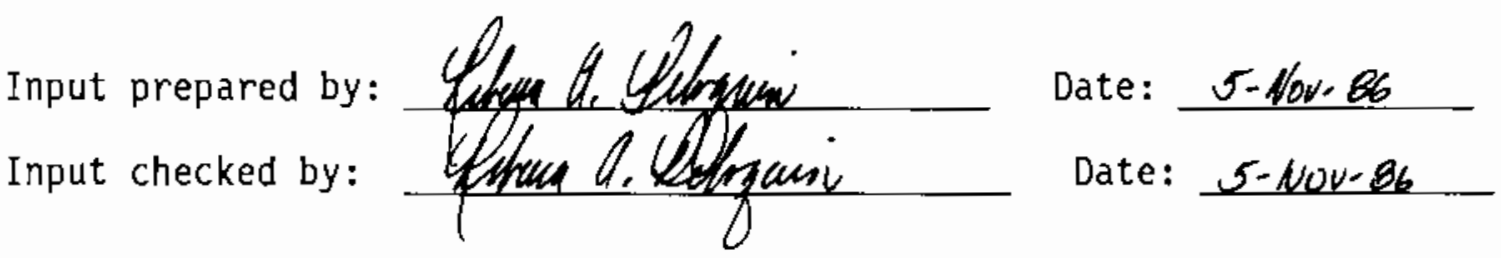

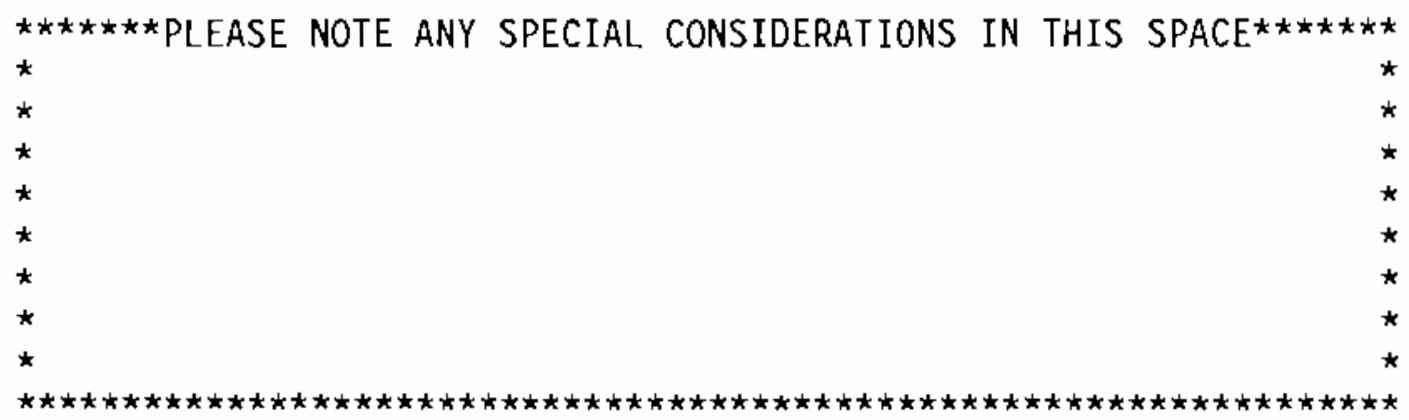

\section{CALCULATED VALUES:}

Ingestion area correction factor:

External/inhalation area correction factor:

.50

Inhalation exposure modification factor:

External exposure modification factor: 
ICRP-2 and ICRP-26/30 Maximum Annual Dose Calculation

(MAXI Version IBM $1.030-J u n-86$ )

Case title: SAMPLE3.IN / MAXI Sample Problem Three

Executed on: $10 / 28 / 1986$ at $16: 33: 46$

Page 4

SOIL, AIR, AND WATER CONCENTRATION SUMMARY FOR YEAR: 10

\begin{tabular}{lccccc}
$\begin{array}{l}\text { Radio- } \\
\text { nuclide }\end{array}$ & $\begin{array}{c}\text { Surface Soil } \\
\mathrm{pCi} / \mathrm{m} 2\end{array}$ & $\begin{array}{c}\text { Deep Soil } \\
\mathrm{pCi} / \mathrm{m3}\end{array}$ & $\begin{array}{c}\mathrm{Air} \\
\mathrm{pCi} / \mathrm{m} 3\end{array}$ & $\begin{array}{c}\text { Irrigation } \\
\mathrm{pCi} / \mathrm{L}\end{array}$ & $\begin{array}{c}\text { Drink Water } \\
\mathrm{pCi} / \mathrm{L}\end{array}$ \\
\hline C0 60 & $-0.0 \mathrm{E}+08$ & $.0 \mathrm{E}+00$ & $5.4 \mathrm{E}+03$ & $.0 \mathrm{E}+00$ & $.0 \mathrm{E}+00$ \\
SR 90 & $2.4 \mathrm{E}+08$ & $.0 \mathrm{E}+00$ & $1.6 \mathrm{E}+03$ & $.0 \mathrm{E}+00$ & $.0 \mathrm{E}+00$ \\
Y 90 & $2.4 \mathrm{E}+08$ & $.0 \mathrm{E}+00$ & $1.6 \mathrm{E}+03$ & $.0 \mathrm{E}+00$ & $.0 \mathrm{E}+00$ \\
CS 137 & $2.4 \mathrm{E}+09$ & $.0 \mathrm{E}+00$ & $1.6 \mathrm{E}+04$ & $.0 \mathrm{E}+00$ & $.0 \mathrm{E}+00$ \\
BA $137 \mathrm{M}$ & $2.3 \mathrm{E}+09$ & $.0 \mathrm{E}+00$ & $1.5 \mathrm{E}+04$ & $.0 \mathrm{E}+00$ & $.0 \mathrm{E}+00$
\end{tabular}

SOIL, AIR, AND WATER CONCENTRATION SUMMARY FOR YEAR: 59

\begin{tabular}{lccccc}
$\begin{array}{l}\text { Radio- } \\
\text { nuclide }\end{array}$ & $\begin{array}{c}\text { Surface Soil } \\
\mathrm{pCi} / \mathrm{m} 2\end{array}$ & $\begin{array}{c}\text { Deep Soil } \\
\mathrm{pCi} / \mathrm{m} 3\end{array}$ & $\begin{array}{c}\text { Air } \\
\mathrm{pCi} / \mathrm{m} 3\end{array}$ & $\begin{array}{c}\text { Irrigation } \\
\mathrm{pCi} / \mathrm{L}\end{array}$ & $\begin{array}{c}\text { Drink Water } \\
\mathrm{pCi} / \mathrm{L}\end{array}$ \\
\hline C0 60 & $-1.3 \mathrm{E}+06$ & $.0 \mathrm{E}+00$ & $8.4 \mathrm{E}-05$ & $.0 \mathrm{E}+00$ & $.0 \mathrm{E}+00$ \\
SR 90 & $7.1 \mathrm{E}+07$ & $.0 \mathrm{E}+00$ & $4.8 \mathrm{E}-03$ & $.0 \mathrm{E}+00$ & $.0 \mathrm{E}+00$ \\
Y 90 & $7.1 \mathrm{E}+07$ & $.0 \mathrm{E}+00$ & $4.8 \mathrm{E}-03$ & $.0 \mathrm{E}+00$ & $.0 \mathrm{E}+00$ \\
CS 137 & $7.7 \mathrm{E}+08$ & $.0 \mathrm{E}+00$ & $5.1 \mathrm{E}-02$ & $.0 \mathrm{E}+00$ & $.0 \mathrm{E}+00$ \\
BA $137 \mathrm{M}$ & $7.3 \mathrm{E}+08$ & $.0 \mathrm{E}+00$ & $4.9 \mathrm{E}-02$ & $.0 \mathrm{E}+00$ & $.0 \mathrm{E}+00$
\end{tabular}

FIGURE 3.6 (Contd) 
ICRP-2 and ICRP-26/30 Maximum Annual Dose Calculation

(MAXI Version IBM 1.0 30-Jun-86)

Case title: SAMPLE3.IN / MAXI Sample Problem Three

Executed on: $10 / 28 / 1986$ at $16: 33: 46$

Page 5

Annual Effective Dose Equivalent (AEDE):

2. $9 E+01$

Maximum Annual Dose (ICRP-2):

To Organ: TOTAL BODY at Year 10

To Organ: BONE at Year 27

To Organ: LUNGS at Year 11

$2.2 \mathrm{E}+01$

$5.6 \mathrm{E}+01$

$1.8 \mathrm{E}+01$

To Organ: THYRDID at Year 10

$1.3 \mathrm{E}+01$

To Organ:

LLI

at Year 10

$1.7 \mathrm{E}+01$

FIGURE 3.6 (Contd)

3.30 
ICRP-2 and ICRP-26/30 Maximum Annual Dose Calculation

(MAXI Version IBM 1.0 30-Jun-86)

Case title: SAMPLE3.IN / MAXI Sample Problem Three

Executed on: $10 / 28 / 1986$ at $16: 33: 46$

Page 6

DOSES FROM 1 YEAR OF EXPOSURE

\begin{tabular}{|c|c|c|c|}
\hline Organ & $\begin{array}{l}\text { Committed } \\
\text { Dose } \\
\text { Equivalent }\end{array}$ & $\begin{array}{r}\text { Weighing } \\
\text { Factors }\end{array}$ & $\begin{array}{l}\text { Weighted } \\
\text { Dose } \\
\text { Equivalent }\end{array}$ \\
\hline $\begin{array}{l}\text { Gonads } \\
\text { Breast } \\
\text { R Marrow } \\
\text { Lungs } \\
\text { Thyroid } \\
\text { Bone Surf } \\
\text { Remainder } 3 \\
\text { LLI Wall } \\
\text { ULI Wal1 } \\
\text { SI Wall } \\
\text { Remainder } 1\end{array}$ & $\begin{array}{l}1.0 \mathrm{E}+01 \\
8.4 \mathrm{E}+00 \\
3.2 \mathrm{E}+01 \\
2.0 \mathrm{E}+01 \\
8.9 \mathrm{E}+00 \\
6.0 \mathrm{E}+01 \\
3.2 \mathrm{E}+01 \\
1.1 \mathrm{E}+01 \\
1.0 \mathrm{E}+01 \\
1.0 \mathrm{E}+01 \\
9.7 \mathrm{E}+00\end{array}$ & $\begin{array}{l}2.5 \mathrm{E}-01 \\
1.5 \mathrm{E}-01 \\
1.2 \mathrm{E}-01 \\
1.2 \mathrm{E}-01 \\
3.0 \mathrm{E}-02 \\
3.0 \mathrm{E}-02 \\
6.0 \mathrm{E}-02 \\
6.0 \mathrm{E}-02 \\
6.0 \mathrm{E}-02 \\
6.0 \mathrm{E}-02 \\
6.0 \mathrm{E}-02\end{array}$ & $\begin{array}{l}2.5 \mathrm{E}+00 \\
1.3 \mathrm{E}+00 \\
3.9 \mathrm{E}+00 \\
2.4 \mathrm{E}+00 \\
2.7 \mathrm{E}-01 \\
1.8 \mathrm{E}+00 \\
1.9 \mathrm{E}+00 \\
6.5 \mathrm{E}-01 \\
6.2 \mathrm{E}-01 \\
6.0 \mathrm{E}-01 \\
5.8 \mathrm{E}-01\end{array}$ \\
\hline $\begin{array}{l}\text { Effective Dose } \\
\text { External Dose }\end{array}$ & Equivalent & & $\begin{array}{l}1.6 \mathrm{E}+01 \\
1.3 \mathrm{E}+01\end{array}$ \\
\hline & & & 2. $9 \mathrm{E}+01$ \\
\hline
\end{tabular}

FIGURE 3.6 (Contd) 
ICRP-2 and ICRP-26/30 Maximum Annual Dose Calculation

(MAXI Version IBM 1.0 30-Jun-86)

Case title: SAMPLE3.IN / MAXI Sample Problem Three

Executed on: $10 / 28 / 1986$ at $16: 33: 46$

Page 7

MAXIMUM ANNUAL DOSE SUMMARY FOR THE YEAR 10 FOR TOTAL BODY-...-.-

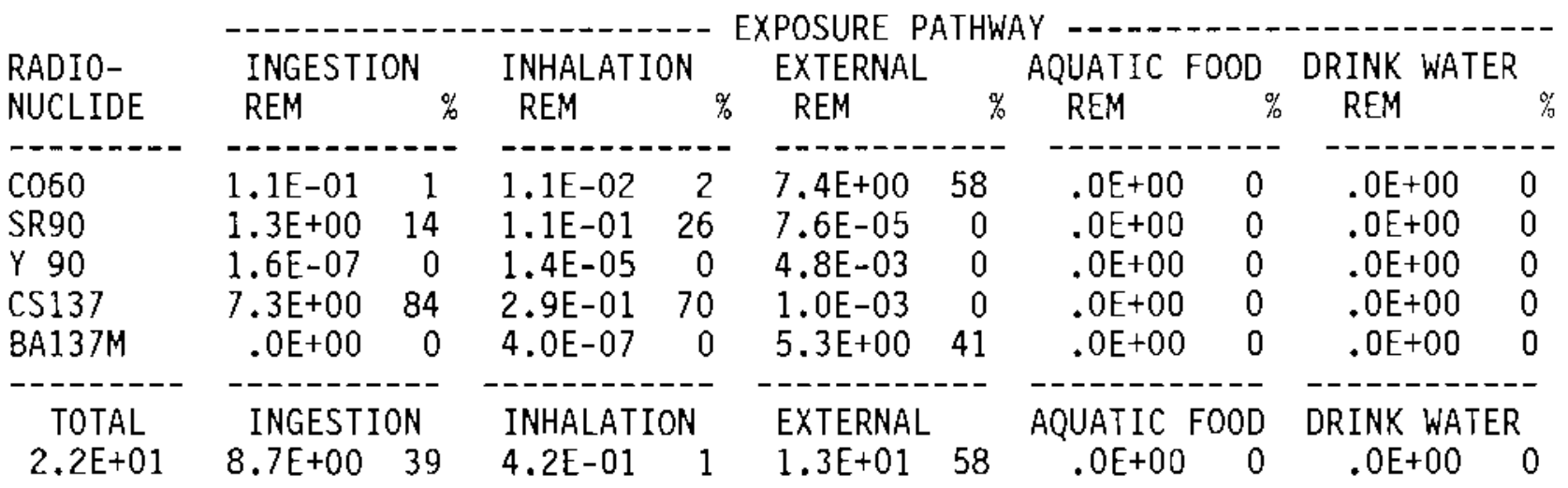

MAXIMUM ANNUAL DOSE SUMMARY FOR THE YEAR 27 FOR BONE

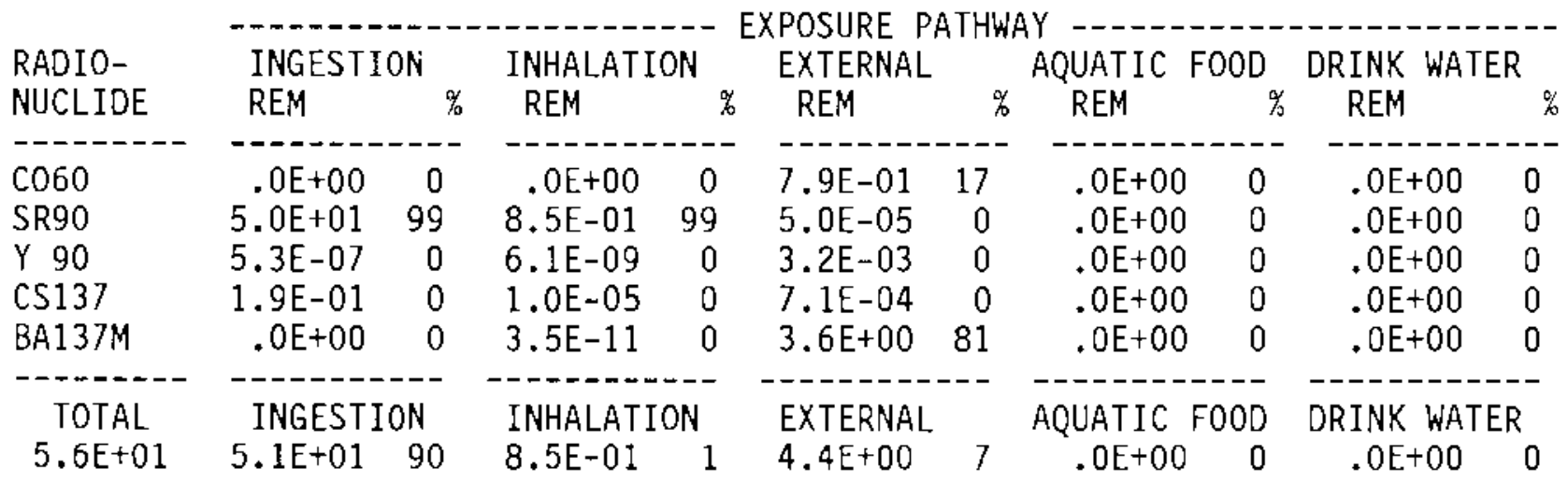

MAXIMUM ANNUAL DOSE SUMMARY FOR THE YEAR 11 FOR LUNGS

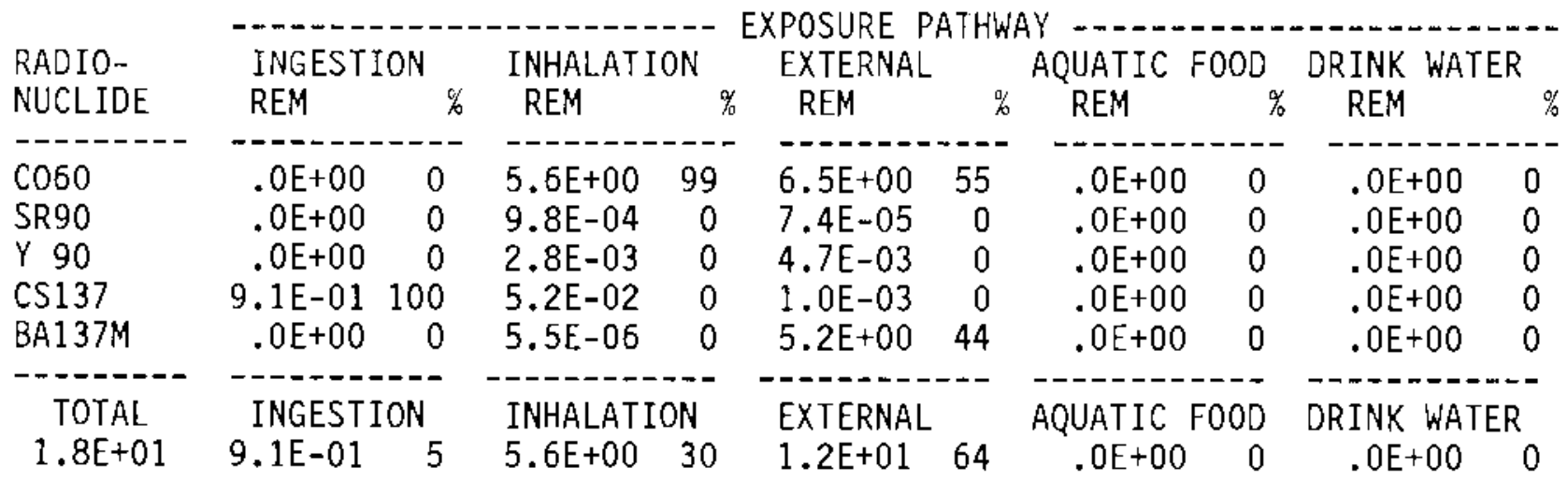

FIGURE 3.6 (Contd) 
ICRP-2 and ICRP-26/30 Maximum Annual Dose Calculation

(MAXI Version IBM 1.0 30-Jun-86)

Case title: SAMPLE3.IN / MAXI Sample Problem Three

Executed on: $10 / 28 / 1986$ at $16: 33: 46$

Page 8

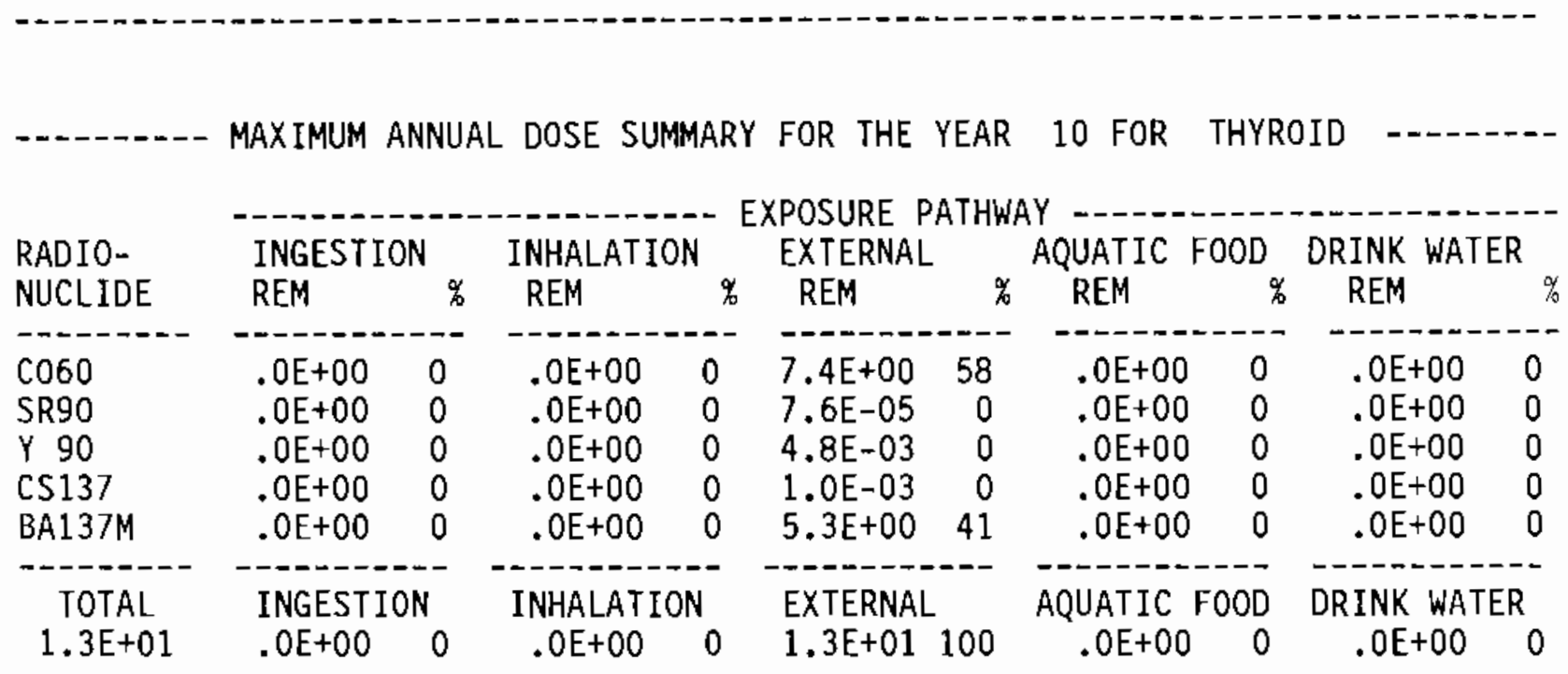

MAXIMUM ANNUAL DOSE SUMMARY FOR THE YEAR 10 FOR LLI

\begin{tabular}{|c|c|c|c|c|c|c|c|c|c|c|}
\hline $\begin{array}{l}\text { RADI0- } \\
\text { NUCLIDE }\end{array}$ & $\begin{array}{l}\text { INGESTIO } \\
\text { REM }\end{array}$ & $\%$ & $\begin{array}{l}\text { INHALATIC } \\
\text { REM }\end{array}$ & ON & $\begin{array}{l}\text { EXTERNAL } \\
\text { REM }\end{array}$ & $\%$ & $\begin{array}{c}\text { AQUATIC } \\
\text { REM }\end{array}$ & $O D$ & $\begin{array}{c}\text { DRINK WATE } \\
\text { REM }\end{array}$ & $\mathbf{R}$ \\
\hline $\begin{array}{l}\text { C060 } \\
\text { SR90 } \\
\text { Y } 90 \\
\text { CS137 } \\
\text { BA137M }\end{array}$ & $\begin{array}{l}.1 \mathrm{E} \\
.2 \mathrm{E} \\
.3 \mathrm{E} \\
.0 \mathrm{E} \\
.0 \mathrm{E}\end{array}$ & $\begin{array}{r}29 \\
58 \\
1 \\
10 \\
0\end{array}$ & $\begin{array}{r}1.1 \\
1.7 \\
6.5 \\
5.5 \\
.0\end{array}$ & $\begin{array}{r}55 \\
8 \\
33 \\
2 \\
0\end{array}$ & $\begin{array}{l}7.41 \\
7.61 \\
4.81 \\
1.01 \\
5.31\end{array}$ & $\begin{array}{r}58 \\
0 \\
0 \\
0 \\
41\end{array}$ & & $\begin{array}{l}0 \\
0 \\
0 \\
0 \\
0\end{array}$ & & $\begin{array}{l}0 \\
0 \\
0 \\
0 \\
0\end{array}$ \\
\hline $\begin{array}{c}\text { TOTAL } \\
1.7 \mathrm{E}+01\end{array}$ & $\begin{array}{l}\text { INGESTIO } \\
3.8 \mathrm{E}+00\end{array}$ & $\begin{array}{l}\text { ON } \\
22\end{array}$ & $.0 \mathrm{E}-01$ & 1 & $\begin{array}{l}\text { EXTERNAL } \\
1.3 \mathrm{E}+01\end{array}$ & 76 & $.0 \mathrm{E}+0 \mathrm{C}$ & $\begin{array}{r}F O 0 D \\
0\end{array}$ & $.0 E+00$ & 0 \\
\hline
\end{tabular}

FIGURE 3.6 (Contd) 


\subsubsection{Sample Problem Four}

The fourth sample problem demonstrates the irrigation/drinking-water scenario. E8r this prablem, a ligensee is assumed to dispose of wastes that result in ${ }^{6} \mathrm{C},{ }^{129} \mathrm{I},{ }^{\mathrm{Sr}}$, and ${ }^{238} \mathrm{U}$ in an offsite well. The water concentrations are $10 \mathrm{pC} \mathrm{C} / \mathrm{L}$ of each radionuclide. In addition, an offsite river-water concentration of $0.1 \mathrm{pCi} / \mathrm{L}$ of each of these radionuclides is assumed. The individual is assumed to use the well water for drinking and the river water for irrigation. The purpose of this sample problem is to determine the doses to this individual from using these contaminated water sources. This sample problem has been expanded in this version of the ONSITE/MAXI1 software package. Both the maximum annual dose (ICRP 1959) and the annual effective dose equivalent, AEDE, (ICRP 1977; ICRP 1979-1982) are to be calculated. The MAXI1 input file for Sample Problem Four is shown in Figure 3.7. Changes from the previous sample problem are uaded on the Sample Problem Four input file to assist the user in understanding the second level of software operation.

The output resulting for this sample problem is shown in Figure 3.8. The calculated maximum annual doses for the scenario in Sample Problem Four are about 0.0016 rem to total body, 0.0077 rem to bone $8.0 \times 10^{-6}$ rem to lungs, 0.028 rem to thyrojd, and $3.5 \times 10^{-4}$ rem to GI(LLI). The annual external dose was $5.7 \times 10^{-6}$ rem, and the internal effective dose equivalent was $1.2 \times 10^{-3}$ rem. Thus, the annual effective dose equivalent was $1.3 \times 10^{-3}$ rem. Results of the radionuclide decay, soil and air concentration, and maximum annual dose estimate calculations were verified by hand using the equations in Section 2 . 
DO YOU WISH TO CONSIDER:

0

1 270.0
External exposure and root penetration for waste modeled as a slab (volume) source? (0-no 1-yes)

Externat exposure to surface contamination modeled as a plane source? (0-no 1-yes)

Inhalation of resuspended material? (0-no 1-yes) INHA

Farm product ingestion? (0-no 1-yes)

IFOD

Aquatic foods ingestion? (0-no 1-yes)

IARG

Drinking water ingestion? (0-no 1-yes)

IWAT

Offsite atmospheric source? (0-no 1-yes)

IAIR

ICRP-26/30 as well as ICRP-2 methodology? (0-no 1-yes)

IEDE

Decay of air release source term? (0-no 1-yes)

Decay of water release source term? (0-no 1-yes)

IDKAIR

Use the One-Year-of-Exposure Model? (0-no 1-yes)

IOKWAT

INTRUD

MONITORING AND OUTPUY OPTIONS

Level of detail in reports (1-least to 3 )

IOUT

Monitor and print hand calculation report

DEBUG (4)

NUMBER OF YEARS AFTER WASTE IS DISPOSED THAT: Scenario begins:

IT1

Scenario ends:

IT2

INVENTORY:

Release Term Input units: (1-pCi 2-uCi 3-mCi 4-Ci) INUT

Soil source units $\left(0-m^{\star \star 2} \quad 1-m^{\star \star} 3 \quad 2-\mathrm{kg}\right)$

Number of radionuclides in inventory:

M3M2

NIN

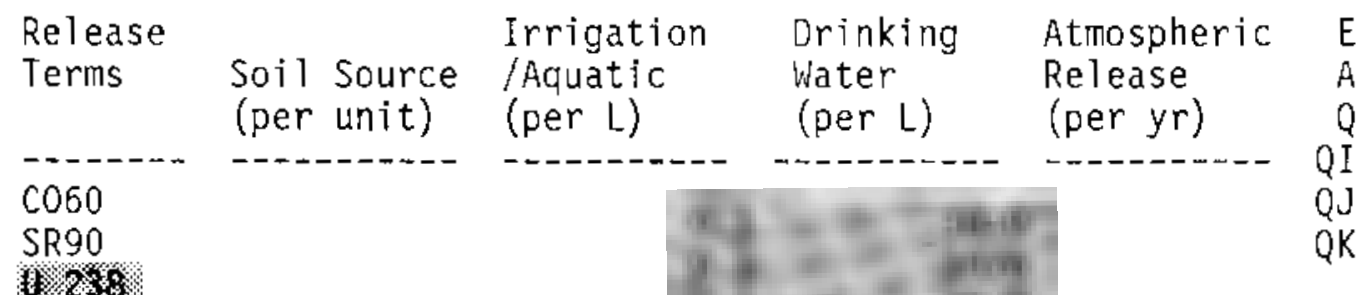

INVENTORY MODIFICATION FACTORS: (multipliers)

Surface inventory modification factor:

SRDIL

Irrigation/aquatic inventory modification factor:

DILF

Size of site (fractional ha):

Fraction of total diet grown on site:

FRSIZ

RPF2

EXTERNAL/INHALATION EXPOSURE:

Hours of external exposure to contamination (h/yr): HREXT

Hours of inhalation of airborne contamination (h/yr): HRINH

Ext. exp. dose rate factors (plane source) filename: PLANAM

Ext. exp. dose rate factors (volume source) filename: VOLNAM

Breathing rate $\left(\mathrm{cm}^{\star \star} 3 / \mathrm{sec}\right)$ :

BRATE

\section{FIGURE 3.7. Sample Problem Four Input}




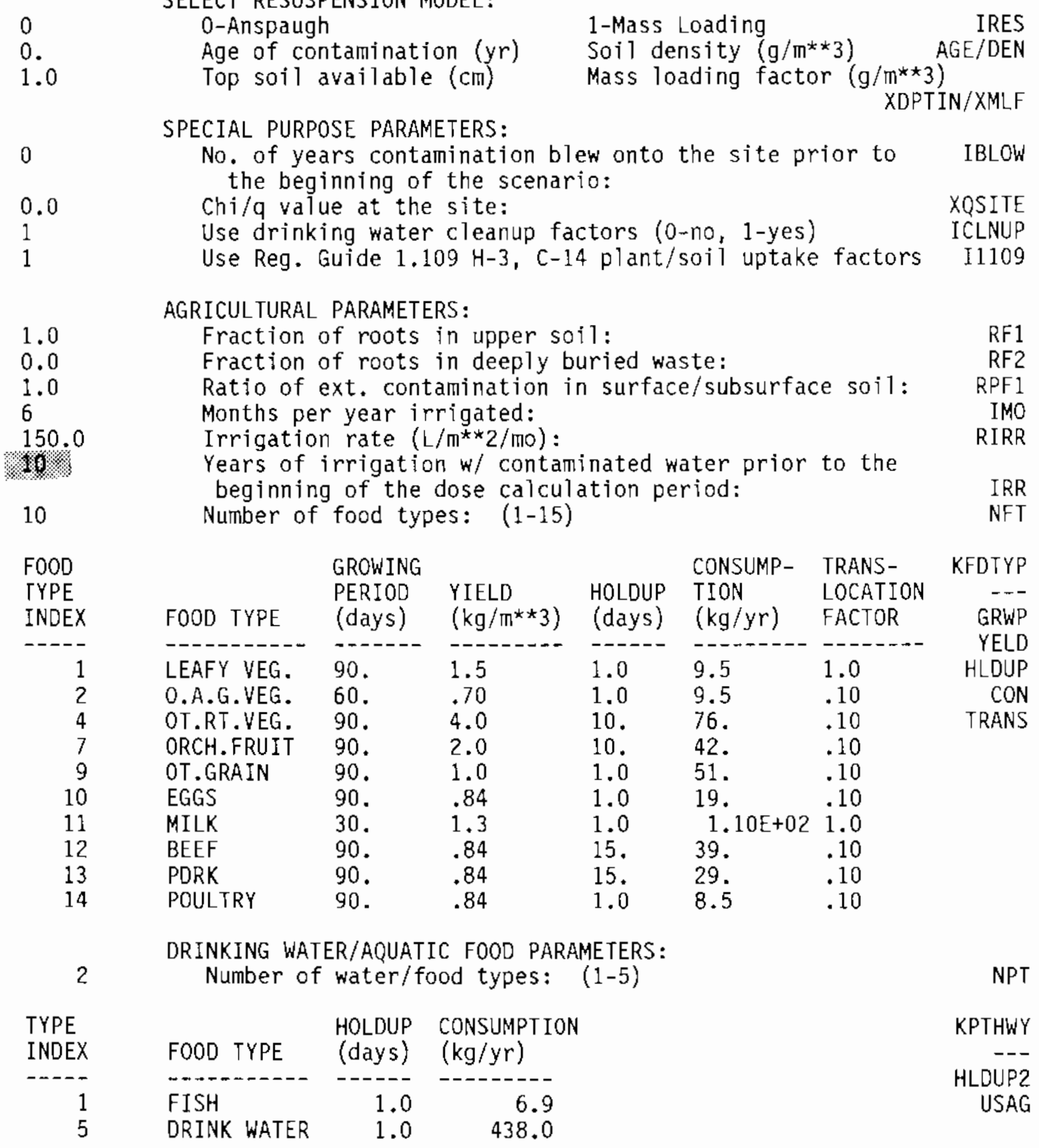

$\underline{\text { FIGURE } 3.7 \text { (Contd) }}$ 
ICRP-2 and ICRP-26/30 Maximum Annual Dose Calculation

(MAXI Version IBM 1.0 30-Jun-86)

Case titie: SAMPLE4. IN / MAXI Sample Problem Four

Executed on: $10 / 28 / 1986$ at $16: 36: 28$

Page 1

PATHWAYS/OPTIONS CONSIDERED:

External exposure to surface contamination modeled as a plane source

Inhalation of resuspended material

Farm product ingestion

Drinking water ingestion

Committed effective dose equivalent (CEDE) calculation

TITLES OF LIBRARY FILES ACCESSED:

10: RMDLIB - Radionuclide Master Library (24-Sep-86 RAP)

11: ORGAN DATA LIBRARY UPDATED BY RA PELDQUIN B-JUT-86

12: FOND TRANSFER COEFFICIENT LIBRARY (RAP/WTF 04-APR-86 Cl Update)

13: Committed Dose Equivalents (Sv/Bq) ICRP Publication 30 (28-0ct-86 RAP)

14: BIOACCUMULATION FACTOR LIBRARY FOR BA NAPIER (04-APR-86/WTF)

22: OVERBURDEN: $0.0 \mathrm{M}$, SOURCE: $0.15 \mathrm{M}$ (DEN: CONCRETE/1.8): MR/HR 8-Jul-86 RAP

23: DACRIN (DIFDOS) DOSE INCREMENT FILE ONSITE/MAXI 26-Aug-86 RAP

NUMBER OF YEARS AFTER WASTE IS DISPOSED THAT:

Scenario begins:

Scenario ends:

INVENTORY:

Release Term Input units: (1-pCi 2-uCi $3-m C i \quad 4-C i)$

Soil source units: $\left(0-m^{\star * 2} 1-m^{* * 3} 2-\mathrm{kg}\right)$

Number of radionuclides in inventory:

Release

Terms

\begin{tabular}{c} 
Soi1 Source \\
(per $\mathrm{m}^{\star *} 3$ ) \\
\hline $.0 \mathrm{E}+00$ \\
$.0 \mathrm{E}+00$ \\
$.0 \mathrm{E}+00$ \\
$.0 \mathrm{E}+00$ \\
$.0 \mathrm{E}+00$ \\
$.0 \mathrm{E}+00$ \\
$.0 \mathrm{E}+00$ \\
$.0 \mathrm{E}+00$
\end{tabular}

Irrigation

/Aquatic

Drinking

Water

Atmospheric

-..--..--

(per L)

(per L) (per

$\mathrm{CO60}$

SR90

Y 90

I 129

U 238

TH234

PA234M

PA234

$.0 \mathrm{E}+00$

$$
\begin{array}{r}
1.0 \mathrm{E}-01 \\
1.0 \mathrm{E}-01 \\
.0 \mathrm{E}+00 \\
1.0 \mathrm{E}-01 \\
1.0 \mathrm{E}-01 \\
.0 \mathrm{E}+00 \\
.0 \mathrm{E}+00 \\
.0 \mathrm{O}+00
\end{array}
$$

(per m3)

1. $0 \mathrm{E}+01$

1. $0 E+01$

$.0 \mathrm{E}+00$

1. $0 \mathrm{E}+01$

1. $0 E+01$

$.0 E+00$

$.0 \mathrm{E}+00$

$.0 \mathrm{E}+00$
1

4

$.0 \mathrm{E}+00$

$.0 \mathrm{E}+00$

$.0 E+00$

$.0 \mathrm{E}+00$

$.0 \mathrm{E}+00$

$.0 E+00$

$.0 E+00$

$.0 E+00$

INVENTORY MODIFICATION FACTORS: (multipliers)

Surface inventory dilution factor:

Irrigation/aquatic inventory modification factor:

Size of site (fractional ha):

Fraction of total diet grown on site:

$i^{2}$
$1.0 E+00$
1.

FIGURE 3.8. Sample Problem Four Output 


\section{ICRP-2 and ICRP-26/30 Maximum Annual Dose Calculation}

(MAXI Version IBM 1.030 -Jun-86)

Case title: SAMPLE4.IN / MAXI Sample Problem Four

Executed on: $10 / 28 / 1986$ at $16: 36: 28$

Page 2

EXTERNAL/INHALATION EXPOSURE:

Hours of external exposure to contamination (h/yr):

Hours of inhalation of airborne contamination $(h / y r)$ :

Breathing rate $\left(\mathrm{cm}^{\star \star} 3 / \mathrm{sec}\right)$ :

2. $0 \mathrm{E}+03$

$2.0 \mathrm{E}+03$

$2.7 \mathrm{E}+02$

RESUSPENSION PARAMETERS:

Model used:

Average age of contamination on ground at Time Zero(yr):

Top soil available for resuspension (cm):

Anspaugh

.0

1 .

1.00

.000

Ratio of ext. Contamination in surface/subsurface soil: 1.00

Months per year irrigated:

Irrigation rate $\left(\mathrm{L} / \mathrm{m}^{\star \star} 2 / \mathrm{mo}\right)$ :

Years of irrigation $\mathrm{w} /$ contaminated water prior to the beginning of the dose calculation period:

Number of food types:

\begin{tabular}{|c|c|c|c|c|c|c|}
\hline $\begin{array}{l}\text { FOOD } \\
\text { TYPE } \\
\text { INDEX }\end{array}$ & FOOD TYPE & $\begin{array}{l}\text { GROWING } \\
\text { PERIOD } \\
\text { (days) }\end{array}$ & $\begin{array}{l}\text { YIELD } \\
\left(\mathrm{kg} / \mathrm{m}^{\star \star} 3\right)\end{array}$ & $\begin{array}{l}\text { HOLDUP } \\
\text { (days) }\end{array}$ & $\begin{array}{l}\text { CONSUMP- } \\
\text { TION } \\
(\mathrm{kg} / \mathrm{yr})\end{array}$ & $\begin{array}{l}\text { TRANS- } \\
\text { LOCATION } \\
\text { FACTOR }\end{array}$ \\
\hline--- & 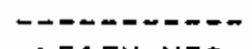 & ------- & $-------x$ & $--m--$ & ------ & ---- \\
\hline 1 & LEAFY VEG. & 90. & 1.50 & 1. & 9.5 & 1.00 \\
\hline 2 & O.A.G.VEG. & 60. & .70 & 1. & 9.5 & .10 \\
\hline 4 & OT.RT.VEG. & 90. & 4.00 & 10. & 76.0 & .10 \\
\hline 7 & ORCH.FRUIT & 90. & 2.00 & 10. & 42.0 & .10 \\
\hline 9 & OT.GRAIN & 90. & 1.00 & 1. & 51.0 & .10 \\
\hline 10 & EGGS & 90. & .84 & 1. & 19.0 & .10 \\
\hline 11 & MILK & 30. & 1.30 & 1. & 110.0 & 1.00 \\
\hline 12 & BEEF & 90 . & .84 & 15. & 39.0 & .10 \\
\hline 13 & PORK & 90 . & .8 & 15. & 29.0 & .10 \\
\hline 14 & POULTRY & 90. & .84 & 1. & 8.5 & .10 \\
\hline
\end{tabular}

FIGURE 3.8 (Contd) 
ICRP-2 and ICRP-26/30 Maximum Annual Dose Calculation

(MAXI Version IBM 1.0 30-Jun-86)

Case title: SAMPLE4. IN / MAXI Sample Problem Four

Executed on: $10 / 28 / 1986$ at $16: 36: 28$

Page 3

DRINKING WATER/AQUATIC FOOD PARAMETERS:

Number of water/food types:

\begin{tabular}{|c|c|c|c|}
\hline $\begin{array}{l}\text { TYPE } \\
\text { INDEX }\end{array}$ & FOOD TYPE & $\begin{array}{l}\text { HOLDUP } \\
\text { (days) }\end{array}$ & $\begin{array}{l}\text { CONSUMPTION } \\
(\mathrm{kg} / \mathrm{yr})\end{array}$ \\
\hline & 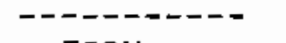 & ----- & \\
\hline $\begin{array}{l}1 \\
5\end{array}$ & $\begin{array}{l}\text { FISH } \\
\text { DRINK WATER }\end{array}$ & $\begin{array}{l}1.0 \\
1.0\end{array}$ & $\begin{array}{r}6.9 \\
438.0\end{array}$ \\
\hline
\end{tabular}

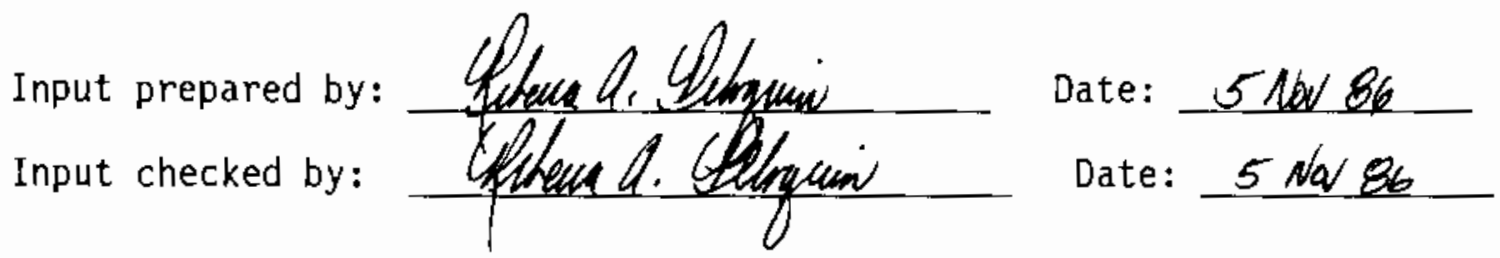

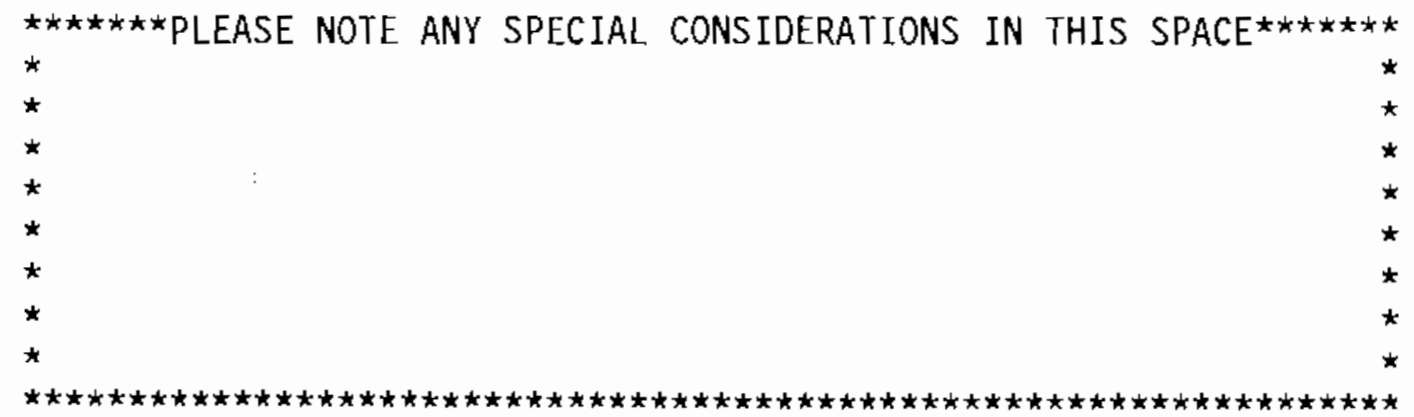

CALCULATED VALUES:

Ingestion area correction factor:

1.0

External/inhalation area correction factor:

1.0

Inhalation exposure modification factor:

.23

External exposure modification factor:

FIGURE 3.8 (Contd) 
ICRP-2 and ICRP-26/30 Maximum Annual Dose Calculation

(MAXI Version IBM 1.0 30-Jun-86)

Case title: SAMPLE4.IN / MAXI Sample Problem Four

Executed on: $10 / 28 / 1986$ at $16: 36: 28$

Page 4

SOIL, AIR, AND WATER CONCENTRATION SUMMARY FOR YEAR: 10

\begin{tabular}{lccccc}
$\begin{array}{l}\text { Radio- } \\
\text { nuclide }\end{array}$ & $\begin{array}{c}\text { Surface Soil } \\
\mathrm{pCi} / \mathrm{m} 2\end{array}$ & $\begin{array}{c}\text { Deep Soil } \\
\mathrm{pCi} / \mathrm{m} 3\end{array}$ & $\begin{array}{c}\text { Air } \\
\mathrm{pCi} / \mathrm{m} 3\end{array}$ & $\begin{array}{c}\text { Irrigation } \\
\mathrm{pCi} / \mathrm{L}\end{array}$ & $\begin{array}{c}\text { Drink Water } \\
\mathrm{pCi} / \mathrm{L}\end{array}$ \\
\hline $\mathrm{C0} 60$ & $4.9 \mathrm{E}+02$ & $.0 \mathrm{E}+00$ & $4.1 \mathrm{E}-07$ & $1.0 \mathrm{E}-01$ & $1.0 \mathrm{E}+01$ \\
SR 90 & $8.6 \mathrm{E}+02$ & $.0 \mathrm{E}+00$ & $7.1 \mathrm{E}-07$ & $1.0 \mathrm{E}-01$ & $1.0 \mathrm{E}+01$ \\
$\mathrm{Y} 90$ & $8.6 \mathrm{E}+02$ & $.0 \mathrm{E}+00$ & $7.1 \mathrm{E}-07$ & $.0 \mathrm{E}+00$ & $.0 \mathrm{E}+00$ \\
$\mathrm{I} 129$ & $9.9 \mathrm{E}+02$ & $.0 \mathrm{E}+00$ & $8.2 \mathrm{E}-07$ & $1.0 \mathrm{E}-01$ & $1.0 \mathrm{E}+01$ \\
$\mathrm{U} 238$ & $9.9 \mathrm{E}+02$ & $.0 \mathrm{E}+00$ & $8.2 \mathrm{E}-07$ & $1.0 \mathrm{E}-01$ & $1.0 \mathrm{E}+01$ \\
TH 234 & $9.9 \mathrm{E}+02$ & $.0 \mathrm{E}+00$ & $8.2 \mathrm{E}-07$ & $.0 \mathrm{E}+00$ & $.0 \mathrm{E}+00$ \\
PA $234 \mathrm{M}$ & $9.9 \mathrm{E}+02$ & $.0 \mathrm{E}+00$ & $8.2 \mathrm{E}-07$ & $.0 \mathrm{E}+00$ & $.0 \mathrm{E}+00$ \\
PA 234 & $1.3 \mathrm{E}+00$ & $.0 \mathrm{E}+00$ & $1.1 \mathrm{E}-09$ & $.0 \mathrm{E}+00$ & $.0 \mathrm{E}+00$
\end{tabular}

SOIL, AIR, AND WATER CONCENTRATION SUMMARY FOR YEAR: 59

\begin{tabular}{|c|c|c|c|c|c|}
\hline $\begin{array}{l}\text { Radio- } \\
\text { nuclide }\end{array}$ & $\begin{array}{c}\text { Surface Soil } \\
\mathrm{pCi} / \mathrm{m} 2\end{array}$ & $\begin{array}{l}\text { Deep Soil } \\
\mathrm{pC} i / \mathrm{m} 3\end{array}$ & $\begin{array}{c}\mathrm{Air} \\
\mathrm{pCi} / \mathrm{m} 3\end{array}$ & $\begin{array}{c}\text { Irrigation } \\
\mathrm{pCi} / \mathrm{L}\end{array}$ & $\begin{array}{c}\text { Drink water } \\
\text { pCi } / L\end{array}$ \\
\hline$-10-1$ & 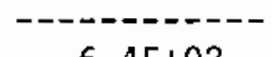 & ------1 & $2-100$ & & \\
\hline $\begin{array}{ll}0 & 60 \\
R & 90\end{array}$ & $6.4 \mathrm{E}+02$ & $.0 E+00$ & $4.3 \mathrm{E}-08$ & $1.0 \mathrm{E}-$ & $1.0 \mathrm{E}$ \\
\hline R 90 & 03 & $.0 \mathrm{E}+00$ & -07 & $1.0 \mathrm{E}-$ & 1. $0 \mathrm{E}+01$ \\
\hline 90 & $2.8 E+03$ & $.0 \mathrm{E}+00$ & $1.9 \mathrm{E}-07$ & $.0 \mathrm{E}$ & $.0 \mathrm{E}+00$ \\
\hline I 129 & $=03$ & $.0 \mathrm{E}+00$ & $3.6 E-07$ & $1.0 \mathrm{E}$ & $1.0 \mathrm{E}+01$ \\
\hline U 238 & $5.4 E+03$ & $.0 E+00$ & $3.6 \mathrm{E}-07$ & 1.0E-01 & $1.0 \mathrm{E}+01$ \\
\hline TH 234 & -03 & $.0 \mathrm{E}+00$ & -07 & $.0 E+00$ & $.0 \mathrm{E}+00$ \\
\hline PA 234M & -03 & $.0 \mathrm{E}+00$ & -07 & $.0 E+00$ & $.0 \mathrm{E}+00$ \\
\hline A 234 & $7.0 \mathrm{E}+00$ & $.0 E+00$ & $4.7 E-10$ & $.0 \mathrm{E}+00$ & $.0 \mathrm{E}+00$ \\
\hline
\end{tabular}

FIGURE 3.8 (Contd) 


ICRP-2 and ICRP-26/30 Maximum Annual Dose Calculation
(MAXI Version IBM 1.0 30-Jun-86)
Case title: SAMPLE4. IN / MAXI Sample Problem Four
Executed on: $10 / 28 / 1986$ at $16: 36: 28$

Annual Effective Dose Equivalent (AEDE): $\quad 1.3 \mathrm{E}-03$

Maximum Annual Dose (ICRP-2):

$\begin{array}{lcll}\text { To Organ: TOTAL BODY at Year } 59 & 1.6 \mathrm{E}-03 \\ \text { To Organ: } & \text { BONE } & \text { at Year } 59 & 7.7 \mathrm{E}-03 \\ \text { To Organ: } & \text { LUNGS } & \text { at Year } 59 & 8.0 \mathrm{E}-06 \\ \text { To Organ: THYROID } & \text { at Year } 59 & 2.8 \mathrm{E}-02 \\ \text { To Organ: } & \text { LLI } & \text { at Year } 59 & 3.5 \mathrm{E}-04\end{array}$

FIGURE 3.8 (Contd) 
ICRP-2 and ICRP-26/30 Maximum Annual Dose Calculation

(MAXI Version IBM 1.0 30-Jun-86)

Case title: SAMPLE4.IN / MAXI Sample Problem Four

Executed on: $10 / 28 / 1986$ at $16: 36: 28$

Page 6

DOSES FROM 1 YEAR OF EXPOSURE

\begin{tabular}{|c|c|c|c|}
\hline Organ & $\begin{array}{c}\text { Committed } \\
\text { Dose } \\
\text { Equivalent }\end{array}$ & $\begin{array}{l}\text { Weighing } \\
\text { Factors }\end{array}$ & $\begin{array}{c}\text { Weighted } \\
\text { Dose } \\
\text { Equivalent }\end{array}$ \\
\hline------- & $-\cdots$ & & \\
\hline $\begin{array}{l}\text { Gonads } \\
\text { Breast }\end{array}$ & $\begin{array}{l}1.1 \mathrm{E}-05 \\
3.8 \mathrm{E}-06\end{array}$ & $\begin{array}{l}2.5 \mathrm{E}-01 \\
1.5 \mathrm{E}-01\end{array}$ & $\begin{array}{l}2.7 \mathrm{E}-06 \\
5.7 \mathrm{E}-07\end{array}$ \\
\hline R Marrow & $8.0 E-04$ & $1.2 \mathrm{E}-01$ & $9.6 \mathrm{E}-05$ \\
\hline Lungs & $4.6 \mathrm{E}-06$ & $1.2 \mathrm{E}-01$ & $5.5 \mathrm{E}-07$ \\
\hline Thyroid & $3.4 \mathrm{E}-02$ & $3.0 E-02$ & $1.0 \mathrm{E}-03$ \\
\hline Bone Surf & $2.1 \mathrm{E}-03$ & $3.0 \mathrm{E}-02$ & $6.4 \bar{E}-05$ \\
\hline LLI Wall & $5.7 \mathrm{E}-04$ & $6.0 \mathrm{E}-02$ & $3.4 \mathrm{E}-05$ \\
\hline Kidneys & $2.0 \mathrm{E}-04$ & $6.0 \mathrm{E}-02$ & 1.2E-05 \\
\hline ULI Wall & $1.9 \mathrm{E}-04$ & $6.0 \mathrm{E}-02$ & 1.2E-05 \\
\hline SI Wall & $1.2 E-05$ & $6.0 \mathrm{E}-02$ & $7.4 \mathrm{E}-07$ \\
\hline Liver & $7.9 \mathrm{E}-06$ & $6.0 \mathrm{E}-02$ & $4.7 \mathrm{E}-07$ \\
\hline - - - - & --- & $-\infty \ldots-\infty$ & \\
\hline $\begin{array}{l}\text { Effective Dose } \\
\text { External Dose }\end{array}$ & Equivalent & & $\begin{array}{l}1.2 E-03 \\
5.7 E-06\end{array}$ \\
\hline------ & 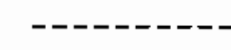 & & \\
\hline nnual Effecti & Dose Equi & $7 a n$ & 1. $3 \mathrm{E}-03$ \\
\hline
\end{tabular}

FIGURE 3.8 (Contd) 
ICRP-2 and ICRP-26/30 Maximum Annual Dose Calculation

(MAXI Version IBM 1.0 30-Jun-86)

Case title: SAMPLE4.IN / MAXI Sample Problem Four

Executed on: $10 / 28 / 1986$ at $16: 36: 28$

Page 7

MAXIMUM ANNUAL DOSE SUMMARY FOR THE YEAR 59 FOR TOTAL BODY----.--

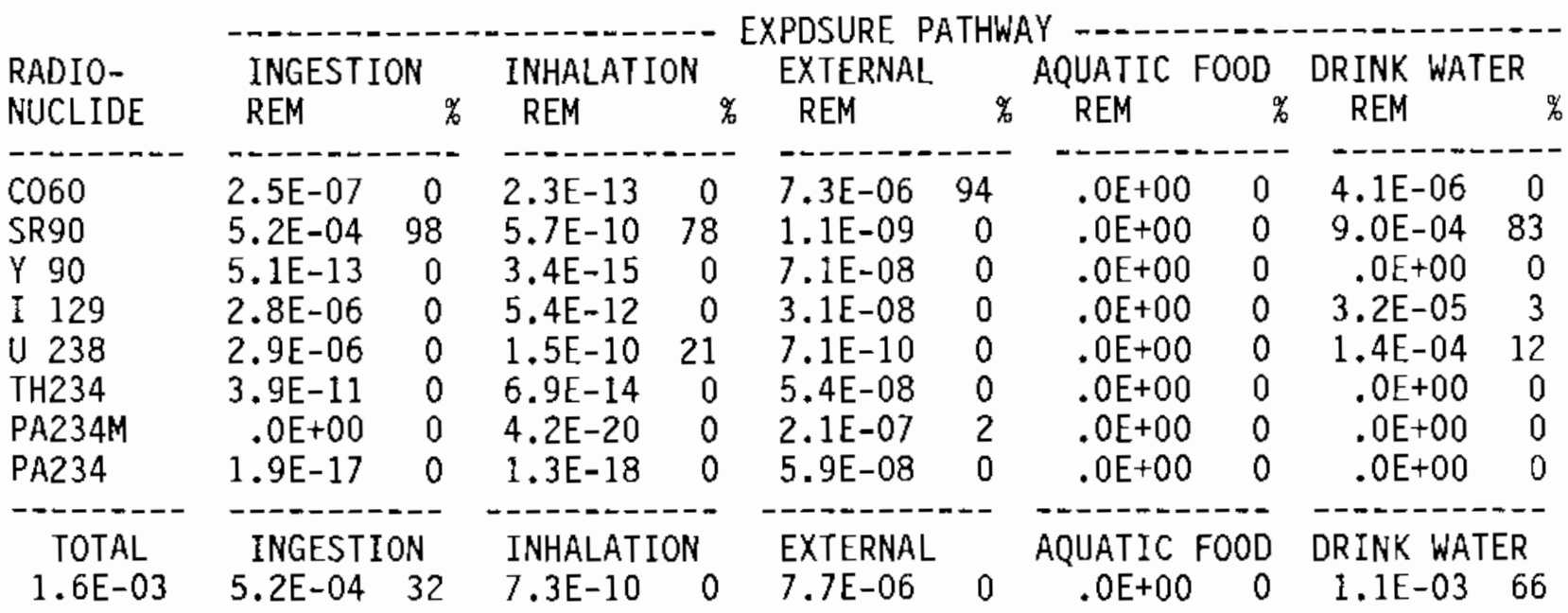

MAXIMUM ANNUAL DOSE SUMMARY FOR THE YEAR 59 FOR BONE

\begin{tabular}{|c|c|c|c|c|c|c|c|c|c|c|}
\hline \multirow{2}{*}{$\begin{array}{l}\text { RADIO- } \\
\text { NUCLIDE }\end{array}$} & \multicolumn{2}{|c|}{ INGESTION } & \multicolumn{2}{|c|}{ INHALATION } & \multicolumn{2}{|l|}{ EXTERNAL } & \multicolumn{2}{|c|}{ AQUATIC FOOD } & \multicolumn{2}{|c|}{ DRINK WATER } \\
\hline & REM & $\%$ & REM & $\%$ & REM & $\%$ & REM & $\%$ & REM & \% \\
\hline & -- & & & & & & & & & \\
\hline $\mathrm{CO6} 0$ & $.0 \mathrm{E}+00$ & 0 & $.0 E+00$ & 0 & $7.3 E-06$ & 94 & $.0 E+00$ & 0 & $.0 E+$ & 0 \\
\hline SR9O & $1.9 \mathrm{E}-03$ & 97 & $8.5 E-09$ & 76 & $1.1 \mathrm{E}$ & 0 & $.0 \mathrm{E}$ & 0 & $3.4 \mathrm{E}$ & 58 \\
\hline Y 90 & $1.9 \mathrm{E}-11$ & 0 & 1. $3 \mathrm{E}-13$ & 0 & $7.1 \mathrm{E}$ & 0 & $.0 \mathrm{E}$ & 0 & .0 & 0 \\
\hline I 129 & $1.0 \mathrm{E}-06$ & 0 & 1. $3 \mathrm{E}-13$ & 0 & 3.10 & 0 & $.0 E+00$ & 0 & 1.1 & 0 \\
\hline U 238 & $4.9 \mathrm{E}-05$ & 2 & $2.6 \mathrm{E}-09$ & 23 & 7.1 & 0 & $.0 \mathrm{E}$ & 0 & 2.3 & 41 \\
\hline $\mathrm{TH} 234$ & $1.3 \bar{E}-09$ & 0 & $2.4 \mathrm{E}-12$ & 0 & 5.4 & 0 & $.0 \mathrm{E}+00$ & 0 & $.0 \mathrm{E}$ & 0 \\
\hline PA234M & $.0 \mathrm{E}+00$ & 0 & $9.1 E-19$ & 0 & $2.1 E-07$ & 2 & $.0 E+00$ & 0 & $.0 \mathrm{E}$ & 0 \\
\hline PA234 & $7.3 \mathrm{E}-17$ & 0 & $5.1 \mathrm{E}-18$ & 0 & $5.9 \mathrm{E}-08$ & 0 & $.0 \mathrm{E}+00$ & 0 & $.0 \mathrm{E}$ & 0 \\
\hline & & & & & & & & & & \\
\hline TOTAL & INGE & & INHALAT & & EXTERNAL & & AQUATIC & FOOD & DRIN & \\
\hline $7.7 \mathrm{E}-03$ & $2.0 \mathrm{E}-03$ & 25 & $1.1 \mathrm{E}-08$ & 0 & $7.7 \mathrm{E}-06$ & 0 & $.0 \mathrm{E}+00$ & 0 & $5.7 E-0$ & 74 \\
\hline
\end{tabular}

FIGURE 3.8 (Contd) 
ICRP-2 and ICRP-26/30 Maximum Annual Dose Calculation

(MAXI Version IBM 1.0 30-Jun-86)

Case title: SAMPLE4.IN / MAXI Sample Problem Four

Executed on: $10 / 28 / 1986$ at $16: 36: 28$

Page 8

MAXIMUM ANNUAL DOSE SUMMARY FOR THE YEAR 59 FOR LUNGS

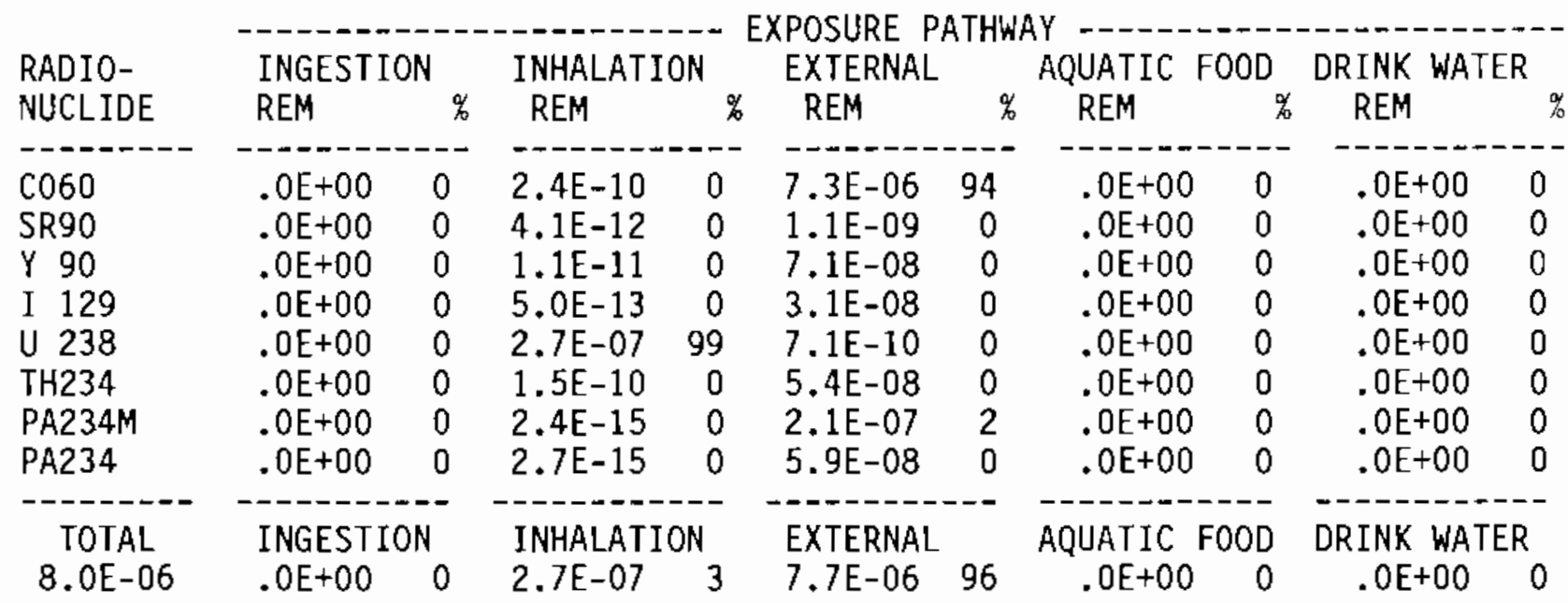

MAXIMUM ANNUAL DOSE SUMMARY FOR THE YEAR 59 FOR THYROID

\begin{tabular}{|c|c|c|c|c|c|c|c|c|c|c|}
\hline \multirow{3}{*}{$\begin{array}{l}\text { RADI0- } \\
\text { NUCLIDE }\end{array}$} & \multicolumn{4}{|c|}{ 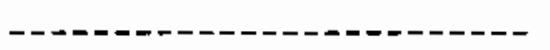 } & \multicolumn{6}{|c|}{ EXPOSURE PATHWAY } \\
\hline & \multirow{2}{*}{\multicolumn{2}{|c|}{$\begin{array}{l}\text { INGESTION } \\
\text { REM } \%\end{array}$}} & \multicolumn{2}{|c|}{ INHALATION } & \multirow{2}{*}{$\begin{array}{l}\text { EXTERNAL } \\
\text { REM }\end{array}$} & \multicolumn{2}{|c|}{ AQUATIC } & FOOD & \multirow{2}{*}{\multicolumn{2}{|c|}{$\begin{array}{l}\text { DRINK WATER } \\
\text { REM }\end{array}$}} \\
\hline & & & REM & $\%$ & & $\%$ & REM & $\%$ & & \\
\hline-4 & 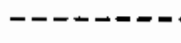 & & & & & & & & & \\
\hline $\mathrm{CO50}$ & $.0 E+00$ & 0 & $.0 E+00$ & 0 & $7.3 \mathrm{E}-06$ & 94 & $.0 E+00$ & 0 & $.0 E+00$ & U \\
\hline SR90 & $.0 \mathrm{E}$ & 0 & & 0 & & 0 & & 0 & $.0 \mathrm{E}+00$ & \\
\hline Y 90 & $.0 \mathrm{E}+00$ & 0 & $.0 \mathrm{E}+00$ & 0 & & 0 & $.0 \mathrm{E}$ & 0 & $.0 \mathrm{E}+00$ & 0 \\
\hline I 129 & $2.2 \mathrm{E}-03$ & 100 & $4.3 E-09$ & 100 & & 0 & & 0 & $2.5 \bar{E}-02$ & 100 \\
\hline U 238 & $.0 \mathrm{E}+00$ & 0 & $.0 E+00$ & 0 & & 0 & $.0 E+00$ & 0 & $.0 E+00$ & 0 \\
\hline TH234 & $.0 \mathrm{E}$ & 0 & $.0 \mathrm{~B}$ & 0 & & 0 & $.0 \mathrm{E}+00$ & 0 & $.0 E+00$ & 0 \\
\hline PA234M & $.0 \mathrm{E}$ & 0 & $.0 \mathrm{E}$ & 0 & & 2 & $.0 \mathrm{E}+00$ & 0 & $.0 E+00$ & 0 \\
\hline PA234 & & 0 & & 0 & & 0 & $.0 E+00$ & 0 & $.0 \mathrm{E}+00$ & 0 \\
\hline TOTA & INC & $\mathrm{N}$ & INH & & FXT & & AOUAT & FOOD & $\mathrm{DR}$ & \\
\hline $2.8 \mathrm{E}-0$ & $2.2 \mathrm{E}-03$ & 7 & 4. $3 \mathrm{E}-09$ & 0 & $7.7 E$ & 0 & $.0 \mathrm{E}+00$ & 0 & $2.5 \mathrm{E}-02$ & 91 \\
\hline
\end{tabular}

FIGURE 3.8 (Contd) 
ICRP-2 and ICRP-26/30 Maximum Annual Dose Calculation

(MAXI Version IBM 1.0 30-Jun-86)

Case title: SAMPLE4.IN / MAXI Sample Problem Four

Executed on: $10 / 28 / 1986$ at $16: 36: 28$

Page 9

MAXIMUM ANNUAL DOSE SLMMARY FOR THE YEAR 59 FOR LLI -

\begin{tabular}{|c|c|c|c|c|c|c|c|c|c|c|}
\hline $\begin{array}{l}\text { RADIO- } \\
\text { NUCLIDE }\end{array}$ & $\begin{array}{l}\text { INGESTION } \\
\text { REM }\end{array}$ & N & $\begin{array}{l}\text { INHALATIOI } \\
\text { REM }\end{array}$ & $\%$ & $\begin{array}{l}\text { EXTERNAL } \\
\text { REM }\end{array}$ & $\%$ & $\begin{array}{l}\text { AQUATIC } \\
\text { REM }\end{array}$ & FOOD & $\begin{array}{c}\text { DRINK WAT } \\
\text { REM }\end{array}$ & ER $\%$ \\
\hline $\begin{array}{l}\text { CO60 } \\
\text { SR90 } \\
\text { Y } 90 \\
\text { I } 129 \\
\text { U } 238 \\
\text { TH234 } \\
\text { PA234M } \\
\text { PA234 }\end{array}$ & $\begin{array}{l}2.3 \mathrm{E}-06 \\
4.3 \mathrm{E}-05 \\
2.2 \mathrm{E}-07 \\
1.5 \mathrm{E}-07 \\
3.9 \mathrm{E}-06 \\
2.1 \mathrm{E}-06 \\
.0 \mathrm{E}+00 \\
7.4 \mathrm{E}-12\end{array}$ & $\begin{array}{r}4 \\
83 \\
0 \\
0 \\
7 \\
4 \\
0 \\
0\end{array}$ & $\begin{array}{r}1.6 \mathrm{E}-11 \\
5.4 \mathrm{E}-14 \\
2.3 \mathrm{E}-11 \\
4.0 \mathrm{E}-11 \\
.0 \mathrm{E}+00 \\
2.7 \mathrm{E}-15\end{array}$ & $\begin{array}{r}2 \\
4 \\
18 \\
0 \\
27 \\
47 \\
0 \\
0\end{array}$ & $\begin{array}{l}7.3 \mathrm{E}-06 \\
1.1 \mathrm{E}-09 \\
7.1 \mathrm{E}-08 \\
3.1 \mathrm{E}-08 \\
7.1 \mathrm{E}-10 \\
5.4 \mathrm{E}-08 \\
2.1 \mathrm{E}-07 \\
5.9 \mathrm{E}-08\end{array}$ & $\begin{array}{r}94 \\
0 \\
0 \\
0 \\
0 \\
0 \\
2 \\
0\end{array}$ & $\begin{array}{l}.0 \mathrm{E}+00 \\
.0 \mathrm{E}+00 \\
.0 \mathrm{E}+00 \\
.0 \mathrm{E}+00 \\
.0 \mathrm{E}+00 \\
.0 \mathrm{E}+00 \\
.0 \mathrm{E}+00 \\
.0 \mathrm{E}+00\end{array}$ & $\begin{array}{l}0 \\
0 \\
0 \\
0 \\
0 \\
0 \\
0 \\
0\end{array}$ & $\begin{array}{r}3.9 \mathrm{E}-05 \\
6.8 \mathrm{E}-05 \\
.0 \mathrm{E}+00 \\
1.7 \mathrm{E}-06 \\
1.9 \mathrm{E}-04 \\
.0 \mathrm{E}+00 \\
.0 \mathrm{E}+00 \\
.0 \mathrm{E}+00\end{array}$ & $\begin{array}{r}13 \\
23 \\
0 \\
0 \\
63 \\
0 \\
0 \\
0\end{array}$ \\
\hline $\begin{array}{c}\text { TOTAL } \\
3.5 \mathrm{E}-04\end{array}$ & $5.2 \mathrm{E}-05$ & 14 & $8.5 E-11$ & 0 & $\begin{array}{l}\text { EXTERNAL } \\
7.7 \mathrm{E}-06\end{array}$ & 2 & $.0 \mathrm{E}+00$ & 0 & $2.9 \mathrm{E}-04$ & $\begin{array}{l}\text { ER } \\
83\end{array}$ \\
\hline
\end{tabular}

FIGURE 3.8 (Contd) 


\subsubsection{Sample Problem Five}

The fifth sample problem demonstrates the user-defined scenario option. This problem is intended to exercise the maximum number of exposure conditions that can be considered by the MAXI1 program in a single run. For this problem, the general exposure conditions described in Sample Problem Four are considered with the addition of ingestion of aquatic foods (fish obtained from a river) and root penetration into deeply buried wastes. The radionuclide concentrations

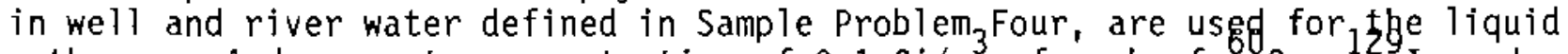
Bathways. A deep waşte copcentration of $0.1 \mathrm{Ci} / \mathrm{m}^{3}$ of each of ${ }^{60} \mathrm{Co},{ }^{129} \mathrm{I}$, and ${ }^{238} \mathrm{U}$ plus $0.001 \mathrm{Ci} / \mathrm{m}^{3}$ of $\mathrm{Sr}$ is also assumed. The site is assumed to cover $1 \mathrm{ha}$. The wastes are assumed to be buried for 10 years prior to the start of farming and they are assumed to be located $1.0 \mathrm{~m}$ from the surface. It is further assumed that about $40 \%$ of the roots from the garden crops penetrate to this depth (based on information in Napier 1982).

For the user-defined scenario, the air concentration used to estimate inhalation dose is calculated using a mass-loading equation instead of the time-dependent resuspension equation. Also, the individual's total diet is assumed to be from foods grown using the contaminated water supply. The purpose of this sample problem is to determine the doses to an individual residing on a site containing deeply buried wastes who also uses contaminated water supplies. This sample problem has been expanded in this version of the ONSITE/MAXIl software package. Both the maximum annual dose (ICRP 1959) and the annual effective dose equivalent, AEDE, (ICRP 1977; ICRP 1979-1982) are to be calculated. The MAXI1 input file for Sample Problem Five is shown in Figure 3.9. Changes from the previous sample problem are oboused on the Sample Problem Five input file to assist the user in understanding the second level of software operation.

The output resulting for the scenario in this sample problem is shown in Figure 3.10. The maximum annual doses for Sample Problem Five are about 1.5 rem to total body, 6.2 rem to bone, $2.1 \times 10^{-4}$ rem to lungs, 240 rem to thyroid, and 0.9 rem to $G I(L L I)$. Ingestion of farm products whose roots penetrate the deeply buried wastes control the doses to total body, bone, thyroid, and GI(LLI). The dose to lungs is control led by the external exposure pathway. The annual external dose was $2.1 \times 10^{-4} \mathrm{rem}$, and the internal effective dose equivalent was 9.8 rem. Thus, the annual effective dose equivalent was 9.8 rem. Radioactive decay, soil and air concentration, and maximum annual dose estimate calculations were verified by hand using the equations in Section 2 . 
DO YOU WISH TO CONSIDER:

External exposure and root penetration

for waste modeled as a slab (voTume) source? (0-no 1-yes)

Externat exposure to surface contamination modeled as a plane source? (0-no 1-yes)

Inhalation of resuspended material? (0-no 1-yes) INHA

Farm product ingestion? (0-no 1-yes)

Aquatic foods ingestion? (0-no 1-yes)

IFOD

Drinking water ingestion? (0-no 1-yes)

IARG

offsite atmospheric source? (0-no 1-yes)

IWAT

ICRP-26/30 as wel] as ICRP-2 methodology? (0-no 1-yes)

Decay of air release source term? (0-no 1-yes)

Decay of water release source term? (0-no 1-yes)

Use the One-Year-of-Exposure Model? (0-no 1-yes)

IDKAIR

IDKWAT

INTRUD

MONITORING AND OUTPUT OPTIONS

Level of detail in reports (1-least to 3 )

IOUT

Monitor and print hand calculation report

DEBUG (4)

NUMBER OF YEARS AFTER WASTE IS DISPOSED THAT:

Scenario begins:

Scenario ends:

IT1

IT2

INVENTORY:

Release Term Input units: (1-pCi $2-4 C i \quad 3-\pi C i \quad 4-C i)$ INUT

Soil source units $\left(0-m^{\star \star 2} 1-m^{\star \star 3} 2-\mathrm{kg}\right)$ M3M2

Number of radionuclides in inventory: NIN

\begin{tabular}{lrrrrr}
$\begin{array}{l}\text { Release } \\
\text { Terms }\end{array}$ & $\begin{array}{l}\text { SoiT Source } \\
\text { (per unit) }\end{array}$ & $\begin{array}{l}\text { Irrigation } \\
\text { Aquatic } \\
\text { (per L) }\end{array}$ & $\begin{array}{l}\text { Drinking } \\
\text { Water } \\
\text { (per L) }\end{array}$ & $\begin{array}{l}\text { Atmospheric } \\
\text { Release } \\
\text { (per yr) }\end{array}$ & $\begin{array}{r}E \\
\text { A }\end{array}$ \\
\hline-060 & & 0.1 & 10.0 & & QI \\
C060 & & 0.1 & 10.0 & QJ \\
SR90 & & 0.1 & 10.0 & \\
U 238 & & 0.1 & 10.0 & \\
I 129 & & & &
\end{tabular}

0.2
1.0
1.0
1.0

2000.0

2000.0

PLANEA.DAT

BUPIA IIT:

270.0

INVENTORY MODIFICATION FACTORS: (multipliers)

Surface inventory modification factor:

Irrigation/aquatic inventory modification factor:

Size of site (fractional ha):

SRDIL

Fraction of total diet grown on site:

DILF

FRSI7

RPF2

EXTERNAL/INHALATION EXPOSURE:

Hours of external exposure to contamination $(\mathrm{h} / \mathrm{yr})$ : HREXT

Hours of inhalation of airborne contamination $(h / y r):$ HRINH

Ext. exp. dose rate factors ( $P$ lane source) filename: PLANAM

Ext. exp. dose rate factors (volume source) filename: VOLNAM

Breathing rate $\left(\mathrm{cm}^{\star *} 3 / \mathrm{sec}\right)$ :

BRATE

FIGURE 3.9. Sample Problem Five Input 


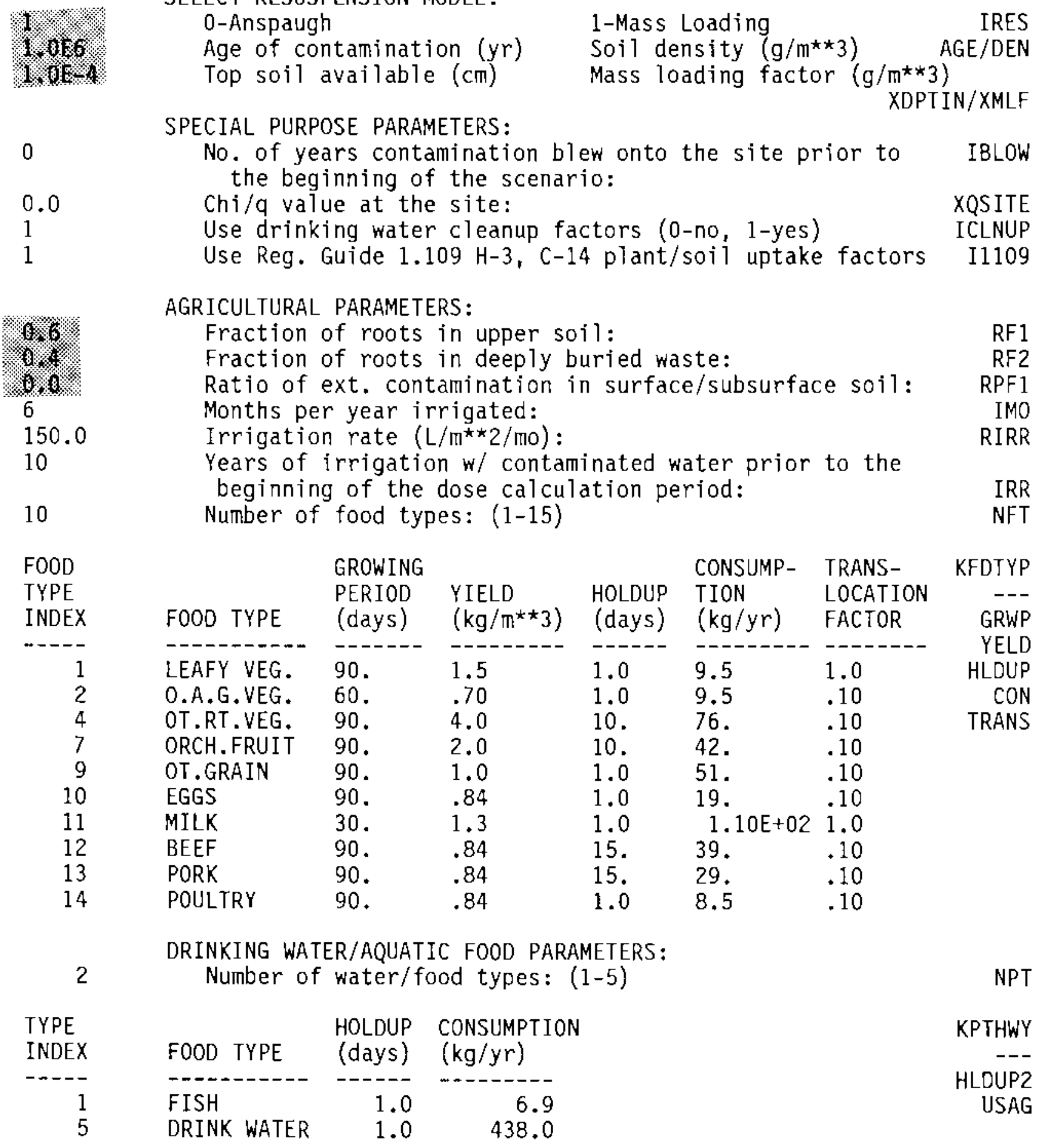

FIGURE 3.9 (Contd) 


\section{ICRP-2 and ICRP-26/30 Maximum Annual Dose Calculation}

(MAXI Version IBM 1.0 30-Jun-86)

Case title: SAMPLE5. IN / MAXI Sample Problem Five

Executed on: $10 / 28 / 1986$ at $16: 41: 21$

Page 1

PATHWAYS/OPTIONS CONSIDERED:

External exposure/root penetration for waste modeled as slab (volume) source External exposure to surface contamination modeled as a plane source Inhalation of resuspended material

Farm product ingestion

Aquatic foods ingestion

Drinking water ingestion

Committed effective dose equivalent (CEDE) calculation

TITLES OF LIBRARY FILES ACCESSED:

10: RMOLIB - Radionuclide Master Library (24-Sep-86 RAP)

11: ORGAN DATA LIBRARY UPDATED BY RA PELOQUIN 8-JU1-86

12: FOOD TRANSFER COEFFICIENT LIBRARY (RAP/WTF 04-APR-86 Cl Update)

13: Committed Dose Equivalents (Sv/Bq) ICRP Publication 30 (28-0ct-86 RAP)

14: BIOACCUMULATION FACTOR LIBRARY FOR BA NAPIER (04-APR-86/WTF)

22: OVERBURDEN: 0.0M, SOURCE: 0.15 M (DEN: CONCRETE/1.8): MR/HR 8-Jul-86 RAP

23: DACRIN (DIFDOS) DOSE INCREMENT FILE ONSITE/MAXI 26-Aug-86 RAP

27: OVERBUROEN: $1.0 \mathrm{~m}$, SOURCE: 1.0M (DEN: CONCRETE/1.8): MR/HR 18-JuT-86 RAP

NUMBER OF YEARS AFTER WASTE IS DISPOSED THAT:

Scenario begins:

Scenario ends:

INVENTORY:

Release Term Input units: (1-pCi 2-uCi $3-m C i \quad 4-C i)$

Soil source units: $\left(0-m^{\star \star 2} \quad 1-m^{\star \star 3} \quad 2-\mathrm{kg}\right)$

Number of radionuclides in inventory:

\begin{tabular}{|c|c|c|c|c|}
\hline $\begin{array}{l}\text { Release } \\
\text { Terms }\end{array}$ & $\begin{array}{l}\text { Soil Source } \\
\text { (per } m^{\star \star 3} \text { ) }\end{array}$ & $\begin{array}{l}\text { Irrigation } \\
\text { /Aquat ic } \\
\text { (per L) }\end{array}$ & $\begin{array}{l}\text { Drinking } \\
\text { Water } \\
\text { (per L) }\end{array}$ & $\begin{array}{l}\text { Atmospheric } \\
\text { Release } \\
\text { (per m3) }\end{array}$ \\
\hline 60 & $1.0 \mathrm{E}+11$ & $1.0 E-01$ & $E+01$ & \\
\hline 90 & & & & \\
\hline & $.0 \mathrm{E}$ & $.0 \mathrm{E}$ & 00 &. \\
\hline 129 & $1.0 \mathrm{E}+$ & 1.0 & $1.0 \varepsilon$ & \\
\hline 238 & $1.0 \mathrm{E}+11$ & $1.0 \mathrm{E}$ & $1.0 \mathrm{E}+01$ & $.0 \mathrm{E}$ \\
\hline TH234 & $.0 \mathrm{E}+00$ & $.0 \mathrm{E}$ & $.0 \mathrm{E}+00$ & .0 \\
\hline 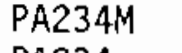 & $.0 \mathrm{E}+$ & & $.0 E+00$ & $.0 \mathrm{~B}$ \\
\hline PA234 & $.0 E+00$ & $.0 \mathrm{E}$ & $.0 \mathrm{E}+00$ & $.0 \mathrm{E}+$ \\
\hline
\end{tabular}

FIGURE 3.10. Sample Problem Five Output 
INVENTORY MODIFICATION FACTORS: (multipliers)

Surface inventory dilution factor:

Irrigation/aquatic inventory modification factor:

1 .

Size of site (fractional ha):

$1.0 \mathrm{E}+00$

Fraction of total diet grown on site:

1.

FIGURE 3.10 (Contd)

3.50 
ICRP-2 and ICRP-26/30 Maximum Annual Dose Calculation

(MAXI Version IBM 1.0 30-Jun-86)

Case title: SAMPLE5.IN / MAXI Sample Problem Five

Executed on: $10 / 28 / 1986$ at $16: 41: 21$

Page 2

EXTERNAL/INHALATION EXPOSURE:

Hours of external exposure to contamination $(h / y r)$ :

2. $0 \mathrm{E}+03$

Hours of inhalation of airborne contamination (h/yr):

2. $0 \mathrm{E}+03$

Breathing rate $\left(\mathrm{cm}^{\star \star} 3 / \mathrm{sec}\right)$ :

2.7E+02

RESUSPENSION PARAMETERS:

Mode1 used:

Soil density $\left(\mathrm{g} / \mathrm{m}^{\star \star} 3\right)$ :

Mass loading factor $\left(\mathrm{g} / \mathrm{m}^{* *} 3\right)$ :

Mass Loading

$1.0 \mathrm{E}+06$

$1.0 \mathrm{E}-04$

AGRICULTURAL PARAMETERS:

Fraction of roots in upper soil: $\quad .600$

Fraction of roots in deeply buried waste: $\quad .400$

Ratio of ext. contamination in surface/subsurface soil: $\quad .000$

Months per year irrigated:

Irrigation rate $\left(\mathrm{L} / \mathrm{m}^{\star \star} 2 / \mathrm{mo}\right)$ :

Years of irrigation $\mathrm{w} /$ contaminated water prior to the beginning of the dose calculation period:

Number of food types:

\begin{tabular}{rlccccc}
$\begin{array}{l}\text { FOOD } \\
\text { TYPE } \\
\text { INDEX }\end{array}$ & FOOD TYPE & $\begin{array}{l}\text { GROWING } \\
\text { PERIOD } \\
\text { (days) }\end{array}$ & $\begin{array}{c}\text { YIELD } \\
\left(\mathrm{kg} / \mathrm{m}^{\star \star} 3\right)\end{array}$ & $\begin{array}{c}\text { HOLDUP } \\
\text { (days) }\end{array}$ & $\begin{array}{l}\text { CONSUMP- } \\
\text { TION } \\
(\mathrm{kg} / \mathrm{yr})\end{array}$ & $\begin{array}{l}\text { TRANS- } \\
\text { LOCATION }\end{array}$ \\
\hline-- & FACTOR \\
\hline 1 & LEAFY VEG. & 90. & 1.50 & 1. & 9.5 & 1.00 \\
2 & O.A.G.VEG. & 60. & .70 & 1. & 9.5 & .10 \\
4 & OT.RT.VEG. & 90. & 4.00 & 10. & 76.0 & .10 \\
7 & ORCH.FRUIT & 90. & 2.00 & 10. & 42.0 & .10 \\
9 & OT.GRAIN & 90. & 1.00 & 1. & 51.0 & .10 \\
10 & EGGS & 90. & .84 & 1. & 19.0 & .10 \\
11 & MILK & 30. & 1.30 & 1. & 110.0 & 1.00 \\
12 & BEEF & 90. & .84 & 15. & 39.0 & .10 \\
13 & PORK & 90. & .84 & 15. & 29.0 & .10 \\
14 & POULTRY & 90. & .84 & 1. & 8.5 & .10
\end{tabular}

FIGURE 3.10 (Contd) 
ICRP-2 and ICRP-26/30 Maximum Annual Dose Calculation

(MAXI Version IBM 1.0 30-Jun-86)

Case title: SAMPLE5.IN / MAXI Sample Problem Five

Executed on: $10 / 28 / 1986$ at $16: 41: 21$

Page 3

DRINKING WATER/AQUATIC FOOD PARAMETERS:

Number of water/food types:

\begin{tabular}{|c|c|c|c|}
\hline $\begin{array}{l}\text { TYPE } \\
\text { INDEX }\end{array}$ & FOOD TYPE & $\begin{array}{l}\text { HOLDUP } \\
\text { (days) }\end{array}$ & $\begin{array}{l}\text { CONSUMPTION } \\
(\mathrm{kg} / \mathrm{yr})\end{array}$ \\
\hline & $-\cdots------0$ & ------ & $---\infty$ \\
\hline 1 & FISH & 1.0 & 6.9 \\
\hline 5 & DRINK WATER & 1.0 & 438.0 \\
\hline
\end{tabular}

Input prepared by:

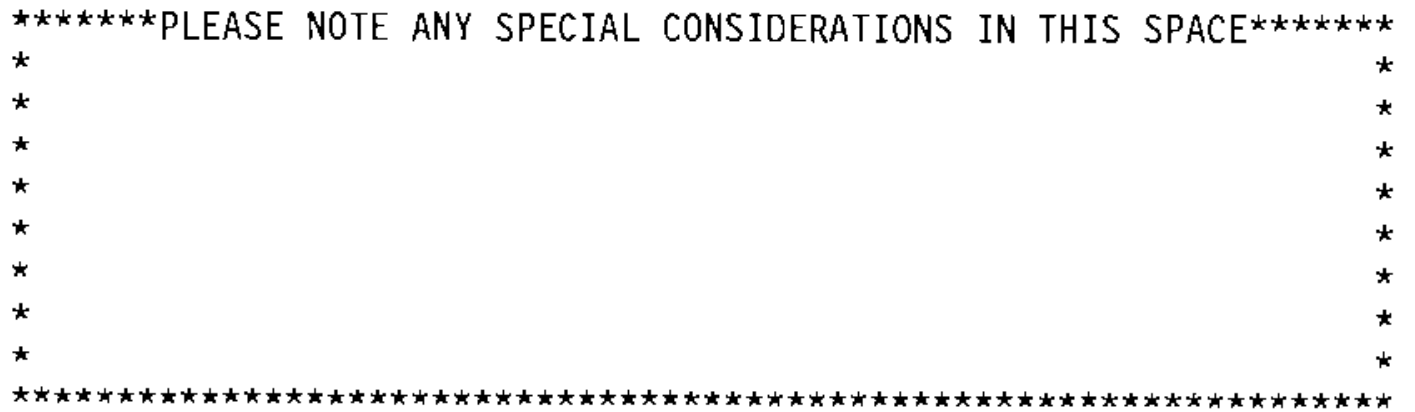

CALCULATED VALUES:

Ingestion area correction factor: $\quad 1.0$

External/inhalation area correction factor: $\quad 1.0$

Inhalation exposure modification factor: $\quad .23$

External exposure modification factor: $\quad .23$

FIGURE 3.10 (Contd) 
ICRP-2 and ICRP-26/30 Maximum Annual Dose Calculation

(MAXI Version IBM 1.0 30-Jun-86)

Case title: SAMPLE5.IN / MAXI Sample Problem Five

Executed on: $10 / 28 / 1986$ at $16: 41: 21$

Page 4

SOIL, AIR, AND WATER CONCENTRATION SUMMARY FOR YEAR: 10

\begin{tabular}{lccccc}
$\begin{array}{l}\text { Radio- } \\
\text { nuclide }\end{array}$ & $\begin{array}{c}\text { Surface Soil } \\
\mathrm{pCi} / \mathrm{m} 2\end{array}$ & $\begin{array}{c}\text { Deep Soil } \\
\mathrm{pCi} / \mathrm{m} 3\end{array}$ & $\begin{array}{c}\text { Air } \\
\mathrm{pCi} / \mathrm{m} 3\end{array}$ & $\begin{array}{c}\text { Irrigation } \\
\mathrm{pCi} / \mathrm{L}\end{array}$ & $\begin{array}{c}\text { Drink Water } \\
\mathrm{pCi} / \mathrm{L}\end{array}$ \\
\hline $\mathrm{C} 060$ & $4.9 \mathrm{E}+02$ & $5.4 \mathrm{E}+09$ & $3.3 \mathrm{E}-07$ & $1.0 \mathrm{E}-01$ & $1.0 \mathrm{E}+01$ \\
SR 90 & $8.6 \mathrm{E}+02$ & $1.6 \mathrm{E}+08$ & $5.7 \mathrm{E}-07$ & $1.0 \mathrm{E}-01$ & $1.0 \mathrm{E}+01$ \\
Y 90 & $8.6 \mathrm{E}+02$ & $1.6 \mathrm{E}+08$ & $5.7 \mathrm{E}-07$ & $.0 \mathrm{E}+00$ & $.0 \mathrm{E}+00$ \\
I 129 & $9.9 \mathrm{E}+02$ & $2.0 \mathrm{E}+10$ & $6.6 \mathrm{E}-07$ & $1.0 \mathrm{E}-01$ & $1.0 \mathrm{E}+01$ \\
U 238 & $9.9 \mathrm{E}+02$ & $2.0 \mathrm{E}+10$ & $6.6 \mathrm{E}-07$ & $1.0 \mathrm{E}-01$ & $1.0 \mathrm{E}+01$ \\
TH 234 & $9.9 \mathrm{E}+02$ & $2.0 \mathrm{E}+10$ & $6.6 \mathrm{E}-07$ & $.0 \mathrm{E}+00$ & $.0 \mathrm{E}+00$ \\
PA 234M & $9.9 \mathrm{E}+02$ & $2.0 \mathrm{E}+10$ & $6.6 \mathrm{E}-07$ & $.0 \mathrm{E}+00$ & $.0 \mathrm{E}+00$ \\
PA 234 & $1.3 \mathrm{E}+00$ & $2.6 \mathrm{E}+07$ & $8.6 \mathrm{E}-10$ & $.0 \mathrm{E}+00$ & $.0 \mathrm{E}+00$
\end{tabular}

SOIL, AIR, AND WATER CONCENTRATION SUMMARY FOR YEAR: 59

\begin{tabular}{lccccc}
$\begin{array}{l}\text { Radio- } \\
\text { nuclide }\end{array}$ & $\begin{array}{c}\text { Surface Soil } \\
\mathrm{DCi} / \mathrm{m} 2\end{array}$ & $\begin{array}{c}\text { Deep Soil } \\
\mathrm{pCi} / \mathrm{m} 3\end{array}$ & $\begin{array}{c}\text { Air } \\
\mathrm{pCi} / \mathrm{m} 3\end{array}$ & $\begin{array}{c}\text { Irrigation } \\
\mathrm{pCi} / \mathrm{L}\end{array}$ & $\begin{array}{c}\text { Drink water } \\
\mathrm{pCi} / \mathrm{L}\end{array}$ \\
\hline C0 60 & $-1.4 \mathrm{E}+02$ & $8.4 \mathrm{E}+06$ & $4.3 \mathrm{E}-07$ & $1.0 \mathrm{E}-01$ & $1.0 \mathrm{E}+01$ \\
SR 90 & $2.8 \mathrm{E}+03$ & $4.8 \mathrm{E}+07$ & $1.9 \mathrm{E}-06$ & $1.0 \mathrm{E}-01$ & $1.0 \mathrm{E}+01$ \\
Y 90 & $2.8 \mathrm{E}+03$ & $4.8 \mathrm{E}+07$ & $1.9 \mathrm{E}-06$ & $.0 \mathrm{E}+00$ & $.0 \mathrm{E}+00$ \\
$\mathrm{I} 129$ & $5.4 \mathrm{E}+03$ & $2.0 \mathrm{E}+10$ & $3.6 \mathrm{E}-06$ & $1.0 \mathrm{E}-01$ & $1.0 \mathrm{E}+01$ \\
U 238 & $5.4 \mathrm{E}+03$ & $2.0 \mathrm{E}+10$ & $3.6 \mathrm{E}-06$ & $1.0 \mathrm{E}-01$ & $1.0 \mathrm{E}+01$ \\
TH 234 & $5.4 \mathrm{E}+03$ & $2.0 \mathrm{E}+10$ & $3.6 \mathrm{E}-06$ & $.0 \mathrm{E}+00$ & $.0 \mathrm{E}+00$ \\
PA 234M & $5.4 \mathrm{E}+03$ & $2.0 \mathrm{E}+10$ & $3.6 \mathrm{E}-06$ & $.0 \mathrm{E}+00$ & $.0 \mathrm{E}+00$ \\
PA 234 & $7.0 \mathrm{E}+00$ & $2.6 \mathrm{E}+07$ & $4.7 \mathrm{E}-09$ & $.0 \mathrm{E}+00$ & $.0 \mathrm{E}+00$
\end{tabular}

FIGURE 3.10 (Contd) 


ICRP-2 and ICRP-26/30 Maximum Annual Dose Calculation
(MAXI Version IBM 1.0 30-Jun-86)
Case title: SAMPLE5. IN / MAXI Sample Problem Five
Executed on: $10 / 28 / 1986$ at $16: 41: 21$

Annual Effective Dose Equivalent (AEDE): $\quad 9.8 E+00$

Maximum Annual Dose (ICRP-2):

$\begin{array}{lclll}\text { To Organ: TOTAL BODY at Year } 30 & 1.5 \mathrm{E}+00 \\ \text { To Organ: } & \text { BONE } & \text { at Year } 30 & 6.2 \mathrm{E}+00 \\ \text { To Organ: } & \text { LUNGS } & \text { at Year } 10 & 2.1 \mathrm{E}-04 \\ \text { To Organ: } & \text { THYROID } \text { at Year } 16 & 2.4 \mathrm{E}+02 \\ \text { To Organ: } & \text { LLI } & \text { at Year } 10 & 9.0 \mathrm{E}-01\end{array}$

FIGURE 3.10 (Contd) 
ICRP-2 and ICRP-26/30 Maximum Annual Dose Calculation

(MAXI Version IBM 1.0 30-Jun-86)

Case title: SAMPLE5.IN / MAXI Sample Problem Five

Executed on: $10 / 28 / 1986$ at $16: 41: 21 \quad$ Page 6

DOSES FROM 1 YEAR OF EXPOSURE

\begin{tabular}{|c|c|c|c|}
\hline Organ & $\begin{array}{l}\text { Committed } \\
\text { Dose } \\
\text { Equivalent }\end{array}$ & $\begin{array}{l}\text { Weighing } \\
\text { Factors }\end{array}$ & $\begin{array}{l}\text { Weighted } \\
\text { Dose } \\
\text { Equivalent }\end{array}$ \\
\hline Gonads & --------- & -------- & $7.7 \mathrm{~F}-03$ \\
\hline Breast & $1.1 \mathrm{E}-02$ & $1.5 \mathrm{E}-01$ & $1.6 \mathrm{E}-03$ \\
\hline R Marrow & $1.2 \mathrm{E}+00$ & $1.2 \mathrm{E}-01$ & $1.5 \mathrm{E}-01$ \\
\hline Lungs & $8.4 \mathrm{E}-03$ & $1.2 \mathrm{E}-01$ & $1.0 \mathrm{E}-03$ \\
\hline Thyroid & $3.1 \mathrm{E}+02$ & $3.0 \mathrm{E}-02$ & $9.4 \mathrm{E}+00$ \\
\hline Bone Surf & $3.0 \mathrm{E}+00$ & $3.0 E-02$ & $9.0 \mathrm{E}-02$ \\
\hline LLI Wall & $1.2 \mathrm{E}+00$ & $6.0 \mathrm{E}-02$ & $7.0 \mathrm{E}-02$ \\
\hline ULI Wall & $4.2 \mathrm{E}-01$ & $6.0 \mathrm{E}-02$ & $2.5 \mathrm{E}-02$ \\
\hline Kidneys & $1.7 E-01$ & $6.0 \mathrm{E}-02$ & $1.0 \mathrm{E}-02$ \\
\hline SI Wall & $3.5 \mathrm{E}-02$ & $E-02$ & 2. $1 \mathrm{E}-03$ \\
\hline Liver & -02 & $E-02$ & $1.3 E-03$ \\
\hline---1 & -- & $--\infty-n-\infty$ & --- \\
\hline $\begin{array}{l}\text { Effective Dose } \\
\text { External Dose }\end{array}$ & Equivalent & & $\begin{array}{l}9.8 \mathrm{E}+00 \\
2.1 \mathrm{E}-04\end{array}$ \\
\hline & & & \\
\hline Annual Effe & Dose Eq & 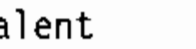 & $9.8 \mathrm{E}+00$ \\
\hline
\end{tabular}

FIGURE 3.10 (Contd) 
ICRP-2 and ICRP-26/30 Maximum Annual Dose Calculation

(MAXI Version IBM 1.0 30-Jun-86)

Case title: SAMPLE5.IN / MAXI Sample Problem Five

Executed on: 10/28/1986 at 16:41:21

Page 7

MAXIMUM ANNUAL DOSE SUMMARY FOR THE YEAR 30 FOR TOTAL BODY

\begin{tabular}{|c|c|c|c|c|c|c|c|c|c|c|}
\hline $\begin{array}{l}\text { RADI0- } \\
\text { NUCLIDE }\end{array}$ & $\begin{array}{l}\text { INGEST } \\
\text { REM }\end{array}$ & $\%$ & $\begin{array}{l}\text { INHALATI } \\
\text { REM }\end{array}$ & $O N$ & $\begin{array}{l}\text { EXTERNAL } \\
\text { REM }\end{array}$ & $\%$ & $\begin{array}{l}\text { AQUATIC } \\
\text { REM }\end{array}$ & OD $\%$ & $\begin{array}{l}\text { DRINK WAT } \\
\text { REM }\end{array}$ & ER \\
\hline $\begin{array}{l}\text { CO60 } \\
\text { SR90 } \\
\text { Y } 90 \\
\text { I } 129 \\
U 2238 \\
\text { TH234 } \\
\text { PA234M } \\
\text { PA234 }\end{array}$ & $\begin{array}{l}8.9 \mathrm{E}-04 \\
1.1 \mathrm{E}+00 \\
1.1 \mathrm{E}-09 \\
3.1 \mathrm{E}-01 \\
1.3 \mathrm{E}-01 \\
8.6 \mathrm{E}-06 \\
.0 \mathrm{E}+00 \\
4.1 \mathrm{E}-12\end{array}$ & $\begin{array}{r}0 \\
71 \\
0 \\
20 \\
8 \\
0 \\
0 \\
0\end{array}$ & $\begin{array}{l}2.3 \mathrm{E}-12 \\
3.0 \mathrm{E}-09 \\
2.4 \mathrm{E}-14 \\
2.8 \mathrm{E}-11 \\
7.4 \mathrm{E}-10 \\
3.6 \mathrm{E}-13 \\
2.2 \mathrm{E}-19 \\
6.7 \mathrm{E}-18\end{array}$ & $\begin{array}{r}0 \\
79 \\
0 \\
0 \\
19 \\
0 \\
0 \\
0\end{array}$ & $\begin{array}{l}2.2 \mathrm{E}-05 \\
7.7 \mathrm{E}-10 \\
5.1 \mathrm{E}-08 \\
1.6 \mathrm{E}-08 \\
3.6 \mathrm{E}-10 \\
2.8 \mathrm{E}-08 \\
1.7 \mathrm{E}-06 \\
4.8 \mathrm{E}-07\end{array}$ & $\begin{array}{r}90 \\
0 \\
0 \\
0 \\
0 \\
0 \\
6 \\
2\end{array}$ & $\begin{array}{r}1.6 \mathrm{E}-07 \\
1.8 \mathrm{E}-05 \\
.0 \mathrm{E}+00 \\
9.5 \mathrm{E}-08 \\
6.3 \mathrm{E}-08 \\
.0 \mathrm{E}+00 \\
.0 \mathrm{E}+00 \\
.0 \mathrm{E}+00\end{array}$ & $\begin{array}{r}0 \\
98 \\
0 \\
0 \\
0 \\
0 \\
0 \\
0\end{array}$ & $\begin{array}{r}4.1 \mathrm{E}-06 \\
7.6 \mathrm{E}-04 \\
.0 \mathrm{E}+00 \\
3.2 \mathrm{E}-05 \\
1.4 \mathrm{E}-04 \\
.0 \mathrm{E}+00 \\
.0 \mathrm{E}+00 \\
.0 \mathrm{E}+00\end{array}$ & $\begin{array}{r}0 \\
81 \\
0 \\
3 \\
14 \\
0 \\
0 \\
0\end{array}$ \\
\hline $\begin{array}{l}\text { TOTAL } \\
1.5 \mathrm{E}+\mathrm{C}\end{array}$ & \multicolumn{2}{|c|}{$\begin{array}{c}\text { INGESTION } \\
1.5 \mathrm{E}+00 \quad 99\end{array}$} & \multicolumn{2}{|c|}{$\begin{array}{l}\text { INHALATION } \\
3.7 \mathrm{E}-09090\end{array}$} & $\begin{array}{l}\text { EXTERNAL } \\
2.4 \mathrm{E}-05\end{array}$ & 0 & \multicolumn{2}{|c|}{$\begin{array}{l}\text { AQUATIC FOOD } \\
1.8 \mathrm{E}-0500\end{array}$} & $\begin{array}{c}\text { DRINK WAT } \\
9.4 \mathrm{E}-04\end{array}$ & 0 \\
\hline
\end{tabular}

MAXIMUM ANNUAL DOSE SUMMARY FOR THE YEAR 30 FOR BONE

\begin{tabular}{|c|c|c|c|c|c|c|c|c|c|c|}
\hline $\begin{array}{l}\text { RADIO- } \\
\text { NUCLIDE }\end{array}$ & $\begin{array}{l}\text { INGESTIOI } \\
\text { REM }\end{array}$ & ON & $\begin{array}{l}\text { INHALAT } \\
\text { REM }\end{array}$ & $\%$ & $\begin{array}{l}\text { EXTERNAL } \\
\text { REM }\end{array}$ & $\%$ & $\begin{array}{l}\text { AQUATIO } \\
\text { REM }\end{array}$ & $000_{\%}$ & $\begin{array}{l}\text { DRINK WAT } \\
\text { REM }\end{array}$ & ER \\
\hline $\begin{array}{l}\text { CO60 } \\
\text { SR90 } \\
\text { Y } 90 \\
\text { I } 129 \\
\text { U } 238 \\
\text { TH234 } \\
\text { PA234M } \\
\text { PA234 }\end{array}$ & $\begin{array}{r}.0 \mathrm{E}+00 \\
4.0 \mathrm{E}+00 \\
3.9 \mathrm{E}-08 \\
1.1 \mathrm{E}-01 \\
2.1 \mathrm{E}+00 \\
3.0 \mathrm{E}-04 \\
.0 \mathrm{E}+00 \\
1.6 \mathrm{E}-11\end{array}$ & $\begin{array}{r}0 \\
64 \\
0 \\
1 \\
33 \\
0 \\
0 \\
0\end{array}$ & $\begin{array}{l}.0 \mathrm{E}+00 \\
4.4 \mathrm{E}-08 \\
8.8 \mathrm{E}-13 \\
6.7 \mathrm{E}-13 \\
1.2 \mathrm{E}-08 \\
1.2 \mathrm{E}-11 \\
4.7 \mathrm{E}-18 \\
2.6 \mathrm{E}-17\end{array}$ & $\begin{array}{r}0 \\
78 \\
0 \\
0 \\
21 \\
0 \\
0 \\
0\end{array}$ & $\begin{array}{l}2.2 \mathrm{E}-05 \\
7.7 \mathrm{E}-10 \\
5.1 \mathrm{E}-08 \\
1.6 \mathrm{E}-08 \\
3.6 \mathrm{E}-10 \\
2.8 \mathrm{E}-08 \\
1.7 \mathrm{E}-06 \\
4.8 \mathrm{E}-07\end{array}$ & $\begin{array}{r}90 \\
0 \\
0 \\
0 \\
0 \\
0 \\
6 \\
2\end{array}$ & $\begin{array}{c}.0 \mathrm{E}+ \\
6.7 \mathrm{E}- \\
.0 \mathrm{E}+ \\
3.4 \mathrm{E}- \\
1.1 \mathrm{E}- \\
.0 \mathrm{E}+ \\
.0 \mathrm{E}+ \\
.0 \mathrm{E}+\end{array}$ & $\begin{array}{r}0 \\
98 \\
0 \\
0 \\
1 \\
0 \\
0 \\
0\end{array}$ & $\begin{array}{r}.0 \mathrm{E}+00 \\
2.8 \mathrm{E}-03 \\
.0 \mathrm{E}+00 \\
1.1 \mathrm{E}-05 \\
2.3 \mathrm{E}-03 \\
.0 \mathrm{E}+00 \\
.0 \mathrm{E}+00 \\
.0 \mathrm{E}+00\end{array}$ & $\begin{array}{r}0 \\
54 \\
0 \\
0 \\
45 \\
0 \\
0 \\
0\end{array}$ \\
\hline $\begin{array}{c}\text { TOTAL } \\
6.2 E+00\end{array}$ & $6.2 \mathrm{E}+00$ & $\begin{array}{l}\text { N } \\
99\end{array}$ & $.6 \mathrm{E}-08$ & 0 & $2.4 \mathrm{E}$ & 0 & $\begin{array}{l}\text { AQUATIC } \\
6.8 \mathrm{E}-05\end{array}$ & $\begin{array}{r}00 \mathrm{D} \\
0\end{array}$ & $5.2 \mathrm{E}-03$ & 0 \\
\hline
\end{tabular}

FIGURE 3.10 (Contd) 
ICRP-2 and ICRP-26/30 Maximum Annual Dose Calculation

(MAXI Version IBM 1.030 -Jun-86)

Case title: SAMPLE5.IN / MAXI Sample Problem Five

Executed on: $10 / 28 / 1986$ at $16: 41: 21$

Page 8

MAXIMUM ANNUAL DOSE SUMMARY FOR THE YEAR 10 FOR LUNGS

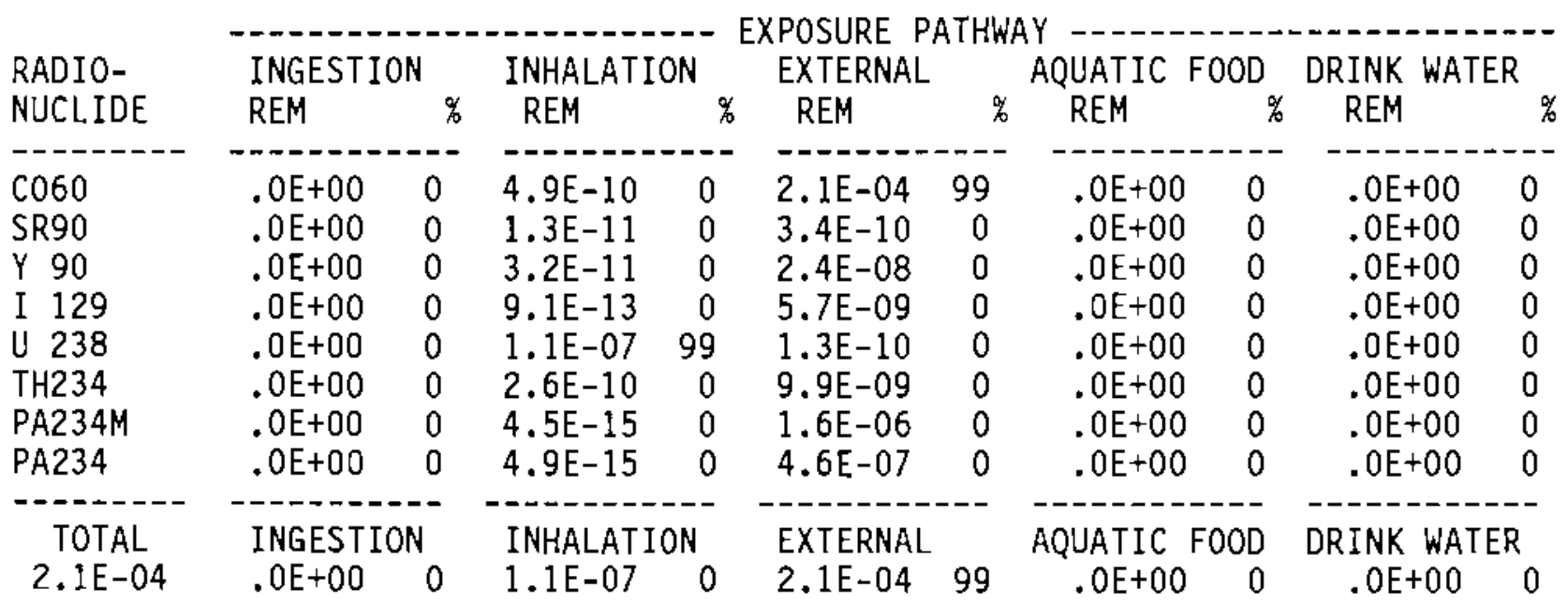

MAXIMUM ANNUAL DOSE SUMMARY FOR THE YEAR 16 FOR THYROID

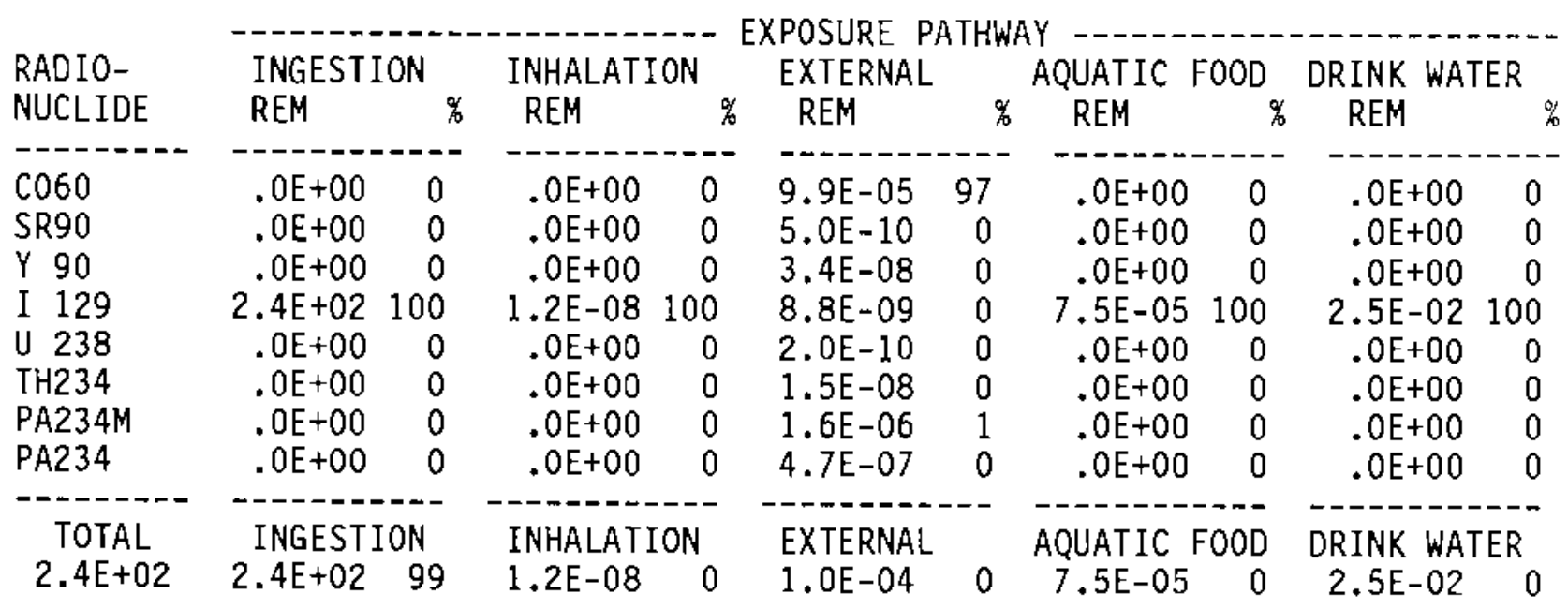

FIGURE 3.10 (Contd) 
ICRP-2 and ICRP-26/30 Maximum Annual Dose Calculation

(MAXI Version IBM 1.0 30-Jun-B6)

Case title: SAMPLE5.IN / MAXI Sample Problem Five

Executed on: $10 / 28 / 1986$ at $16: 41: 21$

Page 9

MAXIMUM ANNUAL DOSE SUMMARY FOR THE YEAR 10 FOR LLI

\begin{tabular}{|c|c|c|c|c|c|c|c|c|c|c|}
\hline \multirow{2}{*}{$\begin{array}{l}\text { RADI0- } \\
\text { NUCLIDE }\end{array}$} & \multicolumn{2}{|c|}{ INGESTION } & \multicolumn{2}{|c|}{ INHALATION } & \multicolumn{2}{|l|}{ EXTERNAL } & \multicolumn{2}{|c|}{ AQUATIC FOOD } & \multicolumn{2}{|c|}{ DRINK WATER } \\
\hline & REM & $\%$ & REM & $\%$ & REM & $\%$ & REM & g & REM & $\%$ \\
\hline 060 & 1. $2 \mathrm{E}-01$ & 12 & $1.3 \mathrm{E}-1$ & 7 & $2.1 \mathrm{E}-04$ & 99 & $1.5 E-06$ & 47 & $3.9 \mathrm{E}$ & 13 \\
\hline RYO & 1.3 & 14 & 1.3 & 6 & $3.4 \mathrm{E}$ & 0 & $1.6 \mathrm{E}$ & 49 & 6.8 & 23 \\
\hline Y 90 & $7.5 \mathrm{E}$ & 0 & $4.8 \mathrm{E}$ & 25 & $2.4 \mathrm{E}$ & 0 & $.0 \mathrm{E}$ & 0 & $.0 \mathrm{E}+00$ & 0 \\
\hline I 129 & 1.7 & 1 & $9.8 \mathrm{E}$ & 0 & $5.7 \mathrm{E}$ & 0 & 5.11 & 0 & 1.7 & 0 \\
\hline U 238 & 1.7 & 18 & 3.7 & 20 & 1.3 & 0 & $8.4 !$ & 2 & $1.9 \mathrm{E}$ & 63 \\
\hline TH234 & 4.7 & 52 & 7.3 & 39 & 9. & 0 & $.0 \mathrm{E}+0 \mathrm{C}$ & 0 & 00 & 0 \\
\hline PA234M & & 0 & .0 & 0 & 1. & 0 & $.0 E+00$ & 0 & $.0 E+00$ & 0 \\
\hline PA234 & 1.7 & 0 & $4.9 E-15$ & 0 & 4. & 0 & .0 & 0 & $.0 E+00$ & 0 \\
\hline TOT & $-\overline{\text { TN }}$ & 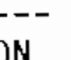 & --1 & & & 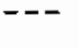 & & & & \\
\hline $9.0 \mathrm{E}-01$ & $9.0 \mathrm{E}-01$ & 99 & $1.8 \mathrm{E}-10$ & 0 & $2.1 E-04$ & 0 & 3. $2 \mathrm{E}-0 \mathrm{E}$ & 0 & $2.9 E-04$ & 0 \\
\hline
\end{tabular}

FIGURE 3.10 (Contd) 


\subsubsection{Sample Problem Six}

This sample problem demonstrates use of the Stored waste Scenario. The Stored Waste Scenario is a sub-set of the External Exposure Scenario (Kennedy et 1 . 1986). For this sample problem, a licensee is assumed to dispose of $100 \mathrm{~m}^{3}$ of noncompacted waste in drums in an aboveground storage facility. The waste is assumed to contain an average of $0.1 \mathrm{Ci} / \mathrm{m}^{3}$ of ${ }^{60} \mathrm{Co}$ and have a density of $1.0 \mathrm{~g} / \mathrm{cm}^{3}$. The resulting dose to an intruder who enters the storage facility a few weeks after the wastes are disposed of and stays there for $500 \mathrm{~h}$ of exposure is determined by running MAXI1 with the input file shown in Figure 3.11. Changes from the previous sample problem are axued on the Sample Problem six input file to assist the user in understanding the second level of software operation.

Note that the ONSITE program asks for an inventory modification factor and that a value of 0.2 is used for the sample problem. This factor accounts for the mixing of the radioactive waste with nonradioactive materials like drums or packaging materials or noncontaminated soil for the case of land disposal. For consistency with the other sample problems given in Napier et al. (1984), the factor of 0.2 is used; however, more appropriate values for stored waste nay be closer to 1.0 and the latter value may be substituted by the user.

The output for this sample problem is shown in Figure 3.12. The calculated total-body dose to the intruder for the scenario in this sample problem is about 6.1 rem. 
SAMPLE6. IN / MAXI Sample Problem Six; Stored Waste Sub-set of Scenario 1

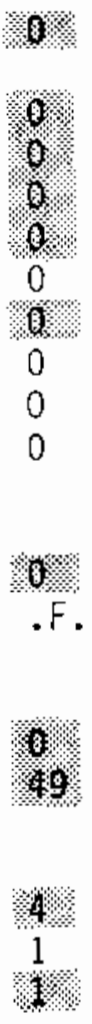

0.2

1.0

1.0

1.0

DO YOU WISH TO CONSIDER:

External exposure and root penetration for waste modeled as a slab (volume) source? (0-no 1-yes)

External exposure to surface contamination modeled as a plane source? (0-no 1-yes)

Inhalation of resuspended material? (0-no 1-yes)

Farm product ingestion? (0-no 1-yes)

Aquatic foods ingestion? (0-no 1-yes)

Drinking water ingestion? (0-no 1-yes)

offsite atmospheric source? (0-no 1 -yes)

ICRP-26/30 as we11 as ICRP-2 methodology? (0-no 1-yes)

Decay of air release source term? (0-no 1-yes)

Decay of water release source term? (0-no 1-yes)

Use the One-Year-of-Exposure Model? (0-no 1-yes)

IEXT

ISUR

INHA

IFOD

IARG

IWAT

IAIR

IEDE

IDKAIR

IDKWAT

INTRUD

MONITORING AND OUTPUT OPTIONS

Level of detail in reports (0-least to 3 )

Monitor and print hand calculation report

IOUT

DEBUG (4)

NUMBER OF YEARS AFTER WASTE IS DISPOSED THAT:

Scenario begins:

IT1

Scenario ends:

IT2

INVENTORY:

Release Term Input units: (1-pCi $2-4 C i \quad 3-m C i \quad 4-C i)$ INUT

Soil source units $\left(0-m^{\star \star 2} \quad 1-m^{\star *} 3 \quad 2-\mathrm{kg}\right) \quad$ M3M2

Number of radionuclides in inventory:

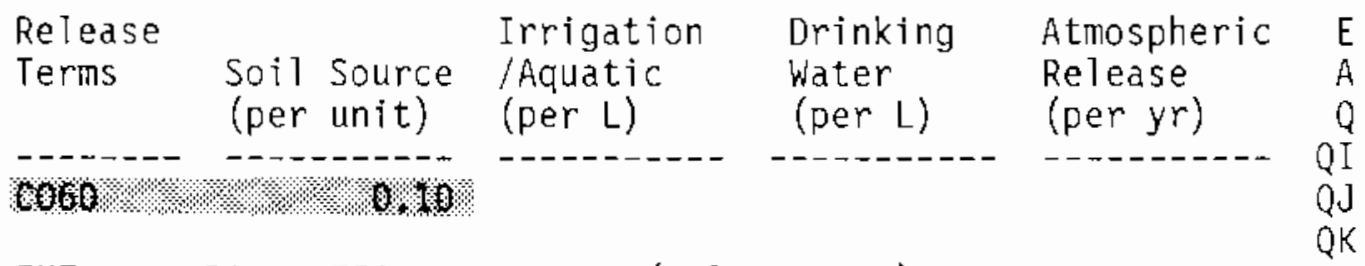

INVENTORY MODIFICATION FACTORS: (multipliers)

Surface inventory modification factor:

SRDIL

Irrigation/aquatic inventory modification factor:

DILF

Size of site (fractional ha):

FRSIZ

Fraction of total diet grown on site:

RPF2

\section{EXTERNAL/INHALATION EXPOSURE:}

500. 月. 0. 0.

PLANEA.DAT STOWFE. WA 270.0
Hours of external exposure to contamination $(h / y r)$ : Hours of inhalation of airborne contamination $(h / y r)$ : Ext. exp. dose rate factors (plane source) filename: Ext. exp. dose rate factors (volume source) filename: Breathing rate $\left(\mathrm{cm}^{\star *} 3 / \mathrm{sec}\right)$ :
HREXT

HRINH

PLANAM

VOLNAM

BRATE

FIGURE 3.11. Sample Problem Six Input 


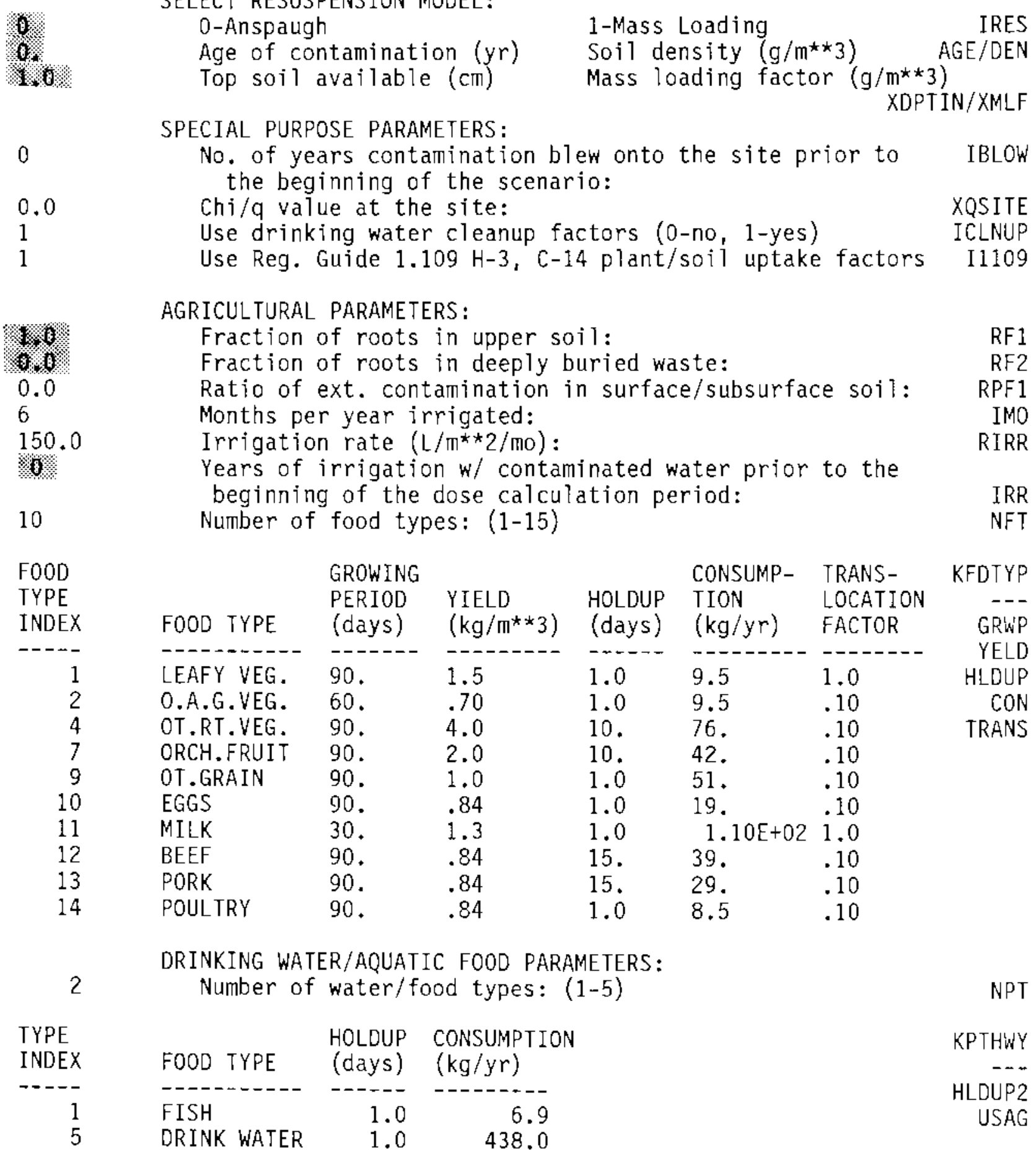

SELECT RESUSPENSION MODEL:

1-Mass Loading IRES

$\begin{array}{ll}\text { Age of contamination ( } \mathrm{yr}) & \text { Soil density }\left(\mathrm{g} / \mathrm{m}^{\star} * 3\right) \\ \text { Top soil available (cm) } & \text { Mass loading factor }\left(\mathrm{g} / \mathrm{m}^{\star *} 3\right)\end{array}$

PECIAL PURPOSE PARAMETERS:

. of years contamination blew onto the site prior to IBLOW

theginning of the scenario:

Use drinking water cleanup factors (0-no, 1-yes) ICLNUP

Use Reg. Guide $1.109 \mathrm{H}-3, \mathrm{C}-14 \mathrm{plant} / \mathrm{so} i 1$ uptake factors I1109

FICULTURAL PARAMETERS:

Fraction of roots in upper soil:

RF1

RF2

IMO

RIRR

IRR

NFT beginning of the dose calculation period: 
ICRP-2 and ICRP-26/30 Maximum Annual Dose Calculation

(MAXI Version IBM 1.0 30-Jun-86)

Case title: SAMPLE6.IN / MAXI Sample Problem Six; Stored Waste Sub-set of Scenario

Executed on: $10 / 2 / 1986$ at $15: 13: 18$

Page 1

PATHWAYS/OPTIONS CONSIDERED:

External exposure/root penetration for waste modeled as slab (volume) source

TITLES OF LIBRARY FILES ACCESSED:

10: RMDLIB - Radionuclide Master Library (24-Sep-86 RAP)

27: ROOM: 1.0 M THICK FINITE SLAB (DEN: H20 1.0): MR/HR 8-JUl-86 RAP

NUMBER OF YEARS AFTER WASTE IS DISPOSED THAT:

Scenario begins:

Scenario ends:

INVENTORY:

Release Term Input units: (1-pCi 2-uCi $3-m C i \quad 4-C i)$

Soil source units: $\left(0-m^{\star \star 2} \quad 1-m^{\star \star} 32-\mathrm{kg}\right)$

Number of radionuclides in inventory:

Release Irrigation Drinking Atmospheric

Terms Soil Source/Aquatic Water Release

$\left(\operatorname{per} m^{* *} 3\right) \quad(\operatorname{per} L) \quad(\operatorname{per} \mathrm{L}) \quad(\operatorname{per} \mathrm{m} 3)$

$\begin{array}{lrrrr} & & & & \\ 0060 & 1.0 \mathrm{E}+11 & .0 \mathrm{E}+00 & .0 \mathrm{E}+00 & .0 \mathrm{E}+00\end{array}$

INVENTORY MODIFICATION FACTORS: (multipliers)

Surface inventory dilution factor:

Irrigation/aquatic inventory modification factor:

.2

size of site (fractional ha):

FIgURE 3.12. Sample Problem Six Output 
ICRP-2 and ICRP-26/30 Maximum Annual Dose Calculation

(MAXI Version IBM 1.0 30-Jun-B6)

Case title: SAMPLE6.IN / MAXI Sample Problem Six; Stored waste Sub-set of Scenario

Executed on: $10 / 2 / 1986$ at $15: 13: 18$

Page 2

EXTERNAL/INHALATION EXPOSURE:

Hours of external exposure to contamination $(h / y r): \quad 5.0 E+02$

RESUSPENSION PARAMETERS:

Model used:

Average age of contamination on ground at Time Zero $(y r)$

Top soil available for resuspension $(\mathrm{cm})$ :

Anspaugh

.0

1 .

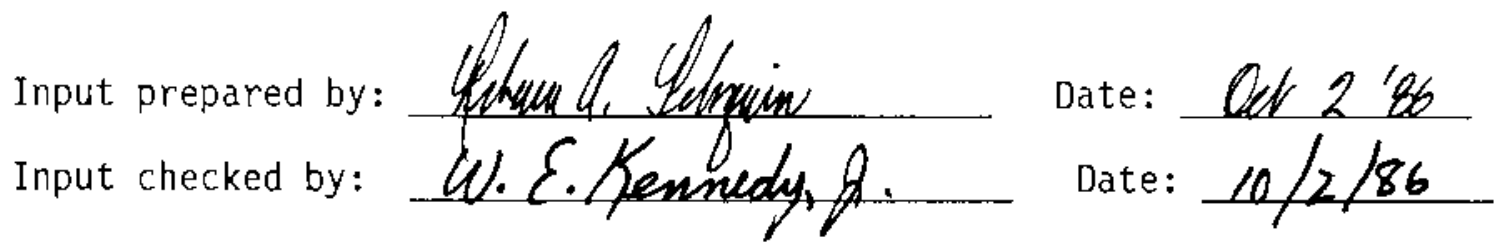

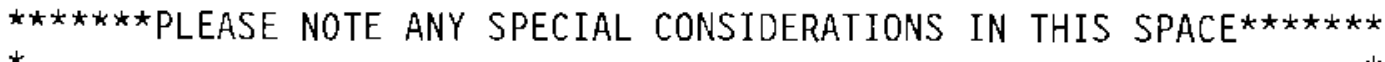

$\star$

$\star$

$\star$

$\star$

$\star$

*

$\star$

*

\section{CALCULATED VALUES:}

Ingestion area correction factor:

External/inhalation area correction factor:

Inhalation exposure modification factor:

External exposure modification factor:

1.0

1.0

.00

5.70E-02

FIGURE 3.12 (Contd) 
ICRP-2 and ICRP-26/30 Maximum Annual Dose Calculation

(MAXI Version IBM 1.0 30-Jun-86)

Case title: SAMPLE6.IN / MAXI Sample Problem Six; Stored Waste Sub-set of Scenario

Executed on: $10 / 2 / 1986$ at $15: 13: 18$

Page 3

SOIL, AIR, AND WATER CONCENTRATION SUMMARY FOR YEAR: 0

\begin{tabular}{lccccc} 
Radio- & Surface Soil & Deep Soil & Air & Irrigation \\
nuclide & $\mathrm{PCi} / \mathrm{m} 2$ & $\mathrm{PCi} / \mathrm{m} 3$ & $\mathrm{PCi} / \mathrm{m} 3$ & $\begin{array}{c}\text { Drink Water } \\
\mathrm{PCi} / \mathrm{L}\end{array}$ & $\mathrm{PC} / \mathrm{L}$ \\
\hdashline $\mathrm{CO} 60$ & $.0 \mathrm{E}+00$ & $2.0 \mathrm{E}+10$ & $.0 \mathrm{E}+00$ & $.0 \mathrm{E}+00$ & $.0 \mathrm{E}+00$
\end{tabular}

SOIL, AIR, AND WATER CONCENTRATION SUMMARY FOR YEAR: 49

\begin{tabular}{lccccc} 
Radio- & Surface Soil & Deep Soil & Air & Irrigation & Drink Water \\
nuclide & $\mathrm{pCi} / \mathrm{m} 2$ & $\mathrm{PCi} / \mathrm{m} 3$ & $\mathrm{PCi} / \mathrm{m} 3$ & $\mathrm{PCi} / \mathrm{L}$ & $\mathrm{PCi} / \mathrm{L}$ \\
\hdashline $\mathrm{CO} 60$ & $.0 \mathrm{E}+00$ & $3.1 \mathrm{E}+07$ & $.0 \mathrm{E}+00$ & $.0 \mathrm{E}+00$ & $.0 \mathrm{E}+00$
\end{tabular}

FIGURE 3.12 (Contd) 
ICRP-2 and ICRP-26/30 Maximum Annual Dose Calculation

(MAXI Version IBM 1.0 30-Jun-86)

Case title: SAMPLE6.IN / MAXI Sample Problem Six; Stored waste Sub-set of Scenario Executed on: $10 / 2 / 1986$ at $15: 13: 18$

Page 4

MAXIMUM ANNUAL DOSE SUMMARY FOR THE YEAR O FOR TOTAL BODY-

\begin{tabular}{|c|c|c|c|c|c|c|c|c|c|}
\hline $\begin{array}{l}\text { RADI0- } \\
\text { NUCLIDE }\end{array}$ & $\begin{array}{l}\text { INGESTION } \\
\text { REM }\end{array}$ & $\%$ & $\begin{array}{l}\text { INHALATION } \\
\text { REM }\end{array}$ & $\%$ & $\begin{array}{l}\text { XPOSURE P } \\
\text { EXTERNAL } \\
\text { REM }\end{array}$ & $\%$ & $\begin{array}{c}\text { AY } \\
\text { AQUATIC } \\
\text { REM }\end{array}$ & $=000$ & $\begin{array}{l}\text { DRINK WATER } \\
\text { REM }\end{array}$ \\
\hline C060 & $.0 E+00$ & 0 & $.0 E+00$ & 0 & $6.1 \mathrm{E}+00$ & 100 & $.0 \mathrm{E}+00$ & 0 & $.0 \mathrm{E}+00$ \\
\hline $\begin{array}{c}\text { TOTAL } \\
6.1 \mathrm{E}+00\end{array}$ & $\begin{array}{l}\text { INGESTION } \\
.0 \mathrm{E}+00\end{array}$ & 0 & $\begin{array}{l}\text { INHALATION } \\
.0 \mathrm{E}+00\end{array}$ & 1 & $\begin{array}{l}\text { EXTERNAL } \\
6.1 \mathrm{E}+00\end{array}$ & & $\begin{array}{l}\text { AQUATIC } \\
.0 \mathrm{E}+00\end{array}$ & $\begin{array}{r}\text { FOOD } \\
0\end{array}$ & $\begin{array}{l}\text { DRINK WATER } \\
.0 E+00\end{array}$ \\
\hline
\end{tabular}

\section{MAXIMUM ANNUAL DOSE SUMMARY FOR THE YEAR O FOR BONE}

\begin{tabular}{|c|c|c|c|c|c|c|c|}
\hline $\begin{array}{l}\text { RADIO- } \\
\text { NUCLIDE }\end{array}$ & $\begin{array}{l}\text { INGESTION } \\
\text { REM } \%\end{array}$ & $\begin{array}{l}\text { INHALATION } \\
\text { REM }\end{array}$ & $\begin{array}{l}\text { EXTERNAL } \\
\% \quad \text { REM }\end{array}$ & $\%$ & $\begin{array}{l}\text { AQUATIC } \\
\text { REM }\end{array}$ & FOOD & $\begin{array}{l}\text { DRINK WATER } \\
\text { REM }\end{array}$ \\
\hline $\mathrm{CO} 060$ & $.0 \mathrm{E}+00$ & $.0 E+00$ & $5.1 E+00$ & 00 & $.0 \mathrm{E}+00$ & 0 & $.0 E+00$ \\
\hline $\begin{array}{c}\text { TOTAL } \\
6.1 \mathrm{E}+00\end{array}$ & $\begin{array}{l}\text { INGESTION } \\
.0 \mathrm{E}+00 \quad 0\end{array}$ & $\begin{array}{l}\text { INHALATION } \\
.0 \mathrm{E}+00\end{array}$ & $\begin{array}{l}\text { EXTERNAL } \\
5.1 \mathrm{E}+00\end{array}$ & & $\begin{array}{l}\text { AQUATIC } \\
.0 \mathrm{E}+00\end{array}$ & $\begin{array}{r}F O O D \\
0\end{array}$ & $\begin{array}{l}\text { DRINK WATER } \\
.0 \mathrm{E}+00 \quad 0\end{array}$ \\
\hline
\end{tabular}

\section{MAXIMUM ANNUAL DOSE SUMMARY FOR THE YEAR O FOR LUNGS}

\begin{tabular}{|c|c|c|c|c|c|c|c|c|c|}
\hline $\begin{array}{l}\text { RADI0- } \\
\text { NUCLIDE }\end{array}$ & $\begin{array}{l}\text { INGESTION } \\
\text { REM }\end{array}$ & $\%$ & $\begin{array}{l}\text { INHALATION } \\
\text { REM }\end{array}$ & $\%$ & $\begin{array}{l}\text { EXTERNAL } \\
\text { REM }\end{array}$ & $\begin{array}{l}\text { AQUATIC } \\
\text { REM }\end{array}$ & $\begin{array}{r}\mathrm{FOOD} \\
\%\end{array}$ & $\begin{array}{l}\text { DRINK WATER } \\
\text { REM }\end{array}$ & $\%$ \\
\hline $\mathrm{CO60}$ & $.0 \mathrm{E}+00$ & 0 & $.0 \mathrm{E}+00$ & 0 & $6.1 E+00100$ & $.0 \mathrm{E}+00$ & 0 & $.0 \mathrm{E}+00$ & 0 \\
\hline $\begin{array}{c}\text { TOTAL } \\
6.1 \mathrm{E}+00\end{array}$ & $\begin{array}{l}\text { INGESTION } \\
.0 \mathrm{E}+00\end{array}$ & $v_{0}$ & $\begin{array}{l}\text { INHALATION } \\
.0 \mathrm{E}+00\end{array}$ & 0 & $\begin{array}{l}\text { EXTERNAL } \\
6.1 \mathrm{E}+00 \mathrm{I} 00\end{array}$ & $\begin{array}{l}\text { AQUATIC } \\
.0 \mathrm{E}+00\end{array}$ & $\begin{array}{r}F O O D \\
0 \quad 0\end{array}$ & $\begin{array}{l}\text { DRINK WATER } \\
.0 \mathrm{E}+00\end{array}$ & $R_{0}$ \\
\hline
\end{tabular}

FIGURE 3.12 (Contd) 
MAXIMUM ANNUAL DOSE SUMMARY FOR THE YEAR O FOR THYROID

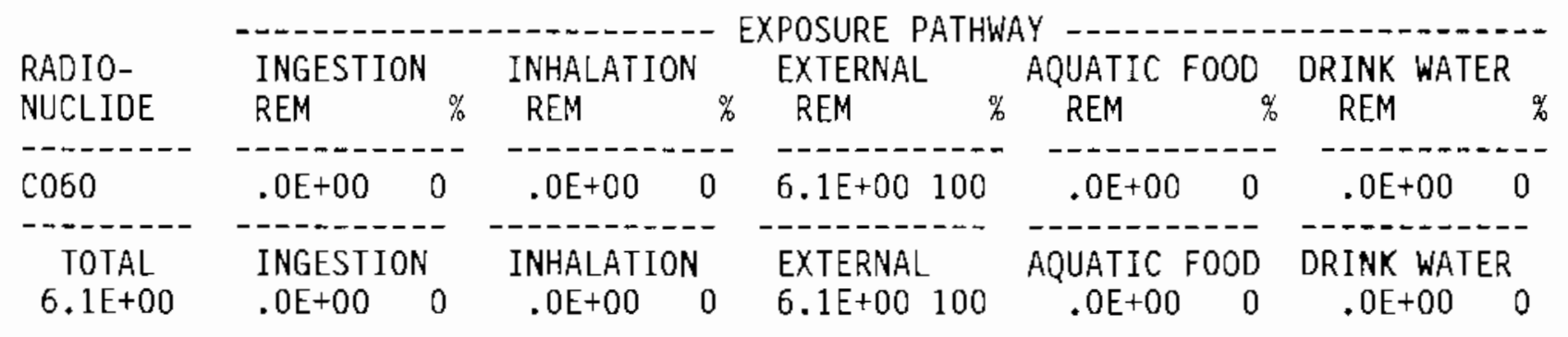

FIGURE 3.12 (Contd) 
ICRP-2 and ICRP-26/30 Maximum Annual Dose Calculation

(MAXI Version IBM $1.030-J u n-86$ )

Case title: SAMPLE6.IN / MAXI Sample Problem Six; Stored Waste Sub-set of Scenario

Executed on: $10 / 2 / 1986$ at $15: 13: 18$

Page 5

\section{MAXIMUM ANNUAL DOSE SUMMARY FOR THE YEAR O FOR LLI}

\begin{tabular}{|c|c|c|c|c|c|c|c|c|c|c|}
\hline $\begin{array}{l}\text { RADI0- } \\
\text { NUCLIDE }\end{array}$ & $\begin{array}{l}\text { INGESTION } \\
\text { REM }\end{array}$ & $\%$ & $\begin{array}{l}\text { INHALATION } \\
\text { REM }\end{array}$ & $\%$ & $\begin{array}{l}\text { XPOSURE P } \\
\text { EXTERNAL } \\
\text { REM }\end{array}$ & W & $\begin{array}{c}\text { AY } \\
\text { AQUATIC } \\
\text { REM }\end{array}$ & F00D & $\begin{array}{l}\text { DRINK WATER } \\
\text { REM }\end{array}$ & R $\%$ \\
\hline 60 & $.0 \mathrm{E}+00$ & 0 & $.0 \mathrm{E}+00$ & 0 & $6.1 E+00$ & 00 & $.0 E+00$ & 0 & $.0 \mathrm{E}+00$ & 0 \\
\hline $\begin{array}{c}\text { TOTAL } \\
6.1 E+00\end{array}$ & $\begin{array}{l}\text { INGEST ION } \\
.0 E+00\end{array}$ & $v_{0}$ & $\begin{array}{l}\text { INHALATION } \\
.0 \mathrm{E}+00\end{array}$ & $v_{0}$ & $\begin{array}{l}\text { EXTERNAL } \\
6.1 E+00\end{array}$ & & $\begin{array}{l}\text { AQUATIC } \\
.0 \mathrm{E}+00\end{array}$ & $\begin{array}{r}F 000 \\
0\end{array}$ & $\begin{array}{c}\text { DRINK WATEF } \\
.0 E+00\end{array}$ & $\mathrm{R}_{0}^{\mathrm{R}}$ \\
\hline
\end{tabular}

FIGURE 3.12 (Contd)

3.67 


\subsection{INPUT PARAMETERS}

This section contains a discussion of input parameters ordered as in the MAXI1 input file. The purpose of this section is to provide more detailed information on each of the parameters than can be contained in the MAXIl input file. The MAXI1 parameter name is listed to the extreme right on the MAXI1 input file.

Attempts were made to simplify MAXIl logic when the IBM PC/XT/AT version was created. Consequently, most multi-purpose parameters have been redefined as two or more separate parameters. This feature simplifies input parameter descriptions. Change in usage from earlier versions of the ONSITE/MAXI1 computer programs will be noted for each parameter as appropriate. Parameter values for the five defined scenarios are presented in Table 3.1. Parameter values for the six sample problems are presented in Table 3.2. Tables 3.1 and 3.2 are located at the end of this subsection.

IEXT Option set to consider the external exposure and terrestrial-crop root penetration pathway for waste modelled as a slab (volume) source. Waste may be stored aboveground, buried at the soil surface, or buried with a soil overburden. Allowable values are: 0-do not consider this pathway, 1-consider this pathway.

ISUR Option set to consider the external exposure to the surface contamination pathway for waste modelled as a plane source. This pathway is selected to consider either a waste spill or agricultural activities as defined in Scenarios 3 and 4. Allowable values are: 0 -do not consider this pathway, 1-consider this pathway.

INHA Option set to consider inhalation of contamination from resuspended material and/or contamination that has blown in from offsite. Definition of this parameter in MAXI1 has simplified option testing. Allowable values: 0-do not consider this pathway, 1-consider this pathway.

IFOD Option set to consider ingestion of terrestrial food products. Allowable values: 0 -do not consider this pathway, 1-consider this pathway.

IARG Option set to consider ingestion of aquatic food products. Allowable values: 0-do not consider this pathway, 1-consider this pathway.

IWAT

Option set to consider ingestion of drinking water. Allowable values: 0-do not consider this pathway, 1-consider this pathway.

IAIR Option to allow an offsite source of continuing deposition via the atmospheric pathway to contaminate the site. The start of the deposition may begin before the start of the dose calculation period at a time IBLOW years earlier. In addition, the external source may be allowed to decay, from its initial value at time 
IT1, if the option IDKAIR is set. For times less than IT1, the source term is reconstructed from the source given for IT1. Allowable values: 0 -do not consider this pathway, 1consider this pathway.

IEDE Option set to consider the dosimetry methodology given in ICRP Publication No. 30 as well as ICRP Publication 2. When this option is selected, the annual effective dose equivalent (AEDE) is calculated in addition to the maximum annual dose to each organ. Allowable values: 0-do not consider this option, 1consider this option.

IDKAIR Dption set to allow decay of the atmospheric source term (QK) starting at time ITl. Allowable values: 0-do not consider this option, 1-consider this option.

IDKWAT Option set to allow decay of river/aquatic release source term (QI) from its initial value at time IT1. Allowable values: $0-$ do not consider this option, 1-consider this option.

INTRUD Option set to consider a special one-year-of-exposure-only intruder model. The intruder in this model resides at the waste disposal site for one year only. Allowable values: 0-do not consider this option, 1-consider this option.

Option set to indicate level of detail desired in output reports.

If option IEDE has not been set, the following reports are indicated by these indices:

1 - tables of maximum annual doses per organ with radionuclide and pathway contribution

2 - the tables listed above and a running summary of the annual doses listed by organ and total only

3 - the above summaries and all annual tables by organ, pathway and radionuclide.

If option IEDE has been set, the following reports are indicated by these indices:

1 - the annual effective dose equivalent (AEDE) and the maximum annual dose (and maximum year) to each organ

2 - the committed dose equivalent by organ, the effective dose equivalent, external dose equivalent, and the annual effective dose equivalent 
3 - the committed dose equivalent to each organ by radionuclide and pathway plus the maximum annual dose to each organ by radionuclide and pathway for the maximum year for each organ.

DEBUG (4)

Logical set if a special report of library and intermediate parameter values is requested. This report is useful when performing hand calculations for verification purposes.

IT1

IT2

The year after waste disposal when intrusion occurs and the annual dose and annual effective dose equivalent calculations begins. This parameter allows for correct chain decay of the source term.

INUT

The year dose calculations cease, normally IT1 +49 .

Release term input units:

$1-p C i$
$2-\mu C i$
$3-m C i$
$4-C i$

M3M2

Soil source input unit, in terms of:

$$
\begin{array}{ll}
0- & \text { INUT } / \mathrm{m}_{3}^{2} \\
1- & \text { INUT } / \mathrm{m}^{3} \\
2- & \text { INUT } / \mathrm{kg}
\end{array}
$$

NIN

Number of radionuclides in the release term. Change this index to correspond to the number of radionuclides in the source term.

$E$

Two-character element symbol for this radionuclide.

A

Mass number of this radionuclide as specified in the file RMDONS. Input may be up to six characters in length and include metastable $(M)$ designation. Radionuclide names $(E+A)$ must be found in the radionuclide master library for ONSITE/MAXI1 named RMDONS, listed in Appendix B. Note: the "including daughters" designation ( $+D)$ has been deleted from the IBM PC/XT/AT version of ONSITE/MAXI1 for consistency with the ICRP Publication No. 30 methodology.

Contamination deposited on the site at start of calculation. This contamination may be:

1 - deposited on the surface (in this case parameter ISUR should be set to 1 ) 
2 - stored at the site (in this case parameter IEXT should be set to 1 )

3 - deeply buried at the site (in this case parameter IEXT should be set to 1 ).

Input units are as specified by the parameters INUT and M3M2.

QI

QJ

Irrigation/river release source in units of activity, as specified by the parameter INUT, per liter. To consider irrigation of terrestrial crops with contaminated water from this source, set the parameter IFOD to 1 . To consider ingestion of aquatic foods grown in this contaminated water, set parameter IARG to 1 .

J Drinking water source term in units of activity, as specified by the parameter INUT, per liter. To consider ingestion of drinking water from this contaminated source, set the parameter IWAT to 1 .

QK Atmospheric release source term in units of activity, as specified by the parameter INUT, per year. This parameter allows consideration of airborne contamination deposited onto the site from offsite sources. To consider air contamination concentration contributions from this source, set the parameter IAIR to 1 . To consider contamination buildup from this source, input a value for the parameter IBLOW.

NOTE: Q, QI, QJ, QK are input

values entered for the above parameters must fall in the columns as designated by the dashed lines in the column headings. Values entered with exponential notation (i.e., FORTRAN E format) must be right-justified in the columns.

SRDIL Inventory dilution/modification factor for the soil source

term. This factor allows the user to:

1 - assume uniform mixing of the contaminated waste with clean soil; the ONSITE/MAXI1 scenarios all assume a value of 0.2 , the default value is 1.0 .

2 - apply a consistent modification factor to all radionuclides in the source term.

DILF Inventory modification factor for the irrigation/aquatic foods source term.

FRSIZ Size of the waste disposal site in terms of fractional ha. This term is meaningful only for site sizes less than 1.0 ha. Area correction factors are calculated for: 
1 - internal pathways (parameter AREAIN)

2 - external pathways (parameter AREAEX)

to account for the limited exposure potential from smaller disposal sites. Note: the parameters AREAIN and AREAEX are now calculated in MAXIl instead of ONSITE to simplify the input process.

RPF2

Fraction of the total terrestrial foods/food products diet that is grown on the waste disposal site.

NOTE on SRDIL, DILF, FRSIZ, and RPF2 parameter interaction:

The parameters SRDIL/DILF, AREAIN (calculated from FRSIZ), AREAEX (calculated from FRSIZ), and RPF2 are all multipliers applied to the exposure/dose calculations. For example, a value of 0.2 for SRDIL and a value of 0.5 for RPF2 will result in a total modification 0.1 to the dose received from the terrestrial foods pathway, assuming the source term was located in the soil $(Q)$.

HREXT Number of hours per year of external exposure to the contamination.

HRINH Number of hours per year that the intruder inhales airborne contamination.

PLANAM Name of the file containing the library of external exposure dose rate factors in units of mrem/yr per $\mathrm{pCi} / \mathrm{m}^{2}$. Three files are available for assignment from the ONSITE/MAXI1 data base. The dose rate factors pertain to an infinite plane of soil contamination with a waste thickness of $15 \mathrm{~cm}$. The three files provide values for three different assumed waste densities:

$$
\begin{array}{lll}
\text { PLANEA.DAT } & - & \text { Waste density: } 1.8 \mathrm{~g} / \mathrm{cm}_{3}^{3} \\
\text { PLANEB.DAT } & - & \text { Waste density: } 1.0 \mathrm{~g} / \mathrm{cm}^{3} \\
\text { PLANEC.DAT } & - & \text { Waste density: } 0.6 \mathrm{~g} / \mathrm{cm}^{3}
\end{array}
$$

This parameter is only used when the parameter ISUR has been set to 1 .

VOLNAM Name of the file containing the library of external exposure dose rate factors in units of mrem/yr per $\mathrm{pCi} / \mathrm{m}^{3}$. Twelve files are available for assignment from the ONSITE/MAXI1 data base. The 12 data files form four data sets describing possible waste configurations. The data sets, their waste configurations, and the associated files are as follows: 
1 - Infinite surface slab source, waste extends to a depth of $1.0 \mathrm{~m}$.

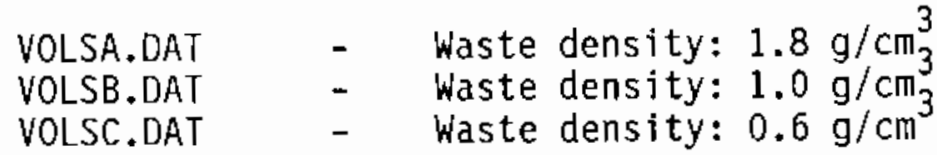

2 - Infinite slab source, buried in soil with an overburden depth of $0.5 \mathrm{~m}$, waste thickness of $1.0 \mathrm{~m}$.

BURHFA.DAT - Waste density: $1.8 \mathrm{~g} / \mathrm{cm}_{3}^{3}$
BURHFB.DAT - Waste density: $1.0 \mathrm{~g} / \mathrm{cm}_{3}$
BURHFC.DAT $\quad$ - Waste density: $0.6 \mathrm{~g} / \mathrm{cm}^{3}$

3 - Infinite slab source, buried in soil with an overburden depth of $1.0 \mathrm{~m}$, waste thickness of $1.0 \mathrm{~m}$.

$$
\begin{array}{lll}
\text { BURIA.DAT } & - & \text { Waste density: } 1.8 \mathrm{~g} / \mathrm{cm}^{3} \\
\text { BUR1B.DAT } & - & \text { Waste density: } 1.0 \mathrm{~g} / \mathrm{cm}^{3} \\
\text { BUR1C.DAT } & - & \text { Waste density: } 0.6 \mathrm{~g} / \mathrm{cm}^{3}
\end{array}
$$

4 - Rectangular slab source, $10.0 \mathrm{~m} \times 3.0 \mathrm{~m}$, waste thickness of $1.0 \mathrm{~m}$.

$\begin{array}{ll}\text { STOREDA.DAT - } & \text { Waste density: } 1.8 \mathrm{~g} / \mathrm{cm}^{3} \\ \text { STOREDB.DAT } & \text { Waste density: } 1.0 \mathrm{~g} / \mathrm{cm}^{3} \\ \text { STOREDB.DAT } & \text { Waste density: } 0.6 \mathrm{~g} / \mathrm{cm}^{3}\end{array}$

This parameter is only used when the parameter IEXT is set to 1 .

NOTE on ISUR, PLANAM, IEXT, and VOLNAM interactions:

The file identified as PLANAM is assigned to logical unit number lun 2 by MAXI. The file identified as VOLNAM is assigned to lun 7 . Files assigned to luns 2 and 7 perform essentially the same functions. Historically, input to PLAMAM was defined as dose factors with units of rem/yr per $\mathrm{pCi} / \mathrm{m}^{2}$, while input to VOLNAM, assumed to be generated by the ISOSHLD computer program (Engel, Greenborg, and Hendrickson 1966), was defined in terms of mrem/yr per $\mathrm{pC} j / \mathrm{m}^{3}$. The MAXI1 computer proggam operates internally in soil concentration units of $\mathrm{pCi} / \mathrm{m}^{2}$, defined by the root and leaf pathway parameters. Whenever the external doses are calculated, the soil concentration units are converted to be compatible with the dose factors. File assignments to luns 2 and 7 are maintained in the present version of MAXII to allow simultaneous consideration of two distinct source geometries (e.g., surface and buried contaminated soit, contaminated soil and a point source, etc.). 

$20 \mathrm{~m} / \mathrm{d}$ is consistent with methodology of ICRP Publication No. 30 .

A breathing rate of $20 \mathrm{~m}^{3} / \mathrm{d}$ (ICRP 1975) was assumed in the DACRIN (Houston, Strenge, and Watson 1974) calculations used to generate the inhalation dose rate factors library FILE23.DAT. For maximum annual dose calculations, the breathing rate is adjusted by BRATE $/ 230.0$.

IRES Option set to select model to use for resuspension calculations: 0-Use Anspaugh Model, 1-Use Mass Loading Model. This parameter will effect inhalation (INHA) and terrestrial food via leaf deposition (IFOD) calculations.

Two input parameters are requested for resuspension calculations. The parameters AGE and XDPTIN are associated with the Anspaugh Mode1, the parameters DEN and XMLF with the Mass Loading Model.

AGE/DEN AGE: The number of years that contamination existed on the surface, to account for environmental weathering, at the start of the scenario. Allowable range: 0.0 to 25.0. Default value: 0.0 .

DEN: The density of the surface soil $\left(\mathrm{g} / \mathrm{m}^{3}\right)$. Allowable range: $1.0 \times 10^{5}$ to $5.0 \times 10^{\circ}$. Default value: $1.0 \times 10^{6}$.

XDPTIN/XMLF XDPTIN: The contaminated surface soil is available for resuspension to a depth of XDPTIN $\mathrm{cm}$. Al Towable range: 0.0 to 15.0. Default value: 1.0 .

XLME: The Mass Loading Factor $\left(\mathrm{g} / \mathrm{m}^{3}\right) \cdot-4^{\text {Allowable range: } 1.0 \times}$ $10^{-7}$ to 0.1 . Default value: $1.0 \times 10^{-4}$.

IBLOW See IAIR; the number of years prior to scenario initiation that the offsite atmospheric source begins. This parameter is only considered when the parameter IAIR is set to 1.

XQSITE The air concentration per unit release (Chi/Q in $\mathrm{sec} / \mathrm{m}^{3}$ ) of the atmospheric release of the source term (QK) at the disposal site. This parameter is only used when the parameter IAIR is set to 1 .

ICLNUP Option set to consider drinking water cleanup factors. This parameter was added to the current version of the MAXI1 program to allow greater flexibility of "environment"/scenario generation. Allowable values: 0 - do not use cleanup factors, 1-use cleanup factors. Default value: 1.

I1109 Option set to consider Regulation Guide 1.109 (U.S. NRC 1977) food transfer coefficjents for plants from soil for the radionuclides ${ }^{3}$ and ${ }^{4} \mathrm{C}$. These coefficients, MAXI1 parameter 
FDTC, [pCi per gram 3 plant (wet) $/ \mathrm{pCi}$ per gram soit (dry)] replace the 0.0 values for ${ }^{3} \mathrm{H}$ and ${ }^{14} \mathrm{C}$ read from the food transfer coefficient library in file FTRANS.DAT. This parameter was added to the current version of the MAXI1 program to eliminate the need for a special version of FTRANS.DAT containing the Regulation Guide 1.109 values. Allowable values: $0 \frac{3}{3}$ do not consider Reg. Guide 1.109 plant up-take ${ }_{3}$ values for ${ }^{3} \mathrm{H}$ and ${ }^{14} \mathrm{C}$, 1-consider Reg. Guide 1.109 values for ${ }^{3} \mathrm{H}$ and ${ }^{14} \mathrm{C}$. Default value: 1 .

RF1 Fraction of terrestrial plant roots in the surface soil layer (assumed to extend to a depth of $15.0 \mathrm{~cm}$ ).

RF2 Fraction of terrestrial plant roots extending below the 15.0-cm-deep surface soil layer.

RPF1

This is the ratio of waste concentration in surface soi? to that in subsurface soils at the waste disposal site. This parameter is used only when contamination is assumed to be present in both surface and subsurface soil. RPF1 allows redistribution of the waste in the soil. Range: 0.0 to 1.0 for buried to surface redistribution. Default value is 1.0 . Option IEXT must be set on.

IMO Months per year that irrigation is applied to terrestrial food crops. Default value: 6 . This parameter is only used when IFOD is set to 1 and a value has been entered for QI.

RIRR Irrigation rate for terrestrial food crops $\left(\mathrm{L} / \mathrm{m}^{2} / \mathrm{mo}\right)$. This parameter is only used when the parameter IFOD is set to 1 and a value has been entered for QI. Default value: 150.0.

IRR

Number of years irrigation source (QI) is irrigated onto the site prior to the beginning of the dose calculation period. Default value: 0 .

NFT

Number of terrestrial food types to be considered for this scenario. Allowable range: 0 to 14.

KFDTYP Numeric index indicating the food types to consider. Allowable entries are as follows:

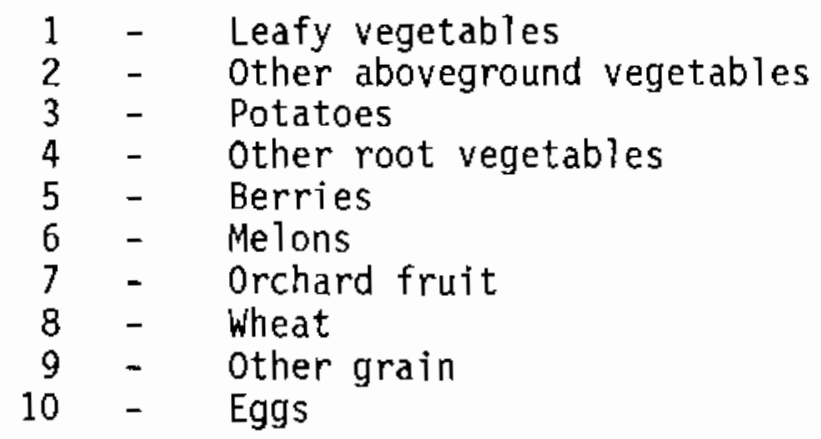




$$
\begin{array}{lll}
11 & - & \text { Milk } \\
12 & - & \text { Beef } \\
13 & - & \text { Pork } \\
14 & - & \text { Poultry }
\end{array}
$$

NOTE: For food types 10,11, 12, 13, and 14; the values of GRWP, YELD, and TRANS apply to the feed and fodder that the animal eats.

NOTE: Be careful to right-justify the indices under the "FOOD TYPE INDEX" column heading.

GRWP The growing period in days of the corresponding food type. For animal products, this is the growing period of the fodder that the animal eats.

YELD The yield in $\mathrm{kg} / \mathrm{m}^{2}$ per year of the corresponding food type.

HLDUP The holdup period in days for the corresponding food type.

CON The consumption rate in $\mathrm{kg} / \mathrm{yr}$ or $\mathrm{L} / \mathrm{yr}$ of the corresponding food type.

TRANS The translocation factor of the corresponding food type.

NPT Number of drinking water/aquatic food types to be considered for this scenario. Range: 1 to 5 .

KPTHWY Numeric index of the drinking water/aquatic food pathways considered. Allowable values are:

$\begin{array}{lll}1 & - & \text { Fish } \\ 2 & - & \text { Crustacea } \\ 3 & - & \text { Mollusks } \\ 4 & - & \text { Aquatic plants } \\ 5 & - & \text { Drinking water }\end{array}$

NOTE: Be careful to right-justify parameter values under the column heading "TYPE INDEX."

HLDUP2

The holdup period in days for the corresponding drinking water/aquatic food type.

USAG

Consumption of drinking water and each aquatic food type; $\mathrm{kg} / \mathrm{yr}$ for edibles, and L/yr drinking water.

NOTE GRWP, YELD, HLDUP, CON, TRANS, HLDUP2, USAG input:

Values entered for the above parameters must fall in the columns as designated by the dashed lines in the column headings. Values entered with exponential notation (i.e., E format) must be right-justified in the columns. 
TABLE 3.1. Scenario Default Parameter values

\begin{tabular}{|c|c|c|c|c|c|c|}
\hline $\begin{array}{l}\text { Parameter } \\
\text { Name }\end{array}$ & $\begin{array}{l}\text { Scenario } \\
\text { No. } 1 \\
\end{array}$ & $\begin{array}{l}\text { Scenario } \\
\text { №. } 2 \\
\end{array}$ & $\begin{array}{l}\text { Scenario } \\
\text { No. } 3 \\
\end{array}$ & $\begin{array}{l}\text { Scenario } \\
\text { No. } 4 \\
\end{array}$ & $\begin{array}{l}\text { Scenario } \\
\text { No. } 5 \\
\end{array}$ & $\begin{array}{l}\text { Waste } \\
\text { Subset }\end{array}$ \\
\hline IEXT & 1 & 1 & 0 & 0 & 1 & 1 \\
\hline ISUR & 0 & 0 & 1 & 1 & 1 & 0 \\
\hline INHA & 0 & 1 & 1 & 1 & 1 & 0 \\
\hline IFOD & 0 & 0 & 1 & 1 & 1 & 0 \\
\hline IARG & 0 & 0 & 0 & 0 & 1 & 0 \\
\hline IWAT & 0 & 0 & 0 & 1 & 1 & 0 \\
\hline IAIR & 0 & 0 & 0 & 0 & 0 & 0 \\
\hline IEDE & 0 & 0 & 0 & 0 & 0 & 0 \\
\hline IDKAIR & 0 & 0 & 0 & 0 & 0 & 0 \\
\hline IDKWAT & 0 & 0 & 0 & 0 & 0 & 0 \\
\hline INTRUD & 0 & 0 & 0 & 0 & 0 & 0 \\
\hline IOUT & 0 & 0 & 0 & 0 & 0 & 0 \\
\hline DEBUG (4) &.$F$. & . F. & . F. &.$F$. & . F. &.$F$. \\
\hline ITI & 1 & 1 & 1 & 1 & 1 & 1 \\
\hline IT2 & 50 & 50 & 50 & 50 & 50 & 50 \\
\hline INUT & 1 & 1 & 1 & 1 & 1 & 1 \\
\hline M3M2 & 1 & 1 & 1 & 1 & 1 & 1 \\
\hline SRDIL & 0.2 & 0.2 & 0.2 & 0.2 & 0.2 & 0.2 \\
\hline DILF & 1.0 & 1.0 & 1.0 & 1.0 & 1.0 & 1.0 \\
\hline FRSIZ & 1.0 & 1.0 & 1.0 & 1.0 & 1.0 & 1.0 \\
\hline RPF2 & 1.0 & 1.0 & 1.0 & 1.0 & 1.0 & 1.0 \\
\hline HREXT & 2000.0 & 2000.0 & 2000.0 & 2000.0 & 8766.0 & 2000.0 \\
\hline HRINH & 0.0 & 2000.0 & 2000.0 & 2000.0 & 8766.0 & 0.0 \\
\hline PLANAM & PLANEA & PLANEA & PLANEA & PLANEA & PLANEA & PLANEA \\
\hline VOLNAM & BURHFA & BURHFA & BURHFA & BURHFA & BURHFA & STOREDA \\
\hline BRATE & 270.0 & 270.0 & 270.0 & 270.0 & 270.0 & 270.0 \\
\hline IRES & & 0 & & & & 0 \\
\hline AGE/DEN & 0. & 0. & 0. & 0. & $1.0 \mathrm{E} 6$ & 0. \\
\hline XPDT IN/XMLF & 1.0 & 1.0 & 1.0 & 1.0 & $1.0 \mathrm{E}-4$ & 1.0 \\
\hline IBLOW & 0 & 0 & 0 & 0 & 0 & 0 \\
\hline XQSITE & 0.0 & 0.0 & 0.0 & 0.0 & 0.0 & 0.0 \\
\hline ICLNUP & 1 & 1 & 1 & 1 & 1 & 1 \\
\hline I1109 & 1 & 1 & 1 & 1 & 1 & 1 \\
\hline RF1 & 1.0 & 1.0 & 1.0 & 1.0 & 1.0 & 1.0 \\
\hline RF2 & 0.0 & 0.0 & 0.0 & 0.0 & 0.0 & 0.0 \\
\hline RPF1 & 0.0 & 1.0 & 1.0 & 1.0 & 0.0 & 0.0 \\
\hline IM0 & & & & & 6 & 6 \\
\hline RIRR & 150.0 & 150.0 & 150.0 & 150.0 & 150.0 & 150.0 \\
\hline IRR & 0 & 0 & 0 & 0 & 0 & 0 \\
\hline
\end{tabular}


TABLE 3.2. Sample Problem Parameter Values

\begin{tabular}{|c|c|c|c|c|c|c|}
\hline $\begin{array}{l}\text { Parameter } \\
\text { Name }\end{array}$ & $\begin{array}{l}\text { Sample } \\
\text { Problem } \\
\text { No. } 1 \\
\end{array}$ & $\begin{array}{l}\text { Sample } \\
\text { Problem } \\
\text { No. } 2 \\
\end{array}$ & $\begin{array}{l}\text { Sample } \\
\text { Problem } \\
\text { No. } 3\end{array}$ & $\begin{array}{l}\text { Sample } \\
\text { Problem } \\
\text { No. } 4\end{array}$ & $\begin{array}{l}\text { Sample } \\
\text { Problem } \\
\text { No. } 5\end{array}$ & $\begin{array}{l}\text { Sample } \\
\text { Problem } \\
\text { No. } 6\end{array}$ \\
\hline IEXT & 1 & 1 & 0 & 0 & 1 & 1 \\
\hline ISUR & 0 & 0 & 1 & 1 & 1 & 0 \\
\hline INHA & 0 & 1 & 1 & 1 & 1 & 0 \\
\hline IFOD & 0 & 0 & 1 & 1 & 1 & 0 \\
\hline IARG & 0 & 0 & 0 & 0 & 1 & 0 \\
\hline IWAT & 0 & 0 & 0 & 1 & 1 & 0 \\
\hline IAIR & 0 & 0 & 0 & 0 & 0 & 0 \\
\hline IEDE & 0 & 0 & 1 & 0 & 0 & 0 \\
\hline IDKAIR & 0 & 0 & 0 & 0 & 0 & 0 \\
\hline IDKWAT & 0 & 0 & 0 & 0 & 0 & 0 \\
\hline INTRUD & 0 & 0 & 0 & 0 & 0 & 0 \\
\hline IOUT & 0 & 0 & 2 & 0 & 0 & 0 \\
\hline DEBUG (4) &.$F$. &.$F$. & . F. &.$F$. &.$F$. &.$F$. \\
\hline IT1 1 & 10 & 10 & 10 & 10 & 10 & 10 \\
\hline IT2 & 59 & 59 & 59 & 59 & 59 & 59 \\
\hline INUT & 4 & 4 & 4 & 1 & 1 & 4 \\
\hline M3M2 & 1 & 1 & 1 & 1 & 1 & 1 \\
\hline SRDIL & 0.2 & 0.2 & 0.2 & 0.2 & 0.2 & 0.2 \\
\hline DILF & 1.0 & 1.0 & 1.0 & 1.0 & 1.0 & 1.0 \\
\hline FRSIZ & 1.0 & 1.0 & 0.05 & 1.0 & 1.0 & 1.0 \\
\hline RPF2 & 1.0 & $\begin{array}{l}1.0 \\
1.0\end{array}$ & 1.0 & 1.0 & $\begin{array}{l}1.0 \\
1.0\end{array}$ & $\begin{array}{l}1.0 \\
1.0\end{array}$ \\
\hline HREXT & 2000.0 & 3000.0 & 2000.0 & 2000.0 & 2000.0 & 500.0 \\
\hline HRINH & 0.0 & 3000.0 & 2000.0 & 2000.0 & 2000.0 & \\
\hline PLANAM & PLANEA & PLANEA & PLANEA & PLANEA & PLANEA & PLANEA \\
\hline VOLNAM & BURHFA & VOLSA & BURHFA & BURHFA & BURIA & STOREDB \\
\hline BRATE & 270.0 & 270.0 & 270.0 & 270.0 & 270.0 & 270.0 \\
\hline IRES & & 0 & 0 & 0 & 1 & \\
\hline AGE/DEN & 0. & 0. & 0. & 0. & 1.0E6 & 0. \\
\hline XPDTIN/XMLF & 1.0 & 1.0 & 1.0 & 1.0 & $1.0 \mathrm{E}-4$ & 1.0 \\
\hline IBLOW & 0 & 0 & 0 & 0 & 0 & 0 \\
\hline XQSITE & 0.0 & 0.0 & 0.0 & 0.0 & 0.0 & 0.0 \\
\hline ICLNUP & 1 & 1 & 1 & 1 & & \\
\hline I1109 & 1 & 1 & 1 & 1 & 1 & 1 \\
\hline RF1 & 1.0 & 1.0 & 1.0 & 1.0 & 0.6 & 1.0 \\
\hline RF2 & 0.0 & 0.0 & 0.0 & 0.0 & 0.4 & 0.0 \\
\hline RPF1 & 0.0 & 1.0 & 1.0 & 1.0 & 0.0 & 0.0 \\
\hline IM0 & & & & & 6 & 6 \\
\hline RIRR & 150.0 & 150.0 & 150.0 & 150.0 & 150.0 & 150.0 \\
\hline IRR & 0 & 0 & & 10 & 10 & 0 \\
\hline
\end{tabular}




\subsection{ERROR AND RECOVERY}

To further ease the user interaction with the ONSITE/MAXI1 computer program, error-checking and recovery capabilities have been added. The following paragraphs describe the error and recovery procedures that have been built into the ONSITE/MAXI1 computer program.

\subsubsection{Error and Recovery - ONSITE}

ONSITE has extensive error-checking and interactive recovery capabilities. Most input values are tested against minimum and maximum allowable limits. If a value does not fall within the allowable limits, the user is asked immediately to re-enter that value.

ONSITE will display error messages if an invalid file name is selected by the user or if the program is unable to locate and access a data base file. The following messages are displayed by ONSITE. Details of the error condition are discussed and appropriate corrective actions are suggested.

"Error opening radionuclide library." The program could not access the radionuclide library, RMDONS. Refer this problem to the person maintaining the ONSITE/MAXI1 computer package.

"Premature end-of-file discovered in library." The program expected additional data in the master radionuclide library, RMDONS. Refer this problem to the person maintaining the ONSITE/MAXI1 computer package.

\subsubsection{Error and Recovery - MAXI1}

MAXII generates the following error messages listed in alphabetical order. Extended explanations and corrective actions are included, if applicable.

1) DECAY CHAIN $n 1$ HAS IMPROPER ORDER. CURRENT MEMBER INDEX IS n2. (RLIBIN).

This message indicates a problem with a decay chain organization while reading the master radionuclide library. Its occurrence indicates that 1) an unsuccessful update was made to the library, or 2) the file has been corrupted. In either case, refer the problem to the person maintaining the data base.

2) END OF FILE ON MASTER LIBRARY UNIT 10. STOP (RLIBIN).

MAXI1 expected additional records in the master radionuclide library. This message indicates that 1) an unsuccessful update was made to the library, or 2) the file has been corrupted. In either case, refer the problem to the person maintaining the data base. 
3) FIL30: Error opening file: nnnnnnn.nnn assigned to LUN mmm.

In this error message, nnnnnnn. nnn is the name of the library file that MAXI1 was not able to open, and mmm is the logical unit to which MAXI1 was attempting to assign the file. This error will occur when the file containing ICRP Publication No. 30 dose rate factors cannot be found. Check that the file named nnnnnnnn. nnn resides in the MAXI subdirectory and that you are executing the MAXI1 program from the MAXI subdirectory.

4) FIL30: Error reading organ index for rrrrrrr in file nnnnnnnn.nnn assigned to LUN mmm.

In this error message, rrrrrrrr is the name of the radionuclide, nnnnnnn. nnn is the name of the library file in which the error occurred, and $\mathrm{mmm}$ is the logical unit to which the file was assigned. This error is most likely to occur when the ICRP Publication No. 30 dose rate factor file has been corrupted. Try recopying the file ORGLIB.DAT from the ONSITE/MAXIl distribution disk.

5) FIL30: Error reading library title of file: nnnnnnnn.nnn assigned to LUN mmm.

In this error message, nnnnnnn.nnn is the name of the library file in which the error occurred, and $\mathrm{mmm}$ is the logical unit to which the file was assigned. This error occurs when the ICRP Publication No. 30 dose rate factor file is empty or corrupted. Try recopying the named file from the ONSITE/MAXI1 distribution disk.

6) File: nnnnnnnn.nnn with plane source dose rate factors was not found. Program terminated.

Based on options selected in your input file, MAXI1 expected to read the file named nnnnnnnn.nnn. If input parameters ISUR was properly set to 1 for this scenario, verify that the file name was not misspelled. This message is printed in module MAXI1.

7) File: nnnnnnnn.nnn with slab source dose rate factors was not found. Program terminated.

Based on options selected in your input file, MAXI1 expected to read the file named nnnnnnnn.nnn. If input parameter IEXT was properly set to 1 for this scenario, verify that the file name was not misspelled. This message is printed in module MAXI1.

8) FILHAN: Error opening file: nnnnnnn. nnn assigned to LUN mmm.

In this error message, nnnnnnn. nnn is the name of the library file that MAXI1 was not able to open, and mmm is the logical unit to which MAXI1 was attempting to assign the file. This error is most likely to occur when a file cannot be found. Check that all library files reside in the MAXI 
subdirectory and that you are executing the MAXI1 program from the MAXI subdirectory.

9) FILHAN: Error reading organ index for rrrrrrr in file nnnnnnnn.nnn assigned to LUN mmm.

In this error message, rrrrrrr is the name of the radionuclide, nnnnnnn. nnn is the name of the library file in which the error occurred, and $m m m$ is the logical unit to which the file was assigned. This error is most likely to occur when the organ data library, ORGLIB.DAT, has been corrupted. Try recopying the file ORGLIB.DAT from the ONSITE/MAXI1 distribution disk.

10) FILHAN: Error reading library title of file: nnnnnnnn.nnn assigned to LUN mmm.

In this error message, nnnnnnn. nnn is the name of the library file that in which the error occurred, and $m$ mm is the logical unit to which the file was assigned. This error is most likely to occur in the event of an empty or corrupted file. Try recopying the named file from the ONSITE/MAXI1 distribution disk.

11) IMPROPER NUMBER OF NUCLIDES IN MASTER LIBRARY, NUC $=\mathrm{n}$ (RLIBIN).

The program expected to find $n$ records in the radionuclide master library. This message indicates that 1) an unsuccessful update was made to the library, or 2) the file has been corrupted. In either case, refer the problem to the person maintaining the data base.

12) INSET: Error opening file nnnnnnnn.nnn assigned to LUN mrnm.

An error occurred while attempting to open the inhalation dose rate factor library file. This usually indicates that the file cannot be found. Check that the file named nnnnnnnn, nnn resides in the MAXI subdirectory and that you are executing the MAXI1 program from the MAXI subdirectory.

13) No inventory or error in subroutine ORDER.

After checking the inventory against the master data file, MAXI1 determined that there were 0 radionuclides in the source term. Check input file inventory data set.

14) No. of radionuclides (including daughters) $=n$. Maximum allowed $=n 2$.

The maximum number of radionuclides that can be considered in a scenario is n2. After including daughters, the source term was determined to have $n$ entries.

15) THERE ARE UNIDENTIFIED NUCLIDES, ISTOP $=n$.

This message is printed by subroutine IDNUC giving the total number ( $n$ ) of radionuclide names that were not identified in the master radionuclide library. The program will not execute until all name discrepancies have 
been resolved. Be sure to change the number of radionuclides (NONUC) if any radionuclides are eliminated at this time.

16) THIS CODE WILL NOT RUN WITHOUT AT LEAST ONE PLANT PATHWAY -- STOP.

If animal products are considered, at least one plant type must be included in the scenario for proper functioning of animal fodder calculations. If you wish to consider the intruder eating animal products only, set consumption (CON) of the plant type to 0.0 .

17) Unidentified radionuclide in input inventory, rrrrrrr

This message is printed by subroutine IDNUC to identify those input radionuclides that MAXI1 does not recognize. Reference the file RMDONS.DAT to determine if rrrrrrr is misspelled, or not included in the master list of radionuclides.

\section{REFERENCES}

Engel, R. L., J. Greenborg, and M. M. Hendrickson. 1966. ISOSHLD - A Computer Code for General Purpose Isotope Shielding Analysis. BNwL-236, Pacific Northwest Laboratory, Richland, Washington.

Houston, J. R., D. L. Strenge, and E, C. Watson. 1974. DACRIN - A Computer Program for Calculating Organ Dose from Acute or Chronic Radionuclide Inhalation. BNWL-B-389, Pacific Northwest Laboratory, Richland, Washington.

International Business Machines (IBM) Corporation. 1983. Disk Operating System Version 2.1 Reference. IBM Corporation, Boca Raton, Florida.

International Business Machines (IBM) Corporation. 1985. Disk Operating System Version 3.1 Reference. IBM Corporation, Boca Raton, Florida.

International Commission on Radiological Protection (ICRP). 1959. Report of ICRP Committee II on Permissible Dose for Internal Radiation. ICRP Publication No. 2, Pergamon Press, Elmsford, New York.

International Commission on Radiological Protection (ICRP). 1975. Report of the Task Group on Reference Man. ICRP Publication No. 23, Pergamon Press, Elmsford, New York.

International Commission on Radiological Protection (ICRP). 1977. Recommendations of the International Commission on Radiological Protection. ICRP Publication No. 26, Pergamon Press, Elmsford, New York.

International Commission on Radiological Protection (ICRP). 1979-1982. Limits for Intakes of Radionuclides by Workers. ICRP Publication No. 30, Parts 1, 2, and 3 (and supplements), Pergamon Press, Elmsford, New York.

Kennedy, W. E., Jr., R. A. Peloquin, B. A. Napier, and S. M. Neuder. 1986. Intruder Dose Pathway Analysis for the Onsite Disposal of Radioactive Wastes: 
The ONSITE/MAXI1 Computer Program. NUREG/CR-3620, Supplement 1, National Technical Information Service, Springfield, Virginia.

Napier, B. A. 1982. A Method for Determining "Allowable Residual Contamination Levels" of Radionuclide Mixtures in Soil. PNL 3852, Pacific Northwest

Laboratory, Richland, Washington.

Napier, B. A., R. A. Peloquin, W. E. Kennedy, Jr., and S. M. Neuder. 1984. Intruder Dose Pathway Analysis for the Onsite Disposal of Radioactive Wastes: The ONSITE/MAXI1 Computer Program. NUREG/CR-3620, National Technical Information Service, Springfield, Virginia. 



\subsection{PROGRAMMING STRUCTURE AND HIERARCHY}

ONSITE/MAXI1 is a package of two separate computer codes and a data base. All the computer codes have been designed in modular fashion and are written to meet ANSI FORTRAN-77 standards. The hierarchical organization of code modules and module functions of ONSITE and MAXI1 are displayed in Figures 4.1 and 4.2, respectively. 


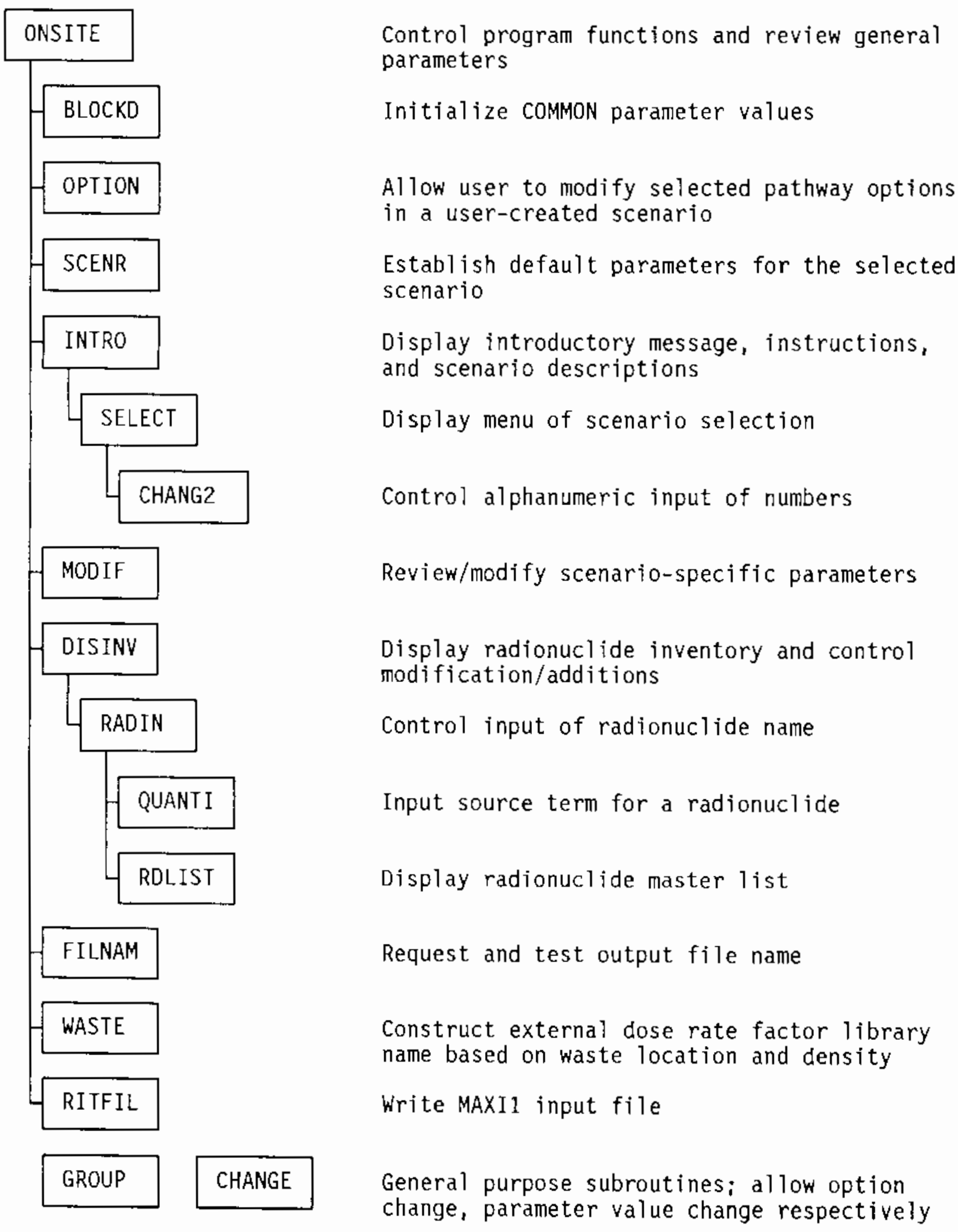

FIGURE 4.1. ONSITE Module Hierarchy and Function 


\begin{tabular}{|c|c|}
\hline MAXI & $\begin{array}{l}\text { Control: parameter and data base input, dose } \\
\text { calculations, output }\end{array}$ \\
\hline BLOCKD & Initialize COMMON parameters \\
\hline MAKDAT & Convert time and date to character strings \\
\hline GETDAT & Get system date \\
\hline GETTIM & Get system time \\
\hline READIN & Read MAXI1 user input file \\
\hline AREA & Calculate area corrections factors \\
\hline IDNUC & Identify radionuclides in the user input \\
\hline RLIBIN & Read master radionuclide library \\
\hline ORDER & $\begin{array}{l}\text { Arrange radionuclides in chain, set parameter } \\
\text { values }\end{array}$ \\
\hline FILHAN & $\begin{array}{l}\text { Read selected library files and set parameter } \\
\text { values }\end{array}$ \\
\hline FIL 30 & Read ICRP Publication 30 dose factor library \\
\hline INSET & $\begin{array}{l}\text { Read ICRP Publication } 2 \text { inhalation dose } \\
\text { factor library }\end{array}$ \\
\hline DUMMY & Read pass unused data sẹts \\
\hline QAPAGE & Write report of input and intermediate \\
\hline HEADNG & Write page heading, case title, date and time \\
\hline
\end{tabular}

FIGURE 4-2. MAXI1 Module Hierarchy and Function 


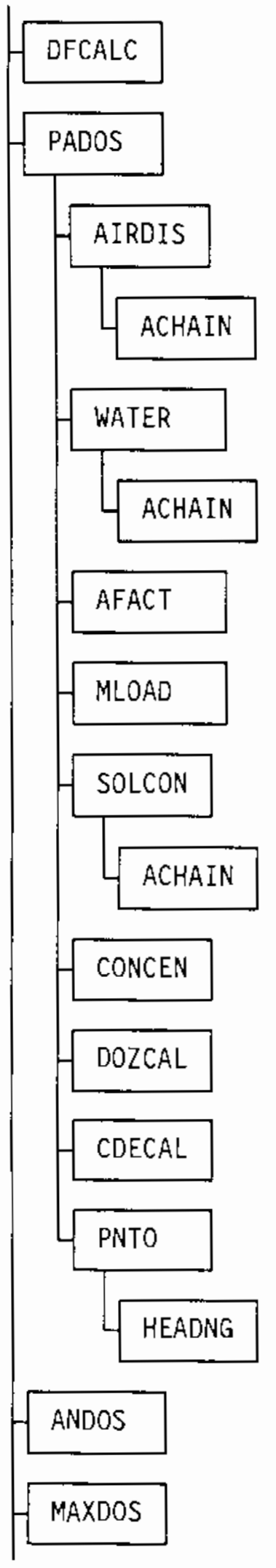

Calculate ingestion dose factors for maximum annual dose calculations

Control environmental buildup and decay as well as dose calculations, main time loop

Decay atmospheric release source term

Decay a chain of radionuclides for a given time period

Decay the water release source term

Decay a chain of radionuclides for a given time period

Calculate resuspension factor using the Anspaugh model

Calculate resuspension factor using the Mass Loading equation

Calculate soil concentration accounting for both decay and deposition in irrigation water

Decay a chain of radionuclides for a given time period

Calculate radionuclide concentration in edible food

Calculate annual dose (ICRP 2)

Calculate committed dose equivalent

Print time-dependent soil, air, irrigation water and drinking water concentrations

Print page heading

Sum annual dose (ICRP Publication 2)

Determine maximum annual dose (ICRP Publication 2)

\section{FIGURE 4.2 (Contd)}




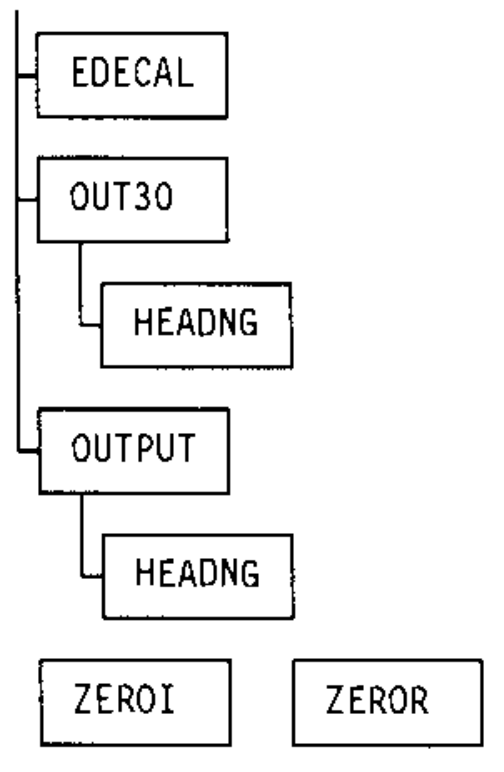

Calculate annual effective dose equivalent (ICRP Publication 30)

Write report of annual effective dose equivalent and committed dose equivalent

Write page heading, title, date, and time

Write maximum annual dose report

Write page heading, title, date, and time

General purpose; zero INTEGER and REAL arrays respectively

FIGURE 4.2 (Contd)

4.5 

APPENDIX A

COMPUTER PROGRAM LISTINGS 


\section{APPENDIX A \\ COMPUTER PROGRAM LISTING - MAXI}

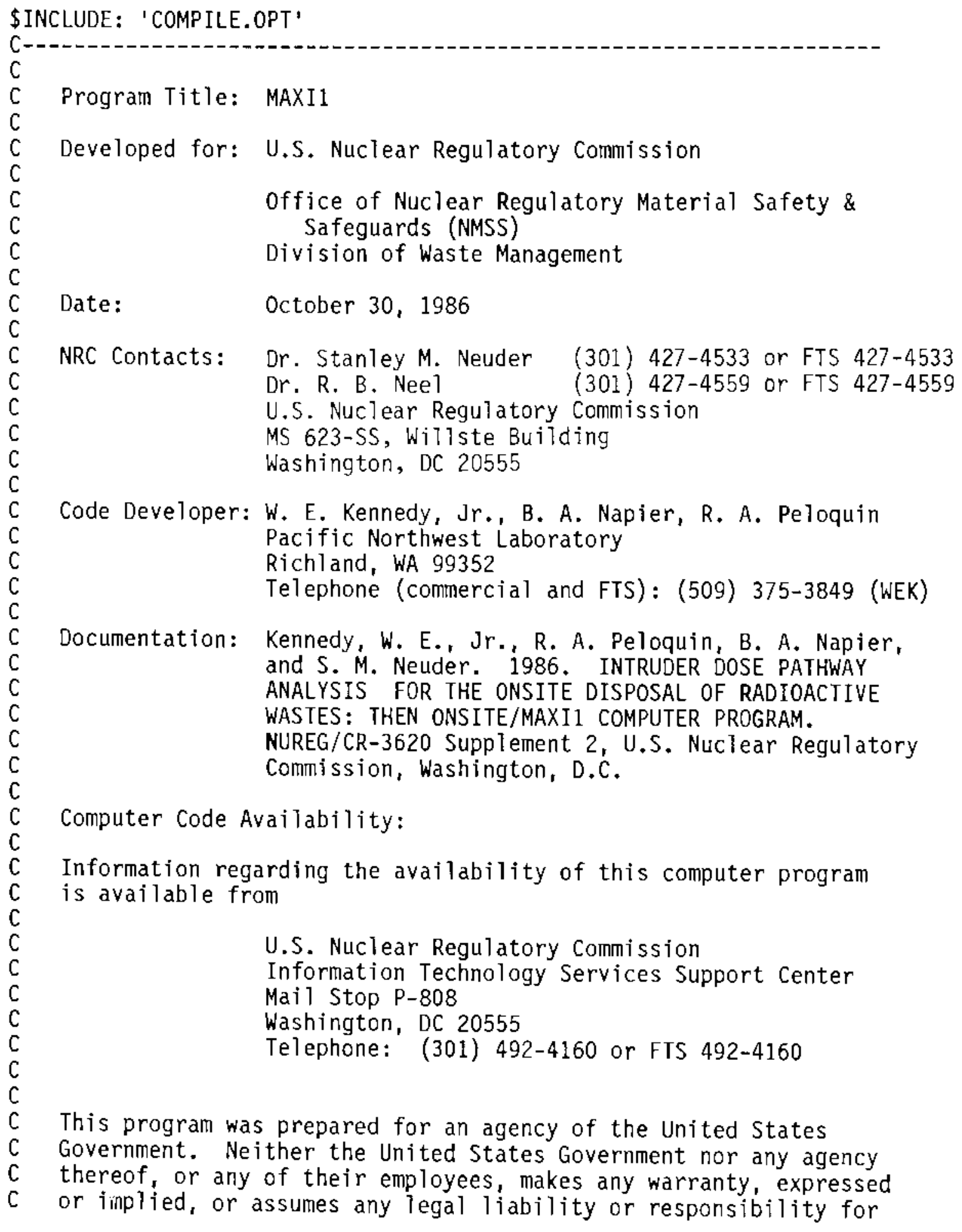

A. 1 
$C$ any third party's use, or the results of such use, of any portion C of this program, or represents that its use by such third party

C would not infringe privately owned rights.

C

C

C

C

\section{PROGRAM MAXI1}

$c$

C This program is designed to calculate annual radiation doses from C ingestion, inhalation, external surface contamination, and aquatic C food and water ingestion resulting from residual radioactivity at C decommissioned nuclear facilities and waste storage sites.

C

C Version of 24-Sep-86 RAP

C

C

C

C

\$INCLUDE: 'INVIN.CMN'

\$INCLUDE: 'INVOUT.CMN'

\$INCLUDE: 'OPT.CMN'

\$INCLUDE: 'INPUT.CMN'

\$INCLUDE: 'PATH.CMN'

\$INCLUDE: 'RAD.CMN'

\$INCLUDE: 'MONITR.CMN'

\$INCLUDE: 'TITL.CMN'

\$INCLUDE: 'DAYPC.CMN'

\$INCLUDE: 'FILNAM.CMN'

$\mathrm{C}$

LOGICAL LIVE

C Get system time and date--

CALL MAKDAT

C

C

C---- Read input for this case and check selected DRF files ------------

C

OPEN $\left(5, F I L E=F I L N(5)\right.$, STATUS $='$ OLD' $\left.^{\prime}\right)$

CALL READIN

CLOSE (5)

C

$\operatorname{OPEN}\left(6, F I L E=F I L N(6)\right.$, STATUS $\left.={ }^{\prime} \mathrm{OLD}^{\prime}\right)$

IF (ISUR .EQ. 1) THEN

LIVE $=$. FALSE.

INQUIRE (FILE $=$ PLANAM， EXIST=LIVE)

IF (LIVE) THEN

$\operatorname{FILN}(2)=$ PLANAM

ELSE

GOTO 92 


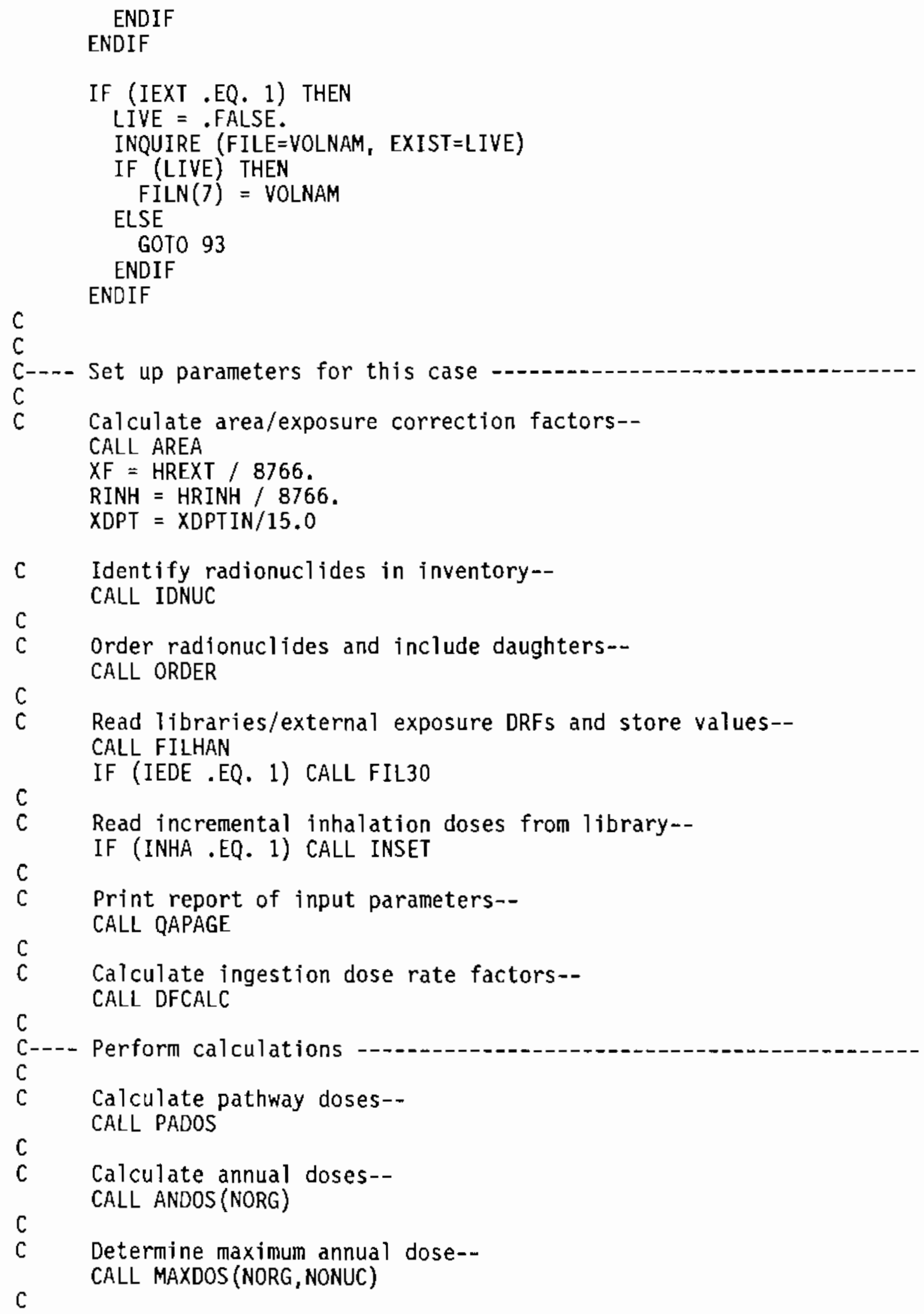




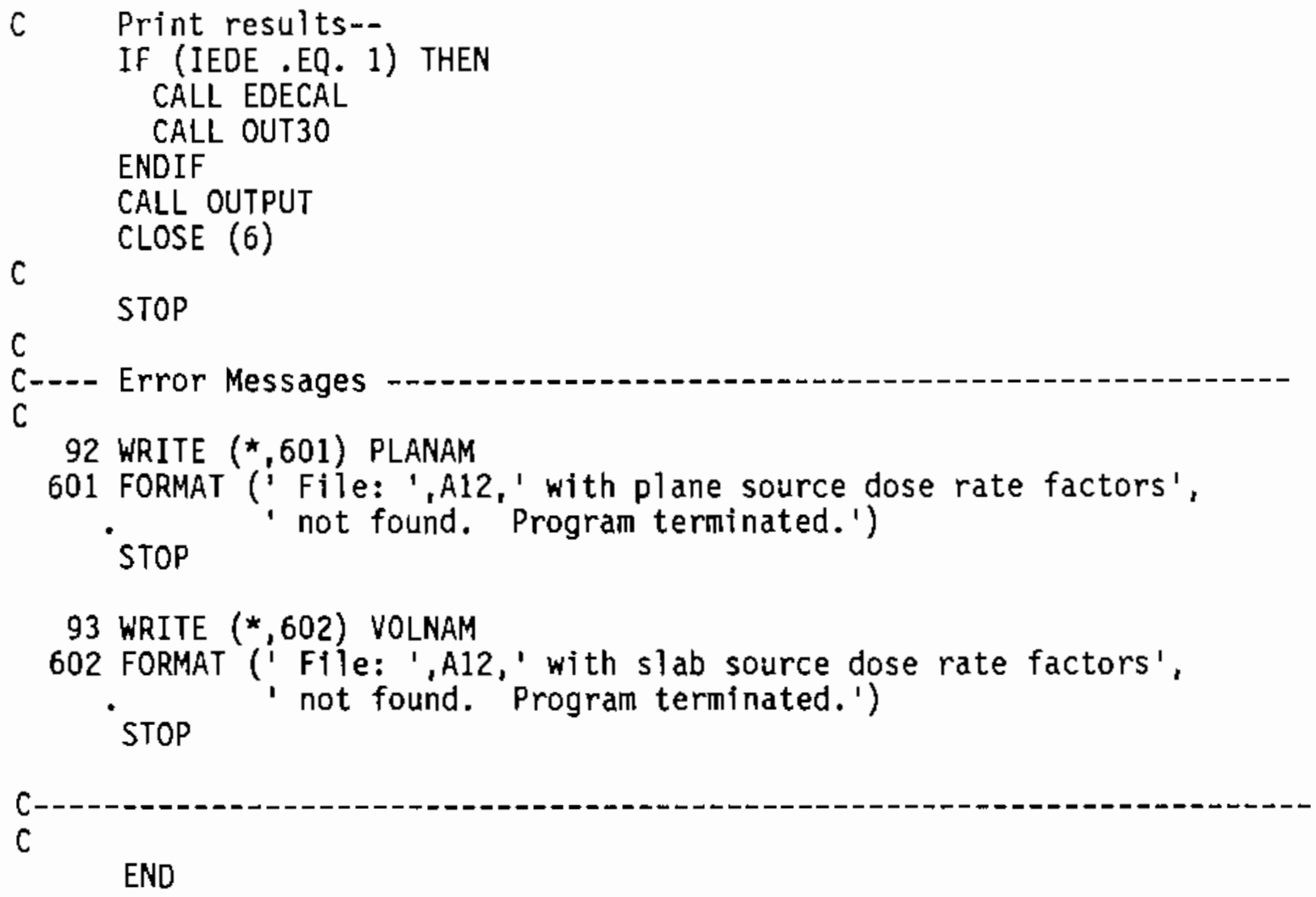




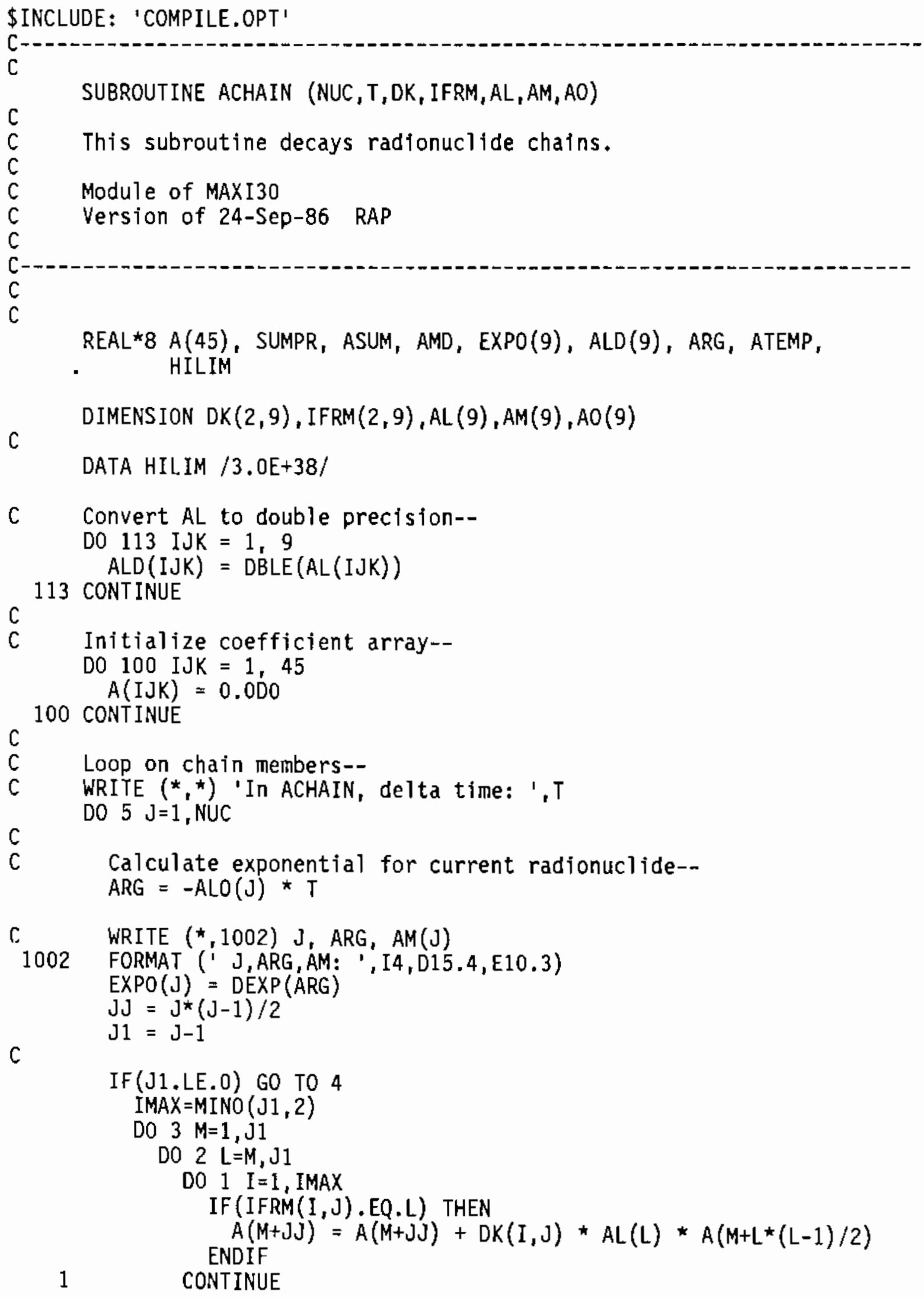




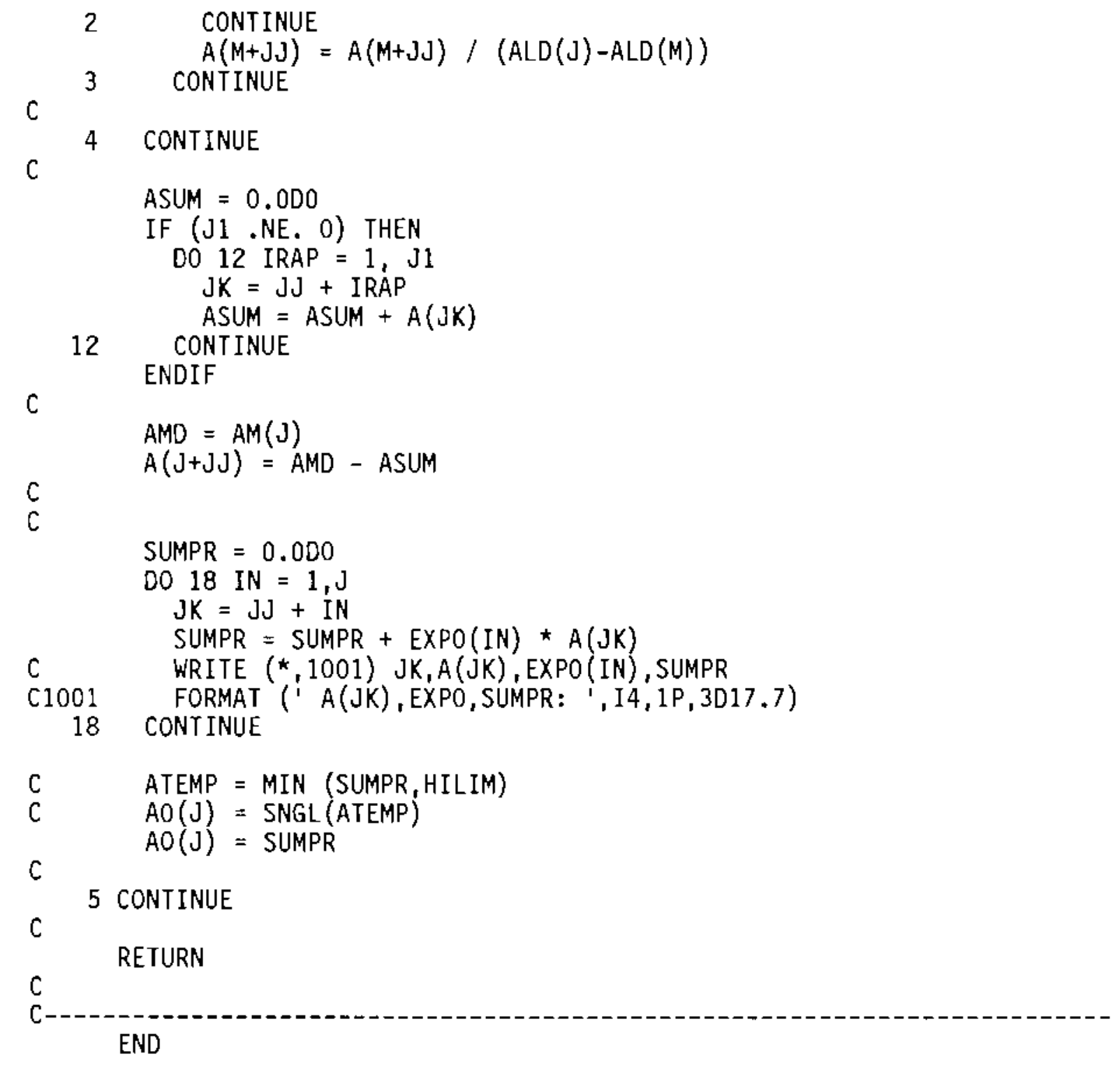


\$INCLUDE: 'COMPILE.OPT'

C-D-n-

C

$c$

SUBROUTINE AFACT (AITIM, ITIME, XDPT)

C THIS SUBROUTINE CALCAULATES A RESUSPENSION FACTOR USING THE ANSPAUGH

C MODEL WITH A LOWER LIMIT OF 1.0E-9.

C Module of MAXI30

C Version of 1-Apr-86 RAP

$\mathrm{C}$

C

$\mathrm{C}$

\$INCLUDE: 'CONC.CMN'

C

IF(AITIM.LE.25) THEN

AIRFA (ITIME) $=1.0 \mathrm{E}-4^{*}(\mathrm{EXP}(-2.87 *(\mathrm{SQRT}(\mathrm{AITIM}))))+1.0 \mathrm{E}-9$

AIRFA (ITIME) $=$ AIRFA (ITIME) $*$ XDPT

ELSE

$\operatorname{AIRFA}($ I TIME) $=1.0 \mathrm{E}-9 \times \mathrm{XDPT}$

c

ENDIF

RETURN

C

C

END 


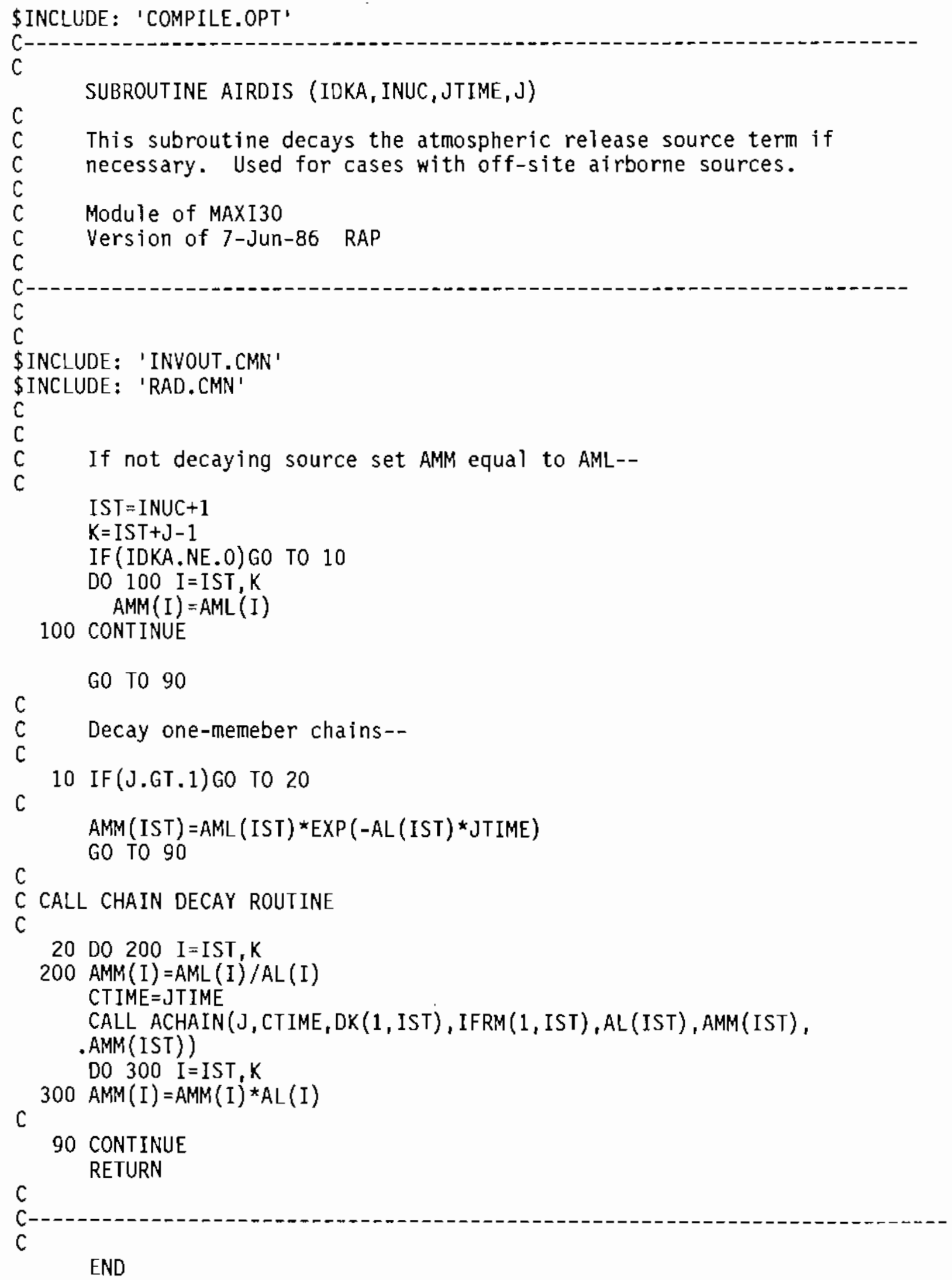




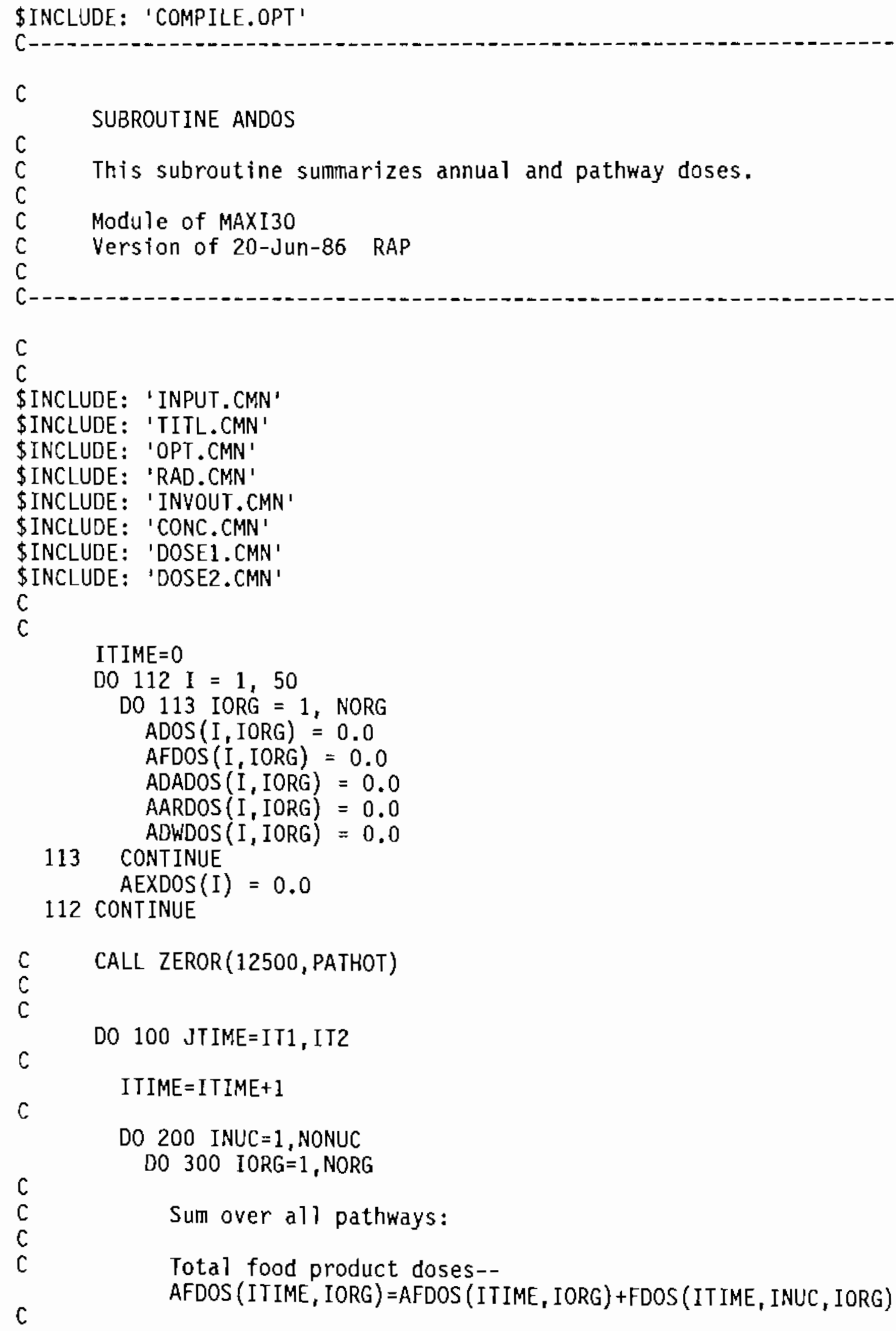

Sum over all pathways:

Total food product doses-AFDOS (ITIME, IORG) =AFDOS (ITIME, IORG)+FDOS (ITIME, INUC, IORG) 


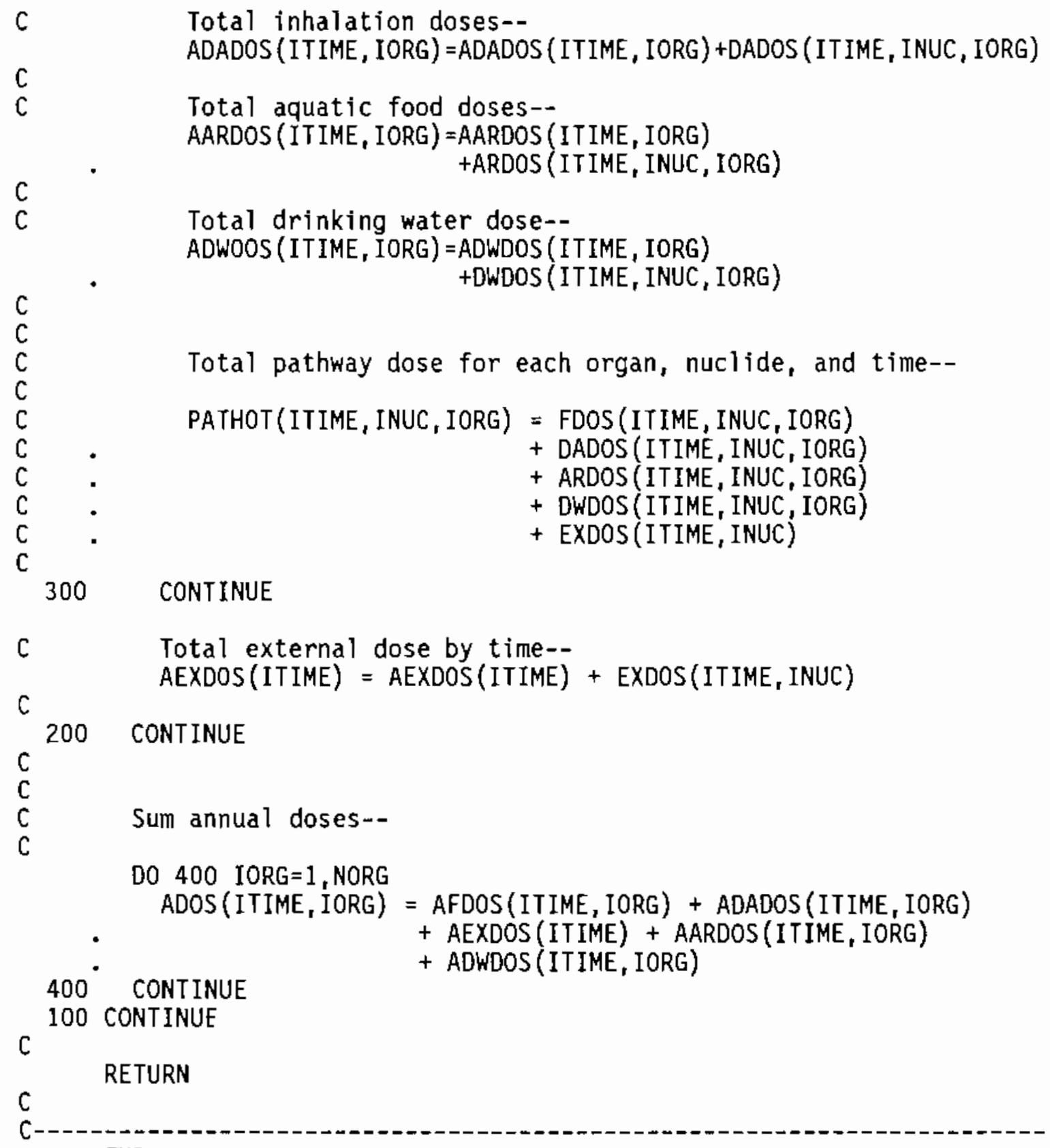

END 


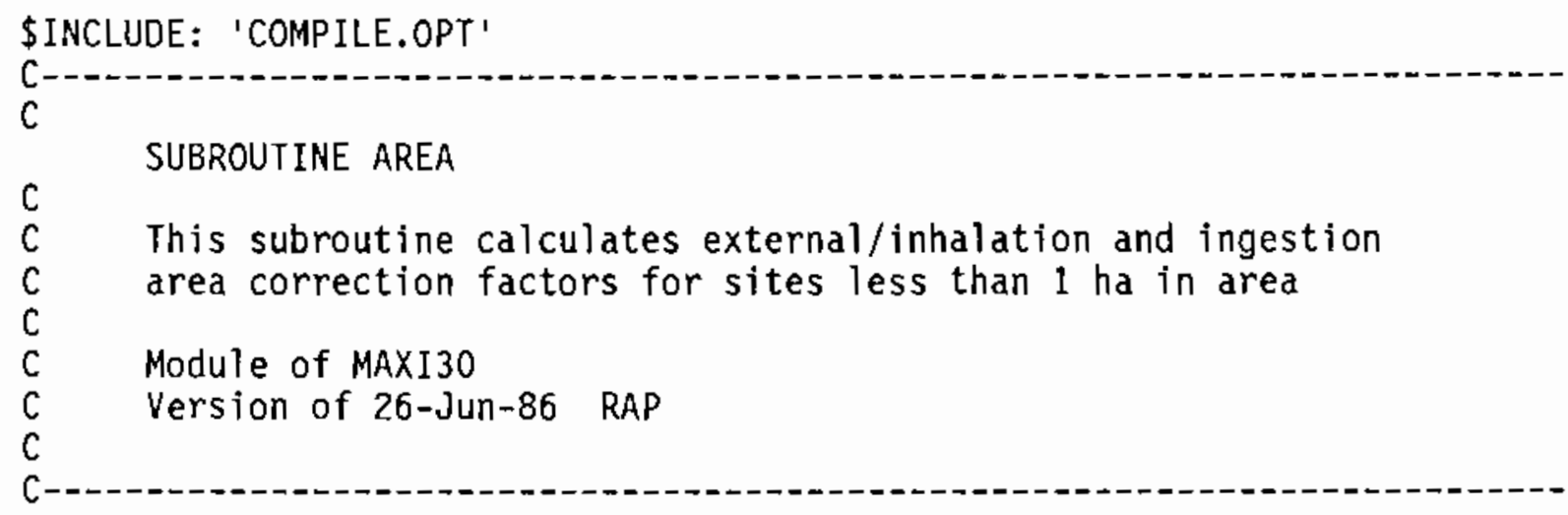

\$INCLUDE: 'INPUT.CMN'

IF (FRSIZ .GE, 1.0) THEN AREAIN $=1.0$

ELSEIF (FRSIZ .GE, 0.1) THEN AREAIN $=0.75$

ELSEIF (FRSIZ .GE. 0.002) THEN AREAIN $=0.50$

ELSEIF (FRSIZ .GE. 0.005) THEN AREAIN $=0.25$

ELSE

AREAIN $=0.10$

c

ENDIF

IF (FRSIZ .GE. 0.125) THEN AREAEX $=1.0$

ELSEIF (FRSIZ .GE. 0.05) THEN AREAEX $=2.7 *$ FRSIZ +0.67

ELSEIF (FRSIZ .GE. 0.01) THEN AREAEX $=6.5 *$ FRSIZ + 0.48

ELSEIF (FRSIZ .GE. 0.0025) THEN AREAEX $=20.0 *$ FRSIZ + 0.35

ELSE

AREAEX $=160.0 * \mathrm{FRSIZ}$

ENDIF

C

RETURN

END 
\$INCLUDE: 'COMPILE.OPT'

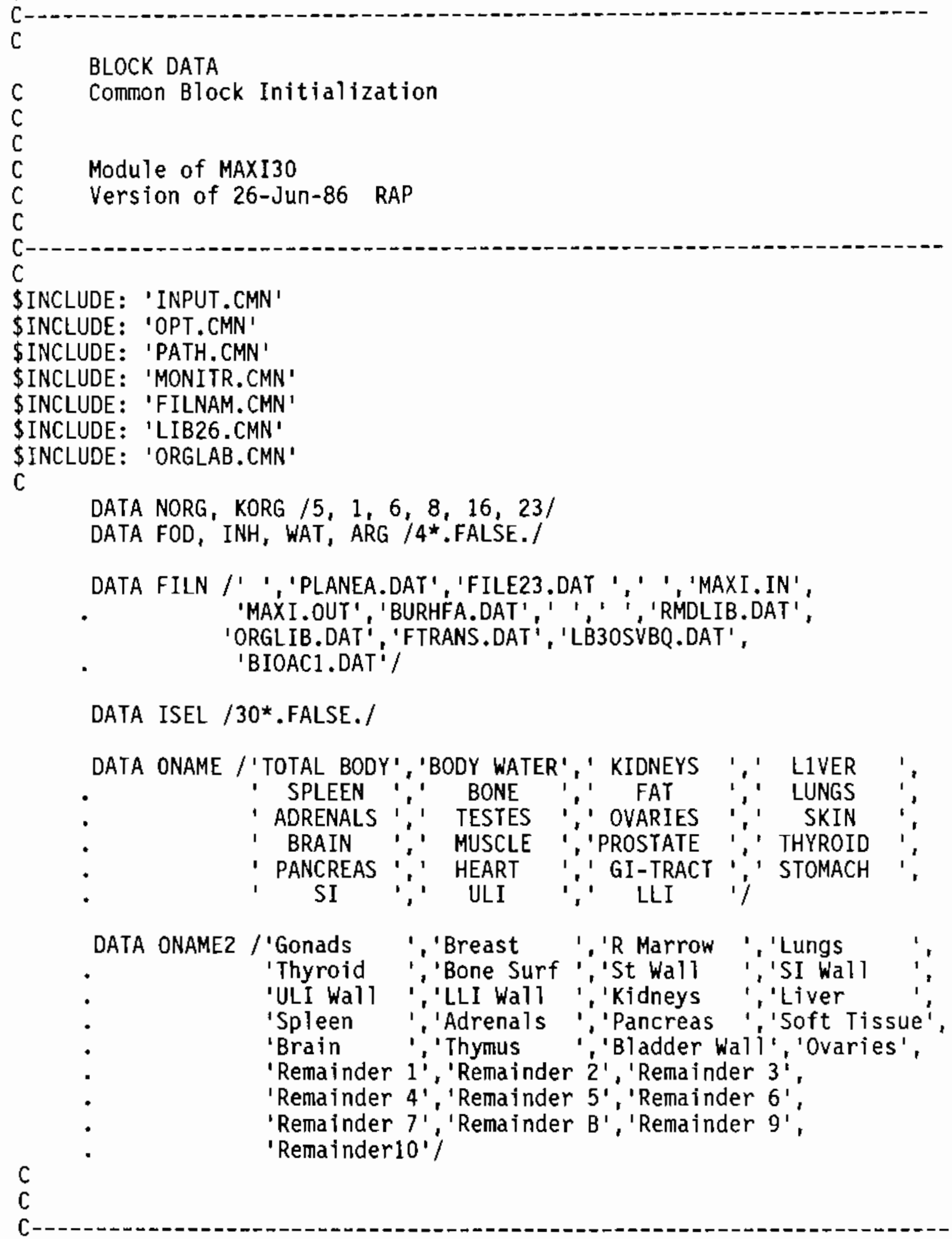

END 
\$INCLUDE: 'COMPILE.OPT'

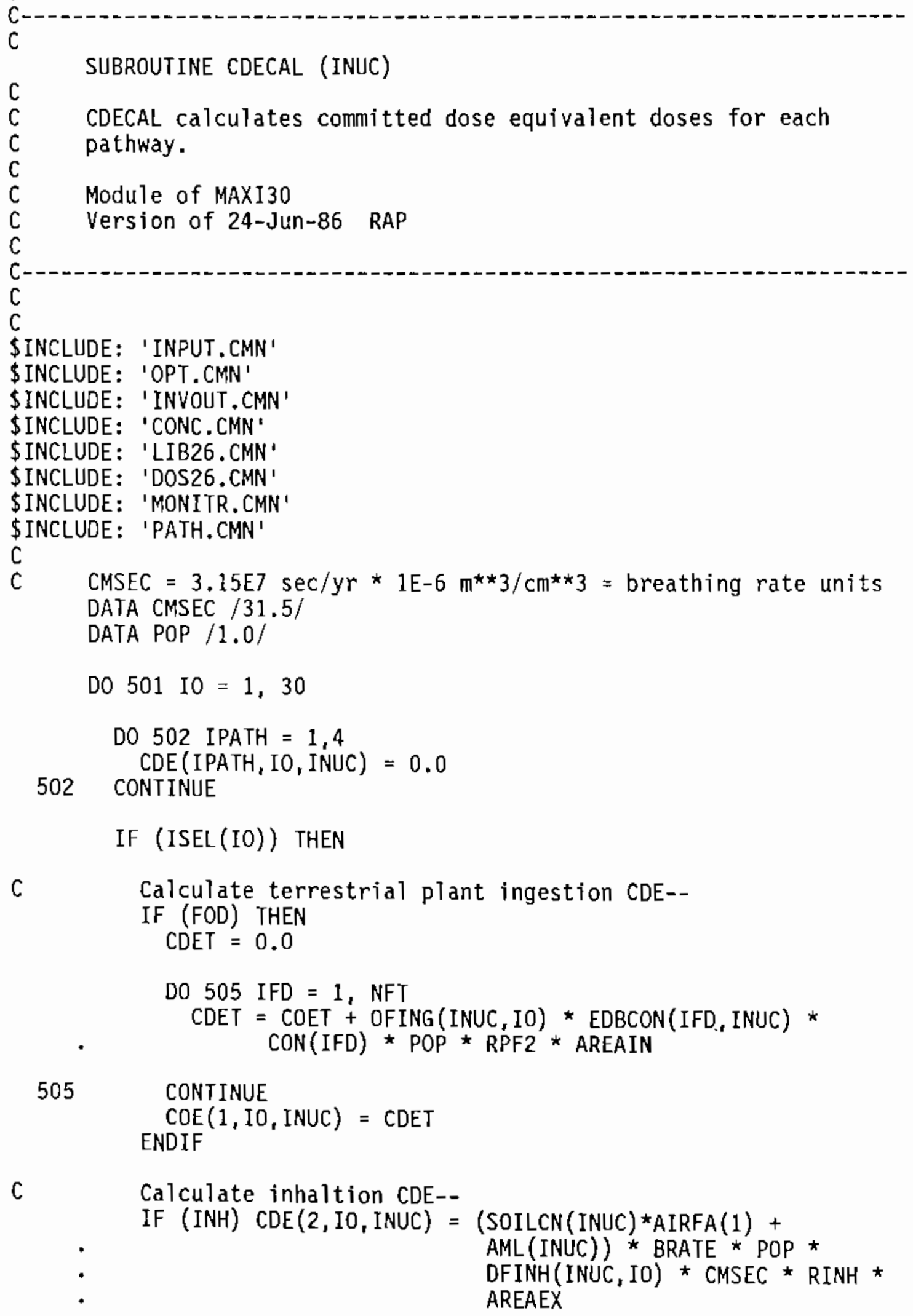




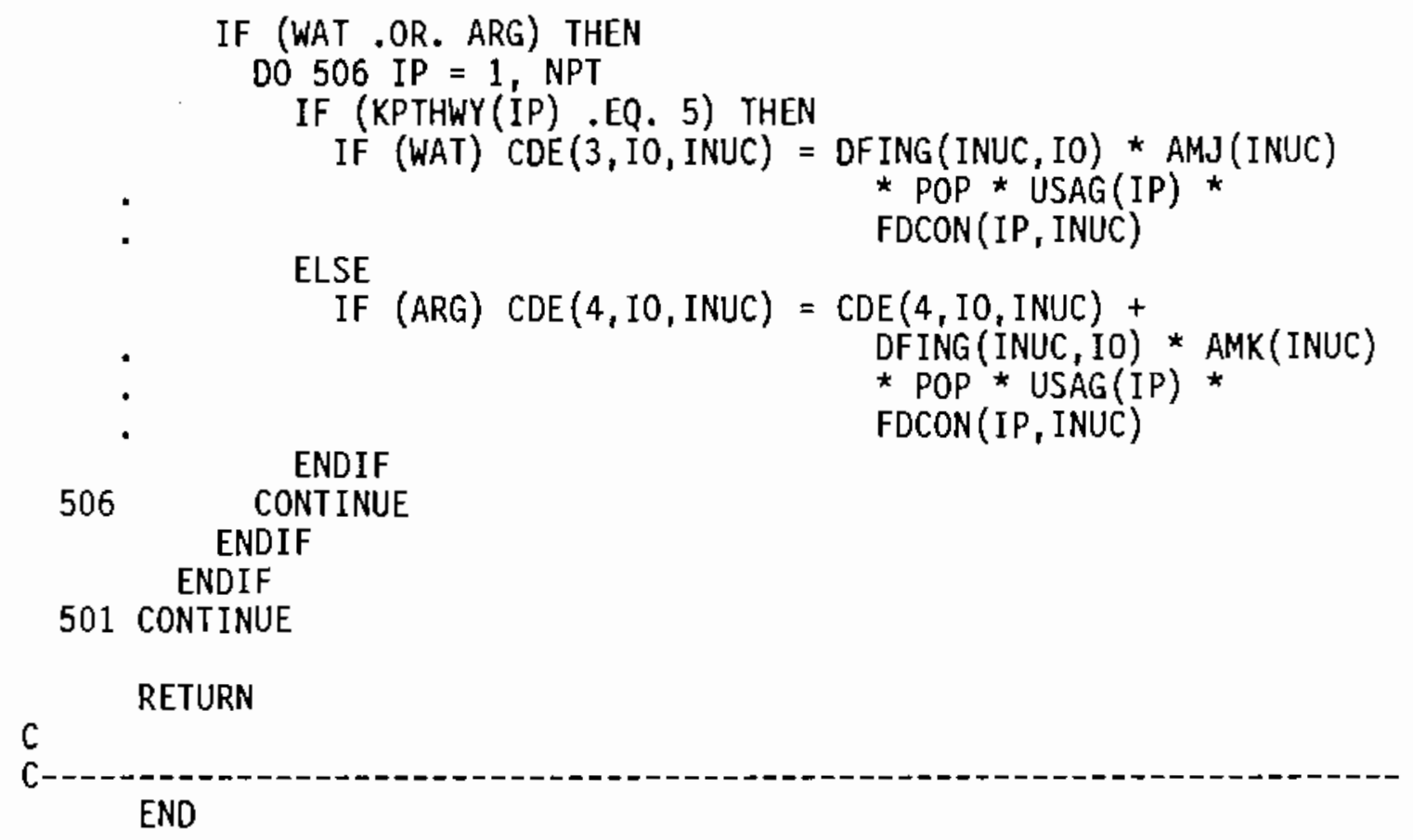




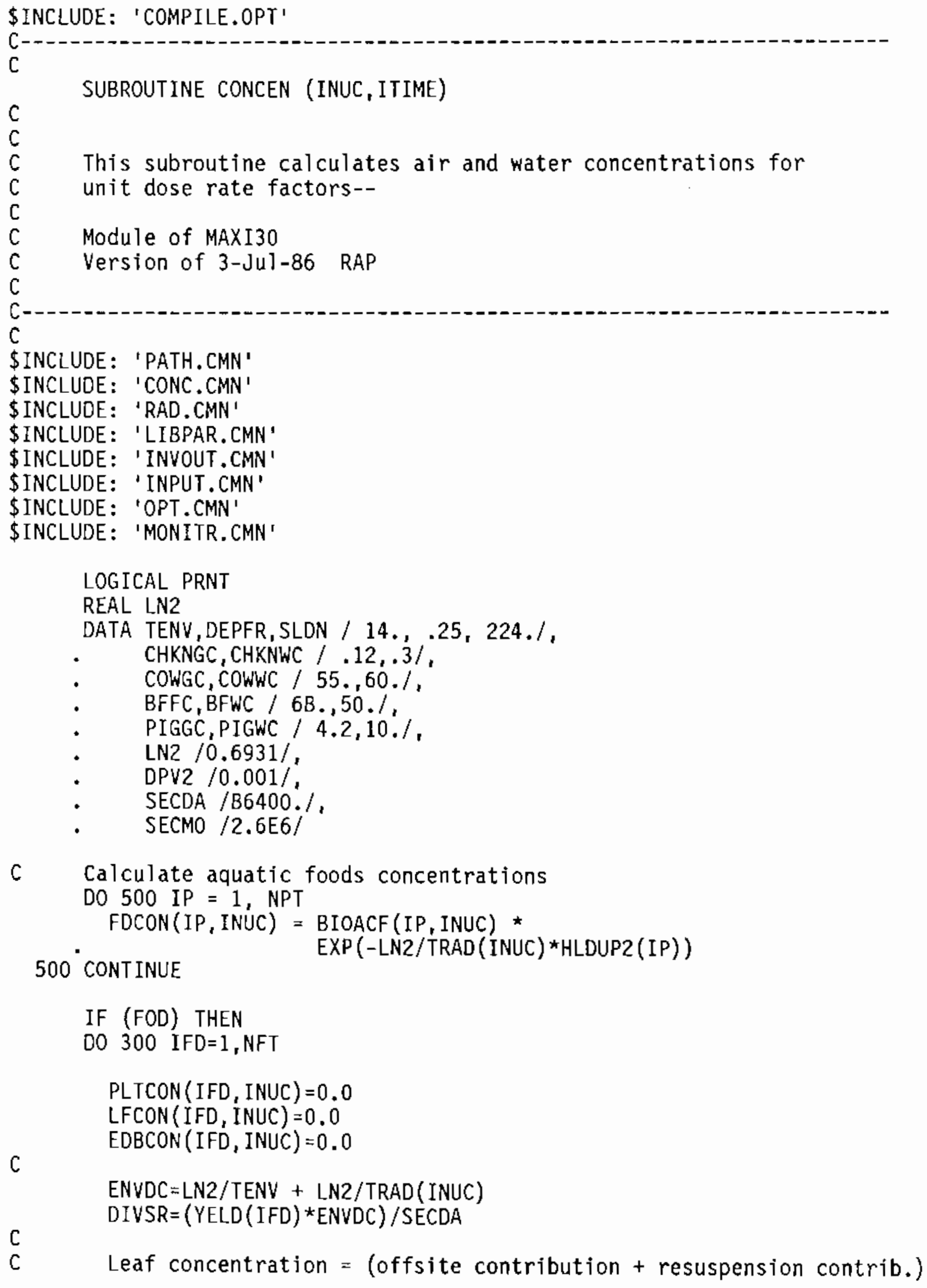




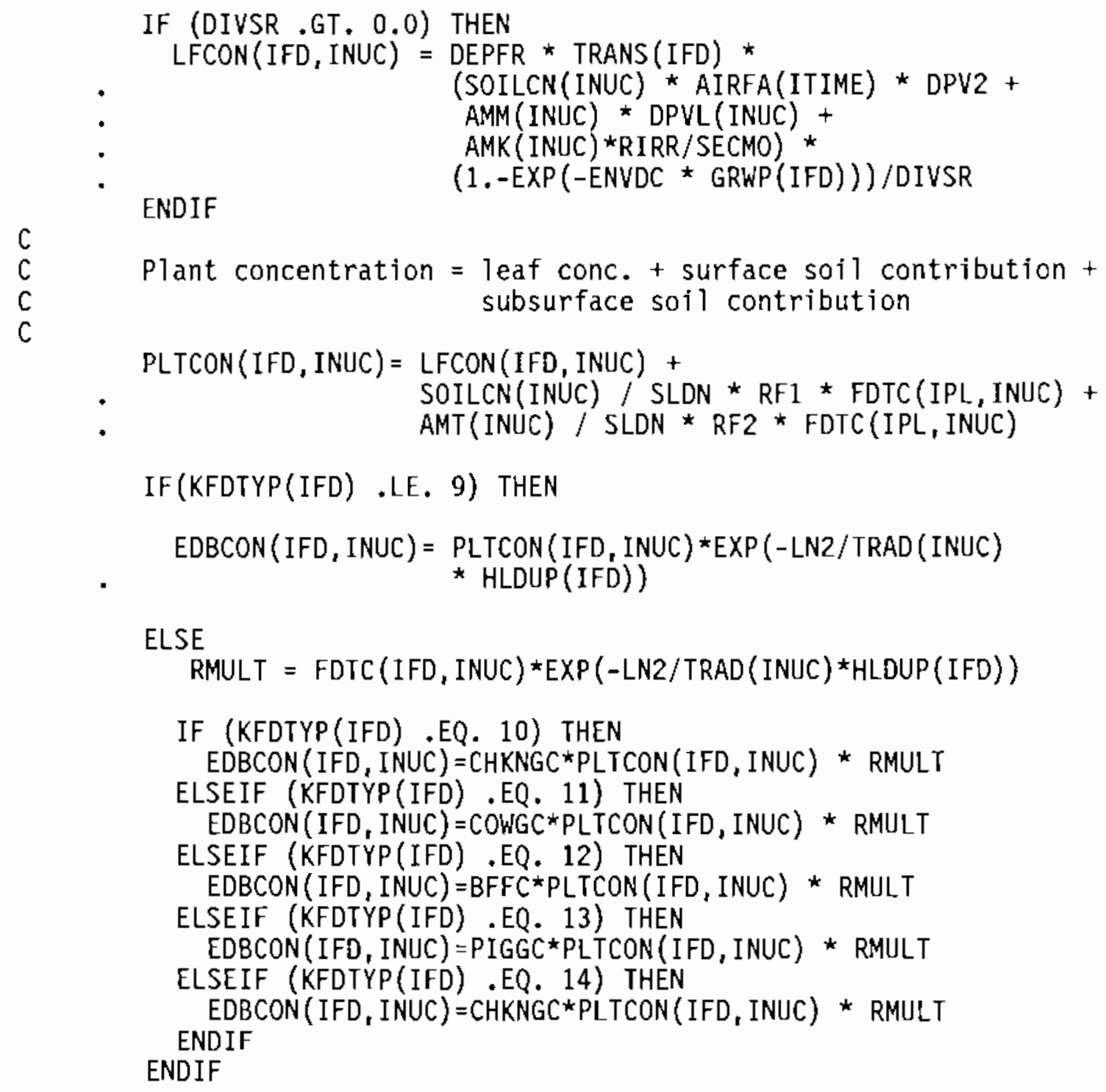

C

C

$\mathrm{C}$

Plant concentration = leaf conc.+ surface soil contribution + subsurface soil contribution

300 CONTINUE

ENDIF

RETURN

END 


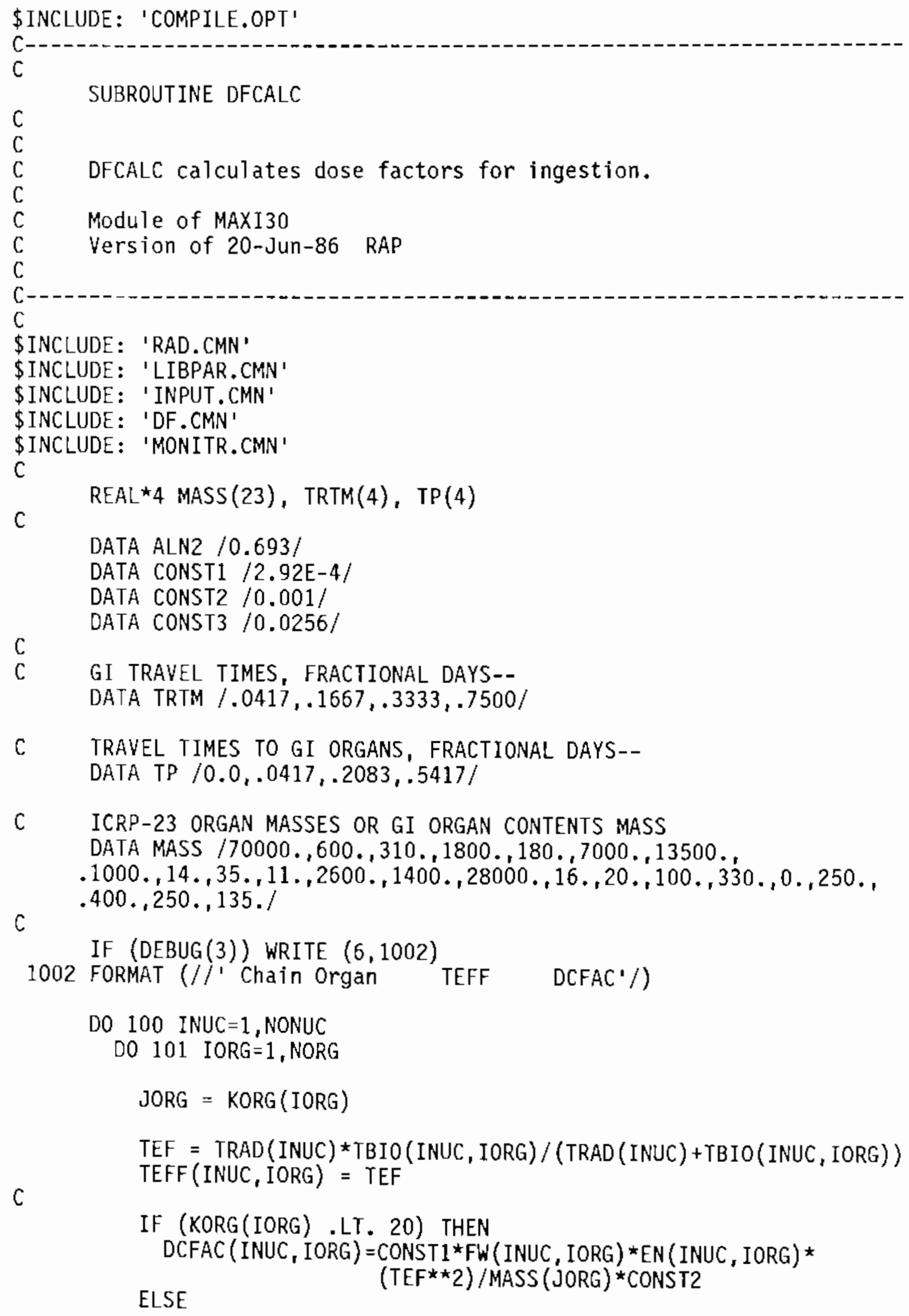




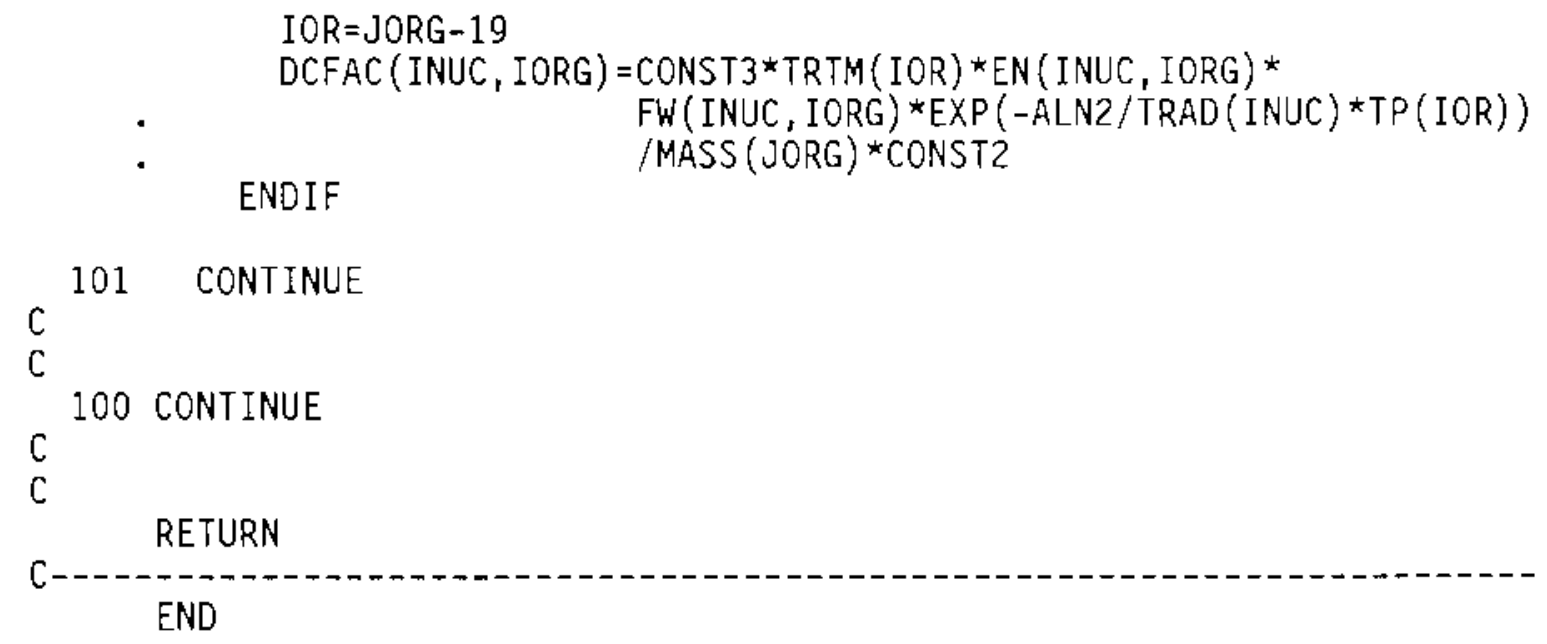

A. 18 


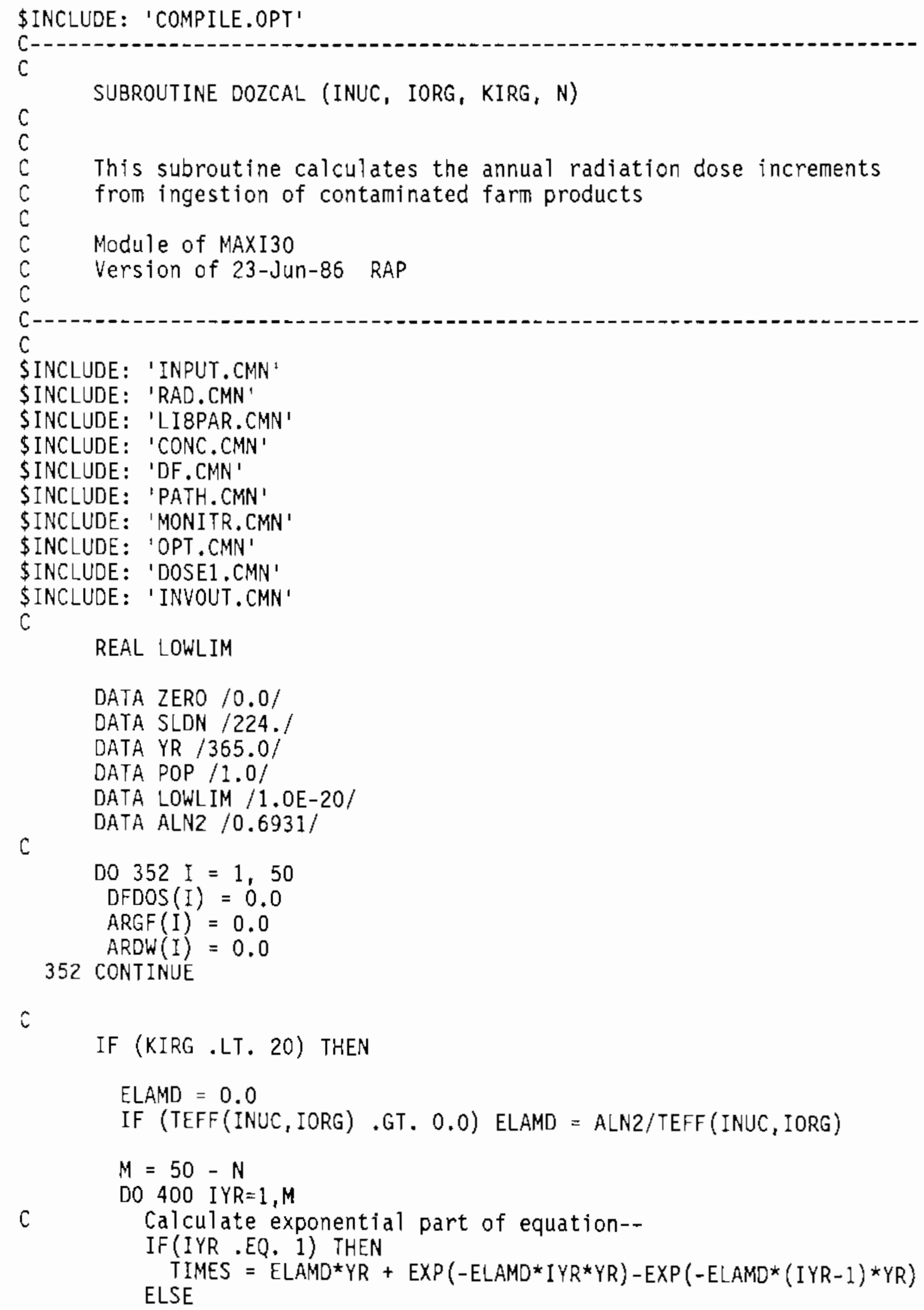




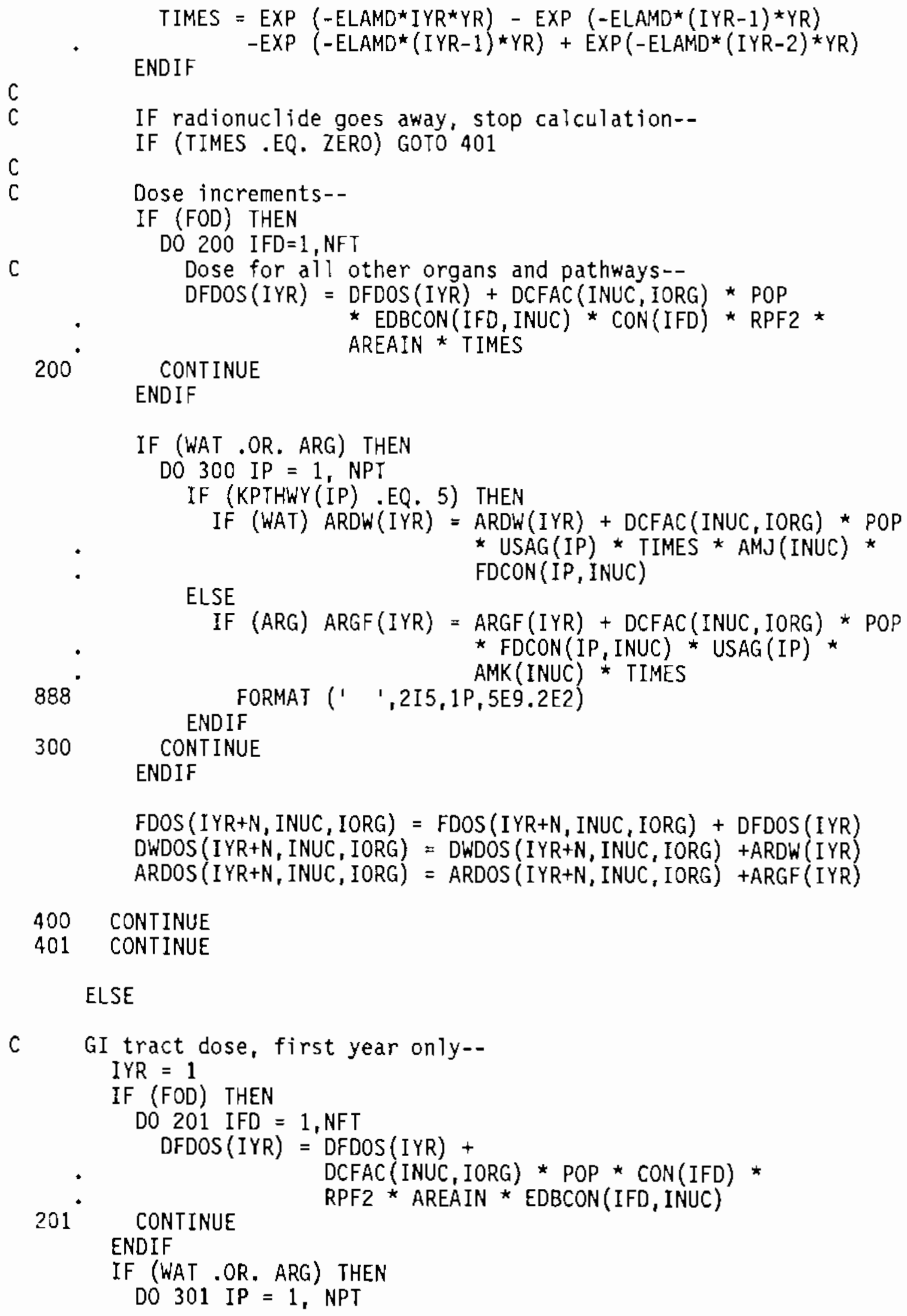

300

400 CONTINUE

401 CONTINUE

ELSE

C GI tract dose, first year only--

$$
\text { IYR }=1
$$




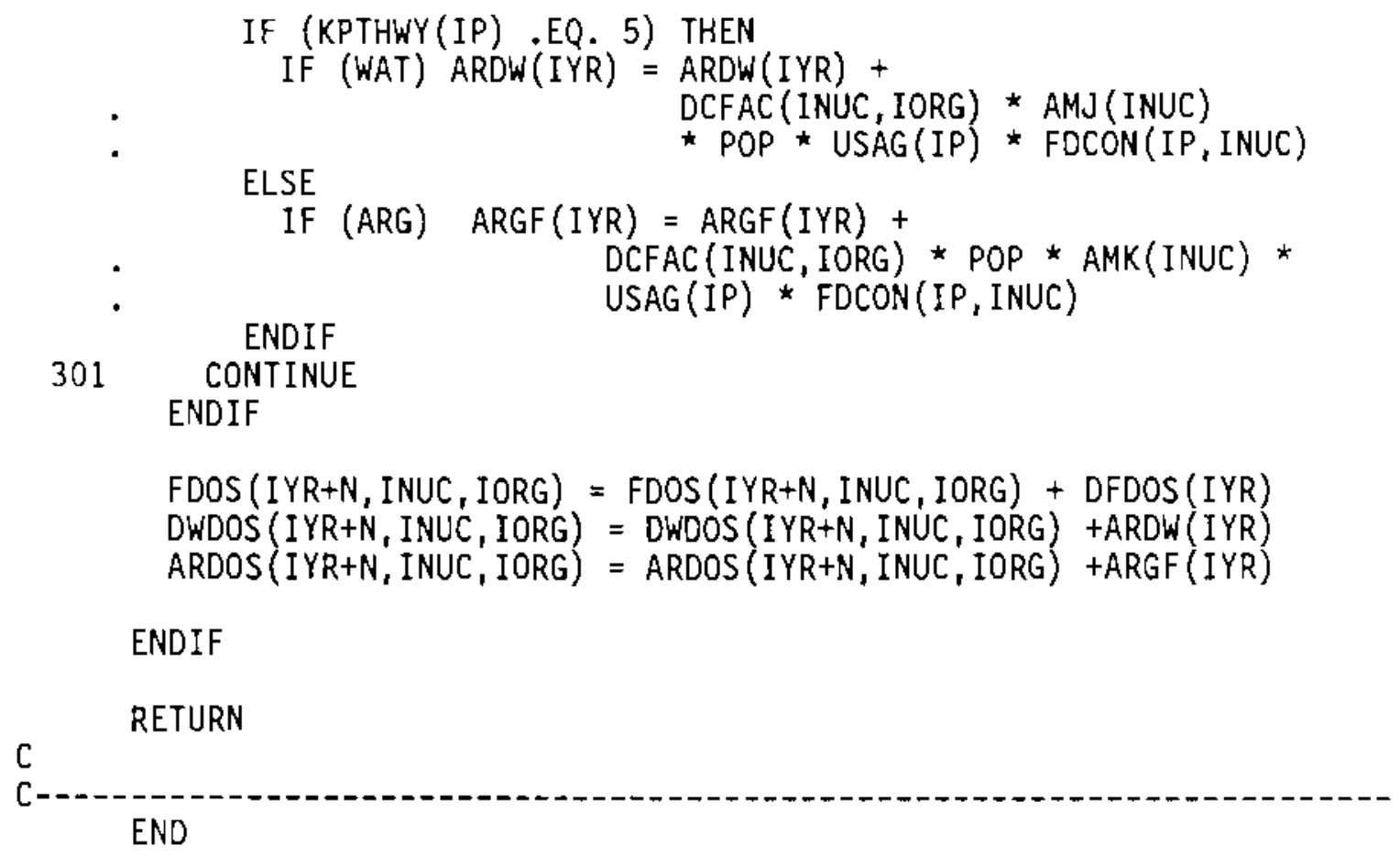


\$INCLUDE: 'COMPILE.OPT'

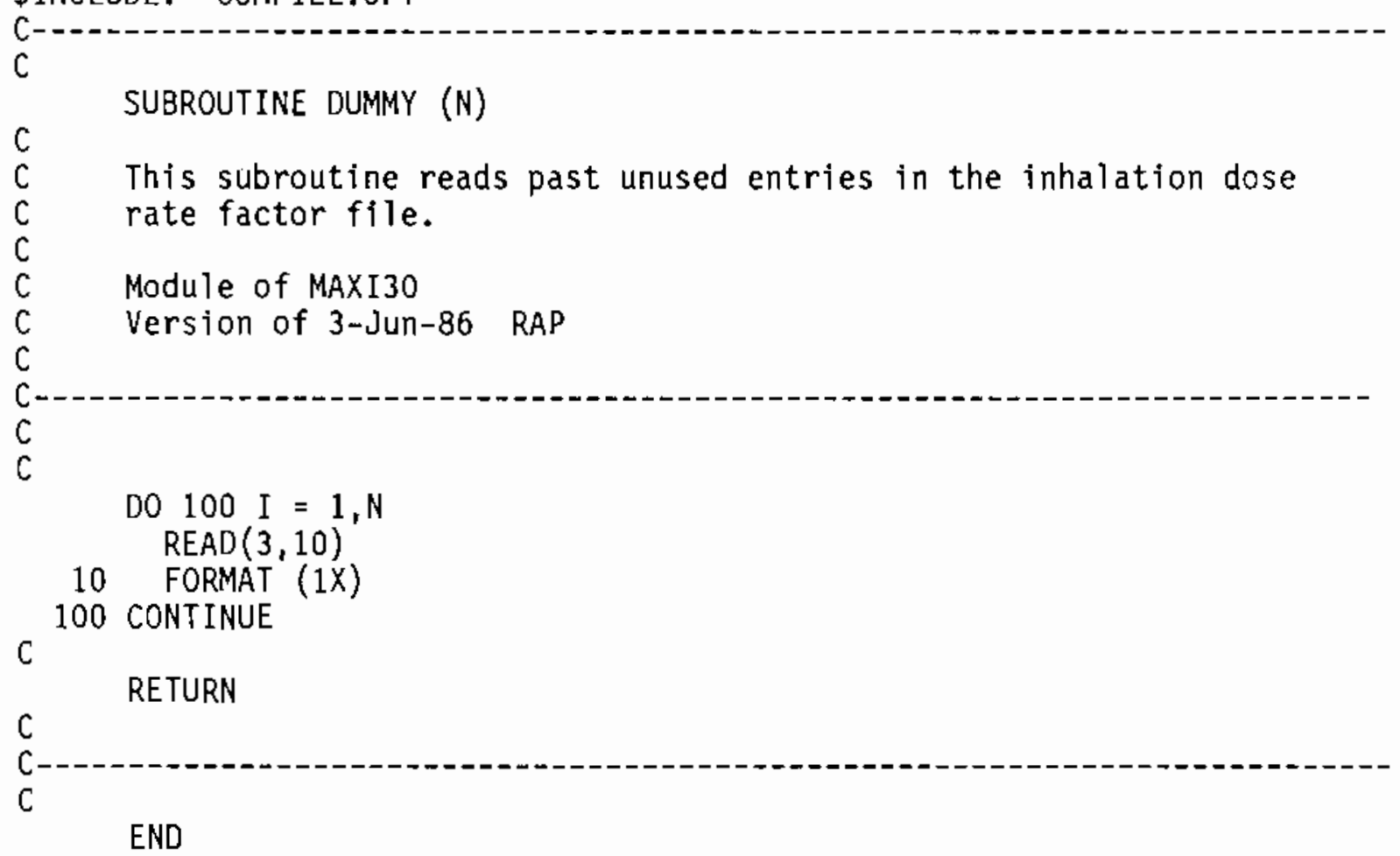




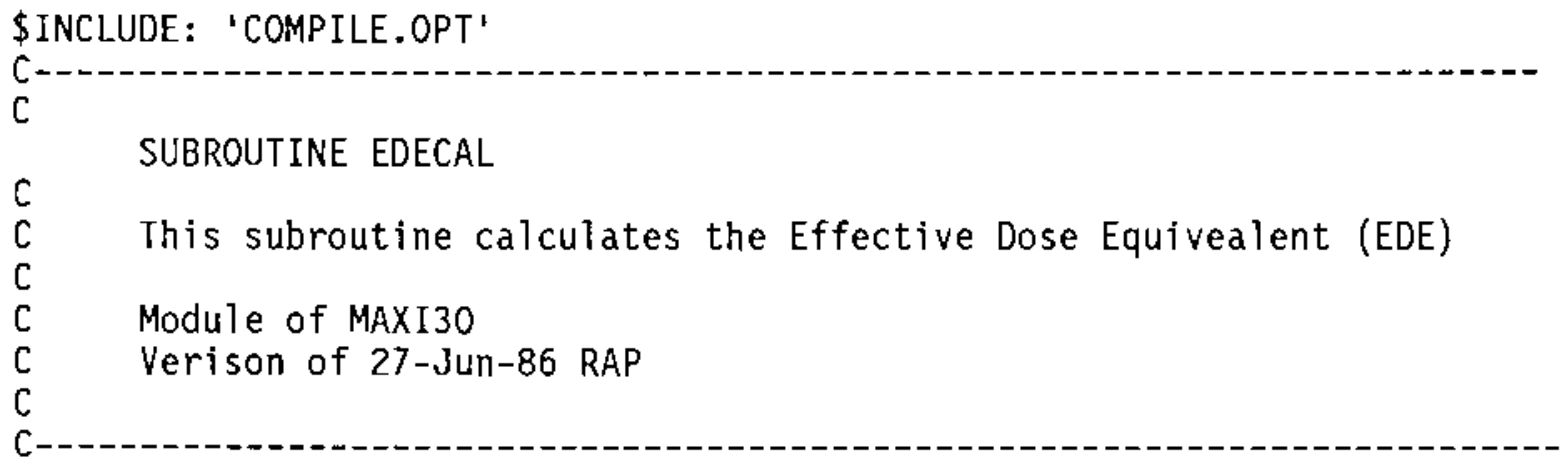

\$INCLUDE: 'DOS26.CMN'

\$INCLUDE: 'LIB26.CMN'

\$INCLUDE: 'INPUT.CMN'

\$INCLUDE: 'DOSE2.CMN'

\$INCLUDE : 'RAD.CMN'

\$INCLUDE: 'MONITR.CMN'

REAL TOTCDE(30), CSORT (24)

INTEGER ICNDX(24)

REAL WT(6)

DATA WT $/ 0.25,0.15,0.12,0.12,0.03,0.03 /$

C Total internal dose--

IF (DEBUG (4)) WRITE $(6,1000)$

1000 FORMAT (/' CDE FOR ALL ORGANS: ')

DO 100 I0 $=1$, NORG30

TOTCDE $(\mathrm{I} 0)=0.0$

IF (ISEL (IO)) THEN

DO 101 INUC $=1$, NONUC

DO 102 IPATH $=1,4$

TOTCDE $($ IO $)=\operatorname{TOTCDE}($ IO $)+\operatorname{CDE}($ IPATH, IO, INUC $)$

102 CONTINUE

101 CONTINUE

ENDIF

IF (DEBUG (4)) WRITE $\left(6,{ }^{\star}\right)$ 'Organ: ', I0, TOTCDE(I0)

100 CONTINUE

C Transfer to sort array

IS $=0$

DO 110 IO $=7,30$

IF (ISEL (IO)) THEN

IS $=$ IS +1

ICNDX (IS) $=$ IO

CSORT(IS) $=$ TOTCDE $(10)$

ENDIF

110 CONTINUE

NIS = IS 
C Sort CSORT array, highest dose first--

$N=N I S-1$

200 CONTINUE

$F L A G=0$

$\mathrm{J}=1$

201 CONTINUE

IF (CSORT (J) .LT. CSORT $(\mathrm{J}+1))$ THEN

CTEMP $=\operatorname{CSORT}(J)$

NTEMP = ICNDX $(\mathrm{J})$

$\operatorname{CSORT}(J)=\operatorname{CSORT}(J+1)$

$\operatorname{ICNDX}(\mathrm{J})=\operatorname{ICNDX}(\mathrm{J}+1)$

$\operatorname{CSORT}(\mathrm{J}+1)=\operatorname{CTEMP}$

$\operatorname{ICNDX}(\mathrm{J}+1)=\mathrm{NTEMP}$

FLAG $=1$

ENDIF

$J=J+1$

IF (J.LE, N) GOTO 201

IF (FLAG , GT, 0) THEN

$N=N-1$

IF (N.GT. 0) G0T0 200

ENDIF

202 CONTINUE

C Transfer values to print arrays--

DO 300 I0 $=1,6$

$\operatorname{CDEPR}(\mathrm{IO})=\operatorname{TOTCDE}(\mathrm{IO})$

$\operatorname{IOP}(I 0)=I 0$

$\operatorname{HWT}(\mathrm{IO})=W T(\mathrm{IO})$

300 CONTINUE

DO 301 IS $=1,5$

$\operatorname{CDEPR}($ IS +6$)=\operatorname{CSORT}($ IS $)$

$\operatorname{IOP}(\mathrm{IS}+6)=\operatorname{ICNDX}$ (IS)

IF (ICNDX (IS) .EQ. 16) THEN

$\operatorname{HWT}(\mathrm{IS}+6)=1.00$

ELSE

HWI $(I S+6)=0.06$

ENDIF

301 CONTINUE

C Calculate weighted values, EDE

$\mathrm{EDE}=0.0$

DO $400 \mathrm{IH}=1,11$

WDE $(I H)=\operatorname{CDEPR}(\mathrm{IH}) * \mathrm{HWT}(\mathrm{IH})$

$\mathrm{EDE}=\mathrm{EDE}+\mathrm{WDE}(\mathrm{IH})$

400 CONTINUE

$\mathrm{WBEDE}=\mathrm{EDE}+\mathrm{AEXDOS}(1)$

RETURN

C

END 
\$INCLUDE: 'COMPILE.OPT'

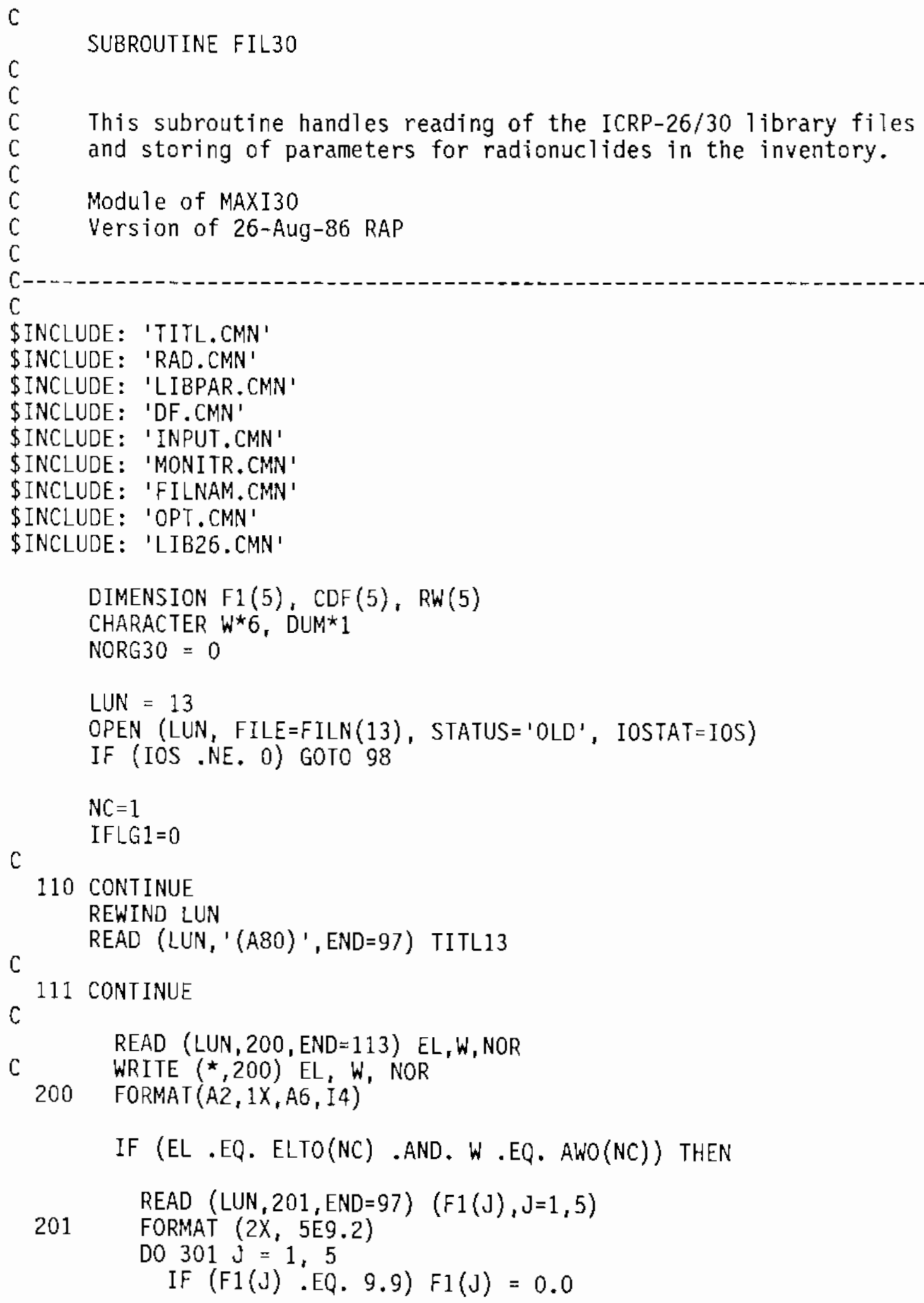




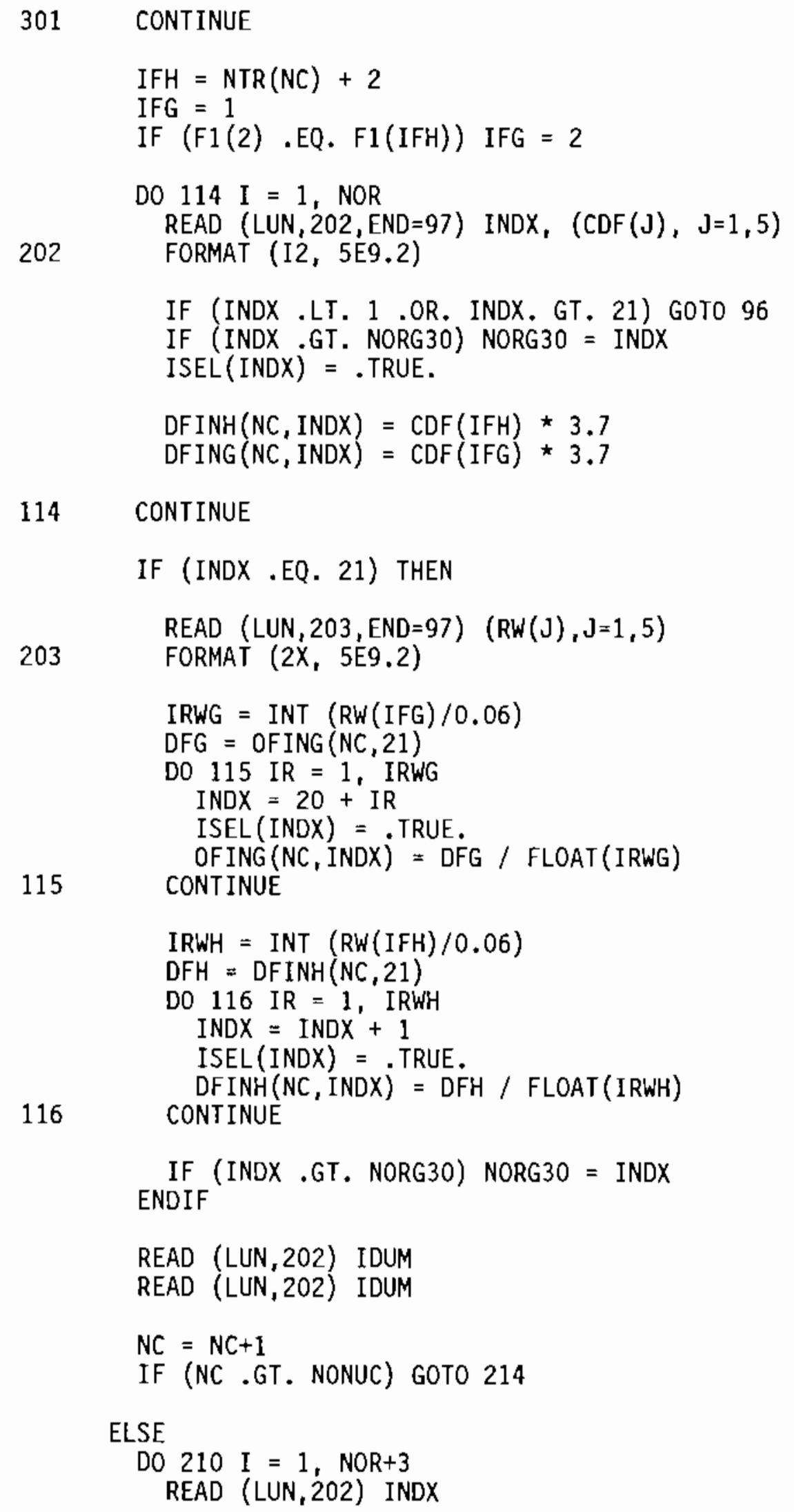




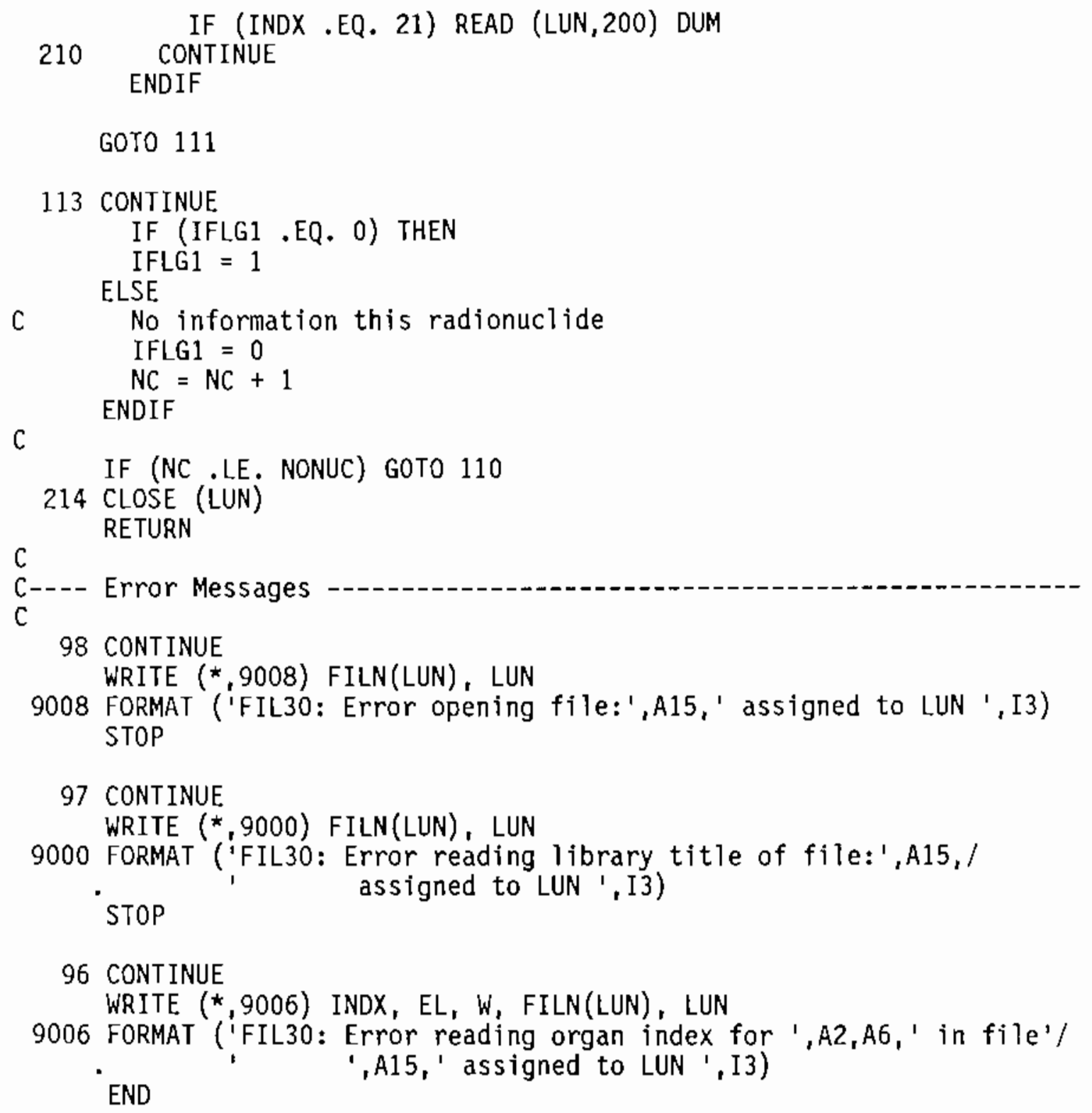


\$INCLUDE: 'COMPILE.OPT'

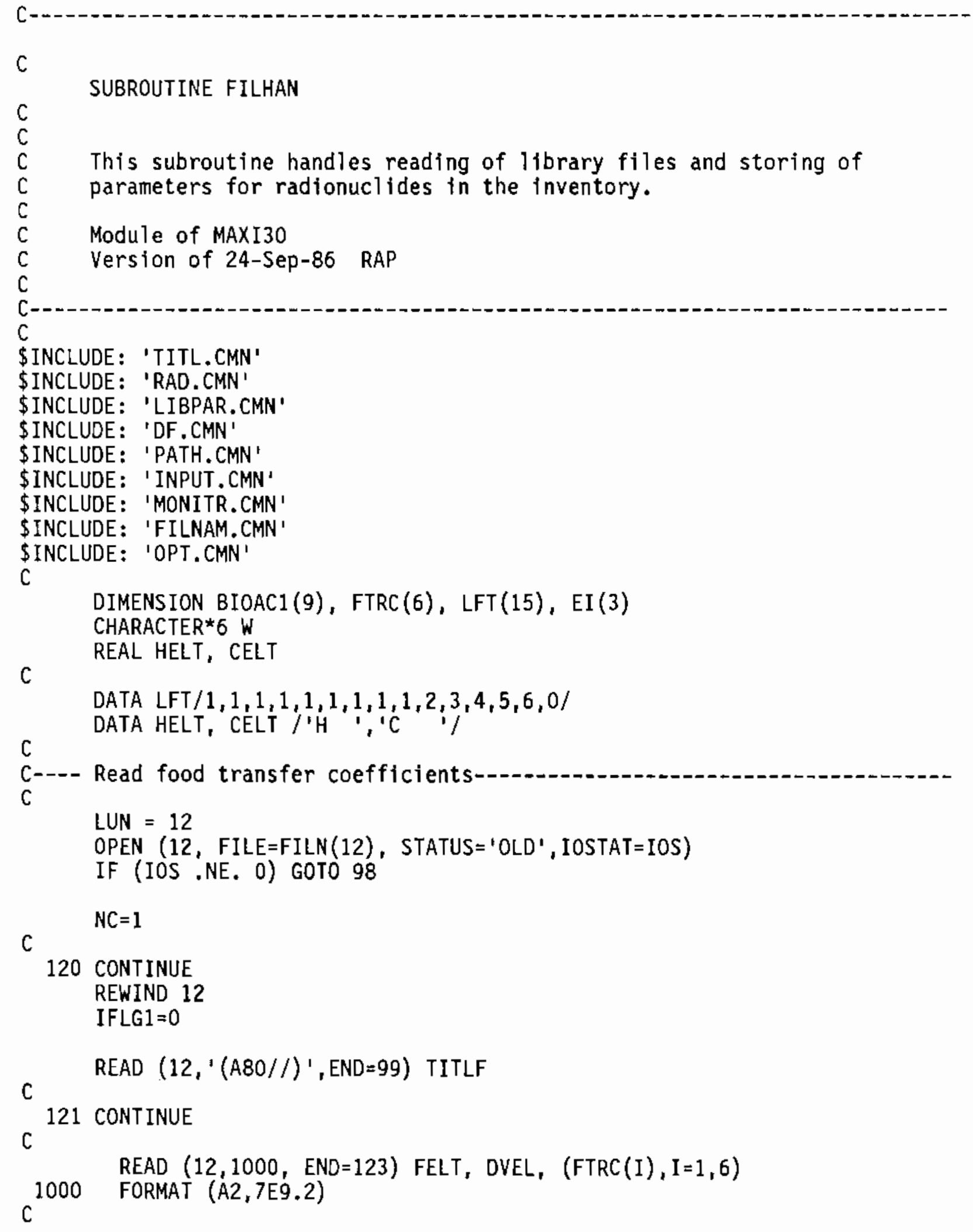




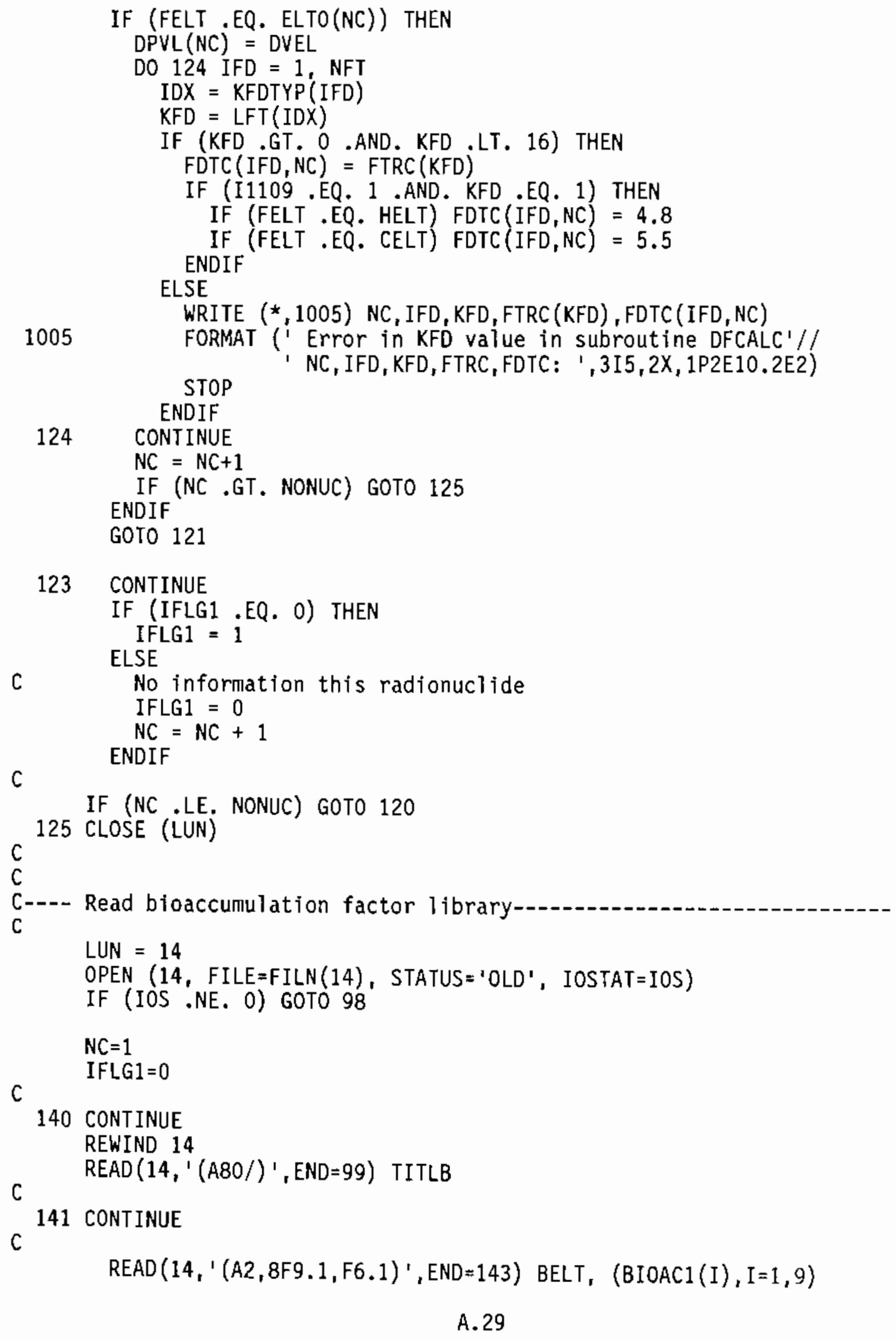

123 CONTINUE

IF (IFLG1 .EQ. 0) THEN 
C

C

IF (BELT . EQ. ELTO(NC)) THEN

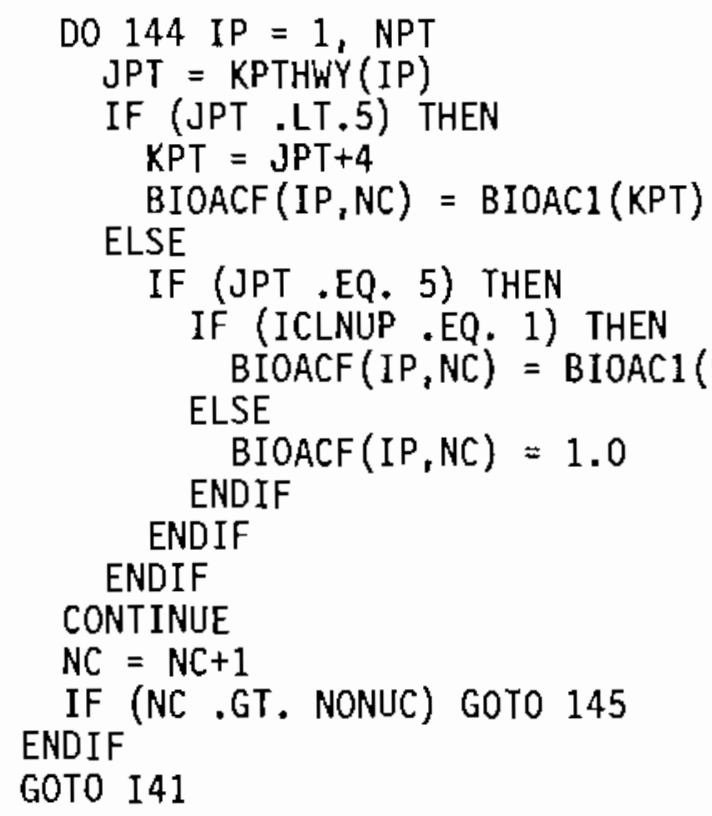

I43 CONTINUE

IF (IFLG1 .EQ. O) THEN

IFLG1 = 1

$$
\text { ELSE }
$$

C

No information this radionuclide IFLG1 = 0 $\mathrm{NC}=\mathrm{NC}+1$

ENDIF

C

C

145 CLOSE (LUN)

C

C---- Read organ data Tibrary

C

LUN $=11$

OPEN (11, FILE=FILN(11), STATUS=' OLD', IOSTAT=IOS)

IF (IOS .NE. O) GOTO 98

$N C=1$

IFLG $1=0$

IFLG2 $=0$

C

I10 CONTINUE

REWIND 11

C

READ $(11, '(A 80)$ ', END $=99)$ TITLO

C

111 CONTINUE 
$\operatorname{READ}(11,200, E N D=113) E L, W, I O R, T B I, F W I, F 2 P I,(E I(I), I=1,3)$

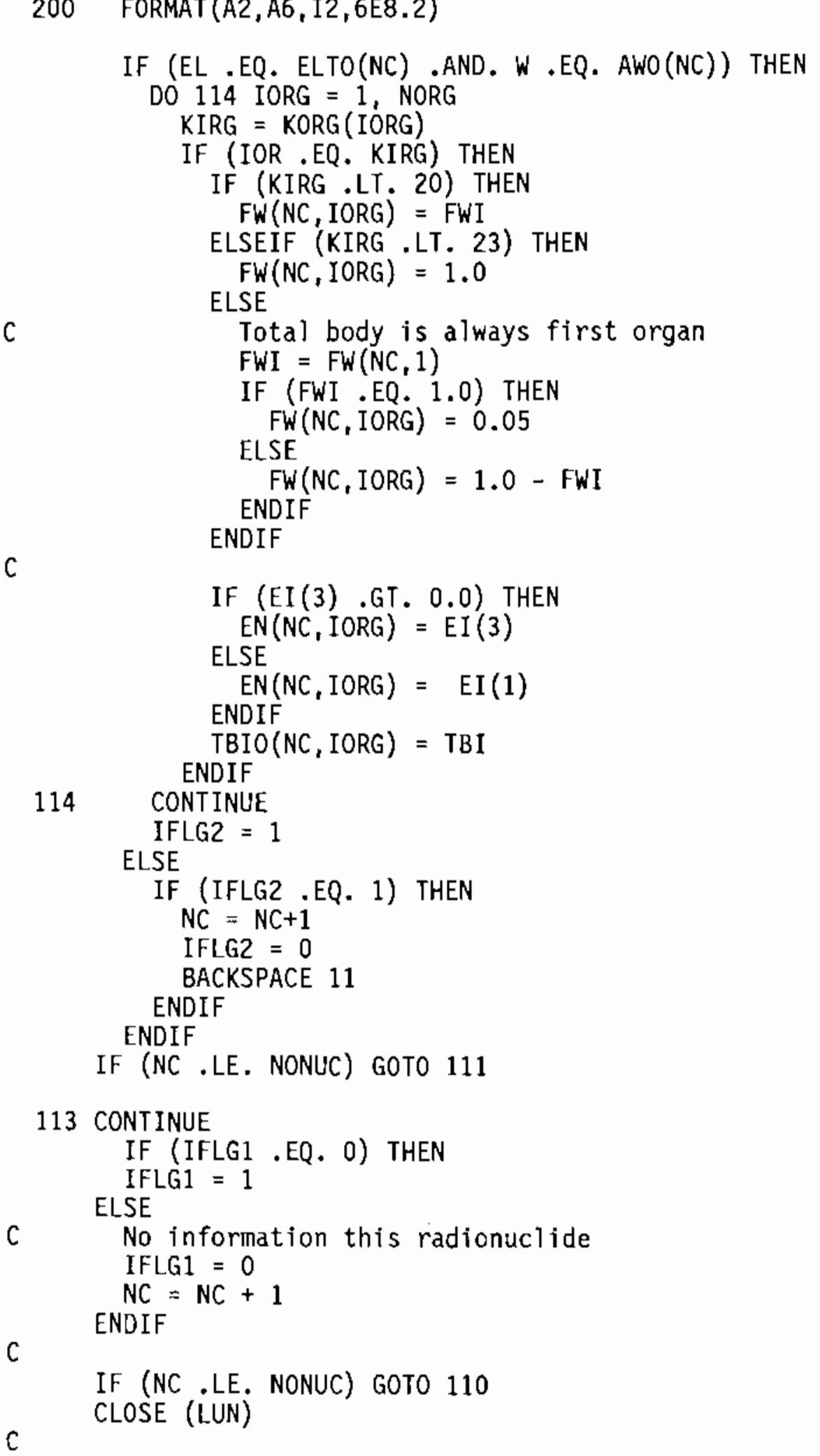


C---- read surface external exposure dose rate factor library-----------------

C

IF (ISUR .EQ. 1) THEN

LUN $=2$

OPEN $\left(2, F I L E=F I L N(2), \quad\right.$ STATUS $={ }^{\prime}$ OLD',$\quad$ IOSTAT $\left.=I O S\right)$

IF (IOS .NE. O) GOTO 98

$N C=1$

IFLG1 $=0$

C

220 CONTINUE

REWIND 2

C

$\operatorname{READ}\left(2,{ }^{\prime}(A 80 / /)\right.$ ', END $\left.=99\right)$ TIT22

c

221 CONTINUE

$\operatorname{READ}(2, '(A 2, A 6, E 9.1)$ ', END=223) E, W, EXSUR

IF (E .EQ. ELTO(NC) .AND. W.EQ. AWO(NC)) THEN $\operatorname{DEXT}(N C)=$ EXSUR

$\mathrm{NC}=\mathrm{NC}+1$

IF (NC .GT. NONUC) GOTO 225

ENDIF

GOTO 221

223 CONTINUE

IF (IFLG1 .EQ. 0) THEN

IFLG1 $=1$

C No information this radionuclide

IFLG1 $=0$

$N C=N C+1$

C

ENDIF

IF (NC .LE. NONUC) GOTO 220

225 CLOSE (LUN)

ENDIF

C

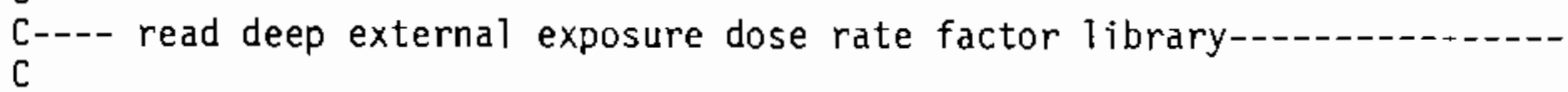

IF (IEXT .EQ. 1) THEN

OPEN (7, FILE $=$ FILN (7), STATUS $=$ 'OLD'， IOSTAT $=$ IOS)

IF (IOS .NE. O) GOTO 98

$N C=1$

IFLG1 $=0$

C

270 CONTINUE 
REWIND 7

C

$\operatorname{READ}(7, '(A 80 / /)$ ', END=99) TIT27

271 CONTINUE

C

$\operatorname{READ}(7, '(A 2, A 6, E 9.1) ', E N D=273) E, W$, EXDEEP

IF (E .EQ. ELTO(NC) .AND. W.EQ. AWO(NC)) THEN $\operatorname{DEDXT}(\mathrm{NC})=$ EXDEEP

$N C=N C+1$

IF (NC .GT. NONUC) GOTO 275

ENDIF

GOTO 271

273 CONTINUE

IF (IFLG1 .EQ. 0) THEN

IFLG $1=1$

C No information this radionuclide IFLG $1=0$ $N C=N C+1$

C ENDIF

IF (NC.LE. NONUC) GOTO 270

275 CLOSE (LUN)

ENDIF

RETURN

C---. Error Messages

$\mathrm{C}$

98 CONTINUE

WRITE $(*, 9008)$ FILN(LUN), LUN

9008 FORMAT ('FILHAN: Error opening file:',A15,' assigned to LUN ', I3) STOP

99 CONTINUE WRITE $\left({ }^{\star}, 9000\right)$ FILN(LUN), LUN

9000 FORMAT ('FILHAN: Error reading library title of file: ', ${ }^{15} 5_{v}$ /

STOP assigned to LUN ', I3)

END 
\$INCLUDE: 'COMPILE.OPT'

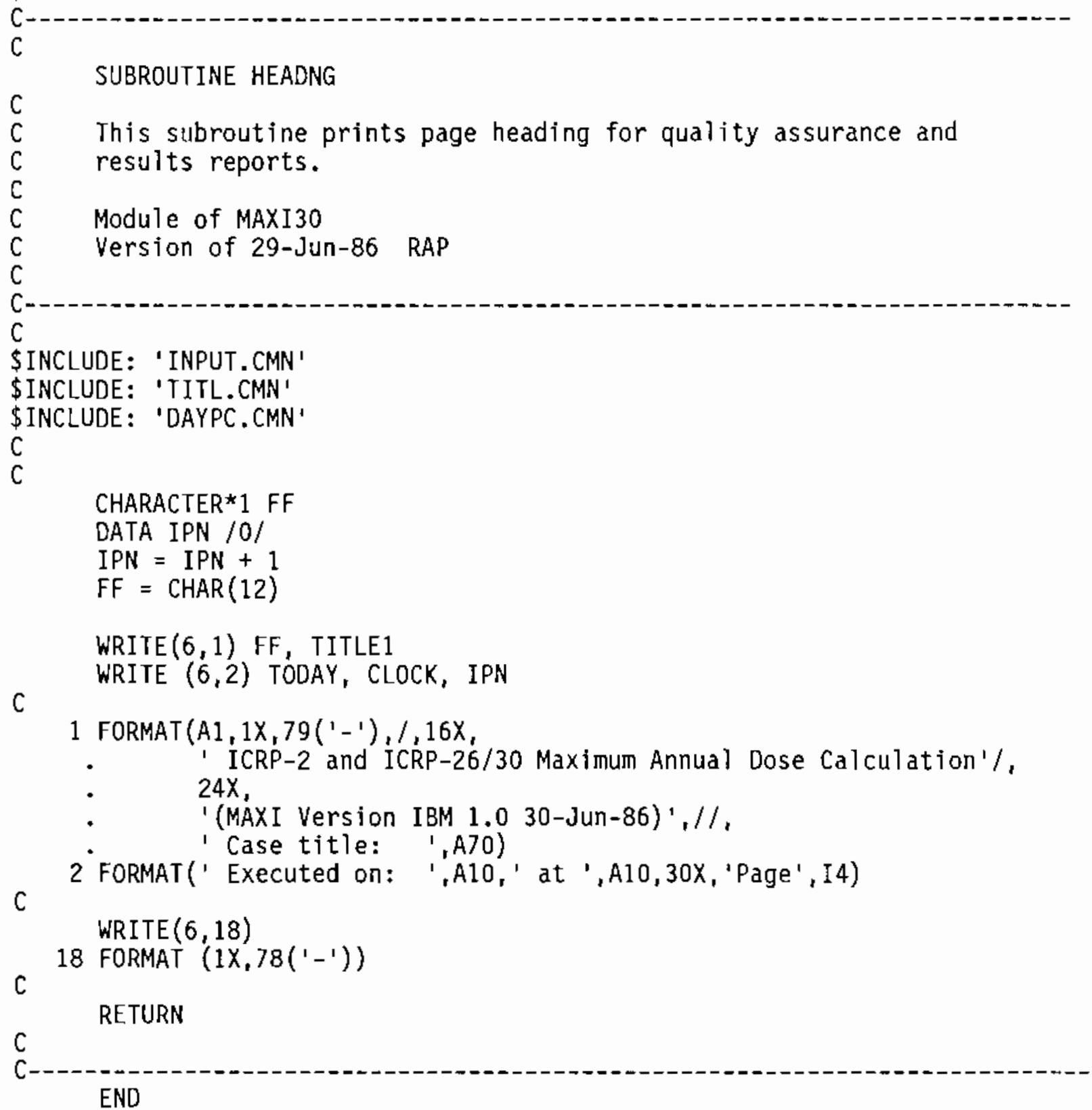


C

SUBROUTINE IDNUC

C

$\mathrm{C}$

C This module controls reading of the master radionuclide library

C and attempts to identify the input inventory.

C

C Module of MAXI30

C Version of 19-Jun-86 RAP

$\mathrm{C}$

$\mathrm{C}$

\$INCLUDE: 'RMD.CMN'

\$INCLUDE: 'RAD. CMN'

C

LOGICAL FOUND

C

C Read Master Radionuclide Library--

CALL RLIBIN

$c$

C Initialize count index on unidentified radionuclides-ISTOP $=0$

C Loop on input radionuclides, test against master library--

C DO 3 IN $=1$, NIN

FOUND $=$. FALSE.

$$
\mathrm{IL}=0
$$

c

1 CONTINUE

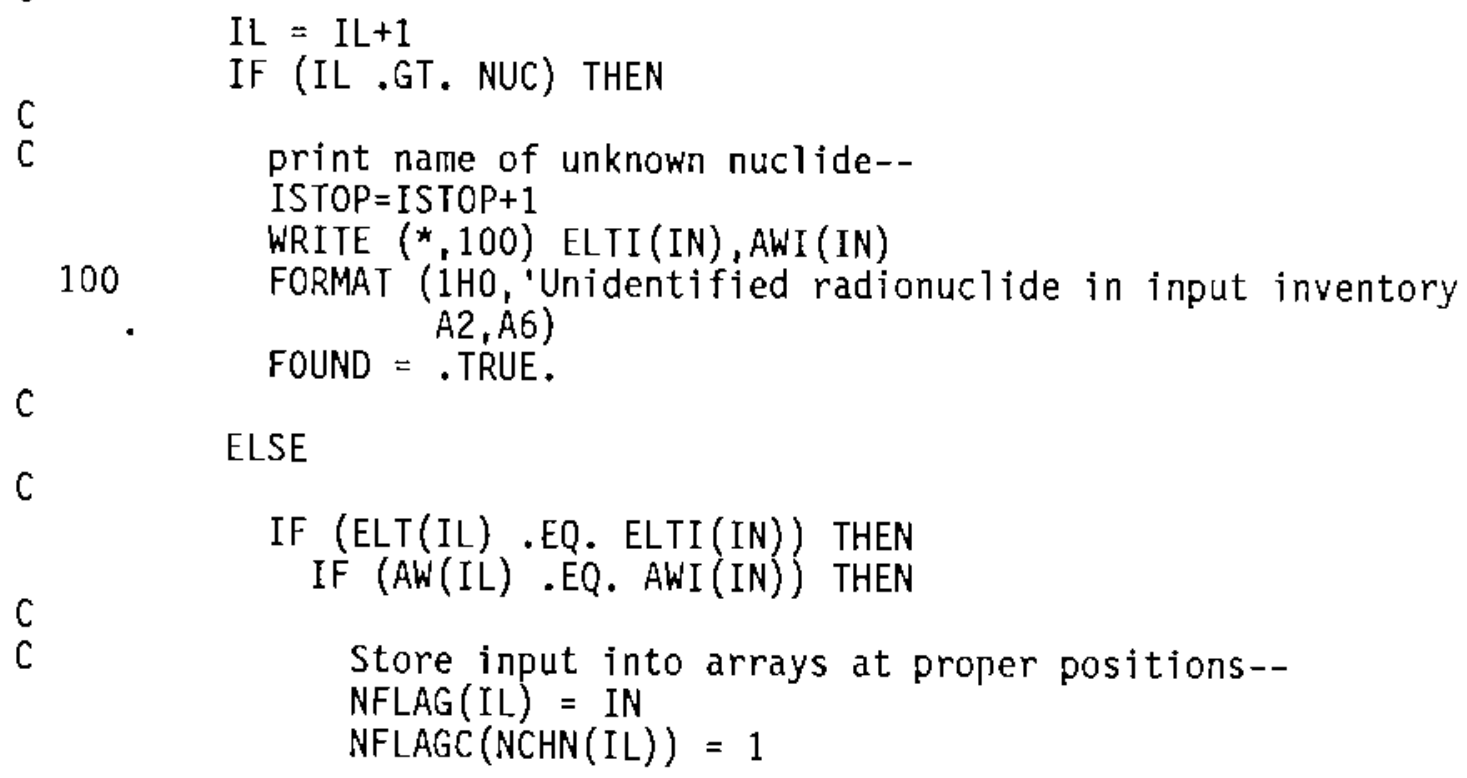

C

ELSE

IF (ELT(IL) .EQ. ELTI(IN)) THEN IF (AW(IL) .EQ. AWI(IN)) THEN

C

Store input into arrays at proper positions-NFLAG (IL) = IN

$\operatorname{NFLAGC}(N C H N(I L))=1$ 


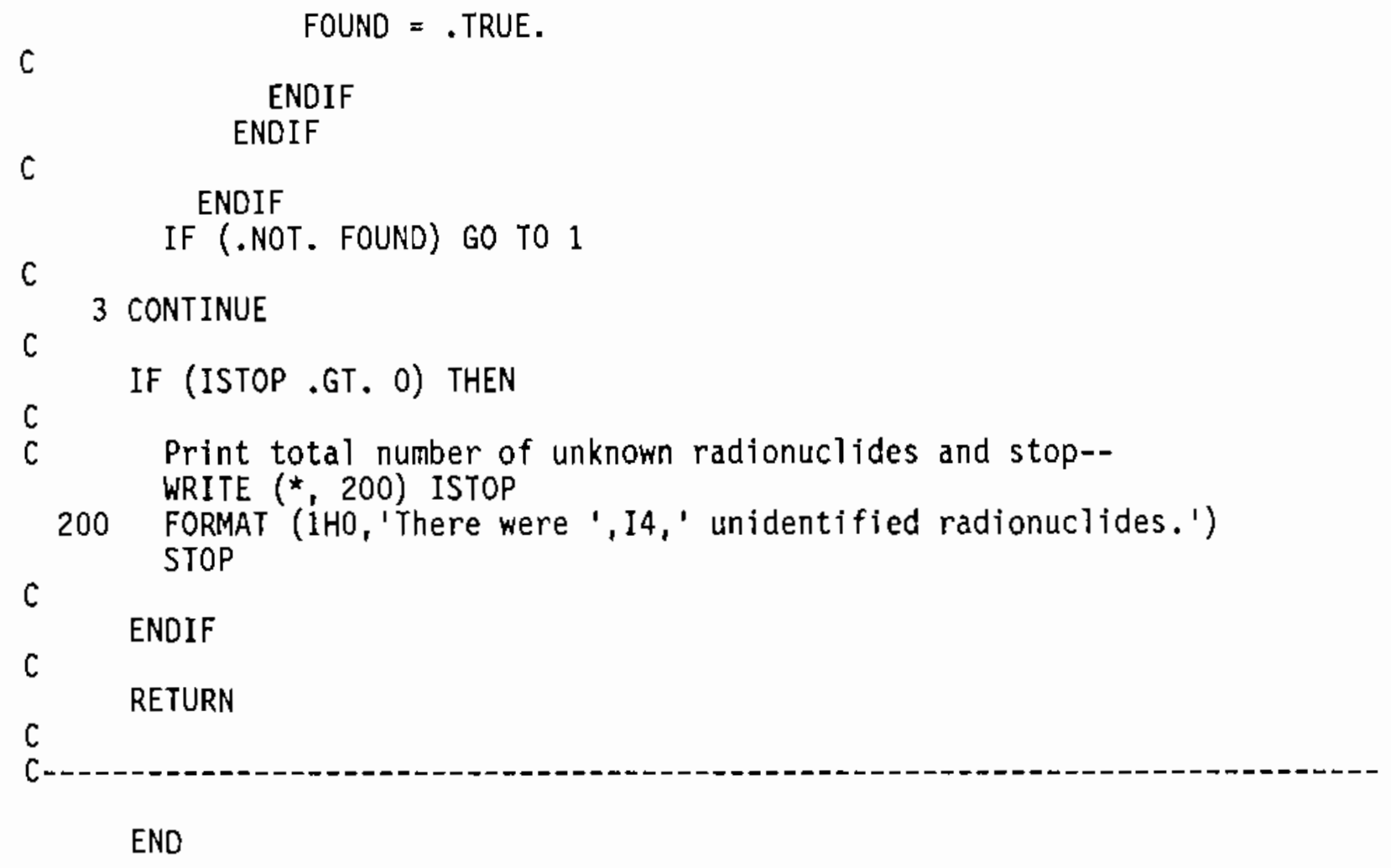




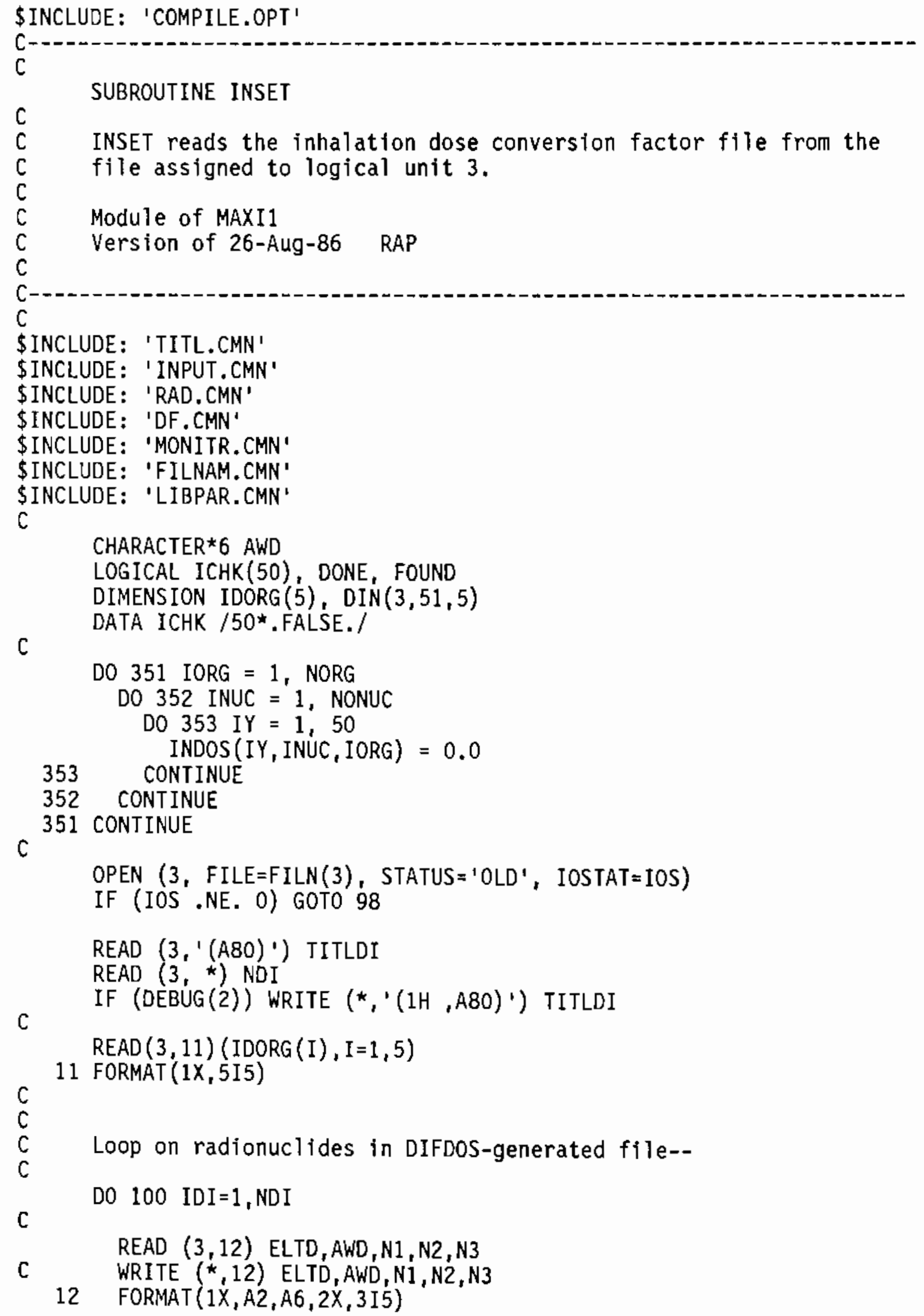




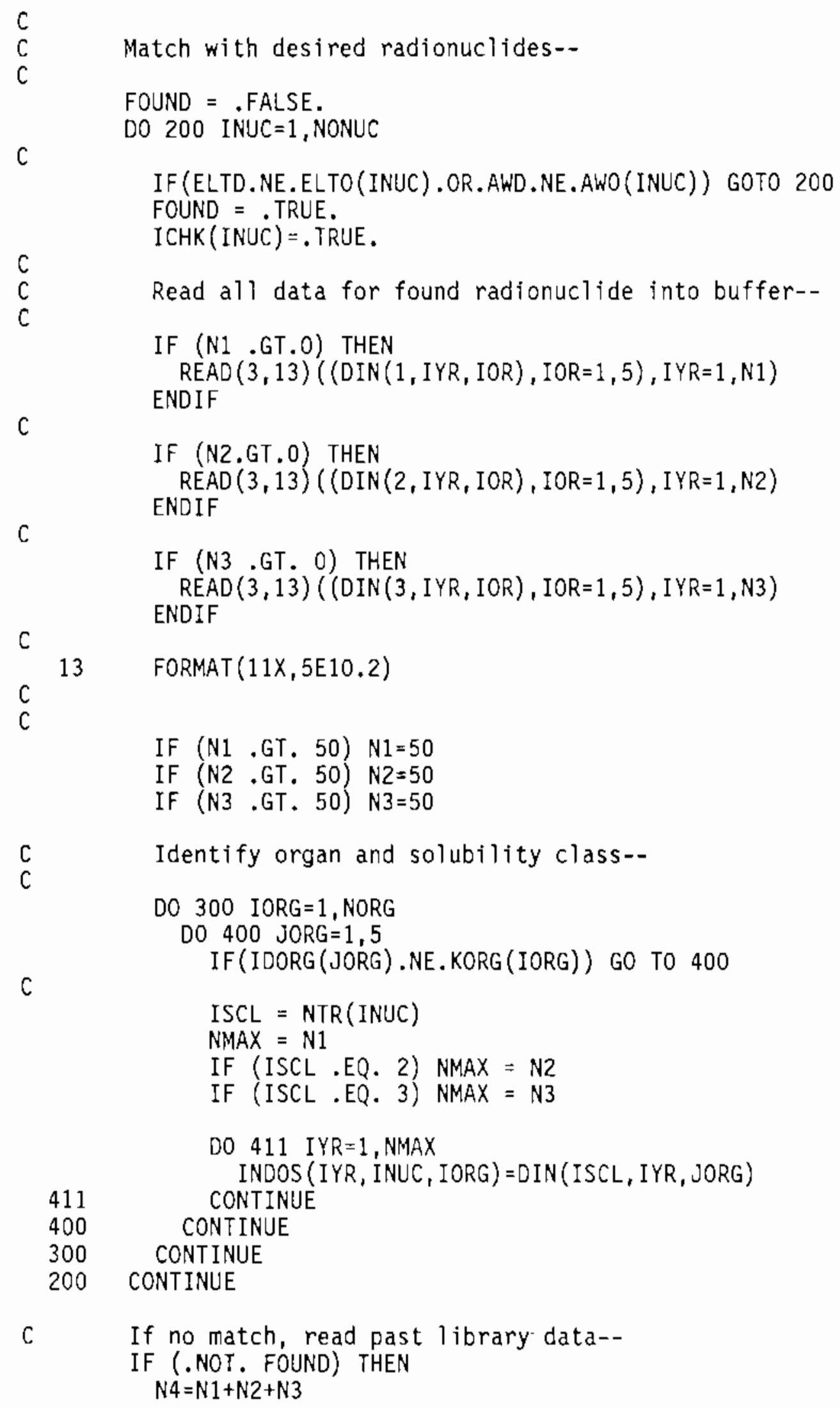




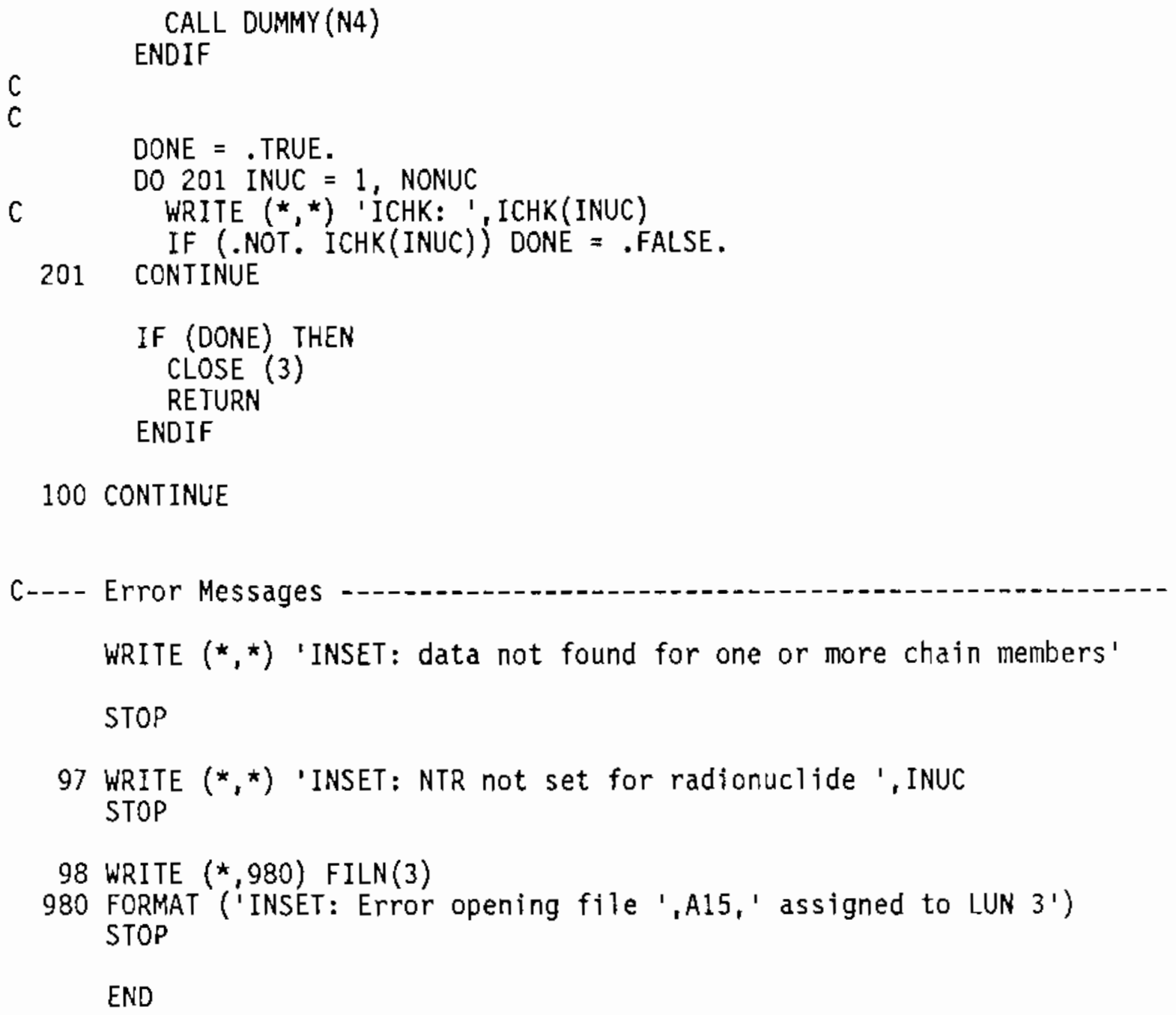

END 


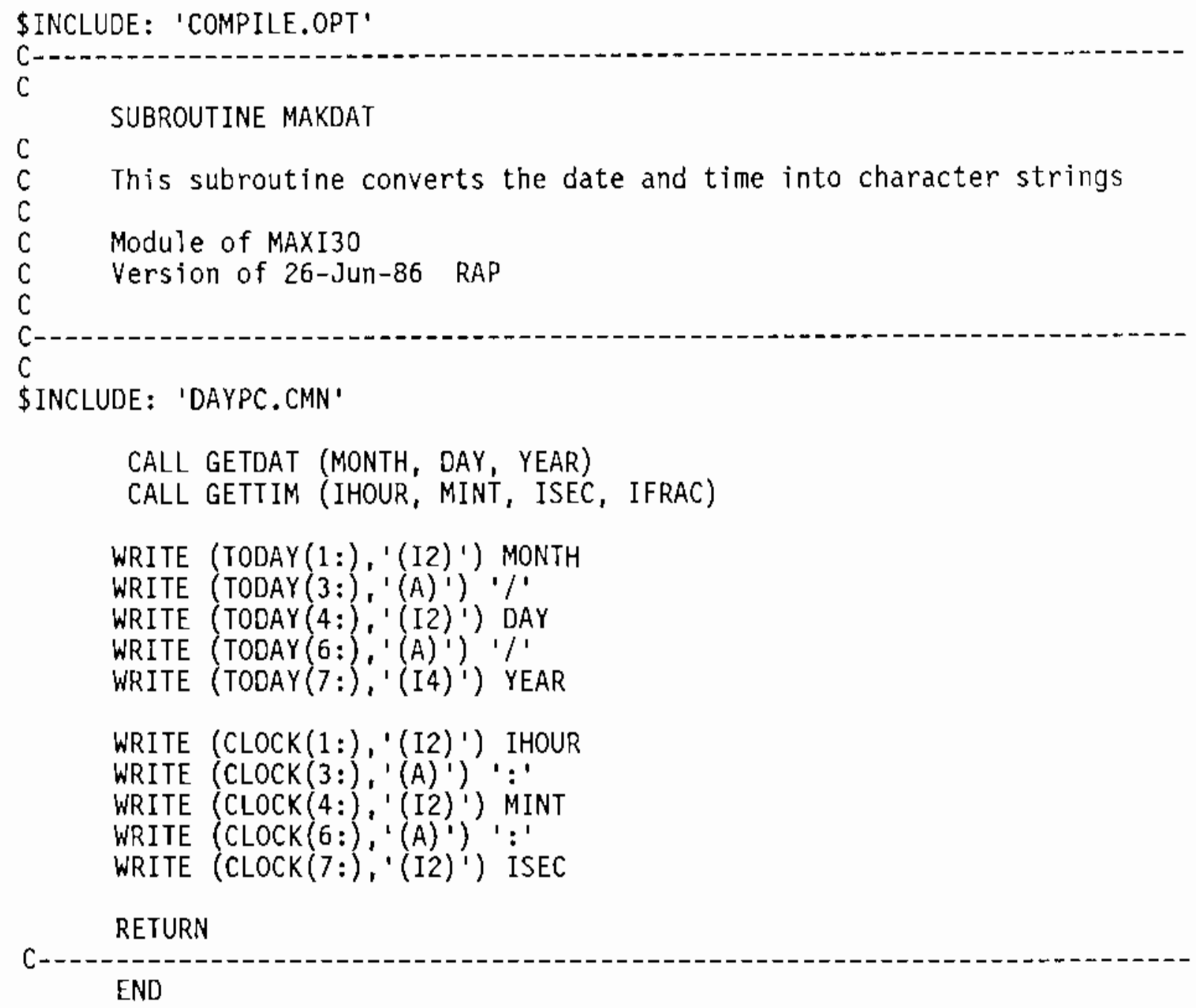


\$INCLUDE: 'COMPILE.OPT'

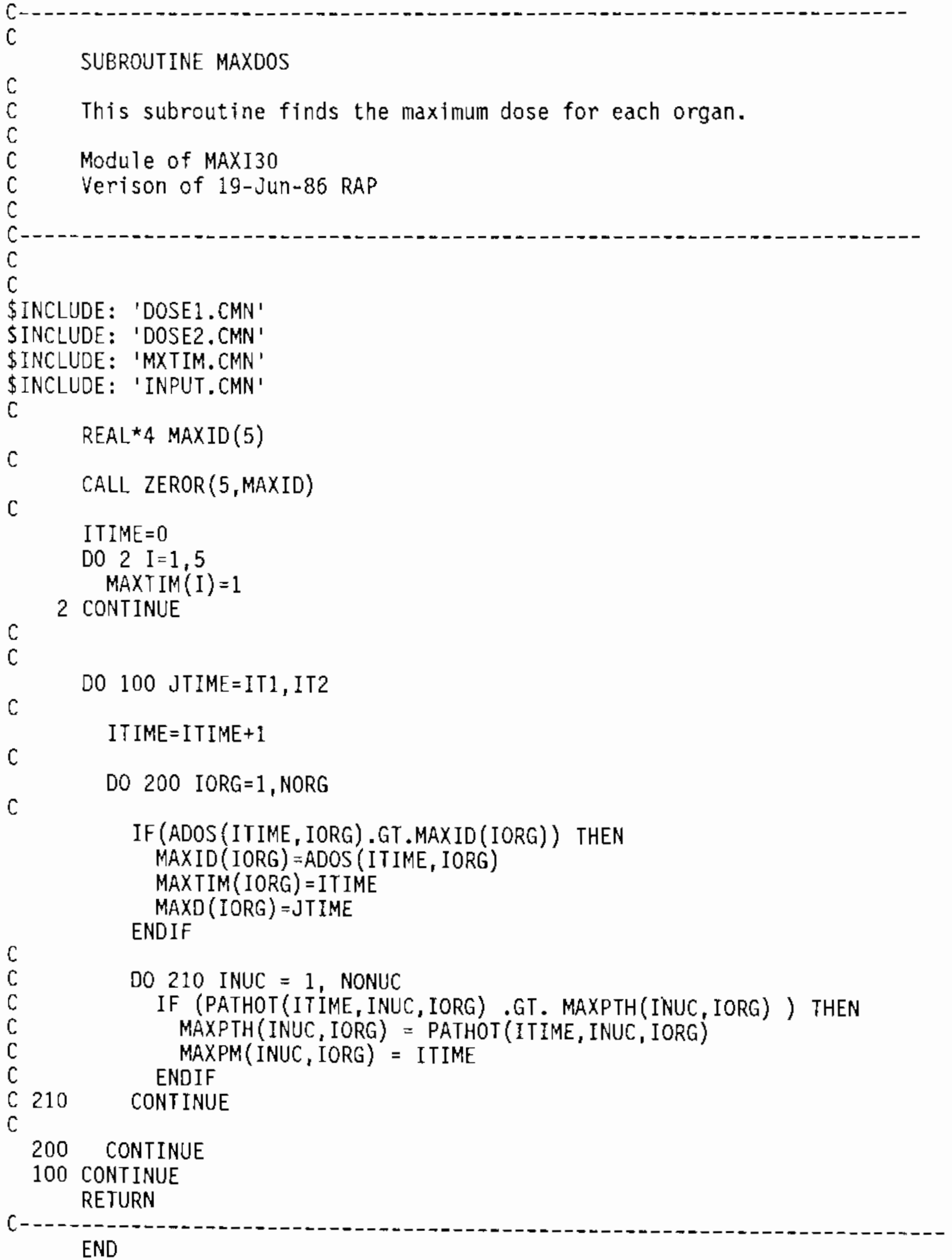




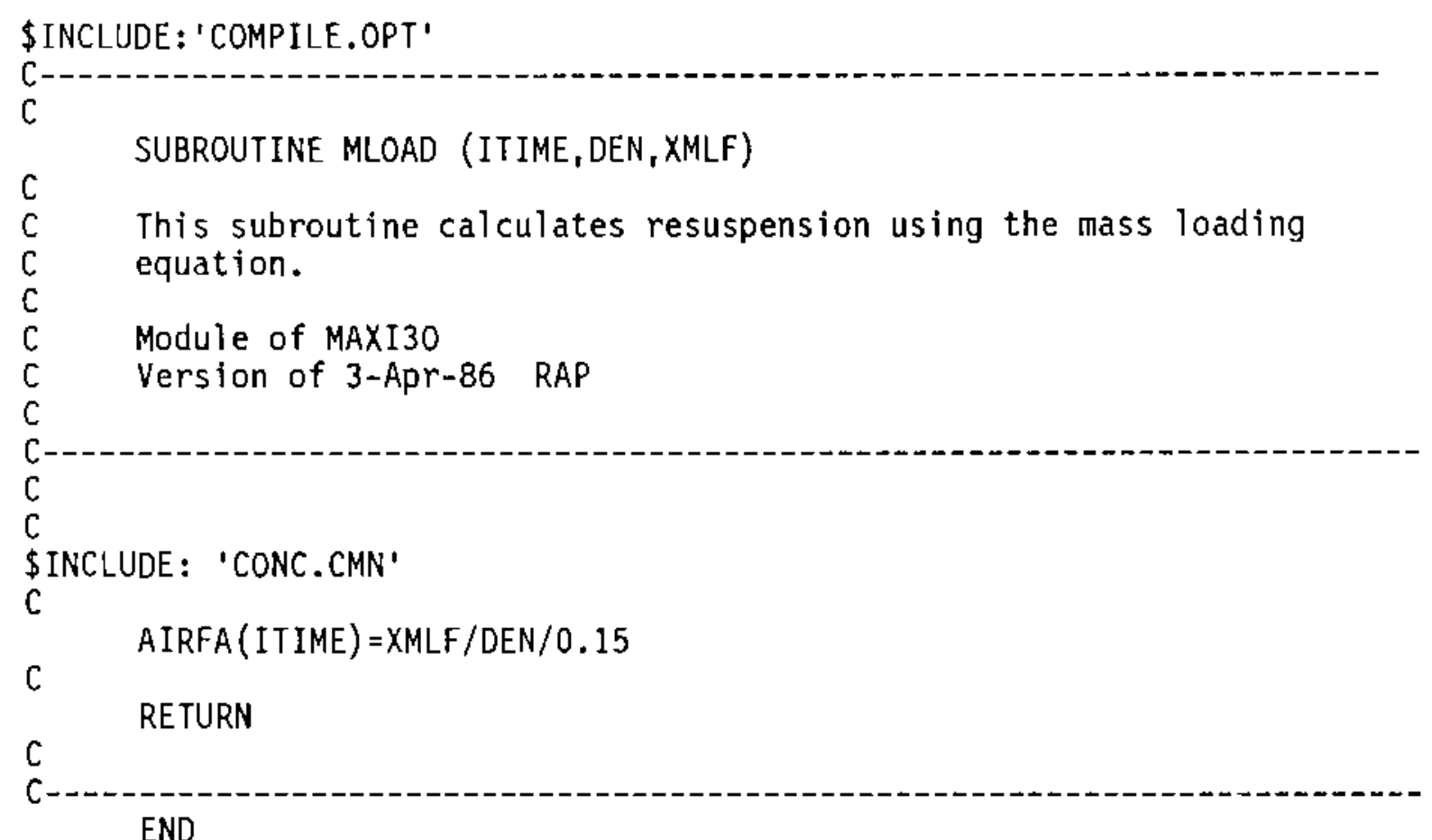




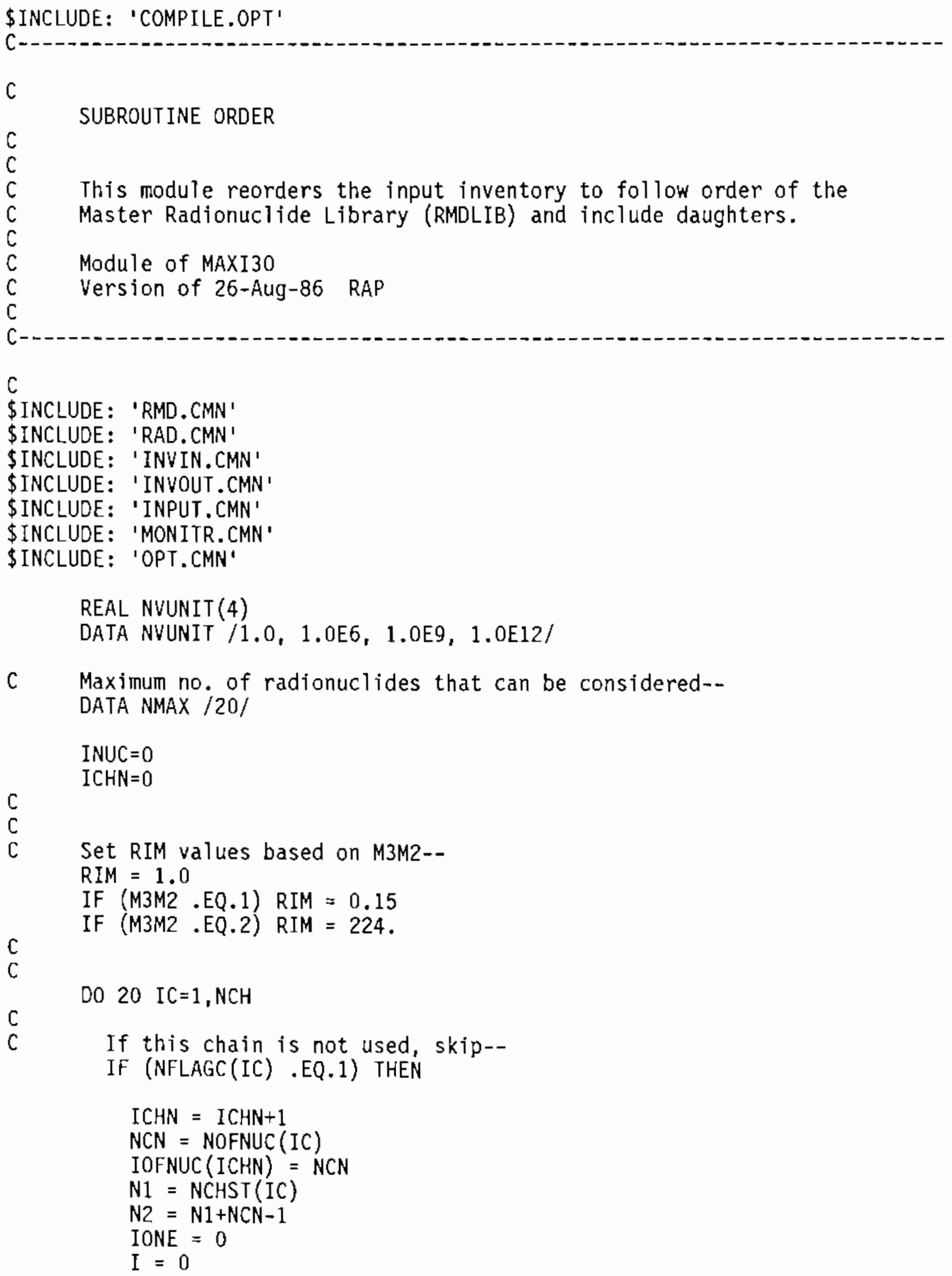




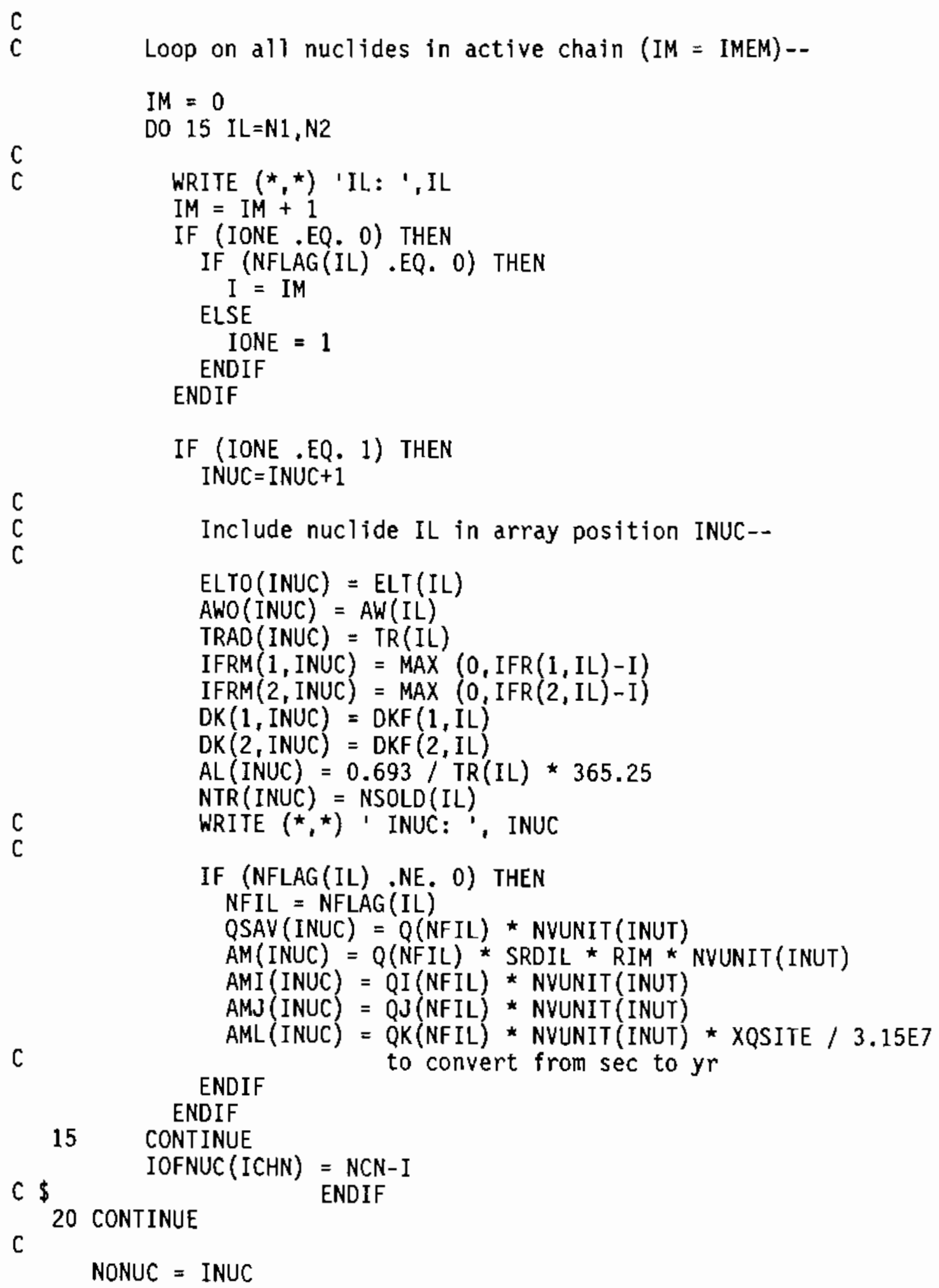




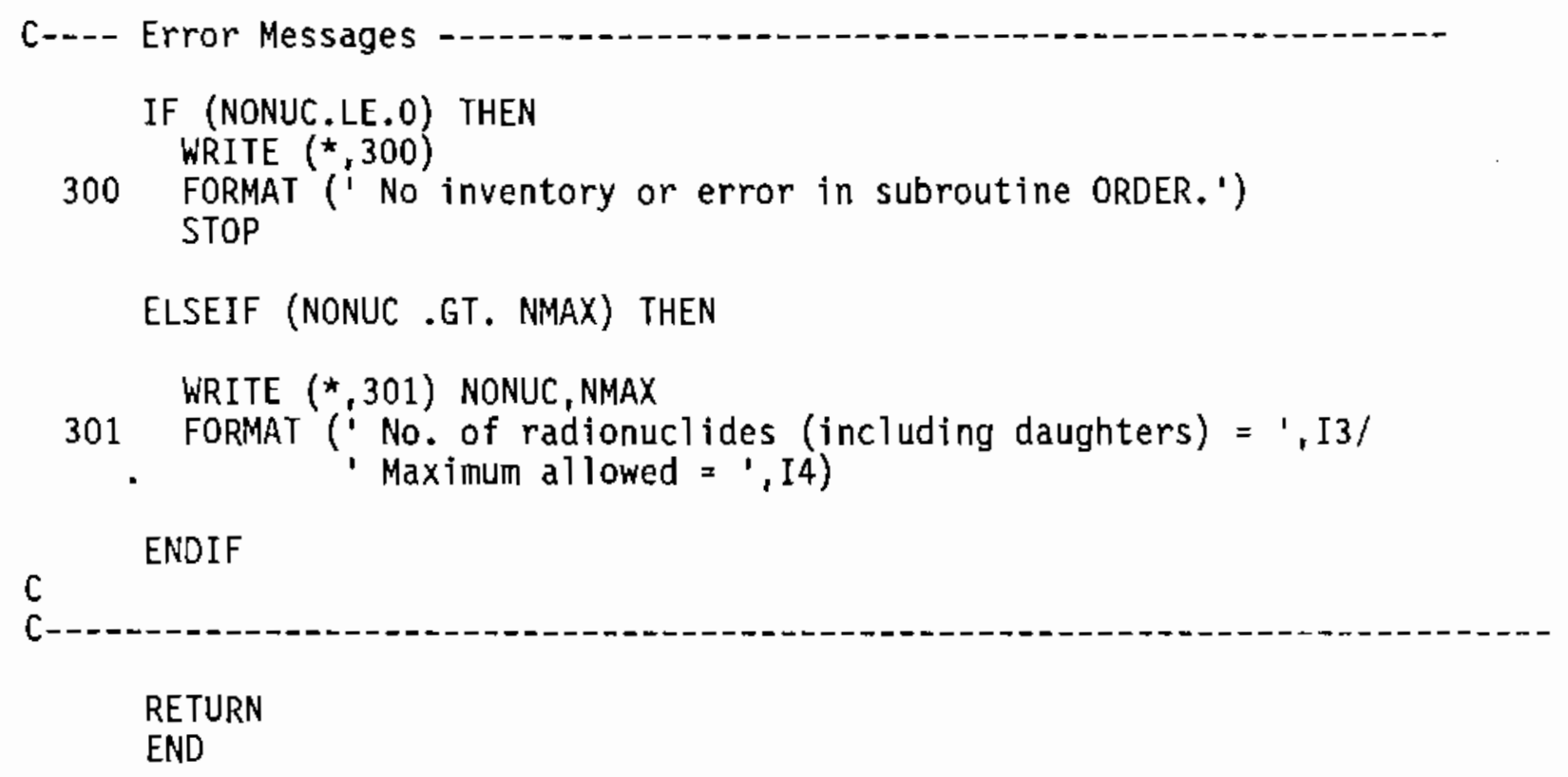




\section{\$INCLUDE: 'COMPILE.OPT'}

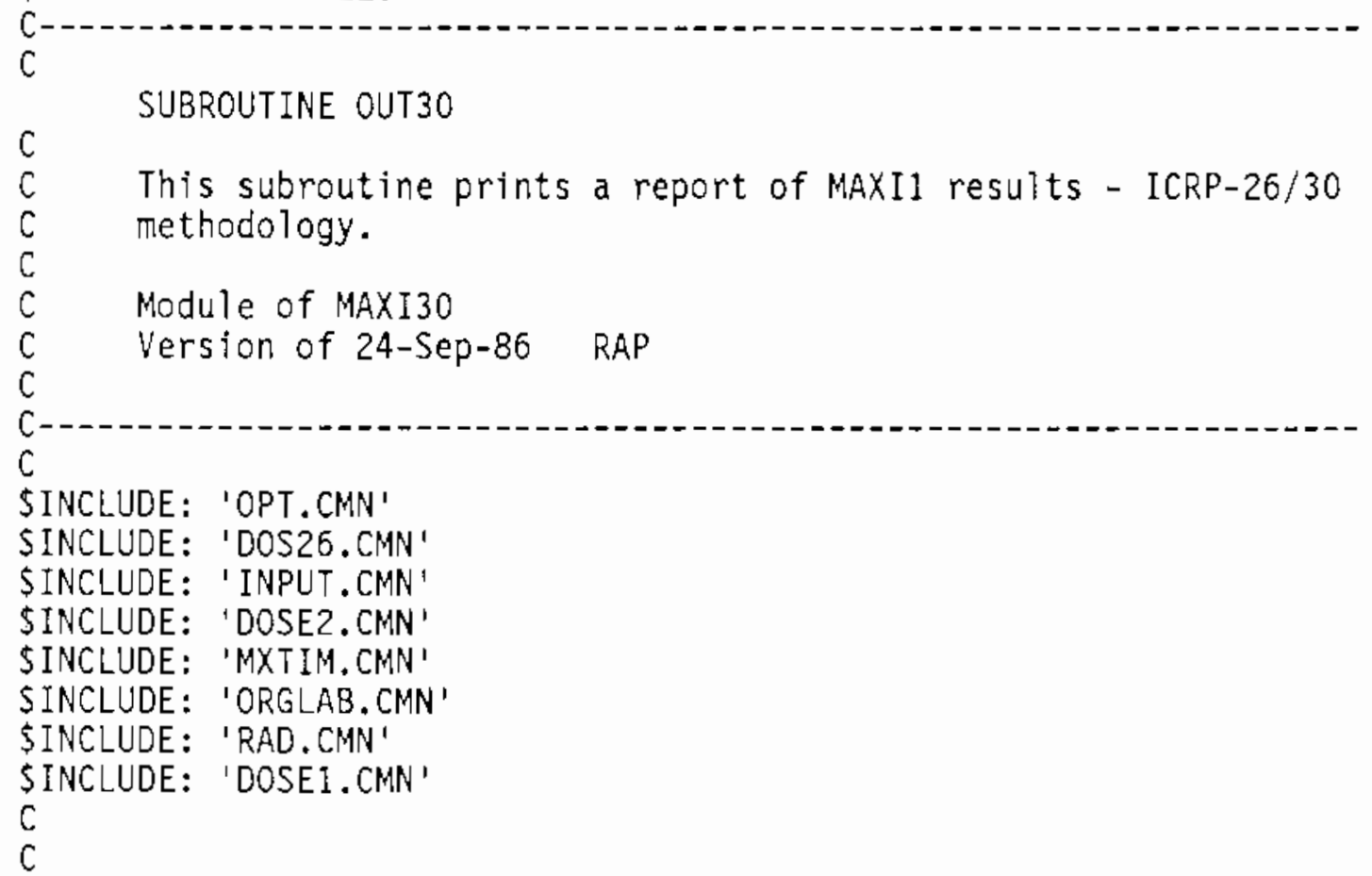

CHARACTER 50 DASH 1

DATA DASH1 /'-.......

C Print tities--

CALL HEADNG

$\mathrm{C}$

WRITE $(6,100)$ WBEDE, (ONAME (KORG(IORG)), MAXD(IORG),

ADOS (MAXTIM(IORG), IORG), IORG=1, NORG)

100 FORMAT (///' Annual Effective Dose Equivalent (AEDE): ${ }^{1}, T 50,1 P$, E9.1E2///

- $\quad$ Maximum Annual Dose (ICRP-2): ' //
(' To Organ: ', Al0,' at Year ',OP, I3, T50,

- $\quad$ IP,E9.1E2/)//)

IF (IOUT .GT. 1) THEN

CALL HEADNG

WRITE $(6,110)$ DASH1

DO 10 IO $=1,11$

WRITE $(6,111)$ ONAME2(IOP(IO)), CDEPR(IO), HWT(IO), WDE(IO)

10 CONTINUE

WRITE $(6,112)$ DASH1, EDE, AEXDOS (1), DASH1, WBEDE

110 FORMAT $(/ / /, T 10, '$ DOSES FROM 1 YEAR OF EXPOSURE',//

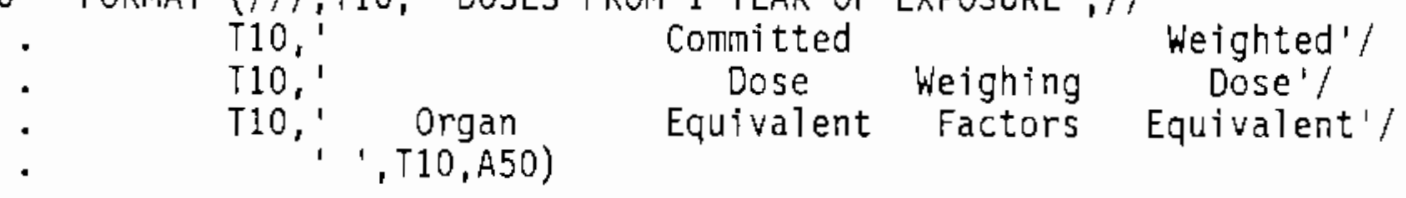




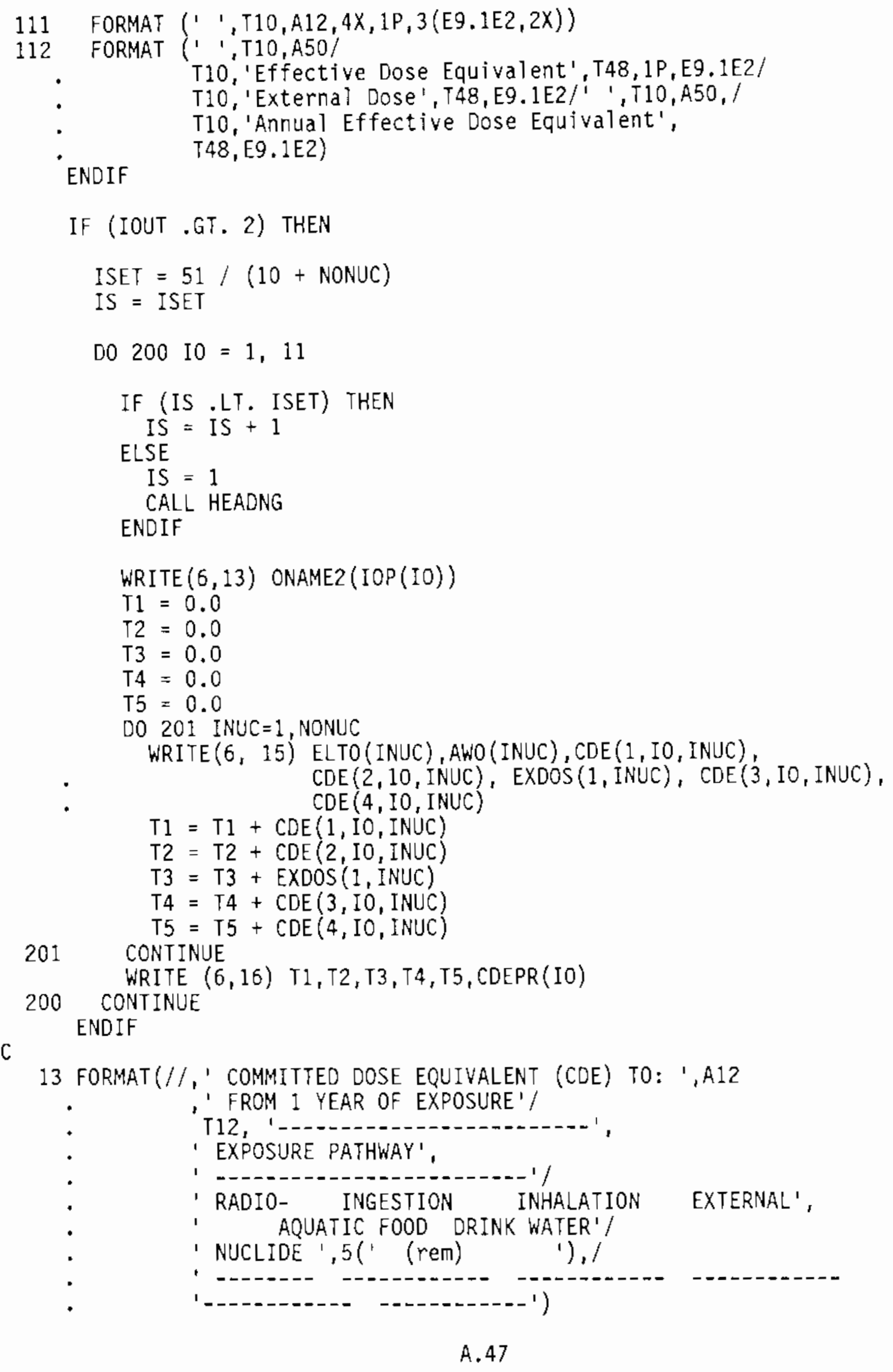




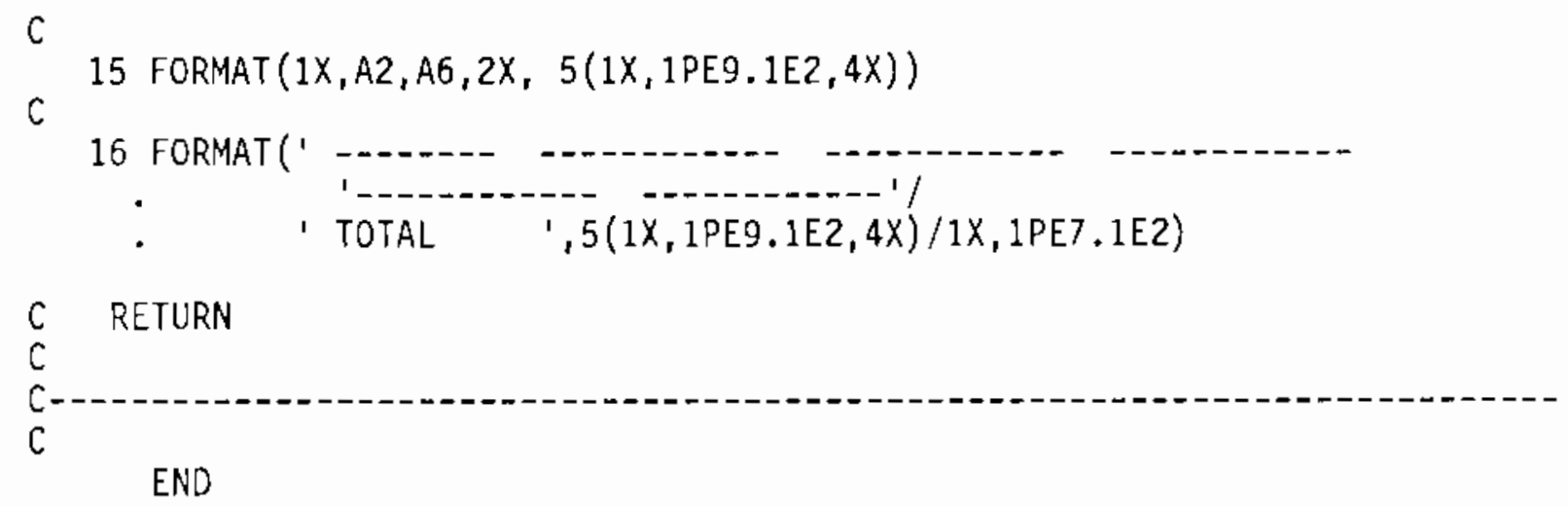


SINCLUDE: 'COMPILE.OPT'

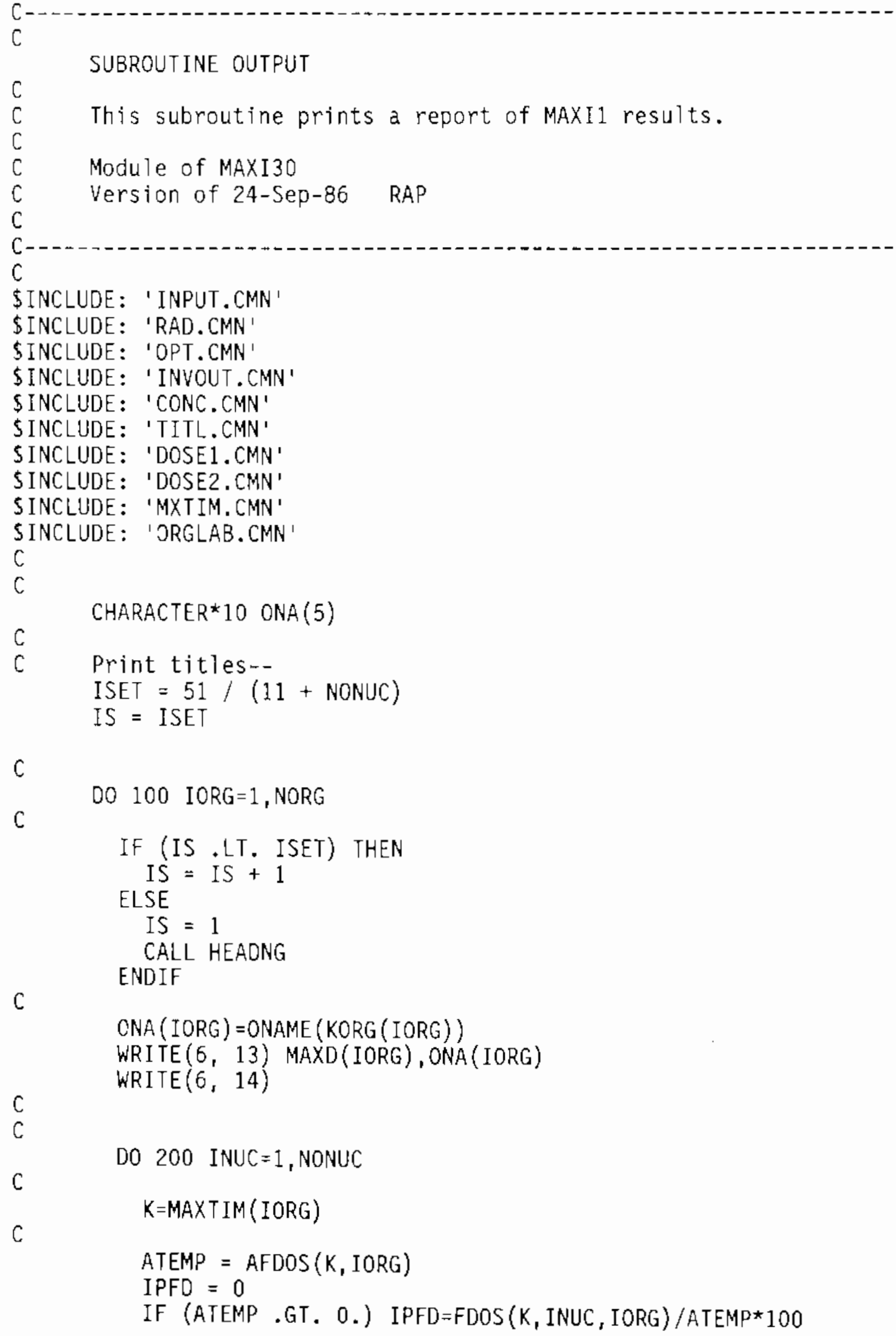




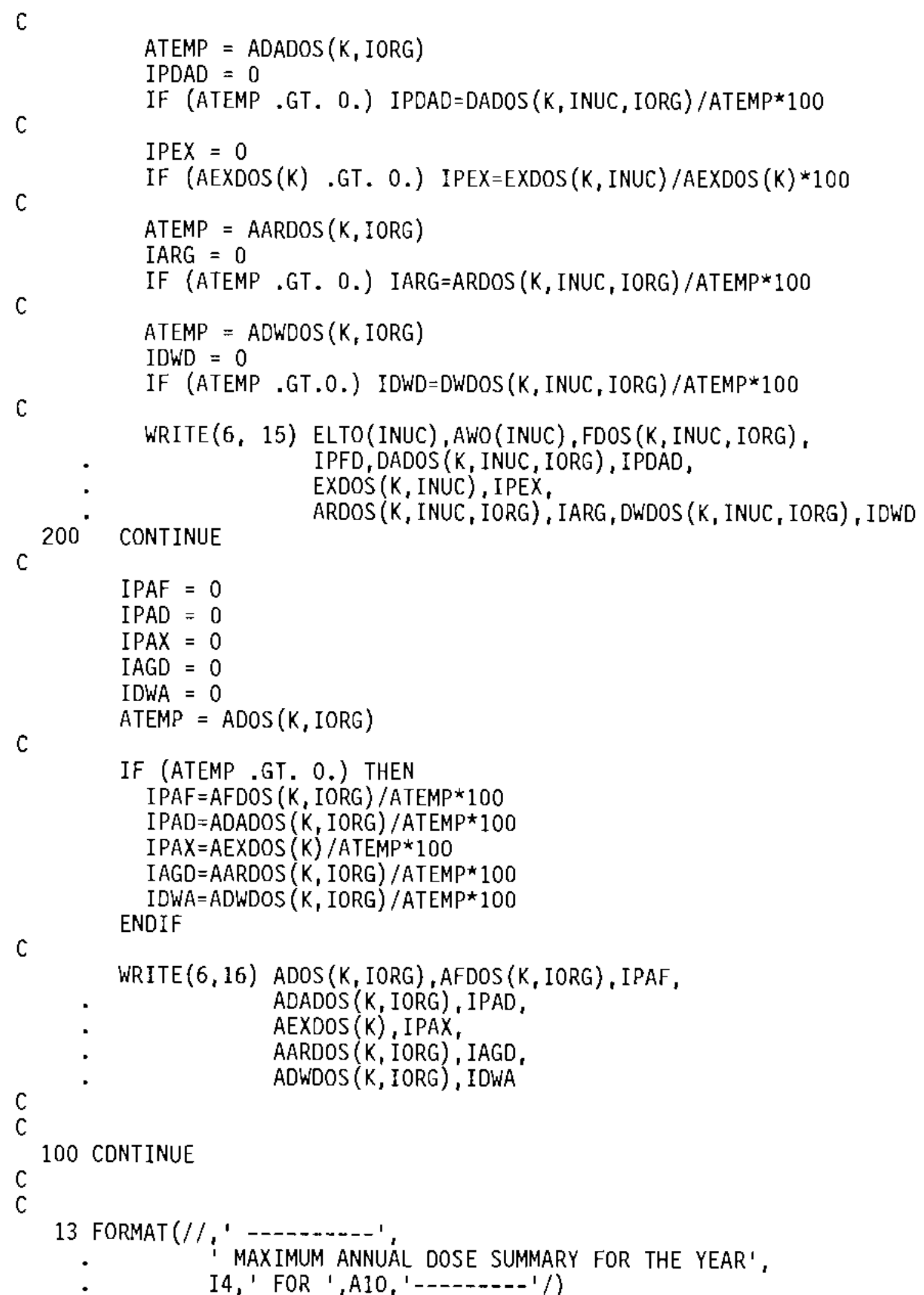

C

IF (ATEMP .GT. 0.) THEN

IPAF $=A F D O S(K$, IORG $) / A T E M P * 100$

IPAD $=A D A D O S(K, I O R G) / A T E M P \star 100$

IPAX $=A$ EXDOS $(K) / A T E M P * 100$

$\mathrm{IAGD}=\mathrm{AARDOS}(K, \mathrm{IORG}) / \mathrm{ATEMP}^{\star} 100$

IDWA $=A D W D O S(K, I O R G) / A T E M P \star 100$ ENDIF

C

WRITE $(6,16) \operatorname{ADOS}(K, \operatorname{IORG}), \operatorname{AFDOS}(K$, IORG), IPAF, $\operatorname{ADADOS}(K, I O R G), I P A D$, $\operatorname{AEXDOS}(K), I P A X$, $\operatorname{AARDOS}(K, I O R G)$, IAGD,

c ADWDOS (K, IORG), IDWA

C

100 CDNTINUE

C

13 FORMAT $\left(/ / \mathbf{i}^{\prime}\right.$ MAXIMUM ANNUAL DOSE SUMMARY FOR THE YEAR', 


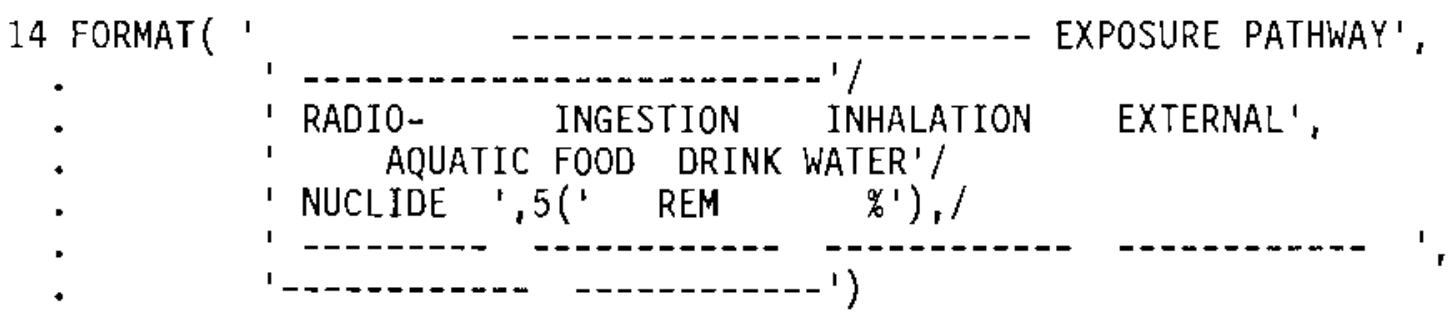

C

15 FORMAT $(1 X, A 2, A 6,5(1 X, 1 P E 9.1 E 2, I 4))$

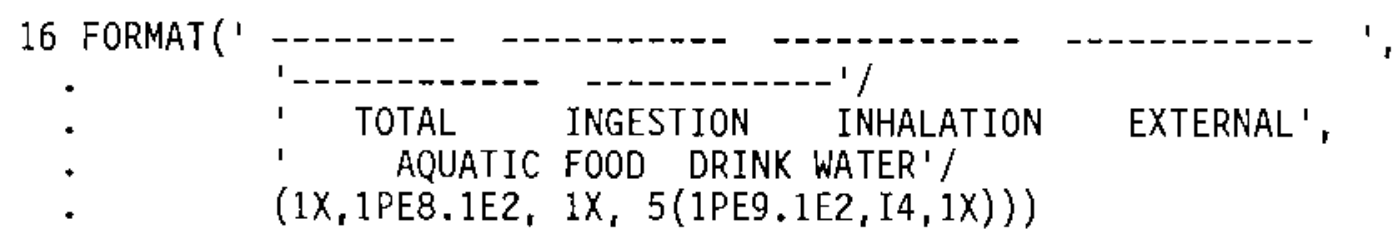

Print maximum dose by all pathways for wach year and organ report-IF (IOUT .EQ. 4) THEN

\section{CALL HEADNG}

WRITE $(6,33)($ ONA (IORG), IORG $=1, N O R G)$

DO 70 INUC $=1$, NONUC WRITE $(6,34)$ ELTO(INUC), AWO(INUC), (MAXPM (INUC, I), PATHOT(MAXPM(INUC, I), INUC, I),

$70^{\circ}$ Continue $I=1, N O R G)$

ENDIF

33 FORMAT (' 'OVER ALL PATHWAYS --.---- '/

'Radio- ',5(', 'Al1),/

1 Nuclide ',5('Year (rem) ')/

'Nuclide is ('year (ren) ')/

C

$c$

C

C

C

$\mathrm{C}$
$\mathrm{C}$
$\mathrm{C}$

Test on output class and continue--

IF (IOUT.EQ.0 .OR. IEDE. EQ. 1) GO TO 999

Print tabulation of annual doses by organ (IOUT=1)

CALL HEADNG

WRITE $(6,20)$ IT2

C

WRITE $(6,21) \quad($ ONA (IORG), IORG $=1$, NORG) 


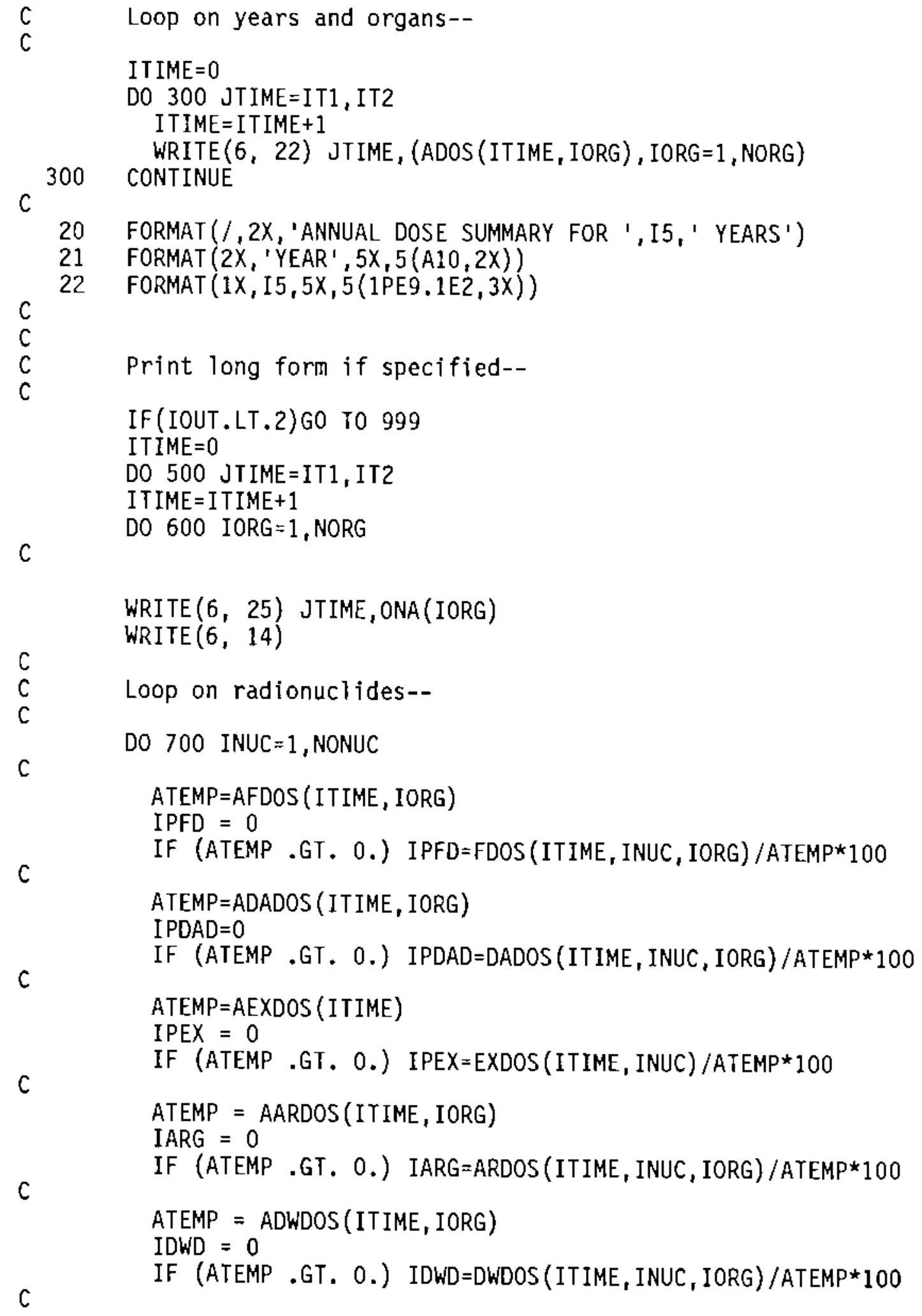




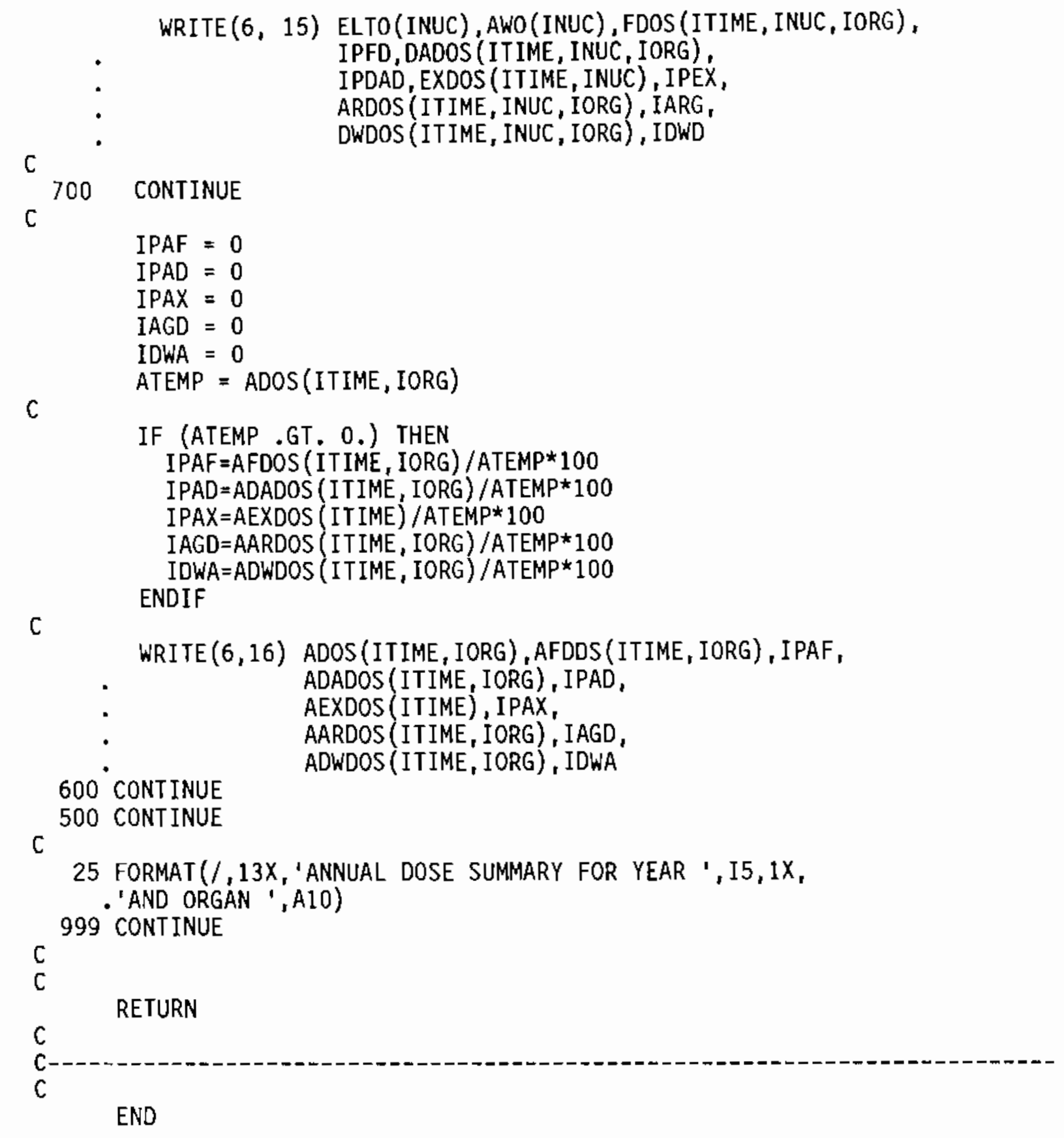




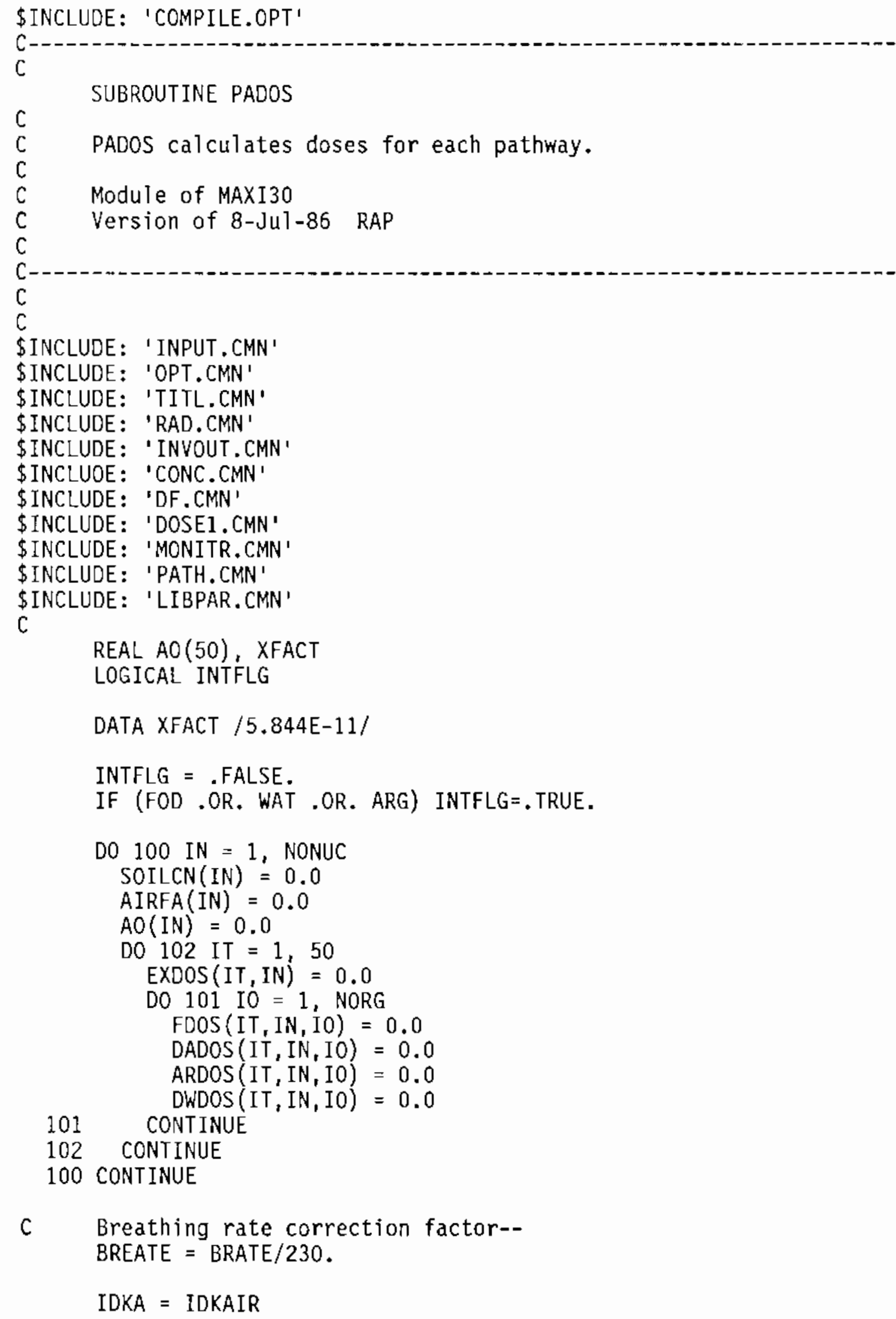




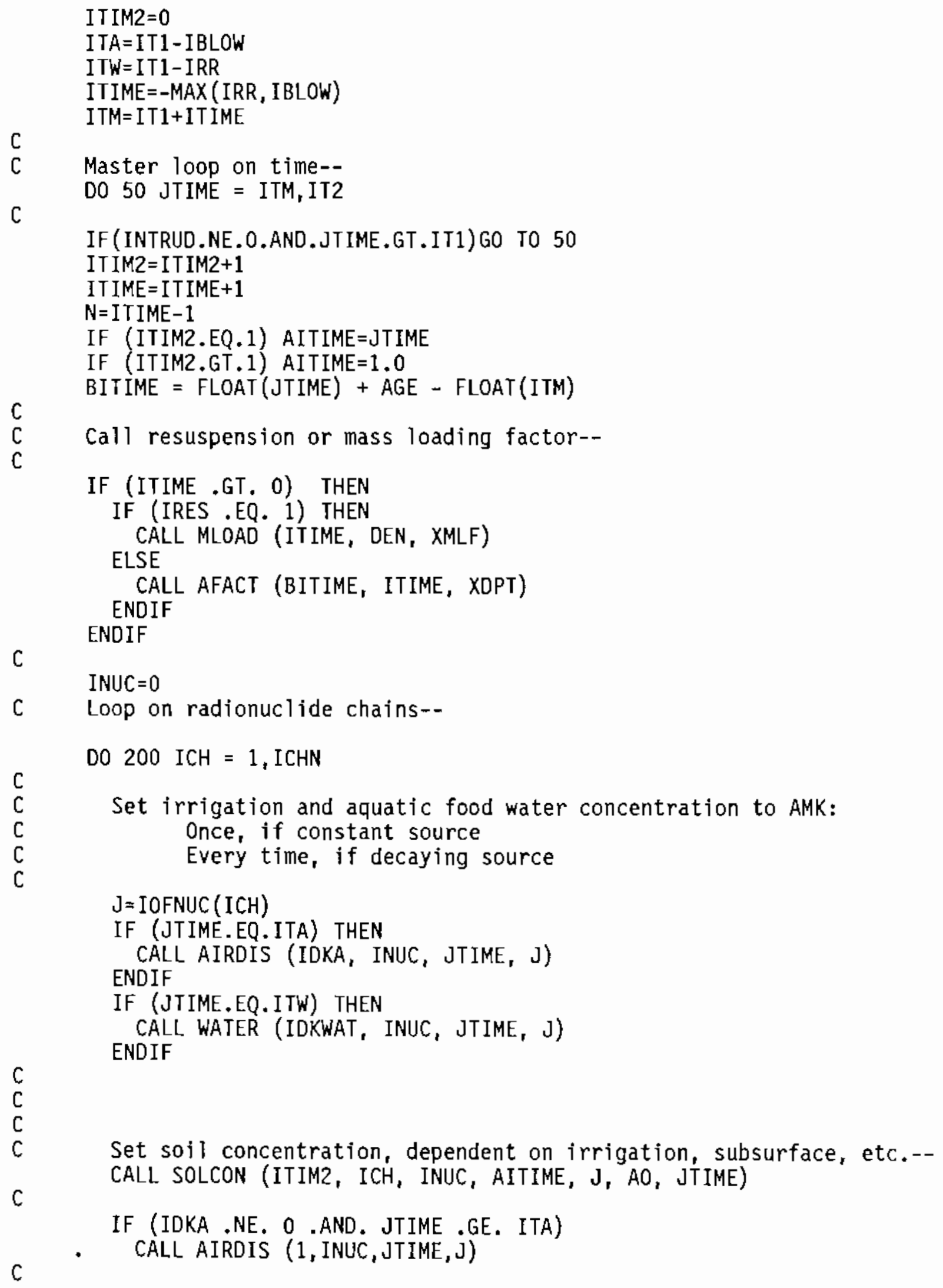




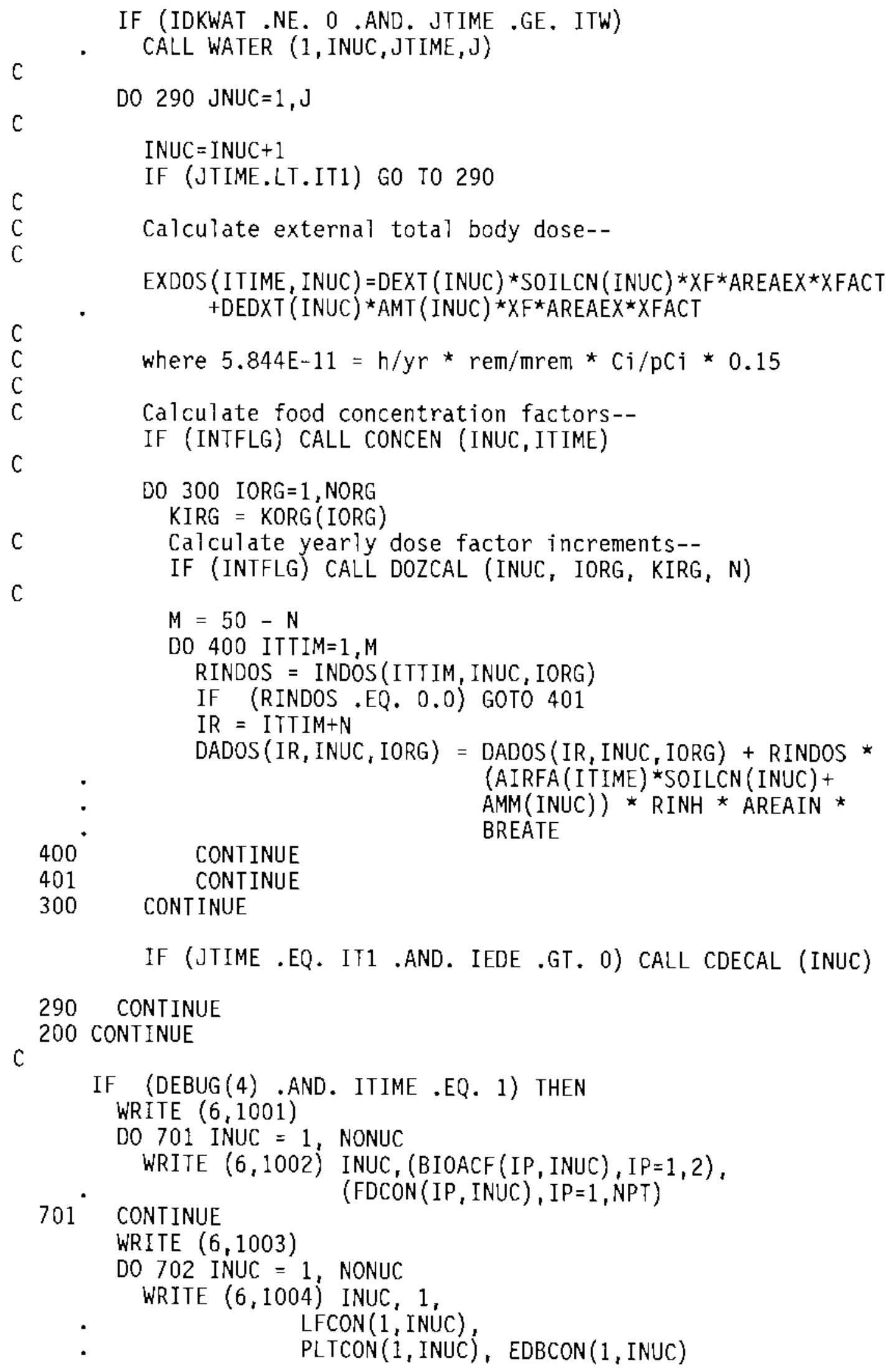




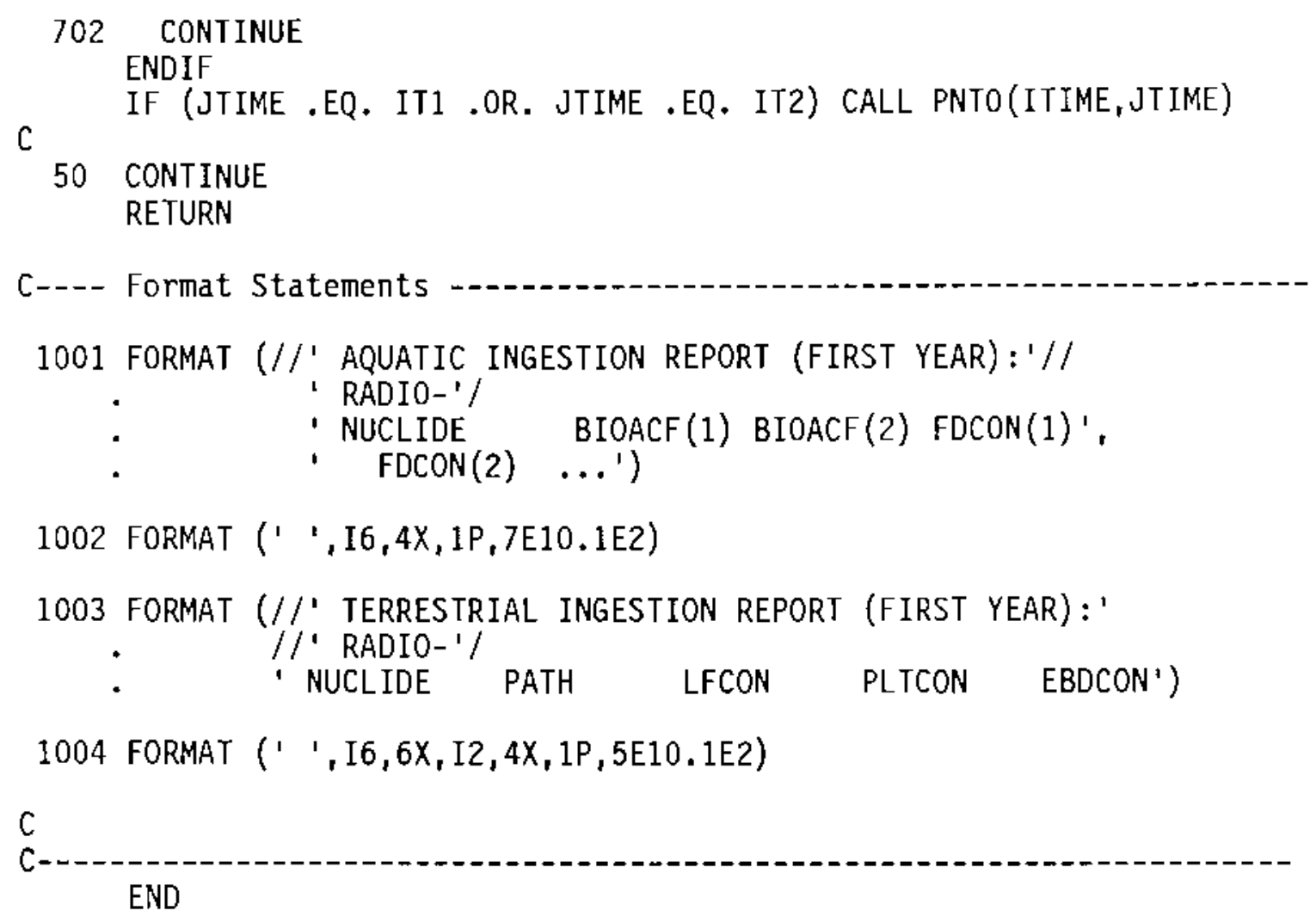




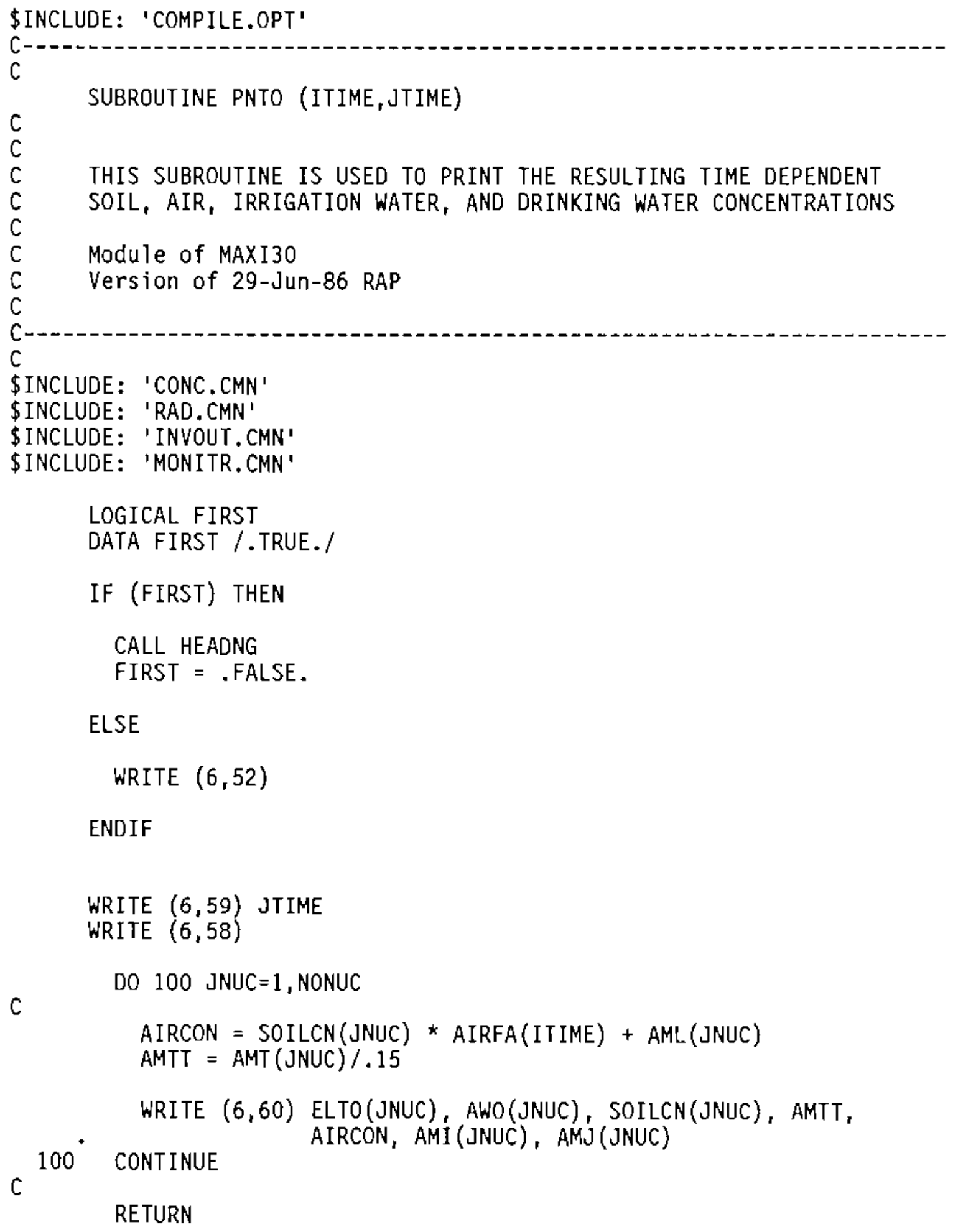




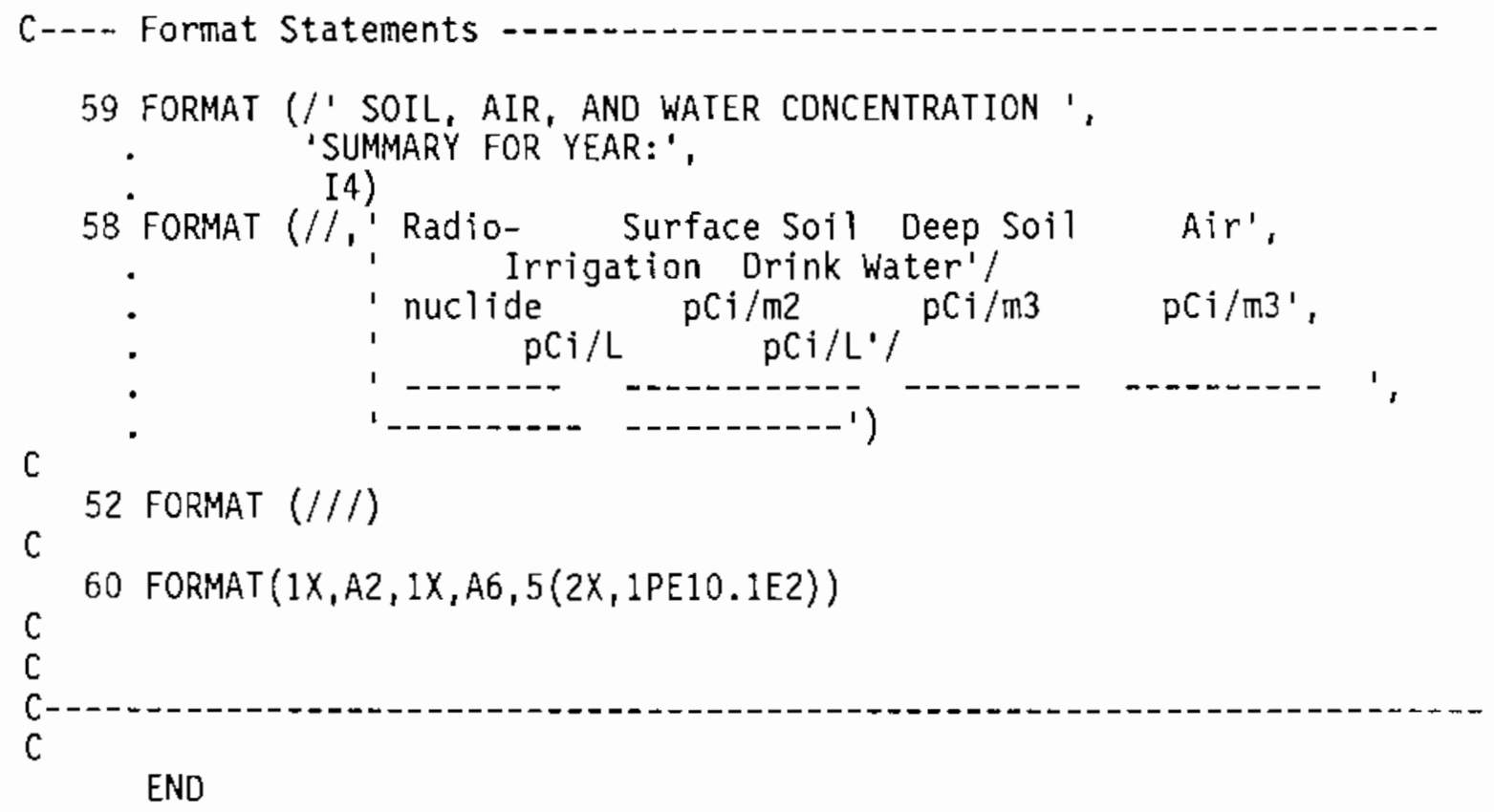




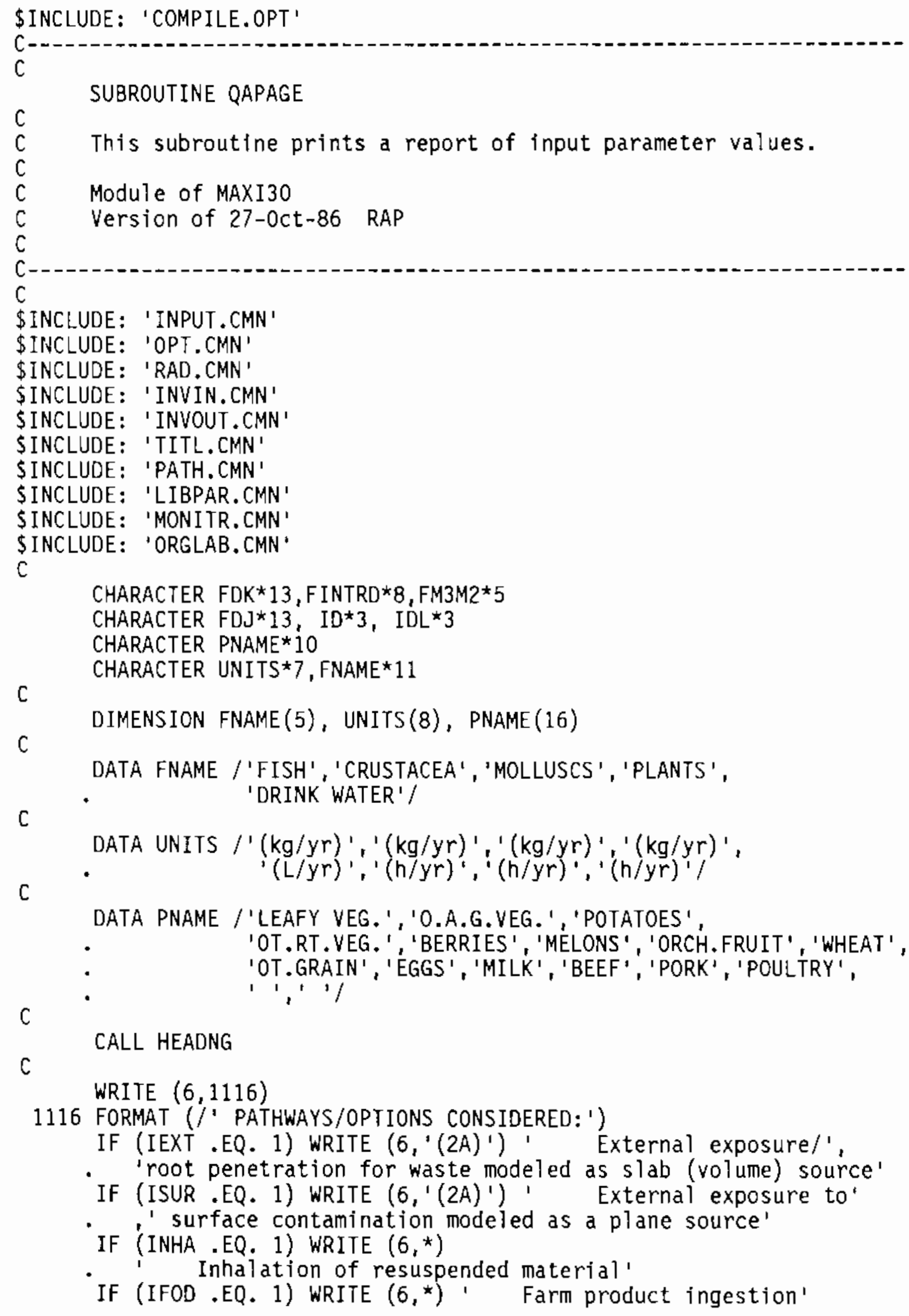




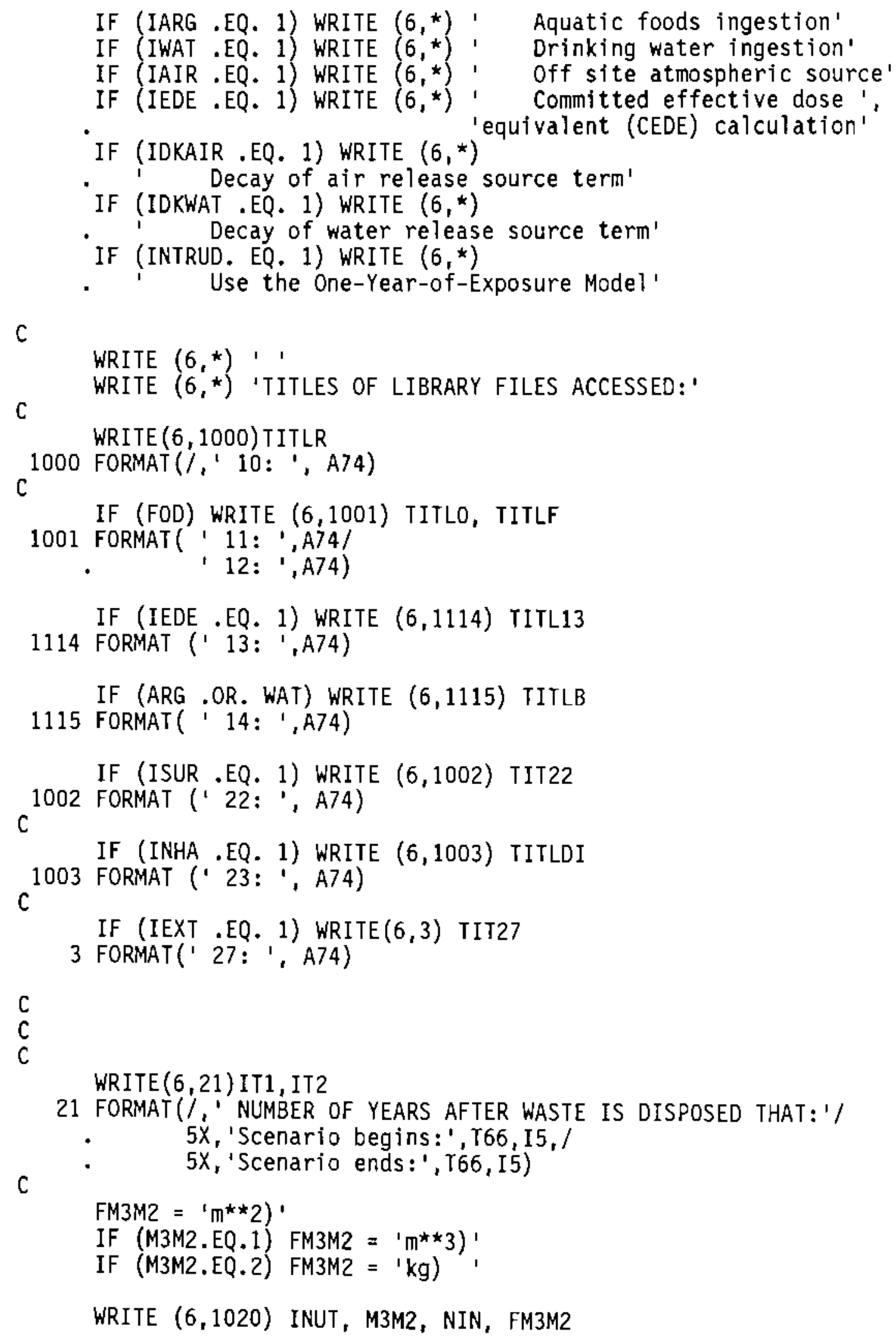


1020 FORMAT $(/, 1$ INVENTORY: ' /

- 5X,'Release Term Input units: (1-pCi 2-uCi 3-mCi 4-Ci)',

- T66, I4/

- 5X,'Soil source units: $\left(0-m^{\star \star 2} \quad 1-m^{\star \star 3} \quad 2-\mathrm{kg}\right)^{\prime}, T 66, \mathrm{I} 4 /$

- $5 x$, 'Number of radionuclides in inventory: ', T66, I4//

- 5X,'Release Irrigation Drinking ',

- $\quad$ 'Atmospheric'/

- 5X,'Terms Soil Source /Aquatic Water Release'/

- 15X,'(per ',A5,' (per L) (per L) (per m3) '1/

DO 13 I $=1$, NONUC WRITE $(6,1021) \operatorname{ELTO}(I), \operatorname{AWO}(I), \operatorname{QSAV}(I), \operatorname{AMI}(I), \operatorname{AMJ}(I), \operatorname{AML}(I)$

13 CONTINUE

1021 FORMAT (5X, A2, A6, 4 (2X,1PE11.1E2))

WRITE $(6,1022)$ SRDIL, DILF, FRSIZ

1022 FORMAT (/' INVENTORY MODIFICATION FACTORS: (multipliers) '/

- 5X, 'Surface inventory dijution factor: ', T66,1PG9.1E2/

- 5X,'Irrigation/aquatic inventory modification factor:',

- T66,G9.1E2/

- 5X,'Size of site (fractional ha):',T66,E9.1E2)

IF (IFOD .EQ. 1) WRITE $(6,1023)$ RPF2

1023 FORMAT (5X, 'Fraction of total diet grown on site: ', T66,G9.1E2)

CALL HEADNG

IF (ISUR.EQ.1.OR.IEXT.EQ.1.OR. INHA.EQ.1) THEN WRITE $(6, *)$, '

WRITE $(6, *)$ ' EXTERNAL/INHALATION EXPOSURE: '

IF (ISUR .EQ. 1 .OR. IEXT .EQ.1) WRITE $(6,1030)$ HREXT

IF (INHA .EQ. 1) WRITE $(6,1031)$ HRINH

WRITE $(6,1032)$ BRATE

ENDIF

1030 FORMAT $\left(5 X_{1}\right.$ 'Hours of external exposure to contamination $(h / y r):$, - T66, 1PG9.1E2)

1031 FORMAT (5X, 'Hours of inhalation of airborne contamination', ' $\left.(\mathrm{h} / \mathrm{yr}):{ }^{\prime}, \mathrm{T} 66,1 \mathrm{PG} 9.1 \mathrm{E} 2\right)$

1032 FORMAT (5X,' 'Breathing rate $\left.\left(\mathrm{cm}^{\star \star} 3 / \mathrm{sec}\right):{ }^{\prime}, T 66,1 \mathrm{PG} 9.1 \mathrm{E} 2\right)$

WRITE $(6, *)$ ' '

WRITE $\left(6,{ }^{*}\right)$ ' RESUSPENSION PARAMETERS:'

IF (IRES .EQ. 0) THEN

WRITE $(6,1041)$ AGE, XDPTIN

ELSE

WRITE $(6,1042)$ DEN, XMLF

ENDIF 
1041 FORMAT (5X,'Model used: ', T66, 'Anspaugh'/

$5 X$, 'Average age of contamination on ground at Time Zero',

- $\quad$ 5X,'Top soil availabie for resuspension $(\mathrm{cm}):$ ',

- T66,G9.1E2)

1042 FORMAT (5X, 'Model used: ', T66, 'Mass Loading'/

$5 X$, 'Soil density $\left(\mathrm{g} / \mathrm{m}^{\star \star} 3\right):{ }^{\prime}, \mathrm{T} 66,1 \mathrm{PE}, 1 \mathrm{E} 2 /$

$5 X$, 'Mass loading factor $\left(g / m^{\star \star} 3\right):$ ', T66, E9.1E2)

IF (IAIR .EQ. 1) WRITE $(6,1050)$ IBLOW， XQSITE

1050 FORMAT (/' SPECIAL PURPOSE PARAMETERS: '/

- 5X, 'No. of years contamination blew onto the site prior to $1 /$

- $7 X_{1}$ ' the beginning of the scenario: ', T66, I9,/

- $5 X,{ }^{\prime} \mathrm{Chi} / \mathrm{q}$ value at the site:', T66,E9.1E2)

IF (IFOD .EQ. 1) WRITE $(6,1060)$ RF1, RF2, RPF1, IM0, RIRR, IRR, NFT

1060 FORMAT (/' AGRICULTURAL PARAMETERS: '/

- $5 X$, 'Fraction of roots in upper soil: ',T66,1PG9.3E2/

- 5X,'Fraction of roots in deeply buried waste: ',T66,G9.3E2/

- 5X,'Ratio of ext. contamination in surface/subsurface soil:',

- T66,G9.3E2/

- $5 X$, 'Months per year irrigated:', T66,0P, 19,1P/

- $5 X_{1}$ 'Irrigation rate $\left(\mathrm{L} / \mathrm{m}^{\star *} 2 / \mathrm{mo}\right):{ }^{\prime}, \mathrm{T} 66, \mathrm{G} 9.1 \mathrm{E} 2 /$

- $5 X$,'Years of irrigation $w /$ contaminated water prior to the $1 /$

- $7 X$, 'beginning of the dose calculation period: ', T66, I9/

- $\quad 5 X,{ }^{\prime}$ Number of food types:', T66, OP, I9)

IF (FOD) THEN

WRITE $(6,1061)$

1061 FORMAT (/' FOOD

- 'CONSUMP- TRANS-'/

- 1 TYPE

- 'TION

- I INDEX

- ' $(\mathrm{kg} / \mathrm{yr})$

LOCATION'/ FOOD TYPE

FACTOR'/
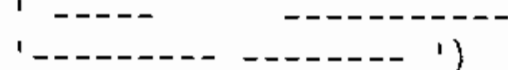

\section{GROWING}

PERIOD YIELD HOLDUP ',

(days) $\left(\mathrm{kg} / \mathrm{m}^{\star \star} 3\right)$ (days) ',

DO $10 \mathrm{I}=1, \mathrm{NFT}$

WRITE $(6,1070) \operatorname{KFDTYP}(I), \operatorname{PNAME}(\operatorname{KFDTYP}(\mathrm{I})), \operatorname{GRWP}(\mathrm{I})$, YELD(I), $\operatorname{HLDUP}(\mathrm{I}), \operatorname{CON}(\mathrm{I}), \operatorname{TRANS}(\mathrm{I})$

10 CONTINUE

1070 FORMAT (1X, I5, 4X,A13,F7.0,2X,F9.2,2X,F6.0,2X,F9.1,2X,F8.2)

CALL HEADNG

ENDIF 


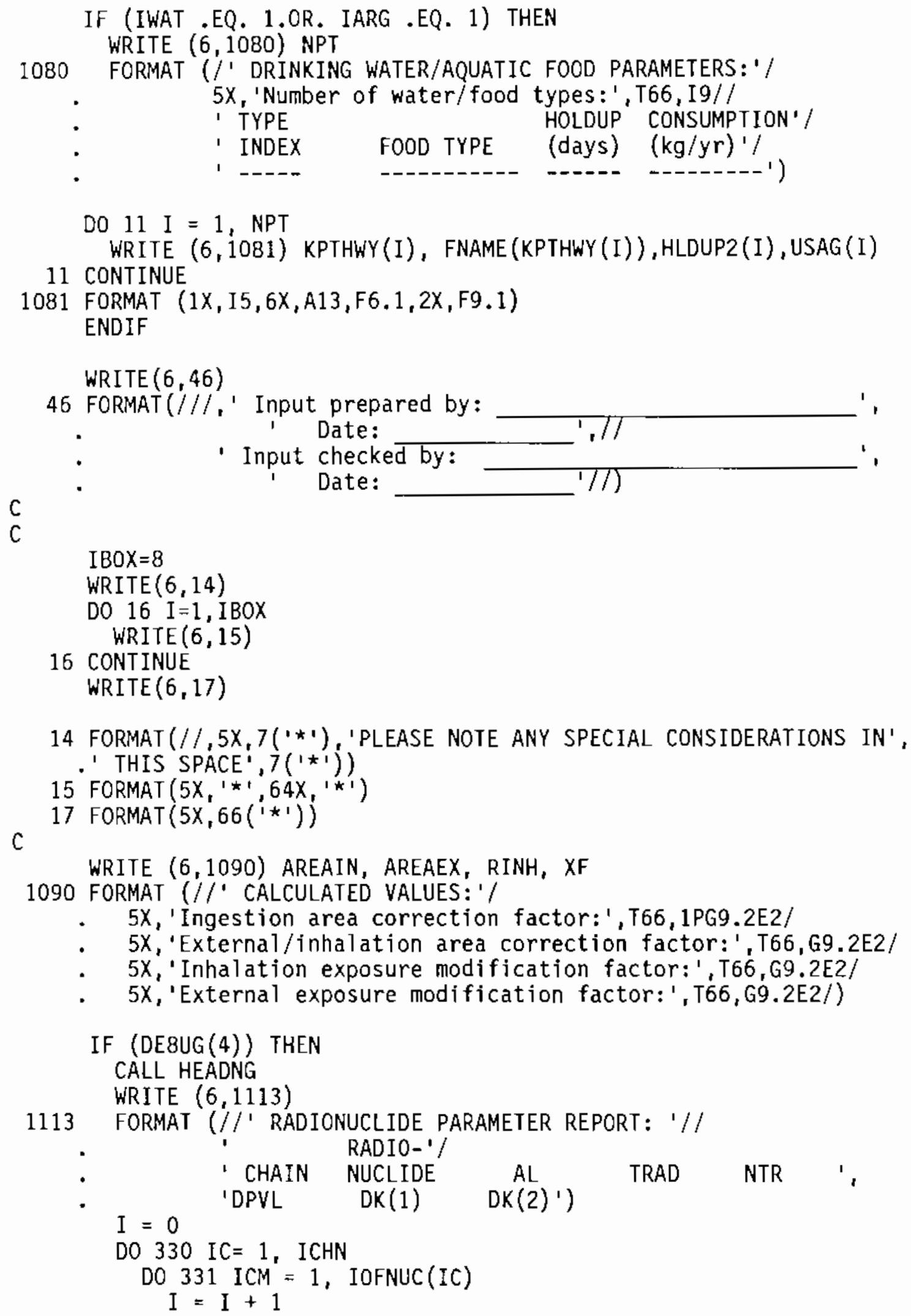

14 FORMAT $(/ /, 5 X, 7(' * ')$, 'PLEASE NOTE ANY SPECIAL CONSIDERATIONS IN', ' THIS SPACE',7(1*i)) 


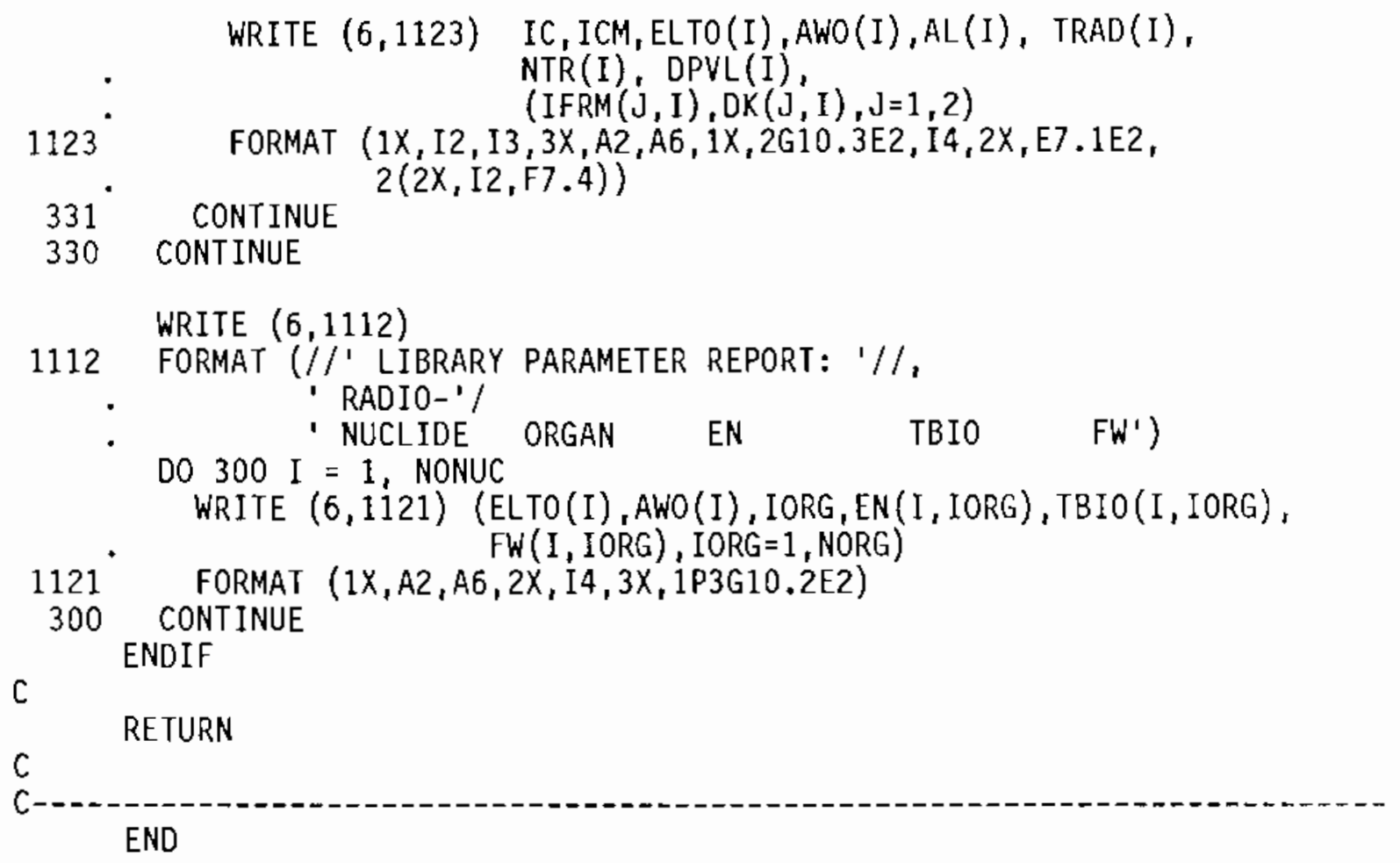




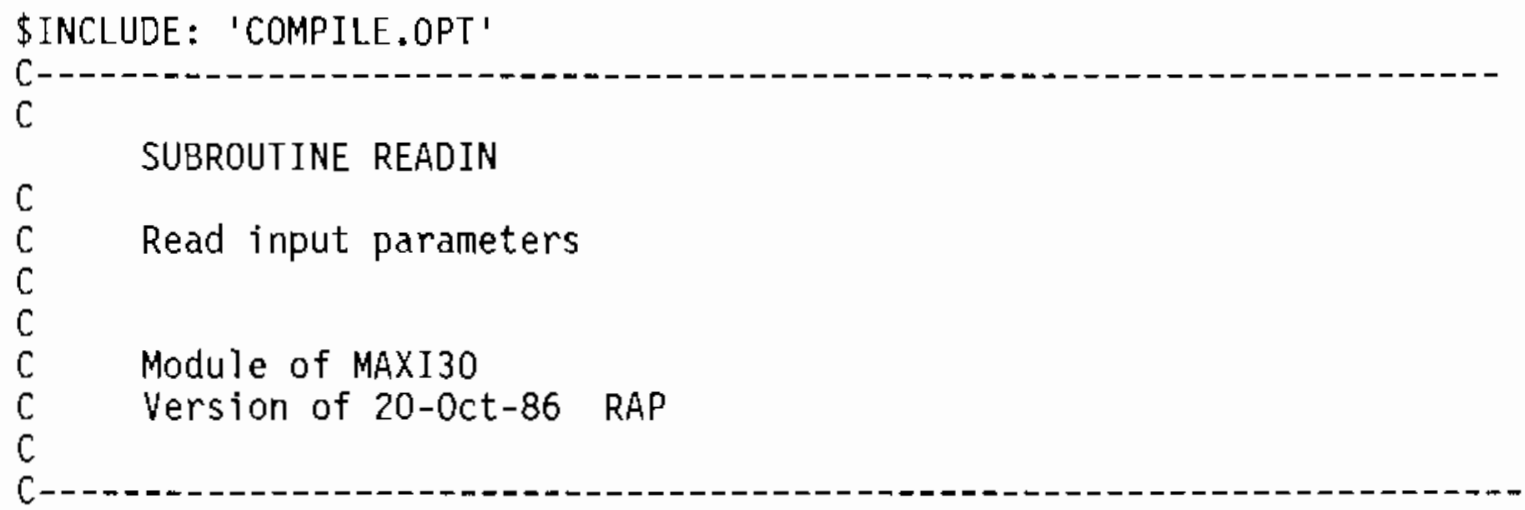

\section{\$INCLUDE: 'INPUT.CMN' \\ \$INCLUDE: 'OPT.CMN' \\ \$INCLUDE: 'PATH.CMN' \\ \$INCLUDE: 'MONITR.CMN' \\ \$INCLUDE: 'INVIN.CMN' \\ \$INCLUDE: 'RAD.CMN' \\ \$INCLUDE: 'TITL.CMN'}

CHARACTER ${ }^{\star} 10$ DUM

READ $(5,1)$ DUM

READ (5, ' (A80)') TITLE1

READ $(5,11)$ DUM, DUM

READ $(5, *)$ IEXT

$\operatorname{READ}(5,1)$ DUM

$\operatorname{READ}(5, *)$ ISUR

READ $(5,1)$ DUM

$\operatorname{READ}(5, *)$ INHA

$\operatorname{READ}(5, \star)$ IFOD

$\operatorname{READ}(5, *)$ IARG

$\operatorname{READ}(5, *)$ IWAT

$\operatorname{READ}(5, *)$ IAIR

$\operatorname{READ}(5, *)$ IEDE

READ $(5, *)$ IDKAIR

READ $\left(5,{ }^{\star}\right)$ IDKWAT

READ $(5, *)$ INTRUD

READ $(5,11)$ DUM, DUM

$\operatorname{READ}(5, *)$ IOUT

$\operatorname{READ}(5, *)$ DEBUG $(4)$

$\operatorname{READ}(5,11)$ DUM, DUM

$\operatorname{READ}(5$, *) IT1

$\operatorname{READ}\left(5,{ }^{*}\right)$ IT2

$\operatorname{READ}(5,11)$ DUM, DUM

$\operatorname{READ}(5, *)$ INUT

$\operatorname{READ}(5, *)$ M3M2

$\operatorname{READ}(5, *)$ NIN

READ $(5,11111)$ DUM, DUM, DUM, DUM, DUM

DO 100 IN $=1$, NIN

READ $(5,2)$ ELTI (IN), AWI (IN), Q(IN), QI (IN), QJ (IN), QK(IN) 


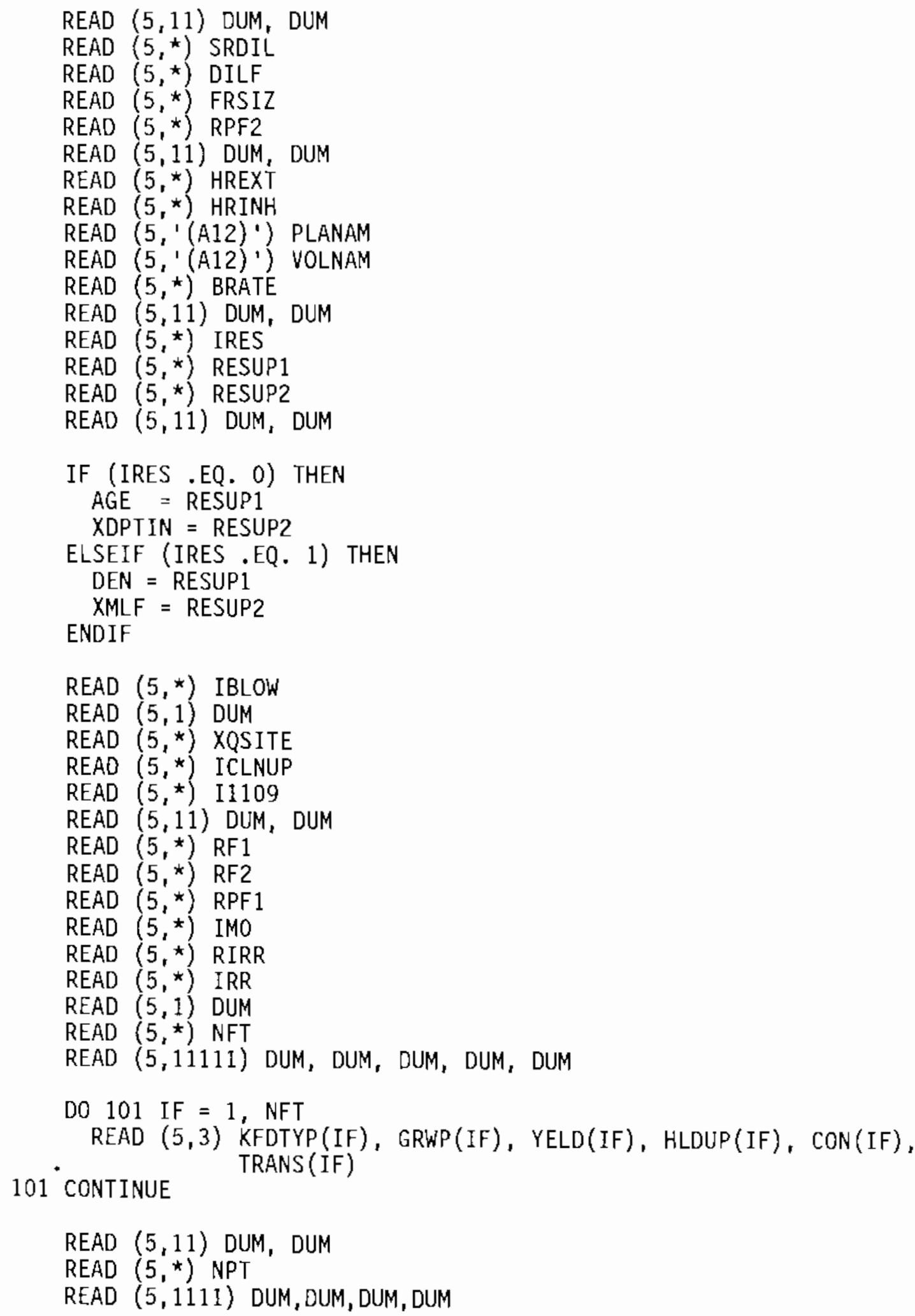


DO 102 IP $=1$, NPT

READ $(5,4)$ KPTHWY (IP), HLDUP2(IP), USAG(IP)

102 CONTINUE

C--- Set option flags -

IF (IFOD .EQ. 1) FOD = .TRUE.

IF (INHA .EQ. 1) INH = .TRUE.

IF (IWAT .EQ. 1) WAT = .TRUE.

IF (IARG .EQ. 1) ARG = . TRUE.

C---- Find an index to give correct FDTC in PLTCON for non-plant paths

$I P L=0$

IF (IFOD .EQ. 1) THEN

DO $10 \quad I=1, N F T$

IF (KFDTYP(I) .GT.9)GO TO 10

$I P L=I$

10 CONTINUE

IF (IPL .EQ. 0) GOTO 98

ENDIF

RETURN

C---- Set RPF1 to proper default value for surface-only case --.-.------

(IF ISUR .EQ. 1 .AND. IEXT .EQ. 0 .AND. RPF1 .EQ . 0.0) RPF1 $=1.0$ WRITE $(*, *)$

WRITE $\left(\star^{*}\right)$ 'The parameter RPF1 has been set to 1.0 assuming'

WRITE $(*, *)$ 'uniform distribution of a surface source.'

C--- Error Messages -

C

C IF THERE ARE NO PLANT PATHWAYS, PRINT MESSAGE AND STOP

98 WRITE $(*, 12)$

12 FORMAT(1H1,' THIS CODE WILL NOT RUN WITHOUT AT LEAST',

- STOP

ONE PLANT PATHWAY--STOP')

C--.- Format Statements

1 FORMAT (AI0)

2 FORMAT (14X, A2, A6, 2X, 4(G11.2, 2X))

3 FORMAT (I5, 19X, G7.2, G11.2, G8.2, G11.2, G10.2)

4 FORMAT (I5, 19X, G6.2, G11.2)

11 FORMAT (A10/A10)

1111 FORMAT (A10/A10/A10/A10)

11111 FORMAT (A10/A10/A10/A10/A10)

END 
\$INCLUDE: 'COMPILE.OPT'

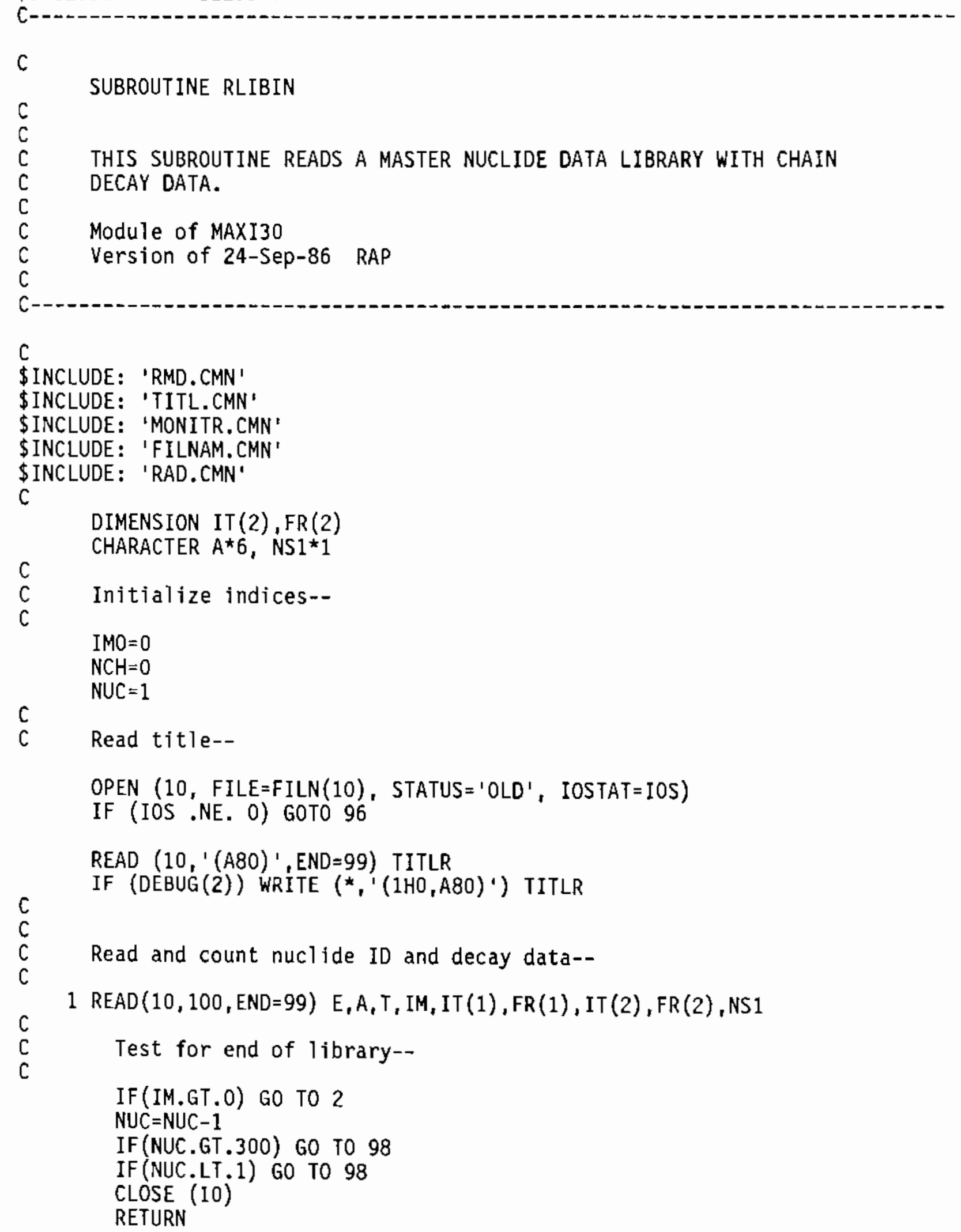

C

C THIS SUBROUTINE READS A MASTER NUCLIDE DATA LIBRARY WITH CHAIN 


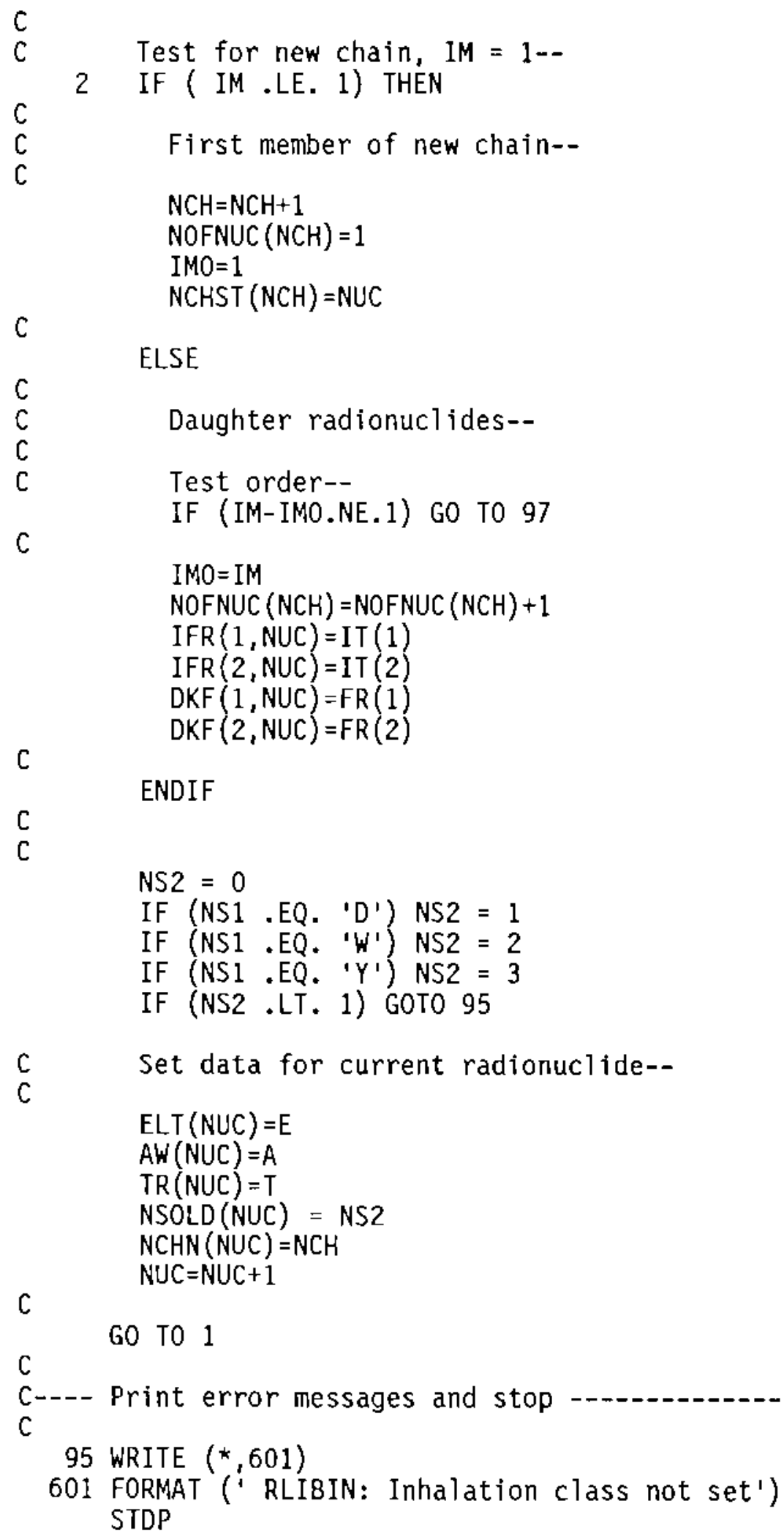




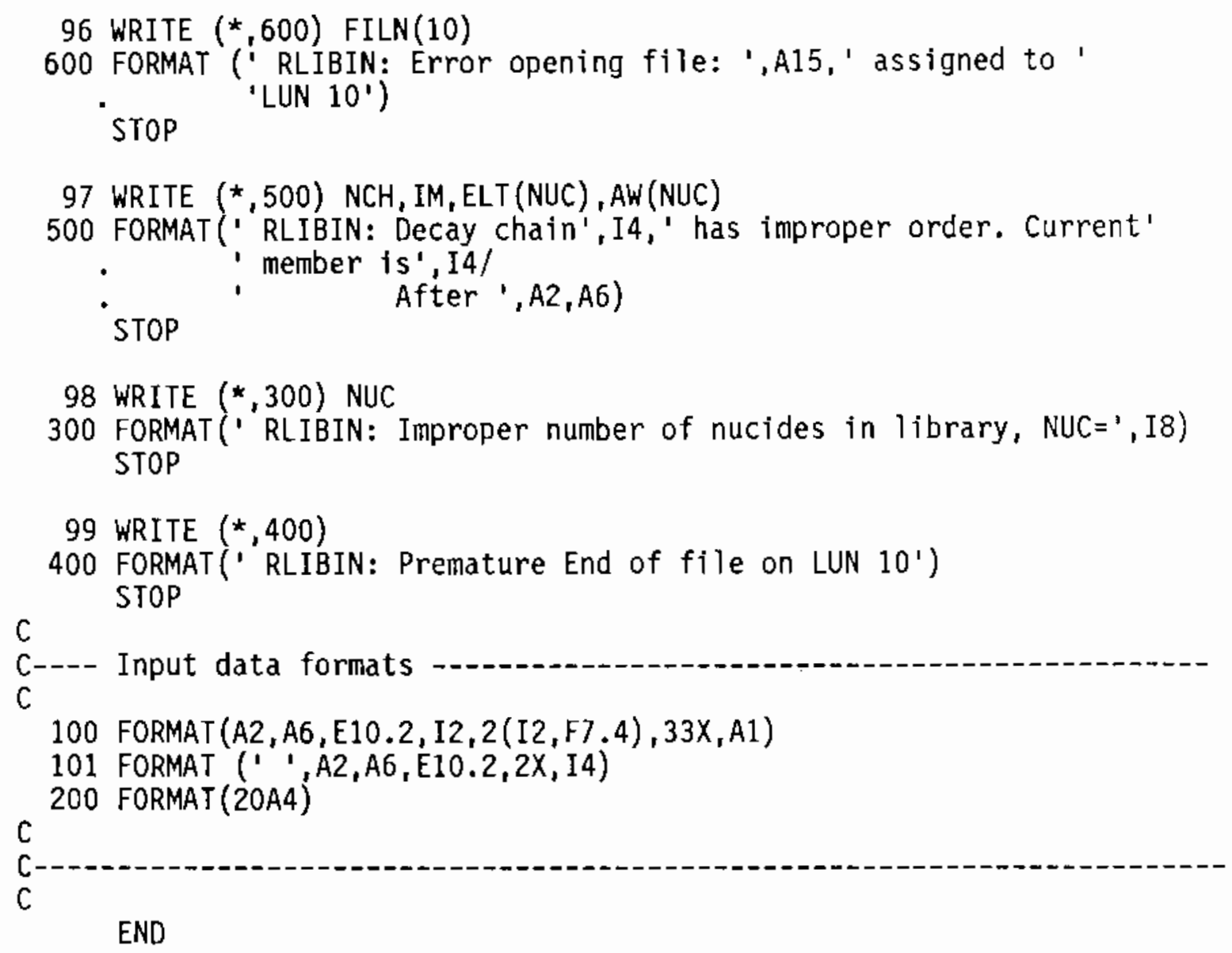




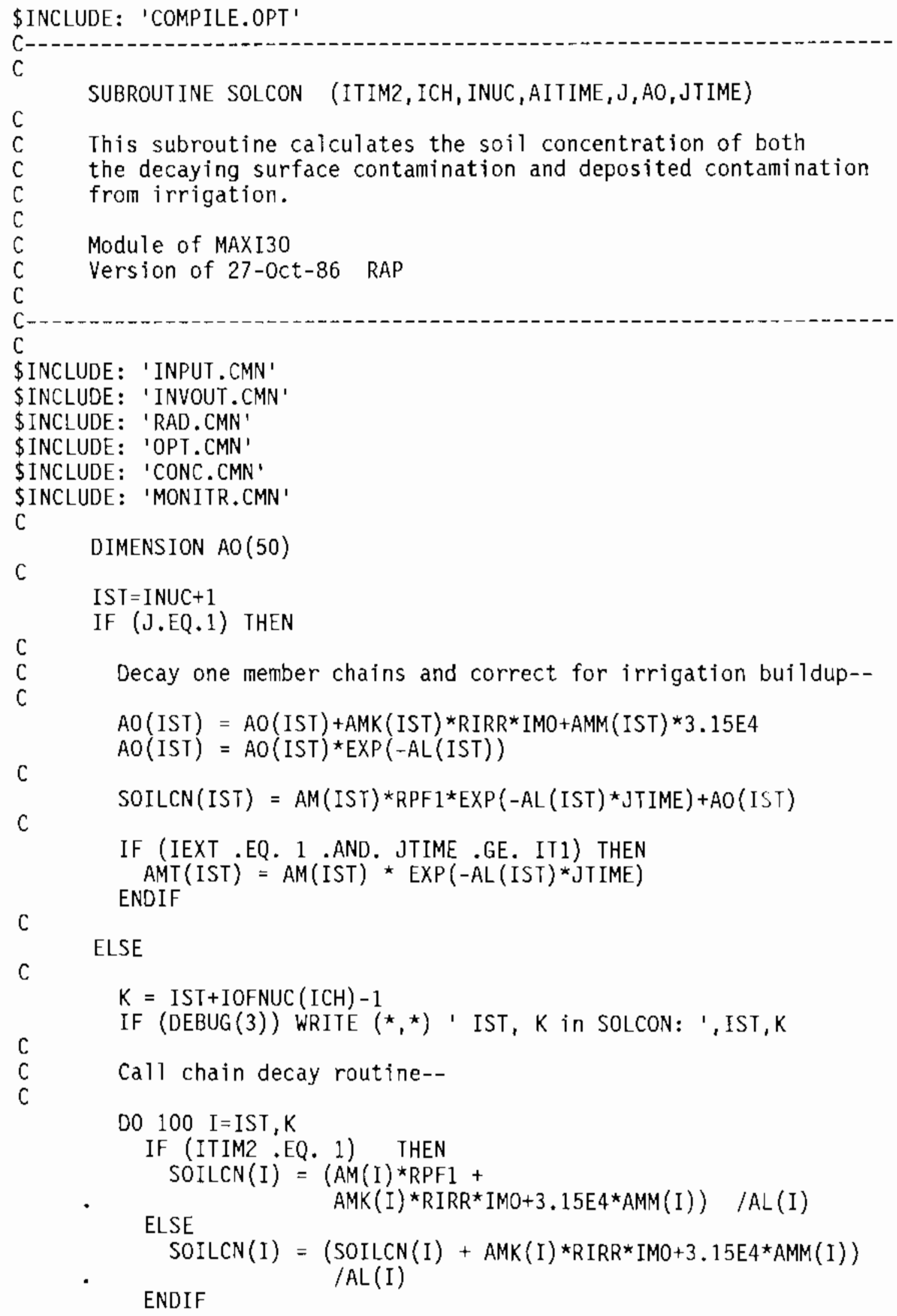




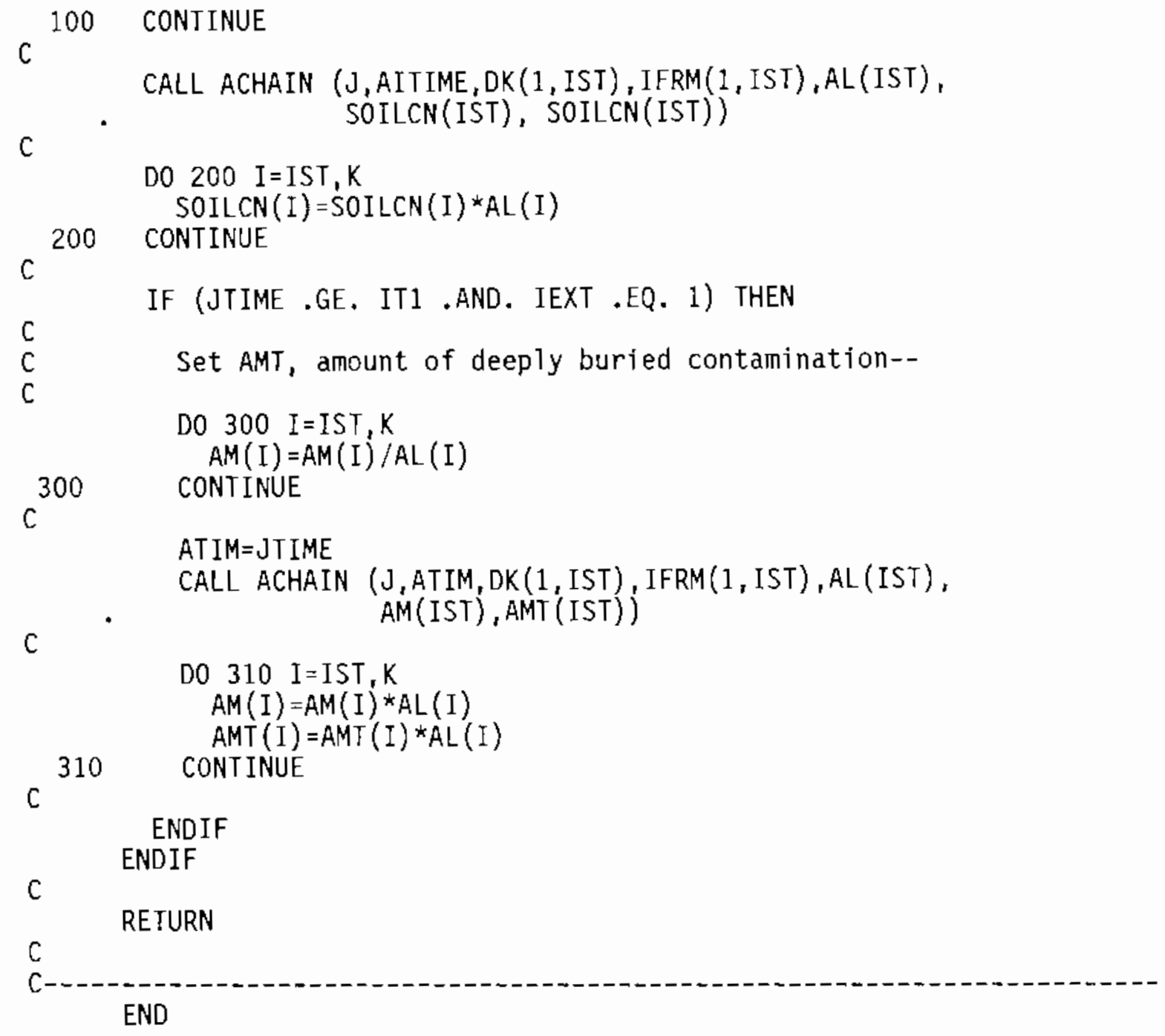




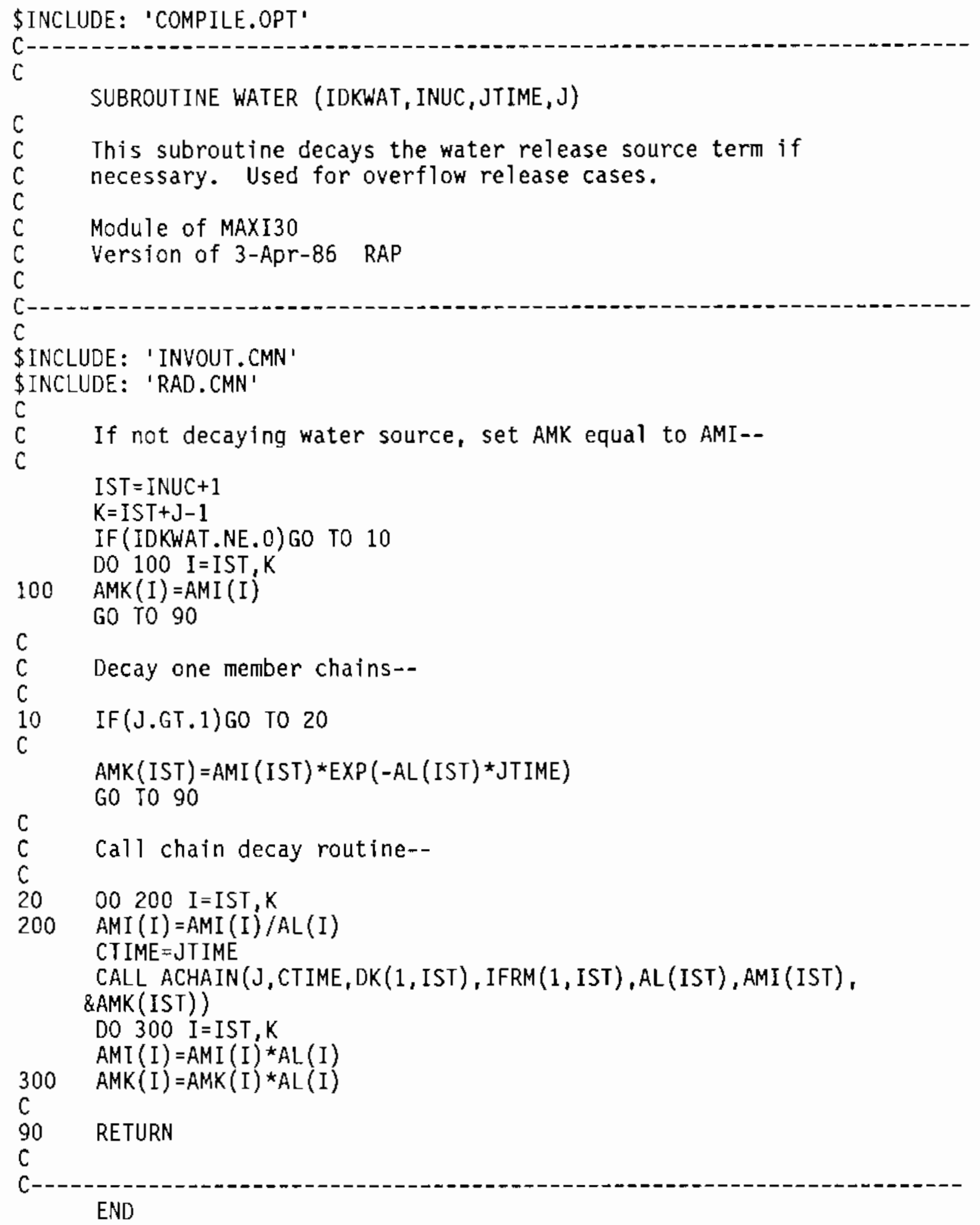




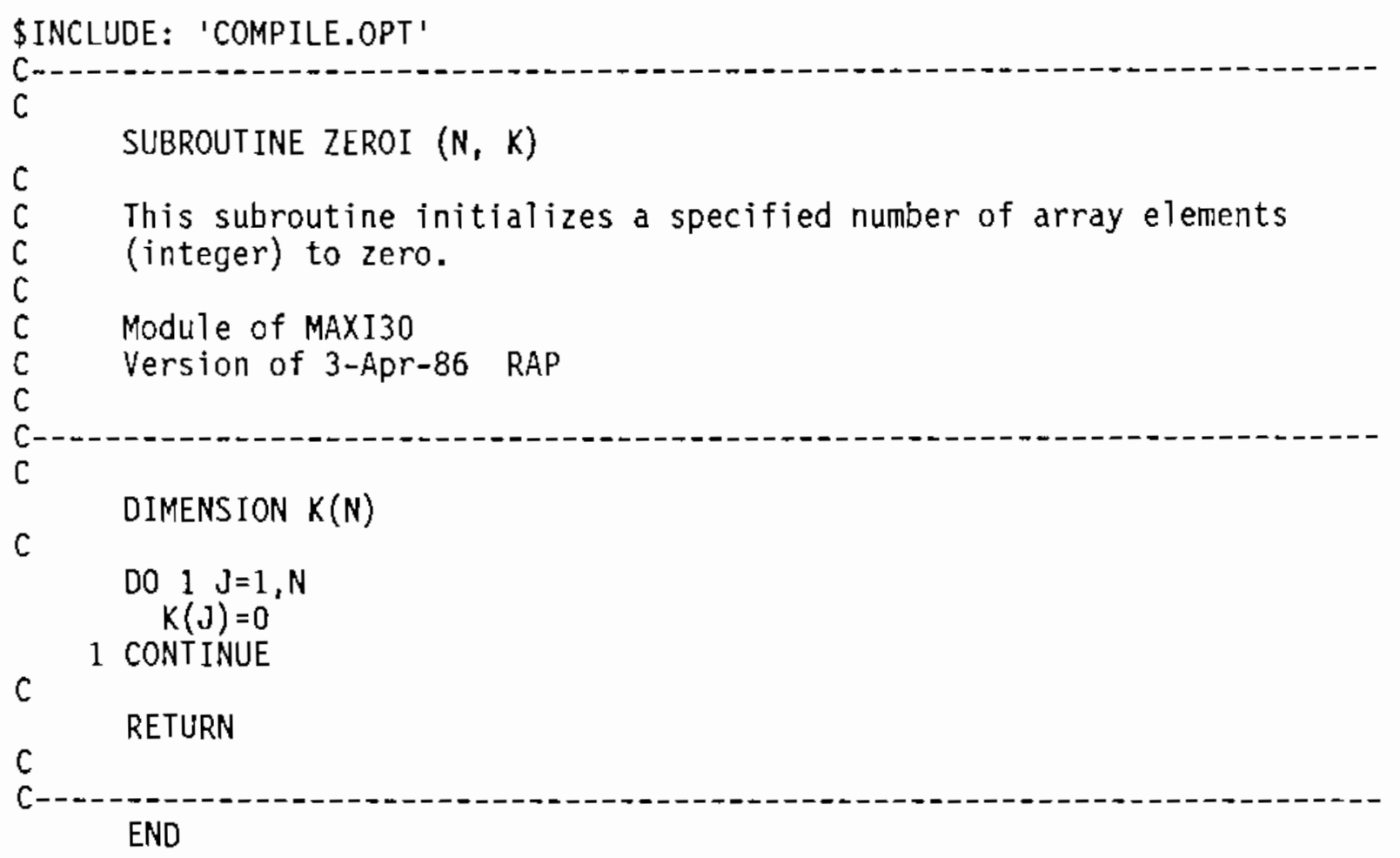


\$INCLUDE: 'COMPILE.OPT'

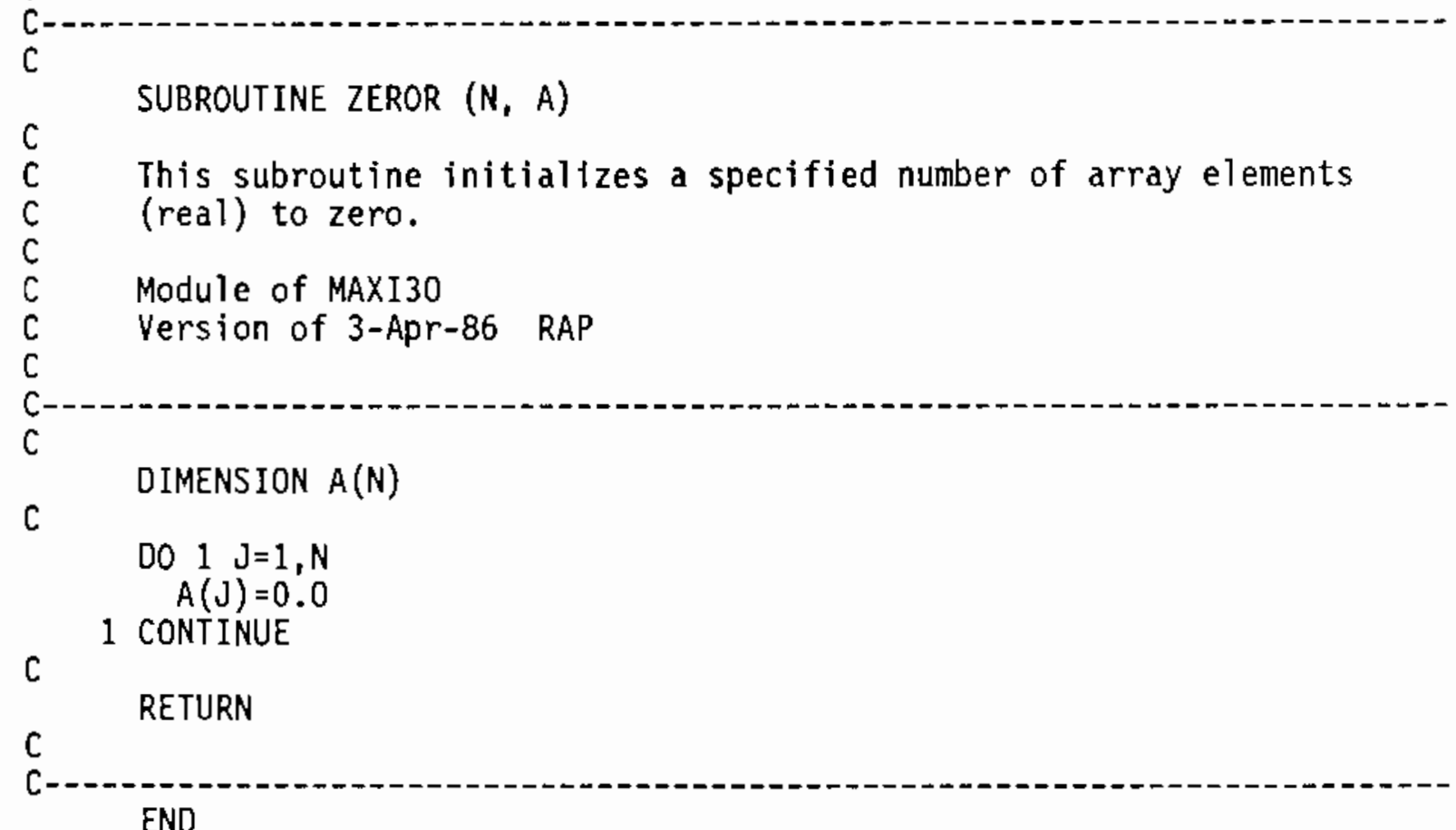




\section{FILE: CONC.CMN}

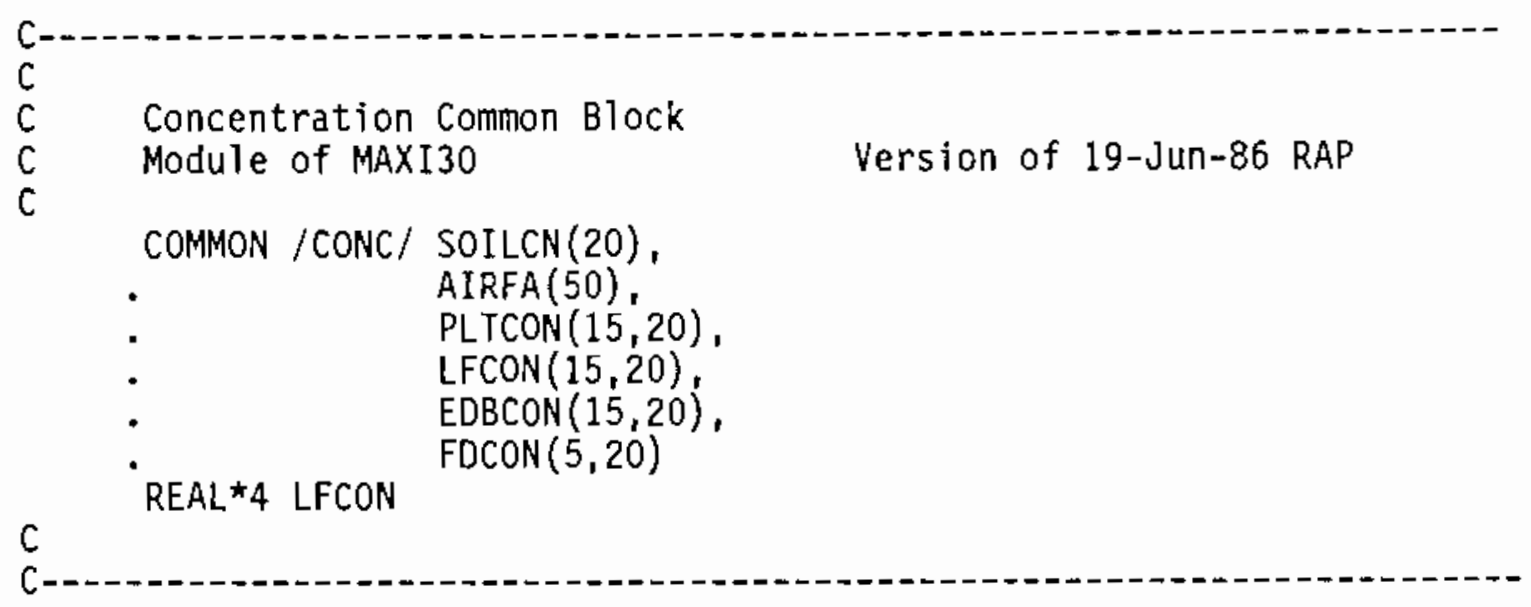

\section{FILE: DAYPC.CMN}

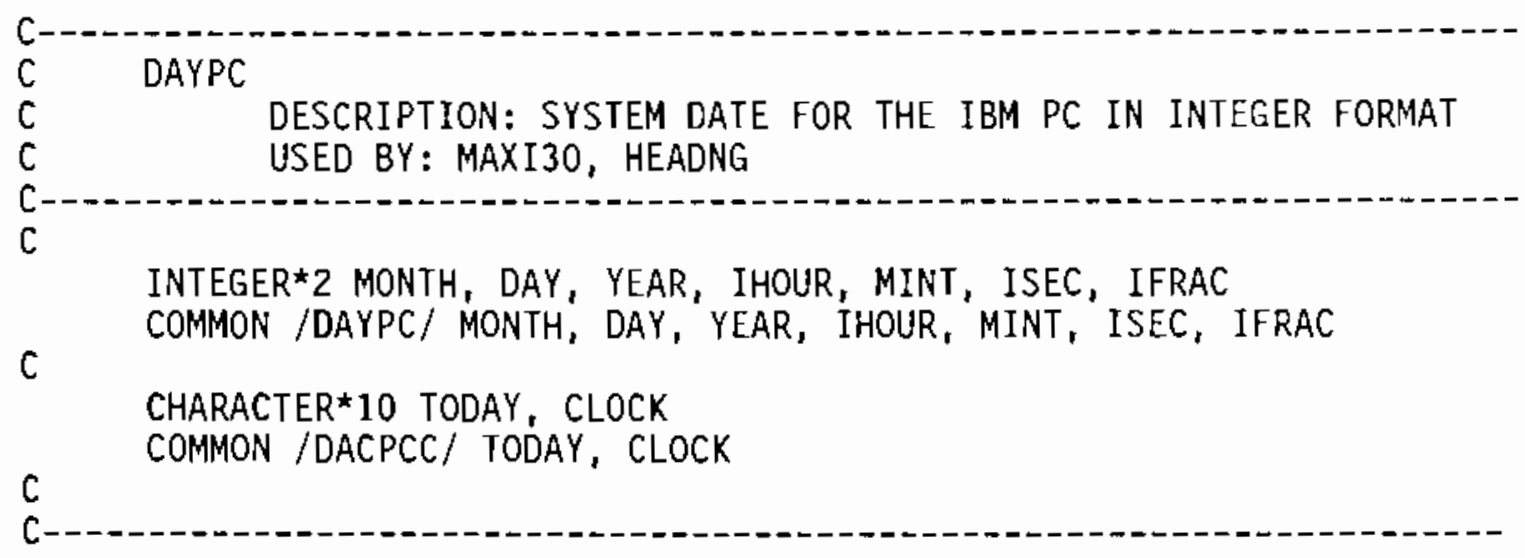

\section{FILE: DF}

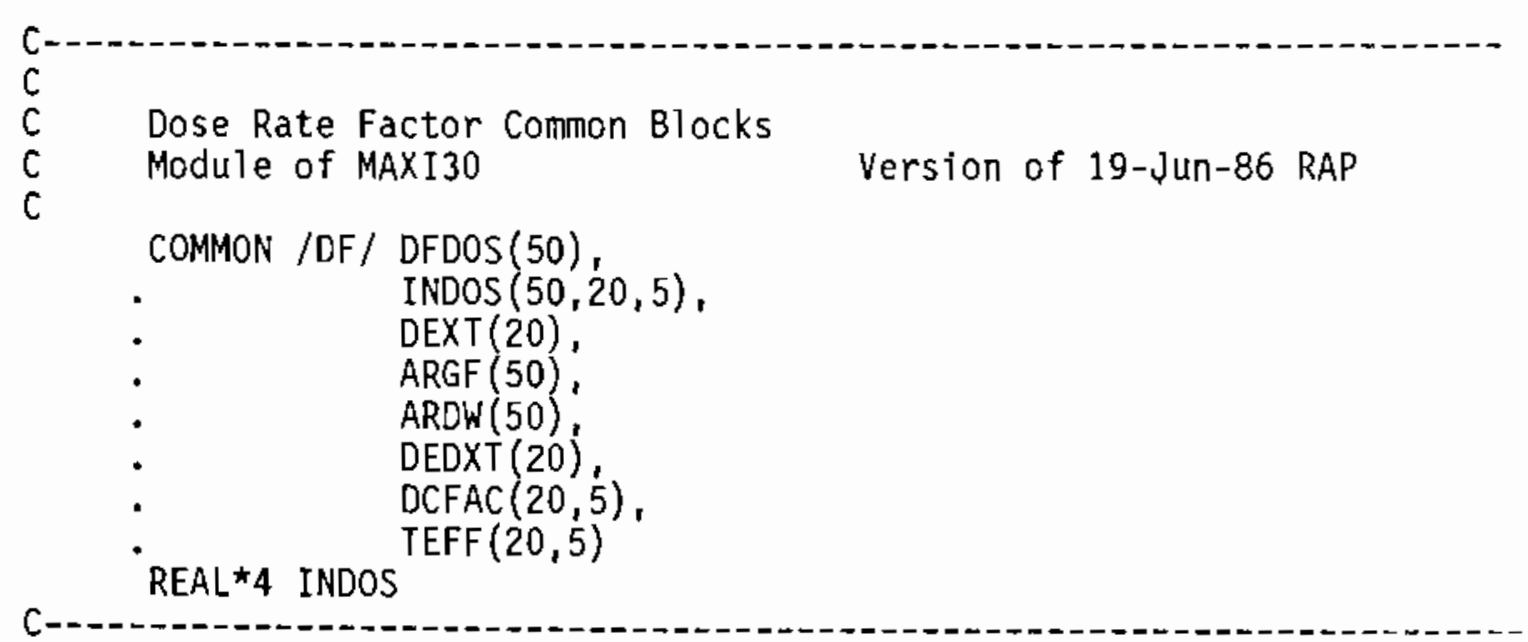




\section{FILE: DOS26.CMN}

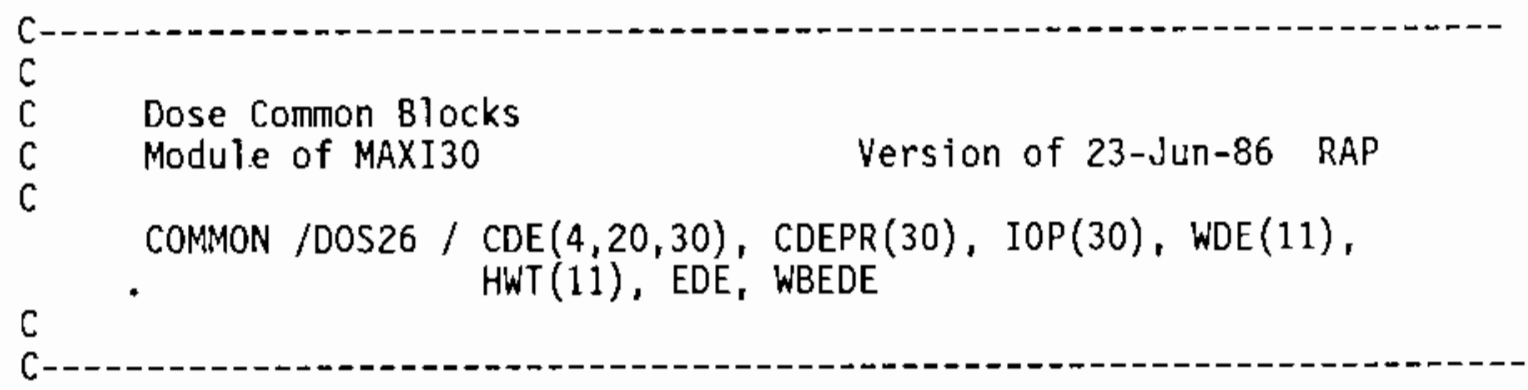

\section{FILE: DOSEI.CMN}

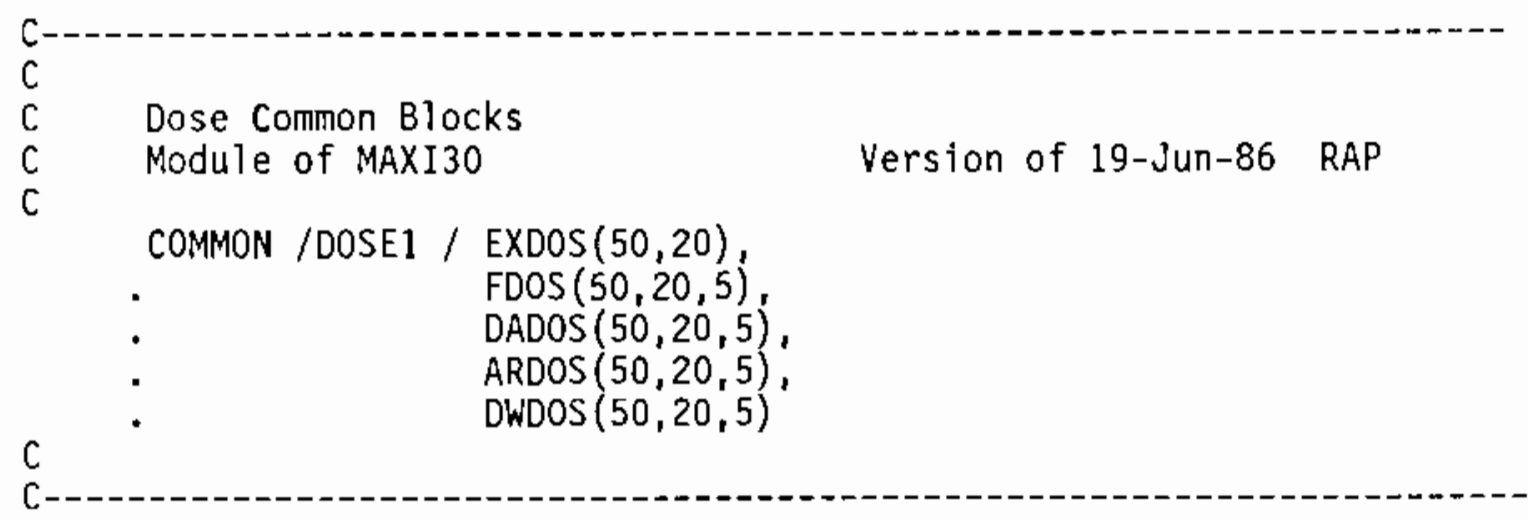

FILE: DOSE2.CMN

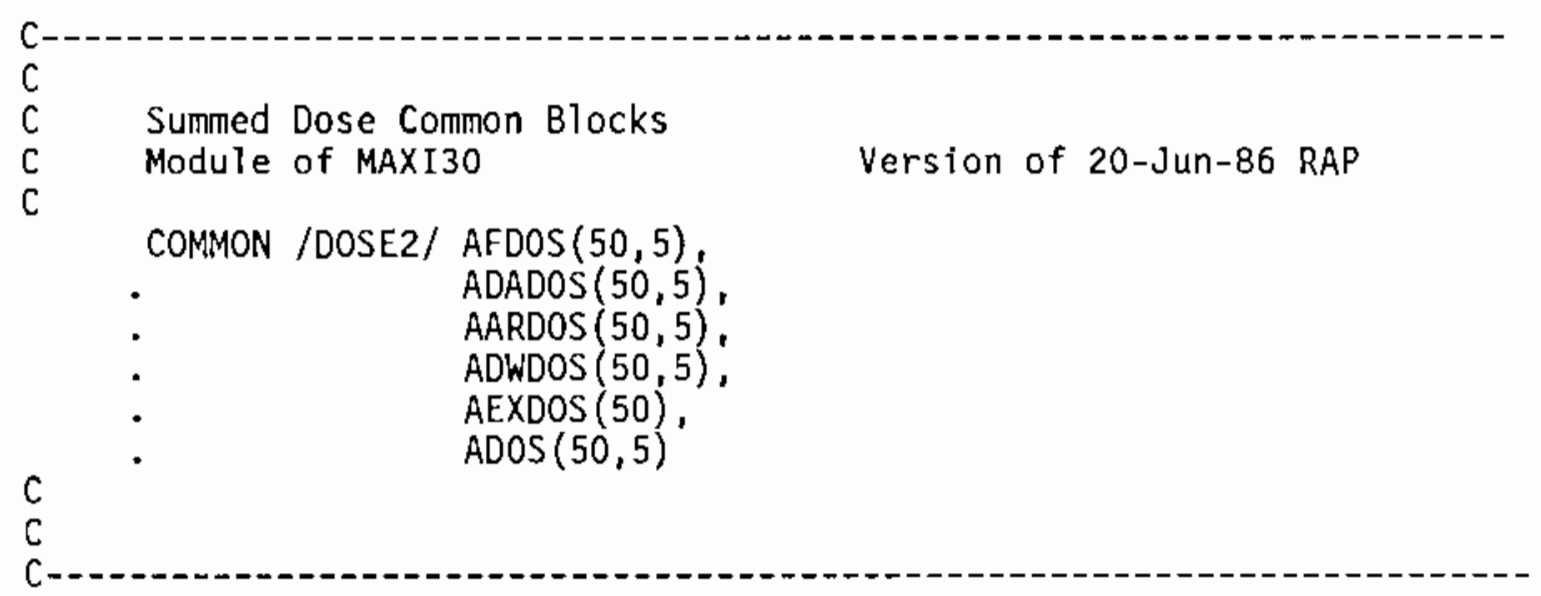




\section{FILE: FILNAM.CMN}

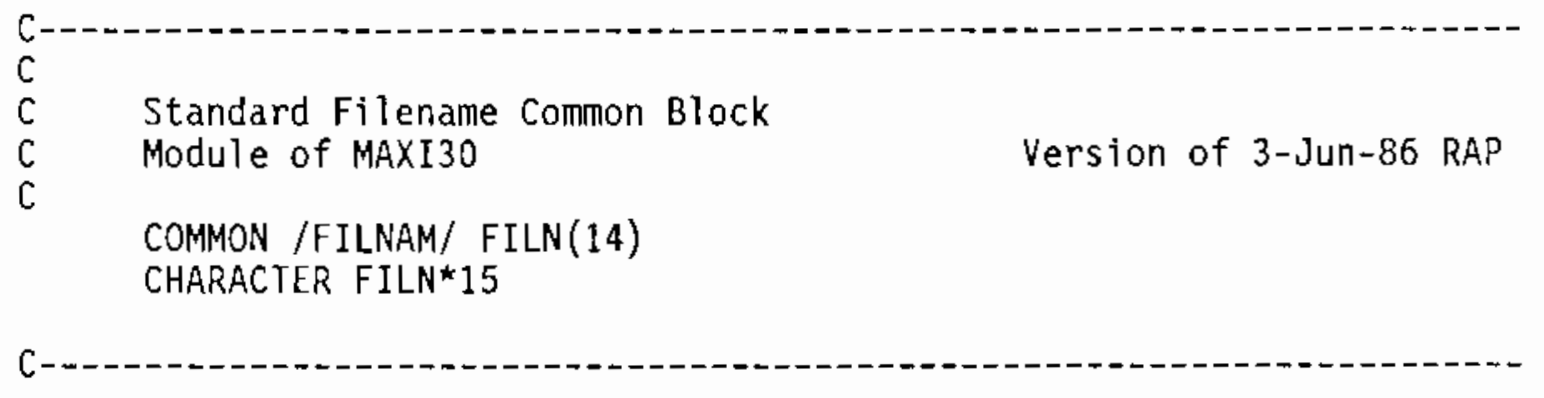

\section{FILE: INPUT.CMN}

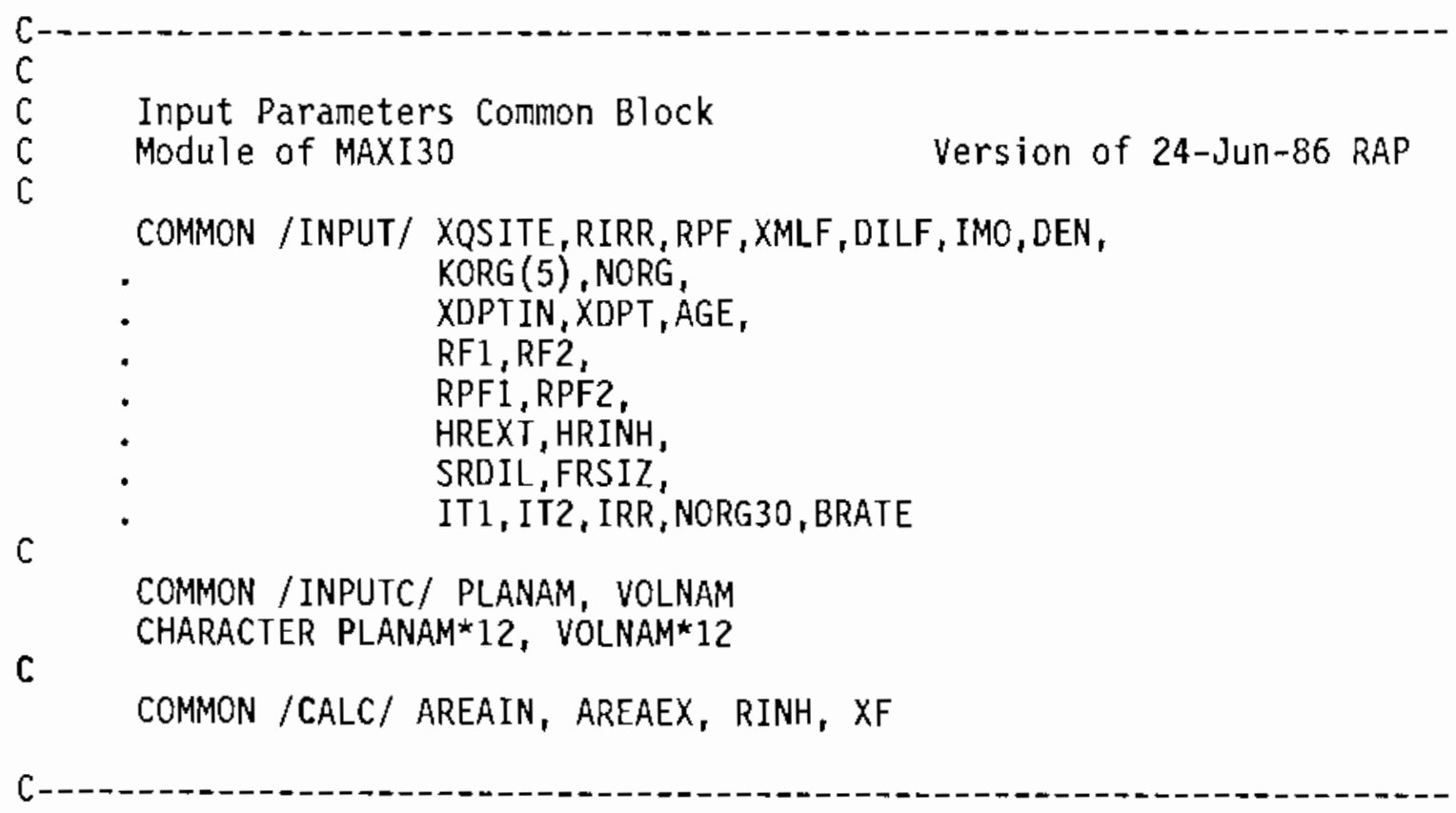

\section{FILE: INVIN.CHN}

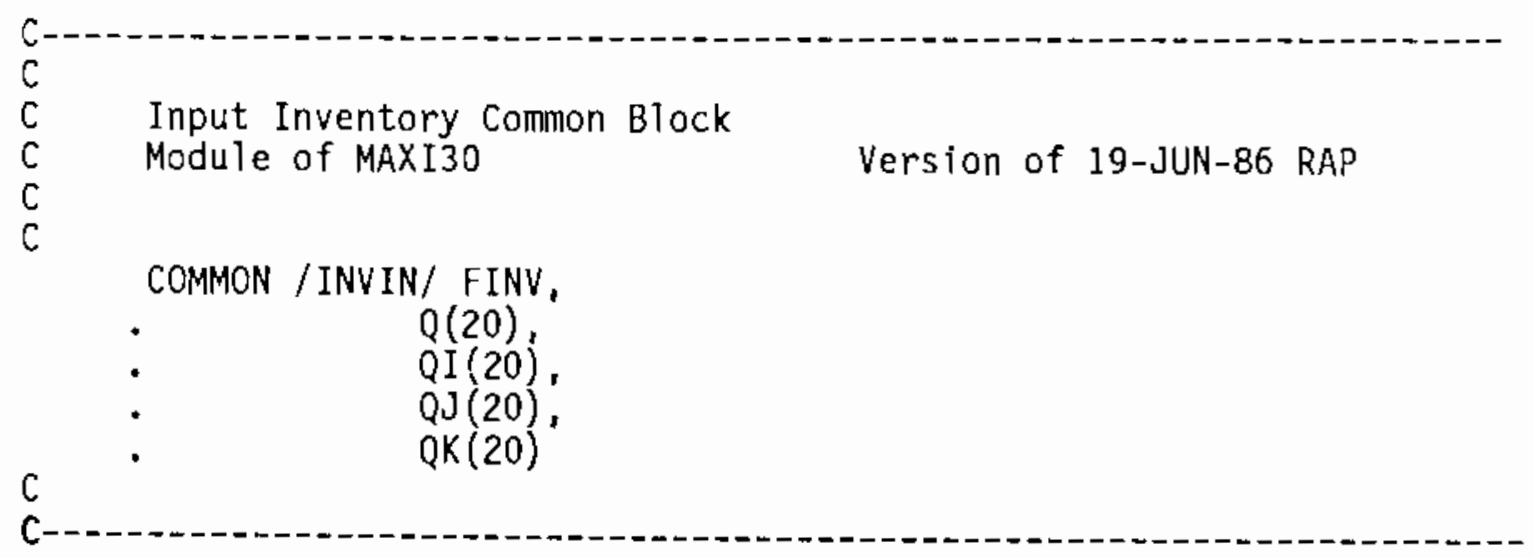




\section{FILE: INVOUT.CMN}

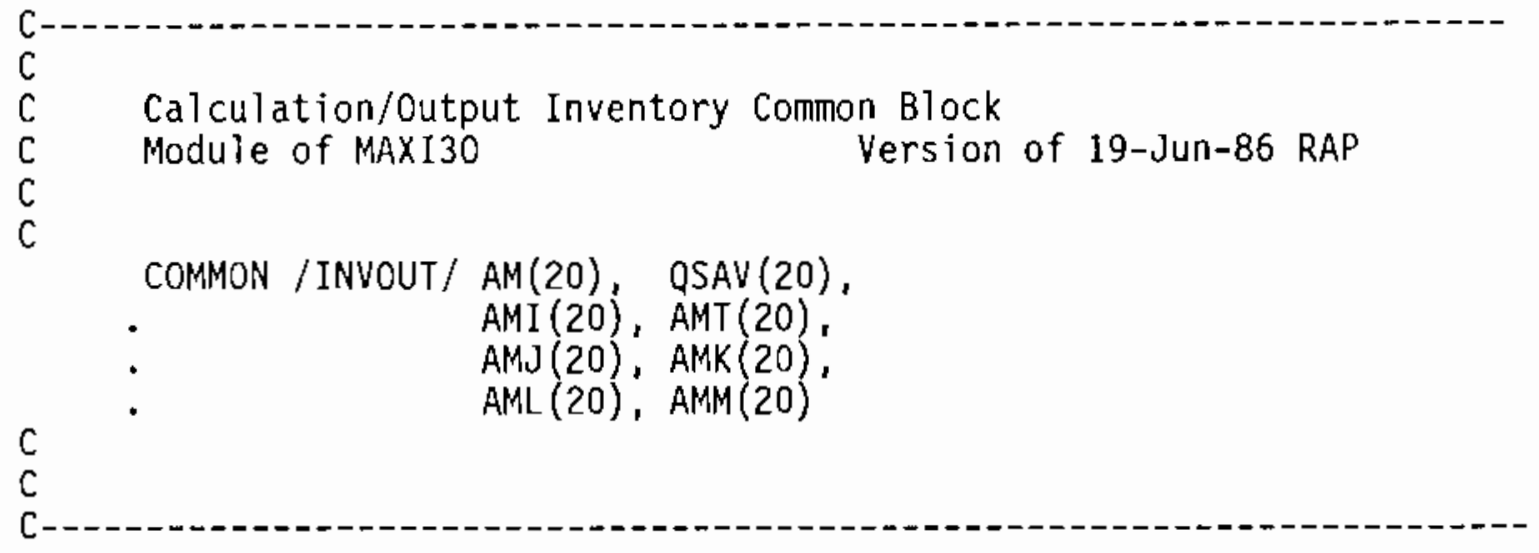

\section{FILE: LIB26.CMN}

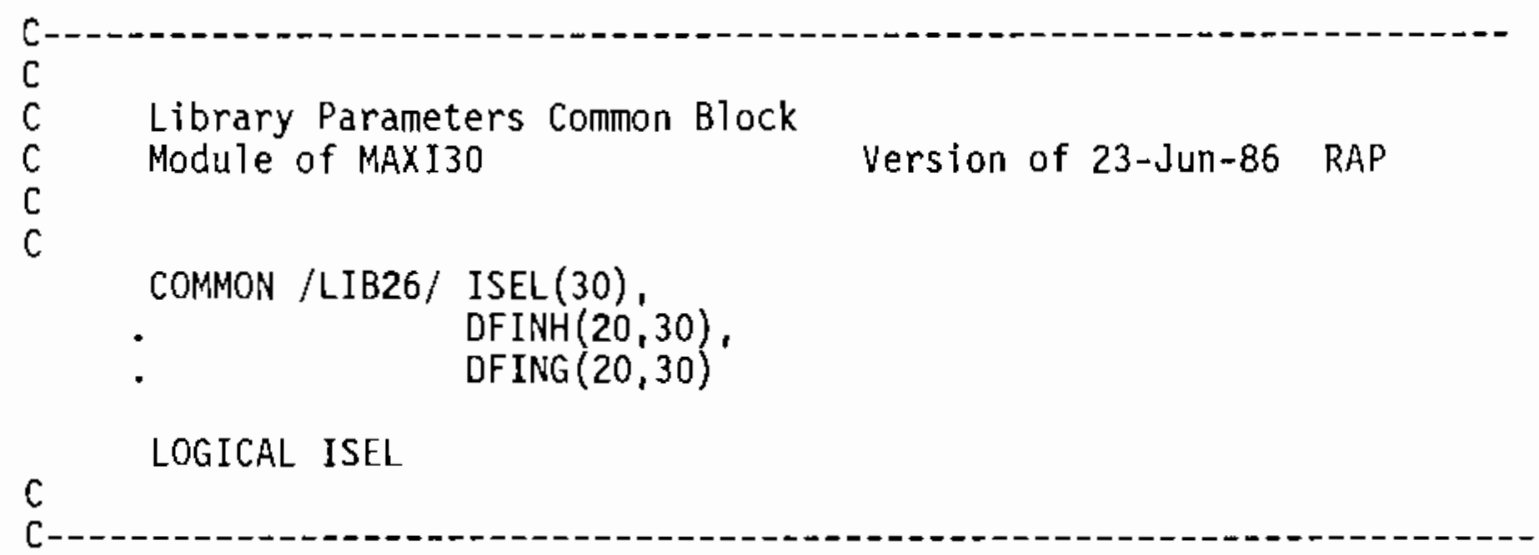

\section{FILE: LIBPAR.CMN'}

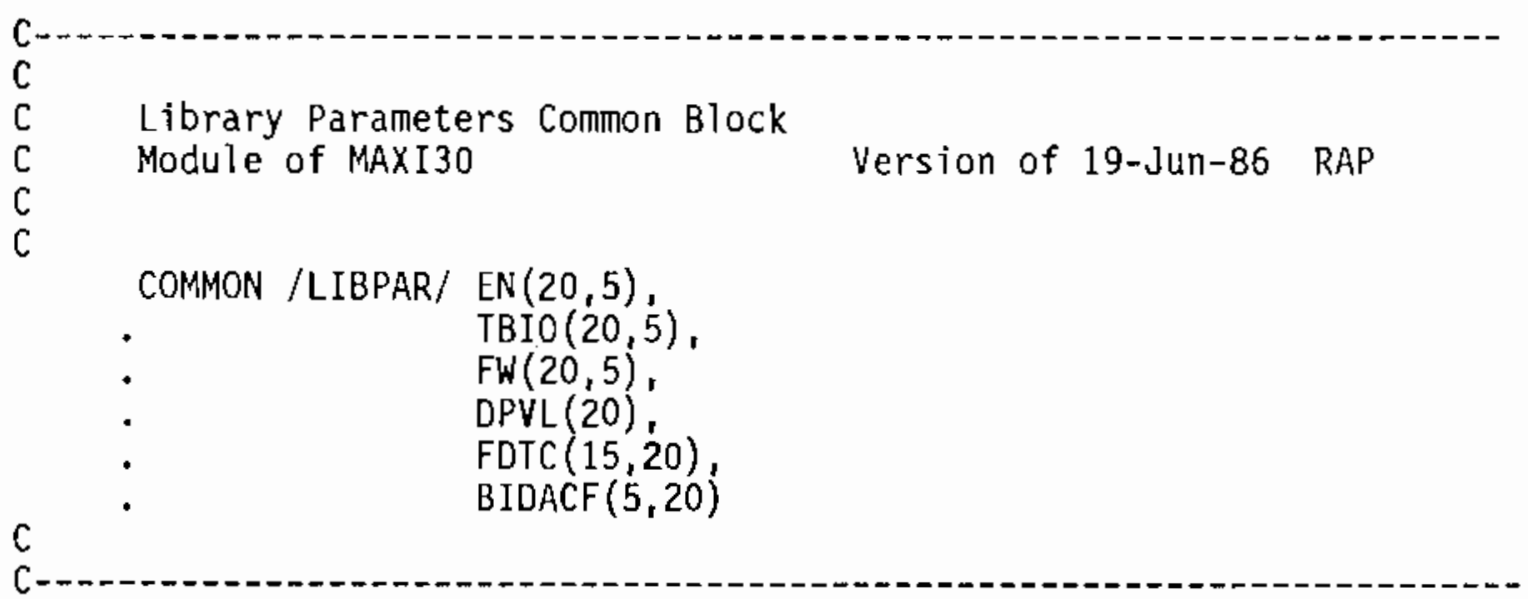




\section{FILE: MONITR.CMN}

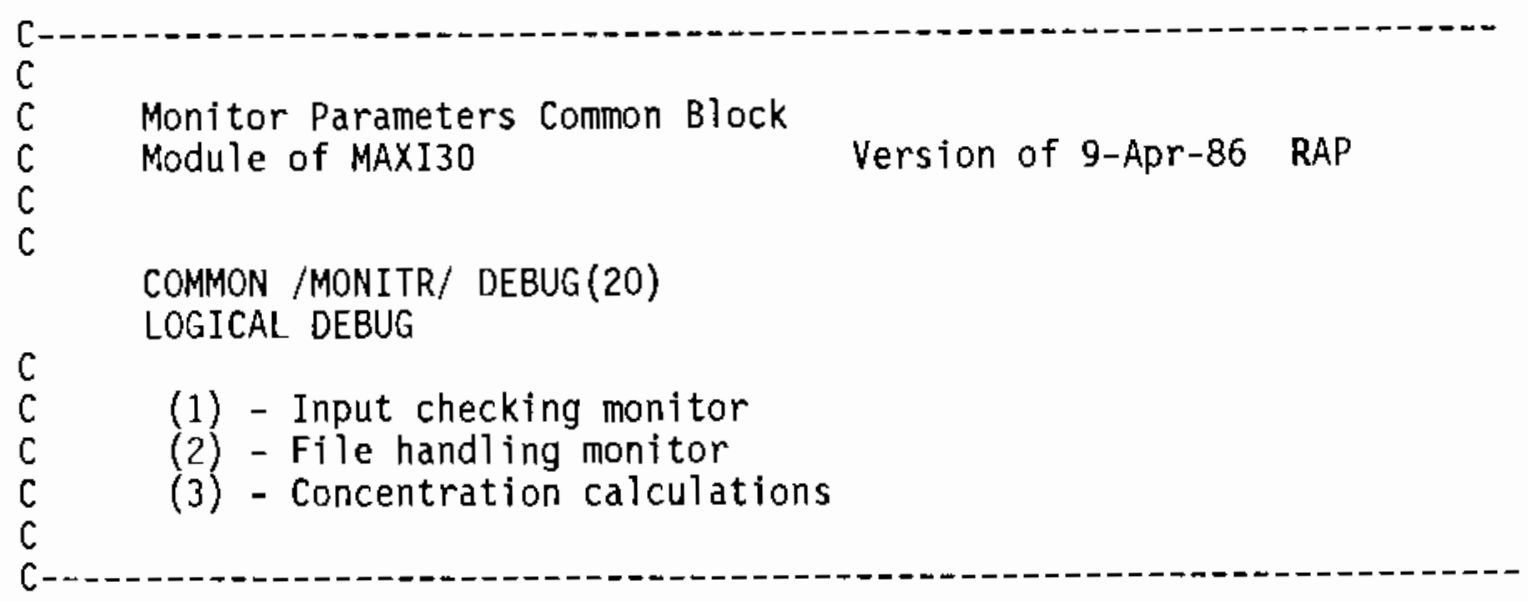

\section{FILE: MXTIM.CMN}

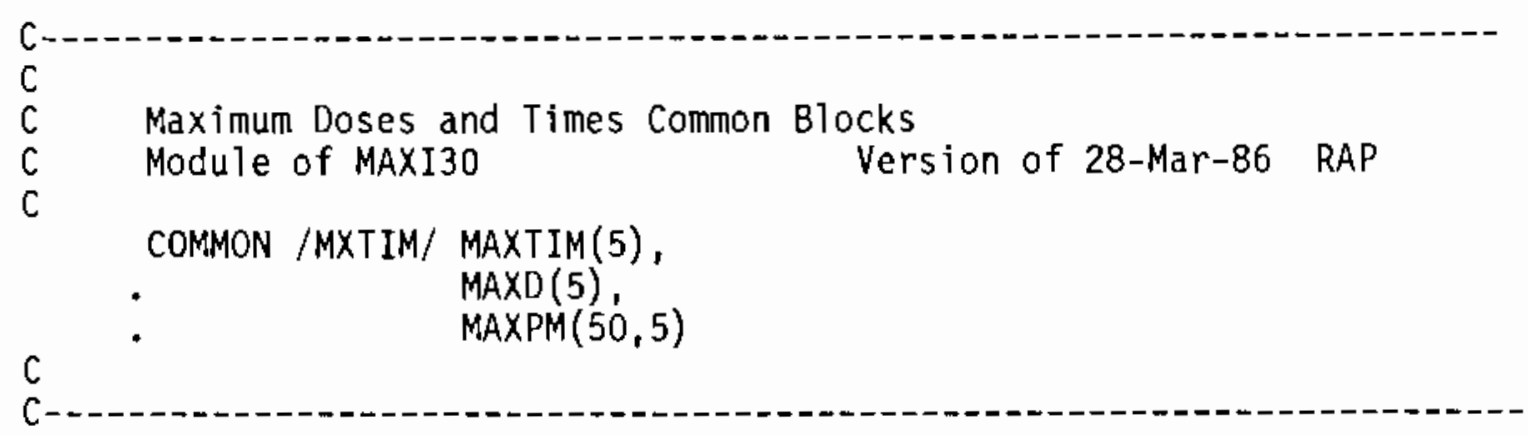

\section{FILE: OPT.CMN}

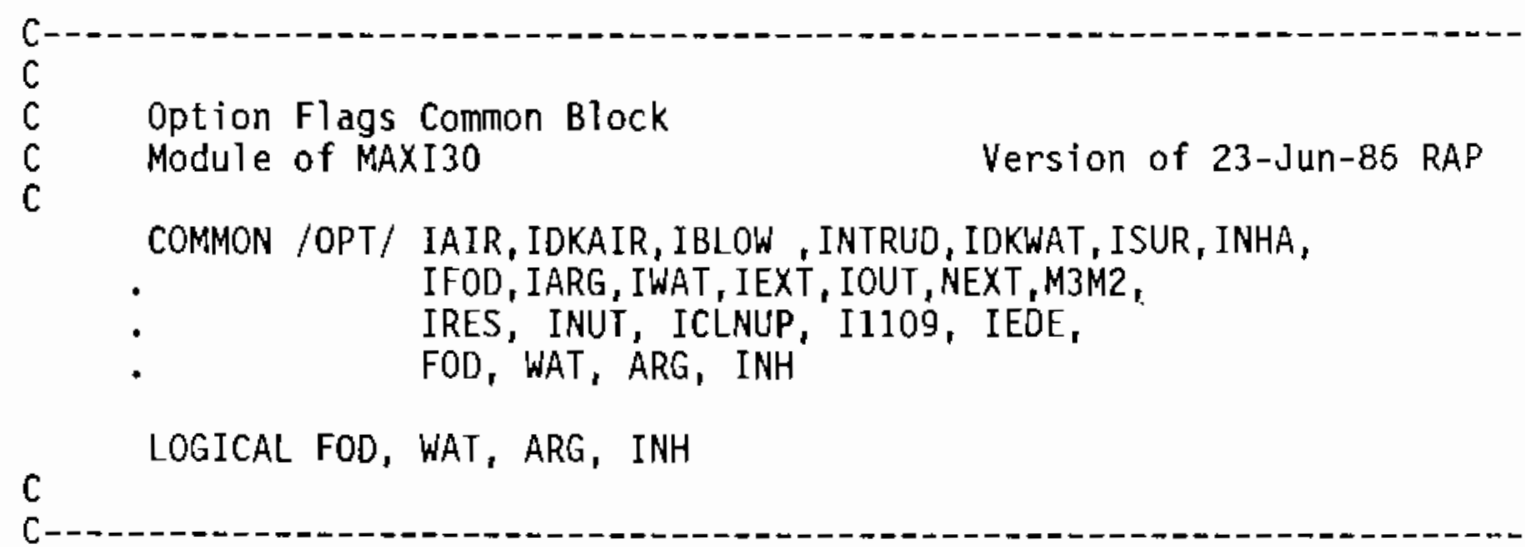




\section{FILE: ORGLAB.CHN}

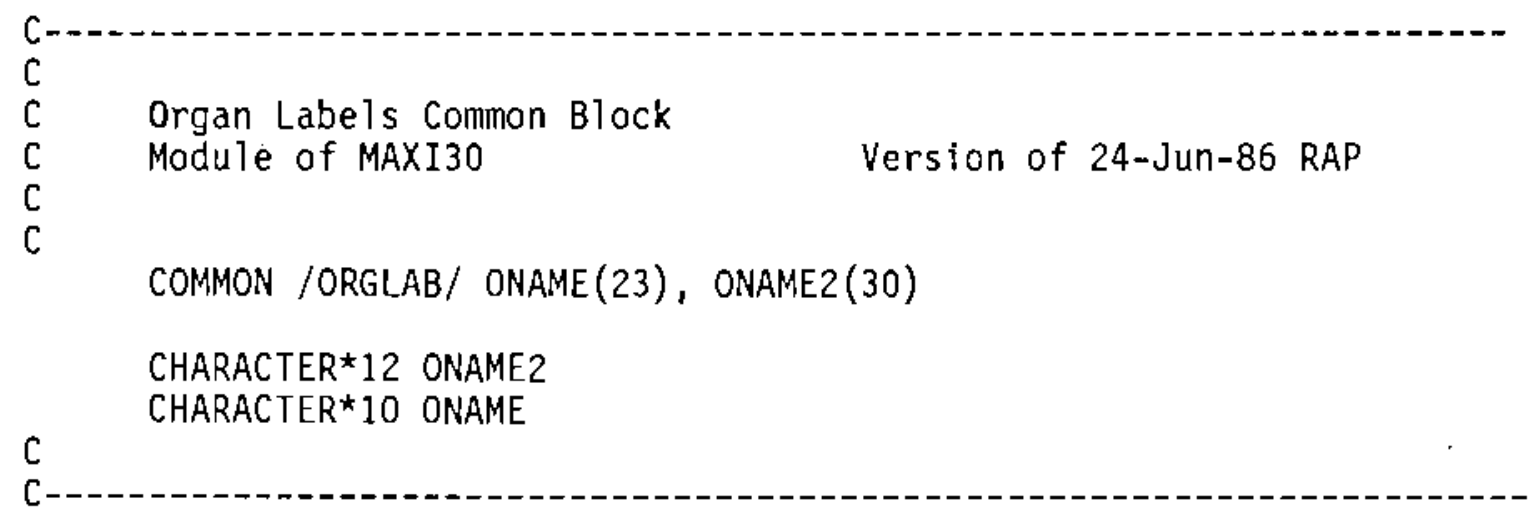

\section{FILE: PATH.CMN}

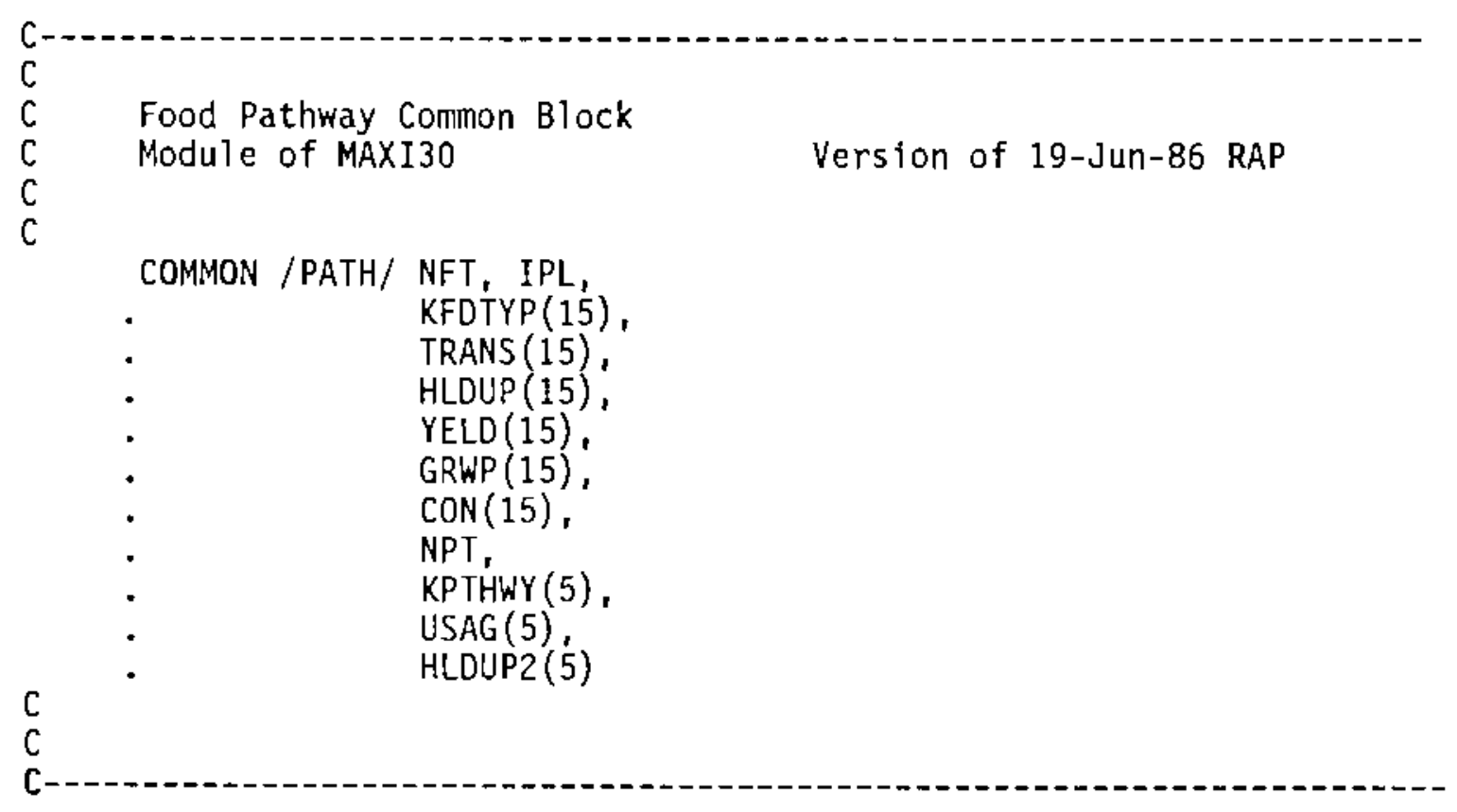




\section{FILE: RAD.CMN}

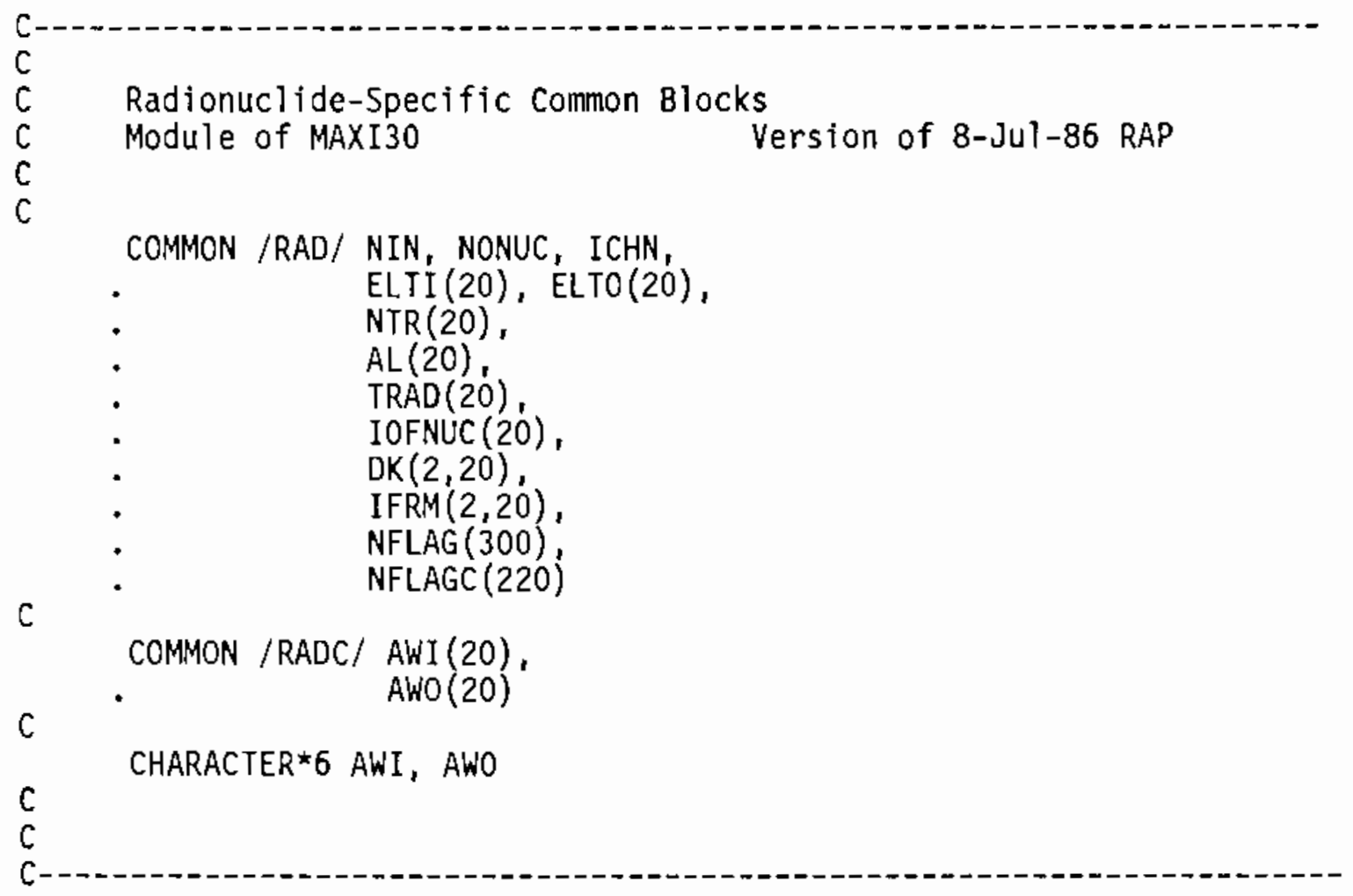

\section{FILE: RMD.CMN}

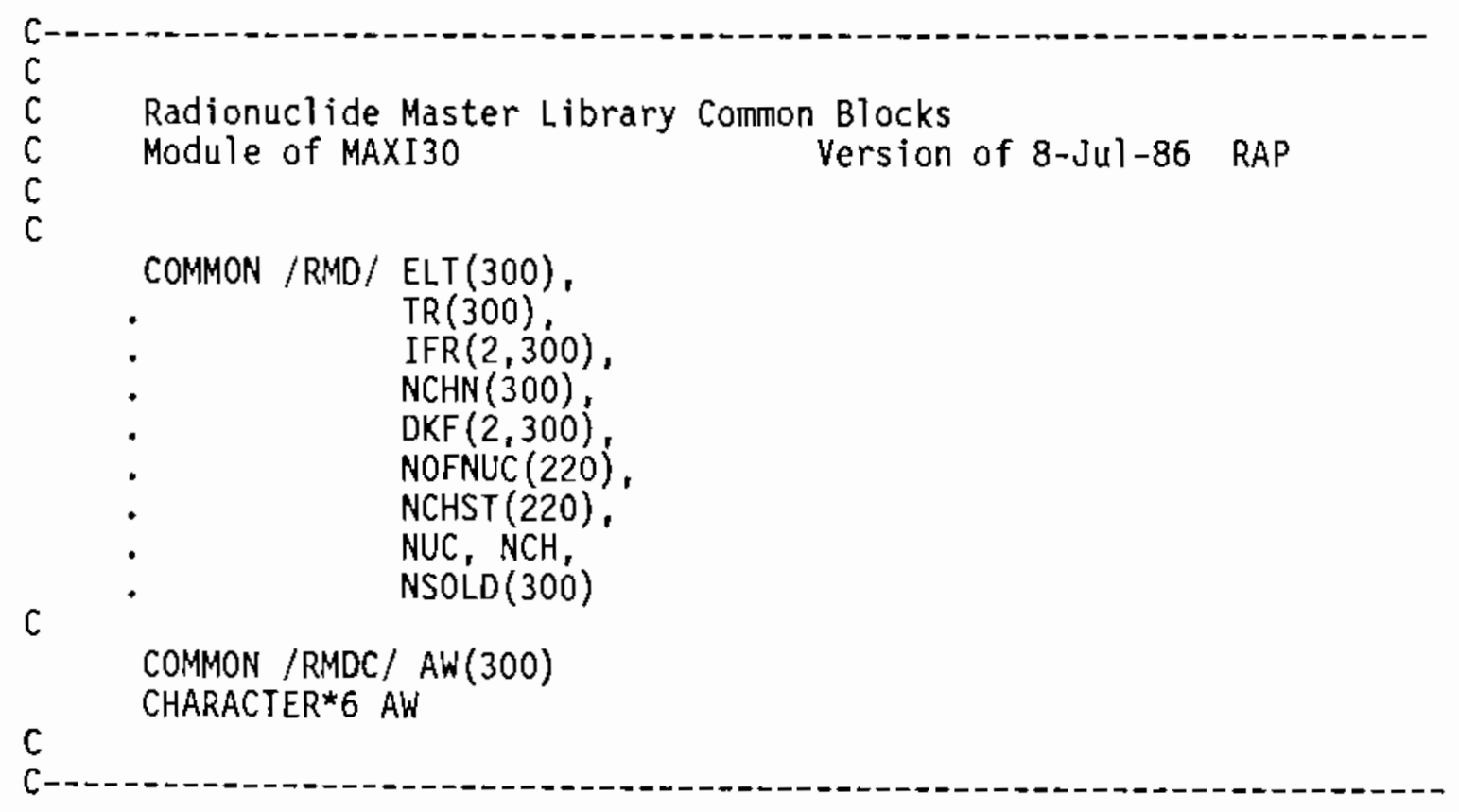




\section{FILE: TITL.CMN}

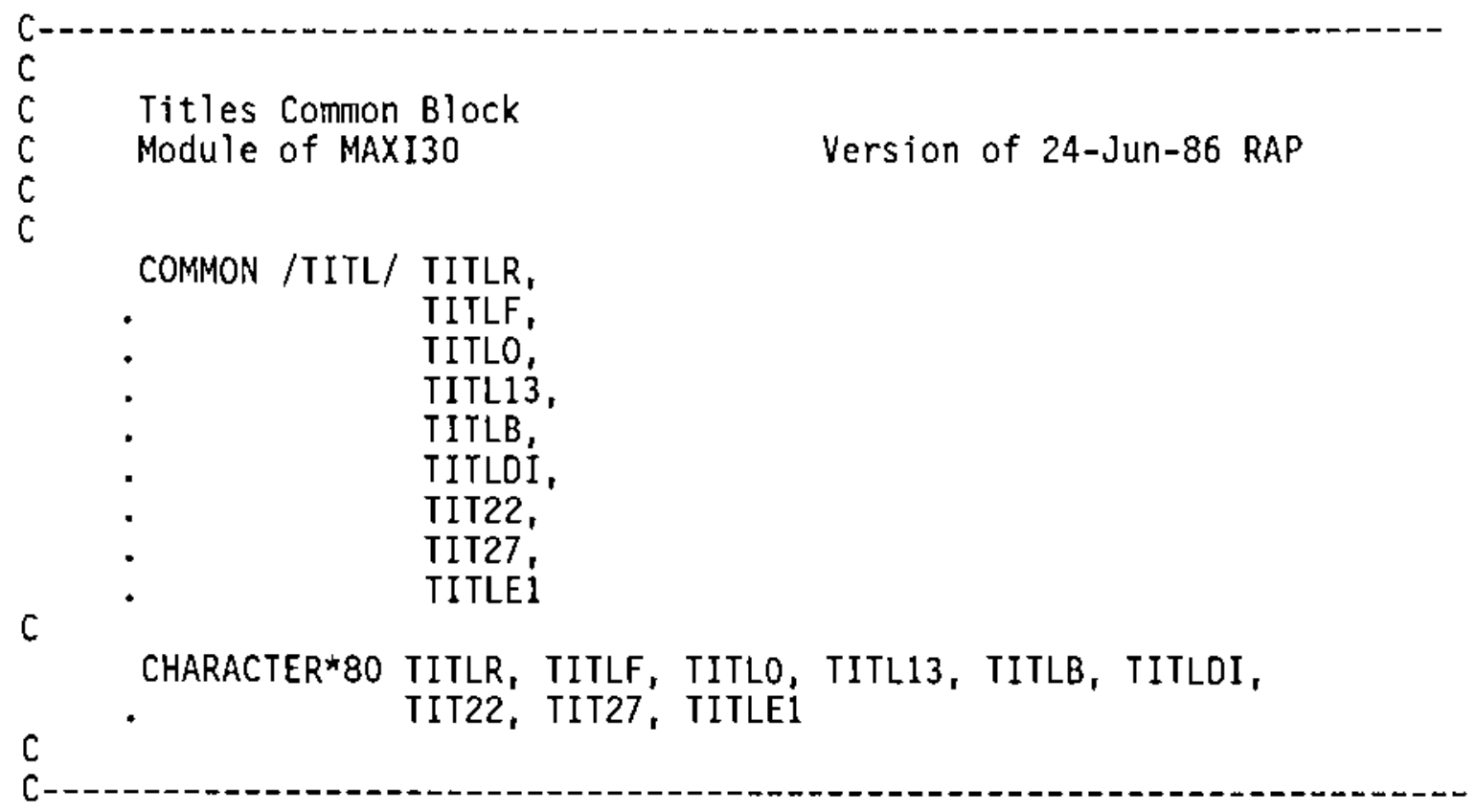

FILE: COMPILE.OPT

\$DEBUG

\$LIST

\$STORAGE: 2

\$PAGESIZE: 63

\$TITLE: 'MAXI1 Source Code'

\$SUBTITLE: 'IBM PC/XT/AT Version 1.0' 


\section{COMPUTER PROGRAM LISTING - ONSITE}

\$INCLUDE: 'COMPILE.OPT'

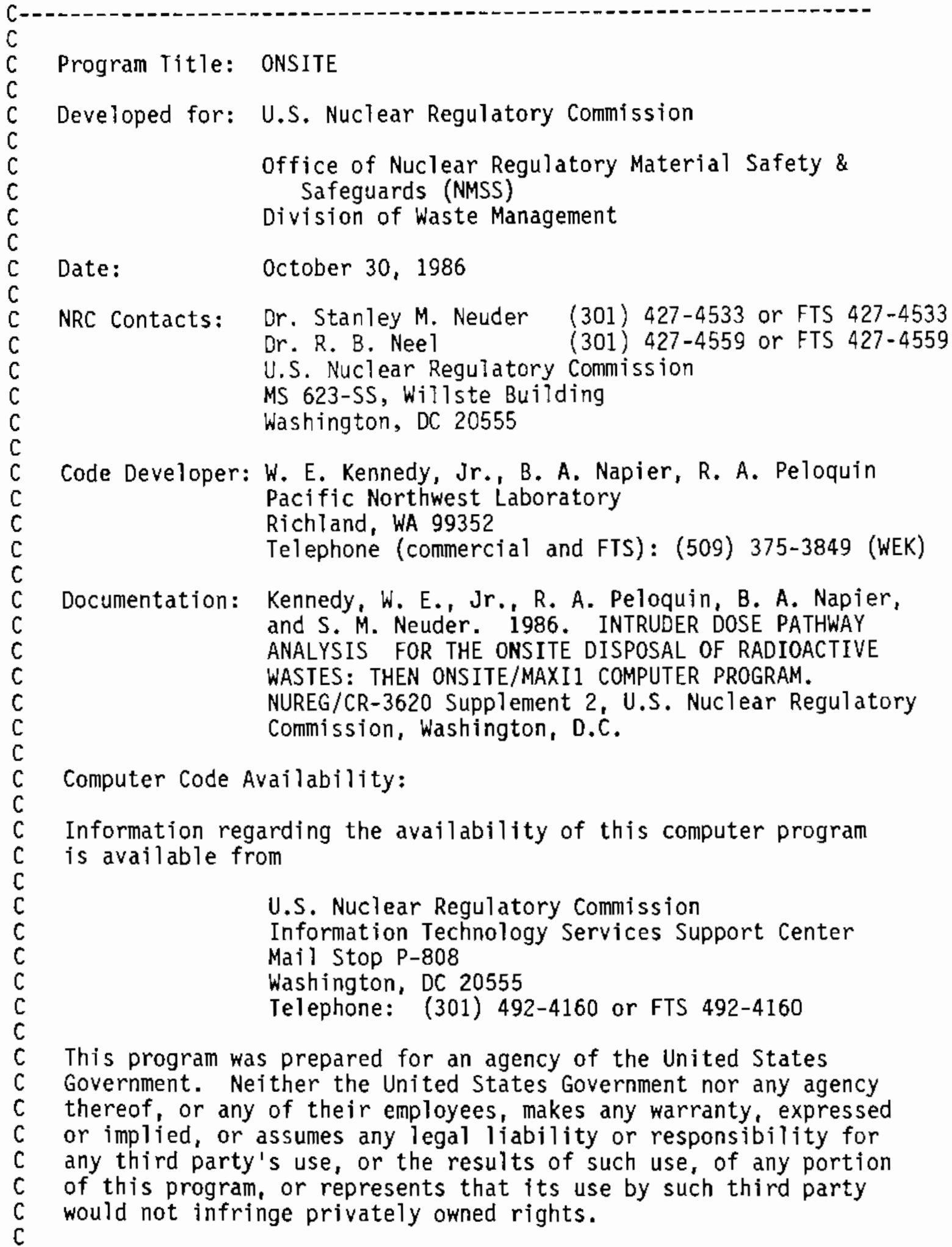




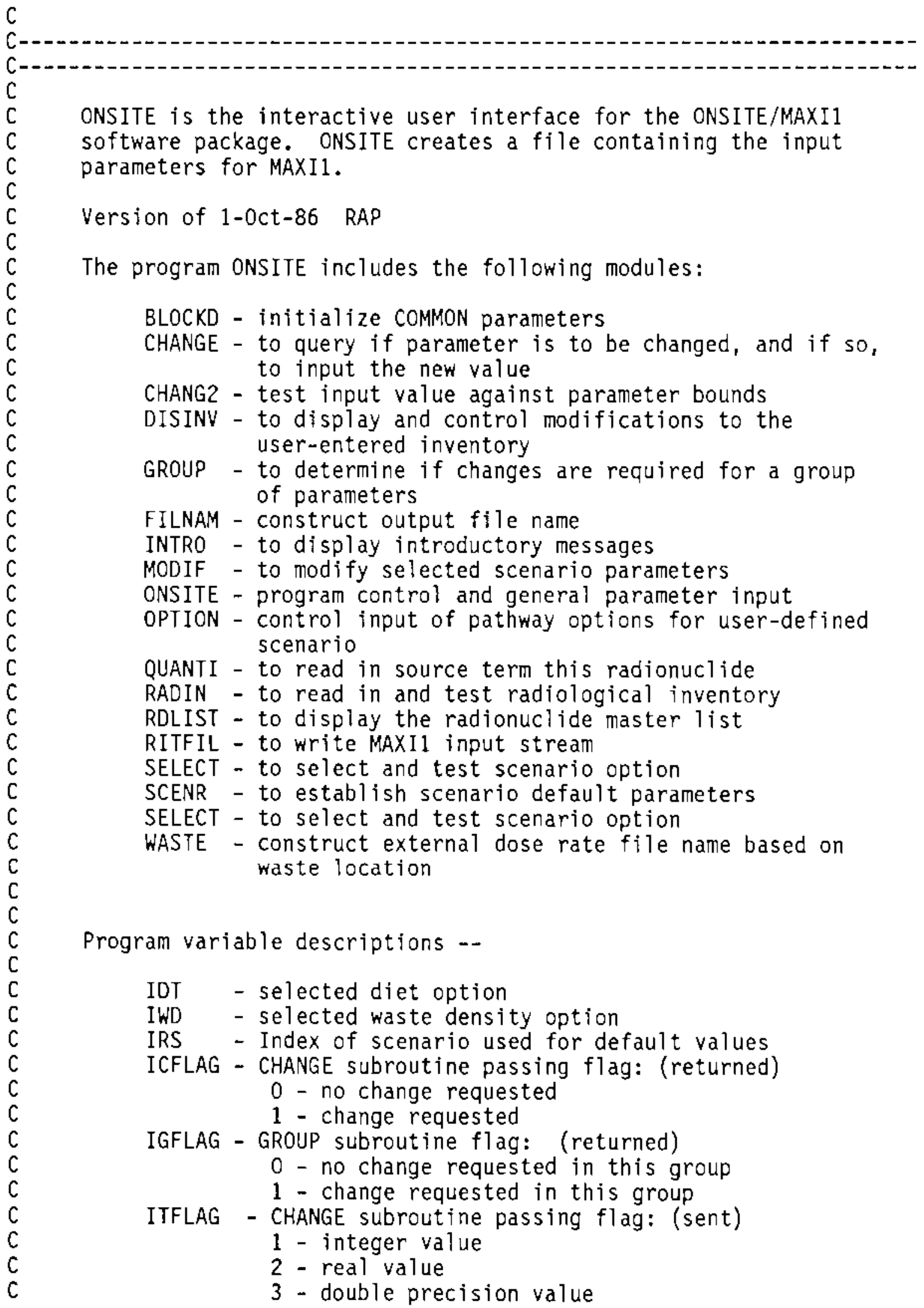




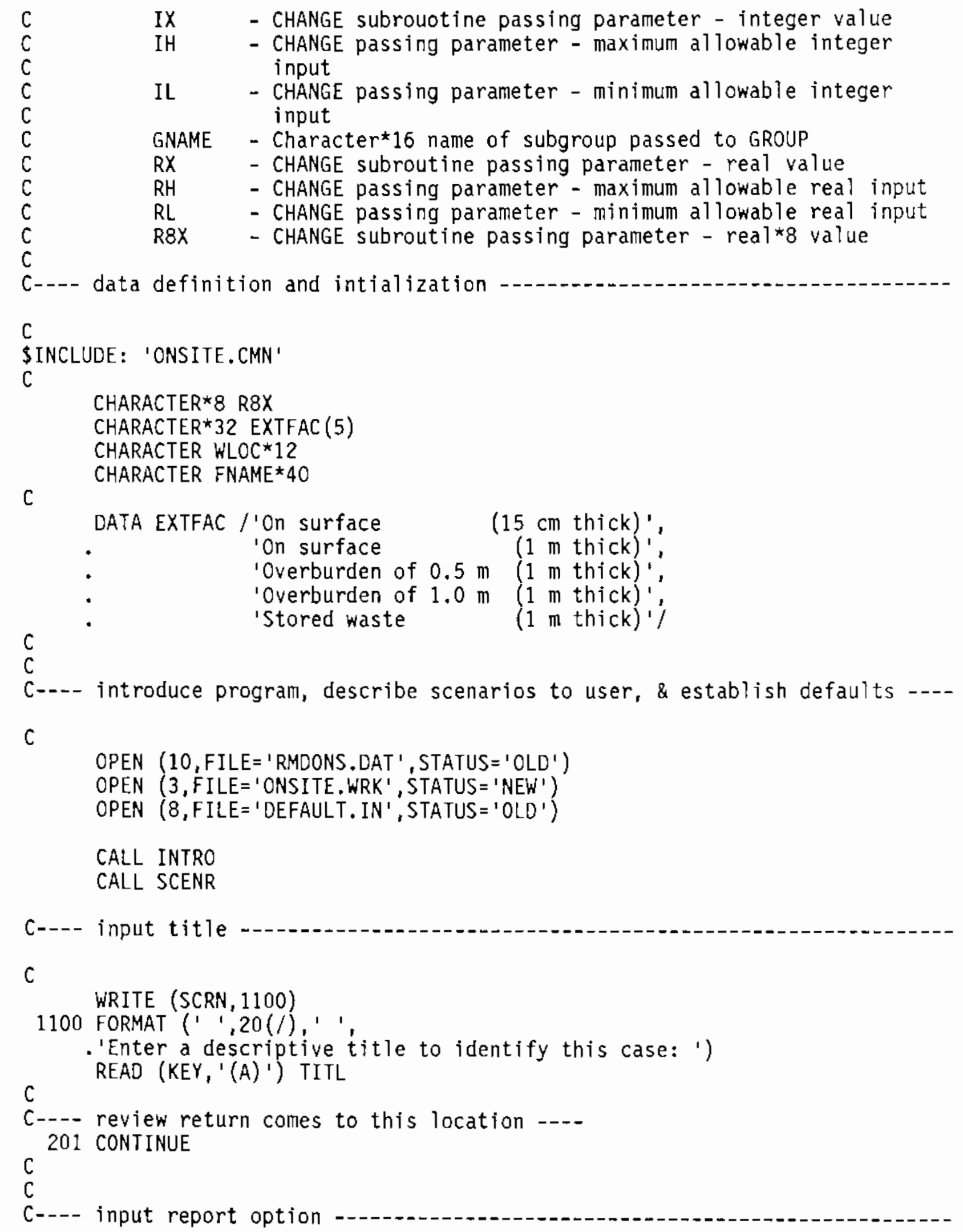


C

WRITE (SCRN, 1101)

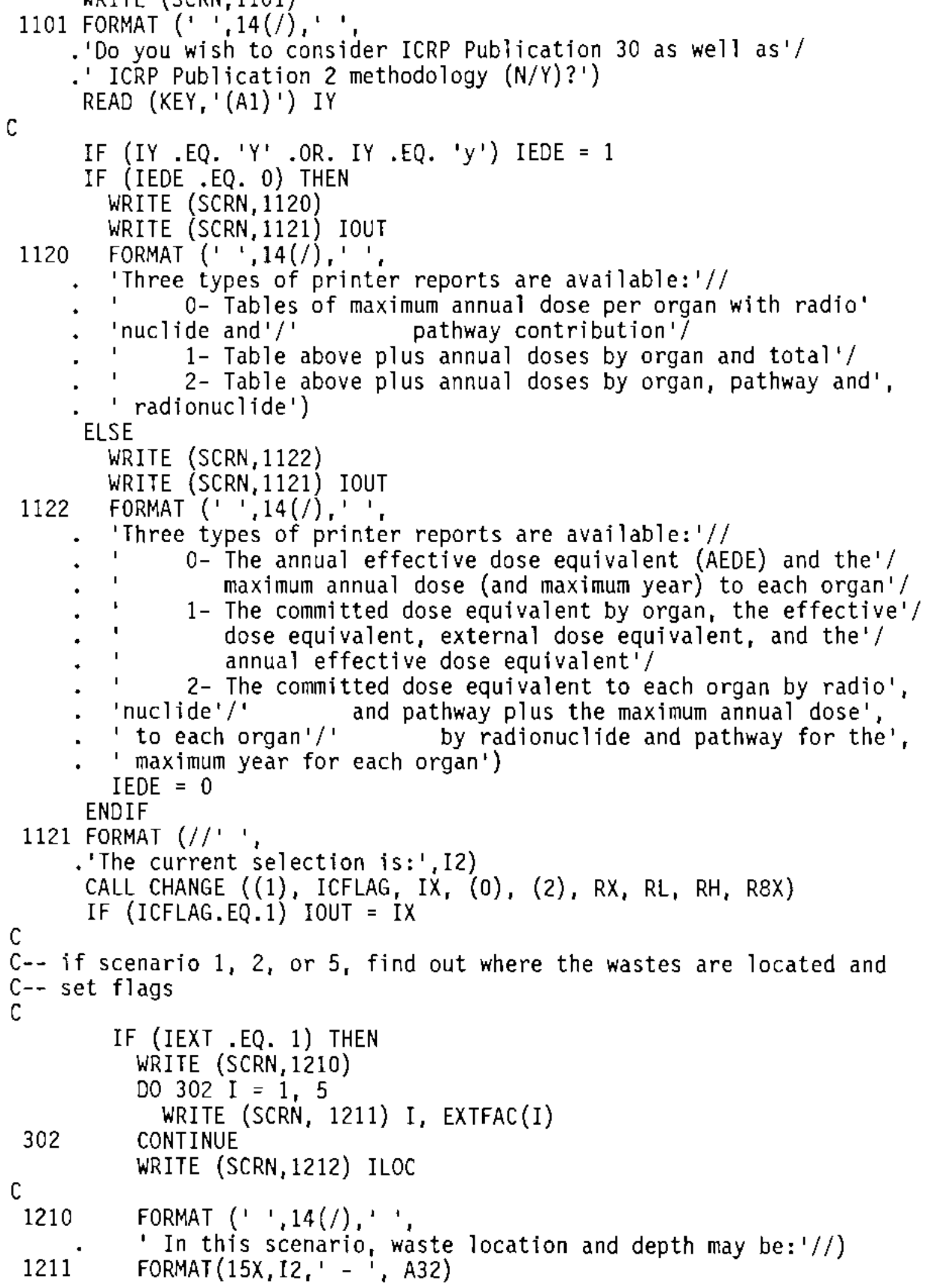


1212 FORMAT (//,' See Table 2.3-1 of NUREG/CR-3620 Supplement.' /

- 'The current selection is: ',I1)

CALL CHANGE ((1), ICFLAG, IX, (1), (5), RX, RL, RH, R8X)

IF (ICFLAG .EQ. 1) ILOC = IX ENDIF

$c$

C--- if user-defined scenario, select pathways -

C

IF (IRS .LT. 5) GO TO 111

IMOD $=1$

CALL OPTION

C

111 CONTINUE

C---- set area correction factor

C

C

400 CONTINUE

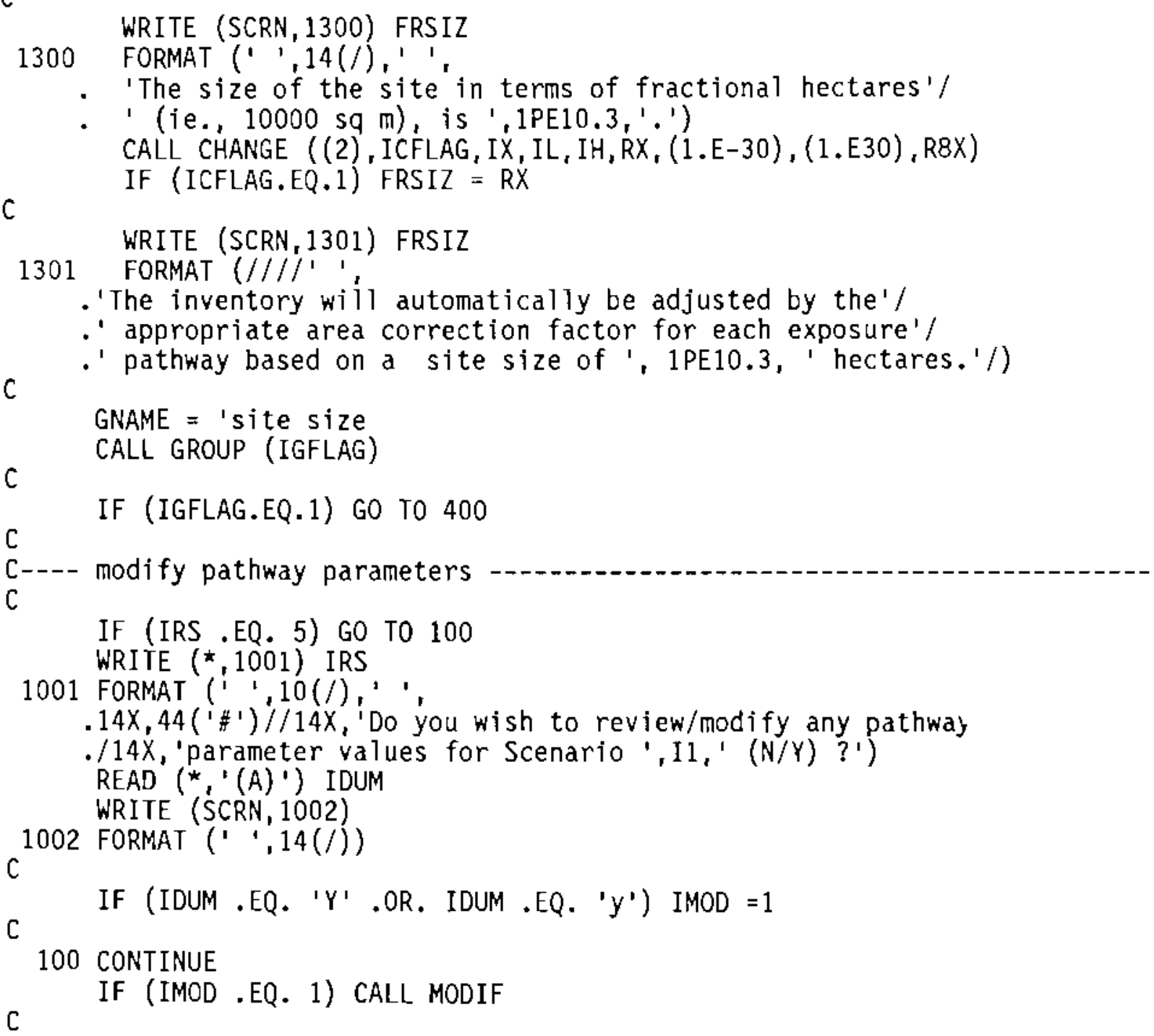




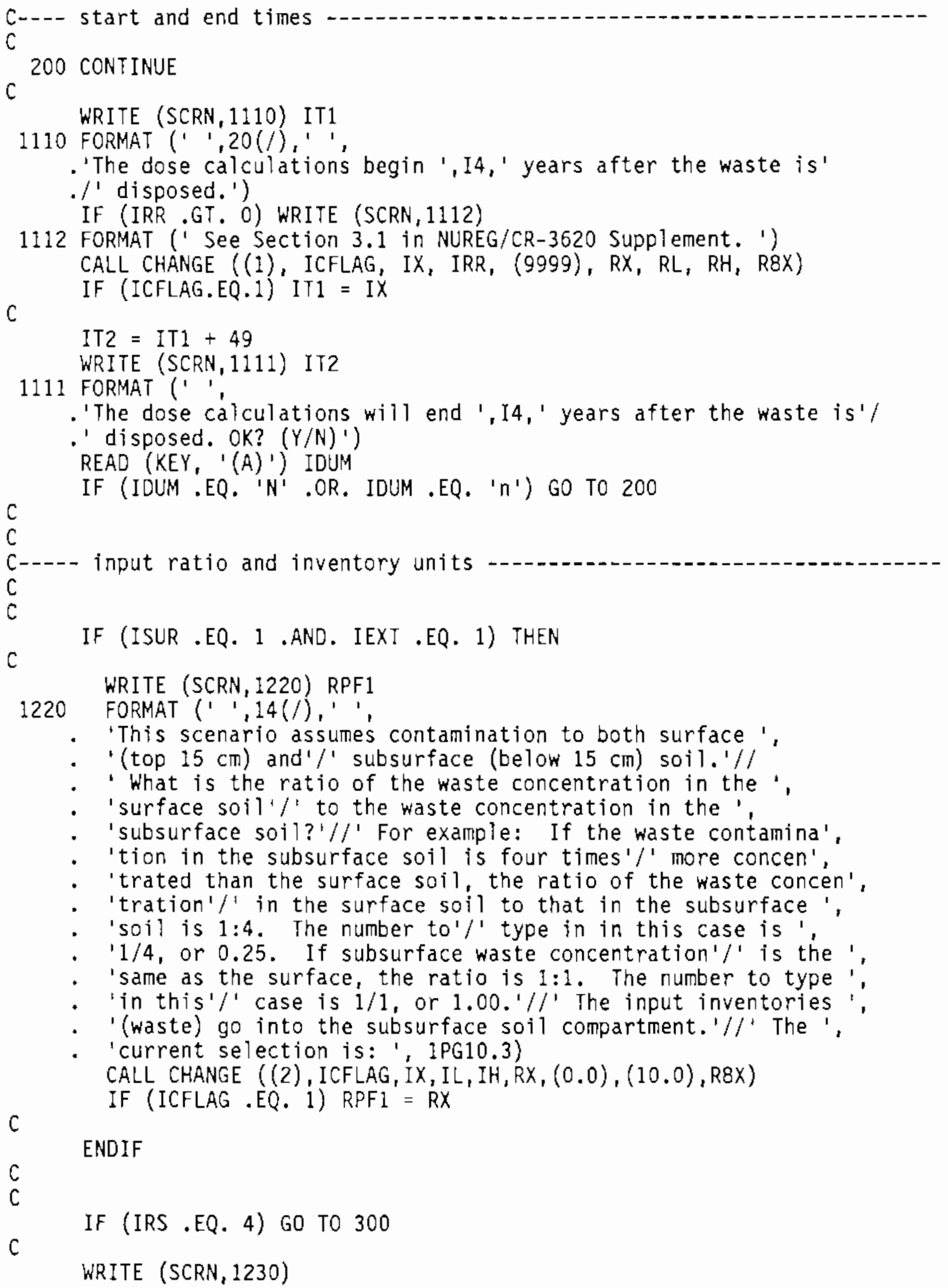




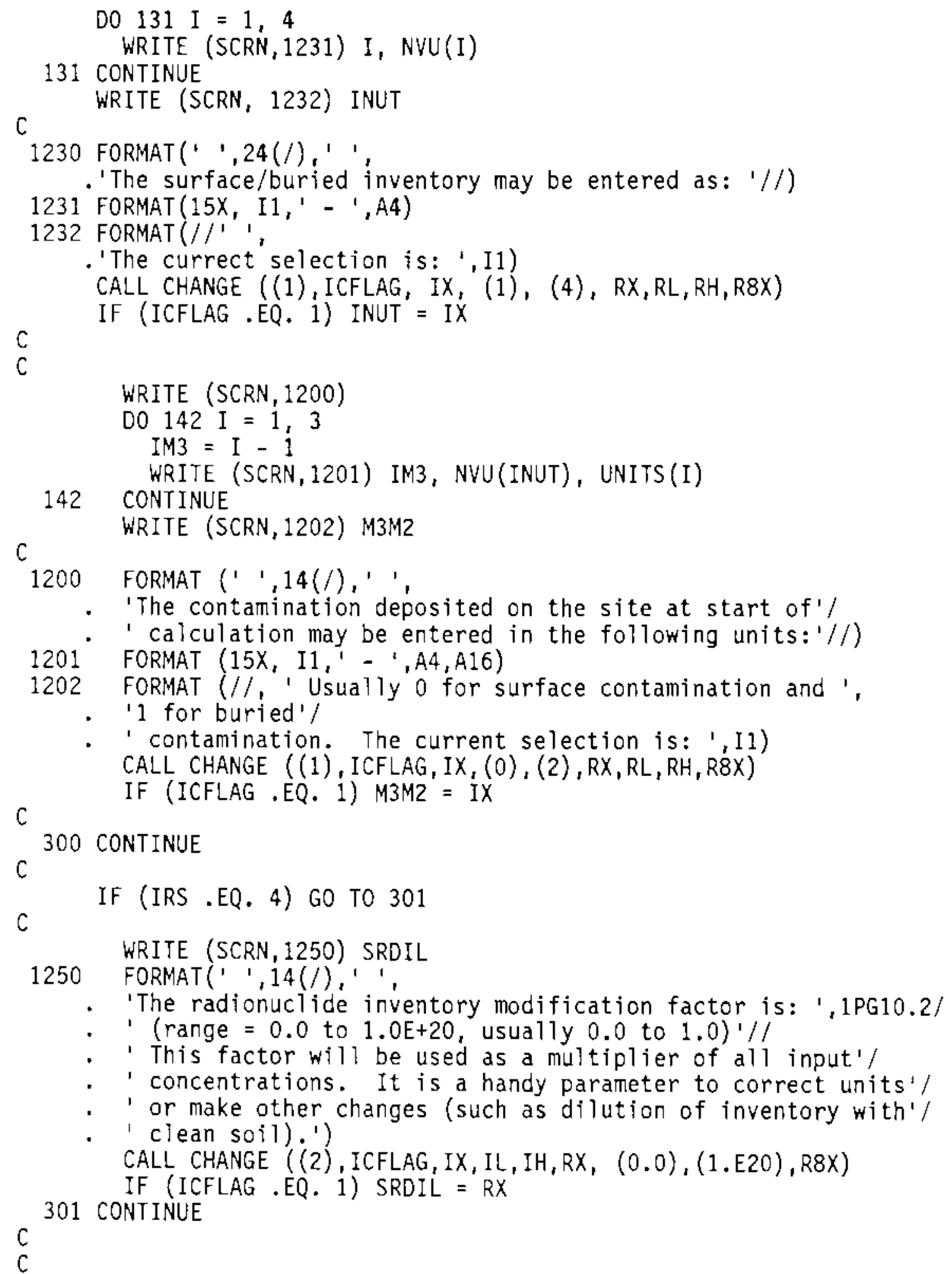


C---- allow user to review parameters before entering the inventory ---------

C

GNAME = 'any of the above'

CALL GROUP (IGFLAG)

IF (IGFLAG .EQ. 1) THEN

$I M O D=0$

GO TO 201

ENDIF

C

C

C---- input inventory and write file

C

CALL RADIN

CALL DISINV

CALL WASTE (WLOC)

CALL FILNAM (FNAME)

C

CALL RITFIL (FNAME)

C.-.- end of program

C

WRITE (SCRN, 8000)

8000 FORMAT $(1,20(/)$,

.10X,'To execute MAXI1 on the IBM PC/XT/AT interactively, type:'// .30X,'MAXI'/)

END 
\$INCLUDE: 'COMPILE.OPT'

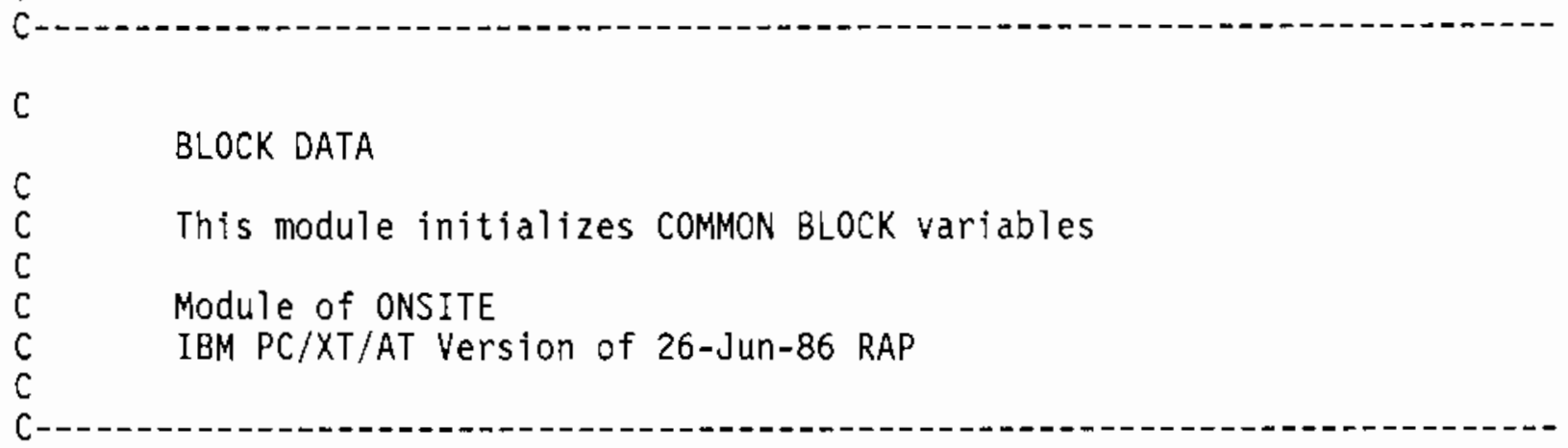

C

\$INCLUDE: 'ONSITE.CMN'

$\mathrm{C}$

C---- set default values per scenario

C

DATA IAIR, IEDE, IDKAIR, IDKWAT, INTRUD, IOUT $/ 6^{\star} 0 /$

DATA IT1, IT2, INUT, M3M2 / $1,50,2 \star 1 /$

DATA SRDIL, DILF, FRSIZ, RPF2, RPF1 / $0.2,44^{\star} 1.0 /$

DATA PLANAM, VOLNAM / 'PLANEA.DAT ", 'BURHFA.DAT ' /

DATA IBLOW, ICLNUP, I1109, XQSITE / 0, 2*I, $0.0 /$

$\mathrm{C}$

DATA RF1, RF2, RIRR, IRR, IMO / 1.0, 0.0, 150.0, 0, $6 /$

C

DATA BRATE, AGE, DEN / 270.0, $0.0,1.0 \mathrm{E} 6 /$

DATA XPDTIN, XMLF / $1.0,1.0 \mathrm{E}-4 /$

DATA IARG, IRES, HREXT, HRINH / $2 * 0,2 * 2000.0 /$

DATA IEXT, INHA, IWAT, ISUR, IFOD / $1,1,0,1,1 /$

DATA DEBUG $/ 4^{\star}$. FALSE. /

DATA IBS, ILOC $/ 1,2 /$

C

DATA IWD, IDT $/ 2{ }^{\star} 1 /$

DATA KEY, SCRN, OUT, IRS, IMOD $/ 22^{\star} 0,7,1,0 /$

DATA NVU / 'PCi ', 'uCi ', 'mCi ', $1 \mathrm{Ci}$ '/

C

DATA NVUNIT / 1.0,1.0E+6, 1.0E+9, $1.0 \mathrm{E}+12 /$

DATA UNITS $/ 1 /$ square meter ',
$1 /$ cubic meter
$1 / \mathrm{kg}$ of soil

C

DATA KFDTYP / $1,2,4,7,9,10,11,12,13,14,5 * 0 /$

DATA GRWP / 90.0, 60.0, $4 * 90.0,30.0,3 \star 90.0,55^{\star} 0.0 /$

DATA YELD / $1.5, .70,4.0,2.0,1.0, .84,1.3,3 * .84,5 * 0.0 /$

DATA HLDUP / $2^{\star} 1.0,2 * 10.0,3 * 1.0,2^{\star} 15.0,1.0,5 * 0.0 /$

DATA CON $/ 2 \star 9.5,76.0,42.0,51.0,19.0,1.10 \mathrm{E}+02,39.0,29.0,8.5,5 * 0.0 /$

C

DATA TRANS / $1.0,5^{\star} .10,1.0,3 * .10,5^{\star} 0.0 /$

DATA KPTHWY / $1,5,3^{\star} 0 /$

DATA HLDUP2 / $2^{*} 1.0,3^{*} 0.0 /$ 


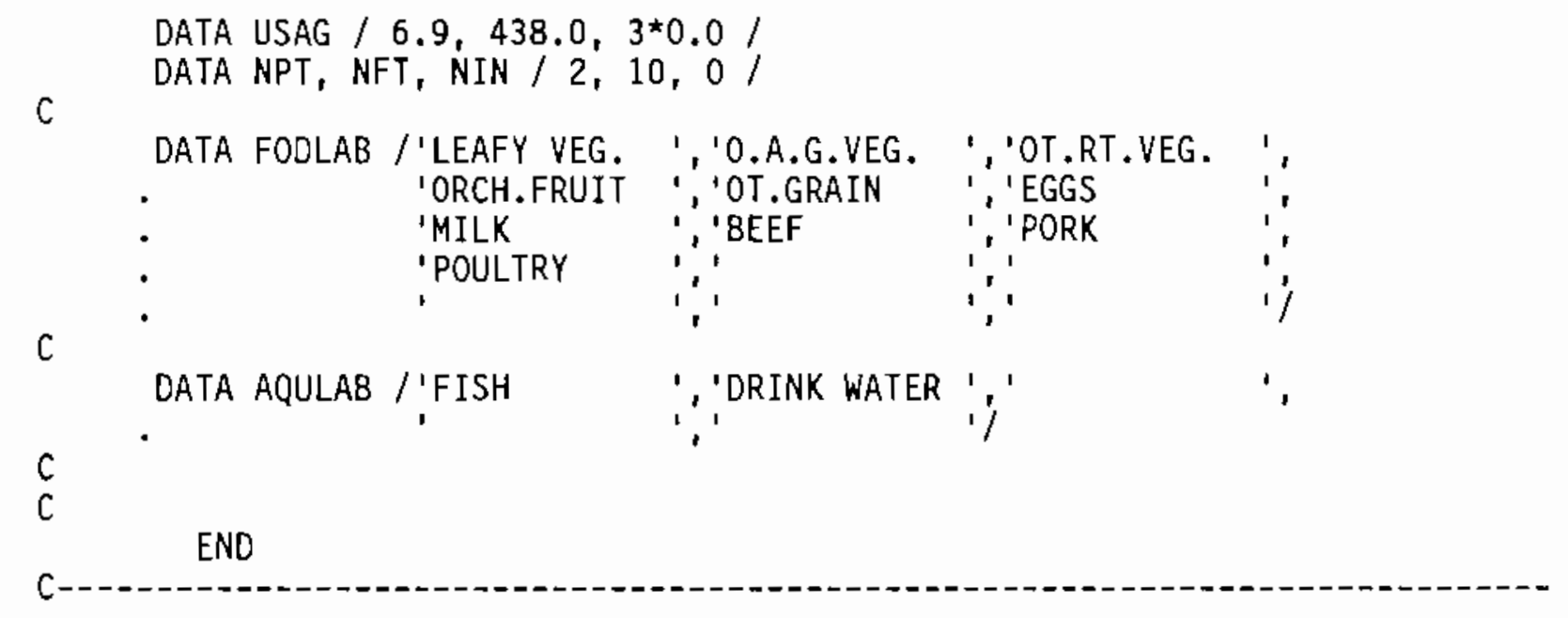


CHANGE is called to query if parameter is to be changed, and if so, to input the new value and test if within the limits.

This subroutine will handle integer, real and alphanumeric input and will test the limits on integer and real input. Alphanumeric input is read into the real*8 variable.

ICFLAG - CHANGE subroutine passing flag: (returned)

0 - no change requested

1 - change requested

2 - input only, not change (received)

ISRT - logical unit number of scratch file used for conversion

IFLAG() - exceptable/not acceptable flag for each character of input string

IH - maximum allowable integer input (received)

IL - minimum allowable integer input (received)

ITFLAG - CHANGE subroutine passing flag: (received)

1 - integer value

2 - real value

3 - alphanumeric input - real*8

IVAL() - ASCII value of each character of input string

IVL - ASCII value of current character of input string

IX - new integer value (returned)

LETTER() - array contianing each character of input string

$\mathrm{RX}$ - new real value (returned)

$\mathrm{RH} \quad$ - maximum allowable real input (received)

$\mathrm{RL} \quad$ - minimum allowable real input (received)

R8X - real*8 alphanumeric string input (returned)

WORD - input string

WRDLEN - number of characters in input string

Module of ONSITE

IBM PC/XT/AT Version of 1-0ct-86 RAP

C

c-

$\mathrm{C}$

\$INCLUDE: 'ONSITE.CMN'

$\mathrm{C}$

CHARACTER $* 8 R 8 X$

C

DIMENSION IVAL(10), IFLAG(10)

C

INTEGER WRDLEN, IVL 


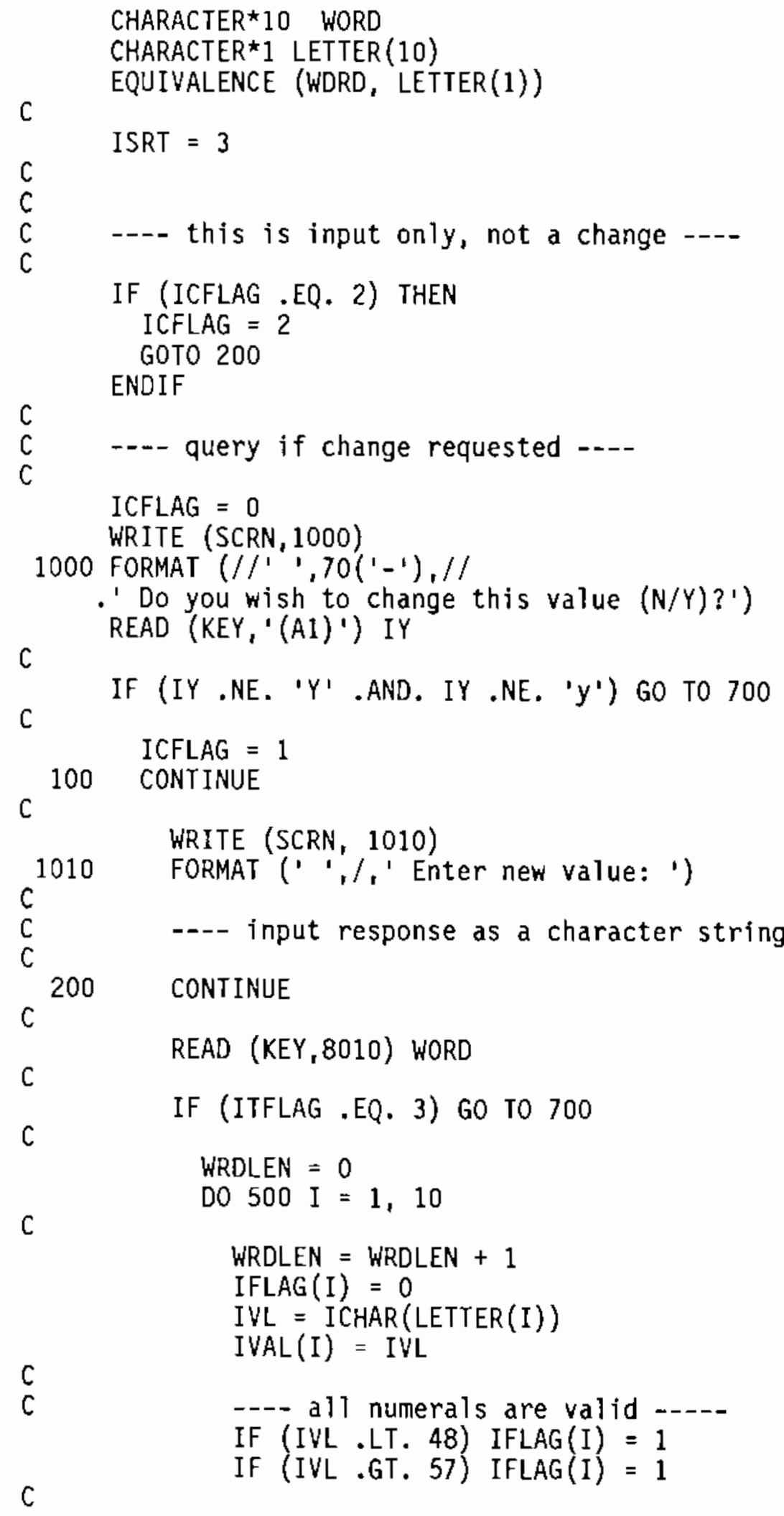


C

$c$
$C$

C

C

C

C

C

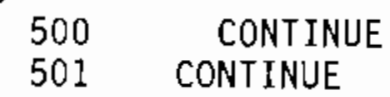

C

C

IF (WRDLEN .LT. 1) WRDLEN $=1$

IF (IVAL (WRDLEN) .EQ. 69) IFLAG(I) $=1$

$$
\mathrm{IGOOD}=1
$$$$
\text { D0 } 502 \text { I = 1, WRDLEN }
$$

502 CONTINUE

C

$$
\begin{aligned}
& \text { IF (IGOOD .EQ. 1) THEN } \\
& \text { WRITE (ISRT, 8010) WORD } \\
& \text { FORMAT (A10) } \\
& \text { BACKSPACE ISRT }
\end{aligned}
$$$$
8010 \text { FORMAT (A10) }
$$

c

$$
\begin{aligned}
& \text { IF (ITFLAG ,EQ. 1) THEN } \\
& \text { READ (ISRT, } \left.{ }^{\star}, \text { ERR }=100, \text { END }=100\right) \text { IX } \\
& \text { IF (IX .LT. IL .OR. IX .GT. IH) GOTO } 100
\end{aligned}
$$

C

C 


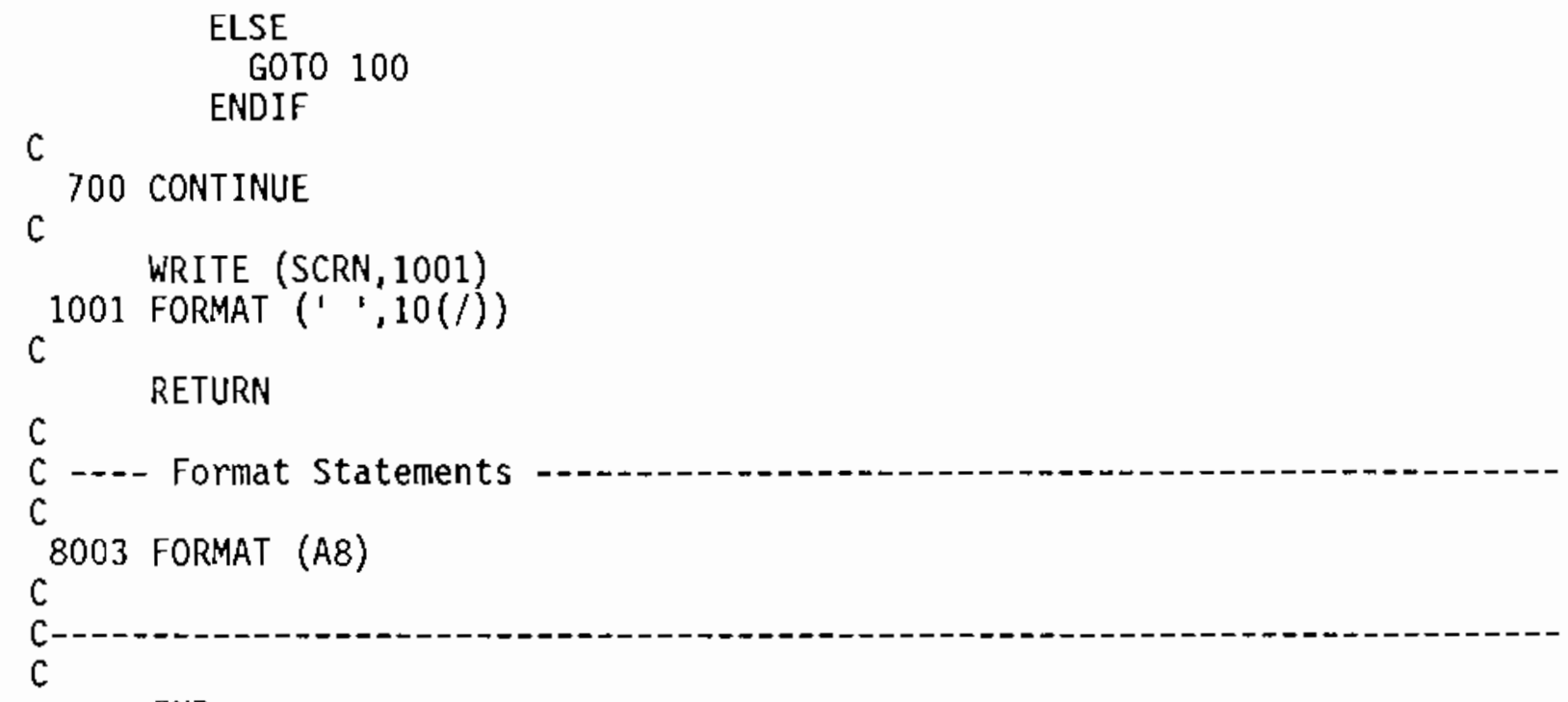

END

A. 98 
\$INCLUDE: 'COMPILE.OPT'

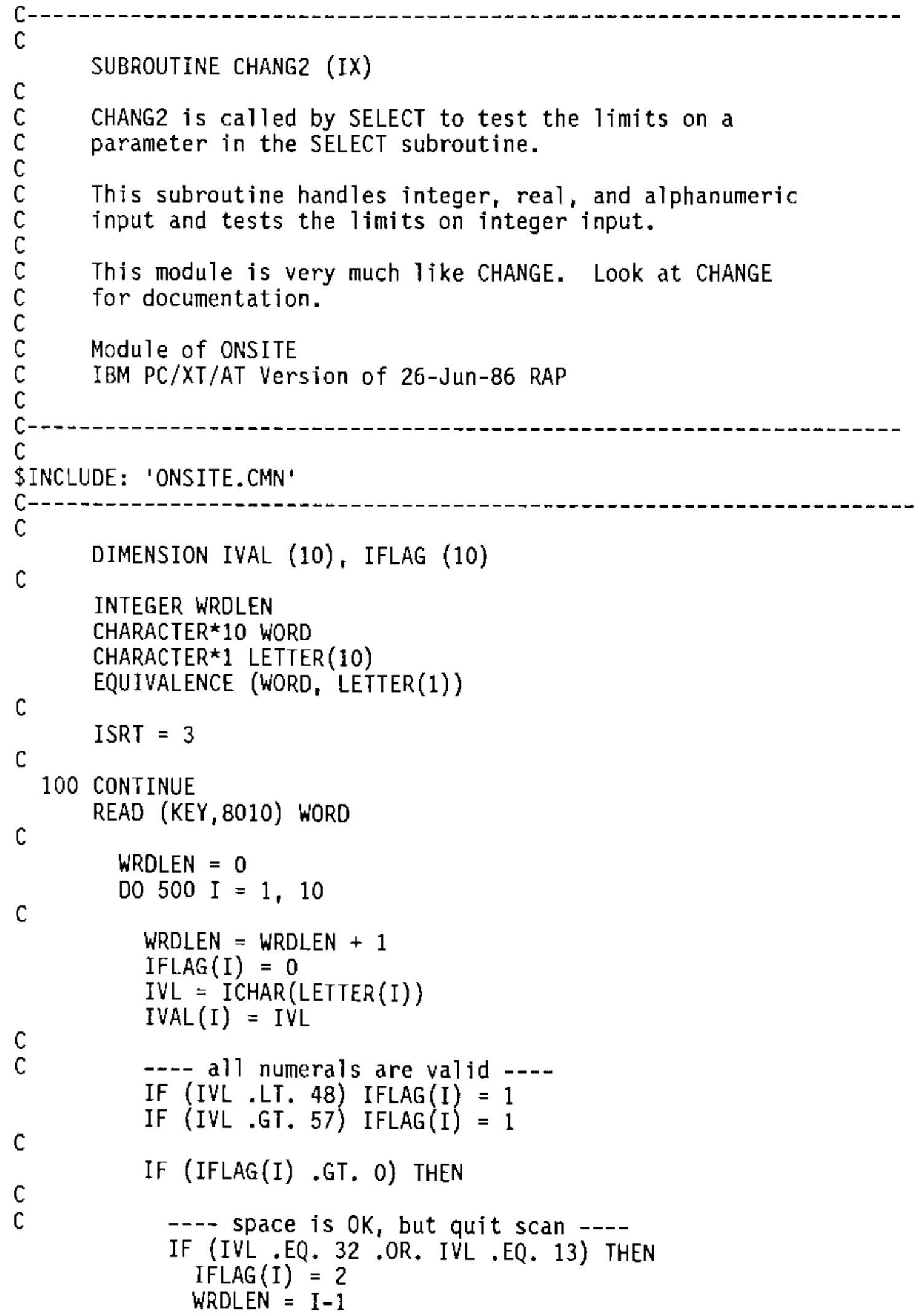




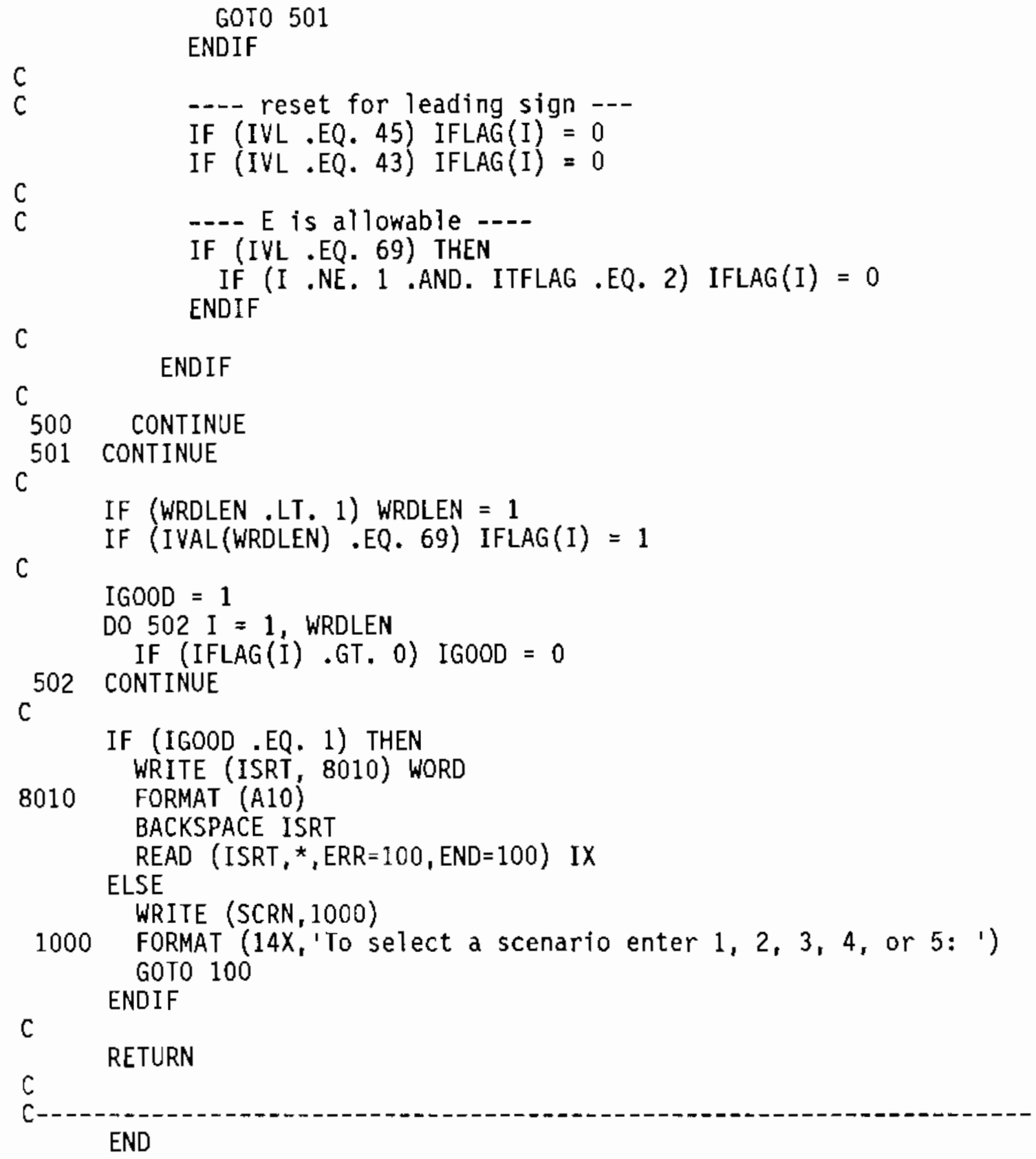


\$INCLUDE: 'COMPILE.OPT'

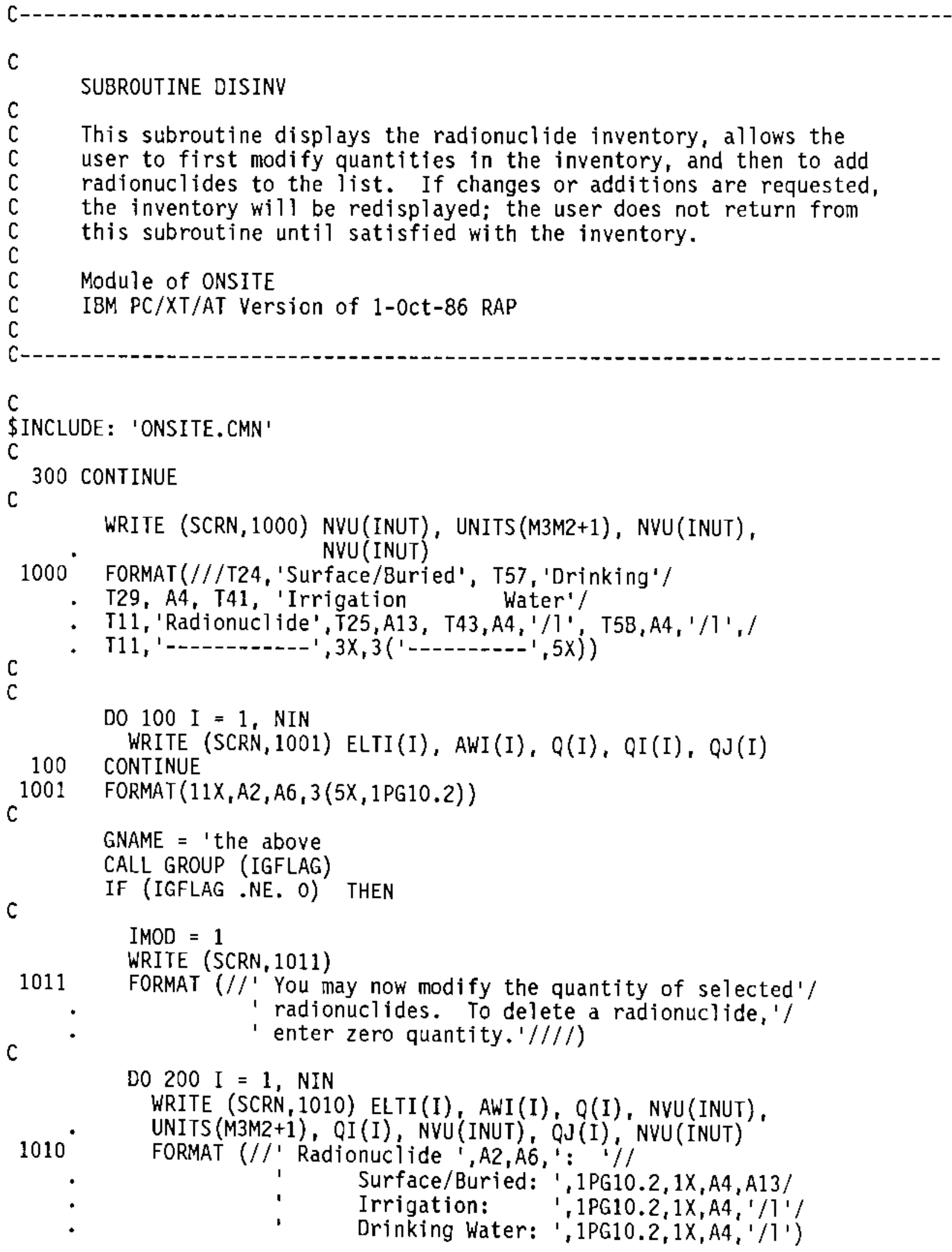

A. 101 


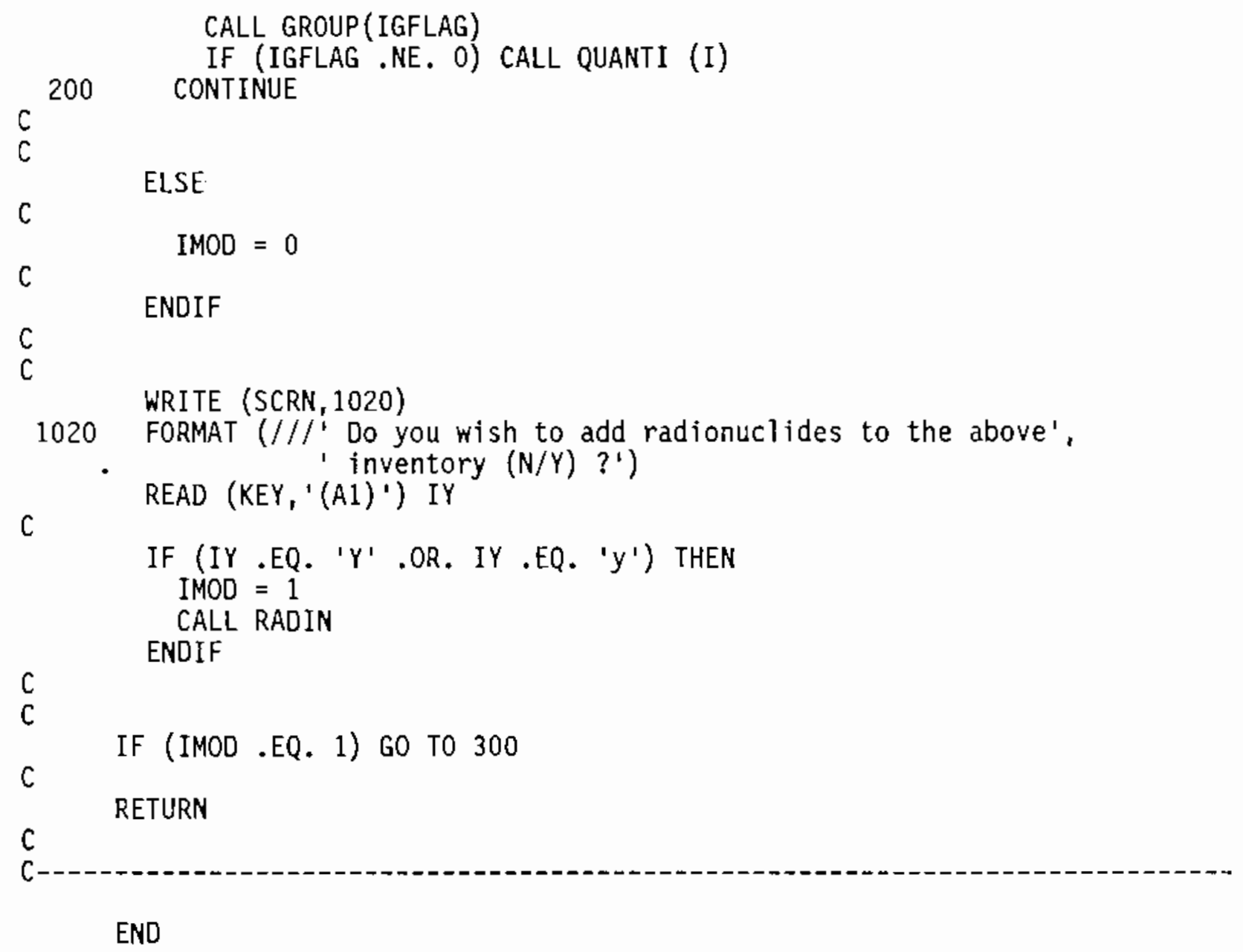

A. 102 
\$INCLUDE: 'COMPILE.OPT'

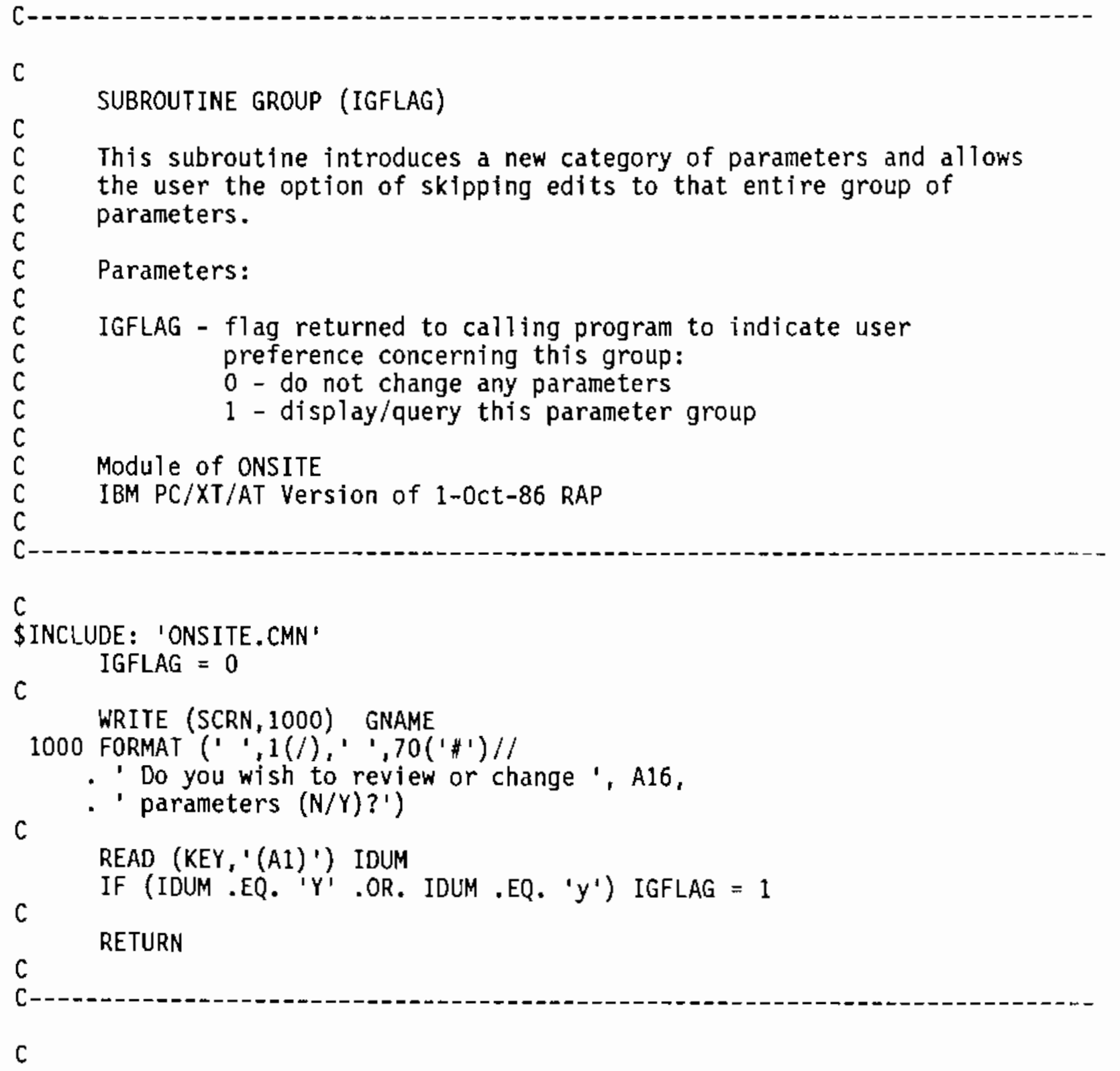

END 


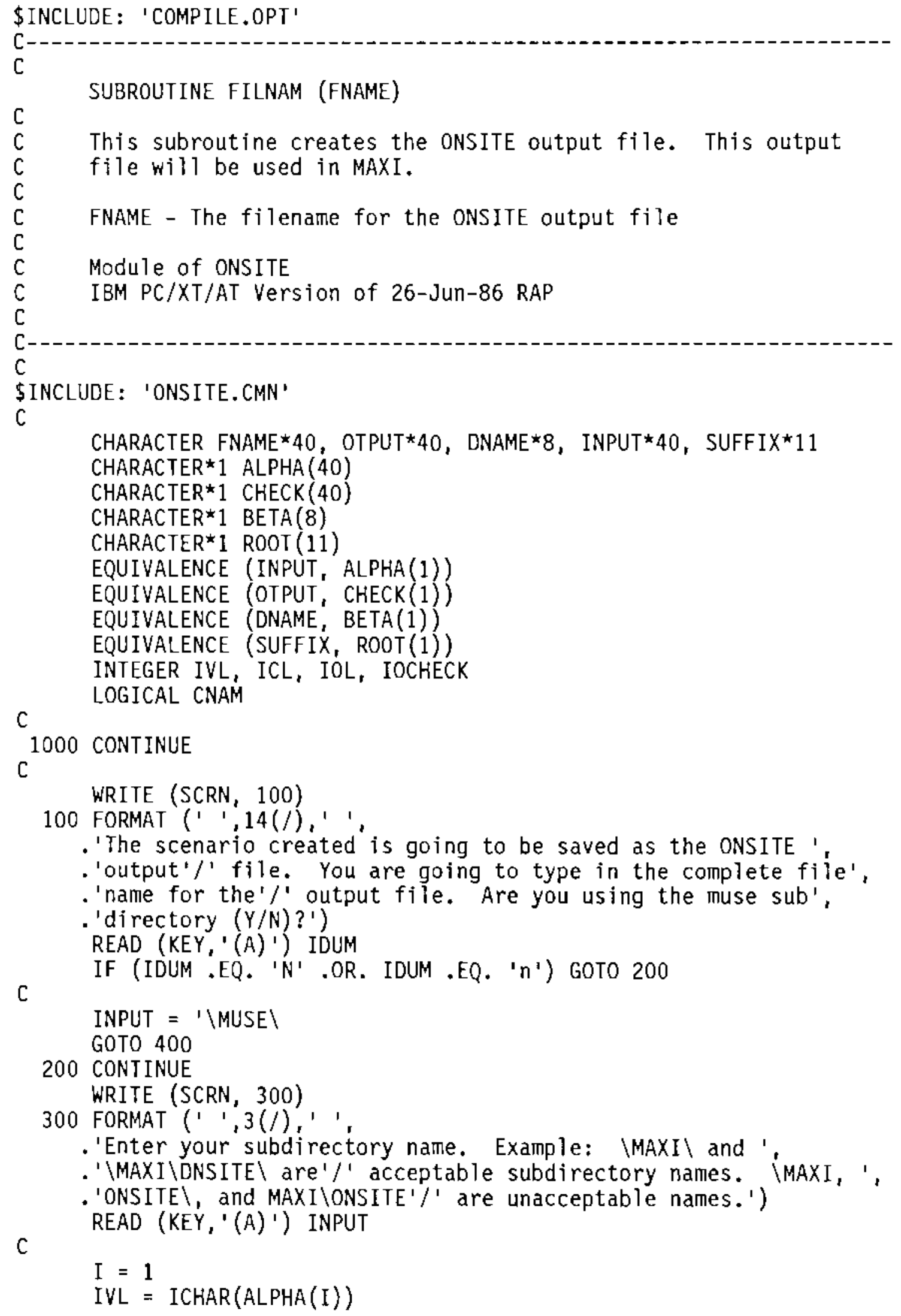




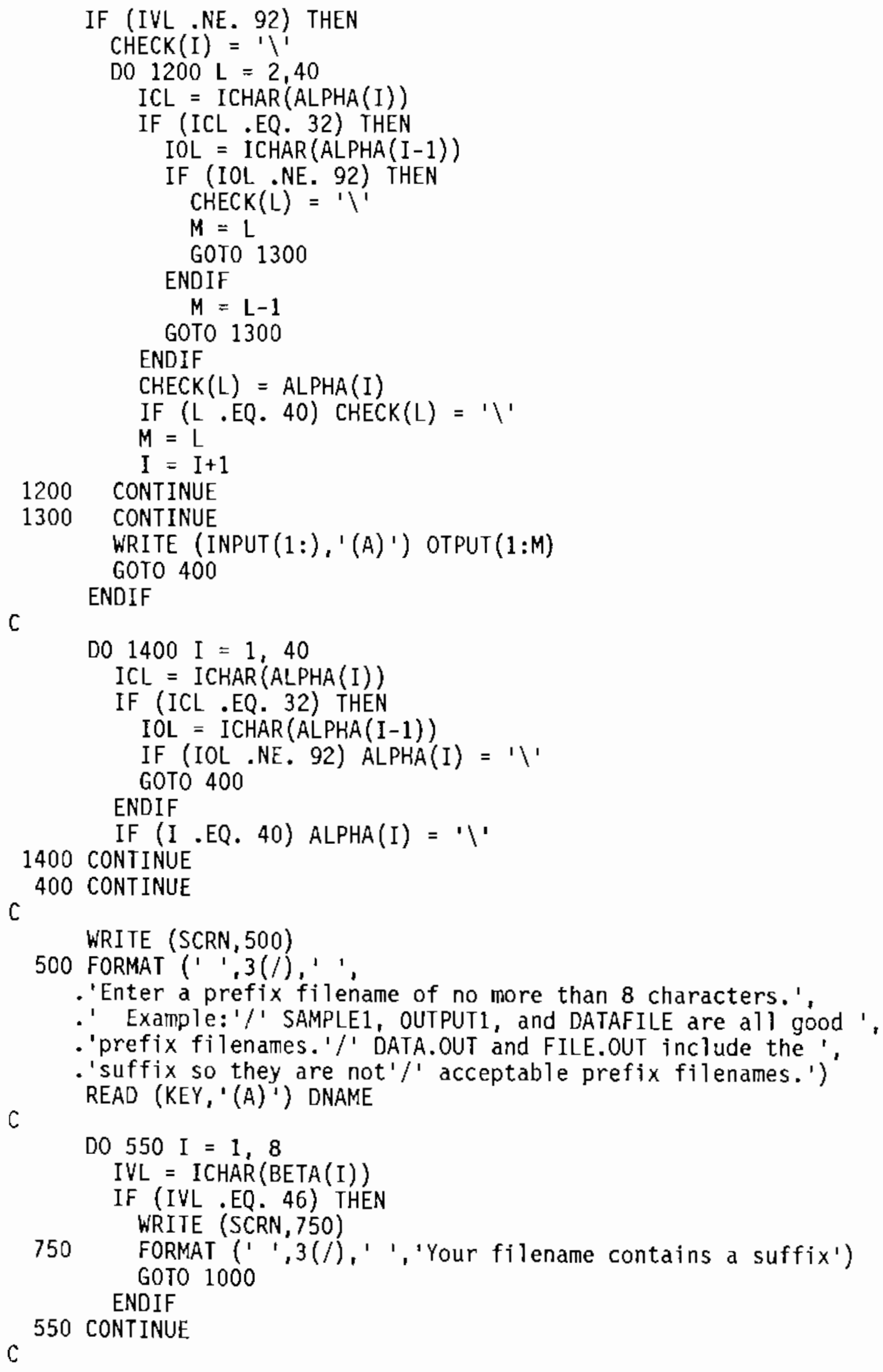




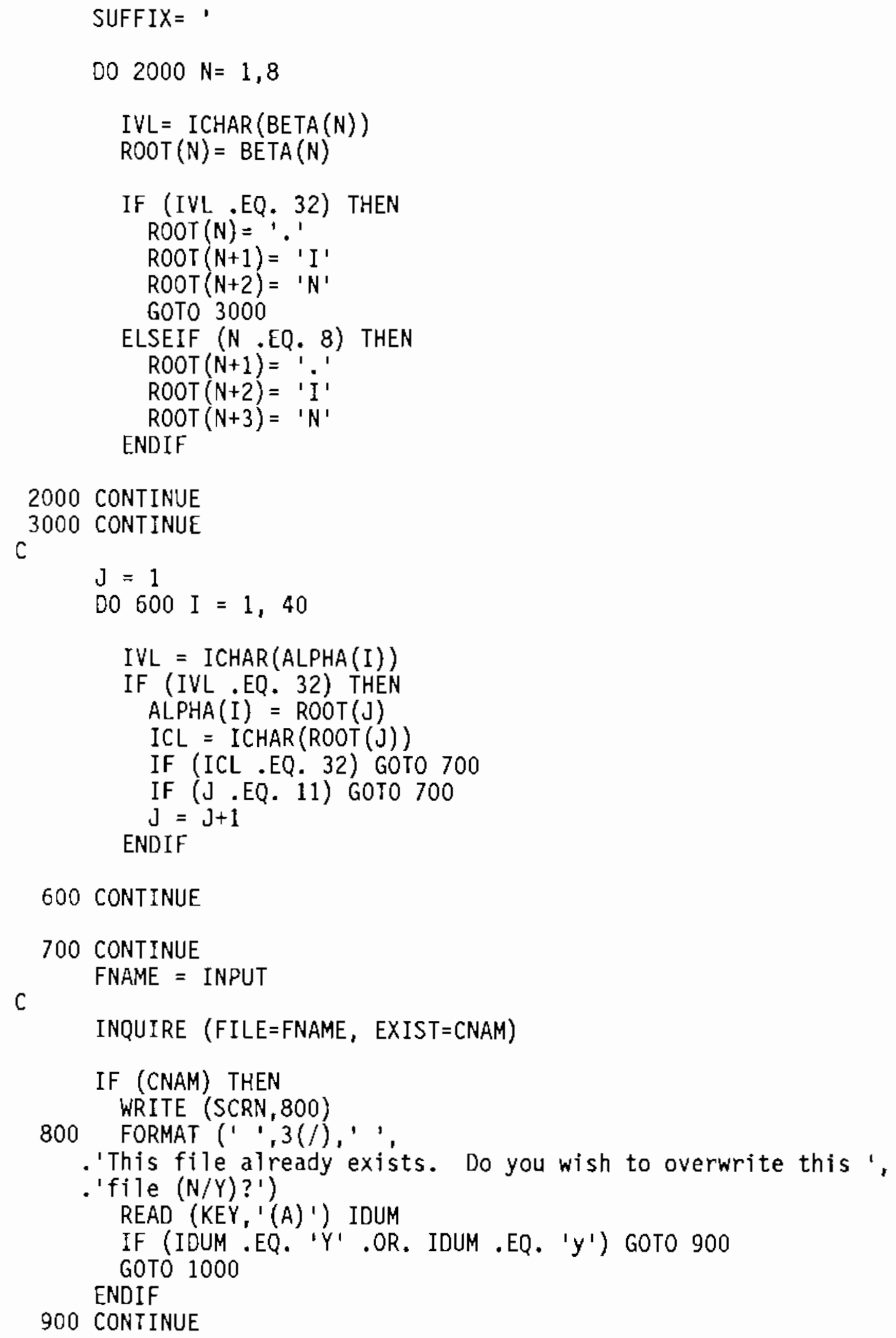




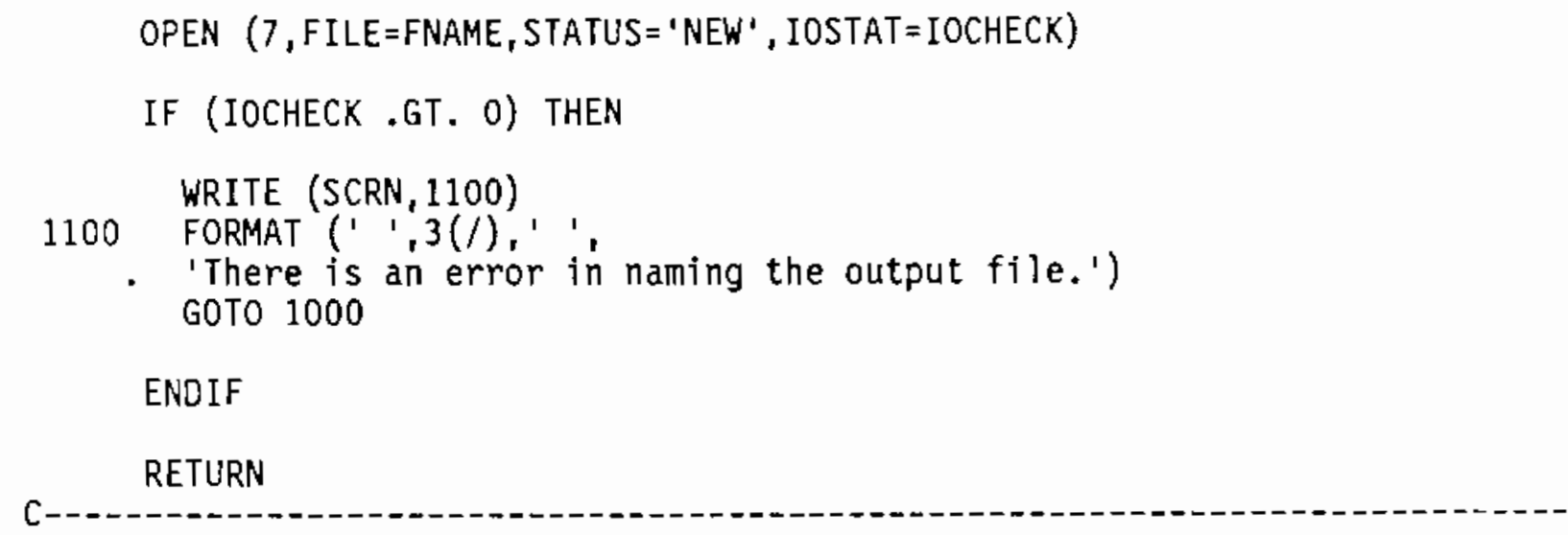

END 
\$INCLUDE: 'COMPILE.OPT'

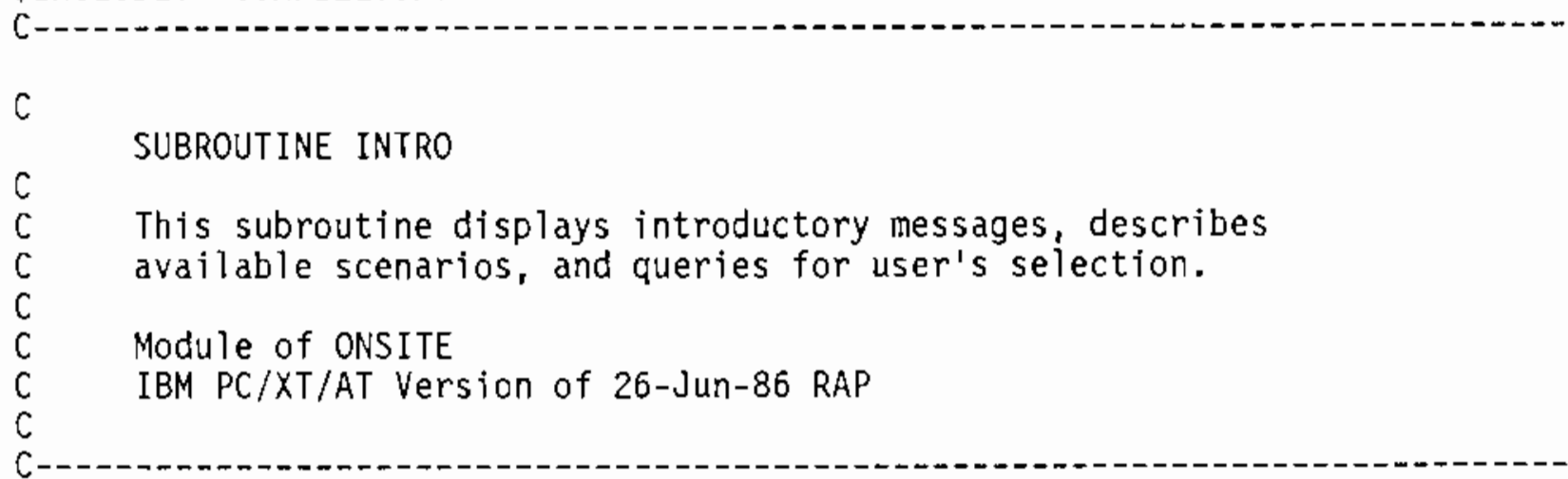

C

\$INCLUDE: 'ONSITE.CMN'

$\mathrm{C}$

C

WRITE (SCRN, 2000)

2000 FORMAT $(' 1,24(/), 30 X, 19('=1) / 33 X, '$ ONSITE/MAXI1 $1 / 30 X$,

C

- $\left.19\left({ }^{\prime}={ }^{\prime}\right), / / / / / /\right)$

WRITE (SCRN, 2011)

2011 FORMAT (14X,'This interactive program will assist you ',

.' in the creation'/

$.14 X_{1}$ ' of scenarios for assessment of onsite disposal of low-1/

.14X,'level waste. Doses to man through the specified pathways'/

$.14 X$, 'will be simulated by the computer program MAXI1. ICRP'/

.14X, 'Publication 30 as well as ICRP Publication 2 methodology'/

$.14 X$, 'may be used to calculate dose. '///

C

$.14 X$, 'The following notes may be of interest: ')

$\mathrm{C}$

WRITE (SCRN, 2010)

READ (KEY, 8003) IDUM

WRITE (SCRN, 2001)

2001 FORMAT ( $1,24(/)$,

$.14 X, 11)$ If the default condition is selected, you need only'/

.14X, 'press 〈return〉. YES-or-NO questions are designated by'/

$.14 X, '(Y / N)$ and should be answered with a $Y$ or $N$. The default'/

C

.14X,' condition is always listed first.' )

WRITE (SCRN, 2002)

2002 FORMAT (' 0 ',

$\left..13 X,{ }^{2} 2\right)$ The values you enter will be tested against reasonable'/

$.14 X, ' 1$ imits and if they are not accepted you will be asked to'/

C

.14X,' supply another value.' )

WRITE (SCRN, 2010)

2010 FORMAT (/14X, 'When you have finished reading, press (return>') READ (KEY, 8003) IDUM

8003 FORMAT (A8) 
C

C---- loop until user is satisfied with scenario selection

$\mathrm{C}$

C

100 CONTINUE

C

CALL SELECT

IF (IRS .EQ. 1) WRITE (SCRN, 2100)

2100 FORMAT (' ', 24(/),

.14X, 'SCENARIO 1: External Exposure'//

.14X.' This scenario can be used alone or as part of Scenarios 2'/

.14X,' and 3. Occupational conditions of $2000 \mathrm{~h} / \mathrm{yr}$ of external'/

.14X, 'exposure are assumed. Waste may be located on the surface, '/

$\mathrm{C}$

$.14 X_{1}$ ' 'buried at $0.5 \mathrm{~m}$, buried at $1.0 \mathrm{~m}$, or stored. ')

IF (IRS .EQ. 2) WRITE (SCRN, 2200)

2200 FORMAT ( $1,24(/)$,

.14X, 'SCENARIO 2: External Exposure plus Inhalation from Resus',

- 'pension'//

$.14 x$, 'This scenario assumes surface contamination results over a'/

$.14 X, ' 1 i m i t e d$ area. The scenario defaults to $2000 \mathrm{~h} / \mathrm{yr}$ exposure'/

.14X,' to surface contamination and inhalation. The Anspaugh')

IF (IRS .EQ. 2) WRITE (SCRN, 2201)

2201 FORMAT (14X,

- 'resuspension model is used. User may define the fraction'/

$.14 X_{1}$, 'of soil in the top $15 \mathrm{~cm}$ containing buried waste (defaults\%

C

$.14 X$, ' to 0.2$).$ ')

IF (IRS .EQ. 3) WRITE (SCRN, 2300)

2300 FORMAT (' $1,24(/)$,

.14X, 'SCENARIO 3: Agricultural Activities'//

-14X,'This scenario assumes surface contamination (as in Scenario'/

$\left..14 X_{1}, 2\right)$ with farming; defaults to $2000 \mathrm{~h} / \mathrm{yr}$ exposure to surface')

IF (IRS .EQ. 3) WRITE (SCRN, 2301)

2301 FORMAT (14X,

- 'contamination and inhalation. Anspaugh resuspension model'/

$.14 x_{1}$ ' 'is used. Scenario defaults to total diet of fruits, veg-"/

$.14 X$, 'atables, and animal products grown on the site. The user'/

.14X.' may specify the percentage of soil containing waste in the'/

$\mathrm{C}$

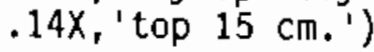

IF (IRS .EQ. 4) WRITE (SCRN, 2400)

2400 FORMAT (' $: 24(/)$,

. 14X, 'SCENARI0 4: Irrigation and Drinking Water'//

-14X. 'This scenario accounts for the use of well or river water'/

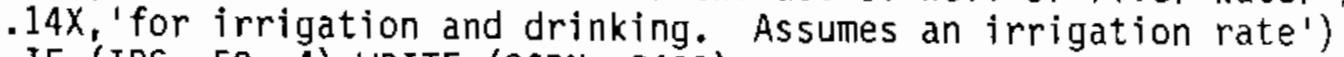

IF (IRS.EQ. 4) WRITE (SCRN, 240I)

2401 FORMAT (14X,

- 'of $1501 / \mathrm{sq} \mathrm{m} / \mathrm{mo}$ for 6 months. Defaults to Anspaugh model'/

$.14 X^{\prime}$, for inhalation. Assumes total diet and 1.21 iters/day of'/

$.14 X$,'drinking water.' $)$ 


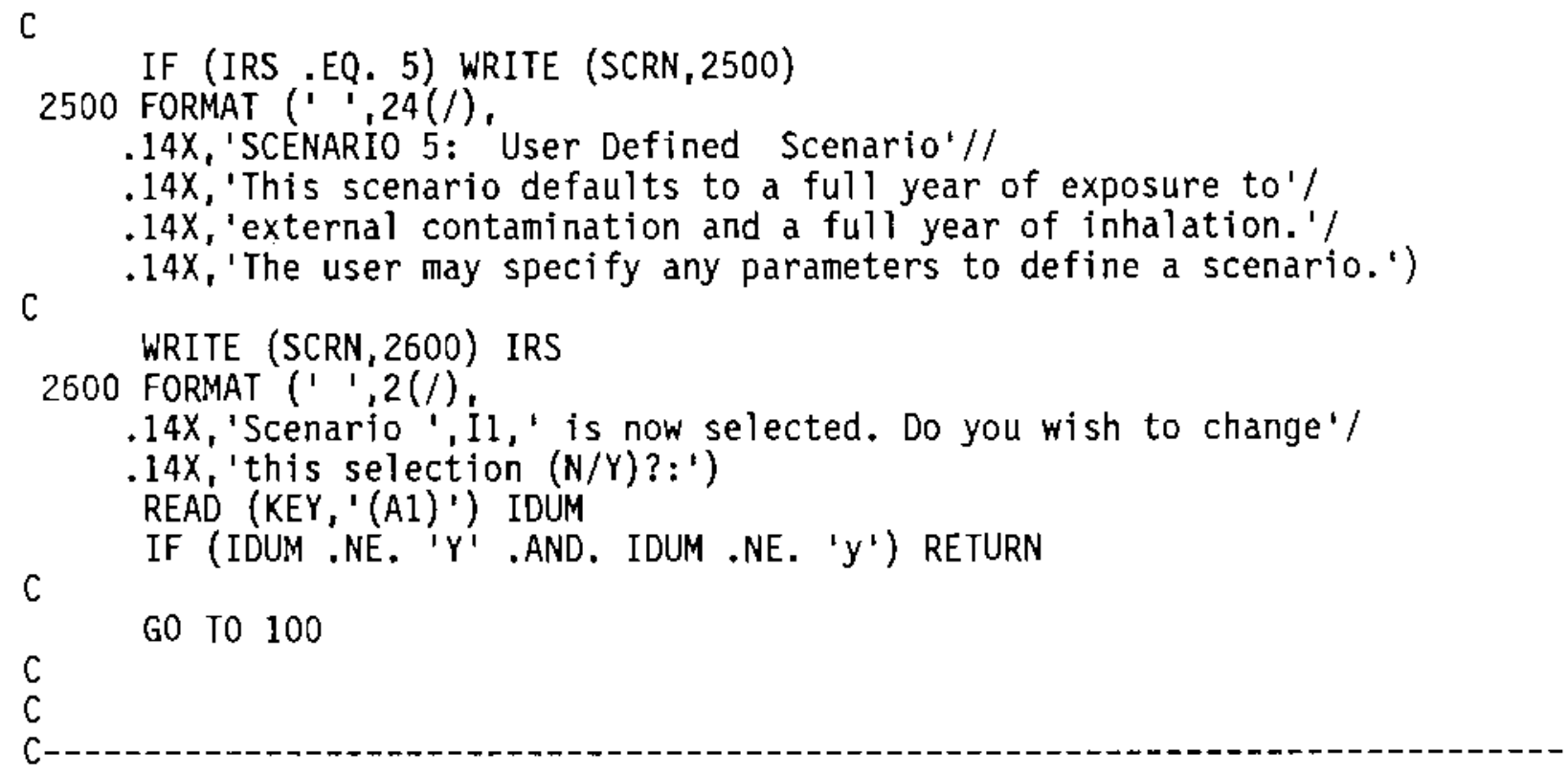

END 
\$INCLUDE: 'COMPILE.OPT'

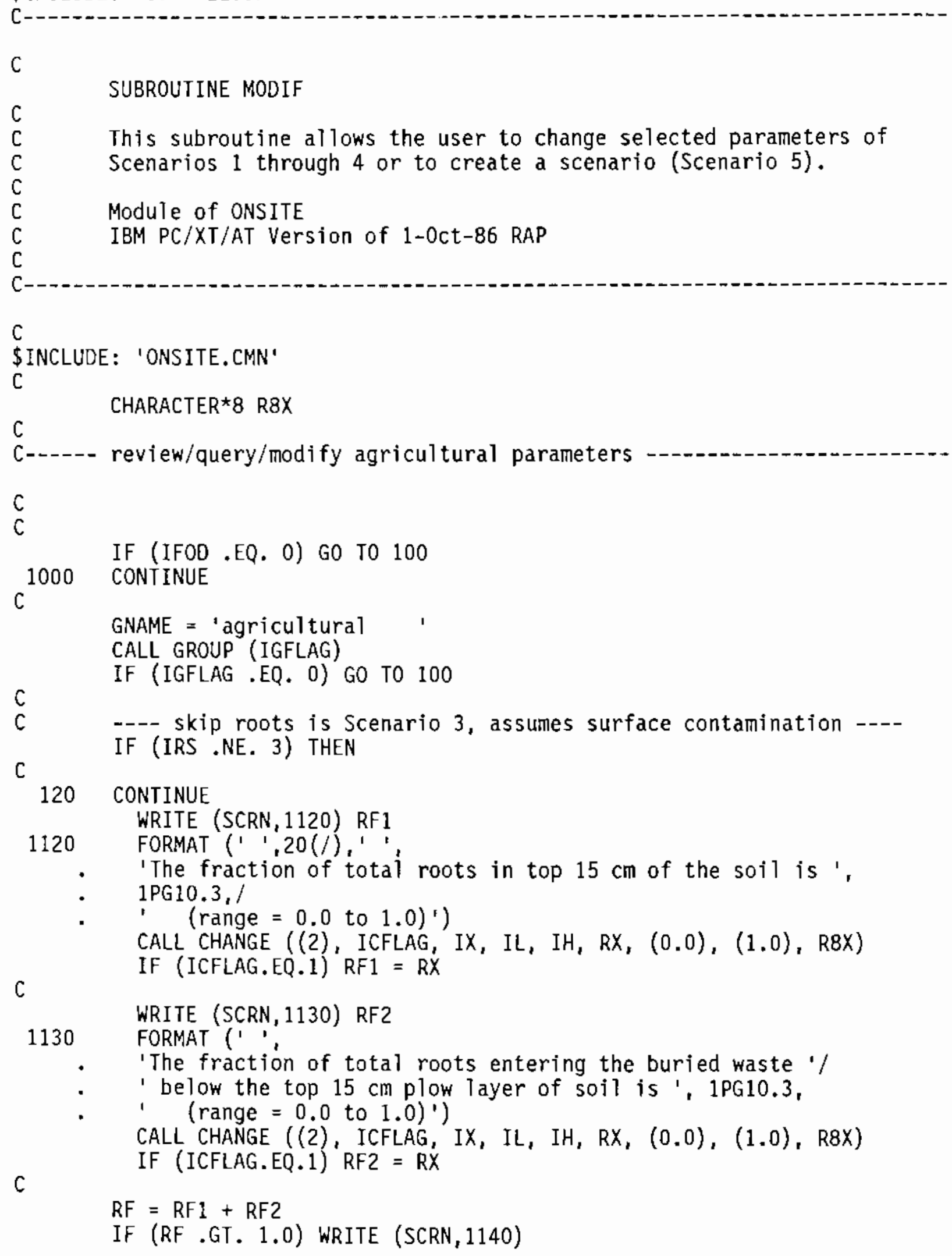

C \$INCLUDE: 'ONSITE.CMN'

C

CHARACTER* 8 R $8 X$

C

C

C $c^{1000}$

IF (IFOO .EQ. O) GO TO 100 CONTINUE

GNAME $=$ 'agricul tural 1

CALL GROUP (IGFLAG)

$c$

IF (IGFLAG .EQ. 0) GO TO 100

C ---- skip roots is Scenario 3, assumes surface contamination ---IF (IRS .NE. 3) THEN

C

120 CONTINUE

WRITE (SCRN, 1120) RF1

1120 FORMAT $(1,20(/), 1$ '

- 'The fraction of total roots in top $15 \mathrm{~cm}$ of the soil is ',

- $1 \mathrm{PG} 10.3,1$

- $\quad$ (range $=0.0$ to 1.0$)^{\prime}$ )

CALL CHANGE ((2), ICFLAG, IX, IL, IH, RX, $(0.0),(1.0), \mathrm{R} 8 X)$

C IF (ICFLAG.EQ.1) RF1 = RX

1130 FORMAT ( 1 ',

- The fraction of total roots entering the buried waste $1 /$

- below the top $15 \mathrm{~cm}$ plow Tayer of soil is ', 1PG10.3,

- $\quad$ (range $=0.0$ to 1.0$)^{\prime}$ )

CALL CHANGE ((2), ICFLAG, IX, IL, IH, RX, $(0.0),(1.0), \mathrm{R} 8 \mathrm{X})$ IF (ICFLAG.EQ.1) RF2 = RX

C

$\mathrm{RF}=\mathrm{RF} 1+\mathrm{RF} 2$

IF (RF.GT. 1.0) WRITE (SCRN, 1140) 
1140 FORMAT (///' !!! The sum of the previous two fractions cannot '/

- ' be greater than 1.0: REENTER !!!')

IF (RF .GT. 1.0) GO TO 120

ENDIF

C

WRITE (SCRN, 1166) IDT

1166 FORMAT (' ',20(/),' ',

- 'The R.G. 1.109 diet raised onsite may be either:'//

- 9x, 1 - Total diet

- $9 x, 12$ - Vegetable product diet (plant only) $\%$

- 9x, ' 3 - Animal product diet (milk, eggs, and meat only)',

- $/ /$, ' The default selection is ', I2,' ')

CALL CHANGE ((1), ICFLAG, IX, (1), (3), RX, RL, RH, R8X)

C

IF (ICFLAG.EQ.1) IDT $=$ IX

IF (IDT .EQ. 2) THEN

DO $400 \mathrm{I}=6,10$

$\operatorname{CON}(I)=0.0$

400 CONTINUE

ELSEIF (IDT .EQ. 3) THEN

DO $450 \mathrm{I}=1,5$

$\operatorname{CON}(\mathrm{I})=0.0$

450

CONTINUE

ENDIF

do $475 i=1,10$

475 continue

write $\left({ }^{*},{ }^{\prime}\right) ' \operatorname{con}(1, i, 1)=1, \operatorname{con}(i)$

C

IF (FRSIZ .LT. 1.0) GOTO 1161

WRITE (SCRN, 1160) RPF2

1160 FORMAT (' ',20(/), ' ',

- 'The fraction of the selected diet grown on the site'.

- ' is ', 1 PG10.3, $/$ (range $=0.0$ to 1.0$)^{\prime}$ ')

CALL CHANGE ((2), ICFLAG, IX, IL, IH, RX, $(0.0),(1.0), \mathrm{R} 8 X)$

IF (ICFLAG.EQ.1) RPF2 = RX

CONT INUE

C

IF (IWAT .EQ. O) GOTO 100

WRITE (SCRN, 1100) RIRR

1100 FORMAT (' ',

- The irrigation rate in liters per square meter per month is ',

- $1 P G 10.3,1$

- ' (range $=0.0$ to 1000.$)^{\prime}$ )

CALL CHANGE((2), ICFLAG, IX, IL, IH, RX, (0.0), (1000.0), R8X)

C

IF (ICFLAG.EQ.1) RIRR = RX

IF (RIRR .EQ. 0.0) GO TO 110 WRITE (SCRN, 1110) IMO

1110 FORMAT (' ',

- 'The number of months per year that crops are irrigated is ',

- $\quad$ I2, $/$

- ${ }^{\prime}$ (range $=0$ to 12$)^{\prime}$ ) 
C

110 CONTINUE

CALL CHANGE ((1), ICFLAG, IX, (0), (12), RX, RL, RH, R8X)

IF (ICFLAG.EQ.1) IMO=IX

1111 WRITE (SCRN, 1111) IRR

- 'The number' of years prior to the beginning of dose '/

- ' calculations that irrigation accumulates radionuclides'/

- ' is ',I4,' (range $=0$ to 1000)' $)$

CALL CHANGE ((1), ICFLAG, IX, (0), (1000), RX, RL, RH, R8X)

C IF (ICFLAG.EQ.1) IRR=IX

100 CONTINUE

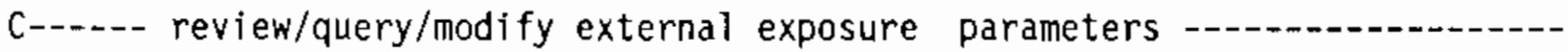

C

C

IF (IEXT .EQ. 0 .ANO. ISUR .EQ. 0) GO TO 200

GNAME $=$ 'ext. exposure,

CALL GROUP (IGFLAG)

IF (IGFLAG .EQ. 0) GO TO 200

WRITE (SCRN, 1200) HREXT

1200 FORMAT (' ',20(/),' ',

- 'The number of hours of exposure to external contamination ',

- /' per year is ', IPG10.3,' (range $=0.0$ to 8766.)')

CALL CHANGE ((2), ICFLAG, IX, IL, IH, RX, $(0.0),(8766$.$) , R8X)$

C IF (ICFLAG.EQ.1) HREXT $=$ RX

WRITE (SCRN, 1201) IWD

1201 FORMAT ( ',20(/),' ',

- 'The waste density may be either: $1 / /$

- 10X, ' $1-1.8 \mathrm{~g} / \mathrm{cu} \mathrm{cm} \mathrm{(soi1)} 1 /$

- 10X, ' $2-1.0 \mathrm{~g} / \mathrm{cu} \mathrm{cm} \mathrm{(water)'/}$

- 10X, $3-0.6 \mathrm{~g} / \mathrm{cu} \mathrm{cm} \mathrm{(0.5} \mathrm{water} \mathrm{+} 0.1$ carbon) $1 / /$

- 'The default selection is ', I2,'.'

CALL CHANGE ((1), ICFLAG, IX, (1), (3), RX,RL, RH, R8X)

IF (ICFLAG.EQ.1) IWD = IX

C

200 CONTINUE

C----- review/query/modify inhalation exposure parameters

C

C

IF (INHA .EQ. 0) GO TO 300

GNAME = 'inhalation

CALL GROUP (IGFLAG)

$\mathrm{C}$

IF (IGFLAG .EQ. 0) GO TO 300

1301 WRITE (SCRN, 1301) HRINH

1301 FORMAT (' ',20(/),' ',

- 'The number of hours of inhalation of contamination ',

- $/$ ' per year is ',1PG10.3,' (range $=0.0$ to 8766.)')

A. 113 


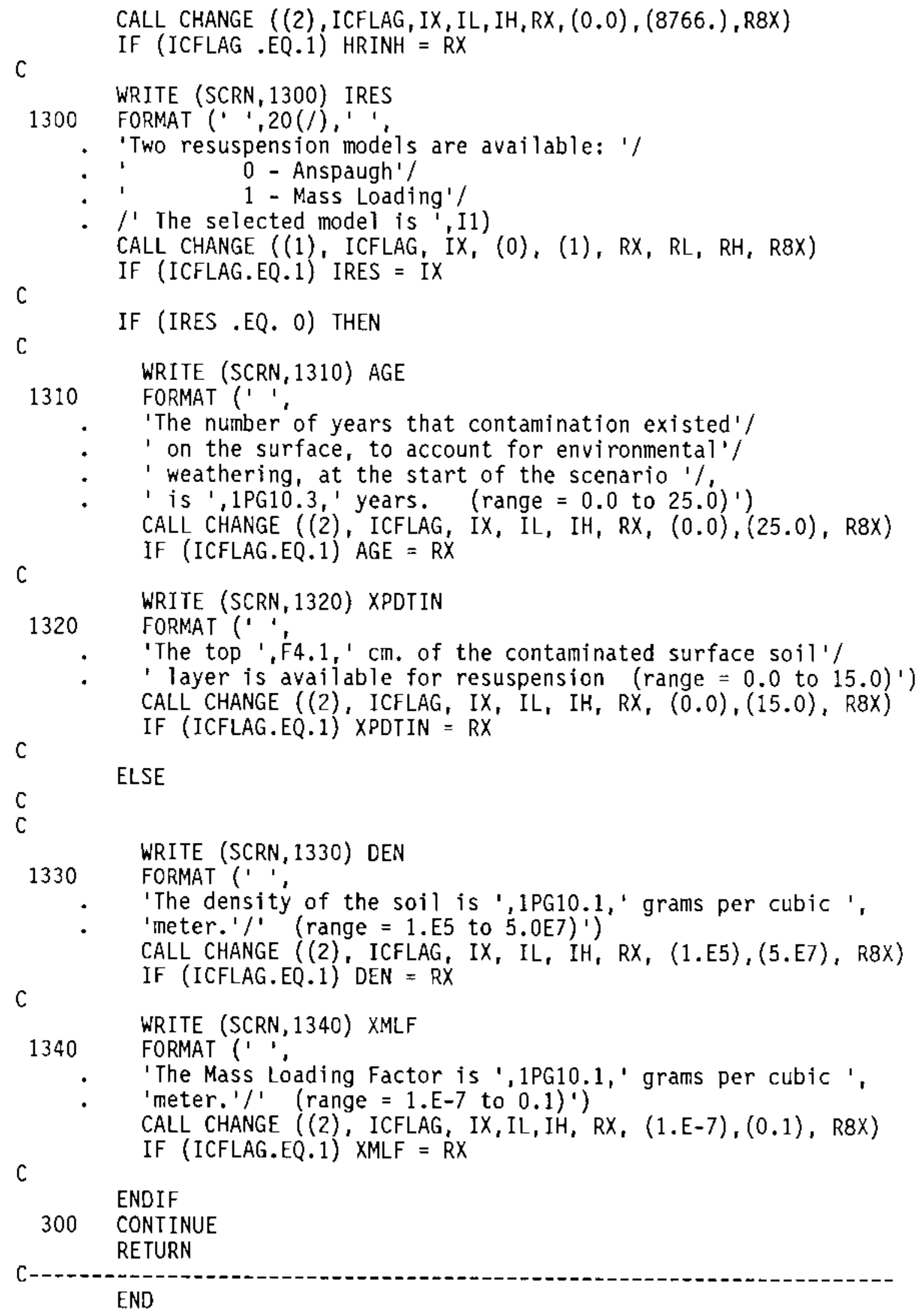


\$INCLUDE: 'COMPILE.OPT'

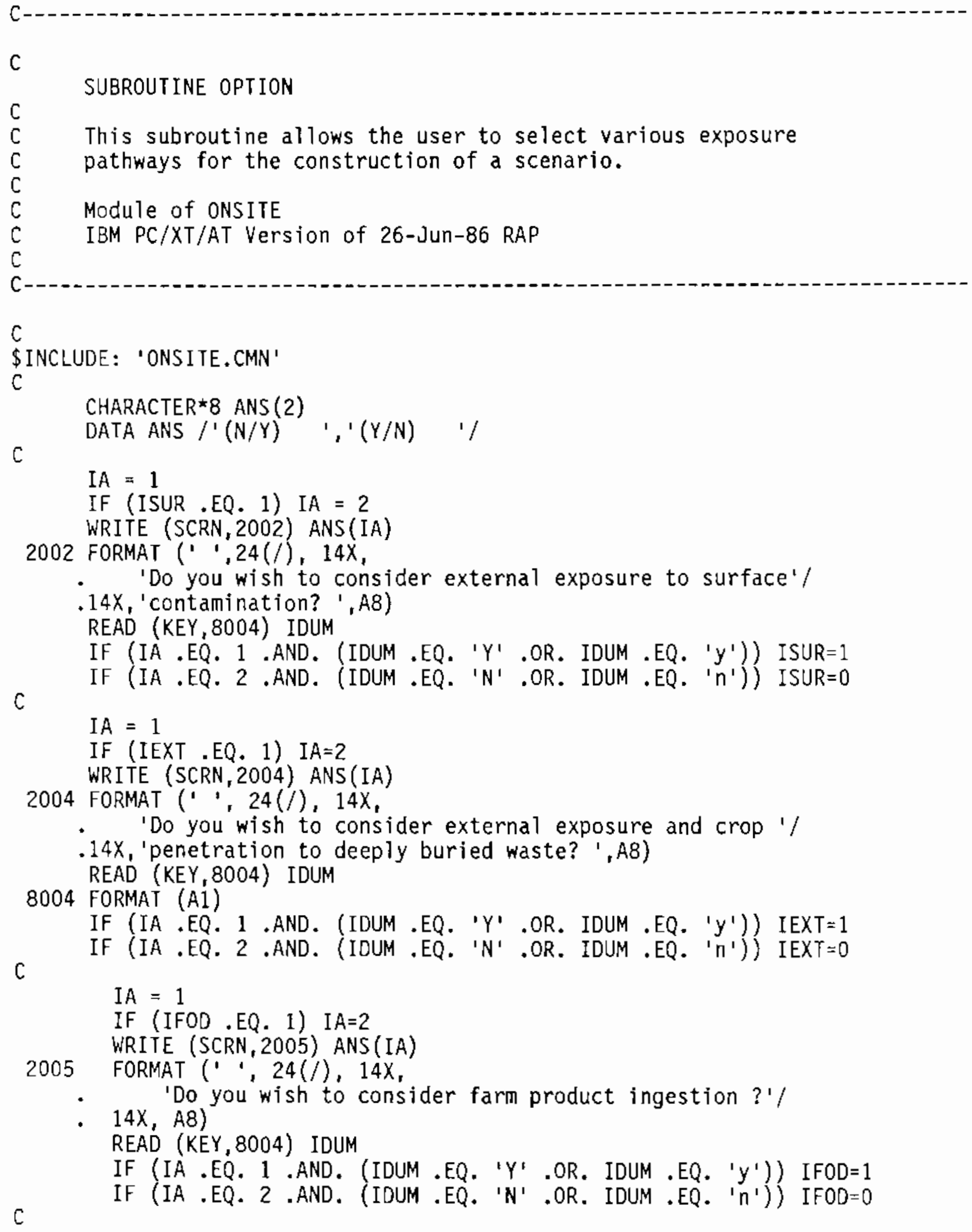




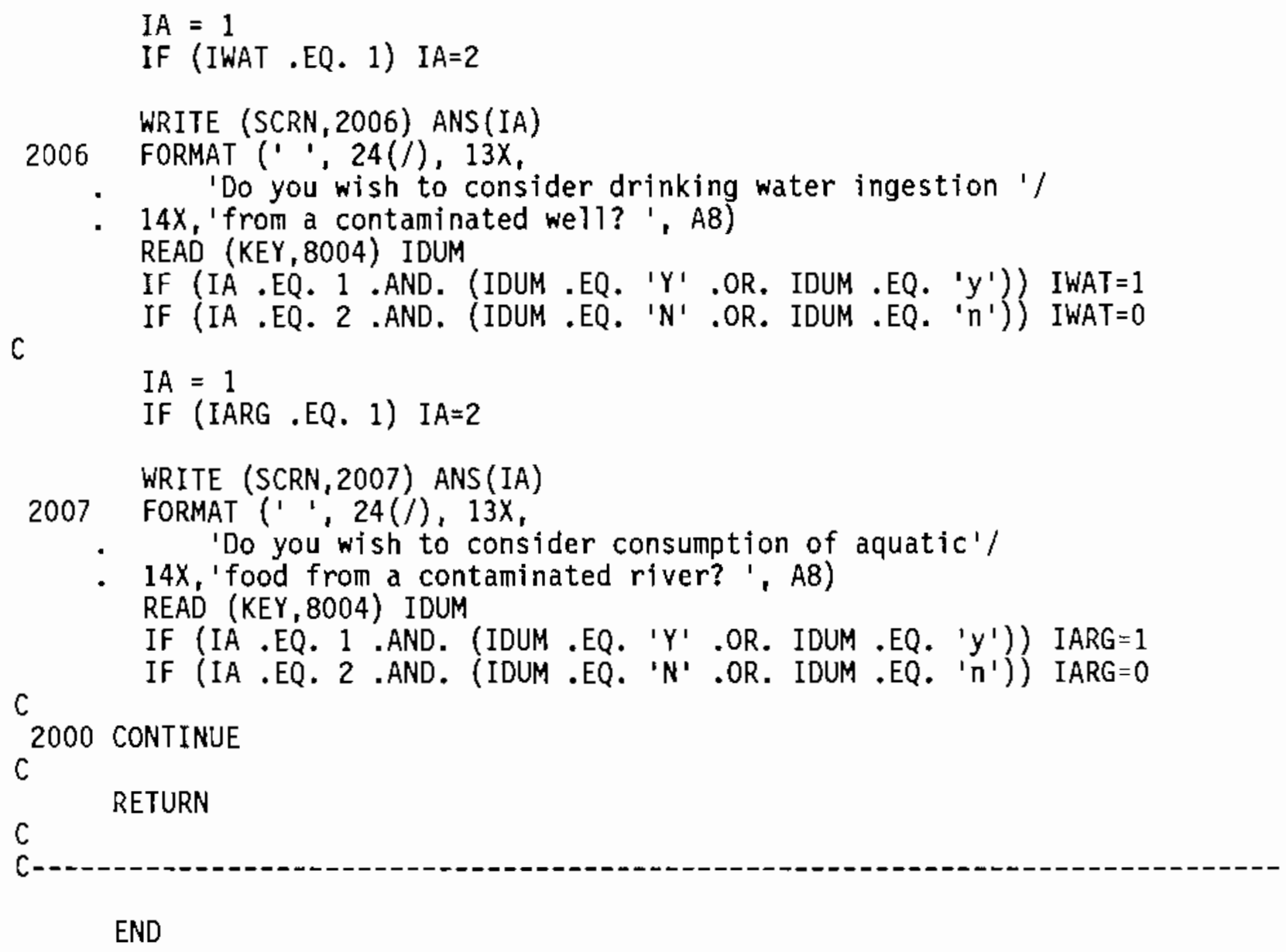


\$INCLUDE: 'COMPILE.OPT'

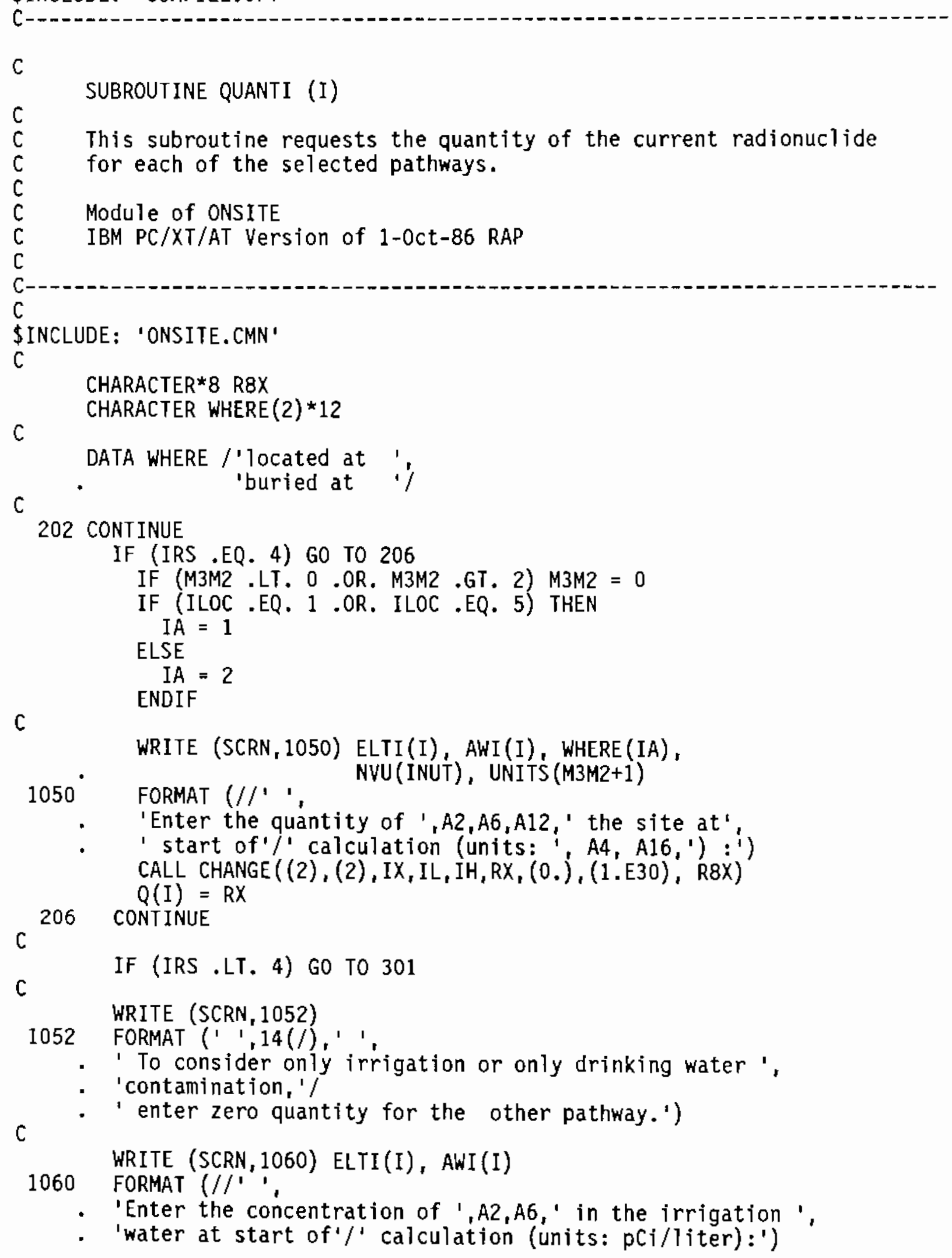

C

SUBROUTINE QUANTI (I)

C This subroutine requests the quantity of the current radionuclide

C for each of the selected pathways.

C Module of ONSITE

C IBM PC/XT/AT Version of 1-0ct-86 RAP

C

$\mathrm{C}$

\$INCLUDE: 'ONSITE.CMN'

$\mathrm{C}$

CHARACTER ${ }^{\star} 8$ R $8 X$

C

CHARACTER WHERE $(2) * 12$

DATA WHERE /'located at ''

C

202 CONTINUE

IF (IRS .EQ. 4) GO TO 206

IF (M3M2 .LT. 0 .OR. M3M2 .GT. 2) M3M2 $=0$

IF (ILOC .EQ. 1 .OR. ILOC .EQ. 5) THEN

$I A=1$$$
\text { ELSE }
$$$$
I A=2
$$

ENDIF

C

$$
\text { WRITE (SCRN, 1050) ELTI(I), AWI(I), WHERE(IA), }
$$

$1050^{\circ}$ FORMAT $\left(/ /{ }^{\prime}\right.$ ', NVU(INUT), UNITS (MBM2+1)

- 'Enter the quantity of ' $A 2, A 6, A 12$, ' the site at',

- 'start of'/' calculation (units: 'A4, A16, ') : ')

CALL CHANGE((2), (2), IX,IL, IH, RX, (0.), (1.E30), R8X) $Q(I)=R X$

206 CONTINUE

C

IF (IRS .LT. 4) GO TO 301

WRITE (SCRN, 1052)

1052 FORMAT (' ', 14(/), ' ',

: ' To consider only irrigation or only drinking water '.

C

- 'enter zero quantity for the other pathway.')

WRITE (SCRN, 1060) ELTI(I), AWI(I)

1060 FORMAT $\left(/ /{ }^{\prime}\right.$,

- 'Enter the concentration of ' $, A 2, A 6$, ' in the irrigation ',

- 'water at start of $/$ ' calculation (units: $p C i / l i t e r): '$ ) 


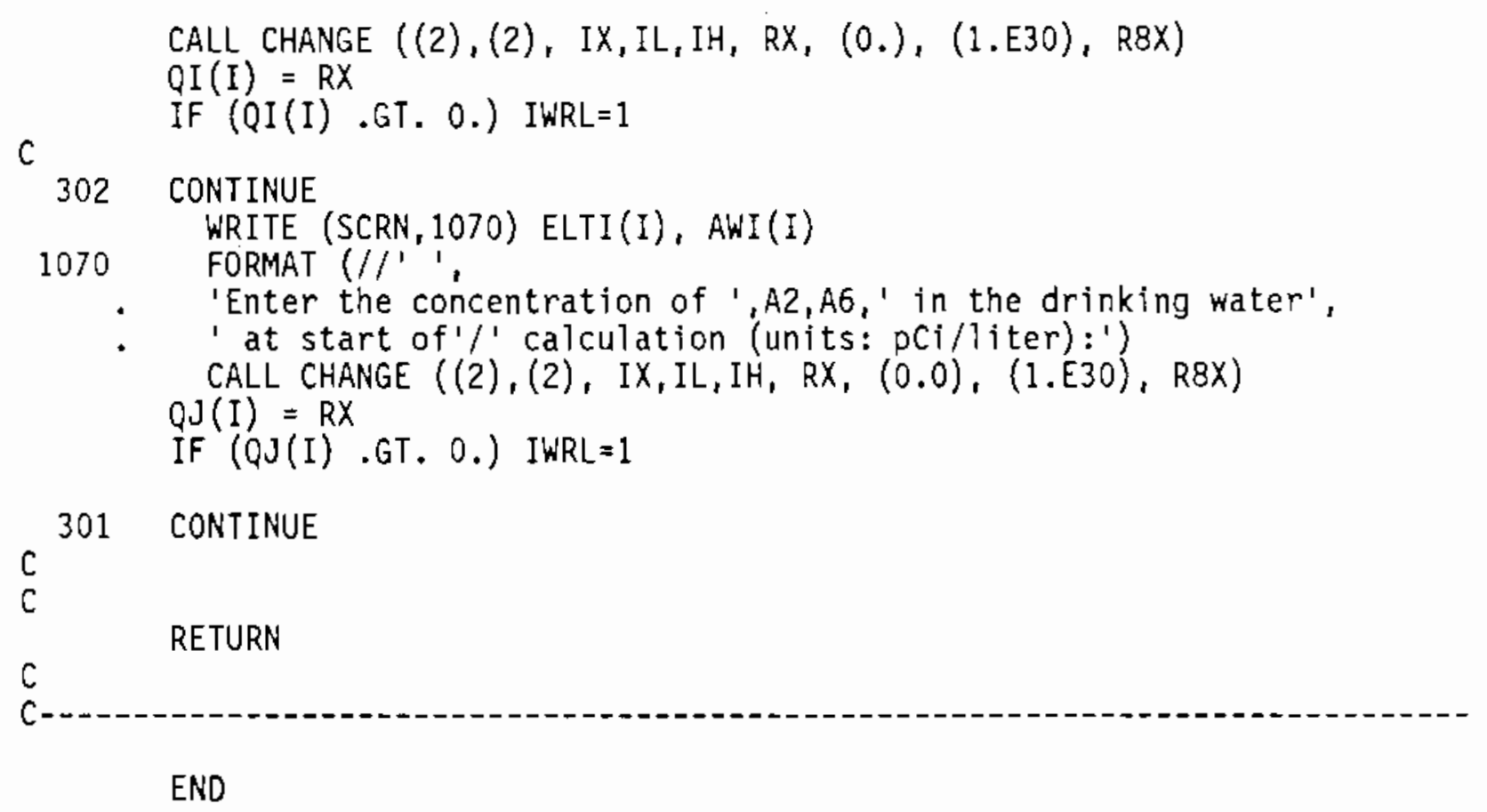




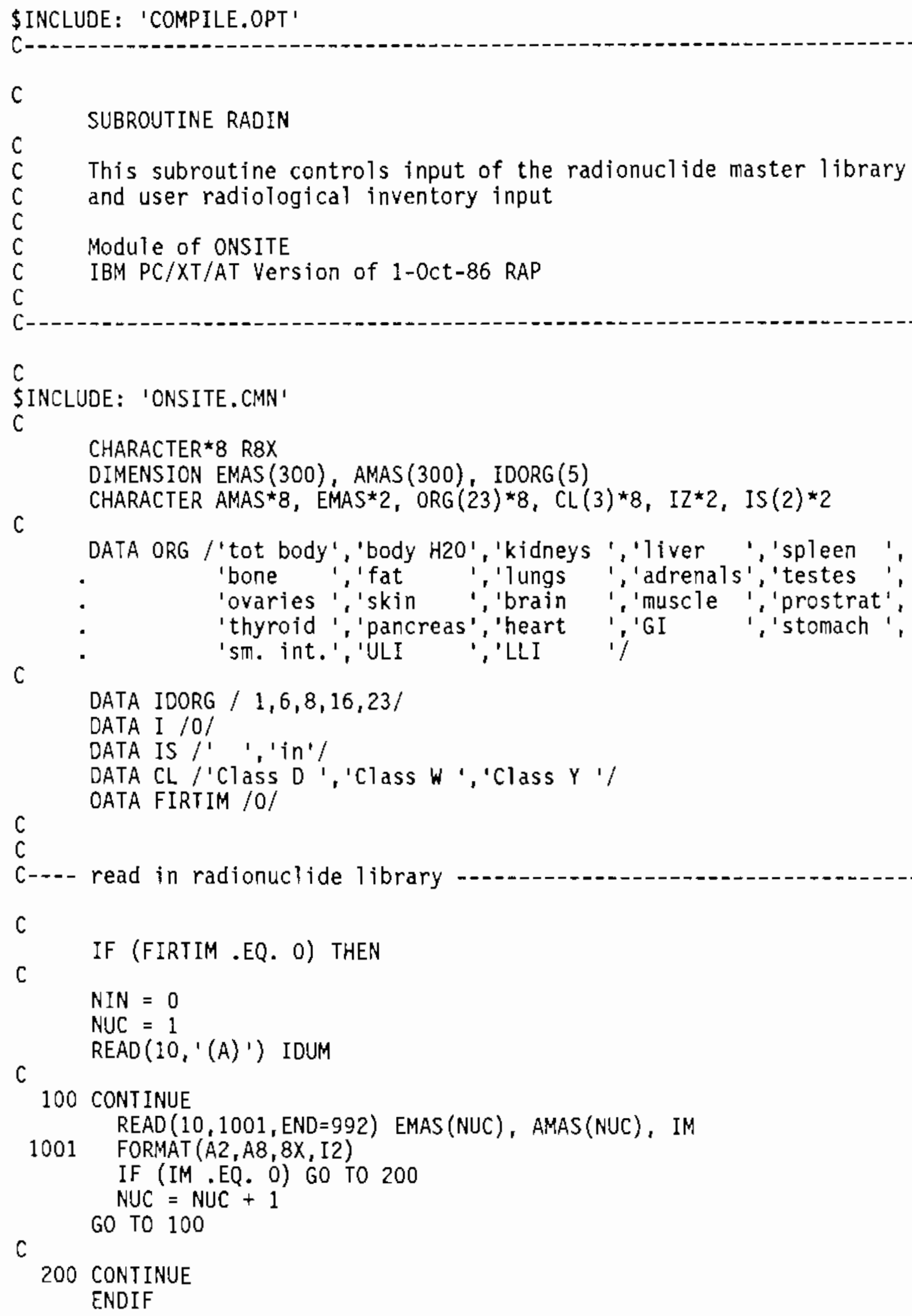


C

C--- test if this is original or additional inventory and set flags -.--.-.-

C

IF (FIRTIM .EQ. O) THEN

$N I N=0$

$I=0$

ELSE

$I=N I N$

ENDIF

$\mathrm{C}$

C---- initialize quantity arrays

C

IF (FIRTIM .EQ. 0) THEN

DO $610 \mathrm{IQ}=1,50$

$Q(I Q)=0$.

$Q I(I Q)=0$.

$Q J(I Q)=0$.

$Q K(I Q)=0$.

610 CONTINUE

ENDIF

C

$\mathrm{C}$

C---- display list of radionuclides

C

c

CALL RDLIST (EMAS, AMAS)

$\mathrm{C}$

C---- signal beginning of inventory input

C

208 CONTINUE

WRITE (SCRN, 1100)

1100 FORMAT (' $1,20(/), 1$ ',

- The following questions pertain to the radionuclide '/

- ' inventory. After inputting the inventory, enter'/

- " "99" for element name to signal to the program that "/

- 'you are finished. '///, ' ',70('\#')//

- 'Press (return> when you have finished reading:')

C READ (KEY, ' (A)') IDUM

C---- input name and source terms for each radionuclide

C

C

149 CONTINUE

2400

300

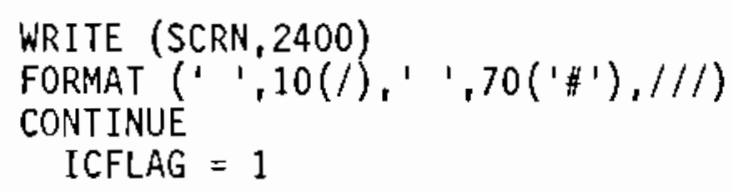




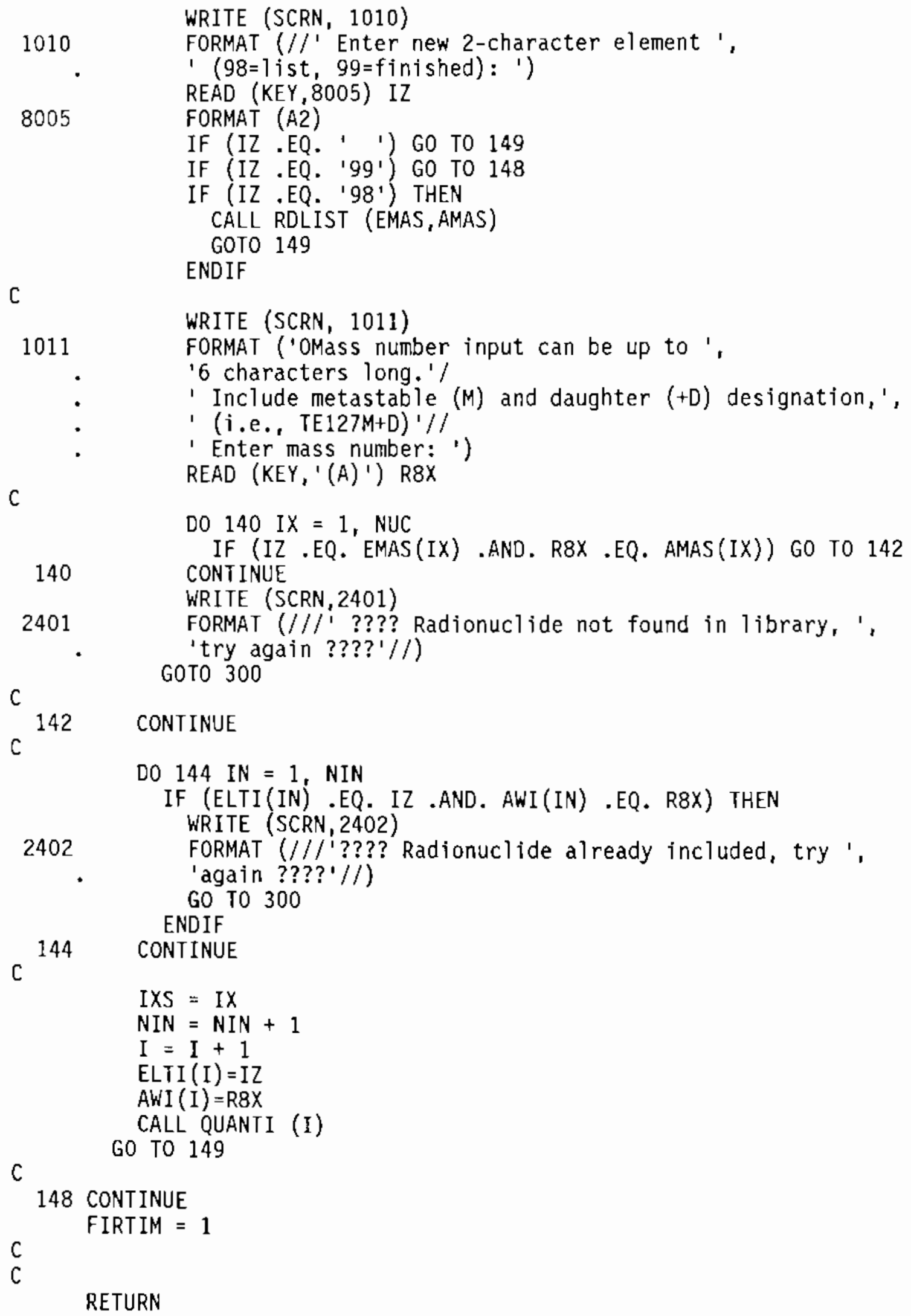

C

DO 140 IX $=1$, NUC

IF (IZ .EQ. EMAS(IX) .AND. R8X .EQ. AMAS(IX)) GO TO 142

$140 \quad$ CONTINUE

WRITE (SCRN, 2401)

2401 FORMAT $\left(/ / /^{\prime}\right.$ ???? Radionuclide not found in library, ', 'try again ????'//)

C GOTO 300

142 CONTINUE

C

DO 144 IN $=1$, NIN

IF (ELTI (IN) .EQ. IZ .AND. AWI(IN) .EQ. R8X) THEN

2402 FORMAT (///'???? Radionuclide already included, try ', 'again ????'//) GO TO 300

144 CONTINUE

C

$I X S=I X$

$N I N=N I N+1$

$I=I+1$

$\operatorname{ELTI}(\mathrm{I})=\mathrm{IZ}$

AWI (I) $=$ R8X

CALL QUANTI (I)

C GO TO 149

148 CONTINUE

FIRTIM $=1$

$\mathrm{C}$

RETURN 


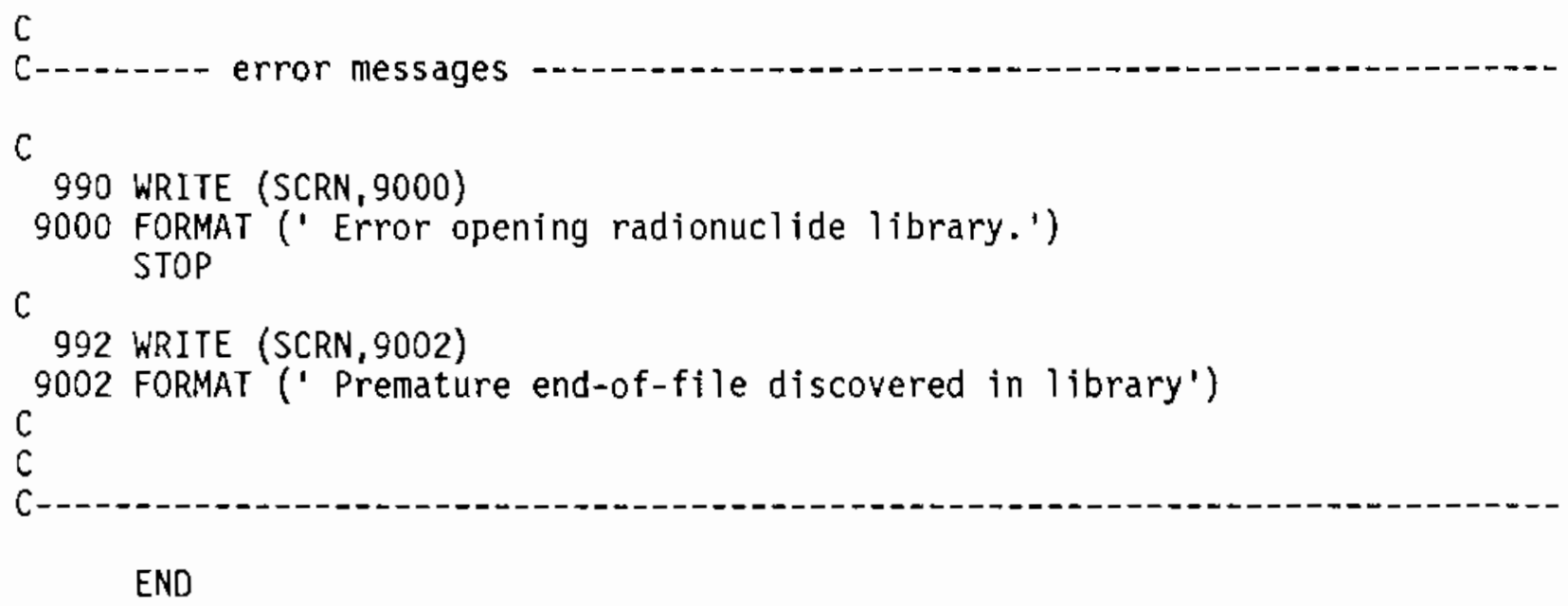




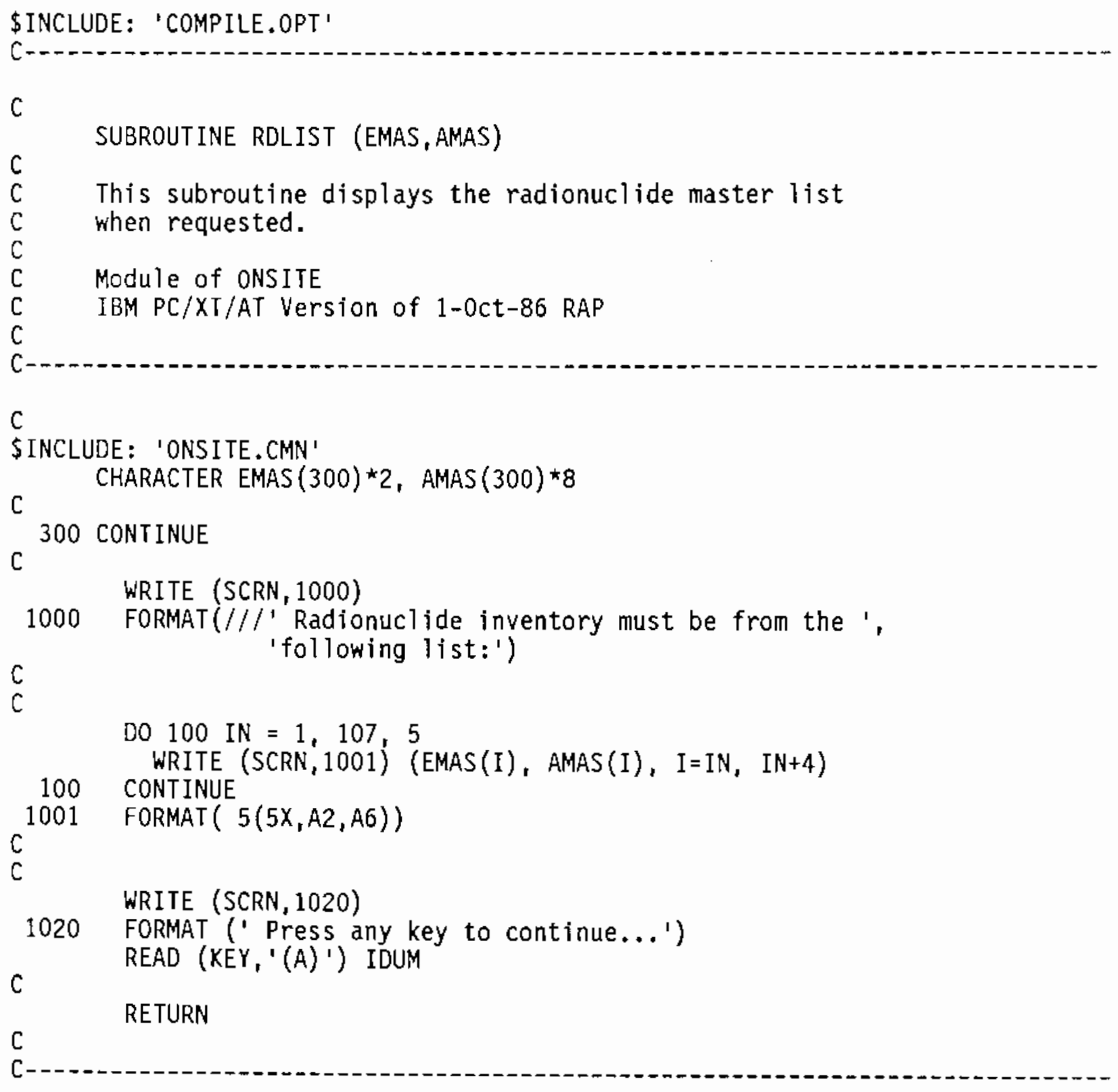

END 
\$INCLUDE: 'COMPILE.OPT'

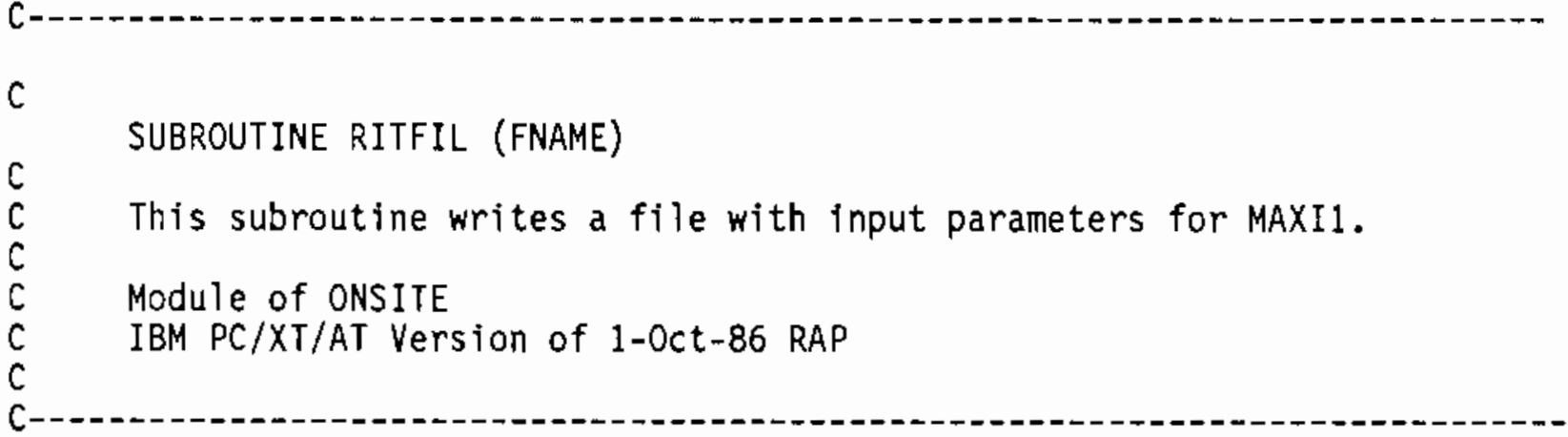

C

\$INCLUDE: 'ONSITE.CMN'

C

CHARACTER LINE*78, NUKE*2, END*5, SEA 4 , BUG* 1, FNAME* 40

C IDUM $=$ ' $N$ '

READ ( $\left.8,{ }^{\prime}(A) '\right)$ LINE

WRITE $(7,1025)$ LINE, FNAME

WRITE $(7,1030)$ TITL

READ ( $8, '$ ' $(A)$ ') LINE

WRITE $(7, '(A) ')$ LINE

$\operatorname{READ}(8, '(A) ')$ LINE

C

WRITE (7,' (A)') LINE

$\operatorname{READ}(8, '$ (A) ') LINE

WRITE $(7,1000)$ IEXT, LINE $(9:)$

READ $\left(8, '(A)^{\prime}\right)$ LINE

WRITE $\left(7, '(A)^{\prime}\right)$ LINE

$\operatorname{READ}\left(8, '(A)^{\prime}\right)$ LINE

WRITE $(7,1000)$ ISUR, LINE(9:)

READ ( $8, '(A) ')$ LINE

WRITE (7,' $\left.(A){ }^{\prime}\right)$ LINE

$\operatorname{READ}(8, '(A) ')$ LINE

WRITE $(7,1000)$ INHA, $\operatorname{LINE}(9:)$

READ ( $\left.8, '(A)^{\prime}\right)$ LINE

WRITE $(7,1000)$ IFOD, $\operatorname{LINE}(9:)$

READ ( $8, '$ ' $\left.(A)^{\prime}\right)$ LINE

WRITE $(7,1000)$ IARG, LINE(9:)

READ ( $8, '(A) ')$ LINE

WRITE (7, 1000) IWAT, LINE(9:)

READ $(8, '$ (A) ') LINE

WRITE $(7,1000)$ IAIR, $\operatorname{LINE}(9:)$

READ ( $\left.8, '(A)^{\prime}\right)$ LINE

WRITE $(7,1000)$ IEDE, LINE (9:)

READ ( $8, '$ (A)') LINE

WRITE $(7,1000)$ IDKAIR, LINE(9:)

READ ( $\left.8, '(A)^{\prime}\right)$ LINE

WRITE $(7,1000)$ IDKWAT, LINE(9:) 


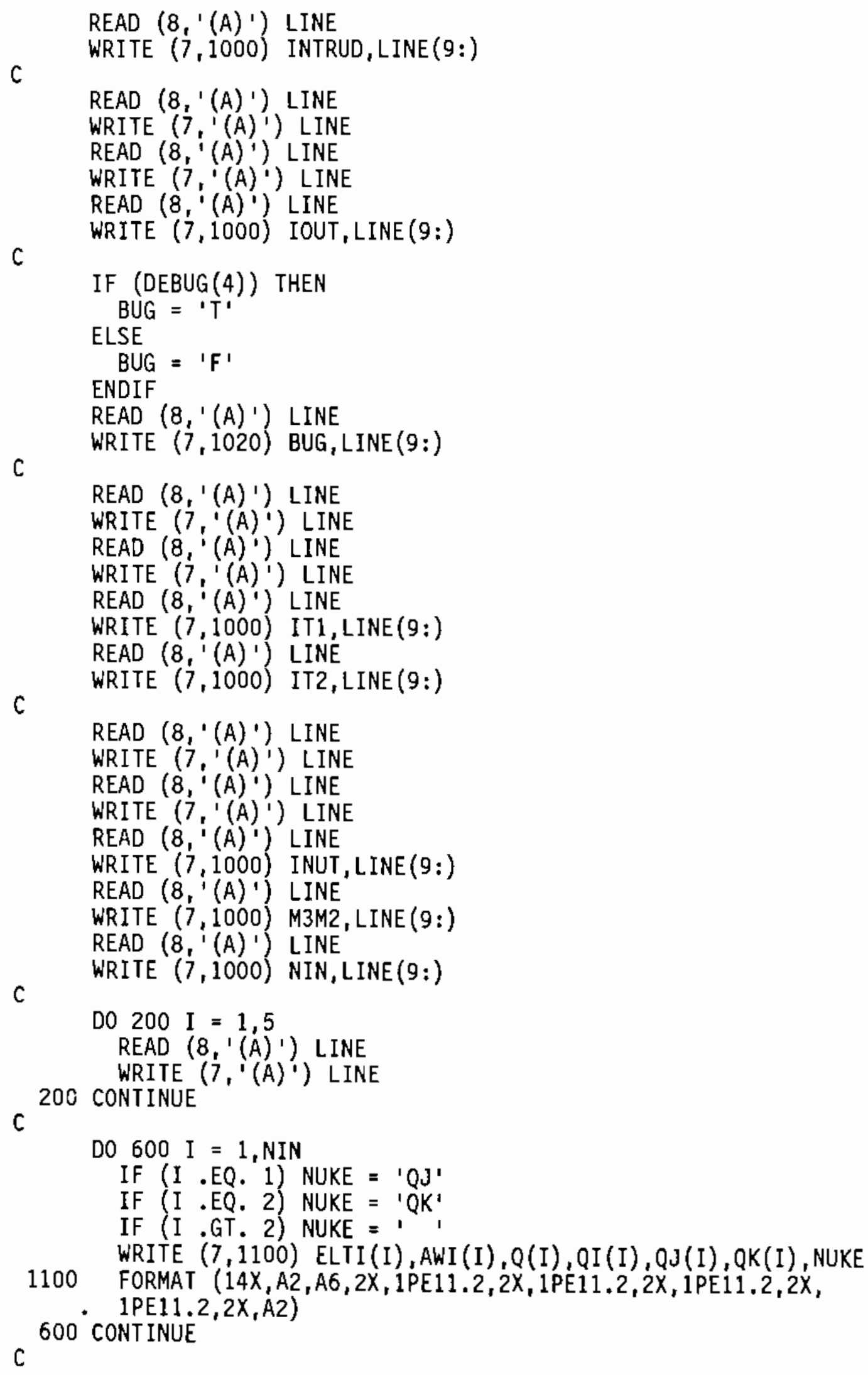


READ $\left(8,{ }^{\prime}(A)^{\prime}\right)$ LINE
WRITE $\left(7, '(A)^{\prime}\right)$ LINE

READ ( $\left.8,1(A)^{\prime}\right)$ LINE

WRITE $\left(7, '(A)^{\prime}\right)$ LINE

READ ( $\left.8,{ }^{\prime}(A) '\right)$ LINE

WRITE $(7,1005)$ SRDIL,LINE(9:)

READ (8,' (A)') LINE

WRITE $(7,1005)$ DILF, $\operatorname{LINE}(9:)$

READ (8,' (A)') LINE

WRITE (7,1005) FRSIZ, LINE(9:)

$\operatorname{READ}(8, '(A) ')$ LINE

C

WRITE $(7,1010)$ RPF2, $\operatorname{LINE}(9:)$

$\operatorname{READ}(8, '(A)$ ') LINE

WRITE $(7, '(A) ')$ LINE

$\operatorname{READ}(8, '(A) ')$ LINE

WRITE (7,' $(A)$ ') LINE

$\operatorname{READ}\left(8, '(A)^{\prime}\right)$ LINE

WRITE $(7,1010)$ HREXT, LINE (9:)

READ (8,' (A)') LINE

WRITE $(7,1010)$ HRINH, LINE $(9:)$

READ ( $8, '$ ' (A) ') LINE

WRITE (7,1015) PLANAM, LINE(13:)

$\operatorname{READ}(8, '$ (A) ') LINE

WRITE $(7,1015)$ VOLNAM, LINE (13:)

READ (8,' (A)') LINE

C

WRITE $(7,1010)$ BRATE, $\operatorname{LINE}(9:)$

READ ( $8, '$ (A) ') LINE

WRITE $\left(7, '(A)^{\prime}\right)$ LINE

$\operatorname{READ}(8, '(A) ')$ LINE

WRITE $\left(7, '(A)^{\prime}\right)$ LINE

$\operatorname{READ}(8, '$ (A) ') LINE

WRITE $(7,1000)$ IRES, LINE $(9:)$

IF (IRES .EQ. 0) THEN READ ( $8, '$ ' A ') ') LINE

WRITE $(7,1010)$ AGE, LINE(9:)

READ (8,' (A)') LINE

WRITE $(7,1010)$ XDPTIN, $\operatorname{LINE}(9:)$

ELSE

$\operatorname{READ}(8, '$ (A) ') LINE

WRITE $(7,1005)$ DEN,LINE(9:)

READ (8,' (A) ') LINE

WRITE $(7,1005)$ XMLF,LINE(9:)

ENDIF

$\operatorname{READ}(8, '(A)$ ') LINE

C

WRITE (7,' (A) ') LINE

READ ( $\left.8,,^{\prime}(A) '\right)$ LINE

WRITE $\left(7, '(A)^{\prime}\right)$ LINE

READ $\left(8, '(A)^{\prime}\right)$ LINE

WRITE $(7,1000)$ I8LOW, $\operatorname{LINE}(9 ;)$ 


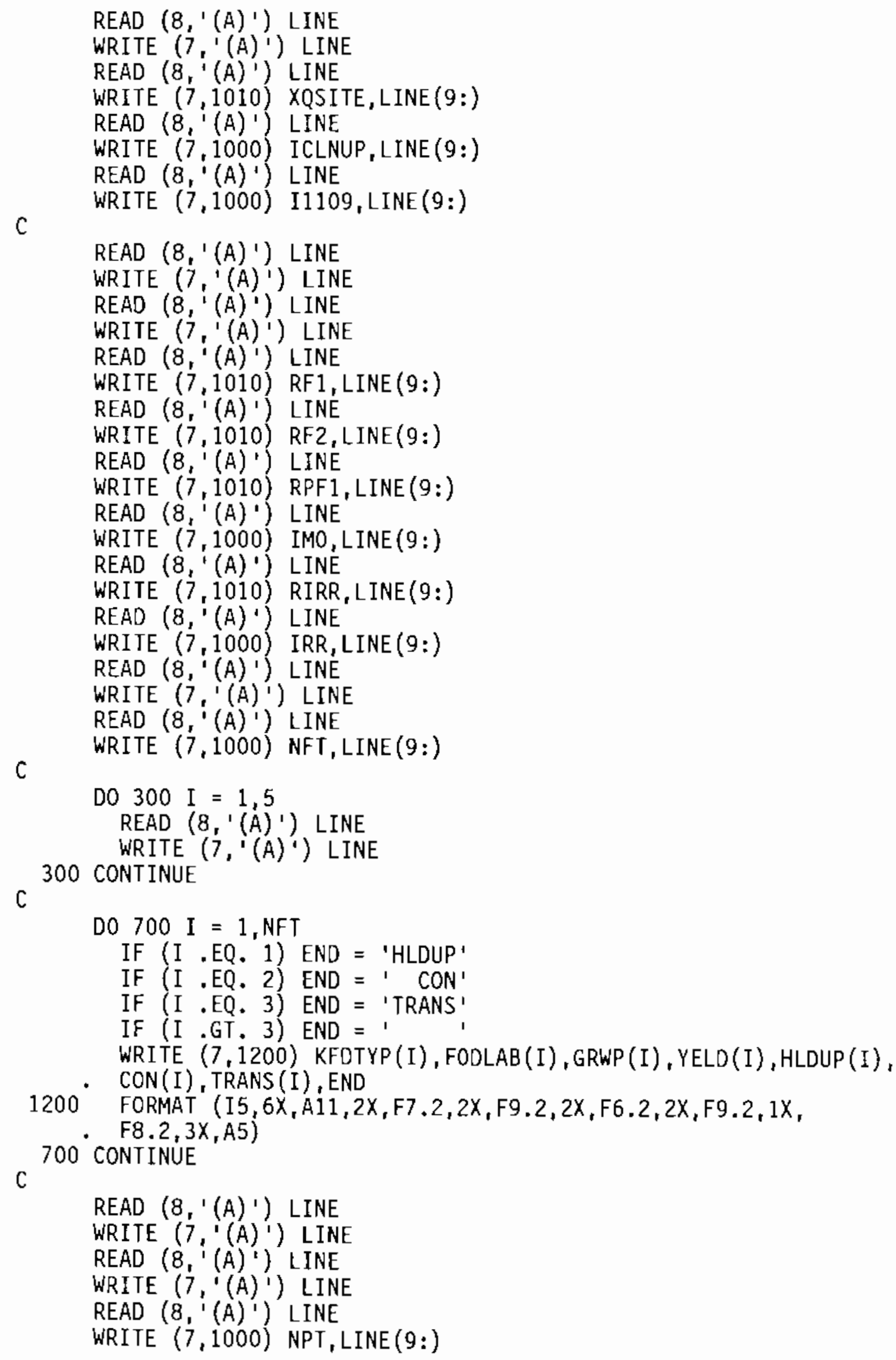


C

$$
\text { DO } 400 \mathrm{I}=1,4
$$

READ (8, ' (A) ') LINE WRITE $\left(7, '(A)^{\prime}\right)$ LINE

C

400 CONTINUE

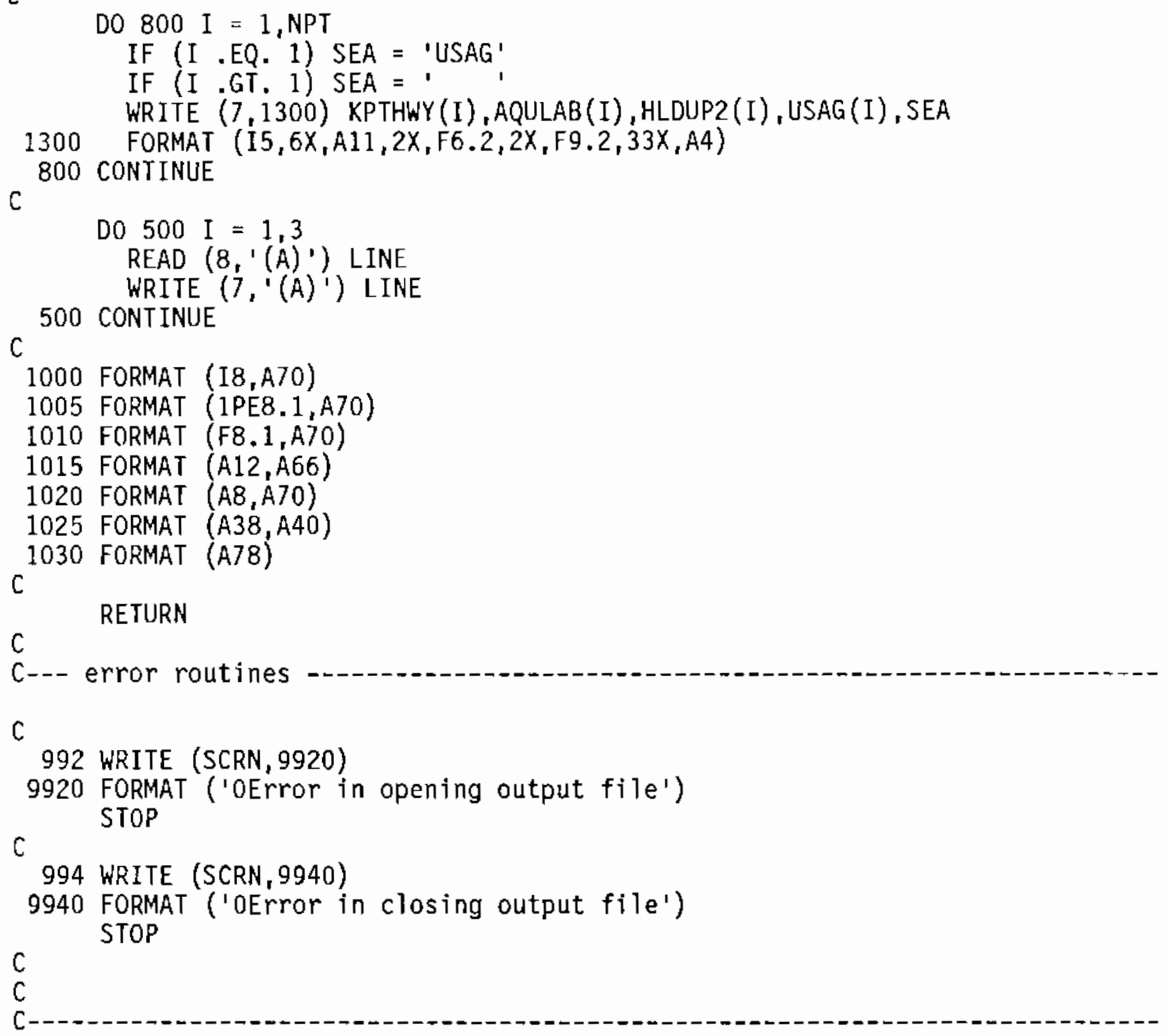

END 


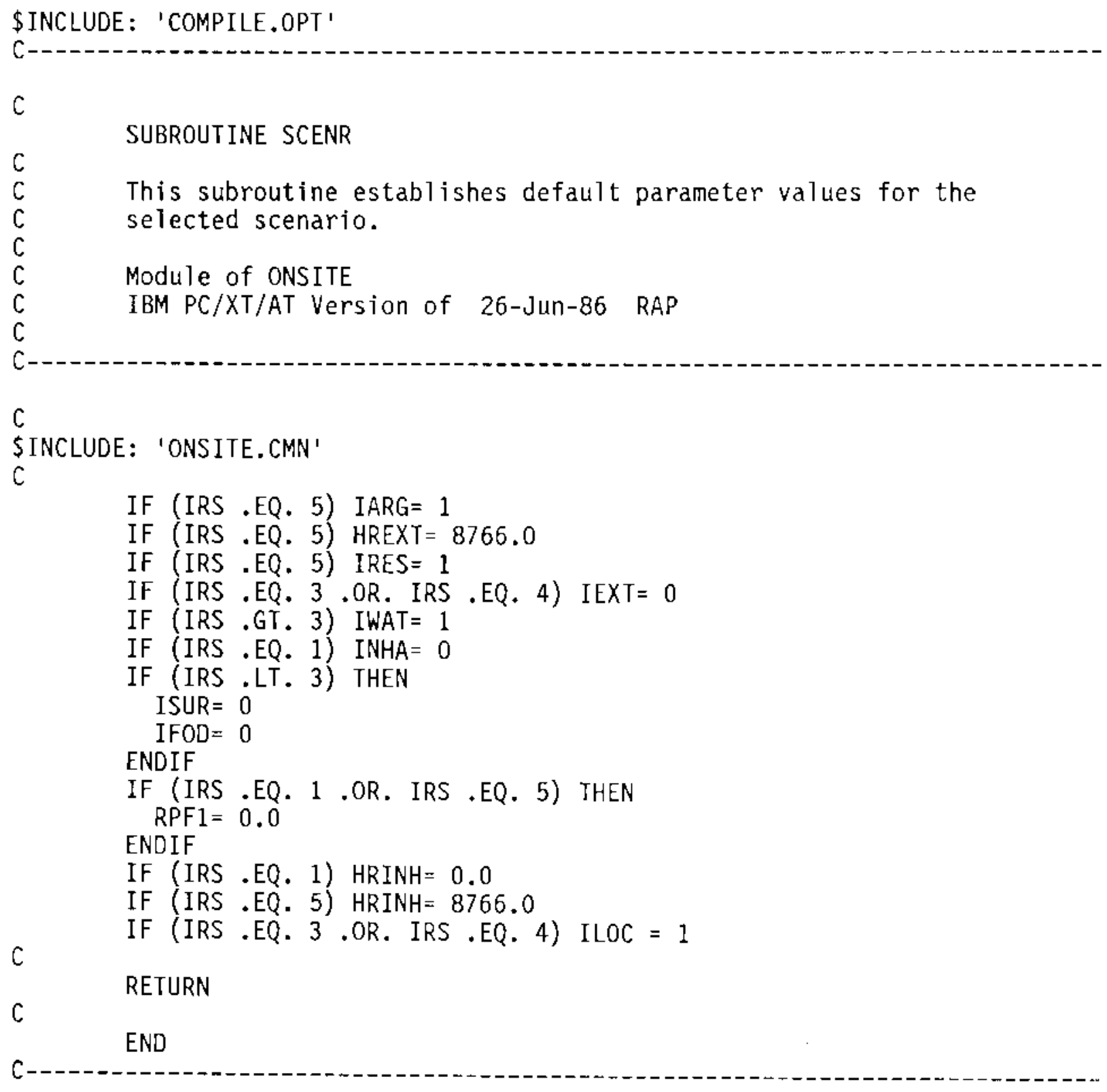


\$INCLUDE: 'COMPILE.OPT'

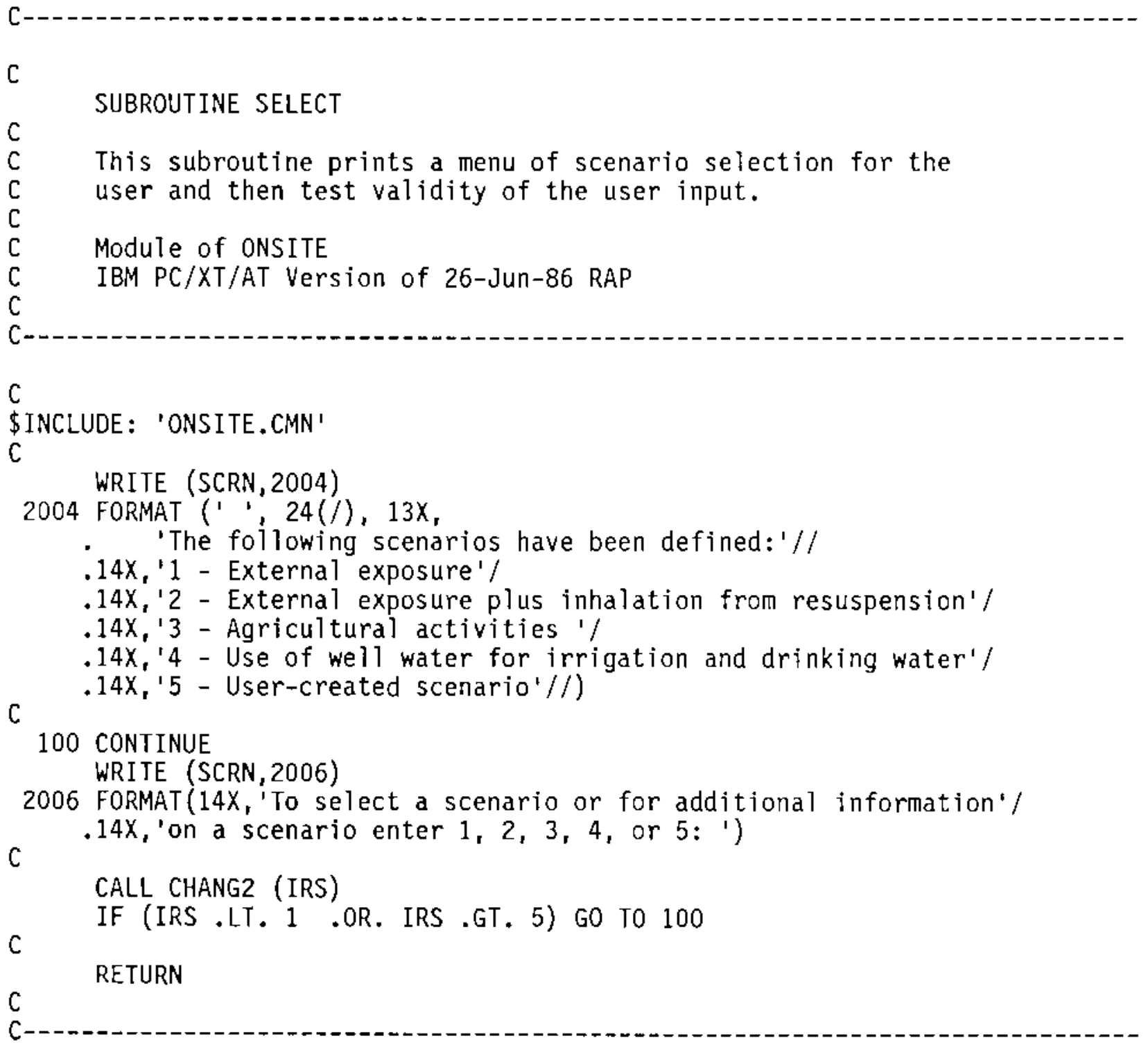

END 


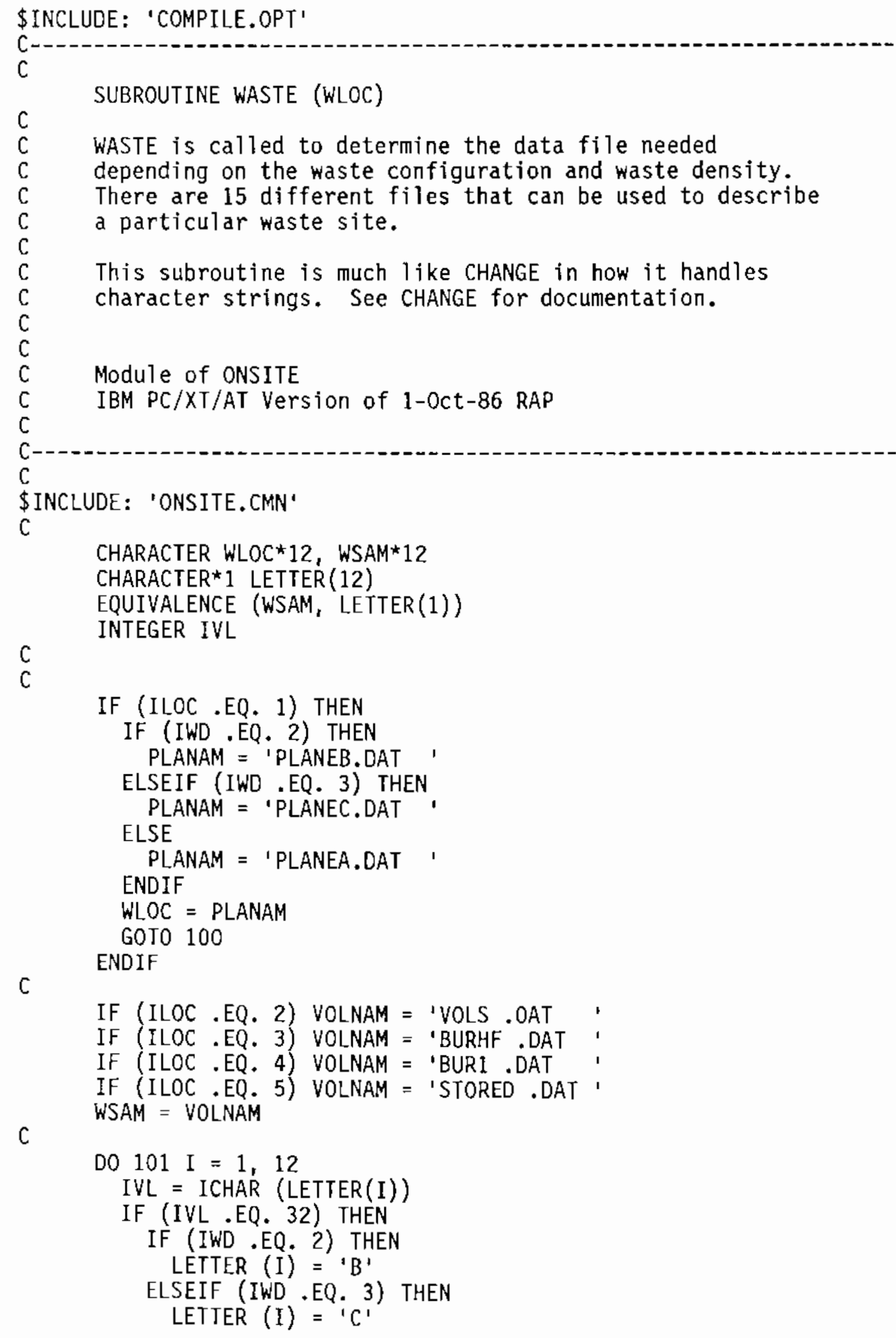




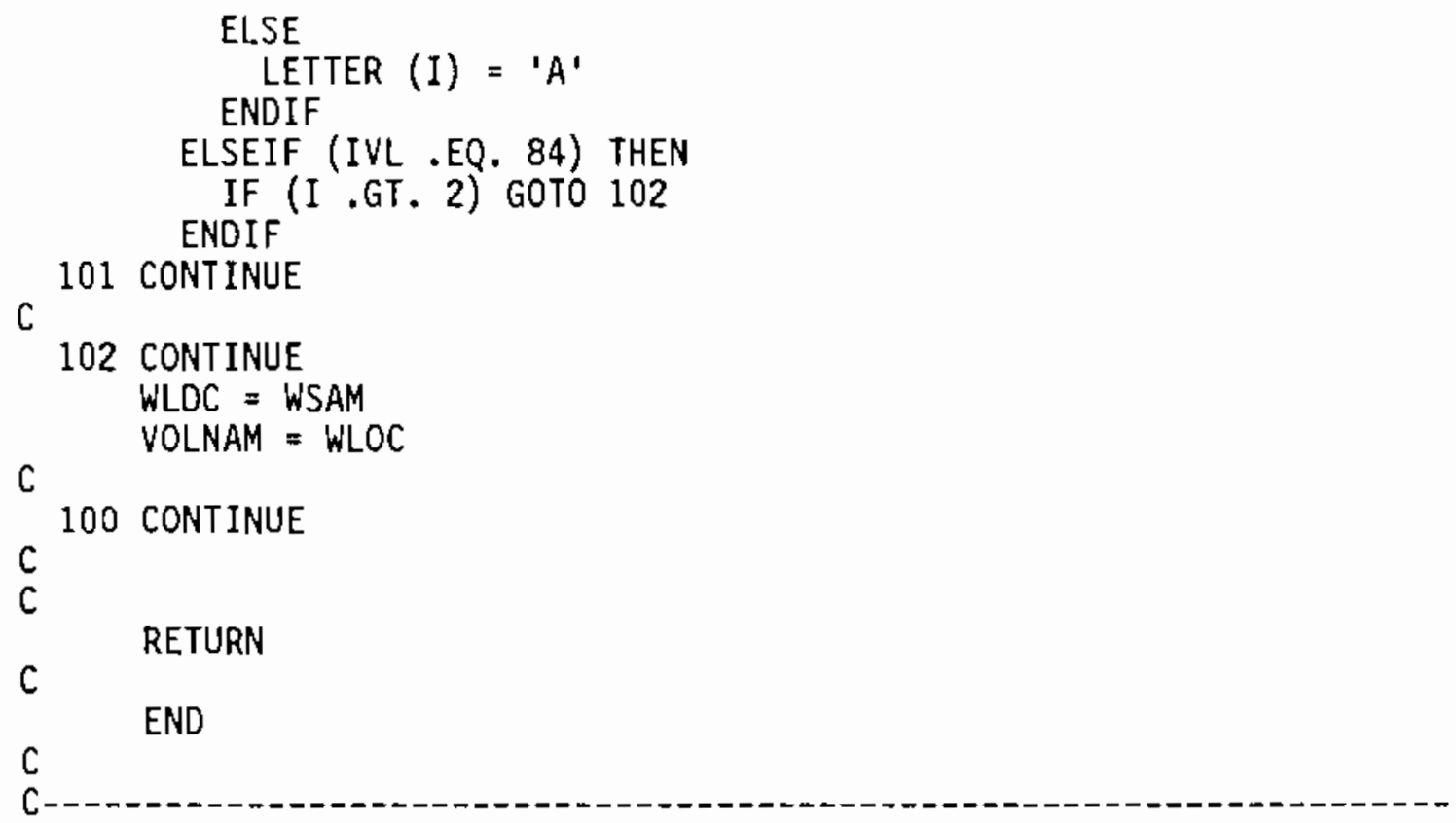




\section{FILE: ONSITE.CMN}

C

COMMON /NARYBL/ IFOD, RIRR, IMO, RF1, RF2, IARG, IWAT,

$$
\text { - }
$$

$\cdot$

$\cdot$

$\cdot$

c IDKWAT, IEXT, IAIR, XQSITE, IDKAIR, ISUR, AGE, DEN, XMLF, DILF, M3M2, INTRUD, IT1, IT2, IOUT,

REAL NVUNIT RPF1, RPF2, IRR, SRDIL, NVUNIT(4)

c

c

C

$c$

C

C

C

C

C

C

C

c

C

C

C

COMMON /INV/ NIN, - $Q(50), Q I(50), Q J(50), Q K(50)$

(1)

COMMON /FLAG/ IRS, ILOC, INUT, IARL, IWRL, IWD, IDT, IMOD, IBS

IRS - INDEX OF SELECTED SCENARIO

ILOC - INDEX OF LOCATION OF THE WASTE:

1) ON SURFACE (15 $\mathrm{cm}$ thick)

2) ON SURFACE ( $1 \mathrm{~m}$ thick)

3) OVERBURDEN OF $0.5 \mathrm{~m}(1 \mathrm{~m}$ thick)

4) OVERBURDEN OF $1.0 \mathrm{~m}$ ( $1 \mathrm{~m}$ thick)

5) STORED ( $1 \mathrm{~m}$ thick)

INUT - INDEX OF RADIOLOGICAL UNITS INPUT FOR ARRAYS NVU (DESCRIPTION) AND NVUNIT (MOD. FACTOR)

IARL - SET IF SURFACE/BURIED CONTAMINATION

IWRL - SET IF LIQUID CONTAMINATION

IWD - INDEX OF SELECTED WASTE DENSITY

IDT - INDEX OF SELECTED DIET

COMMON /AREA/ FRSIZ

FRSIZ - SIZE OF THE SITE IN FRACTIONAL HECTARES

COMMON /IOVAR/ SCRN, OUT, KEY

INTEGER SCRN, OUT

C

$\mathrm{C}$

C

COMMON /CHAR/ TITL, UNITS(3), NVU(4), AWI(50), ELTI(50), OUTFIL,

C GNAME, IDUM, IY

CHARACTER UNITS*16, TITL*80, NVU*4, AWI*8, ELTI*2, OUTFIL*16, C GNAME*16, IDUM*1, IY*1 


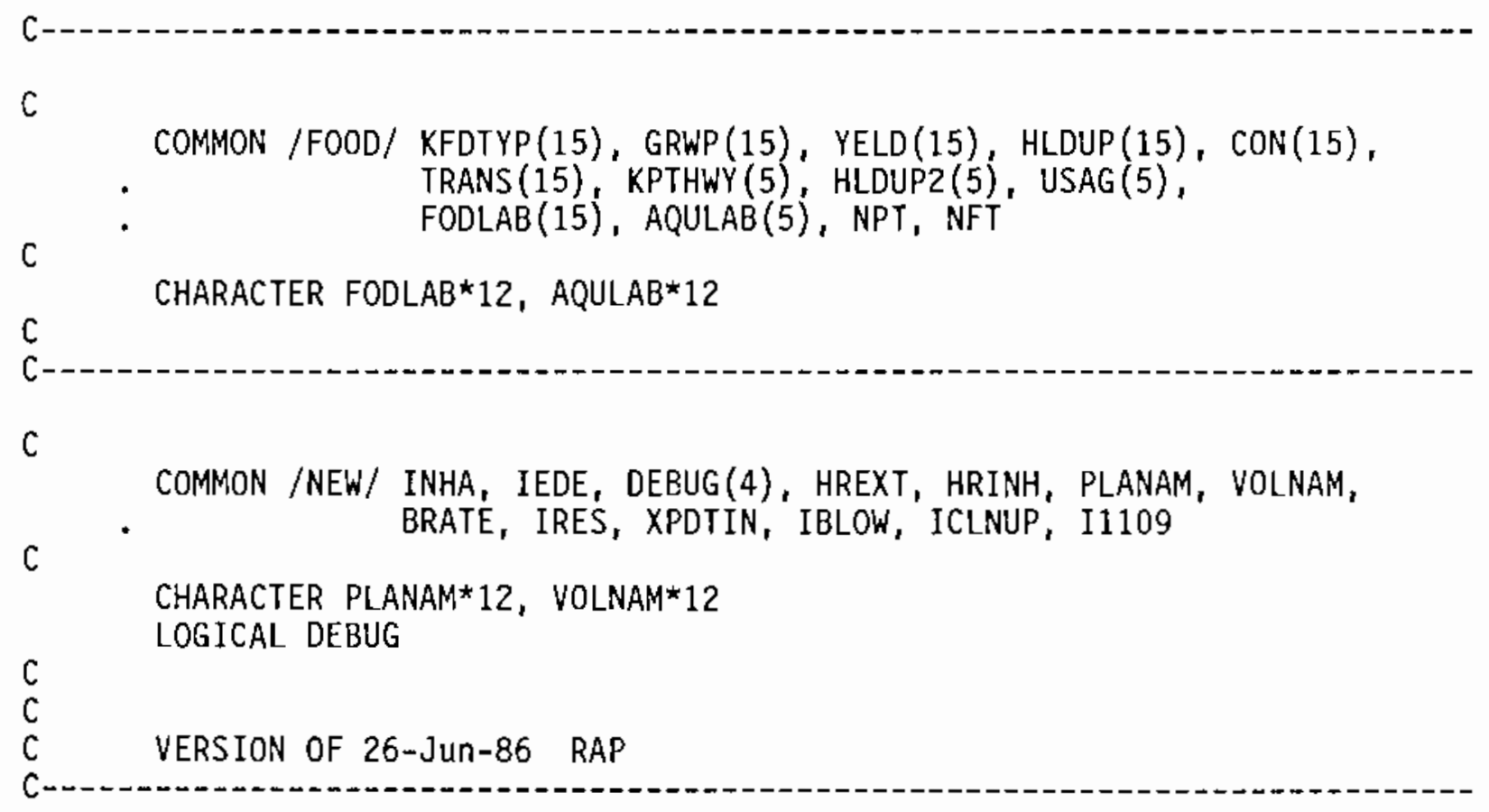




\section{FILE: COMPILE.OPT}

$\$ D E B U G$

\$LIST

\$STORAGE: 2

\$PAGESIZE: 60

\$IITLE: 'ONSITE Source Code'

\$SUBTITLE: 'IBM PC Version' 


\section{FILE: DEFAULT.IN}

MAXI Input File

Filename here/Title on next line:

DO YOU WISH TO CONSIDER:

External exposure and root penetration for waste modeled as a slab (volume) source? (0-no 1-yes)

External exposure to surface contamination modeled as a plane source? (0-no 1-yes)

Inhalation of resuspended material? (0-no 1-yes) INHA

Farm product ingestion? (0-no 1-yes)

Aquatic foods ingestion? (0-no 1 -yes)

Drinking water ingestion? (0-no 1-yes)

off site atmospheric source? (0-no, 1-yes)

ICRP-26/30 as well as ICRP-2 methodology? (0-no 1-yes)

Decay of air release source term? (0-no 1-yes)

Decay of water release source term? (0-no 1-yes)

Use the One-Year-of-Exposure Mode1? (0-no 1-yes)

IEXT

ISUR

IFOD

IARG

IWAT

IAIR

IEDE

IDKAIR

IDKWAT

INTRUD

MONITORING AND OUTPUT OPTIONS

Level of detail in reports (0-least to 3 )

IOUT

Monitor and print hand calculation report

NUMBER OF YEARS AFTER WASTE IS DISPOSED THAT:

Scenario begins:

IT1

Scenario ends:

INVENTORY:

Release Term Input units: (1-pCi 2-uCi 3-mCi 4-Ci) INUT

Soil source units $\left(0-m^{\star *} 21-m^{\star *} 32-\mathrm{kg}\right) \quad$ M3M2

Number of radionuclides in inventory:

NIN

\begin{tabular}{|c|c|c|c|c|}
\hline $\begin{array}{l}\text { Release } \\
\text { Terms }\end{array}$ & $\begin{array}{l}\text { Soil Source } \\
\text { (per unit) }\end{array}$ & $\begin{array}{l}\text { Irrigation } \\
\text { /Aquatic } \\
\text { (per L) }\end{array}$ & $\begin{array}{l}\text { Drinking } \\
\text { Water } \\
\text { (per L) }\end{array}$ & $\begin{array}{l}\text { Atmospheric } \\
\text { Release } \\
\text { (per yr) }\end{array}$ \\
\hline
\end{tabular}

INVENTORY MODIFICATION FACTORS: (muitipliers)

Surface inventory modification factor:

SRDIL

Irrigation/aquatic inventory modification factor:

DILF

Size of site (fractional ha):

FRSIZ

Fraction of total diet grown on site:

RPF2

EXTERNAL/INHALATION EXPOSURE:

Hours of external exposure to contamination (h/yr):

HREXT

Hours of inhalation of airborne contamination (h/yr):

HRINH

Ext. exp. dose rate factors (plane source) filename:

Ext, exp, dose rate factors (volume source) filename:

PLANAM

Breathing rate $\left(\mathrm{cm}^{\star *} 3 / \mathrm{sec}\right)$ :

VOLNAM 
SELECT RESUSPENSION MODEL:

0 -Anspaugh

Age of contamination (yr)

Top soi\} available (cm)

1-Mass Loading IRES

Soil density $\left(\mathrm{g} / \mathrm{m}^{* * 3}\right) \quad$ AGE/DEN

Mass loading factor $\left(\mathrm{g} / \mathrm{m}^{\star *} 3\right)$

XDPTIN/XMLF

SPECIAL PURPOSE PARAMETERS:

No. of years contamination blew onto the site prior to IBLOW the beginning of the scenario:

Chi/g value at the site:

XQSITE

Use drinking water cleanup factors (0-no, 1-yes) ICLNUP

Use Reg. Guide $1.109 \mathrm{H}-3, \mathrm{C}-14 \mathrm{plant} / \mathrm{so} 1 \mathrm{l}$ uptake factors I1109

AGRICULTURAL PARAMETERS:

Fraction of roots in upper soil: RF1

Fraction of roots in deeply burjed waste: RF2

Ratio of ext. contamination in surface/subsurface soil: RPF1

Months per year irrigated:

Irrigation rate $\left(\mathrm{L} / \mathrm{m}^{\star \star} 2 / \mathrm{mo}\right)$ :

Years of irrigation $w /$ contaminated water prior to the beginning of the dose calculation period:

Number of food types: (1-15)

IMO

RIRR

IRR

NFT

FOOD

TYPE

INDEX

GROWING

PERIOD YIELD

CONSUMP- TRANS-

KFDTYP

HOLDUP TION LOCATION

$---$

FOOD TYPE (days) $\left(\mathrm{kg} / \mathrm{m}^{\star \star 3}\right)$ (days) $(\mathrm{kg} / \mathrm{yr})$ FACTOR GRWP

INDE

----------

--o---

-.--n--

(days)

-

YELD

DRINKING WATER/AQUATIC FOOD PARAMETERS:

Number of water/food types: (1-5)

NPT

TYPE

HOLDUP CONSUMPTION

KPTHWY

INDEX

$\begin{array}{lll}\text { FOOD TYPE } & \begin{array}{l}\text { HOLDUP } \\ \text { (days) }\end{array} & \begin{array}{c}\text { CONSUMPTION } \\ (\mathrm{kg} / \mathrm{yr})\end{array} \\ & & \end{array}$

HLDUP2 

APPENDIX B

COMPLETE DATA BASE LISTING 


\section{APPENDIX B \\ COMPLETE DATA BASE LISTING}

CONTENTS - APPENDIX B

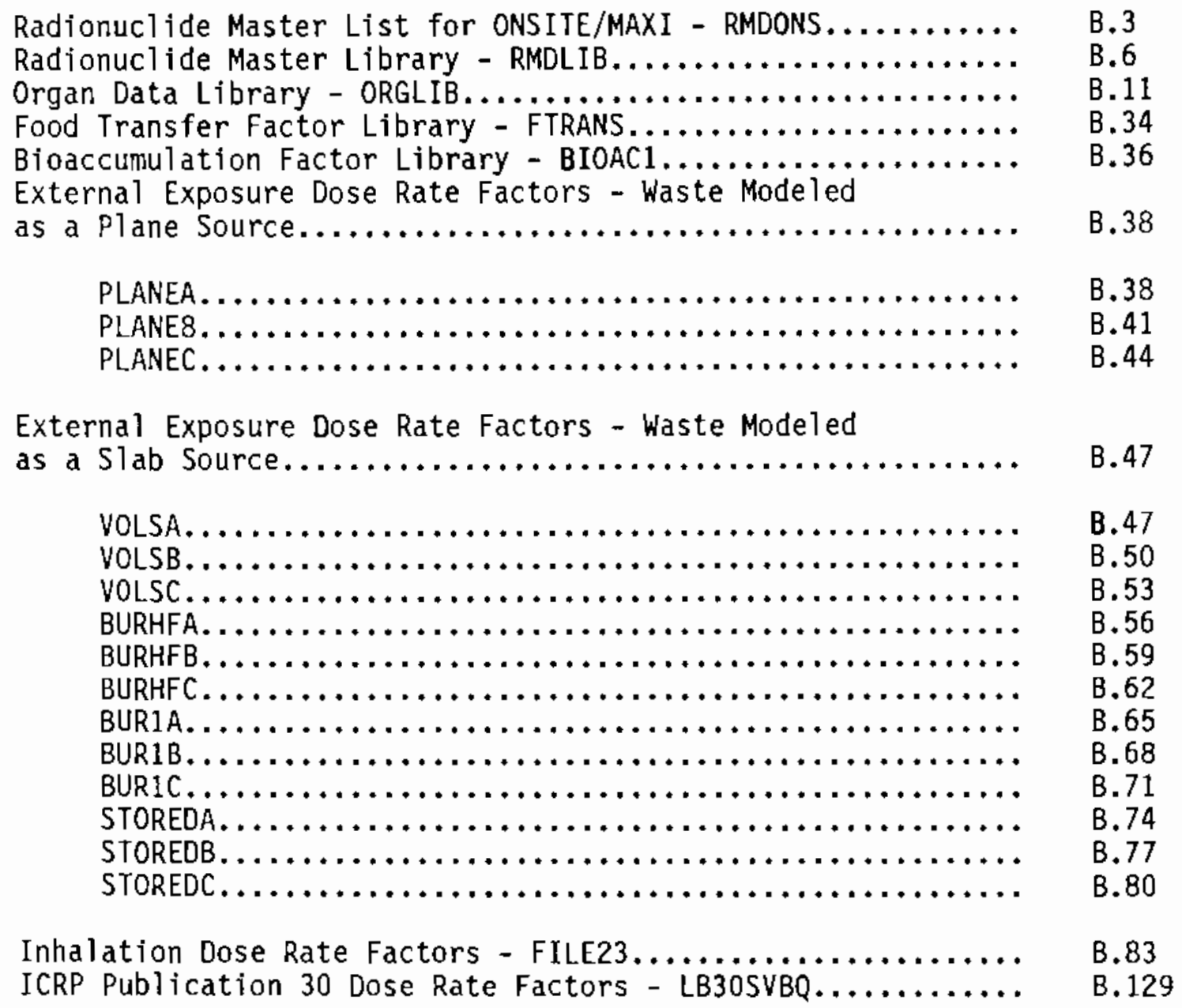



Appendix B - Radionuclide Master List for ONSITE/MAXI - RMDONS

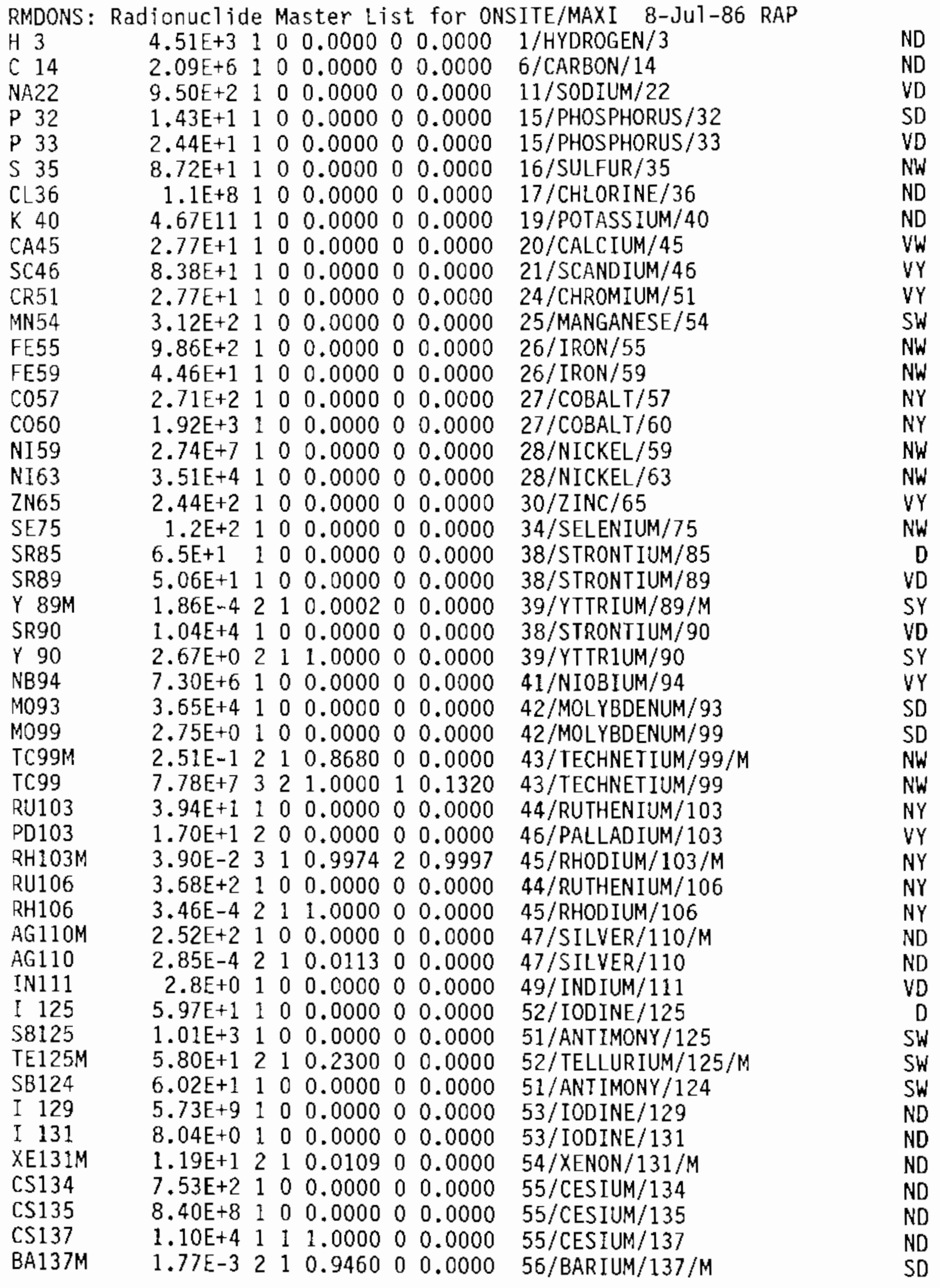


Appendix B - Radionuclide Master List for ONSITE/MAXI - RMDONS

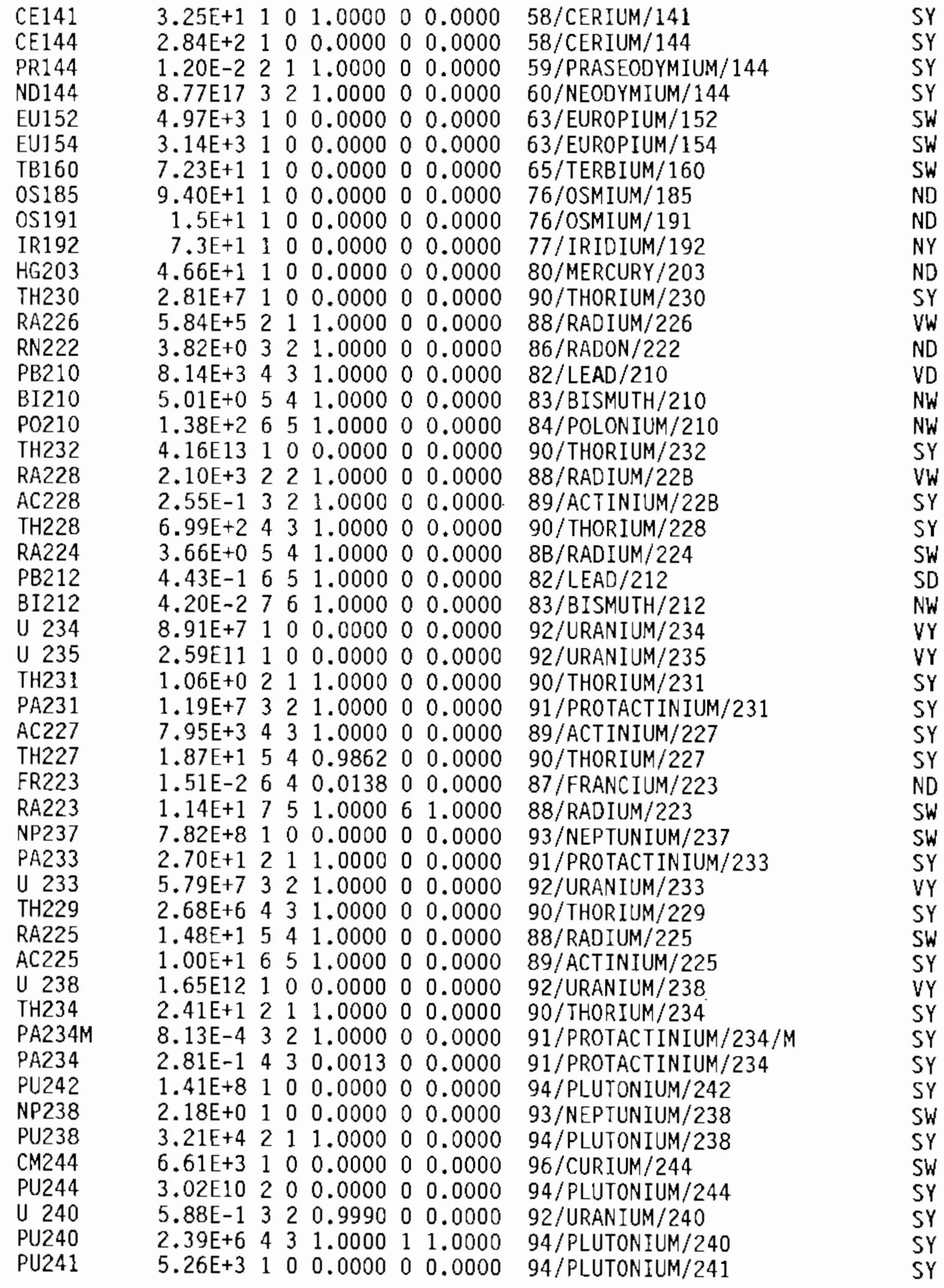




\section{Appendix B - Radionuclide Master List for ONSITE/MAXI - RMDONS}

$\begin{array}{lllllllll}\text { AM241 } & 1.58 E+5 & 2 & 1 & 1.0000 & 0 & 0.0000 & 95 / \text { AMERICIUM/241 } & \text { SW } \\ \text { CM243 } & 1.04 \mathrm{E}+4 & 1 & 0 & 0.0000 & 0 & 0.0000 & 96 / \text { CURIUM/243 } & \mathrm{SW} \\ \text { PU243 } & 2.06 \mathrm{E}-1 & 2 & 0 & 0.0000 & 0 & 0.0000 & 94 / \text { PLUTONIUM/243 } & \mathrm{SY} \\ \text { AM243 } & 2.70 \mathrm{E}+6 & 3 & 2 & 1.0000 & 1 & 0.0024 & 95 / \text { AMERICIUM/243 } & \mathrm{SW} \\ \text { NP239 } & 2.36 \mathrm{E}+0 & 4 & 3 & 1.0000 & 0 & 0.0000 & 93 / \text { NEPTUNIUM } / 239 & \mathrm{SW} \\ \text { PU239 } & 8.91 \mathrm{E}+6 & 5 & 4 & 1.0000 & 1 & 0.9976 & 94 / \text { PLUTONIUM/239 } & \mathrm{SY} \\ & & 0 & 0 & 0.0000 & 0 & 0.0000 & & \end{array}$


Appendix B - Radionuclide Master Library - RMDLIB

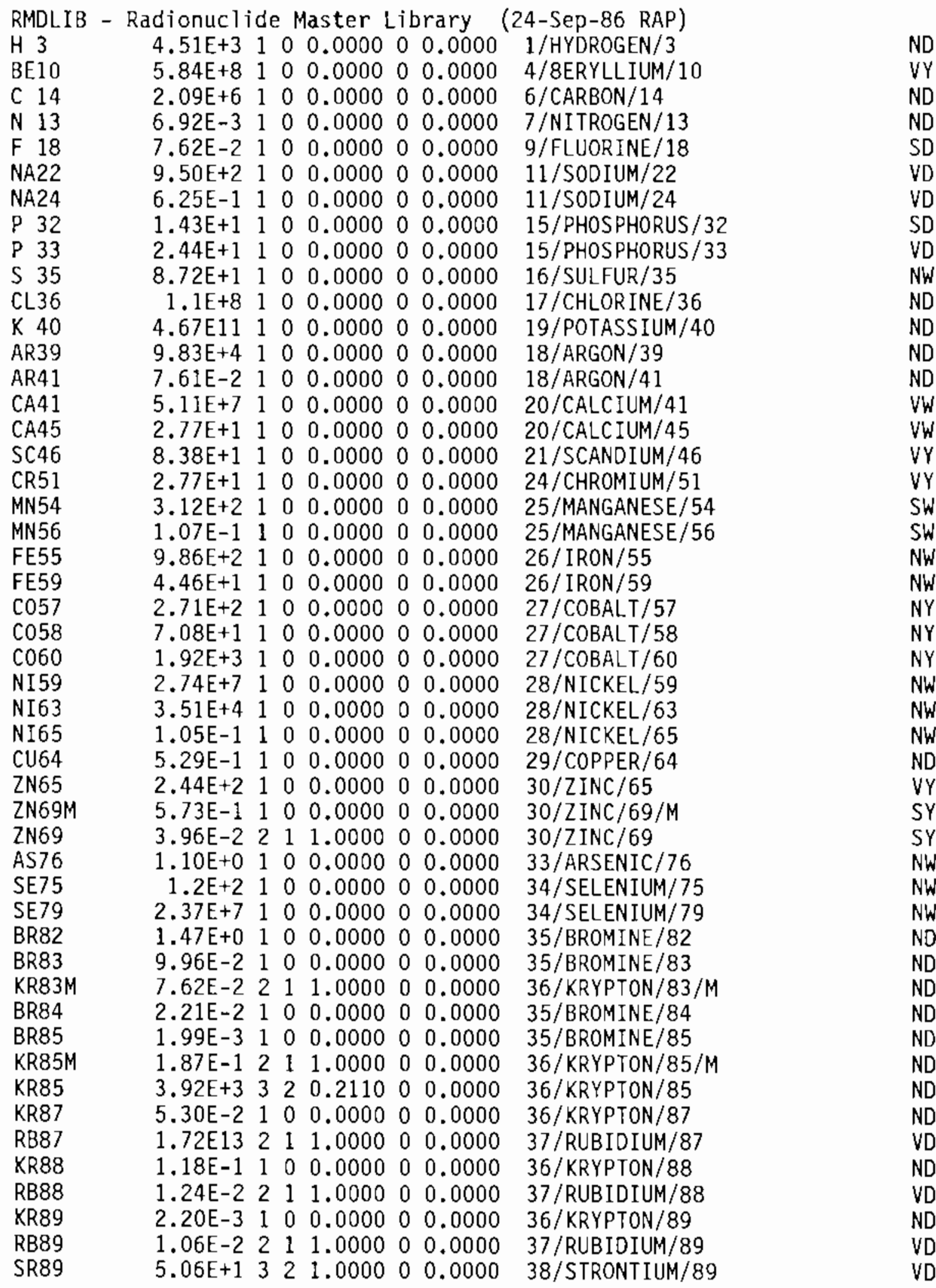




\section{Appendix B - Radionuclide Master Library - RMDLIB}

\begin{tabular}{|c|c|c|c|c|c|c|}
\hline Y $89 M$ & $1.86 \mathrm{E}-4$ & 4 & 0.0002 & 00.0000 & 39/YTTRIUM/89/M & SY \\
\hline KR90 & $3.74 E-4$ & 10 & 0.0000 & 00.0000 & $36 /$ KRYPTON $/ 90$ & ND \\
\hline KR91 & $1.13 \mathrm{E}-4$ & 10 & 0.0000 & 00.0000 & $36 /$ KRYPTON/91 & ND \\
\hline RB86 & $1.87 E+1$ & 10 & 0.0000 & 00.0000 & $37 /$ RUBIDIUM/86 & YU \\
\hline SR85 & $6.5 \mathrm{E}+1$ & 10 & 0.0000 & 00.0000 & 38/STRONT IUM/85 & Vo \\
\hline SR90 & $1.04 \mathrm{E}+4$ & 10 & 0.0000 & 00.0000 & $38 /$ STRONT IUM $/ 90$ & \\
\hline Y 90 & $2.67 E+0$ & 21 & 1.0000 & 00.0000 & 39/YTTRIUM/90 & \\
\hline SR91 & $3.96 \mathrm{E}-1$ & 10 & 0.0000 & 00.0000 & 38/STRONTIUM/91 & \\
\hline Y $91 \mathrm{M}$ & $3.45 \mathrm{E}-2$ & 21 & 0.5800 & 00.0000 & 39/YTTRIUM/91/M & $Y$ \\
\hline Y 91 & $5.85 \bar{E}+1$ & 32 & 1.0000 & 10.4200 & $39 /$ YTTRIUM/91 & \\
\hline SR92 & 1. $13 \mathrm{E}-1$ & 10 & 0.0000 & 00.0000 & 38/STRONTIUM/92 & \\
\hline Y 92 & $1.48 \mathrm{E}-1$ & 21 & 1.0000 & 00.0000 & 39/YTTRIUM/92 & \\
\hline Y 93 & $4.21 E-1$ & 10 & 0.0000 & 00.0000 & $39 /$ YTTRIUM $/ 93$ & \\
\hline ZR93 & $5.59 \mathrm{E}+8$ & 21 & 1.0000 & 00.0000 & 40/ZIRCONIUM/93 & \\
\hline NB93M & $4.97 E+3$ & 32 & 0.2500 & 00.0000 & $41 /$ NIOBIUM $/ 93 / M$ & \\
\hline ZR95 & $6.40 E+1$ & 10 & 0.0000 & 00.0000 & 40/ZIRCONIUM/95 & \\
\hline NB95M & $3.61 \mathrm{E}+0$ & 21 & 0.0070 & 00.0000 & $41 /$ NIOBIUM $/ 95 / M$ & \\
\hline NB95 & $3.52 E+1$ & 32 & 1.0000 & 10.9930 & $41 /$ NIOBIUM $/ 95$ & \\
\hline ZR97 & $7.04 \mathrm{E}-1$ & 10 & 0.0000 & 00.0000 & $40 /$ I IRCONIUM $/ 97$ & \\
\hline NB97M & $6.94 E-4$ & 21 & 0.9460 & 00.0000 & $41 /$ NIOBIUM $/ 97 / M$ & \\
\hline NB97 & $5.01 \mathrm{E}-2$ & 32 & 1.0000 & 10.0540 & $41 /$ NIOBIUM $/ 97$ & \\
\hline NB94 & $7.30 E+6$ & 10 & 0.0000 & 00.0000 & $41 /$ N IOBIUM $/ 94$ & \\
\hline M093 & $3.65 E+4$ & 10 & 0.0000 & 00.0000 & 42/MOL YBDENUM/93 & \\
\hline M099 & $2.75 \mathrm{E}+0$ & 10 & 0.0000 & 00.0000 & 42/MOLYBDENUM/99 & \\
\hline TC99M & $2.51 \mathrm{E}-1$ & 21 & $0.86 \mathrm{BO}$ & 00.0000 & 43/TECHNETIUM/99/M & \\
\hline TC99 & $7.78 \mathrm{E}+7$ & 32 & 1.0000 & 10.1320 & 43/TECHNET IUM/99 & \\
\hline TC101 & $9.86 \mathrm{E}-3$ & 10 & 0.0000 & 00.0000 & 43/TECHNETIUM/101 & \\
\hline RU103 & $3.94 \mathrm{E}+1$ & 10 & 0.0000 & 00.0000 & 44/RUTHENIUM/103 & \\
\hline PD103 & $1.70 \mathrm{E}+1$ & 20 & 0.0000 & 00.0000 & 46/PALLADIUM/103 & \\
\hline RH103M & $3.90 \mathrm{E}-2$ & 31 & 0.9974 & 20.9997 & 45/RHODIUM/103/M & \\
\hline RU105 & $1.85 \mathrm{E}-1$ & 10 & 0.0000 & 00.0000 & 44/RUTHENIUM/ 105 & \\
\hline RH105M & $5.21 E-4$ & 21 & 0.2800 & 00.0000 & 45/RHODIUM/105/M & \\
\hline RH105 & $1.47 \mathrm{E}+0$ & 32 & 1.0000 & 10.7200 & 45/RHODIUM/ 105 & \\
\hline RU106 & $3.68 \mathrm{E}+2$ & 10 & 0.0000 & 00.0000 & 44/RUTHENIUM/106 & \\
\hline RH106 & $3.46 \mathrm{E}-4$ & 21 & 1.0000 & 00.0000 & $45 /$ RHODIUM/ 106 & \\
\hline PD107 & $2.37 E+9$ & 10 & 0.0000 & 00.0000 & 46/PALLADIUM/107 & \\
\hline PD109M & 5.4 & 10 & 0.0000 & 00.0000 & 46/PALLADIUM/109/M & \\
\hline PD109 & 5. & 21 & 1.0000 & 00.0000 & 46/PALLADIUM/109 & \\
\hline AG109M & $3 E-4$ & 32 & 1.0000 & 00.0000 & $47 /$ SILVER $/ 109 / \mathrm{M}$ & \\
\hline AG110M & $2 E+2$ & 10 & 0.0000 & 00.0000 & $47 /$ SILVER $/ 110 / \mathrm{M}$ & \\
\hline AG110 & $E-4$ & 2 & 0.0113 & 00.0000 & $47 /$ SILVER/ 110 & \\
\hline AG111 & $7.45 \mathrm{E}+0$ & 10 & 0.0000 & 00.0000 & $47 /$ SILVER/111 & \\
\hline CD109 & $E+2$ & 10 & 0.0000 & 00.0000 & 48/CADMIUM / 109 & \\
\hline CD113M & $7 E+3$ & 0 & 0.0000 & 00.0000 & $48 /$ CADMIUM $/ 113 / M$ & \\
\hline CD115M & $4.46 \mathrm{E}+0$ & 1 & 0.0000 & 00.0000 & $4 B /$ CADMIUM $/ 115 / M$ & \\
\hline CD115 & $3 \mathrm{E}+0$ & 2 & 0.0000 & 00.0000 & 48/CADMIUM/115 & \\
\hline IN115M & & 3 & 1.0000 & 00.0000 & 49/INDIUM/115/M & \\
\hline IN115 & $2.19 \mathrm{E} 17$ & & 0.9630 & 11.0000 & $49 /$ INDIUM/ 115 & \\
\hline & & & 0.0000 & 00.0000 & 49/INDIUM/111 & \\
\hline IN114M & 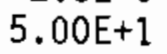 & & 0.0000 & 00.0000 & $49 /$ INDIUM/114/M & \\
\hline
\end{tabular}


Appendix B - Radionuclide Master Library - RMDLIB

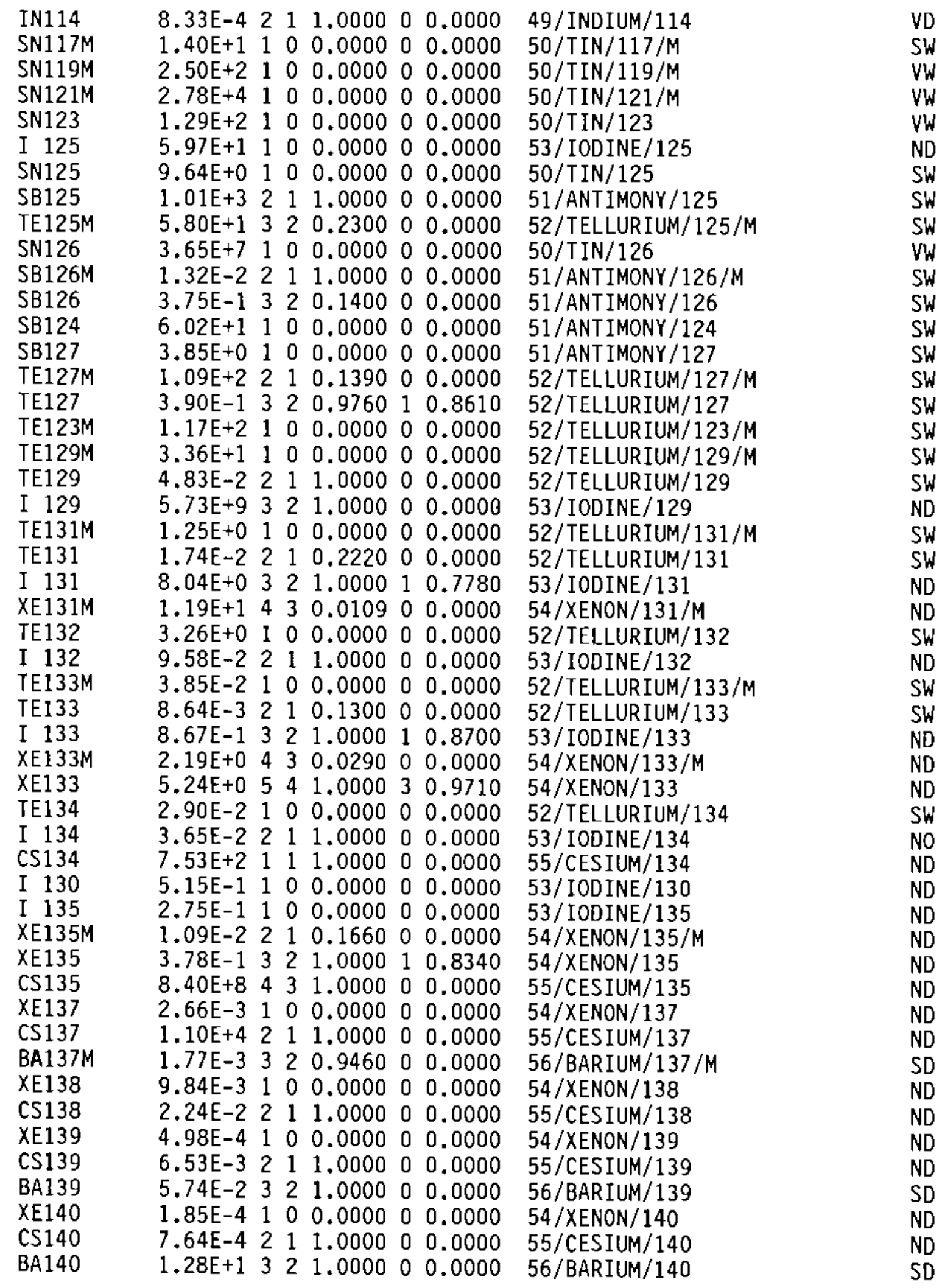




\section{Appendix B - Radionuclide Master Library - RMDLIB}

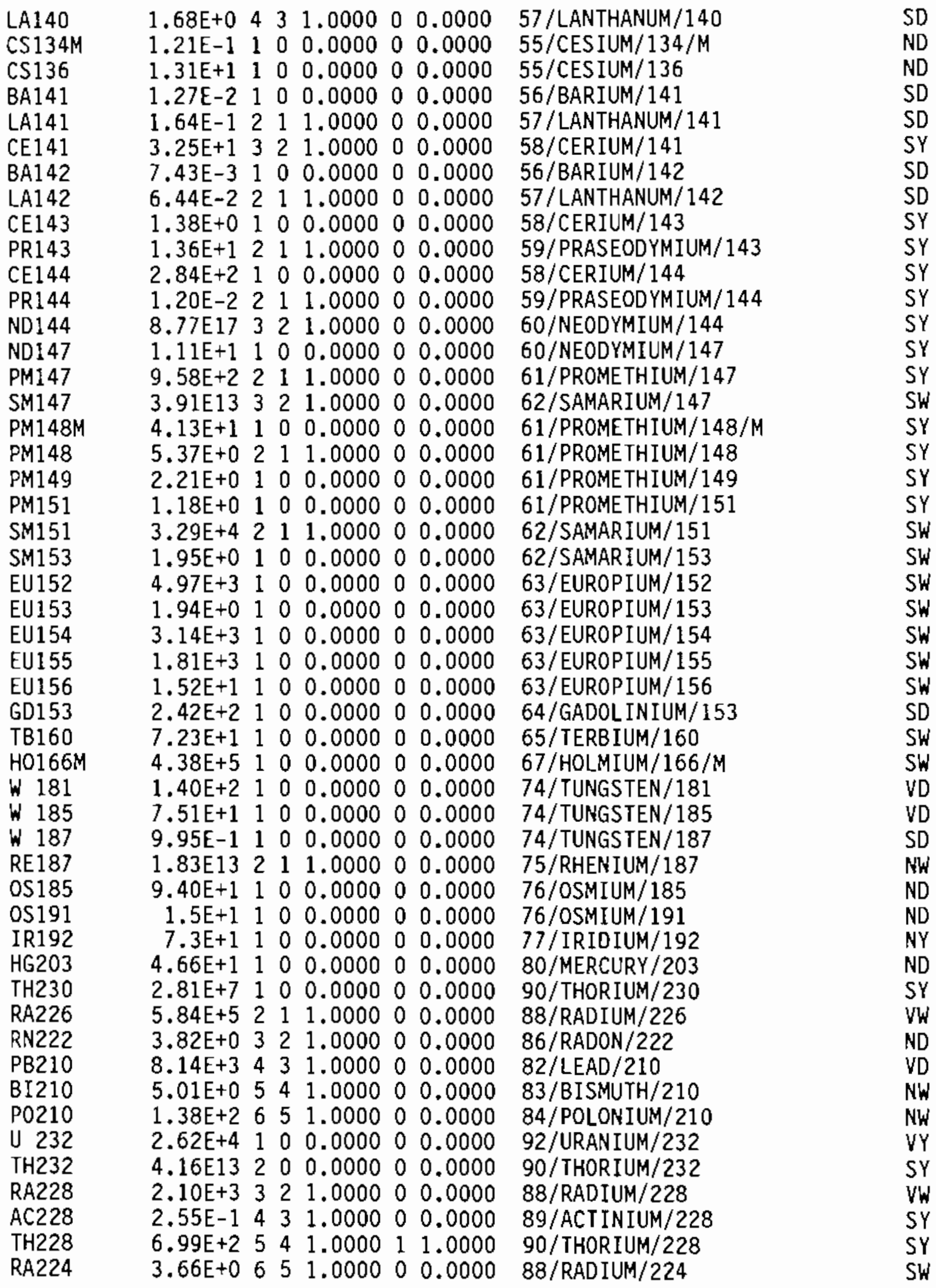




\section{Appendix B - Radionuclide Master Library - RMDLIB}

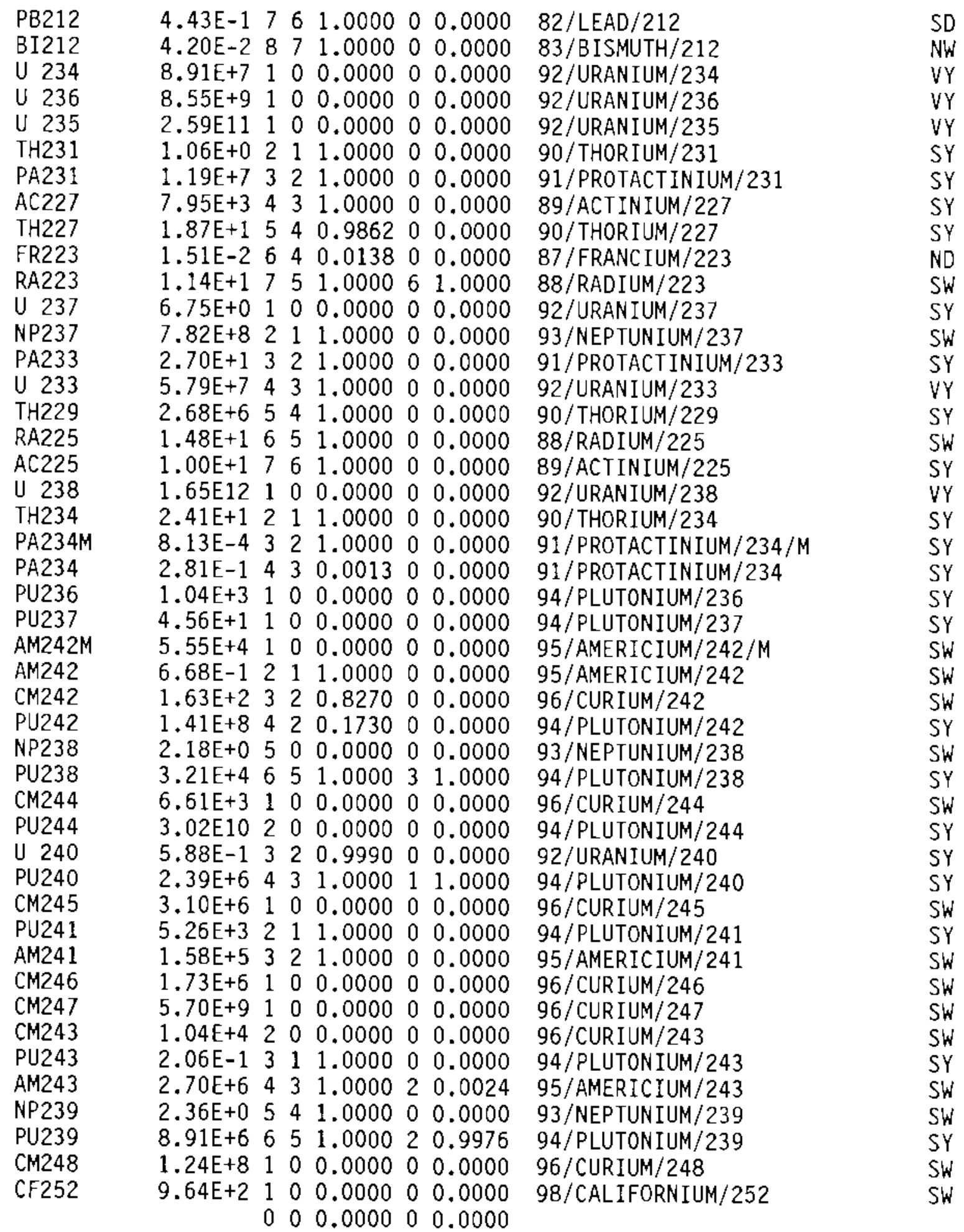




\section{Appendix B - Organ Data Library - ORGLIB}

ORGAN DATA LIBRARY UPDATED BY RA PELOQUIN 8-JUI-86

H 3

H 3

BE10

BE10

BE10

BE10

BE10

BE10

C 14

C 14

C 14

C 14

N 13

F 18

F 18

F 18

F 18

NA22

NA22

NA22

NA24

NA24

NA24

P 32

P 32

P 32

P 32

P 32

P 33

P 33

P 33

P 33

P 33

S 35

S 35

S 35

CL36

CL36

$\mathrm{CL} 36$

K 40

K 40

$\times 40$

$\times 40$

CA41

CA41

CA41

CA41

CA45

CA45

$\begin{array}{rrrrr}1 & 1.0+1 & 1.0 & 1.0 & .0058 \\ 8 & & & & .0058 \\ 1 & 1.8+2 & 2.00-3 & 1.0 & .209 \\ 3 & 1.2+2 & 6.00-5 & 0.03 & .209 \\ 4 & 2.7+2 & 2.00-4 & 0.1 & .209 \\ 6 & 4.5+2 & 6.40-4 & 0.32 & 1.045\end{array}$
8

10.

$40 . \quad 0.025$

12.

0.5

1.0

0.025

.209

.209

7

23

90 .

1.0

$1.45+3 \quad 0.53$

1.0
0.53

0.5

.054

.27

.054

.054

1.143

$1.0 \quad 0.86$

$0.53 \quad 1.462$

8

23

1

$1.10+1 \quad 1.0$

1.0

0.54

0.39

8
23

23
1 (2)

$1.10+1 \quad 1.0$

1.513

$\begin{array}{ll}1.0 & 2.725\end{array}$

1.5

1.0

257.

.75

1.0

0.07

0.50

.696

$1.15+3$

.375

0.50

.696

3.459

.690

23

257.

$18 . \quad .05$

$1.15+3 \quad .38$

1.0

0.07

0.20

.0853

.0853

0.426

.0853

23

90.

$1.0 \quad 1.0$

.0853

600.

$.03 \quad .03$

29.0

1.0

1.0

.0560

.28

.056

1
8

23

1

58.0

1.

1.01.

.26

.26

.251

.843

.200

.182

23

1

$1.64+4$

1.0

.0036

.0036

.0036

.0036

23

1

$1.64+4$

$1.8+4 \quad .54$

1.0

0.9

.086

.430

B. 11 


\section{Appendix B - Organ Data Library - ORGLIB}

\begin{tabular}{|c|c|c|c|c|c|}
\hline $\begin{array}{l}\text { CA45 } \\
\text { CA45 }\end{array}$ & $\begin{array}{l}8 \\
23\end{array}$ & & & & $\begin{array}{r}.086 \\
.086\end{array}$ \\
\hline SC46 & 1 & 30.0 & $.10-3$ & 1.0 & 1.333 \\
\hline $\mathrm{SC} 46$ & 3 & 75.0 & $.20-5$ & .02 & .512 \\
\hline $\mathrm{SC} 46$ & 4 & 36.0 & $.15-4$ & .15 & .654 \\
\hline SC46 & 6 & 33.0 & $.20-4$ & .20 & 1.101 \\
\hline SC46 & 8 & & & & .640 \\
\hline SC46 & 23 & & & & .409 \\
\hline CR51 & 1 & 616. & .005 & 1.0 & .019 \\
\hline CR51 & 3 & 616. & $.13-4$ & $.27-2$ & .007 \\
\hline CR51 & 8 & 616. & $.20-3$ & .04 & .014 \\
\hline CR51 & 15 & 616. & $.45-5$ & $.90-3$ & $.084-1$ \\
\hline CR51 & 16 & 616. & $0.45-5$ & $.90-3$ & $3.6-3$ \\
\hline CR51 & 23 & & & & .0053 \\
\hline MN54 & 1 & 17. & .10 & 1.0 & .512 \\
\hline MN54 & 4 & 25. & .02 & .24 & .227 \\
\hline MN54 & 8 & & & & .230 \\
\hline MN54 & 17 & 5.70 & .003 & .03 & .130 \\
\hline MN54 & 23 & & & & .122 \\
\hline MN56 & 1 & 17. & .10 & 1.0 & 1.810 \\
\hline MN56 & 4 & 25 . & .02 & .24 & 1.236 \\
\hline MN56 & 8 & & & & 1.30 \\
\hline MN56 & 17 & 5.70 & .003 & .03 & 1.10 \\
\hline MN56 & 23 & & & & 1.03 \\
\hline FE55 & 1 & 800 . & .10 & 1.0 & $9.5-3$ \\
\hline FE55 & 4 & 554. & .013 & .13 & $9.5-3$ \\
\hline FE55 & 5 & 600 & .002 & .02 & $6.5-3$ \\
\hline FE55 & 6 & 1680. & .010 & .10 & $4.2-2$ \\
\hline FE55 & 8 & 3200 . & .002 & .02 & $9.5-3$ \\
\hline FE55 & 23 & & & & $9.5-3$ \\
\hline FE59 & 1 & 800 . & .10 & 1.0 & .835 \\
\hline FE59 & 4 & 554. & .013 & .13 & .439 \\
\hline FE59 & 5 & 600 & .002 & .02 & .340 \\
\hline FE59 & 6 & 1680. & .01 & .10 & .946 \\
\hline FE59 & 8 & 3200 . & .002 & .02 & .420 \\
\hline FE59 & 23 & & & & .298 \\
\hline C057 & 1 & 9.50 & .300 & 1.0 & .10 \\
\hline $\mathrm{C} 057$ & 4 & 9.50 & .007 & .04 & .063 \\
\hline C057 & 5 & 9.50 & $.420-3$ & $.14-2$ & .045 \\
\hline C057 & 8 & & & & .053 \\
\hline C057 & 17 & 9.50 & $.600-3$ & .002 & .040 \\
\hline C057 & 23 & & & & $4.96-2$ \\
\hline $\operatorname{co5} 8$ & 1 & 9.50 & .300 & 1.0 & .628 \\
\hline $\operatorname{co58}$ & 4 & 9.50 & .007 & .040 & .292 \\
\hline C058 & 5 & 9.50 & $.420-3$ & $.140-2$ & .220 \\
\hline $\operatorname{co58}$ & 8 & & & & .290 \\
\hline C058 & 17 & 9.50 & $.600-3$ & .002 & .170 \\
\hline $\cos 8$ & 23 & & & & .169 \\
\hline $\mathrm{COSO}$ & 1 & 9.50 & .300 & 1.0 & 1.575 \\
\hline \multirow[t]{2}{*}{$\mathrm{C} 060$} & 4 & 9.50 & .007 & .040 & .745 \\
\hline & & & & & 3.12 \\
\hline
\end{tabular}


Appendix B - Organ Data Library - ORGLIB

\begin{tabular}{|c|c|c|c|c|c|c|c|}
\hline $\begin{array}{l}\text { C060 } \\
\text { C060 }\end{array}$ & $\begin{array}{l}5 \\
8\end{array}$ & 9.50 & $.420-3$ & $.140-2$ & $\begin{array}{l}.560 \\
.720\end{array}$ & & \\
\hline $\mathrm{CO60}$ & 17 & 9.50 & $.600-3$ & $.200-2$ & .440 & & \\
\hline $\mathrm{C} 060$ & 23 & & & & .449 & & \\
\hline NI59 & 1 & 667. & .300 & 1.0 & $7.7-3$ & & \\
\hline NI59 & 4 & 500. & .020 & .070 & $7.7-3$ & & \\
\hline NI59 & 6 & 800. & .150 & .500 & $7.7-3$ & & \\
\hline NI59 & 8 & & & & $.770-2$ & & \\
\hline NI59 & 23 & & & & $7.7-3$ & & \\
\hline NI63 & 1 & 667. & .300 & 1.0 & .021 & & \\
\hline NI63 & 4 & 500 & .020 & .070 & .021 & & \\
\hline NI63 & 6 & 800 & .150 & .500 & .105 & & \\
\hline NI63 & 8 & & & & .021 & & \\
\hline NI63 & 23 & & & & .021 & & \\
\hline NI65 & 1 & 667. & .300 & 1.0 & .940 & & \\
\hline NI65 & 4 & 500 & .020 & .070 & .752 & & \\
\hline NI65 & 6 & 800 & .150 & .500 & 3.176 & & \\
\hline NI65 & 8 & & & & 1.20 & & \\
\hline NI65 & 23 & & & & .686 & & \\
\hline Cu64 & 1 & 80. & .280 & 1.0 & .251 & & \\
\hline CU64 & 3 & 16. & .010 & .050 & .166 & & \\
\hline CU64 & 4 & 150. & .020 & .080 & .181 & & \\
\hline CU64 & 5 & 2. & .020 & .070 & .170 & & \\
\hline CU64 & 8 & & & & .190 & & \\
\hline CU64 & 13 & 800 & .003 & .010 & .210 & & \\
\hline CU64 & 18 & 80. & .003 & .010 & .170 & & \\
\hline CU64 & 23 & & & & .156 & & \\
\hline ZN65 & 1 & 933. & .100 & 1.0 & .341 & & \\
\hline ZN65 & 3 & 149. & .004 & .04 & .113 & & \\
\hline ZN65 & 4 & 91. & .035 & .350 & .153 & & \\
\hline ZN65 & 6 & 1300. & .015 & .150 & .149 & & \\
\hline ZN65 & 8 & & & & .150 & & \\
\hline ZN65 & 10 & 270 & $.900-4$ & $.900-3$ & .056 & & \\
\hline ZN65 & 11 & 107. & $.400-4$ & $.400-3$ & .056 & & \\
\hline ZN65 & 14 & 1960. & .030 & .300 & .320 & & \\
\hline ZN65 & 15 & 14 & .006 & .060 & .056 & & \\
\hline ZN65 & 17 & 25 . & .003 & .030 & .084 & & \\
\hline ZN65 & 23 & & & & .084 & & \\
\hline ZN69M & 1 & 933. & .100 & 1.0 & .610 & & \\
\hline ZN69M & 3 & 149. & .004 & .040 & .435 & & \\
\hline ZN69M & 4 & 91. & .035 & .350 & .466 & & \\
\hline ZN69M & 6 & 1300. & .015 & .150 & 1.852 & & \\
\hline ZN69M & 8 & & & & .487 & .50 & .50 \\
\hline ZN69M & 10 & 270 & $.900-4$ & $.900-3$ & .430 & & \\
\hline ZN69M & 11 & 107. & $.400-4$ & $.400-3$ & .430 & & \\
\hline ZN69M & 14 & 1960. & .030 & .300 & .640 & & \\
\hline ZN69M & 15 & 14 & .006 & .060 & .430 & & \\
\hline ZN69M & 17 & 25 . & .003 & .030 & .450 & & \\
\hline ZN69M & 23 & & & & .412 & & \\
\hline \multirow[t]{2}{*}{ ZN69 } & 1 & 933. & .100 & 1.0 & .328 & & \\
\hline & & & & & 13 & & \\
\hline
\end{tabular}




\section{Appendix B - Organ Data Library - ORGLIB}

\begin{tabular}{|c|c|c|c|c|c|c|c|}
\hline ZN69 & 3 & 149. & .004 & .040 & .328 & & \\
\hline ZN69 & 4 & 91. & .035 & .350 & .328 & & \\
\hline ZN69 & 6 & 1300. & .015 & .150 & 1.638 & & \\
\hline ZN69 & 8 & & & & .370 & & \\
\hline ZN69 & 10 & 270 & $.900-4$ & $.900-3$ & .370 & & \\
\hline ZN69 & 11 & 107. & $.400-4$ & $.400-3$ & .370 & & \\
\hline ZN69 & 14 & 1960. & .030 & .300 & .370 & & \\
\hline ZN69 & 15 & 14. & .006 & .060 & .370 & & \\
\hline ZN69 & 17 & 25. & .003 & .030 & .370 & & \\
\hline ZN69 & 23 & & & & .328 & & \\
\hline SE75 & 1 & 11. & .9 & 1. & .2 & & \\
\hline SE75 & 8 & & & & 9.4 & & \\
\hline AS76 & 1 & 280. & .030 & 1.0 & 1.3 & & \\
\hline AS76 & 3 & 550. & $.300-3$ & .010 & 1.1 & & \\
\hline AS76 & 4 & 550. & $.900-3$ & .030 & 1.1 & & \\
\hline AS76 & 8 & & & & 1.10 & & \\
\hline AS76 & 23 & & & & 1.1 & & \\
\hline SE79 & 1 & 11. & .9 & 1. & .042 & & \\
\hline SE79 & 3 & 11. & .04 & .04 & .042 & & \\
\hline SE79 & 4 & 24. & .06 & .07 & .042 & & \\
\hline SE79 & 8 & & & & .042 & .042 & .042 \\
\hline SE79 & 23 & & & & .042 & & \\
\hline BR82 & 1 & 8.0 & 1.0 & 1.0 & 1.715 & & \\
\hline BR82 & 8 & & & & .850 & & \\
\hline 8R82 & 23 & & $5.0-2$ & & .522 & & \\
\hline SR85 & 1 & $.13 \mathrm{E}+5$ & .300 & 1.0 & .33 & & \\
\hline SR85 & 6 & $.18 \mathrm{E}+5$ & .09 & .175 & .091 & & \\
\hline SR85 & 8 & & & & .16 & & \\
\hline SR85 & 23 & & & & 0. & & \\
\hline RB86 & 1 & 45. & 1.0 & 1.0 & .706 & & \\
\hline RB86 & 4 & 63. & .05 & .05 & .674 & & \\
\hline $\begin{array}{l}\text { RB86 } \\
\text { R886 }\end{array}$ & 5 & 45. & .004 & .004 & .660 & & \\
\hline $\begin{array}{l}\text { R880 } \\
\text { RB86 }\end{array}$ & $\begin{array}{r}8 \\
14\end{array}$ & 80 & .450 & .450 & $\begin{array}{r}.660 \\
700\end{array}$ & & \\
\hline RB86 & 17 & 60. & .003 & .003 & .650 & & \\
\hline RB86 & 23 & & & & .663 & & \\
\hline RB87 & 1 & 45. & 1.0 & 1.0 & .090 & & \\
\hline R887 & 4 & 63. & .050 & .050 & .090 & & \\
\hline R887 & 5 & 45. & .004 & .004 & .090 & & \\
\hline RB87 & 8 & & & & .090 & & \\
\hline RB87 & 14 & 80. & .450 & .450 & .090 & & \\
\hline R887 & 17 & 60. & .003 & .003 & .090 & & \\
\hline R887 & 23 & & & & .090 & & \\
\hline SR89 & 1 & $.130+5$ & .300 & 1.0 & .555 & & \\
\hline SR89 & 6 & $.180+5$ & .210 & .700 & 2.76 & & \\
\hline SR89 & 8 & & & & .550 & & \\
\hline SR89 & 23 & .3 & 1.0 & 0.0 & .555 & & \\
\hline SR90 & 1 & 4000 & .3 & 1.0 & 1.137 & & \\
\hline SR9O & 6 & 4000 & .0225 & .300 & 5.650 & & \\
\hline SR9C & 8 & & & & 1.305 & & \\
\hline & & & & & B. 14 & & \\
\hline
\end{tabular}




\section{Appendix B - Organ Data Library - ORGLIB}

\begin{tabular}{|c|c|c|c|c|c|c|c|}
\hline SR90 & 23 & & & & .777 & & \\
\hline Y 90 & 1 & $.140+5$ & $.100-3$ & 1.0 & .917 & & \\
\hline Y 90 & 6 & $.180+5$ & $.750-4$ & .750 & 4.555 & & \\
\hline Y 90 & 8 & & & & .890 & & \\
\hline Y 90 & 23 & & & & .917 & & \\
\hline SR91 & 1 & $.130+5$ & .300 & 1.0 & 1.834 & & \\
\hline SR91 & 6 & $.180+5$ & .210 & .700 & 6.47 & & \\
\hline SR91 & 8 & & & & 1.15 & 1.4 & 1.7 \\
\hline SR91 & 23 & & & & 0.777 & & \\
\hline Y $91 \mathrm{M}$ & 1 & $.140+5$ & $.100-3$ & 1.0 & .967 & & \\
\hline Y 91M & 6 & $.180+5$ & $.750-4$ & .750 & 3.325 & & \\
\hline Y $91 \mathrm{M}$ & 8 & & & & .160 & .42 & .68 \\
\hline Y $91 \mathrm{M}$ & 23 & & & & .107 & & \\
\hline Y 91 & 1 & $.140+5$ & $.100-3$ & 1.0 & .612 & & \\
\hline Ү 91 & 6 & $.180+5$ & $.750-4$ & .750 & 3.049 & & \\
\hline Y 91 & 8 & & & & .59 & & \\
\hline Y 91 & 23 & & & & .61 & & \\
\hline SR92 & 1 & $.130+5$ & .300 & 1.0 & 2.601 & & \\
\hline SR92 & 6 & $.180+5$ & .210 & .700 & 8.602 & & \\
\hline SR92 & 8 & & & & 1.75 & 1.9 & 1.9 \\
\hline SR92 & 23 & & & & 1.867 & & \\
\hline Y 92 & 1 & $.140+5$ & $.100-3$ & 1.0 & 1.589 & & \\
\hline Y 92 & 6 & $.180+5$ & $.750-4$ & .750 & 7.257 & & \\
\hline Y 92 & 8 & & & & 1.50 & & \\
\hline Y 92 & 23 & & & & 1.475 & & \\
\hline Y 93 & 1 & $.140+5$ & $.100-3$ & 1.0 & 1.648 & & \\
\hline Y 93 & 6 & $.180+5$ & $.750-4$ & .750 & 7.967 & & \\
\hline Y 93 & 8 & & & & 1.50 & 1.50 & 1.50 \\
\hline Y 93 & 23 & & & & 1.602 & & \\
\hline ZR93 & 1 & 450 . & $.100-3$ & 1.0 & .023 & & \\
\hline ZR93 & 3 & 900 . & $.200-5$ & .020 & .020 & & \\
\hline ZR93 & 4 & 320. & $.700-5$ & .070 & .024 & & \\
\hline ZR93 & 5 & 900. & $.600-6$ & .006 & .025 & & \\
\hline ZR93 & 6 & 1000 . & $.360-4$ & .360 & .110 & & \\
\hline ZR93 & 8 & & & & .019 & .020 & .024 \\
\hline ZR93 & 23 & & & & .019 & & \\
\hline NB93M & 1 & 760. & $.100-3$ & 1.0 & .030 & & \\
\hline NB93M & 3 & 760. & $.200-5$ & .020 & .030 & & \\
\hline NB93M & 4 & 845. & $.900-5$ & .090 & .030 & & \\
\hline NB93M & 5 & 95. & $.800-6$ & .008 & .038 & & \\
\hline NB93M & $6 I$ & 000 . & $.380-4$ & .380 & .078 & & \\
\hline NB93M & 8 & & & & .038 & & \\
\hline NB93M & 23 & & & & .030 & & \\
\hline ZR95 & 1 & 450. & $.100-3$ & 1.0 & 1.09 & & \\
\hline ZR95 & 3 & 900. & $.200-5$ & .020 & .510 & & \\
\hline ZR95 & 4 & 320 . & $.700-5$ & .070 & .589 & & \\
\hline ZR95 & 5 & 900. & $.600-6$ & .006 & .460 & & \\
\hline ZR95 & 61 & 000 & $.360-4$ & .360 & 1.30 & & \\
\hline ZR95 & 8 & & & & .327 & .47 & .56 \\
\hline ZR95 & 23 & & & & .238 & & \\
\hline
\end{tabular}

B. 15 


\section{Appendix B - Organ Data Library - ORGLIB}

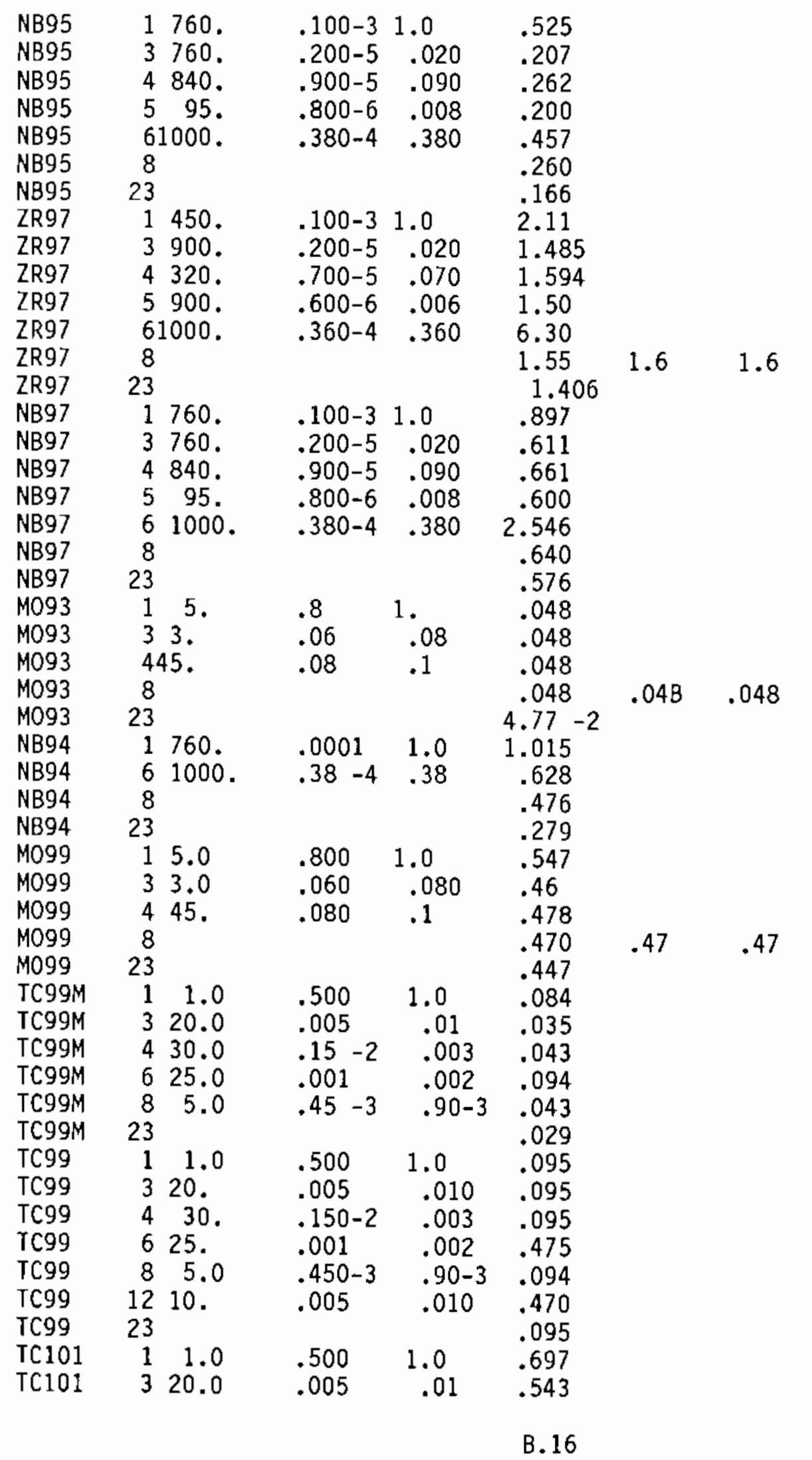




\section{Appendix B - Organ Data Library - ORGLIB}

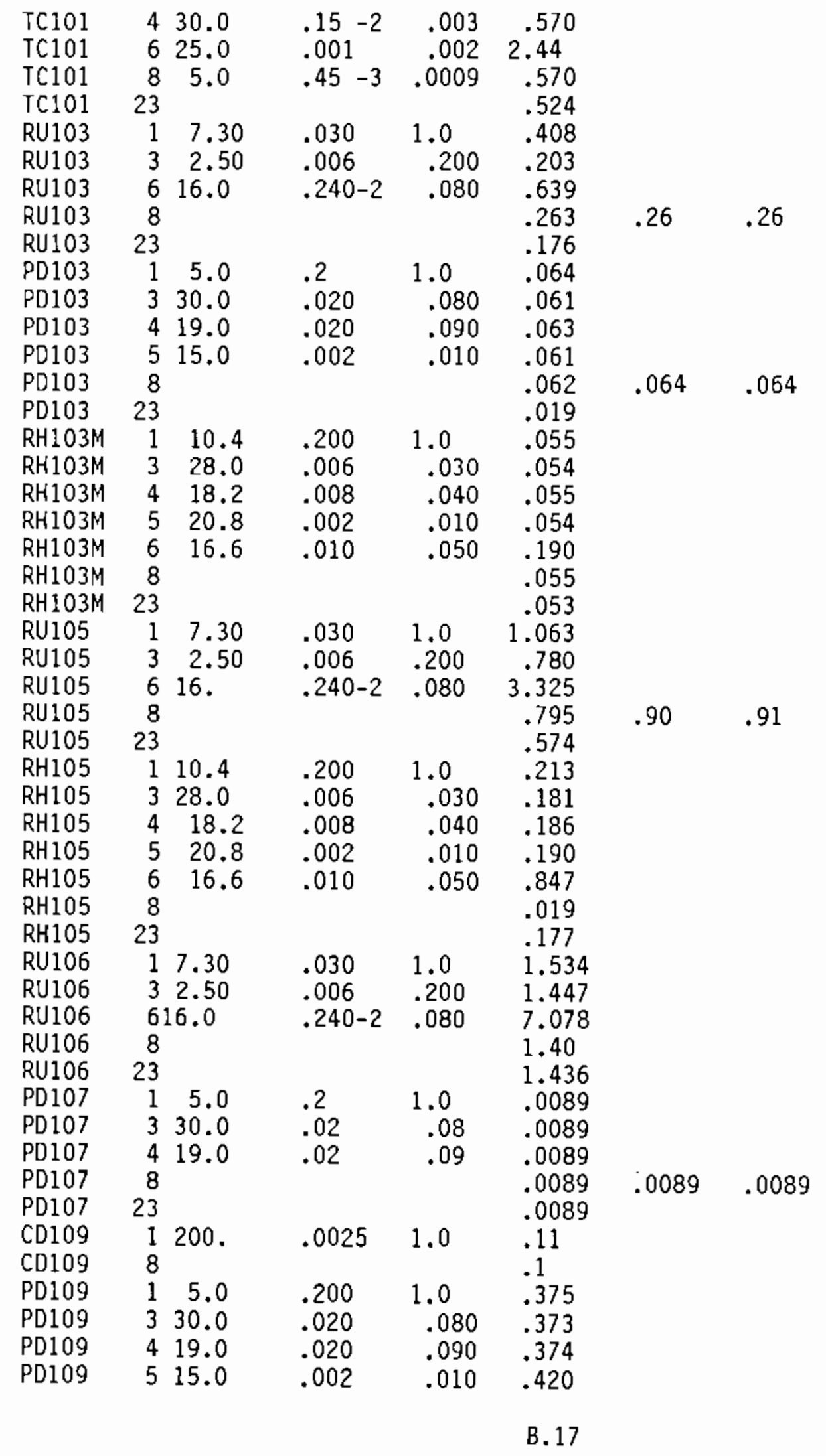




\section{Appendix B - Organ Data Library - ORGLIB}

\begin{tabular}{|c|c|c|c|c|c|c|c|}
\hline $\begin{array}{l}\text { PD109 } \\
\text { PD109 }\end{array}$ & $\begin{array}{r}8 \\
23\end{array}$ & & & & $\begin{array}{l}.42 \\
.373\end{array}$ & & \\
\hline AG $110 M$ & & 5.0 & .010 & 1.0 & 1.697 & & \\
\hline AG110M & & 0.0 & $.200-3$ & .020 & .614 & & \\
\hline AG $110 M$ & & 5.0 & $.300-3$ & .030 & .803 & & \\
\hline AG110M & & 0.0 & $.500-3$ & .050 & 1.129 & & \\
\hline $\mathrm{AG} 110 \mathrm{M}$ & 8 & & & & .840 & & \\
\hline$A G \$ 10 M$ & 23 & & & & .477 & & \\
\hline AG111 & 1 & 5.0 & .010 & 1.0 & .383 & & \\
\hline AG111 & 3 & 10.0 & $.200-3$ & .020 & .372 & & \\
\hline AG111 & 4 & 15.0 & $.300-3$ & .030 & .374 & & \\
\hline AG111 & 6 & 30.0 & $.500-3$ & .050 & 1.84 & & \\
\hline AG111 & 8 & & & & .380 & & \\
\hline AG11I & 23 & & & & .370 & & \\
\hline IN 111 & 1 & 48. & .002 & 1.0 & .286 & & \\
\hline IN111 & 6 & 57. & $.34-03$ & .17 & .302 & & \\
\hline IN111 & 8 & & & & .155 & & \\
\hline IN111 & 16 & 8.4 & $.80 E-6$ & $.40-03$ & .104 & & \\
\hline IN1I1 & 23 & & & & .104 & & \\
\hline CD113M & 1 & 200. & .0025 & 1.0 & .200 & & \\
\hline CD113M & 3 & 300 . & $2.5 \quad-4$ & 0.1 & .200 & & \\
\hline CD113M & 4 & 200 . & $1.9-3$ & 0.75 & .200 & & \\
\hline CD113M & 8 & & & & .184 & .184 & .184 \\
\hline CD113M & 23 & & & & .200 & & \\
\hline IN114M & 1 & 48.0 & .002 & 1.0 & 970 & & \\
\hline IN $114 M$ & 3 & 60.0 & $.800-4$ & .040 & .930 & & \\
\hline IN $114 M$ & 4 & 58.0 & $.280-3$ & .140 & .940 & & \\
\hline IN11 4M & 5 & 48.0 & $.400-4$ & .020 & .930 & & \\
\hline IN1 $14 M$ & 6 & 57.0 & $340-3$ & .170 & 4.50 & & \\
\hline IN $114 M$ & 8 & & & & .940 & & \\
\hline IN114M & 12 & 67.0 & $.360-3$ & .180 & .900 & & \\
\hline IN1 14M & 16 & 8.40 & $.800-6$ & $400-3$ & .920 & & \\
\hline IN1 $14 M$ & 23 & & & & .93 & & \\
\hline CD115M & 1 & 200. & $.250-2$ & 1.0 & .610 & & \\
\hline CD115M & 3 & 300 . & $.250-3$ & .10 & .610 & & \\
\hline CD115M & 4 & 200 . & $.190-2$ & .750 & .610 & & \\
\hline CD115M & 8 & & & & .610 & .610 & .610 \\
\hline CD115M & 23 & & & & .61 & & \\
\hline CD115 & 1 & 200. & $.250-2$ & 1.0 & .710 & & \\
\hline CD115 & 3 & 300 . & $.250-3$ & .1 & .560 & & \\
\hline CD115 & 4 & 200 . & $.190-2$ & .750 & .580 & & \\
\hline CD115 & 8 & & & & $.650-18$ & $.38-16$ & $.39-15$ \\
\hline CD115 & 23 & & & & .36 & & \\
\hline IN1 $15 \mathrm{M}$ & 01 & 48.0 & .002 & 1.0 & .260 & & \\
\hline IN115M & 3 & 60.0 & $.800-4$ & .040 & .190 & & \\
\hline IN $115 \mathrm{M}$ & 4 & 58.0 & $.280-3$ & .140 & .200 & & \\
\hline IN $115 M$ & 05 & 48.0 & $.400-4$ & .020 & .190 & & \\
\hline INI $15 M$ & 6 & 57.0 & $.340-3$ & .170 & .740 & & \\
\hline IN $115 M$ & 8 & & & & $.77-18$ & $.39-16$ & $.39-15$ \\
\hline \multirow[t]{2}{*}{ IN1I5M } & 12 & 67.0 & $.360-3$ & .180 & .140 & & \\
\hline & & & & & B. 18 & & \\
\hline
\end{tabular}




\section{Appendix B - Organ Data Library - ORGLIB}

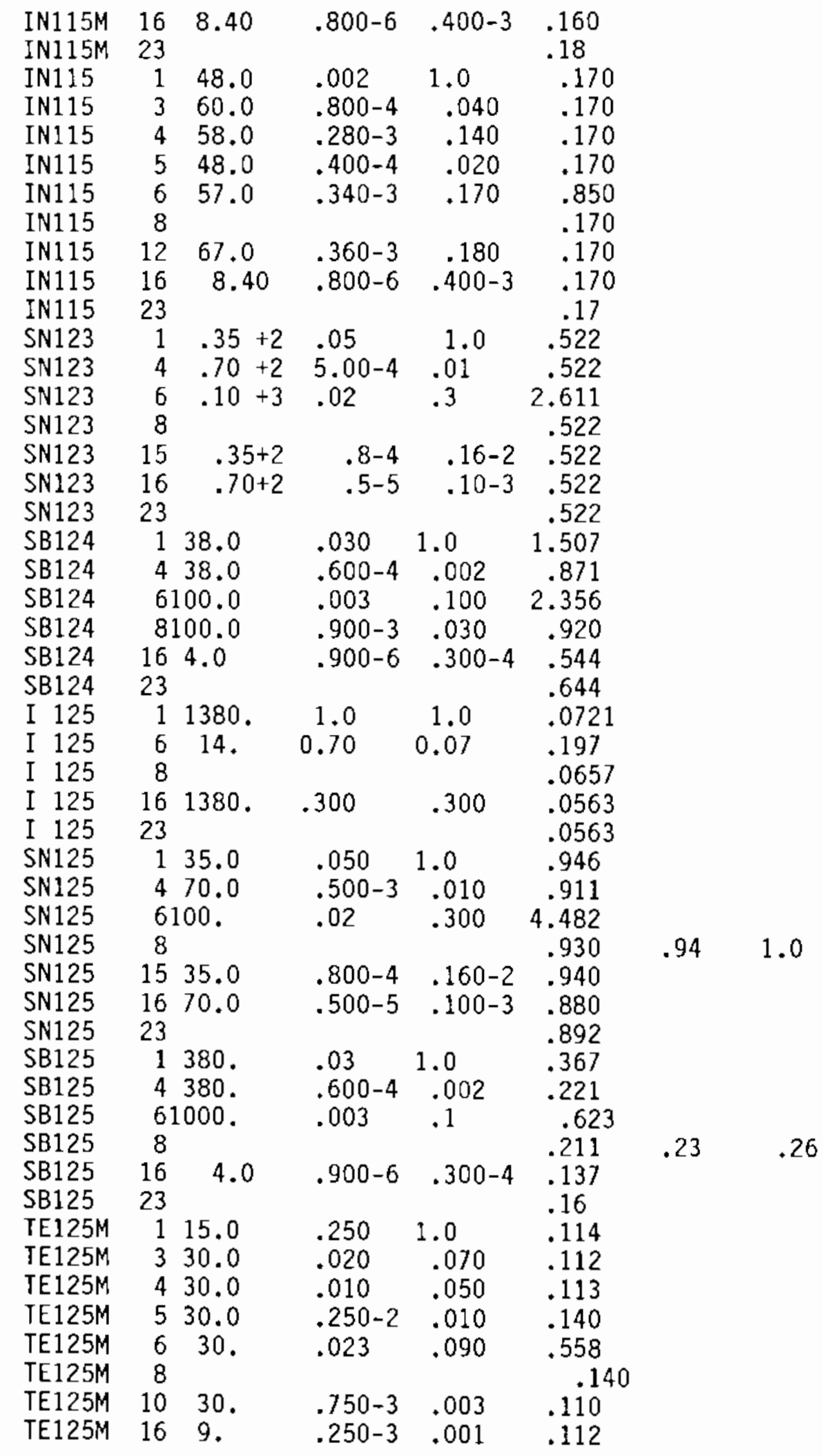

B. 19 


\section{Appendix B - Organ Data Library - ORGLIB}

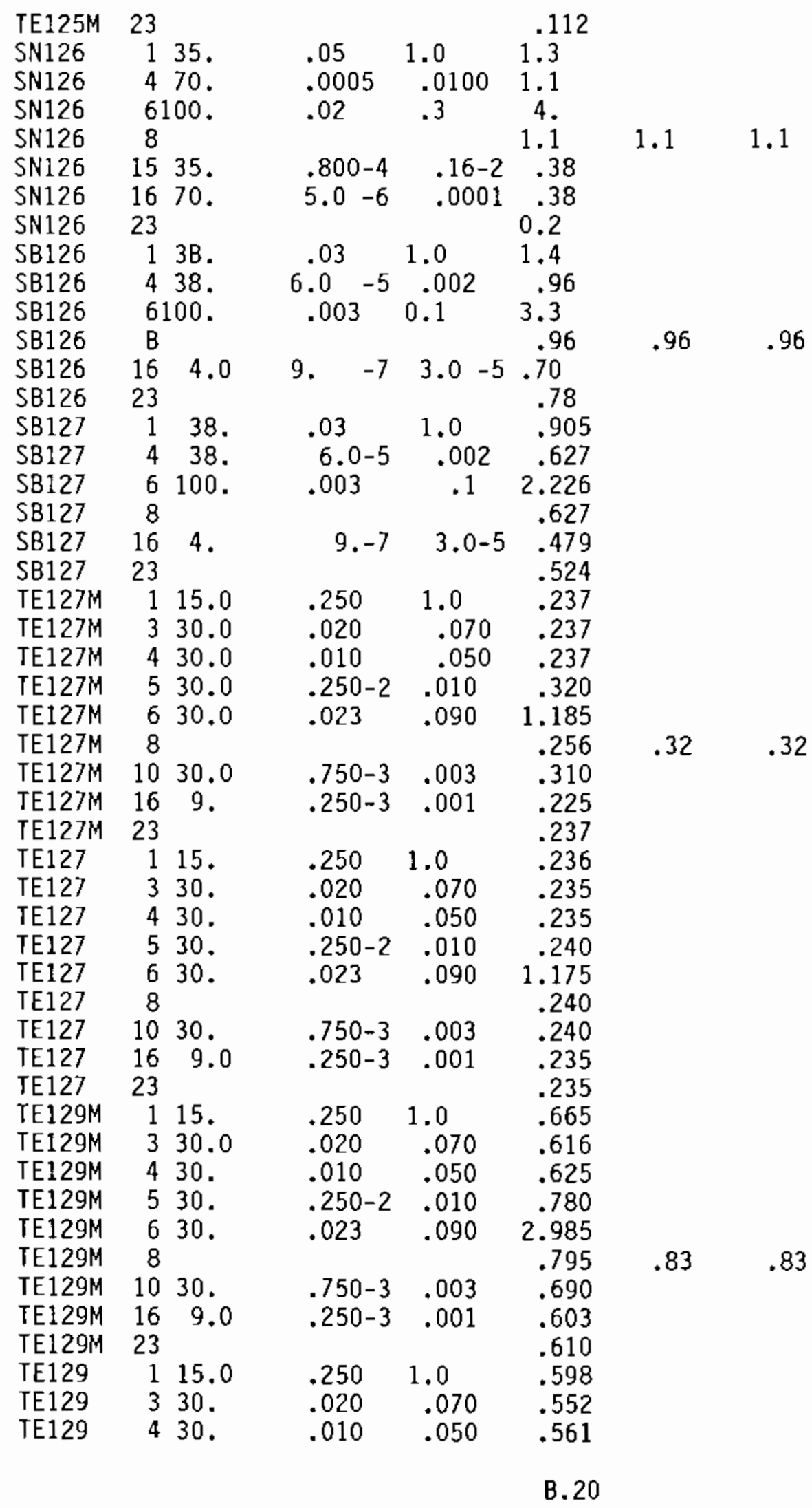




\section{Appendix B - Organ Data Library - ORGLIB}

\begin{tabular}{|c|c|c|c|c|c|c|c|}
\hline $\begin{array}{l}\text { TE129 } \\
\text { TE129 } \\
\text { TE129 }\end{array}$ & $\begin{array}{l}5 \\
6 \\
8\end{array}$ & $\begin{array}{l}30 . \\
30 .\end{array}$ & $\begin{array}{l}.250-2 \\
.023\end{array}$ & $\begin{array}{l}.010 \\
.090\end{array}$ & $\begin{array}{r}.680 \\
2.666 \\
.730\end{array}$ & .73 & .73 \\
\hline TE129 & 10 & 30. & $.750-3$ & .003 & .600 & & \\
\hline TE129 & 16 & 9.0 & $.250-3$ & .001 & .539 & & \\
\hline TE129 & 23 & & & & .546 & & \\
\hline I 129 & 1 & 100. & 1.0 & 1.0 & $8.72-2$ & & \\
\hline I 129 & 3 & 7.0 & .040 & .040 & $8.76-2$ & & \\
\hline I 129 & 4 & 7.0 & .120 & .120 & $7.69-2$ & & \\
\hline I 129 & 5 & 7.0 & .005 & .005 & .077 & & \\
\hline I 129 & 6 & 14.0 & .070 & .070 & .316 & & \\
\hline I 129 & 8 & & & & .082 & & \\
\hline I 129 & 10 & 7.0 & .005 & .005 & .068 & & \\
\hline I 129 & 16 & 100. & .300 & .300 & $6.52-2$ & & \\
\hline I 129 & 23 & & & & .0694 & & \\
\hline TE131M & 1 & 15. & .250 & 1.0 & 2.316 & & \\
\hline TE131M & 3 & 30. & .020 & .070 & 1.449 & & \\
\hline TE131M & 4 & 30. & .010 & .050 & 1.621 & & \\
\hline TE131M & 5 & 30. & $.250-2$ & .010 & .800 & & \\
\hline TE131M & 6 & 30 . & .023 & .090 & 5.953 & & \\
\hline TE131M & 8 & & & & .763 & .99 & 1.0 \\
\hline TE131M & 10 & 30. & $.750-3$ & .003 & 1.317 & & \\
\hline TE131M & 16 & 9. & $.250-3$ & .001 & 1.317 & & \\
\hline TE131M & 23 & & & & 1.181 & & \\
\hline TE131 & 1 & 15. & .25 & 1.0 & 1.358 & & \\
\hline TE131 & 3 & 30. & .02 & .07 & 1.009 & & \\
\hline TE131 & 4 & 30 . & .01 & .05 & 1.091 & & \\
\hline TE131 & 6 & 30 . & .023 & .09 & 4.666 & & \\
\hline TE131 & 8 & & & & 1.13 & & \\
\hline TE131 & 10 & 30 . & $.75-3$ & .003 & 1.01 & & \\
\hline TE131 & 16 & 9. & $.25-3$ & .001 & 1.010 & & \\
\hline IE131 & 23 & & & & .805 & & \\
\hline I 131 & 1 & 100. & 1.0 & 1.0 & .434 & & \\
\hline I 131 & 3 & 7. & .04 & .04 & .277 & & \\
\hline I 131 & 4 & 7. & .12 & .12 & .305 & & \\
\hline I 131 & 5 & 7. & .005 & .005 & 0.27 & & \\
\hline I 131 & 6 & 14. & .07 & .07 & 1.102 & & \\
\hline I 131 & 8 & & & & .300 & & \\
\hline I 131 & 10 & 7. & .005 & .005 & .23 & & \\
\hline I 131 & 16 & 100. & .300 & .300 & .236 & & \\
\hline I 131 & 23 & & $5.0-2$ & & .257 & & \\
\hline TE132 & 1 & 15. & .250 & 1.0 & 2.169 & & \\
\hline TE132 & 3 & 30. & .020 & .070 & 1.09 & & \\
\hline TE132 & 4 & 30. & .010 & .050 & 1.282 & & \\
\hline TE132 & 5 & 30. & $.250-2$ & .010 & .960 & & \\
\hline TE132 & 6 & 30. & .023 & .090 & 3.544 & & \\
\hline TE132 & 8 & & & & 1.04 & 1.1 & 1.1 \\
\hline TE132 & 10 & 30. & $.750-3$ & .003 & .730 & & \\
\hline TE132 & 16 & 9.0 & $.250-3$ & .001 & .814 & & \\
\hline TE132 & 23 & & & & .901 & & \\
\hline
\end{tabular}

B. 21 


\section{Appendix B - Organ Data Library - ORGLIB}

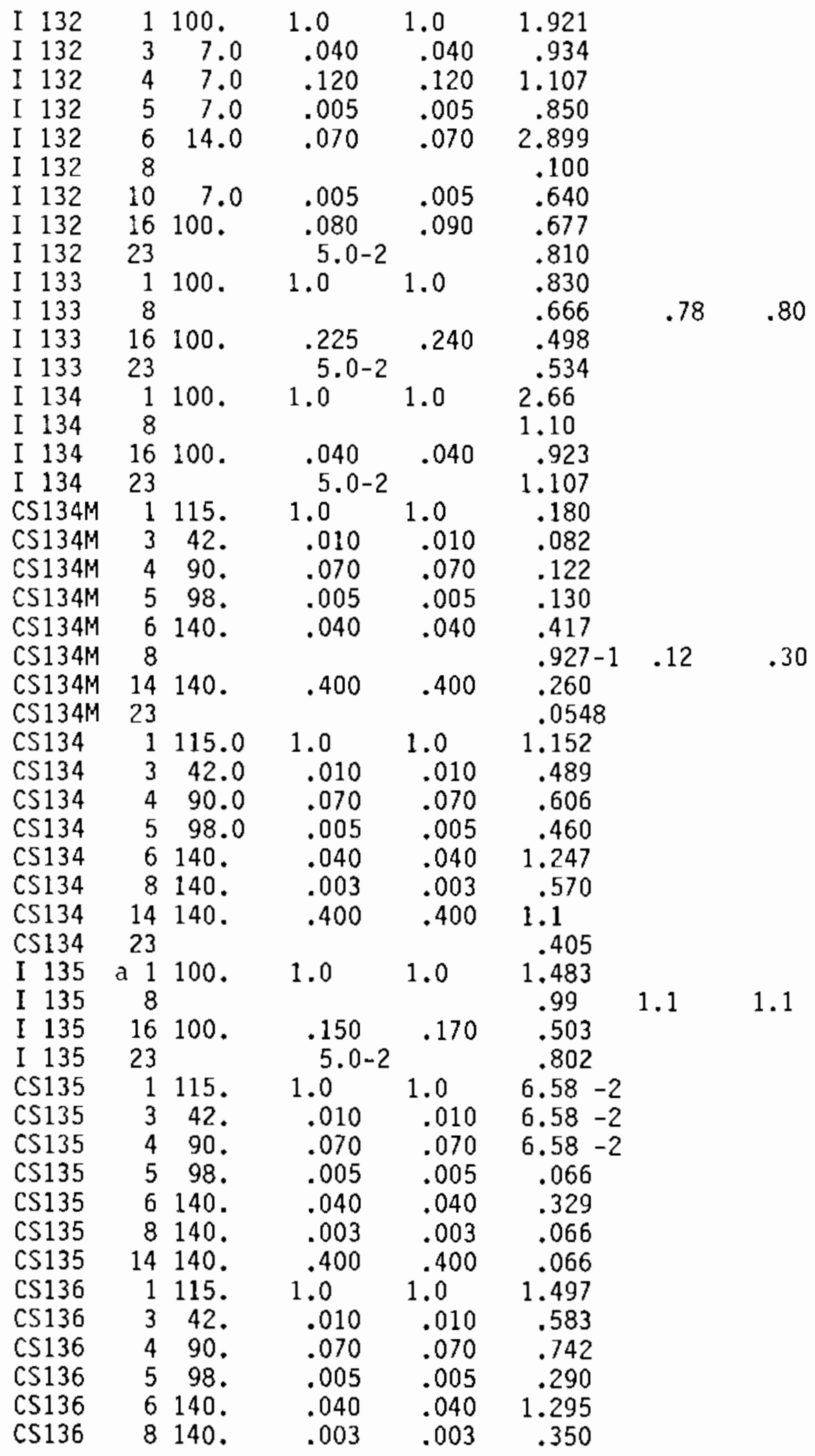

B. 22 


\section{Appendix B - Organ Data Library - ORGLIB}

\begin{tabular}{|c|c|c|c|c|c|c|}
\hline $\begin{array}{l}\text { CS136 } \\
\text { CS136 }\end{array}$ & $\begin{array}{l}14140 . \\
23\end{array}$ & .400 & .400 & $\begin{array}{l}.650 \\
.469\end{array}$ & & \\
\hline CS137 & 1115 & 1.0 & 1.0 & .594 & & \\
\hline CS 137 & 342 & .010 & .010 & .359 & & \\
\hline $\mathrm{CS} 137$ & 490 & .070 & .070 & .400 & & \\
\hline $\operatorname{CS} 137$ & 598 & .005 & .005 & .370 & & \\
\hline $\operatorname{CS} 137$ & 6140 . & .040 & .040 & 1.365 & & \\
\hline CS137 & 8140 . & .003 & .003 & .410 & & \\
\hline CS 137 & 14140 . & .400 & .400 & .590 & & \\
\hline CS137 & 23 & & & .329 & & \\
\hline BA137M & 65. & .05 & 1.0 & .437 & & \\
\hline BA137M & 65. & .035 & .7 & .474 & & \\
\hline BA137M & 8 & & & .231 & & \\
\hline BA137M & 23 & & & .154 & & \\
\hline $\operatorname{CS} 138$ & 1115 & 1.0 & 1.0 & 2.289 & & \\
\hline $\operatorname{CS} 138$ & 342 & .01 & .01 & 1.454 & & \\
\hline $\operatorname{CS} 138$ & 490 & .07 & .07 & 1.596 & & \\
\hline CS138 & 598. & .005 & .005 & 1.45 & & \\
\hline CS138 & 6140. & .04 & .04 & 5.842 & & \\
\hline CS138 & 8 & & & 1.6 & & \\
\hline CS138 & 14140 . & .40 & .40 & 2.29 & & \\
\hline $\begin{array}{l}\operatorname{CS} 138 \\
\operatorname{CS} 139\end{array}$ & 23 & & & 1.352 & & \\
\hline $\begin{array}{l}\text { CS139 } \\
\text { CS139 }\end{array}$ & 342 . & .01 & .01 & $\begin{array}{l}2.111 \\
2.557\end{array}$ & & \\
\hline CS139 & 490. & .07 & .07 & 2.584 & & \\
\hline $\operatorname{CS} 139$ & 598. & .005 & .005 & 2.56 & & \\
\hline $\operatorname{CS} 139$ & 6140 . & .04 & .04 & 12.5 & & \\
\hline $\operatorname{CS} 139$ & 8 & & & 2.58 & & \\
\hline $\operatorname{CS} 139$ & 14140 . & .4 & .4 & 2.71 & & \\
\hline $\operatorname{cs} 139$ & 23 & & & 2.536 & & \\
\hline BA140 & 65.0 & .050 & 1.0 & 2.364 & & \\
\hline BA140 & 8.50 & $.500-5$ & $.100-3$ & 1.379 & & \\
\hline BA140 & 4975. & $.300-4$ & $.600-3$ & 1.548 & & \\
\hline BA140 & $\begin{array}{ll}5 & 13.0\end{array}$ & $.250-5$ & $.500-4$ & 1.20 & & \\
\hline BA140 & $6 \quad 65.0$ & .035 & .700 & 5.139 & & \\
\hline BA140 & 8 & & & .751 & 1.4 & 1.4 \\
\hline BA140 & 142000 & $.150-3$ & .003 & 2.30 & & \\
\hline BA140 & 23 & & & .354 & & \\
\hline LA140 & 1500. & .0001 & 1.0 & 1.889 & & \\
\hline LA140 & 4400 . & $.150-4$ & .150 & 1.160 & & \\
\hline LA140 & 61000 & $.400-4$ & .400 & 3.536 & & \\
\hline LA140 & 8 & & & 1.10 & & \\
\hline LA140 & 23 & & & .903 & & \\
\hline BA141 & 165. & .05 & 1.0 & 2.365 & & \\
\hline BA141 & 8.5 & $.500-5$ & $.10-3$ & 2.116 & & \\
\hline BA141 & 4975. & $.300-4$ & $.60-3$ & 2.147 & & \\
\hline BA141 & 513. & $.250-5$ & $.50-4$ & 2.116 & & \\
\hline BA141 & 665. & .035 & .7 & 10.04 & & \\
\hline BA141 & 8 & & & 2.147 & & \\
\hline BA141 & 142000 & $.150-3$ & .003 & 2.365 & & \\
\hline
\end{tabular}




\section{Appendix B - Organ Data Library - ORGLIB}

\begin{tabular}{|c|c|c|c|c|c|c|}
\hline BA141 & 23 & & & 1.129 & & \\
\hline LA141 & 1500 & .0001 & 1.0 & .951 & & \\
\hline LA141 & 4400 & $.150-4$ & .15 & .942 & & \\
\hline LA141 & 61000 & $.400-4$ & .40 & 4.680 & & \\
\hline $\begin{array}{l}\text { LA141 } \\
\text { LA141 }\end{array}$ & $\begin{array}{r}8 \\
23\end{array}$ & & & $\begin{array}{l}.942 \\
.938\end{array}$ & & \\
\hline CE141 & 1563. & $.100-3$ & 1.000 & .221 & & \\
\hline CE141 & 3563 & $.200-5$ & .020 & .194 & & \\
\hline CE141 & 4293 & $.250-4$ & .250 & .199 & & \\
\hline CE141 & 61500 & $.300-4$ & .300 & .928 & & \\
\hline CE141 & 8 & & & .180 & & \\
\hline CE141 & 23 & & & .191 & & \\
\hline BA142 & 65. & .05 & 1.0 & 3.409 & & \\
\hline BA142 & 8.5 & $.500-5$ & $.100-3$ & 2.022 & & \\
\hline BA142 & 4975 . & $.300-4$ & $.600-3$ & 2.257 & & \\
\hline BA142 & 513 & $.250-5$ & $.500-4$ & 2.022 & & \\
\hline BA142 & 665. & .035 & .7 & 7.755 & & \\
\hline BA142 & 8 & & & 2.257 & & \\
\hline BA142 & 142000 . & $.150-3$ & .003 & 3.409 & & \\
\hline BA142 & 23 & & & 1.855 & & \\
\hline LA142 & 500 . & .0001 & 1.0 & 2.133 & & \\
\hline LA142 & 4400 . & $.15-4$ & .15 & 1.39 & & \\
\hline LA142 & 61000. & $.40-4$ & .40 & 4.718 & & \\
\hline LA142 & 8 & & & 1.39 & & \\
\hline LA142 & 23 & & & 1.135 & & \\
\hline CE143 & 1563 & $.100-3$ & 1.0 & .933 & & \\
\hline CE143 & 3563. & $.200-5$ & .020 & .795 & & \\
\hline CE 143 & 4293 . & $.250-4$ & .250 & .818 & & \\
\hline CE 143 & 61500. & $.300-4$ & .300 & 3.791 & & \\
\hline CE143 & 8 & & & .562 & .79 & .85 \\
\hline $\begin{array}{l}\text { CE143 } \\
\text { PR143 }\end{array}$ & $\begin{array}{r}23 \\
1\end{array} 750$ & & & $\begin{array}{r}.468 \\
324\end{array}$ & & \\
\hline PR143 & $\begin{array}{l}1 \\
3750.0\end{array}$ & $\begin{array}{r}.100-3 \\
.200-5\end{array}$ & $\begin{array}{l}1.0 \\
.020\end{array}$ & $\begin{array}{l}.324 \\
.324\end{array}$ & & \\
\hline PR143 & 4375 . & $.200-4$ & .200 & .324 & & \\
\hline PR143 & 61500 & $.400-4$ & .400 & 1.618 & & \\
\hline PR143 & 8 & & & .320 & & \\
\hline PR143 & 23 & & & .324 & & \\
\hline CE144 & 1563. & $.100-3$ & 1.0 & 1.313 & & \\
\hline CE144 & 3563 . & $.200-5$ & .020 & 1.295 & & \\
\hline CE144 & 4293. & $.250-4$ & .250 & 1.298 & & \\
\hline CE144 & 61500. & $.300-4$ & .300 & 6.443 & & \\
\hline CE144 & 8 & & & 1.30 & 1.30 & 1.30 \\
\hline CE144 & 23 & & & 1.292 & & \\
\hline PR144 & 175 & $.10-3$ & 1. & 1.205 & & \\
\hline PR144 & 3750 . & $.20-5$ & .02 & 1.193 & & \\
\hline PR144 & 4375 . & $.20-4$ & .20 & 1.195 & & \\
\hline PR144 & 61500 & $.40-4$ & .40 & 5.944 & & \\
\hline PR144 & 8 & & & 1.195 & & \\
\hline PR144 & 23 & & & 1.191 & & \\
\hline \multirow[t]{2}{*}{ ND144 } & 1656. & $.100-3$ & 1.0 & 20.0 & & \\
\hline & & & & B. 24 & & \\
\hline
\end{tabular}




\section{Appendix B - Organ Data Library - ORGLIB}

\begin{tabular}{|c|c|c|c|c|c|c|c|}
\hline ND144 & & 656. & $.500-5$ & .050 & 20.0 & & \\
\hline ND144 & & 131. & $.500-4$ & .500 & 20.0 & & \\
\hline ND144 & & 500. & $.350-4$ & .350 & 100. & & \\
\hline ND144 & 8 & & & & 20. & & \\
\hline ND144 & 23 & & & & .19 & & \\
\hline ND147 & 1 & 656. & $.100-3$ & 1.0 & .381 & & \\
\hline ND147 & 3 & 656. & $.500-5$ & .050 & .319 & & \\
\hline ND147 & 4 & 131. & $.500-4$ & .500 & .330 & & \\
\hline ND147 & 61 & 500. & $.350-4$ & .350 & 1.560 & & \\
\hline ND147 & 8 & & & & .290 & .290 & .31 \\
\hline ND147 & 23 & & & & .282 & & \\
\hline PM147 & 1 & 656. & $.100-3$ & 1.0 & $6.980-2$ & & \\
\hline PM147 & 3 & 656. & $.200-5$ & .020 & $6.980-2$ & & \\
\hline PM147 & 4 & 656. & $.600-5$ & .060 & $6.980-2$ & & \\
\hline PM147 & 61 & 500. & $.350-4$ & .350 & .349 & & \\
\hline PM147 & 8 & & & & .069 & .069 & .069 \\
\hline PM1 47 & 23 & & & & $6.980-2$ & & \\
\hline PM148M & 1 & 656. & $.10-3$ & 1.0 & 1.481 & & \\
\hline PM1 48M & 3 & 656. & $.20-5$ & .02 & .629 & & \\
\hline PM148M & 4 & 656 & $.60-5$ & .06 & .784 & & \\
\hline PM148M & 6 & 1500 . & $.35-4$ & .35 & 2.067 & & \\
\hline PM148M & 8 & & & & .784 & & \\
\hline PM148M & 23 & & & & .531 & & \\
\hline PM148 & 1 & 656. & $.10-3$ & 1.0 & 1.065 & & \\
\hline PM148 & 3 & 656. & $.20-5$ & .02 & .857 & & \\
\hline PM148 & 4 & 656 & $.60-5$ & .06 & .857 & & \\
\hline PM148 & 6 & 1500 . & $.35-4$ & .35 & 3.627 & & \\
\hline PM148 & 8 & & & & .857 & & \\
\hline PM148 & 23 & & & & .783 & & \\
\hline PM149 & 1 & 656. & $.100-3$ & 1.0 & .377 & & \\
\hline PM149 & 3 & 656 . & $.200-5$ & .020 & .374 & & \\
\hline PM149 & 4 & 656 . & $.600-5$ & .060 & .374 & & \\
\hline PM149 & 61 & 500. & $.350-4$ & .350 & 1.863 & & \\
\hline PM149 & 8 & & & & .440 & & \\
\hline PM149 & 23 & & & & .373 & & \\
\hline PM151 & 1 & 656. & $.1-3$ & 1. & .474 & & \\
\hline PM151 & & 656. & $.2-5$ & .02 & .359 & & \\
\hline PM151 & & 656. & $.6-5$ & .06 & .379 & & \\
\hline PM151 & 6 & 1500 . & $.35-4$ & .35 & 1.594 & & \\
\hline PM151 & 8 & & & & .379 & & \\
\hline PM151 & 23 & & & & .345 & & \\
\hline SM151 & 1 & 656. & $.100-3$ & 1.0 & .042 & & \\
\hline SM151 & 3 & 656. & $.200-5$ & .020 & .042 & & \\
\hline SM151 & 4 & 187. & $.350-4$ & .350 & .042 & & \\
\hline SM151 & 61 & 500 . & $.350-4$ & .350 & .130 & & \\
\hline SM151 & 8 & & & & .042 & & \\
\hline SM151 & 23 & & & & .041 & & \\
\hline EU152 & 1 & 635. & $.100-3$ & 1.0 & .66 & & \\
\hline EU152 & 31 & 480. & $.300-5$ & .030 & .33 & & \\
\hline EU152 & 4 & 127. & $.250-4$ & .250 & .33 & & \\
\hline & & & & & B. 25 & & \\
\hline
\end{tabular}




\section{Appendix B - Organ Data Library - ORGLIB}

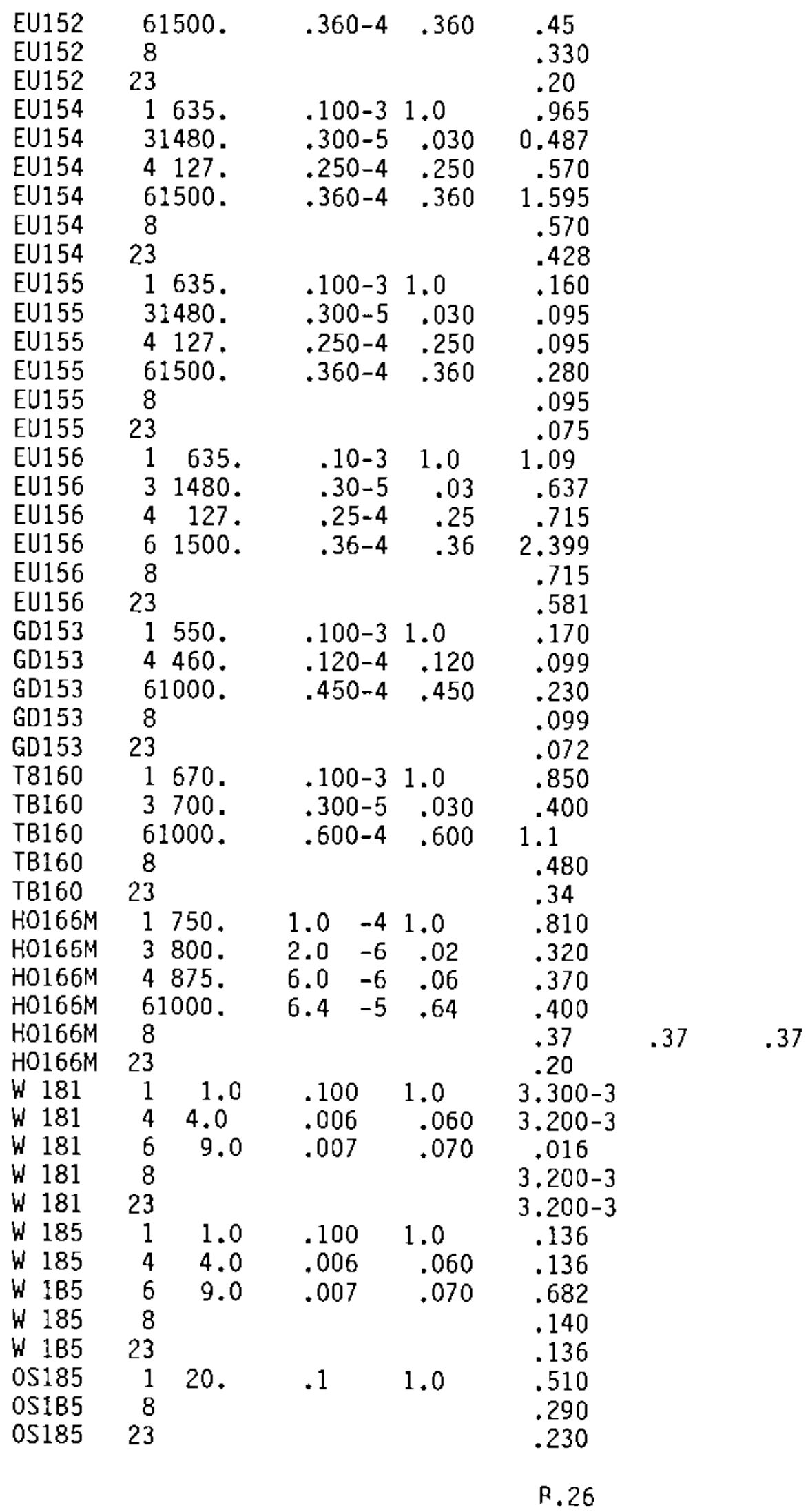




\section{Appendix B - Organ Data Library - ORGLIB}

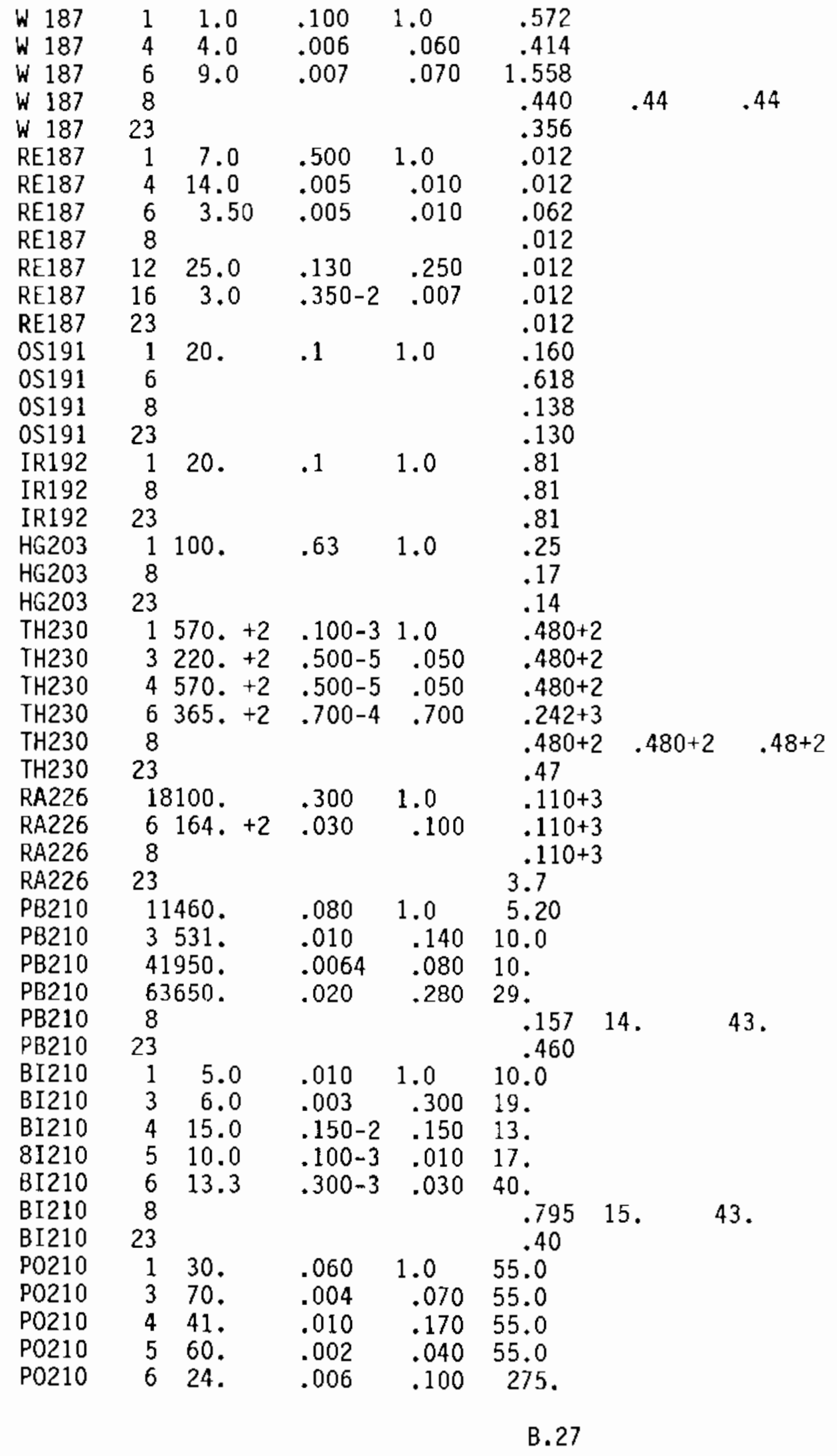


Appendix B - Organ Data Library - ORGLIB

\begin{tabular}{|c|c|c|c|c|c|c|c|}
\hline $\begin{array}{l}\text { P0210 } \\
\text { P0210 }\end{array}$ & $\begin{array}{r}8 \\
23\end{array}$ & & & & $\begin{array}{r}55.0 \\
.53\end{array}$ & & \\
\hline U 232 & 1 & 100. & .010 & 1.0 & $2.80+2$ & & \\
\hline U 232 & 3 & 15.0 & $.110-2$ & .110 & $1.10+2$ & & \\
\hline U 232 & 6 & 300. & $.110-2$ & .110 & $1.20+3$ & & \\
\hline U 232 & 8 & & & & $.552+2$ & $.760+2$ & $.19+3$ \\
\hline U 232 & 23 & & & & .53 & & \\
\hline TH232 & 1 & $570 .+2$ & $.100-3$ & 1.0 & $.62+2$ & & \\
\hline TH232 & 3 & $220 .+2$ & $.500-5$ & .050 & $.41+2$ & & \\
\hline TH232 & 4 & 570 & $.500-5$ & .050 & $.41+2$ & & \\
\hline TH232 & 6 & $365 .+2$ & $.700-4$ & .700 & $.270+3$ & & \\
\hline TH232 & 8 & & & & $.410+2$ & $.410+2$ & $.57+2$ \\
\hline TH232 & 23 & & & & .40 & & \\
\hline RA228 & & 8100 & .300 & 1.0 & $2.3+2$ & & \\
\hline RA228 & 6 & $164 .+2$ & .030 & .100 & $1.9+2$ & & \\
\hline RA228 & 8 & & & & .716 & $.22+2$ & $.14+3$ \\
\hline RA228 & 23 & & & & .63 & & \\
\hline AC228 & 1 & $240 .+2$ & $.100-3$ & 1.0 & $.230+3$ & & \\
\hline AC228 & 3 & $240 .+2$ & $.100-5$ & .010 & $.550+2$ & & \\
\hline$A C 228$ & 4 & $240 .+1$ & $.500-4$ & .500 & $.560+2$ & & \\
\hline AC228 & 6 & $365 .+2$ & $.300-4$ & .300 & $.970+3$ & & \\
\hline AC228 & 8 & & & & .897 & $2.2+1$ & $1.4+2$ \\
\hline AC228 & 23 & & & & .62 & & \\
\hline TH228 & 1 & $570 .+2$ & $.100-3$ & 1.0 & $.230+3$ & & \\
\hline TH228 & 3 & $220 .+2$ & $.500-5$ & .050 & $.56+2$ & & \\
\hline TH228 & 4 & $570 .+2$ & $.500-5$ & .050 & $.56+2$ & & \\
\hline TH228 & 6 & $365 .+2$ & $.700-4$ & .700 & $.970+3$ & & \\
\hline TH228 & 8 & & & & $.750+2$ & $.22+3$ & $.24+3$ \\
\hline TH228 & 23 & & & & 4.4 & & \\
\hline RA224 & & 3100. & .300 & 1.0 & $2.80+2$ & & \\
\hline RA224 & 6 & $164 .+2$ & .150 & .500 & $2.80+2$ & & \\
\hline RA224 & 8 & & & & $.249+3$ & $.28+3$ & $.28+3$ \\
\hline RA224 & 23 & & & & 4.2 & & \\
\hline PB212 & & 1460. & .080 & 1.0 & 82.0 & & \\
\hline P8212 & & 531. & .010 & .140 & 81.0 & & \\
\hline PB212 & & 1950. & $.640-2$ & .080 & 83.0 & & \\
\hline PB212 & & 3650. & .020 & .280 & 410.0 & & \\
\hline P8212 & 8 & & & & 79.9 & 33.0 & 83.0 \\
\hline PB212 & 23 & & & & .22 & & \\
\hline BI212 & 1 & 5.0 & .010 & 1.0 & 83.0 & & \\
\hline BI212 & 3 & 6.0 & .003 & .300 & 82.0 & & \\
\hline BI212 & 4 & 15.0 & $.150-2$ & .150 & 83.0 & & \\
\hline BI212 & 5 & 10.0 & $.100-3$ & .010 & 82.0 & & \\
\hline BI212 & 6 & 13.3 & $.300-3$ & .030 & $.411+3$ & & \\
\hline BI212 & 8 & & & & 83.0 & & \\
\hline BI212 & 23 & & & & 1.7 & & \\
\hline U 234 & 1 & 100. & .010 & 1.0 & 49. & & \\
\hline U 234 & 3 & 15. & $.110-2$ & .110 & 49. & & \\
\hline U 234 & 6 & 300 . & $.110-2$ & .110 & $2.40+2$ & & \\
\hline U 23 & 8 & & & & $.490+2$ & & \\
\hline & & & & & B. 28 & & \\
\hline
\end{tabular}




\section{Appendix B - Organ Data Library - ORGLIB}

\begin{tabular}{|c|c|c|c|c|c|c|c|}
\hline $\begin{array}{l}\text { U } 234 \\
\text { U } 235\end{array}$ & $\begin{array}{r}23 \\
1\end{array}$ & 100. & $\begin{array}{l}1.000 \\
.010\end{array}$ & 1.0 & $46^{.48}$ & & \\
\hline $\begin{array}{ll}U & 235 \\
U & 235\end{array}$ & $\begin{array}{l}1 \\
3\end{array}$ & 15. & $110-2$ & 1.0 & $\begin{array}{l}40 . \\
46 .\end{array}$ & & \\
\hline U 235 & 6 & 300. & $.110-2$ & .110 & $2.30+2$ & & \\
\hline U 235 & 8 & & & & $.461+2$ & $.46+2$ & $.46+2$ \\
\hline U 235 & 23 & & 1.0 & & .61 & & \\
\hline TH231 & 1 & $570 .+2$ & $.100-3$ & 1.0 & .180 & & \\
\hline TH231 & 3 & $220 .+2$ & $.500-5$ & .050 & .140 & & \\
\hline TH231 & 4 & $570 .+2$ & $.500-5$ & .050 & .160 & & \\
\hline TH231 & 6 & $365 .+2$ & $.700-4$ & .700 & .560 & & \\
\hline TH231 & 8 & & & & .110 & .110 & .110 \\
\hline TH231 & 23 & & 1.0 & & .093 & & \\
\hline PA231 & 1 & $410 .+2$ & $.100-3$ & 1.0 & $1.40+2$ & & \\
\hline PA231 & 3 & $510 .+2$ & $.400-5$ & .040 & 79.0 & & \\
\hline PA231 & 4 & $580 .+2$ & $.500-5$ & .050 & $6.3+1$ & & \\
\hline PA231 & 6 & $365 .+2$ & $.450-4$ & .450 & $7.50+2$ & & \\
\hline PA231 & 8 & & & & $.510+2$ & $.52+2$ & $.70+2$ \\
\hline PA231 & 23 & & & & .56 & & \\
\hline $\mathrm{AC} 227$ & 1 & $240 .+2$ & $.100-3$ & 1.0 & $2.0+2$ & & \\
\hline AC 227 & 3 & $240 .+2$ & $.100-5$ & .010 & $6.2+1$ & & \\
\hline AC227 & 4 & 240 & $.500-4$ & .500 & $6.2+1$ & & \\
\hline $\mathrm{AC} 227$ & 6 & $365 .+2$ & $.300-4$ & .300 & $1.0+3$ & & \\
\hline AC227 & 8 & & & & 4.89 & $2.10+2$ & $3.2+2$ \\
\hline AC227 & 23 & & & & .640 & & \\
\hline TH227 & 1 & $570 .+2$ & $.100-3$ & 1.0 & $.200+3$ & & \\
\hline TH227 & 3 & $220 .+2$ & $.500-5$ & .050 & $.61+2$ & & \\
\hline TH227 & 4 & $570 .+2$ & $.500-5$ & .050 & $.61+2$ & & \\
\hline TH227 & 6 & $365 .+2$ & $.700-4$ & .700 & $.990+3$ & & \\
\hline TH227 & 8 & & & & $.750+2$ & $.20+3$ & $.23+3$ \\
\hline TH227 & 23 & & & & 4.3 & & \\
\hline FR223 & 1 & & & & .149 & & \\
\hline FR223 & 6 & & & & .616 & & \\
\hline FR223 & 8 & & & & .135 & & \\
\hline FR223 & 16 & & & & .129 & & \\
\hline FR223 & 23 & & & & .129 & & \\
\hline RA223 & 18 & 3100. & .300 & 1.0 & $2.75+2$ & & \\
\hline RA223 & 3 & 10. & $6.0-4$ & .002 & $2.75+2$ & & \\
\hline RA223 & 4 & 10. & $1.2-4$ & 4.0 & $42.75+2$ & & \\
\hline RA223 & 6 & 164. +2 & .150 & .500 & $2.75+2$ & & \\
\hline RA223 & 8 & & & & $28.0+1$ & & \\
\hline RA223 & 23 & & & & 3.7 & & \\
\hline U 236 & 1 & 100. & .010 & 1.0 & 47. & & \\
\hline U 236 & 3 & 15. & $.110-2$ & .110 & 47.7 & & \\
\hline U 236 & 6 & 300. & $.110-2$ & .110 & $2.30+2$ & & \\
\hline U 236 & 8 & & & & 47. & & \\
\hline U 236 & 23 & & & & .45 & & \\
\hline PU236 & 1 & $650 .+2$ & $.30-4$ & 1.0 & 61. & & \\
\hline PU236 & 3 & $320 .+2$ & $.60-6$ & .02 & 60. & & \\
\hline PU236 & 4 & 146. +2 & $1.35-5$ & .45 & 60. & & \\
\hline PU236 & 6 & $365 .+2$ & $1.35-5$ & .45 & $3.12+2$ & & \\
\hline & & & & & .2 & & \\
\hline
\end{tabular}


Appendix B - Organ Data Library - ORGLIB

\begin{tabular}{|c|c|c|c|c|c|c|c|}
\hline PU236 & 8 & & & & .60 & .60 & .61 \\
\hline PU236 & 23 & $.3-4$ & 1.0 & .0 & .61 & & \\
\hline PU237 & 1 & $650,+2$ & $.300-4$ & 41.0 & .038 & & \\
\hline PU237 & 3 & $320 .+2$ & $.600-6$ & 6.020 & .024 & & \\
\hline PU237 & 4 & $146 .+2$ & $1.35-5$ & 5.450 & .027 & & \\
\hline PU237 & 6 & $365,+2$ & $1.35-5$ & 5.450 & .068 & & \\
\hline PU237 & 8 & & & & .027 & & \\
\hline PU237 & 23 & & & & .022 & & \\
\hline NP237 & & $390 .+2$ & $.100-3$ & 31.0 & 49. & & \\
\hline NP237 & 3 & $640 .+2$ & $.300-5$ & 5.030 & 49. & & \\
\hline NP237 & 4 & $146 .+3$ & $.500-5$ & 5.050 & 49. & & \\
\hline NP237 & 6 & $365 .+2$ & $.450-4$ & 4.450 & $2.50+2$ & & \\
\hline NP237 & 8 & & & & $.490+2$ & $.490+2$ & $.49+2$ \\
\hline NP237 & 23 & & & & .62 & & \\
\hline PA233 & 1 & $410 .+2$ & $.100-3$ & $\begin{array}{ll}31.0 \\
\end{array}$ & .32 & & \\
\hline PA233 & 3 & $510 .+2$ & $.400-5$ & $5 \quad .040$ & .15 & & \\
\hline PA233 & 4 & $580 .+2$ & $.500-5$ & $5 \quad .050$ & .18 & & \\
\hline PA233 & 6 & $365 .+2$ & $.450-4$ & 4.450 & .41 & & \\
\hline PA233 & 8 & & & & .18 & & \\
\hline PA233 & 23 & & & & .13 & & \\
\hline U 233 & 1 & 100. & .010 & 1.0 & 50. & & \\
\hline U 233 & 3 & 15.0 & $.110-2$ & 2.110 & 50. & & \\
\hline U 233 & 6 & 300 & $.110-2$ & 2.110 & $2.50+2$ & & \\
\hline U 233 & 8 & & & & $.500+2$ & & \\
\hline บ 233 & 23 & & & & .49 & & \\
\hline TH229 & 1 & $570 .+2$ & $.100-3$ & 31.0 & $.330+3$ & & \\
\hline TH229 & 3 & 220 & $.500-5$ & 5.050 & $.49+2$ & & \\
\hline TH229 & 4 & $570 .+2$ & $.500-5$ & 5.050 & $.49+2$ & & \\
\hline TH229 & 6 & $730 .+2$ & $.700-4$ & $4 \quad .700$ & $.940+3$ & & \\
\hline TH229 & 8 & & & & $.270+3$ & $.270+3$ & $.27+3$ \\
\hline TH229 & 23 & & & & 4.0 & & \\
\hline RA225 & 18 & 3100. & .30 & 1.0 & $2.80+2$ & & \\
\hline RA225 & 3 & 10. & $6.0-4$ & 4.002 & $2.50+2$ & & \\
\hline RA225 & 4 & 10. & $.12-3$ & $3.040-2$ & $22.50+2$ & & \\
\hline RA225 & 6 & $164 .+2$ & .15 & .50 & $2.80+2$ & & \\
\hline RA225 & 8 & & & & $2.50+2$ & $2.50+2$ & $2.50+2$ \\
\hline RA225 & 23 & & & & 3.5 & & \\
\hline AC225 & 1 & $240 .+2$ & $.001-1$ & 11.0 & $2.80+2$ & & \\
\hline$A C 225$ & 3 & $240 .+2$ & $1.0-6$ & $6 \quad .01$ & $2.80+2$ & & \\
\hline AC225 & 4 & $240 .+1$ & $5.0-5$ & $5 \quad .50$ & $2.80+2$ & & \\
\hline AC225 & 6 & $730 .+2$ & $3.0-5$ & 5.30 & $1.39+3$ & & \\
\hline $\mathrm{AC} 225$ & 8 & & & & $2.70+2$ & $2.70+2$ & $2.70+2$ \\
\hline AC225 & 23 & & & & 3.3 & & \\
\hline U 238 & 1 & 100. & .01 & 1.0 & 43.0 & & \\
\hline U 238 & 3 & 15. & $1.1-3$ & $\begin{array}{ll}30.11 \\
0\end{array}$ & 43.0 & & \\
\hline U 238 & 6 & 300 . & $1.1-3$ & $\begin{array}{ll}3 & 0.11\end{array}$ & 220.0 & & \\
\hline U 238 & 8 & & & & 43.0 & & \\
\hline U 238 & 23 & & 1.0 & & 0.43 & & \\
\hline $\mathrm{TH} 234$ & 1 & $570 .+2$ & $.100-3$ & $\begin{array}{ll}31.0 \\
\end{array}$ & .91 & & \\
\hline TH234 & 3 & $570 .+2$ & $.500-5$ & $5 \quad .050$ & .90 & & \\
\hline & & & & & B. 30 & & \\
\hline
\end{tabular}


Appendix B - Organ Data Library - ORGLIB

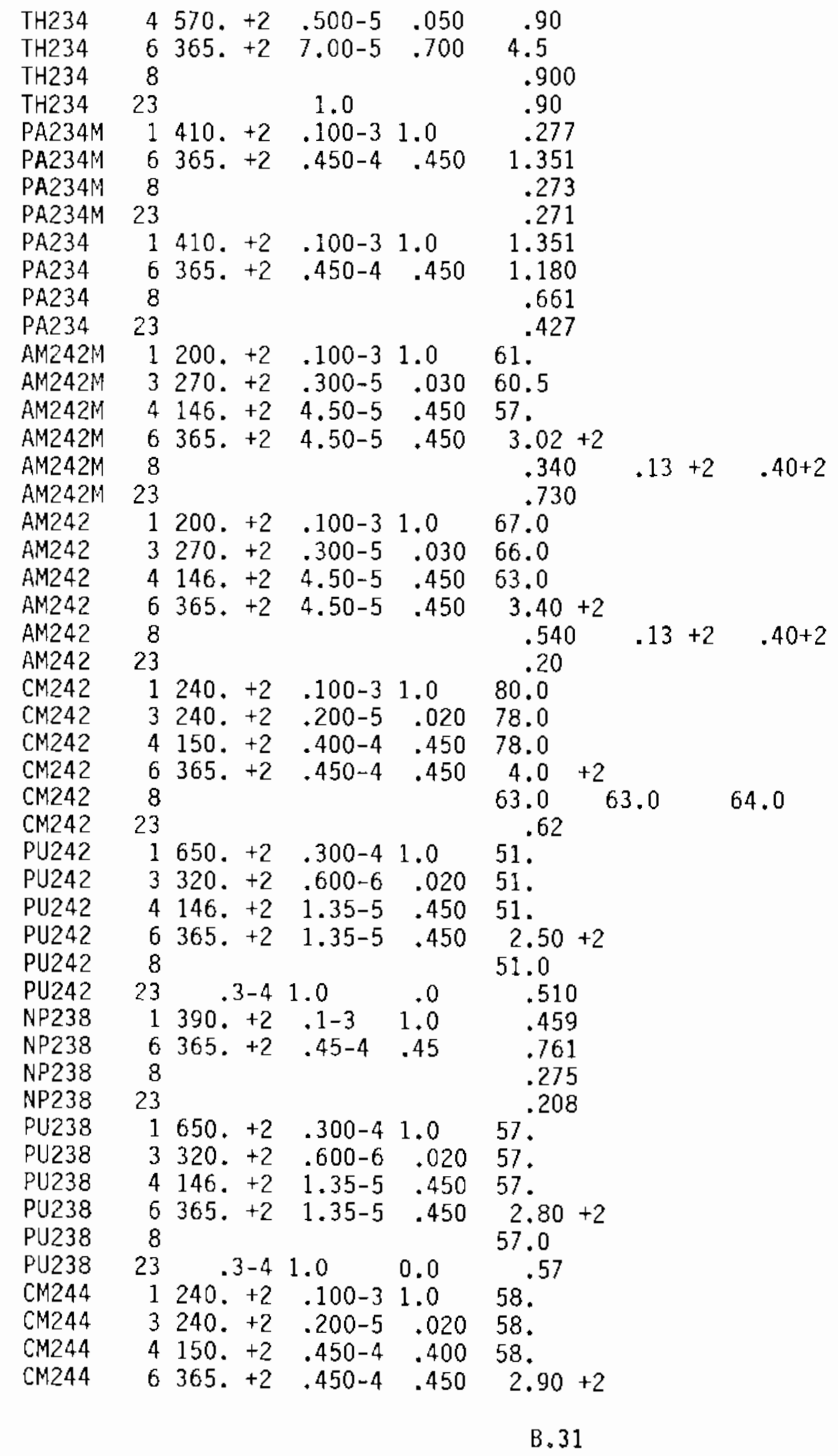




\section{Appendix B - Organ Data Library - ORGLIB}

\begin{tabular}{|c|c|c|c|c|c|c|c|}
\hline CM244 & $\begin{array}{r}8 \\
10\end{array}$ & & & & $\begin{array}{r}60.0 \\
-3\end{array}$ & 60. & 60. \\
\hline $\begin{array}{l}\mathrm{CM} 244 \\
\mathrm{CM} 244\end{array}$ & $\begin{array}{l}10 \\
11\end{array}$ & $\begin{array}{l}1.0+8 \\
1.0+8\end{array}$ & & $\begin{array}{l}0.5 \\
0.1\end{array}$ & $\begin{array}{l}-36.0 \\
-3 \\
-3\end{array}$ & $\begin{array}{l}+1 \\
+1\end{array}$ & \\
\hline CM244 & 23 & & & & .59 & & \\
\hline PU244 & 1 & $650 .+2$ & $.300-4$ & 1.0 & 58.4 & & \\
\hline PU244 & 3 & $320 .+2$ & $.60-6$ & .020 & 58.4 & & \\
\hline PU244 & 4 & $14.6+3$ & $1.35-5$ & .45 & 58.4 & & \\
\hline PU244 & 6 & $365 .+2$ & $1.35-5$ & .45 & 2.92 & +2 & \\
\hline PU244 & 8 & & & & 58.4 & 58.4 & 58.4 \\
\hline PU244 & 23 & & & & .76 & & \\
\hline IJ 240 & 1 & 100. & .01 & 1.0 & .084 & & \\
\hline i) 240 & 6 & 300. & $1.1-3$ & 0.11 & .398 & & \\
\hline U 240 & 8 & & & & .084 & & \\
\hline () 240 & 23 & & & & .084 & & \\
\hline PU240 & 1 & $650 .+2$ & $.300-4$ & 1.0 & 53.0 & & \\
\hline FU 240 & 3 & $320 .+2$ & $.600-6$ & .020 & 53.0 & & \\
\hline PU240 & 4 & 146. +2 & $1.35-5$ & .450 & 53.0 & & \\
\hline PU240 & 6 & $365 .+2$ & $1.35-5$ & .450 & 2.70 & +2 & \\
\hline PU240 & 8 & & & & 53.0 & & \\
\hline PU240 & 23 & $.3-4$ & 1.0 & .0 & .53 & & \\
\hline $\mathrm{CM} 246$ & 1 & $240 .+2$ & $.100-3$ & 1.0 & 56.0 & & \\
\hline CM246 & 3 & $240 .+2$ & $.200-5$ & .020 & 56.0 & & \\
\hline $\mathrm{CM} 246$ & 4 & $150 .+2$ & $.400-4$ & .400 & 56.0 & & \\
\hline $\mathrm{CM} 246$ & 6 & $365 .+2$ & $.300-4$ & .300 & 2.78 & +2 & \\
\hline CM246 & 8 & & & & 56.0 & & \\
\hline CM246 & 23 & & & & .54 & & \\
\hline CM248 & 1 & $240 .+2$ & $.100-3$ & 1.0 & 4.53 & +2 & \\
\hline CM248 & 3 & $240 .+2$ & 2. -6 & .020 & 4.53 & +2 & \\
\hline $\mathrm{CM} 248$ & 4 & $146 .+2$ & $4.0-5$ & .40 & 4.53 & +2 & \\
\hline CM248 & 6 & $365,+2$ & $.30-4$ & .30 & 22.44 & +2 & \\
\hline CM248 & 8 & & & & 4.53 & +2 & \\
\hline CM248 & 23 & & & & 11.45 & & \\
\hline CM247 & 1 & $240 .+2$ & $.100-3$ & 1.0 & 55. & & \\
\hline $\mathrm{CM} 247$ & 3 & $240 .+2$ & $2.0-6$ & .020 & 55. & & \\
\hline CM247 & 4 & $150 .+2$ & $.45-4$ & .450 & 55. & & \\
\hline CM247 & 6 & $365,+2$ & $.45-4$ & .450 & 2.70 & +2 & \\
\hline CM247 & 8 & & & & 55.0 & 55.0 & 55.0 \\
\hline CM 247 & 23 & & & & .71 & & \\
\hline CM243 & 1 & $240 .+2$ & $.100-3$ & 1.0 & 60.0 & & \\
\hline CM243 & 3 & $240 .+2$ & $.200-5$ & .020 & 60.0 & & \\
\hline $\mathrm{CM} 243$ & 4 & $150 .+2$ & $.450-4$ & .450 & 60.0 & & \\
\hline $\mathrm{CM} 243$ & 6 & $365 .+2$ & $.450-4$ & .450 & 2.99 & +2 & \\
\hline $\mathrm{CM} 243$ & 8 & & & & 60. & 60. & 60 \\
\hline CM243 & 23 & & & & .61 & & \\
\hline PU243 & 1 & $650 .+2$ & $.300-4$ & 1.0 & .37 & & \\
\hline P243 & 3 & $320 .+2$ & $.600-6$ & .020 & .37 & & \\
\hline PU243 & 4 & $1.5+4$ & $1.35-5$ & .45 & .25 & & \\
\hline PU243 & 6 & $365 .+2$ & $1.35-5$ & .45 & 2. & & \\
\hline PU243 & 8 & & & & .19 & & \\
\hline Fu & 23 & & & & .18 & & \\
\hline & & & & & B. 32 & & \\
\hline
\end{tabular}




\section{Appendix B - Organ Data Library - ORGLIB}

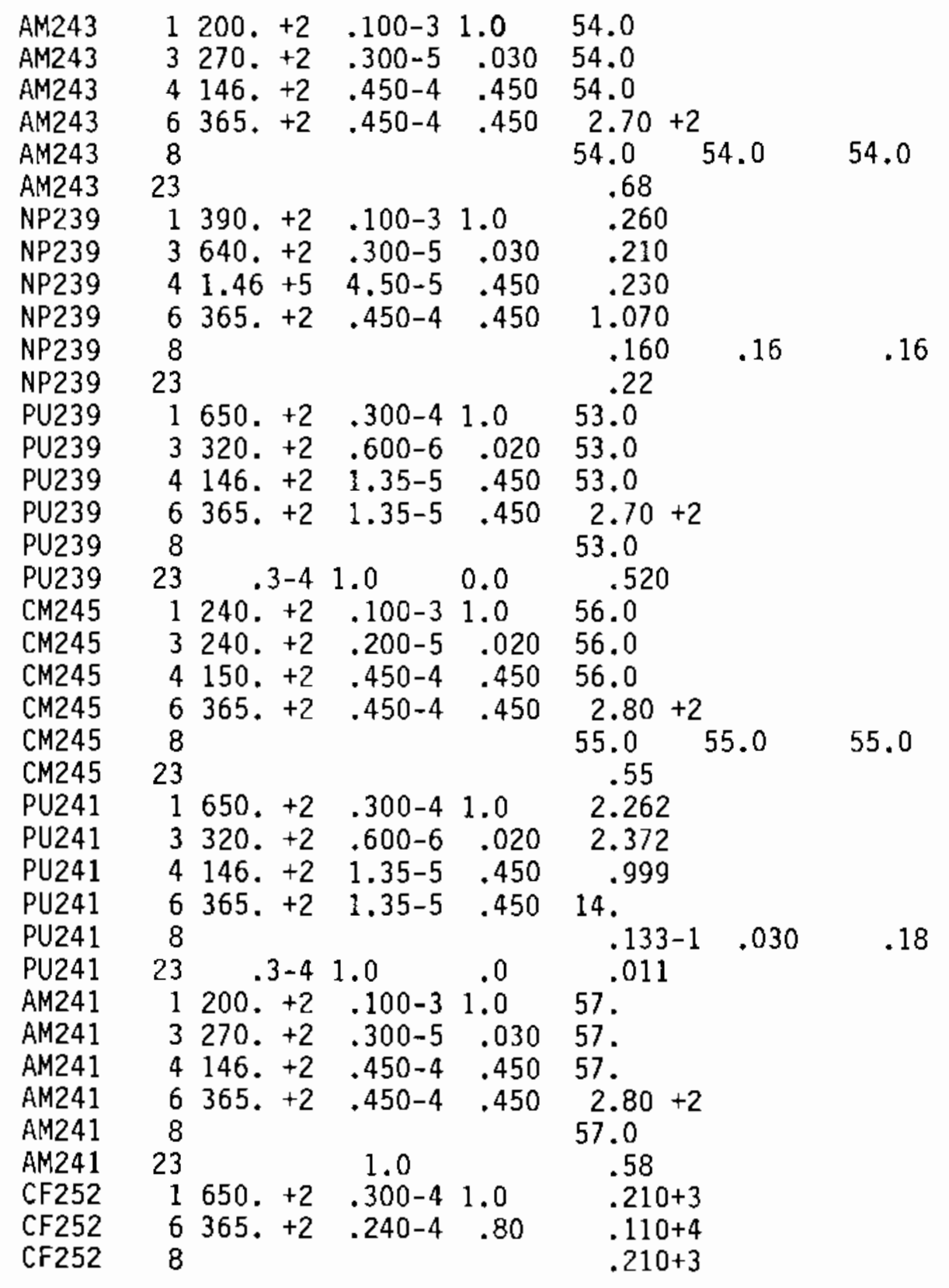




\section{Appendix B - Food Transfer Factor Library - FTRANS}

FOOD TRANSFER COEFFICIENT LIBRARY (RAP/WTF 04-APR-36 Cl Update)

\begin{tabular}{|c|c|c|c|c|c|c|c|c|}
\hline ELT & $\begin{array}{l}\text { DEP. VEL } \\
\mathrm{M} / \mathrm{SEC}\end{array}$ & PLANT & $\begin{array}{c}\mathrm{EGG} \\
\mathrm{DAY} / \mathrm{KG}\end{array}$ & $\begin{array}{l}\text { MILK } \\
\text { DAY/L }\end{array}$ & $\begin{array}{c}\mathrm{BEEF} \\
\mathrm{DAY} / \mathrm{KG}\end{array}$ & $\begin{array}{c}\text { PORK } \\
\text { DAY/KG }\end{array}$ & $\begin{array}{l}\text { POULTRY } \\
\text { DAY/KG }\end{array}$ & \\
\hline $\mathrm{H}$ & 0.0 & 0.0 & 0.0 & 0.0 & 0.0 & 0.0 & 0.0 & $8.0 E-01$ \\
\hline BE & $1.0-03$ & $4.7-04$ & $2.0-02$ & $2.0-06$ & $8.0-04$ & $1.0-02$ & $4.0-01$ & $5.0 E-03$ \\
\hline 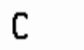 & 0.0 & 0.0 & 0.0 & 0.0 & 0.0 & 0.0 & 0.0 & $8.0 \mathrm{E}-01$ \\
\hline N & $1.0-03$ & $7.5+00$ & $9.9-04$ & $1.1-02$ & $9.9-04$ & $9.9-04$ & $9.9-04$ & \\
\hline$F$ & $1.0-02$ & $2.0-02$ & $9.9-04$ & $7.0-03$ & $2.0-02$ & $9.0-02$ & $9.9-04$ & \\
\hline NA & $1.0-03$ & $5.0-02$ & $2.0-01$ & $4.0-02$ & $5.0-02$ & $1.0-01$ & $1.0-02$ & \\
\hline$p$ & $1.0-03$ & $5.0+01$ & $1.0+01$ & $1.2-02$ & $5.0-02$ & $5.4-01$ & $1.9-01$ & \\
\hline$C L$ & $1.0-03$ & 5.0 & $9.9-04$ & $2.0-02$ & $3.0-02$ & $3.0-02$ & $3.0-02$ & $8.0 \mathrm{E}-01$ \\
\hline AR & 0.0 & 0.0 & 0.0 & 0.0 & 0.0 & 0.0 & 0.0 & \\
\hline $\mathrm{CA}$ & $1.0-03$ & $4.0-02$ & $1.0+00$ & $8.0-03$ & $3.3-03$ & $3.3-03$ & $3.3-03$ & $1.7 E-02$ \\
\hline SC & $1.0-03$ & $1.1-03$ & $9.9-04$ & $2.5-06$ & $6.0-03$ & $1.0-02$ & $4.0-03$ & \\
\hline CR & $1.0-03$ & $2.5-04$ & $9.9-04$ & $1.1-03$ & $9.9-04$ & $9.9-04$ & $9.9-04$ & \\
\hline$M N$ & $1.0-03$ & $3.0-02$ & $1.0-01$ & $1.0-04$ & $5.0-03$ & $2.0-02$ & $1.1-01$ & \\
\hline $\mathrm{FE}$ & $1.0-03$ & $4.0-04$ & $1.0-01$ & $6.0-04$ & $2.0-02$ & $5.0-03$ & $1.0-03$ & \\
\hline $\mathrm{CO}$ & $1.0-03$ & $9.4-03$ & $1.0-01$ & $5.0-04$ & $1.0-03$ & $5.0-03$ & $1.0-03$ & $2.3 E-04$ \\
\hline NI & $1.0-03$ & $1.9-02$ & 1. $0-01$ & $3.4-03$ & $1.0-03$ & $5.0-03$ & $1.0-03$ & $2.3 \mathrm{E}-03$ \\
\hline $\mathrm{CU}$ & $1.0-03$ & $1.3-01$ & 2. $0-01$ & $7.0-03$ & $1.0-02$ & $1.5-02$ & $2.0-03$ & \\
\hline $\mathrm{ZN}$ & $1.0-03$ & $4.0-01$ & $4.0-03$ & $6.0-03$ & $5.0-02$ & $1.4-01$ & $2.0-03$ & \\
\hline AS & $1.0-03$ & $1.0-02$ & $9.9-04$ & $3.0-03$ & $1.47-3$ & $2.38-2$ & $8.33-1$ & \\
\hline ;E & $1.0-03$ & $1.3+00$ & $2.1+00$ & $2.3-02$ & $1.0+00$ & $4.5-01$ & $3.7-01$ & $2.2 \mathrm{E}-02$ \\
\hline R & $1.0-02$ & $7.6-01$ & $1.6+00$ & $2.5-02$ & $2.0-02$ & $9.0-02$ & $4.0-03$ & \\
\hline $\mathrm{KR}$ & 0.0 & 0.0 & 0.0 & 0.0 & 0.0 & 0.0 & 0.0 & \\
\hline $\mathrm{B}$ & $1.0-03$ & $1.3-01$ & $3.0+00$ & $1.0-02$ & $1.5-01$ & $2.0-01$ & $2.0+00$ & \\
\hline ;R & $1.0-03$ & $2.0-01$ & $4.0-01$ & $1.5-03$ & $3.0-04$ & $7.3-03$ & $9.0-04$ & $4.5 E-03$ \\
\hline & $1.0-03$ & $2.5-03$ & $5.0-04$ & $5.0-06$ & $5.0-03$ & $5.0-03$ & $5.0-04$ & \\
\hline $7 \mathrm{R}$ & $1.0-03$ & $1.7-04$ & $1.2-03$ & $2.5-06$ & 5.0 & $1.0-03$ & $1.0-04$ & $1.1 E-04$ \\
\hline NB & $1.0-03$ & $9.4-03$ & $1.2-03$ & $1.2-03$ & 5.0 & $1.0-03$ & $1.0-04$ & \\
\hline 90 & $1.0-03$ & $1.3-01$ & $4.0-01$ & $4.0-03$ & 1. & $2.0-02$ & $2.0-03$ & \\
\hline TC & $1.0-03$ & 1.0 & $9.9-04$ & $1.2-02$ & 9.5 & 04 & $9.9-04$ & $8.0 \mathrm{E}-01$ \\
\hline 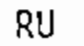 & $1.0-03$ & $1.0-02$ & $4.0-03$ & $5.0-07$ & 1.0 & 03 & 3. & $2.2 \mathrm{E}-02$ \\
\hline 24 & $1.0-03$ & $1.3+01$ & $4.0-03$ & $5.0-03$ & 1.0 & 03 & 3. & $2.2 \mathrm{E}-02$ \\
\hline ח & $1.0-03$ & $5.0+00$ & 4.0 & $5.0-03$ & 1.0 & 03 & 04 & $4.5 E-03$ \\
\hline & $1.0-03$ & $1.5-01$ & 9.9 & 2.5 & 9.9 & 04 & 9 . & \\
\hline 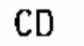 & $1.0-03$ & $3.0-01$ & $9.9-04$ & $6.2-05$ & $1.6-02$ & $1.6-02$ & $1.6-02$ & $7.6 \mathrm{E}-04$ \\
\hline $\mathrm{N}$ & $1.0-03$ & $2.5-03$ & $9.9-04$ & 1.3 & 9.9 & 04 & 9. & -03 \\
\hline & $1.0-03$ & $1.1-02$ & $7.0-02$ & 7.5 & 3. & 03 & 6 . & $7.0 \mathrm{E}-02$ \\
\hline & $1.0-03$ & $1.3+00$ & $4.0-01$ & 5.0 & 5.0 & 1. & 02 & \\
\hline $\mathrm{T}$ & $1.0-02$ & $2.0-02$ & $1.6+00$ & $1.0-02$ & $2.0-02$ & $9.0-02$ & $4.0-03$ & $7.0 \mathrm{E}-02$ \\
\hline r & 0.0 & 0.0 & 0.0 & 0.0 & 0.0 & 0.0 & 0.0 & \\
\hline os & $1.0-03$ & $2.0-03$ & $6.0-01$ & $5.0-03$ & $3.0-02$ & $2.6-01$ & $4.5+00$ & $1,1 \mathrm{E}-04$ \\
\hline & $1.0-03$ & $5.0-03$ & $4.0-01$ & $4.0-04$ & $5.0-04$ & $1.0-02$ & $5.0-04$ & \\
\hline & $1.0-03$ & $2.5-03$ & $2.0-03$ & $2.5-06$ & $5.0-03$ & $5.0-03$ & $4.0-03$ & \\
\hline & $1.0-03$ & $5.0-04$ & $3.0-03$ & $1.0-05$ & $1.0-03$ & $5.0-03$ & $6.0-04$ & $=-04$ \\
\hline & 1. $0-03$ & $2.5-03$ & $4.0-03$ & $2.5-06$ & $5.0-03$ & $5.0-03$ & $0-03$ & $4.6 \mathrm{~F}-04$ \\
\hline & $1.0-03$ & $2.4-03$ & $2.0-04$ & $2.5-06$ & $5.0-03$ & $5.0-03$ & 4. $0-03$ & $4.6 \mathrm{E}-04$ \\
\hline & $1.0-03$ & $2.5-03$ & $7.0-03$ & $2.5-06$ & $5.0-03$ & $5.0-0$ & $1.0-04$ & $4.6 \mathrm{E}-04$ \\
\hline & $1.0-03$ & $2.5-03$ & $7.0-03$ & $2.5-06$ & $5.0-03$ & $5.0-03$ & $4.0-03$ & $4.6 \mathrm{E}-04$ \\
\hline
\end{tabular}




\section{Appendix B - Food Transfer Factor Library - FTRANS}

$\begin{array}{lllllllll}\text { EU } & 1.0-03 & 2.5-03 & 7.0-03 & 2.5-06 & 5.0-03 & 5.0-03 & 4.0-03 & 4.6 \mathrm{E}-04 \\ \text { TB } & 1.0-03 & 2.6-03 & 7.0-03 & 2.5-06 & 5.0-03 & 5.0-03 & 4.0-03 & 4.6 \mathrm{E}-04 \\ \text { HO } & 1.0-03 & 2.6-03 & 7.0-03 & 2.5-06 & 5.0-03 & 5.0-03 & 4.0-03 & 7.6 \mathrm{E}-04 \\ \text { W } & 1.0-03 & 1.8-02 & 9.9-04 & 2.5-04 & 9.9-04 & 9.9-04 & 9.9-04 & \\ \text { IR } & 1.0-03 & 9.9-04 & 9.9-04 & 9.9-04 & 9.9-04 & 9.9-04 & 9.9-04 & \\ \text { HG } & 1.0-03 & 3.8-01 & 9.9-04 & 1.9-02 & 1.0-01 & 3.1+00 & 1.1-02 & \\ \text { PB } & 1.0-03 & 6.8-02 & 9.9-04 & 1.0-05 & 9.9-04 & 9.9-04 & 9.9-04 & 1.1 \mathrm{E}-04 \\ \text { BI } & 1.0-03 & 1.5-01 & 9.9-04 & 2.5-04 & 9.9-04 & 9.9-04 & 9.9-04 & \\ \text { PO } & 1.0-03 & 9.0-03 & 9.9-04 & 1.2-04 & 9.9-04 & 9.9-04 & 9.9-04 & \\ \text { RN } & 0.0 & 0.0 & 0.0 & 0.0 & 0.0 & 0.0 & 0.0 & \\ \text { RA } & 1.0-03 & 1.4-03 & 2.0-05 & 2.0-04 & 9.9-04 & 9.9-04 & 9.9-04 & 2.3 \mathrm{E}-03 \\ \text { AC } & 1.0-03 & 2.5-03 & 2.0-03 & 2.5-06 & 5.0-03 & 1.0-02 & 4.0-03 & \\ \text { TH } & 1.0-03 & 4.2-03 & 2.0-03 & 2.5-06 & 5.0-03 & 1.0-02 & 4.0-03 & 2.3 \mathrm{E}-04 \\ \text { PA } & 1.0-03 & 2.5-03 & 2.0-03 & 2.5-06 & 5.0-03 & 1.0-02 & 4.0-03 & 4.6 \mathrm{E}-04 \\ \text { U } & 1.0-03 & 2.5-03 & 3.4-01 & 6.0-04 & 5.0-03 & 6.0-04 & 1.2-03 & 9.0 \mathrm{E}-03 \\ \text { NP } & 1.0-03 & 2.5-03 & 2.0-03 & 2.5-06 & 5.0-03 & 1.0-02 & 4.0-03 & 2.2 \mathrm{E}-02 \\ \text { PU } & 1.0-03 & 2.5-04 & 2.0-03 & 2.5-08 & 5.0-03 & 1.0-02 & 4.0-03 & 2.3 \mathrm{E}-04 \\ \text { AM } & 1.0-03 & 2.5-04 & 2.0-03 & 2.5-06 & 5.0-03 & 1.0-02 & 4.0-03 & 2.3 \mathrm{E}-04 \\ \text { CM } & 1.0-03 & 2.5-03 & 2.0-03 & 2.5-06 & 5.0-03 & 1.0-02 & 4.0-03 & 2.3 \mathrm{E}-04 \\ \text { CF } & 1.0-03 & 2.5-03 & 2.0-03 & 7.5-07 & 5.0-03 & 1.0-02 & 4.0-03 & \end{array}$




\section{Appendix B - Bioaccumulation Factor Library - BIOAC1}

BIOACCUMULATION FACTOR LIBRARY FOR BA NAPIER (04-APR-86/WTF)

FISH CRUS. MOLL. ALGAE FISH CRUS, MOLL.

\begin{tabular}{|c|c|c|c|c|c|c|c|c|c|}
\hline & FISH & CRUS. & MOLL. & ALGAE & FISH & CRUS. & MOLL. & ALGAE & ORS \\
\hline & 1.0 & 1.0 & 1.0 & 1.0 & .9 & .9 & .9 & .9 & 1.0 \\
\hline E & 1000.0 & 0000.0 & 10000.0 & 10000.0 & 2.0 & 10.0 & 10.0 & 20.0 & .2 \\
\hline & 1.0 & 1.0 & 1.0 & 1.0 & 4600.0 & 9100.0 & 9100.0 & 4600.0 & 1.0 \\
\hline & .0 & .0 & .0 & .0 & .0 & .0 & .0 & .0 & 1.0 \\
\hline & 4.0 & 4.0 & 4.0 & 1.0 & 10.0 & 100.0 & 100.0 & 2.0 & .8 \\
\hline A & 1.0 & 1.0 & 1.0 & 1.0 & 100.0 & 200.0 & 200.0 & 500.0 & .9 \\
\hline & 0000.0 & 10000.0 & 10000.0 & 100000.0 & 00000.0 & 20000.0 & 20000.0 & 00000.0 & .4 \\
\hline L & 1.0 & 1.0 & 1.0 & 1.0 & 1.0 & 1.0 & 1.0 & 1.0 & 1.0 \\
\hline & 1.0 & 1.0 & 1.0 & 1.0 & 1.0 & 1.0 & 1.0 & 1.0 & .0 \\
\hline & 10.0 & 10.0 & 10.0 & 1.0 & 40.0 & 330.0 & 330.0 & 130. & .2 \\
\hline $5 C$ & 100.0 & 300.0 & 300.0 & 1000.0 & 2.0 & 1000.0 & 1000.0 & 10000 & .3 \\
\hline CR & 100.0 & 1000.0 & 1000.0 & 1000.0 & 20.0 & 2000.0 & 2000.0 & 4000 & .9 \\
\hline $\mathbf{N}$ & 3000.0 & 10000.0 & 50000.0 & 10000.0 & 400.0 & 90000.0 & 90000.0 & 10000.0 & .5 \\
\hline & 1000.0 & 4000.0 & 20000.0 & 6000.0 & 100.0 & 3200.0 & 3200.0 & 1000.0 & .2 \\
\hline & 100.0 & 10000.0 & 300.0 & 100.0 & 50.0 & 200.0 & 200.0 & 200.0 & .2 \\
\hline & 500.0 & 100.0 & 100.0 & 100.0 & 100.0 & 100.0 & 100.0 & 50.0 & .2 \\
\hline & 100 & 5000.0 & 5000.0 & 1000.0 & 50.0 & 400.0 & 400.0 & 2000.0 & .6 \\
\hline 7 & 5000.0 & 5000.0 & 50000.0 & 1000.0 & 2000.0 & 10000.0 & 10000.0 & 20000 & .4 \\
\hline & 30 & 300.0 & 300.0 & 300.0 & 300.0 & 300.0 & 300.0 & 300.0 & .7 \\
\hline & 10.0 & 10.0 & 10.0 & 100.0 & 170.0 & 170.0 & 170.0 & 1000.0 & .8 \\
\hline & 3.0 & 10.0 & 10. & 100.0 & 420.0 & 330.0 & 330.0 & 50.0 & .8 \\
\hline & 1.0 & 1.0 & 1. & 1.0 & 1.0 & 1.0 & 1.0 & 1.0 & .0 \\
\hline 28 & 30.0 & 50.0 & 10. & 10.0 & 2000.0 & 1000.0 & 1000.0 & 1000.0 & .9 \\
\hline F & 1.0 & 1.0 & 1 . & 20.0 & 30.0 & 100.0 & 100.0 & 500.0 & .2 \\
\hline & 30.0 & 100.0 & 100. & 300.0 & 25.0 & 1000.0 & 1000.0 & 5000.0 & .2 \\
\hline 71 & 30.0 & 100.0 & 100. & 1000.0 & 3.3 & 6.7 & 6.7 & 1000 . & .7 \\
\hline 4 & 100.0 & 200.0 & 200 . & 100.0 & 30000.0 & 100.0 & 100.0 & 800 . & .7 \\
\hline 10 & 1 & 100.0 & 100. & 100.0 & 10.0 & 10.0 & 10.0 & 1000 . & .9 \\
\hline TC & 10 & 10 & 100 & 1000.0 & 15.0 & 5. & 5.0 & 40.0 & .7 \\
\hline RL & 3 & 10 & 100 & 1000.0 & 10 & 300. & 300.0 & 2000 & .5 \\
\hline $\mathrm{Q}$ & 10 & 10 & 10 & .0 & 10 & 300.0 & 300.0 & 200. & .5 \\
\hline 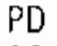 & 1 & 0 & 10 & .0 & 10.0 & 300.0 & 300.0 & 200 . & .5 \\
\hline 40 & 100 & 5000.0 & 500 & 100 & 2 & 770.0 & 770.0 & 200.0 & .7 \\
\hline CD & 100. & 3000.0 & 10000 & 10000.0 & 200.0 & 200 & .0 & 1000.0 & .6 \\
\hline 51 & 3 & 3 & 3 & 10.0 & 3000.0 & 1000.0 & 1000.0 & 100.0 & .7 \\
\hline 58 & 1000. & 1000.0 & 1000. & 10000.0 & n & .0 & 10.0 & 1500.0 & .8 \\
\hline $\mathrm{TE}$ & 10. & 100 & 10 & 1000.0 & 40 & 75 . & 75.0 & 100.0 & .8 \\
\hline I & 20 . & 100.0 & 100. & 10000.0 & 15.0 & 5. & 5.0 & 40.0 & .8 \\
\hline$\langle F$ & & 1.0 & 1 & 1.0 & 1 & .0 & 1.0 & 1.0 & .0 \\
\hline CS & 30. & 50.0 & 10. & 10.0 & 2000.0 & 100.0 & 100.0 & 500.0 & .9 \\
\hline$B A$ & 3 & 3. & 3 & 100.0 & 4.0 & 200.0 & 200.0 & 500.0 & .4 \\
\hline A & 30. & 100.0 & 100. & 300.0 & 25.0 & 1000.0 & 1000.0 & 5000.0 & .2 \\
\hline CE & 30. & 100.0 & 100. & 300.0 & 1.0 & 1000.0 & 1000.0 & 4000.0 & .2 \\
\hline & 100. & 1000.0 & 1000 & 1000.0 & 25.0 & 1000.0 & 1000.0 & 5000.0 & .2 \\
\hline UD & 100. & 1000.0 & 1000 . & 1000.0 & 25.0 & 1000.0 & 1000.0 & 5000.0 & .2 \\
\hline & 100. & 1000.0 & 1000.0 & 1000.0 & 25.0 & 1000.0 & 1000.0 & 5000.0 & .2 \\
\hline & 100.0 & 1000.0 & 1000.0 & 1000.0 & 25.0 & 1000.0 & 1000.0 & 5000.0 & . \\
\hline & 100.0 & 1000.0 & 1000.0 & 1000.0 & 25.0 & 1000.0 & 1000.0 & 500 & .2 \\
\hline
\end{tabular}




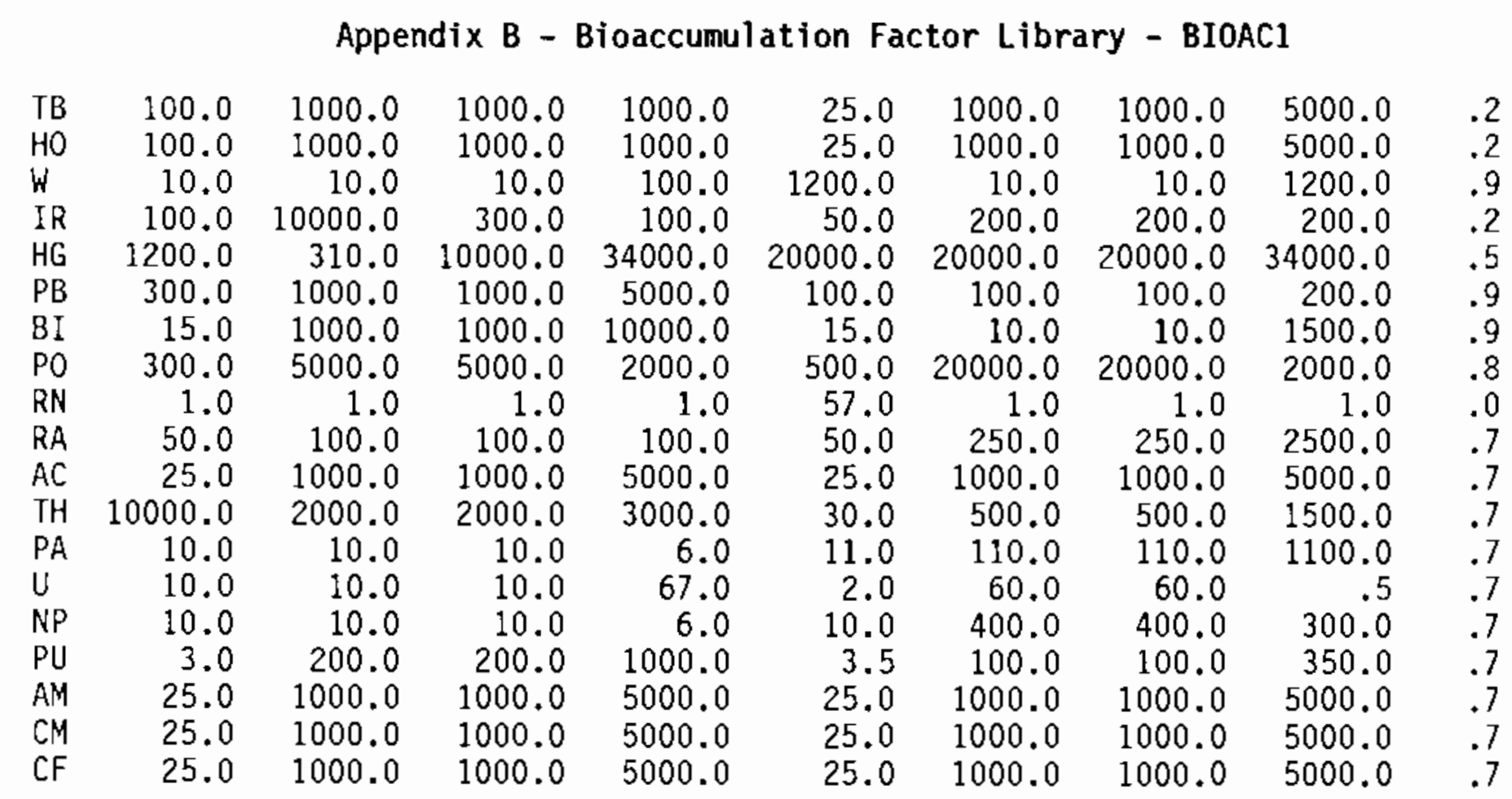




\section{Appendix B - External Exposure Dose Rate Factors - PLANEA}

OVERBURDEN: 0.0M, SOURCE: $0.15 \mathrm{M}$ (DEN: CONCRETE/1.B): MR/HR 8-JUT-86 RAP

$\begin{array}{ll}\text { H } 3 & 0.0 \mathrm{E}+00 \\ \text { C 14 } & 6.2 \mathrm{E}-04 \\ \text { NA22 } & 4.3 \mathrm{E}+02 \\ \text { P 32 } & 9.2 \mathrm{E}-01 \\ \text { P 33 } & 2.8 \mathrm{E}-03 \\ \text { S 35 } & 0.0 \mathrm{E}+00 \\ \text { CL36 } & 7.5 \mathrm{E}-02 \\ \text { K } 40 & 5.7 \mathrm{E}+01 \\ \text { CA45 } & 2.8 \mathrm{E}-03 \\ \text { SC46 } & 7.2 \mathrm{E}+02 \\ \text { CR51 } & 1.1 \mathrm{E}+01 \\ \text { MN54 } & 2.9 \mathrm{E}+02 \\ \text { FE55 } & 0.0 \mathrm{E}+00 \\ \text { FE59 } & 4.0 \mathrm{E}+02 \\ \text { C057 } & 2.1 \mathrm{E}+01 \\ \text { C060 } & 8.6 \mathrm{E}+02 \\ \text { NI59 } & 0.0 \mathrm{E}+00 \\ \text { NI63 } & 1.4 \mathrm{E}-05 \\ \text { ZN65 } & 2.2 \mathrm{E}+02 \\ \text { SE75 } & 9.2 \mathrm{E}+01 \\ \text { SR85 } & 1.8 \mathrm{E}+02 \\ \text { SR89 } & 5.7 \mathrm{E}-01 \\ \text { Y 89M } & 3.7 \mathrm{E}+00 \\ \text { SR90 } & 3.0 \mathrm{E}-02 \\ \text { Y 90 } & 1.9 \mathrm{E}+00 \\ \text { M093 } & 1.4 \mathrm{E}+00 \\ \text { NB94 } & 5.3 \mathrm{E}+02 \\ \text { M099 } & 4.8 \mathrm{E}+01 \\ \text { TC99M } & 1.9 \mathrm{E}+01 \\ \text { TC99 } & 4.0 \mathrm{E}-03 \\ \text { RU103 } & 1.8 \mathrm{E}+02 \\ \text { RH103M } & 5.1 \mathrm{E}-02 \\ \text { P0103 } & 2.8 \mathrm{E}-01 \\ \text { RU106 } & 4.0 \mathrm{E}-07 \\ \text { RH106 } & 4.1 \mathrm{E}+01 \\ \text { CD109 } & 7.6 \mathrm{E}+00 \\ \text { AG110M } & 1.0 \mathrm{E}+03 \\ \text { AG110 } & 1.4 \mathrm{E}+01 \\ \text { IN111 } & 7.6 \mathrm{E}+01 \\ \text { SB124 } & 7.2 \mathrm{E}+02 \\ \text { SB125 } & 1.7 \mathrm{E}+02 \\ \text { TE125M } & 5.5 \mathrm{E}-01 \\ \text { I 125 } & 8.8 \mathrm{E}-01 \\ \text { I 129 } & 4.3 \mathrm{E}-01 \\ \text { I 131 } & 1.2 \mathrm{E}+02 \\ \text { XE131M } & 1.2 \mathrm{E}+00 \\ \text { CS134 } & 6.0 \mathrm{E}+02 \\ \text { CS135 } & 1.3 \mathrm{E}-03 \\ & \\ & \end{array}$




\section{Appendix B - External Exposure Dose Rate Factors - PLANEA}

$\begin{array}{ll}\text { CS137 } & 4.1 \mathrm{E}-02 \\ \text { BA137M } & 2.2 \mathrm{E}+02 \\ \text { CE141 } & 1.0 \mathrm{E}+01 \\ \text { CE144 } & 2.6 \mathrm{E}+00 \\ \text { PR144 } & 1.4 \mathrm{E}+01 \\ \text { ND144 } & 0.0 \mathrm{E}+00 \\ \text { EU152 } & 4.0 \mathrm{E}+02 \\ \text { EU154 } & 4.2 \mathrm{E}+02 \\ \text { TB160 } & 3.8 \mathrm{E}+02 \\ \text { OS185 } & 2.5 \mathrm{E}+02 \\ \text { OS191 } & 7.9 \mathrm{E}+00 \\ \text { IR192 } & 2.5 \mathrm{E}+02 \\ \text { HG203 } & 4.7 \mathrm{E}+01 \\ \text { TH230 } & 1.7 \mathrm{E}-02 \\ \text { RA226 } & 8.0 \mathrm{E}-01 \\ \text { RN222 } & 4.7 \mathrm{E}+02 \\ \text { PB210 } & 8.1 \mathrm{E}-02 \\ \text { BI210 } & 2.6 \mathrm{E}-01 \\ \text { TH228 } & 2.5 \mathrm{E}-01 \\ \text { RA224 } & 2.5 \mathrm{E}+00 \\ \text { PB212 } & 2.9 \mathrm{E}+01 \\ \text { BI212 } & 1.2 \mathrm{E}+03 \\ \text { TH232 } & 7.4 \mathrm{E}-01 \\ \text { RA228 } & 6.5 \mathrm{E}-06 \\ \text { AC228 } & 2.9 \mathrm{E}+02 \\ \text { U 234 } & 6.9 \mathrm{E}-02 \\ \text { U 235 } & 2.0 \mathrm{E}+01 \\ \text { TH231 } & 1.3 \mathrm{E}+00 \\ \text { PA231 } & 8.6 \mathrm{E}+00 \\ \text { AC227 } & 1.3 \mathrm{E}-02 \\ \text { TH227 } & 2.5 \mathrm{E}+01 \\ \text { FR223 } & 7.6 \mathrm{E}+00 \\ \text { RA223 } & 2.6 \mathrm{E}+01 \\ \text { NP237 } & 1.9 \mathrm{E}+00 \\ \text { PA233 } & 6.0 \mathrm{E}+01 \\ \text { U 233 } & 4.2 \mathrm{E}-03 \\ \text { TH229 } & 1.8 \mathrm{E}+00 \\ \text { RA225 } & 6.8 \mathrm{E}-01 \\ \text { AC225 } & 1.7 \mathrm{E}+00 \\ \text { U 238 } & 9.8 \mathrm{E}-03 \\ \text { TH234 } & 7.5 \mathrm{E}-01 \\ \text { PA234M } & 2.9 \mathrm{E}+00 \\ \text { PA234 } & 6.3 \mathrm{E}+02 \\ \text { PU242 } & 8.1 \mathrm{E}-04 \\ \text { NP238 } & 2.0 \mathrm{E}+02 \\ \text { PU238 } & 2.9 \mathrm{E}-03 \\ \text { CM244 } & 1.8 \mathrm{E}-03 \\ \text { PU244 } & 6.8 \mathrm{E}-07 \\ \text { U 240 } & 4.7 \mathrm{E}-02 \\ \text { PU240 } & 2.7 \mathrm{E}-03\end{array}$


Appendix B - External Exposure Dose Rate Factors - PLANEA

$\mathrm{CM} 243 \quad 2.5 \mathrm{E}+01$

PU243 2.7E+00

AM243 $3.6 \mathrm{E}+00$

NP239 2.9E+01

PU239 2.9E-03

PU241 2.9E-12

AM241 $1.1 \mathrm{E}+00$ 


\section{Appendix B - External Exposure Dose Rate Factors - PLANEB}

OVERBURDEN: $0.0 \mathrm{M}$, SOURCE: $0.15 \mathrm{M}$ (DEN: WATER/1.0): MR/HR 8-Ju1-86 RAP

$\begin{array}{ll}\text { H } 3 & 0.0 \mathrm{E}+00 \\ \text { C 14 } & 5.2 \mathrm{E}-03 \\ \text { NA22 } & 6.4 \mathrm{E}+02 \\ \text { P 32 } & 2.3 \mathrm{E}+00 \\ \text { P 33 } & 5.1 \mathrm{E}-02 \\ \text { S 35 } & 0.0 \mathrm{E}+00 \\ \text { CL36 } & 1.8 \mathrm{E}-02 \\ \text { K 40 } & 8.8 \mathrm{E}+01 \\ \text { CA45 } & 1.8 \mathrm{E}-02 \\ \text { SC46 } & 1.1 \mathrm{E}+03 \\ \text { CR51 } & 2.3 \mathrm{E}+01 \\ \text { MN54 } & 4.8 \mathrm{E}+02 \\ \text { FE55 } & 0.0 \mathrm{E}+00 \\ \text { FE59 } & 5.8 \mathrm{E}+02 \\ \text { C057 } & 6.2 \mathrm{E}+01 \\ \text { C060 } & 1.3 \mathrm{E}+03 \\ \text { NI59 } & 0.0 \mathrm{E}+00 \\ \text { NI63 } & 2.9 \mathrm{E}-13 \\ \text { ZN65 } & 3.4 \mathrm{E}+02 \\ \text { SE75 } & 2.2 \mathrm{E}+02 \\ \text { SR85 } & 3.7 \mathrm{E}+02 \\ \text { SR89 } & 1.6 \mathrm{E}+00 \\ \text { Y 89M } & 5.3 \mathrm{E}+00 \\ \text { SR90 } & 1.3 \mathrm{E}-01 \\ \text { Y 90 } & 4.6 \mathrm{E}+00 \\ \text { M093 } & 1.3 \mathrm{E}+01 \\ \text { NB94 } & 9.4 \mathrm{E}+02 \\ \text { M099 } & 9.2 \mathrm{E}+01 \\ \text { IC99M } & 5.5 \mathrm{E}+01 \\ \text { TC99 } & 2.4 \mathrm{E}-02 \\ \text { RU103 } & 3.7 \mathrm{E}+02 \\ \text { RH103M } & 8.7 \mathrm{E}-01 \\ \text { PD103 } & 4.7 \mathrm{E}+00 \\ \text { RU106 } & 7.5 \mathrm{E}-06 \\ \text { RH106 } & 7.9 \mathrm{E}+01 \\ \text { CD109 } & 2.6 \mathrm{E}+01 \\ \text { AG110M } & 1.8 \mathrm{E}+03 \\ \text { AG110 } & 2.8 \mathrm{E}+01 \\ \text { IN111 } & 2.0 \mathrm{E}+02 \\ \text { SB124 } & 1.2 \mathrm{E}+03 \\ \text { SB125 } & 3.3 \mathrm{E}+02 \\ \text { TE125M } & 8.5 \mathrm{E}+00 \\ \text { I 125 } & 1.2 \mathrm{E}+01 \\ \text { I 129 } & 6.2 \mathrm{E}+00 \\ \text { I 131 } & 2.6 \mathrm{E}+02 \\ \text { XE131M } & 8.4 \mathrm{E}+00 \\ \text { CS134 } & 1.1 \mathrm{E}+03 \\ \text { CS135 } & 9.6 \mathrm{E}-03 \\ & \end{array}$


Appendix B - External Exposure Dose Rate Factors - PLANEB

$\begin{array}{ll}\text { CS137 } & 1.6 \mathrm{E}-01 \\ \text { BA137M } & 4.1 \mathrm{E}+02 \\ \text { CE141 } & 3.2 \mathrm{E}+01 \\ \text { CE144 } & 9.1 \mathrm{E}+00 \\ \text { PR144 } & 2.5 \mathrm{E}+01 \\ \text { ND144 } & 0.0 \mathrm{E}+00 \\ \text { EU152 } & 6.5 \mathrm{E}+02 \\ \text { EU154 } & 6.8 \mathrm{E}+02 \\ \text { TB160 } & 6.0 \mathrm{E}+02 \\ \text { OS185 } & 4.7 \mathrm{E}+02 \\ \text { OS191 } & 2.7 \mathrm{E}+01 \\ \text { IR192 } & 5.4 \mathrm{E}+02 \\ \text { HG203 } & 1.2 \mathrm{E}+02 \\ \text { TH230 } & 7.7 \mathrm{E}-02 \\ \text { RA226 } & 2.4 \mathrm{E}+00 \\ \text { RN222 } & 7.6 \mathrm{E}+02 \\ \text { PB210 } & 6.5 \mathrm{E}-01 \\ \text { B1210 } & 7.8 \mathrm{E}-01 \\ \text { TH228 } & 7.2 \mathrm{E}-01 \\ \text { RA224 } & 6.2 \mathrm{E}+00 \\ \text { PB212 } & 7.0 \mathrm{E}+01 \\ \text { BI212 } & 1.8 \mathrm{E}+03 \\ \text { TH232 } & 4.2 \mathrm{E}+00 \\ \text { RA228 } & 1.0 \mathrm{E}-04 \\ \text { AC228 } & 4.4 \mathrm{E}+02 \\ \text { U 234 } & 2.6 \mathrm{E}-01 \\ \text { U 235 } & 5.6 \mathrm{E}+01 \\ \text { TH231 } & 5.4 \mathrm{E}+00 \\ \text { PA231 } & 1.9 \mathrm{E}+01 \\ \text { AC227 } & 4.0 \mathrm{E}-02 \\ \text { TH227 } & 6.0 \mathrm{E}+01 \\ \text { FR223 } & 2.3 \mathrm{E}+01 \\ \text { RA223 } & 6.4 \mathrm{E}+01 \\ \text { NP237 } & 7.8 \mathrm{E}+00 \\ \text { PA233 } & 1.3 \mathrm{E}+02 \\ \text { U 233 } & 3.2 \mathrm{E}-02 \\ \text { TH229 } & 5.8 \mathrm{E}+00 \\ \text { RA225 } & 5.4 \mathrm{E}+00 \\ \text { AC225 } & 5.0 \mathrm{E}+00 \\ \text { U 238 } & 5.6 \mathrm{E}-02 \\ \text { TH234 } & 2.6 \mathrm{E}+00 \\ \text { PA234M } & 4.6 \mathrm{E}+00 \\ \text { PA234 } & 1.0 \mathrm{E}+03 \\ \text { PU242 } & 6.5 \mathrm{E}-03 \\ \text { NP238 } & 2.9 \mathrm{E}+02 \\ \text { PU238 } & 1.3 \mathrm{E}-02 \\ \text { CM244 } & 1.5 \mathrm{E}-02 \\ \text { PU244 } & 1.2 \mathrm{E}-05 \\ \text { U 240 } & 2.7 \mathrm{E}-01 \\ \text { PU240 } & 1.1 \mathrm{E}-02 \\ & \end{array}$


Appendix B - External Exposure Dose Rate Factors - PLANEB

$\mathrm{CM} 243 \quad 6.7 \mathrm{E}+01$

PU243 8.1E+00

AM243 $1.4 \mathrm{E}+01$

NP239 7.6E+01

PU239 1.1E-02

PU241 5.0E-11

AM241 $6.4 E+00$ 


\section{Appendix B - External Exposure Dose Rate Factors - PLANEC}

NO OVERBURDEN, $15 \mathrm{CM}$. SOURCE (DEN: H20:CARBON/.5,.1): MR/HR 8-JUl-86

$\begin{array}{ll}\text { H } 3 & 0.0 \mathrm{E}+00 \\ \text { C } 14 & 8.3 \mathrm{E}-03 \\ \text { NA22 } & 7.3 \mathrm{E}+02 \\ \text { P } 32 & 3.1 \mathrm{E}+00 \\ \text { P } 33 & 2.8 \mathrm{E}-02 \\ \text { S } 35 & 0.0 \mathrm{E}+00 \\ \text { CL36 } & 4.0 \mathrm{E}-01 \\ \text { K } 40 & 1.1 \mathrm{E}+02 \\ \text { CA45 } & 2.8 \mathrm{E}-02 \\ \text { SC46 } & 1.4 \mathrm{E}+03 \\ \text { CR51 } & 2.9 \mathrm{E}+01 \\ \text { MN54 } & 6.0 \mathrm{E}+02 \\ \text { FE55 } & 0.0 \mathrm{E}+00 \\ \text { FE59 } & 7.2 \mathrm{E}+02 \\ \text { C057 } & 8.4 \mathrm{E}+01 \\ \text { C060 } & 1.6 \mathrm{E}+03 \\ \text { NI59 } & 0.0 \mathrm{E}+00 \\ \text { NI63 } & 3.3 \mathrm{E}-04 \\ \text { ZN65 } & 4.1 \mathrm{E}+02 \\ \text { SE75 } & 3.0 \mathrm{E}+02 \\ \text { SR85 } & 4.8 \mathrm{E}+02 \\ \text { SR89 } & 2.1 \mathrm{E}+00 \\ \text { Y 89M } & 6.6 \mathrm{E}+00 \\ \text { SR90 } & 1.9 \mathrm{E}-01 \\ \text { Y 90 } & 6.1 \mathrm{E}+00 \\ \text { M093 } & 2.1 \mathrm{E}+01 \\ \text { NB94 } & 1.2 \mathrm{E}+03 \\ \text { M099 } & 1.1 \mathrm{E}+02 \\ \text { TC99M } & 7.6 \mathrm{E}+01 \\ \text { TC99 } & 3.6 \mathrm{E}-02 \\ \text { RU103 } & 4.6 \mathrm{E}+02 \\ \text { RH103M } & 1.5 \mathrm{E}+00 \\ \text { PD103 } & 8.2 \mathrm{E}+00 \\ \text { RU106 } & 1.3 \mathrm{E}-05 \\ \text { RH106 } & 1.0 \mathrm{E}+02 \\ \text { CD109 } & 3.6 \mathrm{E}+01 \\ \text { AG110M } & 2.2 \mathrm{E}+03 \\ \text { AG110 } & 3.6 \mathrm{E}+01 \\ \text { IN111 } & 2.7 \mathrm{E}+02 \\ \text { SB124 } & 1.5 \mathrm{E}+03 \\ \text { SB125 } & 4.2 \mathrm{E}+02 \\ \text { TE125M } & 1.5 \mathrm{E}+01 \\ \text { I 125 } & 2.0 \mathrm{E}+01 \\ \text { I 129 } & 1.0 \mathrm{E}+01 \\ \text { I 131 } & 3.3 \mathrm{E}+02 \\ \text { XE131M } & 1.3 \mathrm{E}+01 \\ \text { CS134 } & 1.3 \mathrm{E}+03 \\ \text { CS135 } & 1.5 \mathrm{E}-02 \\ & \\ & \end{array}$


Appendix B - External Exposure Dose Rate Factors - PLANEC

$\begin{array}{ll}\text { CS137 } & 2.2 \mathrm{E}-01 \\ \text { BA137M } & 5.1 \mathrm{E}+02 \\ \text { CE141 } & 4.5 \mathrm{E}+01 \\ \text { CE144 } & 1.3 \mathrm{E}+01 \\ \text { PR144 } & 3.1 \mathrm{E}+01 \\ \text { ND144 } & 0.0 \mathrm{E}+00 \\ \text { EU152 } & 8.0 \mathrm{E}+02 \\ \text { EU154 } & 8.4 \mathrm{E}+02 \\ \text { T8160 } & 7.5 \mathrm{E}+02 \\ \text { OS185 } & 5.8 \mathrm{E}+02 \\ \text { OS191 } & 3.8 \mathrm{E}+01 \\ \text { IR192 } & 6.9 \mathrm{E}+02 \\ \text { HG203 } & 1.5 \mathrm{E}+02 \\ \text { TH230 } & 1.1 \mathrm{E}-01 \\ \text { RA226 } & 3.3 \mathrm{E}+00 \\ \text { RN222 } & 9.4 \mathrm{E}+02 \\ \text { PB210 } & 9.8 \mathrm{E}-01 \\ \text { BI210 } & 1.1 \mathrm{E}+00 \\ \text { TH228 } & 9.9 \mathrm{E}-01 \\ \text { RA224 } & 8.3 \mathrm{E}+00 \\ \text { PB212 } & 9.3 \mathrm{E}+01 \\ \text { BI212 } & 2.1 \mathrm{E}+03 \\ \text { TH232 } & 6.1 \mathrm{E}+00 \\ \text { RA228 } & 1.8 \mathrm{E}-04 \\ \text { AC228 } & 5.5 \mathrm{E}+02 \\ \text { U 234 } & 3.7 \mathrm{E}-01 \\ \text { U 235 } & 7.6 \mathrm{E}+01 \\ \text { TH231 } & 8.0 \mathrm{E}+00 \\ \text { PA231 } & 2.6 \mathrm{E}+01 \\ \text { AC227 } & 5.5 \mathrm{E}-02 \\ \text { TH227 } & 7.9 \mathrm{E}+01 \\ \text { FR223 } & 3.1 \mathrm{E}+01 \\ \text { RA223 } & 8.6 \mathrm{E}+01 \\ \text { NP237 } & 1.1 \mathrm{E}+01 \\ \text { PA233 } & 1.7 \mathrm{E}+02 \\ \text { U 233 } & 4.9 \mathrm{E}-02 \\ \text { TH229 } & 8.1 \mathrm{E}+00 \\ \text { RA225 } & 8.1 \mathrm{E}+00 \\ \text { AC225 } & 6.8 \mathrm{E}+00 \\ \text { U 238 } & 8.1 \mathrm{E}-02 \\ \text { TH234 } & 3.6 \mathrm{E}+00 \\ \text { PA234M } & 5.8 \mathrm{E}+00 \\ \text { PA234 } & 1.3 \mathrm{E}+03 \\ \text { PU242 } & 9.8 \mathrm{E}-03 \\ \text { NP238 } & 3.7 \mathrm{E}+02 \\ \text { PU238 } & 1.8 \mathrm{E}-02 \\ \text { CM244 } & 2.2 \mathrm{E}-02 \\ \text { PU244 } & 2.1 \mathrm{E}-05 \\ \text { U 240 } & 5.0 \mathrm{E}-01 \\ \text { PU240 } & 1.6 \mathrm{E}-02 \\ & \\ & \end{array}$




\section{Appendix B - External Exposure Dose Rate Factors - PLANEC}

$\begin{array}{ll}\text { CM243 } & 9.0 \mathrm{E}+01 \\ \text { PU243 } & 1.1 \mathrm{E}+01 \\ \text { AM243 } & 2.1 \mathrm{E}+01 \\ \text { NP239 } & 1.0 \mathrm{E}+02 \\ \text { PU239 } & 1.6 \mathrm{E}-02 \\ \text { PU241 } & 9.1 \mathrm{E}-11 \\ \text { AM241 } & 9.5 \mathrm{E}+00\end{array}$




\section{Appendix B - External Exposure Dose Rate Factors - VOLSA}

OVERBURDEN: $0.0 \mathrm{M}$, SOURCE: $1.0 \mathrm{M}$ (DEN: CONCRETE/1.8): MR/HR 8-JUl-86 RAP

$\begin{array}{ll}\text { H } 3 & 0.0 \mathrm{E}+00 \\ \text { C 14 } & 6.2 \mathrm{E}-04 \\ \text { NA22 } & 5.1 \mathrm{E}+02 \\ \text { P 32 } & 9.8 \mathrm{E}-01 \\ \text { P 33 } & 2.8 \mathrm{E}-03 \\ \text { S 35 } & 0.0 \mathrm{E}+00 \\ \text { CL36 } & 7.7 \mathrm{E}-02 \\ \text { X 40 } & 7.0 \mathrm{E}+01 \\ \text { CA45 } & 2.8 \mathrm{E}-03 \\ \text { SC46 } & 8.4 \mathrm{E}+02 \\ \text { CR51 } & 1.2 \mathrm{E}+01 \\ \text { MN54 } & 3.3 \mathrm{E}+02 \\ \text { FE55 } & 0.0 \mathrm{E}+00 \\ \text { FE59 } & 4.7 \mathrm{E}+02 \\ \text { C057 } & 2.1 \mathrm{E}+01 \\ \text { C060 } & 1.0 \mathrm{E}+03 \\ \text { NI59 } & 0.0 \mathrm{E}+00 \\ \text { NI63 } & 1.4 \mathrm{E}-05 \\ \text { ZN65 } & 2.7 \mathrm{E}+02 \\ \text { SE75 } & 9.6 \mathrm{E}+01 \\ \text { SR85 } & 2.0 \mathrm{E}+02 \\ \text { SR89 } & 6.1 \mathrm{E}-01 \\ \text { Y 89M } & 4.4 \mathrm{E}+00 \\ \text { SR90 } & 3.0 \mathrm{E}-02 \\ \text { Y 90 } & 2.1 \mathrm{E}+00 \\ \text { M093 } & 1.4 \mathrm{E}+00 \\ \text { NB94 } & 6.0 \mathrm{E}+02 \\ \text { M099 } & 5.4 \mathrm{E}+01 \\ \text { TC99M } & 1.9 \mathrm{E}+01 \\ \text { TC99 } & 4.0 \mathrm{E}-03 \\ \text { RU103 } & 2.0 \mathrm{E}+02 \\ \text { RH103M } & 5.1 \mathrm{E}-02 \\ \text { PD103 } & 2.8 \mathrm{E}-01 \\ \text { RU106 } & 4.0 \mathrm{E}-07 \\ \text { RH106 } & 4.6 \mathrm{E}+01 \\ \text { CD109 } & 7.6 \mathrm{E}+00 \\ \text { AG110M } & 1.2 \mathrm{E}+03 \\ \text { AG110 } & 1.6 \mathrm{E}+01 \\ \text { IN111 } & 7.8 \mathrm{E}+01 \\ \text { SB124 } & 8.6 \mathrm{E}+02 \\ \text { SB125 } & 1.9 \mathrm{E}+02 \\ \text { TE125M } & 5.5 \mathrm{E}-01 \\ \text { I 125 } & 8.8 \mathrm{E}-01 \\ \text { I 129 } & 4.3 \mathrm{E}-01 \\ \text { I 131 } & 1.3 \mathrm{E}+02 \\ \text { XE131M } & 1.2 \mathrm{E}+00 \\ \text { CS134 } & 6.7 \mathrm{E}+02 \\ \text { CS135 } & 1.3 \mathrm{E}-03 \\ & \\ & \end{array}$




\section{Appendix B - External Exposure Dose Rate Factors - VOLSA}

$\begin{array}{ll}\text { CS137 } & 4.2 \mathrm{E}-02 \\ \text { BA137M } & 2.4 \mathrm{E}+02 \\ \text { CE141 } & 1.0 \mathrm{E}+01 \\ \text { CE144 } & 2.6 \mathrm{E}+00 \\ \text { PR144 } & 1.7 \mathrm{E}+01 \\ \text { ND144 } & 0.0 \mathrm{E}+00 \\ \text { EU152 } & 4.7 \mathrm{E}+02 \\ \text { EU154 } & 4.9 \mathrm{E}+02 \\ \text { TB160 } & 4.4 \mathrm{E}+02 \\ \text { OS185 } & 2.7 \mathrm{E}+02 \\ \text { OS191 } & 8.0 \mathrm{E}+00 \\ \text { IR192 } & 2.7 \mathrm{E}+02 \\ \text { HG203 } & 4.9 \mathrm{E}+01 \\ \text { TH230 } & 1.7 \mathrm{E}-02 \\ \text { RA226 } & 8.1 \mathrm{E}-01 \\ \text { RN222 } & 5.5 \mathrm{E}+02 \\ \text { PB210 } & 8.1 \mathrm{E}-02 \\ \text { BI210 } & 2.7 \mathrm{E}-01 \\ \text { TH228 } & 2.6 \mathrm{E}-01 \\ \text { RA224 } & 2.6 \mathrm{E}+00 \\ \text { PB212 } & 3.0 \mathrm{E}+01 \\ \text { BI212 } & 1.6 \mathrm{E}+03 \\ \text { TH232 } & 7.4 \mathrm{E}-01 \\ \text { RA228 } & 6.5 \mathrm{E}-06 \\ \text { AC228 } & 3.4 \mathrm{E}+02 \\ \text { U 234 } & 6.9 \mathrm{E}-02 \\ \text { U 235 } & 2.0 \mathrm{E}+01 \\ \text { TH231 } & 1.3 \mathrm{E}+00 \\ \text { PA231 } & 9.3 \mathrm{E}+00 \\ \text { AC227 } & 1.3 \mathrm{E}-02 \\ \text { TH227 } & 2.6 \mathrm{E}+01 \\ \text { FR223 } & 8.0 \mathrm{E}+00 \\ \text { RA223 } & 2.7 \mathrm{E}+01 \\ \text { NP237 } & 2.0 \mathrm{E}+00 \\ \text { PA233 } & 6.5 \mathrm{E}+01 \\ \text { U 233 } & 4.2 \mathrm{E}-03 \\ \text { TH229 } & 1.9 \mathrm{E}+00 \\ \text { RA225 } & 6.8 \mathrm{E}-01 \\ \text { AC225 } & 1.7 \mathrm{E}+00 \\ \text { U 238 } & 9.8 \mathrm{E}-03 \\ \text { TH234 } & 7.6 \mathrm{E}-01 \\ \text { PA234M } & 3.4 \mathrm{E}+00 \\ \text { PA234 } & 7.2 \mathrm{E}+02 \\ \text { PU242 } & 8.1 \mathrm{E}-04 \\ \text { NP238 } & 2.4 \mathrm{E}+02 \\ \text { PU238 } & 2.9 \mathrm{E}-03 \\ \text { CM244 } & 1.8 \mathrm{E}-03 \\ \text { PU244 } & 6.8 \mathrm{E}-07 \\ \text { U 240 } & 4.7 \mathrm{E}-02 \\ \text { PU240 } & 2.7 \mathrm{E}-03 \\ & \\ & \end{array}$


Appendix B - External Exposure Dose Rate Factors - VOLSA

CM243 2.6E+01

PU243 2.8E+00

AM $243 \quad 3.6 \mathrm{E}+00$

NP239 3.0E+01

PU239 2.9E-03

PU24 $12.9 \mathrm{E}-12$

AM241 $1.1 \mathrm{E}+00$ 


\section{Appendix B - External Exposure Dose Rate Factors - VOLSB}

OVERBURDEN: 0.0M, SOURCE: 1. OM (DEN: WATER/1.0): MR/HR 8-JU7-86 RAP

\begin{tabular}{ll} 
H 3 & $0.0 \mathrm{E}+00$ \\
C 14 & $5.4 \mathrm{E}-03$ \\
NA22 & $9.6 \mathrm{E}+02$ \\
P 32 & $2.8 \mathrm{E}+00$ \\
P 33 & $5.9 \mathrm{E}-02$ \\
S 35 & $0.0 \mathrm{E}+00$ \\
CL36 & $1.9 \mathrm{E}-02$ \\
K 40 & $1.5 \mathrm{E}+02$ \\
CA45 & $1.9 \mathrm{E}-02$ \\
SC46 & $1.6 \mathrm{E}+03$ \\
CR51 & $2.8 \mathrm{E}+01$ \\
MN54 & $6.8 \mathrm{E}+02$ \\
FE55 & $0.0 \mathrm{E}+00$ \\
FE59 & $8.7 \mathrm{E}+02$ \\
CO57 & $7.0 \mathrm{E}+01$ \\
CO60 & $1.9 \mathrm{E}+03$ \\
NI59 & $0.0 \mathrm{E}+00$ \\
NI63 & $2.9 \mathrm{E}-13$ \\
ZN65 & $5.1 \mathrm{E}+02$ \\
SE75 & $2.7 \mathrm{E}+02$ \\
SR85 & $4.8 \mathrm{E}+02$ \\
SR89 & $1.9 \mathrm{E}+00$ \\
Y 89M & $7.8 \mathrm{E}+00$ \\
SR90 & $1.4 \mathrm{E}-01$ \\
Y 90 & $5.6 \mathrm{E}+00$ \\
M093 & $1.3 \mathrm{E}+01$ \\
NB94 & $1.3 \mathrm{E}+03$ \\
M099 & $1.3 \mathrm{E}+02$ \\
TC99M & $6.3 \mathrm{E}+01$ \\
TC99 & $2.5 \mathrm{E}-02$ \\
RU103 & $4.8 \mathrm{E}+02$ \\
RH103M & $8.8 \mathrm{E}-01$ \\
PD103 & $4.8 \mathrm{E}+00$ \\
RU106 & $7.5 \mathrm{E}-06$ \\
RH106 & $1.1 \mathrm{E}+02$ \\
CD109 & $2.8 \mathrm{E}+01$ \\
AG110M & $2.6 \mathrm{E}+03$ \\
AG110 & $3.9 \mathrm{E}+01$ \\
IN111 & $2.3 \mathrm{E}+02$ \\
SB124 & $1.8 \mathrm{E}+03$ \\
SB125 & $4.5 \mathrm{E}+02$ \\
TE125M & $8.5 \mathrm{E}+00$ \\
I 125 & $1.2 \mathrm{E}+01$ \\
I 129 & $6.2 \mathrm{E}+00$ \\
I 131 & $3.2 \mathrm{E}+02$ \\
XE131M & $8.7 \mathrm{E}+00$ \\
CS134 & $1.5 \mathrm{E}+03$ \\
CS135 & $1.0 \mathrm{E}-02$ \\
& \\
\hline
\end{tabular}




\section{Appendix B - External Exposure Dose Rate Factors - VOLSB}

$\begin{array}{ll}\text { CS137 } & 1.7 \mathrm{E}-01 \\ \text { BA137M } & 5.7 \mathrm{E}+02 \\ \text { CE141 } & 3.6 \mathrm{E}+01 \\ \text { CE144 } & 1.0 \mathrm{E}+01 \\ \text { PR144 } & 3.7 \mathrm{E}+01 \\ \text { ND144 } & 0.0 \mathrm{E}+00 \\ \text { EU152 } & 9.6 \mathrm{E}+02 \\ \text { EU154 } & 9.8 \mathrm{E}+02 \\ \text { TB160 } & 8.6 \mathrm{E}+02 \\ \text { OS185 } & 6.5 \mathrm{E}+02 \\ \text { OS191 } & 3.0 \mathrm{E}+01 \\ \text { IR192 } & 6.7 \mathrm{E}+02 \\ \text { HG203 } & 1.4 \mathrm{E}+02 \\ \text { TH230 } & 8.3 \mathrm{E}-02 \\ \text { RA226 } & 2.7 \mathrm{E}+00 \\ \text { RN222 } & 1.1 \mathrm{E}+03 \\ \text { PB210 } & 6.8 \mathrm{E}-01 \\ \text { BI210 } & 9.0 \mathrm{E}-01 \\ \text { TH228 } & 8.3 \mathrm{E}-01 \\ \text { RA224 } & 7.4 \mathrm{E}+00 \\ \text { PB212 } & 8.3 \mathrm{E}+01 \\ \text { BI212 } & 3.0 \mathrm{E}+03 \\ \text { TH232 } & 4.4 \mathrm{E}+00 \\ \text { RA228 } & 1.0 \mathrm{E}-04 \\ \text { AC228 } & 6.5 \mathrm{E}+02 \\ \text { U 234 } & 2.9 \mathrm{E}-01 \\ \text { U 235 } & 6.4 \mathrm{E}+01 \\ \text { TH231 } & 5.8 \mathrm{E}+00 \\ \text { PA231 } & 2.4 \mathrm{E}+01 \\ \text { AC227 } & 4.5 \mathrm{E}-02 \\ \text { TH227 } & 7.1 \mathrm{E}+01 \\ \text { FR223 } & 2.6 \mathrm{E}+01 \\ \text { RA223 } & 7.6 \mathrm{E}+01 \\ \text { NP237 } & 8.5 \mathrm{E}+00 \\ \text { PA233 } & 1.6 \mathrm{E}+02 \\ \text { U 233 } & 3.4 \mathrm{E}-02 \\ \text { TH229 } & 6.5 \mathrm{E}+00 \\ \text { RA225 } & 5.6 \mathrm{E}+00 \\ \text { AC225 } & 5.6 \mathrm{E}+00 \\ \text { U 238 } & 5.9 \mathrm{E}-02 \\ \text { TH234 } & 2.8 \mathrm{E}+00 \\ \text { PA234M } & 6.5 \mathrm{E}+00 \\ \text { PA234 } & 1.5 \mathrm{E}+03 \\ \text { PU242 } & 6.8 \mathrm{E}-03 \\ \text { NP238 } & 4.3 \mathrm{E}+02 \\ \text { PU238 } & 1.4 \mathrm{E}-02 \\ \text { CM244 } & 1.5 \mathrm{E}-02 \\ \text { PU244 } & 1.2 \mathrm{E}-05 \\ \text { U 240 } & 2.8 \mathrm{E}-01 \\ \text { PU240 } & 1.2 \mathrm{E}-02 \\ & \\ & \end{array}$


Appendix B - External Exposure Dose Rate Factors - VOLSB

$\begin{array}{ll}\text { CM243 } & 7.7 \mathrm{E}+01 \\ \text { PU243 } & 9.1 \mathrm{E}+00 \\ \text { AM243 } & 1.5 \mathrm{E}+01 \\ \text { NP239 } & 8.8 \mathrm{E}+01 \\ \text { PU239 } & 1.2 \mathrm{E}-02 \\ \text { PU241 } & 5.0 \mathrm{E}-11 \\ \text { AM241 } & 6.8 \mathrm{E}+00\end{array}$


Appendix B - External Exposure Dose Rate Factors - VOLSC

NO OVERBURDEN, $1.0 \mathrm{M}$ SOURCE ON SURFACE (DEN: $.5, .1$ ) MR/HR 8-Ju1-86 RAP

$\begin{array}{ll}\text { H } 3 & 0.0 \mathrm{E}+00 \\ \text { C 14 } & 9.3 \mathrm{E}-03 \\ \text { NA22 } & 1.6 \mathrm{E}+03 \\ \text { P 32 } & 4.7 \mathrm{E}+00 \\ \text { P 33 } & 3.2 \mathrm{E}-02 \\ \text { S 35 } & 0.0 \mathrm{E}+00 \\ \text { CL36 } & 5.2 \mathrm{E}-01 \\ \text { K 40 } & 2.4 \mathrm{E}+02 \\ \text { CA45 } & 3.2 \mathrm{E}-02 \\ \text { SC46 } & 2.7 \mathrm{E}+03 \\ \text { CR51 } & 4.7 \mathrm{E}+01 \\ \text { MN54 } & 1.1 \mathrm{E}+03 \\ \text { FE55 } & 0.0 \mathrm{E}+00 \\ \text { FE59 } & 1.4 \mathrm{E}+03 \\ \text { C057 } & 1.2 \mathrm{E}+02 \\ \text { C060 } & 3.2 \mathrm{E}+03 \\ \text { NI59 } & 0.0 \mathrm{E}+00 \\ \text { NI63 } & 3.5 \mathrm{E}-04 \\ \text { ZN65 } & 8.4 \mathrm{E}+02 \\ \text { SE75 } & 4.5 \mathrm{E}+02 \\ \text { SR85 } & 8.1 \mathrm{E}+02 \\ \text { SR89 } & 3.1 \mathrm{E}+00 \\ \text { Y 89M } & 1.3 \mathrm{E}+01 \\ \text { SR90 } & 2.4 \mathrm{E}-01 \\ \text { Y 90 } & 9.4 \mathrm{E}+00 \\ \text { M093 } & 2.3 \mathrm{E}+01 \\ \text { NB94 } & 2.2 \mathrm{E}+03 \\ \text { M099 } & 2.1 \mathrm{E}+02 \\ \text { TC99M } & 1.1 \mathrm{E}+02 \\ \text { TC99 } & 4.2 \mathrm{E}-02 \\ \text { RU103 } & 8.0 \mathrm{E}+02 \\ \text { RH103M } & 1.5 \mathrm{E}+00 \\ \text { PD103 } & 8.4 \mathrm{E}+00 \\ \text { R106 } & 1.3 \mathrm{E}-05 \\ \text { RH106 } & 1.8 \mathrm{E}+02 \\ \text { CD109 } & 4.7 \mathrm{E}+01 \\ \text { AG110M } & 4.2 \mathrm{E}+03 \\ \text { AG110 } & 6.3 \mathrm{E}+01 \\ \text { IN111 } & 3.9 \mathrm{E}+02 \\ \text { SB124 } & 3.0 \mathrm{E}+03 \\ \text { SB125 } & 7.4 \mathrm{E}+02 \\ \text { TE125M } & 1.5 \mathrm{E}+01 \\ \text { I 125 } & 2.1 \mathrm{E}+01 \\ \text { I 129 } & 1.1 \mathrm{E}+01 \\ \text { I 131 } & 5.4 \mathrm{E}+02 \\ \text { XE131M } & 1.5 \mathrm{E}+01 \\ \text { CS134 } & 2.5 \mathrm{E}+03 \\ \text { CS135 } & 1.7 \mathrm{E}-02 \\ & \\ & \end{array}$




$\begin{array}{ll}\text { CS137 } & 2.9 \mathrm{E}-01 \\ \text { BA137M } & 9.3 \mathrm{E}+02 \\ \text { CE141 } & 6.1 \mathrm{E}+01 \\ \text { CE144 } & 1.7 \mathrm{E}+01 \\ \text { PR144 } & 6.0 \mathrm{E}+01 \\ \text { ND144 } & 0.0 \mathrm{E}+00 \\ \text { EU152 } & 1.6 \mathrm{E}+03 \\ \text { EU154 } & 1.6 \mathrm{E}+03 \\ \text { TB160 } & 1.4 \mathrm{E}+03 \\ \text { OS185 } & 1.1 \mathrm{E}+03 \\ \text { OS191 } & 5.0 \mathrm{E}+01 \\ \text { IR192 } & 1.1 \mathrm{E}+03 \\ \text { HG203 } & 2.3 \mathrm{E}+02 \\ \text { TH230 } & 1.4 \mathrm{E}-01 \\ \text { RA226 } & 4.5 \mathrm{E}+00 \\ \text { RN222 } & 1.9 \mathrm{E}+03 \\ \text { PB210 } & 1.2 \mathrm{E}+00 \\ \text { BI210 } & 1.5 \mathrm{E}+00 \\ \text { TH228 } & 1.4 \mathrm{E}+00 \\ \text { RA224 } & 1.2 \mathrm{E}+01 \\ \text { PB212 } & 1.4 \mathrm{E}+02 \\ \text { BI212 } & 4.9 \mathrm{E}+03 \\ \text { TH232 } & 7.5 \mathrm{E}+00 \\ \text { RA228 } & 1.8 \mathrm{E}-04 \\ \text { AC228 } & 1.1 \mathrm{E}+03 \\ \text { U 234 } & 4.8 \mathrm{E}-01 \\ \text { U 235 } & 1.1 \mathrm{E}+02 \\ \text { TH231 } & 9.8 \mathrm{E}+00 \\ \text { PA231 } & 4.0 \mathrm{E}+01 \\ \text { AC227 } & 7.5 \mathrm{E}-02 \\ \text { TH227 } & 1.2 \mathrm{E}+02 \\ \text { FR223 } & 4.4 \mathrm{E}+01 \\ \text { RA223 } & 1.3 \mathrm{E}+02 \\ \text { NP237 } & 1.4 \mathrm{E}+01 \\ \text { PA233 } & 2.7 \mathrm{E}+02 \\ \text { U 233 } & 5.8 \mathrm{E}-02 \\ \text { TH229 } & 1.1 \mathrm{E}+01 \\ \text { RA225 } & 9.6 \mathrm{E}+00 \\ \text { AC225 } & 9.4 \mathrm{E}+00 \\ \text { U 238 } & 1.0 \mathrm{E}-01 \\ \text { TH234 } & 4.7 \mathrm{E}+00 \\ \text { PA234M } & 1.1 \mathrm{E}+01 \\ \text { PA234 } & 2.5 \mathrm{E}+03 \\ \text { PU242 } & 1.2 \mathrm{E}-02 \\ \text { NP238 } & 7.2 \mathrm{E}+02 \\ \text { PU238 } & 2.3 \mathrm{E}-02 \\ \text { CM244 } & 2.6 \mathrm{E}-02 \\ \text { PU244 } & 2.1 \mathrm{E}-05 \\ \text { U 240 } & 6.0 \mathrm{E}-01 \\ \text { PU240 } & 2.0 \mathrm{E}-02 \\ & \end{array}$


Appendix B - External Exposure Dose Rate Factors - VOLSC

$\begin{array}{ll}\text { CM243 } & 1.3 \mathrm{E}+02 \\ \text { PU243 } & 1.6 \mathrm{E}+01 \\ \text { AM243 } & 2.6 \mathrm{E}+01 \\ \text { NP239 } & 1.5 \mathrm{E}+02 \\ \text { PU239 } & 2.1 \mathrm{E}-02 \\ \text { PU241 } & 9.1 \mathrm{E}-11 \\ \text { AM241 } & 1.2 \mathrm{E}+01\end{array}$




\section{Appendix B - External Exposure Dose Rate Factors - BURHFA}

OVERBURDEN: $0.5 \mathrm{M}$, SOURCE: $1.0 \mathrm{M}$ (DEN: CONCRETE/1.8) MR/HR 8-JU1-86 RAP

$\begin{array}{ll}\text { H } 3 & 0.0 \mathrm{E}+00 \\ \text { C 14 } & 1.2 \mathrm{E}-12 \\ \text { NA22 } & 1.8 \mathrm{E}+00 \\ \text { P } 32 & 4.2 \mathrm{E}-04 \\ \text { P } 33 & 2.5 \mathrm{E}-06 \\ \text { S } 35 & 0.0 \mathrm{E}+00 \\ \text { CL36 } & 1.9 \mathrm{E}-10 \\ \text { K 40 } & 3.9 \mathrm{E}-01 \\ \text { CA45 } & 1.9 \mathrm{E}-10 \\ \text { SC46 } & 2.3 \mathrm{E}+00 \\ \text { CR51 } & 2.8 \mathrm{E}-03 \\ \text { MN54 } & 4.4 \mathrm{E}-01 \\ \text { FE55 } & 0.0 \mathrm{E}+00 \\ \text { FE59 } & 1.6 \mathrm{E}+00 \\ \text { C057 } & 8.4 \mathrm{E}-06 \\ \text { C060 } & 3.7 \mathrm{E}+00 \\ \text { NI59 } & 0.0 \mathrm{E}+00 \\ \text { NI63 } & 0.0 \mathrm{E}+00 \\ \text { ZN65 } & 9.3 \mathrm{E}-01 \\ \text { SE75 } & 1.5 \mathrm{E}-02 \\ \text { SR85 } & 1.1 \mathrm{E}-01 \\ \text { SR89 } & 1.8 \mathrm{E}-04 \\ \text { Y 89M } & 1.4 \mathrm{E}-02 \\ \text { SR90 } & 5.5 \mathrm{E}-07 \\ \text { Y 90 } & 1.7 \mathrm{E}-03 \\ \text { M093 } & 3.5 \mathrm{E}-34 \\ \text { NB94 } & 6.1 \mathrm{E}-01 \\ \text { M099 } & 4.4 \mathrm{E}-02 \\ \text { TC99M } & 7.5 \mathrm{E}-06 \\ \text { TC99 } & 7.8 \mathrm{E}-10 \\ \text { RU103 } & 1.1 \mathrm{E}-01 \\ \text { RH103M } & 2.7 \mathrm{E}-26 \\ \text { PD103 } & 9.3 \mathrm{E}-06 \\ \text { RU106 } & 0.0 \mathrm{E}+00 \\ \text { RH106 } & 6.2 \mathrm{E}-02 \\ \text { CD109 } & 5.5 \mathrm{E}-08 \\ \text { AG110M } & 2.6 \mathrm{E}+00 \\ \text { AG110 } & 1.3 \mathrm{E}-02 \\ \text { IN111 } & 1.3 \mathrm{E}-03 \\ \text { SB124 } & 4.2 \mathrm{E}+00 \\ \text { SB125 } & 1.1 \mathrm{E}-01 \\ \text { TE125M } & 2.5 \mathrm{E}-08 \\ \text { I 125 } & 1.2 \mathrm{E}-34 \\ \text { I 129 } & 6.3 \mathrm{E}-13 \\ \text { I 131 } & 4.0 \mathrm{E}-02 \\ \text { XE13IM } & 1.7 \mathrm{E}-07 \\ \text { CS134 } & 8.0 \mathrm{E}-01 \\ \text { CS135 } & 3.4 \mathrm{E}-11 \\ & \\ & \end{array}$


Appendix B - External Exposure Dose Rate Factors - BURHFA

$\begin{array}{ll}\text { CS137 } & 4.4 \mathrm{E}-06 \\ \text { BA137M } & 1.5 \mathrm{E}-01 \\ \text { CE141 } & 4.3 \mathrm{E}-06 \\ \text { CE144 } & 9.1 \mathrm{E}-07 \\ \text { PR144 } & 1.2 \mathrm{E}-01 \\ \text { ND144 } & 0.0 \mathrm{E}+00 \\ \text { EU152 } & 1.6 \mathrm{E}+00 \\ \text { EU154 } & 1.4 \mathrm{E}+00 \\ \text { TB160 } & 1.1 \mathrm{E}+00 \\ \text { OS185 } & 2.0 \mathrm{E}-01 \\ \text { OS191 } & 2.2 \mathrm{E}-06 \\ \text { IR192 } & 9.0 \mathrm{E}-02 \\ \text { HG203 } & 1.1 \mathrm{E}-03 \\ \text { TH230 } & 3.1 \mathrm{E}-14 \\ \text { RA226 } & 4.4 \mathrm{E}-07 \\ \text { RN222 } & 2.4 \mathrm{E}+00 \\ \text { PB210 } & 1.9 \mathrm{E}-24 \\ \text { BI210 } & 5.0 \mathrm{E}-05 \\ \text { TH228 } & 2.9 \mathrm{E}-06 \\ \text { RA224 } & 1.7 \mathrm{E}-04 \\ \text { PB212 } & 7.7 \mathrm{E}-04 \\ \text { BI212 } & 2.4 \mathrm{E}+01 \\ \text { TH232 } & 3.9 \mathrm{E}-16 \\ \text { RA228 } & 3.4 \mathrm{E}-31 \\ \text { AC228 } & 1.1 \mathrm{E}+00 \\ \text { U 234 } & 1.9 \mathrm{E}-08 \\ \text { U 235 } & 8.7 \mathrm{E}-05 \\ \text { TH231 } & 1.0 \mathrm{E}-07 \\ \text { PA231 } & 1.9 \mathrm{E}-03 \\ \text { AC227 } & 4.3 \mathrm{E}-09 \\ \text { TH227 } & 2.4 \mathrm{E}-03 \\ \text { FR223 } & 2.2 \mathrm{E}-03 \\ \text { RA223 } & 3.9 \mathrm{E}-03 \\ \text { NP237 } & 2.7 \mathrm{E}-08 \\ \text { PA233 } & 1.6 \mathrm{E}-02 \\ \text { U 233 } & 6.5 \mathrm{E}-19 \\ \text { TH229 } & 7.1 \mathrm{E}-07 \\ \text { RA225 } & 4.3 \mathrm{E}-09 \\ \text { AC225 } & 1.4 \mathrm{E}-05 \\ \text { U 238 } & 5.2 \mathrm{E}-18 \\ \text { TH234 } & 4.6 \mathrm{E}-08 \\ \text { PA234M } & 9.1 \mathrm{E}-03 \\ \text { PA234 } & 1.6 \mathrm{E}+00 \\ \text { PU242 } & 2.7 \mathrm{E}-27 \\ \text { NP238 } & 7.4 \mathrm{E}-01 \\ \text { PU238 } & \text { B.4E-10 } \\ \text { CM244 } & 6.2 \mathrm{E}-27 \\ \text { PU244 } & 0.0 \mathrm{E}+00 \\ \text { U 240 } & 1.1 \mathrm{E}-13 \\ \text { PU240 } & 8.4 \mathrm{E}-10 \\ & \\ \end{array}$


Appendix B - External Exposure Dose Rate Factors - BURHFA

$\begin{array}{ll}\text { CM243 } & 3.5 \mathrm{E}-04 \\ \text { PU243 } & 2.1 \mathrm{E}-04 \\ \text { AM243 } & 8.5 \mathrm{E}-10 \\ \text { NP239 } & 7.9 \mathrm{E}-04 \\ \text { PU239 } & 8.4 \mathrm{E}-10 \\ \text { PU241 } & 0.0 \mathrm{E}+00 \\ \text { AM241 } & 2.6 \mathrm{E}-09\end{array}$

B. 58 
Appendix B - External Exposure Dose Rate Factors - BURHFB OVERBURDEN: 0.5M, SOURCE: $1.0 \mathrm{M}$ (DEN: water/1.0) MR/HR 8-Jul-86 RAP

$\begin{array}{ll}\text { H } 3 & 0.0 \mathrm{E}+00 \\ \text { C 14 } & 4.0 \mathrm{E}-12 \\ \text { NA22 } & 3.5 \mathrm{E}+00 \\ \text { P 32 } & 9.5 \mathrm{E}-04 \\ \text { P 33 } & 6.7 \mathrm{E}-06 \\ \text { S 35 } & 0.0 \mathrm{E}+00 \\ \text { CL36 } & 6.2 \mathrm{E}-10 \\ \text { K 40 } & 8.2 \mathrm{E}-01 \\ \text { CA45 } & 6.3 \mathrm{E}-10 \\ \text { SC46 } & 4.5 \mathrm{E}+00 \\ \text { CR51 } & 7.0 \mathrm{E}-03 \\ \text { MN54 } & 9.8 \mathrm{E}-01 \\ \text { FE55 } & 0.0 \mathrm{E}+00 \\ \text { FE59 } & 3.0 \mathrm{E}+00 \\ \text { C057 } & 2.8 \mathrm{E}-05 \\ \text { C060 } & 7.0 \mathrm{E}+00 \\ \text { NI59 } & 0.0 \mathrm{E}+00 \\ \text { NI63 } & 0.0 \mathrm{E}+00 \\ \text { ZN65 } & 1.8 \mathrm{E}+00 \\ \text { SE75 } & 3.9 \mathrm{E}-02 \\ \text { SR85 } & 2.7 \mathrm{E}-01 \\ \text { SR89 } & 4.3 \mathrm{E}-04 \\ \text { Y 89M } & 2.5 \mathrm{E}-02 \\ \text { SR90 } & 1.4 \mathrm{E}-06 \\ \text { Y 90 } & 3.6 \mathrm{E}-03 \\ \text { M093 } & 2.2 \mathrm{E}-33 \\ \text { NB94 } & 1.4 \mathrm{E}+00 \\ \text { M099 } & 1.0 \mathrm{E}-01 \\ \text { TC99M } & 2.5 \mathrm{E}-05 \\ \text { TC99 } & 2.4 \mathrm{E}-09 \\ \text { RU103 } & 2.7 \mathrm{E}-01 \\ \text { RH103M } & 1.8 \mathrm{E}-25 \\ \text { PD103 } & 2.3 \mathrm{E}-05 \\ \text { RU106 } & 0.0 \mathrm{E}+00 \\ \text { RH106 } & 1.3 \mathrm{E}-01 \\ \text { CD109 } & 2.0 \mathrm{E}-07 \\ \text { AG110M } & 5.6 \mathrm{E}+00 \\ \text { AG110 } & 2.8 \mathrm{E}-02 \\ \text { IN111 } & 3.8 \mathrm{E}-03 \\ \text { SB124 } & 8.6 \mathrm{E}+00 \\ \text { SB125 } & 2.6 \mathrm{E}-01 \\ \text { TE125M } & 8.4 \mathrm{E}-08 \\ \text { I 125 } & 7.6 \mathrm{E}-34 \\ \text { I 129 } & 2.2 \mathrm{E}-12 \\ \text { I 131 } & 9.9 \mathrm{E}-02 \\ \text { XE131M } & 5.6 \mathrm{E}-07 \\ \text { CS134 } & 1.8 \mathrm{E}+00 \\ \text { CS135 } & 1.1 \mathrm{E}-10 \\ & \\ & \end{array}$


Appendix B - External Exposure Dose Rate Factors - BURHFB

$\begin{array}{ll}\text { CS137 } & 1.1 \mathrm{E}-05 \\ \text { BA137M } & 3.6 \mathrm{E}-01 \\ \text { CE141 } & 1.4 \mathrm{E}-05 \\ \text { CE144 } & 3.0 \mathrm{E}-06 \\ \text { PR144 } & 2.2 \mathrm{E}-01 \\ \text { ND144 } & 0.0 \mathrm{E}+00 \\ \text { EU152 } & 3.2 \mathrm{E}+00 \\ \text { EU154 } & 2.6 \mathrm{E}+00 \\ \text { TB160 } & 2.2 \mathrm{E}+00 \\ \text { OS185 } & 4.6 \mathrm{E}-01 \\ \text { OS191 } & 7.2 \mathrm{E}-06 \\ \text { IR192 } & 2.3 \mathrm{E}-01 \\ \text { HG203 } & 3.1 \mathrm{E}-03 \\ \text { TH230 } & 1.4 \mathrm{E}-13 \\ \text { RA226 } & 1.4 \mathrm{E}-06 \\ \text { RN222 } & 4.9 \mathrm{E}+00 \\ \text { PB210 } & 1.0 \mathrm{E}-23 \\ \text { 8I210 } & 1.2 \mathrm{E}-04 \\ \text { TH228 } & 8.2 \mathrm{E}-06 \\ \text { RA224 } & 4.3 \mathrm{E}-04 \\ \text { PB212 } & 2.2 \mathrm{E}-03 \\ \text { BI212 } & 4.6 \mathrm{E}+01 \\ \text { TH232 } & 2.1 \mathrm{E}-15 \\ \text { RA228 } & 2.2 \mathrm{E}-30 \\ \text { AC228 } & 2.1 \mathrm{E}+00 \\ \text { U 234 } & 6.4 \mathrm{E}-08 \\ \text { U 235 } & 2.5 \mathrm{E}-04 \\ \text { TH231 } & 3.5 \mathrm{E}-07 \\ \text { PA231 } & 4.8 \mathrm{E}-03 \\ \text { AC227 } & 1.4 \mathrm{E}-08 \\ \text { TH227 } & 6.1 \mathrm{E}-03 \\ \text { FR223 } & 5.1 \mathrm{E}-03 \\ \text { RA223 } & 1.0 \mathrm{E}-02 \\ \text { NP237 } & 9.3 \mathrm{E}-08 \\ \text { PA233 } & 4.0 \mathrm{E}-02 \\ \text { U 233 } & 3.5 \mathrm{E}-18 \\ \text { TH229 } & 2.4 \mathrm{E}-06 \\ \text { RA225 } & 1.3 \mathrm{E}-08 \\ \text { AC225 } & 4.1 \mathrm{E}-05 \\ \text { U 238 } & 2.8 \mathrm{E}-17 \\ \text { TH234 } & 1.5 \mathrm{E}-07 \\ \text { PA234M } & 1.7 \mathrm{E}-02 \\ \text { PA234 } & 3.3 \mathrm{E}+00 \\ \text { PU242 } & 1.8 \mathrm{E}-26 \\ \text { NP238 } & 1.4 \mathrm{E}+00 \\ \text { PU238 } & 2.8 \mathrm{E}-09 \\ \text { CM244 } & 4.1 \mathrm{E}-26 \\ \text { PU244 } & 0.0 \mathrm{E}+00 \\ \text { U 240 } & 3.6 \mathrm{E}-13 \\ \text { PU240 } & 2.8 \mathrm{E}-09 \\ & \\ & \end{array}$

B. 60 
Appendix B - External Exposure Dose Rate Factors - BURHFB

CM243 1.0E-03

PU243 5.3E-04

AM243 3.4E-09

NP239 2.1E-03

PU239 2.8E-09

PU241 $0.0 E+00$

AM241 8.7E-09 


\section{Appendix B - External Exposure Dose Rate Factors - BURHFC}

1.0 M Source buried at $0.5 \mathrm{M}$ (DEN: $0.5 / 0.1$ ) MR/HR 8-Jul-86

$\begin{array}{ll}\text { H } 3 & 0.0 \mathrm{E}+00 \\ \text { C 14 } & 6.7 \mathrm{E}-12 \\ \text { NA22 } & 5.8 \mathrm{E}+00 \\ \text { P 32 } & 1.6 \mathrm{E}-03 \\ \text { P 33 } & 1.0 \mathrm{E}-09 \\ \text { S 35 } & 0.0 \mathrm{E}+00 \\ \text { CL36 } & 1.9 \mathrm{E}-05 \\ \text { K 40 } & 1.3 \mathrm{E}+00 \\ \text { CA45 } & 1.0 \mathrm{E}-09 \\ \text { SC46 } & 7.4 \mathrm{E}+00 \\ \text { CR51 } & 1.2 \mathrm{E}-02 \\ \text { MN54 } & 1.6 \mathrm{E}+00 \\ \text { FE55 } & 0.0 \mathrm{E}+00 \\ \text { FE59 } & 4.9 \mathrm{E}+00 \\ \text { C057 } & 4.7 \mathrm{E}-05 \\ \text { C060 } & 1.2 \mathrm{E}+01 \\ \text { NI59 } & 0.0 \mathrm{E}+00 \\ \text { NI63 } & 1.2 \mathrm{E}-21 \\ \text { ZN65 } & 2.9 \mathrm{E}+00 \\ \text { SE75 } & 6.6 \mathrm{E}-02 \\ \text { SR85 } & 4.6 \mathrm{E}-01 \\ \text { SR89 } & 7.1 \mathrm{E}-04 \\ \text { Y 89M } & 4.2 \mathrm{E}-02 \\ \text { SR90 } & 2.4 \mathrm{E}-06 \\ \text { Y 90 } & 5.9 \mathrm{E}-03 \\ \text { M093 } & 3.9 \mathrm{E}-33 \\ \text { NB94 } & 2.3 \mathrm{E}+00 \\ \text { MO99 } & 1.6 \mathrm{E}-01 \\ \text { TC99M } & 4.2 \mathrm{E}-05 \\ \text { TC99 } & 4.1 \mathrm{E}-09 \\ \text { RU103 } & 4.6 \mathrm{E}-01 \\ \text { RH103M } & 3.1 \mathrm{E}-25 \\ \text { PD103 } & 3.9 \mathrm{E}-05 \\ \text { RU106 } & 0.0 \mathrm{E}+00 \\ \text { RH106 } & 2.1 \mathrm{E}-01 \\ \text { CD109 } & 3.3 \mathrm{E}-07 \\ \text { AG110M } & 9.1 \mathrm{E}+00 \\ \text { AG110 } & 4.6 \mathrm{E}-02 \\ \text { IN111 } & 6.4 \mathrm{E}-03 \\ \text { SB124 } & 1.4 \mathrm{E}+01 \\ \text { SB125 } & 4.3 \mathrm{E}-01 \\ \text { TE125M } & 1.4 \mathrm{E}-07 \\ \text { I 125 } & 1.3 \mathrm{E}-33 \\ \text { I 129 } & 3.6 \mathrm{E}-12 \\ \text { I 131 } & 1.6 \mathrm{E}-01 \\ \text { XE131M } & 9.4 \mathrm{E}-07 \\ \text { CS134 } & 2.9 \mathrm{E}+00 \\ \text { CS135 } & 1.9 \mathrm{E}-10 \\ & \\ & \end{array}$




\section{Appendix B - External Exposure Dose Rate Factors - BURHFC}

$\begin{array}{ll}\text { CS137 } & 1.8 \mathrm{E}-05 \\ \text { BA137M } & 5.8 \mathrm{E}-01 \\ \text { CE141 } & 2.4 \mathrm{E}-05 \\ \text { CE144 } & 5.1 \mathrm{E}-06 \\ \text { PR144 } & 3.6 \mathrm{E}-01 \\ \text { ND144 } & 0.0 \mathrm{E}+00 \\ \text { EU152 } & 5.2 \mathrm{E}+00 \\ \text { EU154 } & 4.3 \mathrm{E}+00 \\ \text { TB160 } & 3.7 \mathrm{E}+00 \\ \text { OS185 } & 7.5 \mathrm{E}-01 \\ \text { OS191 } & 1.2 \mathrm{E}-05 \\ \text { IR192 } & 3.8 \mathrm{E}-01 \\ \text { HG203 } & 5.2 \mathrm{E}-03 \\ \text { TH230 } & 2.4 \mathrm{E}-13 \\ \text { RA226 } & 2.3 \mathrm{E}-06 \\ \text { RN222 } & 2.1 \mathrm{E}-03 \\ \text { PB210 } & 1.8 \mathrm{E}-23 \\ \text { BI210 } & 2.0 \mathrm{E}-04 \\ \text { TH228 } & 1.4 \mathrm{E}-05 \\ \text { RA224 } & 1.2 \mathrm{E}+01 \\ \text { PB212 } & 3.6 \mathrm{E}-03 \\ \text { BI212 } & 6.5 \mathrm{E}-03 \\ \text { TH232 } & 3.6 \mathrm{E}-15 \\ \text { RA228 } & 3.8 \mathrm{E}-30 \\ \text { AC228 } & 3.4 \mathrm{E}+00 \\ \text { U 234 } & 1.1 \mathrm{E}-07 \\ \text { U 235 } & 4.2 \mathrm{E}-04 \\ \text { TH231 } & 5.8 \mathrm{E}-07 \\ \text { PA231 } & 8.0 \mathrm{E}-03 \\ \text { AC227 } & 2.4 \mathrm{E}-08 \\ \text { TH227 } & 1.0 \mathrm{E}-02 \\ \text { FR223 } & 8.4 \mathrm{E}-03 \\ \text { RA223 } & 1.7 \mathrm{E}-02 \\ \text { NP237 } & 1.6 \mathrm{E}-07 \\ \text { PA233 } & 6.7 \mathrm{E}-02 \\ \text { U 233 } & 5.9 \mathrm{E}-18 \\ \text { TH229 } & 4.0 \mathrm{E}-06 \\ \text { RA225 } & 2.1 \mathrm{E}-08 \\ \text { AC225 } & 6.9 \mathrm{E}-05 \\ \text { U 238 } & 4.7 \mathrm{E}-17 \\ \text { TH234 } & 2.6 \mathrm{E}-07 \\ \text { PA234M } & 2.8 \mathrm{E}-02 \\ \text { PA234 } & 5.4 \mathrm{E}+00 \\ \text { PU242 } & 3.1 \mathrm{E}-26 \\ \text { NP238 } & 2.3 \mathrm{E}+00 \\ \text { PU238 } & 4.7 \mathrm{E}-09 \\ \text { CM244 } & 7.0 \mathrm{E}-26 \\ \text { PU244 } & 0.0 \mathrm{E}+00 \\ \text { U 240 } & 3.0 \mathrm{E}-07 \\ \text { PU240 } & 4.7 \mathrm{E}-09 \\ & \end{array}$


Appendix B - External Exposure Dose Rate Factors - BURHFC

CM243 $\quad 1.7 \mathrm{E}-03$

PU243 8.9E-04

AM243 5.7E-09

NP239 3.5E-03

PU239 4.7E-09

PU241 $0.0 \mathrm{E}+00$

AM241 $1.5 \mathrm{E}-08$ 


\section{Appendix B - External Exposure Dose Rate Factors - BUR1A}

OVERBURDEN: $1.0 \mathrm{~m}$, SOURCE: $1.0 \mathrm{M}$ (DEN: CONCRETE/1.8): MR/HR 18-JU1-86 RAP

$\begin{array}{ll}\text { H 3 } & 0.0 \mathrm{E}+00 \\ \text { C 14 } & 1.3 \mathrm{E}-19 \\ \text { NA22 } & 9.6 \mathrm{E}-03 \\ \text { P 32 } & 9.9 \mathrm{E}-07 \\ \text { P 33 } & 1.4 \mathrm{E}-16 \\ \text { S 35 } & 0.0 \mathrm{E}+00 \\ \text { CL36 } & 2.5 \mathrm{E}-09 \\ \text { K 40 } & 3.2 \mathrm{E}-03 \\ \text { CA45 } & 1.5 \mathrm{E}-16 \\ \text { SC46 } & 1.0 \mathrm{E}-02 \\ \text { CR51 } & 1.1 \mathrm{E}-06 \\ \text { MN54 } & 9.1 \mathrm{E}-04 \\ \text { FE55 } & 0.0 \mathrm{E}+00 \\ \text { FE59 } & 7.7 \mathrm{E}-03 \\ \text { C057 } & 6.6 \mathrm{E}-12 \\ \text { C060 } & 1.9 \mathrm{E}-02 \\ \text { NI59 } & 0.0 \mathrm{E}+00 \\ \text { NI63 } & 4.5 \mathrm{E}-34 \\ \text { ZN65 } & 4.8 \mathrm{E}-03 \\ \text { SE75 } & 1.3 \mathrm{E}-05 \\ \text { SR85 } & 9.8 \mathrm{E}-05 \\ \text { SR89 } & 3.1 \mathrm{E}-07 \\ \text { Y 89M } & 6.2 \mathrm{E}-05 \\ \text { SR90 } & 2.0 \mathrm{E}-10 \\ \text { Y 90 } & 8.3 \mathrm{E}-06 \\ \text { M093 } & 0.0 \mathrm{E}+00 \\ \text { NB94 } & 1.1 \mathrm{E}-03 \\ \text { M099 } & 7.3 \mathrm{E}-05 \\ \text { TC99M } & 6.0 \mathrm{E}-12 \\ \text { TC99 } & 1.6 \mathrm{E}-14 \\ \text { RU103 } & 9.9 \mathrm{E}-05 \\ \text { RH103M } & 0.0 \mathrm{E}+00 \\ \text { P0103 } & 3.8 \mathrm{E}-09 \\ \text { RU106 } & 0.0 \mathrm{E}+00 \\ \text { RH106 } & 5.2 \mathrm{E}-04 \\ \text { CD109 } & 8.6 \mathrm{E}-16 \\ \text { AG110M } & 1.5 \mathrm{E}-02 \\ \text { AG110 } & 4.1 \mathrm{E}-05 \\ \text { IN111 } & 5.4 \mathrm{E}-08 \\ \text { SB124 } & 5.1 \mathrm{E}-02 \\ \text { SB125 } & 1.0 \mathrm{E}-04 \\ \text { TE125M } & 2.0 \mathrm{E}-14 \\ \text { I 125 } & 0.0 \mathrm{E}+00 \\ \text { I 129 } & 5.0 \mathrm{E}-20 \\ \text { I 131 } & 2.4 \mathrm{E}-05 \\ \text { XE131M } & 1.3 \mathrm{E}-13 \\ \text { CS134 } & 2.3 \mathrm{E}-03 \\ \text { CS135 } & 2.3 \mathrm{E}-17 \\ & \end{array}$




\section{Appendix B - External Exposure Dose Rate Factors - BUR1A}

$\begin{array}{ll}\text { CS137 } & 4.8 \mathrm{E}-09 \\ \text { BA137M } & 1.4 \mathrm{E}-04 \\ \text { CE141 } & 1.2 \mathrm{E}-10 \\ \text { CE144 } & 7.7 \mathrm{E}-13 \\ \text { PR144 } & 2.1 \mathrm{E}-03 \\ \text { ND144 } & 0.0 \mathrm{E}+00 \\ \text { EU152 } & 1.0 \mathrm{E}-02 \\ \text { EU154 } & 6.9 \mathrm{E}-03 \\ \text { TB160 } & 5.2 \mathrm{E}-03 \\ \text { OS185 } & 2.4 \mathrm{E}-04 \\ \text { OS191 } & 1.7 \mathrm{E}-12 \\ \text { IR192 } & 6.3 \mathrm{E}-05 \\ \text { HG203 } & 4.4 \mathrm{E}-08 \\ \text { TH230 } & 1.6 \mathrm{E}-25 \\ \text { RA226 } & 6.0 \mathrm{E}-12 \\ \text { RN222 } & 2.5 \mathrm{E}-02 \\ \text { PB210 } & 0.0 \mathrm{E}+00 \\ \text { BI210 } & 4.8 \mathrm{E}-08 \\ \text { TH228 } & 1.2 \mathrm{E}-10 \\ \text { RA224 } & 1.1 \mathrm{E}-07 \\ \text { PB212 } & 6.8 \mathrm{E}-08 \\ \text { 8I212 } & 6.9 \mathrm{E}-01 \\ \text { TH232 } & 6.7 \mathrm{E}-31 \\ \text { RA228 } & 0.0 \mathrm{E}+00 \\ \text { AC228 } & 6.1 \mathrm{E}-03 \\ \text { U 234 } & 1.5 \mathrm{E}-14 \\ \text { U 235 } & 3.3 \mathrm{E}-09 \\ \text { TH231 } & 7.3 \mathrm{E}-14 \\ \text { PA231 } & 7.7 \mathrm{E}-07 \\ \text { AC227 } & 3.3 \mathrm{E}-15 \\ \text { TH227 } & 8.3 \mathrm{E}-07 \\ \text { FR223 } & 4.1 \mathrm{E}-06 \\ \text { RA223 } & 2.2 \mathrm{E}-06 \\ \text { NP237 } & 1.9 \mathrm{E}-15 \\ \text { PA233 } & 7.3 \mathrm{E}-06 \\ \text { U 233 } & 1.1 \mathrm{E}-33 \\ \text { TH229 } & 5.6 \mathrm{E}-13 \\ \text { RA225 } & 1.5 \mathrm{E}-13 \\ \text { AC225 } & 5.8 \mathrm{E}-10 \\ \text { U 238 } & 9.0 \mathrm{E}-33 \\ \text { TH234 } & 1.9 \mathrm{E}-14 \\ \text { PA234M } & 3.9 \mathrm{E}-05 \\ \text { PA234 } & 8.7 \mathrm{E}-03 \\ \text { PU242 } & 0.0 \mathrm{E}+00 \\ \text { NP238 } & 3.4 \mathrm{E}-03 \\ \text { PU238 } & 6.6 \mathrm{E}-16 \\ \text { CM244 } & 0.0 \mathrm{E}+00 \\ \text { PU244 } & 0.0 \mathrm{E}+00 \\ \text { P 240 } & 1.4 \mathrm{E}-11 \\ & 6.6 \mathrm{E}-16\end{array}$


Appendix B - External Exposure Dose Rate Factors - BUR1A

CM243 $\quad 1.4 \mathrm{E}-08$

PU243 8.6E-08

AM243 5.0E-19

NP239 1.9E-07

PU239 6.6E-16

PU241 $0.0 \mathrm{E}+00$

AM241 2.0E-15 
Appendix B - External Exposure Dose Rate Ractors - BUR1B

OVERBURDEN: $1.0 \mathrm{~m}$, SOURCE: $1.0 \mathrm{M}$ (DEN: water/1.0): MR/HR 8-Jul-86 RAP

$\begin{array}{ll}\text { H } 3 & 0.0 \mathrm{E}+00 \\ \text { C } 14 & 3.3 \mathrm{E}-19 \\ \text { NA22 } & 1.7 \mathrm{E}-02 \\ \text { P } 32 & 1.8 \mathrm{E}-06 \\ \text { P } 33 & 4.4 \mathrm{E}-09 \\ \text { S } 35 & 0.0 \mathrm{E}+00 \\ \text { CL36 } & 3.6 \mathrm{E}-16 \\ \text { K 40 } & 6.4 \mathrm{E}-03 \\ \text { CA45 } & 3.7 \mathrm{E}-16 \\ \text { SC46 } & 1.9 \mathrm{E}-02 \\ \text { CR51 } & 2.2 \mathrm{E}-06 \\ \text { MN54 } & 1.8 \mathrm{E}-03 \\ \text { FE55 } & 0.0 \mathrm{E}+00 \\ \text { FE59 } & 1.4 \mathrm{E}-02 \\ \text { C057 } & 1.7 \mathrm{E}-11 \\ \text { C060 } & 3.4 \mathrm{E}-02 \\ \text { NI59 } & 0.0 \mathrm{E}+00 \\ \text { NI63 } & 0.0 \mathrm{E}+00 \\ \text { ZN65 } & 8.7 \mathrm{E}-03 \\ \text { SE75 } & 2.5 \mathrm{E}-05 \\ \text { SR85 } & 1.9 \mathrm{E}-04 \\ \text { SR89 } & 5.7 \mathrm{E}-07 \\ \text { Y 89M } & 1.1 \mathrm{E}-04 \\ \text { SR90 } & 3.8 \mathrm{E}-10 \\ \text { Y 90 } & 1.5 \mathrm{E}-05 \\ \text { M093 } & 0.0 \mathrm{E}+00 \\ \text { NB94 } & 2.1 \mathrm{E}-03 \\ \text { M099 } & 1.4 \mathrm{E}-04 \\ \text { TC99M } & 1.5 \mathrm{E}-11 \\ \text { TC99 } & 3.5 \mathrm{E}-14 \\ \text { RU103 } & 1.9 \mathrm{E}-04 \\ \text { RH103M } & 0.0 \mathrm{E}+00 \\ \text { PD103 } & 7.3 \mathrm{E}-09 \\ \text { RU106 } & 0.0 \mathrm{E}+00 \\ \text { RH106 } & 9.3 \mathrm{E}-04 \\ \text { CD109 } & 2.4 \mathrm{E}-15 \\ \text { AG110M } & 3.0 \mathrm{E}-02 \\ \text { AG110 } & 7.8 \mathrm{E}-05 \\ \text { IN111 } & 1.2 \mathrm{E}-07 \\ \text { SB124 } & 9.4 \mathrm{E}-02 \\ \text { SB125 } & 1.9 \mathrm{E}-04 \\ \text { TE125M } & 5.0 \mathrm{E}-14 \\ \text { I 125 } & 0.0 \mathrm{E}+00 \\ \text { I 129 } & 1.3 \mathrm{E}-19 \\ \text { I 131 } & 4.7 \mathrm{E}-05 \\ \text { XE131M } & 3.3 \mathrm{E}-13 \\ \text { CS134 } & 4.6 \mathrm{E}-03 \\ \text { CS135 } & 5.9 \mathrm{E}-17 \\ & \\ & \end{array}$


Appendix B - External Exposure Dose Rate Ractors - BUR1B

\begin{tabular}{|c|c|}
\hline $\operatorname{cs} 137$ & $9.0 E-09$ \\
\hline BA137N & $2.8 \mathrm{E}-04$ \\
\hline CE141 & $2.3 E-10$ \\
\hline CE144 & $1.9 E-12$ \\
\hline PR144 & $3.8 E-03$ \\
\hline ND144 & $0.0 \mathrm{E}+00$ \\
\hline EU152 & $1.9 \mathrm{E}-02$ \\
\hline EU154 & $1.2 \mathrm{E}-02$ \\
\hline T8160 & $9.2 \mathrm{E}-03$ \\
\hline $0 S 185$ & $4.8 E-04$ \\
\hline OS 191 & $4.3 \mathrm{E}-12$ \\
\hline IR192 & $1.2 \mathrm{E}-04$ \\
\hline $\mathrm{HG} 203$ & $9.7 \mathrm{E}-08$ \\
\hline $\mathrm{TH} 230$ & $5.5 E-25$ \\
\hline RA226 & $1.3 \mathrm{E}-11$ \\
\hline RN222 & $4.6 \mathrm{E}-02$ \\
\hline PB210 & $0.0 \mathrm{E}+00$ \\
\hline BI 210 & $9.3 \mathrm{E}-08$ \\
\hline TH 228 & $2.5 E-10$ \\
\hline RA224 & $2.3 E-07$ \\
\hline PB212 & $1.4 \mathrm{E}-07$ \\
\hline BI212 & $1.2 E+00$ \\
\hline TH232 & $2.7 E-30$ \\
\hline RA228 & $0.0 \mathrm{E}+00$ \\
\hline$A C 228$ & $1.1 \mathrm{E}-02$ \\
\hline บ 234 & $3.8 \mathrm{E}-14$ \\
\hline U 235 & 7. $2 \mathrm{E}-09$ \\
\hline TH23i & $1.9 \mathrm{E}-13$ \\
\hline PA231 & $1.5 E-06$ \\
\hline$A C 227$ & $8.4 E-15$ \\
\hline$T\}+227$ & $1.6 \mathrm{E}-06$ \\
\hline FR223 & $8.1 E-06$ \\
\hline RA223 & $4.3 E-06$ \\
\hline NP237 & $4.9 \mathrm{E}-15$ \\
\hline FA? 33 & $1.4 \mathrm{E}-05$ \\
\hline U) 2.33 & $4.5 E-33$ \\
\hline TH?29 & $1.4 \mathrm{E}-12$ \\
\hline RAP25 & $3.3 E-13$ \\
\hline$A C 225$ & $1.3 \mathrm{E}-09$ \\
\hline I) 238 & $3.6 \mathrm{E}-32$ \\
\hline $11: 2 \% 4$ & $4.8 E-14$ \\
\hline 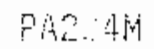 & $6.7 E-05$ \\
\hline PA234 & $1.6 \mathrm{E}-02$ \\
\hline plj?24 & $0.0 \mathrm{E}+00$ \\
\hline ind & $5.8 \bar{E}-03$ \\
\hline picas & $1.7 \mathrm{E}-15$ \\
\hline $\mathrm{CH}_{2} 44$ & $0.0 \mathrm{E}+00$ \\
\hline p:24A & $0.0 E+00$ \\
\hline$\because \quad \therefore \quad \therefore$ & $2.0 E-20$ \\
\hline 80 & $1.7 E-15$ \\
\hline
\end{tabular}




\section{Appendix B - External Exposure Dose Rate Ractors - BUR1B}

CM243 3.0E-08

PU243 1.6E-07

AM243 $1.5 \mathrm{E}-18$

NP239 3.7E-07

PU239 1.7E-15

PU241 $0.0 \mathrm{E}+00$

AM241 $5.0 \mathrm{E}-15$ 
Appendix B - External Exposure Dose Rate Factors - BUR1C

ONSITE/MAXI $1.0 \mathrm{M}$ THICK BURIED AT $1.0 \mathrm{M}$ (DEN: WATER/CARBON 0.5/0.1) :MR/HR

$\begin{array}{ll} & 100 \\ \text { H } 3 & 0.0 \mathrm{E}+00 \\ \text { C } 14 & 5.5 \mathrm{E}-19 \\ \text { NA22 } & 2.8 \mathrm{E}-02 \\ \text { P } 32 & 3.0 \mathrm{E}-06 \\ \text { P } 33 & 5.8 \mathrm{E}-16 \\ \text { S } 35 & 0.0 \mathrm{E}+00 \\ \text { CL36 } & 8.2 \mathrm{E}-09 \\ \text { K } 40 & 1.0 \mathrm{E}-02 \\ \text { CA45 } & 6.2 \mathrm{E}-16 \\ \text { SC46 } & 3.1 \mathrm{E}-02 \\ \text { CR51 } & 3.6 \mathrm{E}-06 \\ \text { MN54 } & 3.0 \mathrm{E}-03 \\ \text { FE55 } & 0.0 \mathrm{E}+00 \\ \text { FE59 } & 2.2 \mathrm{E}-02 \\ \text { C057 } & 2.8 \mathrm{E}-11 \\ \text { C060 } & 5.7 \mathrm{E}-02 \\ \text { NI59 } & 0.0 \mathrm{E}+00 \\ \text { NI63 } & 2.7 \mathrm{E}-33 \\ \text { ZN65 } & 1.4 \mathrm{E}-02 \\ \text { SE75 } & 4.2 \mathrm{E}-05 \\ \text { SR85 } & 3.2 \mathrm{E}-04 \\ \text { SR89 } & 9.5 \mathrm{E}-07 \\ \text { Y 89M } & 1.8 \mathrm{E}-04 \\ \text { SR90 } & 6.4 \mathrm{E}-10 \\ \text { Y 90 } & 2.5 \mathrm{E}-05 \\ \text { M093 } & 0.0 \mathrm{E}+00 \\ \text { NB94 } & 3.5 \mathrm{E}-03 \\ \text { M099 } & 2.3 \mathrm{E}-04 \\ \text { TC99M } & 2.5 \mathrm{E}-11 \\ \text { TC99 } & 5.9 \mathrm{E}-14 \\ \text { RU103 } & 3.2 \mathrm{E}-04 \\ \text { RH103M } & 0.0 \mathrm{E}+00 \\ \text { PD103 } & 1.2 \mathrm{E}-08 \\ \text { RU106 } & 0.0 \mathrm{E}+00 \\ \text { RH106 } & 1.5 \mathrm{E}-03 \\ \text { CD109 } & 4.0 \mathrm{E}-15 \\ \text { AG110M } & 4.8 \mathrm{E}-02 \\ \text { AG110 } & 1.3 \mathrm{E}-04 \\ \text { IN111 } & 2.0 \mathrm{E}-07 \\ \text { SB124 } & 1.5 \mathrm{E}-01 \\ \text { SB125 } & 3.2 \mathrm{E}-04 \\ \text { TE125M } & 8.4 \mathrm{E}-14 \\ \text { I 125 } & 0.0 \mathrm{E}+00 \\ \text { I 129 } & 2.2 \mathrm{E}-19 \\ \text { I 131 } & 7.7 \mathrm{E}-05 \\ \text { XE131M } & 5.6 \mathrm{E}-13 \\ \text { CS134 } & 7.5 \mathrm{E}-03\end{array}$




\section{Appendix B - External Exposure Dose Rate Factors - BUR1C}

$\begin{array}{ll}\text { CS135 } & 1.0 \mathrm{E}-16 \\ \text { CS137 } & 1.5 \mathrm{E}-08 \\ \text { BA137M } & 4.6 \mathrm{E}-04 \\ \text { CE141 } & 3.9 \mathrm{E}-10 \\ \text { CE144 } & 3.2 \mathrm{E}-12 \\ \text { PR144 } & 6.0 \mathrm{E}-03 \\ \text { ND144 } & 0.0 \mathrm{E}+00 \\ \text { EU152 } & 3.1 \mathrm{E}-02 \\ \text { EU154 } & 2.1 \mathrm{E}-02 \\ \text { TB160 } & 1.5 \mathrm{E}-02 \\ \text { OS185 } & 7.9 \mathrm{E}-04 \\ \text { OS191 } & 7.3 \mathrm{E}-12 \\ \text { IR192 } & 2.0 \mathrm{E}-04 \\ \text { HG203 } & 1.6 \mathrm{E}-07 \\ \text { TH230 } & 9.3 \mathrm{E}-25 \\ \text { RA226 } & 2.2 \mathrm{E}-11 \\ \text { RN222 } & 6.3 \mathrm{E}-07 \\ \text { PB210 } & 0.0 \mathrm{E}+00 \\ \text { BI210 } & 1.5 \mathrm{E}-07 \\ \text { TH228 } & 4.2 \mathrm{E}-10 \\ \text { RA224 } & 9.3 \mathrm{E}-02 \\ \text { P8212 } & 2.3 \mathrm{E}-07 \\ \text { BI212 } & 1.6 \mathrm{E}-05 \\ \text { TH232 } & 4.6 \mathrm{E}-30 \\ \text { RA228 } & 0.0 \mathrm{E}+00 \\ \text { AC228 } & 1.8 \mathrm{E}-02 \\ \text { U 234 } & 6.5 \mathrm{E}-14 \\ \text { U 235 } & 1.2 \mathrm{E}-08 \\ \text { TH231 } & 3.1 \mathrm{E}-13 \\ \text { PA231 } & 2.5 \mathrm{E}-06 \\ \text { AC227 } & 1.4 \mathrm{E}-14 \\ \text { TH227 } & 2.7 \mathrm{E}-06 \\ \text { FR223 } & 1.3 \mathrm{E}-05 \\ \text { RA223 } & 7.1 \mathrm{E}-06 \\ \text { NP237 } & 8.3 \mathrm{E}-15 \\ \text { PA233 } & 2.3 \mathrm{E}-05 \\ \text { U 233 } & 7.7 \mathrm{E}-33 \\ \text { TH229 } & 2.4 \mathrm{E}-12 \\ \text { RA225 } & 5.5 \mathrm{E}-13 \\ \text { AC225 } & 2.1 \mathrm{E}-09 \\ \text { U 238 } & 6.2 \mathrm{E}-32 \\ \text { TH234 } & 8.1 \mathrm{E}-14 \\ \text { PA234M } & 1.1 \mathrm{E}-04 \\ \text { PA234 } & 2.6 \mathrm{E}-02 \\ \text { PU242 } & 0.0 \mathrm{E}+00 \\ \text { NP238 } & 9.6 \mathrm{E}-03 \\ \text { PU238 } & 2.8 \mathrm{E}-15 \\ \text { CM244 } & 0.0 \mathrm{E}+00 \\ \text { PU244 } & 0.0 \mathrm{E}+00 \\ \text { U 240 } & 4.5 \mathrm{E}-11\end{array}$




\section{Appendix B - External Exposure Dose Rate Factors - BUR1C}

$\begin{array}{ll}\text { PU240 } & 2.8 \mathrm{E}-15 \\ \text { CM243 } & 5.1 \mathrm{E}-08 \\ \text { PU243 } & 2.8 \mathrm{E}-07 \\ \text { AM243 } & 2.6 \mathrm{E}-18 \\ \text { NP239 } & 6.2 \mathrm{E}-07 \\ \text { PU239 } & 2.8 \mathrm{E}-15 \\ \text { PU241 } & 0.0 \mathrm{E}+00 \\ \text { AM241 } & 8.5 \mathrm{E}-15\end{array}$


Appendix B - External Exposure Dose Rate Factors - STOREDA

ROOM: 1.0 M THICK FINITE SLAB (DEN: soil 1.8): MR/HR 8-Jul-86 RAP

$\begin{array}{ll}\text { H } 3 & 7.6 \mathrm{E}-15 \\ \text { C 14 } & 1.0 \mathrm{E}-04 \\ \text { NA22 } & 1.7 \mathrm{E}+02 \\ \text { P } 32 & 3.4 \mathrm{E}-01 \\ \text { P } 33 & 6.3 \mathrm{E}-04 \\ \text { S } 35 & 0.0 \mathrm{E}+00 \\ \text { CL36 } & 2.5 \mathrm{E}-02 \\ \text { K } 40 & 2.2 \mathrm{E}+01 \\ \text { CA45 } & 6.4 \mathrm{E}-04 \\ \text { SC46 } & 2.8 \mathrm{E}+02 \\ \text { CR51 } & 4.1 \mathrm{E}+00 \\ \text { MN54 } & 1.1 \mathrm{E}+02 \\ \text { FE55 } & 0.0 \mathrm{E}+00 \\ \text { FE59 } & 1.5 \mathrm{E}+02 \\ \text { C057 } & 8.2 \mathrm{E}+00 \\ \text { C060 } & 3.3 \mathrm{E}+02 \\ \text { NI59 } & 0.0 \mathrm{E}+00 \\ \text { NI63 } & 1.2 \mathrm{E}-06 \\ \text { ZN65 } & 8.7 \mathrm{E}+01 \\ \text { SE75 } & 3.6 \mathrm{E}+01 \\ \text { SR85 } & 7.0 \mathrm{E}+01 \\ \text { SR89 } & 2.1 \mathrm{E}-01 \\ \text { Y 89M } & 1.4 \mathrm{E}+00 \\ \text { SR90 } & 9.5 \mathrm{E}-03 \\ \text { Y 90 } & 7.2 \mathrm{E}-01 \\ \text { M093 } & 1.3 \mathrm{E}-01 \\ \text { NB94 } & 2.0 \mathrm{E}+02 \\ \text { M099 } & 1.8 \mathrm{E}+01 \\ \text { TC99M } & 7.4 \mathrm{E}+00 \\ \text { TC99 } & 1.0 \mathrm{E}-03 \\ \text { RU103 } & 6.9 \mathrm{E}+01 \\ \text { RH103M } & 4.0 \mathrm{E}-03 \\ \text { PD103 } & 3.2 \mathrm{E}-02 \\ \text { RU106 } & 3.0 \mathrm{E}-08 \\ \text { RH106 } & 1.6 \mathrm{E}+01 \\ \text { CD109 } & 2.2 \mathrm{E}+00 \\ \text { A6110M } & 4.0 \mathrm{E}+02 \\ \text { AG110 } & 5.4 \mathrm{E}+00 \\ \text { IN111 } & 2.9 \mathrm{E}+01 \\ \text { SB124 } & 2.8 \mathrm{E}+02 \\ \text { SB125 } & 6.4 \mathrm{E}+01 \\ \text { TE125M } & 6.3 \mathrm{E}-02 \\ \text { I 125 } & 7.4 \mathrm{E}-02 \\ \text { I 129 } & 3.8 \mathrm{E}-02 \\ \text { I 131 } & 4.7 \mathrm{E}+01 \\ \text { XE131M } & 2.3 \mathrm{E}-01 \\ \text { CS134 } & 2.3 \mathrm{E}+02 \\ \text { CS135 } & 2.6 \mathrm{E}-04 \\ & \\ & \\ \text { S } & \end{array}$


Appendix B - External Exposure Dose Rate Factors - STOREDA

\begin{tabular}{ll} 
CS137 & $1.4 \mathrm{E}-02$ \\
BA137M & $8.2 \mathrm{E}+01$ \\
CE141 & $4.0 \mathrm{E}+00$ \\
CE144 & $9.5 \mathrm{E}-01$ \\
PR144 & $5.5 \mathrm{E}+00$ \\
ND144 & $0.0 \mathrm{E}+00$ \\
EU152 & $1.6 \mathrm{E}+02$ \\
EU154 & $1.6 \mathrm{E}+02$ \\
TB160 & $1.5 \mathrm{E}+02$ \\
OS185 & $9.3 \mathrm{E}+01$ \\
OS191 & $2.8 \mathrm{E}+00$ \\
IR192 & $9.8 \mathrm{E}+01$ \\
HG203 & $1.8 \mathrm{E}+01$ \\
TH230 & $4.7 \mathrm{E}-03$ \\
RA226 & $3.1 \mathrm{E}-01$ \\
RN222 & $1.8 \mathrm{E}+02$ \\
P8210 & $8.6 \mathrm{E}-03$ \\
BI210 & $9.3 \mathrm{E}-02$ \\
TH228 & $9.0 \mathrm{E}-02$ \\
RA224 & $9.4 \mathrm{E}-01$ \\
PB212 & $1.1 \mathrm{E}+01$ \\
BI212 & $4.8 \mathrm{E}+02$ \\
TH232 & $2.0 \mathrm{E}-01$ \\
RA228 & $4.0 \mathrm{E}-07$ \\
AC228 & $1.1 \mathrm{E}+02$ \\
U 234 & $2.4 \mathrm{E}-02$ \\
U 235 & $7.6 \mathrm{E}+00$ \\
TH231 & $3.9 \mathrm{E}-01$ \\
PA231 & $3.3 \mathrm{E}+00$ \\
AC227 & $5.0 \mathrm{E}-03$ \\
TH227 & $9.5 \mathrm{E}+00$ \\
FR223 & $2.7 \mathrm{E}+00$ \\
RA223 & $9.7 \mathrm{E}+00$ \\
NP237 & $5.6 \mathrm{E}-01$ \\
PA233 & $2.3 \mathrm{E}+01$ \\
U 233 & $6.4 \mathrm{E}-04$ \\
TH229 & $7.0 \mathrm{E}-01$ \\
RA225 & $7.3 \mathrm{E}-02$ \\
AC225 & $5.7 \mathrm{E}-01$ \\
U 238 & $2.6 \mathrm{E}-03$ \\
TH234 & $2.3 \mathrm{E}-01$ \\
PA234M & $1.1 \mathrm{E}+00$ \\
PA234 & $2.4 \mathrm{E}+02$ \\
PU242 & $8.6 \mathrm{E}-05$ \\
NP238 & $7.9 \mathrm{E}+01$ \\
PU238 & $9.1 \mathrm{E}-04$ \\
CM244 & $1.9 \mathrm{E}-04$ \\
UU244 & $7.9 \mathrm{E}-09$ \\
& $7.6 \mathrm{E}-03$ \\
$8.9 \mathrm{E}-04$ \\
\hline
\end{tabular}

B. 75 
Appendix B - External Exposure Dose Rate Factors - STOREDA

$\mathrm{CM} 243 \quad 9.8 \mathrm{E}+00$

PU243 8.8E-01

AM243 $1.0 \mathrm{E}+00$

NP239 1.1E+01

PU239 1.0E-03

PU241 2.9E-14

AM241 3.0E-01 
Appendix B - External Exposure Dose Rate Factors - STOREDB

ROOM: $1.0 \mathrm{M}$ THICK FINITE SLAB (DEN: H20 1.0): MR/HR 8-Ju1-86 RAP

\begin{tabular}{ll} 
H 3 & $7.3 \mathrm{E}-12$ \\
C 14 & $3.4 \mathrm{E}-03$ \\
NA22 & $3.0 \mathrm{E}+02$ \\
P 32 & $1.1 \mathrm{E}+00$ \\
P 33 & $1.1 \mathrm{E}-02$ \\
S 35 & $0.0 \mathrm{E}+00$ \\
CL36 & $1.5 \mathrm{E}-01$ \\
K 40 & $4.5 \mathrm{E}+01$ \\
CA45 & $1.1 \mathrm{E}-02$ \\
SC46 & $5.3 \mathrm{E}+02$ \\
CR51 & $9.8 \mathrm{E}+00$ \\
MN54 & $2.2 \mathrm{E}+02$ \\
FE55 & $0.0 \mathrm{E}+00$ \\
FE59 & $2.8 \mathrm{E}+02$ \\
C057 & $2.7 \mathrm{E}+01$ \\
C060 & $6.1 \mathrm{E}+02$ \\
NI59 & $0.0 \mathrm{E}+00$ \\
NI63 & $1.4 \mathrm{E}-04$ \\
ZN65 & $1.6 \mathrm{E}+02$ \\
SE75 & $9.7 \mathrm{E}+01$ \\
SR85 & $1.7 \mathrm{E}+02$ \\
SR89 & $7.8 \mathrm{E}-01$ \\
Y $89 M$ & $2.5 \mathrm{E}+00$ \\
SR90 & $7.3 \mathrm{E}-02$ \\
Y 90 & $2.2 \mathrm{E}+00$ \\
M093 & $7.9 \mathrm{E}+00$ \\
NB94 & $4.3 \mathrm{E}+02$ \\
M099 & $4.2 \mathrm{E}+01$ \\
TC99M & $2.4 \mathrm{E}+01$ \\
TC99 & $1.5 \mathrm{E}-02$ \\
RU103 & $1.6 \mathrm{E}+02$ \\
RH103M & $6.2 \mathrm{E}-01$ \\
PD103 & $3.4 \mathrm{E}+00$ \\
RU106 & $5.4 \mathrm{E}-06$ \\
RH106 & $3.6 \mathrm{E}+01$ \\
CD109 & $1.7 \mathrm{E}+01$ \\
AG110M & $8.3 \mathrm{E}+02$ \\
AG110 & $1.3 \mathrm{E}+01$ \\
IN111 & $8.8 \mathrm{E}+01$ \\
SB124 & $5.7 \mathrm{E}+02$ \\
SB125 & $1.5 \mathrm{E}+02$ \\
TE125M & $6.0 \mathrm{E}+00$ \\
I 125 & $8.2 \mathrm{E}+00$ \\
I 129 & $4.3 \mathrm{E}+00$ \\
I 131 & $1.1 \mathrm{E}+02$ \\
XE131M & $4.8 \mathrm{E}+00$ \\
CS134 & $4.9 \mathrm{E}+02$ \\
CS135 & $6.2 \mathrm{E}-03$ \\
& \\
\hline
\end{tabular}


Appendix B - External Exposure Dose Rate Factors - STOREDB

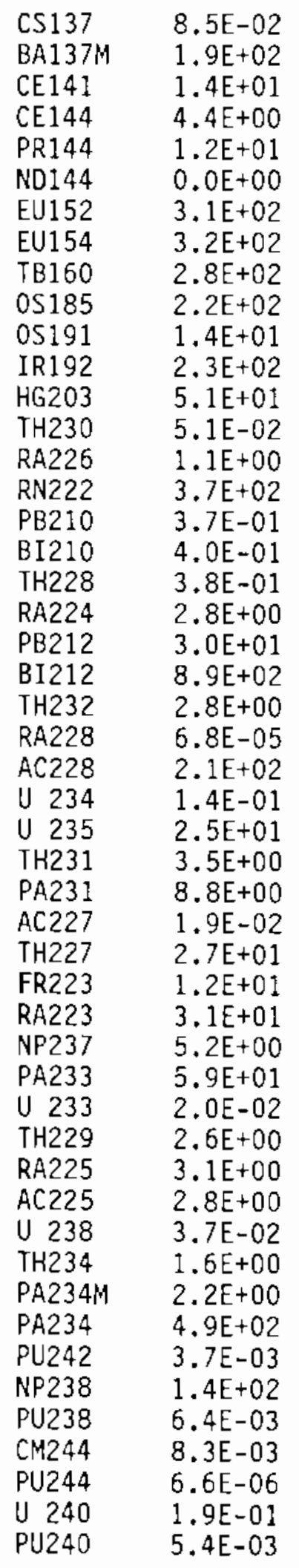

B. 78 
Appendix B - External Exposure Dose Rate Factors - STOREDB

CM243 $2.9 \mathrm{E}+01$

PU243 $4.9 E+00$

AM243 $9.3 \mathrm{E}+00$

NP239 $3.3 \mathrm{E}+01$

PU239 5.9E-03

PU241 2.8E-11

AM241 $\quad 4.3 \mathrm{E}+00$ 


\section{Appendix B - External Exposure Dose Rate Factors - STOREDC}

ROOM: 1.0 M THICK FINITE SLAB (DEN: .5,.1): MR/HR 8-JUl-86 RAP

\begin{tabular}{ll} 
H 3 & $5.6 \mathrm{E}-14$ \\
C 14 & $9.1 \mathrm{E}-04$ \\
NA22 & $4.8 \mathrm{E}+02$ \\
P 32 & $1.5 \mathrm{E}+00$ \\
P 33 & $4.3 \mathrm{E}-03$ \\
S 35 & $0.0 \mathrm{E}+00$ \\
CL36 & $1.3 \mathrm{E}-01$ \\
K 40 & $6.7 \mathrm{E}+01$ \\
CA45 & $4.3 \mathrm{E}-03$ \\
SC46 & $8.3 \mathrm{E}+02$ \\
CR51 & $1.6 \mathrm{E}+01$ \\
MN54 & $3.6 \mathrm{E}+02$ \\
FE55 & $0.0 \mathrm{E}+00$ \\
FE59 & $4.4 \mathrm{E}+02$ \\
C057 & $4.4 \mathrm{E}+01$ \\
CO50 & $9.5 \mathrm{E}+02$ \\
NI59 & $0.0 \mathrm{E}+00$ \\
NI63 & $2.3 \mathrm{E}-05$ \\
ZN65 & $2.5 \mathrm{E}+02$ \\
SE75 & $1.6 \mathrm{E}+02$ \\
SR85 & $2.7 \mathrm{E}+02$ \\
SR89 & $9.3 \mathrm{E}-01$ \\
Y 89M & $3.9 \mathrm{E}+00$ \\
SR90 & $5.2 \mathrm{E}-02$ \\
Y 90 & $3.0 \mathrm{E}+00$ \\
M093 & $1.6 \mathrm{E}+00$ \\
NB94 & $6.9 \mathrm{E}+02$ \\
M099 & $6.6 \mathrm{E}+01$ \\
TC99M & $3.9 \mathrm{E}+01$ \\
TC99 & $6.3 \mathrm{E}-03$ \\
RU103 & $2.6 \mathrm{E}+02$ \\
RH103M & $1.0 \mathrm{E}-01$ \\
PD103 & $5.8 \mathrm{E}-01$ \\
RU106 & $8.5 \mathrm{E}-07$ \\
RH106 & $5.7 \mathrm{E}+01$ \\
CD109 & $9.3 \mathrm{E}+00$ \\
AG110M & $1.3 \mathrm{E}+03$ \\
AG110 & $2.0 \mathrm{E}+01$ \\
IN111 & $1.4 \mathrm{E}+02$ \\
SB124 & $8.9 \mathrm{E}+02$ \\
SB125 & $2.4 \mathrm{E}+02$ \\
TE125M & $1.1 \mathrm{E}+00$ \\
I 125 & $1.4 \mathrm{E}+00$ \\
I 129 & $7.2 \mathrm{E}-01$ \\
I 131 & $1.8 \mathrm{E}+02$ \\
XE131M & $1.7 \mathrm{E}+00$ \\
CS134 & $7.7 \mathrm{E}+02$ \\
CS135 & $2.0 \mathrm{E}-03$ \\
& \\
\hline
\end{tabular}


Appendix B - External Exposure Dose Rate Factors - STOREDC

$\begin{array}{ll}\text { CS137 } & 7.0 \mathrm{E}-02 \\ \text { BA137M } & 3.0 \mathrm{E}+02 \\ \text { CE141 } & 2.1 \mathrm{E}+01 \\ \text { CE144 } & 5.2 \mathrm{E}+00 \\ \text { PR144 } & 1.8 \mathrm{E}+01 \\ \text { ND144 } & 0.0 \mathrm{E}+00 \\ \text { EU152 } & 4.8 \mathrm{E}+02 \\ \text { EU154 } & 5.0 \mathrm{E}+02 \\ \text { TB160 } & 4.4 \mathrm{E}+02 \\ \text { OS185 } & 3.3 \mathrm{E}+02 \\ \text { OS191 } & 1.5 \mathrm{E}+01 \\ \text { IR192 } & 3.8 \mathrm{E}+02 \\ \text { HG203 } & 8.1 \mathrm{E}+01 \\ \text { TH230 } & 2.8 \mathrm{E}-02 \\ \text { RA225 } & 1.6 \mathrm{E}+00 \\ \text { RN222 } & 5.6 \mathrm{E}+02 \\ \text { PB210 } & 8.2 \mathrm{E}-02 \\ \text { BI210 } & 4.3 \mathrm{E}-01 \\ \text { TH228 } & 4.1 \mathrm{E}-01 \\ \text { RA224 } & 4.2 \mathrm{E}+00 \\ \text { PB212 } & 4.9 \mathrm{E}+01 \\ \text { BI212 } & 1.3 \mathrm{E}+03 \\ \text { TH232 } & 1.6 \mathrm{E}+00 \\ \text { RA228 } & 8.6 \mathrm{E}-06 \\ \text { AC228 } & 3.3 \mathrm{E}+02 \\ \text { U 234 } & 1.5 \mathrm{E}-01 \\ \text { U 235 } & 3.9 \mathrm{E}+01 \\ \text { TH231 } & 1.9 \mathrm{E}+00 \\ \text { PA231 } & 1.3 \mathrm{E}+01 \\ \text { AC227 } & 2.5 \mathrm{E}-02 \\ \text { TH227 } & 4.1 \mathrm{E}+01 \\ \text { FR223 } & 1.3 \mathrm{E}+01 \\ \text { RA223 } & 4.1 \mathrm{E}+01 \\ \text { NP237 } & 2.7 \mathrm{E}+00 \\ \text { PA233 } & 9.0 \mathrm{E}+01 \\ \text { U 233 } & 5.8 \mathrm{E}-03 \\ \text { TH229 } & 3.8 \mathrm{E}+00 \\ \text { RA225 } & 6.8 \mathrm{E}-01 \\ \text { AC225 } & 2.5 \mathrm{E}+00 \\ \text { U2238 } & 2.1 \mathrm{E}-02 \\ \text { TH234 } & 9.8 \mathrm{E}-01 \\ \text { PA234M } & 3.3 \mathrm{E}+00 \\ \text { PA234 } & 7.7 \mathrm{E}+02 \\ \text { PU242 } & 8.2 \mathrm{E}-04 \\ \text { NP238 } & 2.2 \mathrm{E}+02 \\ \text { PU238 } & 5.2 \mathrm{E}-03 \\ \text { CM244 } & 1.8 \mathrm{E}-03 \\ \text { PU244 } & 8.4 \mathrm{E}-08 \\ \text { U 240 } & 5.7 \mathrm{E}-02 \\ \text { PU240 } & 5.0 \mathrm{E}-03\end{array}$


Appendix B - External Exposure Dose Rate Factors - STOREDC

$\mathrm{CM} 243 \quad 4.7 \mathrm{E}+01$

PU243 $3.6 E+00$

AM243 $5.0 \mathrm{E}+00$

NP239 5.4E+01

PU239 5.9E-03

PU241 2.1E-13

AM241 2.4E+00 


\section{Appendix B - Inhalation Dose Rate Factors - FILE23}

DACRIN (DIFDOS) DOSE INCREMENT FILE ONSITE/MAXI 26-Aug-86 RAP 121

\begin{tabular}{|c|c|c|c|c|c|c|c|}
\hline \multirow{2}{*}{\multicolumn{8}{|c|}{1}} \\
\hline 3 & & & & & & & \\
\hline C 14 & & & 4 & 0 & & & \\
\hline C & 14 & 11 & $2.39 \mathrm{E}-06$ & $1.05 \mathrm{E}-05$ & $2.39 \mathrm{E}-06$ & $2.39 \mathrm{E}-06$ & $2.06 \mathrm{E}-06$ \\
\hline C & 14 & 12 & $1.01 \mathrm{E}-07$ & $1.97 \mathrm{E}-06$ & $1.01 \mathrm{E}-07$ & $1.01 \mathrm{E}-07$ & $1.23 \mathrm{E}-10$ \\
\hline C & 14 & 13 & .00 & $3.51 \mathrm{E}-09$ & .00 & .00 & .00 \\
\hline C & 14 & 14 & .00 & $6.37 \mathrm{E}-12$ & .00 & .00 & .00 \\
\hline NA22 & & & 2 & 0 & & & \\
\hline NA22 & & 11 & $7.25 \mathrm{E}-05$ & $0.00 \mathrm{E}+00$ & $5.01 \mathrm{E}-05$ & $0.00 \mathrm{E}+00$ & $1.15 \mathrm{E}-05$ \\
\hline NA22 & & 12 & $3.33 \mathrm{E}-06$ & $0.00 E+00$ & $9.90 \mathrm{E}-08$ & $0.00 \mathrm{E}+00$ & $6.88 \mathrm{E}-10$ \\
\hline 32 & & & 2 & 0 & & & \\
\hline 32 & & 11 & $3.79 \mathrm{E}-05$ & $9.79 E-04$ & $4.18 \mathrm{E}-05$ & $0.00 E+00$ & $2.58 \mathrm{E}-05$ \\
\hline 32 & & 2 & $2.18 \mathrm{E}-06$ & $5.89 \mathrm{E}-05$ & $7.98 \mathrm{E}-08$ & $0.00 \mathrm{E}+00$ & $1.53 \mathrm{E}-09$ \\
\hline 33 & & & 3 & 0 & & & \\
\hline 33 & & 11 & $7.41 \mathrm{E}-06$ & $7.88 \mathrm{E}-05$ & $5.23 \mathrm{E}-06$ & $0.00 E+00$ & $3.20 E-06$ \\
\hline 33 & & 12 & $7.24 \mathrm{E}-07$ & $8.31 \mathrm{E}-06$ & $1.01 \mathrm{E}-08$ & $0.00 E+00$ & 1. $90 \mathrm{E}-10$ \\
\hline 33 & & 13 & $8.19 \mathrm{E}-12$ & $2.04 E-10$ & $0.00 \mathrm{E}+00$ & $0.00 E+00$ & $0.00 E+00$ \\
\hline 35 & & & 0 & 0 & & & \\
\hline 35 & & 21 & $8.32 \mathrm{E}-06$ & $1.84 \mathrm{E}-05$ & $1.20 \mathrm{E}-04$ & $0.00 E+00$ & $0.00 \mathrm{E}+00$ \\
\hline 35 & & 22 & $2.03 \mathrm{E}-06$ & $7.99 E-06$ & $168 \mathrm{~F}-05$ & $0.00 F+00$ & $0.00 \mathrm{~F}+00$ \\
\hline 35 & & 23 & $8.52 \mathrm{E}-09$ & $3.03 \mathrm{E}-07$ & $5.81 \mathrm{~F}-09$ & $0.00 F+00$ & $0.00 F+00$ \\
\hline 35 & & 24 & $2.91 \mathrm{~F}-11$ & $1.09 F-08$ & $146 \mathrm{~F}-11$ & $0.00 F+00$ & $0.00 \mathrm{~F}+00$ \\
\hline 35 & & 25 & $0.00 \mathrm{~F}+00$ & $3.91 \mathrm{~F}-10$ & $0.00 \mathrm{~F}+00$ & $0.00 F+00$ & $0.00 F+00$ \\
\hline 35 & & 26 & $0.00 E+00$ & $1.46 \mathrm{E}-11$ & $0.00 \mathrm{E}+00$ & $0.00 E+00$ & $0.00 \mathrm{E}+00$ \\
\hline CL36 & & & 3 & 14 & & & \\
\hline$C L 36$ & & 11 & $3.07 E-05$ & $0.00 E+00$ & $1.63 \mathrm{E}-05$ & $0.00 E+00$ & $1.98 \mathrm{E}-06$ \\
\hline CL.36 & & 12 & $4.01 E-06$ & 0.00 & 3. & 0. & 1.1 \\
\hline CL36 & & 13 & $6.51 \mathrm{E}-10$ & $0.00 \mathrm{E}+00$ & $0.00 \mathrm{E}+00$ & $0.00 E+00$ & $0.00 \mathrm{~F}+00$ \\
\hline K 40 & & & & 0 & & & \\
\hline K 40 & & 11 & $5.18 \mathrm{E}-05$ & $0.00 \mathrm{E}+00$ & $1.25 \mathrm{E}-05$ & $0.00 \mathrm{E}+00$ & $1.39 \mathrm{E}-06$ \\
\hline$\times 40$ & & 12 & $E-05$ & $0.00 \mathrm{E}$ & 2.2 & 0. & 8.3 \\
\hline K 40 & & 13 & 1.9 & $0.00 \mathrm{E}+00$ & $0.00 \mathrm{E}+00$ & $0.00 \mathrm{E}+00$ & $0.00 \mathrm{~F}+00$ \\
\hline K 40 & & 14 & 2.2 & $0.00 E+00$ & $0.00 \mathrm{E}+00$ & $0.00 \mathrm{E}+00$ & $0.00 \mathrm{~F}+00$ \\
\hline K 40 & & 15 & $E-11$ & $0.00 \mathrm{E}+00$ & $0.00 \mathrm{E}+00$ & $0.00 E+00$ & $0.00 \mathrm{E}+00$ \\
\hline CA45 & & & 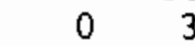 & 0 & & & \\
\hline CA45 & & 21 & $5.36 E-06$ & $2.41 \mathrm{E}-04$ & $0.00 E+00$ & $0.00 E+00$ & $1.46 \mathrm{E}-05$ \\
\hline CA45 & & 22 & $7.18 \mathrm{E}-07$ & 3.23 & 0.0 & 0.0 & 1.09 \\
\hline CA45 & & 23 & 8.5 & 3.84 & $0.00 \mathrm{E}+00$ & $0.00 \mathrm{E}+00$ & 0.00 \\
\hline $5 C 46$ & & & 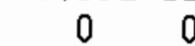 & $c$ & & & \\
\hline SC46 & & 31 & $1.04 \mathrm{E}-06$ & $1.83 \mathrm{E}-06$ & $2.54 \mathrm{E}-03$ & $0.00 E+00$ & 1.95 \\
\hline $\mathrm{SC} 46$ & & 32 & $=-07$ & $4.52 \mathrm{E}-07$ & 9.24 & $0.00 E+00$ & 1.87 \\
\hline $5 r \Delta 6$ & & 33 & $9.21 \mathrm{E}-09$ & $1.68 \mathrm{E}-08$ & $2.71 \mathrm{E}-05$ & $0.00 E+00$ & $4.43 \mathrm{E}-08$ \\
\hline $6 c_{1}$ & & 34 & $4.10 \mathrm{E}-10$ & $7.47 \mathrm{E}-10$ & 7.97 & $0.00 E+00$ & $1.30 \mathrm{E}-0 \mathrm{~g}$ \\
\hline $\mathrm{SC}$ & & 35 & $1.74 \mathrm{E}-11$ & $3.14 \mathrm{E}-11$ & 2.35 & $0.00 E+00$ & $4.37 \mathrm{E}-11$ \\
\hline & & 36 & $6.82 \mathrm{E}-$ & $1.36 \mathrm{E}-12$ & $6.98 \mathrm{E}-10$ & $0.00 E+00$ & $0.00 \mathrm{E}+00$ \\
\hline ותא & & & 0 & 3 & & & \\
\hline$C P$ & 5 & 3 & $2.97 \mathrm{E}-08$ & .00 & 2.56 & 3.1 & 4. \\
\hline 5 & 51 & 32 & $3.95 \mathrm{E}-09$ & .00 & 2.90 & $4.18 \mathrm{E}-09$ & $1.37 \mathrm{E}-08$ \\
\hline
\end{tabular}




\section{Appendix B - Inhalation Dose Rate Factors - FILE23}

\begin{tabular}{|c|c|c|c|c|c|c|}
\hline $\begin{array}{l}\text { CR } \\
\text { MN54 }\end{array}$ & 5133 & $\begin{array}{c}6.33 E-13 \\
0\end{array}$ & .00 & $1.94 \mathrm{E}-10$ & $6.70 E-13$ & $3.41 \mathrm{E}-13$ \\
\hline$M N$ & $\begin{array}{lll}54 & 2 & 1\end{array}$ & $8.51 E-06$ & .00 & $6.28 \mathrm{E}-04$ & .00 & $5.52 \mathrm{E}-05$ \\
\hline MN & 5422 & $1.20 \mathrm{E}-06$ & .00 & $1.25 \mathrm{E}-04$ & .00 & $1.73 \mathrm{E}-06$ \\
\hline$M N$ & 5423 & $4.63 \mathrm{E}-09$ & .00 & $3.39 \mathrm{E}-07$ & .00 & $4.42 \mathrm{E}-09$ \\
\hline$M N$ & 5424 & $1.89 \mathrm{E}-11$ & .00 & $9.24 E-10$ & .00 & 1. $18 \mathrm{E}-11$ \\
\hline MN & 5425 & $1.14 \mathrm{E}-13$ & .00 & .00 & .00 & .00 \\
\hline FE55 & & 050 & 0 & & & \\
\hline $\mathrm{FE}$ & $\begin{array}{lll}55 & 2 & 1\end{array}$ & $7.57 E-07$ & $7.96 \mathrm{E}-07$ & $2.05 E-05$ & .00 & $2.80 \mathrm{E}-06$ \\
\hline $\mathrm{FE}$ & 5522 & $1.23 \mathrm{E}-06$ & $1.41 \mathrm{E}-06$ & $6.49 \mathrm{E}-06$ & .00 & $1.06 \mathrm{E}-07$ \\
\hline $\mathrm{FE}$ & 5523 & $7.30 \mathrm{E}-07$ & $9.90 E-07$ & $1.60 \mathrm{E}-06$ & .00 & $5.07 \mathrm{E}-10$ \\
\hline $\mathrm{FE}$ & $\begin{array}{lll}55 & 24\end{array}$ & $4.23 E-07$ & $6.76 \mathrm{E}-07$ & $1.16 E-06$ & .00 & $2.53 \mathrm{E}-12$ \\
\hline $\mathrm{FE}$ & $\begin{array}{lll}55 & 25\end{array}$ & $2.45 \mathrm{E}-07$ & $4.62 \mathrm{E}-07$ & $8.52 E-07$ & .00 & $2.84 \mathrm{E}-14$ \\
\hline $\mathrm{FE}$ & 5526 & $1.42 \mathrm{E}-07$ & $3.16 \mathrm{E}-07$ & $6.27 \mathrm{E}-07$ & .00 & .00 \\
\hline $\mathrm{FE}$ & 5527 & $8.19 \mathrm{E}-08$ & $2.15 \mathrm{E}-07$ & $4.59 \mathrm{E}-07$ & .00 & .00 \\
\hline $\mathrm{FE}$ & $\begin{array}{lll}55 & 28\end{array}$ & $4.76 E-08$ & $1.48 \mathrm{E}-07$ & $3.38 E-07$ & .00 & .00 \\
\hline $\mathrm{FE}$ & 5529 & $2.75 E-08$ & $1.01 E-07$ & $2.48 E-07$ & .00 & .00 \\
\hline $\mathrm{FE}$ & 55210 & $1.59 \mathrm{E}-08$ & $6.87 \mathrm{E}-08$ & $1.81 E-07$ & .00 & .00 \\
\hline $\mathrm{FE}$ & $\begin{array}{ll}55 & 211\end{array}$ & $9.23 E-09$ & $4.71 E-08$ & $1.34 \mathrm{E}-07$ & .00 & .00 \\
\hline $\mathrm{FE}$ & $55 \quad 212$ & $5.33 \mathrm{E}-09$ & $3.21 \mathrm{E}-08$ & $9.77 \mathrm{E}-08$ & .00 & .00 \\
\hline $\mathrm{FE}$ & 55213 & $3.09 E-09$ & $2.20 \mathrm{E}-08$ & $7.20 E-08$ & .00 & .00 \\
\hline $\mathrm{FE}$ & $55 \quad 214$ & $1.79 \mathrm{E}-09$ & $1.50 E-08$ & $5.27 \mathrm{E}-08$ & .00 & .00 \\
\hline $\mathrm{FE}$ & $55 \quad 215$ & $1.04 \mathrm{E}-09$ & $1.03 \mathrm{E}-08$ & $3.88 \mathrm{E}-08$ & .00 & .00 \\
\hline $\mathrm{FE}$ & 55216 & $6.00 \mathrm{E}-10$ & $7.02 \mathrm{E}-09$ & $2.84 \mathrm{E}-08$ & .00 & .00 \\
\hline $\mathrm{FE}$ & $55 \quad 217$ & $3.46 \mathrm{E}-10$ & $4.78 \mathrm{E}-09$ & $2.08 E-08$ & .00 & .00 \\
\hline $\mathrm{FE}$ & $55 \quad 218$ & $2.01 \mathrm{E}-10$ & $3.28 \mathrm{E}-09$ & $1.53 \mathrm{E}-08$ & .00 & .00 \\
\hline $\mathrm{FE}$ & $55 \quad 219$ & $1.16 \mathrm{E}-10$ & $2.23 \mathrm{E}-09$ & $1.12 \mathrm{E}-08$ & .00 & 00 \\
\hline $\mathrm{FE}$ & $55 \quad 220$ & $6.74 \mathrm{E}-11$ & $1.53 \mathrm{E}-09$ & $8.26 \mathrm{E}-09$ & .00 & .00 \\
\hline $\mathrm{FE}$ & $\begin{array}{ll}55 & 221\end{array}$ & 3. $91 \mathrm{E}-11$ & $1.04 \mathrm{E}-09$ & $6.06 \mathrm{E}-09$ & .00 & .00 \\
\hline $\mathrm{FE}$ & $55 \quad 222$ & $2.25 \mathrm{E}-11$ & $7.12 \mathrm{E}-10$ & $4.43 \mathrm{E}-09$ & .00 & .00 \\
\hline $\mathrm{FE}$ & $55 \quad 223$ & 1. $30 \mathrm{E}-11$ & $4.88 \mathrm{E}-10$ & $3.27 \mathrm{E}-09$ & .00 & .00 \\
\hline $\mathrm{FE}$ & $55 \quad 224$ & $7.56 \mathrm{E}-12$ & 3. $32 \mathrm{E}-10$ & $2.39 \mathrm{E}-09$ & .00 & .00 \\
\hline $\mathrm{FE}$ & $55 \quad 225$ & $4.38 \mathrm{E}-12$ & $2.28 \mathrm{E}-10$ & $1.76 \mathrm{E}-09$ & .00 & .00 \\
\hline $\mathrm{FE}$ & $55 \quad 226$ & $2.53 \mathrm{E}-12$ & $1.55 \mathrm{E}-10$ & $1.29 E-09$ & .00 & .00 \\
\hline $\mathrm{FE}$ & $\begin{array}{ll}55 \quad 227\end{array}$ & $1.48 \mathrm{E}-12$ & $1.06 E-10$ & $9.48 \mathrm{E}-10$ & .00 & .00 \\
\hline $\mathrm{FE}$ & $55 \quad 228$ & $8.81 E-13$ & $7.26 \mathrm{E}-11$ & $6.95 \mathrm{E}-10$ & .00 & .00 \\
\hline $\mathrm{FE}$ & $55 \quad 229$ & $4.55 E-13$ & $4.94 \mathrm{E}-11$ & $5.09 \mathrm{E}-10$ & .00 & .00 \\
\hline FE & $55 \quad 230$ & $3.13 E-13$ & $3.40 E-11$ & $3.75 \mathrm{E}-10$ & .00 & .00 \\
\hline $\mathrm{FE}$ & $\begin{array}{ll}55 \quad 231\end{array}$ & $1.42 \mathrm{E}-13$ & $2.31 \mathrm{E}-11$ & $2.74 \mathrm{E}-10$ & .00 & .00 \\
\hline $\mathrm{FE}$ & $55 \quad 232$ & $8.53 \mathrm{E}-14$ & $1.59 \mathrm{E}-11$ & $2.02 \mathrm{E}-10$ & .00 & .00 \\
\hline $\mathrm{FE}$ & $55 \quad 233$ & $5.68 \mathrm{E}-14$ & $1.07 \mathrm{E}-11$ & $1.49 E-10$ & .00 & .00 \\
\hline $\mathrm{FE}$ & 234 & $2.84 \mathrm{E}-14$ & $7.33 \mathrm{E}-12$ & $1.06 \mathrm{E}-10$ & .00 & .00 \\
\hline $\mathrm{FE}$ & 55235 & $2.84 \mathrm{E}-14$ & $5.12 \mathrm{E}-12$ & $8.14 \mathrm{E}-11$ & .00 & .00 \\
\hline FE & $55 \quad 236$ & .00 & $3.47 \mathrm{E}-12$ & $5.91 \mathrm{E}-11$ & .00 & .00 \\
\hline $\mathrm{FE}$ & $\begin{array}{ll}55 \quad 237\end{array}$ & .00 & $2.27 \mathrm{E}-12$ & $4.23 \mathrm{E}-11$ & .00 & .00 \\
\hline FE & 55238 & .00 & $1.65 \mathrm{E}-12$ & $3.18 \mathrm{E}-11$ & .00 & .00 \\
\hline $\mathrm{FE}$ & $\begin{array}{l}55 \quad 239\end{array}$ & .00 & $1.08 \mathrm{E}-12$ & $2.27 \mathrm{E}-11$ & .00 & .00 \\
\hline 5 & 240 & .00 & $7.96 \mathrm{E}-13$ & $1.73 E-11$ & .00 & .00 \\
\hline F & 241 & .00 & $5.12 \mathrm{E}-13$ & $1.18 \mathrm{E}-11$ & .00 & .00 \\
\hline CE & $\begin{array}{lll}55 & 242\end{array}$ & .00 & $3.41 \mathrm{E}-13$ & $9.55 \mathrm{E}-12$ & .00 & .00 \\
\hline
\end{tabular}




\section{Appendix B - Inhalation Dose Rate Factors - FILE23}

\begin{tabular}{|c|c|c|c|c|c|c|c|}
\hline $\mathrm{FE}$ & 55 & 243 & .00 & $1.71 \mathrm{E}-13$ & $6.37 \mathrm{E}-12$ & .00 & .00 \\
\hline $\mathrm{FE}$ & 55 & 244 & .00 & $2.27 \mathrm{E}-13$ & $5.46 \mathrm{E}-12$ & .00 & .00 \\
\hline $\mathrm{FE}$ & 55 & 245 & .00 & $1.14 \mathrm{E}-13$ & $3.64 \mathrm{E}-12$ & .00 & .00 \\
\hline $\mathrm{FE}$ & 55 & 246 & .00 & $5.68 \mathrm{E}-14$ & $2.27 \mathrm{E}-12$ & .00 & .00 \\
\hline $\mathrm{FE}$ & 55 & 247 & .00 & $5.68 \mathrm{E}-14$ & $1.82 \mathrm{E}-12$ & .00 & .00 \\
\hline FE & 55 & 248 & .00 & .00 & $1.36 \mathrm{E}-12$ & .00 & .00 \\
\hline $\mathrm{FE}$ & 55 & 249 & .00 & .00 & $1.36 \mathrm{E}-12$ & .00 & .00 \\
\hline $\mathrm{FE}$ & 55 & 250 & .00 & .00 & $4.55 \mathrm{E}-13$ & .00 & .00 \\
\hline FE59 & & & 4 & 0 & & & \\
\hline $\mathrm{FE}$ & 59 & 21 & $2.82 E-05$ & .00 & $7.22 \mathrm{E}-04$ & .00 & $1.14 \mathrm{E}-04$ \\
\hline $\mathrm{FE}$ & 59 & 22 & $6.50 \mathrm{E}-06$ & .00 & $7.54 \mathrm{E}-05$ & .00 & $1.32 \mathrm{E}-06$ \\
\hline $\mathrm{FE}$ & 59 & 23 & $1.99 \mathrm{E}-08$ & .00 & $2.10 \mathrm{E}-08$ & .00 & $2.55 \mathrm{E}-11$ \\
\hline $\mathrm{FE}$ & 59 & 24 & $5.28 \mathrm{E}-11$ & .00 & $6.55 \mathrm{E}-11$ & .00 & .00 \\
\hline $\mathrm{CO} 57$ & & & 0 & 14 & & & \\
\hline $\mathrm{CO}$ & 57 & 31 & $8.97 \mathrm{E}-07$ & .00 & $3.32 \mathrm{E}-04$ & .00 & $1.36 \mathrm{E}-05$ \\
\hline $\mathrm{CO}$ & 57 & 32 & $7.77 \mathrm{E}-08$ & .00 & $2.83 \mathrm{E}-04$ & .00 & $4.09 \mathrm{E}-07$ \\
\hline $\mathrm{CO}$ & 57 & 33 & $1.28 \mathrm{E}-08$ & .00 & $6.67 \mathrm{E}-05$ & .00 & $9.04 \mathrm{E}-08$ \\
\hline $\mathrm{CO}$ & 57 & 34 & $3.77 \mathrm{E}-09$ & .00 & $1.57 \mathrm{E}-05$ & .00 & $2.13 \mathrm{E}-08$ \\
\hline $\mathrm{CO}$ & 57 & 35 & $1.12 E-09$ & .00 & $3.71 \mathrm{E}-06$ & .00 & $5.03 \mathrm{E}-09$ \\
\hline $\mathrm{CO}$ & 57 & 36 & $3.33 \mathrm{E}-10$ & .00 & $8.78 \mathrm{E}-07$ & .00 & $1.19 \mathrm{E}-09$ \\
\hline $\mathrm{CO}$ & 57 & 37 & $9.93 \mathrm{E}-11$ & .00 & $2.07 \mathrm{E}-07$ & .00 & $2.80 \mathrm{E}-10$ \\
\hline $\mathrm{CO}$ & 57 & 38 & $2.99 \mathrm{E}-11$ & .00 & $4.89 \mathrm{E}-08$ & .00 & $6.63 \mathrm{E}-11$ \\
\hline $\mathrm{CO}$ & 57 & 39 & $8.98 \mathrm{E}-12$ & .00 & $1.15 \mathrm{E}-08$ & .00 & $1.56 \mathrm{E}-11$ \\
\hline $\mathrm{CO}$ & 57 & 310 & $2.71 \mathrm{E}-12$ & .00 & $2.71 \mathrm{E}-09$ & .00 & 3.75 \\
\hline $\mathrm{CO}$ & 57 & 311 & $8.10 \mathrm{E}-13$ & .00 & $6.40 \mathrm{E}-10$ & .00 & $7.96 \mathrm{E}-13$ \\
\hline $\mathrm{CO}$ & 57 & 312 & $2.56 \mathrm{E}-13$ & .00 & $1.53 \mathrm{E}-10$ & .00 & $2.27 \mathrm{E}-13$ \\
\hline $\mathrm{CO}$ & 57 & 313 & $7.11 \mathrm{E}-14$ & .00 & $3.64 \mathrm{E}-11$ & .00 & $1.14 \mathrm{E}-13$ \\
\hline $\mathrm{CO}$ & 57 & 314 & $2.84 \mathrm{E}-14$ & .00 & $7.28 \mathrm{E}-12$ & .00 & .00 \\
\hline $\mathrm{CO60}$ & & & $\begin{array}{ll}0 & 0\end{array}$ & 40 & & & \\
\hline $\mathrm{CO}$ & 60 & 31 & $1.56 \mathrm{E}-05$ & .00 & $5.64 \mathrm{E}-03$ & .00 & $1.51 \mathrm{E}-04$ \\
\hline $\mathrm{CO}$ & 60 & 32 & $2.06 E-06$ & .00 & $7.49 \mathrm{E}-03$ & .00 & $8.51 \mathrm{~F}-06$ \\
\hline $\mathrm{CO}$ & 60 & 33 & $9.57 \mathrm{E}-07$ & .00 & $3.95 \mathrm{E}-03$ & .00 & $4.34 \mathrm{E}-06$ \\
\hline $\mathrm{CO}$ & 60 & 34 & $6.30 \mathrm{E}-07$ & .00 & $2.08 \mathrm{E}-03$ & .00 & $2.29 \mathrm{E}-06$ \\
\hline $\mathrm{CO}$ & 60 & 35 & $4.17 E-07$ & .00 & $1.10 \mathrm{E}-03$ & .00 & $1.21 \mathrm{E}-06$ \\
\hline $\mathrm{CO}$ & 60 & 36 & $2.79 E-07$ & .00 & $5.81 \mathrm{E}-04$ & .00 & 6.38 \\
\hline $\mathrm{CO}$ & 60 & 37 & $1.86 E-07$ & .00 & $3.05 \mathrm{E}-04$ & .00 & 3.35 \\
\hline $\mathrm{CO}$ & 60 & 38 & $1.25 \mathrm{E}-07$ & .00 & $1.62 \mathrm{E}-04$ & .00 & 1.77 \\
\hline $\mathrm{CO}$ & 60 & 39 & $8.41 E-08$ & .00 & $8.51 E-05$ & .00 & 9.35 \\
\hline $\mathrm{CO}$ & 60 & 310 & $5.65 E-08$ & .00 & $4.48 \mathrm{E}-05$ & .00 & 4.92 \\
\hline $\mathrm{CO}$ & 60 & 311 & $3.83 \mathrm{E}-08$ & .00 & 2.37 & .00 & 2.60 \\
\hline $\mathrm{CO}$ & 60 & 312 & $2.58 \mathrm{E}-08$ & .00 & $1.25 \mathrm{E}-05$ & .00 & 1.37 \\
\hline $\mathrm{CO}$ & 60 & 313 & $1.75 \mathrm{E}-08$ & .00 & $6.59 \mathrm{E}-06$ & .00 & 7.24 \\
\hline $\mathrm{CO}$ & 60 & 314 & $1.18 E-08$ & .00 & $3.47 \mathrm{E}-06$ & .00 & 3.81 \\
\hline $\mathrm{CO}$ & 60 & 315 & $8.06 \mathrm{E} \sim 09$ & .00 & $1.83 \mathrm{E}-06$ & .00 & 2.02 \\
\hline $\mathrm{CO}$ & 60 & 316 & $5.46 \mathrm{E}-09$ & .00 & $9.67 \mathrm{E}-07$ & .00 & 1.06 \\
\hline $\mathrm{CO}$ & 60 & 317 & $3.70 \mathrm{E}-09$ & .00 & $5.09 \mathrm{E}-07$ & .00 & 5.57 \\
\hline $\mathrm{CO}$ & 60 & 318 & $2.52 \mathrm{E}-09$ & .00 & $2.69 \mathrm{E}-07$ & .00 & 2.9 \\
\hline $\mathrm{CO}$ & 60 & 319 & $1.70 \mathrm{E}-09$ & .00 & $1.41 \mathrm{E}-07$ & .00 & 1.55 \\
\hline $\mathrm{CO}$ & 60 & 320 & $1.16 \mathrm{E}-09$ & .00 & $7.50 \mathrm{E}-08$ & .00 & 8.19 \\
\hline $\mathrm{CO}$ & 60 & $3 ? 1$ & $7.88 \mathrm{E}-10$ & .00 & $3.96 \mathrm{E}-08$ & .00 & $4.37 \mathrm{E}-11$ \\
\hline
\end{tabular}




\section{Appendix B - Inhalation Dose Rate Factors - FILE23}

\begin{tabular}{|c|c|c|c|c|c|c|}
\hline $\mathrm{CO}$ & $60 \quad 322$ & $5.33 E-10$ & .00 & $2.07 E-08$ & .00 & $2.36 \mathrm{E}-11$ \\
\hline $\mathrm{CO}$ & 60323 & $3.63 \mathrm{E}-10$ & .00 & $1.09 \mathrm{E}-08$ & .00 & $27 \mathrm{E}-11$ \\
\hline $\mathrm{CO}$ & $60 \quad 324$ & $2.46 \mathrm{E}-10$ & .00 & $5.59 E-09$ & .00 & $46 \mathrm{E}-12$ \\
\hline $\mathrm{CO}$ & $60 \quad 325$ & 1. $68 \mathrm{E}-10$ & .00 & $3.26 \mathrm{E}-09$ & .00 & -12 \\
\hline $\mathrm{CO}$ & $60 \quad 326$ & 1. $14 \mathrm{E}-10$ & .00 & $1.63 \mathrm{E}-09$ & .00 & $1.82 \mathrm{E}-12$ \\
\hline $\mathrm{CO}$ & $\begin{array}{ll}60 & 327\end{array}$ & $7.73 \mathrm{E}-11$ & .00 & $6.98 \mathrm{E}-10$ & .00 & .00 \\
\hline $\mathrm{CO}$ & 60328 & $5.30 \mathrm{E}-11$ & .00 & $2.33 \mathrm{E}-10$ & .00 & $1.82 E-12$ \\
\hline $\mathrm{CO}$ & $60 \quad 329$ & $3.55 \mathrm{E}-11$ & .00 & $4.66 \mathrm{E}-10$ & .00 & .00 \\
\hline $\mathrm{CO}$ & 60330 & $2.41 \mathrm{E}-11$ & .00 & .00 & .00 & .00 \\
\hline $\mathrm{CO}$ & $60 \quad 331$ & $1.64 \mathrm{E}-11$ & .00 & .00 & .00 & .00 \\
\hline $\mathrm{CO}$ & $60 \quad 332$ & $1.14 \mathrm{E}-11$ & .00 & .00 & .00 & .00 \\
\hline $\mathrm{CO}$ & $60 \quad 333$ & $7.50 \mathrm{E}-12$ & .00 & .00 & .00 & .00 \\
\hline $\mathrm{CO}$ & $60 \quad 334$ & $5.23 \mathrm{E}-12$ & .00 & .00 & .00 & .00 \\
\hline $\mathrm{CO}$ & 60335 & $3.64 \mathrm{E}-12$ & .00 & .00 & .00 & .00 \\
\hline $\mathrm{CO}$ & 60336 & $2.50 \mathrm{E}-12$ & .00 & .00 & .00 & .00 \\
\hline $\mathrm{CO}$ & $60 \quad 337$ & $1.59 \mathrm{E}-12$ & .00 & .00 & .00 & .00 \\
\hline $\mathrm{CO}$ & 60338 & $1.14 \mathrm{E}-12$ & .00 & .00 & .00 & .00 \\
\hline $\mathrm{CO}$ & 60339 & $6.82 \mathrm{E}-13$ & .00 & .00 & .00 & .00 \\
\hline $\mathrm{CO}$ & 60340 & $4.55 \mathrm{E}-13$ & .00 & .00 & .00 & .00 \\
\hline NI59 & & 0 & 0 & & & \\
\hline NI59 & 21 & $1.55 \mathrm{E}-06$ & $7.92 E-06$ & $2.37 E-05$ & $0.00 E+00$ & $2.60 \mathrm{E}-\mathrm{C}$ \\
\hline NI59 & 22 & $2.72 \mathrm{E}-06$ & $1.44 \mathrm{E}$ & 5.6 & $0.00 \mathrm{E}+00$ & 1.06 \\
\hline NI59 & 23 & $1.90 \mathrm{E}-06$ & 1.07 & 3.5 & $0.00 \mathrm{E}+00$ & 6.4 \\
\hline NI59 & 24 & $1.30 \mathrm{E}-06$ & 7.7 & 2.2 & $0.00 \mathrm{E}+00$ & 3.8 \\
\hline NI59 & 25 & $8.89 \mathrm{E}-07$ & 5.6 & $1.82 \mathrm{E}-12$ & $0.00 E+00$ & 0.00 \\
\hline NI59 & 26 & $6.09 \mathrm{E}-07$ & 4.1 & $0.00 \mathrm{E}+00$ & $0.00 \mathrm{E}+00$ & 0.00 \\
\hline NI59 & 27 & $4.15 \mathrm{E}-07$ & 3.0 & $0.00 \mathrm{E}+00$ & $0.00 \mathrm{E}+00$ & 0.00 \\
\hline NI59 & 28 & $2.85 \mathrm{E}-07$ & 2.20 & $0.00 \mathrm{E}+00$ & $0.00 \mathrm{E}+00$ & 0.00 \\
\hline NI59 & 29 & $1.95 \mathrm{E}-07$ & 1.60 & $0.00 \mathrm{E}+00$ & $0.00 E+00$ & 0.00 \\
\hline NI59 & 210 & $1.33 \mathrm{E}-07$ & $1.16 \mathrm{~B}$ & $0.00 \mathrm{E}+00$ & $0.00 E+00$ & 0.00 \\
\hline NI59 & 211 & $9.13 \mathrm{E}-08$ & $8.51 \mathrm{l}$ & $0.00 \mathrm{E}+00$ & $0.00 \mathrm{E}+00$ & 0.00 \\
\hline NI59 & 212 & $6.23 \mathrm{E}-08$ & 6.18 & $0.00 \mathrm{E}+00$ & $0.00 \mathrm{E}+00$ & 0.00 \\
\hline NI59 & 213 & $4.27 \mathrm{E}-08$ & 4.52 & $0.00 \mathrm{E}+00$ & $0.00 \mathrm{E}+00$ & 0.008 \\
\hline NI59 & 214 & $2.92 \mathrm{E}-08$ & 3.28 & $0.00 \mathrm{E}+00$ & $0.00 \mathrm{E}+00$ & 0.0 \\
\hline NI59 & 215 & $2.00 \mathrm{E}-08$ & 2.40 & $0.00 \mathrm{E}+00$ & $0.00 \mathrm{E}+00$ & 0.0 \\
\hline & 216 & $1.37 \mathrm{E}-08$ & 1.75 & $0.00 \mathrm{E}+00$ & $0.00 \mathrm{E}+00$ & 0.00 \\
\hline & 217 & 09 & $1.27 \mathrm{E}$ & $0.00 \mathrm{E}+00$ & $0.00 \mathrm{E}+00$ & 0.00 \\
\hline & 218 & $6.40 \mathrm{E}-09$ & 9.28 & $0.00 E+00$ & $0.00 E+00$ & 0.00 \\
\hline & & -09 & $6.74 \mathrm{E}-08$ & $0.00 E+00$ & $0.00 \mathrm{E}+00$ & 0.00 \\
\hline & 22 & -09 & $4.93 \mathrm{E}$ & $0.00 \mathrm{E}+00$ & $0.00 \mathrm{E}+00$ & 0.00 \\
\hline & 2 & -09 & $3.59 \mathrm{E}-08$ & $0.00 E+00$ & $0.00 \mathrm{E}+00$ & 0.0 \\
\hline & 22 & -09 & $2.61 \mathrm{E}-08$ & $0.00 \mathrm{E}+00$ & $0.00 \mathrm{E}+00$ & $0.00 E+00$ \\
\hline $\mathrm{N}]$ & 2 & 9 & $1.91 \mathrm{E}-08$ & $0.00 \mathrm{E}+00$ & $0.00 E+00$ & $0.00 E+00$ \\
\hline $\mathrm{NI}$ & 2 & 6 & 1.39 & $0.00 \mathrm{E}+00$ & $0.00 E+00$ & $0.00 \mathrm{E}+00$ \\
\hline & 2 & & $1.01 \mathrm{E}-08$ & $0.00 \mathrm{E}+00$ & $0.00 \mathrm{E}+00$ & $0.00 \mathrm{E}+00$ \\
\hline & 2 & & $7.36 \mathrm{E}-09$ & $0.00 \mathrm{E}+00$ & $0.00 \mathrm{E}+00$ & $0.00 \mathrm{E}+0$ \\
\hline & 2 & 2. & $5.38 \mathrm{E}-09$ & $0.00 \mathrm{E}+00$ & $0.00 \mathrm{E}+00$ & $0.00 E+00$ \\
\hline & 22 & & 3.93 & $0.00 \mathrm{E}+00$ & $0.00 E+00$ & $0.00 E+00$ \\
\hline & 22 & & 2.8 & $0.00 \mathrm{E}+00$ & $0.00 \mathrm{E}+00$ & $0.00 \mathrm{E}+0$ \\
\hline 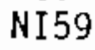 & 23 & $3 E-11$ & $2.08 E-09$ & $0.00 \mathrm{E}+00$ & $0.00 \mathrm{E}+00$ & $0.00 \mathrm{E}+0$ \\
\hline
\end{tabular}




\section{Appendix B - Inhalation Dose Rate Factors - FILE23}

\begin{tabular}{|c|c|c|c|c|c|c|}
\hline NI59 & 231 & $4.55 \mathrm{E}-11$ & $1.51 \mathrm{E}-09$ & $0.00 E+00$ & $0.00 E+00$ & $0.00 \mathrm{E}+00$ \\
\hline NI59 & 232 & $3.18 E-11$ & $1.11 \mathrm{E}-09$ & $0.00 \mathrm{E}+00$ & $0.00 E+00$ & $0.00 \mathrm{E}+00$ \\
\hline NI59 & 233 & $2.18 E-11$ & $8.15 \mathrm{E}-10$ & $0.00 \mathrm{E}+00$ & $0.00 E+00$ & $0.00 \mathrm{E}+00$ \\
\hline NI59 & 234 & $1.46 \mathrm{E}-11$ & $5.68 \mathrm{E}-10$ & $0.00 E+00$ & $0.00 \mathrm{E}+00$ & $0.00 \mathrm{E}+00$ \\
\hline NI59 & 235 & $1.00 \mathrm{E}-11$ & $4.29 \mathrm{E}-10$ & $0.00 E+00$ & $0.00 \mathrm{E}+00$ & $0 E+00$ \\
\hline NI59 & 236 & $7.28 \mathrm{E}-12$ & $3.20 \mathrm{E}-10$ & $0.00 \mathrm{E}+00$ & $0.00 E+00$ & 0 \\
\hline NI59 & 237 & $4.55 \mathrm{E}-12$ & $2.26 \mathrm{E}-10$ & $0.00 E+00$ & $0.00 \mathrm{E}+00$ & 100 \\
\hline NI59 & 238 & $3.64 \mathrm{E}-12$ & $1.67 \mathrm{E}-10$ & $0 E+00$ & $0 E+00$ & 100 \\
\hline NI59 & 239 & $1.82 \mathrm{E}-12$ & 1.0 & $0 \mathrm{E}+00$ & $0 \mathrm{E}+00$ & $0 E+00$ \\
\hline NI59 & 240 & $E-13$ & $6 \mathrm{E}-11$ & $0 E+00$ & $0 \mathrm{E}+00$ & +0 \\
\hline NI59 & 241 & -13 & $5 E-11$ & $0.00 E+00$ & $0 E+00$ & +0 \\
\hline NI59 & 242 & 9. & 11 & $0 \mathrm{E}+00$ & 00 & 0 \\
\hline NI59 & 243 & 9. & 11 & 0. & 0. & 0 \\
\hline NI59 & 244 & $E+00$ & 2 & 0.0 & 0 . & 0 \\
\hline NI59 & 245 & $E+00$ & 1. & 0.0 & 100 & 0 \\
\hline NI59 & 246 & 9. & 1 . & $0.00 E+00$ & $0 E+00$ & 0 \\
\hline NI59 & 247 & $0.00 \mathrm{E}+00$ & $7.28 \mathrm{E}-12$ & $0.00 \mathrm{E}+00$ & $0.00 \mathrm{E}+00$ & 0. \\
\hline NI59 & 248 & $0.00 \mathrm{E}+00$ & $7.28 \mathrm{E}-12$ & $0.00 \mathrm{E}+00$ & $E+00$ & 0. \\
\hline NI59 & 249 & $0.00 \mathrm{E}+00$ & $7.28 \mathrm{E}-12$ & $0.00 \mathrm{E}+00$ & 0.0 & 0. \\
\hline NI59 & 250 & $0.00 \mathrm{E}+00$ & $7.28 \mathrm{E}-12$ & $0.00 \mathrm{E}+00$ & $0.00 \mathrm{E}+00$ & $0.00 E+0$ \\
\hline NI 63 & & 0 & & & & \\
\hline NI63 & 21 & $4.22 E-06$ & $1.08 \mathrm{E}-04$ & $6.45 \mathrm{E}-05$ & $0.00 E+00$ & $7.08 \mathrm{E}-\mathrm{C}$ \\
\hline NI63 & 22 & $7.36 \mathrm{E}-06$ & 1.94 & 1 & $0.00 E+00$ & 2.85 \\
\hline NI63 & 23 & $5.11 \mathrm{E}-06$ & $1.44 \mathrm{E}-04$ & 9.6 & $0.00 \mathrm{E}+00$ & 1.73 \\
\hline NI63 & 24 & 3.47 & 1.0 & 6.0 & $0.00 \mathrm{E}+00$ & 1.0 \\
\hline NI63 & 25 & 2.36 & 7.51 & $0.00 E+00$ & $0.00 \mathrm{E}+00$ & $0.00 E+00$ \\
\hline NI6 & 26 & $1.60 \mathrm{E}-06$ & 5.4 & $0.00 E+00$ & $0.00 \mathrm{E}+00$ & $0.00 E+00$ \\
\hline NI6 & 27 & 1.09 & 3.9 & $0.00 E+00$ & $0.00 \mathrm{E}+00$ & $0.00 E+00$ \\
\hline NI6 & 28 & 7.40 & 2.8 & +00 & $0.00 E+00$ & 0. \\
\hline N16 & 29 & 5.02 & 2. & +00 & $0.00 \mathrm{E}+00$ & $0.00 E+00$ \\
\hline NI6 & 210 & 3.4 & 1.45 & $0.00 E+00$ & $0.00 \mathrm{E}+00$ & $0.00 \mathrm{E}+00$ \\
\hline NI6 & 211 & 2.32 & 1.08 & $0.00 \mathrm{E}+00$ & $0.00 E+00$ & $0.00 E+00$ \\
\hline NI6 & 212 & 1.57 & 7.7 & $0.00 E+00$ & $0.00 E+00$ & $0.00 \mathrm{E}+00$ \\
\hline NI6 & 213 & $1.07 \mathrm{E}-07$ & $5.65 \mathrm{E}-06$ & $0.00 \mathrm{E}+00$ & $0.00 \mathrm{E}+00$ & $0.00 \mathrm{E}+00$ \\
\hline NI6 & 214 & $7.24 \mathrm{E}$ & $4.08 \mathrm{E}-06$ & $0.00 E+00$ & $0.00 E+00$ & $0.00 \mathrm{E}+00$ \\
\hline NIE & 215 & 4.94 & $2.96 \mathrm{E}-06$ & $0.00 \mathrm{E}+00$ & $0.00 \mathrm{E}+00$ & $0.00 \mathrm{E}+00$ \\
\hline NI & 216 & 3.3 & 06 & $0.00 \mathrm{E}+00$ & $0.00 \mathrm{E}+00$ & $0.00 \mathrm{E}+00$ \\
\hline NIt & 217 & 2 & 1. & $0.00 \mathrm{E}+00$ & $0.00 \mathrm{E}+00$ & $0.00 \mathrm{E}+00$ \\
\hline NI & 218 & 1. & 1. & 0.0 & $0.00 \mathrm{E}+00$ & 0.00 \\
\hline NI & 219 & & 8. & 0. & $0.00 \mathrm{E}+00$ & $0.00 \mathrm{E}+\mathrm{C}$ \\
\hline NI6 & 220 & & 07 & 0. & $0.00 \mathrm{E}+00$ & $0.00 E+0$ \\
\hline NIE & 221 & & -07 & 0 & $0.00 E+00$ & 0.00 \\
\hline NIt & 222 & & -07 & 0 . & $0.00 E+00$ & 0.00 \\
\hline NIt & 223 & & -07 & 0. & $0.00 \mathrm{E}+00$ & 0.00 \\
\hline NIE & 224 & & 1 & 0. & $0.00 \mathrm{E}+00$ & $0.00 \mathrm{E}$ \\
\hline NIt & 225 & & -07 & $0 E+00$ & $0.00 E+00$ & 0.00 \\
\hline NI & 226 & & & 0 . & $0.00 \mathrm{E}$ & 0.0 \\
\hline NI & 227 & & & 0.0 & $0.00 E+00$ & $0.00 E-$ \\
\hline NIt & 228 & & & & $0.00 E+00$ & $0.00 \mathrm{E}+0$ \\
\hline NI6 & 229 & 2. & & & $0.00 \mathrm{E}+00$ & $0.00 \mathrm{E}+0$ \\
\hline
\end{tabular}


Appendix B - Inhalation Dose Rate Factors - FILE23

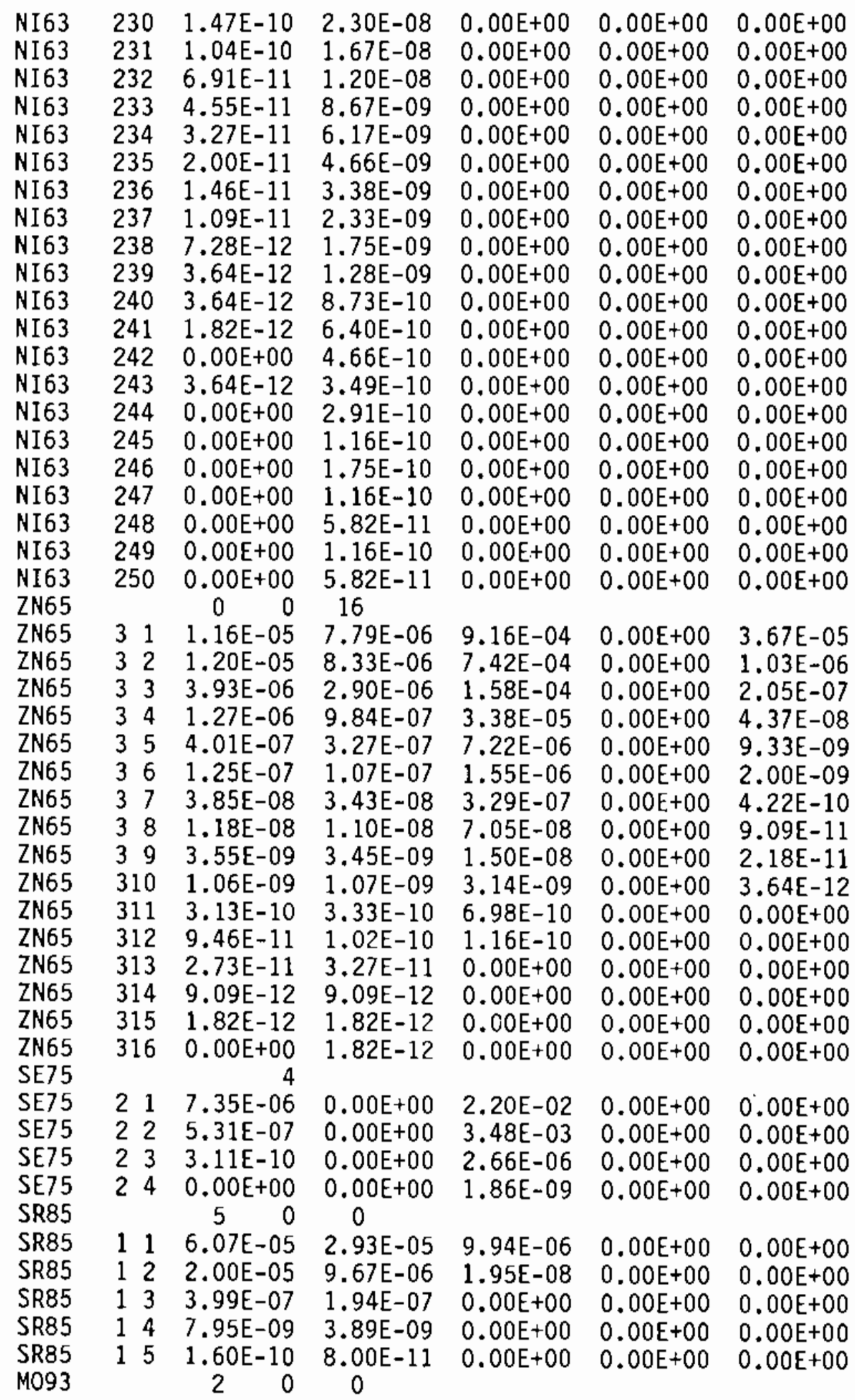


Appendix B - Inhalation Dose Rate Factors - FILE23

\begin{tabular}{|c|c|c|c|c|c|c|}
\hline $\begin{array}{l}\text { M093 } \\
\text { M093 }\end{array}$ & $\begin{array}{ll}1 & 1 \\
1 & 2\end{array}$ & $\begin{array}{l}1.03 \mathrm{E}-06 \\
2.18 \mathrm{E}-08\end{array}$ & $\begin{array}{l}0.00 \mathrm{E}+00 \\
0.00 \mathrm{E}+00\end{array}$ & $\begin{array}{l}3.01 \mathrm{E}-06 \\
5.95 \mathrm{E}-09\end{array}$ & $\begin{array}{l}0.00 E+00 \\
0.00 E+00\end{array}$ & $\begin{array}{l}1.46 \mathrm{E}-06 \\
8.70 \mathrm{E}-11\end{array}$ \\
\hline NB94 & & 0 & 50 & & & \\
\hline NB94 & 31 & $5.14 \mathrm{E}-06$ & $1.21 \mathrm{E}-05$ & $3.88 \mathrm{E}-03$ & $0.00 E+00$ & $1.37 E-04$ \\
\hline NB94 & 32 & $1.55 \mathrm{E}-05$ & $3.85 \mathrm{E}-05$ & $5.59 \mathrm{E}-03$ & $0.00 \mathrm{E}+00$ & $8.66 \mathrm{E}-06$ \\
\hline NB94 & 33 & $2.14 \mathrm{E}-05$ & $5.51 \mathrm{E}-05$ & $3.37 \mathrm{E}-03$ & $0.00 \mathrm{E}+00$ & $5.07 \mathrm{E}-06$ \\
\hline NB94 & 34 & $2.47 \mathrm{E}-05$ & 6. & $2.03 \mathrm{E}-03$ & $0.00 \mathrm{E}+00$ & 3.05 \\
\hline NB94 & 35 & $2.59 \mathrm{E}-05$ & 7.0 & $1.22 \mathrm{E}-03$ & $0.00 \mathrm{E}+00$ & 1.84 \\
\hline NB94 & 36 & $2.54 \mathrm{E}-05$ & $7.18 \mathrm{E}-05$ & $7.38 \mathrm{E}-04$ & $0.00 \mathrm{E}+00$ & 1.11 \\
\hline NB94 & 7 & $2.37 \mathrm{E}-05$ & $6.93 \mathrm{E}-05$ & $4.43 \mathrm{E}-04$ & $0.00 \mathrm{E}+00$ & 6 \\
\hline NB94 & 8 & $2.16 \mathrm{E}-05$ & $6.50 \mathrm{E}$ & $2.68 \mathrm{E}-04$ & $0.00 E+00$ & 4.03 \\
\hline NB94 & 9 & $1.91 \mathrm{E}-05$ & 5.9 & $1.61 \mathrm{E}-04$ & $0.00 \mathrm{E}+00$ & 2 \\
\hline NB94 & 310 & $1.65 \mathrm{E}$ & $5.30 E$ & $9.69 \mathrm{E}-05$ & $0.00 E+00$ & 1.46 \\
\hline NB94 & 311 & $1.42 \mathrm{E}-05$ & $4.69 \mathrm{E}-05$ & $5.86 \mathrm{E}-05$ & $0.00 E+00$ & 8.82 \\
\hline NB94 & 312 & $1.19 \mathrm{E}-05$ & $4.06 \mathrm{t}$ & $3.52 E-05$ & $0.00 \mathrm{E}+00$ & 5. \\
\hline NB94 & 313 & $9.98 \mathrm{E}-06$ & $3.51 \mathrm{E}-05$ & $2.13 E-05$ & $0.00 E+00$ & $3.20 E-08$ \\
\hline NB9 & 314 & $8.23 \mathrm{E}-06$ & $2.98 \mathrm{E}$ & $1.28 \mathrm{E}-05$ & $0.00 E+00$ & 1.5 \\
\hline NB9 & 315 & $6.78 \mathrm{E}-06$ & 2.54 & $7.73 \mathrm{E}-06$ & 0.0 & 1.1 \\
\hline$N B S$ & 316 & $5.53 \mathrm{E}-06$ & $2.13 E-05$ & $4.65 \mathrm{E}-06$ & $0.00 E+00$ & 7 \\
\hline NBS & 317 & $4.47 \mathrm{E}-06$ & 1.78 & $2.80 \mathrm{E}-06$ & 0.0 & 4. \\
\hline NBS & 318 & $3.62 \mathrm{E}-06$ & $1.48 \mathrm{E}-05$ & $1.69 \mathrm{E}-06$ & $0.00 \mathrm{E}+00$ & 2. \\
\hline NBS & 319 & $2.90 \mathrm{E}-06$ & 1.23 & $1.02 \mathrm{E}-06$ & 0.0 & 1 \\
\hline NB9 & 320 & $2.33 \mathrm{E}-06$ & $1.02 \mathrm{E}$ & $6.15 \mathrm{E}$ & $0.00 \mathrm{E}+00$ & 9 \\
\hline NBS & 321 & $1.86 \mathrm{E}-06$ & 8.35 & 3.69 & $0.00 \mathrm{E}+00$ & 5 \\
\hline NBS & 322 & $1.48 \mathrm{E}-06$ & 6.82 & $2.24 \mathrm{E}$ & $0.00 \mathrm{E}+00$ & 3 \\
\hline NB & 323 & $1.18 \mathrm{E}-06$ & $5.60 \mathrm{E}$ & 1.34 & $0.00 E+00$ & 1. \\
\hline NBS & 324 & $9.32 \mathrm{E}-07$ & $4.55 \mathrm{E}$ & $8.20 \mathrm{E}-08$ & $0.00 \mathrm{E}+00$ & 1 \\
\hline NB & 325 & $7.40 \mathrm{E}-07$ & $3.71 \mathrm{l}$ & $4.66 \mathrm{E}$ & $0.00 E+00$ & \\
\hline NBC & 326 & $5.83 \mathrm{E}-07$ & $3.01 \mathrm{E}$ & 2.98 & 0.0 & \\
\hline NB & 327 & $4.61 \mathrm{E}-\mathrm{C}$ & $2.44 \mathrm{E}$ & 1.86 & $0.00 \mathrm{~B}$ & 2 \\
\hline NB & 328 & $E-07$ & 1.9 & 9. & 0.0 & 1. \\
\hline NBS & 329 & 2.8 & 1.59 & 7. & 0.1 & 1. \\
\hline NBS & 330 & $2.24 \mathrm{E}-07$ & 1.29 & 3. & $0 .(1$ & 1 . \\
\hline NBS & 331 & $1.76 \mathrm{E}-07$ & 1.03 & 1. & $E+00$ & 0. \\
\hline NBS & 332 & $1.38 \mathrm{E}-07$ & 8.3 & 1.8 & $E+0$ & 0. \\
\hline NB & & $1.08 \mathrm{E}-07$ & 6.7 & 1.8 & 0.1 & 0. \\
\hline & & $8.32 \mathrm{E}$ & 5.28 & $E+00$ & $0.00 \mathrm{E}+0$ & 0. \\
\hline & & $6.72 \mathrm{E}$ & 4.37 & $0.00 \mathrm{E}+00$ & $0.00 \mathrm{E}+0$ & 0. \\
\hline & & $5.23 \mathrm{E}$ & 3.4 & $0.00 \mathrm{E}+00$ & $0.00 \mathrm{E}+0$ & 0.1 \\
\hline & & 3 & 2.7 & $0.00 \mathrm{E}+00$ & $0.00 \mathrm{E}+0$ & $0.00 \mathrm{E}+00$ \\
\hline & & 3 & 2.2 & $0.00 \mathrm{E}+00$ & $0.00 \mathrm{E}+00$ & 0. \\
\hline & & & 1. & $0.00 \mathrm{E}+00$ & $0.00 \mathrm{E}+00$ & 0 \\
\hline & & & & $0.00 \mathrm{E}+00$ & $0.00 \mathrm{E}+00$ & $00 \mathrm{E}+00$ \\
\hline & & & $1.10 \mathrm{E}$ & $0.00 \mathrm{E}+00$ & $0.00 \mathrm{E}+00$ & $0.00 E+00$ \\
\hline & & 1. & $9.06 \mathrm{E}-08$ & $0.00 \mathrm{E}+00$ & $0.00 \mathrm{E}+00$ & $0.00 \mathrm{E}+00$ \\
\hline & & 8 & $8 E-08$ & $0.00 \mathrm{E}+00$ & $0.00 \mathrm{E}+00$ & $0.00 \mathrm{E}+00$ \\
\hline & & 9 & & $0.00 E+00$ & $0.00 E+00$ & $0.00 E+00$ \\
\hline & & 5. & $4.56 E-$ & $0.00 E+00$ & $0.00 E+00$ & $0.00 E+00$ \\
\hline & & & $1 E-$ & $0.00 E+00$ & $0.00 \mathrm{E}+00$ & $0.00 \mathrm{E}+00$ \\
\hline & & 09 & $2.88 \mathrm{E}-08$ & $0.00 \mathrm{E}+00$ & $0.00 E+00$ & $0.00 \mathrm{E}+00$ \\
\hline
\end{tabular}




\section{Appendix B - Inhalation Dose Rate Factors - FILE23}

\begin{tabular}{|c|c|c|c|c|c|c|}
\hline $\begin{array}{l}\text { NB94 } \\
\text { NB94 } \\
\text { NB94 }\end{array}$ & $\begin{array}{l}348 \\
349 \\
350\end{array}$ & $\begin{array}{l}2.56 \mathrm{E}-09 \\
2.07 \mathrm{E}-09 \\
1.57 \mathrm{E}-09\end{array}$ & $\begin{array}{l}2.21 \mathrm{E}-08 \\
1.82 \mathrm{E}-08 \\
1.40 \mathrm{E}-08\end{array}$ & $\begin{array}{l}0.00 E+00 \\
0.00 E+00 \\
0.00 E+00\end{array}$ & $\begin{array}{l}0.00 E+00 \\
0.00 E+00 \\
0.00 E+00\end{array}$ & $\begin{array}{l}0.00 \mathrm{E}+00 \\
0.00 \mathrm{E}+00 \\
0.00 \mathrm{E}+00\end{array}$ \\
\hline IN111 & & 20 & 0 & & & \\
\hline IN111 & 11 & $2.36 \mathrm{E}-06$ & $4.27 \mathrm{E}-06$ & $8.24 E-06$ & $9.56 \mathrm{E}-07$ & $1.38 \mathrm{E}-\mathrm{c}$ \\
\hline IN111 & 12 & $2.72 E-08$ & $4.96 \mathrm{E}-08$ & $8 E-08$ & & $7.86 \mathrm{E}$ \\
\hline SB124 & & 4 & 0 & & & \\
\hline SB 124 & 21 & $2.63 E-05$ & $5.70 \mathrm{E}-05$ & $1.78 \mathrm{E}-03$ & $1.72 \mathrm{E}-07$ & $2.94 E-04$ \\
\hline SB 124 & 22 & $3.61 \mathrm{E}-06$ & $1.22 \mathrm{E}-05$ & $2.15 \mathrm{E}-04$ & $5 E-09$ & \\
\hline SB 124 & 23 & 8.2 & $2.23 \mathrm{E}-08$ & -08 & $1.21 E-12$ & $3.49 E-10$ \\
\hline SB 124 & 24 & 2.27 & $2.73 \mathrm{E}-11$ & $2.91 \mathrm{E}-11$ & .00 & .00 \\
\hline I 125 & & & 0 & & & \\
\hline I 125 & 11 & $1.47 \mathrm{E}-05$ & $6.85 \mathrm{E}-06$ & $4.08 E-06$ & $1.20 \mathrm{E}-02$ & $4.27 E-07$ \\
\hline I 125 & 12 & $4.20 \mathrm{E}-06$ & $3.28 \mathrm{E}-07$ & $0 E-09$ & -03 & $2.54 \mathrm{E}-11$ \\
\hline I 125 & 13 & $5.03 E-08$ & $0.00 \mathrm{E}+00$ & $0.00 E+00$ & $4.12 E-05$ & $0.00 E+00$ \\
\hline I 125 & 14 & $6.00 E-10$ & $0.00 \mathrm{E}+00$ & $0.00 E+00$ & $E-07$ & $0.00 \mathrm{E}+00$ \\
\hline I 125 & 15 & $7.28 \mathrm{E}-12$ & $0.00 \mathrm{E}+00$ & $0.00 E+00$ & -09 & $0.00 \mathrm{E}+0$ \\
\hline CS136 & & 20 & 0 & & & \\
\hline $\operatorname{CS} 136$ & 11 & $3.08 E-05$ & $1.48 \mathrm{E}-05$ & $2.49 \mathrm{E}-05$ & .00 & $1.78 \mathrm{E}-06$ \\
\hline CS 136 & 12 & $1.43 E-06$ & $7.42 \mathrm{E}-07$ & $2.30 \mathrm{E}-07$ & .00 & 1.05 \\
\hline EU1 & & 0 & 0 & & & \\
\hline $\begin{array}{ll}E 0152 \\
5\end{array}$ & & 5. & 1.4 & $1.01 \mathrm{E}-03$ & $E+00$ & 05 \\
\hline EU. & 22 & 9.65 & $2.89 E-04$ & 2. & 00 & 06 \\
\hline & 2 & 6.39 & $2.39 \mathrm{E}-04$ & $1.42 \mathrm{E}-06$ & 00 & 08 \\
\hline & & 4.0 & $1.92 \mathrm{E}-04$ & $8.50 E-09$ & $E+00$ & 10 \\
\hline & 25 & 2.6 & 1. & $0.00 E+00$ & $E+00$ & \\
\hline & 26 & 1.6 & 1.24 & $0.00 E+00$ & $E+00$ & 00 \\
\hline & 2 & 1. & 9. & $0.00 E+00$ & $E+00$ & 00 \\
\hline & 8 & $6.76 \mathrm{E}$ & 7. & $0.00 \mathrm{E}+00$ & +00 & 0. \\
\hline & 2 & 4.5 & 6. & $0.00 \mathrm{E}+00$ & $E+00$ & 0 . \\
\hline & 21 & 2.74 & 5. & $0 \mathrm{E}+00$ & +00 & 00 \\
\hline & 21. & 1.75 & 4. & $0 E+00$ & 00 & 0. \\
\hline & 21 & 1. & 5 & 00 & 0.0 & 0. \\
\hline & 21 & 7 . & 2 & +00 & 0.0 & 0. \\
\hline & 21 & 4. & & 00 & 0. & 00 \\
\hline EU & 215 & & & 00 & 00 & 00 \\
\hline EU & 216 & & 1. & 10 & 00 & 00 \\
\hline EU & 217 & & & 00 & & \\
\hline EU & 218 & & & -00 & 00 & 00 \\
\hline EU & 219 & 4 & & 00 & 00 & \\
\hline & 220 & 3 & & +00 & +00 & -00 \\
\hline & 221 & 1. & & $E+00$ & +00 & $10 E+00$ \\
\hline El & 222 & 1. & 06 & $E+00$ & $0.00 E+00$ & $0.00 E+00$ \\
\hline & 223 & 09 & -06 & $0.00 E+00$ & $0.00 E+00$ & $0.00 E+00$ \\
\hline & 224 & -09 & $7 \mathrm{E}-06$ & $0.00 \mathrm{E}+00$ & $0.00 E+00$ & $0.00 \mathrm{E}+00$ \\
\hline & 225 & -09 & $0 \mathrm{E}-06$ & $0.00 E+00$ & $0.00 \mathrm{E}+00$ & $0.00 E+00$ \\
\hline & 226 & $2.07 E-09$ & $2 E-06$ & $0.00 E+00$ & $0 E+00$ & 0. \\
\hline & 227 & -09 & -06 & $0.00 \mathrm{E}+00$ & +00 & \\
\hline & 228 & $8.44 \mathrm{E}-10$ & 9. & 0.1 & 0. & 0. \\
\hline & 229 & $=-10$ & & $=00$ & $0.00 E+00$ & $0.00 E+00$ \\
\hline
\end{tabular}


Appendix B - Inhalation Dose Rate Factors - FILE23

\begin{tabular}{|c|c|c|c|c|c|c|}
\hline EU152 & 230 & $2.91 \mathrm{E}-10$ & $6.35 \mathrm{E}-07$ & $0.00 E+00$ & $0.00 E+00$ & $0.00 \mathrm{E}+00$ \\
\hline EU152 & 231 & $2.33 \mathrm{E}-10$ & $5.08 \mathrm{E}-07$ & $0.00 \mathrm{E}+00$ & $0.00 \mathrm{E}+00$ & $0.00 \mathrm{E}+00$ \\
\hline EU152 & 232 & $1.46 \mathrm{E}-10$ & $4.09 E-07$ & $0.00 \mathrm{E}+00$ & $0.00 \mathrm{E}+00$ & $0.00 \mathrm{E}+00$ \\
\hline EU152 & 233 & $8.73 \mathrm{E}-11$ & $3.29 E-07$ & $0.00 E+00$ & $0.00 \mathrm{E}+00$ & $0.00 E+00$ \\
\hline EU152 & 234 & $5.82 \mathrm{E}-11$ & $2.59 \mathrm{E}-07$ & $0.00 \mathrm{E}+00$ & $0.00 \bar{E}+00$ & $0.00 \mathrm{E}+00$ \\
\hline EU152 & 235 & $2.91 \mathrm{E}-11$ & $2.15 \mathrm{E}-07$ & $0.00 E+00$ & $0.00 \mathrm{E}+00$ & $0.00 \mathrm{E}+00$ \\
\hline EU152 & 236 & $2.91 \mathrm{E}-11$ & $1.72 \mathrm{E}-07$ & $0.00 \mathrm{E}+00$ & $0.00 \mathrm{E}+00$ & $0.00 E+00$ \\
\hline EU152 & 237 & $2.91 \mathrm{E}-11$ & $1.34 \mathrm{E}-07$ & $0.00 \mathrm{E}+00$ & $0.00 \mathrm{E}+00$ & $0.00 E+00$ \\
\hline EU152 & 238 & $0.00 \mathrm{E}+00$ & $1.11 \mathrm{E}-07$ & $0.00 \mathrm{E}+00$ & $0.00 \mathrm{E}+00$ & $0.00 E+00$ \\
\hline EU152 & 239 & $0.00 \mathrm{E}+00$ & $8.61 \mathrm{E}-08$ & $0.00 \mathrm{E}+00$ & $0.00 \mathrm{E}+00$ & $0.00 \mathrm{E}+00$ \\
\hline EU152 & 240 & $0.00 \mathrm{E}+00$ & $7.16 \mathrm{E}-08$ & $0.00 \mathrm{E}+00$ & $0.00 \mathrm{E}+00$ & $0.00 \mathrm{E}+00$ \\
\hline EU152 & 241 & $2.91 \mathrm{E}-11$ & $5.55 \mathrm{E}-08$ & $0.00 \mathrm{E}+00$ & $0.00 \mathrm{E}+00$ & $0.00 \mathrm{E}+00$ \\
\hline EU152 & 242 & $0.00 \mathrm{E}+00$ & $4.61 \mathrm{E}-08$ & $0.00 E+00$ & $0.00 E+00$ & $0.00 \mathrm{E}+00$ \\
\hline EU152 & 243 & $0.00 \mathrm{E}+00$ & $3.57 \mathrm{E}-08$ & $0.00 \mathrm{E}+00$ & $0.00 E+00$ & $0.00 \mathrm{E}+00$ \\
\hline EU152 & 244 & $0.00 E+00$ & $2.96 \mathrm{E}-08$ & $0.00 \mathrm{E}+00$ & $0.00 E+00$ & $0.00 \mathrm{E}+00$ \\
\hline EU152 & 245 & $0.00 \mathrm{E}+00$ & $2.39 \mathrm{E}-08$ & $0.00 \mathrm{E}+00$ & $0.00 \mathrm{E}+00$ & $0.00 \mathrm{E}+00$ \\
\hline EU152 & 246 & $0.00 E+00$ & $1.85 \mathrm{E}-08$ & $0.00 E+00$ & $0.00 \mathrm{E}+00$ & $0.00 \mathrm{E}+00$ \\
\hline EU152 & 247 & $0.00 E+00$ & $1.54 \mathrm{E}-08$ & $0.00 \mathrm{E}+00$ & $0.00 E+00$ & $0.00 \mathrm{E}+00$ \\
\hline EU152 & 248 & $0.00 \mathrm{E}+00$ & $1.19 \mathrm{E}-08$ & $0.00 \mathrm{E}+00$ & $0.00 E+00$ & $0.00 \mathrm{E}+00$ \\
\hline EU152 & 249 & $0.00 E+00$ & $9.90 \mathrm{E}-09$ & $0.00 \mathrm{E}+00$ & $0.00 \mathrm{E}+00$ & $0.00 \mathrm{~F}+00$ \\
\hline EU152 & 250 & $0.00 \mathrm{E}+00$ & $7.57 \mathrm{E}-09$ & $0.00 \mathrm{E}+00$ & $0.00 \mathrm{E}+00$ & $0.00 \mathrm{E}$ \\
\hline EU154 & & $0 \quad 50$ & 0 & & & \\
\hline EU 154 & 21 & $1.08 \mathrm{E}-04$ & $8.62 E-04$ & $2.63 \mathrm{E}-03$ & .00 & $3.32 \mathrm{E}$ \\
\hline EU 154 & 22 & $1.91 \mathrm{E}-04$ & $1.75 \mathrm{E}-03$ & $6.21 \mathrm{E}-04$ & .00 & $1.34 \mathrm{E}$ \\
\hline EU 154 & 23 & $1.28 \mathrm{E}-04$ & $1.46 \mathrm{E}-03$ & $3.75 \mathrm{E}-06$ & .00 & $7.73 \mathrm{E}-08$ \\
\hline EU 154 & 24 & $8.21 \mathrm{E}-05$ & $1.18 \mathrm{E}-03$ & $2.27 \mathrm{E}-08$ & .00 & $4.66 \mathrm{E}$ \\
\hline EU 154 & 25 & $5.27 \mathrm{E}-05$ & $9.52 \mathrm{E}-04$ & $1.46 \mathrm{E}-10$ & .00 & $3.64 \mathrm{E}$ \\
\hline EU 154 & 26 & $3.40 \mathrm{E}-05$ & $7.72 \mathrm{E}-04$ & .00 & .00 & .00 \\
\hline EU 154 & 27 & $2.18 \mathrm{E}-05$ & $6.22 \mathrm{E}-04$ & .00 & .00 & .00 \\
\hline EU 154 & 28 & $1.40 \mathrm{E}-05$ & $5.05 E-04$ & .00 & .00 & .00 \\
\hline EU & 29 & $9.01 \mathrm{E}-06$ & $4.08 E-04$ & .00 & .00 & .00 \\
\hline EU 154 & 210 & $5.78 \mathrm{E}-06$ & $3.29 E-04$ & .00 & .00 & .00 \\
\hline EU 154 & 211 & $3.72 \mathrm{E}-06$ & $2.67 \mathrm{E}-04$ & .00 & .00 & .00 \\
\hline EU 154 & 212 & $2.39 \mathrm{E}-06$ & $2.15 \mathrm{E}-04$ & .00 & .00 & .00 \\
\hline EU 154 & 213 & $1.54 \mathrm{E}-06$ & $1.75 \mathrm{E}-04$ & .00 & .00 & .00 \\
\hline EU 154 & 214 & $9.86 \mathrm{E}-07$ & $1.41 \mathrm{E}-04$ & .00 & .00 & 00 \\
\hline EU & 215 & $6.36 \mathrm{E}-07$ & 1.14 & .00 & .00 & .00 \\
\hline EU & 216 & $4.08 \mathrm{E}-07$ & 9.25 & .00 & 00 & 00 \\
\hline EU & 217 & $2.62 \mathrm{E}-07$ & $7.46 \mathrm{E}-05$ & .00 & .00 & 00 \\
\hline EU & 218 & $1.69 \mathrm{E}-07$ & $6.05 \mathrm{E}-05$ & .00 & .00 & .00 \\
\hline$\Gamma_{1}$ & 219 & $1.08 \mathrm{E}-07$ & 4.88 & .00 & .00 & .00 \\
\hline EU & 220 & $6.97 \mathrm{E}-08$ & 3.96 & .00 & .00 & .00 \\
\hline EU & 221 & $4.48 \mathrm{E}-08$ & $3.20 \mathrm{E}-05$ & .00 & .00 & .00 \\
\hline EU & 222 & $2.87 \mathrm{E}-08$ & $2.58 \mathrm{E}-05$ & .00 & .00 & 00 \\
\hline $\mathrm{FI}$ & 223 & $1.85 E-08$ & $2.09 \mathrm{E}-05$ & .00 & 00 & 00 \\
\hline $\mathrm{FI}$ & 224 & $1.19 \mathrm{E}-08$ & $1.69 \mathrm{E}-05$ & .00 & .00 & .00 \\
\hline & 225 & -09 & $1.37 \mathrm{E}-05$ & .00 & .00 & .00 \\
\hline EI & 226 & $4.90 E-09$ & $1.11 \mathrm{E}-05$ & .00 & .00 & 00 \\
\hline & 2 & -09 & $8.97 \mathrm{E}-06$ & .00 & .00 & .00 \\
\hline & $2-$ & $2.03 \mathrm{E}-09$ & $7.25 \mathrm{E}-06$ & .00 & .00 & .00 \\
\hline
\end{tabular}




\section{Appendix B - Inhalation Dose Rate Factors - FILE23}

\begin{tabular}{|c|c|c|c|c|c|c|}
\hline EU 154 & 229 & $1.30 \mathrm{E}-09$ & $5.85 \mathrm{E}-06$ & .00 & .00 & .00 \\
\hline EU 154 & 230 & $8.44 \mathrm{E}-10$ & $4.75 \mathrm{E}-06$ & .00 & .00 & .00 \\
\hline EU 154 & 231 & $5.38 \mathrm{E}-10$ & $3.83 \mathrm{E}-06$ & .00 & .00 & .00 \\
\hline EU 154 & 232 & $3.49 \mathrm{E}-10$ & $3.11 \mathrm{E}-06$ & .00 & .00 & .00 \\
\hline EU 154 & 233 & $2.11 \mathrm{E}-10$ & $2.52 \mathrm{E}-06$ & .00 & .00 & .00 \\
\hline EU 154 & 234 & $1.46 \mathrm{E}-10$ & $1.99 \mathrm{E}-06$ & .00 & .00 & .00 \\
\hline EU 154 & 235 & $9.46 \mathrm{E}-11$ & $1.67 \mathrm{E}-06$ & .00 & .00 & .00 \\
\hline EU 154 & 236 & $5.82 \mathrm{E}-11$ & $1.34 \mathrm{E}-06$ & .00 & .00 & .00 \\
\hline EU 154 & 237 & $3.64 \mathrm{E}-11$ & $1.05 \mathrm{E}-06$ & .00 & .00 & .00 \\
\hline EU 154 & 238 & $2.91 \mathrm{E}-11$ & $8.80 \mathrm{E}-07$ & .00 & .00 & .00 \\
\hline EU 154 & 239 & $1.46 \mathrm{E}-11$ & $6.90 \mathrm{E}-07$ & .00 & .00 & .00 \\
\hline EU 154 & 240 & $7.28 \mathrm{E}-12$ & $5.76 \mathrm{E}-07$ & .00 & .00 & .00 \\
\hline EU 154 & 241 & $1.46 \mathrm{E}-11$ & $4.51 \mathrm{E}-07$ & .00 & .00 & .00 \\
\hline EU 154 & 242 & .00 & $3.77 \mathrm{E}-07$ & .00 & .00 & .00 \\
\hline EU 154 & 243 & .00 & $2.96 \mathrm{E}-07$ & .00 & .00 & .00 \\
\hline EU 154 & 244 & .00 & $2.47 \mathrm{E}-07$ & .00 & .00 & .00 \\
\hline EU 154 & 245 & .00 & $1.99 \mathrm{E}-07$ & .00 & .00 & .00 \\
\hline EU 154 & 246 & .00 & $1.56 \mathrm{E}-07$ & .00 & .00 & .00 \\
\hline EU 154 & 247 & .00 & $1.30 \mathrm{E}-07$ & .00 & .00 & .00 \\
\hline EU 154 & 248 & .00 & $1.02 \mathrm{E}-07$ & .00 & .00 & .00 \\
\hline EU 154 & 249 & .00 & $8.53 \mathrm{E}-08$ & .00 & .00 & .00 \\
\hline EU 154 & 250 & .00 & $6.71 \mathrm{E}-08$ & .00 & .00 & .00 \\
\hline$T 8160$ & & 0 & 0 & & & \\
\hline TB160 & 21 & $2.84 \mathrm{E}-05$ & $2.25 \mathrm{E}-04$ & $9.67 \mathrm{E}-04$ & $0.00 \mathrm{E}+00$ & $1.53 \mathrm{E}-04$ \\
\hline TB160 & 22 & $1.11 \mathrm{E}-05$ & $9.15 \mathrm{E}-05$ & $1.25 \mathrm{E}-04$ & $0.00 \mathrm{E}+00$ & 2.53 \\
\hline TB160 & 23 & $2.61 \mathrm{E}-07$ & $2.40 \mathrm{E}-06$ & $2.36 \mathrm{E}-08$ & $0.00 \mathrm{E}+00$ & $4.37 \mathrm{E}-10$ \\
\hline TB160 & 24 & $5.40 \mathrm{E}-09$ & $5.63 \mathrm{E}-08$ & $0.00 \mathrm{E}+00$ & $0.00 \mathrm{E}+00$ & $0.00 \mathrm{E}+00$ \\
\hline TB160 & 25 & $1.06 \mathrm{E}-10$ & $1.34 \mathrm{E}-09$ & $0.00 \mathrm{E}+00$ & $0.00 E+00$ & $0.00 \mathrm{E}+00$ \\
\hline TB160 & 26 & 3.64 & $2.91 \mathrm{E}-11$ & $0.00 \mathrm{E}+00$ & $0.00 \mathrm{E}+00$ & $0.00 E+00$ \\
\hline $\begin{array}{l}\text { OS } 185 \\
\text { OS } 185\end{array}$ & 11 & $\begin{array}{ll}2 & 0\end{array}$ & 0 & & & \\
\hline OS185 & $\begin{array}{ll}1 & 1 \\
1 & 2\end{array}$ & $2.81 \mathrm{E}-05$ & $0.00 E+00$ & $1.81 \mathrm{E}-05$ & $0.00 \mathrm{E}+00$ & $3.14 \mathrm{E}-05$ \\
\hline OS191 & 12 & $\begin{array}{r}2.00 \\
2\end{array}$ & $\begin{array}{c}0.00 \mathrm{E}+00 \\
0\end{array}$ & $3.55 E-08$ & $0.00 \mathrm{E}+00$ & $1.88 \mathrm{E}-09$ \\
\hline OS191 & 11 & $4.66 \mathrm{E}-06$ & $0.00 E+00$ & $8.36 \mathrm{E}-06$ & $0.00 \mathrm{E}+00$ & $1.74 \mathrm{E}-05$ \\
\hline OS191 & 12 & $1.69 \mathrm{E}-07$ & $0.00 E+00$ & $1.60 \mathrm{E}-08$ & $0.00 \mathrm{E}+00$ & $1.03 \mathrm{E}-09$ \\
\hline IR192 & & 0 & 5 & & & \\
\hline IR192 & 31 & $4.67 \mathrm{E}-06$ & $0.00 \mathrm{E}+00$ & $2.97 E-03$ & $0.00 E+00$ & 3.46 \\
\hline IR192 & 32 & $3.97 \mathrm{E}-07$ & $0.00 \mathrm{E}+00$ & $9.41 \mathrm{E}-04$ & $0.00 \mathrm{E}+00$ & 2.8 \\
\hline IR192 & 33 & $2.58 \mathrm{E}-09$ & $0.00 \mathrm{E}+00$ & $1.77 \mathrm{E}-05$ & $0.00 \mathrm{E}+00$ & 4.07 \\
\hline IR192 & 34 & $6.64 \mathrm{E}-11$ & $0.00 \mathrm{E}+00$ & $3.32 E-07$ & $0.00 \mathrm{E}+00$ & 7.57 \\
\hline IR192 & 35 & $1.82 \mathrm{E}-12$ & $0.00 \mathrm{E}+00$ & $6.52 \mathrm{E}-09$ & $0.00 \mathrm{E}+00$ & $2.91 \mathrm{E}-11$ \\
\hline HG203 & & 0 & 0 & & & \\
\hline HG203 & 11 & $2.89 E-05$ & $0.00 E+00$ & $1.05 E-05$ & $0.00 \mathrm{E}+00$ & $7.84 \mathrm{E}-06$ \\
\hline $\mathrm{HG} 203$ & 12 & $4.19 E-06$ & $0.00 \mathrm{E}+00$ & $2.06 \mathrm{E}$ & $0.00 \mathrm{E}+00$ & 4.67 \\
\hline HG203 & 13 & $1.46 \mathrm{E}-09$ & $0.00 \mathrm{E}+00$ & $0.00 \mathrm{E}+00$ & $0.00 E+00$ & $0.00 E+00$ \\
\hline U 234 & & 0 & 50 & & & \\
\hline 234 & 31 & $2.58 \mathrm{E}-04$ & $2.08 E-03$ & $7.01 \mathrm{E}-01$ & .00 & \\
\hline 234 & 32 & $3.19 \mathrm{E}-04$ & $4.10 \mathrm{E}-03$ & $1.01 \mathrm{E}+00$ & .00 & 1.4 \\
\hline U 23 & 33 & $2.43 \mathrm{E}-04$ & $3.97 \mathrm{E}-03$ & 6.0 & .00 & \\
\hline U 23 & 34 & $2.14 \mathrm{E}-04$ & $3.68 \mathrm{E}-03$ & $3.66 \mathrm{E}-01$ & .00 & \\
\hline
\end{tabular}




\section{Appendix B - Inhalation Dose Rate Factors - FILE23}

\begin{tabular}{|c|c|c|c|c|c|c|c|}
\hline U & 234 & 35 & $1.83 \mathrm{E}-04$ & $3.28 \mathrm{E}-03$ & $2.21 \mathrm{E}-01$ & .00 & $3.13 \mathrm{E}-06$ \\
\hline U & 234 & 36 & $1.53 \mathrm{E}-04$ & $2.83 \mathrm{E}-03$ & $1.33 \mathrm{E}-01$ & .00 & $1.89 E-06$ \\
\hline U & 234 & 37 & $1.25 \mathrm{E}-04$ & $2.37 \mathrm{E}-03$ & $8.00 \mathrm{E}-02$ & .00 & $1.14 \mathrm{E}-06$ \\
\hline U & 234 & 38 & $1.01 \mathrm{E}-04$ & $1.96 \mathrm{E}-03$ & $4.84 \mathrm{E}-02$ & .00 & $6.87 \mathrm{E}-07$ \\
\hline U & 234 & 39 & $8.06 E-05$ & $1.59 \mathrm{E}-03$ & $2.91 \mathrm{E}-02$ & .00 & $4.14 \mathrm{E}-07$ \\
\hline U & 234 & 310 & $6.38 \mathrm{E}-05$ & $1.27 \mathrm{E}-03$ & $1.75 \mathrm{E}-02$ & .00 & $2.49 \mathrm{E}-07$ \\
\hline U & 234 & 311 & $5.05 E-05$ & $1.02 \mathrm{E}-03$ & $1.06 \mathrm{E}-02$ & .00 & $1.50 \mathrm{E}-07$ \\
\hline U & 234 & 312 & $3.96 E-05$ & $8.03 E-04$ & $6.36 \mathrm{E}-03$ & .00 & $9.03 \mathrm{E}-08$ \\
\hline U & 234 & 313 & $3.11 E-05$ & $6.35 \mathrm{E}-04$ & $3.84 \mathrm{E}-03$ & .00 & $5.46 \mathrm{E}-08$ \\
\hline U & 234 & 314 & $2.43 E-05$ & $4.97 \mathrm{E}-04$ & $2.31 E-03$ & .00 & $3.28 E-08$ \\
\hline U & 234 & 315 & $1.90 \mathrm{E}-05$ & $3.91 \mathrm{E}-04$ & $1.40 \mathrm{E}-03$ & .00 & $1.98 \mathrm{E}-08$ \\
\hline U & 234 & 316 & $1.48 \mathrm{E}-05$ & $3.06 \mathrm{E}-04$ & $8.41 \mathrm{E}-04$ & .00 & $1.19 E-08$ \\
\hline $\mathrm{U}$ & 234 & 317 & $1.15 \mathrm{E}-05$ & $2.38 \mathrm{E}-04$ & $5.05 E-04$ & .00 & $7.17 \mathrm{E}-09$ \\
\hline$U$ & 234 & 318 & $8.99 \mathrm{E}-06$ & $1.86 \mathrm{E}-04$ & $3.05 \mathrm{E}-04$ & .00 & $4.33 \mathrm{E}-09$ \\
\hline $\mathrm{U}$ & 234 & 319 & $6.97 \mathrm{E}-06$ & $1.44 \mathrm{E}-04$ & $1.83 \mathrm{E}-04$ & .00 & $2.60 \mathrm{E}-09$ \\
\hline U & 234 & 320 & $5.44 \mathrm{E}-06$ & $1.13 \mathrm{E}-04$ & $1.11 \mathrm{E}-04$ & .00 & $1.58 \mathrm{E}-09$ \\
\hline $\mathrm{U}$ & 234 & 321 & $4.23 \mathrm{E}-06$ & $8.76 \mathrm{E}-05$ & $6.68 \mathrm{E}-05$ & .00 & $9.50 \mathrm{E}-10$ \\
\hline $\mathrm{U}$ & 234 & 322 & $3.27 \mathrm{E}-06$ & $6.79 \mathrm{E}-05$ & $4.01 \mathrm{E}-05$ & .00 & $5.71 \mathrm{E}-10$ \\
\hline U & 234 & 323 & $2.55 \mathrm{E}-06$ & $5.30 E-05$ & $2.43 \mathrm{E}-05$ & .00 & $=-10$ \\
\hline $\mathrm{U}$ & 234 & 324 & $1.98 \mathrm{E}-06$ & $4.10 E-05$ & $1.46 \mathrm{E}-05$ & .00 & -10 \\
\hline U & 234 & 325 & $1.54 \mathrm{E}-06$ & $3.20 \mathrm{E}-05$ & $8.79 E-06$ & .00 & $1.24 \mathrm{E}-10$ \\
\hline U & 234 & 326 & $1.19 \mathrm{E}-06$ & $2.48 \mathrm{E}-05$ & $5.30 \mathrm{E}-06$ & .00 & $7.64 \mathrm{E}-11$ \\
\hline U & 234 & 327 & $9.29 \mathrm{E}-07$ & $1.93 \mathrm{E}-05$ & $3.22 \mathrm{E}-06$ & .00 & $4.73 \mathrm{E}-11$ \\
\hline U & 234 & 328 & $7.21 \mathrm{E}-07$ & $1.50 \mathrm{E}-05$ & $1.91 \mathrm{E}-06$ & .00 & 2.5 \\
\hline U & 234 & 329 & $5.58 \mathrm{E}-07$ & $1.16 \mathrm{E}-05$ & 1.19 & .00 & 1.82 \\
\hline U & 234 & 330 & $4.35 \mathrm{E}-07$ & $9.03 \mathrm{E}-06$ & 6.56 & 00 & 1.00 \\
\hline U & 234 & 331 & $3.36 \mathrm{E}-07$ & $6.99 \mathrm{E}-06$ & $4.47 \mathrm{E}-07$ & .00 & 3.6 \\
\hline U & 234 & 332 & $2.62 \mathrm{E}-07$ & $5.45 \mathrm{E}-06$ & $2.38 \mathrm{E}-07$ & .00 & 3.6 \\
\hline U & 234 & 333 & $2.04 \mathrm{E}-07$ & $4.24 \mathrm{E}-06$ & 1.79 & .00 & $3.64 \mathrm{E}-12$ \\
\hline U & 234 & 334 & $1.55 \mathrm{E}-07$ & $3.22 \mathrm{E}-06$ & 5.96 & .00 & .00 \\
\hline U & 234 & 335 & $1.24 \mathrm{E}-07$ & $2.58 \mathrm{E}-06$ & 5.96 & .00 & .00 \\
\hline U & 234 & 336 & $9.62 \mathrm{E}-08$ & $2.00 \mathrm{E}-06$ & $5.96 \mathrm{E}-08$ & .00 & .00 \\
\hline U & 234 & 337 & $7.24 \mathrm{E}-08$ & $1.50 \mathrm{E}-06$ & .00 & .00 & .00 \\
\hline U & 234 & 338 & $5.80 E-08$ & $1.21 \mathrm{E}-06$ & .00 & .00 & .00 \\
\hline U & 234 & 339 & $4.37 \mathrm{E}-08$ & $9.07 \mathrm{E}-07$ & .00 & .00 & .00 \\
\hline U & 234 & 340 & $3.50 \mathrm{E}-08$ & $7.28 \mathrm{E}-07$ & .00 & .00 & .00 \\
\hline U & 234 & 341 & $2.64 \mathrm{E}-08$ & $5.48 \mathrm{E}-07$ & .00 & .00 & .00 \\
\hline U & 234 & 342 & 2.1 & $4.39 E-07$ & .00 & .00 & .00 \\
\hline U & 234 & 343 & $1.59 E-08$ & $3.30 \mathrm{E}-07$ & .00 & .00 & .00 \\
\hline U & 234 & 344 & $1.27 E-08$ & $2.65 E-07$ & .00 & .00 & .00 \\
\hline U & 234 & 345 & 9.84 & $2.05 \mathrm{E}-07$ & .00 & .00 & .00 \\
\hline U & 234 & 346 & $7.42 \mathrm{E}-09$ & $1.54 \mathrm{E}-07$ & .00 & .00 & .00 \\
\hline U & 234 & 347 & $5.94 \mathrm{E}-09$ & $1.24 \mathrm{E}-07$ & .00 & .00 & .00 \\
\hline U & 234 & 348 & $4.48 \mathrm{E}-09$ & $9.27 \mathrm{E}-08$ & .00 & .00 & .00 \\
\hline U & 234 & 349 & 3.58 & $7.45 \mathrm{E}-08$ & .00 & .00 & .00 \\
\hline & 234 & 250 & 2.68 & $5.63 \mathrm{E}-08$ & .00 & .00 & .00 \\
\hline & & & & 0 & & & \\
\hline & & & $8.50 E-05$ & $2.96 \mathrm{E}-03$ & $3.41 \mathrm{E}-05$ & $0.00 E+00$ & $5.88 \mathrm{E}-05$ \\
\hline & & 2 & $2.10 \mathrm{E}-05$ & $7.31 \mathrm{E}-04$ & $6.68 \mathrm{E}-08$ & $0.00 E+00$ & $3.51 \mathrm{E}-09$ \\
\hline S & & & $1.38 \mathrm{E}-07$ & $4.84 \mathrm{E}-06$ & $0.00 E+00$ & $0.00 E+00$ & $0.00 \mathrm{E}+00$ \\
\hline
\end{tabular}




\section{Appendix B - Inhalation Dose Rate Factors - FILE23}

\begin{tabular}{|c|c|c|c|c|c|c|}
\hline SR89 & 14 & $09 E-10$ & $3.21 E-08$ & $0.00 \mathrm{E}+00$ & $0.00 \mathrm{E}+00$ & $E+00$ \\
\hline SR89 & & $7.28 \mathrm{E}$ & $0.00 E+00$ & $0.00 \mathrm{E}+00$ & $0.00 E+00$ & $\mathrm{~F}+0 \mathrm{n}$ \\
\hline Y $89 M$ & & 0 & 0 & & & \\
\hline SR90 & & 50 & 0 & & & \\
\hline SR90 & 11 & $5.32 \mathrm{E}-04$ & $7.94 \mathrm{E}-03$ & $8.17 \mathrm{E}-05$ & $0.00 E+00$ & $8.30 \mathrm{E}-05$ \\
\hline SR & 2 & $1.01 \mathrm{E}-03$ & $1.50 \mathrm{E}$ & & $0 E+00$ & \\
\hline SRS & 13 & 9.2 & 1.38 & +00 & $0.00 \mathrm{E}+00$ & \\
\hline SRS & 14 & 8.45 & $1.26 \mathrm{E}-02$ & $0.00 \mathrm{E}+00$ & $0.00 \mathrm{E}+00$ & +00 \\
\hline SR90 & 15 & 7.7 & 1.15 & $E+00$ & $E+00$ & \\
\hline$S$ & 16 & $7.10 E-04$ & $1.06 \mathrm{E}$ & $E+00$ & $E+00$ & 00 \\
\hline SR9 & 17 & $6.49 \mathrm{E}-04$ & 9.67 & +00 & +00 & 0 \\
\hline SR9 & 18 & $5.96 E-04$ & 8.8 & +00 & +00 & \\
\hline SR9 & 19 & $5.46 \mathrm{E}-04$ & 8.14 & +00 & +00 & \\
\hline SR90 & 110 & $4.99 E-04$ & 7.4 & +00 & 00 & \\
\hline SR90 & 111 & $4.58 \mathrm{E}-04$ & 6.8 & +00 & +00 & \\
\hline SR9 & 112 & 4.1 & 6.2 & +00 & +00 & \\
\hline SR90 & 113 & $3.85 E-04$ & 5.7 & +00 & $+\infty$ & \\
\hline SR9O & 114 & $3.51 \mathrm{E}-04$ & 5.2 & +00 & $E+00$ & +00 \\
\hline SR90 & 115 & $3.23 \mathrm{E}-04$ & 4.8 & +00 & $E+00$ & $+\infty$ \\
\hline SR90 & 116 & $2.96 E-04$ & 4.4 & $=00$ & $E+00$ & $=00$ \\
\hline SR90 & 117 & $2.70 \mathrm{~B}$ & $4.02 E-03$ & $E+00$ & $E+00$ & +00 \\
\hline SR90 & 118 & 2.48 & $3.70 E-03$ & $E+00$ & $E+00$ & $E+00$ \\
\hline SR90 & 119 & 2.27 & & +00 & $E+00$ & $=+00$ \\
\hline SR90 & 120 & & & $=00$ & $E+00$ & $=00$ \\
\hline SR90 & 121 & & $2 . \varepsilon$ & $\mathrm{E}+00$ & $0 E+00$ & $0 E+00$ \\
\hline SR90 & 122 & 1.7 & & $E+00$ & $0.00 \mathrm{E}+00$ & $E+00$ \\
\hline SR90 & 123 & 1.6 & & $E+00$ & $0 \mathrm{E}+00$ & $0 E+00$ \\
\hline SR90 & 124 & & & $E+00$ & $0 \mathrm{E}+00$ & $0 E+00$ \\
\hline SR90 & 125 & & & $E+00$ & $\mathrm{E}+00$ & $0 E+00$ \\
\hline SR90 & 126 & & & $E+00$ & $0.00 E+00$ & $0.00 E+00$ \\
\hline SR90 & 127 & & & $E+00$ & $0.00 E+00$ & $0.00 \mathrm{E}+00$ \\
\hline SR90 & 128 & 44 & -03 & $E+00$ & $0.00 E+00$ & $10 E+00$ \\
\hline SR90 & 129 & & 03 & $E+\infty$ & $0.00 E+00$ & $0.00 E+00$ \\
\hline SR90 & 130 & & 03 & +00 & $0 \mathrm{E}+00$ & $0 E+00$ \\
\hline SR90 & 131 & 05 & -03 & $E+00$ & $0 \mathrm{E}+00$ & $0.00 \mathrm{E}+00$ \\
\hline SR90 & 132 & -05 & -03 & $E+00$ & $0.00 E+00$ & $0 E+00$ \\
\hline SR90 & 13 & & $9.96 \mathrm{E}-04$ & $E+00$ & $0.00 E+00$ & $O E+00$ \\
\hline SR90 & 13 & 05 & -04 & $E+00$ & $E+00$ & $E+00$ \\
\hline SR90 & 13 & -05 & $E-04$ & $E+00$ & $E+00$ & +00 \\
\hline SR & 13 & -05 & -04 & $E+00$ & $=00$ & +00 \\
\hline SR90 & 13 & -05 & -04 & $E+00$ & $\mathrm{DE}+00$ & +00 \\
\hline SR90 & 13 & $E-05$ & $6.49 E-04$ & $E+00$ & $0 E+00$ & $E+00$ \\
\hline SR90 & & -05 & 5.7 & $0.00 E+00$ & $0.00 E+00$ & $E+00$ \\
\hline SR90 & 14 & & -04 & $0.00 \mathrm{E}+00$ & $0.00 E+00$ & $E+00$ \\
\hline SR90 & 14 & $4 E-05$ & $4.83 E-04$ & $0.00 \mathrm{E}+00$ & $0.00 E+00$ & $E+00$ \\
\hline SR90 & 14 & $3.07 \mathrm{E}-05$ & $4.57 E-04$ & $0.00 \mathrm{E}+00$ & $0.00 E+00$ & $E+00$ \\
\hline SR90 & 14 & $2.72 \mathrm{E}-05$ & 4.06 & $0.00 E+00$ & $0.00 E+00$ & $E+00$ \\
\hline SR90 & 14 & 2. & 3.8 & $0.00 \mathrm{E}+00$ & $0.00 E+00$ & $\mathrm{E}+00$ \\
\hline SR90 & 14 & $2.36 \mathrm{E}-05$ & $3.51 \mathrm{E}-$ & $0.00 \mathrm{E}+00$ & $0.00 \mathrm{E}+00$ & +00 \\
\hline SR90 & 146 & -05 & $3.12 \mathrm{E}-04$ & $0.00 \mathrm{E}+00$ & $0.00 \mathrm{E}+00$ & $0.00 E+00$ \\
\hline
\end{tabular}




\section{Appendix B - Inhalation Dose Rate Factors - FILE23}

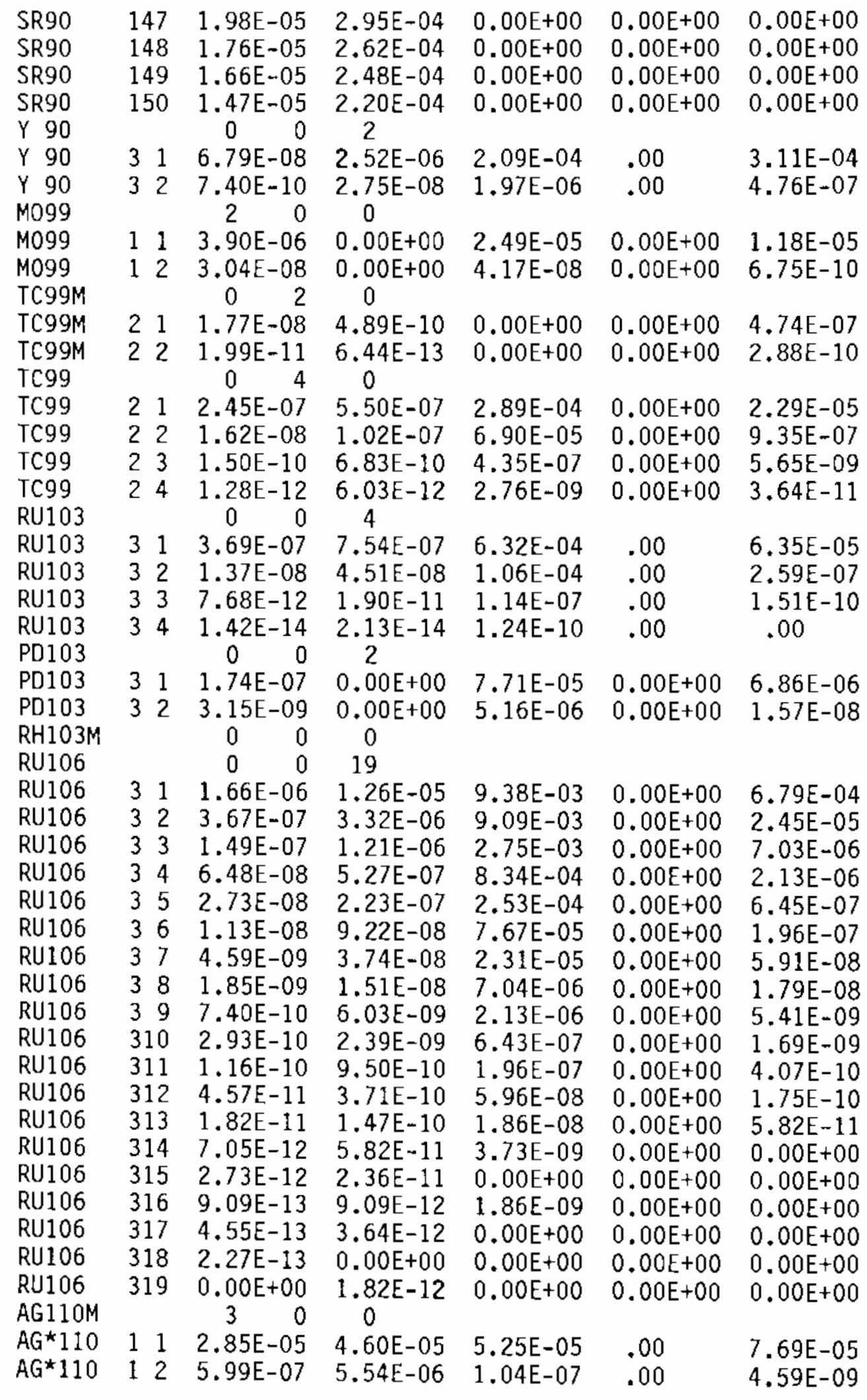




\section{Appendix B - Inhalation Dose Rate Factors - FILE23}

\begin{tabular}{|c|c|c|c|c|c|c|}
\hline $\begin{array}{l}A G \star 110 \\
A G 110 \\
S B 125\end{array}$ & 13 & $\begin{array}{c}.00 \\
0 \\
0\end{array}$ & $\begin{array}{c}4.52 \mathrm{E}-10 \\
0 \\
0\end{array}$ & .00 & .00 & .00 \\
\hline SB & 21 & $2.93 \mathrm{E}-05$ & $5.85 \mathrm{E}-05$ & $6.82 \mathrm{E}-04$ & $5.50 \mathrm{E}-08$ & $7.43 \mathrm{E}-05$ \\
\hline & 22 & $3.95 \mathrm{E}-05$ & $9.83 \mathrm{E}-05$ & $1.53 \mathrm{E}-04$ & 6.0 & \\
\hline & 23 & $1.65 \mathrm{E}-05$ & $6.10 \mathrm{E}-05$ & $7.49 \mathrm{E}-07$ & 5.3 & 1.3 \\
\hline & 24 & $6.56 \mathrm{E}-$ & $3.66 \mathrm{E}-05$ & 3.65 & 3.93 & 6.3 \\
\hline & 25 & $2.61 \mathrm{E}-06$ & $2.20 \mathrm{E}-05$ & $1.46 \mathrm{E}-11$ & $1.78 \mathrm{E}$ & .00 \\
\hline & 26 & $1.04 \mathrm{E}-06$ & $1.32 \mathrm{E}-05$ & .00 & .00 & .00 \\
\hline & 27 & $4.11 E-07$ & $7.92 \mathrm{E}-06$ & .00 & .00 & .00 \\
\hline & 28 & $1.64 \mathrm{E}-07$ & $4.77 \mathrm{E}-06$ & .00 & .00 & .00 \\
\hline & 29 & $6.50 \mathrm{E}-08$ & $2.86 \mathrm{E}-06$ & .00 & .00 & .00 \\
\hline & 210 & $2.57 \mathrm{E}-$ & $1.71 \mathrm{E}-06$ & .00 & .00 & .00 \\
\hline & 211 & 1.0 & $1.03 \mathrm{E}-06$ & .00 & .00 & .00 \\
\hline & 212 & $4.06 \mathrm{E}-$ & $6.18 \mathrm{E}-07$ & .00 & .00 & .00 \\
\hline & 213 & $1.62 \mathrm{E}-$ & -07 & .00 & .00 & .00 \\
\hline & 214 & 6.4 & $2.23 \mathrm{E}-07$ & .00 & .00 & .00 \\
\hline & 215 & 2. & 1.3 & .00 & .00 & .00 \\
\hline & 216 & 1. & 8. & .00 & .00 & .00 \\
\hline & 217 & 4. & 4. & .00 & .00 & .00 \\
\hline & 218 & 1. & 2. & .00 & .00 & .00 \\
\hline & 219 & 6. & 1.7 & .00 & .00 & .00 \\
\hline & 220 & 2.7 & 1.0 & .00 & .00 & .00 \\
\hline & 221 & 9.0 & 6.2 & .00 & .00 & .00 \\
\hline & 222 & .0 & 3.7 & .00 & .00 & .00 \\
\hline & 223 & .0 & 2. & .00 & .00 & .00 \\
\hline & 224 & .0 & 1. & .00 & .00 & .00 \\
\hline & 225 & .0 & 8. & .00 & .00 & .00 \\
\hline & 226 & .0 & 4. & .00 & .00 & .00 \\
\hline & 227 & .00 & 2. & .00 & .00 & .00 \\
\hline & 228 & .00 & 1.7 & .00 & .00 & .00 \\
\hline & 229 & .00 & 1. & .00 & .00 & .00 \\
\hline & 230 & .00 & 6. & .0 & .00 & .00 \\
\hline & 23 & .00 & 3. & .00 & .00 & .00 \\
\hline & 232 & .00 & 2. & .00 & .0 & .00 \\
\hline & 233 & .00 & 1. & .00 & .0 & .00 \\
\hline & 234 & .00 & 7. & .00 & .0 & .00 \\
\hline & 23 & .00 & 3.64 & .00 & .00 & .00 \\
\hline & 2 & $2.56 \mathrm{E}-06$ & & & & \\
\hline & 22 & & 1.3 & 3.0 & 7.1 & 6.1 \\
\hline $\mathrm{TE}^{\star} 125$ & 23 & $1.41 \mathrm{E}-11$ & $1.38 \mathrm{E}-10$ & 2.41 & $5.69 \mathrm{E}$ & $4.27 \mathrm{E}-1$ \\
\hline $\mathrm{TE}^{\star} 125$ & 24 & .00 & .00 & $3.64 \mathrm{E}-12$ & .00 & .00 \\
\hline & & 0 & 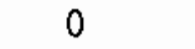 & & & \\
\hline$T E^{\star} 127$ & 21 & $6.22 \mathrm{E}-06$ & $4.46 \mathrm{E}-05$ & $7.23 \mathrm{E}$ & $3.95 \mathrm{E}$ & 2.61 \\
\hline $\mathrm{TE}^{\star} 127$ & 22 & $5.75 \mathrm{E}-07$ & $6.37 \mathrm{E}-06$ & 1.096 & $2.77 \mathrm{E}$ & 5.44 \\
\hline$T E^{\star} 127$ & 23 & $3.46 \mathrm{E}-10$ & $4.66 \mathrm{E}-09$ & $6.17 \mathrm{E}-$ & $1.78 \mathrm{E}-07$ & $2.81 \mathrm{E}-10$ \\
\hline TE* & 24 & 2.8 & $3.64 \mathrm{E}-12$ & $3.64 \mathrm{E}-11$ & $1.16 \mathrm{E}-10$ & $2.27 \mathrm{E}-13$ \\
\hline & & & - & & & \\
\hline & & $7.79 \mathrm{E}-08$ & $3.55 \mathrm{E}-07$ & $1.03 \mathrm{E}-05$ & $8.05 \mathrm{E}-05$ & $1.27 \mathrm{E}-05$ \\
\hline
\end{tabular}




\section{Appendix B - Inhalation Dose Rate Factors - FILE23}

\begin{tabular}{|c|c|c|c|c|c|c|}
\hline $\begin{array}{l}\text { TE127 } \\
\text { TE129M }\end{array}$ & 22 & $\begin{array}{c}1.43 \mathrm{E}-10 \\
0\end{array}$ & $\begin{array}{c}6.60 \mathrm{E}-10 \\
0\end{array}$ & $1.43 E-08$ & $1.46 \mathrm{E}-07$ & $1.01 \mathrm{E}-08$ \\
\hline$T E^{\star} 129$ & 21 & $1.58 \mathrm{E}-05$ & $6.21 E-05$ & $1.20 \mathrm{E}-03$ & $7.13 \mathrm{E}-03$ & $3.00 \mathrm{E}-05$ \\
\hline$T E \star 129$ & 22 & $8.99 \mathrm{E}-07$ & $5.07 \mathrm{E}-06$ & $1.01 \mathrm{E}-04$ & $3.02 \mathrm{E}-04$ & $2.71 \mathrm{E}-07$ \\
\hline$T E \star 129$ & 23 & $2.73 \mathrm{E}-12$ & $2.09 \mathrm{E}-11$ & $3.49 \mathrm{E}-10$ & $9.31 \mathrm{E}-10$ & $9.09 E-13$ \\
\hline TE129 & & 02 & 0 & & & \\
\hline TE129 & 21 & $2.31 \mathrm{E}-08$ & $5.94 \mathrm{E}-08$ & $4.19 \mathrm{E}-06$ & $1.48 \mathrm{E}-05$ & $6.01 \mathrm{E}-\mathrm{C}$ \\
\hline $\begin{array}{l}\text { TE129 } \\
\text { I } 129\end{array}$ & 22 & $\begin{array}{c}5.42 \mathrm{E}-12 \\
12\end{array}$ & $\begin{array}{c}1.40 \mathrm{E}-11 \\
0\end{array}$ & $7.56 \mathrm{E}-10$ & $3.47 \mathrm{E}-09$ & $9.79 \mathrm{E}-$ \\
\hline I 129 & 11 & $3.06 \mathrm{E}-05$ & $1.28 E-06$ & $5.14 \mathrm{E}-06$ & $2.46 \mathrm{E}-02$ & $5.57 \mathrm{E}-0$ \\
\hline I 129 & 12 & $2.18 E-05$ & $7.62 \mathrm{E}-08$ & $1.02 \mathrm{E}-08$ & & 3 \\
\hline I 129 & 13 & $3.48 \mathrm{E}-06$ & .00 & .00 & $2.80 \mathrm{E}-03$ & .00 \\
\hline I 129 & 14 & $5.56 \mathrm{E}-07$ & .00 & .00 & 4.46 & .00 \\
\hline I 129 & 15 & $8.88 \mathrm{E}-08$ & .00 & .00 & $7.13 \mathrm{E}-05$ & .00 \\
\hline I 129 & 16 & $1.42 \mathrm{E}-08$ & .00 & .00 & $1.14 \mathrm{E}-05$ & .00 \\
\hline I 129 & 17 & $2.26 \mathrm{E}-09$ & .00 & .00 & $1.82 \mathrm{E}-06$ & .00 \\
\hline I 129 & 18 & $3.62 \mathrm{E}-10$ & .00 & .00 & $2.91 \mathrm{E}-07$ & .00 \\
\hline I 129 & 19 & $5.87 \mathrm{E}-11$ & .00 & .00 & $6 E-08$ & .00 \\
\hline I 129 & 110 & $8.64 \mathrm{E}-12$ & .00 & .00 & $E-09$ & .00 \\
\hline I 129 & 111 & $1.36 \mathrm{E}-12$ & .00 & .00 & $9.31 \mathrm{E}-10$ & .00 \\
\hline I 129 & 112 & $4.55 \mathrm{E}-13$ & .00 & .00 & .00 & .00 \\
\hline I 131 & & 0 & 0 & & & \\
\hline I 131 & 11 & $1.40 \mathrm{E}-05$ & $1.73 \mathrm{E}-05$ & $1.77 \mathrm{E}-05$ & $8.01 \mathrm{E}-03$ & $1.87 \mathrm{E}-0$ \\
\hline I 131 & 12 & $4.38 E-07$ & $3.71 \mathrm{E}-07$ & $3.29 E-08$ & $2.50 \mathrm{~B}$ & 1.10 \\
\hline XE131M & & 0 & 0 & & & \\
\hline I 133 & & 0 & 0 & & & \\
\hline I 133 & 11 & $2.79 \mathrm{E}-06$ & .00 & $2.66 \mathrm{E}-05$ & $1.88 \mathrm{E}-03$ & $2.79 \mathrm{E}-06$ \\
\hline I 133 & 12 & $1.08 \mathrm{E}-08$ & .00 & $3.34 \mathrm{E}-08$ & $7.30 \mathrm{E}-06$ & $1.46 \mathrm{E}-10$ \\
\hline CS134 & & 10 & 0 & & & \\
\hline CS134 & 11 & $2.44 \mathrm{E}-04$ & $1.27 \mathrm{E}-04$ & $7.40 \mathrm{E}-05$ & .00 & $2.90 \mathrm{E}-06$ \\
\hline $\operatorname{cs} 13$ & 12 & $E-05$ & $7.87 \mathrm{E}-05$ & $2.39 \mathrm{E}-05$ & .00 & $1.73 \mathrm{E}-10$ \\
\hline CS1: & 13 & 1.53 & $9.28 \mathrm{E}-06$ & $2.80 E-06$ & .00 & .00 \\
\hline CS13 & 14 & $2.96 E-08$ & $1.09 \mathrm{E}-06$ & $3.30 \mathrm{E}-07$ & .00 & .00 \\
\hline $\operatorname{CS} 13$ & 15 & $5.71 \mathrm{E}-10$ & $1.29 \mathrm{E}-07$ & $3.89 E-08$ & .00 & .00 \\
\hline CS134 & 16 & $1.09 \mathrm{E}-11$ & $1.52 \mathrm{E}-08$ & $4.60 E-09$ & .00 & .00 \\
\hline CS & 17 & $3.64 \mathrm{E}-12$ & $1.79 E-09$ & $5.40 E-10$ & .00 & .00 \\
\hline CS & 18 & .00 & $2.11 \mathrm{E}-10$ & 6. & .00 & .00 \\
\hline CS & 19 & .00 & 2.55 & 7.2 & .00 & .00 \\
\hline $\operatorname{cs}$ & 110 & .00 & $1.82 \mathrm{E}-12$ & $9.09 \mathrm{E}-13$ & .00 & .00 \\
\hline CS & & $\begin{array}{ll}12 & 0\end{array}$ & 0 & & & \\
\hline $\begin{array}{l}\mathrm{CS} \\
\mathrm{CS}\end{array}$ & $\begin{array}{ll}1 & 1 \\
1 & 2\end{array}$ & $\begin{array}{l}1.55 \mathrm{E}-05 \\
5\end{array}$ & $4.57 \mathrm{E}-05$ & $8.93 \mathrm{E}-06$ & .0 & $\begin{array}{l}5.03 \mathrm{E} \\
3.01 \mathrm{~F}\end{array}$ \\
\hline & $\begin{array}{ll}1 & 2 \\
1 & 3\end{array}$ & $\begin{array}{l}E-06 \\
E-07\end{array}$ & $3.30 \mathrm{E}-05$ & 3.47 & .0 & $\begin{array}{c}3.01 \mathrm{E} \\
00\end{array}$ \\
\hline & 14 & E-09 & $\begin{array}{l}5.40 \mathrm{E}-06 \\
8.86 \mathrm{E}-07\end{array}$ & $\begin{array}{l}5 . \\
9 .\end{array}$ & .0 & $\begin{array}{l}.00 \\
.00\end{array}$ \\
\hline & 15 & $1.09 \mathrm{E}-10$ & $1.45 \mathrm{E}-07$ & $1.52 \mathrm{E}$ & .00 & .00 \\
\hline & 16 & $2.96 \mathrm{E}-12$ & $2.39 \mathrm{E}-08$ & $2.51 \mathrm{E}-09$ & .00 & .00 \\
\hline & 17 & .00 & $3.90 \mathrm{E}-09$ & $4.09 \mathrm{E}-10$ & .00 & .00 \\
\hline & 18 & .00 & $6.41 \mathrm{E}-10$ & 6.73 & .0 & .0 \\
\hline & & .00 & $1.06 \mathrm{E}-10$ & 1.11 & .00 & .0 \\
\hline & 110 & .00 & $1.64 \mathrm{E}-11$ & $1.71 \mathrm{E}-12$ & .00 & .00 \\
\hline
\end{tabular}




\section{Appendix B - Inhalation Dose Rate Factors - FILE23}

\begin{tabular}{|c|c|c|c|c|c|c|}
\hline $\begin{array}{l}\text { CS135 } \\
\text { CS135 } \\
\text { CS } 137\end{array}$ & $\begin{array}{l}111 \\
112\end{array}$ & $\begin{array}{l}.00 \\
.00 \\
12\end{array}$ & $\begin{array}{l}3.64 \mathrm{E}-12 \\
.00 \\
0\end{array}$ & $\begin{array}{l}3.41 \mathrm{E}-13 \\
1.14 \mathrm{E}-13\end{array}$ & $\begin{array}{l}.00 \\
.00\end{array}$ & $\begin{array}{l}.00 \\
.00\end{array}$ \\
\hline CS137 & 11 & $1.38 \mathrm{E}-04$ & $1.93 E-04$ & $5.53 \mathrm{E}-05$ & .00 & $2.59 \mathrm{E}-06$ \\
\hline 5137 & 12 & 4.94 & 1. & $2 E-05$ & .00 & 1.5 \\
\hline 7 & 13 & $1.30 \mathrm{E}$ & 2. & $3.39 \mathrm{E}-06$ & .00 & .00 \\
\hline 7 & 14 & $3.41 \mathrm{E}$ & -06 & $5.43 \mathrm{E}-07$ & .00 & .00 \\
\hline 1 & 15 & 8.9 & & E-08 & .00 & .00 \\
\hline CS137 & 16 & $2.18 \mathrm{E}$ & $9.08 \mathrm{E}-08$ & $1.40 \mathrm{E}-08$ & .00 & .00 \\
\hline CS137 & 17 & .00 & $1.45 \mathrm{E}-08$ & $2.23 \mathrm{E}$ & .00 & .00 \\
\hline $\operatorname{CS} 137$ & 18 & .00 & $2.34 \mathrm{E}-09$ & $3.58 \mathrm{E}$ & .00 & .00 \\
\hline CS13 & 19 & .00 & $3.71 E-10$ & $5.82 \mathrm{E}-11$ & .00 & .00 \\
\hline CS137 & 110 & .00 & $6.18 \mathrm{E}-11$ & $9.09 \mathrm{E}-12$ & .00 & .00 \\
\hline CS137 & 111 & .00 & $7.28 \mathrm{E}-12$ & $1.82 \mathrm{E}-12$ & .00 & .00 \\
\hline $\begin{array}{l}\text { CS137 } \\
\text { BA137M }\end{array}$ & 112 & .00 & $\begin{array}{c}3.64 \mathrm{E}-12 \\
0\end{array}$ & .00 & .00 & .00 \\
\hline BA137M & 11 & 1.97E-10 & $1.50 \mathrm{E}-09$ & $4.89 E-08$ & $0.00 \mathrm{E}+00$ & $0.00 \mathrm{E}+00$ \\
\hline $\begin{array}{l}\text { BA137M } \\
\text { BA140 }\end{array}$ & 12 & $\begin{array}{r}2.55 \mathrm{E}-15 \\
2\end{array}$ & $\begin{array}{c}1.94 E-14 \\
0\end{array}$ & $1 E-13$ & $0.00 \mathrm{E}$ & $0.00 \mathrm{E}+00$ \\
\hline BA140 & 11 & 8. $12 \mathrm{E}-05$ & $1.04 \mathrm{E}-03$ & $4.53 \mathrm{E}-0.5$ & .00 & $4.50 \mathrm{E}-05$ \\
\hline BA140 & 12 & $3.68 \mathrm{E}-06$ & $4.71 \mathrm{E}-05$ & $8.62 \mathrm{E}-08$ & .00 & $2.66 \mathrm{E}-09$ \\
\hline LA140 & & 2 & 0 & & & \\
\hline LA140 & 11 & $4.51 \mathrm{E}-10$ & $2.57 \mathrm{E}-09$ & $2.54 \mathrm{E}-09$ & .00 & $4.63 \mathrm{E}-09$ \\
\hline LA140 & 12 & $3.36 \mathrm{E}-12$ & 1.92 & $3.87 \mathrm{E}-12$ & .00 & $2.57 \mathrm{E}-13$ \\
\hline CE141 & & 0 & 4 & & & \\
\hline CE141 & 3 & $1.90 \mathrm{E}-07$ & $2.27 \mathrm{E}-06$ & $3.73 \mathrm{E}-04$ & .00 & .89 \\
\hline CE & 2 & $3.19 \mathrm{E}-08$ & 3.94 & 4. & .00 & 2.6 \\
\hline Ct & 33 & $2.25 \mathrm{E}-11$ & 3.24 & 1. & .00 & 3.00 \\
\hline CE & 4 & $1.07 \mathrm{E}-14$ & 1.71 & $3.64 \mathrm{E}-12$ & .00 & .00 \\
\hline PR & & 0 & 7 & & & \\
\hline$P R$ & 3 & $1.09 \mathrm{E}-07$ & 2.53 & 3.1 & .00 & 1.43 \\
\hline & 2 & $5.76 \mathrm{E}-09$ & $1.56 \mathrm{~B}$ & 1.7 & .00 & 3.01 \\
\hline PR & & $8.88 \mathrm{E}-16$ & .00 & .0 & .00 & .00 \\
\hline & & & 19 & & & \\
\hline $\mathrm{Cl}$ & & $4.71 \mathrm{E}-06$ & $7.45 \mathrm{E}-05$ & 8.25 & .00 & 6.28 \\
\hline & & $7.63 \mathrm{E}-06$ & $1.33 \mathrm{E}-04$ & $7.19 \mathrm{E}-03$ & $0.00 \mathrm{E}$ & 1.9 \\
\hline & 33 & $4.28 \mathrm{E}-06$ & 8.2 & 1. & $0.00 \mathrm{E}-$ & 4.5 \\
\hline & 34 & $1.98 \mathrm{E}-06$ & $4.38 \mathrm{E}-05$ & $4.39 E-04$ & $0.00 \mathrm{E}$ & 1.12 \\
\hline & 35 & $8.26 \mathrm{E}-07$ & $2.04 \mathrm{E}-05$ & $1.08 \mathrm{E}-04$ & $0.00 \mathrm{E}+00$ & 2.76 \\
\hline & 36 & 3.2 & 8.87 & $2.69 \mathrm{E}-05$ & $0.00 \mathrm{E}+00$ & 6.85 \\
\hline & & 1.2 & & $E-06$ & $0.00 E+00$ & 1.69 \\
\hline & 38 & 4.34 & & $4 E-06$ & $0.00 \mathrm{E}+00$ & $4.13 \mathrm{E}-09$ \\
\hline & & 1.53 & & 4. & $0.00 \mathrm{E}+00$ & $1.05 E-09$ \\
\hline & 31 & 5.25 & 2.21 & $1.01 \mathrm{E}-07$ & $0.00 \mathrm{E}+00$ & $2.91 \mathrm{E}-10$ \\
\hline & 31 & $1.79 \mathrm{E}-0$ & 8.38 & $2.42 \mathrm{E}-08$ & $0.00 E+00$ & 5.8 \\
\hline & 31 & 6.00 & $3.12 \mathrm{E}-08$ & $9 E-09$ & $0.00 E+00$ & $0.00 E+00$ \\
\hline$C E$ & 31 & 2.02 & 1.16 & 0. & $0.00 E+00$ & 0.00 \\
\hline & & & & & $0.00 E+00$ & $0.00 E+00$ \\
\hline & & 2.1 & 1.5 & 0. & $0.00 \mathrm{E}+$ & 0.0 \\
\hline$C F$ & & 7.2 & 5.53 & $=+0$ & $0.00 \mathrm{E}+00$ & $0.00 \mathrm{E}+00$ \\
\hline
\end{tabular}




\section{Appendix B - Inhalation Dose Rate Factors - FILE23}

\begin{tabular}{|c|c|c|c|c|c|c|}
\hline $\begin{array}{l}\text { CE144 } \\
\text { CE144 } \\
\text { CE144 }\end{array}$ & $\begin{array}{l}317 \\
318 \\
319\end{array}$ & $\begin{array}{c}1.82 \mathrm{E}-12 \\
0.00 \mathrm{E}+00 \\
0.00 \mathrm{E}+00 \\
0 \quad 0\end{array}$ & $\begin{array}{l}1.75 \mathrm{E}-10 \\
5.82 \mathrm{E}-11 \\
2.91 \mathrm{E}-11 \\
2\end{array}$ & $\begin{array}{l}0.00 \mathrm{E}+00 \\
0.00 \mathrm{E}+00 \\
0.00 \mathrm{E}+00\end{array}$ & $\begin{array}{l}0.00 \mathrm{E}+00 \\
0.00 \mathrm{E}+00 \\
0.00 \mathrm{E}+00\end{array}$ & $0.00 \mathrm{E}+00$ \\
\hline & 31 & $2.22 \mathrm{E}-10$ & $4.38 \mathrm{E}-09$ & $1.79 \mathrm{E}-06$ & $0.00 E+00$ & $4.41 E-1$ \\
\hline R144 & 32 & $1.53 \mathrm{E}-14$ & $3.02 \mathrm{E}-13$ & 8.45 & & \\
\hline 0144 & & 0 & 50 & & & \\
\hline & 1 & $9.98 \mathrm{E}-05$ & $1.86 \mathrm{E}-03$ & $2.86 \mathrm{E}-01$ & $0 E+00$ & $9.35 \mathrm{E}-0$ \\
\hline & 32 & & & & & \\
\hline & & & & & & \\
\hline & 34 & & & & & \\
\hline & & & 1. & & & \\
\hline & 36 & & 1. & & & \\
\hline & & & & & & \\
\hline & 38 & 3. & & & & \\
\hline & & & & & & \\
\hline & 310 & & & & & \\
\hline & & 2.2 & & & +00 & \\
\hline & 3 & 1 . & & & & \\
\hline & & 1. & & & & \\
\hline & & 1. & & & & \\
\hline & & & & & & \\
\hline & & 8. & & & & \\
\hline & & 6. & & & & \\
\hline & & 5. & & & & \\
\hline & & 4. & & & & \\
\hline & & & & & & \\
\hline & & & & & & \\
\hline & 32 & & & & & \\
\hline & & & & & & \\
\hline & 2 & 1. & & & & \\
\hline & & 1. & & & & \\
\hline & & & & & 00 & \\
\hline & & & & & 00 & \\
\hline & & & & & +00 & \\
\hline & & & & & & \\
\hline & & & & & & \\
\hline & & & & & 00 & \\
\hline & & 1 & & & & \\
\hline & & & & & & \\
\hline & & & & & & \\
\hline & & & & & & \\
\hline & & & & & & \\
\hline & & & & & 00 & \\
\hline & & & & & $E+00$ & \\
\hline & & & & & $E+00$ & \\
\hline & & & & & $E+00$ & \\
\hline & & & & & & \\
\hline & & & & & $0.00 \mathrm{E}+00$ & $E+00$ \\
\hline & & & & 000 & $E+00$ & $0.00 \mathrm{E}+0$ \\
\hline
\end{tabular}




\section{Appendix B - Inhalation Dose Rate Factors - FILE23}

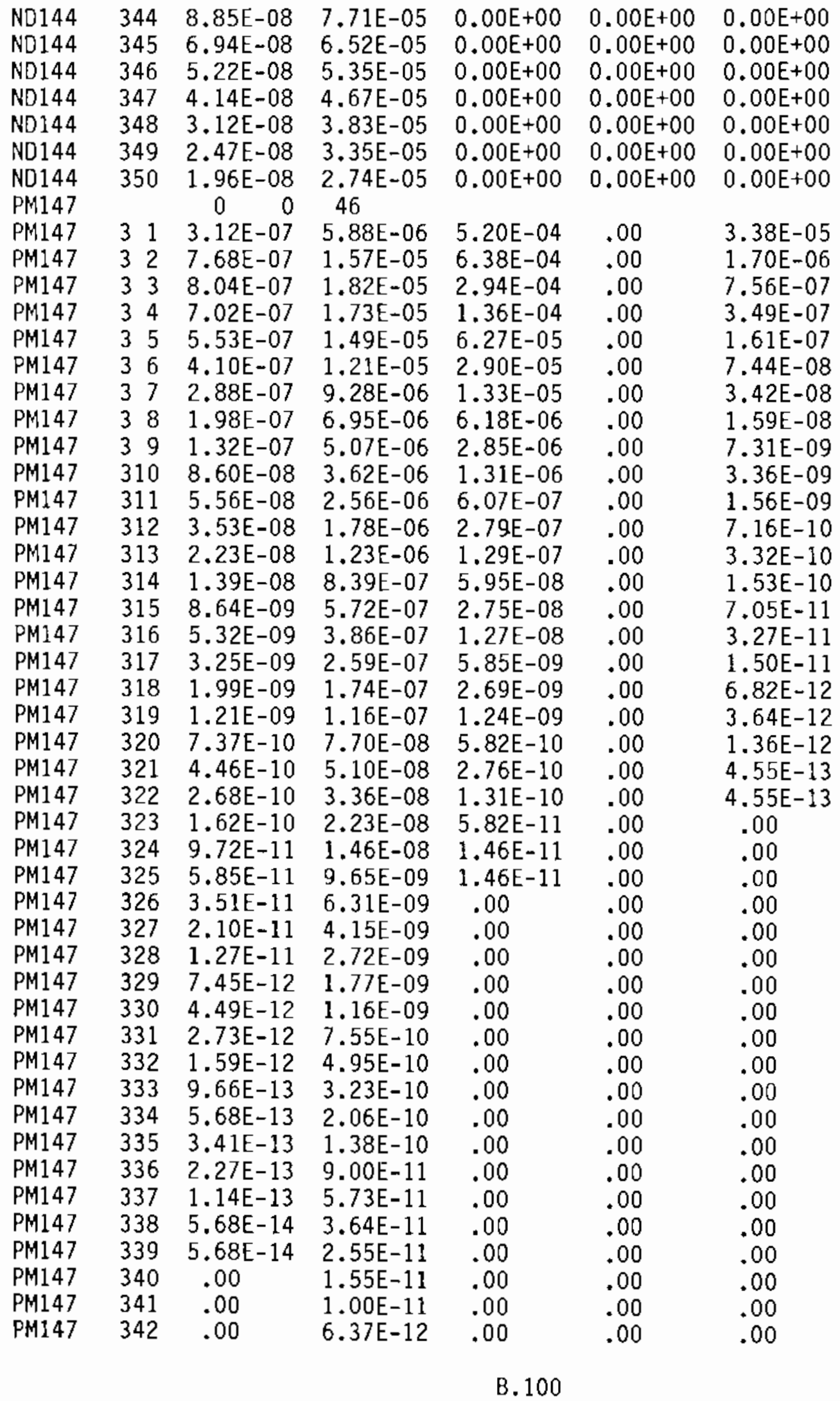




\section{Appendix B - Inhalation Dose Rate Factors - FILE23}

\begin{tabular}{|c|c|c|c|c|c|c|}
\hline S & 343 & .00 & $4.55 \mathrm{E}-12$ & .00 & .00 & .00 \\
\hline PM147 & 344 & .00 & $3.64 E-12$ & .00 & .00 & .00 \\
\hline PM147 & 345 & .00 & $1.82 \mathrm{E}-12$ & .00 & .00 & .00 \\
\hline & 346 & .00 & $9.09 \mathrm{E}-13$ & .00 & .00 & .00 \\
\hline SM15 & & 50 & 0 & & & \\
\hline SM151 & 21 & $3.53 E-06$ & $4.08 \mathrm{E}-05$ & $1.29 \mathrm{E}-04$ & $0.00 \mathrm{E}+00$ & $1.98 \mathrm{E}-05$ \\
\hline & 22 & $6.47 \mathrm{E}-06$ & $8.48 E-05$ & $3.07 \mathrm{E}-05$ & $0.00 \mathrm{E}+00$ & $8.05 \mathrm{E}-07$ \\
\hline M1: & 23 & $4.52 \mathrm{E}-06$ & $7.31 \mathrm{E}-05$ & $1.92 \mathrm{E}-07$ & $0.00 \mathrm{E}+00$ & $4.82 E-09$ \\
\hline SM151 & 24 & $3.05 \mathrm{E}-06$ & $6.13 \mathrm{E}-05$ & $1.21 \mathrm{E}-09$ & $0.00 \mathrm{E}+00$ & $3.09 \mathrm{E}-11$ \\
\hline SM151 & 25 & $2.06 \mathrm{E}-06$ & & $1.46 \mathrm{E}-11$ & $0.00 \mathrm{E}+00$ & $0.00 E+00$ \\
\hline & 26 & $1.39 \mathrm{E}-06$ & & $0.00 \mathrm{E}+00$ & $0.00 E+00$ & $0.00 \mathrm{E}+00$ \\
\hline SM151 & 27 & $9.36 \mathrm{E}-07$ & $3.60 \mathrm{E}-05$ & $0.00 \mathrm{E}+00$ & $0.00 \mathrm{E}+00$ & $0.00 \mathrm{E}+00$ \\
\hline SM1 & 28 & $6.33 E-07$ & $3.03 \mathrm{E}-05$ & $0.00 \mathrm{E}+00$ & $0.00 \mathrm{E}+00$ & $0.00 \mathrm{E}+00$ \\
\hline SM1 & 29 & $4.27 \mathrm{E}-07$ & $2.54 \mathrm{E}-05$ & $0.00 E+00$ & $0.00 \mathrm{E}+00$ & $0.00 E+00$ \\
\hline SM1 & 210 & $2.87 \mathrm{E}-07$ & $2.12 \mathrm{E}-05$ & $0.00 \mathrm{E}+00$ & $0.00 \mathrm{E}+00$ & $0.00 \mathrm{E}+00$ \\
\hline SM1 & 211 & $1.94 \mathrm{E}-07$ & $1.78 \mathrm{E}-05$ & $0.00 E+00$ & $0.00 E+00$ & $0.00 E+00$ \\
\hline SM & 212 & $1.31 \mathrm{E}-07$ & $1.49 \mathrm{E}-05$ & $0.00 \mathrm{E}+00$ & $0.00 E+00$ & $0.00 \mathrm{E}+00$ \\
\hline SM1 & 213 & $8.85 E-08$ & $1.25 \mathrm{E}-05$ & $0.00 \mathrm{E}+00$ & $0.00 \mathrm{E}+00$ & $0.00 \mathrm{E}+00$ \\
\hline$S M$ & 214 & $5.95 \mathrm{E}-08$ & $1.05 \mathrm{E}-05$ & $0.00 \mathrm{E}+00$ & $0.00 E+00$ & $0.00 \mathrm{E}+00$ \\
\hline SM & 215 & $4.03 E-08$ & $8.81 \mathrm{E}-06$ & $0.00 \mathrm{E}+00$ & $0.00 \mathrm{E}+00$ & $0.00 E+00$ \\
\hline SM & 216 & 2. & & $0.00 \mathrm{E}+00$ & $0.00 \mathrm{E}+00$ & $0.00 \mathrm{E}+00$ \\
\hline SM1 & 217 & $E-08$ & $6.17 \mathrm{E}-06$ & $0.00 E+00$ & $0.00 \mathrm{E}+00$ & $0.00 E+00$ \\
\hline SM & 218 & $4 \mathrm{E}-08$ & 5. & $0.00 E+00$ & $0.00 E+00$ & $0.00 \mathrm{E}+00$ \\
\hline SM & 219 & & 4. & $0.00 \mathrm{E}+00$ & $0.00 \mathrm{E}+00$ & $0.00 \mathrm{E}+00$ \\
\hline$S M$ & 220 & 5. & 3. & $0.00 \mathrm{E}+00$ & $0.00 E+00$ & $0.00 \mathrm{E}+00$ \\
\hline SM & 221 & 3. & 3. & $0.00 \mathrm{E}+00$ & $0.00 \mathrm{E}+00$ & 0.00 \\
\hline SM & 222 & 2. & & $0.00 \mathrm{E}+00$ & $0.00 E+00$ & 0. \\
\hline SM1 & 223 & 1. & & $0.00 E+00$ & $0.00 \mathrm{E}$ & 0. \\
\hline SM & 224 & 1. & 1. & $0.00 \mathrm{E}+00$ & $0.00 E+00$ & +00 \\
\hline SM & 225 & 7.8 & 1. & $0.00 \mathrm{E}+00$ & 0. & 0 . \\
\hline SM & 226 & 5.2 & 1. & 0.0 & 0. & 0 . \\
\hline SM & 227 & 3. & 1. & 0.00 & 0. & -00 \\
\hline $\mathrm{SM}$ & 228 & $E-10$ & 8.8 & 0.0 & & 00 \\
\hline SM & 229 & 1 . & 7. & 0. & & 00 \\
\hline SM & 230 & 1. & 6.2 & 0.0 & 0 . & 00 \\
\hline & 231 & & 5. & 0. & & -00 \\
\hline & 232 & & 4. & 0. & & 00 \\
\hline & 233 & 3.2 & 3. & 0. & 0 . & 00 \\
\hline & 234 & 2. & 3. & & 0 . & 100 \\
\hline & 235 & 1.2 & & 0. & 0 . & +00 \\
\hline & 236 & 1. & 2. & 0. & & 0 . \\
\hline & 237 & & 1. & & & 0. \\
\hline & 238 & & & 0 . & 0. & -00 \\
\hline & 239 & 0. & 1.2 & $0.00 \mathrm{E}$ & 0. & -00 \\
\hline & 240 & 5.2 & 1.0 & 0.0 & & 100 \\
\hline SM1 & 241 & 0.0 & 8.7 & 0.0 & 0. & 0. \\
\hline SM1 & 242 & 0.0 & & 0.0 & $0.00 E+00$ & $0.00 \mathrm{E}+00$ \\
\hline SM1 & 243 & 1.8 & & $0.00 E+00$ & $0.00 \mathrm{E}+00$ & $0.00 \mathrm{E}+00$ \\
\hline & 244 & 0 . & & $0.00 \mathrm{E}+00$ & $0.00 \mathrm{E}+00$ & $0.00 E+00$ \\
\hline SM15 & 245 & $0.00 \mathrm{E}+00$ & $4.47 \mathrm{E}-08$ & $0.00 \mathrm{E}+00$ & $0.00 \mathrm{E}+00$ & $0.00 \mathrm{E}+00$ \\
\hline
\end{tabular}


Appendix B - Inhalation Dose Rate Factors - FILE23

\begin{tabular}{|c|c|c|c|c|c|c|}
\hline SM151 & 246 & $0.00 E+00$ & $3.63 E-08$ & $0.00 E+00$ & $0.00 \mathrm{E}+00$ & $0.00 \mathrm{E}+00$ \\
\hline SM151 & 247 & $1.82 \mathrm{E}-12$ & $3.14 \mathrm{E}-08$ & $0.00 \mathrm{E}+00$ & $0.00 \mathrm{E}+00$ & $0.00 \mathrm{E}+00$ \\
\hline SM151 & 248 & $0.00 \mathrm{E}+00$ & $2.56 \mathrm{E}-08$ & $0.00 \mathrm{E}+00$ & $0.00 \mathrm{E}+00$ & $0.00 \mathrm{E}+00$ \\
\hline SM151 & 249 & $0.00 \mathrm{E}+00$ & $2.21 \mathrm{E}-08$ & $0.00 \mathrm{E}+00$ & $0.00 \mathrm{E}+00$ & $0.00 \mathrm{E}+00$ \\
\hline SM151 & 250 & $0.00 \mathrm{E}+00$ & $1.80 \mathrm{E}-08$ & $0.00 E+00$ & $0.00 \mathrm{E}+00$ & $0.00 \mathrm{E}+00$ \\
\hline TH230 & & 0 & 50 & & & \\
\hline TH230 & 31 & $2.68 \mathrm{E}-04$ & $9.37 \mathrm{E}-03$ & $6.87 \mathrm{E}-01$ & $7.90 \mathrm{E}-31$ & $2.31 \mathrm{E}-04$ \\
\hline TH230 & 32 & $9.24 \mathrm{E}-04$ & $3.23 \mathrm{E}-02$ & $9.88 \mathrm{E}-01$ & .00 & 1. \\
\hline TH230 & 33 & $1.49 E-03$ & $5.21 \mathrm{E}-02$ & $5.96 \mathrm{E}-01$ & .00 & $54 E-06$ \\
\hline TH230 & 34 & $2.01 E-03$ & $7.00 \mathrm{E}-02$ & $3.59 \mathrm{E}-01$ & .00 & $5.14 \mathrm{E}-06$ \\
\hline $\mathrm{TH} 230$ & 35 & $2.45 \mathrm{E}-03$ & $8.53 \mathrm{E}-02$ & $2.16 \mathrm{E}-01$ & .00 & $3.10 \mathrm{E}-06$ \\
\hline TH230 & 36 & $2.83 \mathrm{E}-03$ & $9.82 \mathrm{E}-02$ & $1.31 \mathrm{E}-01$ & .00 & $1.87 \mathrm{E}-06$ \\
\hline TH230 & 37 & $3.12 \mathrm{E}-03$ & $1.08 \mathrm{E}-01$ & $7.84 \mathrm{E}-02$ & .00 & $1.12 \mathrm{E}-06$ \\
\hline TH230 & 38 & $3.37 \mathrm{E}-03$ & $1.17 \mathrm{E}-01$ & $4.74 \mathrm{E}-02$ & .00 & $6.80 \mathrm{E}-07$ \\
\hline TH230 & 39 & $3.56 \mathrm{E}-03$ & $1.23 \mathrm{E}-01$ & $2.85 \mathrm{E}-02$ & .00 & $4.09 \mathrm{E}-07$ \\
\hline TH230 & 310 & $3.69 \mathrm{E}-03$ & $1.27 \mathrm{E}-01$ & $1.71 \mathrm{E}-02$ & .00 & $2.46 \mathrm{E}-07$ \\
\hline TH230 & 311 & $3.81 \mathrm{E}-03$ & $1.31 \mathrm{E}-01$ & $1.04 \mathrm{E}-02$ & .00 & $1.49 \mathrm{E}-07$ \\
\hline $\mathrm{TH} 230$ & 312 & $3.88 \mathrm{E}-03$ & $1.33 \mathrm{E}-01$ & $6.23 E-03$ & .00 & $8.93 E-08$ \\
\hline TH230 & 313 & $3.96 \mathrm{E}-03$ & $1.36 \mathrm{E}-01$ & $3.77 \mathrm{E}-03$ & .00 & $5.40 \mathrm{E}-08$ \\
\hline TH230 & $3 i 4$ & $3.99 \mathrm{E}-03$ & $1.36 \mathrm{E}-01$ & $2.26 \mathrm{E}-03$ & .00 & $3.24 \mathrm{E}-08$ \\
\hline TH230 & 315 & $4.03 \bar{E}-03$ & $1.38 \mathrm{E}-01$ & $1.37 \mathrm{E}-03$ & .00 & $1.96 \mathrm{E}-08$ \\
\hline TH230 & 316 & $4.05 \mathrm{E}-03$ & $1.38 \mathrm{E}-01$ & $8.24 \mathrm{E}-04$ & .00 & $1.18 \mathrm{E}-08$ \\
\hline TH230 & 317 & $E-03$ & $1.38 \mathrm{E}-01$ & $4.95 \mathrm{E}-04$ & .00 & $7.09 \mathrm{E}-09$ \\
\hline TH230 & 318 & E-03 & $1.38 \mathrm{E}-01$ & $2.99 \mathrm{E}-04$ & .00 & $4.29 \mathrm{E}-09$ \\
\hline TH230 & 319 & -03 & $1.37 \mathrm{E}-01$ & $1.80 \mathrm{E}-04$ & .00 & $2.58 \mathrm{E}-09$ \\
\hline TH230 & 320 & -03 & $1.37 \mathrm{E}-01$ & $1.09 \mathrm{E}-04$ & .00 & $1.56 \mathrm{E}-09$ \\
\hline TH230 & 321 & -03 & $1.36 \mathrm{E}-01$ & $6.54 \mathrm{E}-05$ & .00 & $9.39 \mathrm{E}-10$ \\
\hline TH230 & 322 & -03 & $E-01$ & $3.93 \mathrm{E}-05$ & .00 & $5.60 \mathrm{E}-10$ \\
\hline TH230 & 323 & 4.0 & -01 & $2.38 \mathrm{E}-05$ & .00 & $3.42 \mathrm{E}-10$ \\
\hline TH230 & 324 & $4.01 \mathrm{E}-03$ & -01 & $1.42 \mathrm{E}-05$ & .00 & $2.04 \mathrm{E}-10$ \\
\hline TH230 & 325 & $4.01 \mathrm{E}$ & 1.3 & $8.67 \mathrm{E}-06$ & .00 & $1.27 \mathrm{E}-10$ \\
\hline TH230 & 326 & 3.9 & -01 & $5.19 E-06$ & .00 & $6.91 \mathrm{E}-11$ \\
\hline TH230 & 327 & 3.9 & $=01$ & $3.10 \mathrm{E}-06$ & .00 & $4.73 \mathrm{E}-11$ \\
\hline TH230 & 328 & 3.9 & -01 & $1.91 \mathrm{E}-06$ & .00 & $2.55 \mathrm{E}-11$ \\
\hline TH230 & 329 & 3. & -01 & $1.13 \mathrm{E}-06$ & .00 & $1.82 \mathrm{E}-11$ \\
\hline TH230 & 330 & 3. & $E-01$ & $6.85 E-07$ & .00 & $1.09 \mathrm{E}-11$ \\
\hline TH230 & 331 & 3. & -01 & 4.17 & .00 & $3.64 \mathrm{E}-12$ \\
\hline TH230 & 332 & 3. & -01 & 2.68 & .00 & $3.64 E-12$ \\
\hline TH230 & 333 & 3. & -01 & 1.4 & .00 & $3.64 \mathrm{E}-1$ \\
\hline TH230 & 334 & 3.7 & 1. & 8.9 & .00 & .00 \\
\hline TH230 & 33 & $E-03$ & -01 & $5.96 \mathrm{E}-08$ & .00 & .00 \\
\hline TH230 & 33 & E-03 & -01 & $2.98 \mathrm{E}-08$ & .00 & \\
\hline TH230 & 33 & 3.7 & -01 & .00 & .00 & .00 \\
\hline TH230 & 33 & -03 & -01 & .00 & .00 & .00 \\
\hline $\mathrm{TH} 230$ & & $E-03$ & $E-01$ & .00 & .00 & .00 \\
\hline & & $E-03$ & $E-01$ & & .00 & .00 \\
\hline $\mathrm{TH} 2$ & & $E-03$ & $1.18 \mathrm{E}-01$ & .00 & .00 & .00 \\
\hline $\mathrm{TH} 2$ & & -03 & $1.21 E-01$ & .00 & .00 & .00 \\
\hline TH2 & & $E-03$ & $1.16 \mathrm{E}-01$ & .00 & .00 & .00 \\
\hline $\mathrm{TH} 2$ & & $3.74 \mathrm{E}-03$ & $1.19 \mathrm{E}-01$ & .00 & .00 & .00 \\
\hline
\end{tabular}




\section{Appendix B - Inhalation Dose Rate Factors - FILE23}

\begin{tabular}{|c|c|c|c|c|c|c|}
\hline TH230 & 345 & $3.72 \mathrm{E}-03$ & $1.18 \mathrm{E}-01$ & .00 & .00 & .00 \\
\hline TH230 & 346 & $3.59 \mathrm{E}-03$ & $1.14 \mathrm{E}-01$ & .00 & .00 & .00 \\
\hline TH230 & 347 & $3.69 \mathrm{E}-03$ & $1.17 \mathrm{E}-01$ & .00 & .00 & .00 \\
\hline TH230 & 348 & $3.56 \mathrm{E}-03$ & $1.12 \mathrm{E}-01$ & .00 & .00 & .00 \\
\hline TH230 & 349 & $3.66 \mathrm{E}-03$ & $1.15 \mathrm{E}-01$ & .00 & .00 & .00 \\
\hline TH230 & 350 & $3.53 \mathrm{E}-03$ & $1.11 \mathrm{E}-01$ & .00 & .00 & .00 \\
\hline RA226 & & $0 \quad 50$ & 0 & & & \\
\hline RA226 & 21 & $2.47 \mathrm{E}-02$ & $2.49 \mathrm{E}-02$ & $5.94 \mathrm{E}-01$ & $7.90 \mathrm{E}-31$ & $1.62 \mathrm{E}-04$ \\
\hline RA226 & 22 & $5.33 \mathrm{E}-02$ & $5.41 \mathrm{E}-02$ & $1.42 \mathrm{E}-01$ & .00 & $6.61 \mathrm{E}-06$ \\
\hline RA226 & 23 & $5.24 \mathrm{E}-02$ & $5.41 \mathrm{E}-02$ & $8.93 \mathrm{E}-04$ & .00 & $3.99 \mathrm{E}$ \\
\hline RA226 & 24 & $5.08 \mathrm{E}-02$ & $5.32 \mathrm{E}-02$ & $5.63 \mathrm{E}-06$ & .00 & $2.51 \mathrm{E}-10$ \\
\hline RA226 & 25 & $4.92 \mathrm{E}-02$ & $5.23 \mathrm{E}-02$ & $3.73 \mathrm{E}-08$ & .00 & $1.82 \mathrm{E}-12$ \\
\hline RA226 & 26 & $4.78 \mathrm{E}-02$ & $5.16 \mathrm{E}-02$ & .00 & .00 & 00 \\
\hline RA226 & 27 & $4.61 \mathrm{E}-02$ & $5.07 \mathrm{E}-02$ & .00 & .00 & .00 \\
\hline RA226 & 28 & $4.48 \mathrm{E}-02$ & $5.00 \mathrm{E}-02$ & .00 & .00 & .00 \\
\hline RA226 & 29 & $4.34 \mathrm{E}-02$ & $4.92 \mathrm{E}-02$ & .00 & .00 & .00 \\
\hline RA226 & 210 & $4.19 \mathrm{E}-02$ & $4.83 \mathrm{E}-02$ & .00 & .00 & .00 \\
\hline RA226 & 211 & $4.08 \mathrm{E}-02$ & $4.77 \mathrm{E}-02$ & .00 & .00 & .00 \\
\hline RA226 & 212 & $3.94 \mathrm{E}-02$ & $4.68 \mathrm{E}-02$ & .00 & .00 & .00 \\
\hline RA226 & 213 & $3.83 \mathrm{E}-02$ & $4.62 \mathrm{E}-02$ & .00 & .00 & .00 \\
\hline RA226 & 214 & $3.69 \mathrm{E}-02$ & $4.53 \mathrm{E}-02$ & .00 & .00 & .00 \\
\hline RA226 & 215 & $3.59 \mathrm{E}-02$ & $4.48 \mathrm{E}-02$ & .00 & .00 & .00 \\
\hline RA226 & 216 & $3.48 \mathrm{E}-02$ & $4.40 \mathrm{E}-02$ & .00 & .00 & .00 \\
\hline RA226 & 217 & $3.36 \mathrm{E}-02$ & $4.32 \mathrm{E}-02$ & .00 & .00 & .00 \\
\hline RA226 & 218 & $3.26 \mathrm{E}-02$ & $4.27 \mathrm{E}-02$ & .00 & .00 & .00 \\
\hline RA226 & 219 & $3.15 \mathrm{E}-02$ & $4.19 \mathrm{E}-02$ & .00 & .00 & .00 \\
\hline RA226 & 220 & $3.06 \mathrm{E}-02$ & $4.13 \mathrm{E}-02$ & .00 & .00 & .00 \\
\hline RA226 & 221 & $2.97 \mathrm{E}-02$ & $4.07 \mathrm{E}-02$ & .00 & .00 & .00 \\
\hline RA226 & 222 & $2.87 \mathrm{E}-02$ & $3.99 \mathrm{E}-02$ & .00 & .00 & .00 \\
\hline RA226 & 223 & $2.79 \mathrm{E}-02$ & $3.94 \mathrm{E}-02$ & .00 & .00 & .00 \\
\hline RA226 & 224 & $2.69 \mathrm{E}-02$ & $3.87 \mathrm{E}-02$ & .00 & .00 & .00 \\
\hline RA226 & 225 & $2.62 \mathrm{E}-02$ & $3.82 \mathrm{E}-02$ & .00 & .00 & .00 \\
\hline RA226 & 226 & $2.53 \mathrm{E}-02$ & $3.75 \mathrm{E}-02$ & .00 & .00 & .00 \\
\hline RA226 & 227 & $2.45 \mathrm{E}-02$ & $3.70 \mathrm{E}-02$ & .00 & .00 & .00 \\
\hline RA226 & 228 & $2.38 \mathrm{E}-02$ & $3.64 \mathrm{E}-02$ & .00 & .00 & .00 \\
\hline RA226 & 229 & 2. $30 \mathrm{E}-02$ & $3.57 \mathrm{E}-02$ & .00 & .00 & .00 \\
\hline RA226 & 230 & $2.23 \mathrm{E}-02$ & $3.53 \mathrm{E}-02$ & .00 & .00 & .00 \\
\hline RA226 & 231 & $2.16 \mathrm{E}-02$ & $3.46 \mathrm{E}-02$ & .00 & .00 & .00 \\
\hline RA226 & 232 & $2.10 \mathrm{E}-02$ & $3.42 \mathrm{E}-02$ & .00 & .00 & .00 \\
\hline RA226 & 233 & $2.04 \mathrm{E}-02$ & $3.37 \mathrm{E}-02$ & .00 & .00 & .00 \\
\hline RA226 & 234 & $1.93 \mathrm{E}-02$ & $3.25 \mathrm{E}-02$ & .00 & .00 & .00 \\
\hline RA226 & 235 & $1.93 \mathrm{E}-02$ & $3.30 \mathrm{E}-02$ & .00 & .00 & .00 \\
\hline RA226 & 236 & $1.87 \mathrm{E}-02$ & $3.25 \mathrm{E}-02$ & .00 & .00 & .00 \\
\hline RA226 & 237 & $1.75 \mathrm{E}-02$ & $3.10 \mathrm{E}-02$ & .00 & .00 & .00 \\
\hline RA226 & 238 & $1.75 \mathrm{E}-02$ & $3.15 \mathrm{E}-02$ & .00 & .00 & .00 \\
\hline RA226 & 239 & $1.65 \mathrm{E}-02$ & $3.00 \mathrm{E}-02$ & .00 & .00 & .00 \\
\hline RA226 & 240 & $1.65 \mathrm{E}-02$ & $3.05 \mathrm{E}-02$ & .00 & .00 & .00 \\
\hline RA226 & 241 & $1.55 \mathrm{E}-02$ & $2.91 \mathrm{E}-02$ & .00 & .00 & .00 \\
\hline & 242 & $1.55 \mathrm{E}-02$ & $2.95 \mathrm{E}-02$ & .00 & .00 & .00 \\
\hline & 243 & $1.45 \mathrm{E}-02$ & $2.82 \mathrm{E}-02$ & .00 & .00 & .00 \\
\hline
\end{tabular}




\section{Appendix B - Inhalation Dose Rate Factors - FILE23}

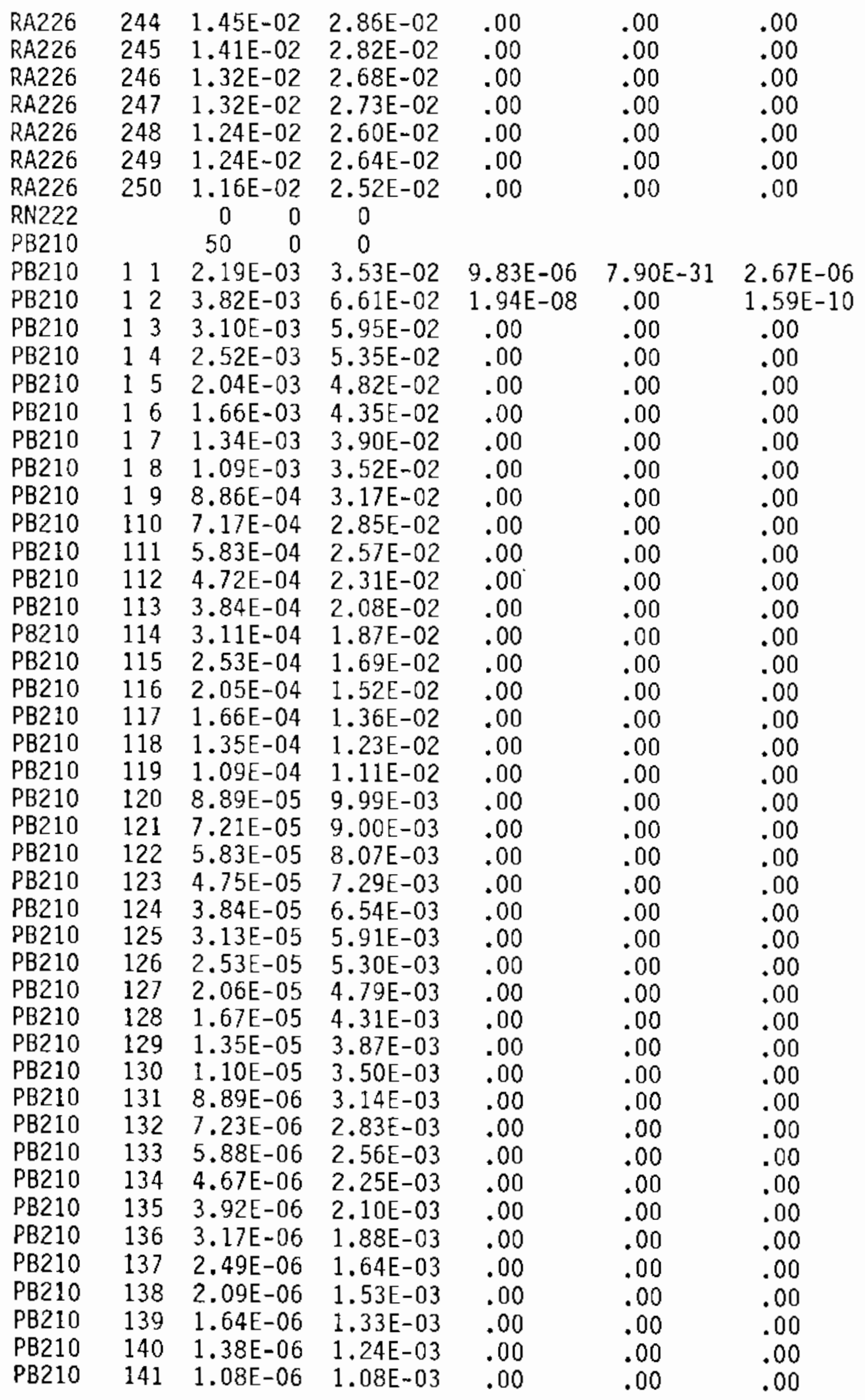




\section{Appendix B - Inhalation Dose Rate Factors - FILE23}

\begin{tabular}{|c|c|c|c|c|c|c|}
\hline PB210 & 142 & $9.06 \mathrm{E}-07$ & $1.00 \mathrm{E}-03$ & .00 & .00 & .00 \\
\hline PB210 & 143 & $7.12 \mathrm{E}-07$ & $8.76 \mathrm{E}-04$ & .00 & .00 & .00 \\
\hline PB210 & 144 & $5.97 \mathrm{E}-07$ & $8.14 \mathrm{E}-04$ & .00 & .00 & .00 \\
\hline PB210 & 145 & 4. B3E-07 & $7.32 \mathrm{E}-04$ & .00 & .00 & .00 \\
\hline PB210 & 146 & $3.79 \mathrm{E}-07$ & $6.39 \mathrm{E}-04$ & .00 & .00 & .00 \\
\hline PB210 & 147 & $3.18 \mathrm{E}-07$ & $5.94 \mathrm{E}-04$ & .00 & .00 & .00 \\
\hline PB210 & 148 & $2.50 E-07$ & $5.18 \mathrm{E}-04$ & .00 & .00 & .00 \\
\hline PB210 & 149 & $2.10 \mathrm{E}-07$ & $4.81 \mathrm{E}$ & .00 & .00 & .00 \\
\hline PB210 & 150 & $1.65 \mathrm{E}-07$ & $4.20 \mathrm{E}-04$ & .00 & .00 & .00 \\
\hline$B 1210$ & & 0 & 0 & & & \\
\hline & 21 & $1.43 \mathrm{E}-05$ & $2.48 \mathrm{E}-05$ & $9.94 \mathrm{E}-03$ & $7.90 \mathrm{E}-31$ & $1.30 \mathrm{t}-04$ \\
\hline & 22 & $1.56 \mathrm{E}-07$ & $3.86 \mathrm{E}-07$ & $1.65 \mathrm{E}-04$ & .00 & $.99 \mathrm{E}-07$ \\
\hline & & 04 & 0 & & & \\
\hline PU & 21 & $1.14 \mathrm{E}-03$ & $4.91 \mathrm{E}-03$ & $2.33 \mathrm{E}-01$ & $7.90 \mathrm{E}-31$ & 2,30 \\
\hline & 22 & $1.94 \mathrm{E}-04$ & & 3.8 & .00 & 5.52 \\
\hline & 23 & $3.41 \mathrm{E}-07$ & 1.1 & 3.91 & .00 & -09 \\
\hline PO & 24 & 5.53 & 1.86 & 4.10 & .00 & 5.4 \\
\hline & & 0 & & & & \\
\hline & 1 & $3.46 \mathrm{E}-04$ & -02 & $8.16 \mathrm{E}-0$ & $0.00 \mathrm{E}+00$ & 1.97 \\
\hline & 32 & $1.19 \mathrm{E}-03$ & 3.6 & 1.17 & $0.00 \mathrm{E}$ & 1.24 \\
\hline & 33 & 1. $93 \mathrm{E}-03$ & 5.8 & 7.07 & $0.00 \mathrm{E}$ & 7.2 \\
\hline & 34 & $2.59 \mathrm{E}-03$ & 7.8 & 4.26 & $0.00 \mathrm{E}$ & 4.3 \\
\hline & 35 & 3.1 & 9. & 2.57 & 0.00 & 2.6 \\
\hline & 36 & 3.6 & 1.1 & 1.5 & $0.00 \mathrm{E}$ & 1.5 \\
\hline & 37 & 4.03 & 1.22 & 9.3 & 0.00 & 9. \\
\hline & 38 & 4. & 1.3 & 5.6 & 0.00 & \\
\hline $\mathrm{TH}$ & 39 & 4. & 1.3 & 3.3 & $0.00 E$ & 3. \\
\hline & 310 & 4 & 1.2 & 2.0 & 0.00 & 2.0 \\
\hline & 311 & 4. & 1.4 & 1.2 & $0.00 \mathrm{E}$ & 1.2 \\
\hline & 312 & 5. & 1.5 & 7.4 & 0.00 & 7.6 \\
\hline & 313 & 5. & 1.5 & 4.4 & 0.00 & 4.6 \\
\hline & 314 & 5. & 1.5 & 2.6 & $0.00 \mathrm{E}$ & 2.7 \\
\hline & 315 & 5.2 & 1. & 1.6 & 0.00 & 1.6 \\
\hline & 316 & 5.2 & 1. & 9.7 & 0.0 & 1. \\
\hline $\mathrm{TH}$ & 317 & 5.2 & 1. & 5.8 & 0.0 & \\
\hline & $218+>$ & 5.2 & 1. & 3. & 0. & 3. \\
\hline & 31 & 5.2 & 1 . & 2. & 0 . & \\
\hline & 32 & 5.2 & 1. & & 0. & \\
\hline & 321 & 5.2 & 1. & 7. & 0. & \\
\hline & 322 & 5.2 & 1.1 & & 0.1 & \\
\hline & 323 & 5.2 & 1. & & 0.8 & \\
\hline & 32 & $5.1 \mathrm{~B}$ & 1. & 1. & 0.0 & \\
\hline & 32 & & & & 0 . & 1. \\
\hline & 326 & & & 6. & 0. & 5. \\
\hline & 32 & & 1.4 & 3.8 & 0.0 & 4. \\
\hline $\mathrm{TH}_{2}$ & 32 & 5.1 & 1.4 & 2. & 0.0 & 2. \\
\hline & 32 & 5. & 1.4 & 1. & 0.0 & \\
\hline & 33 & 5.0 & 1.4 & & 0.00 & \\
\hline & 33 & 5.0 & 1.4 & 2.3 & $0.00 \mathrm{E}$ & 1. \\
\hline TUn & 332 & 5.03 & $1.44 \mathrm{E}-01$ & $2.38 \mathrm{E}-07$ & $0.00 E+00$ & $0.00 \mathrm{E}+0$ \\
\hline
\end{tabular}




\section{Appendix B - Inhalation Dose Rate Factors - FILE23}

\begin{tabular}{|c|c|c|c|c|c|c|}
\hline 1232 & 333 & $5.03 E-03$ & $43 E-01$ & $E-07$ & $0.00 E+00$ & $E+00$ \\
\hline $\mathrm{H} 232$ & 334 & $4.89 \mathrm{E}-03$ & 01 & $E+00$ & $0.00 \mathrm{E}+00$ & +00 \\
\hline $\mathrm{H} 232$ & 335 & $E-03$ & 1. & & $0.00 E+00$ & $O E+00$ \\
\hline $\mathrm{H} 232$ & 336 & 03 & 1. & $E+00$ & $0.00 \mathrm{E}+00$ & $0 E+00$ \\
\hline TH232 & 33 & & 1. & $O E+00$ & $0.00 \mathrm{E}+00$ & +00 \\
\hline TH232 & 33 & $\mathrm{~J}$ & & $10 E+00$ & $0 E+00$ & $0 E+00$ \\
\hline TH232 & & 4. & & $D E+00$ & $0 E+00$ & \\
\hline TH232 & 34 & 4. & & $0.00 E+00$ & $0.00 E+00$ & $=00$ \\
\hline TH232 & 341 & 4 & & $0.00 E+00$ & $0.00 E+00$ & +00 \\
\hline TH232 & & 4 & 01 & $0 E+00$ & $0 E+00$ & +00 \\
\hline TH232 & & & 01 & $E+00$ & $E+00$ & 0 \\
\hline TH2 & & & 01 & $0 E+00$ & $D E+00$ & $10 \mathrm{E}+00$ \\
\hline TH2 & 3 & 4 & 1 & $E+00$ & $0.00 E+00$ & 0 \\
\hline TH2 & 346 & 4. & 1 & $E+00$ & $0.00 E+00$ & 0. \\
\hline TH23 & 347 & 4 & 1 & $E+00$ & $0 \mathrm{E}+00$ & 100 \\
\hline TH232 & 34 & 4. & 1. & $0.00 E+00$ & $E+00$ & -00 \\
\hline TH232 & 3 & 4. & 1. & $0.00 E+00$ & $0.00 E+00$ & +00 \\
\hline TH232 & 350 & 3 & 1. & $0.00 E+00$ & 0.00 & 0.0 \\
\hline RA & & & & & & \\
\hline AA228 & 2 & 4. $96 \mathrm{E}-02$ & $4.12 \mathrm{E}-02$ & $1.17 \mathrm{E}-01$ & $0 E+00$ & 2. \\
\hline 2A228 & & 9. & 8.3 & 2. & $=+00$ & 8 \\
\hline RA228 & & 8. & -02 & $=-04$ & $0 E+00$ & 4 \\
\hline RA228 & 24 & $2 \mathrm{E}-02$ & 6.4 & $8.49 \mathrm{E}-07$ & $0.00 E+00$ & $E-10$ \\
\hline RA228 & 2 & $7 E-02$ & -02 & $E+00$ & $0.00 E+00$ & $0.00 \mathrm{E}+00$ \\
\hline RA228 & 2 & $5.49 \mathrm{E}-02$ & $\mathrm{E}-02$ & $0.00 \mathrm{E}+00$ & $0.00 E+00$ & $0.00 E+00$ \\
\hline RA228 & & $0 \mathrm{E}-02$ & $4.26 \mathrm{E}-02$ & $0.00 \mathrm{E}+00$ & $0.00 \mathrm{E}+00$ & $0.00 E+00$ \\
\hline RA228 & & -02 & 02 & $0.00 E+00$ & $0.00 \mathrm{E}+00$ & $0.00 \mathrm{E}+00$ \\
\hline RA228 & & 3. & -02 & $0.00 \mathrm{E}+00$ & $0.00 E+00$ & $0.00 E+00$ \\
\hline 28 & 210 & & -02 & $0.00 \mathrm{E}+00$ & $0.00 E+00$ & $0.00 \mathrm{E}+00$ \\
\hline & 21 & & -02 & $0.00 \mathrm{E}+00$ & $0.00 E+00$ & $0.00 E+00$ \\
\hline & 212 & & 2 . & $0.00 \mathrm{E}+00$ & $0.00 E+00$ & $0.00 E+00$ \\
\hline & 213 & 1 & .02 & $0.00 \mathrm{E}+00$ & $0.00 E+00$ & $0.00 \mathrm{E}+00$ \\
\hline & 21 & & -02 & $0.00 E+00$ & $0.00 \mathrm{E}+00$ & $0.00 E+00$ \\
\hline & 21 & & .02 & $0.00 \mathrm{E}+00$ & $0.00 E+00$ & $0.00 E+00$ \\
\hline & 21 & & 2 & $0 E+00$ & $0.00 E+00$ & $0.00 E+00$ \\
\hline & 2 & & & +00 & +00 & 0.00 \\
\hline & & & & 0. & 0. & 0 . \\
\hline & 21 & & & & 0 . & 0. \\
\hline & 22 & & & & & \\
\hline & 22 & & & & 0. & \\
\hline & 22 & & & & $E+00$ & -00 \\
\hline & 22 & & & +00 & $0 E+00$ & $0.00 \mathrm{E}+00$ \\
\hline & 22 & & & $\mathrm{E}+00$ & $0.00 \mathrm{E}+00$ & $0.00 E+00$ \\
\hline & 22 & & 03 & $0.00 \mathrm{E}+00$ & $0.00 \mathrm{E}+00$ & $0.00 E+00$ \\
\hline & 226 & & $:-03$ & $0.00 E+00$ & $0.00 E+00$ & $0.00 E+00$ \\
\hline & 227 & & $E-03$ & $0.00 E+00$ & $0.00 E+00$ & $0.00 E+00$ \\
\hline & 228 & & $E-03$ & $0.00 E+00$ & $0.00 E+00$ & $0.00 \mathrm{E}+00$ \\
\hline & 22 & & & $0 E+00$ & $0.00 E+00$ & $0.00 \mathrm{E}+00$ \\
\hline & 23 & & -03 & $0 E+00$ & $0.00 \mathrm{E}+00$ & $0.00 \mathrm{E}+00$ \\
\hline & 23 & 1.2 & -03 & $0.00 E+00$ & $0.00 \mathrm{E}+00$ & $0.00 \mathrm{E}+00$ \\
\hline
\end{tabular}




\section{Appendix B - Inhalation Dose Rate Factors - FILE23}

\begin{tabular}{|c|c|c|c|c|c|c|}
\hline A22 & 232 & $E-03$ & $1.43 E-03$ & $0.00 E+00$ & $0.00 \mathrm{E}+00$ & 0 \\
\hline A228 & 233 & & $1.25 \mathrm{E}-03$ & $0.00 \mathrm{E}+00$ & $0.00 \mathrm{E}+00$ & $00 \mathrm{E}+00$ \\
\hline & 234 & & 03 & $0.00 \mathrm{E}+00$ & $0 \mathrm{E}+00$ & $0 E+00$ \\
\hline & 235 & & & $0 \mathrm{E}+00$ & & $\mathrm{DE}+00$ \\
\hline 28 & 236 & & & & $0 \mathrm{E}+00$ & +00 \\
\hline & 237 & & & & & \\
\hline & 238 & & & & & \\
\hline & 239 & & & & & \\
\hline & 240 & & & & & \\
\hline & 241 & & & & & \\
\hline & 242 & & & & & \\
\hline & 243 & & & & & \\
\hline & 244 & & & & & \\
\hline & 245 & & & & & \\
\hline & 246 & & & & & \\
\hline & 247 & & & +00 & +00 & \\
\hline & 248 & & & & & \\
\hline & 249 & & & +00 & & \\
\hline RA2 & 250 & & 1.21 & +00 & +00 & \\
\hline TH228 & & 0 & 46 & & & \\
\hline TH228 & 1 & $1.12 \mathrm{E}-03$ & -02 & 00 & 00 & \\
\hline TH22 & 2 & 3.0 & 8. & & 0 & \\
\hline $\mathrm{TH}$ & 33 & 3. & & & 00 & \\
\hline & 34 & 3. & & & & \\
\hline & 35 & 2.7 & & & & \\
\hline & 36 & 2.2 & & & & \\
\hline $\mathrm{TH}$ & 37 & 1.7 & & & 0. & \\
\hline & 8 & 1.2 & & & & \\
\hline & 9 & 9.2 & & & & \\
\hline & 310 & & & & & \\
\hline & 311 & & & & & \\
\hline & 312 & & & & & \\
\hline & 313 & & & & & \\
\hline & 314 & & & & & \\
\hline & 315 & & & & & \\
\hline & 3. & 8. & & & & \\
\hline & 31 & & & & & \\
\hline & 31 & & & & & \\
\hline & 31 & & & & & \\
\hline & גר & & & & & \\
\hline & 32 & & & & & \\
\hline & & & & & & \\
\hline & $a r$ & & & & & \\
\hline & 32 & & & & & \\
\hline & מר & & & & & \\
\hline & & & & & & \\
\hline & & & & & & \\
\hline & & & & & & \\
\hline & & & & & & \\
\hline & & & & & & \\
\hline
\end{tabular}




\section{Appendix B - Inhalation Dose Rate Factors - FILE23}

\begin{tabular}{|c|c|c|c|c|c|c|}
\hline $\mathrm{H} 228$ & 331 & $.61 E-07$ & $9.95 E-06$ & $00 \mathrm{E}+00$ & $00 E+00$ & $00 \mathrm{E}+00$ \\
\hline TH228 & 332 & $2.50 \mathrm{E}-07$ & $6.97 \mathrm{E}$ & $0.00 \mathrm{E}+00$ & $0 E+00$ & $0.00 \mathrm{E}+00$ \\
\hline TH228 & 333 & $1.77 \mathrm{E}-07$ & $4.83 E-06$ & $0.00 \mathrm{E}+00$ & $0.00 E+00$ & 0 \\
\hline $\mathrm{H} 228$ & 334 & $1.15 \mathrm{E}$ & $3.22 \mathrm{E}-06$ & $0.00 E+00$ & $10 E+00$ & 0 \\
\hline H228 & 335 & 8.5 & $2.38 \mathrm{E}-06$ & $0.00 E+00$ & +00 & \\
\hline $\mathrm{H} 228$ & 336 & 5. & $1.61 \mathrm{E}-06$ & $0.00 \mathrm{E}+00$ & $E+00$ & 0 \\
\hline TH228 & . & $1 E-08$ & $1 E-06$ & $0.00 \mathrm{E}+00$ & 00 & \\
\hline TH228 & 338 & $2.79 E-08$ & 8. & $E+00$ & $E+00$ & \\
\hline TH228 & 3 & $1.86 \mathrm{E}-08$ & 07 & $E+00$ & $0 \mathrm{E}+00$ & \\
\hline TH228 & 32 & $\mathrm{E}-08$ & 3. & $E+00$ & +00 & \\
\hline TH228 & 3 & -09 & 2.5 & 00 & -00 & $0 E+00$ \\
\hline TH228 & 3 & -09 & 1. & 00 & & \\
\hline TH228 & 3 & -09 & 1. & +00 & 00 & \\
\hline TH228 & 34 & -09 & 5. & +00 & & \\
\hline TH228 & 34 & -09 & 1.1 & 00 & 00 & \\
\hline TH2 & & -09 & 5.96 & $0.00 \mathrm{E}+00$ & $0.00 \mathrm{E}+00$ & 0 . \\
\hline $\mathrm{AC2}$ & & 0 & & & & \\
\hline AC22 & 1 & $1.60 \mathrm{E}-06$ & $2.02 \mathrm{E}-05$ & -03 & +00 & 2 \\
\hline$A C 2$ & 32 & $1.68 \mathrm{E}-09$ & 2.12 & $5 E-06$ & $E+00$ & 1.51 \\
\hline RA22 & & 0 & & & & \\
\hline RA22 & 21 & $1.36 \mathrm{E}-03$ & $6.81 E-03$ & $1.44 \mathrm{E}-01$ & $0 \mathrm{E}+00$ & $9.60 \mathrm{E}-\mathrm{C}$ \\
\hline RA22 & 22 & $2.19 E-05$ & 1.09 & $1.76 \mathrm{E}-03$ & $0.00 \mathrm{E}+00$ & 1 \\
\hline PB21 & & 2 & 0 & & & \\
\hline PB212 & 11 & $9.01 \mathrm{E}-05$ & $1.26 \mathrm{E}-03$ & $4.13 \mathrm{E}-03$ & $0 E+00$ & $1.28 \mathrm{E}-0$ \\
\hline PB212 & 12 & $1.88 \mathrm{E}-07$ & $2.64 \mathrm{E}-06$ & $3.83 \mathrm{E}-06$ & $0.00 E+00$ & $6.19 \mathrm{E}-1$ \\
\hline BI2 & & 0 & 0 & & & \\
\hline BI2 & & $1.48 E-06$ & $2.21 \mathrm{E}-06$ & $7.55 \mathrm{E}-04$ & $0.00 \mathrm{E}+00$ & $8.06 \mathrm{E}-0$ \\
\hline BI2 & 22 & $2.92 \mathrm{E}-10$ & 4.38 & $1.23 \mathrm{E}-07$ & $0.00 \mathrm{E}+00$ & $1.20 \mathrm{E}-1$ \\
\hline U 23 & & 0 & & & & \\
\hline U 23 & & $2.42 E-04$ & $9 E-03$ & $6.58 \mathrm{E}-01$ & .00 & $2.53 E-0$ \\
\hline U 235 & & 2. & -03 & $9.47 \mathrm{E}-01$ & .00 & $1.60 \mathrm{E}-0$ \\
\hline U 235 & & 2. & -03 & $5.71 \mathrm{E}-01$ & .00 & $9.35 \mathrm{E}-0$ \\
\hline U 235 & & -04 & -03 & $3.44 \mathrm{E}-01$ & .00 & 5. \\
\hline U 235 & 3 & 1. & -03 & $\mathrm{E}-01$ & .00 & 3. \\
\hline U 235 & 36 & 1. & -03 & -01 & .00 & 2. \\
\hline U 235 & 37 & & 2. & -02 & .00 & 1. \\
\hline U 235 & 38 & & 1 . & -02 & .00 & 7. \\
\hline U 235 & 39 & & 03 & $=-02$ & .00 & 4.2 \\
\hline U 235 & 310 & & 03 & $E-02$ & .00 & 2. \\
\hline U 235 & 311 & 05 & 04 & -03 & .00 & 1. \\
\hline U 23 & 312 & 05 & 04 & -03 & .00 & 9. \\
\hline U 23 & 313 & 05 & .04 & $E-03$ & .00 & 5. \\
\hline U 23 & 314 & 05 & $4.77 \mathrm{E}-04$ & $2.17 \mathrm{E}-03$ & .00 & 3. \\
\hline U 23 & 315 & -05 & $3.75 \mathrm{E}-04$ & $1.31 \mathrm{E} \sim 03$ & .00 & 2.1 \\
\hline U 23 & 316 & $=-05$ & $2.93 \mathrm{E}-04$ & $7.89 E-04$ & .00 & 1. \\
\hline U 23 & 317 & $E-05$ & $2.28 \mathrm{E}-04$ & $4.74 \mathrm{E}-04$ & .00 & 7.7 \\
\hline U 23 & 318 & -06 & $1.78 E-04$ & $2.87 E-04$ & .00 & 4.7 \\
\hline U 2 & 31 & $6.55 \mathrm{E}-06$ & $1.38 \mathrm{E}-04$ & $1.72 \mathrm{E}-04$ & .00 & \\
\hline 02 & 32 & $5.11 \mathrm{E}-06$ & $1.08 \mathrm{E}-04$ & $1.04 \mathrm{E}-04$ & .00 & \\
\hline & 321 & $3.97 \mathrm{E}-06$ & $8.40 E-05$ & $6.27 \mathrm{E}-05$ & .00 & 1.0 \\
\hline
\end{tabular}




\section{Appendix B - Inhalation Dose Rate Factors - FILE23}

\begin{tabular}{|c|c|c|c|c|c|c|}
\hline U 235 & 322 & $3.07 \mathrm{E}-06$ & $6.51 E-05$ & $3.77 E-05$ & .00 & $6.18 \mathrm{E}-10$ \\
\hline U 235 & 323 & $2.40 E-06$ & $5.08 \mathrm{E}-05$ & $2.28 \mathrm{E}-05$ & .00 & $3.75 E-10$ \\
\hline U 235 & 324 & $1.86 \mathrm{E}-06$ & $3.93 \mathrm{E}-05$ & $1.37 E-05$ & .00 & $2.22 E-10$ \\
\hline U 235 & 325 & $1.45 \mathrm{E}-06$ & $3.06 E-05$ & $8.29 E-06$ & .00 & $1.38 \mathrm{E}-10$ \\
\hline U 235 & 326 & $1.12 \mathrm{E}-06$ & $2.37 \mathrm{E}-05$ & $4.95 \mathrm{E}-06$ & .00 & $8.00 \mathrm{E}-11$ \\
\hline U 235 & 327 & $8.72 \mathrm{E}-07$ & $1.85 \mathrm{E}-05$ & $3.01 \mathrm{E}-06$ & .00 & $5.09 \mathrm{E}-11$ \\
\hline บ 23 & 328 & $6.77 \mathrm{E}-07$ & $1.44 \mathrm{E}-05$ & $1.82 \mathrm{E}$ & .00 & 2.91 \\
\hline U 23 & 329 & $5.24 \mathrm{E}-07$ & $1.11 \mathrm{E}$ & $1.07 \mathrm{E}$ & .00 & 1.8 \\
\hline U 23 & 330 & $4.08 E-07$ & $8.66 \mathrm{E}-06$ & 6.85 & .00 & 1.09 \\
\hline U 23 & 331 & $3.16 \mathrm{E}-07$ & $6.70 \mathrm{E}-06$ & $3.87 \mathrm{E}$ & .00 & 7.28 \\
\hline U 23 & 332 & $2.46 \mathrm{E}-07$ & $5.22 \mathrm{E}$ & $2.38 \mathrm{E}$ & .00 & 3.64 \\
\hline ป 23 & 333 & $1.91 \mathrm{E}-07$ & $4.06 \mathrm{E}-06$ & $1.49 \mathrm{E}-07$ & .00 & .00 \\
\hline U 23 & 334 & $1.46 \mathrm{E}-07$ & $3.09 \mathrm{E}-06$ & $8.94 \mathrm{E}-08$ & .00 & .00 \\
\hline U 23 & 335 & $1.17 \mathrm{E}-07$ & $2.48 \mathrm{E}-06$ & .00 & .00 & .00 \\
\hline U 23: & 336 & $9.03 \mathrm{E}-08$ & $1.92 \mathrm{E}-06$ & .00 & .00 & .00 \\
\hline U 2 & 337 & $6.80 \mathrm{E}-08$ & $1.44 E-06$ & .00 & .00 & .00 \\
\hline U 2 & 338 & $5.45 \mathrm{E}-08$ & $1.16 \mathrm{E}-06$ & .00 & .00 & .00 \\
\hline U 23 & 339 & $4.10 E-08$ & $8.69 E-07$ & $2.98 \mathrm{E}-08$ & .00 & .00 \\
\hline U 23 & 340 & $3.29 \mathrm{E}-08$ & $6.98 \mathrm{E}-07$ & .00 & .00 & .00 \\
\hline U 2. & 341 & $2.47 \mathrm{E}-08$ & 5.25 & .00 & .00 & .00 \\
\hline U 23 & 342 & $1.98 \mathrm{E}-08$ & $4.21 \mathrm{E}-07$ & .00 & .00 & .00 \\
\hline U 23 & 343 & $1.49 \mathrm{E}-08$ & $3.16 \mathrm{E}-07$ & .00 & .00 & .00 \\
\hline U 23 & 344 & $1.19 \mathrm{E}-08$ & 2.54 & .00 & .00 & .00 \\
\hline U 23 & 345 & $9.27 \mathrm{E}-09$ & 1.96 & .00 & .00 & .00 \\
\hline U 23 & 346 & $6.96 \mathrm{E}-09$ & 1.48 & .00 & .00 & .00 \\
\hline U 23 & 347 & $5.59 E-09$ & $1.19 E-07$ & .00 & .00 & .00 \\
\hline U 2 & 348 & $4.21 E-09$ & $8.89 E-08$ & .00 & .00 & .00 \\
\hline$U:$ & 349 & $3.38 \mathrm{E}-09$ & $7.12 E-08$ & .00 & .00 & .00 \\
\hline $\mathrm{U}$ & 350 & $2.53 E-09$ & $5.36 E-08$ & .00 & .00 & .00 \\
\hline & & 0 & 2 & & & \\
\hline $\mathrm{TH}_{2}$ & 31 & $5.43 E-09$ & $1.18 \mathrm{E}-07$ & $1.17 \mathrm{E}-05$ & .00 & $2.18 \mathrm{E}-$ \\
\hline & 32 & $2.35 \mathrm{E}-11$ & $5.11 E-10$ & $4.31 E-08$ & .00 & $2.58 E-08$ \\
\hline & & 0 & 50 & & & \\
\hline PA2 & 31 & $7.81 \mathrm{E}-04$ & $1.88 \mathrm{E}-02$ & $1.00 E+00$ & $7.90 \mathrm{E}$ & $2.76 E-04$ \\
\hline $\mathrm{PA}$ & 32 & $2.69 \mathrm{E}-03$ & $6.48 \mathrm{E}-02$ & $1.44 \mathrm{E}+00$ & .00 & $1.74 E-05$ \\
\hline & 33 & $4.34 E-03$ & $1.05 \mathrm{E}-01$ & $8.69 E-01$ & .00 & $1.02 E-05$ \\
\hline & 34 & $5.84 E-03$ & 1.41 & $5.23 \mathrm{E}-01$ & .00 & $6.13 \mathrm{E}-06$ \\
\hline & 35 & $7.12 \mathrm{E}-03$ & $1.71 \mathrm{E}-01$ & $3.15 \mathrm{E}-01$ & .00 & $3.69 \mathrm{E}-06$ \\
\hline & 36 & $8.20 E-03$ & 1.97 & $1.90 \mathrm{E}-01$ & .00 & $2.23 E-06$ \\
\hline & 37 & $9.04 \mathrm{E}-03$ & 2.17 & $1.14 \mathrm{E}-01$ & .00 & $1.34 E-06$ \\
\hline & 38 & $9.75 E-03$ & 2.34 & $6.91 \mathrm{E}-02$ & .00 & $8.10 E-07$ \\
\hline & 39 & $1.03 E-02$ & 2.47 & $4.16 \mathrm{E}-02$ & .00 & 4.88 \\
\hline & 310 & $1.07 E-02$ & 2.56 & $2.50 \mathrm{E}-02$ & .00 & $2.93 \mathrm{E}-07$ \\
\hline & 311 & $1.10 E-02$ & $2.64 t$ & $1.51 \mathrm{E}-02$ & .00 & 1.77 \\
\hline & 312 & $1.12 \mathrm{E}-02$ & $2.68 \mathrm{E}-01$ & $9.08 \mathrm{E}-03$ & .00 & $1.06 \mathrm{E}-07$ \\
\hline & 313 & $1.14 \mathrm{E}-02$ & $2.72 E-01$ & $5.49 E-03$ & .00 & $6.43 E-08$ \\
\hline & 314 & $1.14 E-02$ & $2.74 \mathrm{E}-01$ & $3.30 E-03$ & .00 & 3.86 \\
\hline & 1 & $1.16 E-02$ & $2.76 E-01$ & $1.99 \mathrm{E}-03$ & .00 & 2.34 \\
\hline & & $1.16 E-02$ & $2.77 \mathrm{E}-01$ & $1.20 \mathrm{E}-0$ & .00 & 1.41 \\
\hline & 317 & $1.16 \mathrm{E}-02$ & $2.76 \mathrm{E}-01$ & $7.21 \mathrm{E}-04$ & .00 & 0.4 \\
\hline
\end{tabular}




\section{Appendix B - Inhalation Dose Rate Factors - FILE23}

\begin{tabular}{|c|c|c|c|c|c|c|}
\hline PA231 & 318 & $1.16 \mathrm{E}-02$ & $2.77 \varepsilon-01$ & $4.36 \mathrm{E}-04$ & .00 & $5.11 \mathrm{E}-09$ \\
\hline PA231 & 319 & $1.15 \mathrm{E}-02$ & $2.75 \mathrm{E}-01$ & $2.52 E-04$ & .00 & $3.07 \mathrm{E}-09$ \\
\hline PA231 & 320 & $1.15 \mathrm{E}-02$ & $2.75 \mathrm{E}-01$ & $1.58 E-04$ & .00 & $35 E-09$ \\
\hline PA231 & 321 & $1.15 \mathrm{E}-02$ & $2.74 \mathrm{E}-01$ & $9.54 \mathrm{E}-05$ & .00 & $12 E-09$ \\
\hline PA231 & 322 & $1.14 \mathrm{E}-02$ & $2.72 \mathrm{E}-01$ & $5.73 \mathrm{E}-05$ & .00 & $6.69 \mathrm{E}-10$ \\
\hline PA231 & 323 & $1.14 \mathrm{E}-02$ & $2.71 \mathrm{E}-01$ & $3.47 \mathrm{E}-05$ & .00 & $07 \mathrm{E}-10$ \\
\hline PA231 & 324 & $1.13 \mathrm{E}-02$ & $2.69 \mathrm{E}-01$ & $2.09 \mathrm{E}-05$ & .00 & $2.44 \mathrm{E}-10$ \\
\hline PA231 & 325 & $1.13 E-02$ & $2.68 \mathrm{E}-01$ & $1.25 \mathrm{E}-05$ & .00 & $1.46 \mathrm{E}-10$ \\
\hline PA231 & 326 & $1.12 \mathrm{E}-02$ & $2.66 \mathrm{E}-01$ & $7.63 E-06$ & .00 & $09 E-11$ \\
\hline PA231 & 327 & $1.12 \mathrm{E}-02$ & $2.65 \mathrm{E}-01$ & $4.53 \mathrm{E}-06$ & .00 & $6 E-11$ \\
\hline PA231 & 328 & $1.11 \mathrm{E}-02$ & $2.53 \mathrm{E}-01$ & $2.74 \mathrm{E}-06$ & .00 & $27 E-11$ \\
\hline PA231 & 329 & $1.10 \mathrm{E}-02$ & $2.61 \mathrm{E}-01$ & $1.67 \mathrm{E}-06$ & .00 & $1.82 \mathrm{E}-11$ \\
\hline PA231 & 330 & $1.10 \mathrm{E}-02$ & $2.60 \mathrm{E}-01$ & $1.01 E-06$ & .00 & $1.09 E-11$ \\
\hline PA231 & 331 & $1.09 \mathrm{E}-02$ & $2.57 \mathrm{E}-01$ & $5.96 \mathrm{E}-07$ & .00 & $7.28 \mathrm{E}-12$ \\
\hline PA231 & 332 & $1.08 \mathrm{E}-02$ & $2.56 \mathrm{E}-01$ & $2.98 \mathrm{E}-07$ & .00 & \\
\hline PA231 & 333 & $1.08 \mathrm{E}-02$ & $2.55 E-01$ & $2.38 \mathrm{E}-07$ & .00 & .00 \\
\hline PA231 & 334 & $1.05 \mathrm{E}-02$ & $2.48 \mathrm{E}-01$ & $1.19 E-07$ & .00 & \\
\hline PA231 & 335 & $1.08 \mathrm{E}-02$ & $2.54 \mathrm{E}-01$ & $1.19 \mathrm{E}-07$ & .00 & .00 \\
\hline PA231 & 336 & $1.07 E-02$ & $2.53 \mathrm{E}-01$ & $5.96 \mathrm{E}-08$ & .00 & .00 \\
\hline PA231 & 337 & $1.03 \mathrm{E}-02$ & $2.43 E-01$ & .00 & .00 & .00 \\
\hline PA231 & 338 & $1.06 \mathrm{E}-02$ & $2.49 \mathrm{E}-01$ & .00 & .00 & .00 \\
\hline PA231 & 339 & $1.02 \mathrm{E}-02$ & $2.40 \mathrm{E}-01$ & & .00 & .00 \\
\hline PA231 & 340 & $1.05 E-02$ & $2.46 \mathrm{E}-01$ & .00 & .00 & .00 \\
\hline PA231 & 341 & $1.01 E-02$ & $2.36 \mathrm{E}-01$ & .00 & .00 & .00 \\
\hline PA231 & 342 & $1.03 \mathrm{E}-02$ & $2.42 \mathrm{E}-01$ & .00 & .00 & .00 \\
\hline PA231 & 343 & $9.95 \mathrm{E}-03$ & $2.33 \mathrm{E}-01$ & .00 & .00 & .00 \\
\hline PA231 & 344 & $1.02 \mathrm{E}-02$ & $2.39 \mathrm{E}-01$ & .00 & .00 & .00 \\
\hline PA231 & 345 & $1.01 \mathrm{E}-02$ & $2.37 \mathrm{E}-01$ & .00 & .00 & .00 \\
\hline PA231 & 346 & $9.76 \mathrm{E}-03$ & $2.28 \mathrm{E}-01$ & .00 & .00 & .00 \\
\hline PA231 & 347 & -02 & $2.34 E=01$ & .00 & .00 & .00 \\
\hline PA231 & 348 & -03 & $2.25 E-01$ & .00 & .00 & .00 \\
\hline PA231 & 349 & -03 & $2.31 \mathrm{E}-01$ & .00 & .00 & .00 \\
\hline PA2 & 350 & $9.52 \mathrm{E}-03$ & $2.22 \mathrm{E}-01$ & .00 & .00 & .00 \\
\hline & & & & & & \\
\hline$A C$ & 31 & $1.10 \mathrm{E}-03$ & $1.65 \mathrm{E}-02$ & $4.54 \mathrm{E}+00$ & $7.90 \mathrm{E}$ & $9.35 \mathrm{t}$ \\
\hline & 32 & $3.70 E-03$ & 5.56 & $6.40 \mathrm{E}+00$ & .00 & $5.74 \mathrm{E}-07$ \\
\hline$A C$ & 33 & -03 & -02 & $3.74 \mathrm{E}+00$ & .00 & $3.25 \mathrm{E}-07$ \\
\hline$A C$ & 34 & 7. & -01 & $2.18 \mathrm{E}+00$ & .00 & $1.90 \mathrm{E}-07$ \\
\hline & 35 & -03 & -01 & $1.27 \mathrm{E}+00$ & .00 & $1.11 E=07$ \\
\hline A & 36 & 9. & -01 & $7.45 E-01$ & .00 & $6.47 \mathrm{E}-08$ \\
\hline & 37 & & -01 & $4.34 \mathrm{E}-01$ & .00 & $3.77 E-08$ \\
\hline & 38 & & -01 & -01 & .00 & $2.21 \mathrm{E}-08$ \\
\hline & 39 & & 1.70 & $1.48 \mathrm{E}-01$ & .00 & $1.29 E-08$ \\
\hline & 310 & 02 & $1.71 E-01$ & $8.62 \mathrm{E}-02$ & .00 & $7.49 \mathrm{E}-09$ \\
\hline & 311 & 02 & -01 & $5.05 \mathrm{E}-02$ & .00 & $4.39 E-09$ \\
\hline & 312 & & -01 & $2.94 \mathrm{E}-02$ & .00 & $2.56 \mathrm{E}-09$ \\
\hline & 313 & 02 & 1. $66 \mathrm{E}-01$ & $1.72 \mathrm{E}-02$ & .00 & $1.50 \mathrm{E}-09$ \\
\hline & 314 & 02 & $1.61 E-01$ & $1.00 E-02$ & .00 & $8.71 \mathrm{E}-10$ \\
\hline & 315 & -02 & 1. & $5.87 E-03$ & .00 & $5.10 \mathrm{E}-10$ \\
\hline 7 & 316 & 9.8 & 1.5 & $3.43 E-03$ & .00 & $2.98 E-10$ \\
\hline
\end{tabular}




\section{Appendix B - Inhalation Dose Rate Factors - FILE23}

\begin{tabular}{|c|c|c|c|c|c|c|}
\hline $\mathrm{AC} 227$ & 317 & $9.46 E-03$ & $1.48 \mathrm{E}-01$ & $1.99 E-03$ & .00 & $1.73 \mathrm{E}-10$ \\
\hline$A C 227$ & 318 & $9.16 \mathrm{E}-03$ & $1.44 \mathrm{E}-01$ & $1.17 \mathrm{E}-03$ & .00 & $1.02 E-10$ \\
\hline AC 227 & 319 & $8.79 \mathrm{E}-03$ & $1.39 \mathrm{E}-01$ & $6.80 \mathrm{E}-04$ & .00 & $5.90 \mathrm{E}-11$ \\
\hline AC 227 & 320 & $8.49 E-03$ & $1.34 \mathrm{E}-01$ & $3.98 \mathrm{E}-04$ & .00 & $3.47 \mathrm{E}-11$ \\
\hline AC 227 & 321 & $8.16 \mathrm{E}-03$ & $1.29 \mathrm{E}-01$ & $2.32 E-04$ & .00 & $2.01 \mathrm{E}-11$ \\
\hline AC227 & 322 & $7.82 \mathrm{E}-03$ & $1.24 \mathrm{E}-01$ & $1.35 \mathrm{E}-04$ & .00 & $1.18 \mathrm{E}-11$ \\
\hline AC227 & 323 & $7.53 \mathrm{E}-03$ & $1.20 \mathrm{E}-01$ & $7.92 \mathrm{E}-05$ & .00 & $6.82 \mathrm{E}-12$ \\
\hline AC227 & 324 & $7.21 \mathrm{E}-03$ & $1.16 \mathrm{E}-01$ & $4.60 \mathrm{E}-05$ & .00 & $4.09 \mathrm{E}-12$ \\
\hline AC227 & 325 & $6.94 \mathrm{E}-03$ & $1.12 E-01$ & $2.72 \mathrm{E}-05$ & .00 & $2.39 \mathrm{E}-12$ \\
\hline AC 227 & 326 & $6.64 \mathrm{E}-03$ & $1.07 \mathrm{E}-01$ & $1.57 \mathrm{E}-05$ & .00 & $1.36 \mathrm{E}-12$ \\
\hline $\mathrm{AC} 227$ & 327 & $6.39 \mathrm{E}-03$ & $1.04 \mathrm{E}-01$ & $9.06 \mathrm{E}-06$ & .00 & 6.82 \\
\hline$A C 227$ & 328 & $6.13 \mathrm{E}-03$ & $9.96 \mathrm{E}-02$ & $5.48 \mathrm{E}-06$ & .00 & $4.55 \mathrm{E}-13$ \\
\hline AC227 & 329 & $5.86 \mathrm{E}-03$ & $9.56 \mathrm{E}-02$ & $3.10 \mathrm{E}-06$ & .00 & $2.27 \mathrm{E}$ \\
\hline $\mathrm{AC} 227$ & 330 & 5. & $9.23 \mathrm{E}-02$ & $1.91 \mathrm{E}$ & .00 & 2.27 \\
\hline AC227 & 331 & 5. & $8.85 \mathrm{E}-02$ & 1.19 & .00 & 1.14 \\
\hline AC227 & 332 & $5.18 \mathrm{E}-03$ & $8.55 \mathrm{E}-02$ & 4.77 & .00 & .00 \\
\hline $\mathrm{AC} 227$ & 333 & $4.98 \mathrm{E}-03$ & 8.2 & 4.77 & .00 & \\
\hline AC227 & 334 & $4.67 \mathrm{E}-03$ & $7.77 \mathrm{E}-02$ & .00 & .00 & .00 \\
\hline AC227 & 335 & $4.63 \mathrm{E}-03$ & 7.7 & & .00 & .00 \\
\hline AC227 & 336 & $4.43 E-03$ & 7.42 & & .00 & .00 \\
\hline$A C$ & 337 & $4.12 \mathrm{E}-03$ & 6.92 & .00 & .00 & .00 \\
\hline$A C$ & 338 & $4.07 \mathrm{E}-03$ & -02 & .00 & .00 & .00 \\
\hline $\mathrm{AC}$ & 33 & -03 & 6.40 & .00 & .00 & .00 \\
\hline $\mathrm{AC}$ & 34 & 3.7 & 6.36 & .00 & .00 & .00 \\
\hline $\mathrm{AC}$ & 34 & -03 & 5. & .00 & .00 & .00 \\
\hline & 34 & -03 & 5.8 & .00 & .00 & .00 \\
\hline$A C$ & 34 & -03 & 5.49 & .00 & .00 & .00 \\
\hline$A C$ & 34 & $3.16 \mathrm{E}-03$ & 5.4 & .00 & .00 & .00 \\
\hline$A C$ & 34 & -03 & 5. & .00 & .00 & .00 \\
\hline$A C$ & 34 & -03 & 4. & .00 & .00 & .00 \\
\hline AC2227 & 34 & 2.7 & 4.8 & .00 & .00 & .00 \\
\hline $\mathrm{AC} 2$ & 34 & 2. & 4. & .00 & .00 & .00 \\
\hline AC2 & 34 & -03 & 4.49 & .00 & .00 & .00 \\
\hline $\mathrm{AC}_{2}$ & 35 & $2.38 \mathrm{E}-03$ & 4.19E-02 & .00 & .00 & .00 \\
\hline $\mathrm{TH}_{2}$ & & 0 & & & & \\
\hline TH227 & 31 & $1.08 \mathrm{E}-04$ & $3.73 \mathrm{E}-03$ & $7.47 \mathrm{E}-01$ & $7.90 \mathrm{E}-31$ & $2.81 E-04$ \\
\hline TH227 & 32 & $9.41 \mathrm{E}-06$ & & $5.43 \mathrm{E}-02$ & .00 & $6.63 \mathrm{E}-07$ \\
\hline TH227 & 33 & $2.27 \mathrm{E}-11$ & 8.15 & $3.73 \mathrm{E}-08$ & .00 & .00 \\
\hline FR223 & & 0 & & & & \\
\hline FR223 & 11 & $0.00 \mathrm{E}+00$ & $0.00 E+00$ & $2.47 \mathrm{E}-07$ & $0.00 \mathrm{E}+00$ & $0.00 \mathrm{E}+00$ \\
\hline FR223 & 12 & $0.00 E+00$ & $0.00 \mathrm{E}+00$ & $1.43 \mathrm{E}-11$ & $0.00 \mathrm{E}+00$ & $4.36 \mathrm{E}-21$ \\
\hline RA223 & & ? & 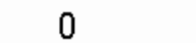 & & & \\
\hline RA223 & 21 & $4.61 E-03$ & $2.31 \mathrm{E}-02$ & $3.60 \mathrm{E}-01$ & $7.90 \mathrm{E}-31$ & $1.02 \mathrm{E}-03$ \\
\hline RA223 & 22 & $2.42 \mathrm{E}-04$ & $1.21 E-03$ & $1.32 E-02$ & .00 & $3.60 \mathrm{E}-06$ \\
\hline & & 50 & 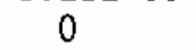 & & & \\
\hline NP2 & & $4.64 \mathrm{E}-03$ & $1.07 \mathrm{E}-01$ & $2.64 \mathrm{E}-01$ & .00 & $2.36 \mathrm{E}-04$ \\
\hline & & $1.07 \mathrm{E}-02$ & $2.45 E-01$ & $6.31 \mathrm{E}-02$ & .00 & $9.64 \mathrm{E}-06$ \\
\hline & 23 & $1.08 \mathrm{E}-02$ & $2.49 \mathrm{E}-01$ & $3.98 \mathrm{E}-04$ & .00 & $5.83 \mathrm{E}-08$ \\
\hline & & $1.08 \mathrm{E}-02$ & $2.47 \mathrm{E}-01$ & $2.51 E-06$ & .00 & $3.71 \mathrm{E}$ \\
\hline & & $1.07 \mathrm{E}-02$ & $2.45 \mathrm{E}-01$ & $1.49 E-08$ & .00 & .00 \\
\hline
\end{tabular}


Appendix B - Inhalation Dose Rate Factors - FILE23

\begin{tabular}{|c|c|c|c|c|c|c|}
\hline NP237 & 26 & $1.07 \mathrm{E}-02$ & $2.44 \mathrm{E}-01$ & .00 & .00 & .00 \\
\hline NP237 & 27 & $1.06 \mathrm{E}-02$ & $2.42 \mathrm{E}-01$ & .00 & .00 & .00 \\
\hline NP237 & 28 & $1.05 \mathrm{E}-02$ & $2.41 \mathrm{E}-01$ & .00 & .00 & .00 \\
\hline NP237 & 29 & $1.04 \mathrm{E}-02$ & $2.39 \mathrm{E}-01$ & .00 & .00 & .00 \\
\hline NP237 & 210 & $1.03 \mathrm{E}-02$ & $2.37 \mathrm{E}-01$ & .00 & .00 & .00 \\
\hline NP237 & 211 & $1.03 \mathrm{E}-02$ & $2.36 \mathrm{E}-01$ & .00 & .00 & .00 \\
\hline NP237 & 212 & $1.02 \mathrm{E}-02$ & $2.33 \mathrm{E}-01$ & .00 & .00 & .00 \\
\hline NP237 & 213 & $1.02 \mathrm{E}-02$ & $2.33 \mathrm{E}-01$ & .00 & .00 & .00 \\
\hline NP237 & 214 & $1.01 \mathrm{E}-02$ & $2.30 \mathrm{E}-01$ & .00 & .00 & .00 \\
\hline NP237 & 215 & $1.00 E-02$ & $2.29 E-01$ & .00 & .00 & .00 \\
\hline NP237 & 216 & $9.98 \mathrm{E}-03$ & $2.28 E-01$ & .00 & .00 & .00 \\
\hline NP237 & 217 & $9.89 \mathrm{E}-03$ & $2.25 \mathrm{E}-01$ & .00 & .00 & .00 \\
\hline NP237 & 218 & $9.86 \mathrm{E}-03$ & $2.25 \mathrm{E}-01$ & .00 & .00 & .00 \\
\hline NP237 & 219 & $9.76 \mathrm{E}-03$ & $2.22 \mathrm{E}-01$ & .00 & .00 & .00 \\
\hline NP237 & 220 & $9.73 \mathrm{E}-03$ & $2.21 \mathrm{E}-01$ & .00 & .00 & .00 \\
\hline NP237 & 221 & $9.67 \mathrm{E}-03$ & $2.20 \mathrm{E}-01$ & .00 & .00 & .00 \\
\hline NP237 & 222 & $9.57 \mathrm{E}-03$ & $2.18 \mathrm{E}-01$ & .00 & .00 & .00 \\
\hline NP237 & 223 & $9.54 \mathrm{E}-03$ & $2.17 \mathrm{E}-01$ & .00 & .00 & .00 \\
\hline NP237 & 224 & $9.45 \mathrm{E}-03$ & $2.15 E-01$ & .00 & .00 & .00 \\
\hline NP237 & 225 & $9.42 E-03$ & 2. $14 \mathrm{E}-01$ & .00 & .00 & .00 \\
\hline NP237 & 226 & $9.33 E-03$ & $2.12 \mathrm{E}-01$ & .00 & .00 & .00 \\
\hline NP237 & 227 & $9.30 \mathrm{E}-03$ & $2.11 \mathrm{E}-01$ & .00 & .00 & .00 \\
\hline NP237 & 228 & $9.24 \mathrm{E}-03$ & 2. $10 \mathrm{E}-01$ & .00 & .00 & .00 \\
\hline NP237 & 229 & $9.15 \mathrm{E}-03$ & $2.07 \mathrm{E}-01$ & .00 & .00 & .00 \\
\hline NP237 & 230 & $9.12 \mathrm{E}-03$ & $2.07 E-01$ & .00 & .00 & .00 \\
\hline NP237 & 231 & $9.03 \mathrm{E}-03$ & $2.05 \mathrm{E}-01$ & .00 & .00 & .00 \\
\hline NP237 & 232 & $9.00 E-03$ & $2.04 \mathrm{E}-01$ & .00 & .00 & .00 \\
\hline NP237 & 233 & $8.97 \mathrm{E}-03$ & $2.03 \mathrm{E}-01$ & .00 & .00 & .00 \\
\hline NP237 & 234 & $8.71 E-03$ & $1.97 \mathrm{E}-01$ & .00 & .00 & .00 \\
\hline NP237 & 235 & $8.94 \mathrm{E}-03$ & $2.02 \mathrm{E}-01$ & .00 & .00 & .00 \\
\hline NP237 & 236 & $8.88 \mathrm{E}-03$ & $2.01 \mathrm{E}-01$ & .00 & .00 & .00 \\
\hline NP237 & 237 & $8.55 E-03$ & $1.93 \mathrm{E}-01$ & .00 & .00 & .00 \\
\hline NP237 & 238 & $8.76 \mathrm{E}-03$ & 1. $98 \mathrm{E}-01$ & .00 & .00 & .00 \\
\hline NP237 & 239 & $8.44 \mathrm{E}-03$ & $1.90 \mathrm{E}-01$ & .00 & .00 & .00 \\
\hline NP237 & 240 & $8.65 \mathrm{E}-03$ & $1.95 \mathrm{E}-01$ & .00 & 00 & 00 \\
\hline NP237 & 241 & $8.33 \mathrm{E}-03$ & $1.88 \mathrm{E}-01$ & .00 & .00 & .00 \\
\hline NP237 & 242 & $8.54 \mathrm{E}-03$ & $1.93 \mathrm{E}-01$ & .00 & .00 & .00 \\
\hline NP237 & 243 & $8.22 E-03$ & $1.85 \mathrm{E}-01$ & .00 & .00 & .00 \\
\hline NP237 & 244 & $8.43 E-03$ & $1.90 \mathrm{E}-01$ & .00 & .00 & .00 \\
\hline NP237 & 245 & $\mathrm{E}-03$ & $1.89 \mathrm{E}-01$ & .00 & .00 & .00 \\
\hline NP237 & 246 & $8.06 \mathrm{E}-03$ & $1.81 \mathrm{E}-01$ & .00 & .00 & .00 \\
\hline NP237 & 247 & $8.27 E-03$ & $1.86 \mathrm{E}-01$ & .00 & .00 & .00 \\
\hline NP237 & 248 & $7.96 \mathrm{E}-03$ & $1.79 \mathrm{E}-01$ & .00 & .00 & .00 \\
\hline NP237 & 249 & $8.16 \mathrm{E}-03$ & $1.83 E-01$ & .00 & .00 & .00 \\
\hline NP237 & 250 & $7.85 \mathrm{E}-03$ & $1.76 \mathrm{E}-01$ & .00 & .00 & .00 \\
\hline PA233 & & & 3 & & & \\
\hline PA233 & 31 & $2.57 \mathrm{E}-07$ & $1.48 \mathrm{E}-06$ & $3.25 E-04$ & .00 & $6.00 \mathrm{E}-05$ \\
\hline PA233 & 32 & $3.65 \mathrm{E}-08$ & $2.11 \mathrm{E}-07$ & $3.62 \mathrm{E}-05$ & .00 & $1.78 \mathrm{E}-07$ \\
\hline PA233 & 33 & $7.95 \mathrm{E}-12$ & $4.58 \mathrm{E}-11$ & $2.11 \mathrm{E}-09$ & .00 & $4.09 \mathrm{E}-12$ \\
\hline U 233 & & & 50 & & & \\
\hline
\end{tabular}




\section{Appendix 8 - Inhalation Dose Rate Factors - FILE23}

\begin{tabular}{|c|c|c|c|c|c|c|}
\hline 23 & 31 & $E-04$ & 03 & $7.16 \mathrm{E}-01$ & $0.00 \mathrm{E}+00$ & \\
\hline 23 & 32 & S & $8 \mathrm{E}-03$ & $1.03 \mathrm{E}+00$ & $0.00 E+00$ & \\
\hline 233 & 33 & $8 \mathrm{E}-04$ & .03 & $0 \mathrm{E}-01$ & $00 \mathrm{E}+00$ & \\
\hline 233 & 34 & $3 E-04$ & & & $00 E+00$ & \\
\hline 23 & 35 & & & & $0 \mathrm{E}+00$ & \\
\hline 23 & 36 & & & & 00 & \\
\hline & 37 & & & & 00 & \\
\hline 233 & 38 & & & & 00 & \\
\hline & 39 & & & & & \\
\hline & 310 & & & & 00 & \\
\hline & & & & & & \\
\hline & 312 & & & & & \\
\hline & 313 & & & & & \\
\hline & 21 & & & & & \\
\hline & 1. & & & & & \\
\hline & & & & & & \\
\hline & $J$ & & & & & \\
\hline & 318 & & & & & \\
\hline 2 & & & & & & \\
\hline-1 & 320 & & & & & \\
\hline 233 & 321 & & & & & \\
\hline L & 322 & & & & & \\
\hline 2 & 323 & & & & & \\
\hline 23 & 324 & & & & & \\
\hline 23 & 325 & & & & & \\
\hline 2 & 326 & 1.2 & & 5. & 00 & \\
\hline 2 & 327 & & & & & \\
\hline 2 & 328 & & & & 00 & \\
\hline 2 & 329 & 5. & & & & \\
\hline & 330 & & & & & \\
\hline & 331 & 3. & 7 & & & \\
\hline & 33 & & & & & \\
\hline 23 & 33 & & & & & \\
\hline & 32 & & & & & \\
\hline & r & & & & & \\
\hline & 3 & & & & & \\
\hline & & & & & & \\
\hline & & & & & & \\
\hline & & & & & & \\
\hline & & & & & & \\
\hline & & & & & & \\
\hline & & & & & & \\
\hline 2 & & & & & & \\
\hline 2 & & & & & & \\
\hline U 23 & 34 & & & & & \\
\hline & 34 & & & & & \\
\hline 20 & 34 & & & & & \\
\hline & 34 & & & & & \\
\hline 2 & & & & & & \\
\hline & & & & & & \\
\hline
\end{tabular}


Appendix B - Inhalation Dose Rate Factors - FILE23

\begin{tabular}{|c|c|c|c|c|c|c|}
\hline$H 229$ & & 0 & 50 & & & \\
\hline TH229 & 31 & $1.84 \mathrm{E}-03$ & $3.67 \mathrm{E}-02$ & $3.86 \mathrm{E}+00$ & $0.00 \mathrm{E}+00$ & $1.97 \mathrm{E}-\mathrm{C}$ \\
\hline TH229 & 32 & $E-03$ & $1.27 \mathrm{E}-01$ & $5.56 \mathrm{E}+00$ & $0.00 \mathrm{E}+00$ & -04 \\
\hline TH229 & 33 & $1.03 E-02$ & $2.05 \mathrm{E}-01$ & $3.35 \mathrm{E}+00$ & $0.00 E+00$ & $7.26 \mathrm{E}-05$ \\
\hline TH229 & 34 & $1.38 \mathrm{E}-02$ & $2.76 \mathrm{E}-01$ & $2.02 \mathrm{E}+00$ & $0.00 \mathrm{E}+00$ & \\
\hline TH229 & 35 & $1.68 \mathrm{E}-02$ & $3.37 \mathrm{E}-01$ & $1.22 \mathrm{E}+00$ & $0.00 \mathrm{E}+00$ & \\
\hline TH229 & 36 & $1.94 \mathrm{E}-02$ & & $7.34 E-01$ & $0.00 \mathrm{E}+00$ & \\
\hline TH229 & 37 & $2.14 \mathrm{E}-02$ & & & $0.00 \mathrm{E}+00$ & \\
\hline TH229 & 38 & 2.3 & & 2.66 & $0.00 E+00$ & \\
\hline TH229 & & & & $1.60 \mathrm{E}-01$ & $\mathrm{OE}+0 \mathrm{O}$ & \\
\hline TH229 & 310 & & & $9.64 \mathrm{E}$ & $\mathrm{OE}+0 \mathrm{O}$ & 2.09 \\
\hline TH229 & 311 & 2.6 & & 5 & +00 & 06 \\
\hline TH229 & 312 & 2.6 & & 02 & $0.00 \mathrm{E}+00$ & \\
\hline TH229 & 313 & 2.72 & & $2.12 \mathrm{E}-02$ & $O E+00$ & 07 \\
\hline TH229 & 314 & 2.7 & & -02 & $E+00$ & 07 \\
\hline TH229 & 315 & 2.77 & & 7. & $E+00$ & 07 \\
\hline TH229 & 316 & 2.78 & & 4.63 & $0.00 \mathrm{E}+00$ & 1. \\
\hline TH229 & 317 & $2.78 \mathrm{E}-02$ & & -03 & $\mathrm{E}+00$ & 6 \\
\hline TH229 & 318 & $2.79 \mathrm{E}-02$ & 5 & $1.68 \mathrm{E}-03$ & $\bar{E}+00$ & 3 \\
\hline TH229 & 319 & 2.78 & 5. & 1. & $\mathrm{E}+00$ & 2 \\
\hline TH229 & 320 & $2.79 \mathrm{E}-02$ & $5.64 \mathrm{E}-01$ & $6.08 \mathrm{E}-04$ & $0.00 \bar{E}+00$ & 1 \\
\hline TH229 & 321 & $2.78 \mathrm{E}-02$ & $5.63 \mathrm{E}-01$ & $3.68 \mathrm{E}-04$ & $E+00$ & 7 \\
\hline TH229 & 322 & $2.77 \mathrm{E}-02$ & 5. & -04 & $0.00 E+00$ & 4 \\
\hline TH229 & 323 & 2.77 & & $1.32 \mathrm{E}-04$ & $0.00 \mathrm{E}+00$ & \\
\hline TH229 & 324 & 2.75 & 1 & 8.0 & +00 & 1 \\
\hline TH229 & 325 & 2.75 & & 4. & +00 & 9 \\
\hline TH229 & 326 & 2.7 & & 2. & $+\infty 0$ & \\
\hline TH229 & 327 & 2.7 & & 1.7 & +00 & 4 \\
\hline TH229 & 32 & 2.72 & & 1. & 0.1 & 10 \\
\hline $\mathrm{TH}$ & 32 & 2.7 & & 3 & +00 & 0 \\
\hline $\mathrm{TH}$ & 33 & 2.6 & & 3 & +00 & 2 \\
\hline TH229 & 331 & 2.6 & & 3 & +00 & $=+00$ \\
\hline $\mathrm{TH}$ & 32 & 2.6 & & +00 & +00 & +00 \\
\hline 29 & & 2.6 & & 1 & +00 & $+\infty$ \\
\hline $\mathrm{TH}$ & 33 & 2.6 & & 00 & +00 & $=00$ \\
\hline 29 & & 2.6 & & +00 & +00 & $E+00$ \\
\hline & & 2. & -01 & +00 & +00 & $E+\infty$ \\
\hline TH229 & 3 & 2. & -01 & 0. & +00 & $E+00$ \\
\hline 29 & 3 & 2. & & 0. & +00 & $E+00$ \\
\hline TH229 & 3 & 2. & & 1. & +00 & $E+00$ \\
\hline & & 2 & & 0. & $E+00$ & $E+00$ \\
\hline TH229 & & -02 & 5 & 0.1 & +00 & $E+00$ \\
\hline TH229 & & 2 & & 0.0 & $E+00$ & $E+00$ \\
\hline TH229 & & -02 & & 0.0 & $E+00$ & $E+00$ \\
\hline TH229 & & 0 & & 0.1 & 0.0 & +00 \\
\hline TH229 & & & & 0.0 & 0.0 & \\
\hline TH229 & & & & & 0.0 & \\
\hline & & & & & 0.1 & \\
\hline & & & & 0. & $0.00 \mathrm{E}+00$ & \\
\hline & & & & . & $0.00 \mathrm{E}+00$ & UU \\
\hline
\end{tabular}


Appendix B - Inhalation Dose Rate Factors - FILE23

\begin{tabular}{|c|c|c|c|c|c|c|}
\hline $\begin{array}{l}\text { TH229 } \\
\text { RA225 }\end{array}$ & 350 & 2. $42 \mathrm{E}-02$ & $\begin{array}{c}5.03 E-01 \\
0\end{array}$ & $0.00 \mathrm{E}+00$ & $0.00 E+00$ & $0.00 \mathrm{E}+00$ \\
\hline RA225 & 21 & $5.86 \mathrm{E}-03$ & 2. $93 \mathrm{E}-02$ & $3.81 \mathrm{E}-01$ & $0.00 \mathrm{E}+00$ & $9.83 E-04$ \\
\hline RA225 & 22 & $3.95 \mathrm{E}-04$ & $1.98 \mathrm{E}-03$ & $1.71 \mathrm{E}-02$ & $0.00 \mathrm{E}+00$ & $4.21 \mathrm{E}-06$ \\
\hline RA225 & 23 & $0.00 \mathrm{E}+00$ & $3.73 \mathrm{E}-09$ & $0.00 \mathrm{E}+00$ & $0.00 \mathrm{E}+00$ & $0.00 E+00$ \\
\hline AC225 & & 0 & 2 & & & \\
\hline AC225 & 31 & $8.11 \mathrm{E}-05$ & $1.21 \mathrm{E}-03$ & $3.58 \mathrm{E}-01$ & $0.00 E+00$ & $1.44 \mathrm{E}-03$ \\
\hline AC225 & 32 & 3.55 & $5.29 \mathrm{E}-05$ & $1.36 \mathrm{E}-02$ & $0.00 E+00$ & $2.80 \mathrm{E}-06$ \\
\hline U 238 & & 0 & 50 & & & \\
\hline U 238 & 31 & $2.26 \mathrm{E}-04$ & 1. $91 \mathrm{E}-03$ & $6.15 \mathrm{E}-01$ & .00 & $2.10 \mathrm{E}-04$ \\
\hline U 238 & 32 & $2.80 \mathrm{E}-04$ & $3.76 \mathrm{E}-03$ & $8.85 \mathrm{E}-01$ & .00 & 1. $32 \mathrm{E}-05$ \\
\hline U 238 & 33 & $2.13 \mathrm{E}-04$ & $3.64 \mathrm{E}-03$ & $5.34 \mathrm{E}-01$ & .00 & $7.73 \mathrm{E}-06$ \\
\hline U 238 & 34 & $1.87 \mathrm{E}-04$ & $3.38 \mathrm{E}-03$ & $3.21 \mathrm{E}-01$ & .00 & $4.66 \mathrm{E}-06$ \\
\hline U 238 & 35 & $1.61 \mathrm{E}-04$ & $3.01 \mathrm{E}-03$ & $1.94 \mathrm{E}-01$ & .00 & $2.81 \mathrm{E}-06$ \\
\hline U 238 & 36 & $1.34 \mathrm{E}-04$ & $2.59 \mathrm{E}-03$ & $1.17 \mathrm{E}-01$ & .00 & $1.69 \mathrm{E}-06$ \\
\hline U 238 & 37 & $1.09 \mathrm{E}-04$ & $2.17 \mathrm{E}-03$ & $7.02 \mathrm{E}-02$ & .00 & $1.02 \mathrm{E}-06$ \\
\hline U 238 & 38 & $8.85 \mathrm{E}-05$ & $1.79 \mathrm{E}-03$ & $4.25 \mathrm{E}-02$ & .00 & $6.16 \mathrm{E}-07$ \\
\hline U 238 & 39 & $7.07 \mathrm{E}-05$ & $1.46 \mathrm{E}-03$ & $2.56 \mathrm{E}-02$ & .00 & $3.71 \mathrm{E}-07$ \\
\hline U 238 & 310 & $5.60 E-05$ & $1.17 \mathrm{E}-03$ & $1.54 \mathrm{E}-02$ & .00 & $2.23 \mathrm{E}-07$ \\
\hline U 238 & 311 & $4.43 E-05$ & $9.32 \mathrm{E}-04$ & $9.29 \mathrm{E}-03$ & .00 & $1.35 \mathrm{E}-07$ \\
\hline U 238 & 312 & $3.47 \mathrm{E}-05$ & $7.36 \mathrm{E}-04$ & $5.58 \mathrm{E}-03$ & .00 & $8.09 \mathrm{E}-08$ \\
\hline U 238 & 313 & $2.73 E-05$ & $5.82 \mathrm{E}-04$ & $3.37 \mathrm{E}-03$ & .00 & $4.89 \mathrm{E}-08$ \\
\hline U 238 & 314 & $2.13 E-05$ & $4.56 \mathrm{E}-04$ & $2.03 \mathrm{E}-03$ & .00 & $2.94 \mathrm{E}-08$ \\
\hline U 238 & 315 & $1.67 \mathrm{E}-05$ & $3.58 \mathrm{E}-04$ & $1.23 \mathrm{E}-03$ & .00 & $1.78 \mathrm{E}-08$ \\
\hline U 238 & 316 & $1.30 \mathrm{E}-05$ & $2.80 \mathrm{E}-04$ & $7.38 \mathrm{E}-04$ & .00 & $1.07 \mathrm{E}-08$ \\
\hline U 238 & 317 & $1.01 \mathrm{E}-05$ & $2.18 \mathrm{E}-04$ & $4.43 E-04$ & .00 & 6.42 \\
\hline U 238 & 318 & $7.89 \mathrm{E}-06$ & $1.70 \mathrm{E}-04$ & $2.68 \mathrm{E}-04$ & .00 & 3.89 \\
\hline U 238 & 319 & $6.12 \mathrm{E}-06$ & $1.32 \mathrm{E}-04$ & $1.61 \mathrm{E}-04$ & .00 & $2.33 \mathrm{E}-09$ \\
\hline U 238 & 320 & $4.77 \mathrm{E}-06$ & $1.03 \mathrm{E}-04$ & $9.74 \mathrm{E}-05$ & .00 & $1.41 \mathrm{E}-09$ \\
\hline U 238 & 321 & $3.71 E-06$ & $8.03 \mathrm{E}-05$ & $5.87 \mathrm{E}-05$ & .00 & $8.49 \mathrm{E}-10$ \\
\hline U 238 & 322 & $2.87 \mathrm{E}-06$ & $6.23 \mathrm{E}-05$ & 3.52 & .00 & $5.09 \mathrm{E}-10$ \\
\hline U 238 & 323 & $2.24 \mathrm{E}-06$ & $4.86 \mathrm{E}-05$ & 2.13 & .00 & $3.09 \mathrm{E}-10$ \\
\hline U 238 & 324 & $1.73 \mathrm{E}-06$ & $3.76 \mathrm{E}-05$ & 1.28 & .00 & $1.87 \mathrm{E}-10$ \\
\hline U 238 & 325 & $1.35 \mathrm{E}-06$ & $2.93 \mathrm{E}-05$ & $7.72 \mathrm{E}-06$ & .00 & $1.11 \mathrm{E}-10$ \\
\hline U 238 & 326 & $1.05 \mathrm{E}-06$ & $2.27 \mathrm{E}-05$ & $4.65 \mathrm{E}-06$ & .00 & $6.91 \mathrm{E}-11$ \\
\hline U 238 & 327 & $8.15 E-07$ & 1.77 & 2.83 & .00 & $3.82 \mathrm{E}-11$ \\
\hline U 238 & 328 & $=-07$ & 1.3 & $1.67 \mathrm{E}-06$ & .00 & 2.55 \\
\hline U 238 & 329 & $E-07$ & 1.0 & $1.04 \mathrm{E}-06$ & .00 & $1.46 \mathrm{~F}-11$ \\
\hline U 238 & 330 & $3.82 E-07$ & 8.2 & $6.26 \mathrm{E}-07$ & .00 & 7.28 \\
\hline U 238 & 331 & $2.95 E-07$ & 6.4 & $3.58 \mathrm{E}-07$ & .00 & $7.28 \mathrm{E}-12$ \\
\hline U 238 & 332 & $2.30 \mathrm{E}-07$ & 4. & $2.38 \mathrm{E}-07$ & .00 & $3.64 \mathrm{E}-12$ \\
\hline U 238 & 333 & 1.7 & 3. & $1.19 \mathrm{E}-07$ & .00 & .00 \\
\hline U 238 & 334 & $1.36 \mathrm{E}-07$ & 2.9 & $5.96 \mathrm{E}-08$ & .00 & 00 \\
\hline 11 & 335 & -07 & 2.3 & $5.96 \mathrm{E}-08$ & .00 & 00 \\
\hline U 2 & 336 & 8.44 & 1.8 & $2.98 \mathrm{E}-08$ & .00 & .00 \\
\hline U 2 & 337 & $6.35 \mathrm{E}-08$ & 1.38 & $2.98 \mathrm{E}-08$ & .00 & .00 \\
\hline 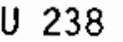 & 338 & $5.09 E-08$ & $1.11 \mathrm{E}-06$ & .00 & .00 & .00 \\
\hline 140 & 339 & $3.83 E-08$ & 8.3 & .00 & 00 & 00 \\
\hline 02 & 340 & $3.07 \mathrm{E}-08$ & $6.68 \mathrm{E}-07$ & .00 & .00 & .00 \\
\hline 110 & 34 & $2.31 \mathrm{E}-08$ & $5.02 \mathrm{E}-07$ & .00 & .00 & .00 \\
\hline
\end{tabular}

B. 115 


\section{Appendix B - Inhalation Dose Rate Factors - FILE23}

\begin{tabular}{|c|c|c|c|c|c|c|}
\hline U 238 & 342 & $1.85 \mathrm{E}-08$ & $4.03 \mathrm{E}-07$ & .00 & .00 & .00 \\
\hline U 238 & 343 & $1.40 \mathrm{E}-08$ & $3.03 E-07$ & .00 & .00 & .00 \\
\hline U 238 & 344 & $1.12 E-08$ & $2.43 \mathrm{E}-07$ & .00 & .00 & .00 \\
\hline U 238 & 345 & $8.66 \mathrm{E}-09$ & $1.88 \mathrm{E}-07$ & .00 & .00 & .00 \\
\hline U 238 & 346 & $6.50 \mathrm{E}-09$ & $1.41 \mathrm{E}-07$ & .00 & .00 & .00 \\
\hline U 238 & 347 & $5.22 \mathrm{E}-09$ & $1.14 \mathrm{E}-07$ & .00 & .00 & .00 \\
\hline U 238 & 348 & $3.91 \mathrm{E}-09$ & $8.48 \mathrm{E}-08$ & .00 & .00 & .00 \\
\hline U 238 & 349 & $3.17 \mathrm{E}-09$ & $6.82 \mathrm{E}-08$ & .00 & .00 & .00 \\
\hline U 238 & 350 & $2.37 E-09$ & $5.15 \mathrm{E}-08$ & .00 & .00 & .00 \\
\hline TH234 & & 0 & 3 & & & \\
\hline TH234 & 31 & $6.43 \mathrm{E}-07$ & $2.22 \mathrm{E}-05$ & $1.46 \mathrm{E}-03$ & .00 & $4.13 E-04$ \\
\hline TH234 & 32 & $7.79 \mathrm{E}-08$ & $2.69 E-06$ & $1.42 \mathrm{E}-04$ & .00 & 1. $12 \mathrm{E}-06$ \\
\hline TH234 & 33 & $4.87 \mathrm{E}-12$ & $1.68 \mathrm{E}-10$ & $2.34 \mathrm{E}-09$ & .00 & $7.28 \mathrm{E}-12$ \\
\hline PA234M & & 0 & 2 & & & \\
\hline PA234M & 31 & 4. $32 \mathrm{E}-13$ & $9.48 \mathrm{E}-12$ & $2.52 E-08$ & $0.00 \mathrm{E}+00$ & $0.00 \mathrm{E}+00$ \\
\hline $\begin{array}{l}\text { PA234M } \\
\text { PA234 }\end{array}$ & 32 & $\begin{array}{c}2.68 \mathrm{E}-18 \\
0\end{array}$ & $\begin{array}{c}5.81 \mathrm{E}-17 \\
2\end{array}$ & $8.17 \mathrm{E}-14$ & $0.00 \mathrm{E}+00$ & $0.00 \mathrm{E}+00$ \\
\hline PA234 & 31 & $1.04 \mathrm{E}-08$ & $4.08 \mathrm{E}-08$ & $2.12 \mathrm{E}-05$ & $0.00 \mathrm{E}+00$ & $2.13 \mathrm{E}-05$ \\
\hline $\begin{array}{l}\text { PA234 } \\
\text { AM242M }\end{array}$ & 32 & 1. $20 \mathrm{E}-11$ & $4.71 \mathrm{E}-11$ & $2.18 \mathrm{E}-08$ & $0.00 \mathrm{E}+00$ & $1.34 \mathrm{E}-08$ \\
\hline$A M * 242$ & 21 & $5.76 \mathrm{~F}-03$ & $1.28 \mathrm{E}-01$ & $7.01 \mathrm{E}-02$ & 0 & \\
\hline$A M * 242$ & 22 & $1.31 \mathrm{E}-02$ & $2.92 \mathrm{E}-01$ & $1.67 \mathrm{~F}-0 ?$ & .00 & $3.30 \mathrm{U}-00$ \\
\hline$A M * 242$ & 23 & $1.32 \mathrm{E}-02$ & $296 \mathrm{~F}-01$ & $1.05 \mathrm{~F}-04$ & .00 & 2.1 \\
\hline $\mathrm{AM} \star 242$ & 24 & $1.30 E-02$ & $2.93 \mathrm{~F}-01$ & $6.60 \mathrm{~F}-07$ & .00 & 1.3 \\
\hline$A M \star 242$ & 25 & $1.28 \mathrm{E}-02$ & $2.89 \mathrm{E}-01$ & $4.66 \mathrm{E}-09$ & .00 & 8.1 \\
\hline$A M * 242$ & 26 & $1.26 \mathrm{E}-02$ & $2.86 \mathrm{E}-01$ & .00 & .00 & 5.0 \\
\hline$A M * 242$ & 27 & 1. $23 \mathrm{E}-02$ & $2.82 \mathrm{E}-01$ & .00 & .00 & .00 \\
\hline$A M \star 242$ & 28 & 1.22E-02 & $2.80 \mathrm{E}-01$ & .00 & .00 & .00 \\
\hline$A M \star 242$ & 29 & $1.19 \mathrm{E}-02$ & $2.77 \mathrm{E}-01$ & .00 & .00 & .00 \\
\hline$A M * 242$ & 210 & $1.17 \mathrm{E}-02$ & $2.73 \mathrm{E}-01$ & .00 & .00 & .00 \\
\hline$A M * 242$ & 211 & $1.15 \mathrm{E}-02$ & $2.70 \mathrm{E}-01$ & .00 & .00 & .00 \\
\hline$A M * 242$ & 212 & $1.13 \mathrm{E}-02$ & $2.66 \mathrm{E}-01$ & .00 & .00 & .00 \\
\hline$A M * 242$ & 213 & $1.12 \mathrm{E}-02$ & $2.64 \mathrm{E}-01$ & .00 & .00 & .00 \\
\hline$A M * 242$ & 214 & $1.09 \mathrm{E}-02$ & $2.60 \mathrm{E}-01$ & .00 & .00 & .00 \\
\hline$A M * 242$ & 215 & $1.08 \mathrm{E}-02$ & $2.58 \mathrm{E}-01$ & .00 & .00 & .00 \\
\hline$A M \star 242$ & 216 & $1.06 \mathrm{E}-02$ & $2.55 \mathrm{E}-01$ & .00 & .00 & .00 \\
\hline$A M * 242$ & 217 & $1.04 E-02$ & $2.52 \mathrm{E}-01$ & .00 & .00 & .00 \\
\hline$A M \star 242$ & 218 & $1.02 \mathrm{E}-02$ & $2.50 \mathrm{E}-01$ & .00 & .00 & .00 \\
\hline$A M * 242$ & 219 & $1.00 \mathrm{E}-02$ & $2.46 \mathrm{E}-01$ & .00 & .00 & .00 \\
\hline$A M \star 242$ & 220 & $9.89 \mathrm{E}-03$ & $2.44 \mathrm{E}-01$ & .00 & .00 & .00 \\
\hline $\mathrm{AM}^{\star} 242$ & 221 & $9.72 \mathrm{E}-03$ & $2.41 \mathrm{E}-01$ & .00 & .00 & .00 \\
\hline$A M * 242$ & 222 & $9.53 \mathrm{E}-03$ & $2.38 \mathrm{E}-01$ & .00 & .00 & .00 \\
\hline$A M^{\star} 242$ & 223 & $9.39 E-03$ & $2.36 \mathrm{E}-01$ & .00 & .00 & .00 \\
\hline$A M \star 242$ & 224 & $9.21 \mathrm{E}-03$ & 2. $32 \mathrm{E}-01$ & .00 & .00 & .00 \\
\hline$A M * 242$ & 225 & $9.08 \mathrm{E}-03$ & $2.30 \mathrm{E}-01$ & .00 & .00 & .00 \\
\hline$A M * 242$ & 226 & $8.89 \mathrm{E}-03$ & $2.27 \mathrm{E}-01$ & .00 & .00 & .00 \\
\hline$A M * 242$ & 227 & $8.77 E-03$ & $2.25 E-01$ & .00 & .00 & 00 \\
\hline$A M^{\star} 242$ & 228 & $8.62 \mathrm{E}-03$ & $2.23 \mathrm{E}-01$ & .00 & .00 & 00 \\
\hline$A M * 242$ & 229 & $8.45 E-03$ & $2.19 \mathrm{E}-01$ & .00 & .00 & .00 \\
\hline$A M * 242$ & 230 & $8.33 \mathrm{E}-03$ & $2.18 E-01$ & .00 & .00 & .00 \\
\hline
\end{tabular}




\section{Appendix B - Inhalation Dose Rate Factors - FILE23}

\begin{tabular}{|c|c|c|c|c|c|c|}
\hline$A M * 242$ & 231 & $8.16 \mathrm{E}-03$ & $2.14 \mathrm{E}-01$ & .00 & .00 & .00 \\
\hline$A M * 242$ & 232 & $8.05 E-03$ & $2.13 \mathrm{E}-01$ & .00 & .00 & .00 \\
\hline$A M * 242$ & 233 & $7.94 \mathrm{E}-03$ & $2.11 \mathrm{E}-01$ & .00 & .00 & .00 \\
\hline$A M * 242$ & 234 & $7.63 \mathrm{E}-03$ & $2.04 \mathrm{E}-01$ & .00 & .00 & .00 \\
\hline$A M \star 242$ & 235 & $7.74 \mathrm{E}-03$ & $2.08 \mathrm{E}-01$ & .00 & .00 & .00 \\
\hline$A M^{*} 242$ & 236 & $7.61 \mathrm{E}-03$ & $2.06 \mathrm{E}-01$ & .00 & .00 & .00 \\
\hline$A M * 242$ & 237 & $7.25 \mathrm{E}-03$ & $1.97 \mathrm{E}-01$ & .00 & .00 & .00 \\
\hline$A M * 242$ & 238 & $7.35 \mathrm{E}-03$ & $2.01 \mathrm{E}-01$ & .00 & .00 & .00 \\
\hline$A M * 242$ & 239 & $7.00 \mathrm{E}-03$ & $1.92 \mathrm{E}-01$ & .00 & .00 & .00 \\
\hline$A M^{*} 242$ & 240 & $7.10 \mathrm{E}-03$ & $1.96 \mathrm{E}-01$ & .00 & .00 & .00 \\
\hline$A M * 242$ & 241 & $6.77 \mathrm{E}-03$ & $1.88 \mathrm{E}-01$ & .00 & .00 & .00 \\
\hline$A M * 242$ & 242 & $6.86 \mathrm{E}-03$ & $1.92 \mathrm{E}-01$ & .00 & .00 & .00 \\
\hline$A M * 242$ & 243 & $6.54 \mathrm{E}-03$ & $1.84 \mathrm{E}-01$ & .00 & .00 & .00 \\
\hline$A M \star 242$ & 244 & $6.63 \mathrm{E}-03$ & $1.88 \mathrm{E}-01$ & .00 & .00 & .00 \\
\hline$A M * 242$ & 245 & $6.52 \mathrm{E}-03$ & $1.85 \mathrm{E}-01$ & .00 & .00 & .00 \\
\hline$A M * 242$ & 246 & $6.21 \mathrm{E}-03$ & $1.78 \mathrm{E}-01$ & .00 & .00 & .00 \\
\hline$A M * 242$ & 247 & $6.30 \mathrm{E}-03$ & $1.81 \mathrm{E}-01$ & .00 & .00 & .00 \\
\hline$A M * 242$ & 248 & $5.00 \mathrm{E}-03$ & $1.74 \mathrm{E}-01$ & .00 & .00 & .00 \\
\hline$A M \star 242$ & 249 & $6.09 \mathrm{E}-03$ & $1.77 \mathrm{E}-01$ & .00 & .00 & .00 \\
\hline$A M \times 242$ & 250 & $5.80 \mathrm{E}-03$ & $1.70 \mathrm{E}-01$ & .00 & .00 & .00 \\
\hline AM242 & & $0 \quad 2$ & 0 & & & \\
\hline AM2 42 & 21 & $2.36 \mathrm{E}-05$ & $5.40 \mathrm{E}-04$ & $1.61 \mathrm{E}-03$ & .00 & 2.75 \\
\hline AM 242 & 22 & $6.47 \mathrm{E}-08$ & $1.48 \mathrm{E}-06$ & 3.76 & .00 & $-v$ \\
\hline CM242 & & $\begin{array}{ll}0 & 14\end{array}$ & 0 & & & \\
\hline CM242 & 21 & $4.59 E-03$ & $1.03 \mathrm{E}-01$ & $2.76 \mathrm{E}-01$ & .00 & $2.83 \mathrm{E}-04$ \\
\hline CM242 & 22 & $4.34 \mathrm{E}-03$ & $9.80 \mathrm{E}-02$ & $4.79 \mathrm{E}-02$ & .00 & $7.28 \mathrm{E}-06$ \\
\hline CM242 & 23 & $9.52 \mathrm{E}-04$ & $2.15 \mathrm{E}-02$ & $6.37 \mathrm{E}-05$ & .00 & $9.01 \mathrm{E}-09$ \\
\hline $\mathrm{CM} 242$ & 24 & $1.98 \mathrm{E}-04$ & $4.51 E-03$ & $8.20 \mathrm{E}-08$ & .00 & $1.09 \mathrm{E}-11$ \\
\hline $\mathrm{CM} 242$ & 25 & $4.13 \mathrm{E}-05$ & $9.43 \mathrm{E}-04$ & .00 & .00 & .00 \\
\hline $\mathrm{CM} 242$ & 26 & $8.64 \mathrm{E}-06$ & $1.98 \mathrm{E}-04$ & .00 & .00 & .00 \\
\hline CM242 & 27 & $1.79 \mathrm{E}-06$ & $4.12 \mathrm{E}-05$ & .00 & .00 & .00 \\
\hline CM242 & 28 & $3.75 E-07$ & $8.65 \mathrm{E}-06$ & .00 & .00 & .00 \\
\hline CM242 & 29 & $7.80 \mathrm{E}-08$ & $1.80 \mathrm{E}-06$ & .00 & .00 & .00 \\
\hline CM242 & 210 & 1.6 & $3.76 \mathrm{E}-07$ & .00 & .00 & .00 \\
\hline $\mathrm{CM} 242$ & 211 & 3.3 & $8.01 \mathrm{E}-08$ & .00 & .00 & .00 \\
\hline $\mathrm{CM} 242$ & 212 & $6.98 \mathrm{E}-10$ & $1.49 \mathrm{E}-08$ & .00 & .00 & .00 \\
\hline $\mathrm{CM} 242$ & 213 & $1.16 \mathrm{E}-10$ & $3.73 \mathrm{E}-09$ & .00 & .00 & .00 \\
\hline $\mathrm{CM} 242$ & 214 & .00 & $1.86 \mathrm{E}-09$ & .00 & .00 & .00 \\
\hline PU242 & & 0 & 50 & & & \\
\hline PU242 & 31 & $2.83 E-04$ & $6.24 \mathrm{E}-03$ & $7.30 E-01$ & $0.00 \mathrm{E}+00$ & $2.51 E-0$ \\
\hline PU242 & 32 & $9.78 \mathrm{E}-04$ & 2.15 & $1.05 \mathrm{E}+00$ & $0.00 E+00$ & $1.58 \mathrm{E}$ \\
\hline PU242 & 33 & $1.58 \mathrm{E}-03$ & $3.48 \mathrm{E}-02$ & $6.33 \mathrm{E}-01$ & $0.00 \mathrm{E}+00$ & $9.26 \mathrm{E}-06$ \\
\hline PU242 & 34 & $2.13 \mathrm{E}-03$ & $4.68 \mathrm{E}-02$ & $3.81 \mathrm{E}-01$ & $0.00 \mathrm{E}+00$ & $5.58 \mathrm{E}-06$ \\
\hline PU242 & 35 & $2.60 E-03$ & $5.70 \mathrm{E}-02$ & $2.30 \mathrm{E}-01$ & $0.00 \mathrm{E}+00$ & $3.36 \mathrm{E}-06$ \\
\hline PU242 & 36 & $3.01 E-03$ & $6.57 \mathrm{E}-02$ & $1.39 \mathrm{E}-01$ & $0.00 \mathrm{E}+00$ & $2.03 \mathrm{E}-06$ \\
\hline PU242 & 37 & $3.32 \mathrm{E}-03$ & $7.23 \mathrm{E}-02$ & $8.33 \mathrm{E}-02$ & $0.00 \mathrm{E}+00$ & $1.22 \mathrm{E}-06$ \\
\hline PU242 & 38 & $3.58 \mathrm{E}-03$ & $7.80 \mathrm{E}-02$ & $5.04 \mathrm{E}-02$ & $0.00 \mathrm{E}+00$ & $7.37 \mathrm{E}-07$ \\
\hline PU242 & 39 & $3.78 \mathrm{E}-03$ & $8.22 \mathrm{E}-02$ & $3.03 \mathrm{E}-02$ & $0.00 \mathrm{E}+00$ & $4.44 \mathrm{E}-07$ \\
\hline PU242 & 310 & -03 & $8.51 E-02$ & $1.82 \mathrm{E}-02$ & $0.00 \mathrm{E}+00$ & $2.67 \mathrm{E}-07$ \\
\hline PU242 & 311 & -03 & $8.78 \mathrm{E}-02$ & $1.10 \mathrm{E}-02$ & $0.00 E+00$ & $1.61 \mathrm{E}-07$ \\
\hline
\end{tabular}


Appendix B - Inhalation Dose Rate Factors - FILE23

\begin{tabular}{|c|c|c|c|c|c|c|}
\hline j242 & 312 & $4.14 \mathrm{E}-03$ & $8.93 E-02$ & $6.62 \mathrm{E}-03$ & $0.00 \mathrm{E}+00$ & 9.6 \\
\hline 242 & 313 & $4.22 \mathrm{E}-03$ & 02 & 03 & $\mathrm{DE}+00$ & \\
\hline & 14 & 03 & 2 & $2.40 \mathrm{E}-03$ & $O E+00$ & \\
\hline & & 4 & & 1 & $0 E+00$ & \\
\hline & & 3 & & & $0 E+00$ & \\
\hline 42 & & & & & $0 E+00$ & \\
\hline 42 & & 3 & & 04 & $0 \mathrm{E}+00$ & \\
\hline 242 & & & & & $\mathrm{E}+00$ & \\
\hline $\mathrm{J} 242$ & 320 & & & & $\mathrm{E}+00$ & \\
\hline U242 & 321 & & & & $0 \mathrm{E}+00$ & \\
\hline$J 242$ & 322 & & & & $0 E+00$ & \\
\hline PU242 & 323 & & 9. & & $0.00 \mathrm{E}+00$ & \\
\hline PU242 & 324 & 4. & $8.96 \mathrm{E}-02$ & $1.53 E-05$ & $0.00 E+00$ & $E-10$ \\
\hline PU242 & 325 & 4. & $8.94 \mathrm{E}-02$ & $9.06 \mathrm{E}-06$ & $0 \mathrm{E}+00$ & $1.16 \mathrm{E}-10$ \\
\hline PU242 & 326 & $4.27 E-03$ & $8.86 \mathrm{E}-02$ & $5.48 \mathrm{E}-06$ & $0.00 E+00$ & \\
\hline PU242 & 327 & $4.27 \mathrm{E}-03$ & $8.83 E-02$ & 3. & $0.00 E+00$ & 5 \\
\hline PU242 & 32 & 4. & $8.77 \mathrm{E}-02$ & $1.67 \mathrm{E}-06$ & $0.00 \mathrm{E}+00$ & \\
\hline PU242 & 329 & $4.23 E$ & 8.6 & & -00 & \\
\hline PU242 & 33 & 4.2 & 8.6 & & & \\
\hline PU242 & 33 & 4.2 & 8. & & 0 & 0 \\
\hline $\mathrm{U} 242$ & 33 & 4.2 & 8. & & & \\
\hline PU & 33 & 4. & 8. & & & \\
\hline 42 & & 4. & & & & \\
\hline & 33 & 4. & & & & \\
\hline & 33 & 4. & & & & \\
\hline & 3 & 4. & & & & \\
\hline & & & & & & .00 \\
\hline & & & & & 00 & \\
\hline & & & & & & \\
\hline & 3 & & & & & \\
\hline & 34 & & & & 00 & \\
\hline & 3 & & & & & \\
\hline & & & & & & \\
\hline 2 & & & & & & \\
\hline & & & & & & \\
\hline & & & & & & \\
\hline & & & & & & \\
\hline & & & 7. & & & \\
\hline & & & & & 0 . & 0 \\
\hline & & & & & & \\
\hline & & $5.31 \mathrm{E}-07$ & 3. & & & \\
\hline NP2 & & 09 & 08 & & & \\
\hline PU238 & & $3.15 \mathrm{E}-04$ & 6.96 & 8.1 & & \\
\hline PU2: & 3 & $1.08 \mathrm{E}$ & & & & \\
\hline & & 1.7 & & & 0. & \\
\hline & & & & & 0. & \\
\hline & & & & & $0.00 \mathrm{E}+00$ & 3.64 \\
\hline & & & & 1.4 & 0.1 & 2.1 \\
\hline & 3 & $3.53 \mathrm{E}-03$ & $7.73 \mathrm{E}-02$ & $8.89 E-02$ & $0.00 E+00$ & $1.30 \mathrm{E}-0$ \\
\hline
\end{tabular}


Appendix B - Inhalation Dose Rate Factors - FILE23

\begin{tabular}{|c|c|c|c|c|c|c|}
\hline PU238 & 38 & $3.79 \mathrm{E}-03$ & $8.27 \mathrm{E}-02$ & $5.33 \mathrm{E}-02$ & $0.00 \mathrm{E}+00$ & $7.80 \mathrm{E}-07$ \\
\hline PU238 & 39 & $3.97 \mathrm{E}-03$ & $8.64 \mathrm{E}-02$ & $3.18 \mathrm{E}-02$ & $0.00 \mathrm{E}+00$ & $66 \mathrm{E}-07$ \\
\hline PU238 & 310 & $4.09 \mathrm{E}-03$ & $8.88 \mathrm{E}-02$ & $1.90 \mathrm{E}-02$ & $0.00 \mathrm{E}+00$ & $2.78 \mathrm{E}-07$ \\
\hline PU238 & 311 & 4.19E-03 & $9.09 \mathrm{E}-02$ & $1.14 \mathrm{E}-02$ & $0.00 \mathrm{E}+00$ & $1.67 \mathrm{E}-07$ \\
\hline PU238 & 312 & $4.24 \mathrm{E}-03$ & $9.17 \mathrm{E}-02$ & $6.79 \mathrm{E}-03$ & $0.00 \mathrm{E}+00$ & $9.94 E-08$ \\
\hline PU238 & 313 & $4.29 E-03$ & $9.25 \mathrm{E}-02$ & $4.07 \mathrm{E}-03$ & $0.00 \mathrm{E}+00$ & $5.96 \mathrm{E}-08$ \\
\hline PU238 & 31 & $4.29 \mathrm{E}$ & $9.23 \mathrm{E}-02$ & $2.43 \mathrm{E}-03$ & $0.00 \mathrm{E}+00$ & 08 \\
\hline PU238 & 31 & $4.31 \mathrm{E}$ & $9.24 \mathrm{E}-02$ & $1.46 \mathrm{E}-03$ & $0.00 E+00$ & .08 \\
\hline PU238 & & $4.30 E-03$ & $9.19 \mathrm{E}-02$ & $8.70 \mathrm{E}-04$ & $0.00 \mathrm{E}+00$ & $1.27 \mathrm{E}$ \\
\hline PU238 & & $4.26 \mathrm{E}-03$ & $9.09 \mathrm{E}-02$ & $5.19 \mathrm{E}-04$ & $0.00 \mathrm{E}+00$ & $7.60 \mathrm{E}-09$ \\
\hline PU238 & 31 & 4. & $9.04 \mathrm{E}-02$ & $\bar{E}-04$ & $0.00 E+00$ & \\
\hline PU238 & & 4. & $8.92 \mathrm{E}-02$ & $1.85 \mathrm{E}-04$ & $0.00 \mathrm{E}+00$ & \\
\hline PU238 & 32 & & 8.84 & 1.1 & $E+00$ & \\
\hline PU238 & 32 & 4. & 2 & 05 & $0.00 E+00$ & \\
\hline PU238 & 24 & $4.09 \mathrm{E}$ & $8.60 \mathrm{E}-02$ & 05 & $0.00 \mathrm{E}+00$ & 10 \\
\hline PU238 & 323 & $4.06 \mathrm{E}$ & 8.521 & 5 & 0. & 10 \\
\hline PU238 & 324 & 4.011 & 02 & 05 & 00 & 2. \\
\hline PU238 & 325 & 3.9 & 8.2 & 8.5 & 00 & 1 \\
\hline PU238 & 326 & 3. & 8.14 & 06 & 0. & 8 \\
\hline PU238 & 327 & & 02 & 3. & 0. & \\
\hline PU2: & 328 & & & 6 & 0. & 0.0 \\
\hline PU2 & 329 & & 02 & & 0. & 2. \\
\hline $\mathrm{P}$ & 330 & & & 07 & +00 & +00 \\
\hline 238 & 331 & & 02 & 97 & 00 & -11 \\
\hline PU238 & 332 & & & 07 & 0. & +00 \\
\hline PU238 & 333 & & 02 & 07 & 0.0 & $0.00 E+00$ \\
\hline PU238 & 334 & & 02 & $0.00 E+00$ & $=00$ & $0.00 \mathrm{E}+00$ \\
\hline PU238 & 335 & & 02 & $0.00 E+00$ & 0.00 & $0.00 E+00$ \\
\hline PU238 & 33 & & -02 & $0.00 E+00$ & $0.00 E+00$ & $0.00 \mathrm{E}+00$ \\
\hline PU238 & 3 & & -02 & $0.00 E+00$ & $E+00$ & $E+00$ \\
\hline PU238 & 3 & & -02 & $0.00 \mathrm{E}+00$ & $E+00$ & +00 \\
\hline PU238 & 339 & & -02 & $0.00 \mathrm{E}+00$ & $=00$ & 0 . \\
\hline PU238 & 34 & & -02 & $0.00 \mathrm{E}+00$ & 0.0 & \\
\hline PU238 & 34 & & -02 & $0.00 E+00$ & 0. & \\
\hline PU238 & 34 & & 02 & $0.00 \mathrm{E}+00$ & 0. & 00 \\
\hline PU238 & 34 & & & $0.00 \mathrm{E}+00$ & $E+00$ & 00 \\
\hline 38 & & & & $0 \mathrm{E}+00$ & $E+00$ & $0.00 \mathrm{E}+00$ \\
\hline Pl & 3 & & & $0 \mathrm{E}+00$ & $\mathrm{E}+00$ & $0.00 \mathrm{E}+00$ \\
\hline Pl & 34 & & & $+\infty$ & 0. & $0.00 \mathrm{E}+00$ \\
\hline & 34 & & & 00 & 00 & $0.00 \mathrm{E}+00$ \\
\hline & 34 & & & & & $0.00 \mathrm{E}+00$ \\
\hline & 34 & & & -00 & & $0.00 \mathrm{E}+00$ \\
\hline & & & & +00 & 0 . & 0.00 \\
\hline & & & & & & \\
\hline & 1 & 2 & 1 & 3. & .00 & 2.79 \\
\hline & & & & $7.62 \mathrm{E}-02$ & .00 & \\
\hline & 2 & 2 & 01 & $4.63 E-04$ & .00 & $6.55 E-08$ \\
\hline & & & 01 & $2.81 E-06$ & .00 & $3.97 E-10$ \\
\hline & & & -01 & $1.86 \mathrm{E}-08$ & .00 & .00 \\
\hline & $\overline{0}$ & -02 & $4 \mathrm{E}-01$ & .00 & .00 & .00 \\
\hline
\end{tabular}




\section{Appendix B - Inhalation Dose Rate Factors - FILE23}

\begin{tabular}{|c|c|c|c|c|c|c|}
\hline & & & & & & \\
\hline $\begin{array}{l}\text { CM244 } \\
\text { CM244 }\end{array}$ & $\begin{array}{ll}2 & 7 \\
2 & 8\end{array}$ & $\begin{array}{l}1.01 \mathrm{E}-02 \\
9.61 \mathrm{E}-03\end{array}$ & $\begin{array}{l}3.47 \mathrm{E}-01 \\
3.33 \mathrm{E}-01\end{array}$ & .00 & $\begin{array}{l}.00 \\
.00\end{array}$ & $\begin{array}{l}.00 \\
.00\end{array}$ \\
\hline CM244 & 29 & $9.16 \mathrm{E}-03$ & $3.18 \mathrm{E}-01$ & .00 & .00 & .00 \\
\hline CM244 & 210 & $8.70 \mathrm{E}-03$ & $3.03 \mathrm{E}-01$ & .00 & .00 & .00 \\
\hline CM244 & 211 & $8.32 \mathrm{E}-03$ & $2.91 \mathrm{E}-01$ & .00 & .00 & .00 \\
\hline CM244 & 212 & $7.90 \mathrm{E}-03$ & $2.77 \mathrm{E}-01$ & .00 & .00 & .00 \\
\hline CM244 & 213 & $7.55 \mathrm{E}-03$ & $2.66 \mathrm{E}-01$ & .00 & .00 & .00 \\
\hline $\mathrm{CM} 244$ & 214 & 7. $17 \mathrm{E}-03$ & $2.54 \mathrm{E}-01$ & .00 & .00 & .00 \\
\hline CM244 & 215 & $6.85 E-03$ & $2.43 \mathrm{E}-01$ & .00 & .00 & .00 \\
\hline CM244 & 216 & $6.53 \mathrm{E}-03$ & $2.33 \mathrm{E}-01$ & .00 & .00 & .00 \\
\hline $\mathrm{CM} 244$ & 217 & $6.20 \mathrm{E}-03$ & $2.22 \mathrm{E}-01$ & .00 & .00 & .00 \\
\hline CM244 & 218 & $5.93 \mathrm{E}-03$ & $2.13 \mathrm{E}-01$ & .00 & .00 & .00 \\
\hline CM244 & 219 & $5.63 \mathrm{E}-03$ & $2.03 \mathrm{E}-01$ & .00 & .00 & .00 \\
\hline CM244 & 220 & $5.38 \mathrm{E}-03$ & $1.94 \mathrm{E}-01$ & .00 & .00 & .00 \\
\hline CM244 & 221 & $5.13 \mathrm{E}-03$ & $1.86 \mathrm{E}-01$ & .00 & .00 & .00 \\
\hline CM244 & 222 & $4.87 \mathrm{E}-03$ & $1.77 \mathrm{E}-01$ & .00 & .00 & .00 \\
\hline CM244 & 223 & $4.66 \mathrm{E}-03$ & $1.70 \mathrm{E}-01$ & .00 & .00 & .00 \\
\hline CM244 & 224 & $4.42 \mathrm{E}-03$ & $1.62 \mathrm{E}-01$ & .00 & .00 & .00 \\
\hline CM244 & 225 & $4.23 E-03$ & $1.56 \mathrm{E}-01$ & .00 & .00 & .00 \\
\hline CM244 & 226 & $4.01 \mathrm{E}-03$ & $1.48 \mathrm{E}-01$ & .00 & .00 & .00 \\
\hline CM244 & 227 & $3.84 \mathrm{E}-03$ & $1.42 \mathrm{E}-01$ & .00 & .00 & .00 \\
\hline CM244 & 228 & $3.66 \mathrm{E}-03$ & $1.36 \mathrm{E}-01$ & .00 & .00 & .00 \\
\hline CM244 & 229 & $3.47 \mathrm{E}-03$ & $1.30 \mathrm{E}-01$ & .00 & .00 & .00 \\
\hline CM244 & 230 & 3. $32 \mathrm{E}-03$ & $1.24 \mathrm{E}-01$ & .00 & .00 & .00 \\
\hline $\mathrm{CM} 244$ & 231 & $3.15 \mathrm{E}-03$ & $1.19 \mathrm{E}-01$ & .00 & .00 & .00 \\
\hline CM244 & 232 & $3.01 \mathrm{E}-03$ & $1.14 \mathrm{E}-01$ & .00 & .00 & .00 \\
\hline CM244 & 233 & $2.88 \mathrm{E}-03$ & $1.09 \mathrm{E}-01$ & .00 & .00 & .00 \\
\hline CM244 & 234 & $2.68 \mathrm{E}-03$ & $1.02 \mathrm{E}-01$ & .00 & .00 & .00 \\
\hline CM244 & 235 & $2.64 \mathrm{E}-03$ & $1.01 \mathrm{E}-01$ & .00 & .00 & .00 \\
\hline CM244 & 236 & $2.51 \mathrm{E}-03$ & $9.63 \mathrm{E}-02$ & .00 & .00 & .00 \\
\hline CM244 & 237 & $2.32 \mathrm{E}-03$ & $8.92 E-02$ & .00 & .00 & .00 \\
\hline CM244 & 238 & $2.28 \mathrm{E}-03$ & $8.80 \mathrm{E}-02$ & .00 & .00 & .00 \\
\hline CM244 & 239 & $2.11 \mathrm{E}-03$ & $8.16 \mathrm{E}-02$ & .00 & .00 & .00 \\
\hline CM244 & 240 & $2.07 \mathrm{E}-03$ & $8.05 \mathrm{E}-02$ & .00 & .00 & .00 \\
\hline CM244 & 241 & $1.91 \mathrm{E}-03$ & $7.46 \mathrm{E}-02$ & .00 & .00 & .00 \\
\hline CM244 & 242 & $1.88 \mathrm{E}-03$ & $7.36 \mathrm{E}-02$ & .00 & .00 & .00 \\
\hline CM244 & 243 & $1.74 \mathrm{E}-03$ & $6.82 \mathrm{E}-02$ & .00 & .00 & .00 \\
\hline CM244 & 244 & $1.71 \mathrm{E}-03$ & $6.73 \mathrm{E}-02$ & .00 & .00 & .00 \\
\hline CM244 & 245 & $1.63 \mathrm{E}-03$ & $6.44 E-02$ & .00 & .00 & .00 \\
\hline CM244 & 246 & $1.50 \mathrm{E}-03$ & $5.96 \mathrm{E}-02$ & .00 & .00 & .00 \\
\hline CM244 & 247 & $1.48 \mathrm{E}-03$ & $5.89 \mathrm{E}-02$ & .00 & .00 & .00 \\
\hline CM244 & 248 & $1.36 \mathrm{E}-03$ & $5.45 \mathrm{E}-02$ & .00 & .00 & .00 \\
\hline CM244 & 249 & 1.3 & $5.38 \mathrm{E}-02$ & .00 & .00 & .00 \\
\hline CM244 & 250 & 1.2 & $4.99 \mathrm{E}-02$ & .00 & .00 & .00 \\
\hline PU244 & & & & & & \\
\hline PU244 & 31 & $3.24 \mathrm{E}-04$ & $7.28 \mathrm{E}-03$ & $8.36 E-01$ & $0.00 \mathrm{E}+00$ & $3.74 \mathrm{E}-04$ \\
\hline PU244 & 32 & 1. $12 \mathrm{E}-03$ & $2.52 \mathrm{E}-02$ & 1.20E+00 & $0.00 \mathrm{E}+00$ & $2.36 \mathrm{E}-0$ \\
\hline PU244 & 33 & $1.81 \mathrm{E}-03$ & $4.06 \mathrm{E}-02$ & 7. $25 \mathrm{E}-01$ & $0.00 \mathrm{E}+00$ & $1.38 \mathrm{E}-0$ \\
\hline & 34 & $2.44 E-03$ & $5.47 \mathrm{E}-02$ & $4.37 \mathrm{E}-01$ & $0.00 E+00$ & 8.32 \\
\hline PU244 & 35 & $2.98 \mathrm{E}-03$ & $6.66 \mathrm{E}-02$ & $2.63 \mathrm{E}-01$ & $0.00 \mathrm{E}+00$ & $5.01 \mathrm{E}-06$ \\
\hline
\end{tabular}


Appendix B - Inhalation Dose Rate Factors - FILE23

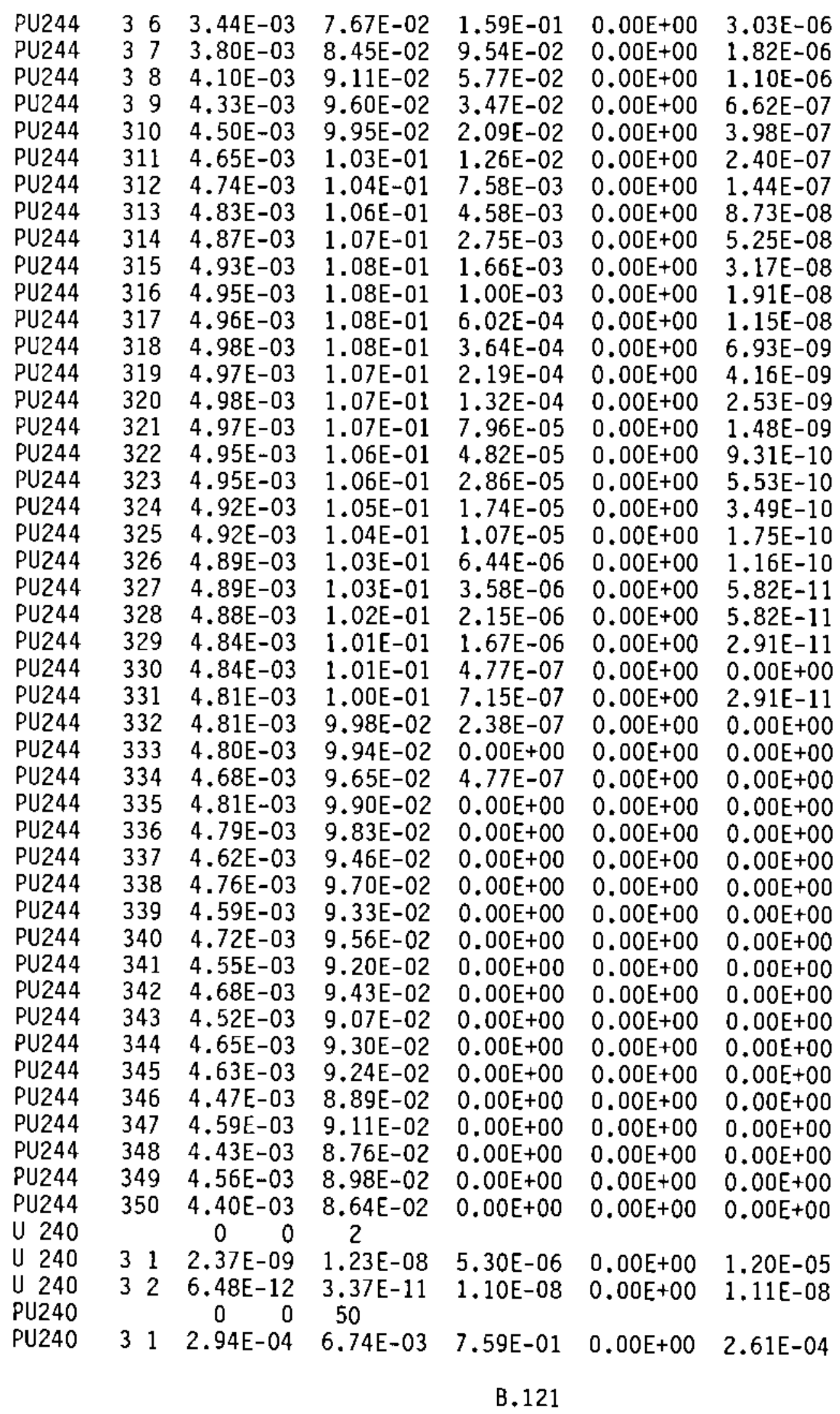


Appendix B - Inhalation Dose Rate Factors - FILE23

\begin{tabular}{|c|c|c|c|c|c|c|}
\hline 2 & 2 & & -02 & $E+00$ & .00 & \\
\hline$J 240$ & & & & & & \\
\hline J240 & & $2.22 \mathrm{E}$ & $5.05 \mathrm{E}-02$ & $3.96 \mathrm{E}-01$ & $0 \mathrm{E}+00$ & \\
\hline 12 & & $2.71 \mathrm{E}-03$ & $6.16 \mathrm{E}-02$ & $2.39 \mathrm{E}-01$ & OF= & \\
\hline & & $3.12 \mathrm{E}$ & $7.09 \mathrm{E}-02$ & & 000 & \\
\hline 12 & & $3.44 \mathrm{E}-03$ & $7.81 \mathrm{E}-02$ & $8.65 \mathrm{E}-02$ & $0.00 \mathrm{E}+00$ & \\
\hline & & & & & & \\
\hline & & 3. & $8.87 \mathrm{E}-$ & $3.15 \mathrm{E}$ & 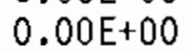 & \\
\hline & & & & & & \\
\hline 12 & & & $9.47 \mathrm{E}-02$ & 1. & & \\
\hline & & & & & & \\
\hline 2 & & & & & & \\
\hline 2 & & & & & & \\
\hline & & & & & & \\
\hline 12 & & & & & & \\
\hline & & & & & & \\
\hline$J 24$ & & & & & & \\
\hline & & & & & & \\
\hline & & & & & & \\
\hline J2 & & & & & & \\
\hline & & & & & & \\
\hline $\mathrm{J} 24$ & & & & & & \\
\hline & & & & & & \\
\hline & & & & & & \\
\hline & & & & & & \\
\hline & & & 9. & & & \\
\hline & & & & & & \\
\hline & & & & & & \\
\hline & & & & & & \\
\hline & & & & & & \\
\hline & & & & & & \\
\hline & & & & & & \\
\hline & & & & & & \\
\hline & & & & & & \\
\hline & & & & & & \\
\hline & & & & & & \\
\hline & & & & & & \\
\hline & & & & & & \\
\hline & & & & & & \\
\hline & & & & & & \\
\hline & 34 & & & & & \\
\hline & & & & & & \\
\hline & 34 & & 8.56 & & & \\
\hline & & & & & & 0 \\
\hline & 34 & & & & & \\
\hline & 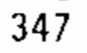 & & & & & \\
\hline & 34 & & & & & \\
\hline & & & & & & \\
\hline & & -1 & $7.95 \mathrm{E}$ & $0.00 E+00$ & $0.00 \mathrm{E}+00$ & \\
\hline & & & & & & \\
\hline
\end{tabular}


Appendix B - Inhalation Dose Rate Factors - FILE23

\begin{tabular}{|c|c|c|c|c|c|c|}
\hline CM243 & 21 & $5.63 \mathrm{E}-03$ & $8.42 \mathrm{E}-02$ & $3.23 \mathrm{E}-01$ & $0.00 \mathrm{E}+00$ & $2.94 \mathrm{E}-04$ \\
\hline CM243 & 22 & $1.27 \mathrm{E}-02$ & $1.90 \mathrm{E}-01$ & $65 \mathrm{E}-02$ & $0.00 \mathrm{E}+00$ & \\
\hline CM243 & 23 & $26 \mathrm{E}-02$ & $1.89 \mathrm{E}-01$ & $1 \mathrm{E}-04$ & $0.00 \mathrm{E}+00$ & -08 \\
\hline CM243 & 24 & $1 E-02$ & & $86 E-06$ & $0.00 \mathrm{E}+00$ & -10 \\
\hline CM243 & 25 & $7 E-02$ & & & $0.00 \mathrm{E}+00$ & \\
\hline CM243 & 26 & & & $E+00$ & $0.00 E+00$ & $0.00 \mathrm{E}+00$ \\
\hline CM243 & 7 & & 1. & $00 \mathrm{E}+00$ & & +00 \\
\hline CM243 & 8 & 02 & & $0 \mathrm{E}+00$ & 00 & \\
\hline & & 2 & & $0 \mathrm{E}+00$ & & \\
\hline & & & & 00 & & \\
\hline & & & 1 & 00 & & \\
\hline & & 3 & 1 & 00 & & \\
\hline & & 3 & & $E+00$ & & \\
\hline $\mathrm{C}$ & 14 & 3 & & $\mathrm{E}+00$ & 00 & \\
\hline & 2 & -03 & & $E+00$ & 00 & \\
\hline & 2 & 03 & & $E+00$ & & \\
\hline & & 7 & & $\$+0$ & & \\
\hline & $L^{2}$ & 7 & & $\$ 00$ & & $=00$ \\
\hline $\mathrm{C}$ & 21 & 7 & & $E+00$ & 00 & $E+00$ \\
\hline & 220 & & & $E+00$ & +00 & +00 \\
\hline & 221 & & 1. & $E+00$ & +00 & +00 \\
\hline & 222 & & & $E+00$ & 00 & 100 \\
\hline - & 223 & & & $E+00$ & 00 & 00 \\
\hline $\mathrm{Cl}$ & 224 & & & $E+00$ & 00 & +00 \\
\hline $\mathrm{Cl}$ & 225 & & & $E+00$ & & $E+00$ \\
\hline $\mathrm{Cl}$ & 226 & & 02 & $E+00$ & 00 & $E+00$ \\
\hline $\mathrm{CP}$ & 227 & & & $0 \mathrm{E}+00$ & 00 & $E+00$ \\
\hline $\mathrm{Cr}$ & 228 & & 02 & $E+00$ & & $E+00$ \\
\hline$C M$ & 229 & & -02 & 0 . & 00 & $E+00$ \\
\hline & 230 & & 02 & 0 . & & $0.00 E+00$ \\
\hline CM & 23 & & 02 & 0. & & $E+00$ \\
\hline & 23 & & 02 & +00 & & $E+00$ \\
\hline $\mathrm{Cr}$ & 23 & & -02 & $E+00$ & 00 & $0 E+00$ \\
\hline $\mathrm{Cr}$ & 23 & & -02 & $E+00$ & 00 & $D O E+00$ \\
\hline $\mathrm{CM}$ & 23 & & -02 & $E+00$ & +00 & $0.00 E+00$ \\
\hline & 23 & & -02 & $O E+00$ & $=00$ & $0.00 E+00$ \\
\hline & 23 & & -02 & $0 \mathrm{E}+00$ & $=00$ & $E+00$ \\
\hline & & & -02 & $E+00$ & +00 & $0.00 E+00$ \\
\hline & & & -02 & $0 \mathrm{E}+00$ & +00 & $0.00 \mathrm{E}+00$ \\
\hline & & & -02 & $0 \mathrm{E}+00$ & +00 & $E+00$ \\
\hline & & & -02 & $0 \mathrm{E}+00$ & +00 & $E+00$ \\
\hline & 2 & & -02 & $0 \mathrm{E}+00$ & +00 & $E+00$ \\
\hline & & & 02 & $0 E+00$ & $0 E+00$ & $E+00$ \\
\hline & & & -02 & $0 E+00$ & $0.00 \mathrm{E}+00$ & $E+00$ \\
\hline & & & -02 & $0 \mathrm{E}+00$ & $0.00 \mathrm{E}+00$ & $E+00$ \\
\hline & & & -02 & $0.00 E+00$ & $0.00 \mathrm{E}+00$ & $E+00$ \\
\hline & & & & $0.00 E+00$ & $0.00 E+00$ & $E+00$ \\
\hline & & & & $0.00 E+00$ & $0.00 \mathrm{E}+00$ & $E+00$ \\
\hline & & & -02 & $E+00$ & & $E+00$ \\
\hline & & 2. & -02 & $E+00$ & $0.00 E+00$ & $0.00 E+00$ \\
\hline
\end{tabular}




\section{Appendix B - Inhalation Dose Rate Factors - FILE23}

\begin{tabular}{|c|c|c|c|c|c|c|}
\hline $\begin{array}{l}\text { PU243 } \\
\text { PU243 }\end{array}$ & 31 & $\begin{array}{cr}0 & 0 \\
2.05 E-09\end{array}$ & $\begin{array}{l}2 \\
4.98 \mathrm{E}-08\end{array}$ & $4.57 \mathrm{E}-06$ & $0.00 E+00$ & $4.59 \mathrm{E}-06$ \\
\hline PU243 & 32 & $1.75 \mathrm{E}-12$ & $4.25 \mathrm{E}-11$ & $3.49 \mathrm{E}-09$ & $0.00 \mathrm{E}+00$ & $2.37 \mathrm{E}-09$ \\
\hline AM 243 & & 50 & 0 & & & \\
\hline AM 243 & 21 & $5.10 \mathrm{E}-03$ & $1.15 \mathrm{E}-01$ & $2.91 \mathrm{E}-01$ & .00 & $2.60 \mathrm{E}-04$ \\
\hline AM243 & 22 & $1.17 \mathrm{E}-02$ & $2.64 E-01$ & $6.95 \mathrm{E}-02$ & .00 & $1.06 \mathrm{E}-05$ \\
\hline AM243 & 23 & $1.18 \mathrm{E}-02$ & $2.69 \mathrm{E}-01$ & $4.39 \mathrm{E}-04$ & .00 & $6.42 \mathrm{E}-08$ \\
\hline AM243 & 24 & $1.17 \mathrm{E}-02$ & $2.67 \mathrm{E}-01$ & $2.77 \mathrm{E}-06$ & .00 & $4.07 E-10$ \\
\hline AM243 & 25 & $1.15 \mathrm{E}-02$ & $2.65 \mathrm{E}-01$ & $1.49 \mathrm{E}-08$ & .00 & $3.64 \mathrm{E}-12$ \\
\hline AM243 & 26 & $1.14 \mathrm{E}-02$ & $2.63 \mathrm{E}-01$ & .00 & .00 & .00 \\
\hline AM243 & 27 & $1.12 \mathrm{E}-02$ & $2.61 \mathrm{E}-01$ & .00 & .00 & .00 \\
\hline $\mathrm{AM} 243$ & 28 & $1.11 \mathrm{E}-02$ & $2.60 \mathrm{E}-01$ & .00 & .00 & .00 \\
\hline AM2 43 & 29 & $1.10 \mathrm{E}-02$ & $2.58 \mathrm{E}-01$ & .00 & .00 & .00 \\
\hline AM243 & 210 & $1.08 \mathrm{E}-02$ & $2.55 \mathrm{E}-01$ & .00 & .00 & .00 \\
\hline AM2.43 & 211 & $1.07 \mathrm{E}-02$ & $2.54 \mathrm{E}-01$ & .00 & .00 & .00 \\
\hline AM243 & 212 & $1.05 \mathrm{E}-02$ & $2.52 \mathrm{E}-01$ & .00 & .00 & .00 \\
\hline AM243 & 213 & $1.04 \mathrm{E}-02$ & $2.51 \mathrm{E}-01$ & .00 & .00 & .00 \\
\hline AM243 & 214 & $1.03 \mathrm{E}-02$ & $2.48 \mathrm{E}-01$ & .00 & .00 & .00 \\
\hline AM243 & 215 & $1.02 \mathrm{E}-02$ & $2.47 \mathrm{E}-01$ & .00 & .00 & .00 \\
\hline AM 243 & 216 & $1.00 \mathrm{E}-02$ & $2.46 \mathrm{E}-01$ & .00 & .00 & .00 \\
\hline AM243 & 217 & $9.87 \mathrm{E}-03$ & $2.43 \mathrm{E}-01$ & .00 & .00 & .00 \\
\hline AM243 & 218 & $9.77 \mathrm{E}-03$ & $2.42 E-01$ & .00 & .00 & .00 \\
\hline AM243 & 219 & $9.62 \mathrm{E}-03$ & $2.40 E-01$ & .00 & .00 & .00 \\
\hline AM243 & 220 & $9.53 \mathrm{E}-03$ & $2.39 E-01$ & .00 & .00 & .00 \\
\hline AM243 & 221 & $9.41 \mathrm{E}-03$ & $2.37 E-01$ & .00 & .00 & .00 \\
\hline AM243 & 222 & $9.26 \mathrm{E}-03$ & $2.35 \mathrm{E}-01$ & .00 & .00 & .00 \\
\hline AM243 & 223 & $9.17 \mathrm{E}-03$ & $2.34 \mathrm{E}-01$ & .00 & .00 & .00 \\
\hline AM243 & 224 & $9.02 \mathrm{E}-03$ & $2.31 \mathrm{E}-01$ & .00 & .00 & .00 \\
\hline AM243 & 225 & $8.94 \mathrm{E}-03$ & $2.31 \mathrm{E}-01$ & .00 & .00 & .00 \\
\hline AM243 & 226 & $8.80 \mathrm{E}-03$ & $2.28 \mathrm{E}-01$ & .00 & .00 & .00 \\
\hline AM243 & 227 & $8.71 \mathrm{E}-03$ & $2.27 \mathrm{E}-01$ & .00 & .00 & .00 \\
\hline AM243 & 228 & $8.60 \mathrm{E}-03$ & $2.26 \mathrm{E}-01$ & .00 & .00 & .00 \\
\hline AM243 & 229 & $8.47 \mathrm{E}-03$ & $2.23 \mathrm{E}-01$ & .00 & .00 & .00 \\
\hline AM243 & 230 & $8.39 \mathrm{E}-03$ & $2.23 \mathrm{E}-01$ & .00 & .00 & .00 \\
\hline AM243 & 231 & $8.25 E-03$ & $2.20 \mathrm{E}-01$ & .00 & .00 & .00 \\
\hline AM243 & 232 & $8.17 \mathrm{E}-03$ & $2.20 \mathrm{E}-01$ & .00 & .00 & .00 \\
\hline AM243 & 233 & $8.10 \mathrm{E}-03$ & $2.19 \mathrm{E}-01$ & .00 & .00 & .00 \\
\hline AM243 & 234 & $7.82 \mathrm{E}-03$ & $2.12 \mathrm{E}-01$ & .00 & .00 & .00 \\
\hline AM243 & 235 & $7.97 \mathrm{E}-03$ & $2.18 \mathrm{E}-01$ & .00 & .00 & .00 \\
\hline AM243 & 236 & $7.87 \mathrm{E}-03$ & $2.16 \mathrm{E}-01$ & .00 & .00 & .00 \\
\hline AM243 & 237 & 7. $52 \mathrm{E}-03$ & $2.08 \mathrm{E}-01$ & .00 & .00 & .00 \\
\hline A.M243 & 238 & $7.67 \mathrm{E}-03$ & $2.13 \mathrm{E}-01$ & .00 & .00 & .00 \\
\hline AM243 & 239 & $7.33 \mathrm{E}-03$ & $2.05 \mathrm{E}-01$ & .00 & .00 & .00 \\
\hline AM243 & 240 & $7.48 \mathrm{E}-03$ & $2.10 \mathrm{E}-01$ & .00 & .00 & .00 \\
\hline AM243 & 241 & $5 \mathrm{E}-03$ & $2.02 \mathrm{E}-01$ & .00 & .00 & .00 \\
\hline & 242 & $7.29 E-03$ & $2.07 E-01$ & .00 & .00 & .00 \\
\hline & 243 & $6.97 \mathrm{E}-03$ & $1.99 \mathrm{E}-01$ & .00 & .00 & .00 \\
\hline & 244 & $7.10 \mathrm{E}-03$ & $2.04 \mathrm{E}-01$ & .00 & .00 & .00 \\
\hline & 245 & $7.01 \mathrm{E}-03$ & $2.03 \mathrm{E}-01$ & .00 & .00 & .00 \\
\hline & 206 & 03 & $1.95 \mathrm{E}-01$ & .00 & .00 & .00 \\
\hline
\end{tabular}




\section{Appendix B - Inhalation Dose Rate Factors - FILE23}

\begin{tabular}{|c|c|c|c|c|c|c|}
\hline $\begin{array}{l}\text { AM243 } \\
\text { AM243 } \\
\text { AM243 } \\
\text { AM243 }\end{array}$ & $\begin{array}{l}247 \\
248 \\
249 \\
250\end{array}$ & $\begin{array}{l}6.84 \mathrm{E}-03 \\
6.54 \mathrm{E}-03 \\
6.67 \mathrm{E}-03 \\
6.38 \mathrm{E}-03 \\
0\end{array}$ & $\begin{array}{l}2.00 \mathrm{E}-01 \\
1.92 \mathrm{E}-01 \\
1.97 \mathrm{E}-01 \\
1.90 \mathrm{E}-01\end{array}$ & $\begin{array}{l}.00 \\
.00 \\
.00 \\
.00\end{array}$ & $\begin{array}{l}.00 \\
.00 \\
.00 \\
.00\end{array}$ & $\begin{array}{l}.00 \\
.00 \\
.00 \\
.00\end{array}$ \\
\hline & 1 & $26 \mathrm{~F}-07$ & $.04 \mathrm{E}-06$ & $25 \mathrm{E}-05$ & $0.00 \mathrm{~F}+00$ & $6.35 \mathrm{E}-0$ \\
\hline & 2 & $\begin{array}{c}3.15 \mathrm{E}-09 \\
0\end{array}$ & 8 & $.57 \mathrm{E}-07$ & & 1.06 \\
\hline & 1 & $2.94 \mathrm{E}-04$ & $6.74 \mathrm{E}-03$ & $7.59 \mathrm{E}-01$ & $0.00 \mathrm{E}+00$ & $2.56 \mathrm{E}-04$ \\
\hline & 2 & $1.02 \mathrm{E}-03$ & 2. & $1.09 \mathrm{E}+00$ & $0.00 \mathrm{E}+00$ & 1.6 \\
\hline PU2 & 33 & $1.65 \mathrm{E}-03$ & $E-02$ & $6.58 \mathrm{E}-01$ & $0.00 \mathrm{E}+00$ & 9. \\
\hline PU239 & 34 & $2.22 \mathrm{E}-03$ & $=-02$ & $3.96 \mathrm{E}-01$ & $0.00 E+00$ & $5.69 \mathrm{E}$ \\
\hline PU239 & 35 & $2.71 \mathrm{E}-03$ & $6.16 \mathrm{E}-02$ & $2.39 \mathrm{E}-01$ & $0.00 E+00$ & $3.43 \mathrm{E}-06$ \\
\hline PU239 & 36 & $3.12 \mathrm{E}-03$ & $7.09 \mathrm{E}-02$ & $1.44 \mathrm{E}-01$ & $0.00 \mathrm{E}+00$ & $2.07 \mathrm{E}-06$ \\
\hline PU239 & 37 & $3.45 \mathrm{E}-03$ & $1 E-02$ & $8.66 \mathrm{E}-$ & $0.00 E+00$ & $1.24 \mathrm{E}-06$ \\
\hline PU239 & 38 & $3.72 \mathrm{E}-03$ & $2 \mathrm{E}-02$ & $5.23 \mathrm{E}-$ & $0.00 \mathrm{E}+00$ & 7.52 \\
\hline PU239 & 39 & $3.93 \mathrm{E}-03$ & $8.88 \mathrm{E}-02$ & $3.15 \mathrm{E}$ & $0.00 \mathrm{E}-$ & 4.5 \\
\hline PU239 & 310 & $4.08 \mathrm{E}$ & 9 & 1.89 & $0.00 \mathrm{E}$ & 2.7 \\
\hline PU239 & 311 & $4.22 \mathrm{E}-03$ & 2 & 1.14 & $0.00 E+00$ & $1.64 \mathrm{E}-07$ \\
\hline PU239 & 312 & 4. & 9. & 6.88 & 0. & 9. \\
\hline$P L$ & 313 & 4 & 9. & 4.16 & 0.8 & 5. \\
\hline $\mathrm{PI}$ & 314 & 4. & 9. & 2.50 & 0. & \\
\hline $\mathrm{Pl}$ & 315 & 4.4 & 9. & 1.51 & 0. & \\
\hline PI & 2 & 4.4 .5 & & 9.1 & 0. & \\
\hline & 31 & 4.4 & & 5.4 & 0. & \\
\hline & 31 & 4. & & 3.3 & 0. & 4. \\
\hline & 31 & 4. & & 1. & 0. & \\
\hline & 320 & 4. & & 1.2 & & \\
\hline & 32 & -03 & & 7. & & \\
\hline & 32 & -03 & 9. & 4. & 0. & \\
\hline & 32 & 4.2 & & 2.0 & 0. & \\
\hline & 7 & 4. & & 1. & & \\
\hline & 2 & 4. & & & 0. & \\
\hline & 2 & 4. & & & & \\
\hline & & 4. & & & & \\
\hline & 32 & 4. & & & & \\
\hline & & & & & & \\
\hline & & & & & & \\
\hline & & & & 4.7 & 0. & \\
\hline & & & & & 0. & \\
\hline & & & & & & \\
\hline & & & & & & 0.0 \\
\hline & & & & & & \\
\hline & & & & & 0. & \\
\hline & & & & & 0 . & \\
\hline & & & & & 0 . & \\
\hline & & & & & & \\
\hline & & & & & & \\
\hline & & & & & & \\
\hline & & & & +00 & $0.00 \mathrm{E}+00$ & 0.0 \\
\hline
\end{tabular}




\section{Appendix B - Inhalation Dose Rate Factors - FILE23}

\begin{tabular}{|c|c|c|c|c|c|c|}
\hline $\begin{array}{l}\text { PU239 } \\
\text { PU239 } \\
\text { PU239 } \\
\text { PU239 } \\
\text { PU239 }\end{array}$ & $\begin{array}{l}343 \\
344 \\
345 \\
346 \\
347 \\
348 \\
349 \\
350\end{array}$ & $\begin{array}{l}4.10 \mathrm{E}-03 \\
4.21 \mathrm{E}-03 \\
4.20 \mathrm{E}-03 \\
4.05 \mathrm{E}-03 \\
4.16 \mathrm{E}-03 \\
4.02 \mathrm{E}-03 \\
4.13 \mathrm{E}-03 \\
3.99 \mathrm{E}-03\end{array}$ & $\begin{array}{l}8.38 \mathrm{E}-02 \\
8.59 \mathrm{E}-02 \\
8.53 \mathrm{E}-02 \\
8.21 \mathrm{E}-02 \\
8.41 \mathrm{E}-02 \\
8.09 \mathrm{E}-02 \\
8.30 \mathrm{E}-02 \\
7.98 \mathrm{E}-02\end{array}$ & $\begin{array}{l}0.00 E+00 \\
0.00 E+00 \\
0.00 E+00 \\
0.00 E+00 \\
0.00 E+00 \\
0.00 E+00 \\
0.00 E+00 \\
0.00 E+00\end{array}$ & $\begin{array}{l}0.00 \mathrm{E}+00 \\
0.00 \mathrm{E}+00 \\
0.00 \mathrm{E}+00 \\
0.00 \mathrm{E}+00 \\
0.00 \mathrm{E}+00 \\
0.00 \mathrm{E}+00 \\
0.00 \mathrm{E}+00 \\
0.00 \mathrm{E}+00\end{array}$ & $\begin{array}{l}+00 \\
+00 \\
+00\end{array}$ \\
\hline & & 17 & & & & \\
\hline PU241 & $\begin{array}{ll}3 & 1 \\
3 & 2\end{array}$ & $\begin{array}{l}1.23 \mathrm{E}-05 \\
4.12 \mathrm{E}-05\end{array}$ & $\begin{array}{l}3.43 \mathrm{E}-04 \\
1.14 \mathrm{E}-03\end{array}$ & $\begin{array}{l}1.45 \mathrm{E}-03 \\
2.02 \mathrm{E}-03\end{array}$ & $\begin{array}{l}0.00 \mathrm{E}+00 \\
0.00 \mathrm{E}+00\end{array}$ & \\
\hline & 3 & $6.36 \mathrm{E}-$ & 1. & & $E+00$ & \\
\hline & 3 & $8.17 \mathrm{E}$ & 2 & & 0. & \\
\hline & 2 & 9. & & & 0 & \\
\hline & 36 & 1. & 2 & 2. & & \\
\hline & 37 & 1. & & & & \\
\hline & 38 & 1. & & & & \\
\hline & 5 & 1. & & & & \\
\hline & 10 & 1. & & & & \\
\hline & [1] & 1. & & & & \\
\hline & 12 & 1. & & & & \\
\hline & 13 & 1 . & & & & \\
\hline & 14 & & & & & \\
\hline & 3 & & & & & \\
\hline & 3 & & & & & \\
\hline & 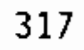 & & & & & \\
\hline & 3 & & & & & \\
\hline & & & & & & \\
\hline & $J$ & & & & & \\
\hline & 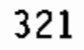 & & & & & \\
\hline & 3 & & & & & \\
\hline & & & & & & \\
\hline & & & & & & \\
\hline & & & & & & \\
\hline & & & & & & \\
\hline & & & & & & \\
\hline & & & & & & \\
\hline & & & & & & \\
\hline & & & & & & \\
\hline & & & & & & \\
\hline & & & & & & \\
\hline & & & & & & \\
\hline & & & & & & \\
\hline & & & & & & \\
\hline & & & & & & \\
\hline & & & & & & \\
\hline & & & & & & \\
\hline & & & & & & \\
\hline & & & & & & \\
\hline & & & & & $0.00 \mathrm{E}+00$ & \\
\hline
\end{tabular}


Appendix B - Inhalation Dose Rate Factors - FILE23

\begin{tabular}{|c|c|c|c|c|c|c|}
\hline PU241 & 342 & $2.52 \mathrm{E}-05$ & $6.29 \mathrm{E}-04$ & $0.00 \mathrm{E}+00$ & $0.00 \mathrm{E}+00$ & $0.00 E+00$ \\
\hline PU241 & 343 & $2.32 \mathrm{E}-05$ & $5.76 \mathrm{E}-04$ & $0.00 \mathrm{E}+00$ & $0.00 \mathrm{E}+00$ & $0.00 \mathrm{E}+00$ \\
\hline PU241 & 344 & $2.27 \mathrm{E}-05$ & $5.63 \mathrm{E}-04$ & $0.00 E+00$ & $0.00 E+00$ & $0.00 \mathrm{E}+00$ \\
\hline PU241 & 345 & $2.16 \mathrm{E}-05$ & $5.33 E-04$ & $0.00 E+00$ & $0.00 \mathrm{E}+00$ & $0.00 \mathrm{E}+00$ \\
\hline PU241 & 346 & $1.98 \mathrm{E}-05$ & $4.88 E-04$ & $0.00 \mathrm{E}+00$ & $0.00 \mathrm{E}+00$ & $0.00 \mathrm{E}+00$ \\
\hline PU241 & 347 & $1.94 \mathrm{E}-05$ & $4.77 \mathrm{E}-04$ & $0.00 E+00$ & $0.00 \mathrm{E}+00$ & $0.00 E+00$ \\
\hline PU241 & 348 & $1.79 \mathrm{E}-05$ & $4.38 \mathrm{E}-04$ & $0.00 E+00$ & $0.00 \mathrm{E}+00$ & $0.00 \mathrm{E}+00$ \\
\hline PU241 & 349 & $1.75 \mathrm{E}-05$ & $4.28 \mathrm{E}-04$ & $0.00 \mathrm{E}+00$ & $0.00 \mathrm{E}+00$ & $0.00 \mathrm{E}+00$ \\
\hline PU241 & 350 & $1.61 \mathrm{E}-05$ & $3.92 \mathrm{E}-04$ & $0.00 \mathrm{E}+00$ & $0.00 \mathrm{E}+00$ & $0.00 \mathrm{E}+00$ \\
\hline AM241 & & 50 & 0 & & & \\
\hline AM241 & 21 & $5.39 E-03$ & 1. $19 \mathrm{E}-01$ & $3.08 \mathrm{E}-01$ & .00 & $2.70 \mathrm{E}-04$ \\
\hline AM241 & 22 & $1.23 E-02$ & $2.74 \mathrm{E}-01$ & $7.34 \mathrm{E}-02$ & .00 & $1.10 \mathrm{E}-05$ \\
\hline AM241 & 23 & $1.24 \mathrm{E}-02$ & $2.78 \mathrm{E}-01$ & $4.62 \mathrm{E}-04$ & .00 & $6.65 \mathrm{E}-08$ \\
\hline AM241 & 24 & $1.23 \mathrm{E}-02$ & $2.76 \mathrm{E}-01$ & $2.92 \mathrm{E}-06$ & .00 & $4.18 \mathrm{E}-10$ \\
\hline AM241 & 25 & $1.21 \mathrm{E}-02$ & $2.73 \mathrm{E}-01$ & $1.86 \mathrm{E}-08$ & .00 & $3.64 \mathrm{E}-12$ \\
\hline AM241 & 26 & $1.19 \mathrm{E}-02$ & $2.71 \mathrm{E}-01$ & .00 & .00 & .00 \\
\hline AM241 & 27 & $1.17 \mathrm{E}-02$ & $2.68 \mathrm{E}-01$ & .00 & .00 & .00 \\
\hline AM241 & 28 & $1.16 \mathrm{E}-02$ & $2.67 \mathrm{E}-01$ & .00 & .00 & .00 \\
\hline AM241 & 29 & $1.14 \mathrm{E}-02$ & $2.65 \mathrm{E}-01$ & .00 & .00 & .00 \\
\hline AM241 & 210 & $1.12 \mathrm{E}-02$ & $2.62 \mathrm{E}-01$ & .00 & .00 & .00 \\
\hline AM241 & 211 & $1.11 \mathrm{E}-02$ & $2.60 \mathrm{E}-01$ & .00 & .00 & .00 \\
\hline AM241 & 212 & $1.09 \mathrm{E}-02$ & $2.57 \mathrm{E}-01$ & .00 & .00 & .00 \\
\hline AM241 & 213 & $1.08 \mathrm{E}-02$ & $2.56 \mathrm{E}-01$ & .00 & .00 & .00 \\
\hline AM241 & 214 & $1.06 \mathrm{E}-02$ & $2.53 \mathrm{E}-01$ & .00 & .00 & .00 \\
\hline AM241 & 215 & $1.05 \mathrm{E}-02$ & $2.52 \mathrm{E}-01$ & .00 & .00 & .00 \\
\hline AM241 & 216 & $1.04 \mathrm{E}-02$ & $2.49 \mathrm{E}-01$ & .00 & .00 & .00 \\
\hline AM241 & 217 & $1.02 E-02$ & $2.47 \mathrm{E}-01$ & .00 & .00 & .00 \\
\hline AM241 & 218 & $1.01 \mathrm{E}-02$ & $2.45 E-01$ & .00 & .00 & .00 \\
\hline AM241 & 219 & $9.90 \mathrm{E}-03$ & $2.42 \mathrm{E}-01$ & .00 & .00 & .00 \\
\hline AM241 & 220 & $9.79 \mathrm{E}-03$ & $2.41 \mathrm{E}-01$ & .00 & .00 & .00 \\
\hline AM241 & 221 & $9.65 \mathrm{E}-03$ & $2.39 \mathrm{E}-01$ & .00 & .00 & .00 \\
\hline AM241 & 222 & $9.49 \mathrm{E}-03$ & $2.36 \mathrm{E}-01$ & .00 & .00 & .00 \\
\hline AM241 & 223 & $9.38 \mathrm{E}-03$ & $2.35 \mathrm{E}-01$ & .00 & .00 & .00 \\
\hline AM241 & 224 & $9.22 \mathrm{E}-03$ & $2.32 E-01$ & .00 & .00 & .00 \\
\hline AM241 & 225 & $9.12 \mathrm{E}-03$ & $2.31 \mathrm{E}-01$ & .00 & .00 & .00 \\
\hline AM241 & 226 & $8.97 \mathrm{E}-03$ & $2.29 \mathrm{E}-01$ & .00 & .00 & .00 \\
\hline AM 241 & 227 & $8.87 \mathrm{E}-03$ & $2.27 \mathrm{E}-01$ & .00 & .00 & .00 \\
\hline AM241 & 228 & $8.74 \mathrm{E}-03$ & $2.25 \mathrm{E}-01$ & .00 & .00 & .00 \\
\hline AM241 & 229 & $8.59 E-03$ & $2.23 \mathrm{E}-01$ & .00 & .00 & .00 \\
\hline AM241 & 230 & $8.50 E-03$ & $2.22 \mathrm{E}-01$ & .00 & .00 & .00 \\
\hline AM241 & 231 & $8.35 \mathrm{E}-03$ & $2.19 \mathrm{E}-01$ & .00 & .00 & .00 \\
\hline AM241 & 232 & 8.2 & $2.18 \mathrm{E}-01$ & .00 & .00 & .00 \\
\hline AM241 & 233 & 8.1 & $2.17 \mathrm{E}-01$ & .00 & .00 & .00 \\
\hline AM241 & 234 & 7.8 & $2.10 \mathrm{E}-01$ & .00 & .00 & .00 \\
\hline AM241 & 235 & $8.02 E-03$ & $2.15 \mathrm{E}-01$ & .00 & .00 & .00 \\
\hline AM241 & 236 & $7.91 \mathrm{E}-03$ & 2. $13 \mathrm{E}-01$ & .00 & .00 & .00 \\
\hline AMZ & 237 & $7.55 \mathrm{E}-03$ & $2.05 \mathrm{E}-01$ & .00 & .00 & .00 \\
\hline $\mathrm{AM}$ & 238 & $7.69 \mathrm{E}-03$ & $2.10 \mathrm{E}-01$ & .00 & .00 & .00 \\
\hline & 239 & 7.34 & $2.02 \mathrm{E}-01$ & .00 & .00 & .00 \\
\hline & 240 & $7.47 \bar{E}-03$ & $2.06 \mathrm{E}-01$ & .00 & .00 & .00 \\
\hline
\end{tabular}




\section{Appendix B - Inhalation Dose Rate Factors - FILE23}

$\begin{array}{lllllll}\text { AM241 } & 241 & 7.14 \mathrm{E}-03 & 1.98 \mathrm{E}-01 & .00 & .00 & .00 \\ \text { AM241 } & 242 & 7.26 \mathrm{E}-03 & 2.03 \mathrm{E}-01 & .00 & .00 & .00 \\ \text { AM241 } & 243 & 6.94 \mathrm{E}-03 & 1.95 \mathrm{E}-01 & .00 & .00 & .00 \\ \text { AM241 } & 244 & 7.06 \mathrm{E}-03 & 2.00 \mathrm{E}-01 & .00 & .00 & .00 \\ \text { AM241 } & 245 & 6.96 \mathrm{E}-03 & 1.98 \mathrm{E}-01 & .00 & .00 & .00 \\ \text { AM241 } & 246 & 6.65 \mathrm{E}-03 & 1.90 \mathrm{E}-01 & .00 & .00 & .00 \\ \text { AM241 } & 247 & 6.77 \mathrm{E}-03 & 1.95 \mathrm{E}-01 & .00 & .00 & .00 \\ \text { AM241 } & 248 & 6.46 \mathrm{E}-03 & 1.87 \mathrm{E}-01 & .00 & .00 & .00 \\ \text { AM241 } & 249 & 6.58 \mathrm{E}-03 & 1.91 \mathrm{E}-01 & .00 & .00 & .00 \\ \text { AM241 } & 250 & 6.28 \mathrm{E}-03 & 1.84 \mathrm{E}-01 & .00 & .00 & .00\end{array}$




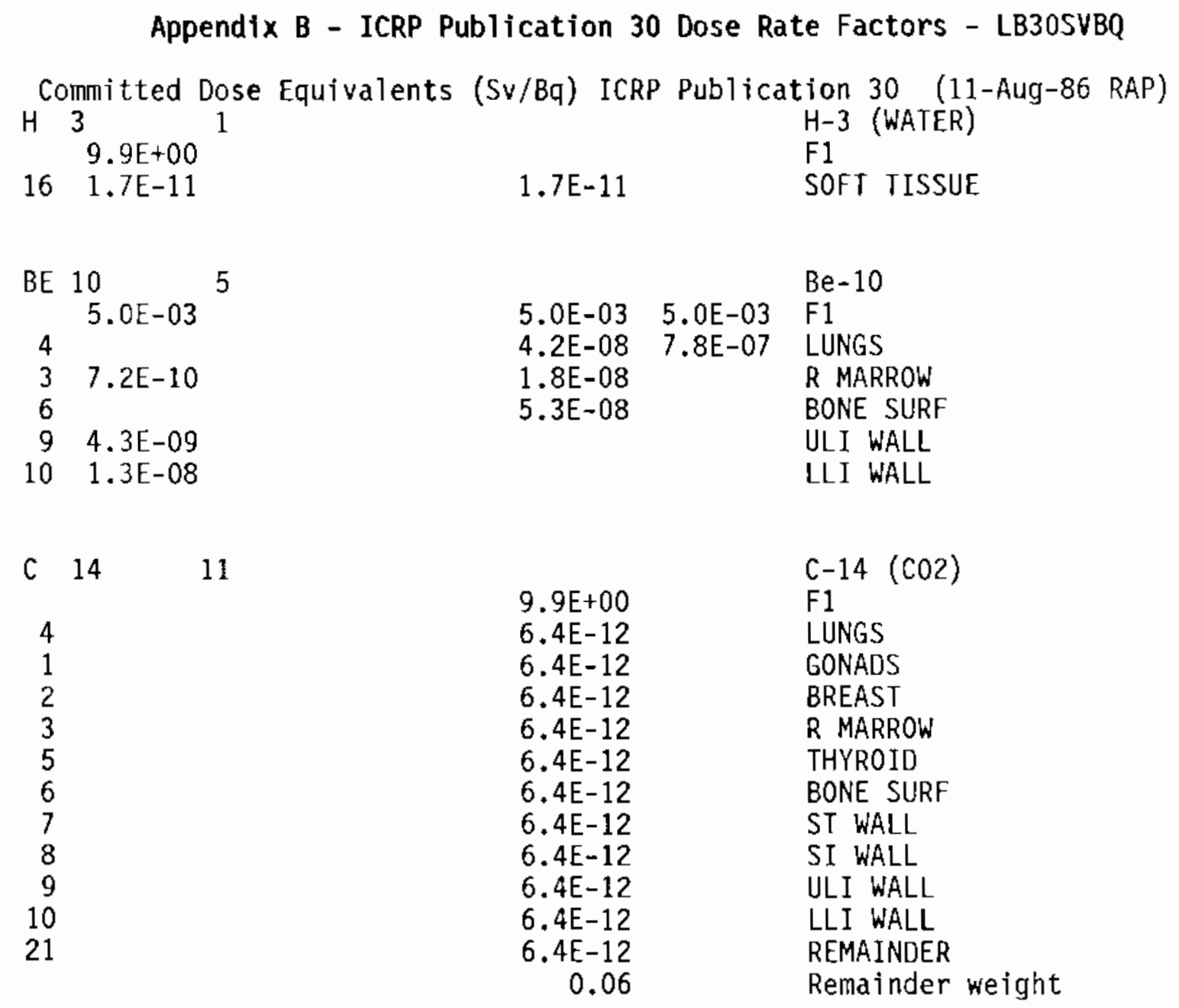

$\begin{array}{lll}N & 13 & 0\end{array}$

F 18 1.0E+00

4

3 5.9E-11

6 6.0E-11

$7 \quad 2.9 \mathrm{E}-10$

$212.9 \mathrm{E}-11$

0.06
1. $0 \mathrm{E}+00$

1. $1 \mathrm{E}-10$

2. $8 \mathrm{E}-11$

4. IE-11

1. $0 \mathrm{E}+00$

1. $3 \mathrm{E}-10$
1. $0 E+00$

$1.4 \mathrm{E}-10$

$\mathrm{F}-18$

F1

LUNGS

R MARROW

BONE SURF

ST WALL

REMAINDER

Remainder weight

$\mathrm{Na}-22$

$\mathrm{F} 1$

LUNGS

GONADS

BREAST

B. 129 
Appendix B - ICRP Publication 30 Dose Rate Factors - LB30SVBQ

$\begin{array}{rrr}3 & 4.3 \mathrm{E}-09 & 2.7 \mathrm{E}-09 \\ 6 & 5.5 \mathrm{E}-09 & 3.5 \mathrm{E}-09 \\ 5 & 2.5 \mathrm{E}-09 & 1.6 \mathrm{E}-09 \\ 7 & 2.9 \mathrm{E}-09 & \\ 8 & 3.0 \mathrm{E}-09 & 1.9 \mathrm{E}-09 \\ 10 & 3.1 \mathrm{E}-09 & 1.9 \mathrm{E}-09 \\ 21 & 4.0 \mathrm{E}-09 & 2.6 \mathrm{E}-09 \\ & 0.12 & 0.18\end{array}$

R MARROW

BONE SURF

THYROID

ST WALL

SI WALL

LLI WALL

REMAINDER

Remainder weight

\begin{tabular}{|c|c|c|}
\hline & & 8 \\
\hline & $1.0 \mathrm{E}+00$ & $1.0 \mathrm{E}+00$ \\
\hline & $2.6 \mathrm{E}-10$ & $1.2 \mathrm{E}-09$ \\
\hline & $3.4 \mathrm{E}-10$ & $1.8 \mathrm{E}-10$ \\
\hline & 2.7E-10 & $1.6 \mathrm{E}-10$ \\
\hline & 3.7E-10 & $2.1 \mathrm{E}-10$ \\
\hline & $\begin{array}{l}\text { 4. } 7 \mathrm{E}-10 \\
1.2 \mathrm{~F}-0 \mathrm{0}\end{array}$ & \\
\hline & $3.4 \mathrm{E}-10$ & $3.1 E-10$ \\
\hline & $4.4 E-10$ & \\
\hline
\end{tabular}

$\mathrm{Na}-24$

F1

LUNGS

GONADS

BREAST

$R$ MARROW

BONE SURF

ST WALL

LLI WALL

REMAINDER

Remainder weight

$\begin{array}{rc}\mathrm{P} & 32 \\ & 8.0 \mathrm{E}-01 \\ 4 & \\ 1 & 6.5 \mathrm{E}-10 \\ 2 & 6.5 \mathrm{E}-10 \\ 3 & 8.1 \mathrm{E}-09 \\ 6 & 7.9 \mathrm{E}-09 \\ 9 & 3.0 \mathrm{E}-09 \\ 10 & 7.2 \mathrm{E}-09\end{array}$

8.0E-01

8.0E-01

$\mathrm{P}-32$

F1

2.5E-09 2.6E-08

LUNGS

GONADS

BREAST

4. $8 \mathrm{E}-10$

6.0E-09 4.2E-09

R MARROW

BONE SURF

ULI WALL

1.5E-09

LLI WALL

$$
\text { P } 33.9
$$

8.0E-01

8. $0 \mathrm{E}-01$

$P-33$

3.0 $\mathrm{E}-10$

4.2E-09

F1

7.0E-11

7.0E-11

LUNGS

GONADS

BREAST

3.7E-10

$R$ MARROW

9. $8 \mathrm{E}-10$

BONE SURF

$8.3 E-11$

ST WALL

7.6E-11

SI WALL

$1.1 \mathrm{E}-10$

ULI WALL

1. $9 \mathrm{E}-10$

LLI WALL

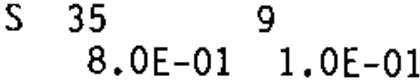
8.0E-01
8.0E-01
S-35
F1
LUNGS 


\section{Appendix B - ICRP Publication 30 Dose Rate Factors - LB30SVBQ}

$\begin{array}{rr}1 & 7.6 \mathrm{E}-11 \\ 2 & 7.6 \mathrm{E}-11 \\ 3 & 7.6 \mathrm{E}-11 \\ 7 & 1.3 \mathrm{E}-10 \\ 8 & 1.0 \mathrm{E}-10 \\ 9 & 2.4 \mathrm{E}-10 \\ 10 & 5.7 \mathrm{E}-10 \\ 21 & 7.6 \mathrm{E}-11 \\ & 0.06\end{array}$

S 35

11

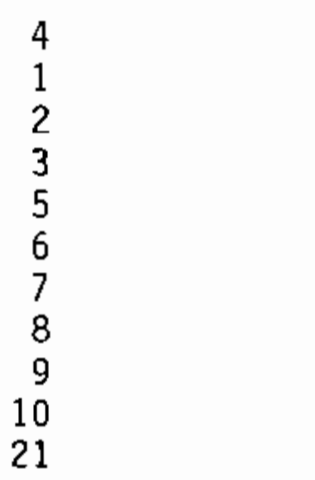

$\mathrm{CL} 36 \quad 11$

$\begin{array}{rr} & 1.0 \mathrm{E}+00 \\ 4 & 8.0 \mathrm{E}-10 \\ 1 & 8.0 \mathrm{E}-10 \\ 2 & 8.0 \mathrm{E}-10 \\ 3 & 8.0 \mathrm{E}-10 \\ 6 & 8.0 \mathrm{E}-10 \\ 5 & 8.0 \mathrm{E}-10 \\ 7 & 1.1 \mathrm{E}-09 \\ 8 & 8.0 \mathrm{E}-10 \\ 9 & 8.0 \mathrm{E}-10 \\ 10 & 8.0 \mathrm{E}-10 \\ 21 & 8.0 \mathrm{E}-10 \\ & 0.06\end{array}$

AR 39

0

AR 41 0

$$
\begin{array}{r}
5.7 \mathrm{E}-11 \\
5.7 \mathrm{E}-11 \\
5.7 \mathrm{E}-11 \\
6.6 \mathrm{E}-11 \\
6.1 \mathrm{E}-11 \\
8.2 \mathrm{E}-11 \\
1.3 \mathrm{E}-10 \\
5.7 \mathrm{E}-11 \\
0.06
\end{array}
$$

GONADS

BREAST

R MARROW

ST WALL

SI WALL

ULI WALL

LLI WALL

REMAINDER

Remainder weight

S-35 (GASES)

F1

LUNGS

GONADS

BREAST

R MARROW

THYROID

BONE SURF

ST WALL

SI WALL

ULI WALL

LLI WALL

REMAINDER

Remainder weight

C1-36

F1

LUNGS

GONADS

BREAST

$R$ MARROW

BONE SURF

THYROID

ST WALL

SI WALL

ULI WALL

LLI WALL

REMAINDER

Remainder weight 


\section{Appendix B - ICRP Publication 30 Dose Rate Factors - LB30SVBQ}

$\begin{array}{rrr}\mathrm{K} & 40 \quad 10 & \\ & 1.0 \mathrm{E}+00 & 1.0 \mathrm{E}+00 \\ 4 & 4.9 \mathrm{E}-09 & 4.7 \mathrm{E}-09 \\ 1 & 5.1 \mathrm{E}-09 & 3.2 \mathrm{E}-09 \\ 2 & 4.9 \mathrm{E}-09 & 3.1 \mathrm{E}-09 \\ 3 & 4.9 \mathrm{E}-09 & 3.1 \mathrm{E}-09 \\ 6 & 4.9 \mathrm{E}-09 & 3.1 \mathrm{E}-09 \\ 5 & 4.9 \mathrm{E}-09 & 3.1 \mathrm{E}-09 \\ 7 & 5.5 \mathrm{E}-09 & 3.2 \mathrm{E}-09 \\ 8 & 5.0 \mathrm{E}-09 & 3.2 \mathrm{E}-09 \\ 10 & 5.0 \mathrm{E}-09 & 3.2 \mathrm{E}-09 \\ 21 & 5.4 \mathrm{E}-09 & 3.4 \mathrm{E}-09 \\ & 0.12 & 0.12\end{array}$

$\mathrm{K}-40$

F1

LUNGS

GONADS

BREAST

R MARROW

BONE SURF

THYROID

ST WALL

SI WALL

LLI WALL

REMAINDER

Remainder weight

$\mathrm{Ca}-41$

F1

LUNGS

R MARROW

BONE SURF

$$
\begin{array}{rlr}
\text { CA } & 45 & 5 \\
& 3.0 \mathrm{E}-01 \\
4 & \\
3 & 3.5 \mathrm{E}-09 \\
6 & 5.2 \mathrm{E}-09 \\
9 & 9.9 \mathrm{E}-10 \\
10 & 2.8 \mathrm{E}-09
\end{array}
$$

3. $0 \mathrm{E}-01$

9.7E-09

2. $9 \mathrm{E}-09$

4. $4 \mathrm{E}-09$
Ca-45

F1

LUNGS

$R$ MARROW

BONE SURF

ULI WALL

LLI WALL

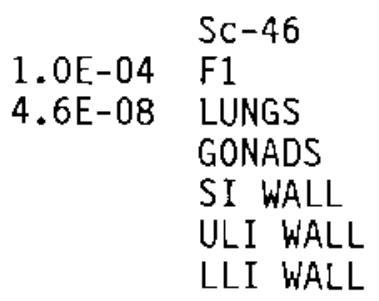

Cr-51

$F 1$

LUNGS

GONADS

BREAST

R MARROW

BDNE SURF

SI WALL

ULI WALL

B. 132 
Appendix B - ICRP Publication 30 Dose Rate Factors - LB30SVBQ

\begin{tabular}{|c|c|c|c|c|c|}
\hline $\begin{array}{ll}10 & 2.5 E-10 \\
21 & \end{array}$ & $2.7 \mathrm{E}-10$ & $\begin{array}{r}5.9 \mathrm{E}-11 \\
2.5 \mathrm{E}-11 \\
0.12\end{array}$ & $1.1 \mathrm{E}-10$ & 1. $2 \mathrm{E}-10$ & $\begin{array}{l}\text { LLI WALL } \\
\text { REMAINDER } \\
\text { Remainder weight }\end{array}$ \\
\hline
\end{tabular}

$\begin{array}{rl}\text { MN } & 54 \\ & 1.0 \mathrm{E}-01 \\ 4 & 2.3 \mathrm{E}-10 \\ 1 & 9.5 \mathrm{E}-10 \\ 2 & 2.8 \mathrm{E}-10 \\ 3 & 4.9 \mathrm{E}-10 \\ 6 & \\ 12 & 1.0 \mathrm{E}-09 \\ 8 & 9.8 \mathrm{E}-10 \\ 9 & 1.4 \mathrm{E}-09 \\ 10 & 2.2 \mathrm{E}-09 \\ 21 & 5.0 \mathrm{E}-10 \\ & 0.06\end{array}$

$1.0 \mathrm{E}-01 \quad 1.0 \mathrm{E}-01$

$1.2 \mathrm{E}-09 \quad 6.7 \mathrm{E}-09$

$8.9 \mathrm{E}-10 \quad 7.1 \mathrm{E}-10$

9.1E-10 8.6E-10

$1.7 \mathrm{E}-09 \quad 1.1 \mathrm{E}-09$

$2.6 \mathrm{E}-09$

4.6E-09 2.5E-09

$1.3 \mathrm{E}-09$

$1.8 \mathrm{E}-09 \quad 1.8 \mathrm{E}-09$

$0.18 \quad 0.18$

Mn-54
FI
LUNGS
GONADS
BREAST
R MARROW
BONE SURF
LIVER
SI WALL
ULI WALL
LLI WALL
REMAINDER wight
Remainder weight

MN $56{ }_{1.0 \mathrm{E}-01}^{6}$

$1.0 \mathrm{E}-01$

$1.0 \mathrm{E}-01$

4. $4 \mathrm{E}-10 \quad 5.4 \mathrm{E}-10$

$\mathrm{Mn}-56$

F1

2.1E-11

1. $4 \mathrm{E}-10$

$1.6 \mathrm{E}-10$

2. $0 \mathrm{E}-10$

LUNGS

GONADS

ST WALL

SI WALL

ULI WALL

LLI WALL

$\begin{array}{rlrl}\text { FE } & 55 \quad 10 & \\ & 1.0 \mathrm{E}-01 & 1.0 \mathrm{E}-01 & 1.0 \mathrm{E}-01 \\ 4 & 1.0 \mathrm{E}-10 & 5.2 \mathrm{E}-10 & 1.1 \mathrm{E}-09 \\ 1 & 1.1 \mathrm{E}-10 & 5.2 \mathrm{E}-10 & 1.8 \mathrm{E}-10 \\ 2 & 1.0 \mathrm{E}-10 & 5.1 \mathrm{E}-10 & 1.7 \mathrm{E}-10 \\ 3 & 1.1 \mathrm{E}-10 & 5.2 \mathrm{E}-10 & 1.8 \mathrm{E}-10 \\ 12 & 3.4 \mathrm{E}-10 & 1.7 \mathrm{E}-09 & 5.8 \mathrm{E}-10 \\ 13 & 5.6 \mathrm{E}-10 & 2.8 \mathrm{E}-09 & 9.5 \mathrm{E}-10 \\ 8 & 1.2 \mathrm{E}-10 & & \\ 9 & 1.7 \mathrm{E}-10 & 5.2 \mathrm{E}-10 & \\ 10 & 3.0 \mathrm{E}-10 & 5.4 \mathrm{E}-10 & 2.8 \mathrm{E}-10 \\ 21 & & 5.2 \mathrm{E}-10 & \\ & & 0.06 & \end{array}$

$\mathrm{Fe}-55$

F1

LUNGS

GONADS

BREAST

$R$ MARROW

LIVER

SPLEEN

SI WALL

ULI WALL

LLI WALL

REMAINDER

Remainder weight

$\begin{array}{lllll}\text { FE } & 59 \quad 12 & & & \text { Fe-59 } \\ & 1.0 \mathrm{E}-01 & 1.0 \mathrm{E}-01 & 1.0 \mathrm{E}-01 & \text { F1 } \\ 4 & 6.4 \mathrm{E}-10 & 3.5 \mathrm{E}-09 & 1.4 \mathrm{E}-08 & \text { LUNGS } \\ 1 & 1.7 \mathrm{E}-09 & 3.3 \mathrm{E}-09 & 1.4 \mathrm{E}-09 & \text { GONADS } \\ 2 & 7.4 \mathrm{E}-10 & 3.0 \mathrm{E}-09 & 1.3 \mathrm{E}-09 & \text { BREAST }\end{array}$

B. 133 
Appendix B - ICRP Publication 30 Dose Rate Factors - LB30SVBO

$\begin{array}{rl}3 & 8.5 \mathrm{E}-10 \\ 6 & \\ 5 & \\ 13 & 1.8 \mathrm{E}-09 \\ 12 & 1.5 \mathrm{E}-09 \\ 8 & 2.1 \mathrm{E}-09 \\ 9 & 3.9 \mathrm{E}-09 \\ 10 & 8.4 \mathrm{E}-09 \\ 21 & \end{array}$
$3.2 E-09$
2. $9 \mathrm{E}-09$
2. $9 \mathrm{E}-09$
$8.3 \mathrm{E}-09$
7. $1 \mathrm{E}-09$
4. $1 \mathrm{E}-09$
$4.8 \mathrm{E}-09$
4.7E-09
0.06
2.9E-09
$4.5 E-09$

R MARROW

BONE SURF

THYROID

SPLEEN

LIVER

SI WALL

ULI WALL

LLI WALL

REMAINDER

Remainder weight \begin{tabular}{rlr} 
C0 & 57 & \multicolumn{2}{l}{9} \\
& $5.0 \mathrm{E}-02$ & $3.0 \mathrm{E}-01$ \\
4 & & $1.6 \mathrm{E}-10$ \\
1 & $1.8 \mathrm{E}-10$ & $2.9 \mathrm{E}-10$ \\
2 & & $1.6 \mathrm{E}-10$ \\
3 & $8.8 \mathrm{E}-11$ & $2.7 \mathrm{E}-10$ \\
12 & & $4.6 \mathrm{E}-10$ \\
8 & $2.3 \mathrm{E}-10$ & $3.4 \mathrm{E}-10$ \\
9 & $5.3 \mathrm{E}-10$ & $5.6 \mathrm{E}-10$ \\
10 & $1.3 \mathrm{E}-09$ & $1.1 \mathrm{E}-09$ \\
21 & & $2.5 \mathrm{E}-10$ \\
& & 0.06
\end{tabular}

CO $58 \quad 9$

5.0E-02 3.0E-01

$4 \quad 4.1 \mathrm{E}-10$

$1 \quad 1.0 \mathrm{E}-09 \quad 1.1 \mathrm{E}-09$

$2 \quad 1.8 \mathrm{E}-10 \quad 4.5 \mathrm{E}-10$

$3 \quad 2.6 \mathrm{E}-10 \quad 5.4 \mathrm{E}-10$

$121.0 \mathrm{E}-09$

$8 \quad 1.1 \mathrm{E}-09 \quad 1.3 \mathrm{E}-09$

$9 \quad 1.9 \mathrm{E}-09$ 1.9E-09

10 4.0E-09 3.3E-09

$21 \quad 4.8 \mathrm{E}-10 \quad 7.8 \mathrm{E}-10$

$0.06 \quad 0.06$

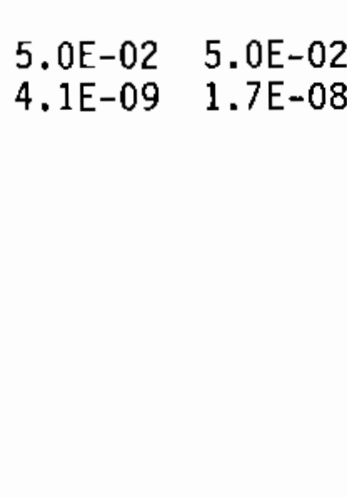

Co-57

$F 1$

LUNGS

GONADS

BREAST

R MARROW

LIVER

SI WALL

ULI WALL

LLI WALL.

REMAINDER

Remainder weight

5. $0 \mathrm{E}-02$

7. $9 \mathrm{E}-09$

$6.5 \mathrm{E}-10$

5. $0 \mathrm{E}-02$

Co-58

$F 1$

1.6E-08 LUNGS

GONADS

BREAST

R MARROW

LIVER

SI WALL

ULI WALL

2.0E-09

LLI WALL

REMAINDER

Remainder weight

Co-60

5. $0 \mathrm{E}-02$

$3.6 \mathrm{E}-08$

4. $0 \mathrm{E}-09$

4.2E-09

4. $2 \mathrm{E}-09$

9. 2E-09

5.0E-02 F1

3.4E-07 LUNGS

GONADS

BREAST

R MARROW

LIVER

SI WALL

ULI WALL

8.2E-09 LLI WALL 
Appendix B - ICRP Publication 30 Dose Rate Factors - LB30SVBQ

$\begin{array}{rrrrl}21 & 2.1 \mathrm{E}-09 & 8.7 \mathrm{E}-09 & 8.0 \mathrm{E}-09 & \text { REMAINDER } \\ & 0.06 & 0.06 & 0.06 & \text { Remainder weight }\end{array}$

$\begin{array}{rc}\text { NI } & 59 \\ & 5.0 \mathrm{E}-02 \\ 4 & 3.5 \mathrm{E}-11 \\ 1 & 3.8 \mathrm{E}-11 \\ 2 & 3.6 \mathrm{E}-11 \\ 3 & 3.7 \mathrm{E}-11 \\ 6 & \\ 5 & \\ 7 & 4.1 \mathrm{E}-11 \\ 8 & 4.1 \mathrm{E}-11 \\ 9 & 1.2 \mathrm{E}-10 \\ 10 & 2.7 \mathrm{E}-10 \\ 21 & 3.7 \mathrm{E}-11 \\ & 0.06\end{array}$

$\begin{array}{ll}5.0 \mathrm{E}-02 & 5.0 \mathrm{E}-02 \\ 3.6 \mathrm{E}-10 & 1.2 \mathrm{E}-09 \\ 3.6 \mathrm{E}-10 & 1.1 \mathrm{E}-10 \\ 3.5 \mathrm{E}-10 & 1.1 \mathrm{E}-10 \\ 3.5 \mathrm{E}-10 & \\ 3.5 \mathrm{E}-10 & \\ 3.8 \mathrm{E}-10 & \end{array}$

Ni-59(INORGANIC)

F1

LUNGS

GONADS

BREAST

R MARROW

BONE. SURF

THYROID

ST WALL

SI WALL

3.6E-10 ULI WALL

3. $8 \mathrm{E}-10$

3. $6 \mathrm{E}-10$

LLI WALL

REMAINDER

Remainder weight

$\begin{array}{rc}\text { NI } & 63 \\ & 5.0 \mathrm{E}-02 \\ 4 & 8.5 \mathrm{E}-11 \\ 1 & 8.5 \mathrm{E}-11 \\ 2 & 8.5 \mathrm{E}-11 \\ 3 & 8.5 \mathrm{E}-11 \\ 6 & \\ 11 & \\ 5 & \\ 7 & 1.0 \mathrm{E}-10 \\ 8 & 1.3 \mathrm{E}-10 \\ 9 & 3.6 \mathrm{E}-10 \\ 10 & 9.2 \mathrm{E}-10\end{array}$

5. $0 \mathrm{E}-02$

8.7E-10

5.0E-02

8. $2 \mathrm{E}-10$

8.2E-10

3. $1 \mathrm{E}-09$

$2.5 \mathrm{E}-10$

8.2E-10

2. $5 E-10$

8.2E-10

8.2E-10

8.2E-10

8. $3 \mathrm{E}-10$

8. $3 \mathrm{E}-10$

8.7E-10

9.5E-10 6.7E-10

Ni-63 (INORGANIC)

F1

LUNGS

GONADS

BREAST

$R$ MARROW

BONE SURF

KIDNEYS

THYROID

ST WALL

SI WALL

ULI WALL

LLI WALL
NI $65 \quad 6$

$\begin{array}{rl} & 5.0 \mathrm{E}-02 \\ 4 & \\ 1 & 2.4 \mathrm{E}-11 \\ 7 & 6.2 \mathrm{E}-10 \\ 8 & 7.3 \mathrm{E}-10 \\ 9 & 9.3 \mathrm{E}-10 \\ 10 & 3.7 \mathrm{E}-10\end{array}$

5.0E-02

$3.1 \mathrm{E}-10$

5.0E-02

Ni-65 (INORGANIC)

F1

LUNGS

9. $2 \mathrm{E}-11$

1.1 $\mathrm{E}-10$

GONADS

ST WALL

SI WALL

ULI WALL

LLI WALL

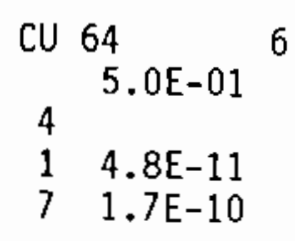

5. $0 \mathrm{E}-01$

2. $0 \mathrm{E}-10$

5. $0 \mathrm{E}-01$

5. $0 \mathrm{E}-01$

3. $5 \mathrm{E}-10$

Cu-64

F1

1. $6 \mathrm{E}-11$

LUNGS

GONADS

ST WALL 
Appendix B - ICRP Publication 30 Dose Rate Factors - LB30SVBQ

$\begin{array}{rr}8 & 2.1 \mathrm{E}-10 \\ 9 & 6.1 \mathrm{E}-10 \\ 10 & 7.5 \mathrm{E}-10 \\ & \\ & \\ \text { ZN } & 65 \quad 10 \\ & 5.0 \mathrm{E}-01 \\ 4 & 3.1 \mathrm{E}-09 \\ 1 & 3.5 \mathrm{E}-09 \\ 2 & 3.3 \mathrm{E}-09 \\ 3 & 4.5 \mathrm{E}-09 \\ 5 & 3.2 \mathrm{E}-09 \\ 6 & 4.5 \mathrm{E}-09 \\ 8 & 4.3 \mathrm{E}-09 \\ 9 & 4.2 \mathrm{E}-09 \\ 10 & 5.0 \mathrm{E}-09 \\ 21 & 4.8 \mathrm{E}-09 \\ & 0.12\end{array}$

$\begin{array}{llll}4.2 \mathrm{E}-11 & & & \text { SI WALL } \\ 1.0 \mathrm{E}-10 & 1.3 \mathrm{E}-10 & 1.5 \mathrm{E}-10 & \text { ULI WALL } \\ 1.2 \mathrm{E}-10 & 1.6 \mathrm{E}-10 & 1.9 \mathrm{E}-10 & \text { LLI WALL }\end{array}$

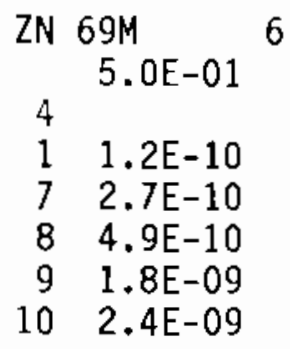

$\begin{aligned} & \text { Zn-65 } \\ 5.0 \mathrm{E}-01 & \text { F1 } \\ 2.1 \mathrm{E}-08 & \text { LUNGS } \\ 2.0 \mathrm{E}-09 & \text { GONADS } \\ 3.1 \mathrm{E}-09 & \text { BREAST } \\ 3.6 \mathrm{E}-09 & \text { R MARROW } \\ & \text { THYROID } \\ & \text { BONE SURF } \\ & \text { SI WALL } \\ & \text { ULI WALL } \\ & \text { LLI WALL } \\ 5.9 \mathrm{E}-09 & \text { REMAINDER } \\ 0.18 & \text { Remainder weight }\end{aligned}$

$\begin{array}{cc}\text { ZN } & 69 \\ & 5.0 \mathrm{E}-01 \\ 4 & 4 \\ 7 & 2.1 \mathrm{E}-10 \\ 8 & 1.1 \mathrm{E}-10 \\ 9 & 6.1 \mathrm{E}-11\end{array}$

$$
\begin{array}{rl}
\text { AS } & 76 \\
& 5.0 \mathrm{E}-01 \\
4 & 5 \\
7 & 1.4 \mathrm{E}-09 \\
8 & 1.7 \mathrm{E}-09 \\
9 & 6.6 \mathrm{E}-09 \\
10 & 1.2 \mathrm{E}-08
\end{array}
$$

$5.0 \mathrm{E}-01$

$5.0 \mathrm{E}-09$

$1.8 \mathrm{E}-09$

3. $3 \mathrm{E}-09$
$\mathrm{Zn}-69 \mathrm{M}$

5.0E-01 F1

1.0E-09 LUNGS

GONADS

ST WALL

4.8E-10 ULI WALL

$6.3 \mathrm{E}-10$ LLI WALL
SE 75
$\quad 8.0 \mathrm{E}-01 \quad 5.0 \mathrm{E}-02$
8.0E-01

$1.4 \mathrm{E}-09$
8.0E-01
$5.4 \mathrm{E}-09$

Se -75

FI

LUNGS

\section{B. 136}




\section{Appendix B - ICRP Publication 30 Dose Rate Factors - LB30SVBQ}

$\begin{array}{rrrrr}1 & 1.8 \mathrm{E}-09 & 5.9 \mathrm{E}-10 & 1.3 \mathrm{E}-09 & 1.1 \mathrm{E}-09 \\ 2 & 1.5 \mathrm{E}-09 & 1.4 \mathrm{E}-10 & 1.1 \mathrm{E}-09 & 1.1 \mathrm{E}-09 \\ 3 & 2.1 \mathrm{E}-09 & 2.4 \mathrm{E}-10 & 1.5 \mathrm{E}-09 & 1.5 \mathrm{E}-09 \\ 6 & 1.7 \mathrm{E}-09 & & 1.3 \mathrm{E}-09 & \\ 11 & 7.2 \mathrm{E}-09 & 5.0 \mathrm{E}-10 & 5.4 \mathrm{E}-09 & 4.5 \mathrm{E}-09 \\ 12 & 5.7 \mathrm{E}-09 & 3.9 \mathrm{E}-10 & 4.3 \mathrm{E}-09 & 3.9 \mathrm{E}-09 \\ 15 & 4.0 \mathrm{E}-09 & & 3.0 \mathrm{E}-09 & 2.8 \mathrm{E}-09 \\ 13 & 3.6 \mathrm{E}-09 & & 2.7 \mathrm{E}-09 & 2.6 \mathrm{E}-09 \\ 8 & & 6.0 \mathrm{E}-10 & & \\ 9 & & 9.4 \mathrm{E}-10 & & \\ 10 & & 1.8 \mathrm{E}-09 & & \\ 21 & 2.8 \mathrm{E}-09 & & 2.1 \mathrm{E}-09 & 2.1 \mathrm{E}-09 \\ & 0.06 & & 0.06 & 0.06\end{array}$

GONADS

BREAST

R MARROW

BONE SURF

KIDNEYS

LIVER

PANCREAS

SPLEEN

SI WALL

ULI WALL

LLI WALL

REMAINDER

Remainder weight

\begin{tabular}{|c|c|c|c|}
\hline 79 & 10 & & \\
\hline $8.0 \mathrm{E}-01$ & $5.0 \mathrm{E}-02$ & 8. $0 E-01$ & $8.0 \mathrm{E}-01$ \\
\hline $9.1 \mathrm{E}-10$ & & $8.5 E-10$ & $9.8 \mathrm{E}-09$ \\
\hline $9.1 E-10$ & & $6.8 \mathrm{E}-10$ & $6.0 \mathrm{E}-10$ \\
\hline $9.1 \mathrm{E}-10$ & & $6.8 \mathrm{E}-10$ & \\
\hline 9.1 & & 6. & \\
\hline 1. $3 E-08$ & $7.8 \mathrm{E}-10$ & $9.4 \mathrm{E}-09$ & $8.3 E-09$ \\
\hline 6.5 & $4.1 \mathrm{E}-10$ & 4. & $4.3 E-09$ \\
\hline 30 & & & $2.6 E-09$ \\
\hline & 9. & & \\
\hline & & & \\
\hline
\end{tabular}

Se-79

F1

LUNGS

GONADS

BREAST

R MARROW

KIDNEYS

LIVER

PANCREAS

SPLEEN

ULI WALL

LLI WALL

$\begin{array}{rrrr}\text { BR } & 82 & 8 & \\ & 1.0 \mathrm{E}+00 & 1.0 \mathrm{E}+00 & 1.0 \mathrm{E}+00 \\ 4 & 3.8 \mathrm{E}-10 & 7.8 \mathrm{E}-10 & 1.7 \mathrm{E}-09 \\ 1 & 4.5 \mathrm{E}-10 & 2.5 \mathrm{E}-10 & 1.7 \mathrm{E}-10 \\ 2 & 3.8 \mathrm{E}-10 & 2.4 \mathrm{E}-10 & 2.1 \mathrm{E}-10 \\ 3 & 4.1 \mathrm{E}-10 & 2.5 \mathrm{E}-10 & 2.2 \mathrm{E}-10 \\ 6 & 3.8 \mathrm{E}-10 & & \\ 7 & 8.3 \mathrm{E}-10 & 3.4 \mathrm{E}-10 & 3.8 \mathrm{E}-10 \\ 8 & 5.0 \mathrm{E}-10 & & \\ 21 & 5.6 \mathrm{E}-10 & 3.5 \mathrm{E}-10 & 3.5 \mathrm{E}-10 \\ & 0.18 & 0.24 & 0.06\end{array}$

$\mathrm{Br}-82$

F1

LUNGS

GONADS

BREAST

$R$ MARROW

BONE SURF

ST WALL

SI WALL

REMAINDER

Remainder weight

$\begin{array}{llll}\text { BR } & 83 & & \\ & 1.0 \mathrm{E}+00 & 1.0 \mathrm{E}+00 & 1.0 \mathrm{E}+00 \\ 4 & & 1.5 \mathrm{E}-10 & 1.8 \mathrm{E}-10 \\ 1 & 7.3 \mathrm{E}-12 & & \\ 7 & 3.0 \mathrm{E}-10 & 4.3 \mathrm{E}-11 & \end{array}$

$\mathrm{Br}-83$

F1

LUNGS

GONADS

ST WALL

BR 84

$\mathrm{Br}-84$ 


\begin{tabular}{rrrrr}
\multicolumn{5}{c}{ Appendix B - ICRP Publication 30 Dose Rate Factors - LB30SVBQ } \\
\multicolumn{1}{r}{$1.0 \mathrm{E}+00$} & $1.0 \mathrm{E}+00$ & $1.0 \mathrm{E}+00$ & F1 \\
4 & & $1.6 \mathrm{E}-10$ & $1.7 \mathrm{E}-10$ & LUNGS \\
7 & $6.8 \mathrm{E}-10$ & $7.4 \mathrm{E}-11$ & & ST WALL
\end{tabular}

BR $85 \quad 0$

KR 83M 0

KR $85 \quad 0$

KR 85M $\quad 0$

KR $87 \quad 0$

KR $88 \quad 0$

KR $89 \quad 0$

KR $90 \quad 0$

$\begin{array}{ll}\text { KR } & 91 \quad 0\end{array}$

$\begin{array}{llll}\text { RB } & 86 & & \\ & 1.0 \mathrm{E}+00 & 1.0 \mathrm{E}+00 & \mathrm{Rb}-86 \\ 4 & 2.1 \mathrm{E}-09 & 3.3 \mathrm{E}-09 & \mathrm{~F} 1 \\ 1 & 2.2 \mathrm{E}-09 & 1.3 \mathrm{E}-09 & \text { LUNGS } \\ 2 & 2.1 \mathrm{E}-09 & 1.3 \mathrm{E}-09 & \text { GONADS } \\ 3 & 3.7 \mathrm{E}-09 & 2.3 \mathrm{E}-09 & \text { BREAST } \\ 6 & 6.9 \mathrm{E}-09 & 4.3 \mathrm{E}-09 & \text { R MARROW } \\ 5 & 2.1 \mathrm{E}-09 & 1.3 \mathrm{E}-09 & \text { BONE SURF } \\ 7 & 2.9 \mathrm{E}-09 & 1.5 \mathrm{E}-09 & \text { THYROID } \\ & & & \text { ST WALL }\end{array}$

B. 138 
Appendix B - ICRP Publication 30 Dose Rate Factors - LB3OSVBQ

$\begin{array}{rr}10 & 2.2 \mathrm{E}-09 \\ 21 & 2.2 \mathrm{E}-09 \\ 0.18\end{array}$

R8 87

$\begin{array}{rrr} & 1.0 \mathrm{E}+00 & 1.0 \mathrm{E}+00 \\ 4 & 1.1 \mathrm{E}-09 & 1.1 \mathrm{E}-09 \\ 1 & 1.1 \mathrm{E}-09 & 7.2 \mathrm{E}-10 \\ 2 & 1.1 \mathrm{E}-09 & 7.2 \mathrm{E}-10 \\ 3 & 2.0 \mathrm{E}-09 & 1.3 \mathrm{E}-09 \\ 6 & 3.8 \mathrm{E}-09 & 2.4 \mathrm{E}-09 \\ 5 & 1.1 \mathrm{E}-09 & 7.2 \mathrm{E}-10 \\ 7 & 1.3 \mathrm{E}-09 & 7.4 \mathrm{E}-10 \\ 8 & 1.1 \mathrm{E}-09 & 7.2 \mathrm{E}-10 \\ 9 & 1.1 \mathrm{E}-09 & 7.2 \mathrm{E}-10 \\ 10 & 1.1 \mathrm{E}-09 & 7.2 \mathrm{E}-10 \\ 21 & 1.1 \mathrm{E}-09 & 7.2 \mathrm{E}-10 \\ & 0.06 & 0.06\end{array}$

LLI WALL

REMAINDER

Remainder weight

$\mathrm{Rb}-87$

F1

LUNGS

GONADS

BREAST

R MARROW

BONE SURF

THYROID

ST WALL

SI WALL

ULI WALL

LLI WALL

REMAINDER

Remainder weight

$\mathrm{Rb}-88$

F1

LUNGS

ST WALL

$\mathrm{Rb}-89$

F1

LUNGS

ST WALL

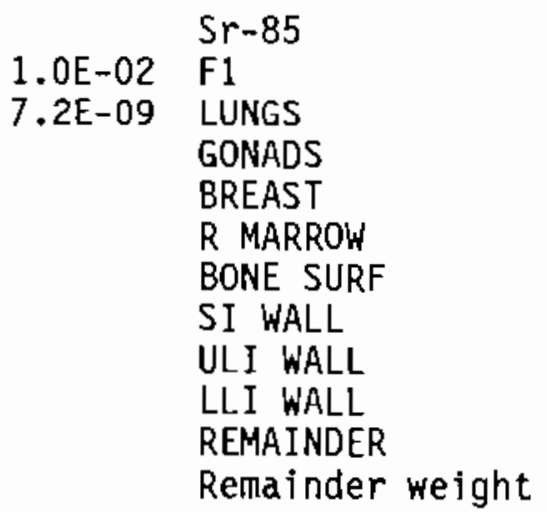
1. $0 \mathrm{E}-02$
$5 r-89$
$F 1$
8.4E-08 LUNGS
SR 89
4
3. $0 \mathrm{E}-01 \quad 1.0 \mathrm{E}-02 \quad 3.0 \mathrm{E}-01$
2.2E-09 


\begin{tabular}{|c|c|c|c|c|}
\hline $\begin{array}{r}1 \\
3 \\
6 \\
9 \\
10\end{array}$ & $\begin{array}{l}3.2 \mathrm{E}-09 \\
4.8 \mathrm{E}-09 \\
7.3 \mathrm{E}-09 \\
2.1 \mathrm{E}-08\end{array}$ & $\begin{array}{l}1.0 \mathrm{E}-08 \\
2.9 \mathrm{E}-08\end{array}$ & $\begin{array}{l}4.2 \mathrm{E}-10 \\
5.6 \mathrm{E}-09 \\
8.4 \mathrm{E}-09 \\
1.5 \mathrm{E}-09 \\
3.6 \mathrm{E}-09\end{array}$ & $\begin{array}{l}\text { GONADS } \\
\text { R MARROW } \\
\text { BONE SURF } \\
\text { ULI WALL } \\
\text { LLI WALL }\end{array}$ \\
\hline
\end{tabular}

\begin{tabular}{rrrr} 
SR 90 & \multicolumn{3}{c}{5} \\
& $3.0 \mathrm{E}-01$ & $1.0 \mathrm{E}-02$ & $3.0 \mathrm{E}-01$ \\
4 & & & \\
3 & $1.9 \mathrm{E}-07$ & $6.4 \mathrm{E}-09$ & $3.3 \mathrm{E}-07$ \\
6 & $4.2 \mathrm{E}-07$ & $1.4 \mathrm{E}-08$ & $7.3 \mathrm{E}-07$ \\
9 & & $6.1 \mathrm{E}-09$ & \\
10 & & $2.6 \mathrm{E}-08$ &
\end{tabular}

$\begin{aligned} 1.0 E-02 & \text { Sr-90 } \\ 2.9 E-06 & \text { LUNGS } \\ & \text { R MARROW } \\ & \text { BONE SURF } \\ & \text { ULI WALL } \\ & \text { LLI WALL }\end{aligned}$

\begin{tabular}{rrrr} 
SR & 91 & \multicolumn{3}{l}{7} \\
& $3.0 \mathrm{E}-01$ & $1.0 \mathrm{E}-02$ & $3.0 \mathrm{E}-01$ \\
4 & & & $9.2 \mathrm{E}-10$ \\
1 & $2.1 \mathrm{E}-10$ & $2.5 \mathrm{E}-10$ & $6.4 \mathrm{E}-11$ \\
3 & & & $1.2 \mathrm{E}-10$ \\
7 & $8.6 \mathrm{E}-10$ & $8.5 \mathrm{E}-10$ & \\
8 & $1.4 \mathrm{E}-09$ & $1.8 \mathrm{E}-09$ & $2.4 \mathrm{E}-10$ \\
9 & $3.7 \mathrm{E}-09$ & $4.9 \mathrm{E}-09$ & $5.9 \mathrm{E}-10$ \\
10 & $3.9 \mathrm{E}-09$ & $5.1 \mathrm{E}-09$ & $6.1 \mathrm{E}-10$
\end{tabular}

$\begin{aligned} 1.0 \mathrm{E}-02 & \text { Sr-91 } \\ 2.1 \mathrm{E}-09 & \text { LUNGS } \\ & \text { GONADS } \\ & \text { R MARROW } \\ & \text { ST WALL } \\ & \text { SI WALL } \\ 1.1 \mathrm{E}-09 & \text { ULI WALL } \\ 1.2 \mathrm{E}-09 & \text { LLI WALL }\end{aligned}$

\begin{tabular}{rrrrrl} 
SR & 92 & \multicolumn{2}{c}{6} & & \\
& $3.0 \mathrm{E}-01$ & $1.0 \mathrm{E}-02$ & $3.0 \mathrm{E}-01$ & & \\
4 & & & $7.1 \mathrm{E}-10$ & $1.0 \mathrm{E}-02$ & F1 \\
1 & $8.0 \mathrm{E}-11$ & & & $1.1 \mathrm{E}-09$ & LUNGS \\
7 & $5.3 \mathrm{E}-10$ & $5.2 \mathrm{E}-10$ & & & GONADS \\
8 & $1.1 \mathrm{E}-09$ & $1.4 \mathrm{E}-09$ & $1.9 \mathrm{E}-10$ & & ST WALL \\
9 & $3.0 \mathrm{E}-09$ & $3.9 \mathrm{E}-09$ & $4.7 \mathrm{E}-10$ & $2.8 \mathrm{E}-10$ & SI WALL \\
10 & $2.1 \mathrm{E}-09$ & $2.7 \mathrm{E}-09$ & $3.3 \mathrm{E}-10$ & $6.1 \mathrm{E}-10$ & ULI WALL \\
& & & & $3.8 \mathrm{E}-10$ & LLI WALL
\end{tabular}

Y 89M 0

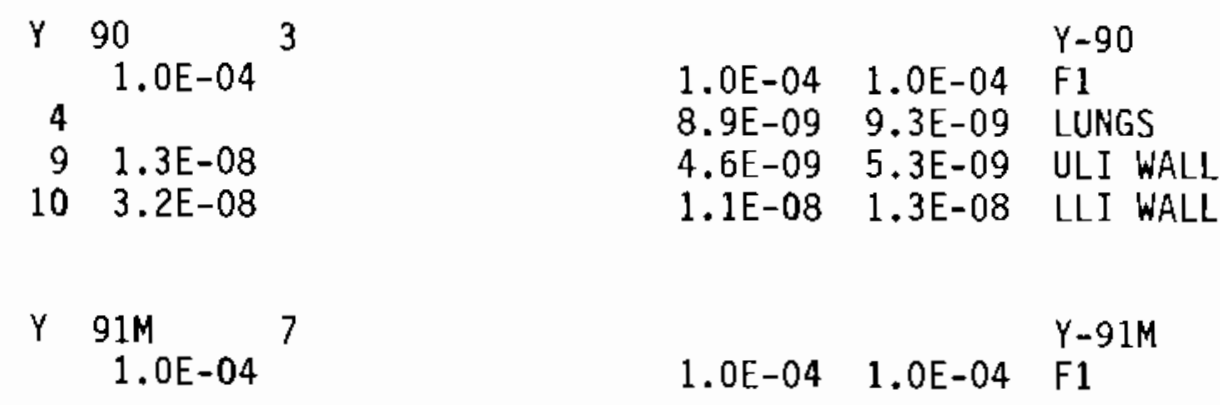

B. 140 


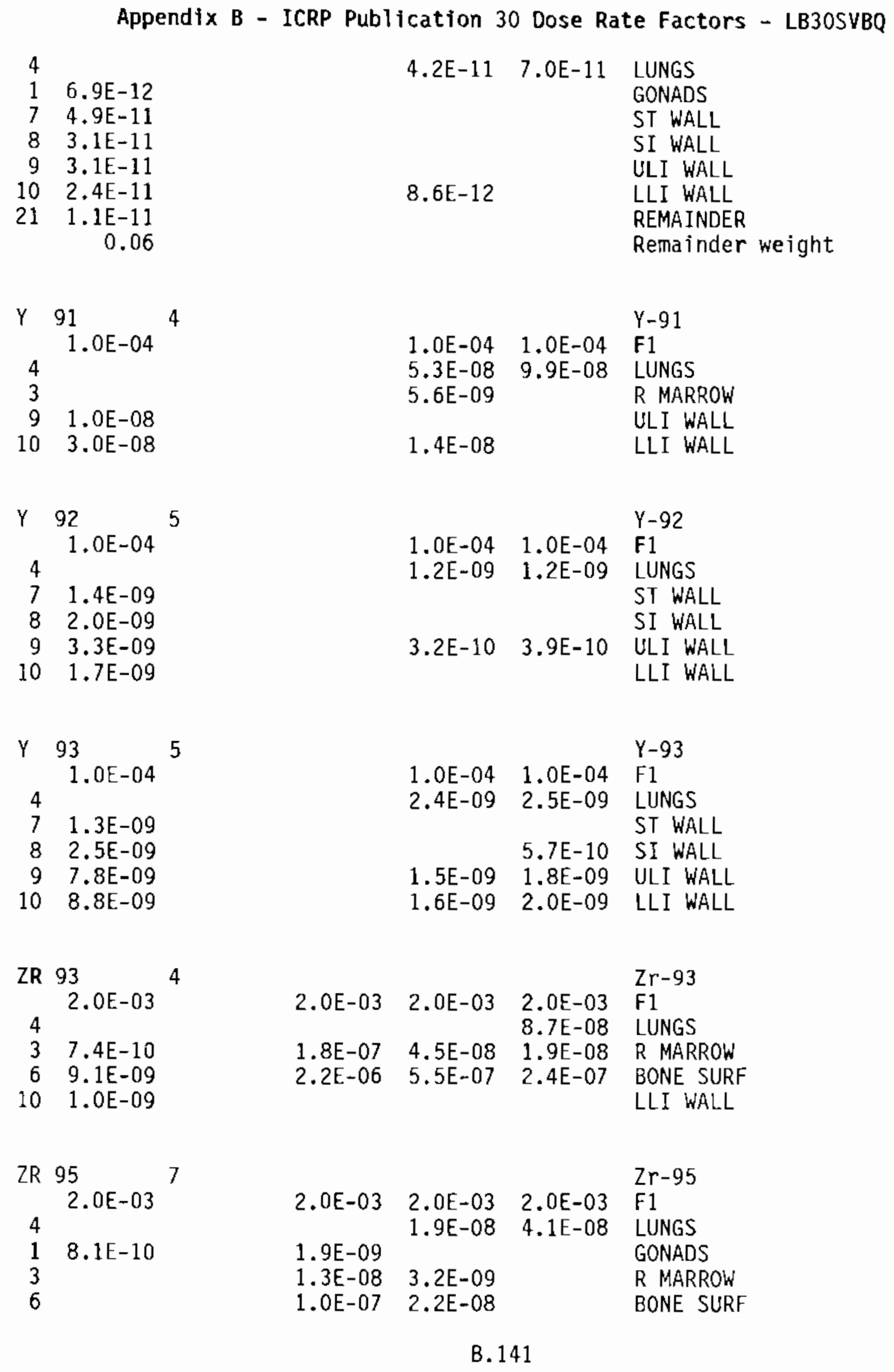




\section{Appendix B - ICRP Publication 30 Dose Rate Factors - LB30SVBQ}

$$
\begin{array}{rr}
8 & 1.1 \mathrm{E}-09 \\
9 & 3.0 \mathrm{E}-09 \\
10 & 7.8 \mathrm{E}-09
\end{array}
$$

2. $0 \mathrm{E}-03$

2. $1 \mathrm{E}-09$

1. $8 \mathrm{E}-10$

5. $0 \mathrm{E}-10$

$6.1 \mathrm{E}-10$

1.9E-09

2. $8 \mathrm{E}-09$
SI WALL

ULI WALL

4.2E-09

LLI WALL

2.0E-03

2.0E-03

4.1E-09

4. $0 \mathrm{E}-09$

4.1E-09

8.9E-10 1.0E-09

$2.9 E-09 \quad 3.5 E-09$

4.3E-09 5.1E-09

2r-97

$F 1$

LUNGS

GONADS

R MARROW

SI WALL

ULI WALL

LL.I WALL.

$N b-93 M$
$1.0 \mathrm{E}-02$

4. $9 \mathrm{E}-09$

4. $2 \mathrm{E}-10$

$8.8 \mathrm{E}-10$

$1.0 \mathrm{E}-08$

1. $0 \mathrm{E}-02$

$6.4 \mathrm{E}-08$

LUNGS

GONADS

R MARROW

BONE SURF

ULI WALL

LLI WALL

Nb-94
1.0E-02 1.0E-02

4.2E-08 7.5E-07

4. $8 \mathrm{E}-09$

$6.3 \mathrm{E}-09$

F1

LUNGS

GONADS

$R$ MARROW

SI WALL

ULI WALL

LLI WALL

$\mathrm{Nb}-95 \mathrm{M}$

1. $0 \mathrm{E}-02$

1.0E-02

2.6E-09 3.1E-09 LUNGS

$9.5 \mathrm{E}-10 \quad 1.1 \mathrm{E}-09$ ULI WALL

2.4E-09 2.7E-09 LLI WALL

$F 1$

$$
\begin{array}{cc}
\text { N8 } & 95 \\
& 1.0 \mathrm{E}-02 \\
4 & \\
1 & 8.0 \mathrm{E}-10 \\
3 & \\
6 & \\
8 & 9.1 \mathrm{E}-10 \\
9 & 1.8 \mathrm{E}-09
\end{array}
$$

$\mathrm{Nb}-95$

$\mathrm{F} 1$

LUNGS

GONADS

R MARROW

BONE SURF

SI WALL

ULI WALL 


\section{Appendix B - ICRP Publication 30 Dose Rate Factors - LB30SVBQ}

10

4.0E-09

NB 97M $\quad 0$

1.9E-09 1.9E-09 LLI WALL

$$
\begin{array}{rc}
\text { NB } & 97 \\
& 1.0 \mathrm{E}-02 \\
4 & \\
1 & 1.5 \mathrm{E}-11 \\
7 & 3.9 \mathrm{E}-10 \\
8 & 3.0 \mathrm{E}-10 \\
9 & 2.2 \mathrm{E}-10 \\
10 & 4.7 \mathrm{E}-11
\end{array}
$$

MO $93 \quad 10$

$\begin{array}{rccc} & 8.0 \mathrm{E}-01 & 5.0 \mathrm{E}-02 & 8.0 \mathrm{E}-01 \\ 4 & 1.1 \mathrm{E}-10 & & 1.2 \mathrm{E}-10 \\ 1 & 1.3 \mathrm{E}-10 & 2.5 \mathrm{E}-11 & 9.3 \mathrm{E}-11 \\ 2 & 1.0 \mathrm{E}-10 & & 7.5 \mathrm{E}-11 \\ 3 & 2.8 \mathrm{E}-10 & & 2.1 \mathrm{E}-10 \\ 6 & 1.1 \mathrm{E}-09 & & 3.6 \mathrm{E}-10 \\ 12 & 1.8 \mathrm{E}-09 & 1.1 \mathrm{E}-10 & 1.3 \mathrm{E}-09 \\ 11 & 1.6 \mathrm{E}-09 & 1.0 \mathrm{E}-10 & 1.2 \mathrm{E}-09 \\ 8 & & 4.7 \mathrm{E}-11 & \\ 9 & & 1.7 \mathrm{E}-10 & \\ 10 & 1.8 \mathrm{E}-10 & 4.4 \mathrm{E}-10 & \end{array}$

MO $99 \quad 10$

$\begin{array}{rrrr} & 8.0 \mathrm{E}-01 & 5.0 \mathrm{E}-02 & 8.0 \mathrm{E}-01 \\ 4 & & & 1.2 \mathrm{E}-09 \\ 1 & 2.2 \mathrm{E}-10 & & 1.3 \mathrm{E}-10 \\ 2 & 1.8 \mathrm{E}-10 & & 1.3 \mathrm{E}-10 \\ 3 & 5.3 \mathrm{E}-10 & & 3.7 \mathrm{E}-10 \\ 6 & 7.7 \mathrm{E}-10 & & 5.4 \mathrm{E}-10 \\ 12 & 2.7 \mathrm{E}-09 & & 1.9 \mathrm{E}-09 \\ 11 & 2.5 \mathrm{E}-09 & & 1.7 \mathrm{E}-09 \\ 7 & 6.7 \mathrm{E}-10 & & \\ 9 & 1.4 \mathrm{E}-09 & 5.7 \mathrm{E}-09 & 3.3 \mathrm{E}-10 \\ 10 & 3.1 \mathrm{E}-09 & 1.4 \mathrm{E}-08 & 5.8 \mathrm{E}-10\end{array}$
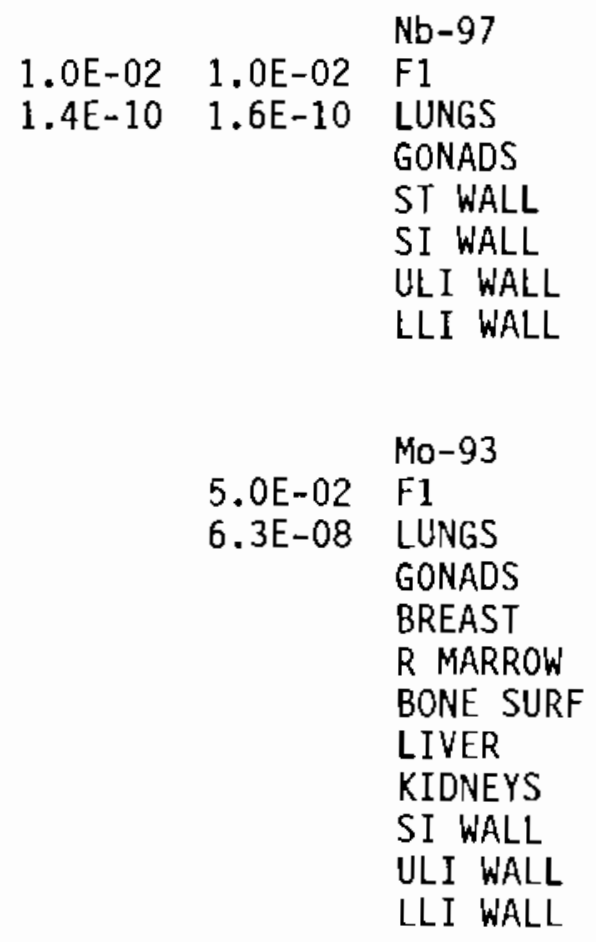

Mo-99

5.0E-02 F1

4.3E-09 LUNGS

GONADS

BREAST

R MARROW

BONE SURF

LIVER

KIDNEYS

ST WALL

2.3E-09 ULI WALL

5.5E-09 LLI WALL

TC-99M

F1

LUNGS

GONADS

BREAST 


\begin{tabular}{rrrr}
\multicolumn{4}{c}{ Appendix B - ICRP Publication 30} \\
3 & $6.3 \mathrm{E}-12$ & $3.4 \mathrm{E}-12$ & \\
5 & $8.5 \mathrm{E}-11$ & $5.0 \mathrm{E}-11$ & $2.1 \mathrm{E}-11$ \\
7 & $7.2 \mathrm{E}-11$ & $2.9 \mathrm{E}-11$ & $1.5 \mathrm{E}-11$ \\
8 & $2.2 \mathrm{E}-11$ & $4.8 \mathrm{E}-12$ & \\
9 & $3.7 \mathrm{E}-11$ & $7.0 \mathrm{E}-12$ & \\
10 & $2.5 \mathrm{E}-11$ & $5.0 \mathrm{E}-12$ & \\
21 & $1.1 \mathrm{E}-11$ & $5.0 \mathrm{E}-12$ & \\
& 0.06 & 0.06 &
\end{tabular}

$\begin{array}{rc}\text { TC } & 99 \\ & 8.0 \mathrm{E}-01 \\ 4 & 5 \\ 5 & 1.6 \mathrm{E}-09 \\ 7 & 3.4 \mathrm{E}-09 \\ 9 & 4.1 \mathrm{E}-10 \\ 10 & 1.1 \mathrm{E}-09\end{array}$

$$
\begin{array}{ll}
8.0 \mathrm{E}-01 & 8.0 \mathrm{E}-01 \\
3.5 \mathrm{E}-10 & 1.7 \mathrm{E}-08 \\
1.2 \mathrm{E}-09 & \\
2.5 \mathrm{E}-09 &
\end{array}
$$

R MARROW

THYROID

ST WALL

SI WALL

ULI WALL

LLI WALL

REMAINDER

Remainder weight

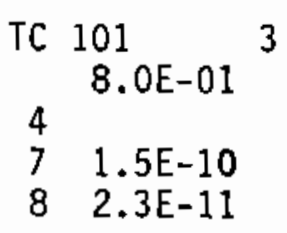

$$
\begin{array}{ll}
8.0 \mathrm{E}-01 & 8.0 \mathrm{E}-01 \\
2.8 \mathrm{E}-11 & 3.0 \mathrm{E}-11 \\
1.4 \mathrm{E}-11 &
\end{array}
$$

Tc-99

F1

LUNGS

THYROID

ST WALL

ULI WALL

LLI WALL

$$
\begin{array}{rl}
\text { RU } & 103 \\
& 5.0 E-02 \\
4 & \\
1 & 5.7 E-10 \\
2 & \\
3 & \\
6 & \\
7 & \\
8 & 8.5 E-10 \\
9 & 2.5 E-09 \\
10 & 6.5 E-09 \\
21 &
\end{array}
$$$$
\text { 5.0E-02 }
$$$$
\text { 5.0E-02 }
$$$$
5.0 \mathrm{E}-02
$$$$
\text { 1. } 0 \mathrm{E}-09
$$$$
7.3 \mathrm{E}-10
$$$$
6.1 \mathrm{E}-10
$$$$
6.7 E-10
$$$$
6.2 \mathrm{E}-10
$$$$
\text { 7. } 2 \mathrm{E}-10
$$$$
\text { 8. } 4 \mathrm{E}-10
$$$$
1.1 \mathrm{E}-09
$$$$
\text { 1.7E-09 }
$$$$
9.9 \mathrm{E}-09 \quad 1.6 \mathrm{E}-08
$$

Tc-101

F1

LUNGS

ST WALL

SI WALL

$$
\begin{array}{r}
\text { 8. } 1 E-10 \\
0.06
\end{array}
$$

$\mathrm{Ru}-103$

$F 1$

LUNGS

GONADS

BREAST

R MARROW

BONE SURF

ST WALL

SI WALL

ULI WALL

3.1E-09

3.1E-09

LLI WALL

REMAINDER

Remainder weight

RU 1056

5. OE-02

$3.7 \mathrm{E}-10$

2.7E-11

8.9E-11

1.3E-10

2. $5 \mathrm{E}-10$

$5.0 E-02$

$5.4 E-10$

5.0E-02

Ru-105

F1

LUNGS

GONADS

7 5.0E-10

$8 \quad 7.9 \mathrm{E}-10$

$9 \quad 1.6 \mathrm{E}-09$

$10 \quad 1.3 E-09$

$2.1 \mathrm{E}-10$

\subsection{E-10}

1.2E-10 SI WALL

$2.6 E-10$ 2.8E-10 ULI WALL 


\section{Appendix B - ICRP Publication 30 Dose Rate Factors - LB30SVBQ}

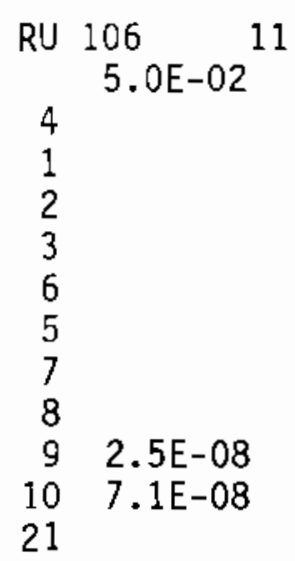

RH $\underset{5.0 \mathrm{E}-02}{103 \mathrm{M}} 4$

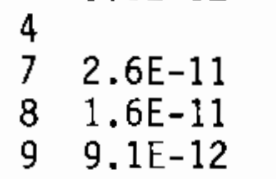

$\begin{array}{lll}5.0 \mathrm{E}-02 & 5.0 \mathrm{E}-02 & 5.0 \mathrm{E}-02 \\ 1.8 \mathrm{E}-08 & 2.1 \mathrm{E}-07 & 1.0 \mathrm{E}-06 \\ 1.4 \mathrm{E}-08 & & \\ 1.4 \mathrm{E}-08 & & \\ 1.4 \mathrm{E}-08 & & \\ 1.4 \mathrm{E}-08 & & \\ 1.4 \mathrm{E}-08 & & \\ 1.4 \mathrm{E}-08 & & \\ 1.5 \mathrm{E}-08 & & \\ 1.7 \mathrm{E}-08 & & \\ 2.5 \mathrm{E}-08 & & \\ 1.4 \mathrm{E}-08 & & \\ 0.06 & & \end{array}$

$\mathrm{Ru}-106$

F1

LUNGS

GONADS

BREAST

R MARROW

BONE SURF

THYROID

ST WALL

SI WALL

ULI WALL

LLI WALL

REMAINDER

Remainder weight

$\mathrm{Rh}-103 \mathrm{M}$

F1

LUNGS

ST WALL

SI WALL

ULI WALL

$R h-105$

F1

LUNGS

GONADS

SI WALL

ULI WALL

LLI WALL

RH 105M 0

RH $106 \quad 0$

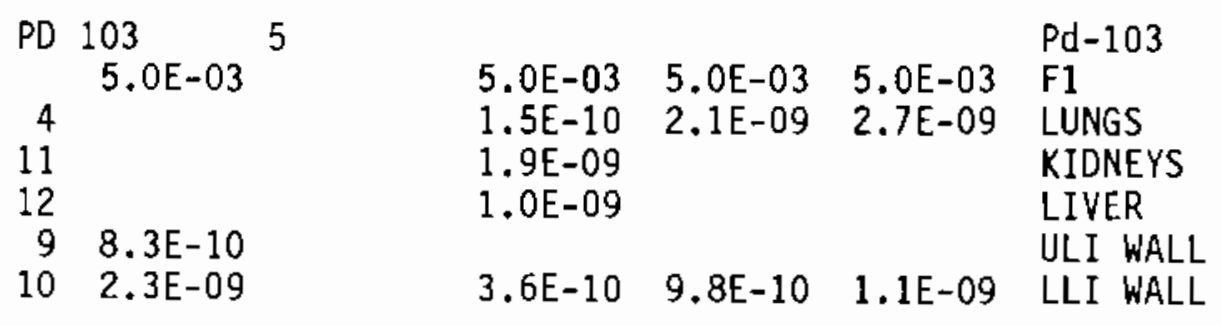




\section{Appendix B - ICRP Publication 30 Dose Rate Factors - LB30SVBQ}

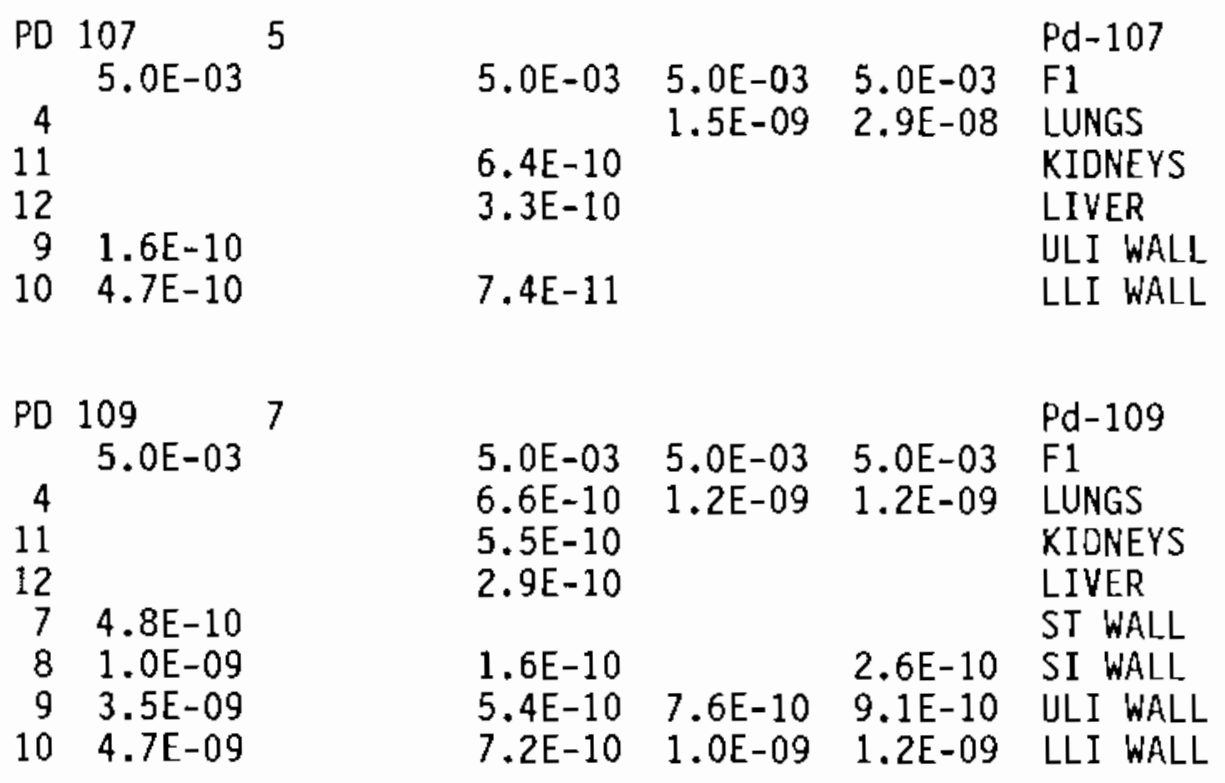

PD $109 \mathrm{M} \quad 0$

AG $109 \mathrm{M} \quad 0$

AG $110 \quad 0$

$$
\begin{array}{rl}
\text { AG } & 110 \mathrm{M} \\
& 5.0 \mathrm{E}-02 \\
4 & 8.3 \mathrm{E}-10 \\
1 & 3.0 \mathrm{E}-09 \\
2 & 7.5 \mathrm{E}-10 \\
3 & 9.4 \mathrm{E}-10 \\
12 & 8.6 \mathrm{E}-09 \\
8 & 3.5 \mathrm{E}-09 \\
9 & 5.9 \mathrm{E}-09 \\
10 & 1.1 \mathrm{E}-08 \\
21 & 1.6 \mathrm{E}-09 \\
& 0.06
\end{array}
$$

$5.0 \mathrm{E}-02$

$8.1 \mathrm{E}-09$

$3.3 \mathrm{E}-09$

4.1E-09

$5.0 \mathrm{E}-02$

$3.2 \mathrm{E}-08$

2.3E-09

2. $9 \mathrm{E}-09$

8.1E-08 2.6E-08

$8.4 \mathrm{E}-09$

$\begin{array}{rr}1.4 \mathrm{E}-08 & 6.9 \mathrm{E}-09 \\ 0.18 & 0.12\end{array}$

$5.0 \mathrm{E}-02$
$1.2 \mathrm{E}-07$

$\mathrm{Ag}-110 \mathrm{M}$

$\mathrm{F} 1$

LUNGS

GONADS

BREAST

R MARROW

LIVER

SI WALL

ULI WALL

LLI WALL

REMAINDER

Remainder weight
AG $111 \quad 4$
4
$5.0 \mathrm{E}-02$

$5.0 \mathrm{E}-02$

$5.0 \mathrm{E}-02$

5.0E-02

Ag-111

F1

$1.1 \mathrm{E}-09$

$7.8 \mathrm{E}-09$

8.7E-09

LUNGS

\section{B. 146}




\section{Appendix B - ICRP Publication 30 Dose Rate Factors - LB3OSVBQ}

\begin{tabular}{|c|c|c|c|c|c|}
\hline & $\begin{array}{l}5.4 \mathrm{E}-09 \\
1.5 \mathrm{E}-08\end{array}$ & $\begin{array}{l}8.7 \mathrm{E}-09 \\
9.1 \mathrm{E}-10 \\
2.3 \mathrm{E}-09\end{array}$ & $\begin{array}{l}1.8 \mathrm{E}-09 \\
2.1 \mathrm{E}-09 \\
5.8 \mathrm{E}-09\end{array}$ & $\begin{array}{l}2.4 \mathrm{E}-09 \\
6.6 \mathrm{E}-09\end{array}$ & $\begin{array}{l}\text { LIVER } \\
\text { ULI WALL } \\
\text { LLI WALL }\end{array}$ \\
\hline 1 & $\begin{array}{l}109 \\
5.0 \mathrm{E}-02 \\
4.1 \mathrm{E}-08 \\
7.4 \mathrm{E}-09 \\
4.6 \mathrm{E}-09\end{array}$ & $\begin{array}{l}5.0 \mathrm{E}-02 \\
3.9 \mathrm{E}-07 \\
7.1 \mathrm{E}-08\end{array}$ & $\begin{array}{l}5.0 \mathrm{E}-02 \\
1.5 \mathrm{E}-08 \\
1.1 \mathrm{E}-07 \\
2.1 \mathrm{E}-08\end{array}$ & $\begin{array}{l}5.0 \mathrm{E}-02 \\
7.8 \mathrm{E}-08 \\
3.4 \mathrm{E}-08\end{array}$ & $\begin{array}{l}\text { Cd-109 } \\
\text { FI } \\
\text { LUNGS } \\
\text { KIDNEYS } \\
\text { LIVER } \\
\text { LLI WALL }\end{array}$ \\
\hline 4 & $\begin{array}{c}113 \mathrm{M} \\
5.0 \mathrm{E}-02 \\
5.6 \mathrm{E}-07 \\
9.7 \mathrm{E}-08\end{array}$ & $\begin{array}{l}5.0 \mathrm{E}-02 \\
5.5 \mathrm{E}-06 \\
9.4 \mathrm{E}-07\end{array}$ & $\begin{array}{l}5.0 \mathrm{E}-02 \\
1.6 \mathrm{E}-06 \\
2.8 \mathrm{E}-07\end{array}$ & $\begin{array}{l}5.0 \mathrm{E}-02 \\
4.1 \mathrm{E}-07 \\
7.8 \mathrm{E}-07 \\
1.3 \mathrm{E}-07\end{array}$ & $\begin{array}{l}\text { Cd-113M } \\
\text { F1 } \\
\text { LUNGS } \\
\text { KIDNEYS } \\
\text { LIVER }\end{array}$ \\
\hline & $\begin{array}{l}115 \mathrm{M} \\
5.0 \mathrm{E}-02 \\
\\
2.6 \mathrm{E}-08 \\
4.5 \mathrm{E}-09 \\
9.9 \mathrm{E}-09 \\
2.9 \mathrm{E}-08\end{array}$ & $\begin{array}{l}5.0 \mathrm{E}-02 \\
2.5 \mathrm{E}-07 \\
4.3 \mathrm{E}-08\end{array}$ & $\begin{array}{l}5.0 \mathrm{E}-02 \\
4.7 \mathrm{E}-08 \\
5.9 \mathrm{E}-08 \\
1.0 \mathrm{E}-08 \\
1.3 \mathrm{E}-08\end{array}$ & $\begin{array}{l}5.0 \mathrm{E}-02 \\
7.8 \mathrm{E}-08\end{array}$ & $\begin{array}{l}\text { Cd-115M } \\
\text { F1 } \\
\text { LUNGS } \\
\text { KIDNEYS } \\
\text { LIVER } \\
\text { ULI WALL } \\
\text { LLI WALL }\end{array}$ \\
\hline 2 & $\begin{array}{l}6.2 E-09 \\
1.5 E-08\end{array}$ & $\begin{array}{l}5.0 \mathrm{E}-02 \\
1.2 \mathrm{E}-09 \\
8.7 \mathrm{E}-09 \\
1.7 \mathrm{E}-09 \\
1.1 \mathrm{E}-09 \\
2.4 \mathrm{E}-09\end{array}$ & $\begin{array}{l}5.0 \mathrm{E}-02 \\
4.1 \mathrm{E}-09 \\
1.8 \mathrm{E}-09 \\
2.2 \mathrm{E}-09 \\
5.1 \mathrm{E}-09\end{array}$ & $\begin{array}{l}5.0 \mathrm{E}-02 \\
4.2 \mathrm{E}-09 \\
2.6 \mathrm{E}-09 \\
5.9 \mathrm{E}-09\end{array}$ & $\begin{array}{l}\text { Cd-115 } \\
\text { F1 } \\
\text { LUNGS } \\
\text { KIDNEYS } \\
\text { LIVER } \\
\text { ULI WALL } \\
\text { LLI WALL }\end{array}$ \\
\hline 3 & $\begin{array}{l}111 \\
2.0 \mathrm{E}-02 \\
4.2 \mathrm{E}-10 \\
1.1 \mathrm{E}-10\end{array}$ & $\begin{array}{l}2.0 \mathrm{E}-02 \\
2.2 \mathrm{E}-10 \\
1.3 \mathrm{E}-10 \\
6.4 \mathrm{E}-11 \\
3.3 \mathrm{E}-10 \\
1.7 \mathrm{E}-10 \\
4.6 \mathrm{E}-10 \\
3.3 \mathrm{E}-10 \\
1.7 \mathrm{E}-10 \\
2.4 \mathrm{E}-10 \\
3.8 \mathrm{E}-10\end{array}$ & $\begin{array}{l}2.0 \mathrm{E}-02 \\
6.2 \mathrm{E}-10 \\
1.6 \mathrm{E}-10 \\
1.1 \mathrm{E}-10\end{array}$ & & $\begin{array}{l}\text { In-111 } \\
\text { Fl } \\
\text { LUNGS } \\
\text { GONADS } \\
\text { BREAST } \\
\text { R MARROW } \\
\text { BONE SURF } \\
\text { KIDNEYS } \\
\text { LIVER } \\
\text { SPLEEN } \\
\text { SI WALL } \\
\text { UL I WALL } \\
\text { LLI WALL }\end{array}$ \\
\hline
\end{tabular}


IN $114 \quad 0$

$\begin{array}{rllll}\text { IN } \begin{array}{c}114 \mathrm{M} \\ 2.0 \mathrm{E}-02\end{array} & 2.0 \mathrm{E}-02 & 2.0 \mathrm{E}-02 & \text { In-114M } \\ 4 & & & 7.2 \mathrm{E}-08 & \text { F1 } \\ 3 & 3.5 \mathrm{E}-09 & 8.3 \mathrm{E}-08 & 1.8 \mathrm{E}-08 & \text { LUNGS } \\ 6 & & 4.3 \mathrm{E}-08 & & \text { R MARROW } \\ 11 & & 9.5 \mathrm{E}-08 & 2.0 \mathrm{E}-08 & \text { BONE SURF } \\ 12 & & 4.7 \mathrm{E}-08 & & \text { KIDNEYS } \\ 13 & & 2.3 \mathrm{E}-08 & & \text { LIVER } \\ 9 & 1.5 \mathrm{E}-08 & & & \text { SPLEEN } \\ 10 & 4.4 \mathrm{E}-08 & & \text { ULI WALL } \\ & & & 2.0 \mathrm{E}-08 & \text { LLI WALL }\end{array}$

$\begin{array}{rllll}\text { IN } & 115 \mathrm{M} & 7 & & \\ & 2.0 \mathrm{E}-02 & 2.0 \mathrm{E}-02 & 2.0 \mathrm{E}-02 & \text { In-115M } \\ 4 & & 1.4 \mathrm{E}-10 & 1.8 \mathrm{E}-10 & \text { F1 } \\ 1 & 2.2 \mathrm{E}-11 & & & \text { LUNGS } \\ 3 & & 1.9 \mathrm{E}-11 & & \text { GONADS } \\ 7 & 1.9 \mathrm{E}-10 & 3.2 \mathrm{E}-11 & & \text { R MARROW } \\ 8 & 3.0 \mathrm{E}-10 & 4.7 \mathrm{E}-11 & & \text { ST WALL } \\ 9 & 5.7 \mathrm{E}-10 & 8.6 \mathrm{E}-11 & 6.6 \mathrm{E}-11 & \text { SI WALL } \\ 10 & 3.6 \mathrm{E}-10 & 5.4 \mathrm{E}-11 & 4.1 \mathrm{E}-11 & \text { ULI WALL } \\ & & & \text { LLI WALL }\end{array}$

$\begin{array}{rllll}\text { IN } 115 & 5 & & \\ & 2.0 \mathrm{E}-02 & 2.0 \mathrm{E}-02 & 2.0 \mathrm{E}-02 & \text { In-115 } \\ 3 & 1.5 \mathrm{E}-07 & 3.7 \mathrm{E}-06 & 1.0 \mathrm{E}-06 & \text { F1 } \\ 6 & 7.9 \mathrm{E}-08 & 1.9 \mathrm{E}-06 & 5.1 \mathrm{E}-07 & \text { R MARROW } \\ 11 & 1.7 \mathrm{E}-07 & 4.1 \mathrm{E}-06 & 1.1 \mathrm{E}-06 & \text { BONE SURF } \\ 12 & 8.5 \mathrm{E}-08 & 2.0 \mathrm{E}-06 & 5.5 \mathrm{E}-07 & \text { KIDNEYS } \\ 13 & 4.3 \mathrm{E}-08 & 1.0 \mathrm{E}-06 & 2.8 \mathrm{E}-07 & \text { LIVER } \\ & & & \text { SPLEEN }\end{array}$

$\begin{array}{rllll}\text { SN } 117 M & 6 & & \text { Sn-117M } \\ & 2.0 \mathrm{E}-02 & 2.0 \mathrm{E}-02 & 2.0 \mathrm{E}-02 & \text { F1 } \\ 4 & & 5.8 \mathrm{E}-10 & 6.1 \mathrm{E}-09 & \text { LUNGS } \\ 1 & 2.2 \mathrm{E}-10 & & & \text { GONAOS } \\ 3 & & 1.1 \mathrm{E}-09 & & \text { R MARROW } \\ 6 & & 1.1 \mathrm{E}-08 & & \text { BONE SURF } \\ 9 & 2.9 \mathrm{E}-09 & & & \text { ULI WALL } \\ 10 & 7.9 \mathrm{E}-09 & 1.3 \mathrm{E}-09 & 3.3 \mathrm{E}-09 & \text { LLI WALL }\end{array}$

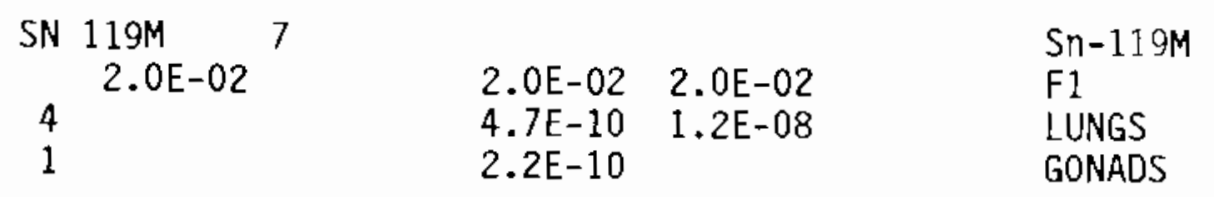




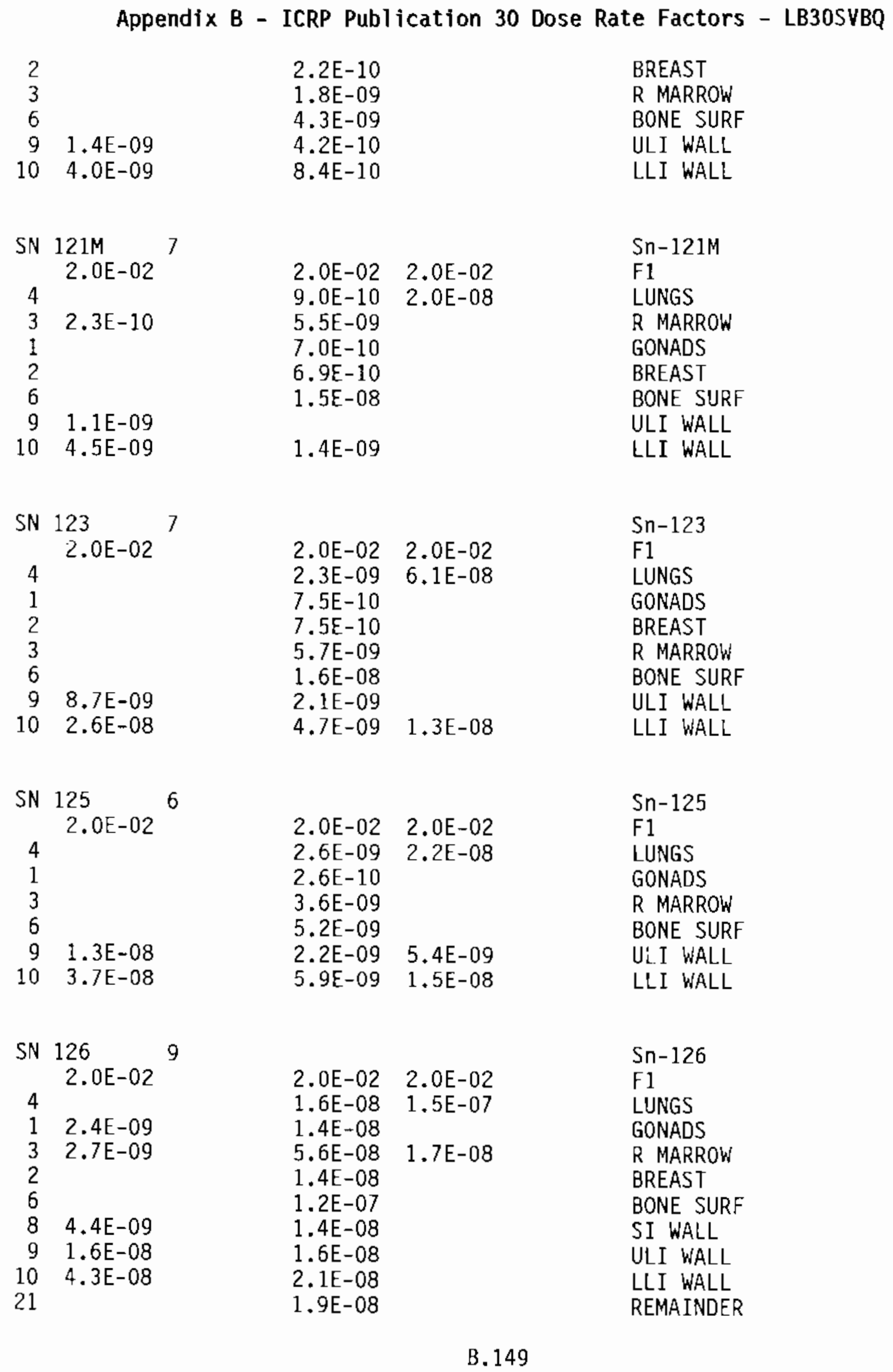




\section{Appendix B - ICRP Publication 30 Dose Rate Factors - LB3OSVBQ}

0.12

SB 124

$\begin{array}{rrrrr} & 1.0 \mathrm{E}-01 & 1.0 \mathrm{E}-02 & 1.0 \mathrm{E}-01 & 1.0 \mathrm{E}-02 \\ 4 & & & 2.0 \mathrm{E}-09 & 4.1 \mathrm{E}-08 \\ 1 & 1.7 \mathrm{E}-09 & 1.8 \mathrm{E}-09 & 9.1 \mathrm{E}-10 & \\ 2 & & & 6.5 \mathrm{E}-10 & \\ 3 & & & 1.5 \mathrm{E}-09 & \\ 6 & & & 3.4 \mathrm{E}-09 & \\ 12 & & & 2.5 \mathrm{E}-09 & \\ \mathrm{~B} & 2.7 \mathrm{E}-09 & 2.8 \mathrm{E}-09 & 1.1 \mathrm{E}-09 & \\ 9 & 8.2 \mathrm{E}-09 & 8.8 \mathrm{E}-09 & 1.9 \mathrm{E}-09 & \\ 10 & 2.1 \mathrm{E}-08 & 2.3 \mathrm{E}-08 & 4.0 \mathrm{E}-09 & 1.1 \mathrm{E}-08 \\ 21 & & & 9.9 \mathrm{E}-10 & \\ & & & 0.06 & \end{array}$

SB $125 \quad 10$

$\begin{array}{rrrrr} & 1.0 \mathrm{E}-01 & 1.0 \mathrm{E}-02 & 1.0 \mathrm{E}-01 & 1.0 \mathrm{E}-02 \\ 4 & & & 6.4 \mathrm{E}-10 & 2.2 \mathrm{E}-08 \\ 1 & 5.2 \mathrm{E}-10 & 5.3 \mathrm{E}-10 & 3.2 \mathrm{E}-10 & \\ 2 & & & 2.5 \mathrm{E}-10 & \\ 3 & & & 6.5 \mathrm{E}-10 & \\ 6 & & & 2.7 \mathrm{E}-09 & \\ 12 & & & 1.1 \mathrm{E}-09 \\ 8 & 7.6 \mathrm{E}-10 & 7.8 \mathrm{E}-10 & 3.9 \mathrm{E}-10 \\ 9 & 2.2 \mathrm{E}-09 & 2.4 \mathrm{E}-09 & 6.2 \mathrm{E}-10 \\ 10 & 5.8 \mathrm{E}-09 & 6.3 \mathrm{E}-09 & 1.1 \mathrm{E}-09 \\ 21 & & & 3.6 \mathrm{E}-10 \\ & & & 0.06\end{array}$

SB 126M 3

$\begin{array}{lllll} & 1.0 \mathrm{E}-01 & 1.0 \mathrm{E}-02 & 1.0 \mathrm{E}-01 & 1.0 \mathrm{E}-02 \\ 4 & & & 5.0 \mathrm{E}-11 & 5.6 \mathrm{E}-11 \\ 7 & 2.6 \mathrm{E}-10 & 2.6 \mathrm{E}-10 & 2.4 \mathrm{E}-11 & \\ 8 & 6.9 \mathrm{E}-11 & 7.0 \mathrm{E}-11 & & \end{array}$

SB $126 \quad 10$

$\begin{array}{rll} & 1.0 \mathrm{E}-01 & 1.0 \\ 1 & 2.7 \mathrm{E}-09 & 2.9 \\ 2 & & \\ 3 & & \\ 6 & & \\ 12 & & \\ 8 & 3.4 \mathrm{E}-09 & 3.6 \\ 9 & 7.8 \mathrm{E}-09 & 8.5\end{array}$

$1.0 \mathrm{E}-02$

$1.0 \mathrm{E} \sim 01$

$1.8 \mathrm{E}-09$

$1.0 \mathrm{E}-02$

$1.4 \mathrm{E}-08$

9. $1 \mathrm{E}-10$

5. $9 \mathrm{E}-10$

$1.1 \mathrm{E}-09$

$1.7 \mathrm{E}-09$

$1.9 \mathrm{E}-09$

$1.3 \mathrm{E}-09$

$1.1 \mathrm{E}-09$

3. $6 \mathrm{E}-09$

$1.8 \mathrm{E}-09$
Remainder weight

Sb-124

F1

LUNGS

GONADS

BREAST

$R$ MARROW

BONE SURF

LIVER

SI WALL

ULI WALL

LLI WALL

REMAINDER

Remainder weight

Sb-125

F1

LUNGS

GONADS

BREAST

$R$ MARROW

BONE SURF

LIVER

SI WALL

ULI WALL

LLI WALL

REMAINDER

Remainder weight

$\mathrm{Sb}-126 \mathrm{M}$

F1

LUNGS

ST WALL

SI WALL

Sb-126

F1

LUNGS

GONADS

BREAST

R MARROW

BONE SURF

LIVER

SI WALL

ULI WALL 


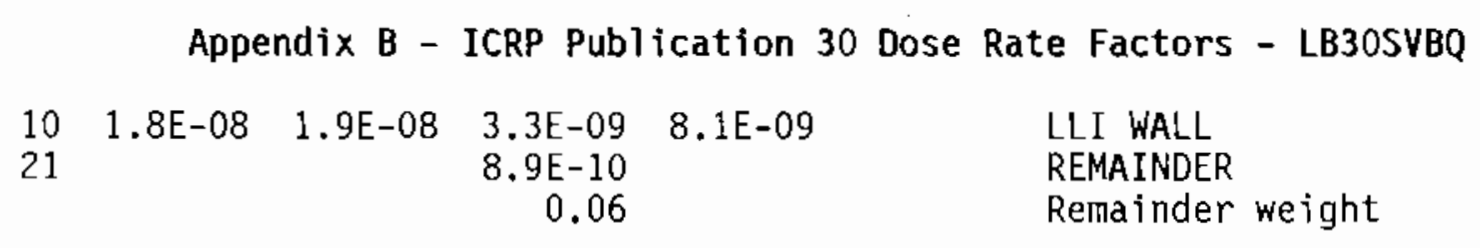

$\begin{array}{rlcll}\text { SB } & 127 & & & \\ & 1.0 \mathrm{E}-01 & 1.0 \mathrm{E}-02 & 1.0 \mathrm{E}-01 & 1.0 \mathrm{E}-02 \\ 4 & & & 1.4 \mathrm{E}-09 & 6.9 \mathrm{E}-09 \\ 1 & 5.9 \mathrm{E}-10 & 6.1 \mathrm{E}-10 & 2.3 \mathrm{E}-10 & \\ 2 & & & 1.6 \mathrm{E}-10 & \\ 3 & & & 4.9 \mathrm{E}-10 & \\ 12 & & & 7.4 \mathrm{E}-10 & \\ 8 & & & 3.9 \mathrm{E}-10 & \\ 9 & 6.7 \mathrm{E}-09 & 7.3 \mathrm{E}-09 & 1.2 \mathrm{E}-09 & 2.9 \mathrm{E}-09 \\ 10 & 1.8 \mathrm{E}-08 & 2.0 \mathrm{E}-08 & 2.9 \mathrm{E}-09 & 7.4 \mathrm{E}-09\end{array}$

Sb-127

F1

LUNGS

GONADS

BREAST

$R$ MARROW

LIVER

SI WALL

ULI WALL

LLI WALL

$\begin{array}{rrlll}\text { TE } & 123 M & 5 & & \text { Te-123M } \\ & 2.0 \mathrm{E}-01 & 2.0 \mathrm{E}-01 & 2.0 \mathrm{E}-01 & \text { F1 } \\ 4 & & & 1.3 \mathrm{E}-08 & \text { LUNGS } \\ 3 & 2.3 \mathrm{E}-09 & 5.8 \mathrm{E}-09 & 2.4 \mathrm{E}-09 & \text { R MARROW } \\ 6 & 2.4 \mathrm{E}-08 & 6.1 \mathrm{E}-08 & 2.4 \mathrm{E}-08 & \text { BONE SURF } \\ 9 & 1.7 \mathrm{E}-09 & & & \text { ULI WALL } \\ 10 & 4.5 \mathrm{E}-09 & & & \text { LLI WALL }\end{array}$

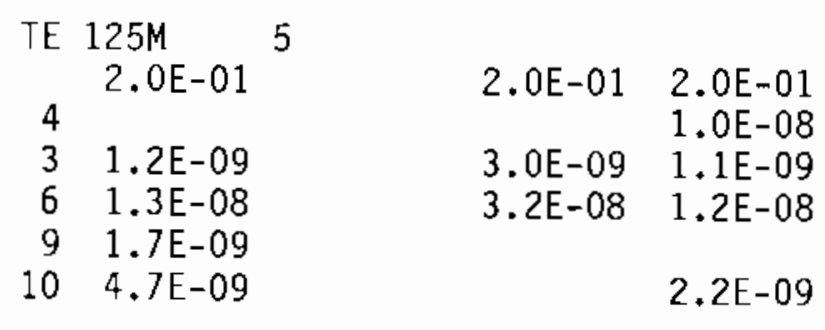

$\mathrm{Te}-125 \mathrm{M}$

F1

LUNGS

R MARROW

BONE SURF

ULI WALL

LLI WALL

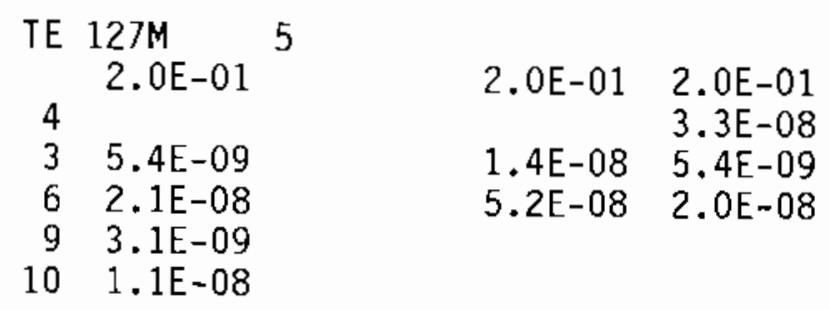

$\mathrm{Te}-127 \mathrm{M}$

F1

LUNGS

R MARROW

BONE SURF

ULI WALL

LLI WALL

$\begin{array}{ll}\text { TE } & 127 \\ & 2.0 \mathrm{E}-01 \\ 4 & \\ 7 & 2.4 \mathrm{E}-10 \\ 8 & 3.9 \mathrm{E}-10 \\ 9 & 1.2 \mathrm{E}-09\end{array}$

$\begin{array}{ll}2.0 \mathrm{E}-01 & 2.0 \mathrm{E}-01 \\ 2.8 \mathrm{E}-10 & 4.3 \mathrm{E}-10 \\ 6.4 \mathrm{E}-11 & \\ 1.8 \mathrm{E}-10 & 2.1 \mathrm{E}-10\end{array}$

Te-127

F1

LUNGS

ST WALL

SI WALL

ULI WALL 


\section{Appendix B - ICRP Publication 30 Dose Rate Factors - LB30SVBQ}

10

1. $3 \mathrm{E} \sim 09$

TE $129 \mathrm{M}$

$$
\text { 2. } 0 \mathrm{E}-01
$$

4

$3 \quad 3.5 \mathrm{E}-09$

$6 \quad 8.0 \mathrm{E}-09$

$9 \quad 8.4 \mathrm{E}-09$

$10 \quad 2.5 \mathrm{E}-08$

TE $129 \quad 4$

$\begin{array}{ll} & 2.0 \mathrm{E}-01 \\ 4 & \\ 7 & 4.0 \mathrm{E}-10 \\ 8 & 2.7 \mathrm{E}-10 \\ 9 & 1.9 \mathrm{E}-10\end{array}$

TE $131 \mathrm{M} 5$

$\begin{array}{rr}4 & \\ 1 & 7.3 \mathrm{E}-10 \\ 5 & 4.3 \mathrm{E}-08 \\ 9 & 4.6 \mathrm{E}-09 \\ 10 & 8.2 \mathrm{E}-09\end{array}$

TE $131 \quad 5$

$$
2.0 \mathrm{E}-01
$$

4

$5 \quad 4.2 \mathrm{E}-09$

$7 \quad 6.3 \mathrm{E}-10$

$8 \quad 5.5 \mathrm{E}-10$

$9 \quad 5.3 \mathrm{E}-10$
2.0E-01

2.2E-09

2. $0 \mathrm{E}-01$

$8.8 \mathrm{E}-09$

2. $0 \mathrm{E}-08$

$4.2 \mathrm{E}-09 \quad 1.1 \mathrm{E}-08$

2.0 $\mathrm{E}-01$

2.0E-01

$1.3 \mathrm{E}-10 \quad 1.5 \mathrm{E}-10$

5. $1 \mathrm{E}-11$

3. $6 \mathrm{E}-11$
LLI WALL

Te-129M

F1

LUNGS

R MARROW

80NE SURF

ULI WALL

LLI WALL

Te-129

F1

LUNGS

ST WALL

SI WALL

ULI WALL

$\mathrm{Te}-131 \mathrm{M}$

F1

LUNGS

GONADS

THYROID

ULI WALL

LLI WALL

Te-131

F1

LUNGS

THYROID

ST WALL

SI WALL

ULI WALL

Te-132

F1

LUNGS

THYROID

LLI WALL

$\mathrm{Te}-133 \mathrm{M}$

F1

LUNGS

THYRO ID

ST WALL 


\section{Appendix B - ICRP Publication 30 Dose Rate Factors - LB30SVBQ}

\begin{tabular}{|c|c|c|c|c|c|}
\hline $\begin{array}{l}8 \\
9\end{array}$ & $\begin{array}{l}4.1 \mathrm{E}-10 \\
2.7 \mathrm{E}-10\end{array}$ & & & & $\begin{array}{l}\text { SI WALL } \\
\text { ULI WALL }\end{array}$ \\
\hline $\begin{array}{l}\text { TE } \\
\\
4 \\
5 \\
7\end{array}$ & $\begin{array}{l}133 \\
2.0 \mathrm{E}-01 \\
9.4 \mathrm{E}-10 \\
2.4 \mathrm{E}-10\end{array}$ & 3 & $\begin{array}{l}2.0 \mathrm{E}-01 \\
4.4 \mathrm{E}-11 \\
5.9 \mathrm{E}-10\end{array}$ & $\begin{array}{l}2.0 \mathrm{E}-01 \\
4.6 \mathrm{E}-11 \\
5.9 \mathrm{E}-10\end{array}$ & $\begin{array}{l}\text { Te-133 } \\
\text { F1 } \\
\text { LUNGS } \\
\text { THYROID } \\
\text { ST WALL }\end{array}$ \\
\hline $\begin{array}{l}\text { TE } \\
\\
4 \\
1 \\
5 \\
7 \\
8 \\
9\end{array}$ & $\begin{array}{l}134 \\
2.0 \mathrm{E}-01 \\
2.0 \mathrm{E}-11 \\
8.8 \mathrm{E}-10 \\
2.3 \mathrm{E}-10 \\
1.2 \mathrm{E}-10 \\
7.3 \mathrm{E}-11\end{array}$ & 6 & $\begin{array}{l}2.0 \mathrm{E}-01 \\
6.0 \mathrm{E}-11 \\
8.9 \mathrm{E}-12 \\
5.5 \mathrm{E}-10 \\
3.4 \mathrm{E}-11\end{array}$ & $\begin{array}{l}2.0 \mathrm{E}-01 \\
6.6 \mathrm{E}-11 \\
7.5 \mathrm{E}-12 \\
5.6 \mathrm{E}-10\end{array}$ & $\begin{array}{l}\text { Te-134 } \\
\text { F1 } \\
\text { LUNGS } \\
\text { GONADS } \\
\text { THYROID } \\
\text { ST WALL } \\
\text { SI WALL } \\
\text { ULI WALL }\end{array}$ \\
\hline $\begin{array}{l}I \\
5\end{array}$ & $\begin{array}{l}125 \\
1.0 \mathrm{E}+00 \\
3.4 \mathrm{E}-07\end{array}$ & 1 & $\begin{array}{l}1.0 \mathrm{E}+00 \\
2.2 \mathrm{E}-07\end{array}$ & & $\begin{array}{l}\text { I-125 } \\
\text { F1 } \\
\text { THYROID }\end{array}$ \\
\hline $\begin{array}{l}\mathrm{I} \\
5\end{array}$ & $\begin{array}{l}129 \\
1.0 \mathrm{E}+00 \\
2.5 \mathrm{E}-06\end{array}$ & 1 & $\begin{array}{l}1.0 \mathrm{E}+00 \\
1.6 \mathrm{E}-06\end{array}$ & & $\begin{array}{l}\text { I-129 } \\
\text { F1 } \\
\text { THYROID }\end{array}$ \\
\hline $\begin{array}{l}\text { I } \\
\\
4 \\
5\end{array}$ & $\begin{array}{l}130 \\
1.0 E+00 \\
3.9 E-08\end{array}$ & 2 & $\begin{array}{l}1.0 E+00 \\
6.0 E-10 \\
2.0 E-08\end{array}$ & & $\begin{array}{l}\text { I-130 } \\
\text { F1 } \\
\text { LUNGS } \\
\text { THYROID }\end{array}$ \\
\hline $\begin{array}{l}\text { I } \\
5\end{array}$ & $\begin{array}{r}131 \\
1.0 \mathrm{E}+00 \\
4.8 \mathrm{E}-07\end{array}$ & 1 & $\begin{array}{l}1.0 \mathrm{E}+00 \\
2.9 \mathrm{E}-07\end{array}$ & & $\begin{array}{l}\text { I-131 } \\
\text { F1 } \\
\text { THYROID }\end{array}$ \\
\hline $\begin{array}{l}\text { I } \\
\\
4 \\
5 \\
7\end{array}$ & $\begin{array}{l}132 \\
1.0 \mathrm{E}+00 \\
3.9 \mathrm{E}-09 \\
6.3 \mathrm{E}-10\end{array}$ & 3 & $\begin{array}{l}1.0 \mathrm{E}+00 \\
2.7 \mathrm{E}-10 \\
1.7 \mathrm{E}-09 \\
9.9 \mathrm{E}-11\end{array}$ & & $\begin{array}{l}\text { I-132 } \\
\text { F1 } \\
\text { LUNGS } \\
\text { THYROID } \\
\text { ST WALL }\end{array}$ \\
\hline$I$ & 133 & 1 & & & $1-133$ \\
\hline
\end{tabular}




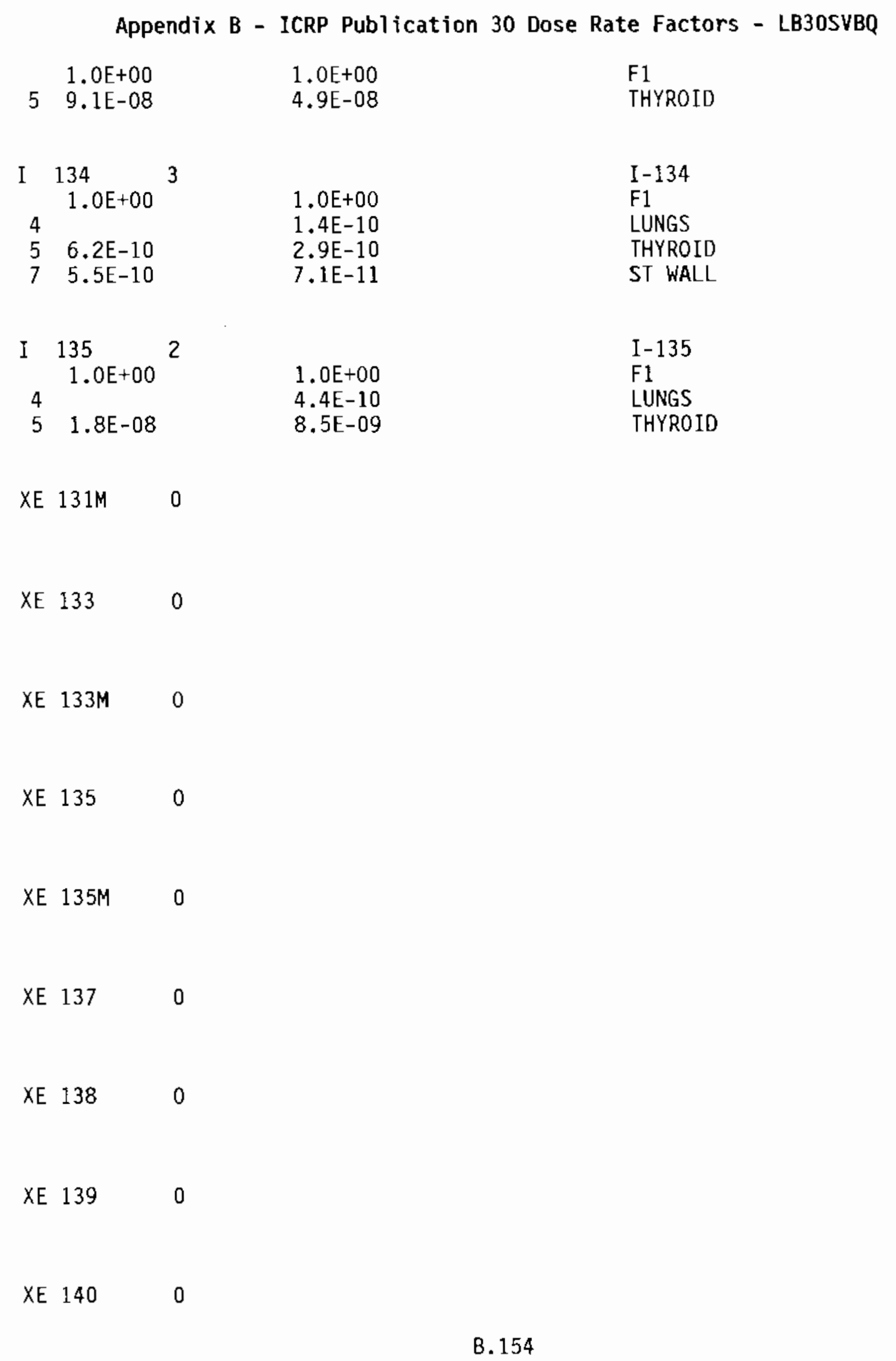




\section{Appendix B - ICRP Publication 30 Dose Rate Factors - LB30SVBQ}

$\begin{array}{ll}\text { CS } & 134 \mathrm{M} \\ & 1.0 \mathrm{E}+00 \\ 4 & 6.4 \mathrm{E}-12 \\ 1 & 6.7 \mathrm{E}-12 \\ 2 & 6.3 \mathrm{E}-12 \\ 3 & 6.9 \mathrm{E}-12 \\ 7 & 1.2 \mathrm{E}-10\end{array}$

CS 134 1.0E+00

$4 \quad 1.8 \mathrm{E}-08$

$12.1 \mathrm{E}-08$

$2 \quad 1.7 \mathrm{E}-08$

$3 \quad 1.9 \mathrm{E}-08$

$5 \quad 1.8 \mathrm{E}-08$

$6 \quad 1.7 \mathrm{E}-08$

$8 \quad 2.2 \mathrm{E}-08$

$10 \quad 2.2 \mathrm{E}-08$

$212.3 \mathrm{E}-08$

0.18

CS $135 \quad 11$

1. $0 \mathrm{E}+00$

$4 \quad 1.9 \mathrm{E}-09$

1 1.9E-09

$2 \quad 1.9 \mathrm{E}-09$

$3 \quad 1.9 \mathrm{E}-09$

5 1.9E-09

$6 \quad 1.9 \mathrm{E}-09$

$72.0 \mathrm{E}-09$

8 1.9E-09

$9 \quad 1.9 \mathrm{E}-09$

$10 \quad 1.9 \mathrm{E}-09$

21 1.9E-09

0.06
1. $0 \mathrm{E}+00$

6. $4 \mathrm{E}-11$

3. $6 \mathrm{E}-12$

$1.9 \mathrm{E}-11$

9

1. $0 \mathrm{E}+00$

1. $2 \mathrm{E}-08$

1. $3 \mathrm{E}-08$

$1.1 \mathrm{E}-08$

$1.2 \mathrm{E}-08$

$1.1 \mathrm{E}-08$

$1.1 \mathrm{E}-08$

1. $4 \mathrm{E}-08$

$1.4 \mathrm{E}-08$

$1.5 \mathrm{E}-08$

0.18

1. $0 \mathrm{E}+00$

$1.4 \mathrm{E}-09$

1. $2 \mathrm{E}-09$

$1.2 \mathrm{E}-09$

1. $2 \mathrm{E}-09$

1. $2 \mathrm{E}-09$

1. $2 \mathrm{E}-09$

1. $2 \mathrm{E}-09$

1.2E-09

$1.2 \mathrm{E}-09$

$1.2 \mathrm{E}-09$

1. $2 \mathrm{E}-09$

0.06
Cs-134M

F1

LUNGS

GONADS

BREAST

R MARROW

ST WALL

CS -134

F1

LUNGS

GONADS

BREAST

R MARROW

THYROID

BONE SURF

SI WALL

LLI WALL

REMAINDER

Remainder weight

Cs-135

F1

LUNGS

GONADS

BREAST

R MARROW

THYROID

BONE SURF

ST WALL

SI WALL

ULI WALL

LLI WALL

REMAINDER

Remainder weight

Cs -136

F1

LUNGS

GONADS

BREAST

R MARROW

THYROID

BONE SURF

SI WALL

LLI WALL 


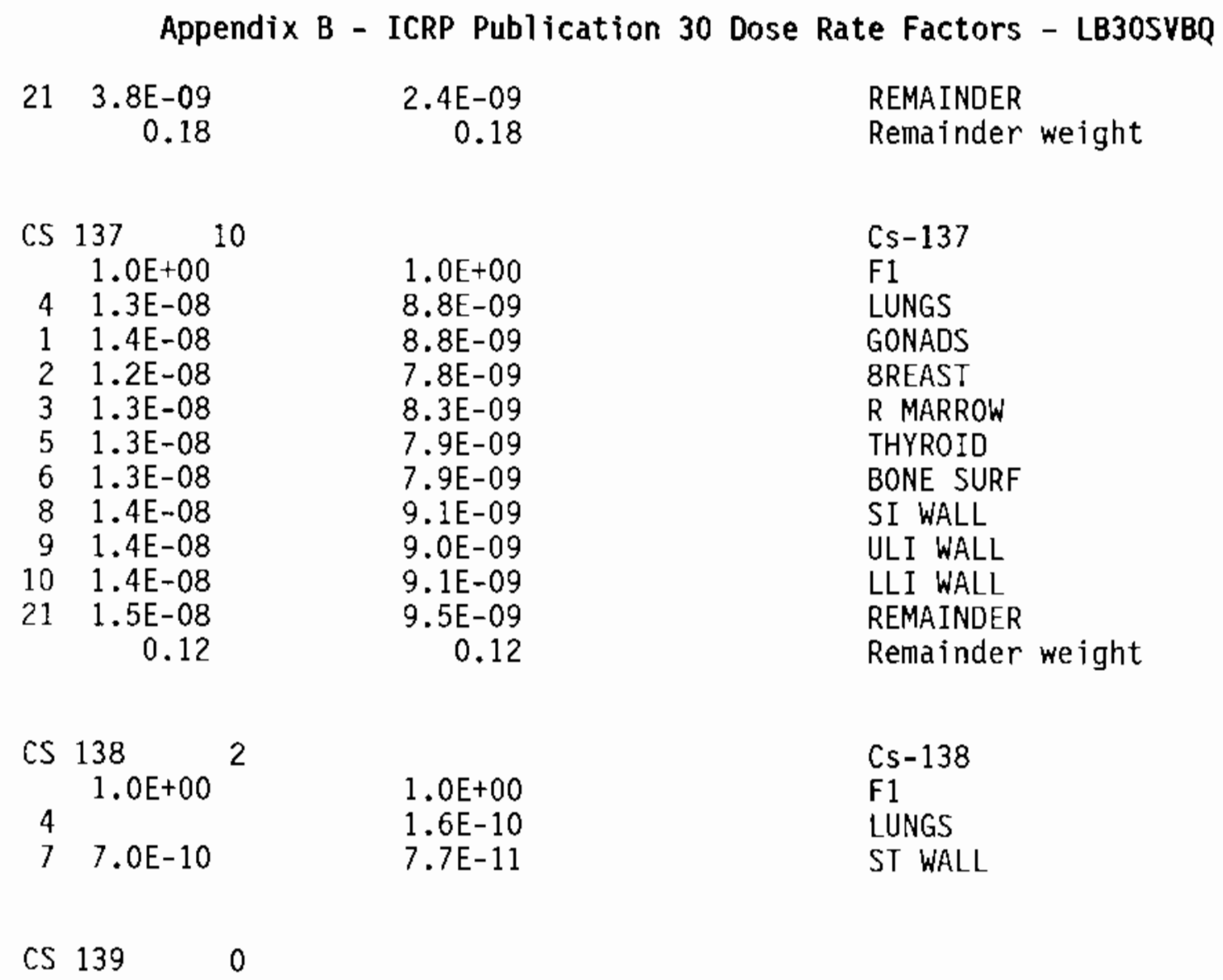

CS $140 \quad 0$

BA 137M 0

$\begin{array}{rlll}\text { BA } & 139 & & \text { Ba-139 } \\ & 1.0 \mathrm{E}-01 & 1.0 \mathrm{E}-01 & \text { F1 } \\ 4 & & 2.5 \mathrm{E}-10 & \text { LUNGS } \\ 7 & 6.9 \mathrm{E}-10 & 9.1 \mathrm{E}-11 & \text { ST WALL } \\ 8 & 5.5 \mathrm{E}-10 & 7.4 \mathrm{E}-11 & \text { SI WALL } \\ 9 & 4.4 \mathrm{E}-10 & 5.8 \mathrm{E}-11 & \text { ULI WALL } \\ 10 & 9.9 \mathrm{E}-11 & & \text { LLI WALL }\end{array}$
BA $140 \quad 8$
1.0E-01
Ba- 140
F1
1.7E-09
LUNGS
1 1. $0 \mathrm{E}-09$
4. $3 \mathrm{E}-10$
GONADS 


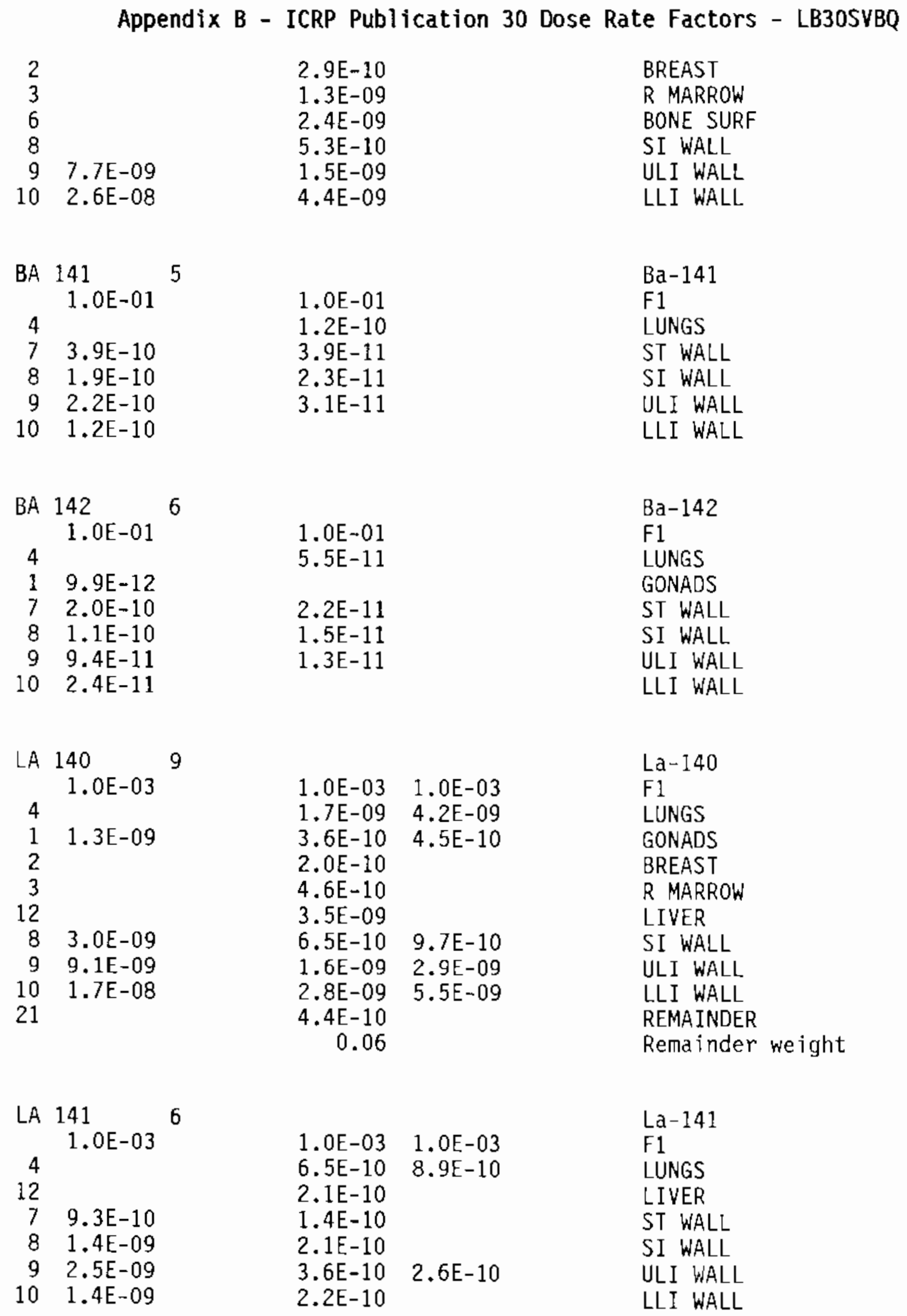




\section{Appendix B - ICRP Publication 30 Dose Rate Factors - LB30SVBQ}

$$
\begin{array}{rl}
\text { LA } & 142 \\
& 1.0 \mathrm{E}-03 \\
4 & \\
1 & 7.0 \mathrm{E}-11 \\
7 & 8.5 \mathrm{E}-10 \\
8 & 7.8 \mathrm{E}-10 \\
9 & 7.2 \mathrm{E}-10 \\
10 & 1.9 \mathrm{E}-10
\end{array}
$$
La-142
F1
LUNGS
GONADS
ST WALL
SI WALL
ULI WALL
LLI WALL

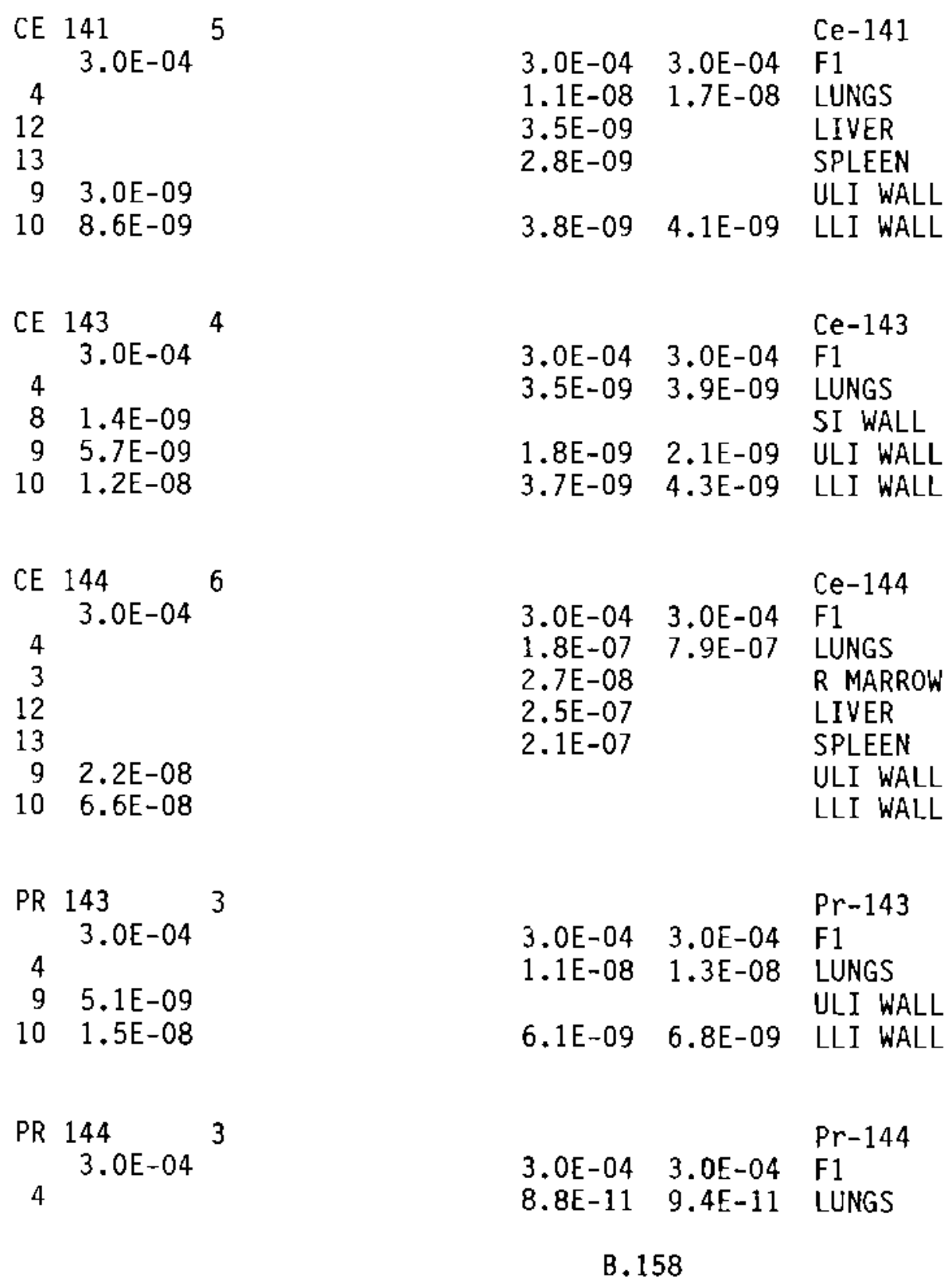




\section{Appendix B - ICRP Publication 30 Dose Rate Factors - LB30SVBQ}

$\begin{array}{ll}7 & 4.1 \mathrm{E}-10 \\ 8 & 9.6 \mathrm{E}-11\end{array}$

ND $144 \quad 0$
ST WALL

SI WALL

\section{ND $144-0$}

$$
\begin{array}{rlr}
\text { ND } & 147 & 3 \\
& 3.0 \mathrm{E}-04 \\
4 & \\
9 & 4.6 \mathrm{E}-09 \\
10 & 1.3 \mathrm{E}-08
\end{array}
$$

$\begin{array}{rlr}\text { PM } & 147 & \\ & 3.0 \mathrm{E}-04 \\ 4 & \\ 3 & \\ 6 & \\ 12 & \\ 9 & 1.1 \mathrm{E}-09 \\ 10 & 3.2 \mathrm{E}-09\end{array}$

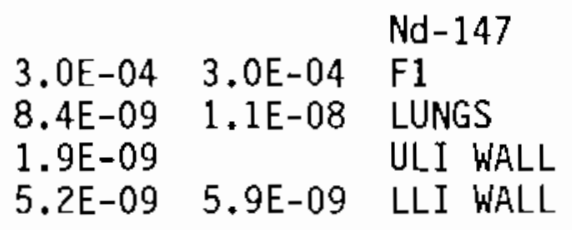

$\begin{array}{lll} & & \text { Pm-147 } \\ 3.0 \mathrm{E}-04 & 3.0 \mathrm{E}-04 & \text { F1 } \\ 9.7 \mathrm{E}-09 & 7.7 \mathrm{E}-08 & \text { LUNGS } \\ 8.2 \mathrm{E}-09 & & \text { R MARROW } \\ 1.0 \mathrm{E}-07 & & \text { BONE SURF } \\ 2.7 \mathrm{E}-08 & & \text { LIVER } \\ & & \text { ULI WALL } \\ & & \text { LLI WALL }\end{array}$

$3.0 \mathrm{E}-04 \quad 3.0 \mathrm{E}-04$

$2.2 \mathrm{E}-08 \quad 3.6 \mathrm{E}-08$

$1.4 \mathrm{E}-09$

2. $9 \mathrm{E}-09$

9.0 -09

1. $1 \mathrm{E}-08$

$6.6 \mathrm{E}-09$

2. $6 \mathrm{E}-09$

$5 . \mathrm{BE}-09$

$1.4 \mathrm{E}-08$

$$
\begin{array}{rlr}
\text { PM } & 148 & 3 \\
& 3.0 \mathrm{E}-04 \\
4 & \\
9 & 1.2 \mathrm{E}-08 \\
10 & 3.1 \mathrm{E}-08
\end{array}
$$

$\begin{array}{rlr}\text { PM } & 149 & 3 \\ & 3.0 \mathrm{E}-04 \\ 4 & \\ 9 & 5.0 \mathrm{E}-09 \\ 10 & 1.1 \mathrm{E}-08\end{array}$

$\begin{array}{lll} & & \text { Pm-148 } \\ 3.0 \mathrm{E}-04 & 3.0 \mathrm{E}-04 & \text { F1 } \\ 1.3 \mathrm{E}-08 & 1.4 \mathrm{E}-08 & \text { LUNGS } \\ 4.5 \mathrm{E}-09 & 5.2 \mathrm{E}-09 & \text { ULI WALL } \\ 1.2 \mathrm{E}-08 & 1.3 \mathrm{E}-08 & \text { LLI WALL }\end{array}$

3.0E-04 3.0E-04

3.0E-09 3.1E-09 LUNGS

$1.7 \mathrm{E}-09$ 1.9E-09 ULI WALL

3.8E-09 4.4E-09 LLI WALL 


\section{Appendix B - ICRP Publication 30 Dose Rate Factors - LB30SVBQ}

$$
\begin{array}{rl}
\text { PM } & 151 \\
& 3.0 \mathrm{E}-04 \\
4 & \\
1 & 2.1 \mathrm{E}-10 \\
8 & 1.0 \mathrm{E}-09 \\
9 & 3.8 \mathrm{E}-09 \\
10 & 7.1 \mathrm{E}-09
\end{array}
$$

$\begin{array}{lll}3.0 \mathrm{E}-04 & 3.0 \mathrm{E}-04 & \text { Pm-151 } \\ 1.6 \mathrm{E}-09 & 1.6 \mathrm{E}-09 & \text { LUNGS } \\ & & \text { GONADS } \\ & 3.4 \mathrm{E}-10 & \text { SI WALL } \\ 1.1 \mathrm{E}-09 & 1.3 \mathrm{E}-09 & \text { ULI WALL } \\ 2.0 \mathrm{E}-09 & 2.4 \mathrm{E}-09 & \text { LLI WALL }\end{array}$

Sm-147

F1

R MARROW

BONE SURF

LIVER

Sm-151

3. $0 \mathrm{E}-04$

$1.4 \mathrm{E}-07$

1. $1 \mathrm{E}-08$

3.7E-08

3. $0 \mathrm{E}-04$

2. $0 \mathrm{E}-09$

1.2E-09

2. $6 \mathrm{E}-09$

1. $0 \mathrm{E}-03$

$5.8 \mathrm{E}-08$

$1.3 \mathrm{E}-08$

$1.7 \mathrm{E}-08$

7. $9 \mathrm{E}-08$

2. $4 \mathrm{E}-07$

3.7E-08

3. $5 \mathrm{E}-07$

5. $0 \mathrm{E}-08$

0.12
$\mathrm{F} 1$

BONE SURF

R MARROW

LIVER

ULI WALL

LLI WALL

Sm-153

F1

LUNGS

ULI WALL

LLI WALL

Eu-152

F1

LUNGS

GONADS

BREAST

R MARROW

BONE SURF

KIDNEYS

LIVER

SI WALL

ULI WALL

LLI WALL

REMAINDER

Remainder weight

EU 153 


\section{Appendix B - ICRP Publication 30 Dose Rate Factors - LB30SVBQ}

$\begin{array}{rl}\text { EU } & 154 \\ & 1.0 \mathrm{E}-03 \\ 4 & 9 \\ 1 & 1.4 \mathrm{E}-09 \\ 3 & 1.1 \mathrm{E}-09 \\ 6 & 4.5 \mathrm{E}-09 \\ 12 & 3.7 \mathrm{E}-09 \\ 8 & 2.2 \mathrm{E}-09 \\ 9 & 6.9 \mathrm{E}-09 \\ 10 & 1.8 \mathrm{E}-08 \\ 21 & \end{array}$

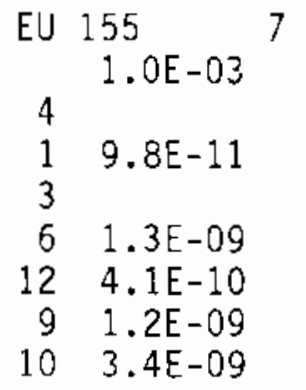

1.0E-03

$7.9 \mathrm{E}-08$

$1.2 \mathrm{E}-08$

$1.1 \mathrm{E}-07$

$5.2 \mathrm{E}-07$

$4.3 \mathrm{E}-07$

$4.4 \mathrm{E}-08$

0.06

$1.0 \mathrm{E}-03$

$1.2 \mathrm{E}-08$

$1.4 \mathrm{E}-08$

$1.5 \mathrm{E}-07$

$4.8 \mathrm{E}-08$
Eu-154

F1

LUNGS

GONADS

$R$ MARROW

BONE SURF

LIVER

SI WALL

ULI WALL

LLI WALL

REMAINDER

Remainder weight

Eu-155

F1

LUNGS

GONADS

$R$ MARROW

BONE SURF

LIVER

ULI WALL

LLI WALL

Eu-156

F1

LUNGS

GONADS

SI WALL

ULI WALL

LLI WALL

Gd-153

$\mathrm{F} 1$

LUNGS

GONADS

R MARROW

BONE SURF

LIVER

SI WALL

ULI WALL

LLI WALL
TB 160
$3.0 E-04$

3. $0 \mathrm{E}-04$

3. $0 \mathrm{E}-08$

Tb -160

FI

LUNGS 


\section{Appendix B - ICRP Publication 30 Dose Rate Factors - LB30SVBQ}

$\begin{array}{rl}1 & 1.2 \mathrm{E}-09 \\ 3 & \\ 6 & \\ 12 & \\ 8 & 1.9 \mathrm{E}-09 \\ 9 & 5.9 \mathrm{E}-09 \\ 10 & 1.6 \mathrm{E}-08\end{array}$

\section{4. $4 \mathrm{E}-09$ \\ $2.5 \mathrm{E}-08$ \\ 9.1E-09 \\ $7.6 \mathrm{E}-09$}

3. $0 E-04$

1. $1 \mathrm{E}-07$

3. $0 \mathrm{E}-08$

$4.8 \mathrm{E}-08$

$1.6 \mathrm{E}-07$

$8.9 \mathrm{E}-07$

$7.5 \mathrm{E}-07$

1.1E-06

$1.5 \mathrm{E}-07$

0.06
GONADS

R MARROW

BONE SURF

LIVER

SI WALL

ULI WALL

LLI WALL

Ho- 166

F1

LUNGS

GONADS

BREAST

$R$ MARROW

BONE SURF

LIVER

PANCREAS

SI WALL

ULI WALL

LLI WALL

REMAINDER

Remainder weight

W-181

F1

LUNGS

GONADS

BREAST

R MARROW'

BONE SURF

KIDNEYS

LIVER

SPLEEN

SI WALL

ULI WALL

LLI WALL

$W-185$

F1

LUNGS

R MARROW'

BONE SURF

KIDNEYS

LIVER

SPLEEN

ULI WALL 
Appendix B - ICRP Publication 30 Dose Rate Factors - LB30SVBQ

$\begin{array}{rlll}\text { W } & 187 & 5 & \\ & 1.0 \mathrm{E}-02 & 3.0 \mathrm{E}-01 & 3.0 \mathrm{E}-01 \\ 4 & & & 6.0 \mathrm{E}-10 \\ 1 & 2.6 \mathrm{E}-10 & 1.9 \mathrm{E}-10 & 2.9 \mathrm{E}-11 \\ 8 & 1.0 \mathrm{E}-09 & 7.5 \mathrm{E}-10 & \\ 9 & 3.6 \mathrm{E}-09 & 2.6 \mathrm{E}-09 & 3.9 \mathrm{E}-10 \\ 10 & 6.0 \mathrm{E}-09 & 4.4 \mathrm{E}-09 & 6.6 \mathrm{E}-10\end{array}$

RE $187 \quad 5$

$\begin{array}{rr} & 8.0 \mathrm{E}-01 \\ 4 & \\ 5 & 1.1 \mathrm{E}-11 \\ 7 & 2.2 \mathrm{E}-11 \\ 9 & 2.6 \mathrm{E}-12 \\ 10 & 7.2 \mathrm{E}-12\end{array}$

OS $185 \quad 11$

$\begin{array}{rr} & 1.0 \mathrm{E}-02 \\ 4 & 8.3 \mathrm{E}-10 \\ 2 & \\ 3 & 2.1 \mathrm{E}-10 \\ 11 & \\ 12 & \\ 13 & \\ 8 & 8.7 \mathrm{E}-10 \\ 9 & 1.4 \mathrm{E}-09 \\ 10 & 2.8 \mathrm{E}-09 \\ 21 & 3.6 \mathrm{E}-10 \\ & 0.06\end{array}$

8.0E-01

2. $3 \mathrm{E}-12$

$7.9 E-12$

1. $6 \mathrm{E}-11$
$W-187$

F1

LUNGS

GONADS

SI WALL

ULI WALL

LLI WALL

Re-187

F1

LUNGS

THYROID

ST WALL

ULI WALL

LLI WALL

0s-185

F1

LUNGS

GONADS

BREAST

R MARROW

KIDNEYS

LIVER

SPLEEN

SI WALL

ULI WALL

LLI WALLL

REMAINDER

Remainder weight

$\begin{array}{rlllll}\text { OS } 191 & & & & \text { OS }-191 \\ & 1.0 \mathrm{E}-02 & & & \\ 4 & & 1.0 \mathrm{E}-02 & 1.0 \mathrm{E}-02 & 1.0 \mathrm{E}-02 & \text { F1 } \\ 1 & 6.0 \mathrm{E}-10 & 5.6 \mathrm{E}-09 & 6.8 \mathrm{E}-09 & \text { LUNGS } \\ 3 & & 1.9 \mathrm{E}-10 & & & \text { GONADS } \\ 2 & & 2.3 \mathrm{E}-10 & & & \text { R MARROW } \\ 11 & & 1.7 \mathrm{E}-10 & & & \text { 8REAST } \\ 12 & & 2.2 \mathrm{E}-09 & & & \text { KIDNEYS } \\ 13 & & 2.0 \mathrm{E}-09 & & & \text { LIVER } \\ 9 & 2.3 \mathrm{E}-09 & 1.9 \mathrm{E}-09 & & & \text { SPLEEN } \\ 10 & 6.6 \mathrm{E}-09 & 5.5 \mathrm{E}-10 & & & \text { ULI WALL } \\ & & 1.2 \mathrm{E}-09 & 2.8 \mathrm{E}-09 & 3.1 \mathrm{E}-09 & \text { LLI WALL }\end{array}$

IR $192 \quad 11$
1. $0 \mathrm{E}-02$
$1.0 \mathrm{E}-02$
$1.0 E-02 \quad 1.0 E-02$
Ir -192
F1 


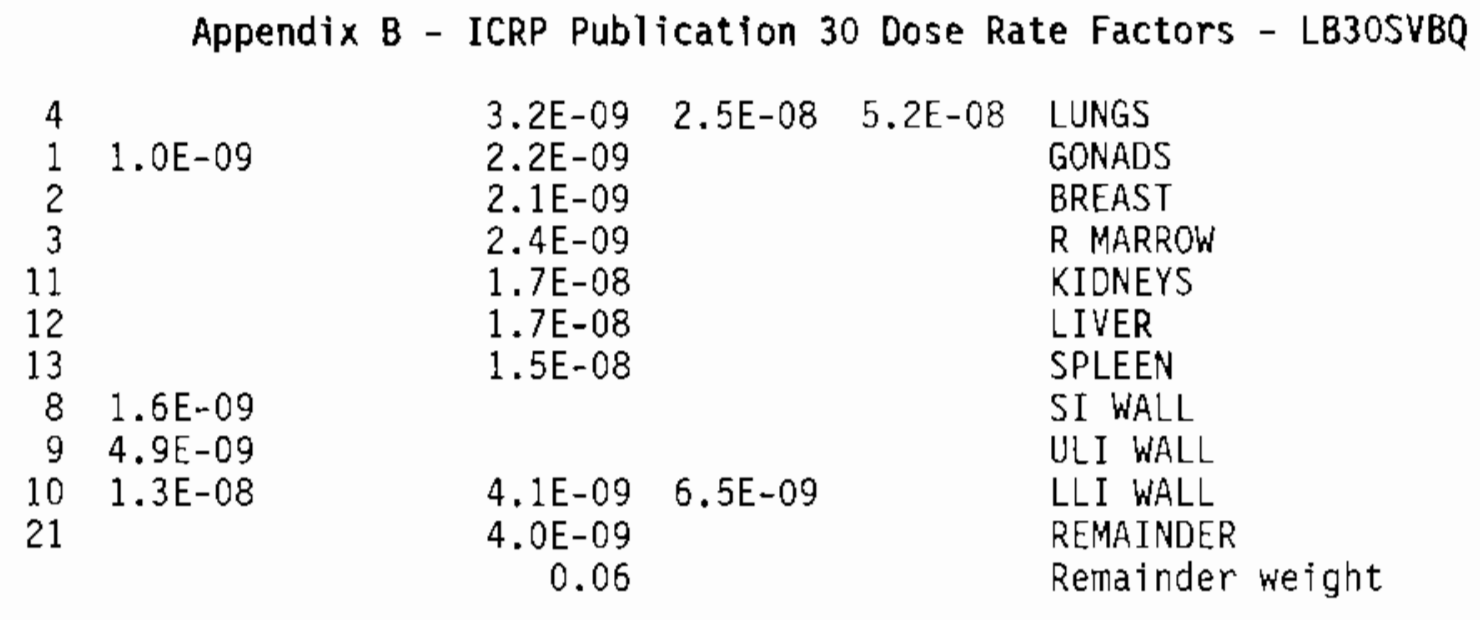

$\begin{array}{rlll}\mathrm{HG} & 203 & 9 & \\ & 1.0 \mathrm{E}+00 & 4.0 \mathrm{E}-01 & 1.0 \mathrm{E}+00 \\ 4 & 1.2 \mathrm{E}-09 & 4.9 \mathrm{E}-10 & 1.1 \mathrm{E}-09 \\ 1 & 1.4 \mathrm{E}-09 & 7.3 \mathrm{E}-10 & 8.6 \mathrm{E}-10 \\ 2 & 1.2 \mathrm{E}-09 & 5.1 \mathrm{E}-10 & 7.8 \mathrm{E}-10 \\ 3 & 1.7 \mathrm{E}-09 & 7.1 \mathrm{E}-10 & 1.1 \mathrm{E}-09 \\ 11 & 1.9 \mathrm{E}-08 & 7.5 \mathrm{E}-09 & 1.2 \mathrm{E}-08 \\ 17 & 1.2 \mathrm{E}-08 & 4.6 \mathrm{E}-09 & 7.3 \mathrm{E}-09 \\ 8 & & 9.3 \mathrm{E}-10 & \\ 9 & & 1.8 \mathrm{E}-09 & \\ 10 & & 3.9 \mathrm{E}-09 & \end{array}$

$\mathrm{Hg}-203$ (ORG)

F1

LUNGS

GONADS

BREAST

$R$ MARROW

KIDNEYS

BRAIN

SI WALL

ULI WALL

LLI WALL

$\begin{array}{rlr}\text { PB } & 210 & \\ & 2.0 \mathrm{E}-01 & \\ 3 & 1.5 \mathrm{E}-06 & 2.0 \mathrm{E}-01 \\ 6 & 2.2 \mathrm{E}-05 & 3.7 \mathrm{E}-06 \\ 11 & 2.8 \mathrm{E}-06 & 5.5 \mathrm{E}-05 \\ 12 & 6.1 \mathrm{E}-06 & 7.1 \mathrm{E}-06 \\ & & 1.5 \mathrm{E}-05\end{array}$

$\mathrm{Pb}-210$

F1

R MARROW

BONE SURF

KIDNEYS

LIVER

$\begin{array}{rll}\text { PB } & 212 & 7 \\ & 2.0 \mathrm{E}-01 & 2.0 \mathrm{E}-01 \\ 4 & & 2.0 \mathrm{E}-07 \\ 3 & 1.5 \mathrm{E}-08 & 3.3 \mathrm{E}-08 \\ 6 & 1.7 \mathrm{E}-07 & 3.7 \mathrm{E}-07 \\ 12 & 2.2 \mathrm{E}-08 & 4.9 \mathrm{E}-08 \\ 11 & 1.1 \mathrm{E}-08 & \\ 9 & 1.7 \mathrm{E}-08 & \\ 10 & 2.0 \mathrm{E}-08 & \end{array}$

$\mathrm{Pb}-212$

F1

LUNGS

$R$ MARROW

BONE SURF

LIVER

KIDNEYS

ULI WALL

LLI WALL
BI $210 \quad 4$
$5.0 \mathrm{E}-02$
5. $0 \mathrm{E} \sim 02$
$\mathrm{Bi}-210$
4
11
5. $8 \mathrm{E}-08$
F1
LUNGS
KIDNEYS

\section{B. 164}




\section{Appendix B - ICRP Publication 30 Dose Rate Factors - LB30SVBQ}

$\begin{array}{rr}9 & 5.7 \mathrm{E}-09 \\ 10 & 1.5 \mathrm{E}-08\end{array}$

$\begin{array}{rl}\text { BI } & 212 \\ & 5.0 \mathrm{E}-02 \\ 4 & 5 \\ 11 & 1.1 \mathrm{E}-09 \\ 7 & 1.6 \mathrm{E}-09 \\ 8 & 1.1 \mathrm{E}-09 \\ 9 & 6.6 \mathrm{E}-10\end{array}$

$5.0 \mathrm{E}-02$

$3.4 \mathrm{E}-08$

2.7E-08

$5.0 \mathrm{E}-02$

$3.9 E-08$

$1.0 \mathrm{E}-01 \quad 1.0 \mathrm{E}-01$

1.3E-05

$1.2 \mathrm{E}-05 \quad 3.9 \mathrm{E} \rightarrow 06$

2.2E-06

2.2E-05
ULI WALL LLI WALL

Bi-212

F1

LUNGS

KIDNEYS

ST WALL

SI WALL

ULI WALL

Po-210

F1

LUNGS

KIDNEYS

LIVER

SPLEEN

RN $222 \quad 0$

$\begin{array}{rcr}\text { FR } & 223 & 10 \\ & 1.0 \mathrm{E}+00 & 1.0 \mathrm{E}+00 \\ 4 & 2.3 \mathrm{E}-09 & 3.4 \mathrm{E}-09 \\ 1 & 2.3 \mathrm{E}-09 & 1.4 \mathrm{E}-09 \\ 2 & 2.3 \mathrm{E}-09 & 1.4 \mathrm{E}-09 \\ 3 & 2.3 \mathrm{E}-09 & 1.4 \mathrm{E}-09 \\ 5 & 2.3 \mathrm{E}-09 & 1.4 \mathrm{E}-09 \\ 6 & 2.3 \mathrm{E}-09 & 1.4 \mathrm{E}-09 \\ 7 & 2.5 \mathrm{E}-09 & 1.5 \mathrm{E}-09 \\ 8 & 2.3 \mathrm{E}-09 & \\ 9 & 2.3 \mathrm{E}-09 & \\ 21 & 2.3 \mathrm{E}-09 & 1.4 \mathrm{E}-09 \\ & 0.12 & 0.24\end{array}$

$\mathrm{Fr}-223$

F1

LUNGS

GONADS

BREAST

$R$ MARROW

THYROID

BONE SURF

ST WALL

SI WALL

ULI WALL

REMAINDER

Remainder weight
RA 2235
4 1 $4.2 E-08$
$3 \quad 2.8 \mathrm{E}-07$
$6 \quad 2.9 \mathrm{E}-06$
$10 \quad 2.8 \mathrm{E}-07$

2. $0 \mathrm{E}-01$

$1.7 \mathrm{E}-05$
$\mathrm{Ra}-223$

F1

LUNGS

GONADS

R MARROW

BONE SURF

LLI WALL

RA 224

$R a-224$ 


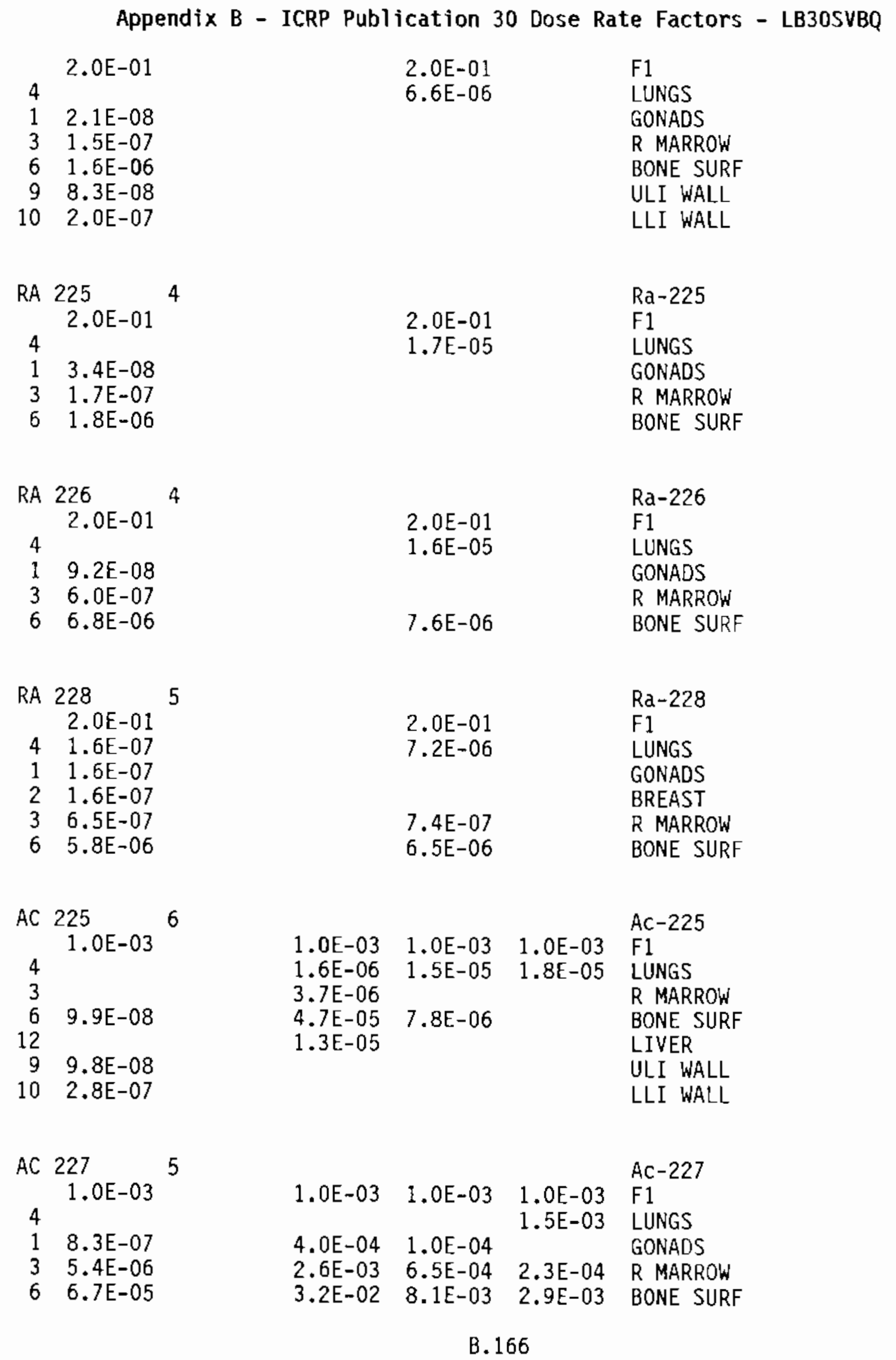


Appendix B - ICRP Publication 30 Dose Rate Factors - LB30SVBQ

$\begin{array}{rlllll}12 & 1.5 \mathrm{E}-05 & 7.3 \mathrm{E}-03 & 1.9 \mathrm{E}-03 & 6.7 \mathrm{E}-04 & \text { LIVER } \\ & & & & \\ \mathrm{AC} & 228 & & & \\ & 1.0 \mathrm{E}-03 & & & & \text { AC-228 } \\ 4 & & 1.0 \mathrm{E}-03 & 1.0 \mathrm{E}-03 & 1.0 \mathrm{E}-03 & \mathrm{~F} 1 \\ 1 & 1.8 \mathrm{E}-10 & & 3.5 \mathrm{E}-08 & 2.5 \mathrm{E}-07 & \text { LUNGS } \\ 3 & 2.7 \mathrm{E}-10 & & & & \text { GONADS } \\ 6 & 3.0 \mathrm{E}-09 & 1.1 \mathrm{E}-07 & 2.8 \mathrm{E}-08 & \text { R MARROW } \\ 12 & \mathrm{~B} .2 \mathrm{E}-10 & 1.4 \mathrm{E}-06 & 3.5 \mathrm{E}-07 & \text { BONE SURF } \\ 7 & 6.2 \mathrm{E}-10 & 3.8 \mathrm{E}-07 & 9.2 \mathrm{E}-08 & \text { LIVER } \\ 8 & 1.1 \mathrm{E}-09 & & & \text { ST WALL } \\ 9 & 2.4 \mathrm{E}-09 & & & \text { SI WALL } \\ 10 & 1.9 \mathrm{E}-09 & & & & \text { ULI WALL } \\ & & & & & \text { LLI WALL }\end{array}$

$\begin{array}{rl}\text { TH } & 227 \\ & 2.0 \mathrm{E}-04 \\ 4 & \\ 3 & 5.7 \mathrm{E}-09 \\ 6 & 6.8 \mathrm{E}-08 \\ 9 & 2.5 \mathrm{E}-08 \\ 10 & 9.1 \mathrm{E}-08\end{array}$

2.0E-04

$2.4 \mathrm{E}-05$

2. $4 \mathrm{E}-06$

2. $9 \mathrm{E}-05$

$\begin{array}{ll} & \text { Th-227 } \\ 2.0 \mathrm{E}-04 & \text { F1 } \\ 3.6 \mathrm{E}-05 & \text { LUNGS } \\ & \text { R MARROW } \\ & \text { BONE SURF } \\ & \text { ULI WALL } \\ & \text { LLI WALL }\end{array}$

Th-228

2. $0 \mathrm{E}-04$

9.5E-05

1.1E-04

1. $4 \mathrm{E}-03$
2.0E-04

6. $9 \mathrm{E}-04$

LUNGS

R MARROW

BONE SURF

LLI WALL
TH 229 3
4
$\begin{array}{ll}3 & 1.9 \mathrm{E}-06 \\ 6 & 2.4 \mathrm{E}-05\end{array}$
TH $230 \quad 3$
$\begin{array}{ll}4 & 2.0 \mathrm{E}-04 \\ 3 & 2.9 \mathrm{E}-07 \\ 6 & 3.6 \mathrm{E}-06\end{array}$
TH $231 \quad 6$ 4
$2.0 \mathrm{E}-04 \quad 2.0 \mathrm{E}-04$
2. $0 \mathrm{E}-03$
$1.1 \mathrm{E}-03$
$1.4 \mathrm{E}-02$
$5.7 \mathrm{E}-03$
$R$ MARROW
BONE SURF
Th-229
F1

BONE SURF

Th-230

$F 1$

$\begin{array}{ll}3.0 \mathrm{E}-04 & \text { LUNGS } \\ 7.0 \mathrm{E}-05 & \text { R MARROW }\end{array}$

$\begin{array}{lll}1.7 \mathrm{E}-04 & 7.0 \mathrm{E}-05 & \text { R MARROW } \\ 2.2 \mathrm{E}-03 & 8.7 \mathrm{E}-04 & \text { BONE SURF }\end{array}$

$\begin{array}{lll}2.0 \mathrm{E}-04 & 2.0 \mathrm{E}-04 & \text { Th-231 } \\ 7.8 \mathrm{E}-10 & 8.8 \mathrm{E}-10 & \text { LUNGS }\end{array}$

B. 167 


\section{Appendix B - ICRP Publication 30 Dose Rate Factors - LB30SVBQ}

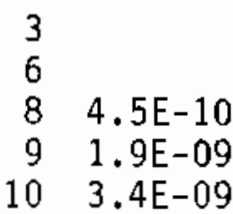

$7.9 \mathrm{E}-11$

9.2E-10

$5.3 \mathrm{E}-10$

$9.4 \mathrm{E}-10$

$6.2 \mathrm{E}-10$

$1.1 \mathrm{E}-09$

$R$ MARROW

BONE SURF

SI WALL

ULI WALL

LLI WALL

$\begin{array}{lll}2.0 \mathrm{E}-04 & 2.0 \mathrm{E}-04 & \text { F1 } \\ & 9.4 \mathrm{E}-04 & \text { LUNGS } \\ 8.9 \mathrm{E}-04 & 4.0 \mathrm{E}-04 & \text { R MARROW } \\ 1.1 \mathrm{E}-02 & 5.0 \mathrm{E}-03 & \text { BONE SURF }\end{array}$

$\begin{array}{lll} & & \text { Th-234 } \\ 2.0 \mathrm{E}-04 & 2.0 \mathrm{E}-04 & \text { F1 } \\ 4.6 \mathrm{E}-08 & 6.4 \mathrm{E}-08 & \text { LUNGS } \\ & & \text { ULI WALL } \\ 1.9 \mathrm{E}-08 & 2.0 \mathrm{E}-08 & \text { LLI WALL }\end{array}$

$\begin{array}{rlr}\text { PA } & 233 & 5 \\ & 1.0 \mathrm{E}-03 \\ 4 & \\ 1 & 2.6 \mathrm{E}-10 \\ 6 & \\ 9 & 3.6 \mathrm{E}-09 \\ 10 & 1.0 \mathrm{E}-08\end{array}$

PA $234 \quad 6$

$1.0 \mathrm{E}-03$

4

$1 \quad 3.3 \mathrm{E}-10$

$7 \quad 7.8 \mathrm{E}-10$

$8 \quad 1.5 \mathrm{E}-09$

$9 \quad 3.1 \mathrm{E}-09$

$10 \quad 2.5 \mathrm{E}-09$
$1.0 \mathrm{E}-03 \quad 1.0 \mathrm{E}-03$

$1.2 \mathrm{E}-08 \quad 1.7 \mathrm{E}-08$

$7.4 \mathrm{E}-09$

4. 5E-09

$4.8 \mathrm{E}-09$

$\mathrm{Pa}-233$

F1

LUNGS GONADS BONE SURF ULI WALL

LLI WALL

$\mathrm{Pa}-234$

$\begin{array}{lll}1.0 \mathrm{E}-03 & 1.0 \mathrm{E}-03 & \mathrm{~F} 1 \\ 8.4 \mathrm{E}-10 & 9.0 \mathrm{E}-10 & \text { LUNGS }\end{array}$

5.0E-11 6.1E-11 GONADS

$2.2 \mathrm{E}-10 \quad 2.7 \mathrm{E}-10$ SI

4.6E-10 5.7E-10 ULI WALL

$3.6 \mathrm{E}-10 \quad 4.5 \mathrm{E}-10$ LLI WALL

PA 234M 0 


\section{Appendix B - ICRP Publication 30 Dose Rate Factors - LB30SVBO}

\begin{tabular}{|c|c|c|c|c|c|c|}
\hline $\begin{array}{r}4 \\
3 \\
6 \\
11 \\
9 \\
10\end{array}$ & $\begin{array}{l}232 \\
5.0 \mathrm{E}-02 \\
4.2 \mathrm{E}-07 \\
6.6 \mathrm{E}-06 \\
1.6 \mathrm{E}-06\end{array}$ & $\begin{array}{l}6 \\
2.0 \mathrm{E}-03 \\
1.7 \mathrm{E}-08 \\
2.7 \mathrm{E}-07 \\
6.3 \mathrm{E}-08 \\
1.8 \mathrm{E}-08 \\
5.6 \mathrm{E}-08\end{array}$ & $\begin{array}{l}5.0 E-02 \\
4.1 E-06 \\
6.4 E-05 \\
1.5 E-05\end{array}$ & $\begin{array}{l}5.0 E-02 \\
2.5 E-05 \\
1.9 E-05\end{array}$ & $\begin{array}{l}2.0 E-03 \\
1.5 E-03\end{array}$ & $\begin{array}{l}\text { U-232 } \\
\text { F1 } \\
\text { LUNGS } \\
\text { R MARROW } \\
\text { BONE SURF } \\
\text { KIDNEYS } \\
\text { ULI WALL } \\
\text { LLI WALL }\end{array}$ \\
\hline $\begin{array}{r}4 \\
3 \\
6 \\
11 \\
9 \\
10\end{array}$ & $\begin{array}{l}233 \\
5.0 \mathrm{E}-02 \\
7.4 \mathrm{E}-08 \\
1.2 \mathrm{E}-06 \\
4.7 \mathrm{E}-07\end{array}$ & $\begin{array}{l}6 \\
2.0 \mathrm{E}-03 \\
2.9 \mathrm{E}-09 \\
4.6 \mathrm{E}-08 \\
1.9 \mathrm{E}-08 \\
1.6 \mathrm{E}-08 \\
5.0 \mathrm{E}-08\end{array}$ & $\begin{array}{l}5.0 E-02 \\
3.2 E-07 \\
7.1 E-07 \\
1.1 E-05 \\
4.6 E-06\end{array}$ & $\begin{array}{l}5.0 \mathrm{E}-02 \\
1.6 \mathrm{E}-05\end{array}$ & $\begin{array}{l}2.0 E-03 \\
3.0 E-04\end{array}$ & $\begin{array}{l}\text { U-233 } \\
\text { F1 } \\
\text { LUNGS } \\
\text { R MARROW } \\
\text { BONE SURF } \\
\text { KIDNEYS } \\
\text { ULI WALL } \\
\text { LLI WALL }\end{array}$ \\
\hline $\begin{array}{r}\text { U } \\
4 \\
4 \\
3 \\
6 \\
11 \\
9 \\
10\end{array}$ & $\begin{array}{l}234 \\
5.0 \mathrm{E}-02 \\
7.2 \mathrm{E}-08 \\
1.1 \mathrm{E}-06 \\
4.7 \mathrm{E}-07\end{array}$ & $\begin{array}{l}6 \\
2.0 \mathrm{E}-03 \\
2.9 \mathrm{E}-09 \\
4.5 \mathrm{E}-08 \\
1.9 \mathrm{E}-08 \\
1.6 \mathrm{E}-08 \\
4.9 \mathrm{E}-08\end{array}$ & $\begin{array}{l}5.0 E-02 \\
3.2 E-07 \\
7.0 E-07 \\
1.1 E-05 \\
4.5 E-06\end{array}$ & $\begin{array}{l}5.0 \mathrm{E}-02 \\
1.6 \mathrm{E}-05\end{array}$ & $\begin{array}{l}2.0 E-03 \\
3.0 E-04\end{array}$ & $\begin{array}{l}\text { U-234 } \\
\text { F1 } \\
\text { LUNGS } \\
\text { R MARROW } \\
\text { BONE SURF } \\
\text { KIDNEYS } \\
\text { ULI WALL } \\
\text { LLI WALL }\end{array}$ \\
\hline $\begin{array}{r}4 \\
3 \\
6 \\
11 \\
10 \\
9\end{array}$ & $\begin{array}{l}235 \\
5.0 \mathrm{E}-02 \\
6.8 \mathrm{E}-08 \\
1.0 \mathrm{E}-06 \\
4.3 \mathrm{E}-07 \\
5.3 \mathrm{E}-08\end{array}$ & $\begin{array}{l}6 \\
2.0 \mathrm{E}-03 \\
2.8 \mathrm{E}-09 \\
4.2 \mathrm{E}-08 \\
1.7 \mathrm{E}-08 \\
5.3 \mathrm{E}-08 \\
1.7 \mathrm{E}-08\end{array}$ & $\begin{array}{l}5.0 E-02 \\
2.9 E-07 \\
6.6 E-07 \\
1.0 E-05 \\
4.2 E-06\end{array}$ & $\begin{array}{l}5.0 \mathrm{E}-02 \\
1.5 \mathrm{E}-05\end{array}$ & $\begin{array}{l}2.0 \mathrm{E}-03 \\
2.8 \mathrm{E}-04\end{array}$ & $\begin{array}{l}\text { U-235 } \\
\text { F1 } \\
\text { LUNGS } \\
\text { R MARROW } \\
\text { BONE SURF } \\
\text { KIDNEYS } \\
\text { LLI WALL } \\
\text { ULI WALL }\end{array}$ \\
\hline $\begin{array}{r}4 \\
3 \\
6 \\
11 \\
9 \\
10\end{array}$ & $\begin{array}{l}236 \\
5.0 \mathrm{E}-02 \\
6.8 \mathrm{E}-08 \\
1.1 \mathrm{E}-06 \\
4.4 \mathrm{E}-07\end{array}$ & $\begin{array}{l}6 \\
2.0 \mathrm{E}-03 \\
2.7 \mathrm{E}-09 \\
4.3 \mathrm{E}-08 \\
1.8 \mathrm{E}-08 \\
1.5 \mathrm{E}-08 \\
4.7 \mathrm{E}-08\end{array}$ & $\begin{array}{l}5.0 E-02 \\
3.0 E-07 \\
6.6 E-07 \\
1.0 E-05 \\
4.3 E-06\end{array}$ & $\begin{array}{l}5.0 E-02 \\
1.5 E-05\end{array}$ & $\begin{array}{l}2.0 \mathrm{E}-03 \\
2.8 \mathrm{E}-04\end{array}$ & $\begin{array}{l}\text { U-236 } \\
\text { F1 } \\
\text { LUNGS } \\
\text { R MARROW } \\
\text { BONE SURF } \\
\text { KIDNEYS } \\
\text { ULI WALL } \\
\text { LLI WALL }\end{array}$ \\
\hline
\end{tabular}




\section{Appendix B - ICRP Publication 30 Dose Rate Factors - LB3OSVBQ}

$\begin{array}{rll}\text { U } & 237 & 6 \\ & 5.0 \mathrm{E}-02 & 2.0 \mathrm{E}-03 \\ 4 & & \\ 3 & & \\ 6 & & \\ 11 & & \\ 9 & 3.2 \mathrm{E}-09 & 3.4 \mathrm{E}-09 \\ 10 & 8.5 \mathrm{E}-09 & 8.9 \mathrm{E}-09\end{array}$

U 2386

$\begin{array}{rrr} & 5.0 \mathrm{E}-02 & 2.0 \mathrm{E}-03 \\ 4 & & \\ 3 & 6.8 \mathrm{E}-08 & 2.7 \mathrm{E}-09 \\ 6 & 1.0 \mathrm{E}-06 & 4.0 \mathrm{E}-08 \\ 11 & 4.1 \mathrm{E}-07 & 1.7 \mathrm{E}-08 \\ 9 & & 1.5 \mathrm{E}-08 \\ 10 & & 4.6 \mathrm{E}-08\end{array}$

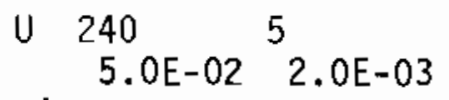

4
11
8
9

10

1. $9 \mathrm{E}-09$

2. $0 \mathrm{E}-09$

$6.7 \mathrm{E}-09$

9.1E-09

$7.0 \mathrm{E}-09$

9.5E-09

$5.0 \mathrm{E}-02$

$2.8 \mathrm{E}-07$

$6.6 \mathrm{E}-07$

$9.8 \mathrm{E}-06$

$4.0 \mathrm{E}-06$

$5.0 \mathrm{E}-02$

$1.4 \mathrm{E}-05$

$2.0 \mathrm{E}-03$

$2.7 \mathrm{E}-04$

1.3E-09 1.5E-09

3.3E-09 3.9E-09

2.0E-03

4.7E-09

$-237$

F1

LUNGS

R MARROW

BONE SURF

KIDNEYS

ULI WALL

LLI WALL

$\mathrm{U}-238$

F1

LUNGS

R MARROW

8ONE SURF

KIDNEYS

ULI WALL

LLI WALL

$U-240$

$\mathrm{F} 1$

5. $0 \mathrm{E}-02$

5. $0 \mathrm{E}-02$

2. $0 \mathrm{E}-03$

2. $4 \mathrm{E}-09$

8. $9 \mathrm{E}-10$

2.3E-09

$5.4 \mathrm{E}-10$

LUNGS

KIDNEYS

3. $2 E-10$

1. $0 E-09$

1. $5 \mathrm{E}-09$

$1.8 \mathrm{E}-09$

SI WALL

$1.4 \mathrm{E}-09$
2. $5 \mathrm{E}-09$

ULI WALL

LLI WALL

$\begin{array}{rc}\text { NP } & 237 \\ & 1.0 \mathrm{E}-02 \\ 1 & 2.5 \mathrm{E}-06 \\ 3 & 1.5 \mathrm{E}-05 \\ 6 & 1.9 \mathrm{E}-04 \\ 12 & 4.1 \mathrm{E}-05\end{array}$

$\begin{array}{rl}\mathrm{NP} & 238 \\ & 1.0 \mathrm{E}-02 \\ 1 & 5.2 \mathrm{E}-10 \\ 3 & 1.1 \mathrm{E}-09 \\ 6 & 1.2 \mathrm{E}-08 \\ 12 & 2.7 \mathrm{E}-09 \\ 8 & 1.1 \mathrm{E}-09 \\ 9 & 4.2 \mathrm{E}-09 \\ 10 & 8.9 \mathrm{E}-09\end{array}$

NP 239
1.0E-02

3. $1 \mathrm{E}-05$

1. $9 \mathrm{E}-04$

2. $4 \mathrm{E}-03$

5. $1 \mathrm{E}-04$

$1.0 \mathrm{E}-02$

2. $1 E-09$

1. $2 \mathrm{E}-08$

1. $5 \mathrm{E}-07$

3. $3 \mathrm{E}-08$
Np-237

F1

GONADS

$R$ MARROW

BONE SURF

LIVER

Np - 238

F1

GONADS

$R$ MARROW

BONE SURF

LIVER

SI WALL

ULI WALL

LLI WALL

Np-239

B. 170 
Appendix B - ICRP Publication 30 Dose Rate Factors - LB3OSVBQ

$\begin{array}{rr} & 1.0 \mathrm{E}-02 \\ 4 & \\ 6 & \\ 8 & 8.7 \mathrm{E}-10 \\ 9 & 3.8 \mathrm{E}-09 \\ 10 & 8.6 \mathrm{E}-09\end{array}$

$\begin{array}{ll}1.0 \mathrm{E}-02 & \text { F1 } \\ 2.4 \mathrm{E}-09 & \text { LUNGS } \\ 1.4 \mathrm{E}-09 & \text { BONE SURF } \\ 1.3 \mathrm{E}-09 & \text { SI WALL } \\ 2.9 \mathrm{E}-09 & \text { ULI WALL } \\ & \text { LLI WALL }\end{array}$

$\begin{array}{rll}\text { PU } & 236 & 7 \\ & 1.0 \mathrm{E}-04 & 1.0 \mathrm{E}-05 \\ 4 & & \\ 1 & 7.8 \mathrm{E}-09 & \\ 3 & 5.1 \mathrm{E}-08 & 5.1 \mathrm{E}-09 \\ 6 & 6.3 \mathrm{E}-07 & 6.3 \mathrm{E}-08 \\ 12 & 1.4 \mathrm{E}-07 & 1.4 \mathrm{E}-08 \\ 9 & & 1.9 \mathrm{E}-08 \\ 10 & 6.0 \mathrm{E}-08 & 6.0 \mathrm{E}-08\end{array}$

$\begin{array}{lll} & & \text { PU-236 } \\ 1.0 \mathrm{E}-04 & 1.0 \mathrm{E}-05 & \text { F1 } \\ & 1.9 \mathrm{E}-04 & \text { LUNGS } \\ 9.3 \mathrm{E}-06 & & \text { GONADS } \\ 6.0 \mathrm{E}-05 & 2.0 \mathrm{E}-05 & \text { R MARROW } \\ 7.5 \mathrm{E}-04 & 2.6 \mathrm{E}-04 & \text { BONE SURF } \\ 1.7 \mathrm{E}-04 & 5.8 \mathrm{E}-05 & \text { LIVER } \\ & & \text { ULI WALL } \\ & & \text { LLI WALL }\end{array}$

$\begin{array}{rll}\text { PU } & 237 & 8 \\ & 1.0 \mathrm{E}-04 & 1.0 \mathrm{E}-05 \\ 4 & & \\ 1 & 7.2 \mathrm{E}-11 & 7.2 \mathrm{E}-11 \\ 3 & & \\ 6 & & \\ 12 & & \\ \mathrm{~B} & 1.2 \mathrm{E}-10 & 1.2 \mathrm{E}-10 \\ 9 & 4.0 \mathrm{E}-10 & 4.0 \mathrm{E}-10 \\ 10 & 1.0 \mathrm{E}-09 & 1.0 \mathrm{E}-09\end{array}$

$\begin{array}{lll} & & \text { PU-237 } \\ 1.0 \mathrm{E}-04 & 1.0 \mathrm{E}-05 & \text { F1 } \\ 2.2 \mathrm{E}-09 & 3.7 \mathrm{E}-09 & \text { LUNGS } \\ & & \text { GONADS } \\ 2.5 \mathrm{E}-10 & & \text { R MARROW } \\ 1.8 \mathrm{E}-09 & & \text { BONE SURF } \\ 8.0 \mathrm{E}-10 & & \text { LIVER } \\ & & \text { SI WALL } \\ & \text { ULI WALL } \\ 4.7 \mathrm{E}-10 & & \text { LLI WALL }\end{array}$

\begin{tabular}{rll} 
PU & 238 & \multicolumn{1}{l}{} \\
& $1.0 \mathrm{E}-04$ & $1.0 \mathrm{E}-05$ \\
4 & & \\
1 & $2.3 \mathrm{E}-08$ & $2.3 \mathrm{E}-09$ \\
3 & $1.5 \mathrm{E}-07$ & $1.5 \mathrm{E}-08$ \\
6 & $1.8 \mathrm{E}-06$ & $1.8 \mathrm{E}-07$ \\
12 & $4.0 \mathrm{E}-07$ & $4.0 \mathrm{E}-08$ \\
9 & & $1.8 \mathrm{E}-08$ \\
10 & & $5.7 \mathrm{E}-08$
\end{tabular}

$\begin{array}{lll}1.0 \mathrm{E}-04 & 1.0 \mathrm{E}-05 & \text { PU1 } \\ & 3.2 \mathrm{E}-04 & \text { LUNGS } \\ 2.8 \mathrm{E}-05 & & \text { GONADS } \\ 1.8 \mathrm{E}-04 & 6.6 \mathrm{E}-05 & \text { R MARROW } \\ 2.2 \mathrm{E}-03 & 8.3 \mathrm{E}-04 & \text { BONE SURF } \\ 4.8 \mathrm{E}-04 & 1.8 \mathrm{E}-04 & \text { LIVER } \\ & & \text { ULI WALL } \\ & & \text { LLI WALL }\end{array}$

\begin{tabular}{rrr} 
PU & \multicolumn{2}{l}{239} \\
& $1.0 \mathrm{E}-04$ & $1.0 \mathrm{E}-05$ \\
4 & & \\
1 & $2.6 \mathrm{E}-08$ & $2.6 \mathrm{E}-09$ \\
3 & $1.6 \mathrm{E}-07$ & $1.6 \mathrm{E}-08$ \\
6 & $2.1 \mathrm{E}-06$ & $2.1 \mathrm{E}-07$ \\
12 & $4.4 \mathrm{E}-07$ & $4.4 \mathrm{E}-08$ \\
9 & & $1.7 \mathrm{E}-08$
\end{tabular}

$\begin{array}{lll}1.0 \mathrm{E}-04 & 1.0 \mathrm{E}-05 & \text { PU-239 } \\ & 3.2 \mathrm{E}-04 & \text { LUNGS } \\ 3.2 \mathrm{E}-05 & & \text { GONADS } \\ 2.0 \mathrm{E}-04 & 7.6 \mathrm{E}-05 & \text { R MARROW } \\ 2.5 \mathrm{E}-03 & 9.5 \mathrm{E}-04 & \text { BONE SURF } \\ 5.3 \mathrm{E}-04 & 2.1 \mathrm{E}-04 & \text { LIVER } \\ & & \text { ULI WALL }\end{array}$

B. 171 


\section{Appendix B - ICRP Publication 30 Dose Rate Factors - LB30SVBQ}

10

\begin{tabular}{rll} 
PU & 240 & \multicolumn{1}{l}{7} \\
& $1.0 \mathrm{E}-04$ & $1.0 \mathrm{E}-05$ \\
4 & & \\
1 & $2.6 \mathrm{E}-08$ & $2.6 \mathrm{E}-09$ \\
3 & $1.6 \mathrm{E}-07$ & $1.6 \mathrm{E}-08$ \\
6 & $2.1 \mathrm{E}-06$ & $2.1 \mathrm{E}-07$ \\
12 & $4.4 \mathrm{E}-07$ & $4.4 \mathrm{E}-08$ \\
9 & & $1.7 \mathrm{E}-08$ \\
10 & & $5.3 \mathrm{E}-08$
\end{tabular}

PU $241 \quad 6$

$1.0 \mathrm{E}-04 \quad 1.0 \mathrm{E}-05$

4

$1 \quad 5.7 \mathrm{E}-10 \quad 5.7 \mathrm{E}-11$

$3 \quad 3.4 \mathrm{E}-09 \quad 3.4 \mathrm{E}-10$

$6 \quad 4.2 \mathrm{E}-08 \quad 4.2 \mathrm{E}-09$

$128.6 \mathrm{E}-09 \quad 8.6 \mathrm{E}-10$

10
LLI WALL

$\begin{array}{lll}1.0 \mathrm{E}-04 & 1.0 \mathrm{E}-05 & \text { PU-240 } \\ & 3.2 \mathrm{E}-04 & \text { LUNGS } \\ 3.2 \mathrm{E}-05 & & \text { GONADS } \\ 2.0 \mathrm{E}-04 & 7.6 \mathrm{E}-05 & \text { R MARROW } \\ 2.5 \mathrm{E}-03 & 9.5 \mathrm{E}-04 & \text { BONE SURF } \\ 5.3 \mathrm{E}-04 & 2.1 \mathrm{E}-04 & \text { LIVER } \\ & & \text { ULI WALL } \\ & & \text { LLI WALL }\end{array}$

$\mathrm{Pu}-241$

$1.0 \mathrm{E}-04 \quad 1.0 \mathrm{E}-05 \quad \mathrm{~F} 1$ 3.2E-06 LUNGS

$\begin{array}{lll}6.8 \mathrm{E}-07 & 2.8 \mathrm{E}-07 & \text { GONADS } \\ 4.1 \mathrm{E}-06 & 1.7 \mathrm{E}-06 & \text { R MARROW }\end{array}$

5.1E-05 2.1E-05 BONE SURF

1.0E-05 4.4E-05 LIVER

LLI WALL $\begin{array}{rll}\text { PU } & 243 & 7 \\ & 1.0 \mathrm{E}-04 & 1.0 \mathrm{E}-05 \\ 4 & & \\ 6 & & \\ 12 & & \\ 7 & 1.8 \mathrm{E}-10 & 1.8 \mathrm{E}-10 \\ 8 & 2.9 \mathrm{E}-10 & 2.9 \mathrm{E}-10 \\ 9 & 6.0 \mathrm{E}-10 & 6.0 \mathrm{E}-10 \\ 10 & 4.1 \mathrm{E}-10 & 4.1 \mathrm{E}-10\end{array}$

$$
\begin{array}{ccc}
\text { PU } & 244 & 7 \\
& 1.0 \mathrm{E}-04 & 1.0 \mathrm{E}-05 \\
4 & & \\
1 & 2.5 \mathrm{E}-08 & 2.9 \mathrm{E}-09
\end{array}
$$

$1.0 \mathrm{E}-04$

1. $9 \mathrm{E}-10$

$2.2 \mathrm{E}-10$

$4.8 \mathrm{E}-11$

1. $0 \mathrm{E}-05$

2. $3 \mathrm{E}-10$

F1

LUNGS

BONE SURF

LIVER

ST WALL

SI WALL

7.3E-11 9.0E-11 ULI WALL

5.0E-11 6.2E-11 LLI WALL$$
\text { 1.0E-04 1.0E-05 FI }
$$$$
\text { 3.0E-04 LUNGS }
$$$$
\text { 3.0E-05 GONADS }
$$

$\mathrm{Pu}-244$

GONADS

B. 172 


\section{Appendix B - ICRP Publication 30 Dose Rate Factors - LB30SVBQ}

$\begin{array}{rrr}3 & 1.6 \mathrm{E}-07 & 1.6 \mathrm{E}-08 \\ 6 & 1.9 \mathrm{E}-06 & 1.9 \mathrm{E}-07 \\ 12 & 4.2 \mathrm{E}-07 & 4.2 \mathrm{E}-08 \\ 9 & & 2.4 \mathrm{E}-08 \\ 10 & & 8.3 \mathrm{E}-08\end{array}$

$\begin{array}{lll}1.9 \mathrm{E}-04 & 7.2 \mathrm{E}-05 & \text { R MARROW } \\ 2.3 \mathrm{E}-03 & 8.9 \mathrm{E}-04 & \text { BONE SURF } \\ 5.0 \mathrm{E}-04 & 2.0 \mathrm{E}-04 & \text { LIVER } \\ & & \text { ULI WALL } \\ & & \text { LLI WALL }\end{array}$

$\begin{array}{rlr}\text { AM } & 241 & 4 \\ & 5.0 \mathrm{E}-04 \\ 1 & 1.4 \mathrm{E}-07 \\ 3 & 8.4 \mathrm{E}-07 \\ 6 & 1.1 \mathrm{E}-05 \\ 12 & 2.3 \mathrm{E}-06\end{array}$

$\begin{array}{ll} & \text { Am-241 } \\ 5.0 \mathrm{E}-04 & \text { F1 } \\ 3.2 \mathrm{E}-05 & \text { GONADS } \\ 2.0 \mathrm{E}-04 & \text { R MARROW } \\ 2.5 \mathrm{E}-03 & \text { BONE SURF } \\ 5.5 \mathrm{E}-04 & \text { LIVER }\end{array}$

$\begin{array}{rlr}\text { AM } & 242 \mathrm{M} & 4 \\ & 5.0 \mathrm{E}-04 \\ 1 & 1.3 \mathrm{E}-07 \\ 3 & 8.3 \mathrm{E}-07 \\ 6 & 1.0 \mathrm{E}-05 \\ 12 & 2.2 \mathrm{E}-06\end{array}$

$5.0 \mathrm{E}-04$

3. $2 \mathrm{E}-05$

2. $0 \mathrm{E}-04$

2. $5 \mathrm{E}-03$

5. 3E-04

Am-242M
F1
GONADS
R MARROW
BONE SURF
LIVER

$\begin{array}{rl}\text { AM } & 242 \\ & 5.0 \mathrm{E}-04 \\ 4 & \\ 6 & 8.3 \mathrm{E}-10 \\ 3 & \\ 12 & \\ 8 & 4.4 \mathrm{E}-10 \\ 9 & 1.7 \mathrm{E}-09 \\ 10 & 2.6 \mathrm{E}-09\end{array}$

$5.0 \mathrm{E}-04$

$5.5 \mathrm{E}-08$

1. $8 \mathrm{E}-07$

1. $4 \mathrm{E}-08$

4. $6 \mathrm{E}-08$

$\begin{array}{rlr}\text { AM } & 243 & 4 \\ & 5.0 \mathrm{E}-04 \\ 1 & 1.4 \mathrm{E}-07 \\ 3 & 8.4 \mathrm{E}-07 \\ 6 & 1.1 \mathrm{E}-05 \\ 12 & 2.3 \mathrm{E}-06\end{array}$

5.0E-04

3. $2 \mathrm{E}-05$

2. $0 \mathrm{E}-04$

2. $5 \mathrm{E}-03$

$5.4 \mathrm{E}-04$

Am-242

F1

LUNGS

BONE SURF

R MARROW

LIVER

SI WALL

ULI WALL

LLI WALL

$\begin{array}{rl}\text { CM } & 242 \\ & 5.0 \mathrm{E}-04 \\ 4 & \\ 3 & 1.8 \mathrm{E}-08 \\ 6 & 2.3 \mathrm{E}-07 \\ 12 & 6.0 \mathrm{E}-08 \\ 9 & 2.0 \mathrm{E}-08 \\ 10 & 6.3 \mathrm{E}-08\end{array}$
5.0E-04
1. 5E-05
4. $0 \mathrm{E}-06$
5.0E-05
1. 3E-05

Am-243

$\mathrm{F}$ ?

GONADS

R MARROW'

BONE SURF

LIVER

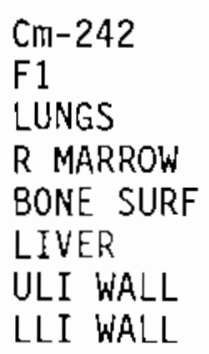

B. 173 


\section{Appendix B - ICRP Publication 30 Dose Rate Factors - LB3OSVBQ}

$\begin{array}{rl}\text { CM } & 243 \\ & 5.0 \mathrm{E}-04 \\ 1 & 8.6 \mathrm{E}-08 \\ 3 & 5.6 \mathrm{E}-07 \\ 6 & 6.9 \mathrm{E}-06 \\ 12 & 1.6 \mathrm{E}-06\end{array}$

$\begin{array}{ll} & \text { Cm-243 } \\ 5.0 \mathrm{E}-04 & \text { F1 } \\ 2.1 \mathrm{E}-05 & \text { GONADS } \\ 1.3 \mathrm{E}-04 & \text { R MARROW } \\ 1.7 \mathrm{E}-03 & \text { BONE SURF } \\ 3.8 \mathrm{E}-04 & \text { LIVER }\end{array}$

$\mathrm{Cm}-244$

F1

GONADS

$R$ MARROW

BONE SURF

LIVER

$\mathrm{Cm}-245$

$5.0 \mathrm{E}-04$

3. $4 \mathrm{E}-05$

2. $1 E-04$

$2.6 \mathrm{E}-03$

$5.6 \mathrm{E}-04$

$12 \quad 2.3 \mathrm{E}-05$
4

$$
\begin{aligned}
& 5.0 \mathrm{E}-04 \\
& 3.3 \mathrm{E}-05 \\
& 2.1 \mathrm{E}-04 \\
& 2.6 \mathrm{E}-03 \\
& 5.6 \mathrm{E}-04
\end{aligned}
$$

$5.0 \mathrm{E}-04$

$3.1 \mathrm{E}-05$

1. $9 \mathrm{E}-04$

2. $4 \mathrm{E}-03$

$5.1 \mathrm{E}-04$

5. $0 \mathrm{E}-04$

1.2E-04

7. $6 \mathrm{E}-04$

9.5E-03

2. $0 \mathrm{E}-03$
$\mathrm{F} 1$

GONADS

$R$ MARROW

BONE SURF

LIVER

Cm-246

F1

GONADS

$R$ MARROW

BONE SURF

LIVER

Cm-247

F1

GONADS

R MARROW

BONE SURF

LIVER

$\mathrm{Cm}-248$

F1

GONADS

R MARROW

BONE SURF

LIVER 


\section{Appendix B - ICRP Publication 30 Dose Rate Factors - LB30SVBQ}

$\begin{array}{rlr}\text { CF } & 252 & \\ & 5.0 \mathrm{E}-04 \\ 4 & \\ 1 & 2.5 \mathrm{E}-08 \\ 3 & 1.7 \mathrm{E}-07 \\ 6 & 2.1 \mathrm{E}-06 \\ 12 & 5.4 \mathrm{E}-07 \\ 10 & 1.5 \mathrm{E}-07\end{array}$

$\begin{array}{lll}5.0 \mathrm{E}-04 & 5.0 \mathrm{E}-04 & \text { Cf }-252 \\ 3.7 \mathrm{E}-05 & 3.0 \mathrm{E}-04 & \text { LUNGS } \\ & & \text { GONADS } \\ 3.9 \mathrm{E}-05 & & \text { R MARROW } \\ 4.9 \mathrm{E}-04 & & \text { BONE SURF } \\ 1.3 \mathrm{E}-04 & & \text { LIVER } \\ & & \text { LLI WALL }\end{array}$



NUREG/CR-3620

PNL-4054 Suppl. 2

$R E, R H$, RW

$W L, A X, A N$

\section{$\underline{\text { DISTRIBUTION }}$}

No. of

Copies

OFFSITE

U.S. Nuclear Regulatory Commission

Division of Technical

Information and Document

Control

7920 Norfolk Avenue

Bethesda, MD 20014

10 R. B. Neel

U.S. Nuclear Regulatory Commission

Mail Stop 623 SS

office of NMSS

Washington, DC 20555

10 S. M. Neuder

U.S. Nuclear Regulatory Commission

Division of Waste Management

office of NMSS

Washington, DC 20555

Director, Office of NMSS

U.S. Nuclear Regulatory Commission

Washington, DC 20555

Director, Division of Waste Management

U.S. Nuclear Regulatory Commission

Washington, DC 20555
No. of

Copies

ONS ITE

$50 \quad$ Pacific Northwest Laboratory

P. E. Bramson

D. W. Dragnich

W. T. Farris

J. M. Hales

D. G. Huizenga

P. C. Hays

G. R. Hoenes

W. E. Kennedy, Jr. (10)

B. A. Napier (10)

T. L. Page

R. A. Peloquin (10)

R. G. Schreckhise

J. K. Soldat

J. A. Stottlemyre

E. C. Watson

R. E. Wildung

Publishing Coordination (2)

Technical Information (5) 



\begin{tabular}{|c|c|}
\hline 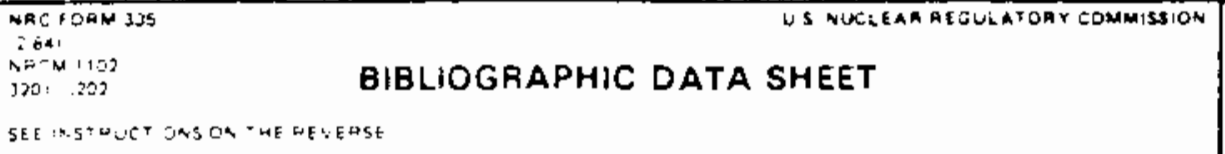 & $\begin{array}{l}\text { NUREG/CR-3620, SUPp. No. } 2 \\
\text { PNL-4054 }\end{array}$ \\
\hline \multirow[t]{2}{*}{$\begin{array}{l}\text { Intruder Dose Pathway Analysis for the Onsite Disposal of } \\
\text { Radioactive Wastes: The ONSITE/MAXI1 Computer Program }\end{array}$} & 3 LEAVE BLANA \\
\hline & \begin{tabular}{l|r} 
Wontm & EiR \\
November & 1986
\end{tabular} \\
\hline \multirow{2}{*}{$\begin{array}{l}\text { W. E. Kennedy, Jr., R. A. Peloquin, B. A. Napier/PNL } \\
\text { S. M. Neuder/NRC }\end{array}$} & 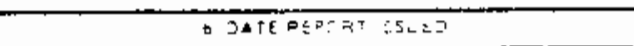 \\
\hline & \begin{tabular}{l|r} 
MONiM & 1987 \\
February & 1987
\end{tabular} \\
\hline 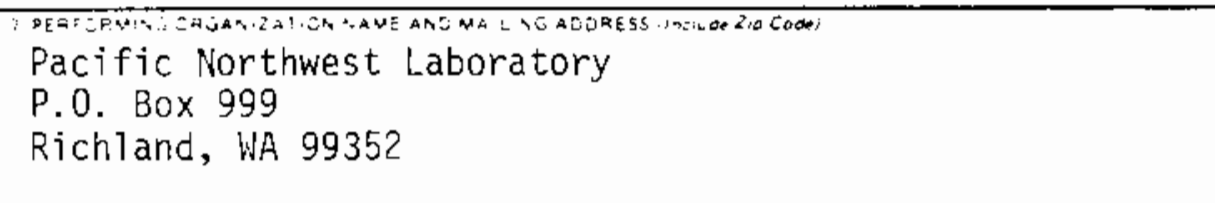 & 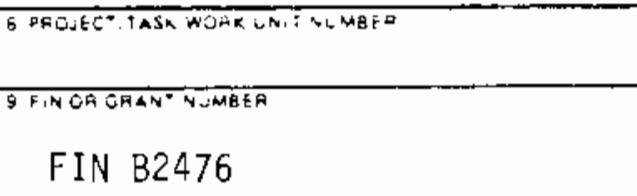 \\
\hline $\begin{array}{l}\text { Division of Waste Management } \\
\text { office of Nuclear Material Safety and Safeguards } \\
\text { U.S. Nuclear Regulatory Commission } \\
\text { Washington, DC } 20555\end{array}$ & i. TYOE OF AEROFT \\
\hline \multicolumn{2}{|l|}{ 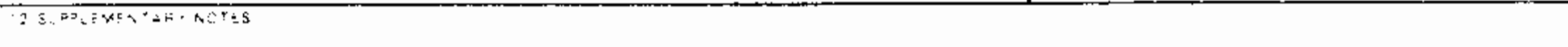 } \\
\hline \multicolumn{2}{|c|}{$\begin{array}{l}\text { The document entitled Intruder Dose Pathway Analysis of the Onsite Disposal of Radio- } \\
\text { active Wastes: The 0NSITE/MAXI1 Computer Program (1984) by Napier et al. summarizes } \\
\text { our initial efforts to develop human-intrusion SCenarios and a modified version of the } \\
\text { MAXI computer program for potential use by the VRC in reviewing applications for onsite } \\
\text { radioactive waste disposal. Supplement } 1 \text { of NUREG/CR-3620 (1986) by Kennedy et al. } \\
\text { summarized modifications and improvements to the ONSITE/MAXI1 software package. This } \\
\text { document summarizes a modified version of the 0NSITE/MAXI1 computer program. This } \\
\text { modified version of the computer program operates on a personal computer and permits } \\
\text { the user to optionally select radiation dose conversion factors published by the } \\
\text { International Commission on Radiological Protection (ICRP) in their Publication No. 30 } \\
\text { (ICRP 1979-I982) in place of those published by the ICRP in their Publication No. } 2 \\
\text { (ICRP 1959) (aS implemented in the previous versions of the ONSITE/MAXI1 computer pro- } \\
\text { gram). The pathway-to-human models used in the computer program have not been changed } \\
\text { frcm those described previously (Napier et al. 1984; Kennedy et al. 1986). Computer } \\
\text { listings of the ONSITE/MAXII computer program and supporting data bases are included } \\
\text { in the appendices of this document. }\end{array}$} \\
\hline \multirow[t]{3}{*}{$\begin{array}{l}\text { ONSITE/MAXI1 computer program } \\
\text { onsite radioactive waste disposal }\end{array}$} & 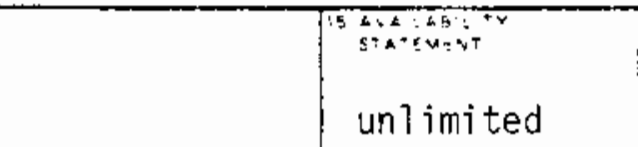 \\
\hline & 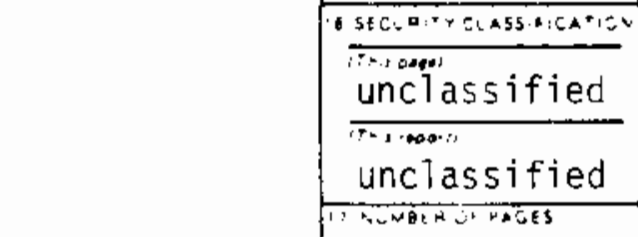 \\
\hline & \\
\hline
\end{tabular}


